


$$
\text { ชิ }
$$




THE NUTRITION OF FARM ANIMALS 
The $>$ X

THE MACMILLAN COMPANY

NEW YORK - BOSTON - CHICAGO - DALLAS

ATLANTA - SAN FRANCISCO

MACMILLAN \& CO., LiMited

LONDON - BOMBAY - CALCUTTA

MELBOURNE

THE MACMILLAN CO. OF CANADA, LTD. TORONTO 


\section{THE NUTRITION OF}

\section{F A R M A N I M A L S}

BY

HENRY PRENTISS ARMSBY, Ph.D., LL.D.

DIRECTOR OF THE INSTITUTE OF ANIMAL NUTRITION OF THE PENNSYLVANIA STATE COLLEGE; EXPERT IN ANIMAL NUTRITION, UNITED STATES DEPARTMENT OF

' AGriculture; FOREIGN MEMber, ROYAL ACADEMY OF AGRICULTURE OF SWEDEN

Y̛ew 19ark

THE MACMILLAN COMPANY I9I7

All rights reserved 


\section{$S F 95$

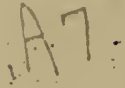

COPYRIGHT, 1917,

By THE MACMILLAN COMPANY.

Set up and electrotyped. Published June, 1917.

\section{JUN 211917}

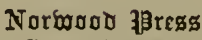

J. S. Cushing Co. - Berwick \& Smith Co.

Norwood, Mass., U.S.A.

\section{(C) Cl. 4470024}

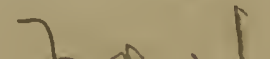




\section{PREFACE}

THE manner in which the subject of the nutrition of farm animals is presented to the student will naturally differ according to the ultimate end in view. If the prime purpose is to impart practical skill in the feeding of live stock, the study of the principles of nutrition is likely to be regarded as preliminary and to partake of the nature of an information course, and chief stress will be laid upon familiarity with the results of experience, particularly as related to the business aspects of the subject, and to the acquisition of practical skill.

But while by no means disposed to minimize the significance of this aspect of the subject, the writer is nevertheless convinced that for the students of our agricultural colleges a somewhat different procedure is desirable. He believes that greater emphasis than they sometimes receive may wisely be laid upon the chemical and physiological laws which underlie the practice of feeding, both on account of their intrinsic importance and because the subject may thus be made a real collegiate discipline which shall contribute to the training as well as to the information of the student.

Accordingly, the present volume attempts to deal primarily with the natural laws governing the nutrition of farm animals, as distinguished from the broader field of animal husbandry, and only secondarily with the specific details of practice. It seeks to avoid so far as may be mere dogmatic statements, and, although not attempting complete citation of literature even upon important points, to present the experimental evidence with sufficient fullness to indicate something of the limitations of present knowledge and of the opportunities for further investigation. Its aim is to discuss the fundamental principles upon which successful stock feeding is consciously or unconsciously based in the firm persuasion of the truth so pithily expressed almost half a century ago by the father of agricultural science in the United States, Professor Samuel William 
Johnson, that, "Other qualifications being equal, the more advanced and complete the theory of which the farmer is the master, the more successful must be his farming. The more he knows, the more he can do. The more deeply, comprehensively, and clearly he can think, the more economically and advantageously can he work," and that "A true theory is the surest guide to a successful practice."

In short, the book is intended for the student rather than directly for the farmer and assumes a certain degree of preliminary training on the part of the reader, including an elementary knowledge of chemistry and physics.

The author is under obligations to The Honorable Secretary of Agriculture, for permission to reproduce, in Chapter XVIII, a part of Bulletin No. 459 of the United States Department of Agriculture; to the Macmillan Company for the similar use in Chapter XV of material from Bailey's "Cyclopedia of American Agriculture"; and to Messrs. Henry and Morrison for permission to base the tables of the net energy values of feeding stuffs contained in the Appendix upon their extensive compilations in the fifteenth edition of "Feeds and Feeding." He is likewise indebted to the following publishers for the use of the cuts named:

The Carnegie Institution of Washington, Figure 24.

The F. A. Davis Company, Figure 17 .

Ginn \& Company, Figures 2, 3, 6, 7, 8, 16, I9, 20, and 22.

The Macmillan Company, Figures 4, 15, 29, 31, 32, 33, 34, $37,43,44$, and 45 .

The W. B. Saunders Company, Figures I, 5, and I4.

John Wiley \& Sons, Inc., Figures 18 and 40.

State College, Pa.,

May, 1917. 


\section{CONTENTS}

INTRODUCTION $\quad \cdot \quad \cdot \quad \cdot \quad \cdot \quad \cdot \quad \cdot \quad \cdot \quad \cdot \quad \cdot \quad \cdot \quad \cdot \quad$ vii

\section{PART I}

\section{THE MATERIALS OF NUTRITION}

\section{CHAPTER I}

The Components of Plants and Animals . . . . . 3

$\S$ I. Dry matter; organic matter; ash . . . . . . 3

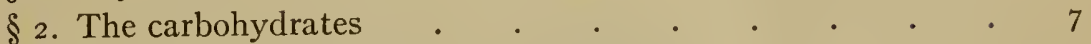

$\S 3$. Fats and related bodies. - The Lipoids . . . . . 16

§. The proteins. . . . . . . . . . . 24

5. The non-proteins . . . . . . . . . . 36

§6. Sundry ingredients . . . . . . . . . . 39

\section{CHAPTER II}

The Composition of Animals and of Feeding Stuffs a $\cdot 42$

$\S$ I. The cell . . . . . . . . . . . 42

$\S 2$ 2. Animal tissues and organs . . . . . . . . . 45

$\S 3$. The composition of the animal as a whole . . . . . 6I

$\S 4$. The composition of feeding stuffs . . . . . . 66

\section{PART II}

\section{THE PROCESSES OF NUTRITION}

\section{CHAPTER III}

Digestion AND RESORPTION

$\S$ I. The organs of digestion

$\S 2$. The chemistry of digestion . . . . . . . . 89

\$3. Resorption - The feces . . . . . . . IO5

$\S 4$. The determination of digestibility . . . . . . III 


\section{CHAPTER IV}

Circulation, Respiration, and Excretion . . . . . . i 23

$\S$ I. Circulation

$\S 2$. Respiration

$\S 3$. Excretion

\section{CHAPTER V}

\section{METABOLISM}

$\S$ I. General conception . . . $\quad$. . . . . . I 44

§ 2. Enzyms as agents in metabolism . . . . . . I48

$\S 3$. The metabolism of the carbohydrates . . . . . 152

$\S 4$. The metabolism of the simple proteins . . . . . . I60

$\S 5$. The metabolism of the nucleoproteins . . . . . . 168

§6. The metabolism of the fats . . . . . . . I7I

$\S 7$. Metabolism of ash ingredients . . . . . . 178

$\S 8$. Functions of the nutrients . . . . . . . . I 82

\section{CHAPTER VI}

The Balance of Nutrition

$\S \mathrm{I}$. General conception

$\S 2$. Methods of investigation

§. The balance of matter. . . . . . . . 202

$\S 4$. The balance of energy . . . . . . . . . . 216

$\S 5$. Significance of results . . . . . . . . . . $24 \mathrm{I}$

PART III

THE FEED REQUIREMENTS

\section{CHAPTER VII}

The Fasting Katabolism

$\S \mathrm{I}$. The protein katabolism in fasting

$\S 2$. The energy katabolism in fasting .

$\S 3$. Conditions affecting the fasting katabolism . 


\section{CHAPTER VIII}

Maintenance - The Energy Requirements .

$\S$ I. Net energy values for maintenance . . . . . 27 I

$\S 2$. Maintenance requirements of farm animals . . . . 280

$\S 3$. Factors affecting the maintenance requirement . . . 304

$\S 4$. The relation of the maintenance requirement to external temperature . . . . . . . . . 308

\section{CHAPTER IX}

Maintenance (Continued) - The Requirements of Matter . 3I3

$\S \mathrm{I}$. The protein requirements for maintenance . . . . 3I 3

$\S 2$. The ash requirements for maintenance . . . . . 332

§. Accessory substances . . . . . . . . . 348

\section{CHAPTER X}

The Fattening of Mature Animals . . . . . . 350

$\S$ I. Composition of the increase in fattening . . . . 350

§ 2. Feed requirements for fattening . . . . . . . 359

\section{CHAPTER XI}

Growth

$\S$ I. General nature of growth . . . . . . . 37 I

$\S 2$. The utilization of feed in growth . . . . . . 38I

$\S 3$. The feed requirements for growth . . . . . 396

\section{CHAPTER XII}

Meat Production

$\S$ I. Nature of meat production

424

meat production . 424

$\S 2$. The animal as a factor in meat production . . . . 428

$\S 3$. Feeding for meat production . . . . . . 444

$\S 4$. Influence of external conditions . . . . . . . 453

\section{CHAPTER XIII}

Milk Production

$\S$ I. The physiology of milk production . . . . . $\quad$. 459

$\S 2$. The animal as a factor in milk production . . . . 470

$\S 3$. The influence of environment on milk production . . 478

$\S 4$. The utilization of feed in milk production . . . . . 488

§5. Feeding for milk production . . . . . . . . . . 500 


\section{CHAPTER XIV}

WORK Production . . . . . . . . . . 53I

$\S$ I. The physiology of work production . . . . . 53I

$\S$ 2. The efficiency of the body as a motor . . . . . 544

$\S 3$. Feed requirements for work . . . . . . 560

\section{PART IV}

THE FEED SUPPLY

\section{CHAPTER XV}

The Feeding Stuffs . . . . . . . . . $57 \mathrm{I}$

$\S$ I. Roughages, or coarse fodders $\quad . \quad$. $\quad . \quad . \quad$. $\quad . \quad 572$

$\S 2$. Roots, tubers and fruits . . . . . . . . . 578

$\S 3$. The concentrates . . . . . . . . . $\quad$. 579

CHAPTER XVI

Relative Values of Feeding Stuffs . . . . . . 59I

$\S$ I. Direct comparisons of feeding stuffs . . . . . . 59 I

$\S 2$. Relative values based on composition and digestibility . 597

$\S 3$. Conditions affecting digestibility . . . . . . 60I

\section{- CHAPTER XVII}

The Production Values of Feeding Stuffs . . . . 630 § I. General considerations . . . . . . . . . 630

$\S 2$. Production values as regards energy - Net energy values . 634

$\S 3$. The computation of net energy values . . . . . 667

$\S 4$. Production values as regards protein . . . . . $\quad .678$

\section{CHAPTER XVIII}

The Computation of Rations

$\S$ I. Feeding standards

$\S 2$. Feed requirements

$\S 3$. Method of computation

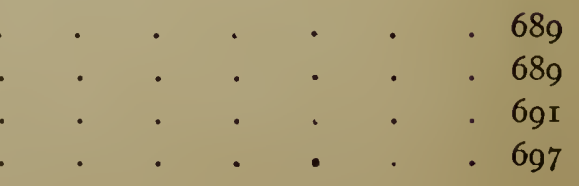




\section{APPENDIX}

PAGE

Estimated Protein and Energy Requirements of Farm Animals 7 II

Table I. Maintenance requirements of cattle and horses, per day and head . . . . . . 7II

Table II. Maintenance requirements of sheep and swine, per day and head . . . . . . . 7II

Table III. Requirements for fattening with no considerable growth - all species - in addition to the maintenance requirement . . . . 712

Table IV. Requirements for growth with no considerable fattening . . . . . . . . 712

Table V. Requirements for milk production . . . . 7 II4

Table VI. Requirements for work production by the horse . 7I4

Average Dry Matter, Digestible Protein and Net Energy

Values of Feeding Stuffs per too Pounds . . . $7 \mathrm{I} 4$

Table VII. Values per roo pounds for ruminants . . . 7I5

Table VIII. Values per roo pounds for the horse . . . $72 \mathrm{I}$

Table IX. Values per roo pounds for swine . . . . 722

Table X. Mineral elements of feeding stuffs - per roo pounds of dry substance . . . . . 723

\section{REFERENCES}

The full-face numbers in parenthesis in the body of the text refer to the numbered paragraphs and not to pages. 



\section{ILLUSTRATIONS}

FIG.

I. Different types of cells composing the body

PAGE

2. One end of a muscle fiber

3. Part of a muscle fiber . . . . . . . . . . 5 I

4. Fat cells in muscles . . . . . . . . . 52

5. Scheme of a fat cell . . . . . . . . . . ${ }_{58}^{8}$

6-8. Successive stages in the formation of adipose tissue . . 59

9. Sheep's stomach . . . . . . . . . 80

IO. Stomach and duodenum of horse . . . . . . . 8 I

II. Stomach of hog . . . . . . . . . . 8 I

I2. Intestines of cattle . . . . . . . . . 84

13. Cœcum of horse . . . . . . . . . . . 85

I4. Section of villi . $. \quad . \quad . \quad . \quad . \quad . \quad . \quad$ I05

15. Steer in digestion stall . . . . . . . . . . . II3

I6. Blood corpuscles . . . . . . . . . . . 124

17. Diagram of mammalian heart . . . . . . . . 125

18. Scheme of circulation of blood . . . . . . . . 127

I9. Relation of cells to blood vessels and lymphatics . . . I30

20. Main lymphatic trunks . . . . . . . . . . I3I

2I. Alveoli of lung . . . . . . . . . . . . . I33

22. Section of two alveoli . . . . . . . . . . I33

23. Diagrammatic scheme of metabolism . . . . . . I 82

24. Scheme of closed-circuit respiration apparatus . . . . . 209

25. Original Regnault-Reiset apparatus . . . . . . 2 I0

26. Regnault-Reiset apparatus as used by Zuntz . . ' . . 2 II

27. Scheme of Pettenkofer respiration apparatus . . . . 2 I3

28. Pettenkofer respiration apparatus, explanatory sketch . . 2 I3

29. The Möckern respiration apparatus . . . . . . 2 I4

30. Horse equipped for experiments with Zuntz apparatus . . 2 I5 $_{5}$

3I. Lavoisier's ice calorimeter . . . . . . . . . 222

32. Section of bomb calorimeter . . . . . . . 224

33. The Zuntz tread power dynamometer . . . . . . 226

34. Dulong's water calorimeter . . . . . . . . 237

35. The respiration calorimeter at The Pennsylvania State College . ${ }_{2} 38$

36. Rubner's calorimeter . . . . . . . . . . 239

37. The marbling of meat . . . . . . . . . . 356

38. Rate of gain of protein per ıooo pounds live weight . . . 379

xiii 


\section{ILLUSTRATIONS}

39. Rate of gain of energy per 1000 pounds live weight $\cdot \quad \cdot \quad 398$

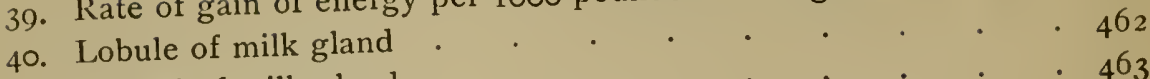

41. Alveoli of milk gland . . . . . . . . . . . 463

42. Structure of milk gland . . . . . . . . . . 463

43. Partial section of wheat grain . $\cdot{ }^{-} \cdot{ }^{-} \cdot{ }^{882}$

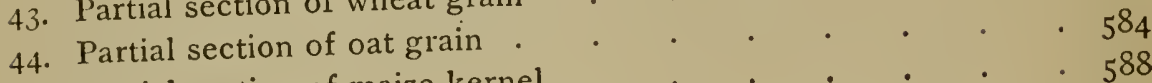

45. Partial section of maize kernel • • • • • 


\section{INTRODUCTION}

THE problems of nutrition concern the farmer both directly and indirectly - indirectly because his function in society is to furnish the materials for the nutrition of man ; directly, because an essential part of that function consists in the economical conversion of vegetable into animal products by means of farm animals. Particularly is this true regarding the inedible products of the farm. It is a well-recognized fact that only the smaller portion of the solar energy or of the proteins which are stored up in the farmer's crops is directly available for man's use. Even in distinctively food crops, such as wheat, for example, more than two-thirds of the energy which they contain may be unavailable for human nutrition, while the grasses and legumes, so important in all systems of agriculture, are of no direct value as food for man. The essential function of the animal in a permanent system of agriculture is the conversion of as large a proportion as possible of these inedible products into forms whose matter and energy can be utilized by the human body. It is true that animal products contribute largely to our supply of clothing and also that, as a motor, the work animal plays an important part in agriculture and industry. In both respects, however, substitution is possible to a greater or less extent. Vegetable fibers may to a degree replace animal fibers in our textiles, while inanimate motors seem destined to fill an increasing rôle in power production in all its aspects. But for the conversion of the by-products of the farm and factory into human food, there is as yet no suggestion of an agency which can take the place of the animal body.

With the growth of the non-agricultural population it is increasingly important that this function of conserving the food supply through the utilization of inedible soil products shall be performed with a maximum of efficiency. This requires, on the one hand, as intimate a knowledge as possible of the fundamental laws governing the nutrition of farm animals, so that 
the transformation may be effected with the least possible waste, and, on the other hand, the ability so to apply these laws as to secure the greatest economic return, since it must never be forgotten that the criterion of success in agriculture is not a maximum production but a maximum profit. It is with the former portion of this complex problem that the present work attempts primarily to deal.

Without entering into the controversy between the vitalist and the mechanist, the nutrition of the animal, whatever its guiding principle, may be regarded as a physico-chemical process, including the entire complex of reactions by which the crude materials of the feed are converted into substances suited to maintain the activities of the body cells or capable of being built up into living structures. In other words, the study of nutrition is a study of the chemistry and physics of the changes through which the crude products of the soil yield animal tissues or secretions on the one hand and excretory products on the other.

The earlier investigators dealt with the food as a supply of matter, dividing it into inorganic and organic constituents and distinguishing among the latter between the nitrogenous and non-nitrogenous substances. In other words, they studied the problems of nutrition substantially as problems of biological chemistry. Rubner's fundamental investigations went far to shift the emphasis to the physical side of the problem. It has come to be clearly recognized that the animal body is essentially a transformer of energy - a mechanism for the conversion of the chemical energy of its feed into motion energy while more or less incidentally a reserve of energy-containing material may be stored up which can be utilized for human food. It is this capacity of the animal body to store up in itself or in its secretions a part of the matter and energy of the feed it consumes which gives the animal its economic significance as a conserver of the food supply. Its value in this respect depends upon the proportion of its feed which it is able thus to set aside - i.e. upon the balance between the income and outgo of matter and of energy - and it is from this point of view that the present volume undertakes to present the nutrition of farm animals. From this standpoint, the subject naturally falls into four principal divisions. 
First, since nutrition involves chemical changes by which feed substances are converted into body substances, there is required some knowledge of the chemical compounds concerned and of their occurrence and proportions in plants and animals.

Second, the conversion of feed substances into body substances is a function of the living organism and it becomes necessary, therefore, to learn something of the processes by which the body effects these changes or, in other words, to study the physiology of nutrition.

Third, in order to apply the principles of the chemistry and physiology of nutrition to the practical problems arising in the feeding of farm animals it is requisite to determine quantitatively the amounts of matter and of energy which are required by different species of animals for their support and for the production of meat, milk or work.

Fourth, to supply the feed requirements as thus ascertained in the most economical manner demands a knowledge of the available feed resources, both as to the nature and quantity of nutriment which they contain and as to the proportion of this nutriment which can be utilized by the body.

Accordingly, the general subject of the nutrition of farm animals is treated of under four general heads, viz.: -

Part I, The Materials of Nutrition.

Part II, The Processes of Nutrition.

Part III, The Feed Requirements.

Part IV, The Feed Supply. 



\section{PART I}

THE MATERIALS OF NUTRITION 



\section{NUTRITION OF FARM ANIMALS}

\section{CHAPTER I \\ THE COMPONENTS OF PLANTS AND ANIMALS}

§ I. Dry Matter; Organic Matter; Ash

1. Dry matter. - The material composing the plant or animal may be regarded as consisting of water and dry matter. The two are ordinarily separated by maintaining the material at or above the boiling point of water until it ceases to lose weight. The loss in weight is regarded as consisting solely of water, while the residue is, of course, the dry matter.

2. Water. - Water is by no means to be regarded as an accidental or incidental component of plants or animals. The necessity for an adequate water supply to living beings is too well known to require mention, while very little reflection is needed to show that the water is as essential a part of the organism as any other ingredient. In the supporting tissues of the plant or animal it has a mechanical function, lending elasticity combined with strength. It acts as a solvent and carrier of food materials and waste products and the osmotic pressures of the solutes are an important factor in physiological processes. Finally, its action in dissociating electrolytes appears to be very intimately related to the chemistry of living matter.

Water is usually abundantly supplied to live stock. The study of animal nutrition, therefore, deals chiefly with the dry matter, its supply and transformations, not because this is fundamentally any more essential than the water but because ordinarily it is economically more important. 
3. Organic matter. - By the action of oxygen at a high temperature, the dry matter of plants or animals may be separated into two portions, one being converted into the gaseous state, while the other remains behind in the solid form. Following the older nomenclature, it is customary to distinguish these two portions as "organic" and "inorganic," or "ash," ingredients. The terms, however, are to some extent misnomers, since no such sharp distinction exists as was once supposed between organic and inorganic compounds. Organic matter in the sense in which the term is commonly used may be said to be broadly equivalent to the carbon compounds of the organism, but even this definition is inexact and the same element may be volatilized during oxidation or may appear in the ash according to circumstances.

For example, the element sulphur is an essential ingredient of the proteins. When these are burned in air part of the sulphur escapes in the gaseous form, but a part also combines with any bases present and appears in the ash as sulphates. Even the element carbon, distinctive of so-called organic matter, may appear in part in the ash of the plant or animal in the form of carbonates when the bases of the ash are in excess of the acid radicles. These examples serve to show that an element may be an integral part of the molecules which make up the organic matter and yet appear after incineration in the ash. Thus it has recently been shown that the phosphorus of wheat bran and other feeding stuffs is present chiefly in the form of a complex carbon compound, yet when these materials are burned the phosphorus appears in the ash in the form of phosphates.

Organic matter is usually regarded as consisting of the elements carbon, hydrogen, oxygen, nitrogen and sulphur, phosphorus being sometimes added to the list, but doubtless other elements like potassium, sodium, chlorin, etc., also enter into the structure of the "organic" molecules.

4. Subdivision of organic matter. - The number of individual organic compounds found in the animal body or in the plant is very great. For the present purpose, however, it is not necessary to consider separately each individual substance but only the general properties of the important groups into which they may be classified.

The organic constituents of the body may be subdivided into 
non-nitrogenous and nitrogenous substances. Under the former are included the carbohydrates, the fats, the organic acids and various other minor groups. The nitrogenous substances include the proteins and a variety of simpler nitrogenous substances sometimes classed together as the non-proteins. In the following sections these various groups will be considered as far as is requisite for an intelligent study of their behavior in the animal body, it being assumed that the reader has already some knowledge of their general properties, both chemical and physical.

5. Mineral matter, or ash. - To what extent the elements found in the ash and commonly reckoned as the mineral elements, namely, potassium, sodium, calcium, magnesium, iron, phosphorus, sulphur, chlorin, silicon, etc., are actually present in the living plant or animal as electrolytes and to what extent as ingredients of complex organic molecules, it is at present impossible to state with any definiteness. In ordinary usage the term ash is equivalent to the residue remaining after incineration at as low a temperature as possible, usually not exceeding a dull red heat.

The proportion of ash in ordinary feeding stuffs varies considerably according to the kind of plant, the portion of the plant used (seeds, stems, leaves, roots, etc.), the maturity of the plant and various other conditions. Wolff gives the following as general averages for the proportion of ash in the dry matter:-

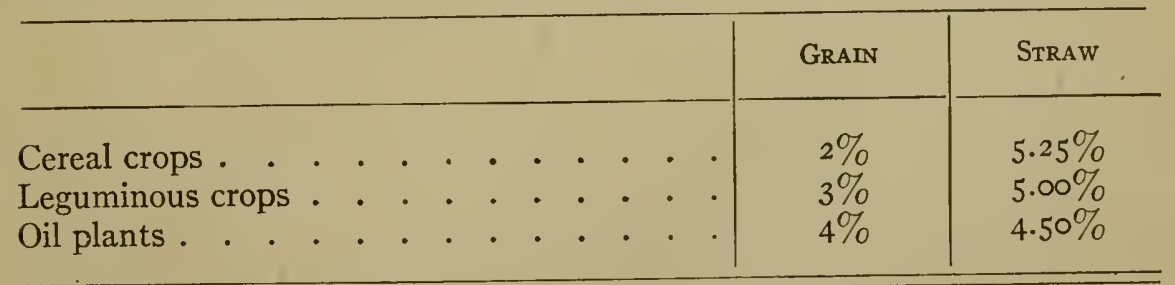

The proportion varies most in the straw and least in the grain. In the animal, the presence of ash is most evident in the bones. About two-thirds of the dry matter of the clean bone (free from fat) consists of ash. Ash is by no means absent from the soft tissues of the body, however, of which it forms an essential ingredient. The proportion varies in different organs, but as a rough general average the body, inclusive of the skeleton, contains about 3.5 per cent of ash in the fresh substance, 
equivalent to about 7.I per cent of the dry matter. The proportion of ash to dry matter is greater in the young than in the mature animal and greater in the lean than in the fat condition.

The more important elements found in the ash are as follows:-

Potassium. - This metal is indispensable to plant growth and is found in all parts of the plant, but especially in the active, growing parts. In the animal body it is found abundantly in the tissues, such as the muscles, glands, nerves, etc., while the fluids (blood, plasma, lymph, etc.) contain relatively small amounts of it.

Sodium. - Unlike potassium, sodium is not indispensable to plant growth, although it apparently is useful to the plant under some conditions. It is found especially in the stems and leaves of plants, although not so abundantly as potassium. Seeds contain but little of it. In the animal body it is especially abundant in the fluids, which, as just noted, contain relatively little potassium.

Calcium. - Like potassium, calcium is necessary for the growth of plants. It is found especially in the leaves and stems of plants and to a much less extent in the seeds. It appears to be equally essential to the animal and is found in all parts and organs of the body. Its most striking use, however, is in the formation of the skeleton, the mineral portion of which (81) consists chiefly of calcium phosphate and carbonate: Both these compounds being scarcely at all soluble in water, they are well adapted to form the framework of the body. In the skeletons of the higher animals calcium phosphate is the chief mineral ingredient, while in the lower animals like shellfish and crustacea, the shell, which corresponds to the bones of domestic animals, contains chiefly calcium carbonate.

Magnesium. - Magnesium is also one of the elements essential for plant growth. It is found throughout the plant in smaller amounts than calcium, but is more abundant than the latter in the seeds and seems to aid in seed formation. In the animal body, magnesium usually accompanies calcium, but in much smaller amounts.

Iron. - A small amount of iron is required by the higher plants for the formation of the green coloring matter (chlorophyl) by means of which they assimilate the carbon dioxid of the air. In the animal, iron in small quantity is necessary for the formation of the red coloring matter (hæmoglobin) of the blood which is the agent for conveying the oxygen of the air to the tissues. While, therefore, but a very small amount of iron is required by either plants or animals, ${ }^{1}$ it is nevertheless essential to the most fundamental processes of life.

${ }^{1}$ It is estimated that the blood of an adult man contains about 3 grams of iron. 
Phosphorus. - Phosphorus is another of the elements essential to plant growth, its chief function seeming to be to aid in the production and transportation of the proteins. It is found in all parts of the plant but accumulates especially in the seeds.

Plants may contain more or less phosphorus in the form of phosphates, especially in their vegetative organs. Even in the latter, however, a considerable share of it is in "organic" combination, while in the seeds but very small amounts of "inorganic" phosphorus are found. The "organic" phosphorus of plants is contained chiefly in three classes of compounds, viz., the phosphatids $(37,38)$, or socalled phosphorized fats, the nucleo- and phospho-proteins $(52,55)$, and phytin, the latter being the chief phosphorus compound of seeds. Phytin is a compound of phosphoric acid and inosit and may be split up into these constituents by hydrolysis and also by an enzym found in seeds.

In the animal, the great store of phosphorus is found in the skeleton, where it exists, as already stated, chiefly in the form of calcium phosphate. It is also found somewhat abundantly in the soft tissues of the body, of which it is an essential ingredient. Here it seems to exist largely in "organic" combination in the phosphatids and the nucleo- and phospho-proteins.

Sulphur. - Sulphur is taken up by the roots of the plant in the form of sulphates, and when plant or animal substances are burned, more or less of the sulphur which they contain is found as sulphates in the ash. For these reasons, sulphur has been commonly regarded as one of the ash ingredients of plants and animals. As a matter of fact, however, as already pointed out, it is usually as truly an "organic" ingredient as nitrogen or carbon. In particular, it is one of the elements of which the proteins are composed, and seems to exist in the plant and animal chiefly in this form.

Chlorin. - Chlorin is found in plants associated with sodium. It does not seem to be necessary to plant life. In the animal it is an essential element in the gastric juice.

Small amounts of fluorine and traces of iodin and of manganese and other catalysts also occur, but their specific functions are obscure except that fluorin is an ingredient of the enamel of the teeth.

\section{§ 2. The Carbohydrates}

6. Occurrence. - Although substances belonging to this group of compounds are found in the bodies of animals, they are especially characteristic of plants. Starch, one of the most familiar of them, is the first visible product of the assimilation 
of carbon dioxid by chlorophyl-bearing plants, and the great mass of vegetable tissue is composed either of carbohydrates or of their nearly related derivatives.

The more common carbohydrates have been known for a long time. Starch is familiar to us in the mealy portion of grains and in certain tubers, and cellulose in cotton and linen and, in impure forms, in the woody fiber of plants. Of the sugars, cane sugar has been known since almost prehistoric times, while the presence of this and other sugars in plant juices, in sweet fruits, honey, etc., is a familiar fact. The more common sugars were separated and identified quite early in the history of chemistry.

7. Classification. - The carbohydrates contain hydrogen and oxygen in exactly the proportions to form water, and their name is derived from this fact, although compounds exist which contain two atoms of hydrogen to one of oxygen and yet are not carbohydrates, such, for example, as acetic acid, $\mathrm{C}_{2} \mathrm{H}_{4} \mathrm{O}_{2}$. The simplest of the carbohydrates are the simple sugars, more exactly designated as the monosaccharids. By polymerization, with elimination of water, the monosaccharids yield more complex carbohydrates which are conveniently classified as di-, tri-, and polysaccharids.

\section{Monosaccharids, or simple sugars}

8. Composition. - The monosaccharids may be represented by the general formula $\mathrm{C}_{n} \mathrm{H}_{2 n} \mathrm{O}_{n}$. Substances having this general formula are known whose molecules contain from one to nine carbon atoms and which, from a chemical point of view, may be called carbohydrates. The simplest of these is formaldehyde, $\mathrm{CH}_{2} \mathrm{O}$, which is believed by many to be the first step in the synthesis of carbohydrates by the green plant. Only the $\mathrm{C}_{6}$ and $\mathrm{C}_{5}$ compounds, however, known respectively as the hexose and pentose carbohydrates, are of importance in their relations to nutrition.

9. Hexoses. - The most important hexose monosaccharids are dextrose, levulose, galactose and mannose.

Dextrose, $d$-glucose, or grape sugar, is generally regarded as an aldose of the hexatomic alcohol sorbite.

$$
\begin{aligned}
& \text { Sorbite: } \mathrm{CH}_{2} \mathrm{OH}-(\mathrm{CH} \cdot \mathrm{OH})_{4}-\mathrm{CH}_{2} \mathrm{OH} \\
& \text { Dextrose: } \mathrm{CH}_{2} \mathrm{OH}-(\mathrm{CH} \cdot \mathrm{OH})_{4}-\mathrm{CHO}
\end{aligned}
$$


It occurs almost universally in the juices of plants along with levulose and cane sugar, and is found also in small amounts in the blood of mammals. Sixteen isomers of this compound are possible, twelve of which are known.

Galactose and mannose are isomers of dextrose, occurring in nature only in combination as di- or polysaccharids.

Levulose, or fruit sugar, is a ketose of sorbite, having the formula $\mathrm{CH}_{2} \mathrm{OH}-(\mathrm{CH} \cdot \mathrm{OH})_{3}-\mathrm{CO}-\mathrm{CH}_{2} \mathrm{OH}$, eight isomers being theoretically possible. It occurs mixed with dextrose in plant juices and in honey.

The hexose monosaccharids are all soluble in water and readily diffusible and have a more or less sweet taste. All those found in nature are optically active, rotating the plane of polarized light. Thus dextrose, as its name implies, has a right-handed rotation and levulose a left-handed rotation. They reduce an alkaline solution of metallic salts, especially of copper, and this fact is utilized both as a qualitative test for them and as a means of quantitative determination. They are fermented by yeast, yielding as the chief products ethyl alcohol and carbon dioxid.

10. Pentoses. - The pentoses are simple sugars, corresponding to the hexoses but having the general formula $\mathrm{C}_{5} \mathrm{H}_{10} \mathrm{O}_{5}$. Those occurring in nature are aldoses. Like the hexoses, they reduce metallic oxids, but unlike them they are not fermentable by yeast.

Arabinose. - By the hydrolysis of gum-arabic or cherry gum, there is produced dextro-rotatory arabinose ( $l$-arabinose). Levorotatory arabinose ( $d$-arabinose) has been prepared artificially. The inactive or racemic form ( $i$-arabinose) has been found in human urine in small amounts.

Xylose. - By the hydrolysis of wood gum there is produced a dextro-rotatory pentose known as $l$-xylose. The levo-rotatory form of the same sugar ( $d$-xylose) is obtained in the hydrolysis of certain nucleo-proteins, the pentose group seeming to be a constituent of the molecule of those compounds.

Rhamnose is a derivative of the pentose sugars in which an atom of hydrogen has been replaced by methyl. It occurs somewhat widely in the vegetable kingdom. 


\section{Glucosids}

11. The monosaccharids not only occur in the free state but also in combination with a great variety of substances in the so-called glucosids. The glucosids readily undergo hydrolytic cleavage into their two (or more) constituents, either by the action of chemical reagents or of enzyms. For example, the amygdalin of the bitter almond yields two molecules of dextrose, one of benzaldehyd and one of hydrocyanic acid, and cerebron, a constituent of the brain, splits up into cerebronic acid, sphingosin and galactose. Among other more or less familiar glucosids may be mentioned salicin, saponin, phloridzin and digitalin.

\section{Disaccharids}

12. The hexose group. - The disaccharids may be regarded as polymers or anhydrids of the monosaccharids, formed by the union of two molecules of the latter with the elimination of one molecule of water. The only disaccharids at present known belong to the hexose group and their formation may be represented by the equation $\mathrm{C}_{6} \mathrm{H}_{12} \mathrm{O}_{6}+\mathrm{C}_{6} \mathrm{H}_{12} \mathrm{O}_{6}=\mathrm{C}_{12} \mathrm{H}_{22} \mathrm{O}_{11}+\mathrm{H}_{2} \mathrm{O}$. From another point of view they are termed by some writers glucosids of the monosaccharids.

Sucrose. - Sucrose, or cane sugar, has probably been longest known of the more familiar carbohydrates. It is found in the juices of the sugar cane and sorghum, in the sugar beet and in the sap of the maple, all of which are utilized as commercial sources of sugar. In smaller amounts it is present in a large number of plants.

By the action of heat, aided by a dilute acid or alkali, or by the action of certain enzyms, notably the invertase of yeast, the reverse of the general reaction for the formation of the disaccharids may be brought about, one molecule of sucrose combining with one molecule of water to yield one molecule each of dextrose and levulose.

$$
\mathrm{C}_{12} \mathrm{H}_{22} \mathrm{O}_{11}+\mathrm{H}_{2} \mathrm{O}=\mathrm{C}_{6} \mathrm{H}_{12} \mathrm{O}_{6}+\mathrm{C}_{6} \mathrm{H}_{12} \mathrm{O}_{6}
$$

Sucrose rotates the plane of polarized light to the right, while, owing to the fact that the rotatory power of levulose is greater than that of dextrose, the mixture of equal parts of the two which is formed in the foregoing reaction rotates to the 
left. On account of this fact, this breaking up of cane sugar has been called inversion and the use of this term has been extended to designate in general the hydrolytic cleavage of disaccharids into their constituent monosaccharids.

Lactose. - Lactose, or milk sugar, is a characteristic ingredient of the milk of mammals. Like sucrose, it may be broken up, with the addition of one molecule of water, into two molecules of monosaccharids, in this case dextrose and galactose. It is less soluble than sucrose and therefore less sweet to the taste, having a gritty feel in the mouth. It is not found in plants.

Maltose. - By the action of certain ferments upon starch during the germination of seeds and also in the digestive tract of animals, a disaccharid known as maltose is produced. It is therefore present abundantly in malt, whence its name. This sugar when hydrolyzed yields two molecules of dextrose.

13. General properties. - The disaccharids are crystalline, soluble in water and optically active. Sucrose does not reduce an alkaline copper solution, but lactose and maltose do. The disaccharids are not fermentable. Any cases in which they are apparently fermented are found to be preceded by some action which inverts or breaks up the disaccharids into their constituent monosaccharids.

\section{Trisaccharids}

14. By the union of three molecules of $\mathrm{C}_{6} \mathrm{H}_{12} \mathrm{O}_{6}$ with the elimination of two molecules of water, there may be formed the compound $\mathrm{C}_{18} \mathrm{H}_{32} \mathrm{O}_{16}$, called a trisaccharid. One such, known as raffinose, is present in the sugar beet, the cotton seed, in barley and in wheat. Upon hydrolysis it yields one molecule each of dextrose, levulose and galactose.

\section{Polysaccharids}

15. Chemical structure. - The polysaccharids, like the disaccharids, are anhydrids, but are formed by the combination of many molecules of the monosaccharids and have a correspondingly high molecular weight. The general formula of the hexose polysaccharids is $\left(\mathrm{C}_{6} \mathrm{H}_{10} \mathrm{O}_{5}\right)_{n}$, the value of $n$ doubtless varying 
through a wide range, but the molecular weights of the individual polysaccharids have not been finally determined.

The polysaccharids are tasteless and usually amorphous substances which, with the exception of cellulose, are more or less soluble in water. They are optically active but in general are not diffusible through membranes. They are hydrolyzed easily, especially by the action of heat and acids and by enzyms, yielding ultimately monosaccharids.

In addition to their common names, they are designated by terms derived from the monosaccharids out of which they are built up. Thus starch, which is an anhydrid of dextrose and yields only this sugar upon hydrolysis, is a dextran. Similarly, there are levulans, galactans, mannans, arabans, xylans, etc., yielding the corresponding sugars when hydrolyzed. In the same manner, it is customary to distinguish between the hexosans, derived from the hexoses, and the pentosans, the anhydrids of the pentoses.

16. The hexosans. - This group of carbohydrates includes those which are most abundant in the vegetable kingdom and of the greatest significance as sources of nutriment for man and animals, viz., starch, the dextrins and gums, and cellulose and its various derivatives. It will be convenient to consider the more important hexosans somewhat in the order of their resistance to solvents.

17. Cellulose. - Cellulose constitutes the basis of the cell walls of plants and is also found in certain lower animals (tunicates). Clean cotton consists of nearly pure cellulose, each fiber being a single cell from which the contents (protoplasm) have nearly disappeared. Linen and the best qualities of paper are other examples of nearly pure cellulose. A crystalline form has also been described.

Cellulose is insoluble in water and comparatively resistant to reagents in general. Plants, however, contain enzyms (cytases) which are able to bring it into solution in the processes of plant growth, and apparently these enzyms play some part in its digestion by animals. It is also attacked and dissolved by some species of bacteria. Concentrated sulphuric acid dissolves it, and the solution, on dilution and boiling, undergoes hydrolysis, yielding dextrose. Cellulose is therefore a dextran. Its molecular weight is unknown. 
18. Hemicelluloses. - These polysaccharids differ from true cellulose in being hydrolyzed by comparatively short boiling with dilute acids and further in the fact that the hydrolysis, instead of yielding only dextrose, as in the case of cellulose, produces a variety of both hexose and pentose sugars, the former including galactose, mannose and levulose, as well as dextrose, and the latter arabinose and xylose. The hemicelluloses must be regarded, therefore, as containing both hexosans and pentosans, but whether in mixture or chemical union is uncertain. While true cellulose constitutes the framework of the plant, the hemicelluloses serve to a greater or less extent as reserve material. In the conventional method of feeding stuffs analysis, the hemicelluloses are found both in the "crude fiber" (109) and in the "nitrogen-free extract" (110).

19. Lignin. - In the young plant, the cell walls consist of nearly pure cellulose. With advancing maturity they become thickened, not only by the formation of additional cellulose and of hemicelluloses but by the deposition of numerous " incrusting substances," the most important group of which has received the collective name of lignin. These substances contain a considerably higher percentage of carbon than cellulose ( 54 to 60 per cent) and may be separated from the latter by oxidizing agents. The substances of the lignin group contain methoxyl $\left(-\mathrm{O} \cdot \mathrm{CH}_{3}\right)$ and ethoxyl $\left(-\mathrm{O} \cdot \mathrm{C}_{2} \mathrm{H}_{5}\right)$ groups in considerable amount, and by some are regarded as substituted celluloses.

20. Crude fiber. - The so-called "crude fiber" (109) of plants contains most of the cellulose and lignin of the cell walls and in addition a third group - the cutin group ${ }^{1}$ - whose percentage of carbon is still higher (6o-75 per cent). Cutin appears to be indigestible.

21. Starch. - Starch is one of the most common and important of the vegetable carbohydrates. In the growth of plants, starch is formed in the green leaves by the aid of light, and is the first visible product of assimilation. In the mature plant, it is stored up in large quantities in the seed or in the tuber to supply the needs of the new plant. Hence the common grains, corn, wheat, oats, barley, etc., as well as potatoes, are rich in starch and form commercial sources of it. The seeds of most legumes contain it in less amounts but still abundantly. In

${ }^{1}$ Compare König : Landw. Vers. Stat., 65 (1907), 55. 
the oil seeds it is replaced by oil. It is not found in the animal body.

Starch occurs in plants in the form of microscopic granules, which have a peculiar form for each species, so that we may speak of the starches rather than of starch. These grains consist of a surrounding envelope consisting of a variety of cellulose inclosing a more soluble substance or substances known as granulose. When treated with much hot water the starch grain swells and bursts the envelope and the enclosed granulose dissolves, probably after undergoing more or less hydration.

Starch may be hydrolyzed readily by dilute acids or alkalies or by heat. The final product of its hydrolysis is dextrose, which in an impure form constitutes commercial glucose or starch sugar. Starch is therefore a dextran. As already noted, certain enzyms, notably those formed in germinating seeds and others secreted in the digestive tract of animals, act upon starch readily with the production of maltose. Starch is also acted upon by some species of bacteria with the formation of lactic, butyric and other acids, methan and in some cases hydrogen.

22. Galactans. - Galactans occur more particularly in leguminous plants, other feeding stuffs being comparatively free from them.

23. Inulin. - The roots of the artichoke, dahlia, dandelion, chicory and other composite contain instead of starch a quite similar carbohydrate, inulin, which on hydrolysis yields levulose instead of dextrose, i.e., it is a levulan.

24. The dextrins. - In the hydrolysis of starch a series of ill-defined, intermediate compounds is produced, collectively called dextrins. Commercial dextrin is made by heating moist starch to about $235^{\circ}$ Fahrenheit. It is likewise produced in the cooking of starchy materials, the brown crust of bread, for example, consisting largely of dextrin. Various dextrins have been separated and described, but it seems questionable whether the investigators have worked with definite chemical individuals. For the present, it seems wiser to speak collectively of the dextrins as intermediate products between starch and the simpler di- and mono-saccharids.

25. Glycogen. - In the liver and muscles of animals, and to a less degree in other parts of the body, there is found in rather 
small amounts a carbohydrate called glycogen. Glycogen has the same percentage composition as starch and has sometimes been called animal starch, although improperly, since its properties are quite different from those of starch. It has important functions in the animal, as will appear later. It is not found in the plant. It is readily soluble in water, yielding an opalescent solution. The empirical formula of glycogen is the same as that of starch. When hydrolyzed it yields only dextrose, and is therefore a dextran.

26. The gums. - Familiar examples of this class of substances are gum arabic and the gums of the cherry, peach and plum. The mucilage of flax seed closely resembles the gums, and other seeds also contain gum-like bodies. Upon hydrolysis, the gums yield hexoses, especially galactose, showing that they contain galactans. In addition to hexoses, however, they yield sugars belonging to the pentose group.

27. The pentosans. - The pentosans may be regarded as polymers or anhydrids of the pentoses, corresponding in this respect to the polysaccharids of the hexose group. Their general formula is $\left(\mathrm{C}_{5} \mathrm{H}_{8} \mathrm{O}_{4}\right)_{n}$, but their molecular structure is unknown.

Araban. - This is a constituent of gum arabic and other gums, as shown by the fact that these gums, as already noted (10), yield $l$-arabinose when hydrolyzed.

Xylan. - This compound is also known as wood gum. It can be extracted from various woods, from the cob of maize and from various other vegetable materials by the action of dilute alkalies, and yields $l$-xylose when hydrolyzed. In the plant, araban and xylan appear to be in a more or less close chemical combination with hexosans, especially in the cell walls of the more mature plant, constituting the so-called hemicelluloses (18).

Pectins. - Most ripe fruits, as well as the flesh of beets, turnips and similar roots, contain a group of substances called the pectin group. As they exist in the roots or fruits they are insoluble in water, but by cooking they are converted into substances which form the basis of fruit jellies. On hydrolysis they yield pentoses, chiefly arabinose. 


\section{§ 3. Fats and Related Bodies - the Lipoids}

28. Classification. - Under the rather vague term "lipoids," or fat-like substances, there are included, besides true fats, a large number of chemical individuals of varied and complex molecular structure. Chemically, these substances (with the exception of the cholesterins) are characterized by containing radicles of the so-called fatty acids, principally the higher ones of the series. Physically, the lipoids have been defined, principally from the standpoint of the physiological chemist, as substances which are soluble in organic solvents, such as ether, alcohol, chloroform or benzol. This latter definition, however, includes substances, such as the cholesterins, which would be excluded by the chemical definition just given. For the present purpose, the principal lipoids may be conveniently grouped under five heads: (I) fats, (2) waxes, (3) cholesterins, (4) phosphatids or phospholipins, (5) cerebrosids or galactolipins.

\section{The Fats}

29. Occurrence. - It is a familiar fact that the bodies of animals contain a not inconsiderable amount of fat, the percentage seldom falling below six in the very lean animal while it may rise as high as forty in the very fat animal. The fat is the reserve material of the body and is contained in what is called adipose tissue (94), consisting of cells of connective tissue more or less filled with fat. Larger or smaller amounts of adipose tissue are found in all parts of the body but especially in the subcutaneous tissues, the tissues surrounding the intestines, and, particularly in fat animals, in the muscles.

In plants, fats are usually less abundant. They occur in all parts of the plant but are especially stored up in the seeds, where they serve as reserve material which is metabolized during germination. Some seeds, like those of cotton, flax and rape, contain fat so abundantly that they are commercial sources of oil. In the plant, the fat is not deposited in special tissues but is usually distributed through the protoplasm of the cell. Both animal and vegetable fats are mixtures of various simple fats, often containing also small amounts of free fatty acids. 
30. Molecular structure. - The simple neutral fats are triglycerids, that is, they are esters of the triatomic alcohol glycerol with monobasic fatty acids, the hydrogen atoms of the three hydroxyls being replaced by the acid radicles. Their general formula is as follows, $R_{1}, R_{2}$ and $R_{3}$ representing the acid radicles, which may or may not be the same:-

$$
\begin{aligned}
& \text { Glycerol } \mathrm{CH}_{2} \cdot \mathrm{OH}-\mathrm{CH} \cdot \mathrm{OH}-\mathrm{CH}_{2} \cdot \mathrm{OH} \\
& \text { Neutral fat } \mathrm{CH}_{2} \cdot \mathrm{OR}_{1}-\mathrm{CH} \cdot \mathrm{OR}_{2}-\mathrm{CH}_{2} \cdot \mathrm{OR}_{3}
\end{aligned}
$$

The fatty acids may be divided into the saturated and the unsaturated. The saturated fatty acids have the general formula $\mathrm{C}_{n} \mathrm{H}_{2 n} \mathrm{O}_{2}$ and are the normal acids of the aliphatic series, the two lower members of which are familiar as formic and acetic acids. The general formula of these acids is as follows, each carbon atom being united to the adjacent ones by a single bond.

\section{$\mathrm{CH}_{3}-\left(\mathrm{CH}_{2}\right)_{n}-\mathrm{COOH}$}

The two principal saturated acids contained in the animal fats are stearic acid, $\mathrm{C}_{18} \mathrm{H}_{36} \mathrm{O}_{2}$, and palmitic acid, $\mathrm{C}_{16} \mathrm{H}_{32} \mathrm{O}_{2}$. Besides these two, however, others are also found in small amounts. In butter fat, especially, several of the lower acids of the series are present, the principal ones being butyric, $\mathrm{C}_{4} \mathrm{H}_{8} \mathrm{O}_{2}$, caproic, $\mathrm{C}_{6} \mathrm{H}_{12} \mathrm{O}_{2}$, caprylic, $\mathrm{C}_{8} \mathrm{H}_{16} \mathrm{O}_{2}$, capric, $\mathrm{C}_{10} \mathrm{H}_{20} \mathrm{O}_{2}$, lauric, $\mathrm{C}_{12} \mathrm{H}_{24} \mathrm{O}_{2}$ and myristic, $\mathrm{C}_{14} \mathrm{H}_{28} \mathrm{O}_{2}$. In the body fats there have been found also higher acids of the same series, particularly arachnic acid, $\mathrm{C}_{20} \mathrm{H}_{40} \mathrm{O}_{2}$.

The unsaturated fatty acids differ from the saturated acids in containing two or more carbon atoms united by two bonds instead of one and consequently in containing less hydrogen than the saturated acids. Of the unsaturated acids, the most abundant in animal fats is oleic acid, having the formula

$$
\mathrm{CH}_{3}-\left(\mathrm{CH}_{2}\right)_{7}-\mathrm{CH}=\mathrm{CH}-\left(\mathrm{CH}_{2}\right)_{7}-\mathrm{COOH}
$$

The eruic acid of rape oil also belongs to this series, and the linoleic acid, $\mathrm{C}_{18} \mathrm{H}_{32} \mathrm{O}_{2}$, of linseed oil and other drying oils belongs to a related series of unsaturated acids of the general formula $\mathrm{C}_{n} \mathrm{H}_{2 n-4} \mathrm{O}_{2}$ with two double unions of carbon atoms.

It is a noteworthy fact that nearly all the fatty acids occurring in the animal body contain an even number of carbon atoms. 
31. Chemical reactions. - Of the chemical reactions of the fats, the one of most importance physiologically is that known as saponification, or more strictly as hydrolysis. It consists of a cleavage and hydration of the molecule, yielding glycerol and fatty acids. The most familiar instance of this reaction is in the process of soap making. For example, if tri-stearin is acted upon by potassium hydrate the final result is as represented by the following equation:-

\section{$\mathrm{C}_{3} \mathrm{H}_{5}\left(\mathrm{C}_{18} \mathrm{H}_{35} \mathrm{O}_{2}\right)_{3}+(\mathrm{KOH})_{3}=\left(\mathrm{KC}_{18} \mathrm{H}_{35} \mathrm{O}_{2}\right)_{3}+\mathrm{C}_{3} \mathrm{H}_{8} \mathrm{O}_{3}$ \\ Tristearin Potassium hydrate Potassium tristearate Glycerol}

In this reaction, the alkali salt of the fatty acid, that is, a soap, is obtained. By the action of water at temperatures considerably above $100^{\circ} \mathrm{C}$., essentially the same result is reached except that the free acid is obtained instead of the salt. The same decomposition may also be effected by means of acids, which probably act as catalyzers.

Of most importance physiologically is the hydrolysis of fat by means of enzyms. Such enzyms are produced by certain plants and are also found in various digestive juices, notably in the secretion of the pancreas. These enzyms have received the general name of lipases. The hydrolysis of fats by enzyms appears to be a reversible reaction, at least with the glycerids of low molecular weight. In other words, the same enzym may effect the cleavage of a glycerid or the combination of glycerol and the fatty acid, the reaction in either case reaching an equilibrium at a certain stage.

32. Physical properties. - Certain general properties are common to all the fats. Their specific gravity is in all cases less than one, so that they float on water. They have a fatty feel and leave a permanent grease spot on paper or fabric. They are almost insoluble in water, although water is soluble to a not inconsiderable extent in fats. They are readily soluble in ether, benzol, carbon disulphid and most of them in petroleum ether, but only sparingly in alcohol.

The melting point of the fatty acids increases with the molecular weight. The exact melting point of a fat is difficult to determine, but for the three common glycerids and the corresponding acids it may be stated approximately as follows:- 
Melting Points

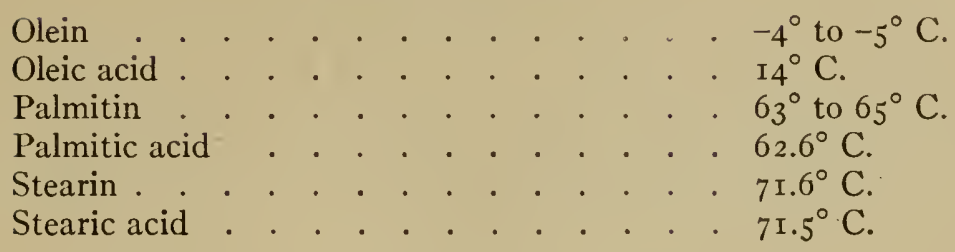

A distinction is commonly made between fats and oils, the fats being solid at ordinary temperatures and the oils liquid. The difference depends largely upon the proportion in which the various simple fats are present. Olein and other fats containing unsaturated acids are usually liquid at room temperature and their presence increases the softness of the fat.

The fatty acids of higher molecular weight are volatile only at comparatively high temperatures and at reduced pressure. Those of lower molecular weight, notably those contained in butter fat, can be readily distilled in a current of steam and their proportion serves to distinguish butter fat from other animal fats.

An important physical property of the fats, which, however, is by no means peculiar to them, is that of forming what is known as an emulsion. Fat is said to be emulsified when, in the liquid state, it is distributed in minute droplets or globules throughout some other liquid; for example, if fat be violently shaken with water an emulsion is formed. Such an emulsion is not permanent, however, the fat droplets very soon coalescing and rising to the surface. The presence of small amounts of certain other substances dissolved in the water, however, will prevent this separation and give rise to a permanent emulsion. The most common substance producing this effect is soap.

Certain gums and also proteins likewise serve to retain fat in the emulsified state. The most familiar example of such an emulsion is milk, the fat being held in suspension in this case by the action of the proteins of the milk. This effect of various substances in retaining fat in the emulsified form depends upon their effect upon the surface tension of the contact layer between fat and water, but a full discussion of this point would be out of place in this connection.

33. Native fats. - As has already been stated, the reserve fats. of the animal body are triglycerids, chiefly of stearic, oleic 
and palmitic acids, although small quantities of esters of lauric, myristic and arachnic acids and frequently free fatty acids are also found, as well as minute amounts of esters of the higher alcohols, coloring matter, etc. Since stearin and palmitin are solid at ordinary temperatures, while olein is liquid, the consistency of a fat depends largely upon the proportion of olein which it contains and varies not only between different species of animals but often in different parts of the body of the same animal. The fats of cold-blooded animals contain more olein than those of warm-blooded animals and therefore remain liquid at lower temperatures.

The vegetable fats contain a greater variety of fatty acids than the animal fats, notably unsaturated acids like linoleic and eruic, as well as oxy-acids and esters of the higher alcohols (waxes), while the so-called crude fat, or ether extract (108) of vegetable materials contains a great variety of ether-soluble substances, including waxes, resins, chlorophyl, etc., some of which are but remotely related to the true fats.

34. Elementary composition. - The three principal triglycerids, stearin, palmitin and olein, while differing in formula and molecular weight, differ but little in their elementary composition, as the following table shows:-

TABle I. - CoMposition OF TRIglycerids

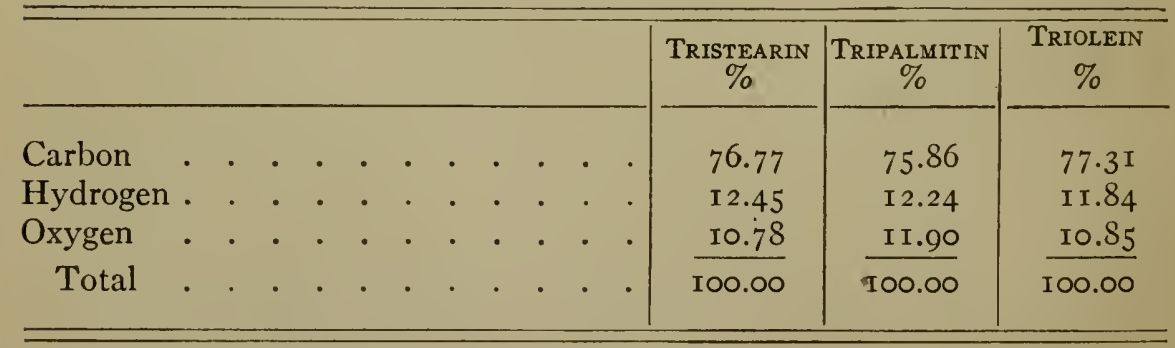

Naturally, therefore, the composition of the ordinary mixed animal fats varies but little, either in different individuals or in different species of animals. The classic investigations of Schulze and Reinecke ${ }^{1}$ upon the composition of animal fats gave the following results.

${ }^{1}$ Landw. Vers. Stat., 9 (1867), 97. 
Table 2. - Composition of Animal Fats

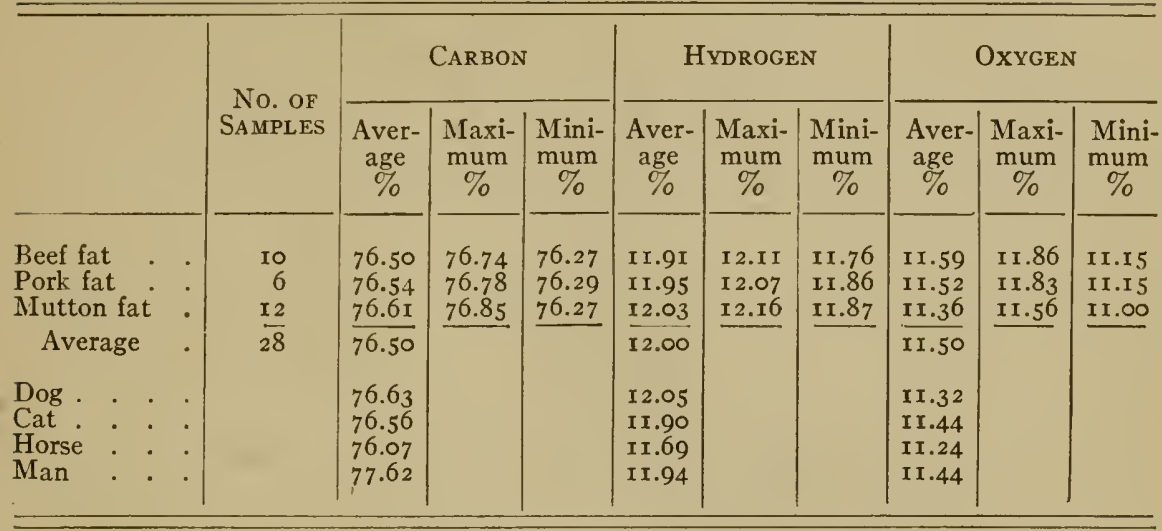

Benedict and Osterberg ${ }^{1}$ obtained the following for the composition of human fat:-

Table 3. - Composition of Human fat

\begin{tabular}{|c|c|c|c|c|c|c|c|c|c|c|c|}
\hline & & & & & & & & & & $\begin{array}{c}\text { CARBON } \\
\%\end{array}$ & $\begin{array}{c}\text { HYDROGEN } \\
\%\end{array}$ \\
\hline Sample No. I & . & . & . & . & . & . & . & . & . & 76.29 & I 1.80 \\
\hline Sample No. 2 & . & . & . . & . . & . & . & . . & . & . & 76.36 & II. 72 \\
\hline Sample No. 3 & . & . & . & . . & . & . & . . & . . & . . & 75.85 & I I. 87 \\
\hline Sample No. 4 & . & . & . . & .. & . & . & . . & . . & - . & 75.95 & I 1.85 \\
\hline Sample No. 5 & . & . & . . & .. & . & . & . . & . . & . . & 75.94 & I I.74 \\
\hline Sample No. 6 & . & . & . . & .. & . & . & . . & . . & . . & 76.07 & I I. 69 \\
\hline Sample No. 7 & . & . & . . & . . & . & . & . . & . . & . . & 76.13 & I 1.84 \\
\hline Sample No. 8 & . & - & . & . . & . & . & . . & . . & - . & 76.05 & I I. $8 \mathrm{I}$ \\
\hline Average & . & . & $\ldots$ & . . & . & . & . . & . . & . . & $\overline{76.08}$ & $\overline{11.78}$ \\
\hline
\end{tabular}

The average carbon content of animal fat is commonly considered to be 76.5 per cent.

\section{Waxes}

35. In popular usage, the distinction between fats and waxes is based upon their obvious physical properties, substances having the well-known greasy feel being called fats or oils according to their consistency at ordinary temperatures while the waxes are solid, can be kneaded and lack largely or wholly the greasy feel.

1 Amer. Jour. Physiol., 4 (Igor), 69. 
Chemically, waxes are defined as fatty acid esters of alcohols other than glycerol, while the fats have already been defined as the fatty esters of glycerol. This distinction is far from according with common usage. Under it many substances popularly known as waxes are technically fats, as for example, Japan wax and in part beeswax. On the other hand, numerous materials ordinarily regarded as oils or fats must be designated as waxes. One of the most familiar bodies of this class is spermaceti, commonly regarded as a fat, which consists chiefly of the palmitic ester of cetyl alcohol, $\mathrm{CH}_{3}\left(\mathrm{CH}_{2}\right)_{14} \mathrm{CH}_{2} \mathrm{OH}$, and sperm oil, which contains no glycerids, would also be regarded as a liquid wax. Similarly wool fat is chemically a mixture of waxes, including the stearic esters of cholesterin and isocholesterin. Beeswax is likewise in part a true wax, containing the palmitic ester of myricyl alcohol, $\mathrm{CH}_{3}\left(\mathrm{CH}_{2}\right)_{28} \mathrm{CH}_{2} \mathrm{OH}$. The secretion of the anal glands of certain birds contains esters of octodeckyl alcohol, $\mathrm{C}_{18} \mathrm{H}_{37} \mathrm{OH}$.

\section{Cholesterins}

36. Substances of this group are found in the nonsaponifiable residue of various fats. In the animal organism they are found widely distributed through the tissues in small amounts and are apparently normal constituents of protoplasm. As just noted, they are especially abundant in wool fats in combination with stearic acid. They are also widely distributed in plants. Their exact constitution is still unknown, but they contain a single alcohol hydroxyl and apparently belong to the terpene group. Their formula is $\mathrm{C}_{27} \mathrm{H}_{44} \mathrm{OH}$, or $\mathrm{C}_{27} \mathrm{H}_{46} \mathrm{OH}$, more probably the latter. From the chemical point of view, they are entirely unrelated to the other groups classified as lipoids, but biologically their functions appear to be closely related to those of the other ether-soluble cell constituents.

\section{Phosphatids or Phospholipins}

37. Lecithins. - Quite closely related to the fats are the substances known as lecithins, which are sometimes, although inexactly, called phosphorized fats. Like the fats, the lecithins are esters of glycerol. They differ from the fats in that only two of the hydroxyls of the glycerol are replaced by fatty acid radicles, the third being replaced by phosphoric acid which is also in combination with the nitrogenous base cholin, a derivative of glycol. The lecithins, therefore, contain, in addition to carbon, hydrogen and oxygen, both phosphorus and nitrogen. 
The molecular structure of the lecithins is illustrated by the following formula for distearyl lecithin :-

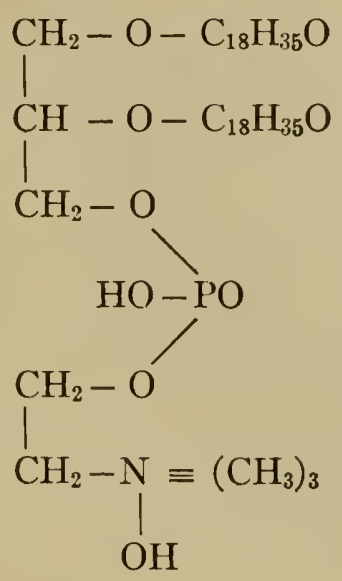

The lecithins resemble fats in their general properties. ' They are soluble in ether but, unlike the fats, readily form permanent emulsions or colloidal solutions with water. On hydrolysis, they yield fatty acids, glycero-phosphoric acid and cholin. They are found widely distributed both in animals and plants and appear to be essential constituents of protoplasm.

38. Other phosphatids. - A variety of other lipoids of the type of the lecithins, but differing in both the fatty acid and the nitrogenous base which they contain and likewise in the ratio of phosphorus to nitrogen, have been described, but the chemistry of this group is still in a very unsatisfactory state. The various phosphatid preparations obtained from vegetable 'materials, especially seeds, by E. Schulze and his associates and designated as lecithins are held by other authors to be such only in a generic sense and in some cases are regarded as more analogous to the cerebrosids or galactolipins of the succeeding paragraph.

\section{Cerebrosids or Galactolipins}

39. This group of substances, found especially in the brain and in nerve tissue in general, belongs chemically to the lipoids, since its members yield fatty acids on hydrolysis. The other products of hydrolysis are galactose and nitrogenous substances but no phosphoric acid, but the constitution of these compounds is still unknown. 


\section{§ 4. The Proteins}

40. Importance. - By far the larger share of the organic matter of the animal body, aside from fat, consists of substances belonging to the well-defined group of the proteins, these compounds, according to the results of analyses recorded on subsequent pages (98), making up from 17.5 to 21 per cent of the fat-free body. These substances are characteristic of the animal body, as the carbohydrates are of plants. Biologically, they are of prime importance to both plants and animals, since they form the basis of the cytoplasm and nucleus of every living cell.

41. Nomenclature. - The chemical structure of the protein molecule has until quite recently been almost entirely unknown and even yet has been but very partially unraveled. Accordingly, the basis for a scientific classification of these substances has been lacking. As a matter of necessity, therefore, the nomenclature hitherto followed has been based chiefly on their physical properties, more particularly their solubilities and coagulation temperatures. Naturally, such a classification has been far from satisfactory and this has been the more true on account of the difficulty of accurately separating the different proteins either by precipitation or crystallization.

Accordingly, there has existed a great and confusing diversity in the nomenclature of the proteins, and uniformity is still far from having been reached. For the present, it seems desirable to follow the classification and nomenclature which has been adopted provisionally by the American Physiological Society ${ }^{1}$ and the American Society of Biological Chemists. ${ }^{2}$ This nomenclature rejects entirely the term proteid as ambiguous on account of the wide diversity in its use, and employs protein as a general term to signify the group of substances which, according to the nomenclature adopted by the Association of American Agricultural Colleges and Experiment Stations in I $898,{ }^{3}$ was called proteids. In other words, protein under the new plan excludes altogether the non-protein nitrogenous substances of plants and animals.

1 Proceedings, Amer. Physiol. Soc., Amer. Jour. Physiol., 21 (I908), xxvii.

2 Proceedings, Amer. Soc. Biol. Chemists, 1, I42.

${ }^{3}$ U. S. Dept. Agr., Office of Expt. Stas., Bul. 65, pp. 1 I 7-123. 
The proteins in this sense are subdivided into:-

I. Simple proteins

2. Conjugated proteins

3. Derived proteins

Simple proteins are defined as those yielding only $a$ amino acids or their derivatives upon hydrolysis. Conjugated proteins are those which contain the protein molecule united to some other molecule or molecules otherwise than as a salt. Derived proteins are the products of the hydrolytic cleavage of the protein molecule and include a wide range of substances, from slightly altered protein to the peptids.

42. Physical properties. - In the dry state, the proteins are in general white or slightly tinted substances. They are usually amorphous, but a number of them have also been obtained in the crystalline form and some are found crystallized in nature. Some of the proteins are soluble in water, others only in salt solutions or in acids or alkalies. They are insoluble in-most other ordinary solvents.

The proteins belong to the class of colloids, i.e., they do not diffuse through membranes and are claimed to have no osmotic pressure when free from electrolytes. Colloids in general exist in two forms, a liquid form, technically known as a sol, and a solid form called a gel, the difference being well illustrated by the familiar substance gelatin. When a colloid is distributed through water so as to form an appareht solution the latter is known as a hydrosol. Whether the proteins are to be regarded as soluble in water, or whether their apparent solution is in reality a suspension, has been much discussed. It has been shown, however, that these solutions are conductors of electricity and it has been concluded that they are true solutions. It may be said, however, that no sharp boundary exists between a true solution and a suspension but that an indefinite number of intermediate stages is possible. As a matter of convenience, however, we may speak of solutions of the proteins.

Different proteins may be precipitated from their solutions by various reagents, particularly acids, alkalies and metallic salts. Ammonium sulphate, especially, has been largely used for the purpose of separating different proteins by means of fractional precipitation. 
43. Coagulation. - An important property of the proteins is that of coagulation. For instance, if a solution of ordinary egg albumin be heated to $55^{\circ} \mathrm{C}$. the albumin begins to separate in an insoluble form and at about $60^{\circ} \mathrm{C}$. the precipitation is complete. This change differs from the change in the case of gelatin solutions from liquid to solid in being irreversible, i.e., coagulated protein cannot be changed back to the soluble form. It should be noted that this change is entirely distinct from the precipitation of proteins by means of ammonium sulphate for example. The exact nature of the change is unknown, but it would seem to be in part chemical in character.

All forms of protein appear to be subject to coagulation in the chemical sense of the word. Thus the precipitated proteins obtained from solutions are at first in the colloidal form but on standing pass more or less rapidly into the coagulated or "denatured" form. The same is true of the solid proteins like fibrin, etc. The coagulated proteins are insoluble in water and salt solutions, but may be dissolved in acids or alkalies.

\section{The simple proteins}

44. Composition. - The simple proteins differ from the compounds considered in the previous sections in containing, in addition to carbon, hydrogen and oxygen, the elements nitrogen and sulphur. Notwithstanding the considerable variation in the properties of the different simple proteins and the notable differences which have been shown to exist in their chemical structure, their elementary composition differs but little. Cohnheim ${ }^{1}$ quotes the following figures from Michel for the composition of serum albumin, which is in many respects a typical animal protein.

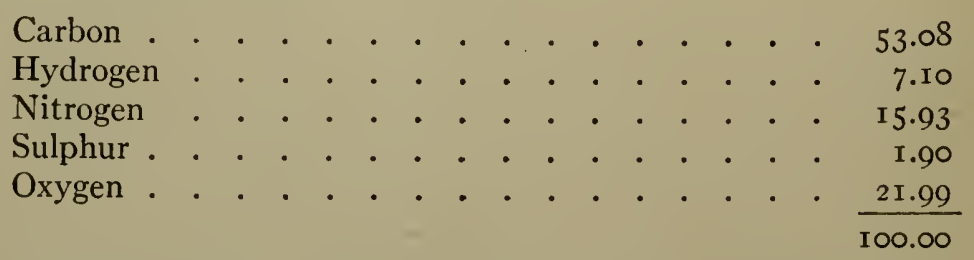

The variations in the percentages of the principal elements as stated by Cohnheim ${ }^{1}$ and by Plimmer ${ }^{2}$ are :-

1 Chemie der Eiweisskörper, 2d Ed., p. I5I.

z The Chemical Constitution of the Proteins, Part I, p. 2. 
46. Hydrolysis of proteins. - The simple proteins readily undergo hydrolysis when acted upon by strong acids or alkalies, or by various enzyms such as the pepsin of the gastric juice, the trypsin of the pancreatic juice, etc. These various agents effect a succession of cleavages and hydrations resulting in a series of products of decreasing molecular complexity and increasing solubility, ranging from very slightly modified proteins through the so-called proteoses and peptones to still simpler substances.

47. Cleavage products of proteins. - When the hydrolysis, especially acid hydrolysis, of the simple proteins is pushed as far as possible, there result a number of comparatively simple crystalline substances which are qualitatively the same for all proteins with a few exceptions, although the proportions of the various products obtained from different proteins vary materially. It is believed, therefore, that the protein molecule is built up of these final products of hydrolysis, the so-called "building stones."

These primary cleavage products of the simple proteins are all $a$ amino acids. One of the first of them to be isolated was glycin or aminoacetic acid, represented by the following formula : -
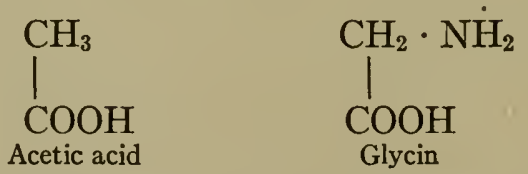

The other cleavage products of the simple proteins may be regarded as derived from glycin by the replacement of one atom of hydrogen in the $\mathrm{CH}_{2}$ group by various atomic groupings. In all of them the $\mathrm{NH}_{2}$ group occupies the same position in the molecule relative to the group $\mathrm{COOH}$ as in glycin, the so-called a position. The atomic grouping

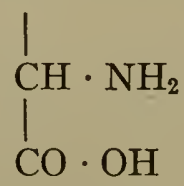

is therefore common to all of these bodies and determines their general chemical behavior as well as that of the proteins from which they are derived. 
The amino acids derived from the proteins may be divided into two classes; the monamino acids, of which glycin is typical, containing one $\mathrm{NH}_{2}$ group, and the diamino acids, containing two $\mathrm{NH}_{2}$ groups. To these there are to be added certain heterocyclic compounds. Plimmer ${ }^{1}$ gives the following list of the amino acids which have been identified with certainty among the cleavage products of the proteins. The presence of others has been claimed by several investigators.

\section{A. Monoaminomonocarboxylic acids}

I. Glycin, $\mathrm{C}_{2} \mathrm{H}_{5} \mathrm{NO}_{2}$, or aminoacetic acid.

$$
\mathrm{CH}_{2} \cdot\left(\mathrm{NH}_{2}\right) \cdot \mathrm{COOH}
$$

2. Alanin, $\mathrm{C}_{3} \mathrm{H}_{7} \mathrm{NO}_{2}$, or $\alpha$-aminopropionic acid.

$$
\mathrm{CH}_{3} \cdot \mathrm{CH}\left(\mathrm{NH}_{2}\right) \cdot \mathrm{COOH}
$$

3. Valin, $\mathrm{C}_{5} \mathrm{H}_{11} \mathrm{NO}_{2}$, or $\alpha$-aminoisovalerianic acid.

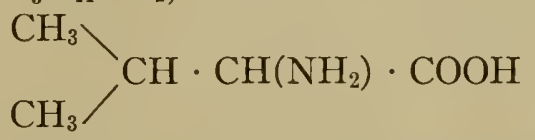

4. Leucin, $\mathrm{C}_{6} \mathrm{H}_{13} \mathrm{NO}_{2}$ or $\alpha$-aminoisocaproic acid.

$$
\mathrm{CH}_{3}
$$

$\mathrm{CH}_{3} /$

$\mathrm{CH} \cdot \mathrm{CH}_{2} \cdot \mathrm{CH}\left(\mathrm{NH}_{2}\right) \cdot \mathrm{COOH}$

5. Isoleucin, $\mathrm{C}_{6} \mathrm{H}_{13} \mathrm{NO}_{2}$, or $\alpha$-amino- $\beta$-methyl- $\beta$-ethyl-propionic acid.

$$
\mathrm{C}_{2} \mathrm{H}_{5} / \mathrm{CH} \cdot \mathrm{CH}\left(\mathrm{NH}_{2}\right) \cdot \mathrm{COOH}
$$

6. Phenylalanin, $\mathrm{C}_{9} \mathrm{H}_{11} \mathrm{NO}_{2}$, or $\beta$-phenyl- $\alpha$-aminopropionic acid.

$$
\mathrm{C}_{6} \mathrm{H}_{5} \cdot \mathrm{CH}_{2} \cdot \mathrm{CH}\left(\mathrm{NH}_{2}\right) \cdot \mathrm{COOH}
$$

7. Tyrosin, $\mathrm{C}_{9} \mathrm{H}_{11} \mathrm{NO}_{3}$, or $\beta$-parahydroxyphenyl- $\alpha$-aminopropionic acid. $\quad \mathrm{HO} \cdot \mathrm{C}_{6} \mathrm{H}_{4} \cdot \mathrm{CH}_{2} \cdot \mathrm{CH}\left(\mathrm{NH}_{2}\right) \cdot \mathrm{COOH}$

8. Serin, $\mathrm{C}_{3} \mathrm{H}_{7} \mathrm{NO}_{3}$, or $\beta$-hydroxy- $\alpha$-aminopropionic acid.

$\mathrm{CH}_{2}(\mathrm{OH}) \cdot \mathrm{CH}\left(\mathrm{NH}_{2}\right) \cdot \mathrm{COOH}$

9. Cystin, $\mathrm{C}_{6} \mathrm{H}_{12} \mathrm{~N}_{2} \mathrm{O}_{4} \mathrm{~S}_{2}$, or dicysteine, or di- ( $\beta$-thio- $\alpha$-aminopropionic acid) $\mathrm{HOOC} \cdot \mathrm{CH}\left(\mathrm{NH}_{2}\right) \cdot \mathrm{CH}_{2} \cdot \mathrm{S}-\mathrm{S} \cdot \mathrm{CH}_{2} \cdot \mathrm{CH}\left(\mathrm{NH}_{2}\right) \cdot \mathrm{COOH}$

\section{B. Monoaminodicarboxylic acids}

Io. Aspartic acid, $\mathrm{C}_{4} \mathrm{H}_{7} \mathrm{NO}_{4}$, or $\alpha$ aminosuccinic acid.

$$
\mathrm{HOOC} \cdot \mathrm{CH}_{2} \cdot \mathrm{CH}\left(\mathrm{NH}_{2}\right) \cdot \mathrm{COOH}
$$

rr. Glutamic acid, $\mathrm{C}_{5} \mathrm{H}_{9} \mathrm{NO}_{4}$, or $\alpha$-aminoglutaric acid. $\mathrm{HOOC} \cdot \mathrm{CH}_{2} \cdot \mathrm{CH}_{2} \cdot \mathrm{CH}\left(\mathrm{NH}_{2}\right) \cdot \mathrm{COOH}$

1 The Chemical Constitution of the Proteins, Part I, 2d Ed., I9r 2. 
C. Diaminomonocarboxylic acids

I2. Arginin, $\mathrm{C}_{6} \mathrm{H}_{14} \mathrm{~N}_{4} \mathrm{O}_{2}$, or $\alpha$-amino- $\gamma$-guanidin valerianic acid.

$$
\mathrm{HN}=\mathrm{C}\left\langle\begin{array}{l}
\mathrm{NH}_{2} \\
\mathrm{NH} \cdot \mathrm{CH}_{2} \cdot \mathrm{CH}_{2} \cdot \mathrm{CH}_{2} \cdot \mathrm{CH}\left(\mathrm{NH}_{2}\right) \cdot \mathrm{COOH}
\end{array}\right.
$$

I3. Lysin, $\mathrm{C}_{6} \mathrm{H}_{14} \mathrm{~N}_{2} \mathrm{O}_{2}$ or $\alpha$, $\epsilon$-diaminocaproic acid.

$\mathrm{H}_{2} \mathrm{~N} \cdot \mathrm{CH}_{2} \cdot \mathrm{CH}_{2} \cdot \mathrm{CH}_{2} \cdot \mathrm{CH}_{2} \cdot \mathrm{CH}\left(\mathrm{NH}_{2}\right) \cdot \mathrm{COOH}$

D. Heterocyclic compounds

I4. Histidin, $\mathrm{C}_{6} \mathrm{H}_{9} \mathrm{~N}_{3} \mathrm{O}_{2}$, or $\beta$-imidazol- $\alpha$-aminopropionic acid.

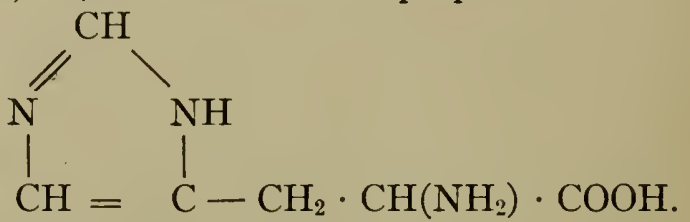

I 5. Prolin, $\mathrm{C}_{5} \mathrm{H}_{9} \mathrm{NO}_{2}$, or -pyrrolidin carboxylic acid

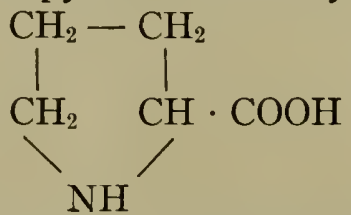

r6. Oxyprolin, or oxypyrrolidine carboxylic acid.

$$
\mathrm{C}_{5} \mathrm{H}_{9} \mathrm{NO}_{3}
$$

I 7. Tryptophan, $\mathrm{C}_{11} \mathrm{H}_{12} \mathrm{~N}_{2} \mathrm{O}_{2}$, or $\beta$-indol- $\alpha$-aminopropionic acid.

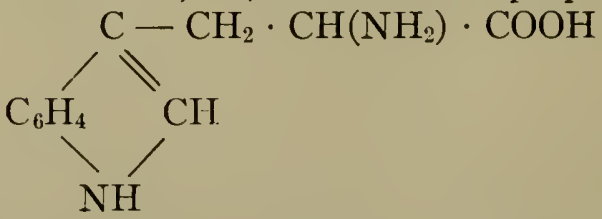

48. Synthesis of proteins. - Peptids. - Fischer and others have shown that the amino acids which result from the cleavage of the simple proteins may combine with each other, the $\mathrm{NH}_{2}$ of one uniting with the $\mathrm{COOH}$ group of the other with the elimination of one molecule of water. As many as 18 molecules of amino acids have been combined in this way, although the exact structure of the resulting compounds is still more or less uncertain.

The compounds of the amino acids which have been prepared artificially have received the general name of peptids, the prefixes di-, tri-, etc., being used to indicate the number of amino acid molecules entering into the compound. The term poly- 
peptids is also commonly used as a general term for the more complex substances of this group. The latter show many of the reactions of the proteins or of their less modified derivatives. For example, many of them give the biuret reaction characteristic of the proteins, are precipitated by phosphotungstic acid and undergo cleavage by appropriate proteolytic ferments. Moreover, some of the artificial polypeptids of known composition have also been isolated from the mixture of products resulting from the action of ferments upon the proteins.

49. Conclusions. - Since, therefore, the same comparatively simple crystalline products are obtained as the final result of the complete hydrolysis of all the simple proteins, viz., the various amino acids enumerated in a previous paragraph (47), and since, on the other hand, these cleavage products may be synthesized to form substances closely resembling the proteins, it is believed that the protein molecule is built up of these amino acids, united in substantially the same way as in the artificially prepared polypeptids. In other words, it is believed that the latter are the first steps toward the synthesis of proteins, or indeed that they may, from a systematic point of view, be regarded as the simplest of the proteins.

It should be noted, however, that while the foregoing method of combination of the amino acids appears to be characteristic of the protein molecule, it is not the only form of combination in which nitrogen enters into it. For example, arginin, apparently a constituent of all proteins, contains an atom of imid nitrogen, HN. The proteins also contain amid nitrogen (i.e., $\mathrm{NH}_{2}$ substituted for the $\mathrm{OH}$ of the carboxyl group) which yields ammonia on hydrolysis. Furthermore, the proteins are capable of acting as polyacid bases and therefore the molecule must contain numerous $\mathrm{NH}_{2}$ end-groups such as that of the amids just mentioned or those of the diamino-acids like lysin and arginin.

50. Proportions of cleavage products in different proteins. While all the simple proteins yield, with a few exceptions, qualitatively the same cleavage products, the relative proportions of these "building stones" vary widely in proteins from different sources. This is strikingly illustrated by the following tabulation of the percentages of the various amino acids yielded by a number of proteins according to the researches of Osborne and his associates. 
Table 4. - Cleavage Products of Proteins ${ }^{1}$

\begin{tabular}{|c|c|c|c|c|c|c|c|c|c|c|c|c|c|c|}
\hline & & & & & & 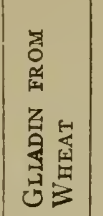 & 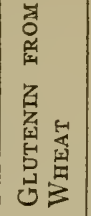 & 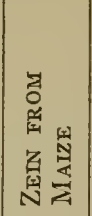 & 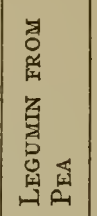 & 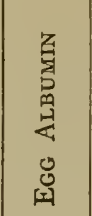 & 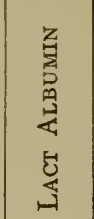 & 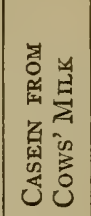 & 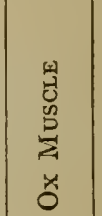 & 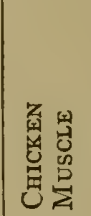 \\
\hline Glycin & • & . & $\bullet$ & . & & $\begin{array}{c}\% \\
0.00\end{array}$ & $\begin{array}{c}\% \\
0.89\end{array}$ & $\begin{array}{c}\% \\
0.00\end{array}$ & $\begin{array}{c}\% \\
0.38\end{array}$ & $\begin{array}{l}\% \\
0.0\end{array}$ & $\begin{array}{l}\% \\
0.0\end{array}$ & $\begin{array}{c}\% \\
0.00\end{array}$ & $\begin{array}{c}7 \% \\
2.06\end{array}$ & $\begin{array}{c}\% \\
0.68\end{array}$ \\
\hline Alanin & . & & & 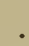 & . & 2.00 & 4.65 & I3.39 & 2.08 & 2.22 & 2.50 & I. 50 & 3.72 & 2.28 \\
\hline Valin . & . & & & . & . & $3 \cdot 34$ & 0.24 & I.8S & - & 2.50 & 0.90 & 7.20 & $0.8 \mathrm{I}$ & $t$ \\
\hline Leucin & & & & & & 6.62 & $5 \cdot 95$ & I9.55 & 8.00 & $10.7 \mathrm{I}$ & 19.40 & $9 \cdot 35$ & II.65 & II.I9 \\
\hline Phenylala & nin & & . & - & . & 2.35 & I.97 & 6.55 & $3 \cdot 75$ & 5.07 & 2.40 & 3.20 & 3.15 & 3.53 \\
\hline Tyrosin & . & & & & . & $\mathrm{I} .50$ & 4.25 & $3 \cdot 55$ & I. 55 & I. 77 & 2.20 & 4.50 & 2.20 & 2.16 \\
\hline Serin . & . & & . & - & . & 0.13 & 0.74 & 1.02 & 0.53 & ? & ? & 0.50 & ? & ? \\
\hline Cystin . & . & & & & . & 0.45 & 0.02 & $?$ & - & ? & ? & ? & 一 & - \\
\hline Prolin . & . & & . & & . & 13.22 & 4.23 & 9.04 & 3.22 & $3 \cdot 56$ & 4.00 & 6.70 & 5.82 & 4.74 \\
\hline Aspartic a & acic & & & & & $0.5^{8}$ & $0.9 \mathrm{I}$ & $1.7 \mathrm{I}$ & $5 \cdot 30$ & 2.20 & I.OO & I.39 & $4.5 \mathrm{I}$ & $3.2 \mathrm{I}$ \\
\hline Glutamic & ac & & . & & & 43.66 & 23.42 & 26.17 & I3.80 & 9.10 & 10.10 & I 5.55 & I 5.49 & 16.48 \\
\hline Tryptoph & Ian & . & & & & 1.00 & + & 0.00 & + & + & $t$ & I. 50 & + & + \\
\hline Arginin & . & $\cdot$ & & & & 3.16 & 4.72 & I. 55 & IO.I2 & $4.9 I$ & 3.01 & $3.8 \mathrm{I}$ & $7 \cdot 47$ & 6.50 \\
\hline Lysin . & . & & & & & ? & I. 92 & 0.00 & 4.98 & 3.76 & 8.10 & 7.61 & $7 \cdot 59$ & 7.24 \\
\hline Histidin & . & $\cdot$ & 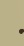 & . & . & I. 49 & x.76 & 0.82 & 2.42 & $\mathrm{I} .7 \mathrm{I}$ & I.53 & 2.50 & 1.76 & 2.47 \\
\hline Ammonia & . & 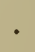 & & 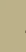 & & 5.22 & 4.01 & 3.64 & I.99 & I. 34 & I. 32 & I. $6 \mathrm{I}$ & 1.07 & I. 67 \\
\hline Total & . & & & & & 84.72 & 59.68 & 88.87 & $5^{8.12}$ & 48.85 & $5^{6.46}$ & 66.92 & 67.29 & 15 \\
\hline
\end{tabular}

The results shown in the foregoing table are typical. In a few proteins, certain amino acids have not been found at all. For example, no glycin has been found among the products of the hydrolysis of gliadin, zein, albumin or casein and no lysin among those of gliadin or zein. Furthermore, the proportion of the various cleavage products is quite variable in the different proteins, one of the most striking instances being that of glutamic acid which ranges from nearly 44 per cent in the gliadin of wheat to a little over 9 per cent in egg albumin, and is notably more abundant in vegetable than in animal proteins.

51. Classification. - For the present purpose, it seems superfluous to enter into a full description of the various simple pro-

1 The sign + signifies that the substance was present but was not quantitatively determined. A blank simply indicates that the ingredient in question was not determined but does not show that it was not present. 
teins. The principal groups into which they are subdivided are designated as follows:-

a. Albumins. - These are simple proteins soluble in pure water and coagulable by heat. Besides the familiar egg albumin, they include the albumins of blood serum and of milk serum. Albumins have also been found in small amounts in a great variety of seeds, including those of wheat, rye, barley, pea, vetch, soybean and cowpea.

b. Globulins. - The globulins are simple proteins insoluble in pure water but soluble in neutral solutions of salts of strong bases with strong acids. Globulins are found in the lymph and the blood serum and in the muscles and other organs, but they appear to be especially characteristic of the vegetable kingdom, occurring in considerable amounts in a large number of seeds. Osborne ${ }^{1}$ gives a list of ${ }^{5} 5$ globulins occurring in 24 different species of seeds and enumerates 12 additional species which contain globulins to which no distinctive names have yet been given.

c. Glutelins. - These are defined as simple proteins insoluble in all neutral solvents but readily soluble in very dilute acids and alkalies. The only well-defined members of this group at present known are the glutenin of wheat and the oryzenin of rice, although there seems reason to believe that similar proteins exist in the seeds of other cereals.

d. Prolamins, or alcohol-soluble proteins. - The typical member of this group is the gliadin of wheat and the name has been applied by some authors to the entire group, but the term prolamins, proposed by Osborne, seems preferable. The prolamins are soluble in relatively strong alcohol (70-8o per cent) but insoluble in water, absolute alcohol and other neutral solvents. They are characteristic of the seeds of the cereals, the principal prolamins being the gliadin of wheat and rye, the hordein of barley, the zein of maize and the bynin of malt.

e. Albuminoids. - This name, formerly used to a considerable extent as practically synonymous with proteins, is now applied to two groups of nitrogenous substances which have been otherwise designated as the collagens, or gelatinoids, and the keratins. Albuminoids are defined as simple proteins which possess essentially the same chemical structure as the other ${ }_{1}^{1}$ The Vegetable Proteins, p. 78. 
proteins but are characterized by great insolubility in all neutral solvents. They form the principal organic constituents of the skeletal structures of animals and of their external covering and its appendages and hence have also been called scleroproteins. This definition does not provide for gelatin, which is, however, an artificial derivative of collagen. Besides gelatin the more important members of this group are chondrin, or collagen, which constitutes the organic basis of cartilage and bone; elastin, the characteristic component of the ligaments; and the keratins of the epidermal tissues such as hair, wool, feathers, horns, hoofs, etc.

\section{The conjugated proteins}

52. Nucleoproteins. - In the scheme of classification here followed (41), the nucleoproteins are defined as follows: "These proteins are especially characteristic of the nucleus of the vegetable and animal cell (74). They consist of protein molecules united with one or more of the compounds known as nucleic acids. These are complex organic compounds containing a phosphoric acid radicle and also a xanthin group."

The simple proteins of the nucleoproteins apparently may be of quite diverse nature and belong to various groups of the simple proteins. The special interest of the nucleoproteins attaches to the nucleic acids entering into their composition.

53. Nucleic acids. - These compounds contain in addition to carbon, hydrogen, nitrogen and oxygen the element phosphorus. Their constitution has not yet been fully worked out, but their cleavage yields four classes of products, viz.,

I. Xanthin, or purin, bases

2. Pyrimidin bases

3. A pentose carbohydrate

4. Phosphoric acid

According to the recent investigations of Levene and others, the nucleic acid molecule may be regarded as built up from nucleosids, or glucosid-like combinations of a pentose carbohydrate with a purin or pyrimidin base. By the union of such a nucleosid with phosphoric acid there is formed a nucleotid. Finally, the most common nucleic acids are tetranucleotids. 
which seem always to contain both purin and pyrimidin nucleosids.

54. Glycoproteins. - The glycoproteins are defined as " Compounds of the protein molecule with a substance or substances containing a carbohydrate group other than a nucleic acid. The principal compounds of this group are the mucins and the mucoids."

55. Phosphoproteins. - These are defined as compounds of the protein molecule with some, as yet undefined, phosphoruscontaining substance other than a nucleic acid or lecithin. The casein, or rather caseinogen, of milk is one of the most familiar and important of this group.

56. Hæmoglobins. - The hæmoglobins are compounds of the protein molecule with hæmatin or some similar substance, and constitute the red coloring matter of the blood.

57. Lecithoproteins. - Compounds of the protein molecule with lecithins.

\section{The derived proteins}

58. Primary protein derivatives. - Derivatives of protein apparently formed through hydrolytic changes which involve only slight alterations of the molecule.

Proteans. - Insoluble products which apparently result from the incipient action of water, very dilute acids or enzyms.

Metaproteins. - Products of the further action of acids and alkalies whereby the molecule is so far altered as to form products soluble in very weak acids and alkalies but insoluble in neutral fluids. This group will thus include the familiar "acid proteins" and "alkali proteins," not the salts of proteins with acids.

Coagulated proteins. - Insoluble products which result from (I) the action of heat on their solutions, or (2) the action of alcohols on the protein.

59. Secondary protein derivatives. - Products of the further hydrolytic cleavage of the protein molecule.

Proteoses. - Soluble in water, uncoagulated by heat, and precipitated by saturating their solutions with ammonium or zinc sulphate.

Peptones. - Soluble in water, uncoagulated by heat but not precipitated by saturating their solutions with ammonium sulphate.

Peptids. - Definitely characterized combinations of two or more amino acids, the carboxyl group of one being united with the amino group of the other with the elimination of a molecule of water (48). 


\section{§ 5. The Non-Proteins}

60. Occurrence.- In addition to the proteins, both plants and animals contain a great variety and sometimes relatively considerable amounts of nitrogenous compounds of the most diverse nature. While the occurrence of such compounds, especially in feeding stuffs, was known from an early day, it was long assumed that the amounts present were relatively insignificant and that no material error was involved in regarding all the nitrogen of a feeding stuff as existing in the form of protein. Accordingly, the total nitrogen multiplied by the conventional factor 6.25 and designated as "crude protein " was taken as representing the true protein content of the material. The researches of Scheibler, E. Schulze and Kellner in the seventies, however, showed that this was far from being the case. It was found that nitrogenous substances other than protein were very widely distributed and that sometimes as much as one-third or even one-half of the total nitrogen of feeding stuffs existed in these non-protein compounds. These results have been fully confirmed by subsequent investigations and it has therefore become necessary to distinguish between these substances and the true proteins.

61. Definition. General properties. - While these nitrogenous compounds other than protein are of the most varied nature, they all differ from the proteins in having a much less complex molecular structure. Many are comparatively simple, crystalline substances, most of them readily soluble in water and diffusible, and they appear distinctly inferior in nutritive value to the proteins. It is a matter of practical convenience, therefore, to have a group name by which to distinguish them and for this purpose the term non-proteins has been proposed. It is, of course, a contraction for non-protein nitrogenous substances and means simply substances which contain nitrogen but are not proteins. It therefore includes a great variety of compounds and may be considered as in a sense a cover for our ignorance of their exact nature. The more important groups of non-proteins are:-

The nitrogenous muscle extractives

The nitrogenous lipoids 
The nitrogenous glucosids

Alkaloids and organic bases

Amino acids and amids

Nitrates and ammonium salts

62. The muscle extractives. - The more important nitrogenous muscle extractives are creatin, creatinin and the purin bases xanthin'and hypoxanthin.

63. Nitrogenous lipoids. - As noted (37-39), the lipoid group includes a number of compounds, classed as phosphatids and cerebrosids, which contain a nitrogenous group in combination with fatty acid radicles. The most familiar members of this group are the lecithins. The actual amounts of these substances contained either in the animal or plant are small and their nitrogen does not constitute any important fraction of the total nitrogen of the body or of the feed.

64. Alkaloids and organic bases. - Alkaloids are comparatively rare in agricultural plants, the seeds of the lupine forming the principal exception. The organic bases, on the other hand, appear to be somewhat widely distributed. In addition to the so-called "hexon bases" arginin, lysin and histidin, derived from the proteins and nucleo-proteins, the bases cholin, betain, trigonellin and stachydrin have been found in a variety of plants.

65. Nitrogenous glucosids. - The substances of this group are characteristic of the vegetable kingdom. They contain a variety of nitrogenous compounds coupled with simple sugars. The nitrogenous glucosids do not appear to be especially abundant in the ordinary feeding stuffs of domestic animals and where they do occur are distinguished rather by their specific physiological effects than by their nutritive value in the ordinary sense. E. Schulze ${ }^{1}$ mentions seven bodies of this class which have been found in various plants.

66. Amino acids and amids. - These substances are by far the most abundant forms of non-protein in vegetable materials. The first one to be discovered was asparagin, in 1805 , in asparagus shoots, and this substance has since been found in a large number of plants or parts of plants. Glutamin, a second amid, is also of frequent occurrence in plants.

I Jour. Landw., 52 (I 904), 305. 
Asparagin and glutamin are respectively the amids of aspartic and glutamic acids, both of which are constituents of the protein molecule.
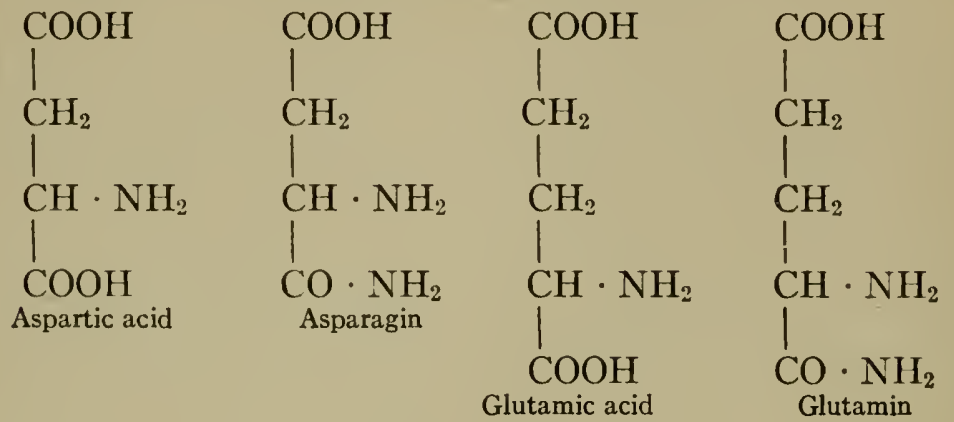

It has thus come about that the term amids has been more or less commonly used as a general designation for the non-protein nitrogenous substances found in feeding stuffs. The usage, however, is unfortunate. The word amid denotes a distinct class of chemical substances of which only asparagin and glutamin appear to be especially common in plants, while the latter contain a variety of nitrogenous substances which are not amids at all. The general term non-protein proposed above, therefore, seems preferable.

In addition to asparagin and glutamin there have been found in feeding stuffs a large number of the cleavage products of the proteins. E. Schulze $\mathrm{e}^{1,2,3}$ enumerates ten amino acids, viz., valin, leucin, isoleucin, phenylalanin, tyrosin, prolin, tryptophan, arginin, lysin and histidin, besides the purin bases xanthin, hypoxanthin, adenin and guanin, as well as guanidin, allantoin and carnin, as having been isolated from various vegetable materials. Hart and Bentley ${ }^{4}$ found that from 50 to 70 per cent of the water-soluble nitrogen of a variety of feeding stuffs existed as amino acids or peptids, while the amid nitrogen proper amounted to only io to 20 per cent.

Occurrence. - These substances evidently stand in a close relation to the protein metabolism of the plant. They appear to be in part intermediate products in the synthesis of protein from nitrates and ammonium salts and in part to be formed in the cleavage of proteins necessary for their translocation and resynthesis during the processes of growth. They are especially

1 Jour. f. Landw., 52 (1904), 305. $\quad 2$ Ztschr. Physiol. Chem., 45 (1905), 38.

${ }^{3}$ Ztschr. Physiol. Chem., 47 (I906), 507. ${ }^{4}$ Jour. Biol. Chem., 22 (I915), 477. 
abundant, therefore, where growth is going on most rapidly. Young and succulent feeding stuffs, such as pasture grass, green soiling crops and the like, accordingly contain a considerable proportion of their nitrogen in the non-protein form. As plants approach ripeness, the proportion of non-protein to protein nitrogen becomes less, so that mature hay, straw and the like are relatively poor in non-proteins. This is especially true of seeds, whose nitrogen is contained chiefly in the reserve proteins, although small amounts of various non-proteins are also found. One class of feeding stuffs relatively rich in non-protein is the roots and tubers, in which the conversion of inorganic nitrogen into protein seems to be incomplete and in which the non-protein serves as a nitrogenous reserve for the growth of the succeeding year. Finally, feeding stuffs which have undergone fermentation, such as silage, show a relative increase of the non-protein nitrogen over that of the original material.

67. Nitrates and ammonium salts. - Occasionally somewhat considerable amounts of nitrates or of ammonium compounds are found in vegetable material, especially when the supply of soluble nitrogen compounds in the soil is abundant. In such cases they are to be regarded as materials taken up in excess of the immediate needs of the plant.

\section{$\S$ 6. SUNDRY INGREDIENTS}

68. Number and significance. - In the foregoing sections the groups of substances which constitute the animal body or, in the form of feed, supply the matter and energy for its growth and maintenance have been considered. It is hardly necessary to say, however, that these four groups, the carbohydrates, fats, proteins and non-proteins, are very far from comprising all the constituents of animals or plants.

In the animal body the physiological chemist has recognized relatively small amounts of a vast number of substances of the most varied nature. Some of these are derived quite directly from the proteins, fats or carbohydrates and these will be considered to a greater or less extent in studying the changes which these substances undergo in the body. Others, while of great physiological importance, have little direct relation to the processes of nutrition. 
Similarly, plants contain a great variety of ingredients not strictly belonging to any of the four main groups. In the aggregate, these substances do not often add greatly to the potential food value of feeding stuffs, but, on the other hand, they may in some cases considerably modify their palatability or the activity of the various processes of nutrition and so affect the actual results of feeding. Until recently these secondary ingredients of feeding stuffs have received comparatively little attention.

69. Organic acids. - Aside from the small amounts of free fatty acids occurring in most native fats, both animal and vegetable $(29,33)$, the acids of this series are seldom or never found in native feeding stuffs. In those feeding stuffs which have undergone bacterial fermentation, however, notably in the case of silage, more or less acetic and butyric acids occur, but the principal acid product of such fermentations is lactic acid, $\mathrm{C}_{3} \mathrm{H}_{6} \mathrm{O}_{3}$. The same acids, along with formic and propionic acids and minute amounts of ethyl aldehyde, likewise result from the bacterial fermentation of the carbohydrates of the feed in the paunch of ruminants and thus constitute a not unimportant portion of the non-nitrogenous material resorbed from the feed (128-132). The principal organic acids found in native feeding stuffs are malic, tartaric, citric and oxalic, usually as the potassium, sodium or calcium salts.

70. Ethereal oils. - The so-called ethereal oils are substances of complex molecular nature, somewhat resembling the true oils in their physical properties but which can readily be distilled in a current of steam. Familiar examples are the so-called oils of peppermint, lemon, anise, and the like. It is not known that they have any direct nutritive value themselves but they add to the flavor and aroma of feeds and in some cases are believed to stimulate the digestive processes. The agreeable odor of good hay, for example, and doubtless in part its favorable dietetic effect, is due to substances resembling in properties the ethereal oils. To the same class of ethereal oils belong the oils of mustard, onion and garlic, whose deleterious effect upon the flavor of dairy products is so well known.

71. Flavoring substances in general. - What is called the flavor of a food or feeding stuff depends largely upon the action on the sense of smell of a great variety of substances either con- 
tained in the material originally or, especially in the case of human foods, artificially added or developed during cooking. Besides ethereal oils, stock feeds contain a great variety of bitter or astringent substances, gums, waxes, resins, etc., etc., of whose properties and physiological effects little or nothing is known. The flavor and palatability of feed, as already indicated, are usually dependent upon these accessory ingredients, while the fact that palatability is an important factor in nutrition aside from any direct nutritive effect will appear in later discussions.

72. Vitamins: Growth substances. - Much attention has been devoted during the past few years to an important but as yet rather ill-defined group of food constituents called by some investigators vitamins and by others growth substances. These substances, however, are known by their effects rather than by their chemical properties and may therefore be more appropriately considered in their relations to the requirements for maintenance and growth (438, 498, 738). 


\section{CHAPTER II}

THE COMPOSITION OF ANIMALS AND OF FEEDING STUFFS

\section{$\S$ I. The CeLL}

73. Definition. - The cell may be defined as the biological unit of all life. It is the simplest form in which living matter can exist. It might be regarded as bearing somewhat the same relation to the animal or plant that the atom does to a complex organic molecule such as that of one of the proteins for example. It is seen in its simplest form in unicellular organisms (protozoa) in which all the functions of life are performed by a single cell. As we ascend in the scale of organization a number of cells are united to form one individual, the various vital functions being to a greater or less extent distributed among different cells or cell groups. In the higher organisms the cells are numbered by myriads, while the physiological division of labor and the corresponding differentiation of form reach an extreme. The organization of such an individual has been likened to that of a state or nation, in which the functions of the single citizen are highly specialized. A few of the diverse forms of animal cells are represented in Fig. $\mathbf{I}$.

74. Structure of cells. - The typical cell consists of the cell body, or cytoplasm, within which is the nucleus. The peripheral portion of the cytoplasm is often somewhat more compact than the remainder and serves to separate the cell from its surroundings. Sometimes a distinct membrane, or cell wall, is developed, especially in plants, although this is not a necessary part of the cell. The name protoplasm is often applied to the entire active part of the cell, i.e., to cytoplasm plus nucleus. All forms of life, vegetable as well as animal, are indissolubly associated with and manifested through the activities of protoplasm, which was called by Huxley the physical basis of life. It should be understood, however, that the word protoplasm is not a chemical but a biological term. It is a struc- 
ture rather than a substance. Moreover, there is not one protoplasm, common to all cells, but as many protoplasms as there are kinds of cells.

There is a more or less sharp differentiation between the functions of the nucleus and those of the cytoplasm. The nucleus appears to be especially concerned in cell reproduction,

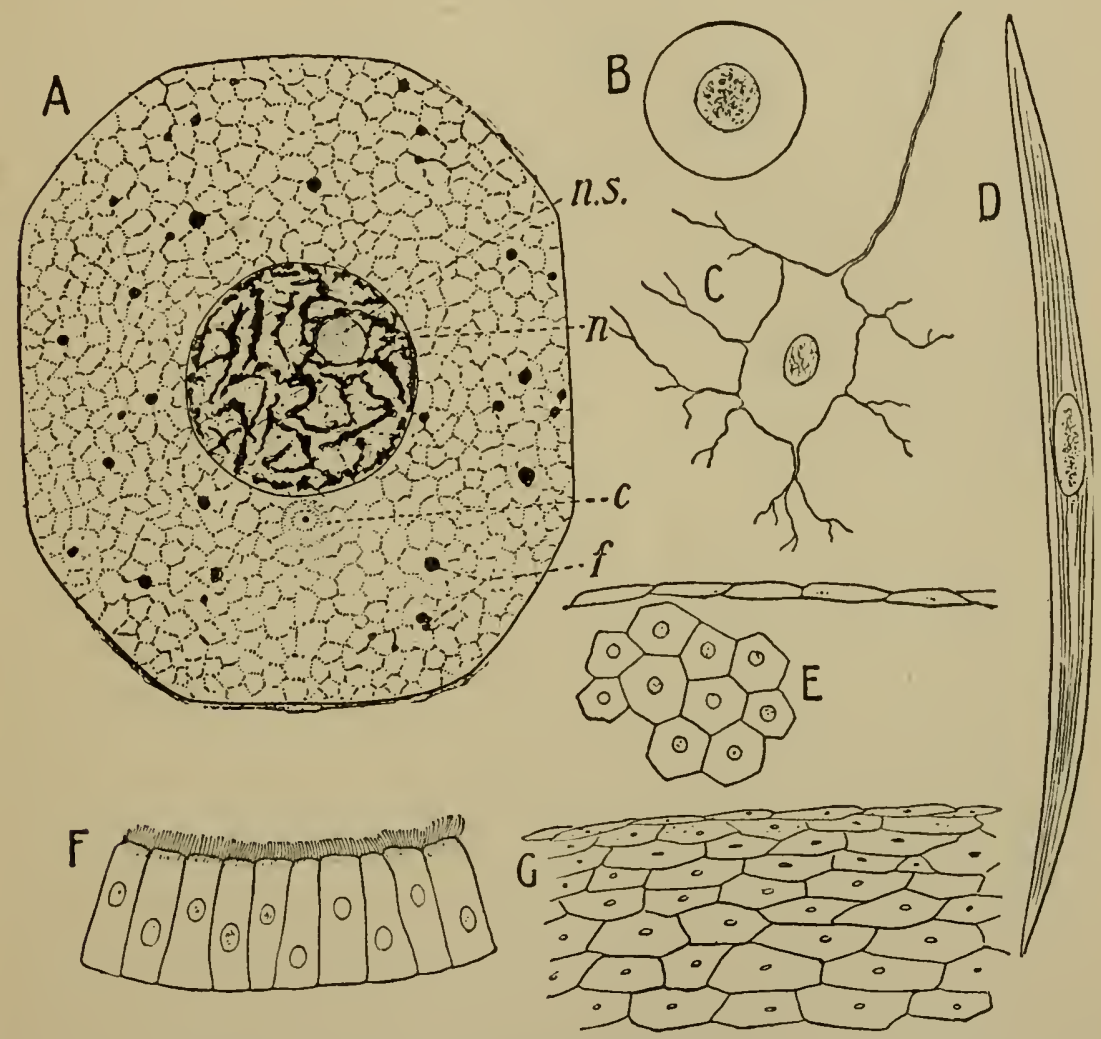

FIG. I. - Different types of cell composing the body. (Hadley, The Horse in Health and Disease.)

the formation of a new cell beginning with a division of the nucleus of an existing coll and being followed by a division of its cytoplasm. The main function of the cytoplasm, on the other hand, seems to be the nutrition of the cell, and the presence of at least a minimum amount of it is essential to the continued existence of the nucleus. For the present purpose, it is unnecessary to attempt a further discussion of those finer details of the structure of the cell which have been worked out by the labors of the histologist and physiologist. 
75. Composition of protoplasm. - The chemical constitution of living protoplasm is unknown, partly because it is undoubtedly very complex but chiefly because of its instability and the impossibility of isolating it without at the same time destroying its life. Moreover, it doubtless varies materially in cells of different types. The proteins, perhaps combined with each other into "giant molecules," undoubtedly constitute the basis and predominating ingredient of protoplasm, but certain lipoids (lecithins and cholesterins), ash ingredients (electrolytes), and perhaps glycogen and other carbohydrates, in addition, of course, to water, appear to be also essential constituents. In the cytoplasm, the simple proteins (41) seem to predominate, while the nucleus is especially characterized by the presence of the nucleoproteins (52).

76. The cell wall. - As already indicated, the protoplasm often develops a cell wall. So far as concerns the species of plants which serve as feed for farm animals, it may be said that a vegetable cell is always surrounded by a cell wall the basic ingredient of which is the carbohydrate cellulose, a substance not found in the bodies of the higher animals.

In the young and growing parts of plants, the cell wall is thin and consists substantially of cellulose only. In certain parts of plants, such as the cotyledons and endosperms of seeds or the tissues of succulent roots and tubers, the cell wall remains comparatively thin even in mature tissue. In other parts of the plant, on the contrary, it becomes very much thickened by the deposition of additional cellulose and especially of substances other than cellulose. These other substances, which appear to be essentially carbohydrates or their derivatives, are of two general kinds. The first of these is the hemicelluloses (18), which are more readily attacked by hydrolyzing agents than pure cellulose and which constitute to a large extent a deposit of reserve material and include both hexosans and pentosans. The second consists of substances belonging to the lignin and cutin groups $(19,20)$, which serve to impart strength and rigidity along with more or less impermeability to the cell wall. They are, therefore, particularly abundant in older plants as compared with younger ones and in those organs which serve to support the plant, such as the stem. The extreme form of the thickened cell wall is seen in wood. A few of the numerous 
forms of vegetable cells are illustrated in Figs. $43-45$ of Chapter XV.

77. Cell enclosures. - In addition to the essential constituents of the cytoplasm and nucleus there are observed in cells a variety of other substances designated as subsidiary ingredients or cell enclosures. These may consist of food material which has entered the cell and is on its way to being incorporated into the molecules of protoplasm, or, on the other hand, of waste products of cell activity on their way to being excreted from the cell into the surrounding medium. Moreover, many cells have the power of storing up surplus food, especially non-nitrogenous substances, as reserve material. Such material is not usually regarded as constituting a part of the protoplasm but as being simply included in it mechanically.

The most common cell enclosure in the animal is fat, which is contained in large quantity in certain connective tissue cells and constitutes the reserve fuel material of the animal body, the storage of carbohydrates (glycogen) being much more limited in amount. While some important groups of plants also store up large amounts of fat in their seeds, nevertheless the predominating reserve materials in the vegetable kingdom are carbohydrates, including the reserve carbohydrates of the cell wall and, as a cell enclosure, starch. Starch is found in all parts of plants, but is especially abundant in seeds and in the starchy roots and tubers, where large amounts of this substance are stored up. Illustrations of plant cells containing starch are afforded by Figs. $43-45$ of Chapter XV.

Both because of the chemical composition of the cell wall and the nature of the cell enclosures, carbohydrates are quantitatively the predominating ingredients of most plants, while animal cells and tissues are chiefly proteid or fatty in character.

\section{$\S$ 2. Animal Tissues and ORgans}

78. Classification. - Not only do the cells of higher animals show extreme differentiation of form and function, but cells having the same general nature and office are associated together to form what are called tissues, such as nerve tissue, muscular tissue, connective tissue and the like, each serving its own specific purpose. These tissues, again, are grouped together 
to form organs, such as the muscles, heart, lungs, stomach, liver and the like, each performing its special part in the complex interplay of activities necessary for the life of the organism as a whole.

Since this is not a treatise on anatomy, it is unnecessary to consider in detail all the diverse types of tissue or all the various organs making up the body. It is desirable, however, that the student of nutrition should acquire some notion of the chemical make-up of the various parts of the body. For this purpose it will be convenient to use the following scheme, based chiefly on the functions performed by the different groups of tissues, which ignores to some extent the distinction between tissues and organs and which does not pretend to be an exact or exhaustive classification.

First : The supporting tissues, including bone, tendon, cartilage, ligament, elastic tissue, etc.

Second: The tissues of motion, including the muscular tissues and the nerve tissues or the nervous system.

Third: The tissues of alimentation, including the tissues and organs concerned in digestion, resorption, circulation, respiration and excretion.

Fourth: The epidermal tissues.

Fifth: The reserve tissues, including, besides adipose tissue, those tissues in which glycogen is more or less abundantly stored.

\section{The supporting tissues}

79. Intercellular substance. - In the bodies of the higher animals certain tissues show an enormous development of the so-called intercellular substance, so that the cells, instead of closely adjoining each other, are imbedded in a mass of noncellular material which may vary greatly in consistency. Sometimes this intercellular substance is entirely homogeneous but it usually contains a greater or less number of fibers imbedded in a homogeneous basis. By virtue of the special properties of the intercellular substance, tissues of this sort perform primarily mechanical functions, maintaining the form of the body or serving to connect and support other tissues, while the cells themselves serve principally to produce and maintain the intercellular substance. The organic basis of the latter is the group 
of proteins called in Chapter I the albuminoids or the scleroproteins $(51 e)$ accompanied by varying amounts of mineral matter, and, of course, a considerable proportion of water.

80. Bone. - Bone is the most familiar example of the supporting tissues of the animal body. In the young embryo the bones first appear as cartilaginous structures consisting of rounded cells imbedded in a homogeneous intercellular substance containing also fibers and consisting mainly of collagen (51 e). As development advances, the process of ossification begins, the homogeneous substance of the cartilage taking up inorganic salts, chiefly calcium phosphate, while the fibers of the cartilage are stated not to take part in this process. In addition to mineral matter, the bones store up also a variable amount of fat. Mature bone, therefore, aside from its fat, consists of a basis of organic matter largely impregnated with mineral matter. The presence of these two classes of constituents is readily demonstrated by the familiar experiments in which, on the one hand, the mineral matter is removed by immersion in dilute acid leaving behind the flexible cartilage, or, on the other hand, the organic basis of the bone is destroyed by heating, leaving the so-called bone ash.

Ossification has not been completed at birth but continues to a greater or less extent up to full maturity Moreover, it is not carried to the same extent in all bones nor in different parts of the same bone. Consequently, both the percentages of ash and of fat and the proportion of water to dry matter in bones may vary within wide limits, so that it is impossible to state an average composition. The extremes of $\mathrm{I} 5$ per cent and 44 per cent have been found for the average water content of the entire skeleton of the dog and even wider variations have been reported in the case of man. Compact bones contain less water than more spongy ones.

In general it may be said that from one-half to two-thirds of the dry, fat-free bone consists of ash. About three-fourths of the remainder is stated to consist essentially of albuminoids, or collagens, yielding gelatin when treated with hot water, especially under pressure. It is evident, therefore, that the skeleton of an animal contains not only a large share of the total ash of the body but a not inconsiderable portion of its nitrogenous constituents as well. On the average of the ten animals analyzed by Lawes and Gilbert (97), 77.78 per cent of 
the total ash of the entire animal and 83.0I per cent of the ash of the carcass was contained in the bones. Of the total nitrogen of the carcasses of eight of these animals I8.04 per cent was contained in the bones. Corresponding data for the entire animal are not recorded.

81. Bone ash. - But while the composition of bone itself is quite variable, that of the bone ash is notably constant even in different species. The predominant ingredient is tri-calcic phosphate but it contains, also, calcium carbonate as well as phosphates and carbonates of magnesium and other bases. The average composition given by Zalesky $^{\mathbf{1}}$ is as follows : -

Table 5. - Composition of Bone Ash of Different Species

\begin{tabular}{|c|c|c|c|c|}
\hline & MAN & Cattle & Guinea Pig & TurtLe \\
\hline & $\%$ & $\%$ & $\%$ & $\%$ \\
\hline Calcium phosphate $\cdot$. & 83.89 & 86.09 & $87 \cdot 32$ & 85.98 \\
\hline Magnesium phosphate.. & I.O4 & 1.02 & 1.05 & I. 36 \\
\hline $\begin{array}{l}\text { Calcium combined with } \mathrm{CO}_{2} \text {, } \\
\mathrm{Cl}, \mathrm{Fl}\end{array}$ & 7.65 & $7 \cdot 36$ & 7.03 & 6.32 \\
\hline 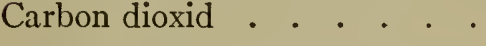 & $5 \cdot 73$ & 6.20 & - & 5.27 \\
\hline
\end{tabular}

More detailed analyses by Gabriel $^{2}$ yielded the following results :

Table 6. - Composition of Bone Ash

\begin{tabular}{|c|c|c|c|c|c|c|c|c|c|c|}
\hline & & & & & & & $\begin{array}{c}\text { Teeth of } \\
\text { Cattle }\end{array}$ & $\begin{array}{l}\text { BONES OF } \\
\text { MAN }\end{array}$ & $\begin{array}{c}\text { Bones of } \\
\text { CATtLE }\end{array}$ & $\begin{array}{c}\text { BONES of } \\
\text { GEESE }\end{array}$ \\
\hline & & & & & & & $\%$ & $\%$ & $\%$ & $\%$ \\
\hline $\mathrm{CaO}$ & . & . & $\ddots$ & . & . & . & 50.76 & $5^{I} \cdot 3 \mathrm{I}$ & 51.28 & 5I.OI \\
\hline $\mathrm{MgO}$ & . & . & . & . & . & . & I. 52 & 0.77 & I.05 & I. 27 \\
\hline $\mathrm{K}_{2} \mathrm{O}$ & . & . & . & . & . & . & 0.20 & $0.3^{2}$ & 0.18 & 0.19 \\
\hline $\mathrm{Na}_{2} \mathrm{O}$ & . & . & . & . & . & . & I.I 6 & I.04 & 1.09 & I.I I \\
\hline $\mathrm{H}_{2} \mathrm{O}$ & . & . & . & . & . & . & $2.2 \mathrm{I}$ & 2.46 & 2.33 & 3.05 \\
\hline $\mathrm{P}_{2} \mathrm{O}_{5}$ & . & . & . & . & . & . & 38.88 & 36.65 & 37.46 & $3^{8.19}$ \\
\hline $\mathrm{CO}_{2}$ & . & . & . & . & . & . & 4.09 & 5.86 & 5.06 & 4. I I \\
\hline \multirow[t]{2}{*}{$\mathrm{Cl}$. } & \multirow[t]{2}{*}{. } & \multirow[t]{2}{*}{. } & \multirow[t]{2}{*}{. } & \multirow[t]{2}{*}{. } & \multirow[t]{2}{*}{. } & \multirow[t]{2}{*}{$\cdot$} & 0.05 & 0.01 & 0.04 & 0.06 \\
\hline & & & & & & & 98.87 & 98.43 & 98.49 & 98.99 \\
\hline
\end{tabular}

1 Neumeister; Lehrbuch der Physiologischen Chemie, I897, p. 456.

${ }^{2}$ Ztschr. Physiol. Chem., 18 (I 894), 257. 
The small amounts of magnesium, sodium, potassium, carbon dioxid and chlorin appear to be as essential ingredients of bone ash as its calcium or phosphorus.

82. Cartilage, ligament, tendon, elastic tissue. - Not all of the cartilaginous ground work of the skeleton as laid down in the embryo is converted into bone. In particular, the end surfaces of bones at a joint consist of cartilage, which in other cases forms a connecting link between adjoining bones, as, for example, the cartilage connecting the ribs with the breast bone, thus allowing a limited degree of motion. At the joints proper, the bones are held in place, and the direction and extent of their motions limited, by the ligaments, while the muscles which serve to impart motion to the various parts of the body are attached to the bones by means of tendons. In many cases the intercellular substance of the supporting tissue contains fibers of elastin. When these fibers are abundant the tissue is elastic in contrast to the ligaments and tendons of the joints, which are almost inextensible. A striking instance is afforded by the elastic band (Ligamentum nuche) which runs along the back of the neck of quadrupeds and supports the weight of the head. Another example is furnished by the layer of elastic tissue contained in the walls of the arteries which gives them a certain degree of resilience to the pressure of the blood pumped by the heart.

The "organic" portion of all these forms of supporting tissue, like the organic portion of the bones, consists essentially of different proteins belonging to the group of albuminoids.

83. Connective tissue. - This name is sometimes applied to all the various forms of supporting tissue, since they also serve to connect the various organs of the body. In a more ordinary and limited sense, however, it is used to designate a form of supporting tissue of which the most familiar example is the tissue lying between the skin and the underlying muscles, or lean meat, and serving to connect them together. A more careful examination shows that this subcutaneous connective tissue is continuous with other similar tissue which extends between the single muscles and serves at the same time to delimit them and connect them. Not only so, but this sheath of connective tissue extends into the muscle itself, dividing it into muscular bundles or fasciculi and these again into secondary 
fasciculi. The connective tissue of the interior of the muscle unites at the ends and is continuous with a form of connective

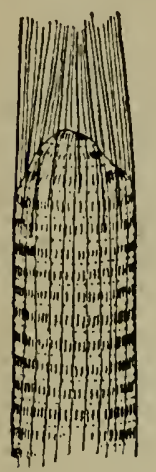

Fig. 2. One end of a muscle fiber. (Hough and S e d g wi ck, The Human Mechanism.) tissue already mentioned, viz., the tendons, by means of which the muscles are attached to the bones (Fig. 2).

A similar sheath of connective tissue surrounds the internal organs of the body and extends into them, forming a framework which supports the active tissues of these organs as well as the blood vessels, nerves, lymphatics, etc., so that it may be said in a broad general way that the body of a higher animal consists of a variety of active tissues and organs contained in and supported by connective tissue and the other forms of supporting tissue already described.

Like other forms of supporting tissue, the connective tissue consists of cells which have produced a relatively large amount of intercellular substance, which in connective tissue consists chiefly of fibers. Chemically, it is composed of collagen. Cells of connective tissue, however, may also store up within themselves large amounts of fat (94).

\section{Tissues of motion}

84. The muscles. - Both the external movements of an animal and those of the internal organs are effected by means of the muscles, and the muscular tissue is preëminently the tissue of motion. Moreover, the muscles make up a large part of the entire mass of the body of a lean animal and furnish nearly all the protein contained in the edible portion of the carcass. The composition of muscle and muscular tissue, therefore, is of special interest.

85. Structure of muscles. - The smallest anatomical element of muscular tissue is the single muscle fiber. This is a highly specialized and greatly elongated, thread-like cell one to one and a half inches long and having a diameter of from .0004 to .004 inch. It is enclosed in a very thin transparent membrane and contains many nuclei. A large number of these fibers - hundreds or even thousands - are bound together to form a fasciculus, the fibers running lengthwise and overlapping each other, being generally shorter than the 
fasciculus. These fasciculi, as stated in a previous paragraph (83), are surrounded by connective tissue and united into larger fasciculi, or bundles, each with its envelope of connective tissue, these bundles again being united to form the individual muscles. The connective tissue serves also to carry the blood vessels, nerves and lymphatics with which the muscle is abundantly supplied, and, moreover, may contain larger or smaller accumulations of fat. Evidently, then, the muscle as a whole, and even more the collective muscles making up the lean meat of an animal, are far from constituting a homogeneous material.

86. Composition of muscles. - If the term muscular tissue be limited to the ultimate muscular fibers which are the active agents in producing motion, considering the other structural elements of the muscle as accessory, it may probably be said in a general way that it consists essentially of water, protein, meat extractives and the various lipoids and electrolytes found in greater or less amounts in all protoplasm. But such a limitation of the term muscular tissue, however rational from an anatomical standpoint, is little suited to the present purpose. In the nutrition of the animal, material is required to build up the entire muscular system, with all

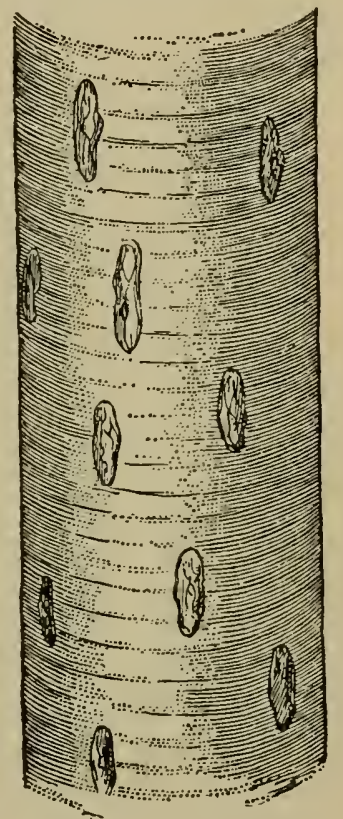

FIG. 3. - Part of a muscle fiber. (Hough and Sedgwick, The Human Mechanism.) its accessory structures, and not merely for the production of the muscle fibers, and we are concerned, therefore, with the composition of the muscles as a whole - i.e., of the lean meat rather than with that of the ultimate muscle-fibers.

Since, however, the lean meat contains a variety of tissues aside from muscular tissue in the narrower sense - connective tissue, nerves, blood and lymph vessels, etc. (85), with more or less of the fluid contents of the latter - it is evident that its composition is likely to be variable. Moreover, the lean meat, especially of fat animals, contains a considerable and variable amount of fat even after all the fat tissue which it is practicable to separate mechanically has been removed. This fat, however, forms no part of the muscle proper but is simply a deposit 
of reserve material. It is contained in minute masses of adipose tissue (94) developed between the muscle bundles or even between the individual muscular fibers and differing only in size from the larger masses which may be trimmed off or removed with the scalpel. It is necessary to distinguish, therefore, between lean meat in the commercial sense, with its varying content of fat, and lean meat in the stricter scientific sense,

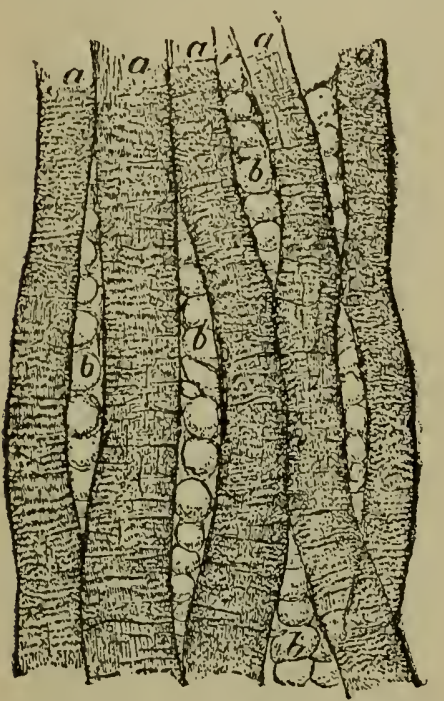

FIG. 4. - Fat cells in muscle. (Bailey's Cyclopedia of American Agriculture.)

meat of the entire carcasses of four steers. i.e., the fat-free muscle. The composition of the latter may be ascertained either by actually removing the fat from the ordinary trimmed meat by means of a.solvent and analyzing the residue or, more conveniently, by analyzing the fresh meat and removing the fat arithmetically, i.e., by calculating the composition of the fat-free muscle.

87. Composition of fat-free muscle. - The composition of the fat-free lean meat of butchers' cuts has been determined by Henneberg, Kern and Wattenberg ${ }^{1}$ for two old sheep and six younger ones ranging from $6 \frac{1}{2}$ to 28 months old, and Jordan ${ }^{2}$ has determined the composition of the lean

Table 7. - Average Composition of Fat-free Lean Meat of Sheep

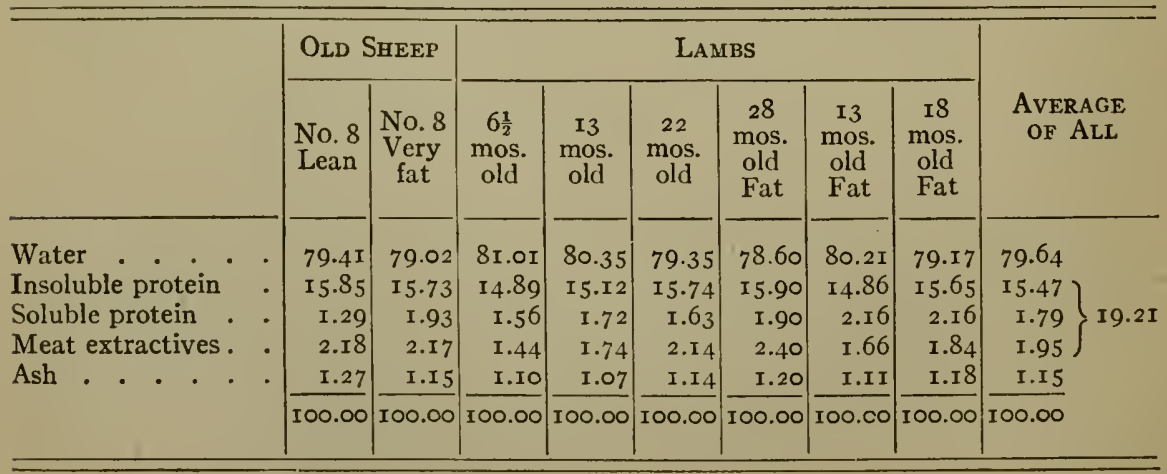

1 Jour. Landw., 26 (I878), 549; 28 (I881), 289.

2 Maine Expt. Sta. Rpt. I 895 , II, 36. 
Tabile 8. - Average Composition of Fat-free lean Meat of Steers

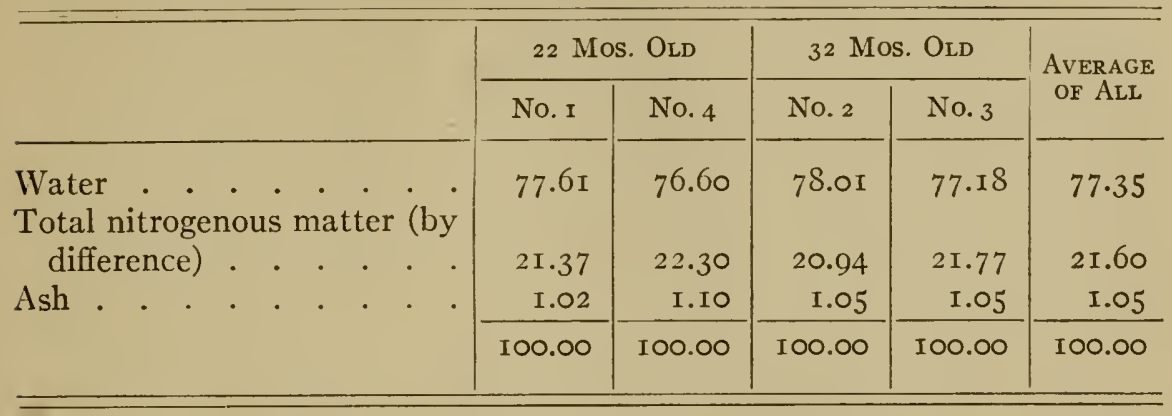

The figures of the foregoing tables indicate but very slight differences in the composition of the fat-free lean meat of the different animals, aside from a slightly greater water content in that of the sheep. An approximate average is 95 per cent total nitrogenous matter and 5 per cent ash in the dry, fat-free substance.

In the course of investigations upon human nutrition, numerous analyses have been made of the various commercial cuts of meat which in general confirm the foregoing figures and show relatively small differences in this respect between the different cuts.

88. Elementary composition of fat-free meat. - The following analyses by Rubner, Stohmann and Langbein, and Argutinsky show the ultimate composition of ash-free muscular tissue after prolonged extraction with ether:-

Table 9. - Composition of Fat- and Ash-free Muscular Tissue

\begin{tabular}{|c|c|c|c|c|c|}
\hline & $\begin{array}{c}\text { Carbon } \\
\%\end{array}$ & $\begin{array}{c}\text { HYDRO- } \\
\text { GEN } \\
\%\end{array}$ & $\begin{array}{c}\text { NITRO- } \\
\text { GEN } \\
\%\end{array}$ & $\begin{array}{c}\text { OXYGEN } \\
\%\end{array}$ & $\begin{array}{c}\text { HEAT OF } \\
\text { COMBUSTION } \\
\text { PER GRAM. } \\
\text { CALS. }\end{array}$ \\
\hline Rubner . . . . & 53.40 & & 16.30 & & $5.656 \mathrm{I}$ \\
\hline Stohmann and Langbein . & 52.02 & $7 \cdot 30$ & I6.36 & $24 \cdot 32$ & 5.6409 \\
\hline Argutinsky . . . . . . & 52.33 & $7 \cdot 30$ & I6. 15 & 24.22 & \\
\hline
\end{tabular}

Köhler ${ }^{1}$ has investigated the elementary composition of the muscular tissue of cattle, sheep, swine, horses, rabbits and hens. The material was prepared with much care, the fat being

${ }^{1}$ Ztschr. Physiol. Chem., 31 (IgoI), 479. 
removed as fully as possible by prolonged extraction with ether. The residual fat which cannot be removed in this way was determined by Dornmeyer's digestion method and a corresponding correction made in the analytical results. The following are his averages for the fat- and ash-free substance:--

Table io. - Composition of Fat- and Ash-free Lean Meat

\begin{tabular}{|c|c|c|c|c|c|c|c|c|c|c|}
\hline & & & & $\begin{array}{l}\text { No. } \\
\text { OF SAM- } \\
\text { PLES }\end{array}$ & $\begin{array}{c}\text { CaRBoN } \\
\%\end{array}$ & $\begin{array}{c}\text { HYDRO- } \\
\text { GEN } \\
\%\end{array}$ & $\begin{array}{c}\text { Nitro- } \\
\text { GEN } \\
\%\end{array}$ & $\begin{array}{c}\text { SULPHUR } \\
\%\end{array}$ & $\begin{array}{c}\text { OxyGen } \\
\%\end{array}$ & $\begin{array}{c}\text { HEAT OF } \\
\text { COMBUS- } \\
\text { TION PER } \\
\text { GRAM. } \\
\text { CALS. }\end{array}$ \\
\hline Cattle. & - & - & . & 4 & 52.54 & 7.14 & I 6.67 & 0.52 & 23.12 & 5.6776 \\
\hline Sheep . & . & • & . & 2 & $5^{2} .53$ & 7.19 & I6.64 & 0.69 & 22.96 & $5.6_{3} 87$ \\
\hline Swine . & . & • & . & 2 & $52.7 \mathrm{I}$ & 7.17 & I6.60 & 0.59 & 22.95 & 5.6758 \\
\hline Horse . & . & . & . & 3 & 52.64 & 7.10 & I 5.55 & 0.64 & 24.08 & $5 \cdot 5990$ \\
\hline Rabbit & - & - & . & 2 & 52.83 & 7.10 & I6.90 & 一 & - & 5.6166 \\
\hline Hen & • & . & . & 2 & 52.36 & 6.99 & I6.88 & 0.50 & 23.28 & 5.6173 \\
\hline
\end{tabular}

All the samples were tested for glycogen, but only traces were found, except in the horseflesh, for the two samples of which an average of 3.65 per cent was obtained, a result which accounts for the low figure for nitrogen.

\section{The tissues of alimentation}

89. Definition. - Under this heading may be grouped the organs and tissues directly concerned with supplying food to the organism, with its distribution through the body, and with the removal of waste products of cell activity. That is, it includes the organs of digestion, resorption, circulation, respiration and excretion, which constitute what are ordinarily spoken of as the entrails of slaughtered animals. So far as most of the familiar internal organs of the animal are concerned they may be considered as made up to a large extent of the classes of tissues already considered. In addition, however, the internal organs include a somewhat distinct type of tissue, viz., glandular tissue, which plays an especially important part in the digestive processes, while it is also of the highest significance for other bodily functions. 
Glands, like many other organs, have as their basis a rather loose and soft framework of connective tissue serving to support cells whose function it is to prepare certain fluids or chemical substances required in the body. The largest gland is the liver, which secretes the bile and has other important functions. Other examples are the pancreas, spleen, salivary glands, etc. Less conspicuous but equally important are the smaller glands imbedded in the walls of the stomach and intestines which secrete such important fluids as the gastric juice, intestinal juices, etc.

90. Chemical composition. - From the standpoint of human nutrition, the tissues of alimentation of farm animals, as here broadly defined, are largely waste products. While certain organs, like the liver, kidneys, heart, etc., are utilized as food, the larger portion of the entrails passes into the offal and the feed consumed in its growth and maintenance is a part of the necessary cost of production of animal foods.

An idea of the composition of the offal and of the proportion of total protein, fat and ash of the body which it contains is afforded by Lawes and Gilbert's analyses of entire animals (97), although the offal in their experiments included, in the case of cattle and sheep (but not of pigs), the head, feet and skin, while the kidneys and kidney fat were in all cases included in the carcass. On the average of the ten animals the percentage composition of the carcass and of the offal was:-

Table il. - Composition of Carcass and Offal

\begin{tabular}{|c|c|c|c|c|c|c|c|c|c|c|c|c|}
\hline & & & & & & & & & & & $\begin{array}{c}\text { Carcass } \\
\%\end{array}$ & $\begin{array}{c}\text { Offal } \\
\%\end{array}$ \\
\hline \multicolumn{13}{|l|}{ In the fresh state } \\
\hline Water . . . & & . & • & . & . & . & . & . & - & . $\quad$. & 48.4 & $5^{8.8}$ \\
\hline Ash . . . . . & . & . & . & . & . & . & - & . & . & .. & $3 \cdot 7$ & 3.0 \\
\hline Nitrogenous matter & . & . & . & . & - & - & - & . & . & .. & I $3 \cdot 5$ & I 7.2 \\
\hline \multirow{2}{*}{\multicolumn{2}{|c|}{ Fat . . . . }} & & \multirow[t]{2}{*}{. } & \multirow[t]{2}{*}{ - } & \multirow[t]{2}{*}{. } & \multirow[t]{2}{*}{. } & \multirow[t]{2}{*}{. } & \multirow[t]{2}{*}{. } & \multirow{2}{*}{\multicolumn{2}{|c|}{$\cdot \quad \cdot$}} & $34 \cdot 4$ & 21.0 \\
\hline & & & & & & & & & & & 100.0 & 100.0 \\
\hline \multicolumn{13}{|l|}{ In the fat-free dry matter } \\
\hline Ash . . . . . & . & . & . & . & . & . & . & . & . & & 21.5 & 14.9 \\
\hline \multirow[t]{2}{*}{ Nitrogenous matter } & \multirow[t]{2}{*}{ • } & \multirow[t]{2}{*}{. } & \multirow[t]{2}{*}{$\cdot$} & \multirow[t]{2}{*}{. } & \multirow[t]{2}{*}{$\cdot$} & \multirow[t]{2}{*}{$\cdot$} & \multirow[t]{2}{*}{$\cdot$} & \multirow[t]{2}{*}{. } & \multirow[t]{2}{*}{ - } & & 78.5 & 85.1 \\
\hline & & & & & & & & & & & 100.0 & I00.0 \\
\hline
\end{tabular}


Table i2. - Percentage Distribution of Ash, Protein and Fat Between Carcass and Offal

\begin{tabular}{|c|c|c|c|c|c|c|c|c|c|c|c|c|}
\hline & & & • & & & & & & & AsH & $\begin{array}{c}\text { PROTEIN } \\
(\mathrm{N} \times 6.25)\end{array}$ & FAT \\
\hline \multicolumn{13}{|l|}{ Fat calf } \\
\hline In carcass & - & . & . & - & - & • & - & - & . . & 73.2 & $65 \cdot 9$ & 70.5 \\
\hline \multirow[t]{2}{*}{ In offal } & . & . & . & . & . & . & . & . & . . & 26.8 & $34 . \mathrm{I}$ & 29.5 \\
\hline & & & & & & & & & & $\overline{100.0}$ & $\overline{100.0}$ & 100.0 \\
\hline \multicolumn{13}{|l|}{ Half-fat ox } \\
\hline In carcass & . & . & . & . & . & . & · & . & . . & $77 \cdot 3$ & 66.8 & 78. I \\
\hline \multirow{2}{*}{ In offal } & . & . & . & . & . & - & . & . & . . & 22.7 & 33.2 & 21.9 \\
\hline & & & & & & & & & & 100.0 & 100.0 & $\overline{100.0}$ \\
\hline \multicolumn{13}{|l|}{ Fat ox } \\
\hline In carcass & . & . & . & . & . & . & . & . & . . & 77.0 & 67.2 & 77.0 \\
\hline \multirow{2}{*}{ In offal } & - & . & . & . & . & . & . & . & $\cdot \quad$. & 23.0 & 32.8 & 23.0 \\
\hline & & & & & & & & & & $\overline{100.0}$ & $\overline{100.0}$ & $\overline{100.0}$ \\
\hline \multicolumn{13}{|l|}{ Fat lamb } \\
\hline In carcass & . & - & - & - & - & • & - & - & $\cdot$ & 74.0 & 52.1 & $78 . \mathbf{I}$ \\
\hline \multirow{2}{*}{ In offal } & . & . & . & . & . & - & - & . & . . & 26.0 & 47.9 & 21.9 \\
\hline & & & & & & & & & & $\overline{100.0}$ & 100.0 & 100.0 \\
\hline \multicolumn{13}{|l|}{ Store sheep } \\
\hline In carcass & . & . & . & - & - & • & · & - & . . & $73 \cdot 5$ & 52.8 & 67.2 \\
\hline \multirow{2}{*}{ In offal } & . & . & . & . & . & . & . & . & .. & 26.5 & 47.2 & 32.8 \\
\hline & & & & & & & & & & 100.0 & 100.0 & 100.0 \\
\hline \multicolumn{13}{|c|}{ Half-fat old sheep } \\
\hline In carcass & . & . & . & - & - & - & • & • & . $\quad \cdot$ & 69.8 & $54 \cdot 3$ & 72.0 \\
\hline \multirow[t]{2}{*}{ In offal } & . & . & . & $\therefore$ & . & . & . & . & $\cdot$ & 30.2 & $45 \cdot 7$ & 28.0 \\
\hline & & & & & & & & & & 100.0 & 100.0 & 100.0 \\
\hline \multicolumn{13}{|l|}{ Fat sheep } \\
\hline In carcass & . & . & . & . & . & . & . & . & . . & 70.5 & 52.5 & $73 \cdot 5$ \\
\hline \multirow[t]{2}{*}{ In offal } & · & . & . & . & . & . & . & $\cdot$ & $\cdot$. & 29.5 & 47.5 & 26.5 \\
\hline & & & & & & & & & & 100.0 & 100.0 & 100.0 \\
\hline \multicolumn{13}{|l|}{ Extra-fat sheep } \\
\hline In carcass & . & . & . & - & . & . & • & • & . . & 60.2 . & 50.0 & $75 \cdot 9$ \\
\hline \multirow[t]{2}{*}{ In offal } & . & • & . & . & . & . & . & . & . . & $39.8^{\circ}$ & 50.0 & $24 . I$ \\
\hline & & & & & & & & & & 100.0 & 100.0 & 100.0 \\
\hline \multicolumn{13}{|l|}{ Store pig } \\
\hline In carcass & . & . & . & . & . & . & . & . & . & 64.0 & 69.4 & 80.3 \\
\hline \multirow[t]{2}{*}{ In offal } & . & . & . & . & . & . & . & . & . & 36.0 & 30.6 & I9.7 \\
\hline \multirow{2}{*}{\multicolumn{13}{|c|}{ Fat pig }} \\
\hline & & & & & & & & & & & & \\
\hline In carcass & . & . & . & . & - & • & · & · & . & 64.4 & 74.0 & $89 \cdot 3$ \\
\hline In offal & $\cdot$ & $\cdot$ & · & • & - & $\cdot$ & $\cdot$ & $\cdot$ & $\cdot \cdot$ & 35.6 & 26.0 & 10.7 \\
\hline Mean of all & & & & & & & & & & 100.0 & 100.0 & 100.0 \\
\hline In carcass & . & . & - & - & • & • & $\cdot$ & • & . & 71.4 & 60.8 & 77.2 \\
\hline In offal & . & . & . & . & . & . & . & 。 & & 28.6 & 39.2 & 22.8 \\
\hline & & & & & & & & & & $\overline{100.0}$ & 100.0 & 100.0 \\
\hline
\end{tabular}


It thus appears that the offal contained relatively more protein and water and less ash and fat than the carcass. From the weights of the carcass and offal, respectively, may be computed the percentage distribution of the ingredients between the two with the results shown in Table I2, from which it appears that on the average 39 per cent of the protein, 28 per cent of the ash and 23 per cent of the fat of the entire animal was contained in the offal.

\section{Epidermal tissues}

91. Functions. - The epidermis, or outer layer of the skin, consists of numerous layers of cells of which those nearer the true skin are alive and capable of multiplication while towards the outer surface they are gradually transformed to flattened, horny scales which serve as a protective layer and gradually slough off. Both the epidermis and the protective covering of animals, - hair, wool, feathers, etc., - as well as the hoofs and horns, corresponding to the nails in man, are modified forms of epidermal tissue, their characteristic ingredients being the class of albuminoids designated as keratins (51e).

92. Composition. - Except for their high and variable sulphur content, the keratins differ little in elementary composition from the simple proteins, but they are much more resistant to chemical reagents, being, for example, insoluble in alkalies in the cold and unattacked by either pepsin or trypsin. These properties fit them well for the outer covering of the animal. The following table shows the elementary composition of some of the more important epidermal tissues:-

Table i3. - Composition of Epidermal Tissues

\begin{tabular}{|c|c|c|c|c|c|c|c|c|}
\hline & & & & $\begin{array}{c}\text { CARBON } \\
\%\end{array}$ & $\begin{array}{c}\text { HYDRO- } \\
\text { GEN } \\
\%\end{array}$ & $\begin{array}{c}\text { NITRO- } \\
\text { GEN } \\
\%\end{array}$ & $\underset{\%}{\text { OxyGEN }}$ & $\underset{\%}{\text { SULPHUR }}$ \\
\hline Epidermis of man & . & . & . & 50.28 & 6.76 & $\mathrm{I} 7.2 \mathrm{I}$ & $25.0 I$ & 0.74 \\
\hline Hair & . & . & . & 50.65 & 6.36 & I 7.I 4 & 20.85 & 5.00 \\
\hline Nails . . . . & • & . & . & $51 . \infty 0$ & 6.94 & $\mathrm{I} 7 \cdot 5 \mathrm{I}$ & $2 \mathrm{I} .75$ & 2.80 \\
\hline Horn of cow . . & . & . & . & 51.03 & 6.80 & I6.24 & $22.5 \mathrm{I}$ & 3.42 \\
\hline Hoof of horse & . & . & . & $5 \mathrm{I} .4 \mathrm{I}$ & 6.96 & $\mathrm{I} 7.46$ & I9.49 & 4.23 \\
\hline Pure dry wool & . & . & . & 49.67 & 7.26 & I6.0I & 23.65 & $3.4 \mathrm{I}$ \\
\hline Pure dry wool & . & . & . & 49.89 & $7 \cdot 36$ & I6.०8 & 23.10 & $3 \cdot 57$ \\
\hline
\end{tabular}




\section{The reserve tissues}

93. Food storage. - The classes of tissue considered in the foregoing paragraphs may be said in a general way to constitute the working machinery of the body. They are composed of cells which either serve the organism through specific activities of their protoplasm, as by producing motion of one sort or another, transmitting stimuli or secreting enzyms or other products, or which, by means of the extraordinary development of their intercellular substance, support and protect the various organs of the body as a whole.

As previously stated, however (77), many cells have the power of storing up surplus food in the form of cell enclosures, especially as fat or glycogen, which apparently constitute no part of the protoplasm itself but which are simply reserve material. This is more or less true of all cells, but certain tissues show this property to a marked degree so that they may properly be spoken of as preëminently the reserve tissues.

94. Adipose tissue. - The most familiar and most important form of reserve tissue is adipose tissue, in which the stored material consists of fat and which constitutes the great store of reserve material in the animal body.

Fat in the form of minute droplets may be deposited in the cytoplasm of all body cells but the presence of more than minute

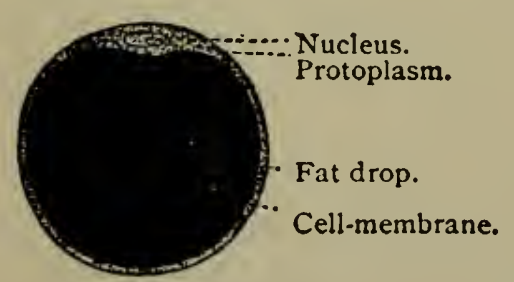

Fig. 5. - Scheme of a fat cell. (Bohm, Davidorf, Huber, Text Book of Histology.) amounts in normal cells of muscles, nerves, glands, etc., is unusual.

It is particularly in certain cells of the connective tissue that the large accumulations of visible fat in the body take place. At the outset these cells present no special characters, but in a well-nourished animal globules of fat begin to accumulate in them, the cells enlarge, the globules of fat coalesce into larger ones and finally the cell substance is reduced to a mere envelope, cytoplasm and nucleus being pushed to one side and almost the whole volume of the cell occupied by fat. Masses of connective tissue thus loaded with fat constitute adipose tissue. 
The increase of adipose tissue, according to Waters and Bell, ${ }^{1}$ takes place in two ways : first by the formation of new cells and second by an increase in the size of existing cells as the storage of fat proceeds. They observed fat cells ranging from $20 \mu$ in diameter in an emaciated animal to about $60 \mu$ in an animal in ordinary thrifty condition and to as much as $200 \mu$ in a very fat animal. The corresponding relative volumes, therefore, are $1: 27: 1000$.

There are two regions in particular in which fat tends to accumulate, viz., in the subcutaneous connective tissue and in the connective tissue surrounding the internal organs, especially that of the mesentery and omentum, although all the looser forms of connective tissue, including, as already noted, the connective tissue lying between and within the muscles, may serve for the storage of fat.

95. Composition of adipose tissue. - What is here called adipose tissue is commonly spoken of as fat, but it is evident that only a portion of it is fat in the strict sense, the remainder consisting of connective tissue, made up of albuminoids, or collagens, together with their accompanying water. It is this nitrogenous material

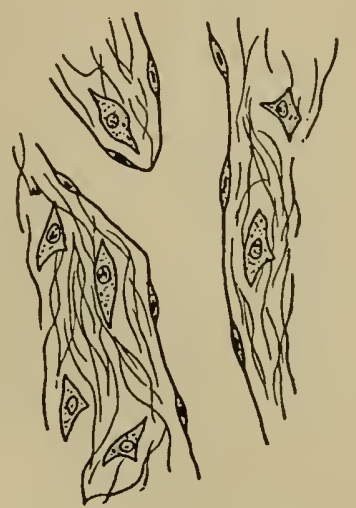

FIG. 6

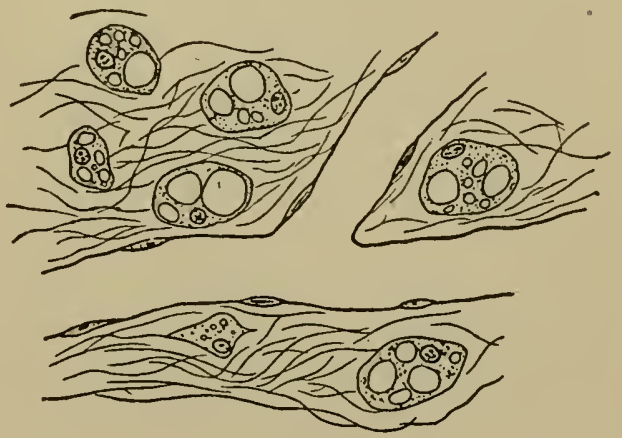

FIG. 7

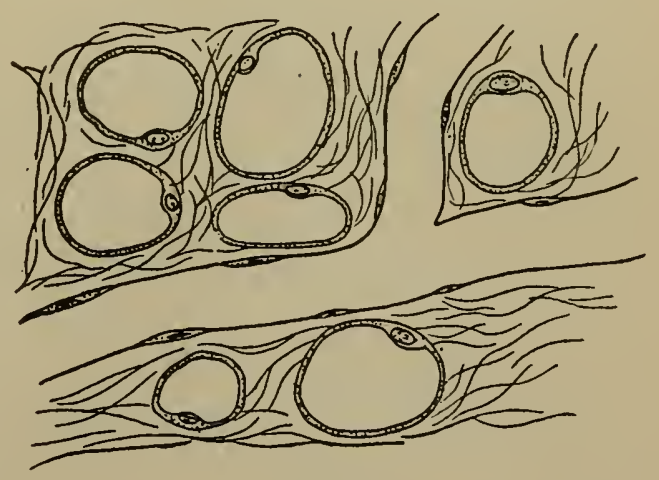

FIG. 8

FIGs. 6-8. - Successive stages in the formation of adipose tissue. (Hough and Sedgwick, The Human Mechanism.)

1 Proceedings, Soc. Prom. Agri. Science, I909, pp. 20-24. 
which forms the "cracklings" when the fat is melted out as in making lard or tallow.

It is evident that the composition of adipose tissue must vary according to the extent to which the deposition of fat in the cells has been carried. When the cells are enlarged and well filled with fat, as in the fattened animal, the percentage of fat will be high and that of protein, water and ash correspondingly low. When there has been little deposition of fat, or when fat previously present has been withdrawn by starvation, the fat content will be low and the percentage of protein, water and ash will be high. The following figures reported by Beythien for the extremes of composition of the adipose tissue of commercial beef, pork and mutton serve to give a general idea of the composition of such deposits in well-fed animals.

Table i4. - Range of Composition of Adipose Tissue of Commercial MEAT

\begin{tabular}{|c|c|c|c|c|c|c|c|c|c|c|c|c|c|c|c|}
\hline & & & & & & & & & & & & & & $\begin{array}{c}\text { MinimuM } \\
\%\end{array}$ & $\begin{array}{c}\text { MaXimuM } \\
\%\end{array}$ \\
\hline Water & . & • & . & - & - & - & - & 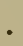 & 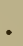 & . & . & . & - & 5.04 & I 8.73 \\
\hline Fat & . & . . & . & . & . & . & . & . & . & . & . & 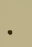 & . & 76.10 & 93.02 \\
\hline Nitroge & nous & $\mathrm{s} \mathrm{ma}$ & tter & & - & . & . & . & . & . & • & . & $\therefore$ & I. 84 & 4.97 \\
\hline Ash . & . . & . . & . & . & . & . & . & . & . & . & . & 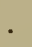 & . & 0.10 & 0.23 \\
\hline
\end{tabular}

As an illustration of the variations in the composition of adipose tissue in different regions of the body the following average figures found by Henneberg, Kern and Wattenberg for the composition of the fat tissues of five lambs may be presented.

Table i 5. - Composition of Adipose Tissue of Different Regions

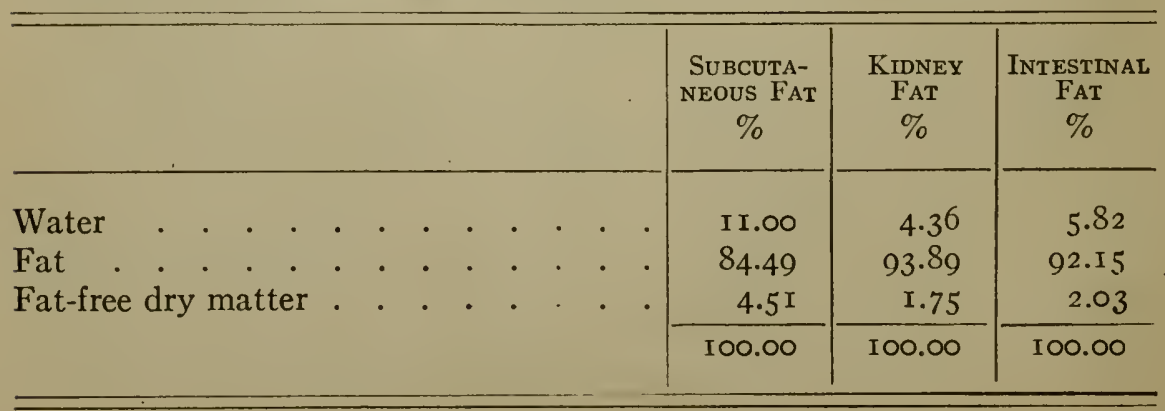


At the other extreme stand the figures reported by Trowbridge ${ }^{1}$ for the composition of the kidney fat of a steer which had received a submaintenance ration for about eleven months and was in a very reduced condition:-

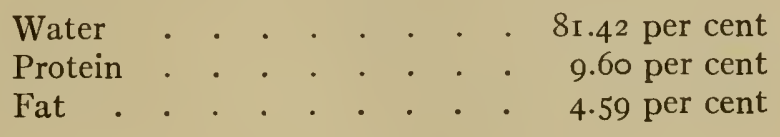

96. Glycogen storage. - In addition to the large accumulations of fat which the body sometimes contains, a much more limited storage of reserve material may occur in the form of the carbohydrate glycogen, especially in the muscles and in the liver.

Neumeister estimates that the liver of the average man may store up approximately 150 grams of glycogen and the muscles and other tissues approximately the same amount, making a total of about 300 grams for the entire body. Estimating the weight of the liver of a 1200 pound steer at 16 pounds and that of the muscles at 800 pounds, and assuming a content of ro per cent of glycogen in the liver and one of 0,4 per cent in the muscles, the total amount of glycogen containèd in the body would be approximately 2200 grams, but naturally this amount would vary greatly at different times according to the conditions of feeding and exercise.

\section{§ 3. The Composition of the Antmal as a Whole}

97. Composition of entire body. - In view of the great number of individual chemical compounds already discovered in the animal body and of the lack of accurate quantitative methods for the determination of many of them, any complete and detailed estimate of the composition of the body as a whole is manifestly impossible. The most that can be done is to determine the proportions of the principal groups of compounds enumerated in Chapter I. Several such investigations have been made at different times. In all of them water and dry matter, as well as the fat content of the latter, have been determined, while sometimes determinations of the total nitrogen

${ }^{1}$ Proc. Amer. Soc. Animal Nutrition, I910, p. I3. 
or of the ash or of both have been added. From such investigations a general idea may be reached of what might be called the gross composition of the body.

\section{Table i6. - Composition of Entire Bodies of Animals - Empty WEIGHT}

\begin{tabular}{|c|c|c|c|c|c|c|c|c|}
\hline & \multirow[b]{2}{*}{$\begin{array}{l}\text { SPE- } \\
\text { CIES }\end{array}$} & \multirow[b]{2}{*}{ AGE } & \multirow[b]{2}{*}{ Condition } & \multicolumn{5}{|c|}{ Percentage Composition } \\
\hline & & & & Ash & $\begin{array}{l}\text { Pro- } \\
\text { tein }\end{array}$ & Fat & $\begin{array}{c}\text { Dry } \\
\text { Mat- } \\
\text { ter }\end{array}$ & Water \\
\hline \multirow{11}{*}{$\begin{array}{l}\text { Lawes and Gilbert } \\
\text { Phil. Trans., Part } \\
\text { II (I859), p. } 493\end{array}$} & \multirow{5}{*}{ Cattle } & 9-ro wks. & Fat & 3.9 & 15.9 & 15.3 & 34.9 & 05.1 \\
\hline & & 4 yrs. & Half-fat & 5.0 & 18.4 & 20.8 & 43.9 & 56.1 \\
\hline & & $4 \mathrm{y}$ & Fat & 4.2 & 15.4 & 32.0 & 51.6 & 48.4 \\
\hline & & 6 mos. & & 3.2 & 13.4 & 31.2 & 47.8 & 52.2 \\
\hline & & t yr. & Store & 3.4 & I 5.8 & I9.9 & 39.0 & 61.0 \\
\hline & \multirow[t]{3}{*}{ Sheep } & $3 \frac{1}{4}$ yrs. & Half-fat & 3.5 & 15.5 & 25.9 & 44.8 & 55.2 \\
\hline & & I $\frac{1}{4}$ yrs. & Fat & 3.0 & 13.0 & 37.8 & 53.8 & 46.2 \\
\hline & & I $\frac{3}{4}$ yrs. & Extra fat & 3.1 & II 1.6 & 48.3 & 62.9 & $37 . \pi$ \\
\hline & \multirow{3}{*}{ Swine } & I I-I 2 mos. & Store & 2.8 & 14.6 & 24.6 & 41.9 & 58.1 \\
\hline & & I I-I 2 mos. & Fat & I. 7 & 11.4 & 43.9 & 57.0 & 43.0 \\
\hline & & $16 \frac{1}{2}$ & - & 2.63 & $12.7 \mathrm{I}$ & 40.56 & $56 . \mathrm{II}$ & 43.89 \\
\hline \multirow{5}{*}{$\begin{array}{l}\text { Soxhlet } \\
\text { Centbl. Agr. Chem., } \\
\quad \text { I0 (J88I), } 674\end{array}$} & \multirow{5}{*}{ Swine } & I9 mos. & - & 2.44 & 12.92 & 35.69 & 51.56 & 48.44 \\
\hline & & rg mos. & - & 2.17 & 10.88 & 44.59 & 58.55 & 41.45 \\
\hline & & 9 mos. & Unfattened & $3.94^{1}$ & $22.76^{2}$ & 19.03 & $45 \cdot 73$ & 54.27 \\
\hline & & 9 mos. & Unfattened & 3.861 & $23.24^{2}$ & 15.80 & 42.90 & 57.10 \\
\hline & & Io mos. & Fattened & $3.2 \mathrm{I}^{1}$ & 19.012 & 24.49 & $46.7 x$ & 53.29 \\
\hline \multirow{6}{*}{$\begin{array}{l}\text { B. Schulze } \\
\text { Landw. Jahrb., I I } \\
\text { (I882), } 57 \text {. . }\end{array}$} & \multirow{6}{*}{ Geese } & Io & Fattened & $3.59^{1}$ & $17.82^{2}$ & 26.78 & 48.19 & $51.8 \mathrm{I}$ \\
\hline & & Io mos. & Fattened & 3.381 & $19.19^{2}$ & 26.82 & 49.39 & $50.6 \mathrm{I}$ \\
\hline & & Io mos. & Fattened & $3.4 \mathrm{I}^{1}$ & 18.782 & 29.22 & $5 \mathrm{I} .4 \mathrm{I}$ & 48.59 \\
\hline & & Io mos. & & $2.99^{1}$ & $18.53^{2}$ & $25 \cdot 36$ & 46.88 & 53.12 \\
\hline & & os. & Fattened & 3.181 & $18.93^{2}$ & 26.21 & 48.32 & 51.68 \\
\hline & & to wks. & Unfattened & $3.5 \mathrm{I}^{3}$ & $14.30^{4}$ & 10.27 & 28.08 & 71.92 \\
\hline \multirow{4}{*}{$\begin{array}{l}\text { Tschirwinsky } \\
\text { Landw. Vers. Stat., } \\
29(1883), 317 . \\
\text { Chaniewski }\end{array}$} & \multirow{4}{*}{ Swine } & & Unfat & $4.14^{3}$ & $15.21^{4}$ & 10.39 & 29.74 & 70.26 \\
\hline & & $28 \mathrm{wks}$. & Fattened & $2.62^{3}$ & II.084 & 40.92 & 54.62 & $45 \cdot 38$ \\
\hline & & 23 wks. & Fattened & $3.90^{3}$ & $11.70^{4}$ & 27.77 & 43.37 & 56.63 \\
\hline & & Mat & $\mathrm{TH}$ & 3.35 & $26.93^{2}$ & 6.65 & 36.85 & 63.15 \\
\hline Ztschr. Biol., 20 & \multirow{4}{*}{ Geese } & $\mathrm{Ma}$ & $\mathrm{Fa}$ & 3.19 & $23.90^{2}$ & 12.68 & 39.67 & 60.33 \\
\hline$(\mathrm{I} 884)$, I 79 & & Mature & Fat & 2.79 & $21.74^{2}$ & 19.89 & 44.36 & 55.64 \\
\hline (Compute & & Mature & Faste & $5 . \mathrm{II}^{1}$ & $21.37^{2}$ & 3.26 & 29.74 & 70.26 \\
\hline live wei & & Mature & Fat & $3.94^{1}$ & 19.981 & 16.01 & 39.93 & 60.07 \\
\hline Jordan & \multirow{4}{*}{ Cattle } & 23 mos. & & $4 \cdot 45$ & 17.381 & 18.80 & 40.63 & 59.37 \\
\hline Maine Expt. Sta., & & 23 mos. & & 5.17 & $17.51^{1}$ & 20.19 & 42.87 & 57.13 \\
\hline Rept. I895, II, 36 & & 33 mos. & & 5.14 & $16.59^{1}$ & 25.18 & $46.9 \mathrm{I}$ & 53.09 \\
\hline \multirow[t]{3}{*}{ (Hides not included) } & & 33 mos. & & 5.24 & $16.73^{1}$ & 24.62 & 46.59 & $53.4 \mathrm{I}$ \\
\hline & \multirow{6}{*}{ Swine } & $\circ$ & & $6.15^{1}$ & I2.19 & I.3I & 19.65 & 80.35 \\
\hline & & $\circ$ & & $6.36^{1}$ & I1.92 & 1.55 & 19.83 & 80.17 \\
\hline Wilson & & ० & & $6.36^{1}$ & I $2.5 \mathrm{I}$ & 1.60 & 20.46 & 79.54 \\
\hline \multirow{3}{*}{$\begin{array}{l}\text { Amer. Jour. Physiol., } \\
8 \text { (1903), I } 97\end{array}$} & & I 6 days & & $3.92^{1}$ & 14.57 & 1.29 & 19.78 & 80.22 \\
\hline & & & & $4.15^{1}$ & 14.78 & 1.43 & 20.36 & 79.64 \\
\hline & & I 6 days & & $4.56^{1}$ & I 4.13 & I. 35 & 20.04 & 79.96 \\
\hline
\end{tabular}

1 By difference.

* By difference in soft parts.
2 Includes feathers.

${ }^{4} \mathrm{By}$ difference in bones. 
The earliest investigation of this sort was that of Lawes and Gilbert ${ }^{1}$ in 1859 , already several times referred to, in which analyses were made of both the carcass and the offal parts of ten animals, viz., a fat calf, a half-fat ox, a moderately fat ox, a fat lamb, a store sheep, a half-fat sheep, a fat sheep, a very fat sheep, a store pig and a fat pig. The determinations made included total dry matter, ash, fat and total nitrogen. Several later investigators have also reported analyses of the entire bodies of animals, including cattle, swine and geese.

Table 16 contains the results of these various investigations up to I903 arranged chronologically. In all cases where the data given permit, the results have been computed upon the "empty" weight of the animals, that is, upon the live weight minus the contents of the digestive tract. On account of this recalculation, the figures for Lawes and Gilbert's results differ somewhat from those usually cited. In all cases where nitrogen was determined the "protein" equals $\mathrm{N} \times 6.25$. In those cases in which nitrogen was not determined the protein is equivalent to fat- and ash-free dry matter.

Haecker, ${ }^{2}$ as the result of analyses of the bodies of sixty wellfed steers, has reported the following average composition at various weights.

\section{Table i7. - Composition of Steers at Various Stages - Empty WEIGHT}

\begin{tabular}{|c|c|c|c|c|c|}
\hline $\begin{array}{c}\text { NoRMAL WEIGHT } \\
\text { LBS. }\end{array}$ & $\begin{array}{l}\text { WATER } \\
\%\end{array}$ & DRY $\underset{\%}{\text { MATTER }}$ & $\begin{array}{c}\text { Protein } \\
\%\end{array}$ & $\begin{array}{c}\mathrm{FAT}_{\mathrm{AT}} \\
\%\end{array}$ & $\underset{\%}{\text { AsH }}$ \\
\hline IOO & 71.85 & 28. I 5 & I 9.90 & 3.99 & 4.26 \\
\hline 200 & 69.47 & 30.53 & x 9.63 & 6.26 & 4.64 \\
\hline 300 & $66.3 \mathrm{I}$ & 33.69 & I9.35 & 9.84 & 4.50 \\
\hline 400 & 65.76 & 34.24 & I 9.31 & $10.5^{6}$ & $4 \cdot 37$ \\
\hline 500 & $62.9 \mathrm{I}$ & 37.09 & I9.I 5 & I 3.73 & $4.2 I$ \\
\hline 600 & $62.2 \mathrm{I}$ & 37.79 & 19.22 & 13.97 & 4.60 \\
\hline 700 & 60.75 & 39.25 & x 8.83 & I 5.9 I & $4.5 \mathrm{I}$ \\
\hline 800 & 57.88 & 42.12 & I8.69 & 19.23 & 4.20 \\
\hline 900 & 54.09 & 45.90 & I 7.66 & 24.08 & 4. 16 \\
\hline 1000 & 53.09 & 46.91 & 17.57 & 25.53 & $3.8 \mathrm{I}$ \\
\hline I I OO & 48.02 & 51.98 & I6. I9 & $31.9 \mathrm{I}$ & 3.88 \\
\hline I 200 & 48.64 & 51.36 & I 5.66 & 31.10 & 3.67 \\
\hline
\end{tabular}

${ }^{1}$ Phil. Trans., Part II, I 859, p. 493.

${ }^{2}$ Amer. Soc. Animal Produc., Proc., I9I 4, p. I 8. 
It appears from the foregoing figures that the most abundant single constituent, although one which is subject to marked variations, is water, its percentage ranging from over 80 in very young pigs to 37 in a very fat sheep. Only in this latter case and two others does the percentage of water fall below that of fat. Relatively, the greatest variations are in the fat, as would be expected, since fat is the reserve material of the

Table i8. - Composition of Fat-free Body - Empty Weight

\section{Cattle}

Lawes and Gilbert . Jordan · ·

\section{Sheep}

Lawes and Gilbert.

$\left|\begin{array}{c}\text { LIVE } \\ \text { WEIGHT } \\ \text { LBS. }\end{array}\right|$ AGE

Lawes and Gilbert.

Lawes and Gilbert. Swine

Wilson

Wilson

Tschirwinsky

Tschirwinsky

Lawes and Gilbert

Soxhlet

Geese

B. Schulze

Chaniewski

\begin{tabular}{|c|c|c|c|}
\hline $\begin{array}{c}\text { AsH } \\
\%\end{array}$ & $\begin{array}{c}\text { Pro- } \\
\text { TEIN } \\
\%\end{array}$ & $\begin{array}{c}\text { DRY } \\
\text { MATTER } \\
\%\end{array}$ & $\begin{array}{c}\text { WATER } \\
\%\end{array}$ \\
\hline & & & \\
\hline 4.60 & I 8.80 & 23.10 & 76.90 \\
\hline 6.45 & 21.92 & 28.37 & 71.63 \\
\hline 6.30 & 23.20 & 29.00 & 71.00 \\
\hline 4.44 & 20.73 & 25.17 & 74.83 \\
\hline 4.95 & 20.94 & 25.89 & 74.I I \\
\hline 4.99 & 21.46 & 26.45 & 73.55 \\
\hline 4.89 & 21.59 & 26.48 & $73.5^{2}$ \\
\hline 4.88 & 22.20 & 27.08 & 72.92 \\
\hline $5 \cdot 35$ & 22.34 & 27.69 & 72.31 \\
\hline $5 \cdot 3^{6}$ & 22.39 & 27.76 & 72.2 \\
\hline 5.20 & 23.14 & 28.34 & 71.6 \\
\hline 5.48 & 23.76 & 28.74 & 71.20 \\
\hline 5.12 & 23.59 & $28.7 \mathrm{I}$ & 71.29 \\
\hline 5.70 & 23.78 & 29.48 & 70.52 \\
\hline $5 \cdot 33$ & 24.08 & $29.4 \mathrm{I}$ & 70.5 \\
\hline 4.60 & I9.60 & 24.10 & 75.90 \\
\hline 5.00 & 21.20 & 25.90 & $74 . \mathrm{I}$ \\
\hline 4.70 & $2 \mathrm{I} .10$ & $25 \cdot 50$ & 74.5 \\
\hline 6.38 & I 2.39 & I 8.77 & 81.23 \\
\hline 4.27 & I 4.69 & I8.96 & 81.0 \\
\hline 4.27 & I6.45 & 20.72 & 79.2 \\
\hline 4.92 & I 7.47 & 22.39 & 77.6 \\
\hline 3.40 & I9.90 & 23.10 & 76.9 \\
\hline 4.04 & 20.37 & $25 \cdot 34$ & 74.6 \\
\hline & 26.06 & 30.60 & $69.4 c$ \\
\hline 4.14 & 25.85 & 29.93 & 70.0 \\
\hline
\end{tabular}


body and may be stored up in large quantities, reaching in one instance 48 per cent, or, on the other hand, may be almost lacking in the insufficiently fed or fasted animal.

98. Composition of fat-free body. - Since the adipose tissue of the animal body represents substantially a storage of reserve material $(93,94)$ temporarily set aside from the physiological activities of the organism, a better idea of the composition of the working machinery of the body is obtained by computing its composition fat-free as in Table 18.

When this is done, it appears that the composition of the fatfree body is much less variable than that of the body as a whole, the chief difference being due to variations in the water content, which in turn depends chiefly upon the age of the animal, as the preceding table shows. So far as can be concluded from these few cases, however, the fat-free bodies of mature cattle would appear to contain three to four per cent less water than those of mature sheep or swine. In the case of geese, the percentage of water is probably low on account of the relatively small amount in the feathers.

99. Composition of fat- and ash-free dry matter. - In some of the foregoing investigations, viz., in Lawes and Gilbert's, Soxhlet's and three of Chaniewski's, the total nitrogen was determined and the protein has been calculated by multiplying by the factor 6.25. These experiments permit a computation of the percentage of nitrogen contained in the fat-free dry matter which in the other experiments has been regarded as protein. For example, in the case of Lawes and Gilbert's fat calf the figures are as follows: -

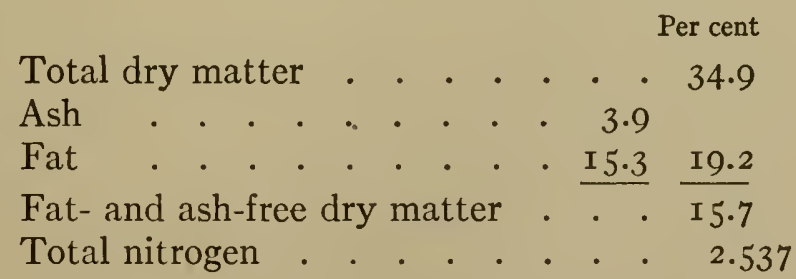

$2.537 \div 15.7=16.16 \%$ nitrogen in ash- and fat-free dry matter.

The results of such a computation for all of the experiments in which the published data permit it are contained in Table I9. 
Table i9. - Nitrogen in Fat- and Ash-free Dry Matter

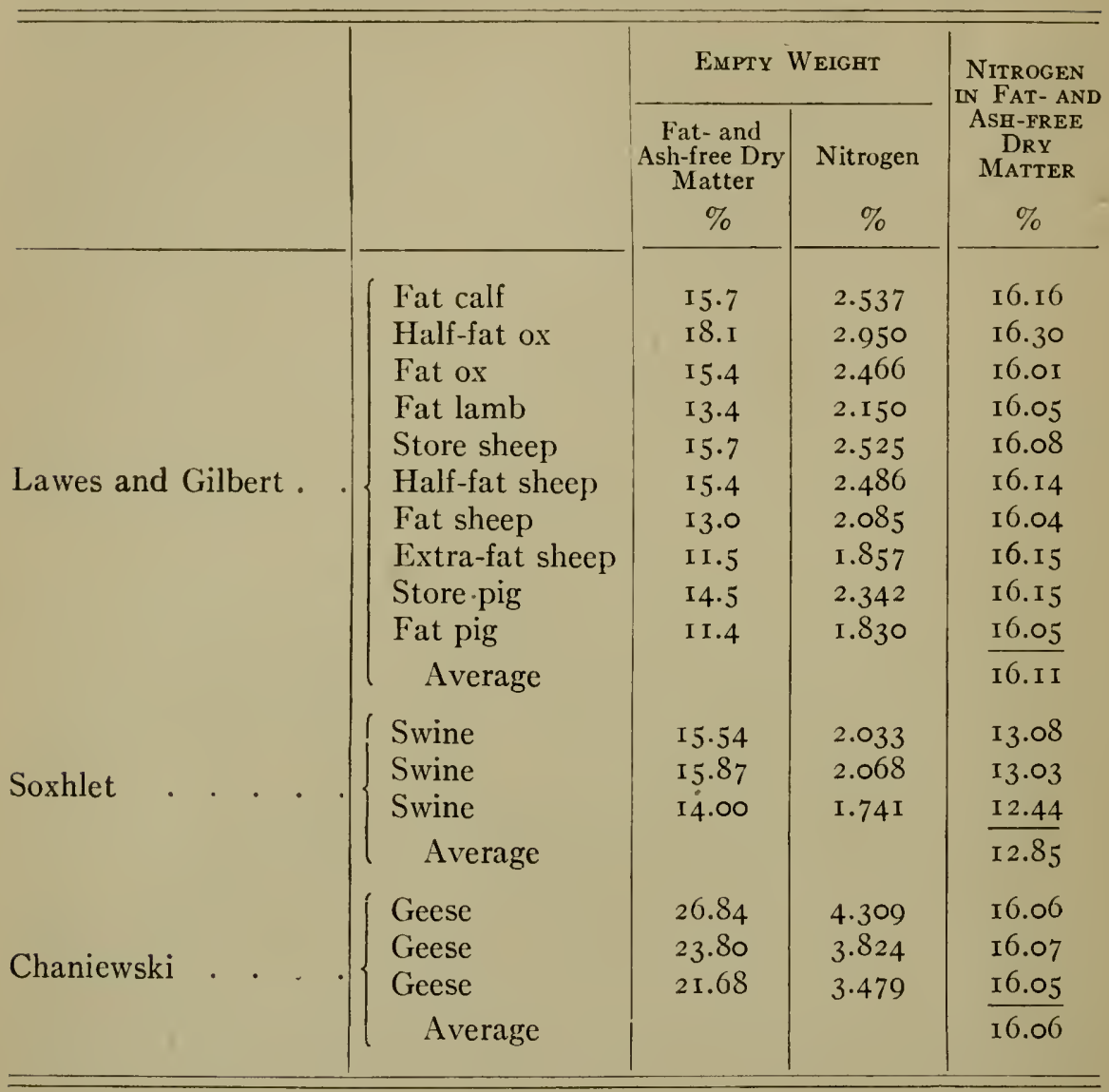

With the exception of Soxhlet's experiments, the percentage of nitrogen approximates closely to that of animal proteins. If account be taken of the fact that the ether-extraction method used in these investigations does not completely remove the fat from dried animal tissue, the conclusion appears justified that the organic matter other than fat contained in the animal body has substantially the composition of protein.

\section{$\S$ 4. The Composition of Feeding Stuffs}

100. Groups of ingredients. - As in the case of the animal body, the vast number of single chemical compounds found in the plant, as well as the lack of accurate quantitative methods for the determination of many of them, renders it practically 
necessary to be content in most cases with a separation of the plant substances into a few major groups or sub-groups of ingredients. As ordinarily carried out, feeding stuffs analysis recognizes seven of these categories, viz., water, ash, protein, non-protein, ether extract, crude fiber and nitrogen-free extract.

101. Water. - The amount of water in a feeding stuff is commonly inferred from the loss of weight which the substance undergoes at a temperature above the boiling point of water. There is also a possibility, however, of a loss of other volatile matter besides water, while, on the other hand, some substances tend to absorb oxygen and thus increase in weight, especially when dried in air at a high temperature. The exact determination of water and dry matter, therefore, is by no means an easy problem, but the results obtained by the ordinary methods are sufficiently exact for almost all purposes related to stock feeding. Commonly, the residue is weighed and regarded as dry matter, the amount of water being obtained by difference.

102. Ash. - In the ordinary feeding stuffs analysis, ash is equivalent to the residue left after complete incineration of the substance in air or oxygen, the process being carried out at as low a temperature as practicable in order to avoid volatilization of part of the alkalies present.

That this method fails entirely to distinguish between those elements which were originally present as electrolytes and those which were in organic combination has already been pointed out (5), as has also the fact that certain elements, notably sulphur and phosphorus, are only partially recovered in the ash by the ordinary method of preparation. As the study of the functions of the ash ingredients progresses, it may be anticipated that we shall come to determine the several elements involved in the way most appropriate to each rather than simply to determine the ash as a whole.

103. Nitrogenous constituents. - As yet no methods exist for the quantitative separation of the nitrogenous constituents from the other ingredients of plants. While much labor has been expended upon a study of the individual proteins of a comparatively few vegetable materials, and while in some instances it is possible to state with approximate accuracy the amounts of the several proteins present, nevertheless the only available methods for the determination of the nitrogenous compounds 
of feeding stuffs in general are indirect ones based upon a determination of their characteristic element nitrogen.

104. Crude protein. - In the method of feeding stuffs analysis inherited from the early investigations of Henneberg and Stohmann, the protein is estimated from the amount of total nitrogen upon two assumptions: first, that all the proteins contain 16 per cent of nitrogen and, second, that all the nitrogen of feeding stuffs exists in the protein form. On the basis of these assumptions, the protein is, of course, equal to total nitrogen multiplied by 6.25 . The protein as thus determined is designated as crude protein to indicate the approximate nature of the determination.

Subsequent investigations by Scheibler, E. Schulze, Kellner and others have shown the presence in many feeding stuffs of relatively large amounts of non-protein nitrogenous compounds, so that it is desirable to distinguish at least between the nitrogen present as true protein and that present in the simpler compounds grouped under the general term non-protein (60-67), and all analyses of feeding stuffs for scientific purposes should at least make this distinction. Logically, too, the term crude protein should be dropped altogether, but when, as in the case of the older analyses, this is impracticable, care should be taken to retain the adjective, reserving the term "protein" for use in the sense given it in the next paragraph.

105. True protein. - As a means of effecting an approximate separation of the true protein from the other nitrogenous compounds present in plants, advantage is taken of the fact that most of the latter class of substances are soluble in water. An aqueous extract of a feeding stuff, therefore, contains by far the larger share of its non-protein. Such an extract, however, contains also any water-soluble proteins existing in the substance. These are removed in part by coagulation by heating, i.e., by boiling the solution, and in part by the addition of some reagent with which they form insoluble compounds. Various substances have been used for this purpose but the present official method of analysis, based upon Stutzer's investigations, uses copper hydrate as the precipitant. In practice, the feeding stuff is boiled with water, the precipitant added and the soluble matter filtered off. The nitrogen of the insoluble residue is regarded as being protein nitrogen and from 
it by multiplication by 6.25 (or some other agreed factor) the amount of protein is calculated.

It is obvious that this method of determining protein is substantially a conventional method and that the adjective true is employed in a somewhat Pickwickian sense. The result probably includes all of the proteins of the feed but may also include other insoluble nitrogenous compounds.

106. Non-protein. - The non-protein in feeding stuffs analysis includes all the nitrogenous compounds which remain in solution when the material is treated in the manner just described for the determination of protein. The nitrogen may be determined in the solution but ordinarily it is obtained by subtracting the protein nitrogen from the total nitrogen. The difference, multiplied by some conventional factor, equals the non-protein. Obviously, the non-protein is a heterogeneous mixture, varying as between different feeding stuffs and even in the same feeding stuff grown or harvested under different conditions.

107. Nitrogen factors. - Evidently the accuracy with which the protein and the non-protein in a feeding stuff are determined depends not only upon the accuracy with which the protein and non-protein nitrogen can be separated and determined but also on the correctness of the factors used for converting nitrogen into protein or non-protein respectively.

For protein the usual factor has been 6.25 as already stated, based upon the assumption of 16 per cent of nitrogen in average protein. As was stated in Chapter I (44), however, different proteins vary in their nitrogen content, and in particular the vegetable proteins run higher in nitrogen than the animal proteins, which is, of course, equivalent to a smaller conversion factor. But while it is easily shown that the present factor is incorrect in many cases, it is not so easy to find a substitute. There is a rather wide range in the nitrogen content of the individual vegetable proteins, while most feeding stuffs contain two or more proteins in unknown proportions. Moreover, the proteins of the majority of feeding stuffs, especially of the roughages, have not yet been separated and studied.

Ritthausen ${ }^{1}$ has suggested the use of the factor 5.7 for the majority of cereal grains and leguminous seeds, 5.5 for the oil 
seeds and for lupines, and 6.0 for barley, maize, buckwheat, soybean, white bean, and rape and other brassicas.

For various classes of human foods, Atwater and Bryant ${ }^{1}$ have proposed the following factors for the computation of protein from protein nitrogen :-

Animal foods

6.25

Wheat, rye, barley and their manufactured products . . . 5.70 Maize, oats, buckwheat and rice, and their manufactured products . . . . . . . . . . . . . . . . . 6.00

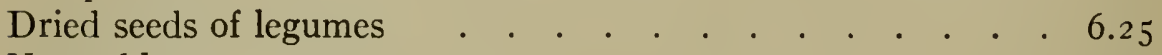
Vegetables . . . . . . . . . . . . . . . 5.65 Fruits

For feeding stuffs whose proteins have not yet been studied, there seems to be no reason for changing from the present usage.

With the non-proteins the case is even more perplexing in view of the greater variety of substances included under this term and the wide range of their nitrogen content. The writer has used tentatively 4.7 , the factor for asparagin (66), one of the most widely distributed substances of this class, but the choice of this factor is substantially arbitrary.

108. Crude fat. Ether extract. - The methods for determining the fat content of feeds are based upon its extraction by means of some solvent which dissolves as little as possible of the other ingredients. A variety of solvents has been used for this purpose, such as carbon disulphid, carbon tetrachlorid, petroleum ether and the like, but the one most commonly employed is ethyl ether, or the so-called "sulphuric" ether commonly used as an anæsthetic.

All the various solvents used, however, remove other substances besides neutral fats and fatty acids, including more or less of the more complex lipoids. In particular the ether extract obtained from coarse fodders contains a variety of waxes, resins, etc., as well as the chlorophyl of the leaves, and a relatively small proportion of true fats. It is customary, therefore, to designate the extracted material as "crude fat" or, since ether is the reagent ordinarily used, as "ether extract."

${ }^{1}$ Storrs (Conn.) Agr. Expt. Sta., Rpt., 12, 79. 
If a different solvent is used, this should be specified in the statement of the analysis.

109. Crude fiber. - The so-called crude fiber of feeding stuffs is determined by boiling them first with dilute acid and then with dilute alkali under strictly defined conditions of concentration and time, and washing the undissolved residue with alcohol and ether. The residue, after deducting the small amount of ash remaining in it, constitutes the crude fiber.

Crude fiber as thus obtained contains most of the cellulose, lignin and cutin of the feeding stuff, along with more or less of the more difficultly soluble hemicelluloses, particularly those containing pentosans. The proportion of pentosans contained in the crude fiber naturally varies according to the nature of the feeding stuff. Tollens, ${ }^{1}$ for example, obtained the following figures for the crude fiber of meadow hay and of brewers' grains :-

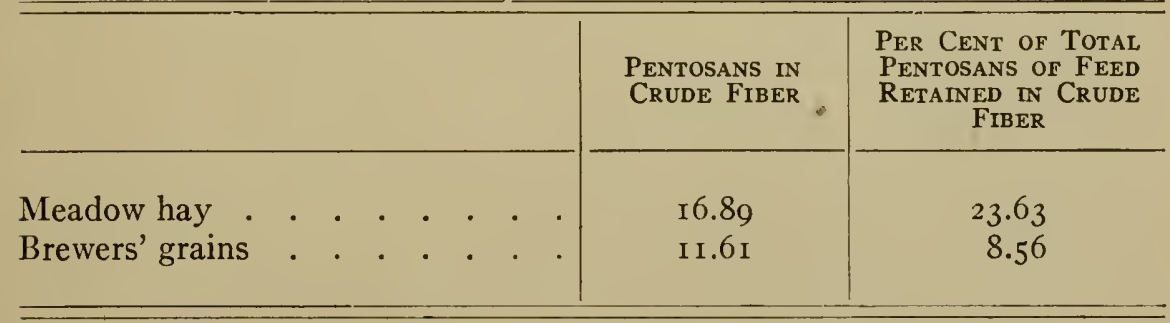

110. Nitrogen-free extract. - All the ingredients of feeding stuffs which are not included in the foregoing six categories, viz., water, ash, protein, non-protein, ether extract and crude fiber, are usually grouped together under the collective name of nitrogen-free extract. The significance of the name is evident. By definition the nitrogen-free extract includes all those nonnitrogenous organic constituents, other than fat, which are extracted from the feeding stuff in the process of determining the crude fiber. The amount of nitrogen-free extract in a feeding stuff is not ascertained by any process of direct determination but simply by subtracting the sum of the other six groups from roo per cent. Such a residual group naturally includes a great variety of substances of very diverse nature, ${ }^{2}$

1 Jour. Landw., 45 (1897), ro3.

2 For an enumeration of the principal ingredients of the nitrogen-free extract, compare Tollens, Jour. Landw., 45 (1897), 295. 
but as a rule the nitrogen-free extract consists to a considerable extent of carbohydrates of one sort or another. Indeed, it has sometimes been designated by the latter name, but the use of the word in this sense is misleading and undesirable.

The nitrogen-free extract includes not only hexose but also pentose carbohydrates, these latter substances being, therefore, by the ordinary method of feeding stuffs analysis, divided between the crude fiber and nitrogen-free extract. Some of these various carbohydrates can be determined separately with a reasonable degree of accuracy, while others, including unfortunately starch, can be determined only more or less approximately. That the nitrogen-free extract is far from consisting exclusively of carbohydrates has been strikingly shown by Stone. $^{1}$ He determined the content of the various classes of carbohydrates in samples of wheat and maize as accurately as possible and found that the sum in both cases was considerably less than the amount of nitrogen-free extract as determined by the conventional method. Much greater differences in this respect have been shown to exist in roughages.

111. Classes of feeding stuffs. - The composition and characteristics of the principal classes of feeding stuffs are considered in Chapter $\mathrm{XV}$, but it seems desirable to anticipate that discussion here to the extent of indicating the three major classes into which the feeding stuffs are commonly divided. This classification is based primarily on botanical characteristics with which, however, are associated corresponding differences in chemical composition.

Concentrates or concentrated feeds. - As the name implies, these are feeding stuffs which contain much nutriment in a. small bulk. They include primarily the grains and other seeds and, secondarily, a wide range of technical by-products derived from them as well as certain by-products of animal origin. Chemically, they are characterized by their relatively low content of crude fiber, ranging from practically zero in certain byproducts to perhaps Io or I 2 per cent in grains having a considerable proportion of hulls, like oats or buckwheat, and in certain by-products.

Coarse fodders or roughage. - Botanically, these consist of the vegetative organs of the plant, $i$. e., substantially of stalks

${ }^{1}$ Jour. Amer. Chem. Soc., 19 (I897), I83. 
and leaves. They include hay, straw and other forms of forage either fresh, ensiled or dried. Chemically, they are characterized by their relatively high percentage of crude fiber, which, however, naturally varies within quite wide limits. As compared with the concentrates they are bulky feeds and contain a larger proportion of difficultly soluble ingredients.

Roots and tubers. - These feeding stuffs contain a large percentage of water, resembling in this respect the fresh or ensiled roughages. Their dry matter, on the other hand, resembles that of the concentrates in containing relatively little crude fiber and a large proportion of ingredients which are easily soluble. They might be briefly characterized as dilute concentrates. 



\section{PART II}

THE PROCESSES OF NUTRITION 



\section{CHAPTER III}

\section{DIGESTION AND RESORPTION}

112. The first step in nutrition. - The facts considered in Part I have served incidentally to show some particulars of those differences between the feed of herbivora and the animal body which it serves to nourish which are, in a general way, familiar to every one. The former contains many ingredients not found in the latter, and it is plain that, for example, substances like starch and cellulose must undergo considerable modification before they can be used in the animal organism. One need not be a chemist, however, to reach this conclusion. A simple comparison of the feeds given farm animals with the products which they manufacture out of them convinces one that profound changes are necessary to convert hay and grain into meat or milk. The first step in this process is the digestion of the feed. In all but the lowest animals, special tissues are set apart for this work, together constituting the organs of digestion, or the alimentary canal with its appendages.

\section{$\S$ I. The Organs of Digestion}

113. General plan. - The process of digestion is seen in its simplest form in unicellular animals like the ameba. When the ameba comes in contact with a particle of feed, a depression forms in its outer surface which finally closes around the particle, forming a cavity which serves as a temporary digestive organ. Undigested residues are rejected by the reverse process. In animals slightly higher in the scale, this temporary cavity becomes a permanent one, the same opening serving for the entrance of feed and the exit of waste. The next step in the evolution is the provision of a separate exit for the waste matter, thus giving the typical form of digestive apparatus, of which that of the higher animals is a development, consisting 
of a cavity or cavities communicating with the external world by two openings, one for the reception of feed and the other for the rejection of waste. In domestic animals, the digestive tract is large and of very complex structure, but in all cases it is built upon the general plan just outlined. Always, from the ameba up to man, the inner surface of the digestive cavity is morphologically simply a continuation of the external surface of the body, turned in as one might a glove finger. Consequently, the material contained in the digestive cavity, strictly speaking, is still outside the body. ${ }^{1}$

Finally, as an essential part of the digestive apparatus, there must be such organs as the cilia, tentacles, proboscis, lips, etc., by which feed is grasped and introduced into the digestive cavity, and likewise means by which it may be mechanically ground to fit it for the process of digestion, as, for example, the teeth of mammals, the bills and gizzards of birds, etc.

For the present purpose, it is unnecessary to enter into any elaborate consideration of the anatomy of the digestive organs, since we are concerned chiefly with the chemical rather than the physical processes of digestion, and this section may be confined to a very general description of the digestive organs of domestic animals. In these animals, the digestive apparatus may be described briefly as a tube having various enlargements, folds and diverticula.

114. Digestive fluids and enzyms. - In the ameba, whatever changes are effected in the substances which it takes as feed are accomplished by the cells of the introverted surface or by their secretions. As the digestive apparatus becomes more complicated, however, a division of cellular labor takes place and certain groups of cells are set apart to produce the digestive juices which act upon the feed. In the higher animals, these cells become the numerous secreting glands which are an essential part of the organs of digestion. The principal active agents in digestion are certain enzyms secreted by these glands, the more important digestive enzyms in the higher animals being:-

I. The amylases, ptyalin (in the saliva) and amylopsin (in the pancreatic juice), acting. upon starch.

1 For a more complete discussion of the development of the digestive apparatus see R. Meade Smith, The Physiology of the Domestic Animals, pp. 20.3-226. 
2. The invertases, sucrase, maltase and lactase (in the intestinal juice), acting upon di-saccharids.

3. The proteases, pepsin (in the gastric juice), trypsin (in the pancreatic juice) and erepsin (in the intestinal juice), acting upon proteins.

4. The lipase, steapsin (in the pancreatic juice), acting upon the lipoids, or specifically the fats.

115. The mouth. - The mouth is the organ of prehension and mastication. Chiefly by means of the tongue, lips and teeth, feed is seized and introduced into the digestive cavity, while the teeth serve also to grind it up, rendering it capable of being swallowed and also exposing more surface to the action of the digestive fluids. The mouth also receives the secretion of three pairs of glands called the salivary glands whose product is known as the saliva. These three pairs are called, respectively, the parotid, the submaxillary and the sublingual glands.

The mixed saliva, consisting of the secretion of all three pairs of salivary glands, together with the comparatively insignificant amounts secreted by the various smaller glands of the mouth, is a thin, colorless, watery, slightly viscid liquid of alkaline reaction. The organic matter of the saliva includes a trace of albumin, more or less mucus, and the enzym ptyalin, ${ }^{1}$ which is its active constituent.

The saliva has both physical and chemical functions. - The presence of feed in the mouth, its taste, odor or sometimes even sight, causes active secretion of saliva, which is mixed with the feed in the act of mastication and moistens and lubricates it so that it can be swallowed. With dry feeding stuffs, the amount of saliva required for this purpose is very large. The total secretion has been estimated at about 84 pounds per day for the horse and at least II 2 pounds per day for the ox, although varying greatly with the dryness of the feed. Besides moistening and lubricating the feed, the saliva has also a chemical action upon it. In a slightly alkaline medium and at body temperature, the ptyalin acts upon the starch of the feed, converting it ultimately into maltose.

116. The stomach. - From the mouth, the feed in the act of swallowing passes through the esophagus, or gullet, to the stomach which, except in fowls, is the first enlargement of the 
alimentary canal. The horse and hog, as well as carnivorous animals like the dog and cat, have a single stomach cavity, while ruminants, such as cattle, sheep and goats, have a socalled compound stomach consisting, in the farm animals, of four divisions, called respectively the rumen, or paunch, the

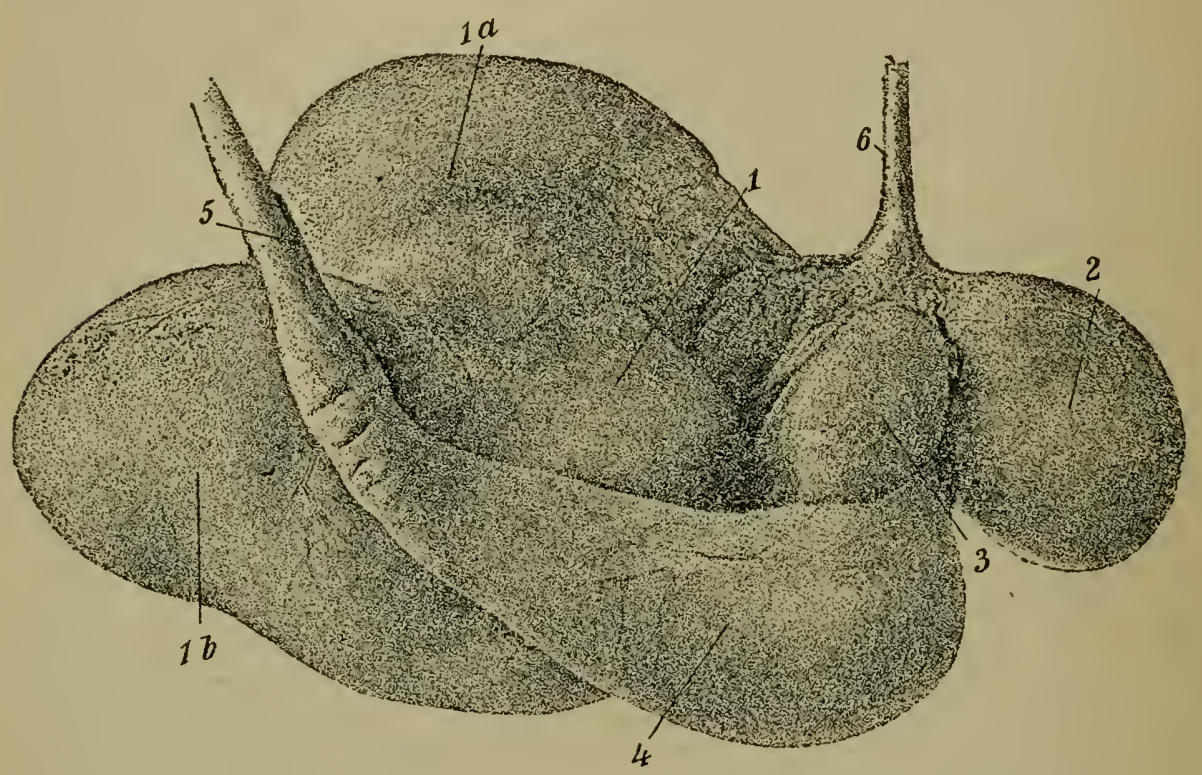

FIG. 9. - Sheep's stomach. (Hagemann, Anatomie und Physiologie der Haus-Säugetiere.)

I, Rumen. 2, Reticulum. 3, Omasum. 4, Abomasum. 5, Duodenum. 6, Esophagus.

reticulum, the omasum, or manifolds, and the abomasum, or true stomach.

In reality the first three divisions of the ruminant stomach are to be regarded as dilatations of the esophagus in which the feed undergoes a softening and fermentation preliminary to true gastric digestion, while only the fourth division is a stomach in the strict sense. In domestic fowls a similar dilatation of the esophagus at the base of the neck constitutes the crop.

Moreover, even the so-called single stomachs of the horse and hog, while they have but a single cavity, are in reality compound stomachs. In the case of the horse three quite distinct parts may be distinguished, viz., the left or cardiac portion, the fundus region and the pyloric region, the two latter having the functions of the true stomach. In the case of the 


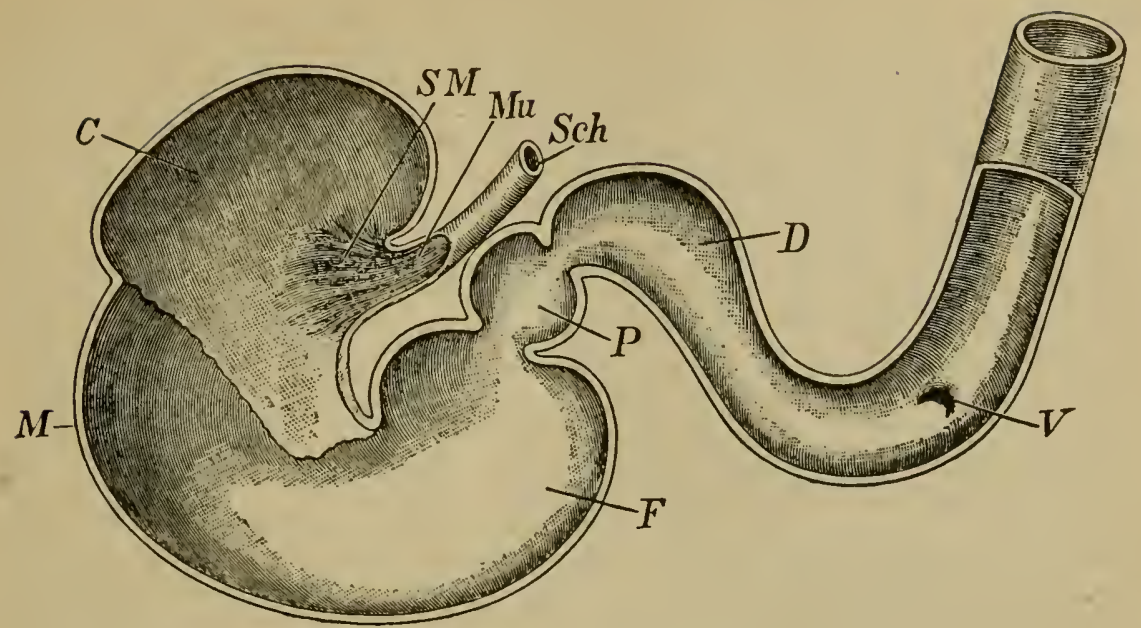

Fig. 10. - Stomach and duodenum of horse. (Hagemann, Anatomie und Physiologie der Haus-Säugetiere.)

Sch., Esophagus. $\quad C$, Cardiac portion. $M$, Fundus. $F$, Pyloric region. $\quad D$, Duodenum.

hog the cardiac portion is comparatively small and the remainder of the organ is to be regarded as constituting the stomach proper.

117. Rumination. - In the ruminant, water and liquid feeds may pass quite directly to the abomasum, although as a matter of fact, they seem to reach all four divisions of the stomach. The more bulky feeds, however, fail to pass through the esophageal canal but enter the rumen and reticulum. This is especially the case because the ruminant masticates its feed very imperfectly at the time of eating. In the reticulum and especially

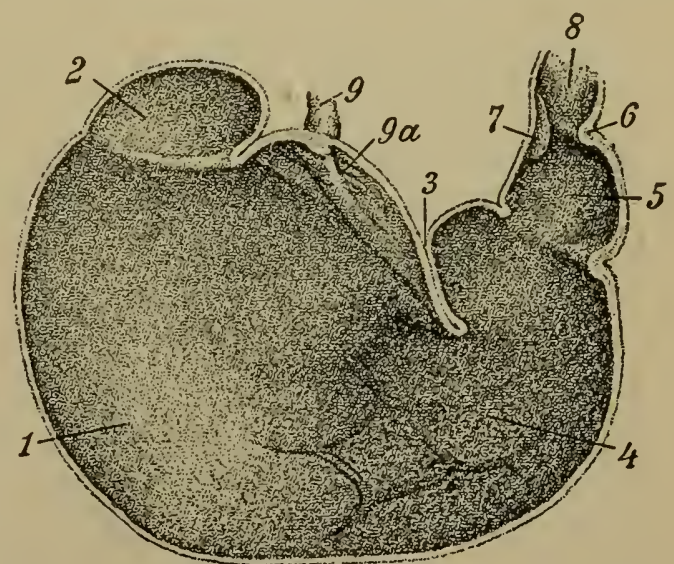

FIG. II. - Stomach of hog. (Hagemann, Anatomie und Physiologie der Haus-Säugetiere.) I-4, Fundus. 2, Cardiac portion. 5, Pylorus. 8, Duodenum. in the capacious rumen, the partially masticated feed remains for some time in contact with the saliva and such 
portion of the drink as reaches this stomach and is thoroughly softened and prepared for further action. The rumen is so large that it always contains a considerable amount of material and the new feed when swallowed is more or less completely mixed with that already in the rumen by the peristaltic action of the latter, thus tending to prolong its stay. The liquid or finely comminuted portions probably pass on directly to the omasum, or manifolds, and the abomasum, but the bulk of the feed undergoes the process of rumination.

After the animal has completed feeding, and if it is left in quiet, small portions of the feed are raised again to the mouth from the rumen and reticulum by contraction of these organs, aided by the action of the abdominal muscles and of the diaphragm, thoroughly chewed and swallowed a second time. This time they pass to a considerable extent, though not entirely, the esophageal canal and enter the third stomach, the manifolds, and from this pass into the fourth or true stomach.

The ruminants are animals which in the wild state depend on speed and cunning to escape from their enemies. Hence it is an advantage to them to be able to consume hastily large amounts of feed, and then to retire to some safe concealment to remasticate and prepare it for digestion. Rumination also enables these animals to utilize more thoroughly coarse rough fodders, the long stay in the paunch softening and fermenting the material and helping especially to destroy or dissolve the carbohydrates of the cell walls and thus render the cell contents accessible to the digestive fluids.

118. The gastric juice. - The mucous membrane lining the true stomach contains numerous simple glands (tubular glands) differing in appearance in different portions of the stomach. Those of the fundus region contain two kinds of secreting cells, commonly designated as "chief" and "parietal " cells. The glands of the pyloric end contain " chief " cells similar to those of the fundus glands, but only an occasional "parietal" cell. The parietal cells secrete as their essential product hydrochloric acid. The "chief" cells produce the enzym pepsin, or rather a precursor of pepsin called pepsinogen. The mixed secretion of these different glands constitutes the gastric juice, which is a thin, clear acid liquid having a 
specific gravity of $\mathrm{I} .005$ to $\mathrm{I} . \mathrm{OI}$ and containing a maximum of about 2.5 per cent of solids. The combined action of the pepsin and hydrochloric acid of the gastric juice splits the proteins of the feed into derived proteins, especially proteoses and peptones, and to some extent into polypeptids. ${ }^{1}$ The hydrochloric acid of the gastric juice has also an important antiseptic action and likewise serves to dissolve more or less of the ash of the feed.

In addition to its digestive action on proteins, the gastric juice contains an enzym which brings about the coagulation of the caseinogen of milk - the rennet ferment, or chymosin. According to some investigators, chymosin is identical with pepsin, but the weight of opinion seems to be in favor of its independent existence.

119. The passage of feed from the stomach. - The lower or posterior end of the stomach is closed by a sphincter muscle called the pylorus, which prevents the ingested feed from passing into the next division of the alimentary canal, the duodenum, or being forced into it by the contractions of the stomach. When in the course of gastric digestion, however, the difference between the acid reaction of the stomach contents and the alkaline reaction which normally prevails in the duodenum reaches a certain level, the pylorus relaxes and allows the peristaltic contraction of the stomach to press a portion of its acid contents into the duodenum. The partial neutralization of the duodenal contents which results causes the pylorus to close again until the alkaline reaction is restored, when the cycle may be repeated.

The mechanism of this process has been especially studied by Cannon in carnivora, but it may be presumed that what is true of these animals is also substantially true of herbivora, although experimental proof of this is lacking.

While both protein and carbohydrates undergo considerable digestion in the stomach, it is evident that one important function which the stomach performs is that of a receptacle which prevents too rapid passage of the feed into the duodenum and at the same time initiates chemical changes and prepares

${ }^{1}$ By prolonged peptic digestion in vitro amino acids may also be produced but it is not believed that this occurs in natural digestion. 
the feed for the more vigorous action of the intestinal enzyms. Moreover, the setting free of cell contents by the fermentation of the cell walls of vegetable feeds, as well as the liberation of the fat of animal feeds by the solution of the protein of the adipose tissue, render these materials more accessible to the action of the digestive juices.

120. The small intestine. - On leaving the stomach through the pylorus, the feed enters the small intestine, which may briefly

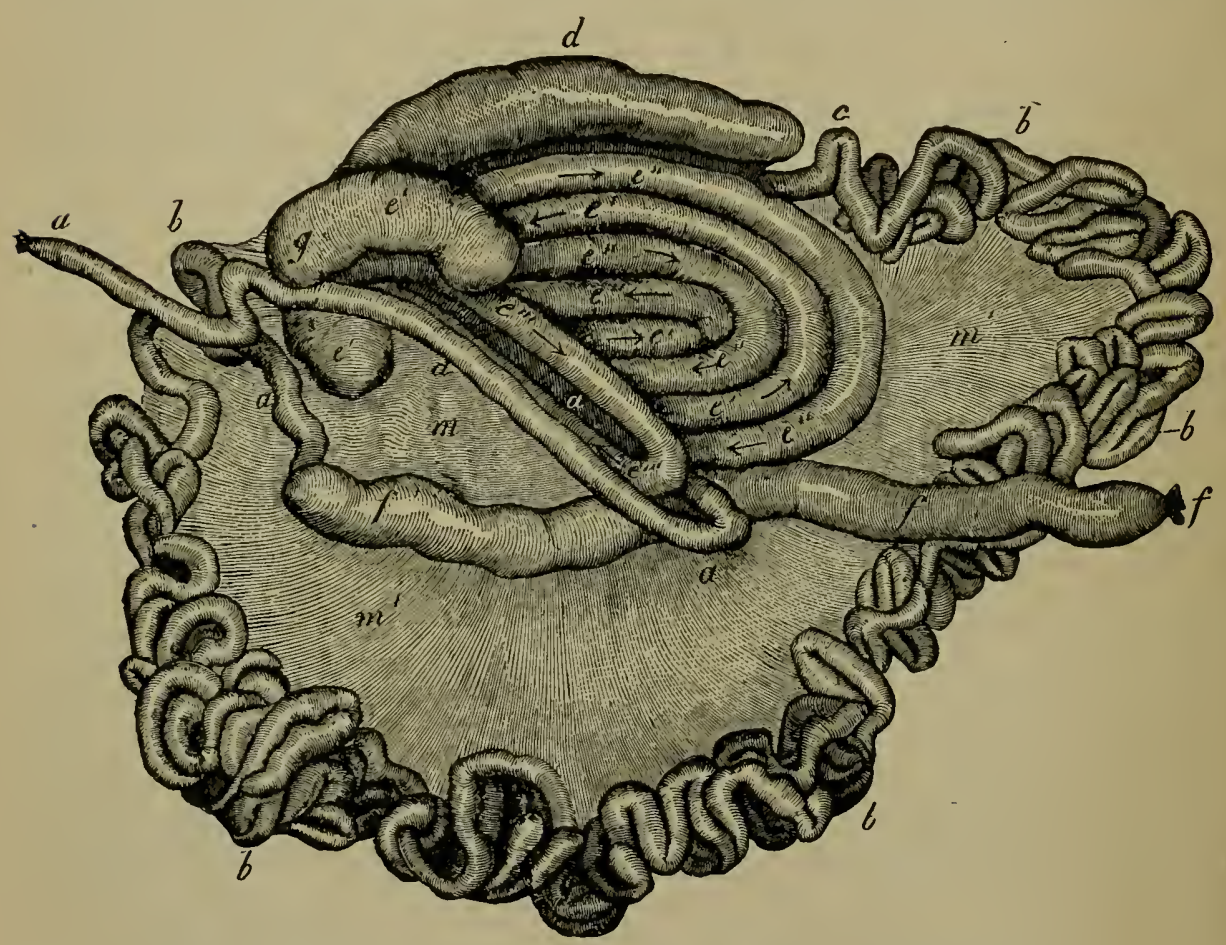

FIg. I2. - Intestines of cattle. (Leisering, Die Rindviehzucht.)

be described as a long, comparatively narrow tube. Its average length is, according to Colin, about nine times that of the body in the horse, sixteen times in the ox and sheep and eleven times in the hog. It is suspended in the abdominal cavity by a reflection of the peritoneum called the mesentery, and as shown in Fig. I 2 is much convoluted. It is commonly subdivided into duodenum, jejunum and ileum.

121. The cœcum. - From the small intestine the contents of the digestive tract pass, through the ileo-cœcal valve, into the cœecum, which is a diverticulum of the digestive canal, the point 
of entrance from the small intestine and that of exit into the colon being near together and in the upper part of the cocum. Anatomically, it might almost be called a second stomach. Its functions, however, resemble those of the first stomach of ruminants and not those of the true stomach, the feed stagnating, so to speak, in the cœcum and undergoing extensive fermentation and putrefaction. The size of the cocum, in a general way, varies inversely as that of the stomach. Thus in the horse it is very large, having about I6 per cent of the total capacity of the digestive canal. In the ox, on the other hand, it has only about 3 per cent and in the sheep less than 2.5 per cent of the total capacity and in the hog about 5.5 per cent.

\section{The large} intestine. - The alimentary canal is continued from the

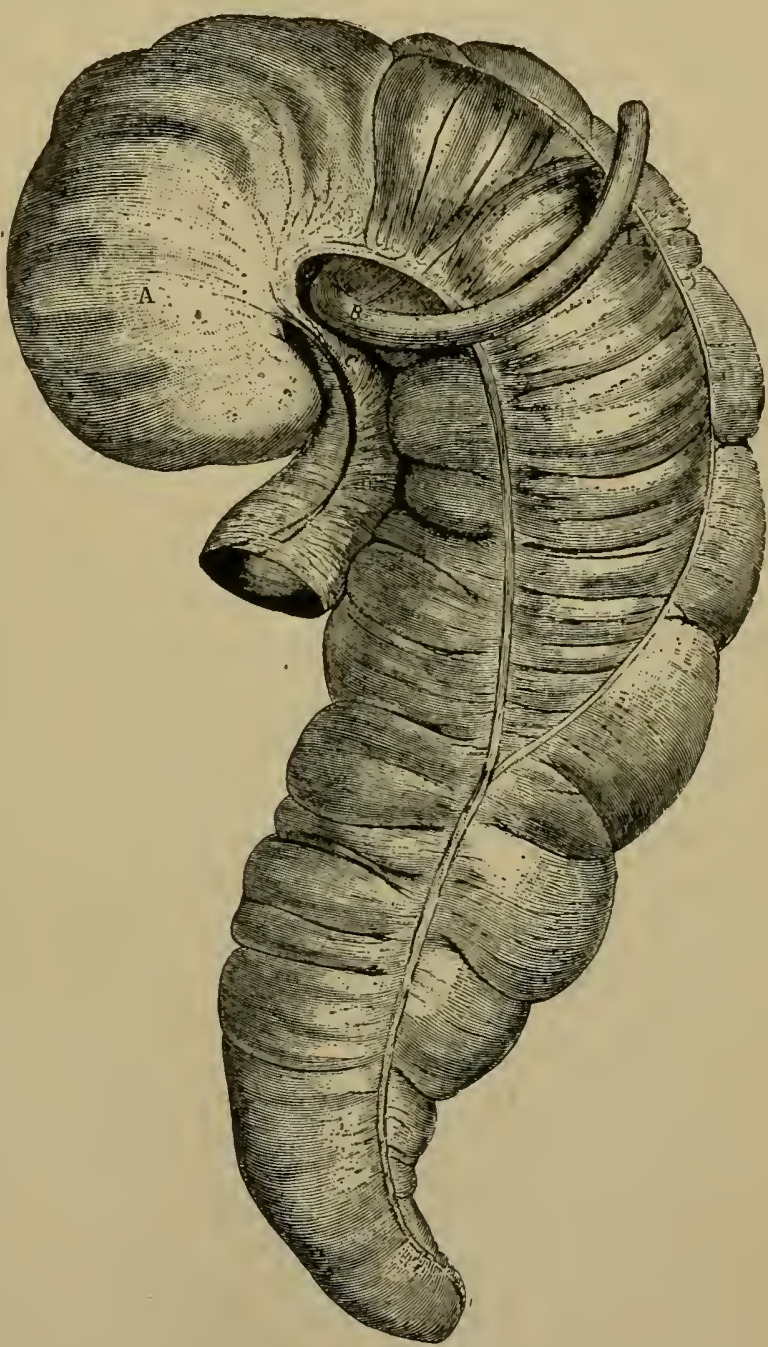

FIG. 13. - Cœcum of horse. (Colin, Physiologie comparée des Animaux.)

cœcum as the large intestine, which, as its name implies, is generally of greater diameter than the small intestine but also shorter. It is subdivided into the colon and the rectum and serves rather as a resorbent than as a digestive organ. The colon 
is enormously developed in the horse, having about 45 per cent of the total capacity of the digestive tract, and serves, like the cœcum, to continue the digestion of the less soluble portions of the feed.

123. The pancreas. - In the stomach, the glands which secrete the gastric juice are located in the mucous lining of the organ. In the case of the intestines, the glands which supply the various digestive juices, like the salivary glands of the mouth, lie in part entirely outside the alimentary canal proper. One of the most important of these is the pancreas. This is a large gland located near the stomach, liver and duodenum, its duct opening into the latter either by a common exit with that of the bile duct (horse, sheep), or somewhat lower down (cattle, swine). The secretory action of the pancreas, like that of the salivary and gastric glands, is intermittent, the gland being active only when feed is present in the duodenum.

The pancreatic juice is a clear, viscid liquid, having an alkaline reaction due to its content of sodium salts. It contains in the neighborhood of eight to ten per cent of solid matter and has a specific gravity of approximately r.030. It differs from other digestive juices in containing a relatively large amount of protein. As in the case of all the other digestive fluids, the essential active ingredients are enzyms, of which the pancreatic juice contains three in particular, viz., a protease, trypsin, acting upon the proteins, an amylase, amylopsin, acting upon starch, and a lipase, steapsin, acting upon fats. Small amounts of chymosin and of a lactase have also been found. In the juice as secreted by the pancreas, the trypsin at least, if not the other enzyms, exists in the form of a pro-enzym, trypsinogen, which is converted into trypsin (" activated ") after the secretion reaches the duodenum.

124. The liver. - This, the largest gland in the body, is located immediately below the diaphragm and lies chiefly on the right side of the body. It is relatively small in ruminants and large in the hog.

The liver has other important functions in nutrition, as will appear in Chapter V, but as related to digestion it secretes the bile. This fluid, produced by the hepatic cells, passes from them into the bile capillaries, which unite to form small ducts, the latter finally coalescing and constituting the bile duct. In 
the horse, this empties directly into the duodenum a short distance from the stomach. In cattle, sheep and swine, the bile is stored up in the gall bladder, a reservoir from which a duct leads to the duodenum.

The chief action of the bile is upon fats of the feed. To a small extent, it saponifies them and it also assists in emulsifying them. Its digestive action may, however, be more conveniently considered along with that of the pancreatic juice $(126,135)$. In addition to its action upon the fats, an antiseptic effect and also a stimulating effect upon peristalsis have been ascribed to the bile.

125. The intestinal juice. - In addition to the external glands (pancreas and liver), already mentioned, the walls of the small intestine contain a large number of small glands of two kinds, Brunner's and Lieberkühn's glands, which yield an intestinal juice containing a number of enzyms. Prominent among these are the invertases maltase, sucrase and lactase, which act upon the corresponding disaccharids, the maltose resulting from the digestion of starch being converted into dextrose, sucrose into a mixture of dextrose and levulose, and lactose, in suckling animals at least, into dextrose and galactose.

There may also be extracted from the mucous membrane of the small intestine a protease called erepsin. This enzym does not act upon the native proteins, with the exception of casein, but acts powerfully upon the derived proteins (proteoses and peptones), hydrolyzing them and breaking them down very completely to their constituent amino acids. The presence of erepsin has also been demonstrated in the intestinal juice, but its action in this case was weaker than in the extracts of the intestinal wall and it may be that a portion of its action in the living animal takes place within the cells in which it is produced.

The presence in the intestinal juice of an amylase, a lipase and of ferments (nucleinases and nucleotidases), which act upon the nucleic acids has also been demonstrated.

126. Intestinal digestion. - In the duodenum the neutralization of the acid material coming from the stomach is effected by the alkalies of the bile and pancreatic juice, while the bile also precipitates proteins and partly digested proteins in combination with the bile acids and this precipitate carries down with it mechanically the pepsin which is present. In these 
two ways, namely by neutralization and precipitation, the pepsin is prevented from digesting the enzyms of the pancreatic juice and bile, an action which would otherwise take place, since these enzyms appear to be substantially protein in their nature. ${ }^{1}$ In the small intestine, the neutralized contents of the stomach are subjected to the combined action of the pancreatic juice, the bile and the intestinal juice, while they are moved along through the successive divisions of the small and large intestines by means of the peristaltic movements of the latter. These movements serve also to mix the contents of the intestines and to bring them into intimate contact with the intestinal walls.

The fats of the feed, under the action of the steapsin of the pancreatic juice, undergo a cleavage into glycerol and fatty acids and this change is considerably accelerated by the bile, which also aids in emulsifying the fats and so exposing vastly more surface to the action of the enzyms. The fatty acids thus set free unite to a greater or less extent with the alkali of the pancreatic juice and bile, forming soaps, while both soaps and free fatty acids are soluble in bile in the presence of sodium carbonate. The presence of soaps in solution also aids, as was pointed out in Chapter I, in producing a permanent emulsion of the fats.

Starch, if any escapes digestion in the stomach, is acted upon by the pancreatic amylopsin substantially in the same manner as by the ptyalin of the saliva but much more energetically, yielding maltose, while both maltose and any other disaccharid present in the feed are acted upon by the invertases of the intestinal juice, yielding monosaccharids.

Any proteins which escape digestion in the stomach, and likewise the proteoses and peptones resulting from peptic digestion, are hydrolyzed by trypsin and erepsin much more energetically than by pepsin and yield not only proteoses and peptones, but a whole series of progressively simpler polypeptids and finally are largely or wholly split up into their constituent amino acids.

1 The foregoing statements describe what takes place when the materials are mixed in the laboratory. The actual importance of the precipitation of the pepsin in the intestine is somewhat in doubt. 


\section{§ 2. The Chemistry of Digestion}

127. Digestion a chemical process. - The foregoing paragraphs have dealt chiefly with those more general facts regarding the organs of digestion which are necessary for an understanding of their functions and only incidentally and in outline with the chemical processes involved. It is now time to revert to the statement made at the beginning of the chapter, namely, that digestion is the first step in the conversion of feed substances into body substances, and specifically in the case of farm animals the conversion of vegetable into animal substances. These, however, are chemical transformations and from this point of view a knowledge of the structure of the digestive apparatus is of significance chiefly as an aid to the understanding of these processes. In taking up this aspect of the subject, it will be convenient to consider the three chief groups of nutrients separately.

\section{The digestion of carbohydrates}

By far the larger proportion of the carbohydrates contained in the feed of farm animals consists of polysaccharids, especially starch, cellulose and the various pentosans and hexo-pentosans. The disaccharids, especially sucrose and lactose, probably stand next in importance, while comparatively small amounts of monosaccharids are consumed.

128. Cellulose. - The cellulose of feeding stuffs was long assumed to be indigestible. Haubner was the first to show the incorrectness of this assumption and to prove that even the cellulose of such substances as paper pulp and sawdust, as well as that of ordinary feeds, was digested by cattle. The subsequent investigations of Henneberg and Stohmann (158, 707) showed that the crude fiber of feeding stuffs was digested to a considerable extent by cattle and sheep, and later digestion experiments have proved this to be true not only of ruminants but to a varying degree of other animals, both herbivora and omnivora, including domestic fowls. Even man is capable of digesting the tenderer forms of cellulose to a considerable extent.

None of the digestive enzyms of the higher animals, however, have been shown to have any action upon cellulose and the small 
amounts of cellulose-dissolving enzyms (cytases) found in some feeds appear quite inadequate to account for its solution, so that the manner of its digestion was long a puzzle. The investigations of Wildt ${ }^{1}$ in 1874 upon the digestive process in sheep, however, showed, as Zuntz ${ }^{2}$ subsequently pointed out, that the solution of cellulose occurs chiefly in those portions of the alimentary canal where the feed stagnates, - that is, in the paunch of the ruminant and in the cœcum and colon. This fact tended to confirm the view already advanced that the solution of cellulose in the digestive tract was due to a process of fermentation, and this hypothesis also served to explain the presence of methane and hydrogen in the digestive tract. Tappeiner, ${ }^{3}$ however, seems to have been the first to show experimentally that the disappearance of cellulose in the digestive tract is effected by a fermentation brought about by the microorganisms inhabiting the alimentary canal.

Tappeiner's conclusions have been fully confirmed by more recent investigations, notably those of Markoff ${ }^{4}$ in Zuntz's laboratory, while Kellner ${ }^{5}$ has shown that the consumption of crude fiber (straw pulp) by cattle causes a marked increase in the amount of methane eliminated. In the light of these results it may be regarded as established that the disappearance of cellulose during its passage through the alimentary canal of herbivora is not due to a digestion in the sense of a simple hydrolytic cleavage, like that of starch or protein, but to a destructive fermentation. The products of this fermentation are large quantities of carbon dioxid and methane and small amounts of hydrogen, which are excreted, and various organic acids of the aliphatic series which combine with the alkalies of the saliva or other digestive fluids. The salts thus formed are resorbed and constitute the sole contribution which cellulose makes to the nutrition of the body. The principal acids formed appear to be acetic and butyric, although others are also present. In ruminants, the chief seat of this fermentation is the capacious first stomach, while in the horse, with his relatively small, simple stomach, it takes place principally or wholly in the enormous cœcum and colon.

1 Jour. Landw., 22 (1874), I.

${ }^{2}$ Landw. Jahrb., 8 (I879), Ior.

3 Ztschr. Biol., 20 (I 884 ), 52.

${ }^{4}$ Biochem. Ztschr., 34 (I9II), 2 I I ; 57 (I9I3), I.

${ }^{5}$ Landw. Vers. Stat., 53 (1900), I93, 300. 
129. Pentosans. - The pentosans are widely distributed in the vegetable kingdom and appear to be contained chiefly or wholly in the cell walls of plants, probably in combination to a greater or less extent with hexosans. If the ordinary methods of feeding stuffs analysis are followed, both the crude fiber and nitrogen-free extract contain them $(109,110)$.

Stone,$^{1}$ who was the first to show that they were digestible, found a percentage digestibility of about 60 for the pentosans in the ordinary feed of the rabbit. Later, ${ }^{2}$ in conjunction with Jones, he showed that in I4 different samples of roughages from 48 to 90 per cent of the pentosans were digested by sheep, while in mixed rations the corresponding figures were from 46 to $7 \mathrm{I}$ per cent. Weiske ${ }^{3}$ about the same time obtained similar results in experiments with sheep and rabbits. The digestibility of pentosans has been fully confirmed by later experiments.

But while pentosans are digestible, or at least disappear in the digestive tract, the manner of their digestion is not certainly known. Up to the present time no enzyms have been discovered either in the digestive organs or elsewhere, which have been proved to be capable of hydrolyzing them. On the other hand, however, the pentosans are attacked by bacteria much like other carbohydrates and yield similar products, especially the acids of the aliphatic series. That the pentosans are to a considerable extent subject to the methane fermentation in the digestive tract seems clear from Kellner's investigations upon straw pulp (128), in which over one-third of the digested organic matter consisted of pentosans, so that it is difficult to resist the conclusion that these, as well as the cellulose, underwent fermentation. Moreover, in a large number of similar experiments, the methane fermentation has been found in a general way to be proportional to the total digestible crude fiber and nitrogen-free extract, including the pentosans. Of course these results do not preclude the possibility of a hydrolysis of the pentosans in the digestive tract, converting them into pentose sugars, but as yet there is no direct evidence that such a process takes place. If it does not, then the products of the digestion of the pentosans are substantially the same as those from cellulose.

1 Amer. Chem. Jour., 14 (1 892), 9.

${ }^{2}$ Agricultural Science, 7 (1893), 6.

${ }^{3}$ Ztschr. Physiol. Chem., 20 (I895), 489. 
130. Hemicelluloses. - What is true specifically of the pentosans appears to hold also for the reserve carbohydrates of the cell wall - the so-called hemicelluloses (18) - as a whole. No animal enzyms are known which hydrolyze the galactans, levulans, etc., or which break up their union, if it exists, with the pentosans, but nevertheless these substances disappear in part in the digestive tract of herbivora. Pending more exact knowledge on this point, the assumption seems warranted that they as well as the pentosans undergo bacterial fermentation and yield substantially the same products.

131. Starch. - The first agent to act upon starch is the ptyalin of the saliva (115). As is the case with the other enzyms, ptyalin has never been isolated, but its effects and the conditions governing its action have been extensively studied, in part owing to the ease with which saliva can be procured. The most important of these conditions are that ptyalin acts most efficiently in the neighborhood of $40^{\circ} \mathrm{C}$., that is, at about blood temperature, in a neutral solution, while more than a trace of free acid or alkali inhibits its action. To acids or alkalies combined with proteins, even though they show an acid or alkaline reaction to ordinary indicators, ptyalin is much less sensitive and it is also less sensitive to organic than to inorganic acids. In brief, the action of ptyalin is inhibited by a very low concentration of either hydrogen or hydroxyl ions.

The action of ptyalin on starch consists of a succession of cleavages and hydrations resulting in the formation of the various dextrins (24) and finally of sugar. With cooked starch, the action is fairly rapid; upon raw starch ptyalin acts more slowly, the rate varying somewhat with the kind of starch and being apparently determined by the degree of resistance of the cellulose envelope of the starch grains. Chemically, the action is analogous to that of acids, but is less vigorous and is not carried so far. The action of acids yields dextrose as a final product; that of ptyalin is usually stated to stop with the production of maltose. ${ }^{1}$

The action of ptyalin in the mouth is necessarily very brief. In the stomach the feed comes into contact with the gastric juice containing free hydrochloric acid. At first, this acid combines with the proteins contained in the feed, but as soon

1 Carlson and Luckhart (Amer. Jour. Physiol., 23 (I908-9), I49) state that both ptyalin and amylopsin produce dextrose from starch. 
as more than a trace of free acid accumulates, or to speak more exactly, as soon as the concentration of the hydrogen ions exceeds a certain small limit, the action of the ferment is not only inhibited, but the ptyalin is digested by the pepsin.

This, however, is far from happening immediately upon the entry of the feed into the stomach. The secretion of the gastric juice requires a certain length of time. Moreover, the contents of the stomach are semi-solid rather than liquid and while the muscular contractions of the stomach serve to mix the feed to some extent, this effect is less than is often assumed. Frozen sections of animals to which variously colored feeds have been given show the contents of the stomach to be distinctly stratified some time after the ingestion of feed. Furthermore, the gastric juice is secreted only in the pyloric portion of the stomach (116). Time is required, therefore, for the acid to penetrate and acidify the whole mass and consequently the action of the ptyalin may continue for a considerable period.

Extensive investigations, especially by Ellenberger and Hofmeister, upon gastric digestion in the horse and hog have demonstrated that in these animals the action of the saliva in the stomach upon the starch of the feed plays an important part in digestion. In the horse (116), the left end of the stomach is really a dilation of the esophagus. In the hog, while nearly the entire surface of the stomach is lined with mucous membrane, the left-hand end contains no peptic glands. When the stomach is filled with feed, therefore, it is evident that the action of the hydrochloric acid will begin along the walls of the fundus of the stomach and only gradually spread to the rest of the contents. It is true that lactic fermentation usually sets in during this period, aiding to acidify the stomach contents but, as already stated, ptyalin is less sensitive to organic than to inorganic acids. It has been found that the solution of starch may continue to a greater or less extent for as much as four or five hours both in the horse and hog. In ruminants, the conditions are even more favorable for salivary action, since the feed remains in contact with the saliva in the rumen for a considerable time, the contents of this stomach being maintained slightly alkaline by the large amount of saliva secreted by these animals (115). It may be assumed, therefore, in spite of the 
fact that the saliva of ruminants contains but little ptyalin, that a. considerable digestion of starch is effected.

In the duodenum, any starch not digested in the stomach, as well as any dextrins, etc., produced by the action of the ptyalin, are subjected to the action of the amylopsin of the pancreatic juice. This enzym, if not identical with ptyalin, is very similar to it but appears to act more energetically. As in the case of ptyalin, the final product of its action is maltose. ${ }^{1}$

The further fate of the maltose resulting from the digestion of starch is more conveniently considered along with that of other disaccharids in a succeeding paragraph.

132. Fermentation of starch. - The organisms producing the methane fermentation in the digestive tract were believed by Tappeiner to attack cellulose specifically and not to act upon other carbohydrates. As regards ruminants, however, this has been shown to be an error. In G. Kühn's ${ }^{2}$ extensive respiration experiments with cattle upon the formation of fats from carbohydrates, considerable amounts of starch were added to basal rations. Invariably this resulted in an increased excretion of methane. Moreover, there was no increase, but on the other hand, more or less decrease in the amount of crude fiber digested, showing that the additional methane must have had its source in the starch consumed. This conclusion is confirmed by the fact that the total excretion of methane was quite closely proportional to the sum of the digested crude fiber and nitrogen-free extract. On the average four parts of methane were produced for each one hundred parts of starch digested. Kellner's subsequent investigations ${ }^{3}$ have fully confirmed these results, although giving a lower average, viz., 3.07 parts of methane per one hundred parts of digestible starch. Moreover, Kellner's investigations have shown that the methane fermentation is not confined to cellulose and starch but that, as already indicated, the complex of compounds grouped under the head of nitrogen-free extract, including the sugars, is subject to this process. His experiments also show that the proportion of methane produced is somewhat variable, depending upon conditions not yet fully investigated.

As already stated (128), the chief seat of fermentation in the

\footnotetext{
1 See footnote on p. $92 . \quad{ }^{2}$ Kellner; Landw. Vers. Stat., 44 (I 894), 257.

${ }^{3}$ Landw. Vers. Stat., 53 (I900), 423.
} 
horse is the cœcum and colon. Before the feed reaches these, however, it has been acted upon by the amylases of the saliva and the pancreatic juice and its starch and soluble carbohydrates pretty thoroughly extracted. Consequently, the methane production of the horse is substantially at the expense of the crude fiber of his feed, although if starch for any reason escapes digestion and reaches the cœcum it is doubtless also attacked by the bacteria.

133. The disaccharids. - At first thought, it would seem that the carbohydrates of this group need no digestive change, since they are already soluble and diffusible and seemingly ready to pass into the circulation. But while this is true, they are not assimilable by the organism. Disaccharids are nowhere found in the normal body fluids and if injected into the circulation in any considerable amount are voided in the urine. In other words, the disaccharids are treated in the organism as foreign substances which the cells cannot use.

In the small intestine the disaccharids are inverted, that is, hydrolyzed to monosaccharids. Cane sugar taken in the food appears to be inverted to some extent by the acid of the gastric juice, but the principal action is by the inverting enzym sucrase of the intestinal juice, which splits up the cane sugar into dextrose and levulose. Similarly, the maltose resulting from the digestion of starch is split up by the maltase of the intestinal juice, yielding dextrose, while lactose, at least in suckling animals, is split up by lactase into dextrose and galactose. These inversions appear to take place to a considerable extent in the epithelial cells lining the intestines, and this seems to be the normal method of assimilation of lactose in many mature animals. The epithelial cells are also stated to convert levulose and galactose into dextrose.

Finally it should be added that the sugars, like other carbohydrates, may undergo the methane fermentation in the first stomach of ruminants.

\section{The digestion of fats}

134. Emulsification. - As already indicated, the digestion of fats includes two processes, namely, emulsification and saponification, effected chiefly by the action of the bile and 
pancreatic juice. The two processes go hand in hand. As explained in Chapter I, the presence of free fatty acids favors the formation of a permanent emulsion. As there noted, most native fats contain small amounts of such acids which exist dissolved in the natural fat. Furthermore, there seems to be good evidence that some cleavage of fat into fatty acids and glycerol takes place in the stomach of carnivora, while the digestion of protein in the stomach helps to liberate any enclosed fat. When the acid fats come in contact with the alkaline pancreatic juice, the molecules of the free acid in solution are saponified and in this way the mass of fat is broken up into an emulsion. The action of the steapsin of the pancreatic juice, which splits fat into glycerol and fatty acids, would obviously tend to aid in the emulsification, while, on the other hand, the latter, by vastly increasing the amount of surface exposed by the fats, tends to aid the action of the enzyms.

135. Saponification. - The saponification of fat is accomplished essentially by the lipase steapsin of the pancreatic juice. As just noted, the saponification is facilitated by the previous emulsification, while the presence of the bile is also an important factor. It is claimed that the presence of bile is necessary to activate the steapsin, while it has also been shown that the cleavage of the fats is much accelerated by the presence of bile, the effect being ascribed to the lecithins which it contains. The presence of bile also assists in the process of digestion by its power of dissolving large quantities of fatty acids and of their calcium and magnesium soaps. It appears also that the bile aids in some way in the resorption of the fat, but just how is not clear.

Fats do not seem to be fermented to any extent in the digestive tract and when administered to cattle in the form of emulsions have been found to produce no effect upon the excretion of methane. When given in substance, they have in some instances had the effect of diminishing the excretion of that gas.

\section{The digestion of the proteins and non-proteins}

136. Peptic digestion. - In digestion the proteins are first subjected in the stomach to the action of the pepsin and hydrochloric acid of the gastric juice. 
The products of peptic digestion are usually substances belonging to the group of derived proteins $(58,59)$. The first product or products are substances called metaproteins, or, according to the older terminology, syntonin or acid proteins. By still further action there is formed a succession of proteoses. and from these, by subsequent cleavage, peptones. Undoubtedly the products resulting from peptic digestion contain a large number of chemical individuals but for the present purpose it is sufficient to say that the action of pepsin and hydrochloric acid gives rise to the formation of a series of progressively simpler, more soluble and more diffusible substances. In natural digestion, the action extends in the main only as far as the production of peptones, although polypeptids seem to be also formed to some extent. Amino acids are not found among the products of natural peptic digestion, although they may be produced by the long continued action of pepsin-hydrochloric acid in artificial digestion.

The conjugated proteins are split into their two constituents and the protein portion is then acted upon like other proteins. The gastric juice has no action upon the nucleic acids of the nucleoproteins.

137. Tryptic digestion. - In the duodenum, the proteins and the products of their peptic digestion are subjected to the action of the trypsin of the pancreatic juice. This is produced in the pancreas in the form of a pro-ferment or zymogen, called trypsinogen. The presence of pancreatic juice in the duodenum stimulates the glands of the latter to the production of the intestinal juice which Pawlow has found to contain a substance, enterokinase, which activates the trypsinogen, or converts it into trypsin, in some unknown manner.

The action of trypsin, like that of pepsin, has been largely studied in laboratory experiments either with extracts of the pancreas or with its secretion as obtained from fistulæ. Trypsin, especially in a neutral or alkaline solution, acts upon proteins substantially in the same manner as pepsin, causing a hydrolytic cleavage and producing at first proteoses and peptones. It acts much more energetically than pepsin, however, and carries the cleavage much further. The action of pepsin substantially stops with the production of peptones. Trypsin, on the other hand, produces a relatively large amount of the 
simple amino acids out of which the protein molecule is built up. Even the most prolonged action of trypsin, however, leaves a considerable residue in which no free amino acids are found but which on hydrolysis with strong mineral acids yields them in abundance.

Conjugated proteins seem to be acted upon by trypsin in the same manner as by pepsin but much more energetically.

138. Erepsin. - The presence of a proteolytic enzym in the intestinal juice and in the epithelial cells of the small intestine has already been noted (135). This enzym does not act on unaltered proteins, with the exception of casein, but it hydrolyzes proteoses and peptones energetically, yielding crystalline cleavage products. It is of special interest to note that, according to Cohnheim, ${ }^{1}$ erepsin is capable of effecting the cleavage of that part of the protein molecule which is not attacked by pepsin and trypsin and that in artificial digestion experiments almost complete conversion into comparatively simple crystalline products may be obtained in a relatively short time.

139. Extent of protein cleavage in natural digestion. When it was first shown by Kühne and Chittenden that the action of trypsin upon proteins yielded among other products such simple crystalline substances as leucin and tyrosin, comparatively little importance was attached to the fact from the physiological standpoint. While the fact was interesting as throwing light upon the chemical structure of the proteins, it was believed that in natural digestion the soluble proteoses and peptones were promptly removed from the digestive tract by resorption and that at most but a small proportion of the feed protein underwent any profound cleavage. This belief was the stronger because it was believed that only proteins or their slightly altered derivatives, the proteoses and peptones, could supply the demands of the organism for proteins. Whatever protein was broken down into crystalline products was looked upon as wasted. With the progress of investigation, however, it has become increasingly clear that the processes of hydrolytic cleavage go further and play a much more important part than was formerly supposed. While it is admitted that peptones, or even soluble proteins, may be resorbed, the weight

${ }^{3}$ Ztschr. Physiol. Chem. 49 (I906), 64; 51 (I907), 415. 
of opinion is that, as a matter of fact, proteins are largely resorbed in the form of comparatively simple cleavage products; not necessarily in every case as simple amino acids but at least in the form of comparatively simple peptids.

The nucleic acids derived from the peptic or tryptic digestion of the nucleoproteins are split by the nucleases of the intestinal juice into mononucleotids and these again by the nucleotidases of the same secretion into nucleosids (53). No digestive enzyms attacking the latter class of compounds are known, but they are split to some extent by intestinal bacteria into pentoses and purin or pyrimidin bases. Furthermore, it has been found that extracts of the intestinal mucous membrane (epithelial cells) possess the power of bringing about the same cleavages which are accomplished by the enzyms of the intestinal juice, and in addition are able to split the resulting nucleosids into pentose and base. It appears, then, that the final digestive products of the nucleic acids are, as in the case of the simple proteins, relatively simple substances, viz., phosphoric acid, pentoses, and purin and pyrimidin bases.

140. Putrefaction of proteins. - Attention has already been called, in connection with the digestion of the carbohydrates, to the bacterial flora of the digestive tract. The carbohydrates, as was shown, are acted upon chiefly by the organisms producing the methane fermentation. Proteins and their derivatives, on the other hand, have been shown by Kellner to contribute practically nothing to this fermentation in the case of cattle. They are, however, especially subject to the action of the organisms producing putrefaction. The action of such organisms is prevented in the stomach by the hydrochloric acid of the gastric juice. In the small intestine they become more active, especially as the feed reaches the lower portion, while their activity lessens again as the lower portion of the large intestine is reached, owing to the progressive resorption of water from the intestinal contents. The characteristic products of the putrefaction are ammonia and certain aromatic compounds derived from the heterocyclic components of the proteins (47).

The aromatic products of putrefaction (indols and phenols) are found in part in the feces but are in large part resorbed. They cannot, however, be utilized by the organism but, on the contrary, are poisonous and are therefore combined with other 
substances which render them innocuous. In particular, they unite with sulphates to form the so-called ether-sulphates which are excreted in the urine. The amount of these substances in the urine furnishes a convenient index to the extent of intestinal putrefaction.

141. The non-proteins. - As ordinarily determined (61, 106), the non-proteins constitute a group of nitrogenous substances soluble in water, many of which are identical with or closely related to the final products of the digestion of the proteins. Accordingly, they have generally been assumed to be ready for resorption without further action by the digestive juices and therefore to be entirely digestible.

It has been shown, however, that, in ruminants at least, the matter is by no means so simple as the mere resorption of watersoluble substances. In the capacious first stomach of these animals, the non-proteins play an important rôle as a supply of nitrogenous food for the organisms which are so abundant there. This has several consequences.

In the first place, it appears that these soluble compounds are more readily attacked and utilized by the organisms than are the true proteins of the feed. The presence of non-proteins, therefore, tends to protect the proteins from bacterial decomposition.

In the second place, an abundant supply of soluble nitrogenous matter stimulates the multiplication and activity of the organisms and so brings about a more extensive fermentation of the carbohydrates of the feed, as is evidenced by an increase in the methane given off and in the proportion of the carbohydrates apparently digested.

Third, it seems to be fairly well made out that the nitrogen which these organisms assimilate is utilized to build up their protoplasm and thus, by a sort of symbiosis, becomes a source of protein to their host. It has been claimed that this bacterial protein is indigestible, but the evidence on which this claim is based is capable of a different interpretation and there seems to be good reason for believing that it may be acted on in the stomach and intestines by the digestive enzyms like other proteins and serve as a source of protein to the body. Some of the evidence in favor of this view is presented in a subsequent discussion of the nutritive value of the non-proteins (786-789). 


\section{The digestion of ash}

The various digestive enzyms whose action has been considered in the foregoing pages bring about extensive chemical changes in the organic nutrients of feeding stuffs by means of which they are prepared to enter into the nutritive processes in the tissues. At the same time, the so-called "inorganic" ingredients of feed are also prepared for resorption, but the digestion of these substances has been less extensively studied than that of the organic nutrients.

142. Sulphur and phosphorus. - As regards the sulphur of the proteins, it does not appear that this element is separated from its union with carbon, nitrogen and hydrogen in the processes of protein digestion. The sulphur of the proteins is contained in the amino-acid cystin, which, so far as known, is resorbed without further change. As regards the phosphorus of the nucleo-proteins, opinions still differ as to whether it is split off as phosphoric acid in the course of digestion or resorbed, still in "organic" combination, as a nucleosid. To what extent other ash ingredients are taken up, like sulphur and phosphorus, in organic combination, it is difficult to say, but that such resorption takes place is to be regarded as probable.

143. Electrolytes. - As regards those ash ingredients of feeds which are present as electrolytes, it may be assumed that they are brought into solution to a greater or less extent by the hydrochloric acid of the gastric juice, but how much reprecipitation may occur in the more or less alkaline contents of the intestine it is difficult to say. The whole subject of the digestion of the ash ingredients of feeding stuffs, however, is so intimately related to the question of the paths of excretion and to the problems of ash metabolism that it can be more profitably considered in that connection.

\section{Summary of changes in digestion}

144. Solution of nutrients. - The substances which make up the larger part of the feed of domestic animals (and of man as well) are comparatively insoluble in water. Some of them, such as cellulose and the fats, may be regarded as practically entirely insoluble. Others, like starch and the proteins, are 
very sparingly so. While small amounts of soluble proteins and somewhat larger quantities of soluble carbohydrates occur, they ordinarily play but a subordinate rôle in nutrition. One obvious result of the chemical changes brought about by the enzyms and organized ferments of the digestive tract is to convert these insoluble substances into soluble ones. Thus starch yields sugar, cellulose the organic acids, fats form soaps and protein yields peptones and amino acids. It was natural, therefore, that digestion should be looked upon as a process of solution and compared to the preparation of extracts in a pharmaceutical laboratory by means of various solvents.

The solvent action of the digestive juices is important, since the animal, like the plant, absorbs its real food substances substantially in aqueous solution. The mere dissolving of the ingredients of the feeds, however, is far from being the only or even the chief function of the digestive juices, as is clearly indicated, for example, by the existence of a coagulating enzym like chymosin, which precipitates the soluble casein, or the presence of the various invertases, which attack substances already soluble.

145. Colloids converted into crystalloids. - The principal nutrients belong to the class of substances called colloids. Gelatin is a typical colloid as are, indeed, all the proteins and the more abundant carbohydrates, while the sugars, organic acids, etc., are classed as crystalloids.

As related to digestion, the most important distinction between colloids and crystalloids is the difference in the osmotic pressures of their solutions by virtue of which crystalloids diffuse readily through permeable membranes. This diffusibility plays an important part in the resorption of the digested material into the blood and lymph current, as will appear in the next section, although it is by no means the only factor concerned.

A review of the chemical changes which take place in digestion shows that they are all in the direction of molecular simplification. They are substantially processes of cleavage by which large molecules are split into two or more smaller ones. Such a change, however, is in the direction from the colloid to the crystalloid condition. The final products of digestion are 
mostly substances of comparatively low molecular weight, readily soluble in water and having a relatively high osmotic pressure and therefore readily diffusible. This difference is most marked in some of the more simple cleavage products of the proteins and least so in the case of the digestive products of the fats.

146. Uniformity in nutritive material. - The feed consumed, especially by herbivora, is of a very heterogeneous character. The proteins and carbohydrates in particular are present in great variety, so that the nature and proportions of the substances out of which the body must draw the material for the construction and maintenance of its tissues may vary greatly at different times. Under the action of the digestive enzyms, however, these diverse substances all yield substantially the same products so that the nutritive solution supplied to the body proper is qualitatively of a very uniform composition, containing chiefly monosaccharids, various acids of the aliphatic series, amino acids derived from the proteins, and the soluble ash ingredients. By this preliminary action upon the feed in the digestive canal, - i.e., practically outside the body, - the organism is rendered independent of the particular kinds of feed available, its cells being constantly supplied with uniform nutritive material.

147. Molecular simplification. - It has just been pointed out (145) that digestion from the chemical standpoint consists substantially of a series of hydrolytic cleavages of the nutrients, yielding compounds of lower molecular weight and greater solubility and diffusibility. This molecular simplification has, however, a more important aspect which is most strikingly illustrated in the case of the proteins. It was shown in Chapter I that the proteins, although very similar in many physical properties, may differ widely from each other as regards molecular structure. This is shown in the first place by the wide variations in the proportions of the constituent amino acids which they yield on hydrolysis (50). Moreover, even were these cleavage products present in the same proportions, the existence of optical isomers and the possible variations in the order and manner of linkage of the amino acids afford almost endless possibilities of isomerism. Studies in immunity have in fact revealed striking specific differences between proteins 
bearing the same name but derived from different species or different individuals of the same species, the proteins of one animal often being toxic to another.

The body proteins, then, are specific both as to composition and structure and differ in both respects from those of the feed. In order that the latter may nourish the organism they must be converted into the specific proteins of the body. This is accomplished through their cleavage in the digestive tract into their constituent "building stones" which the body may then reassemble to form proteins constructed according to its own specific pattern. Not only so, but the proteins of different tissues or even cells must be regarded as specific. The body proteins are built not after a single pattern but after numerous ones. It is only by a very extensive, even if not complete (139), breaking down of the structure of the feed proteins that it becomes possible for the body to build up out of the fragments the various proteins which it requires: "Its protein molecules have a different architecture from those of the plant." This fact throws an interesting light upon the coagulation of the soluble casein of milk in the stomach. Although present in soluble form, it is not a body protein and its coagulation serves to retain it in the digestive tract and give the proteolytic enzyms an opportunity to break it up into its constituent amino acids.

What is so strikingly true of the proteins is likewise true of other nutrients. The digestive cleavages serve not merely, or perhaps not chiefly, to render them soluble and diffusible but to reduce the molecular complexes to forms which the body cells can assimilate. The carbohydrates, e.g., are converted into monosaccharids, even the somewhat larger molecules of the disaccharids appearing to be too large or to have an unsuitable molecular structure for the body cells to use. In general the complex compounds of the feed are split up by the enzyms of the digestive fluids into their constituent atomic groupings or "building stones" which supply the material out of which the body by selection and rearrangement builds up the proteins, carbohydrates and fats peculiar to itself, and the value of a feed depends upon the nature and amounts of the cleavage products which it yields in digestion rather than upon the specific substances which it contains. 


\section{§ 3. Resorption - The FeCES}

148. Definition. - As was stated at the beginning of this chapter (113), the digested feed contained in the alimentary canal is really outside the body, just as in the case of the ameba. In order to enter the body, the digested material must pass through or be taken up by the cells surrounding the digestive cavity. The process by which the products of the digestion of the feed are transferred from the digestive organs to the circulating media (blood and lymph) of the body is called resorption.

149. Epithelium. Villi. - The inner, or mucous, membrane of the digestive tract bears on its surface a layer of epi-

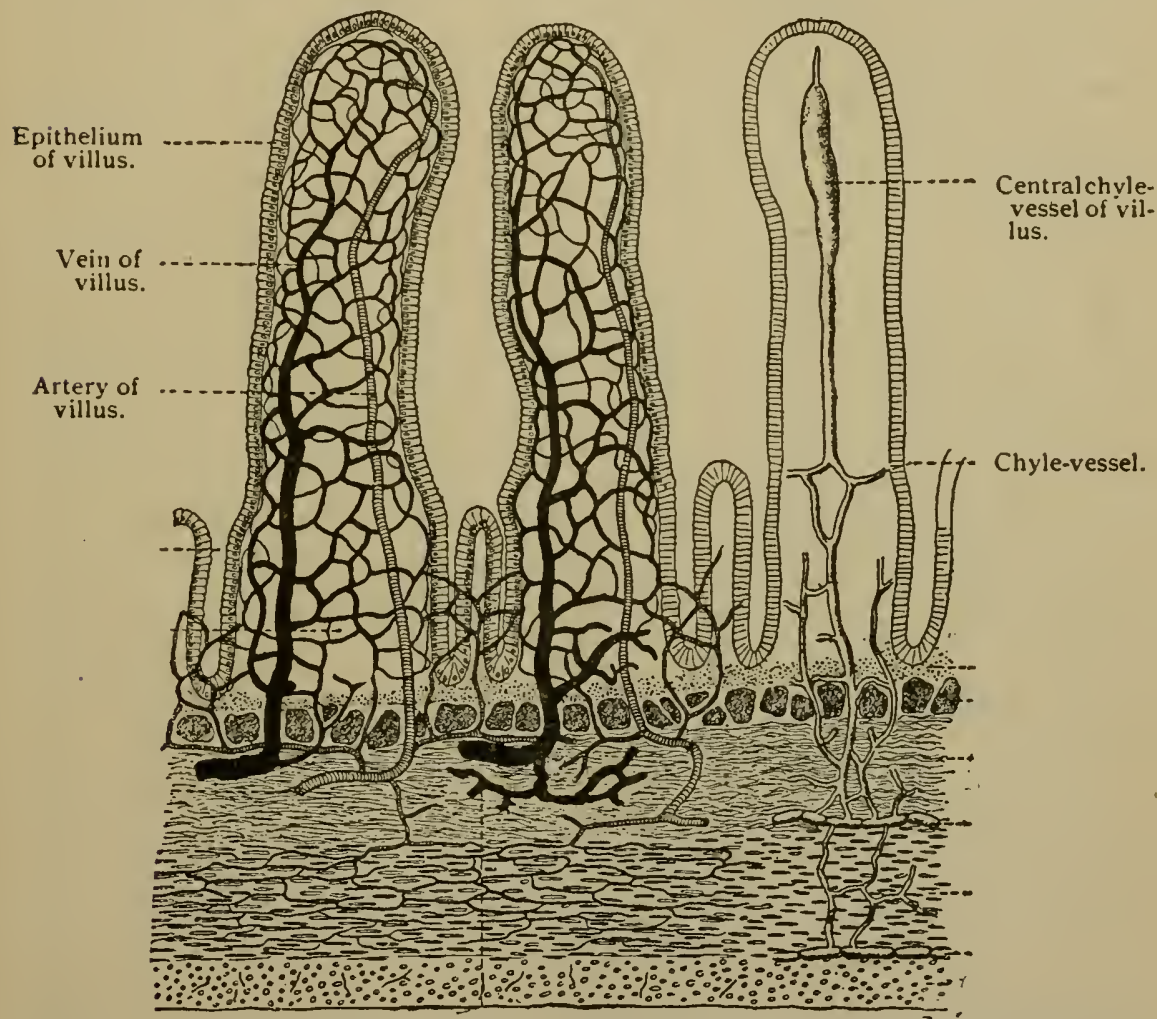

FIG. I4. - Section of villi. (Bohm, Davidorf, Huber, Text Book of Histology.)

thelial cells, more or less resembling those lining the mouth, which is closely underlaid with a network of blood capillaries and lymph vessels. It is these epithelial cells which are the active agents in resorption. 
In the higher animals the extent of resorbing surface is greatly increased by certain projections of the interior surface of the small intestine known as the villi. Those are round or clubshaped protuberances of the inner surface of the intestine. They are covered, like all parts of the intestinal surface, with the epithelial cells just described, which are underlaid by a delicate membrane, beneath which are found numerous minute capillary blood-vessels, a layer of smooth (involuntary) muscular fibers and a network of nerves. In the center of each villus ends a vessel called a lacteal, belonging to the lymphatic system. Figure I4 shows a longitudinal section of three villi.

The villi are absent in the stomach and in the large intestine. Although some resorption takes place in the stomach, and while a considerable amount of water and more or less of the fermentation products are resorbed in the large intestine, the small intestine is the special resorptive organ.

150. Mechanism of resorption. - Since the processes of digestion are apparently directed toward the conversion of feed substances into soluble and diffusible forms, it was quite natural that resorption should be regarded as an osmotic process. In this conception of it, the epithelial cells and other tissues between the cavity of the digestive organs and the blood and lymph vessels constituted a membrane through which osmosis took place. On the one side of this membrane were the contents of the digestive tract, containing the soluble products of digestion, while on the other side were the blood and lymph, containing little or none of these products. Under these conditions, osmosis was assumed to set in and transfer the digested nutrients to the blood and lymph.

Undoubtedly osmosis plays an important part in resorption, but its effects are profoundly modified by the properties of the resorbing cells of the intestinal epithelium in ways which as yet are but very partially understood, and resorption can by no means be explained by a simple analogy with the parchment dialyzing tube of the laboratory.

Differences in the permeability of the epithelial cells and of the intercellular substance for the various dissolved substances in the digestive tract doubtless play their part in bringing about the phenomena of selective resorption, while variations in the affinity of the cell colloids for water may be assumed to influence the resorp- 
tion of that substance as well as of salts. There are other facts, however, for which it is difficult at present to offer any physicochemical explanation. Notable among these is the predominant permeability of the intestinal epithelium in one direction, viz., from the intestinal lumen towards the blood and lymph vessels.

For the present, it is necessary to be content with the statement that resorption is a function of the living epithelial cells, although such a statement, of course, explains nothing but simply means that it is impossible at present to form an adequate conception of the intimate mechanism of the process.

Resorption might be characterized briefly as a reverse secretion. In secretion the active cells of a gland take materials from the blood or lymph, transform them into the specific substances characteristic of the cells, and then eject the latter into the duct of the gland. The epithelial cells of the digestive tract, on the other hand, take up digested materials from the contents of the alimentary canal, modify them more or less chemically and transmit the products to the blood or lymph.

151. Paths of resorption. - Most of the resorbed substances seem to pass from the epithelial cells to the blood in the capillaries and thus finally through the portal vein (182) to the liver. This is true of the cleavage products of the proteins, of carbohydrates, organic acids, salts and water. Fats, on the other hand, enter the circulation chiefly or wholly through the lymph in the form of minute droplets which are secreted by the epithelium into the lacteals of the villi, whence they pass through the lymphatics to the thoracic duct (186).

152. Chemical changes in resorption. - It is somewhat generally believed that the products of digestion, especially of the proteins and fats, undergo rather extensive chemical changes in the epithelial cells during the process of resorption. This question is considered more particularly in Chapter $\mathrm{V}$ but may be briefly referred to here for the sake of completeness.

Proteins. - In digestion the proteins yield comparatively simple cleavage products. It has been maintained, especially by Abderhalden and his school, that these cleavage products are resynthesized in the epithelial cells into serum albumin, which is regarded as the common source of all the body proteins. This view has been based chiefly on the failure to detect amino acids or other protein cleavage products in the blood coming 
from the intestines even during the height of protein resorption. Folin and Denis and also Van Slyke and Meyer have recently demonstrated, however, that sufficiently delicate tests show the presence of such products in the portal blood in amounts as large as could be expected in view of the gradual nature of digestion and resorption and of the large volume of blood passing through the intestinal capillaries. The prevailing opinion seems to be at present that the digestive products of the proteins undergo relatively little modification before entering into the circulation.

Fats. - The mechanism of fat resorption has been the subject of heated controversy. Some physiologists have maintained that it is chiefly a physical process; that globules of emulsified feed fat are taken up bodily by the epithelial cells and excreted again by them into the lacteals. This view is based largely on microscopical observations which show the presence of apparently unaltered fat globules of the intestinal emulsion in the protoplasm of the epithelial cells and in the lymph of the lacteals after the ingestion of fat. Other no less eminent physiologists, however, have as stoutly held that fats are not resorbed unaltered but only after cleavage into glycerol and fatty acids (or their salts), which are soluble in bile (135), and that the fat globules observed in the epithelial cells are the product of a resynthesis. At present the weight of scientific opinion is strongly in favor of this latter view. It is perhaps true that unaltered fat droplets may be taken up by the epithelial cells but that any considerable amount of fat is resorbed in this fashion is to say the least very questionable.

At any rate, the digested fat reaches the lacteals almost entirely in the form of fat, so that after a meal containing much fat the lacteals are filled with a milky fluid and the lymph is found to contain relatively large amounts of neutral fats. It is clear, then, that the resorbed soaps and fatty acids are speedily synthesized to fat again. This synthetic power is still further and strikingly demonstrated by the fact that free fatty acids are readily digested but are transmitted to the lacteals in the form of the corresponding neutral fats, having evidently been combined with glycerol in the process of resorption, although the source from which the body derives its glycerol is still uncertain. Evidently, then, from this point of view, nothing 
stands in the way of the supposition that the digested fats are completely split up into glycerol and fatty acids in the process of digestion and synthesized again in the epithelial cells, although, on the other hand, of course, it does not prove that such is the case.

153. The feces. - As the contents of the digestive tract move forward through the small and large intestines they become. progressively more and more impoverished in digestible material and also, in the lower portion of the large intestine, are deprived of part of their water, so that there accumulates in the rectum a more or less solid residue which is voided at intervals as the feces.

The feces are to be regarded as both an excretory product (198) and a feed residue.

154. The feces as an excretory product. - The fact that the feces are an excretory product is most obvious in the carnivora, whose normal feed consists of substances almost wholly digestible, but it is evident also in man. On a pure meat diet, for example, feces continue to be produced in which undigested feed residues are either absent entirely or present in minimal amounts only. Even a fasting animal continues to produce feces, while an empty loop of the intestine, separated from the remainder of the digestive tract, soon fills up with fecal-like material.

The excretory ingredients of the feces include unresorbed digestive juices and their decomposition products, intestinal mucus, worn-out epithelial cells and cell fragments, leucocytes and excretions of the intestinal mucosa. Especially notable among the latter are salts of calcium and of iron and in herbivora the phosphates of calcium and magnesium. The feces also include a not inconsiderable proportion of intestinal microorganisms.

155. The feces as a feed residue. - The ordinary mixed diet of man, and to a much more marked degree the ordinary feed of herbivorous animals, contains relatively considerable amounts of materials which are either indigestible or which for one reason or another escape digestion and therefore reappear in the feces. Among these, some, like lignin, cutin, the waxes, chlorophyl and other non-fatty ingredients of the "ether extract," and the insoluble ash ingredients, may be regarded 
as wholly indigestible. Of more importance, however, are such carbohydrates as cellulose and the various hemicelluloses, the levulans, galactans, mannans, pentosans, etc., which may be said to be practically only partially digestible. By this is not meant, of course, that one molecule of cellulose, e.g., is any less digestible per se than another, but only that part of the cellulose of ordinary feeds does as a matter of fact escape digestion, largely because the length of time during which it is exposed to the action of the organisms which attack it is insufficient to allow of its complete solution. The feces of herbivorous animals, therefore, contain amounts of these diverse carbohydrates varying with the character of the feed and the activity of the fermentation processes in the digestive tract. Since these compounds are especially abundant in the roughages, the feces from these feeds are bulky and especially rich in undigested " crude fiber."

Other ingredients, particularly of vegetable feeding stuffs, partially escape digestion not on account of any lack of the appropriate digestive enzyms but because they are mechanically protected from the action of the latter. If granules of starch, e.g., are contained within a cell which has not been ruptured during the mastication of the feed, the cell wall tends to protect them from the action of the digestive juices, and they may escape digestion although per se entirely digestible. The extent to which such a nutrient will actually be digested, therefore, will depend to a considerable degree upon whether the cellulose of the cell wall is attacked and destroyed by the organisms of the alimentary canal. What is true of starch in this respect is obviously true of all cell enclosures, and explains why more or less matter intrinsically digestible may be rejected in the feces. For a like reason, seeds which escape mastication are but imperfectly digested, being protected by the relatively insoluble seed coats. Similarly, cellulose itself may be so impregnated with lignin and cutin substances that the "crude fiber" may be attacked only with difficulty or not at all by the methane fermentation.

Finally, there is to be considered the possibility of a misproportion between digestion and resorption. In heavy rations, especially, substances which are actually digested may perhaps escape resorption through insufficient contact with the 
intestinal walls or from lack of time, and so be found in the feces.

156. Composition of feces. - Evidently the feces are a very complex and variable mixture, including, on the one hand, the various excretory products just enumerated and, on the other hand, indigestible feed substances and digestible materials which have for one reason or another escaped actual digestion or which, having been digested, have failed of resorption. Among the latter may be included unresorbed products of the putrefaction of the proteins, especially skatol, which impart to the feces their offensive odor.

The proportions of these two groups - the excretory products and the feed residues - in the feces vary widely with the nature of the feed consumed. In the carnivora the body wastes predominate, so that the feces of these animals are to be regarded as primarily an excretory product. To a considerable degree the same thing is true of man, especially when living on a concentrated diet. With the herbivora, on the contrary, the indigestible or undigested feed residues constitute the bulk of the feces, although the amount of true excretory products is by no means insignificant. Omnivora like the hog occupy an intermediate position in this respect.

\section{§4. The Determination of Digestibility}

157. Definition of digestibility. - The words digestible and digestibility are used in more than one sense. Sometimes, for example, a food is said to be digestible because it is easily digested - that is, causes no unpleasant sensation after it is eaten - while by an indigestible food is meant one that is apt to cause gastric or intestinal disturbances. Again, it is not uncommon to judge of the digestibility of stock feeds by their effects and to regard that one as the more digestible which causes or seems to cause the greater gain in weight.

The word digestibility as used in the study of animal nutrition, however, has a definite and limited meaning. It denotes the percentage of the feed, or of any single ingredient of the feed, which is dissolved or otherwise acted on in the digestive canal so that it can be resorbed and thus put at the disposal of the body cells. For example, the digestion experiment with a steer 
described in a subsequent paragraph (160) showed that out of each roo grams of protein in the clover hay eaten by the animal 53 grams were apparently digested and resorbed. The digestibility of the protein in this case, therefore, is said to be 53 per cent. Digestibility in this sense is obviously a conception entirely distinct from that of rapidity or ease of digestion. A feed may have no injurious nor disagreeable effects and yet may have a low percentage digestibility - straw, for example.

Neither does the percentage digestibility alone determine the effect produced by a feed. Two feeds may be equally digestible and yet one may be more valuable than the other because its digested matter can be used to better advantage by the body. Nevertheless, it is clear that the indigestible portion of the feed can make no contribution to the nutrition of the body. The first step, therefore, although by no means the last one, in comparing the values of different feeds or rations is to determine as accurately as possible what proportion of each ingredient is capable of digestion. In other words, we shall seek to add to the qualitative knowledge of the processes of digestion and resorption outlined in the preceding sections a quantitative knowledge of the extent of digestion.

158. Method of digestion experiments. - The percentage digestibility of feeding stuffs can, as a rule, be determined only by trial with an animal. Such trials are called digestion experiments, and a brief outline of the way in which they are made will aid in understanding just what is meant by digestibility.

The method is substantially that originated by Henneberg and Stohmann in their early investigations (707). Since it is obviously impossible to collect and measure the substances digested and resorbed from the feed, it is necessary to have recourse to an indirect method, viz., to determine what portions of the feed are not digested and compute by difference the amounts digested. As already stated (155), the undigested portions of the feed are excreted in the feces. A digestion experiment really consists in determining as exactly as may be how much of the feed is thus rejected, any portions of it which disappear during its passage through the alimentary canal being regarded as digested.

If the feces consisted only of undigested feed residues, the matter would be very simple, but they also include a greater or 
less proportion of excretory products (154). In the case of herbivora, the proportion of the latter is relatively small and in the digestion experiment as ordinarily conducted is neglected, it being assumed, in other words, that the feces are equivalent to undigested feed residues. The same method is pursued in digestion experiments with swine, although in these animals the proportion of excretory products in the feces is larger. The error thus introduced into digestion experiments is not negligible, especially as regards certain ingredients. It will be convenient, however, to take up first the methods of digestion experiments as ordinarily conducted and to consider later the nature and extent of the errors introduced by neglecting the presence of the excretory products.

159. Time required for digestion experiments. - It is essential that a digestion experiment be preceded by a prelimi-

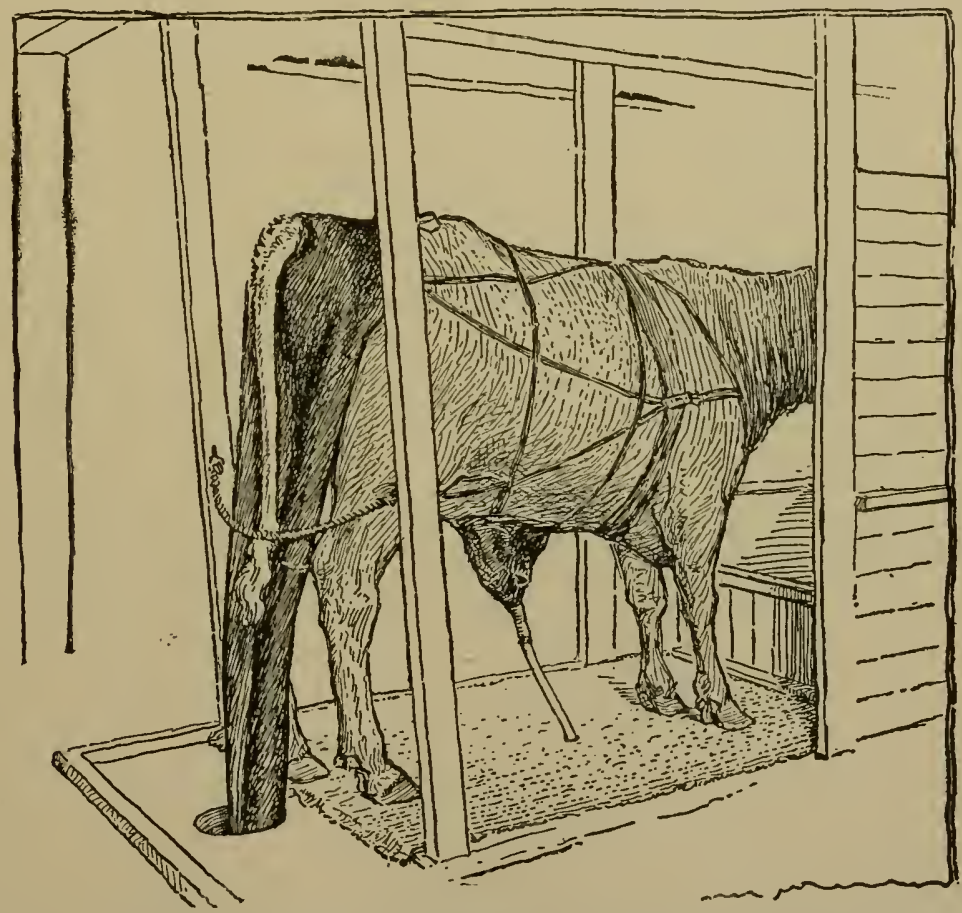

FIG. I5. - Steer in digestion stall. (Bailey's Cyclopedia of American Agriculture.)

nary period in which the feed to be investigated is fed in the same weighed amounts daily as in the actual experiment. This is for the purpose of removing from the digestive tract residues 
of previous feeds and also of establishing as uniform a rate of excretion of feces as practicable. In the case of ruminants, such a preliminary period should extend over one or two weeks, while with swine it may be made somewhat less. In the succeeding digestion experiment proper, the same feeding is continued and the feces are collected quantitatively for a number of days (seven to ten or more), in order to eliminate the error due to the irregularity of the excretion from day to day. From the weights of feeds and feces and their composition as determined by analysis, the digestibility is computed as illustrated in the following paragraphs.

160. Example of a digestion experiment. - A steer was fed 3.7 kilograms of clover hay per day for three weeks. During the last ten days of this time, the average weight of the daily feces was 5.662 kilograms. Samples of each were analyzed and found to contain the following percentages of dry matter.

$$
\begin{aligned}
& \text { Clover hay . . . . . . . } 84.97 \text { per cent } \\
& \text { Feces . . . . . . . } 22.36 \text { per cent }
\end{aligned}
$$

The 3.7 kilograms of hay, therefore, contained 3.144 kilograms of dry matter while the 5.662 kilograms of feces excreted contained only 1.267 kilograms of dry matter. The difference, I.877 kilograms, which did not appear in the feces, is regarded as having been digested by the steer. This amount is 59.7 per cent of the 3.144 kilograms eaten; we say, then, that the percentage digestibility of the dry matter of this hay was 59.7 and this number is sometimes called its "digestion coefficient."

In precisely the same way the percentage digestibility of each ingredient may be computed from the results of analyses of the hay

\begin{tabular}{|c|c|c|c|c|c|c|c|c|c|c|}
\hline & & & & & & & & & $\begin{array}{c}\mathrm{HAY} \\
\%\end{array}$ & $\begin{array}{c}\text { FECES } \\
\%\end{array}$ \\
\hline Water . & . . . & & & & & & & & 15.03 & 77.64 \\
\hline Ash . . & . . . & . & . & . & . & • & . & . & 5.49 & I.92 \\
\hline Protein & . . . & . & . & . & . & . & . & . & 10.24 & $3.13^{1}$ \\
\hline Non-protein & . . . & . & . & . & . & . & . & . & I. 36 & - \\
\hline Crude fiber & . . & . & . & . & . & . & & & $28.6 \mathrm{I}$ & 9.29 \\
\hline Nitrogen-free & extract & & . & . & . & . & . & . & 36.98 & $7 \cdot 50$ \\
\hline \multirow{2}{*}{ Ether extract } & 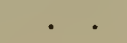 & & & . & . & & & & 2.29 & $0.5^{2}$ \\
\hline & & & & & & & & & 100.00 & 100.00 \\
\hline
\end{tabular}
and of the feces, which in this case gave the following results:-

1 All the nitrogen of the feces is here assumed to exist in the form of protein, an assumption which, as will appear later, is far from being true (166), but which does not affect the method of computation. 
These figures, together with the weights of hay eaten and feces excreted per day, yield the following results :-

Table 20. - Results of a Digestion Experiment

\begin{tabular}{|c|c|c|c|c|c|c|c|}
\hline & \begin{tabular}{|} 
DRY \\
MATTER \\
Kgs.
\end{tabular} & $\begin{array}{l}\text { Ash } \\
\text { Kgs. }\end{array}$ & $\begin{array}{l}\text { Pro- } \\
\text { TẸIN } \\
\text { Kgs. }\end{array}$ & $\begin{array}{c}\text { Non- } \\
\text { PROTEIN } \\
\text { Kgs. }\end{array}$ & $\begin{array}{l}\text { CRude } \\
\text { FIBer } \\
\\
\text { Kgs. }\end{array}$ & $\begin{array}{c}\text { NITRO- } \\
\text { GEN } \\
\text { FREE } \\
\text { I EX- } \\
\text { TRACT } \\
\text { Kgs. }\end{array}$ & $\begin{array}{c}\text { ETHER } \\
\text { Ex- } \\
\text { TRACT } \\
\text { Kgs. }\end{array}$ \\
\hline $\begin{array}{l}\text { In hay eaten } . \\
\text { In feces excreted. }\end{array}$ & $\begin{array}{l}3.144 \\
\mathrm{I} .267 \\
\end{array}$ & $\begin{array}{l}0.203 \\
0.109 \\
\end{array}$ & $\begin{array}{l}0.379 \\
0.177 \\
\end{array}$ & $\stackrel{0.050}{-}$ & $\begin{array}{l}1.059 \\
0.526 \\
\end{array}$ & $\begin{array}{l}1.368 \\
0.425 \\
\end{array}$ & $\begin{array}{l}0.085 \\
0.030 \\
\end{array}$ \\
\hline $\begin{array}{l}\text { Difference }=\text { digested } \\
\text { Percentage digesti- } \\
\text { bility . . . . . }\end{array}$ & $\begin{array}{l}1.877 \\
59.70\end{array}$ & $\begin{array}{l}0.094 \\
46.48\end{array}$ & $\begin{array}{l}0.202 \\
53.19\end{array}$ & $\begin{array}{l}0.050 \\
100.00\end{array}$ & $\begin{array}{l}0.533 \\
50.27\end{array}$ & $\begin{array}{l}0.943 \\
68.94\end{array}$ & $\begin{array}{l}0.055 \\
65.02\end{array}$ \\
\hline
\end{tabular}

161. Digestibility of concentrates. - The method just outlined for determining the digestibility of a roughage or of a total ration is in conception very simple. The determination of the digestibility of concentrates by herbivora is somewhat more complicated, since they cannot be made the sole feed of these animals. They must, therefore, be fed along with a known amount of a roughage whose digestibility by the same animal is likewise determined in a preceding or following period. From the digestibility of the total ration and the known digestibility of the roughage that of the concentrate is obtained by means of a second calculation by difference.

Thus, the same steer used in the experiment of the preceding paragraph received per day in a subsequent period the same amount, 3.7 kilograms, of clover hay and in addition 4 kilograms of maize meal. The average daily excretion of feces on this mixed ration was 8.7 I5 kilograms. An analysis of the clover hay used in this period showed but slight variations from that of the preceding period. The composition of the maize meal and of the feces was:-

\begin{tabular}{cc} 
MaIZE MEAL & FECES \\
$\%$ & $\%$ \\
I 3.73 & 81.91 \\
1.25 & 1.77 \\
8.80 & 3.66 \\
0.25 & - \\
I.89 & $6.5 \mathrm{I}$ \\
70.44 & $5.7 \mathrm{I}$ \\
3.64 & 0.44 \\
\hline IO0.00 & IO0.00
\end{tabular}


The digestible matter contained in the total ration, computed exactly as in the previous example, was as shown in the first part of Table 2I. If, now, it be assumed that the digestibility of the clover hay was unaltered by the addition of the maize meal, it is possible to compute how much of each kind of digestible matter (protein, crude fiber, nitrogen-free extract, etc.) in the total ration was derived from the hay; the remainder, therefore, must have come from the maize meal and by comparison with the total amounts present in the latter the percentage digestibility is computed.

It is evident that the determination of the digestibility of a concentrate in this way is less accurate than that of a feed which can be given by itself. The assumption that the digestibility of the roughage is not changed is unproved and probably not strictly correct. Moreover, any errors arising from this source and likewise all the errors in weighing and analysis are, by the method of calculation, assigned to the concentrate. The writer has shown ${ }^{1}$ that the range of uncertainty thus introduced may be very wide. It will evidently be greatest when the proportion of concentrate to roughage is least and will affect most those ingredients of the concentrate which are present in the smallest proportion, such as crude fiber and often ether extract. In extreme cases, absurd results are sometimes obtained, such as negative digestibility or a digestibility greater than roo per cent.

\section{Laboratory determination of digestibility. - Actual} digestion experiments upon animals according to the method just outlined, while simple in principle, require special facilities and a considerable expenditure of time. It would obviously be very desirable to possess methods by which the action of the digestive fluids of the body could be imitated in the laboratory and the digestibility of feeds thus determined in a simpler and more expeditious manner.

Numerous attempts have been made to solve this problem, but as yet a satisfactory method has been worked out only for protein, while attempts to devise similar methods for the non-nitrogenous ingredients of feeding stuffs have not yet proven successful. The method for protein is based upon suggestions made long ago by Stöckhardt and by Hofmeister, but was first put into practical form by Stutzer. ${ }^{2}$ It consists in treating the feed with a solution of pepsin and hydrochloric acid under specified conditions and determining the undissolved nitrogen in the residue. The difference between this and the

${ }^{1}$ Amer. Jour. Sci., 29 (I885), 355.

${ }^{2}$ Jour. Landw., 28 (I880), 195 and 435. 
Table 21. - Computation of the Digestibility of a Concentrate

\begin{tabular}{|c|c|c|c|c|}
\hline 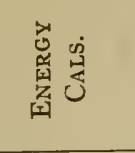 & 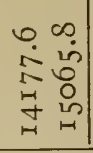 & 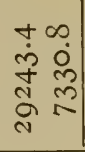 & 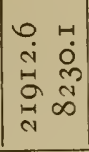 & 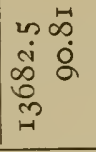 \\
\hline 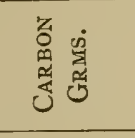 & 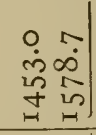 & $\begin{array}{ll} & 0 \\
\dot{H} & 0 \\
0 & 0 \\
0 & 1\end{array}$ & 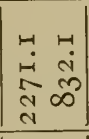 & 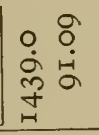 \\
\hline 产兽兽 & \begin{tabular}{cc|}
0 & 0 \\
& 0 \\
0 & 0
\end{tabular} & $\left|\begin{array}{cc|}0 & -4 \\
\dot{m} & - \\
\ddot{m} & \text { n. }\end{array}\right|$ & $\left|\begin{array}{cc}9 & 0 \\
\dot{d} & \dot{f} \\
\infty & \end{array}\right|$ & 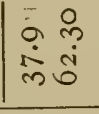 \\
\hline 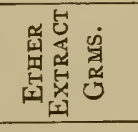 & $\begin{array}{ll}+ & 0 \\
\dot{厶} & \dot{\varphi} \\
0 & \end{array}$ & 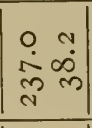 & 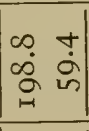 & $\mid \begin{array}{l}+ \\
\dot{j} \\
\dot{j} \\
\dot{m}\end{array}$ \\
\hline 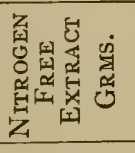 & 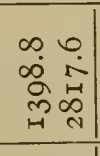 & 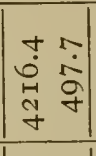 & $\left|\begin{array}{ll}0 & ? \\
0 & \dot{d} \\
0 & 0 \\
\tilde{m} & 0\end{array}\right|$ & 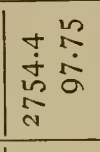 \\
\hline 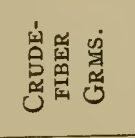 & 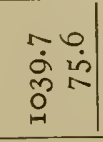 & 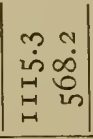 & 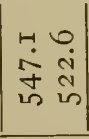 & 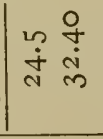 \\
\hline 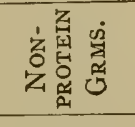 & 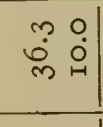 & $\left|\begin{array}{ll}\tilde{b} & 1 \\
\dot{b} & 1\end{array}\right|$ & $\ddot{q} \stackrel{m}{\dot{q}}$ & $\mid \begin{array}{ll}0 & 0 \\
0 & \dot{0} \\
0 & 0 \\
& 0 \\
\end{array}$ \\
\hline 宽总兽 & $\begin{array}{cc}20 & 0 \\
0 & 0 \\
0 & 0 \\
+ & n\end{array}$ & $\left|\begin{array}{ll}n & m \\
0 & 0 \\
0 & 0 \\
1 & m\end{array}\right|$ & \begin{tabular}{|ll|} 
& 2 \\
\cline { 1 - 1 } & 0 \\
& 0 \\
& 0 \\
\end{tabular} & 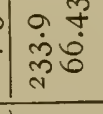 \\
\hline 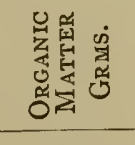 & \begin{tabular}{cc}
$\infty$ & $\infty$ \\
$\dot{1}$ & $\dot{0}$ \\
$\hat{\alpha}$ & \multicolumn{1}{c}{} \\
& 0
\end{tabular} & 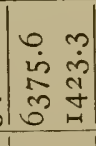 & $\begin{array}{ll}m & 0 \\
i & 0 \\
2 & 0 \\
2 & 0 \\
\dot{a} & 0 \\
& 4\end{array}$ & $\mid \begin{array}{ll}0 & 8 \\
\dot{j} & 0 \\
\dot{+} & \alpha \\
m\end{array}$ \\
\hline 累密 & 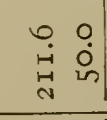 & 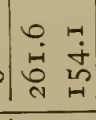 & 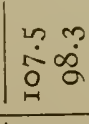 & 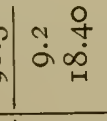 \\
\hline 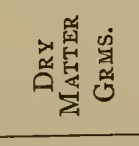 & $\begin{array}{cc}+\infty \\
\dot{0} \\
\dot{0} \\
\infty & 0 \\
1 & \stackrel{n}{1} \\
n & m\end{array}$ & 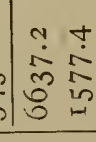 & 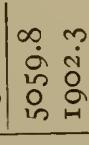 & 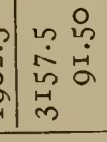 \\
\hline & 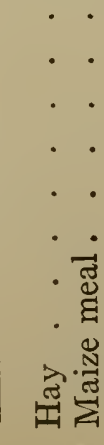 & 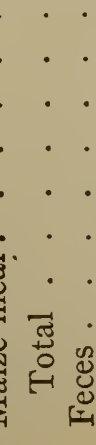 & 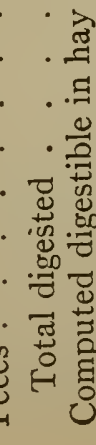 & 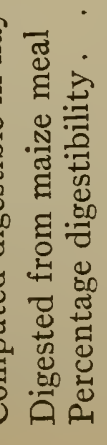 \\
\hline
\end{tabular}


total nitrogen of the feed represents, of course, the amount of nitrogenous matter which has been dissolved and which, therefore, is regarded as digestible.

Comparisons by Kellner, ${ }^{1}$ Pfeiffer, ${ }^{2}$ G. Kühn ${ }^{3}$ and others between the natural and artificial digestion of protein have shown that the former method gives lower results on account of the presence in the feces of nitrogenous excretory products $(\mathbf{1 5 4}, \mathbf{1 5 8})$, but that when a correction is made for the latter in the manner indicated on a subsequent page (166) the results of the two methods show a substantial agreement. In other words, the method of artificial digestion shows with a good degree of accuracy the true as compared with the apparent digestibility $(163,167)$ of the protein.

163. Influence of excretory products on apparent digestibility. - Since the digestion experiment as ordinarily conducted ignores the presence in the feces of excretory products, the results obtained by its use will necessarily be too low, since substances are reckoned as undigested ingredients which really are not such. Obviously, the ingredients most affected by this error will be those which, on the one hand, are contained in the feed in the smallest proportion and which, on the other hand, are relatively most abundant among the excretory products in the feces. These ingredients are, when the ordinary scheme of feeding stuffs analysis is followed, ash, ether extract and nitrogenous substances. As regards the crude fiber, on the other hand, this error is absent, since obviously no crude fiber is included among the excretory products, and it seems probable that substantially the same thing is true of the nitrogen-free extract.

164. Digestibility of ash ingredients. - Certain ash ingredients, particularly iron, calcium, magnesium and phosphorus, are largely or wholly excreted from the body in the feces (199). Furthermore, the resorption of the ash ingredients of the digestive juices may not be complete and these residues may be added to the ash content of the feces. The ordinary digestion experiment, therefore, affords little information as to the extent to which the ash ingredients of the feed are actually

1 Centbl. Agr. Chem., 9 (1880), 763.

2 Jour. Landw., 33 (I 885 ), I 49; 34 (I 886), 425.

${ }^{3}$ Landw. Vers. Stat., 44 (1 894 ), r 88. 
digested and resorbed and this fact constitutes a serious difficulty in the study of the ash metabolism.

165. Digestibility of ether extract. - Among the excretory products contained in the feces are included ether-soluble substances, especially those derived from unresorbed bile constituents. While their total amount is small, the feed of farm animals is also usually poor in ether extract and consequently the error in the computation of the percentage digestibility may be relatively large. Indeed, not a few instances are on record in which the ether extract of the feces has exceeded that of the feed. Little definite knowledge is available, however, as to the actual extent of the error thus introduced, but it is of relatively less importance in view of the small rôle which fat plays in the ordinary rations of farm animals.

166. Digestibility of nitrogenous substances. - Most of the excretory products in the feces (154) are nitrogenous substances and it is particularly with reference to their influence upon the determination of the digestibility of the nitrogenous constituents of feeding stuffs that investigation has been active. That they may seriously affect it is evident from the results obtained in numerous experiments upon feeding stuffs poor in protein, such as straw, in which a negative digestibility of the crude protein has been observed, - i.e., in which the feces have contained more nitrogen than the feed. Moreover, experiments upon rations containing no nitrogen at all have shown that under these conditions nitrogen continues to be excreted in the feces.

Various methods for distinguishing between the nitrogen of feed residues and the nitrogen of excretory products have been proposed at different times, but the one which has proved most satisfactory and which is generally accepted at present is based upon the solubility of the nitrogenous excretory products in the solution of pepsin and hydrochloric acid employed in Stutzer's method for the laboratory determination of the digestibility of protein described in a previous paragraph (162).

By treatment of a sample of the fresh feces with such a solution under proper conditions the excretory nitrogenous products are dissolved, and it has been shown that very close agreement can be obtained between the artificial and natural digestion of protein if the comparison in the latter case be made upon the pepsin-insoluble nitrogen of the feces. In other words, the 
pepsin-insoluble nitrogen of the feeds appears quantitatively in the feces, where it may be regarded as representing indigestible feed protein, while the pepsin-soluble nitrogen of the feces is contained in the excretory products, part of which are protein (mucus, epithelium, etc.) and part non-protein (residues of digestive fluids, etc.). An approximate correction for the amount of nitrogenous excretory products may also be computed by the use of Pfeiffer's factor of 0.4 gram nitrogen per Ioo grams digested dry matter.

167. Apparent digestibility. - When the results of the ordinary digestion experiment are corrected, in the manner just outlined, for the nitrogenous excretory products in the feces we get an approximation to the true percentage digestibility of the protein, while, as regards the carbohydrates, the error, as has been shown, is probably not serious, at least for herbivora.

There is another way of looking at the matter, however. The intestinal products found in the feces are, in effect, part of the cost of digesting the feed. They represent the "wear and tear" of the digestive organs. The difference, then, between feed and feces will show the net gain to the animal from the digestion of the feed, that is, it will show how much more proteins, carbohydrates, etc., the body has at its disposal than it would have had if the feed had not been given. From this point of view, we may speak of the digestibility as ordinarily determined as the apparent digestibility, and regard it as a measure (approximately at least) of the matter gained by the body from the consumption of the feed. For many purposes, therefore, the apparent digestibility gives a better basis for comparing the values of feeding stuffs than does the real digestibility. It was from this point of view that Atwater ${ }^{1}$ proposed the use of the term availability as the equivalent of what is here called apparent digestibility.

168. Composition of digested crude fiber. - The crude fiber (109) consists of the cellulose of the plant together with varying amounts of pentosans and of lignin and other incrusting substances, the ratio of which to the cellulose increases with the maturity of the plant. Cellulose itself seems to be attacked and dissolved with comparative ease by the organisms of the rumen and the cœcum, and the same is probably true of the

${ }^{1}$ Rpt. Conn. (Storrs) Expt. Sta., I897, p. 156. 
pentosans, but lignin appears to be much less readily digested and some of the other incrusting materials not at all. As a consequence, a computation based on the elementary composition of the crude fiber of the feed and of the feces respectively and on the percentage of the former which is digestible shows the digested portion to have approximately the ultimate composition and heat of combustion of cellulose.

This is by no means equivalent to saying that the digested crude fiber consists only of cellulose. The variations between the results in individual experiments show clearly that this cannot be the case and doubtless more or less of the pentosans and other ingredients of the crude fiber are attacked to some extent, but it is nevertheless evident that the cellulose is the chief constituent digested. Neither is the heat of combustion of the digested portion in any sense a measure of the energy which it can supply for the bodily activities, as will appear more clearly later.

169. Composition of digested nitrogen-free extract. - By a difference calculation identical in principle with that employed for crude fiber but somewhat more complicated in its details and involving certain assumptions, it has been shown that the digested portion of the nitrogen-free extract has also approximately the composition and heat of combustion of starch or cellulose. Even less than in the case of crude fiber does this fact serve to fix with any definiteness the nutritive value of the digested portion. We know that the nitrogenfree extract of feeding stuffs includes a great variety of substances (110), some of which, like starch, are digested in the narrower sense of the word while many others, like the hemicelluloses, pentosans, etc., are fermented rather than digested. The data as to the composition of the digested portion indicate, it is true, that it consists chiefly of carbohydrates, but on account of the small range of ultimate composition shown by these substances no indications are afforded of the specific carbohydrates present.

170. Digestible carbohydrates. - Since both the digested crude fiber and the digested nitrogen-free extract have approximately an ultimate composition corresponding to the formula $\mathrm{C}_{6} \mathrm{H}_{10} \mathrm{O}_{5}$, it has become customary in estimating the nutritive values of feeding stuffs to add together the digestible portions 
of these two groups and to designate the sum as the " digestible carbohydrates." The practice dates from the early experiments of Henneberg and Stohmann, but in the light of our present knowledge has little justification.

In the first place, as just stated, the agreement in composition is but approximate and variable. The essential point, however, is that a digestion experiment can show simply that a certain amount of material of a certain ultimate composition has failed to reappear in the feces of the animal, and by itself affords no information as to the changes which it has undergone nor as to the nature of the products actually resorbed. As a matter of fact, a large share of the "digested " portion of these two groups, especially in the case of ruminants, has been fermented rather than digested. A considerable proportion of it has been excreted in gaseous form as carbon dioxid and methane and only a residue of organic acids has been resorbed. Such being the case, the term digestible carbohydrates is a palpable misnomer.

171. Digested ether extract. - No determinations of the composition of the digested ether extract similar to those on crude fiber have been made, but a few determinations of the heat of combustion of the digested extract are reported by Kellner. ${ }^{1}$ The presence in the feces of ether soluble excretory products (165) interferes with the accuracy of such a comparison and its results must be regarded as approximations. The ether extract of the feces was found to have a higher heat of combustion than that of the hay fed, doubtless on account of the presence in the former of the indigestible waxes, etc., while the computed heat of combustion of the digested portion was distinctly lower than that for pure fats, which average about 9.5 Cals. per gram. The heats of combustion per gram on the average of five trials were:-

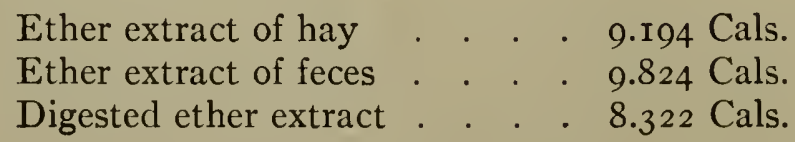

${ }^{1}$ Landw. Vers. Stat., 47 (I 896), 30 I. 


\section{CHAPTER IV}

\section{CIRCULATION, RESPIRATION AND EXCRETION ${ }^{1}$}

\section{$\S$ I. Circulation}

172. Distribution of nutrients. - The digestive changes by which the ingredients of the feed are prepared for the nutrition of the organism take place outside the body proper (113). In order that the products formed shall serve their purpose they must not only be taken up into the body by the processes of resorption described in the preceding chapter but they must be distributed through it, so that each of its myriad cells may receive the substances which it requires. The chief vehicle of this distribution is the blood, into which the resorbed nutrients are transferred, directly or indirectly, and the distribution is accomplished by means of the circulation of the blood, discovered by Harvey in I62I.

173. The blood. - This familiar but highly complex fluid serves a variety of purposes, being not only the carrier of the resorbed feed ingredients to the tissues and cells but transmitting to them the equally necessary oxygen and carrying away the products of their activity to be used in other parts of the body or to be excreted.

The blood of the higher animals is a thickish, somewhat viscid fluid, having a faint but peculiar odor, a slightly salt taste and a color varying from bright to dark red. It is somewhat heavier than water (sp. gr. I.045-I.075), and contains about 2I per cent of total solids. Under the microscope it is seen to consist of a clear fluid, the plasma, holding in suspension a vast number of small, solid bodies, the corpuscles. The latter are of two kinds, known as the red corpuscles, or erythrocytes, and the white corpuscles, or leucocytes.

1 Only such a very general consideration of the outlines of these functions as seems essential for a proper comprehension of the phenomena of metabolism and of the processes of nutrition is attempted here. For a more complete elementary discussion, the reader is referred to Hough and Sedgwick's The Human Mechanism, Chapters IX, X and XI, and for further details to the larger treatises on physiology. 
174. Red blood corpuscles. - These are by far the more numerous of the two kinds. In man they are round like a coin but thicker at the edges than in the center, and have a diameter of $0.0060-0.0085$ millimeter. Their number is enormous, being estimated at 4 to $5 \frac{1}{2}$ millions per cubic millimeter of blood. To them the color and opacity of the blood are due.

The corpuscles of each species of animal are peculiar to it, both

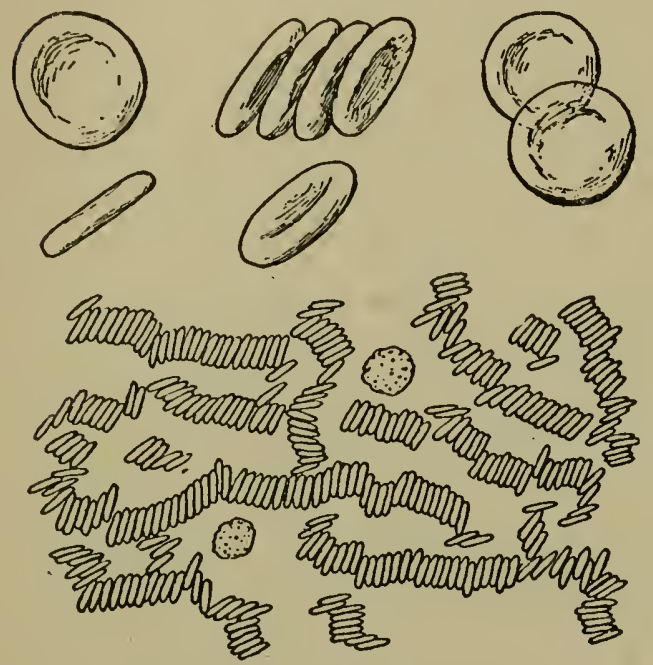

Fig. I6. - Blood corpuscles.

Above are shown nine red corpuscles, highly magnified; below, less highly magnified, the appearance of the blood soon after being drawn. (Hough and Sedgwick, The Human Mechanism.)

as to shape and size, but their general characteristics are the same in all. Those of most animalsaresmaller than those of man. Each corpuscle is a cell, having no nucleus but containing as its characteristic ingredient the conjugated protein hæmoglobin to which the red color of the blood is due. Hæmoglobin is a crystalline substance and it has recently been shown by Reichert that the hæmoglobin of each species of animal has its specific crystalline form and properties.

175. White blood corpuscles. - The white corpuscles are colorless, nucleated cells which are not confined to the blood but which, by means of ameboid movements, are able to pass through the walls of the blood vessels and the lymph spaces of connective tissue as the so-called "wandering cells." They have important functions, especially in protecting the body from disease, but need not be further considered here.

176. Blood platelets. - In addition to the two kinds of corpuscles, the blood contains more minute nucleated cells, ranging in diameter from $0.0003-0.0005$ millimeter, called blood platelets, or thrombocytes. They are much more abundant than the white corpuscles and are thought to be concerned in the coagulation of the blood. 
177. Blood plasma. - This very complex fluid contains, besides about 90 per cent of water, a great variety of substances, the most prominent of which are the proteins, of which two groups are recognized, viz., two or more serum globulins and the so-called serum albumin, which is probably not a single chemical individual. Plasma contains also approximately O.I-O.I5 per cent of dextrose, from O.I to as much as I.O per cent of fat, usually in some soluble form (243), a great variety of so-called extractives which are in part waste products of cell action, and about I per cent of mineral ingredients.

178. Coagulation. - When blood is drawn from the body it usually coagulates or clots within a few minutes. The coagulating substance is a globulin called fibrinogen and its coagulation is an enzymatic reaction brought about by a ferment, thrombin, believed to be derived from the blood platelets by a very complicated process. The coagulated protein constitutes the so-called blood fibrin, which entangles within itself the corpuscles, producing the familiar blood clot. While the clot is very bulky the dry blood fibrin amounts to only $0.2-$ 0.3 per cent of the weight of the blood.

179. The heart. The blood is distributed to all parts of the body by means of

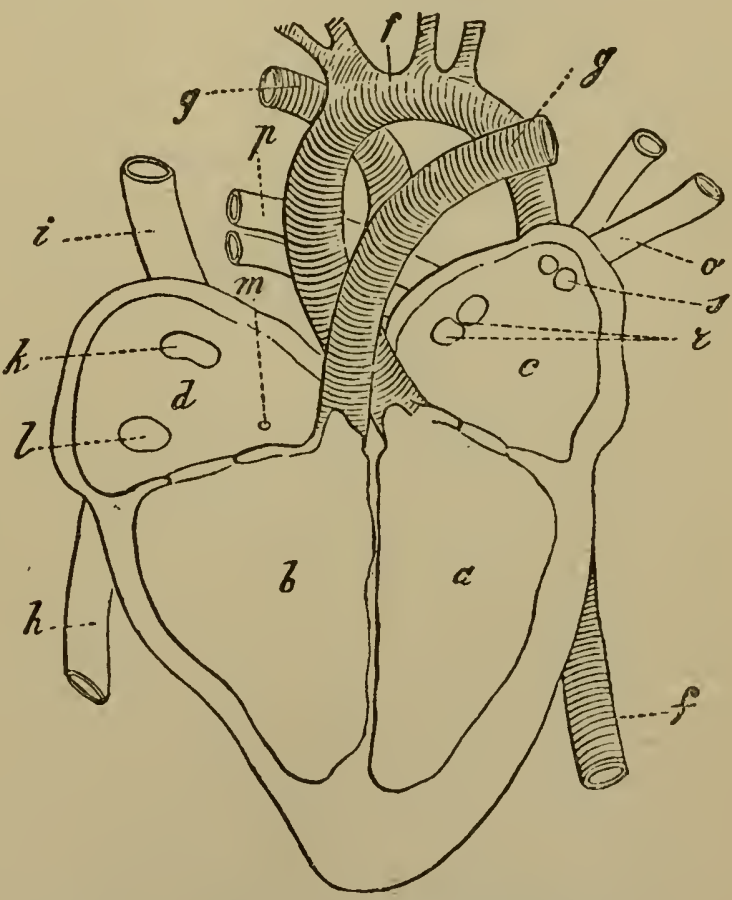

Frg. I7. - Diagram of mammalian heart.

$a$, Left ventricle. $b$, Right ventricle. $c$, Left auricle. $d$, Right auricle. $f$, Aorta. $g g$, Pulmonary arteries. $o p$. . Pulmonary veins. (Smith, Physiology of the Domestic Animals.)

a most interesting organ, the heart, which is in effect a living force pump. Figure 17 shows diagrammatically the structure of the mam- 
malian heart, which is substantially the same in all farm animals.

It is divided by an impervious partition into a right and left half, and each of these is subdivided by a cross partition into two chambers, communicating with each other by a valve in the dividing wall. The upper and smaller of these divisions are known as the right and left auricles, and the lower and larger as the right and left ventricles. Into these cavities of the heart open several large blood vessels, whose mouths are closed with valves so arranged that the blood can only flow into the auricles and out of the ventricles.

180. Arteries. - The blood vessels which conduct the blood from the heart to the various organs of the body are called arteries and may be described as tubes with strong, elastic and contractile walls, to withstand the force with which the blood is pumped into them by the heart. Their walls consist of an outer layer of elastic and connective tissue, a middle layer of muscular tissue and an inner layer of epithelium. The arteries originate in the aorta ( $h$, Fig. 18$)$, which receives the blood from the left ventricle, and as they extend farther and farther from the heart subdivide and throw off branches to the various organs, the more minute of which are called arterioles, finally ending in the capillaries.

181. Capillaries. - The capillaries are exceedingly minute blood vessels which penetrate all the tissues of the body and form the connecting link between the arteries and veins. Their walls are thin and delicate, and through them the nutritive mattars of the blood pass out into the tissues while the waste products of cell activity pass from the tissues into the blood. In Fig. I $8, n$ represents the capillaries of the posterior part of the body, $o$ those of the stomach and intestines, $t$ those of the kidneys, $p$ those of the liver, and $m$ those of the anterior part of the body. The capillaries gradually unite again into larger vessels, the veins, which convey the blood back to the heart and lungs.

182. Veins. - The veins are tubular vessels somewhat similar to the arteries but with weaker and non-elastic walls, the pressure of the blood on them being slight, owing to the interposition of the capillaries between them and the arteries and to the fact that their total cross section is greater than that of the 
arteries. To prevent any possible flowing back of the blood, the veins are provided at intervals with valves which permit the blood to pass toward the heart but not in the opposite direction. The smaller veins unite to form larger ones, and finally empty their contents through two branches into the right auricle of the heart. From the capillaries of the intestines the blood carrying the resorbed nutrients passes through the portal vein, $s$, to the liver, $p$, is there distributed through another system of capillaries and then rejoins the blood from the extremities through the hepatic vein, $u$. Into the branch, $k$, coming from the head and anterior parts of the body, the nutrients which are resorbed by the lacteals enter by way of the thoracic duct.

183. Course of the blood. The blood returning through the veins from the extremities of the body to the heart enters first the right auricle ( $a$, Fig. I8), through two large veins, $k$ and $l$, coming from the anterior and posterior parts of the body. The auricle then contracts and the blood, being prevented from returning into the veins by the valves at their mouths, is forced through the valve in the partition wall into the right ventricle, $b$. This,

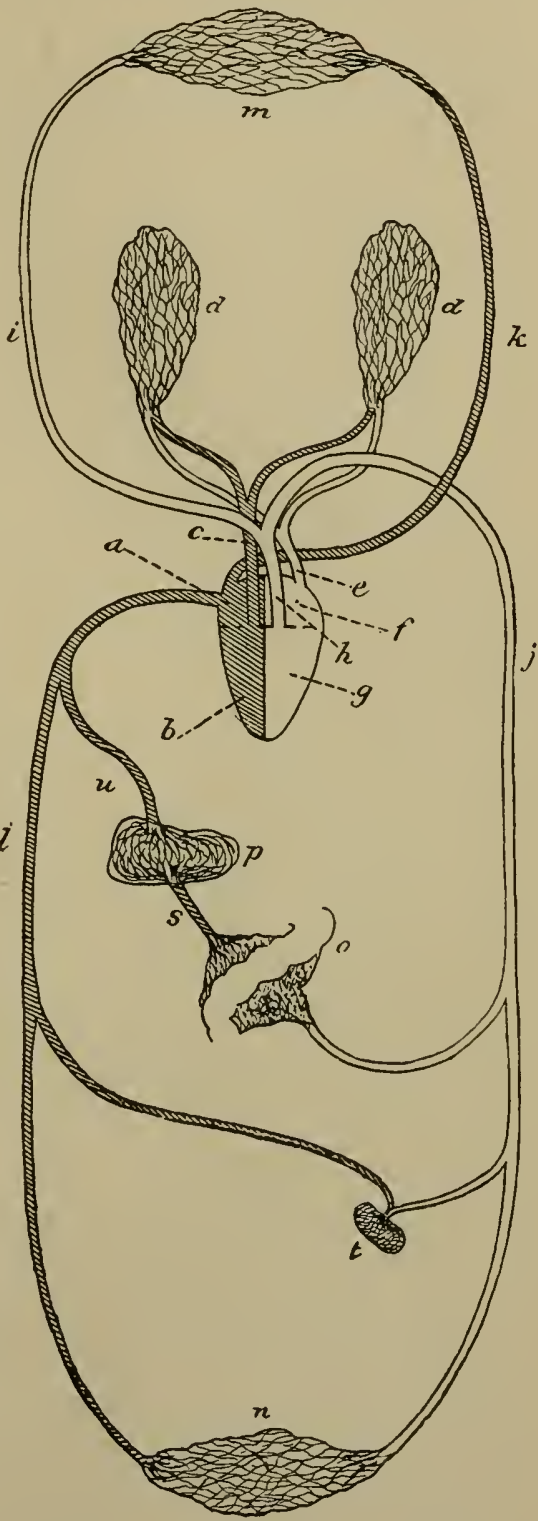

FIG. I8. - Scheme of circulation of blood. (Armsby, Manual of Cattle Feeding.) in turn, contracting, the blood, prevented as before by a valve from turning back in its course, is forced out of the 
ventricle into the pulmonary artery, $c$, which divides into two branches leading to the capillaries of the right and left lungs, $d, d$. The entrance to this blood vessel, like that of the others, is provided with a valve which prevents the return of the blood. The blood, after passing through the lung capillaries, returns to the left auricle, $f$, through the pulmonary veins, represented by $e$. The auricle then contracting, sends the blood into the left ventricle, $g$, which, in its turn, contracts powerfully and expels the blood into one -large vessel, the aorta, $h$. The aorta, soon after leaving the heart, divides into two branches, $i$ and $j$, and these repeatedly subdivide, forming the arteries which carry the blood to the arterioles and capillaries, whence it returns again through the veins to the right side of the heart.

The passage of the blood from the left side of the heart through the body capillaries and back to the right side is called the greater or systemic circulation; that from the right side of the heart through the lung capillaries, the pulmonary circulation.

The appearance of the blood in the veins and arteries is strikingly different. In the veins it has a dark, cherry-red color, but after it has passed through the lungs and is sent out by the heart to the arteries it has a bright scarlet color. The former is called venous, the latter, arterial blood. An exception to this rule, that the arteries carry bright red blood and the veins dark, is found in the pulmonary circulation, where, of course, the vessels leading from the heart to the lungs carry venous blood, and those leading from the lungs to the heart, arterial. Nevertheless, the general nomenclature is adhered to, and the former are called arteries and the latter veins. Arteries conduct the blood from the heart, veins toward it.

184. Mechanics of circulation. - While it is not uncommon to speak of the flow of the blood, or of the blood stream, suggesting an analogy to a brook or river, the circulation is not in reality a flow of this sort but resembles rather the movement of the water pumped into a hose by a force pump. The heart constitutes the force pump and the arteries correspond to the hose. The powerful muscular contraction of the ventricle drives the blood into the arteries by successive impulses, as the water is driven into the hose by the pump. If the end of the hose were left open the water would issue in a series of spurts 
corresponding to the strokes of the pump. By the addition of a nozzle of smaller diameter than the hose this intermittent outflow is converted into a steady stream. The resistance of the nozzle to the passage of the water gives rise to a pressure which stretches the walls of the hose, and their elastic force maintains the flow between the strokes of the pump.

Substantially the same conditions exist in the body. The walls of the arteries are elastic while the capillaries in which the arteries terminate may be compared to the nozzle of the hose. The resistance caused to the flow of the blood by these minute channels tends to hold it back and produces a pressure in the arteries which, like the pressure in the hose, causes a steady movement of blood through the capillaries. In other words, the immediate cause of the motion of the blood through the capillaries is the elasticity of the arterial walls. If the latter become weakened and lose their tone or become hardened as in old age (arteriosclerosis), the driving force is lessened and the circulation slows down, since the veins can return blood to the heart only as fast as it is forced through the capillaries by the arterial pressure. The blood pressure in the arteries, therefore, is an important indicator of the activity of the circulatory system. The veins serve substantially as a return system, the blood being pushed along them by the residual pressure from the capillaries, perhaps aided somewhat by the expansion of the auricle of the heart, while valves prevent any backward flow. As compared with the arterial pressure, therefore, the blood pressure in the veins is low.

185. The lymph. - The body cells are not closely packed together but are imbedded more or less loosely in connective tissue (83) leaving spaces between them (intercellular spaces). These spaces contain a colorless transparent fluid called the lymph which is the real nutritive medium in which the cells live. From it, by means of osmosis through their outer membranes and perhaps in other ways, the cells derive the substances required for their vital activities and into it they discharge the waste products of their action.

The lymph in its turn stands in relation to the blood, from which it is separated by the thin walls of the capillaries. While the minute capillaries penetrate all the tissues and convey blood to all parts of the body, it should be understood that the cir- 
culatory apparatus is a closed system. Even the very thin delicate walls of the capillaries are continuous and the blood does not come into direct contact with the living cells, except, of course, those lining the blood vessels. The accompanying diagram (Fig. I9) illustrates schematically the anatomical relations of the cells, intercellular spaces, capillaries and lymphatics, $A$ representing a minute artery, or arteriole, subdividing into capillaries which are reunited to form the small vein $V$. Through the capillary walls the nutritive substances contained in the blood pass, partly by osmosis and partly by

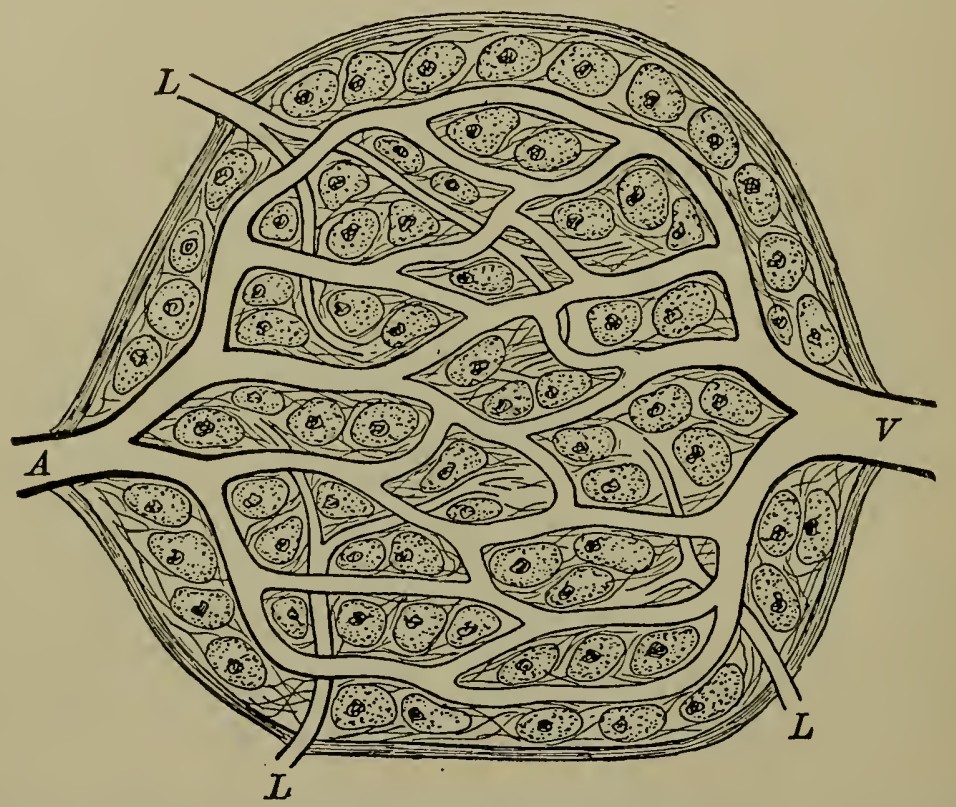

Fig. 19. - Relation of cells to blood vessels and lymphatics. (Hough and Sedgwick, The Human Mechanism.)

filtration, in to the lymph to maintain its stock, while the waste products of cell action pass in the opposite direction into the blood and are carried off.

186. Lymphatics. - In the intercellular spaces there originates another set of minute vessels, the lymphatics, which unite like the capillaries to form larger ones ( $L$ in Fig. I9) and finally form two main lymphatic trunks, the thoracic duct and the small lymphatic trunk, which empty into the great veins near the heart. The lacteals of the intestinal villi, through which the fats are chiefly resorbed, belong to the lymphatic system. 
In the lymphatics there is a continuous slow movement of the lymph from the tissues towards the main trunks, the lymphatics, like the veins, being provided at intervals with valves preventing a backward flow. This lymph flow is sustained in part by a slightly greater pressure in the lymphatic spaces but largely by the rhythmic motions of breathing, and is aided by muscular activity. Thus, in addition to the exchange of substances between the lymph and the blood through the walls of the capillaries, there is a general movement of the lymph itself over the surface of the cells which tends to facilitate the exchanges between it and the protoplasm.

187. Adjustment of circulation. - The activity of the various tissues varies at different times. A muscle, for example, is sometimes at rest and sometimes actively contracting. Consequently, a greater or less supply of food material and of oxygen will suffice according to circumstances, and the blood supply needs to be regulated accordingly.

This regulation is effected in substantially two ways. First, when the cells of any particular tissue increase their activity they consume more oxygen and give off more waste products than be-

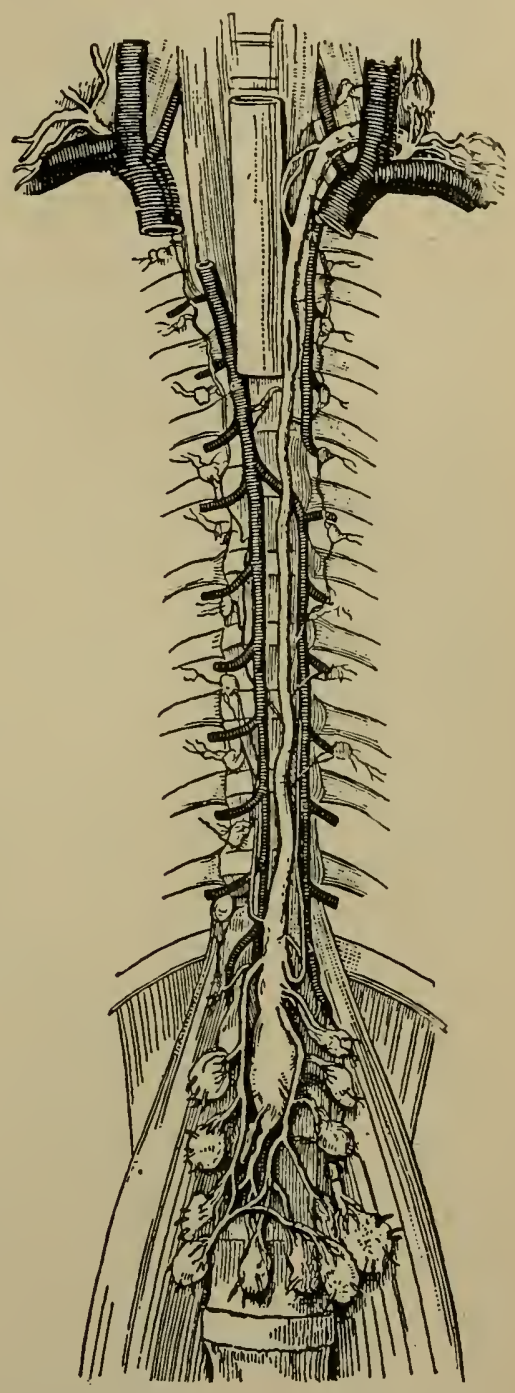

FIG. 20. - Main lymphatic trunks (in white). (Hough and Sedgwick, fore, tending to produce a relative The Human Mechanism.) deficiency of the one and an excess of the other in the lymph and blood. These conditions bring about an increase in the heart action (194), probably by means of a nerve stimulus, so that the amount of blood 
passing through the heart is increased and a more abundant supply of it reaches the active tissues. Second, there may be a partial shunting of the blood supply from one region of the body to another as one set of organs or another calls for a larger amount. This is accomplished through the agency of the middle or muscular coat of the arterioles, controlled by the so-called vaso-motor nerves. When a larger supply of blood is called for in the muscles, for example, these fibers relax and allow the arterioles to enlarge, thus reducing the resistance offered to the blood flow and allowing the arterial pressure to force blood into the capillaries more rapidly. To compensate for this there is a contraction of the arterioles of the internal organs, especially of the abdominal organs, resulting in a diminished blood supply. The effect of the performance of work upon digestion, discussed in Chapter XVI (721), is possibly connected with this effect upon the blood flow. On the other hand, after a hearty meal the arterioles of the digestive tract relax, while the superficial blood vessels tend to contract and the blood supply is partially diverted from the surface tissues to the internal organs. This power of the body to regulate the supply of blood to different regions is of special importance, as will appear later (321), in connection with the regulation of the body temperature.

\section{$\S$ 2. RESPIRATION}

188. The oxygen supply. - By means of the processes described in the preceding section the nutritive materials derived from the feed and taken up by the intestinal capillaries and lacteals are distributed to the various tissues and cells. Equally necessary with a supply of feed materials to the living cells, however, is a supply of oxygen, and this another set of organs, those of respiration, are engaged in furnishing to the blood through another set of capillaries for transmission to the cells.

1.89. The lungs. - The transfer of oxygen from the air to the blood is effected in the lungs, which, with the heart and large blood vessels, fill the cavity of the thorax, or chest. This cavity is enclosed on the sides by the ribs and their connections, forming the chest walls, and is separated from the abdominal cavity, containing the digestive organs, by a strong, arched, mus- 
cular partition, convex toward the chest, the diaphragm. The air enters the lungs through the trachea, or windpipe, from the mouth and nostrils. The trachea, after reaching the chest, divides into two branches, or bronchi, one leading to the right and one to the left lung. Each bronchus subdivides repeatedly into a multitude of fine tubes, the smallest of which are called bronchioles (little bronchi), each of which finally ends in an alveolus, the inner surface of which is much increased by being arranged in

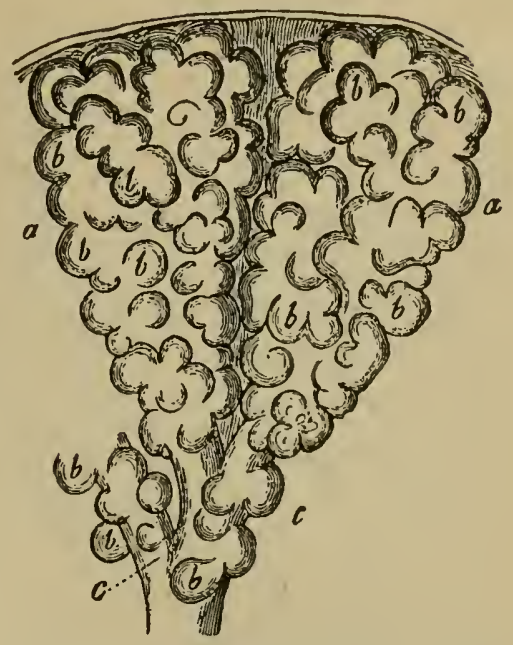

FIG. 21. - Alveoli of lung. (Wilckens, Form und Leben der Landwirthschaftlichen Hausthiere.) the form of pits or air cells.

In Fig. 2I, $c$ represents a bronchiolus, $a a$ two alveoli and $b b$ the air cells. Figure 22 shows diagrammatically on a large scale a cross section of two alveoli.

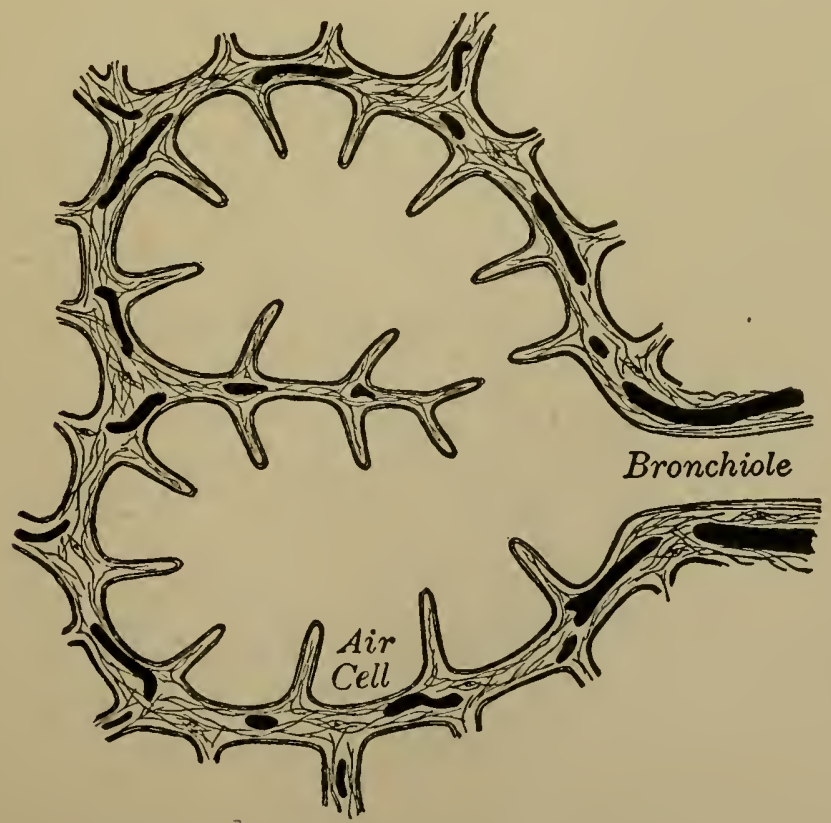

Fig. 22. - Section of two alveoli. (Hough and Sedgwick, The Human Mechanism.) 
The walls of the trachea and bronchi consist of cartilaginous rings which prevent them from collapsing. The alveoli and bronchioles are surrounded and bound together by connective tissue consisting largely of elastic fibers so that the minute air cavities of the lungs are extensible and their walls elastic. In this connective tissue are found the larger branches of the pulmonary artery and pulmonary vein, connected by a network of capillaries which are spread out over the inside of the alveoli in direct contact with their lining membrane. Each lung is enclosed in a double-walled sack, the pleura, one wall of which covers the lungs and the other the chest walls and diaphragm, the narrow cavity between the two being filled with a liquid.

190. Mechanics of breathing. - In breathing, the lungs themselves play an essentially passive rôle, the movement of air into and out of them being effected by changes in the capacity of the chest brought about by the movements of the diaphragm and ribs.

Since the diaphragm is convex toward the chest its contraction tends to pull the apex of the dome toward the abdomen, thus increasing the volume of the chest cavity and by pressure on the digestive organs distending the abdominal walls. When the diaphragm relaxes again the volume of the chest is reduced and the abdominal walls return to their former position. This type of breathing is what is called abdominal breathing.

The ribs pass obliquely around the chest from the spine to the breast bone (sternum). By means of the intercostal muscles, located between them, the ribs can be elevated, turning on their attachments to the spine and sternum, thus increasing the diameter of the chest both from side to side and from front to back and so increasing the capacity of the chest cavity. This type of breathing is called costal, or rib, breathing.

The two types of breathing are ordinarily combined. By their joint action the size of the closed pleural cavity containing the lungs is increased and the atmospheric pressure forces more air into the extensible alveoli of the lungs, so that the latter expand along with the chest cavity, the whole constituting the act of inspiration, or breathing in. When the diaphragm and the intercostal muscles relax, the elasticity of the 
chest walls causes them to return to their original position and this, together with the elasticity of the lung tissue itself, compresses the air in the alveoli and forces part of it out through the trachea, this constituting the movement of expiration.

Inspiration is an active process, while expiration is chiefly passive. The respiratory movements are ordinarily what are called involuntary, i.e., they go on independent of consciousness, being governed by automatic nerve impulses, conveyed by nerves of various origin but controlled by the so-called " respiratory center," although the movements can be accelerated or retarded or even suspended entirely for a few moments by an effort of the will.

From the foregoing, it is plain that the ventilation of the lungs does not consist in the passage of air through them but of a surging or tidal movement in and out. The alveoli are never entirely emptied of air even in forced expiration. In inspiration the new or tidal air enters the trachea and bronchi, gives up by diffusion some of its oxygen to the residual air in the alveoli and receives from the latter some of the carbon dioxid which it contains. In this way, by the ebb and flow of the tidal air and by diffusion between it and the residual air, fresh oxygen is being continually introduced into the lungs and carbon dioxid continually removed.

191. Absorption of oxygen. - The oxygen introduced into the alveoli of the lungs in the manner just described is still outside the body proper, just as is the feed in the digestive tract. In order to fulfill its functions it, like the feed, must be transmitted to the blood for distribution to the tissues. This transfer is accomplished in the lung capillaries as is that of the feed in the intestinal capillaries. In the lung capillaries the blood is separated from the air of the alveoli only by a thin membrane. The coloring matter of the red corpuscles, hæmoglobin, has the power of entering into combination with oxygen, of which it can take up a maximum of about r.66 c.c. per gram, forming a loose chemical compound known as oxyhæmoglobin. The red corpuscles of the venous blood as it comes to the lungs contain chiefly hæmoglobin. In their passage through the lung capillaries they are exposed to the oxygen of the alveolar air and, aided by the relatively large surface of the blood corpuscles, their hæmoglobin takes up more or less oxygen and is 
converted partly or wholly into oxyhæmoglobin, the amount of the oxygen taken up ranging from eight to twelve volume per cent. The color of hæmoglobin is a dark red or purplish, while that of oxyhæmoglobin is bright scarlet. To this difference of color is due the marked difference in appearance between venous and arterial blood.

192. Respiration of tissues. - The term respiration is very commonly applied to the mechanical processes of breathing just described or to the exchange of gases in the lungs. In reality all these are preliminary to the real respiration, which takes place in the tissues. The vital processes in the body cells consist, broadly speaking, as will appear in detail in the next chapter, of a series of oxidations. The requisite oxygen is necessarily drawn from the lymph in which the cells exist(185), while the carbon dioxid produced by oxidation is discharged into it. The lymph, therefore, tends continually to become richer in carbon dioxid and poorer in oxygen. In the manner just described the blood takes up oxygen in the lungs and acts as a carrier through the body. Through the capillary blood vessels of the body generally, therefore, there are continually passing red blood corpuscles charged with loosely combined oxygen, while on the other side of the capillary wall is a fluid (the lymph) in which the partial pressure of oxygen is relatively low. Accordingly, the combination of oxygen and hæmoglobin is dissociated to a greater or less extent and oxygen passes into the lymph as required to supply the needs of the cells. At the same time the excess of carbon dioxid in the lymph passes in the opposite direction into the blood and is thus removed from the neighborhood of the cell. ${ }^{1}$ It is this continual consumption of oxygen and elimination of carbon dioxid by the cells which constitutes the real act of respiration, while the complex structure of the lungs and the elaborate mechanism of breathing and of the blood corpuscles are simply means for providing oxygen to the cells and taking away carbon dioxid. That the movements of breathing are not an essential part of respiration is strikingly shown by the fact that it is perfectly possible by suitable devices to maintain oxygenation of the blood

${ }^{1}$ In these exchanges, as in other similar ones, while diffusion doubtless plays a large part, its effects are no doubt modified by the special properties of the living cells. 
of an animal in the absence of any respiratory movements whatever.

193. Respiration regulated by cell activity. - It is apparent from the foregoing that the amount of oxygen taken up by the blood in the lungs depends in the first instance upon the amount of this element consumed by the body cells. When they are relatively inactive they take up correspondingly little oxygen from the lymph and the tension of oxygen in the latter is lowered but little. As a consequence there is less dissociation of the oxyhæmoglobin in the blood and the corpuscles tend to return to the lungs still carrying more or less oxygen and therefore capable of taking up relatively less. On the other hand, as the tissues become more active they consume more oxygen, the oxyhæmoglobin in the corpuscles is more extensively dissociated and the corpuscles tend to come back to the lungs relatively exhausted of oxygen and ready to take up the maximum amount.

Any considerable degree of tissue activity, however, calls for a more rapid supply of oxygen than can be provided for in this way and this need is met by a nerve stimulus to the heart, causing it to beat faster and more powerfully, thus increasing the arterial pressure and therefore the amount of blood passing through the capillaries in a given time. In these two ways the amount of oxygen absorbed in the lungs is very accurately adjusted to the needs of the organism. It is impossible to stimulate the body oxidations by a free supply of air as, for example, by deep and rapid breathing, as one might blow up a fire with a bellows, or to get more intense combustion by replacing air with pure oxygen. In the body such additional air or oxygen never reaches the fire. Each corpuscle is a receptacle which can carry only a definite amount of oxygen and if it comes back to the source still partly filled it takes up so much the less on its next trip, or if it travels slowly it is less efficient than if it returns more frequently. The respiration of the tissues can no more be affected by increasing the ventilation of the lungs than the amount of water delivered by a pump is by the volume of the stream from which the water is taken.

194. Regulation of the rhythm of breathing. - The illustration just used is true, of course, only on the condition that the stream carries at least as much water as the pump can handle. So, too, the amount of oxygen available in the lungs 
must at least equal the amount required by the tissues. It is a familiar observation that the rate of ventilation of the lungs varies with the varying activity of the body cells. This is true of all these activities, but is most familiar in the case of muscular work which, as everyone knows, promptly increases the rate and depth of breathing, so that severe exercise, such as rapid running, for example, brings into play all the reserve resources of the breathing mechanism. As already stated (190), the muscles which are used in breathing are ordinarily controlled from the so-called "respiratory center" and it is through this center that the regulation is effected. If, for example, an animal be supplied with air largely diluted with some indifferent gas, such as nitrogen or hydrogen, the partial pressure of the oxygen in the alveoli is so reduced that the hæmoglobin of the blood is only partially saturated with oxygen. Such a deficiency of oxygen stimulates the respiratory center and produces more active breathing and a corresponding increase in the rapidity with which the air in the alveoli (residual air) is renewed.

Under ordinary conditions, however, the stimulus to the respiratory center is not a lack of oxygen in the blood but an excess of carbon dioxid. As has already been implied, the lungs serve not only for the absorption of oxygen but for the elimination of the carbon dioxid produced by respiration, which passes by way of the lymph to the blood and thence to the air in the alveoli of the lungs. Any increase in the activity of the tissues by which more carbon dioxid is produced tends to increase the content of this substance in the blood. Even a very slight increase, however, promptly stimulates the respiratory center and so causes greater activity of the muscles concerned, resulting especially in deeper and to some extent more rapid breathing. By this means the ventilation of the lungs is augmented and so provision is made for the removal of a greater amount of carbon dioxid.

It is plain, however, that a simple increase in the lung ventilation alone is not sufficient, except in a limited degree, to carry away more carbon dioxid from the tissues. Along with the increased ventilation there must be an increase in the rapidity of the blood current which is the medium by which the transfer of gases between the lungs and the lymph takes place. Accordingly, 
we find that substantially the same stimuli which cause more active breathing also stimulate the heart action and vice versa.

Lack of oxygen or excess of carbon dioxid are the two principal factors in regulating the breathing rhythm but by no means the only ones. They are, however, the ones of most importance in the present connection.

195. Gaseous exchange through the skin. - In addition to the exchange of gases between the air and the blood which goes on in the lungs, a similar process takes place, though to a much smaller extent, through the skin. The true skin, underlying the cuticle or scarf-skin, is penetrated by capillary blood vessels, and in its passage through these capillaries the blood gives off some carbon dioxid and takes up some oxygen by diffusion through the skin. The amounts given off and taken up are small compared with the corresponding amounts in the lungs, but still are not inconsiderable, and must be taken into account in accurate experimental work.

\section{§3. EXCRETION}

196. Excretory products. - As already implied, the vital activities of the body cells lead to the formation of products which must be removed from the cells and some of which must ultimately be discharged from the body. The next chapter will be concerned with the nature of the more important of these products and with some of the steps by which they are formed. For the present, it suffices to say that the gradual oxidations of non-nitrogenous material taking place in the cells give rise substantially to the production of carbon dioxid and water, while the proteins and related substances yield in addition certain comparatively simple nitrogenous substances of which the most abundant is urea. In addition to these substances, more or less of the mineral ingredients also pass into the excreta.

197. Excretion of carbon dioxid. - As stated in the previous section, the carbon dioxid produced by the tissue respiration passes by way of the lymph into the blood and is excreted through the lungs and to a minor degree through the skin. In the blood the carbon dioxid is carried by both the corpuscles and the plasma, but chiefly (two-thirds or more) by the latter, in combination with proteins and hæmoglobins, but especially 
with the alkalies. As in the case of oxygen, the amount of carbon dioxid contained in the blood depends upon the partial pressure of this gas in the surrounding medium. Since the tension of the carbon dioxid in the alveolar air is less than that in the blood of the alveolar capillaries, the carbon dioxid passes from the latter to the former. If the air were stationary the process would continue until an equilibrium was reached. Since the air is being continually renewed by breathing, the tension of carbon dioxid in it is kept permanently lower than that in the blood and there is, therefore, a continual passage of carbon dioxid from the blood to the alveolar air.

It is by means of this tendency to equilibrium that the mechanism for the regulation of breathing is set in motion. Increased tissue respiration discharges more carbon dioxid into the blood, where its tension increases. This causes a more rapid diffusion of the gas into the alveolar air and tends to raise its carbon dioxid tension also, so that with an unchanged rate of lung ventilation the carbon dioxid level of both the alveolar air and the blood would be raised. Even a very slight rise in the carbon dioxid tension in the blood, however, as already stated, acts promptly upon the respiratory center and stimulates the muscles of breathing, resulting in an increased lung ventilation and consequently a more rapid excretion. At the same time the rapidity of circulation is increased and in these two ways the level of carbon dioxid tension in the blood and in the alveolar air is maintained very constant. On the other hand, if the lung ventilation be artificially increased, as by artificial respiration or by the use of oxygen, the carbon dioxid excretion may be so facilitated that the amount in the blood falls below the normal and the movements of breathing may be temporarily suspended (apnœa).

198. Excretion of nitrogenous products. - The urea and other nitrogenous products of cell action, like the non-nitrogenous products, pass ultimately into the blood. In its course through the body the blood passes through a capillary system in two bean-shaped organs, the kidneys, indicated by $t$ in Fig. I8, situated in the abdominal cavity on either side of the spine near the loins. In these organs the urine is being continually secreted, passing thence through the ureters into the bladder from whence it is voided at intervals. 
The chief stimulus to the secretion of water by the kidneys is the water content of the blood, the kidneys acting as regulators of this important factor and eliminating more or less water as the blood contains a larger or smaller percentage of it.

As regards the excretion of dissolved matter, very interesting relations exist. With one important exception (hippuric acid) the kidneys do not manufacture the excretory products. Their essential function is to maintain the composition of the blood constant. For each substance capable of being excreted at all in the urine there exists a certain minimum concentration in the blood above which it begins to pass through the kidneys into the urine. For the normal excretory products, as well as for foreign substances, this minimum approaches zero, that is, only very minute amounts of these substances can be retained in the blood. For dextrose the limit is approximately $0.2-0.3$ per cent, for sodium chlorid 0.6 per cent, etc. So long as the percentage of one of these substances in the blood does not exceed its own particular limit, none of it is excreted through the kidneys. On the other hand, a slight rise above this limit causes an excretion of the substance concerned. This function of the kidneys has been likened to the working of an overflow valve on a tank. It should be added that each particular substance has its own minimum,.independent to a large degree of all the others.

The functions of the kidneys, however, in this respect are not so simple as those of an overflow valve for the reason that the concentration of the excreted substances is greater in the urine than in the blood. In other words, the kidney does its work by transferring substances from a fluid of lower to a fluid of higher osmotic pressure and the expenditure of energy in this work is not inconsiderable. This is notably true of urea and the other nitrogenous waste products, of which only traces can be detected in the blood.

In addition to the nitrogenous substances excreted in the urine there are present in the feces, as already noted (154), excretory products which represent a certain fraction of the organic body waste. Finally, small amounts of urea and other nitrogenous substances are excreted in the perspiration.

199. Excretion of ash ingredients. - Being non-volatile the ash ingredients are excreted chiefly through the feces or 
urine according as the intestines or kidneys form the normal path of excretion, although they are contained to a small extent also in the perspiration.

The intestines are the usual path of excretion for certain mineral substances, notably iron, calcium and to some extent magnesium. To these must be added in the case of the herbivora phosphoric acid, which, under ordinary conditions, is excreted in the feces. The urine of herbivora, especially when they consume roughage freely, or in more general terms when the basic predominate over the acid ingredients of the ash, is alkaline and contains but minute amounts of phosphoric acid. On the other hand, during fasting or upon a ration having an acid ash, the urine may have an acid reaction and then, like the acid urine of carnivora or omnivora, may contain phosphoric acid. The urine is the normal vehicle for the excretion of sulphur, chlorin and the alkalies.

200. Excretion of water. - The motions of air in and out of the lungs are the means of removing from the body more or less incidentally large amounts of water by simple evaporation. The presence of water vapor in the expired air is a familiar fact, shown by its condensation on a cold surface or in cold air. The skin likewise acts, by means of its sweat-glands, as a channel for the removal of water from the system, considerable being continually evaporating from the skin in the form of the "insensible perspiration." Under certain circumstances the excretion of water is so rapid as to give rise to the formation of visible drops (sweating).

The amounts of water excreted in these two ways are larger than are sometimes realized. For example, a thousand pound ox at ordinary temperature and on light feed may easily excrete through the lungs and skin eight to ten pounds of water in twenty-four hours, the amount depending to a considerable extent upon the temperature and amount of movement of the surrounding air. The feces also contain a large percentage of water and in the case of herbivorous animals the amount thus eliminated is very considerable.

Finally, water is excreted in the urine, serving as a solvent for the nitrogenous products of cell activity which are removed through this channel. The amount of water thus excreted depends in part upon the amount consumed, in part upon the 
quantity of nitrogenous material which must be dissolved and in part upon the amount eliminated through the lungs and skin.

Most of the water excreted by animals is, of course, consumed as such, but it includes also that formed by the oxidation of organic hydrogen - the so-called metabolic water. Babcock has shown that in some classes of animals, notably insects, this metabolic water suffices for all the needs of the organism, so that they are not dependent upon a supply of drinking water. 


\section{CHAPTER V}

\section{METABOLISM}

\section{General Conception}

201. Assimilation and excretion. - The cell has already been defined (73) as the biological unit of life. It is the living protoplasm of the body cells which is the seat of the multifarious activities of the organism.

Every such activity requires an expenditure of energy, derived from the breaking down of constituents of the protoplasm itself or of cell enclosures and solutes and their transformation into other forms. The presence of oxygen is essential to these changes and while, as will appear, they seldom are primarily direct oxidations; nevertheless, they yield products which are ultimately oxidized to carbon dioxid, water and other comparatively simple compounds.

Two things, then, are necessary for the continued life of the cell: first, a supply of material from without to replace that consumed and, second, the removal of the waste products of its activities. Both conditions are fulfilled in the higher animals by the circulation of the blood and lymph. In the processes of digestion, the heterogeneous nutritive materials contained in the feed are gradually brought into solution by a series of molecular cleavages, so that the resorptive organs transmit to the blood and lymph current a qualitatively uniform material consisting of substances of comparatively simple molecular structure $(146,147)$, while oxygen is supplied to the blood corpuscles through the lung capillaries. The mechanism of circulation is continually distributing to each tissue and cell oxygen from the lungs and nutritive material from the digestive tract and carrying away the waste products of cell action to the various organs of excretion which remove them from the body.

202. Definition of metabolism. - It is clear from the foregoing that the body is the seat of extensive chemical trans- 
formations. On the one hand, molecules of resorbed digestion products are being assimilated by the body cells and built up into the structure of their protoplasm, while, on the other hand, molecules of protoplasm or of cell enclosures are being broken down and oxidized, yielding finally the relatively simple excretory products.

The term metabolism is commonly used to designate the totality of the chemical changes which the constituents of the resorbed feed undergo in the course of their conversion into the corresponding excretory products. Similarly, one may speak in a more restricted sense of the metabolism of single ingredients of the feed, as of the proteins, carbohydrates or fats, protein metabolism, for example, signifying the chemical changes undergone by the digestion products of the proteins of the feed during their assimilation and subsequent transformation into excretory products. The adjective metabolic is also used to describe these chemical changes.

203. Anabolism and katabolism. - The term metabolism, as just defined, includes processes of two distinct kinds, viz., those by which molecules of sugars, organic acids, amino acids, etc., are built up into more complex compounds in the body and those by which these complex compounds are broken down again into simpler substances and finally into the excretory products.

The building up metabolism has received the name anabolism, while the breaking down or oxidative phase is called katabolism. Any change in the direction of greater molecular complexity is spoken of as an anabolic change, while one in the direction of greater molecular simplicity is a katabolic change.

It must not be inferred from what has been said that anabolism always precedes katabolism. Neither is the breaking down of cell constituents by any means a process of uninterrupted katabolism. On the contrary, many instances are known in which it is interrupted at various stages by anabolic changes of one sort or another. While the general direction of the change is towards simplification, there are eddies in the current. Moreover, it is by no means probable that all the resorbed substances are actually built up into protoplasm before being katabolized. It is true that, to the best of our knowledge, the metabolic processes take place within the cells but it appears unlikely that the relatively large amounts of material some- 
times katabolized must first become integral parts of the protoplasm. In other words, it is probable that the cells have the power to katabolize substances present within them but not structurally a part of them.

204. Synthetic processes in the body. - The foregoing conception of metabolism implies that the body has power to carry out extensive chemical syntheses, contrary to the idea still current that the course of chemical change in the organic world is toward the building up of complex compounds in the plant and their breaking down to simpler ones in the animal. Synthethic chemical changes were long regarded as peculiar to the vegetable kingdom, while the reactions in the animal body were supposed to be exclusively analytic. The first synthetic action to be recognized in the animal was the formation of hippuric acid from benzoic acid, discovered by Keller and Wöhler in I824, and which attracted wide attention. More recent physiological investigations have shown that this is by no means an isolated case, but that syntheses in great variety are executed in the animal body. No such sharp distinction between animal and vegetable organisms exists as was formerly supposed. The fundamental laws of metabolism are the same for both and both execute synthetic as well as analytic processes. It is only the special synthetic activity of the chlorophyl in green plants which tends to obscure this fundamental likeness. The conception, then, that the digestive cleavages supply to the body cells comparatively simple "building stones" which are synthesized to produce the complex ingredients of cells and tissues is quite in harmony with our general knowledge of the nature of metabolism.

205. Metabolism oxidative and analytic. - Metabolism regarded as a whole may be characterized chemically as an oxidation. Oxygen is introduced into the system through the blood and reacts with the feed or tissue materials or with the products of their breaking down, and the final excretory products are either completely oxidized substances, like carbon dioxid and water, or substances approaching this condition, like urea, etc.

From a slightly different point of view, metabolism as a whole may be characterized as an analytic as opposed to a synthetic process. The general tendency is toward the formation of simpler molecules. For example, the molecule of dextrose or levulose contains 24 atoms and those of the three most common fats, respectively, I55, I67 and I73 atoms, while the molecules of carbon dioxid and water resulting from their metab- 
olism contain but 3 atoms each. Even the cleavage products of protein which are resorbed from the digestive tract are, with few exceptions, much more complex than the final products which result from their metabolism.

206. Metabolism a gradual process. - While metabolism has just been characterized as an oxidative process, and is often loosely spoken of as a burning of the feed or tissue ingredients, it is in fact radically different from what is commonly understood by these terms. The building up and breaking down of materials in metabolism is a gradual, i.e., a step by step, process.

Metabolism is the sum of the chemical reactions through which the life of the cells is manifested. These reactions, however, differ from tissue to tissue and from cell to cell, and even in the same cell from time to time, and the resulting products are correspondingly numerous and varied. Between the nutrients supplied to the cells by the blood and the final products of metabolism as excreted from the body there are innumerable intermediate products, comparatively few of which, in all probability, have been recognized. We know the first and last terms of the series and thus are able to measure, as it were, the algebraic sum of the changes, but of the single factors making up the so-called intermediary metabolism as well as of the specific tissues concerned in the changes, we are largely ignorant, although we know that they are numerous.

Furthermore, while metabolism results in the formation of highly oxidized products, it does not consist primarily in the direct union of oxygen with feed materials, i.e., the step by step processes of which it is made up do not consist of a series of partial oxidations. The primary processes of metabolism are of the nature of cleavages and hydrations and it is only the comparatively simple molecules resulting from these which unite directly with oxygen. Correspondingly, the extent of metabolism is determined by the amount of functional activity of the various cells and not, as in the case of direct oxidation in a fire, by the supply of oxygen (193). The somewhat common notion that an increased proportion of oxygen in the air or a voluntary increase in the rate and depth of breathing may cause more material to be oxidized in the body is without foundation, except so far as increased breathing involves increased muscular exertion. 
207. Purpose of metabolism. - As implied at the opening of this chapter, the vital activities of the body are essentially transformations of energy. The living body is continually doing work upon its surroundings and continually loosing heat to them and the energy for the production of work and the maintenance of the body temperature is derived, as already stated, from the transformation of the chemical energy contained in the substances broken down, this transformation being indeed the essence of the whole process. This fact is familiarly, if not altogether accurately, expressed in the statement that the feed is the fuel of the body.

There will be occasion later to consider this aspect of the matter in detail, but it is important at the outset to grasp the conception that the final end and aim of metabolism is to supply energy for the vital activities and that the demand for energy is the controlling factor in all its processes. It is these transformations of energy which, if not synonymous with life, are at least its objective manifestation.

But while it is essential to hold fast to this broad general conception of metabolism, it is also important to understand clearly that the processes by which this end is reached are exceedingly complex. A volume would be required for any adequate discussion even of existing knowledge regarding the details of the metabolic processes. Such a discussion lies outside the scope of the present work. All that is attempted in this chapter is to outline the metabolism of the principal groups of feed substances and, as preliminary to a subsequent consideration of their values as sources of matter and energy to the body, to indicate the functions which they perform in the building up and maintenance of the organism and the support of its activities.

\section{§ 2. Enzyms as Agents in Metabolism}

Enzym action has come to play so large a part, even if a more or less hypothetical one, in the current conceptions of the processes of metabolism that a brief outline of the prevailing views seems called for.

208. Extracellular enzyms. - The enzyms of the digestive tract are those which are most familiar in physiology. As has been seen (114), the digestion of all three of the chief classes 
of feed ingredients is brought about largely or wholly by their agency and is often effected by different enzyms in successive stages. Thus the ptyalin of the saliva converts starch into maltose while the further conversion of the latter into dextrose is effected by the maltase of the intestine. Quite similar are the successive actions of pepsin, trypsin and erepsin on the proteins. In all these cases, as well as in the even more familiar case of the diastase of germinating seeds, the enzyms act at a distance from the cells which produce them and have, therefore, been called extracellular enzyms.

209. Intracellular enzyms. - From the fact that the most obvious cases of enzym action were those in which the ferment acted at a distance from the cells producing it, enzyms came to be regarded as substances whose action belonged in a different category from that of living cells. A sharp distinction was drawn between unorganized substances, acting substantially as chemical reagents, and organisms producing chemical changes by virtue of their life. The action of the yeast plant upon sugar afforded a typical example of this distinction. It was shown that yeast secreted an enzym (invertase) which was capable of inverting sucrose independently of the action of the yeast cell,. while, on the other hand, the alcoholic fermentation of monosaccharids was held to be a vital function of the living yeast cells.

Buchner, however, in 1897 , showed that by suitable means there could be extracted from yeast a substance (zymase) which fermented the simple sugars exactly like yeast in the absence of any living organism whatever; i.e., it acted as an enzym. It became evident, then, that the yeast cell ferments monosaccharids not because it is alive but because it contains zymase. The only essential difference between the yeast fermentation and that, for example, produced by diastase or by the invertase of yeast is that the enzym normally acts within the cell which produces it. Later it was shown that what is true of the yeast fermentation is true also of the fermentation caused by the lactic acid bacillus. It, too, is due to an intracellular enzym which can be separated from the cell and act independently. Investigators are inclined, therefore, to regard all fermentation as the work of enzyms, some of which, like the digestive enzyms, are excreted by the cells and may 
act at a considerable distance from their point of origin, while others normally produce their effect within the secreting cell.

210. Intracellular enzyms in the body. - Still more recently the presence of intracellular enzyms in all parts of the animal body has been recognized. It has been shown that a very considerable variety of reactions which are known to take place in the body may also be brought about outside the body by the action of extracts of various tissues and organs under conditions apparently excluding the action of any living organisms. Consequently, they have been ascribed to the action of enzyms originally present in the cells, and the reactions in the body have been regarded as due to these same enzyms. The idea of intracellular enzyms has thus been extended to account for the metabolic activities of the organism, and this explanation has been very generally accepted by physiologists. According to this view, the body cells bring about metabolic changes substantially in the same way as do the cells of yeast or of the lactic acid bacillus, viz., by the formation of appropriate enzyms which act upon the substances to be metabolized. This phase of the subject is a comparatively new one and unanimity as to individual cases has by no means been reached, but of the value of the general conception as a working hypothesis there can be little question.

The word explanation is used above, of course, in a limited sense. It is not known how the cell produces enzyms, nor with any degree of certainty how an enzym acts. Nevertheless, this hypothesis, if confirmed, is a real explanation as far as it goes, in that it enables related phenomena to be grouped together from a broader standpoint, as will be apparent from the following paragraphs.

211. Enzym reactions reversible. - A chemical reaction is said to be reversible when it may progress in either direction according to the conditions. For example, if a mixture of hydrogen and iodin in molecular proportions be heated to $448^{\circ} \mathrm{C}$. hydrogen iodid is produced. If, however, hydrogen iodid be heated to the same temperature it yields hydrogen and iodin. The reaction between these two elements, then, is represented by the equation

$$
\mathrm{H}_{2}+\mathrm{I}_{2} \rightleftarrows(\mathrm{HI})_{2}
$$


At the temperature of $448^{\circ} \mathrm{C} ., 79$ per cent of the matter exists as $\mathrm{HI}$ and the remainder as free $\mathrm{H}_{2}$ and $\mathrm{I}_{2}$; the $\mathrm{HI}$ is dissociated at the same rate at which the $\mathrm{H}_{2}$ and $\mathrm{I}_{2}$ unite, and a condition of chemical equilibrium exists. At a different temperature, the point of equilibrium is different, but otherwise the result is the same. In theory, all chemical reactions are regarded as reversible, but in many cases the reverse action is so slight as to be incapable of detection under attainable experimental conditions, and such reactions are often spoken of as irreversible.

In addition to the temperature, the position of the point of equilibrium in a reversible reaction is affected by the relative mass of the ingredients. Thus if, in the example just given, the iodin be removed from the field of chemical action (as, for example, by condensing it to the solid form in a cold portion of the apparatus) the dissociation of the hydrogen iodid will proceed until it is practically complete. On the other hand, if the hydrogen iodid be removed (as by allowing it to react with calcium carbonate) the reaction may be pushed to completion in the reverse direction. Similarly, an increase in the concentration of one of the reacting substances tends to displace the equilibrium in the opposite direction.

It is a matter of much interest that at least some enzym reactions have been shown to be reversible. One of the best authenticated cases appears to be that of the action of lipase on fats. It has been shown by Kastle and Loevenhart ${ }^{1}$ that this enzym acts on ethyl butyrate according to the equation

$$
\mathrm{C}_{2} \mathrm{H}_{5} \cdot \mathrm{C}_{4} \mathrm{H}_{7} \mathrm{O}_{2}+\mathrm{H}_{2} \mathrm{O} \rightleftarrows \mathrm{C}_{2} \mathrm{H}_{5} \mathrm{OH}+\mathrm{C}_{4} \mathrm{H}_{8} \mathrm{O}_{2}
$$

A similar reaction has also been shown to take place with monobutyrin, the glycerol ester of butyric acid, which may be regarded as a simple fat, while it is at least very probable that the higher fats are acted on in the same way. Another example of reversible enzym reaction is claimed to be that of the conversion of maltose into dextrose by the action of the ferment maltase, it appearing, according to the researches of Croft Hill, ${ }^{2}$ that the same ferment may also convert dextrose into maltose. Similar, although less decisive, results have also been reported regarding the action of the proteases.

1 Amer. Chem. Jour., 24 (rgoo), 49r.

2 Jour. Chem. Soc., Trans., 73 (1898), 634. 
212. Reversibility of metabolic reactions. - It would appear, then, that the action of the intracellular enzyms which are believed to play such an important part in metabolism may be synthetic as well as analytic, and that the metabolic processes may be conceived of as a complex of reversible chemical reactions, now accelerated and now retarded by appropriate enzyms. ${ }^{1}$ The idea that each cell of the body thus exists in a state of constantly shifting chemical equilibrium, according as the concentration of one or another substance in its domain changes, is an attractive one in its breadth and comparative simplicity, and there seems to be little doubt that it contains elements of truth and will prove an important aid to research. As yet, however, it is to be regarded as a probable hypothesis rather than as a fully established fact.

\section{§ 3. The Metabolism of the Carbohydrates}

\section{The hexose carbohydrates}

213. Glycogenic function of the liver. - The monosaccharids (principally dextrose) produced in the digestive cleavage of the carbohydrates are resorbed chiefly or wholly by the blood capillaries of the intestines. These capillaries unite into the portal vein leading to the liver, where it subdivides into a capillary system in which the blood is brought into intimate contact with the cells of that organ and from whence it passes by way of the hepatic vein into the posterior vena cava, thus entering the general circulation (182).

The proportion of dextrose found in the blood of the general circulation is remarkably constant, and if any considerable excess be introduced it is promptly excreted through the kidneys (198). On the other hand, the supply of carbohydrates from the digestive tract may be more or less intermittent or fluctuating, so that there is evidently need for some regulatory mechanism to prevent a waste of sugar by excretion in the urine. This regulation is effected chiefly in two localities, viz., in the muscles and in the liver. The function of the liver

1 That syntheses can be effected by the agency of enzyms seems established, but that enzym reactions in general are reversible is questioned by good authorities. For example, the ferment maltase, acting on dextrose, is stated to produce not maltose but isomaltose, and it is claimed that a different enzym is required to reconvert isomaltose into dextrose. 
in this respect was the earliest to be discovered and may be appropriately considered first.

When dextrose is being freely resorbed from the digestive tract, it undergoes dehydration and polymerization in the liver, yielding the polysaccharid glycogen (25), which is stored up in the liver cells. If, on the other hand, the resorption of dextrose from the intestines is insufficient to maintain the supply in the blood, glycogen previously formed may undergo the reverse process of hydration and cleavage, giving rise to a production of dextrose. This regulatory activity, discovered by Claude Bernard in 1853 , by which carbohydrates are held back or released according to the demands of the body, is called the glycogenic function of the liver. While this function has other aspects, as will appear later, as respects the digested carbohydrates the liver may be likened to a storage reservoir by which the flow of a stream is controlled.

214. Mechanism of regulation. - It is of interest to note that this phase of carbohydrate metabolism illustrates two of the general conceptions formulated on preceding pages.

First, the formation of glycogen is a synthetic reaction. The comparatively simple molecules of dextrose are built up temporarily into the more complex molecules of the polysaccharid. In other words, almost the first step in carbohydrate metabolism is an anabolic change (203).

Second, the process of the formation and destruction of glycogen is susceptible of explanation as a reversible enzym reaction (212). It is known that the conversion of glycogen into dextrose is effected by an enzym or enzyms which may be extracted from the liver and which, it would seem, must be similar to those of the digestive tract. The action of one of the latter, maltase, however, is claimed to be reversible (211), and one is naturally tempted to infer that the synthesis of the liver glycogen is effected by the same enzym which brings about its cleavage, although experimental proof that such is the case is lacking. According to this hypothesis, the changes taking place in the liver would be represented by the equation

$$
{ }_{n}\left(\mathrm{C}_{6} \mathrm{H}_{12} \mathrm{O}_{6}\right) \rightleftarrows \mathrm{C}_{6 n} \mathrm{H}_{10 n} \mathrm{O}_{5 n}+{ }_{n}\left(\mathrm{H}_{2} \mathrm{O}\right)
$$

An excess of dextrose in the blood would have the effect of displacing the point of equilibrium in the direction of the for- 
mation of glycogen, while a deficiency of dextrose would have the contrary effect. If it be supposed further that the glycogen as soon as formed combines with the protoplasm of the liver cells, forming compounds which withdraw a considerable portion of it from the sphere of action of the enzym, after the analogy of the precipitation of an insoluble compound, we have a plausible, even if chiefly hypothetical, scheme of the chemical mechanism of the process. Whether or not it adequately represents the actual facts, it may at least serve as a concrete illustration of the manner in which the conception of enzym action may be applied to metabolic processes.

215. Muscle glycogen. - While the glycogenic function of the liver has been the subject of very extensive investigation, the presence of glycogen is by no means confined to this organ. Indeed, glycogen seems to be a normal constituent of animal protoplasm. It is found in greater or less amounts in practically all tissues, being particularly abundant where rapid cell multiplication is taking place, as in embryonic tissues or in rapidly growing tumors. It is estimated that in an animal in normal condition roughly one-half of the glycogen of the body is contained in the liver. Of the other half by far the larger proportion. is found in the muscles (96).

The glycogen of the muscles (and other organs) is not simply glycogen which has been formed in the liver and transported to the muscles, but is produced independently from the dextrose of the blood, apparently in much the same manner as in the liver. That this is true is shown by the fact that glycogen is still formed in the muscles when, by surgical interference (Eck fistula), the blood is prevented from passing through the liver. In fact, the formation of glycogen in the muscles, etc., appears to be the primary process, while the liver serves rather as a secondary reservoir which may be eliminated without seriously affecting the general carbohydrate metabolism. With the liver excluded from the circulation, the dextrose resorbed from the digestive tract is still converted into glycogen and the animal is still able to digest considerable quantities of carbohydrates without the appearance of sugar in the urine.

Even in the normal animal, however, the power to dispose of surplus sugar is not unlimited. If large quantities of sugar are consumed, the conversion into glycogen, together with the normal katabolism, 
may not keep pace with the resorption and there occurs an excretion of sugar in the urine - the so-called "alimentary glycosuria." The amount of sugar which can be resorbed without producing alimentary glycosuria, - i.e., the limit of tolerance for sugar - varies with the kind of sugar, being highest with dextrose (220).

216. Carbohydrates formed in the body. - In their relation to the carbohydrates of the feed, the muscles and liver act, as has been seen, as a sort of storage reservoir or regulator of the sugar supply to the blood. The total withdrawal of carbohydrates from the feed, however, by no means results in the disappearance of these substances from the body. The carbohydrates appear to be essential to the normal course of metabolism and if they are absent from the feed, they are manufactured in the body from other materials. A carnivorous animal, e.g., fed exclusively on meat or fat, shows a normal percentage of dextrose in its blood, while its liver and muscles contain a normal amount of glycogen. It is true that in such an experiment small quantities of glycogen are contained in the meat consumed, but their amount is entirely insignificant as compared with the quantities of dextrose which there is reason to believe are produced and katabolized in the organism. This dextrose must obviously have its origin either in the proteins or the fats. Which of the two is the source or whether both can be thus utilized will be considered later in connection with the metabolism of those substances $(235,253)$.

217. Formation of fat. - The mutual transformations of sugar and glycogen tend to keep the dextrose content of the blood approximately constant, while holding a supply of readily available carbohydrate material at hand to meet promptly any sudden demand. The amount of carbohydrates which can be disposed of in this way is, however, limited. For man it is estimated at about 300 grams and for cattle at about 2 kilograms (96). It is evident, then, that if the feed contains a permanent excess of carbohydrates over the needs of the body the capacity to store them up as glycogen will soon be exhausted. A surplus of carbohydrates over the amount which can be disposed of in this way is applied by the organism to the production of fat, which may be stored up in very large amounts in the cells of connective tissue through the body, but especially in those immediately beneath the skin and about the abdominal 
organs, constituting the adipose tissue (94). This tissue constitutes a reserve of non-nitrogenous material which may be mobilized later if need arises.

Of the chemistry of the conversion of carbohydrates into fats, as well as of the organ or organs where it is effected, our knowledge is still meager, but the fact of such a change is undisputed and it is perhaps the most notable example of a synthetic and anabolic process in the animal body. The physiological evidence for this fact and the quantitative relations of the process may be taken up more conveniently later (249).

218. Katabolism of carbohydrates. - The physiological significance of the dextrose of the blood and the glycogen of the muscles and liver appears most clearly when they are regarded, not in the light of a more or less temporary storage of matter in the body, but rather as carriers of energy for the physiological processes. Of these processes, the most obvious one, which vastly predominates over all others, is the performance of work by the muscles, external and internal, but what is true of muscular work is in the main true also of the subordinate forms of glandular and cellular activity. The former, therefore, may be taken as typical.

In the performance of muscular work, as will appear later, there is a rapid katabolism of non-nitrogenous material and especially of carbohydrates, largely, it would appear, in the form of dextrose. The resulting impoverishment of the blood in dextrose causes a conversion of stored up glycogen into dextrose to supply the lack. If the view of the formation of glycogen which regards it as a reversible reaction may be accepted, we may say that the chemical equilibrium between the dextrose and the glycogen is disturbed by the removal of the former during muscular work. As long as the work is continued, the process of conversion of glycogen into dextrose also continues, and by prolonged work it is possible to reduce the glycogen content of an animal to a very low limit.

It should be clearly understood that the foregoing is only a highly schematic view of the chemistry of muscular contraction as related to the katabolism of the carbohydrates. Some further consideration is given in Chapter XIV (630) to the very complicated chemical mechanism of the process. 
219. Intermediary katabolism. - Regarding the intermediary katabolism of the carbohydrates, not very much is known. It appears probable, however, that dextrose undergoes preliminary cleavage with the formation of glyceric aldehyde, pyruvic aldehyde (methyl glyoxal) and either lactic or pyruvic acid, which is then further oxidized to acetic acid, carbon dioxid and water. Many facts, including especially those derived from a study of the fermentation of sugar, seem to point to the possibility of such reactions. Lactic acid is also widely distributed in the body, although its presence is also susceptible of explanation as arising in the katabolism of protein (233), and, moreover, it has been shown that lactic acid may give rise to glycogen or dextrose in the animal body. Accordingly, these changes, like the mutual transformations of glycogen and dextrose, may be conceived of as constituting a series of reversible reactions.

Glycogen

$\mathrm{CH}_{2} \mathrm{OH}(\mathrm{CHOH})_{4} \mathrm{CHO}$

$\mathrm{CH}_{2} \mathrm{OH} \cdot \mathrm{CHOH} \cdot \mathrm{CHO}-$
$\mathrm{CH}_{3} \cdot \mathrm{CO} \cdot \mathrm{CHO}+\mathrm{H}_{2} \mathrm{O}$
or

$\mathrm{CH} \cdot \mathrm{CO} \cdot \mathrm{CHO} \cdot+\mathrm{O}$

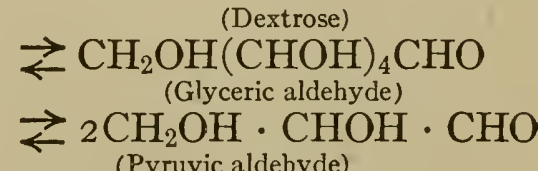

$\stackrel{\text { (Pyruvic aldehyde) }}{\leftarrow \mathrm{CH}_{3} \cdot \mathrm{CO} \cdot \mathrm{CHO}}$

(Lactic acid)

$\gtrless \mathrm{CH}_{3} \mathrm{CHOH} \cdot \mathrm{COOH}$

(Pyruvic acid)

$\rightleftarrows \mathrm{CH}_{3} \mathrm{CO} \cdot \mathrm{COOH}$

The conversion of dextrose into lactic acid is a nearly isothermic process, the resulting lactic acid containing almost the same amount of chemical energy as the dextrose. If, then, these cleavages occur in the katabolism of carbohydrates they are obviously preparatory to the actual oxidation in which the principal portion of the energy is liberated.

\section{The pentose carbohydrates}

The foregoing paragraphs have treated of the metabolism of the hexoses, which constitute the chief carbohydrate supply of man, and of the carnivora so far as the latter consume carbohydrates. The feed of herbivora, however, contains also considerable amounts of various pentose carbohydrates which are in part 
digestible, or at least disappear from the feed during its passage through the alimentary canal.

220. Pentose sugars. - In general, it may be stated that the pentose sugars (in particular arabinose and xylose), whether administered by the stomach or injected into the blood, are at least partially oxidized in the body. The pentoses differ from the hexoses chiefly in the fact that the limit of tolerance in the blood (215) is lower. Excessive amounts of hexose carbohydrates cause an excretion of sugar in the urine. The same effect is produced by the pentoses, but much smaller quantities, relatively, are required to bring it about.

Most, although not all, investigators have found an increase in the glycogen of the liver consequent upon the ingestion of pentoses, but in every case it has been the ordinary $\mathrm{C}_{6}$ glycogen, indicating that the effect is an indirect one.

221. Pentosans. - The investigations upon the soluble pentose sugars or their derivatives just referred to have shown that they are to a greater or less extent assimilable. The pentose carbohydrates in the feed of herbivora, however, exist to a very limited extent, if at all, in this form. They are chiefly polysaccharids, being either pure pentosans or combinations of pentosans and hexosans. In discussing the nutritive value of these pentosans, it seems to have been frequently assumed that they are converted into pentoses during digestion. As a matter of fact, however, there is no direct evidence that such is the case, while Kellner's results (129) afford reason to believe that they are largely fermented along with cellulose, yielding, besides gaseous products, chiefly organic acids. If this is the case, farm animals do not acquire from their feed any considerable amounts of pentoses and conclusions drawn from experiments with the pentose sugars regarding the nutritive value of these substances are inapplicable to ordinary stock feeds. Their true value in the latter would be simply that of the products of their fermentation.

\section{The organic acids}

222. Formation in digestion. - As was shown in Chapter IV (128-130, 132), a considerable proportion of both the hexose and pentose carbohydrates contained in the feed of herbivora undergoes fermentation in the digestive tract, giving rise, in 
addition to gaseous products, to the formation of various organic acids. In particular, the constituents of the cell walls of plants appear to owe their apparent digestibility chiefly to this action of the organized ferments of the alimentary canal. While, therefore, the organic acids are chemically distinct from the carbohydrates, and while some of these acids are contained as such in the feed, the amounts produced from carbohydrates are so considerable that this would appear an appropriate point at which to consider their metabolism.

Unfortunately, however, little is known of the metabolism of the simpler organic acids, beyond the fact that such of them as have been subjected to experiment are katabolized to carbon dioxid and water, not more than traces of them at most appearing in the excreta. A portion of the carbon dioxid produced unites with alkalies and appears in the urine as carbonates.

223. Analogy with carbohydrates. - It is interesting to recall in this connection that the carbohydrates themselves undergo cleavage, producing lactic or even acetic and formic acids, before their final oxidation (219). If it be true that these latter comparatively simple substances are those whose oxidation yields most of the energy supplied by the carbohydrates, there would seem to be no reason why the same acids resorbed directly from the digestive tract should not follow the same general course of metabolism and have substantially the same nutritive value. If this view be correct, there is after all a considerable similarity between the metabolism of the carbohydrates and that of their fermentation products.

\section{The non-nitrogenous matter of the urine}

224. Products of incomplete katabolism. - It has been implied in the foregoing pages that the digested carbohydrates of the feed, whatever the intermediate stages through which they may pass, are ultimately oxidized to carbon dioxid and water. Of the ordinary hexose carbohydrates this is doubtless true, but with some of the large variety of substances ordinarily grouped together in the conventional scheme of feeding stuffs analysis as "carbohydrates and related bodies," or as "crude fiber" and " nitrogen-free extract," the case appears to be otherwise.

It has been shown that the urine, in addition to the nitrogenous 
products of protein katabolism which will be considered in the following section, contains also non-nitrogenous materials, presumably arising from the incomplete katabolism of ingredients of the feed. In the urine of man and of the carnivora these non-nitrogenous substances are chiefly or wholly such as might be derived from the katabolism of proteins (phenols and other compounds of the aromatic series), and their amount is comparatively small. In the urine of herbivora, particularly of ruminants, however, their quantity is relatively very considerable, and it seems impossible to regard any large portion of them as products of protein katabolism.

225. Origin. - Apparently these non-nitrogenous organic substances originate in some way from the roughages. Their proportion in the urine is relatively large when the ration consists exclusively of roughage, and the addition of such feeding stuffs to a basal ration causes a marked increase in their amount, while, on the other hand, such concentrates as have been investigated do not produce this effect to any very considerable extent. Furthermore, their amount seems to bear no fixed relation to the protein of the feed. When the amount of the latter ingredient is small, the total organic matter of the urine has in some cases exceeded the digested protein of the feed, thus demonstrating that a portion at least of the non-nitrogenous urinary constituents must have had some other source. As the proportion of protein in the feed increases, the amount of nitrogenous products in the urine likewise increases, while that of the non-nitrogenous products appears to be more constant, so that the ratio of urinary nitrogen to carbon increases. The most plausible explanation of these facts seems to be that the substances in question are derived from some of the non-nitrogenous ingredients of the roughages, but from what ones, or what is the nature of the products, we are still ignorant.

\section{$\S$ 4. The Metabolism of the Simple Proteins}

\section{Anabolism}

226. Synthesis of proteins from digestive products. - The simple proteins are resorbed $(139,152)$ in the form of comparatively simple cleavage products; largely as amino acids but in part perhaps as more or less complex polypeptids. Out 
of these substances the body builds up the great variety of specific proteins which are peculiar to itself and which differ in properties and chemical structure from the proteins of the feed, especially from those of the vegetable kingdom (147). This process of building animal proteins from the fragments of vegetable proteins is the most conspicuous example at once of the synthetic powers of the animal organism and of the object of the digestive cleavage.

227. Seat of protein synthesis. - As regards the place where this synthesis of proteins occurs, opinions are divided. Until recently, most experimenters have not been able to detect the products of digestive cleavage with certainty in the blood, either in the general circulation or in the portal vein, and the current view has been, therefore, that of Abderhalden, viz., that the "building stones" of the proteins are synthesized in the epithelial cells of the intestine and that the resulting proteins - in particular serum albumin - are passed on to the blood to serve as nourishment to the protein tissues of the body.

Various investigators, however, have reported the presence in the blood of greater or less amounts of non-protein nitrogen and with the aid of more refined chemical methods Folin and Denis ${ }^{1}$ and Van Slyke and Meyer ${ }^{2}$ seem to have shown beyond question that amino acids may pass through the resorbing epithelium unchanged and be found in the blood and tissues in amounts sufficient to account for practically all that was administered (152). The latter experimenters have likewise shown that after meat feeding the proportion of amino acids in the blood may be doubled and that the increase affects the blood of the entire circulatory system and not that of the portal vein only, while Abel, ${ }^{3}$ by a diffusion method, has been able to secure considerable amounts of amino acids from the circulating blood of living animals. The evidence at the present time, therefore, seems decisively in favor of the view that the fragments into which the protein molecule is split during digestion pass without material change into the blood current and serve as a common source from which the proteins, both of the blood and the various tissues, are built up and that every living cell, each in its own measure, has this anabolic power.

1 Jour. Biol. Chem., 11 (1912), 87. 2 Jour. Biol. Chem., 12 (IgI 2), 399.

3 Jour. Pharmacol, and Expt'l Therap., 5 (IgI4), 275. 


\section{Katabolism}

228. Nitrogenous end products. - The total katabolism of the proteins results in the elimination of all their nitrogen through the kidneys in the form of the various relatively simple crystalline products found in the urine. Of the nitrogenous excretory products of man and the carnivora, urea is the most prominent, while others, such as uric acid, creatin, creatinin, ammonia, etc., are of subordinate importance quantitatively. Traces of hippuric acid are also found in the urine of man and carnivora, while it is present in relatively large amounts in that of herbivora along with considerable quantities of ammonia and apparently but little urea.

The nitrogenous ingredients of the urine of mammals other than those just mentioned are either derived chiefly from the nucleoproteins, whose metabolism will be considered later, or are present in such small amounts as to call for no special consideration from the present very general point of view. Finally, it should be noted for completeness that a small amount of nitrogenous products is eliminated in the perspiration and also that from one point of view the incompletely katabolized nitrogenous excretory products of the feces (154) may also be regarded as products of protein katabolism.

Urea, or dicarbamid, $\mathrm{CO}\left(\mathrm{NH}_{2}\right)_{2}$, is the chief nitrogenous product of the katabolism of the simple proteins in carnivora and omnivora. In human urine from 80 to 90 per cent of the nitrogen is ordinarily present in this form, although the proportion may be considerably diminished under special conditions, notably on a low protein diet. Urea, however, is not simply split off as such from the proteins as some earlier schematic statements have sometimes been taken to imply. The immediate antecedent of urea is ammonium carbonate, which undergoes a dehydration in the liver or elsewhere, while there is evidence in favor of the view that the ammonia is brought to the liver in the form of ammonium lactate. At any rate it is an accepted fact that most, if not all, of the nitrogen of the simple proteins passes through the ammonia stage on its way to excretion as urea. ${ }^{1}$ That the formation of urea from ammonia is not exclusively a function of the liver is shown by the fact that it still continues when this organ is excluded from the circulation by means of an Eck fistula.

I It has been, shown that the liver, kidneys and other organs contain an enzym which splits off the guanidin group from arginin (47) producing urea and ornithin. 
Hippuric acid in small amounts is a normal constituent of the urine of mammals but is especially abundant in that of herbivora. Its formation is the result of a synthesis (204). When benzoic acid or other compounds containing the benzoyl radicle are introduced into the circulation they are paired with glycin, one of the cleavage products of the proteins, in the kidneys and excreted as hippuric acid, which, chemically, is benzoyl-glycin, or benzamidoacetic acid, $\left(\mathrm{C}_{6} \mathrm{H}_{5} \cdot \mathrm{CO}\right) \mathrm{NHCH}_{2} \cdot \mathrm{COOH}$. The normal presence of small quantities of hippuric acid in the urine arises from the fact that the putrefaction of the proteins in the intestines yields compounds containing the benzoyl radicle which are resorbed and combine with glycin to form hippuric acid. But a small proportion of the hippuric acid produced by herbivora can be thus accounted for, however. Most of it appears to owe its origin to the roughages consumed by these animals, especially those derived from plants of the graminex, while, on the other hand, concentrates do not seem to increase its amount. Apparently its formation bears some relation to some of the ingredients of the cell walls, but to what ones in particular is not clear.

229. The non-nitrogenous residue. - In addition to the nitrogenous products eliminated in the urine, the complete oxidation of the protein molecule gives rise to the production of considerable amounts of carbon dioxid and water, which are excreted through the same channels as those derived from the katabolism of carbohydrates or fats. To put the matter in the reverse way, while the urinary products account for all the nitrogen of the protein, they contain but a relatively small part of its carbon, hydrogen and oxygen. This is clearly shown by comparing the average amounts of these elements in 100 parts of protein with the quantities contained in the urea corresponding to the total nitrogen of the protein. Disregarding the sulphur of the proteins, the results of such a computation are as follows : -

\begin{tabular}{|c|c|c|c|c|c|c|c|c|c|c|c|c|c|}
\hline & & & & & & & & & & & Proterns & UREA & Residue \\
\hline Carbon & . & . & . & . & . & . & . & . & . & . & 53.0 & 6.86 & 46.16 \\
\hline Hydrogen & . & . & . & . & . & . & . & . & . & . & 7.0 & 2.29 & $4.7 \mathrm{I}$ \\
\hline Oxygen & . & . & . & . & . & . & . & . & . & . . & 24.0 & 9.14 & I 4.86 \\
\hline \multirow[t]{2}{*}{ Nitrogen } & . & . & . & . & . & . & . & . & . & . . & 26.0 & I6.00 & \\
\hline & & & & & & & & & & & 100.0 & 34.29 & $65.7 \mathrm{I}$ \\
\hline
\end{tabular}


After abstracting the elements of urea, there remains considerably over half the hydrogen and oxygen of the protein and the larger part of its carbon. A substantially similar result is reached in case of the other nitrogenous metabolic products. The splitting off of these products from the proteins leaves a non-nitrogenous residue.

230. Two stages of protein katabolism. - Two general stages in the katabolism of the proteins may be distinguished. The first is a hydrolysis by which the proteins are split up into their constituent amino acids. The second is a deaminization of the amino acids in which the nitrogen of these acids is split off as ammonia.

231. Protein hydrolysis. - The first stage in the katabolism of the body proteins is a hydrolytic cleavage, more or less similar to that effected in digestion and like the latter brought about by enzyms, which in this case are contained in the body cells - the intracellular enzyms (209).

The truth of this view is attested by the facts that the presence of proteases in almost all of the tissues and organs of the body has been demonstrated and that under proper conditions they effect a rapid solution of the tissue proteins - the so-called autolysis. Further confirmation is afforded by the known facts regarding the transformation of one protein into another in the body, while finally the production in the organism of some of the cleavage products of the proteins, presumably as products of katabolism, may be indirectly shown.

232. Is protein hydrolysis a reversible process? - If the katabolism of body proteins is initiated by an enzymatic cleavage in the body cells, this is precisely the reverse of the synthetic action by which it is believed that body proteins are built up out of the products of digestive cleavage (226), and the question at once arises whether we have to do here with a reversible enzym reaction, analogous to that which has been suggested as occurring in the case of the carbohydrates (214), the general nature of which may be represented by the formula

\section{Protein $\rightleftarrows$ Amino acids}

It must be freely admitted that proof of the reversibility of the action of proteolytic enzyms is as yet lacking, such phenomena as the formation of the plasteins discovered by Okunew 
and the alleged formation of paraneuclein by the action of pepsin as reported by Robertson being apparently due to adsorption phenomena. ${ }^{1}$ On the other hand, however, many authorities $^{2}$ are inclined to regard reversibility as a general characteristic of enzym action and mere negative evidence cannot, of course, disprove this belief. At any rate the conception of a reversible reaction between the amino acids of the blood and lymph and the proteins of the cells affords a comparatively simple and unforced explanation of the facts outlined in the foregoing paragraphs, as well as of others relating to the influence of the supply of feed protein on metabolism which will be considered later (402). In particular, it may be observed that, according to this view, by no means all the amino acids resorbed. into the blood stream would undergo synthesis to proteins but that, especially if the amino acid supply were liberal, a large part of them might pass directly to the second stage of protein katabolism, viz., deaminization.

Finally, since the proportions of the single amino acids supplied from the digestive tract vary, one must conceive, not of a single reaction between protein and amino acid, but, speaking broadly, of as many independent reversible reactions as there are amino acids concerned.

233. Deaminization. - The second general stage of protein katabolism seems to be the splitting off of the $\mathrm{NH}_{2}$ group from the amino acids, the products being the corresponding or closely related non-nitrogenous organic acids on the one hand and ammonia on the other. This also, it would appear, is a case of enzym action, although the discovery of deaminizing enzyms in various tissues is comparatively recent and its biological importance is still to some extent speculative.

The ammonia resulting from the deaminization of the amino acids is believed to be the immediate antecedent of urea, into which it is rapidly converted, chiefly although not exclusively in the liver (228). In this way the nitrogen of any amino acids resorbed in excess of the immediate demands of the body cells for protein building material is promptly converted into excretory products and so disposed of, while the larger part of their carbon and hydrogen remains in a series of substances

1 Compare Rohonyi; Biochem. Ztschr., 53 (I913), I79.

2 Compare Bayliss; The Nature of Enzym Action (1908), Chapter V. 
bearing a more or less close relation to the fatty acids and together constituting the "non-nitrogenous residue" of the proteins (229).

It is important to note that these non-nitrogenous products contain the larger share of the chemical energy of the original proteins, the ammonia carrying off but little and both the digestive cleavage and the deaminization being nearly isothermic processes. The cleavage and deaminization of proteins, therefore, do not necessarily involve a destruction of their nutritive value and the excretion of a given amount of nitrogen in the urine is not to be regarded as indicating the total destruction of a corresponding amount of protein. It has ceased to exist $a s$ protein, but its non-nitrogenous residue is made up of substances which are closely related chemically to both the carbohydrates and fats, and which, like these, may be katabolized to supply energy.

234. Deaminization reversible. - Since deaminization in the body appears to be an enzymatic reaction, it is natural to inquire whether in this case, as in the other enzymatic reactions already considered, there is any evidence that the reaction is a reversible one.

So far as direct experiments with deaminizing enzyms are concerned, no such evidence has been produced, but Knoop ${ }^{1}$ and Embden and Schmitz ${ }^{2}$ have demonstrated a fact of fundamental significance in metabolism, viz., that amino acids may be formed in the body from ketonic or hydroxy acids and ammonium salts. In other words, the animal body can manufacture some at least of the "building stones" of the proteins, and from the latter presumably the proteins themselves (226), out of the ammonium salts of the corresponding ketonic or hydroxy acids. The full significance of this comparatively recent discovery is not yet fully apparent. The question of the utilization of ammonium salts will be considered later. In this connection the important fact is that these results indicate that the reaction, or series of reactions, by which deaminization takes place is reversible, so that the whole process of protein metabolism may be represented schematically as follows:-

$$
\text { Proteins } \rightleftarrows \text { Amino acids } \rightleftarrows\left\{\begin{array}{l}
\text { Organic acids } \\
\text { Ammonia }
\end{array}\right.
$$

${ }^{1}$ Ztschr. Physiol. Chem., 67 (1910), $489 . \quad 2$ Biochem. Ztschr., 29 (1910), 423. 
235. Formation of carbohydrates from proteins. - It has already been stated (216) that carbohydrates may be manufactured, in the bodies of carnivorous animals at least and probably in those of other species, but the question whether the proteins or the fats or both serve as the source was left open.

Without entering into experimental details, it may be stated, as the general result of many trials in which the possibility of a production from fats was excluded as completely as possible, that carbohydrates have been produced in such large amounts, and in quantities so closely paralleling the quantities of protein katabolized, as to amount to a proof of their formation from the latter. The acceptance, however, of the view that carbohydrates may be a product of protein katabolism by no means excludes the possibility of their formation also from fats. Indeed, in view of the importance of carbohydrates in metabolism it seems altogether likely that the body has the power to manufacture them from both fats and proteins, while, as already stated (217), the reverse process of the formation of fats from carbohydrates has been demonstrated.

With the increasing knowledge of the details of protein katabolism afforded by recent investigations, the question under consideration has assumed a somewhat different aspect, the discussion shifting from the fate of the proteins as a whole to that of the single amino acids and of the non-nitrogenous products of their katabolism. It has been shown, especially by the work of Lusk and his associates, that some at least of the amino acids (glycin, alanin, aspartic acid, glutamic acid, histidin), after deaminization may yield dextrose. In the case of glycin and alanin all the carbon of the amino acid could be recovered in the form of dextrose. In the case of aspartic and glutamic acids, on the other hand, only three out of the four or five carbon atoms respectively were found in the dextrose produced. Still other amino acids, notably leucin and tyrosin, apparently do not yield dextrose, but instead compounds like $\beta$ hydroxybutyric acid and aceto-acetic acid which are the distinctive products of the katabolism of the higher fatty acids (252).

In the case of some, then, but apparently not all, of the products of protein katabolism, the relations between protein and 
carbohydrate metabolism may be schematically expressed thus : -

Proteins $\rightleftarrows$ Amino acids $\rightleftarrows\left\{\begin{array}{l}\text { Lower fatty acids } \rightarrow \mathrm{CO}_{2} \text { and } \mathrm{H}_{2} \mathrm{O} \\ \mathrm{NH}_{3} \longrightarrow \text { Urea }\end{array}\right.$

236. Formation of fat from proteins. - Since the non-nitrogenous products of protein katabolism appear to consist largely of comparatively simple substances closely related to the lower members of the fatty acid series, and since some at least of these may in all probability be synthesized to carbohydrates, while the latter can undoubtedly give rise to fats, it is natural to conclude that the non-nitrogenous products of protein katabolism may serve as a source of fat, either by direct synthesis of the simpler fatty acid chains or possibly by way of the carbohydrates. The conclusion is one which has been hotly debated and much of the earlier evidence in its favor has been shown to be inconclusive. The experimental evidence may be more conveniently considered in connection with a discussion of the sources of animal fat (247-249). For the present, it may suffice to say that the formation of fat from protein seems altogether probable, but that on the other hand the amount of fat thus formed under normal conditions is usually unimportant.

\section{$\S$ 5. The Metabolism of the Nucleoproteins}

The metabolism of the conjugated proteins, with the exception of the nucleoproteins, offers few features of special interest. In general it may be said that they are split up into their constituents during digestion and that the cleavage products undergo substantially the same metabolic changes as if consumed by the animal in the uncombined form. In the case of the nucleoproteins, however, the metabolism of the nucleic acid portion of the molecule calls for more specific consideration.

\section{Anabolism}

237. Fate of digestive products. - The nucleic acids undergo extensive enzymatic cleavages in digestion (139), the products 
passing into the circulation being essentially phosphoric acid, pentoses, and purin and pyrimidin bases. By analogy with the simple proteins, one might expect, therefore, to find that these fragments of the nucleic acid molecule are rebuilt into nucleoproteins in the body cells, of which they constitute such an indispensable ingredient (75).

The occurrence of such a synthesis, however, has been seriously questioned. One argument against it is the fact that the ingestion of nucleoproteins, or more specifically purin bases, results in a prompt excretion in the urine of end products of their katabolism which, although it has not been proved to be quantitative, is certainly large, while the amounts excreted on a purin free diet are small and notably uniform. It has been argued, therefore, that the so-called "exogenous" purins, i.e., the nucleic acid constituents derived from the feed, are simply katabolized and excreted without serving to rebuild nucleic acids in the cells. Precisely the same argument might be made, however, against the synthesis of the simple proteins from their cleavage products, since in this case also an increase in the supply causes a prompt and almost quantitative increase in the excretion of the end product, urea (402).

238. Autogenesis. - It is true that the formation of nucleoproteins differs from that of the simple proteins in that the latter is a reconstruction of the molecule from its fragments ${ }^{1}$ rather than a synthesis in the stricter sense, while it has been demonstrated that the body can build up nucleic acids out of a feed supply containing neither purins, pyrimidins nor pentoses. One of the most striking instances of this is seen in the development of the embryo of birds and insects. The eggs contain practically none of the substances just mentioned, yet the bodies of the young animals contain normal amounts of nucleic acid. Equally significant is the case of the suckling mammal,which receives in the milk a food very poor in purins, pyrimidins and pentoses, yet which maintains a rapid growth and cell multiplication with its accompanying formation of nucleoproteins. So, too, in Osborne and Mendel's extensive investigations ${ }^{2}$ upon the nutritive values of the proteins, normal

1 The possibility of the formation of proteins from ammonia (234) is of little significance under ordinary conditions of nutrition.

${ }^{2}$ Carnegie Institution of Washington, Publication No. 156, p. 85. 
growth of rats through two generations was secured on purinand pyrimidin-free feed. Another fact pointing in the same direction is that the body does not appear to require a supply of phosphorus in organic combination but can build up its organic compounds from phosphates (258).

239. Regeneration from cleavage products. - In view of the capacity of the body to produce nucleoproteins in the entire absence of their constituent "building stones" may it be supposed that when the latter are supplied in the feed they may be recombined in the cells somewhat as are the amino acids of the simple proteins?

No positive answer can be given to this question. It would seem, however, that the first steps in the autogenesis of the nucleoproteins must be the formation of pentoses and of the purin and pyrimidin bases, i.e., of precisely those substances which result from the digestive cleavages. Even though it be assumed that, in the former case, they are produced within the cells where they are further synthesized to nucleic acid, it is not altogether clear why the same substances brought to the cell by the blood current should not be available for the same purpose. Provisionally, at least, it seems perfectly possible to regard the entire stock of these " building stones" contained in the body, whether derived from the feed or produced by the body cells, as potentially available for the regeneration of nucleic acids. From this point of view, the increased excretion of purins which results from their ingestion would be considered as a consequence of their increased concentration in the blood and as analogous to the increased excretion of urea which follows the ingestion of simple proteins or of amino acids (402).

\section{Katabolism}

240. Cleavages. - The katabolism of the nucleic acids bears a close general resemblance to that of the simple proteins. As in the case of the latter, the first general stage of the process consists of a series of enzymatic cleavages. These cleavages are quite analogous to those of the simple proteins and yield as final products the comparatively simple " building stones" of the nucleic acids. Since it is to be supposed that the autogenesis (238) of these compounds is via these same 
"building stones" it would appear that we have here, as in the case of the simple proteins, a complex of reversible enzym reactions.

$$
\text { Nucleic acid } \rightleftarrows\left\{\begin{array}{l}
\text { Phosphoric acid } \\
\text { Pentose } \\
\text { Purin or pyrimidin bases }
\end{array}\right.
$$

241. Deaminization. - The phosphoric acid which is split off from the nucleic acids is, of course, added to the general stock of this substance in the body. The pentose may be presumed to be katabolized or possibly built up into a hexose.

The bases, on the other hand, like the amino acids derived from the proteins, undergo, as the second general stage of their katabolism, an enzymatic deaminization and oxidation. The $\mathrm{NH}_{2}$ groups are split off as ammonia and converted into urea, while the ring formations are largely unbroken, the principal end products of purin katabolism being uric acid in man and allantoin in most other mammals. Of the katabolism of the pyrimidin bases little is known. The deaminization is never complete, however, purin and pyrimidin bases appearing in the urine along with the end products of katabolism.

242. Synthesis of uric acid. - In birds and reptiles, uric acid is the principal nitrogenous constituent of the semi-solid urine. Since no considerable portion of its nitrogen can have existed as preformed purins in the feed, it is evident that these animals must synthesize uric acid. This synthesis appears to take place in the liver, the antecedents probably being lactic acid and urea.

\section{$\S 6$. The Metabolism of the Fats}

\section{Anabolism}

243. Resynthesis of feed fat. - In considering the resorption of the fats (152) it was shown that, while the products of their digestion are glycerol and fatty acids (or their salts), after resorption only neutral fats have been recognized in the epithelial cells and in the lymph of the intestinal lacteals. The cleavage of the fats in digestion is reversed in the epithelial cells. It seems altogether plausible to ascribe this resynthesis to the action of an intracellular lipase, the more since the action 
of lipase has been shown to be reversible in some cases (211). Whether this resynthesis be regarded as part of the process of resorption or be classed as one of the metabolic processes is a matter of indifference. In either case the material transmitted to the blood current consists substantially of fats.

The digested fats are contained in the lymph in the emulsified form and in this state pass from the thoracic duct into the blood of the subclavian vein. The blood itself, however, although sometimes containing as much as I per cent of fat, does not normally carry emulsified fats, and the fat globules entering it from the thoracic duct do not long persist. The nature of the change is still uncertain; by some, it has been regarded as a cleavage into fatty acids and glycerol and by others as a union with proteins. But whatever the nature of the change it seems to be well established that the fat of the blood exists in some sort of combination which is soluble in water and diffusible and which may be called for convenience "soluble fat."

244. Storage of fat. - A liberal supply of fat to the blood from the digestive tract may give rise to a storage of reserve fat in the adipose tissues (94) of the body. It is to be presumed that this deposition of reserve fat is substantially a reversal of the process, whatever it is, by which it was brought into solution in the blood, the "soluble fat" of the latter passing into the cells and being there reconverted into the emulsified form and so giving rise to the globules characteristic of fat cells.

245. Formation of cell lipoids. - The fats deposited in the adipose tissues, as already implied, are a store of reserve material, laid aside temporarily from the body metabolism when the feed supply is more than adequate for immediate needs.

The various more complex lipoids (37-39, 75), however (cholesterins, lecithins and other phosphatids, cerebrosids, etc.), appear to be essential ingredients of protoplasm and to perform specific functions in the cell. All these substances have as their basis fatty acid molecules coupled with other groups and it is a reasonable assumption that the former are derived from the "soluble fat" of the blood and synthesized in the cells into the specific lipoids as required.

246. Manufacture of fats. - But while the feed fats may serve as a source of body fats, the organism is by no means dependent upon the former for its supply of these substances, 
but may, as has already been indicated (217, 236), manufacture fats from other ingredients of its feed.

This view, first propounded by Liebig in I843, was contrary to the opinion then prevailing and led to a lively controversy which, however, was definitely resolved in favor of the newer belief. Indeed, the feed fats, especially in case of herbivorous animals, are usually of subordinate importance as sources of body fat, a large share of the latter being produced de novo in the body. This fact explains in part the general uniformity of composition of the body fat of each species. The steer produces beef fat and the sheep mutton fat on substantially identical rations largely because the fat deposited in the body is derived only in small part from the feed fat, most of it being produced by the specific metabolic activities of the body cells. The seat of this synthetic production of fat, however, as well as the manner in which it is deposited in the reserve tissues, are still unknown.

\section{The sources of animal fat}

247. Experimental evidence. - The sources of animal fat have been already indicated. Aside from whatever feed fat may be stored up in the adipose tissues, the body can produce fat from the carbohydrates of the feed (217) and in all probability from the non-nitrogenous residue of the proteins (236). In view of the historic interest attaching to the long controversy over this question, however, as well as of its intrinsic importance, an outline of the experimental evidence seems appropriate.

That the feed fat is a source of body fat was never seriously questioned. When the correctness of Liebig's contention that the animal body can also manufacture fat had been demonstrated, it was assumed that the source of this new-formed fat was to be found in the carbohydrates of the feed and this was for years the accepted view. Following Liebig's terminology, the proteins were designated as the "plastic materials," serving to build up tissue, while the carbohydrates and fats were " respiratory materials," serving as sources of heat and of fat.

248. Fat from protein. - Several earlier investigators observed facts pointing to the formation of fat from protein in the animal body, but Carl Voit ${ }^{1}$ was the first to distinctly ad-

1 Ztschr. Biol., 5 ( 1869 ), 79-169. 
vocate the belief that protein constitutes an important source of animal fat, this conclusion being based largely on the famous respiration experiments of Pettenkofer and Voit at Munich in which a dog was fed lean meat freed from visible fat as carefully as possible (this being the nearest practicable approach to a pure protein diet) and the balance of nitrogen and carbon (287, 292) determined. The results showed in many cases a retention of carbon by the animal greater than corresponded to the quantity of protein gained, and this difference was interpreted, according to the methods described in Chapter VI (293), as showing a production of fat.

Pettenkofer and Voit's experiments were long accepted as conclusive until Pfluger ${ }^{1}$ subjected them to destructive criticism, showing the possibility of material errors in the estimates of the carbon of both feed and visible excreta.

It scarcely need be said that this result does not prove that fat is not formed from protein, but simply that Pettenkofer and Voit's experiments fail to demonstrate it. Of later experiments on the subject, a number seem to show clearly the formation of a small amount of fat from protein, even after every allowance has been made for the objections raised by Pfluger in his criticisms of the experiments. A number of negative results have, it is true, also been reported, but naturally negative results are of much less value than positive ones.

Moreover, the indirect evidence in favor of the possibility of the formation of fat from protein seems practically conclusive. As already stated (235), it has been established beyond reasonable doubt that carbohydrates may be produced from protein in the body. If this is true, however, it almost necessarily involves the possibility of the formation of fats from protein, since carbohydrates are undoubtedly a source of fat.

249. Fat from carbohydrates. - Pettenkofer and Voit, ${ }^{2}$ however, went further than to demonstrate, as they believed, the formation of fat from protein. Their experiments included a number in which carbohydrates were added to a ration of protein (lean meat). Assuming with Henneberg ${ }^{3}$ that roo grams of protein might yield $5^{1.4}$ grams of fat, they computed that all the fat produced by the animal in these experiments

1 Arch. Physiol. (Pflüger), 51 (I892), 229.

${ }^{2}$ Ztschr. Biol., 9 ( 1873 ), 435. ${ }^{3}$ Landw. Vers. Stat., 10 (I868), 455. 
could, with only one or two exceptions, be accounted for by the fat and protein of the feed. They, therefore, characterized the formation of fats from carbohydrates as improbable. The somewhat general impression that Voit absolutely denied the production of fat from carbohydrates is incorrect, although he regarded it as improbable and unproved. Indeed, he came later to admit the truth of the opposite view and even furnished from his own laboratory experimental evidence in its support. Nevertheless, his earlier opinion as to its improbability obtained wide currency and in the hands of his followers became almost a dogma, so that protein was given a vital and preponderant importance the effect of which has been unfortunate both for the development of the science of nutrition in general and upon the theory of stock feeding in particular.

Henneberg's estimate of the maximum fat production from protein was soon virtually accepted as an established fact and with the use of this high figure it was easy to compute from most of the experiments on fat production then on record that the fat and protein of the feed were sufficient to account for the fat produced. Similar computations upon a large number of later feeding experiments ${ }^{1}$ yielded similar results, so that belief in the non-formation of fat from carbohydrates was further strengthened.

One notable exception to the rule, however, were the experiments made by Lawes and Gilbert in 1850 upon the fattening of swine. These were comparative slaughter tests (284) in which the gain of fat was determined by comparing the weight and composition of similar animals, one before and the other after fattening. They were, accordingly, subject to a somewhat considerable range of error, but even on the most extreme assumptions it was impossible in six out of the nine experiments to account for the fat actually produced by the supply of fat and protein in the feed. These investigators, therefore, continued to maintain, in spite of much adverse criticism, the formation of fat from carbohydrates, although their experiments hardly secured the recognition which they deserved.

As time went on, however, results began to accumulate which, like Lawes and Gilbert's showed a much larger production of fat than could possibly be ascribed to the fat and protein of

See the author's Manual of Cattle Feeding, p. I77. 
the feed. This was particularly the case as it came to be more clearly recognized that Henneberg's estimate of a production of 51.4 grams of fat from roo grams of protein was in all probability too high, and especially after it was shown that what had been regarded as digested protein in many of these experiments (i.e., digestible $\mathrm{N} \times 6.25$ ) consisted in part of much simpler nitrogenous compounds. The ready formation of fat by the hog rendered this animal a very suitable subject for experiment, and the great majority of investigations on this animal have supported the view that fat is produced from carbohydrates, but similar results upon other species have not been lacking, while respiration experiments upon swine, geese, dogs, and especially the extensive investigations by G. Kühn ${ }^{1}$ upon cattle have completed the demonstration. ${ }^{2}$

In the light of all these results, the formation of fat from carbohydrates in the animal body is now universally admitted, while its production from protein is still questioned by a few and in any case is of little economic significance, so that we have come back by a curious reversal of views almost to Liebig's classification of the nutrients into plastic and respiratory.

This conclusion applies specifically to the pure hexose carbohydrates, particularly starch. In many of the experiments cited, however, the non-nitrogenous material digested by the animal consisted to a not inconsiderable extent of those substances of uncertain chemical nature included in the terms crude fiber and nitrogen-free extract. Postponing for the present any discussion of the nutritive value of these groups, it may suffice to say here that Kellner's investigations ${ }^{3}$ in particular show that both of them, including the pentosans, may serve as sources of fat.

\section{Katabolism}

250. Body fat a reserve. - The stored fat of the adipose tissues, aside from its mechanical functions, constitutes the great reserve of energy-yielding material in the body. In the lack of an adequate feed supply, common observation shows that this

${ }^{1}$ Kellner: Landw. Vers. Stat.; 44 (1894), 257.

${ }^{2}$ Compare the author's Principles of Animal Nutrition, pp. I65-184.

${ }^{3}$ Landw. Vers. Stat.; 51 (1900). 
reserve is drawn upon for the support of the internal activities of the body and as a source of energy for the performance of external work.

251. Mobilization of reserve fat. - In order that the stored fat may be used for the general metabolism of the body it must first be transferred from the adipose tissue cells to the localities where it is needed. Presumably this is accomplished by its reconversion into "soluble fat" and its passage through the walls of the cells into the blood, that is, by a reversal of the process by which it was laid down. Since the transfer of fat through the epithelial cells in resorption is effected by a hydrolytic cleavage (152), one is tempted to, imagine a similar reversible enzymatic process in this case. Direct evidence of this is lacking, but apparently such a cleavage takes place somewhere at an early stage in the katabolism of the fats, the resulting glycerol perhaps serving as a source of dextrose. From that point on the katabolism is that of the fatty acids.

252. Oxidation at the $\beta$ carbon atom. - The oxidation of the fatty acids, either saturated or unsaturated, to carbon dioxid and water, like the other katabolic processes already considered, is a step by step process. The researches of Knoop, Embden, Dakin and others ${ }^{1}$ have rendered it highly probable, if not certain, that the oxidation, at least in the case of the normal saturated acids, begins at the $\beta$ carbon atom (i.e., at the second carbon atom from the $\mathrm{COOH}$ group) and results in the splitting off of two carbon atoms at a time. The products are carbon dioxid, water and a fatty acid containing two less carbon atoms than the original one and with which the same process of erosion is repeated.

If it be true that the fatty acids thus undergo katabolism in the body by stages of two carbon atoms each, and particularly if it may be regarded as probable that they may be built up again in a similar manner from simpler atomic chains, there is afforded a plausible explanation of the rather striking fact that nearly all of these compounds found in the animal body contain an even number of carbon atoms.

This scheme does not provide for the oxidation of the three lower acids of the series, propionic, acetic and formic, and in

${ }^{1}$ Compare, Dakin, Oxidations and Reductions in the Animal Body, I9I2, pp. I7-47. 
fact, while these acids are known to be freely oxidized in the body, the chemical mechanism of the process is little understood.

253. Formation of carbohydrates from fats. - In discussing the probability of the formation of carbohydrates from proteins (235), it was pointed out that their origin might often be ascribed to either proteins or fats or both. It was there shown that in many cases the probabilities strongly favored a formation from proteins. In other instances, however, the probabilities seem equally strong that fats give rise to carbohydrates. In particular, experiments upon phloridzin diabetes of the dog have shown the production of more sugar than could be formed from the quantity of protein katabolized during the same time, while the stock of glycogen in the animals experimented on had been so exhausted by fasting and muscular work. that it seems scarcely possible to interpret the results otherwise than as showing the formation of sugar from fat. It should be added, however, that it has been seriously questioned whether the conditions of the experiments were sufficiently controlled to warrant the conclusions drawn.

The very low values for the respiratory quotient (296) which have been reported in some cases for hibernating animals have also been interpreted as indicating a production of carbohydrates from fat. In the conversion of fat into sugar, there must obviously be an absorption of oxygen with no corresponding evolution of carbon dioxid, the tendency of which would be to lower the respiratory quotient. The value of the latter for the direct oxidation of fat is 0.7 . In hibernating animals, however, figures as low as 0.3 have been reported, while the weight of the fasting animal increased. While these facts, of course, do not demonstrate the formation of sugar from fat, they are quite compatible with that interpretation and seem to indicate a storage of oxygen. The more recent experiments on hibernating animals, however, have failed to give such low quotients as were obtained by earlier observers.

\section{§ 7. Metabolism of Ash Ingredients}

254. Certain chemical elements of the body and of the feed are found wholly or in part in their ash when these materials 
are burned and are therefore spoken of as ash ingredients, although, as already pointed out $(\mathbf{3}, \mathbf{5})$, this does not necessarily imply that they existed in the original material in "inorganic" combination. Most of these elements are as essential to the vital processes as the more abundant elements carbon, nitrogen, hydrogen and oxygen of the so-called "organic compounds," although unfortunately the laws regulating their metabolism have been much less extensively studied. Among these elements sulphur and phosphorus are of special importance in this connection.

\section{Sulphur}

255. Sources. - While feeding stuffs may contain small amounts of sulphur in the form of sulphates, by far the greater part of this element in the feed of animals exists in organic compounds. Such, for instance, are the allyl sulphid $\left(\mathrm{C}_{3} \mathrm{H}_{5}\right)_{2} \mathrm{~S}$, contained in garlic and other members of the genus allium, and the allyl sulphocyanat, $\mathrm{C}_{3} \mathrm{H}_{5} \cdot \mathrm{CNS}$, found in mustard and other genera of the cruciferæ. Ordinarily, however, the chief carriers of organic sulphur, both in feeding stuffs and animals, are the proteins, which contain the element in the form of the di-amino acid cystin (47).

256. Katabolism. - The question whether the animal body can build up its sulphur compounds from inorganic sulphur does not appear to have been investigated.

The katabolism of the cystin component of proteins presumably follows the same general course as that of the other amino acids, i.e., it is split off from the proteins by hydrolytic cleavage and subsequently deaminized. One of the products of the katabolism of cystin appears to be taurin, $\mathrm{CH}_{2} \cdot \mathrm{NH}_{2} \cdot \mathrm{CH}_{2} \cdot \mathrm{SO}_{3} \mathrm{H}$, contained in the taurocholic acid of the bile. To the extent, therefore, to which the latter contpound escapes resorption in the lower intestine, it carries small amounts of sulphur into the feces. Both cystin and taurin, however, are readily oxidized in the body, the larger part of their sulphur taking ultimately the form of sulphuric acid and being excreted in the urine. The sulphuric acid of the urine exists in combination in part with aromatic radicles derived from the putrefaction of the proteins in the lower intestine and in part with bases. In human urine about one-fifth of 
the total sulphur exists in a less completely oxidized form known as neutral sulphur, the nature and origin of which is obscure.

\section{Phosphorus}

257. Forms ingested. - The phosphorus supply of the body is received substantially in the four forms indicated in Chapter I (5), viz., as phosphates, as phosphatids, as phospho- and nucleo-proteins and as phytin. Of these the various "organic" forms usually predominate.

It appears probable, however, that all these various forms of phosphorus are resorbed into the blood stream in the form of phosphoric acid. Of the phosphates ingested as such this is certainly true. There seems good reason for believing that the phosphoric acid radicle contained in the nucleic acid of the nucleoproteins is quite completely split off by the digestive enzyms and reaches the blood as phosphoric acid (139), and the same thing is presumably true of the phosphoproteins. The phosphatids are probably acted on by the lipases of the digestive tract, but whether the glycerophosphoric acid resulting from their cleavage is further split up is unknown. The ready cleavage of phytin in seeds would suggest that probably its phosphorus also is resorbed as phosphoric acid.

258. Anabolism and katabolism. - The animal body contains a large store of phosphorus in the "inorganic" form, especially in the skeleton. For the maintenance or increase of this store the resorbed phosphoric acid is naturally available.

The body also contains, however, organic phosphorus compounds, which, although less in amount than the inorganic, are of the highest significance for the vital functions. The belief that the phosphorus supply of the body is resorbed chiefly in the form of phosphoric acid necessarily implies, therefore, that the organism is able to utilize inorganic phosphorus for the synthesis of nucleic acids, phosphoproteins, phosphatids, etc., and the experimental evidence is strongly in favor of this belief (497): In this respect, as in many others, the synthetic power of the organism appears to be greater than was long supposed.

Little is known regarding the course followed by the phosphorus in the katabolism of the nucleoproteins, phosphatids, 
etc. Ultimately it takes the form of phosphoric acid and is excreted in the feces or urine (199), but whether any intermediate compounds are formed is not known.

\section{Other elements}

While the other so-called ash ingredients are no less important than the two just considered, little is known regarding their katabolism in the ordinary sense, i.e., of the chemical changes which they undergo in the body. That they may exist in feeding stuffs in organic as well as in inorganic forms is probable. That they enter into organic combination in the animal body is likewise to be assumed but is positively known in only a few instances like that of the iron of the hæmoglobin and the iodin of the thyroid glands.

259. Sodium and potassium. - Both sodium and potassium are contained in the ordinary foods and feeding stuffs and in addition man and farm animals consume not inconsiderable amounts of common salt, although it appears probable that this serves to a considerable extent as a condiment and that the amount actually necessary is less than is often supposed. Babcock, e.g., was able to keep cows for over a year without access to salt, except that contained in their feed, without any obvious ill consequences.

Both potassium and sodium, as well as the chlorin combined with the latter in the form of salt, are excreted in the urine.

260. Calcium and magnesium. - These elements, calcium in particular, are especially important in their relations to the growth and maintenance of the skeleton, but they are not lacking in the soft tissues also, where they perform important functions. Of their intermediary metabolism little is known. As noted in Chapter IV (199), the normal path of excretion of calcium, and to some extent of magnesium, is through the lower intestine, so that the apparent digestibility of these elements is no measure of the amount actually resorbed and utilized in the body processes.

261. Iron. - A long controversy has been carried on over the question whether inorganic iron may be resorbed and if so whether it can be utilized for the synthesis of the hæmoglobins 
and for other purposes in the body. Both questions, however, may be regarded as settled in the affirmative. The matter is of importance in its relations to the use of iron in medicine but is of no special significance in stock feeding.

The excretion of iron takes place almost exclusively through the intestines and this fact led to the earlier conclusion that inorganic iron cannot be resorbed.

\section{§ 8. Functions of the Nutrients}

262. General scheme of metabolism. - In considering the metabolism of the several classes of nutrients in the foregoing paragraphs, it was found that the main features of the process

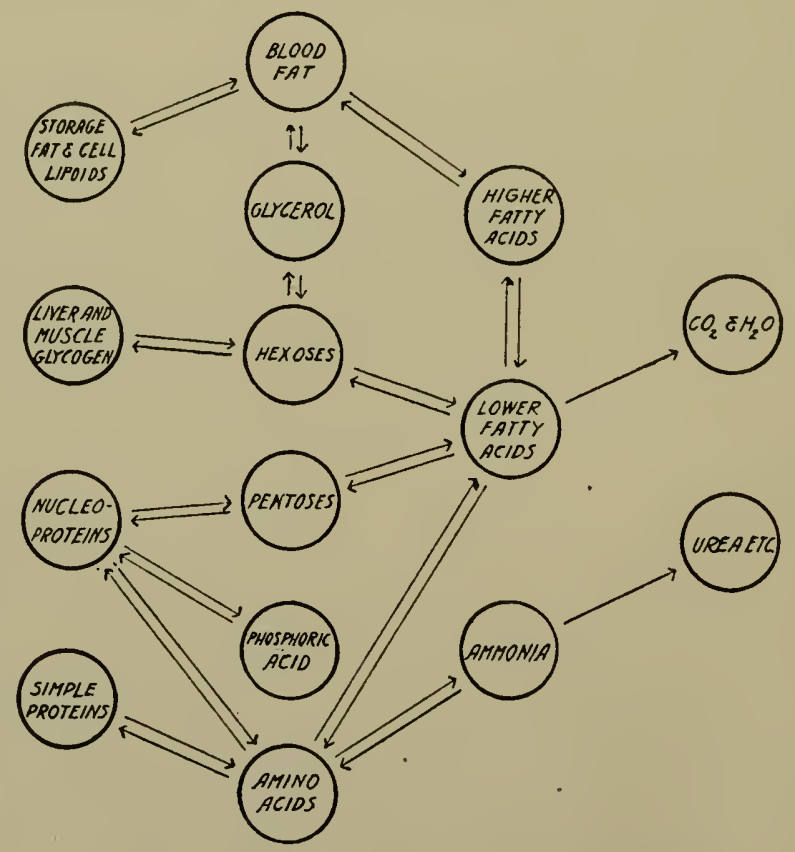

FIG. 23. - Diagrammatic scheme of metabolism.

in each case might be conceived of in accordance with the ideas suggested in $\S 2$ (210-212) as consisting of a complex of reversible reactions accelerated or retarded by intracellular enzyms. By combining the equations used to represent those reactions, it appears possible to take a further step and formulate the following highly generalized scheme for the total metabolism which may serve to show the interrelations between the metabolism 
of the chief classes of organic nutrients. For the sake of simplicity some of the intermediate steps mentioned on previous pages have been omitted. The central portion of the diagram includes the feed substances taken up into the blood. At the extreme left are shown the main groups of tissue ingredients, and at the extreme right the excretory products.

It cannot be too strongly emphasized that any such diagram as the foregoing is of necessity in the highest degree schematic.

For one thing, neither the enzymatic nature nor the reversibility of the changes indicated in the diagram has been established except in a few cases. As already pointed out (212), this conception of the nature of metabolism is still to a large extent hypothetical, although the hypothesis harmonizes well with the present state of our knowledge.

Moreover, aside from the mere omission from the diagram of certain recognized products of the intermediary metabolism, the chemical processes in the body are doubtless infinitely more complex than can be indicated in any such way. A vast number of different substances have been identified in the animal body, many of which are known to have important functions in keeping the organism in running order but which are not even hinted at in this scheme.

In brief, the scheme is concerned with the results of the metabolic processes so far as they are related to nutrition rather than with the mechanism by which these results are brought about. It seeks to show in outline how the principal groups of nutrients are related, on the one hand, to the building up of body tissues, and, on the other hand, to the formation of excretory products, and to indicate the mutual relations of the several groups. For this purpose it may perhaps serve a useful end as an aid to memory, provided its limitations are clearly understood.

263. Dual function of feed. - As pointed out in $\S$ I of this chapter (207), the animal body may be regarded in the light of a transformer of energy. By the agency of the protoplasm of its cells, in ways largely hidden from us, it converts the chemical energy supplied in its feed into the various forms characteristic of living matter. From this point of view the feed has a twofold function.

First, the feed ingredients are carriers of energy. The higher 
plants transform the radiant energy of the sun into the chemical energy of their various constituents, to be yielded up by the latter to the animal organism through the processes of metabolism. This conception has become a familiar one and much emphasis has been laid upon it in recent years.

Second, the feed supplies the specific materials required for building and maintaining all the complex structures of the body and for their harmonious functioning, i.e., it is the source of structural and repair material.

That the proteins, fats and mineral ingredients which make up by far the larger part of the dry matter of the body $(99,280)$ are derived ultimately from the feed needs no special demonstration, but the importance of many substances present in the body in only minute amounts tends to be overlooked. For example, the enzyms of the body, both extra- and intra-cellular, form no considerable portion of its mass, yet they are essential to its vital activities. So, too, the various hormones and secretions of the ductless glands, while ignored in the broad scheme of metabolism just presented, are essential to the vital processes. Clearly, the feed must supply material for the production of these and other similar substances.

In other words, no amount of energy-yielding material will suffice to support life in the absence of those specific substances which are necessary in order that the machinery of conversion shall operate properly, much as no amount of coal under the boiler will enable an electric plant to furnish a normal amount of current if the insulation of the generator is defective. For example, if tryptophan is necessary for the formation of some essential internal secretion, a diet lacking that substance, however much energy it might furnish, would fail to support the - organism permanently unless the body can manufacture tryptophan from other substances.

The latter qualification is a very important one. The animal is very far from being dependent upon the presence in its feed of all the varied chemical compounds required for its operation. Indeed, quite the reverse is the case. As has appeared in previous sections, the actual substances resorbed are comparatively simple and uniform and upon them the animal body executes a great variety of chemical changes, both analytic and synthetic. What is necessary is that the resorbed feed shall include sub- 
stances out of which the body can manufacture the compounds which it requires.

264. Functions of the proteins. - The proteins furnish at once the most familiar and the most striking example of this dual function of the feed.

Since the proteins may be katabolized in the body with the formation of products (carbon dioxid, water, urea, etc.) containing either no available energy or but a small fraction of that found in the original proteins, it is clear that the latter serve as carriers of energy. In fact, it has been shown to be possible to maintain a carnivorous animal in normal activity for an indefinite time on a diet containing substantially nothing but protein as a source of energy.

But proteins serve also as building material. Aside from water, the working machinery of the body is composed largely of proteins, while very many at least of the special substances already mentioned are nitrogenous and probably derived from the proteins. These protein tissues and other substances must be built up in the growing animal and maintained in the mature one, and for this purpose only proteins or their cleavage products can be utilized, and their presence in the feed is indispensable.

A point which sometimes causes perplexity is that the same portion of protein may not only serve as structural material but also yield energy for the vital processes, so that in estimating the energy supplied by a feeding stuff that of its protein as well as that of its other ingredients is included. The difficulty disappears, however, when it is remembered that any given portion of protein does not perform both these functions at the same time. If a gram of protein in the feed of a mature animal is used for structural purposes it practically takes the place of an equal amount of tissue protein, while the latter is katabolized and yields substantially the same amount of energy as would have been available from the gram of feed protein had that been katabolized instead. The latter, with its store of energy, has been temporarily set aside from the katabolic process but at some later time may itself be replaced by another gram of feed protein and katabolized in its turn, liberating the corresponding amount of energy. The repairing of a wooden building may serve as an illustration. The old wood taken out to make way 
for new material, as well as any surplus of new wood over that immediately required, may be used indifferently as fuel for warming the building. The case of the young animal, in which protein is permanently set aside for growth, is a trifle more complex but substantially the same considerations hold good.

265. Functions of fats. - In the case of the fats the energybearing function is the predominant and obvious one. Fats are a concentrated form of fuel, containing much more energy per unit than any of the other nutrients. They supply much energy in a small bulk and are, therefore, well adapted for the storage of reserve energy in the body.

The fats and closely related bodies (the lipoids), however, are also important and apparently essential constituents of protoplasm (75). The lipoids, therefore, have important structural functions and an adequate supply of them in the body is indispensable. From this point of view, some interest attaches to the results obtained by a number of investigators who claim to have shown that a certain minimum supply of lipoids in the feed is essential, especially for growing animals. The evidence, however, is negative evidence, $i$. e., experimental animals failed to grow normally on a lipoid-free diet. In view of the positive results obtained by Osborne and Mendel, ${ }^{1}$ as well as of the fact that both the simple fats and the phosphatids, at least, can be synthesized freely in the organism, and taking into consideration the extensive synthetic power of the body in general, it is difficult to believe that the presence of lipoids in the feed is indispensable, and more recent investigations have afforded a different explanation of the observed facts (498). On the other hand, it has been shown that the lecithins stimulate growth and also that the fats appear, within certain limits, to favor the production of milk fat.

266. Functions of carbohydrates. - The carbohydrates even more distinctly than the fats serve chiefly as carriers of energy. While containing less energy per unit than fats, they can, on the other hand, be consumed in larger quantities and they practically supply the greater part of the energy in the diet of man and of farm animals. While the presence of carbohydrates (dextrose) in the blood and lymph is essential, this appears to be chiefly on account of their ready availability as fuel material.

${ }^{1}$ Jour. Biol. Chem., 12 (rgI2), 8r. 
The carbohydrates seem, however, to have a specific function in relation to the katabolism of fats. When the body is compelled to draw its energy supply chiefly from the fats, as in fasting or in diabetes (in which the power of katabolizing carbohydrates is lost), or when carbohydrates are absent from the diet, the katabolism of the fats fails to be complete and considerable amounts of beta-oxybutyric acid as well as the abnormal katabolic product aceton are excreted unoxidized in the urine.

267. Non-nitrogenous nutrients in general. - While it thus appears that both the fats (or lipoids) and carbohydrates may serve special purposes in the body, it is, nevertheless, clear that their chief function is to supply energy. Their amounts in ordinary rations are so abundant that as compared with their functions as carriers of energy any specific purposes which they serve in the body are amply provided for. As related to the nutrition of farm animals in particular, it is of special interest to note that not only the fats and carbohydrates digested as such but also the products of the bacterial fermentation of the insoluble carbohydrates are available as sources of energy.

268. Functions of ash ingredients. - While the non-nitrogenous organic nutrients serve chiefly as carriers of energy and only in a minor degree to provide the compounds necessary for the performance of specific bodily functions, the so-called ash ingredients represent the other extreme in this respect. They introduce practically no available energy into the organism but, on the other hand, they are not only essential structural components of the body tissues but likewise supply and maintain certain conditions indispensable to the performance of the bodily functions.

The structural importance of the ash ingredients is most manifest in the case of the skeleton, which, in the higher animals, contains relatively large amounts of calcium and phosphoric acid and small quantities of magnesium, sodium and carbonic acid (81) which impart to it certain necessary mechanical qualities of strength and rigidity. The necessity for a supply of these substances in the feed, especially in that of growing animals, is too obvious to require discussion. The ash ingredients, however, have other equally important functions 
in providing the necessary conditions for the chemical and physical activities of the various tissues.

269. Osmotic pressure. - The cells of the various tissues draw their nourishment from the lymph which constitutes their immediate nutritive environment (185) and from which they are separated by cell walls which partake of the nature of semipermeable membranes. In order to maintain normal conditions in the protoplasm of the cells the osmotic pressure of the lymph, and therefore that of the blood from which it is derived, must be maintained approximately constant. The osmotic pressure of the blood is stated to be approximately about 8 atmospheres, due largely to the ash ingredients contained in solution. With an adequate supply in the feed the concentration of mineral matter in the blood is regulated chiefly by the excretory activity of the kidneys. Thus, in the case of sodium chlorid, for example, it is estimated that the blood of an average man contains approximately 30 grams of this substance, of which hardly half a gram is excreted daily when none is consumed. If, however, salt is added to the diet, the excess is promptly excreted in the course of the next twentyfour hours. What is true of salt in this respect is true also of other diffusible ingredients of the blood.

270. Ionic concentration. - The variqus salts are contained in the body largely in dilute aqueous solution. In such solutions, however, it is believed that salts are largely dissociated into their constituent ions, a dilute solution of common salt, for example, containing in addition to some unchanged $\mathrm{NaCl}$ the ions $\mathrm{Na}$ and $\mathrm{Cl}$, one of calcium sulphate the ions $\mathrm{Ca}$ and $\mathrm{SO}_{4}$, etc. Acids are similarly dissociated, yielding hydrogen ions $\left(\mathrm{H}_{2} \mathrm{SO}_{4} \rightleftarrows \mathrm{H}+\mathrm{SO}_{4}\right)$, while alkalies yield $\mathrm{OH}$ ions ( $\mathrm{KOH} \rightleftarrows \mathrm{K}+\mathrm{OH})$. Some of these ions have been shown to have specific effects on certain cellular activities. For example, a frog muscle kept in 0.7 per cent $\mathrm{NaCl}$ solution retains its irritability for one or two days. In a solution of a non-electrolyte, like sugar, asparagin, etc., having the same osmotic pressure, the muscle soon loses its irritability, but if $\mathrm{NaCl}$ be added to the solution it regains it. Since a number of other sodium salts produce the same effect, while chlorids of other metals do not, it is apparent that the effect is due to the $\mathrm{Na}$ ions. On the other hand, $\mathrm{Na}$ ions alone cause long continued rhythmic con- 
traction of muscles, which, however, is suspended by the presence in the solution of certain (not all) dyad ions like $\mathrm{Ca}$ or $\mathrm{Mg}$. Numerous other examples of such antagonistic actions of ions are known, such as those observed by Loeb, for example, in the development of the egg. In general it may be said that cell activities are dependent among other things upon a suitable ionic concentration of various elements in their surroundings, and it is a striking and interesting fact that the so-called physiological salt solutions in which living organs may be kept functionally active for a longer or shorter time contain the various salts in approximately the same proportions as are found in sea water.

Another example of the influence of ionic concentration is afforded in the case of the digestive enzyms. Ptyalin, for example, is sensitive to a very slight excess of hydrogen ions. Pepsin, on the other hand, is most active in the presence of hydrogen ions, while trypsin acts best in the presence of an excess of $\mathrm{OH}$ ions.

271. Maintenance of neutrality. - Closely connected with the foregoing topic and constituting indeed a special case of it, is that of the maintenance of neutrality in the body fluids. A fluid is neutral in the chemical sense when it contains no excess of $\mathrm{H}$ nor of $\mathrm{OH}$ ions, an excess of the former being equivalent to acidity and an excess of the latter to alkalinity. It has been shown that the blood serum, as a representative of the body fluids, is very nearly neutral, its content of $\mathrm{H}$ and $\mathrm{OH}$ ions being approximately $0.4 \times \mathrm{10}^{-7}$ and $7.2 \times 10^{-7}$, i.e., it has an alkalinity equivalent to about $0.0000 \mathrm{I} 2$ gram $\mathrm{NaOH}$ per liter. ${ }^{1}$

The body katabolism is continually producing acids, especially carbonic, phosphoric and sulphuric acids $(256,259)$, which tend to increase the acidity of the blood. These acids are in part neutralized by the ammonia produced in the katabolism of protein (233), but it has been shown by the investigations of L. J. Henderson that the salts of the blood serum, especially the sodium phosphates and bicarbonates, play an important part in maintaining its neutrality. They are present in such

1 Blood is commonly said to be alkaline because it gives an alkaline reaction to ordinary indicators, such as litmus. Such a reaction, however, gives no definite measure of the true alkalinity or acidity. 
proportions that their solution possesses nearly the maximum capacity for the preservation of neutrality, while they also, particularly the phosphates, serve as a means of elimination of an excess of acid through the kidneys in the form of the acid phosphates of the urine.

272. Other functions of ash. - The three general functions just enumerated by no means exhaust the list of offices performed by the ash ingredients. Iron, for example, is an essential ingredient of hæmoglobin, the coloring matter of the red blood corpuscles, which is the vehicle by which oxygen is distributed throughout the body (191). Although contained in the body in relatively minute amounts, this element is, therefore, one of prime necessity. Iodin appears to be an essential ingredient of the thyroid glands, and although we are ignorant of its exact functions it is known that the absence of these glands, or their failure to function, gives rise to serious disturbances (goitre, myxœdema). Recent investigations seem to indicate that manganese and boron, and perhaps other elements not heretofore regarded as essential, may have important functions as catalysts in plants and perhaps, therefore, in animals also, although this is at present a conjecture. It is likewise possible that other elements present in small amounts may later be shown to have physiological functions.

273. Functions of water. - Its very familiarity tends to make us overlook the striking nature of the fact that life as we know it is impossible in the absence of water. If protoplasm may be regarded as a collodial solution, one may almost say that life is possible only in aqueous solutions.

Some reasons for this are fairly obvious. The phenomena of osmotic pressure and ionization, for example, whose importance has just been indicated, are substantially solution phenomena. It is possible also that there are more fundamental reasons for this striking fact. Certainly the larger share of our present chemical knowledge relates to the chemistry of either aqueous solutions or gases, two states resembling each other in many respects and in which chemical action seems to occur most readily, if indeed it ever takes place in the solid state. Moreover, it has been shown that some reactions, at least, in which water is not commonly regarded as concerned, are dependent upon the presence of minute amounts of this sub- 
stance, or at least proceed with extreme slowness in its absence. Such, for example, is the action of chlorin on metallic copper or iron, or of oxygen upon many of the elements even at high temperatures.

Aside from these considerations, however, the importance of the part played by water in the animal economy is sufficiently obvious, while it constitutes in most cases more than half of the weight of the body (97) and therefore may be regarded as having structural importance. 


\section{CHAPTER VI}

\section{THE BALANCE OF NUTRITION}

\section{§. General Conception}

274. The animal as a prime motor. - The living animal constitutes what is known as a prime motor; that is, it generates power for its own operation and is able to produce a surplus which may be applied to do external work. In particular, a fairly close analogy may be drawn between the animal body and what are known as internal combustion motors. In such motors, a fuel (gas, gasoline, alcohol, etc.) is burned in the cylinder of the engine itself and its available chemical energy is transformed in part into motion and in part into heat. In a somewhat similar manner the compounds supplied to the cells of the body by the processes of digestion, resorption and circulation are katabolized, combine with the oxygen introduced through the lungs, and yield energy for the various activities of the organism. It should be noted that these activities include not merely external work done by the animal but likewise a variety of internal work, such as that of circulation, respiration, digestion, resorption, secretion, etc. In other words, the animal machine is always in operation, even when performing no external work.

275. Expenditure by the body. - When in operation, a mechanical prime motor (a gasoline engine, for example) consumes two things. First, the material of which the working parts are composed is gradually worn away so that ultimately repairs are necessary, and second, fuel is consumed in amount depending upon the work done. Substantially the same thing is true of the animal body.

The working machinery of the body may be regarded as composed essentially of water, ash and protein. This machinery, like that of the engine, is continually wearing out; that is, the protein in particular is being continually katabolized and 
the products of its oxidation excreted. In addition, the activities of the body, like those of the engine, require a supply of fuel material containing available chemical energy equivalent to the work to be done. For this purpose the body utilizes in the first instance the substances contained in its own cells and tissues. As shown in Chapter V, all the organic ingredients of the body - protein, fat and carbohydrates - undergo katabolism, giving rise to carbon dioxid, water and comparatively simple nitrogenous products, accompanied by a transformation of their chemical energy into other forms. In other words, the body is a storehouse of chemical energy as well as a mechanism. This stored-up energy of the body is contained particularly in its fat, and to a minor degree in its glycogen, while the body protein, although it likewise yields energy when katabolized, especially through the oxidation of its non-nitrogenous residue (229), usually plays a small part quantitatively. The fat of the body constitutes its great reserve of energy. The store of reserve material in the body may be compared, for the sake of illustration, to the gasoline in the tank of an automobile, with the difference, however, that the body derives more or less energy from the combustion of the material (protein) of the engine itself.

276. The feed. - Neither the automobile nor the animal can long depend entirely upon its own stock of material without disaster. Sooner or later it must obtain supplies from the outside. The supplies required in both cases are obviously of two classes, corresponding to the two classes of materials consumed in the operation of the machine, and may be briefly designated as repair material and fuel.

In the automobile, parts of the machinery, the tires, etc., as they wear out must be replaced by new ones of the same kind, while the gasoline tank must be filled at intervals and the working parts must be suitably lubricated. The case of the animal is precisely similar. In the first place, it must be supplied in its feed with materials from which, by the processes of digestion and resorption, it can secure the particular atomic groupings (amino acids, peptids, ash ingredients, etc.) which will exactly fit into its protoplasm and replace those eliminated by the vital activities. In the second place it must also derive from its feed molecules which it may, according to circumstances, break 
down (katabolize) at once for the sake of their energy or store up as a reserve of energy (fat, glycogen) for future use. Finally, to carry the analogy a step further, it must obtain from its feed such amounts and proportions of the several ash ingredients as will maintain the necessary working conditions of osmotic pressure, ionic concentration and the like, somewhat as the engine must be lubricated.

277. Balance of income and expenditure. - It is evident from the foregoing considerations that the body exhibits two sets of activities, those concerned in its actions as a prime motor, tending to destroy it, and those of nutrition, tending to build up and increase it. Whether the body gains, is maintained or falls away depends upon the balance between these two sets of activities.

In a broad general way, of course, this fact is perfectly obvious. We do not need a physiologist to teach us that the horse or cow cannot long continue to do work or to yield milk unless supplied with sufficient feed to make good the resulting loss of body material. Similarly, we are familiar with the fact that those operations of the body which go on in a state of so-called rest likewise require material for their support, so that the mere maintenance of an animal calls for an expenditure of feed. What is needed in a scientific study of nutrition is something more than the mere general knowledge of these familiar facts; namely, a quantitative measure of the extent to which the various feeding stuffs or their single ingredients contribute to the nutritive functions of the body under varying conditions.

\section{§ 2. Methods of Investigation}

278. Investigation of details of metabolism. - One method of attacking the problem just stated is by investigating the details of the metabolic processes. In the study of metabolism (including the chemical changes in digestion and resorption) the attempt is made to follow the various ingredients of the feed through the body and to trace in detail how, where and to what extent they contribute to the maintenance or growth of tissue or supply energy for the use of the organism. Such studies are of fundamental importance. They reveal to us how the animal mechanism operates. When carried to their ultimate con- 
clusion, and when accompanied by a complete knowledge of the chemical ingredients found in feeding stuffs, they will make it possible to give an exhaustive account of nutrition as a physicochemical process. It is hardly necessary to say that the realization of this ideal lies in the distant future.

279. Total nutritive effect. - Meanwhile, students of stock feeding are interested primarily in a somewhat different aspect of the subject, viz., in the aggregate effect of the varied and complex metabolic processes in reducing, maintaining or increasing the stock of matter and of chemical energy in the body. Is the body under any given regimen maintaining itself and making due growth, or is the animal doing work or yielding milk or other products at the expense of its own tissues? This is evidently a question of balance. Is the income of the body equal to its outgo?

280. The schematic body. - The idea of the organism as dependent upon a balance between constructive and destructive activities may be made more specific by means of the conception of the schematic body, which regards the body of the animal, aside from water, as consisting essentially of ash, protein and fat, together with an amount of glycogen so small that it may for many purposes be neglected.

The justification for this conception is found in the data contained in Chapter II, $\S_{3}$, regarding the composition of the animal as a whole. It will be recalled that in the investigations there recorded the water, ash and fat were determined directly, the difference between the sum of these and the total weight of the animal, of course, showing the amount of fat- and ash-free dry matter. In those cases in which the total nitrogen contained in the body was also determined, it appeared (99) that, with one exception, the percentage of nitrogen in this fat-and ash-free dry matter closely approximated that in the animal proteins. In other words, the amount of glycogen and other substances included in the fat-and ash-free dry matter is so small as to be negligible and the latter may be considered to consist essentially of protein.

From this point of view, it is evident that the effect of any feeding stuff or ration in causing a gain or preventing a loss of ash, protein and fat (and glycogen) shows its aggregate nutritive effect. Or, since the organic matter of the body may be 
looked upon in the light of stored energy, a still simpler expression of the nutritive effect may be obtained by determining the effect of the feed upon the store of protein and of chemical energy in the body. ${ }^{1}$

Experiments directed to the determination of the gain or loss of matter and of energy by the body have been of two general kinds, viz., comparative slaughter tests and what are called balance experiments. Both have played an important rôle in the study of nutrition.

281. Live weight as a measure of nutritive effect. - At the very outset, however, the question arises whether the simple and obvious method of weighing an experimental animal is not sufficient to determine the aggregate effect of a ration, without the necessity for any elaborate experimental devices.

The answer to this question depends largely upon the object of the experiment. If it be one undertaken to answer a commercial question, the increase in live weight during a considerable period, when determined with the necessary precautions, may be entirely adequate as a measure of the results obtained. If, for example, the question under investigation is the relative profits of two methods of fattening cattle, the gains made by a considerable number of animals, together with the judgment of the market regarding the quality of the finished animals, will substantially determine which method is to be preferred. The use of more elaborate experimental methods would not only be a needless refinement but might actually interfere with the settlement of the economic question involved. So, too, in the handling of young stock or in milk production, the general appearance and condition of the animals, together with the gain in live weight or the yield of milk, furnishes a sufficiently accurate indication of the practical results obtained, provided a sufficient number of individuals be employed.

If, however, the purpose of the investigation is to study some question relating to the fundamental principles of nutrition,

1 To make the demonstration absolutely complete, of course, it would be necessary to show that the stock of each different kind of protein in the body had been maintained and that all the energy containing material derived from the feed was actually capable of yielding up its energy to the organism. Usually, however, especially on a mixed diet, it may be assumed that if the body maintains its stock of protein, each particular kind is practically maintained, while no considerable storage of unavailable energy in the body has been recognized. 
such, for example, as the relative values of the carbohydrates and fats, the changes of live weight are of little value as indicators. For this there are two principal reasons.

282. Fluctuations in live weight. - In the first place the live weight of an animal fluctuates considerably from day to day, even when taken under what seem to be identical conditions, chiefly on account of variations in the weight of the contents of the digestive tract. This is true of all animals, but especially of herbivora on account of their comparatively bulky feed, and reaches the extreme in ruminants.

For example, a steer which had been receiving daily for two months a uniform ration of $6.35 \mathrm{Kgs}$. of timothy hay and which was kept under as uniform conditions as possible was weighed daily 24 hours after watering. On February I9 he weighed 4I9.0 Kgs. and on March 6 practically the same amount, 4I8.6 Kgs. The intermediate weights, however, were as follows:-

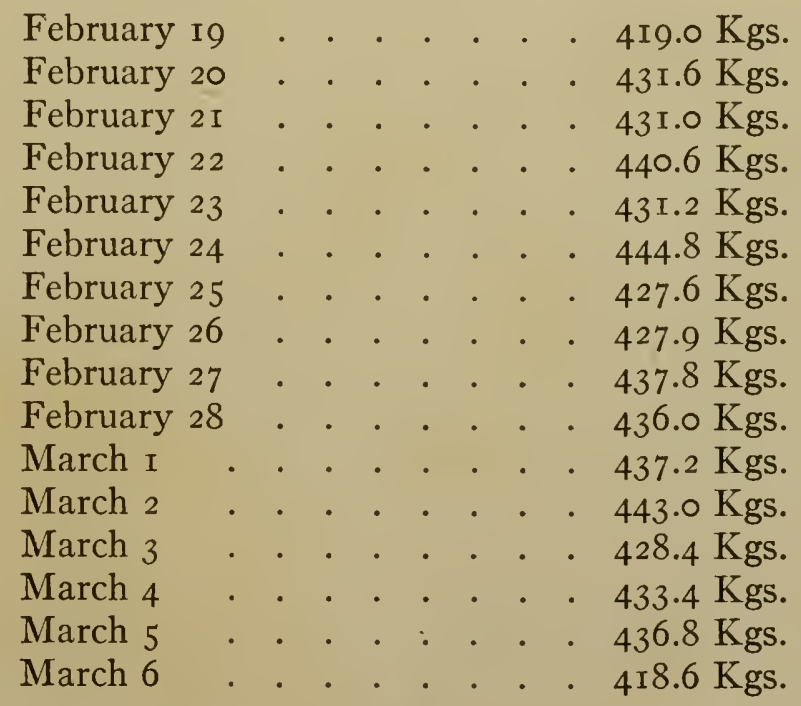

It is evident that conclusions based upon a comparison of single weighings would have been entirely untrustworthy. Thus a comparison of the live weight of February I9 with that of March 6 would have led to the conclusion that the animal was being practically maintained. If, however, the initial weight had chanced to be taken on February 20, a comparison with that of March 6 would have shown a loss of 13 Kgs., while on the other hand, a comparison of February I9 with March 5 would have shown a gain of $17.8 \mathrm{Kgs}$. Even aver- 
aging two or three successive daily weighings, as is often done, while it reduces the error does not eliminate it. For example, a comparison of the average of February I9-2 $\mathrm{I}$ with that of March $3-5$ shows a gain of $8.7 \mathrm{Kgs}$., while if each average be taken a day later, viz., February 20-22 and March 4-6, the comparison shows a loss of 4.8 Kgs. By increasing the number of single weighings averaged, the uncertainty may, of course, be further reduced but not entirely eliminated, even ten-day averages varying materially, as is illustrated by the following figures.

February 24-March 5, inclusive, $435.3 \mathrm{Kgs}$. February 25-March 6, inclusive, 432.7 Kgs. February 26-March 7, inclusive, $432.6 \mathrm{Kgs}$. February 27-March 8, inclusive, $434.2 \mathrm{Kgs}$.

A similar reduction of the error may be obtained by the use of a number of animals combined into a group which is treated as an individual, the fluctuations in the single animals tending to balance each ọther.

These fluctuations are such as to preclude the use of the gain in live weight as a measure of the nutritive effect in exact scientific investigations, while it is evident that they must also be considered in the planning and interpreting of commercial experiments, as well as in judging the effects of rations in practice. Such experiments should extend over a considerable length of time and include a considerable number of animals, while the weights on which comparisons are based should be the average of as many single weighings as possible.

283. Composition of increase. - In the second place, even were it possible to ascertain the gain or loss in weight by the body tissues proper, exclusive of the contents of the digestive tract, i.e., the empty weight, the composition of the material gained would still be unknown. An increase of a kilogram in tissue weight, for example, might consist chiefly of adipose tissue containing Io or I 2 per cent of water (95), or it might be largely muscular tissue with 75 or 80 per cent of water (87). Moreover, aside from the difference in water content, the dry matter of adipose tissue carries more chemical energy than that of muscular tissue, so that a gain of a kilogram in the former case would be equivalent to the storage of seven or eight times as much energy as in the latter. Finally, a knowledge of the 
kind of material gained or lost is necessary. In the study of growth, for example, it is important to know how much of the increase in weight is due to a storage of protein, ash, etc., i.e., to real growth, and how much to a mere storage of fat or water, or both.

For all these reasons the increase or decrease in live weight, while not valueless, is by itself an entirely inadequate measure of nutritive effect in investigations into the principles of nutrition. In such experiments it is essential to determine at least the gain or loss of the great groups of substances of which the body is composed, viz., water, ash, protein, fat and if possible carbohydrates, by one of the two general methods already mentioned as available for this purpose, viz., the comparative slaughter test or the balance experiment.

284. The comparative slaughter test. - This method seeks to determine by analysis the actual weights of water, protein, fat, etc., or the quantities of chemical energy, contained in the body of the experimental animal at the beginning and at the end of the experiment. Since, however, it is obviously impossible to analyze the same animal twice, its original stock of protein, etc., is ascertained by the use of a check animal as exactly like the other in age, weight, condition, conformation, etc., as it is possible to select, which is slaughtered and analyzed at the beginning of the experiment. Assuming initial identity of percentage composition for the two animals, the results of this analysis are used to compute the weights of the several ingredients contained in the body of the experimental animal at the outset of the experiment, while the animal itself is analyzed at its close.

The method of comparative slaughter tests has the advantage of being a direct determination of the amounts of each ingredient gained and of requiring comparatively simple appliances. Furthermore, it may be applied not only to the conventional groups of protein, fat, etc., but to any substance capable of accurate analytical determination. Finally, in addition to the total amount of any substance, its distribution between different parts of the body may be ascertained. On the other hand, the method has certain drawbacks.

In the first place, it requires relatively long experimental periods. Assuming the work of weighing, sampling and analysis to be correctly performed, the accuracy of the results evidently depends upon the 
care and skill exercised in the choice of the check animal. The assumed identity of composition of the two animals cannot in the nature of things be proved and is very unlikely to be absolute. In a short experiment, therefore, the error thus possibly introduced may be relatively large. Its importance diminishes the greater the increase made over the original weight, i.e., the longer the period covered by the experiment. Furthermore, an experiment by this method can be divided into periods only by the use of additional check animals, involving additional assumptions as to identity of composition at different times, while even these subdivisions, for the reason just stated, must be fairly long. Finally, the method is laborious, especially with the larger animals. The different parts of the carcass must be separated, the weight of each part accurately determined, avoiding mechanical losses and making due allowance for evaporation of water. A correct sample of each part must be taken promptly and at once so treated as to preclude any changes previous to its analysis. The task of analyzing the carcass of a hog or sheep, and still more that of a steer, with the degree of accuracy required in a scientific investigation is not one to be undertaken lightly.

285. The balance experiment. - The comparative slaughter test attempts to determine the weights of the several ingredients contained in the body at two different times. The balance experiment, on the contrary, consists of a comparison of income and outgo and does not attempt to determine the original stock in the body. If I know that I have a balance of $\$ 50$ in bank at the beginning of the month and $\${ }_{1} \circ$ at the end, it is clear that I have gained \$1oo in the meantime. This is the principle of the comparative slaughter test. On the other hand, if I know that my deposits during the month were $\$ 500$ and my drafts $\$ 400$, I am equally sure that I have gained $\$ 100$, even if $I$ do not know whether my balance at the beginning was $\$ 50$ or $\$ 500$. This is the principle of the balance experiment. If, for example, a steer digests 750 grams of protein out of his daily ration and if the amount of nitrogenous products excreted in 24 hours shows that he has katabolized 500 grams of protein, it is evident that his original stock of protein, whatever its amount may have been, has been increased by 250 grams. By comparisons based on the same general principle, although more complicated as to details, the increase or decrease of the body's stock of fat, glycogen, ash and water or of chemical energy may 
be determined. The specific methods used for such comparisons are described in the two following sections.

A great advantage of the balance experiment is the comparatively short time which it requires. A period sufficiently long for the determination of the digestibility of a ration (159) is in general sufficient also for a balance experiment, while for the requisite determination of the respiratory products or of the heat produced twenty-four to forty-eight hours suffice, and even this short period may be divided into a number of subperiods of a few hours each. For this reason, and also because the animal is not injured in the process, repeated experiments may be made on the same subject, so that the effect of various rations or conditions may be compared on the same individual, while the method of comparative slaughter tests necessarily involves comparisons between two different animals.

On the other hand, the complete balance experiment requires elaborate and expensive apparatus, while opinions as to the relative amount of labor involved in the two classes of experiments would perhaps depend largely upon the previous experience of the experimenter. Furthermore, the balance experiment shows only the amounts of the constituent groups - protein, fat, etc. - gained or lost. It affords no opportunity to subdivide these and determine the fate of single chemical compounds nor does it give any clue to the particular region of the body where the gains have been deposited.

286. The balance of nutrition. - The phrase "balance of nutrition " used as the title of this chapter refers in a general way to the balance between income and outgo of matter and energy in the body as determined by the methods of the balance experiment.

Logically, of course, the comparative slaughter test, if combined with determinations of the feed consumed, may also be. regarded as a balance experiment. In it the income of the body and the resulting gain are determined, leaving the outgo to be inferred, while in a balance experiment in the technical sense, the income and outgo are determined and the gain is inferred. Nevertheless, the latter type of experiment has played so large a part in the study of the balance of nutrition, both for physiological and for agricultural purposes, that a clear conception of its methods and postulates is essential for a comprehension of many of the results to be considered in subsequent chapters. The subject may be conveniently considered under the two heads of the balance of matter and the balance of energy. 


\section{§3. The Balance of Matter}

\section{The gain or loss of protein}

287. The nitrogen balance. - Feed protein which fails to be stored up in the body is not excreted as protein but in the form of the various products of its katabolism. The gain or loss of protein, therefore, cannot be determined by a direct comparison of its income and outgo because there is no outgo of protein as such. Since, however, the protein of the schematic body (280) is equivalent to total nitrogenous matter, the gain or loss of protein may be inferred from that of its characteristic element, nitrogen, and this is readily ascertained by a comparison of the total nitrogen of the feed with the total nitrogen of the excreta, i.e., by a determination of the nitrogen balance.

288. Free nitrogen not excreted. - In Chapter V (228) it was stated that all the nitrogen of the protein katabolized is found in the urea and other organic compounds which are excreted in the urine. Obviously this is a point of fundamental importance. If nitrogen leaves the body only as combined nitrogen in the urine and in the feed residues and nitrogenous excretory products found in the feces, it is a comparatively simple matter to compare the income and outgo. If, however, the metabolic processes or the fermentations of the feed in the digestive tract yield also gaseous nitrogen, then the nitrogen of the respiratory products must also be determined, a task of no small difficulty.

The question of the excretion of gaseous nitrogen has been the subject of a vast amount of investigation and controversy. Substantially two general methods of experimentation have been followed, viz., a comparison of the income and outgo of combined nitrogen and direct investigation of the respiratory products, and the results of both have been in substantial accord. The cumulative force of the great number of experiments in which substantial equality between income and outgo of combined nitrogen has been observed under conditions which precluded the possibility of any considerable gain or loss of body protein, together with the fact that the very careful and accurate investigations of recent years upon the respiratory excretion of free nitrogen have given negative results, amount to 
a demonstration that nitrogen leaves the body only in the combined form in the visible excreta.

289. Determination of nitrogen balance. - There being no excretion of gaseous nitrogen, a determination of the nitrogen balance requires simply a determination of the amounts of this element contained in the feed and in the visible excreta. Evidently this end is already partially attained in a digestion experiment (158). It is only necessary in addition to provide for the quantitative collection and analysis of the urine and, in very accurate experiments, of the perspiration and of the epidermal excreta, in order to obtain data for a comparison of the income and outgo of nitrogen, and the same precautions as to length of period, uniformity of feeding, etc., which are necessary in a digestion experiment, suffice also to render the results of a balance experiment representative.

290. Example of a nitrogen balance experiment. - The digestion experiment with clover hay used as an example in Chapter III (160) may serve also to illustrate the nature of a nitrogen balance experiment. In that experiment the hay consumed daily contained 3.I44 Kgs. of dry matter and the daily feces $1.267 \mathrm{Kgs}$., while the average daily weight of the urine for 9 days was $5.449 \mathrm{Kgs}$. Analysis showed the following percentages of nitrogen :-

\begin{tabular}{|c|c|c|c|c|}
\hline In dry matter of hay & & & & \\
\hline n dry matter of feces & & . & & \\
\hline fresh urine & 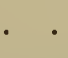 & & & \\
\hline
\end{tabular}

The brushings of the steer (hair, dandruff, etc.) were found to contain 1.87 grams of nitrogen per day. The daily nitrogen balance may accordingly be computed as follows, showing a loss from the body which, of course, must be placed in the income column to complete the balance.

Table 22. - Example of a Nitrogen Balance

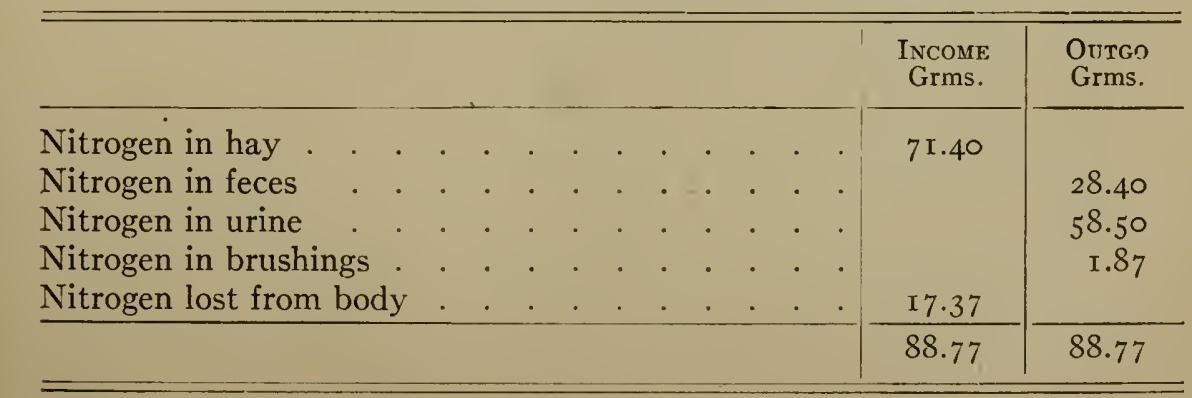


291. Computation of protein. - The conception of the schematic body (280) upon which balance experiments are based regards the total nitrogenous matter of the animal as consisting substantially of protein. All the vast number of other substances containing this element which have been identified as constituents of the body are insignificant in amount as compared with the great mass of protein which it contains. Accordingly, a gain or loss of nitrogen is interpreted as signifying a gain or loss of protein and the amount of the latter may be computed from the former just as the protein of a feeding stuff is computed from its protein nitrogen, it being only necessary to fix upon a suitable factor or factors, $i$. e., to know the average percentage of nitrogen in body protein.

From the results of analyses of entire bodies of animals cited in Chapter II, the average nitrogen content of the fat- and ash-free dry matter was computed (99) to be :-

In Lawes and Gilbert's experiments . . . . I6.II \%

In Chaniewski's experiments . . . . . $16.06 \%$

It is probable that in both cases the supposedly fat-free matter still contained some fat, it having been subsequently shown that extraction with ether does not remove the last traces of it from animal tissues.

Köhler's analyses (88) of the fat-and ash-free lean meat of various species, after correction for the glycogen content of the horse flesh, show an average nitrogen content of $\mathrm{r} 6.64$ per cent. Since the material of Lawes and Gilbert's and of Chaniewski's experiments doubtless included some residual fat and other non-nitrogenous substance, and since the larger share of the protein of the body is contained in the muscular tissues, it appears justifiable to regard Köhler's figures as representing with substantial accuracy the average elementary composition of body protein as a whole, especially since they are the results of direct analysis while the others are derived from slaughter experiments in which the limits of error are somewhat wide.

Assuming, on the basis of Köhler's results, that average body protein contains 16.64 per cent of nitrogen, the corresponding protein factor is 6.0 , and the gain or loss of nitrogen observed in a nitrogen balance experiment multiplied by this factor gives the gain or loss of protein. This is, of course, an approximation, since protein is not the only nitrogenous substance contained 
in the body and since not all the animal proteins contain exactly 16.67 per cent of nitrogen, but the error involved is insignificant in most cases so far as it relates to the question of the balance between income and outgo.

On this basis, the steer in the foregoing example was losing daily $17.37 \times 6.0=104.22$ grams of body protein. Evidently the results of an experiment in which a gain of nitrogen occurs can be computed in precisely the same way.

\section{The gain or loss of fat and glycogen}

292. The carbon balance. - By a method quite similar in principle to that just described for protein, it is possible to compute approximately the gain or loss of body fat from the combined income and outgo of nitrogen and carbon, while if the balance of hydrogen and of oxygen can also be determined the computation may be made considerably more exact and may include glycogen also. The experimental methods, however, are necessarily much more elaborate than those required for a simple determination of the nitrogen balance, since it is evident that, in addition to the carbon of the feed and of the visible excreta, it is necessary to determine the amount of this element contained in the gaseous excreta, viz., carbon dioxid and methane, while if the balance of hydrogen and oxygen is to be included, the hydrogen of the feed, the water excreted and the amount of oxygen taken up from the air must also be ascertained. An outline of the experimental methods employed for these purposes is given in a succeeding paragraph (297), but at the outset it seems desirable to confine attention to the principles involved.

293. Computation of gain or loss of fat. - According to the conception of the schematic body (280) on which the whole scheme of the balance experiment is based, substantially all the carbon of the body is regarded as existing in the two forms of protein and fat. Evidently if a comparison of the income and outgo of carbon shows a gain of that element it can, according to the fundamental assumption, have been only in one or the other or both of these two forms. The nitrogen balance, however, shows the amount of protein gained and the carbon content of protein is known. If the carbon of the protein gained 
be subtracted from the total gain of carbon, the remainder can have been gained only in the form of fat and the corresponding amount of this substance can be readily computed.

294. Example of a carbon balance. - In a respiration experiment on a steer a complete statement of the nitrogen and carbon balances is as follows:-

Table 23. - Nitrogen and Carbon Balances of a Steer

\begin{tabular}{|c|c|c|c|c|c|c|c|c|}
\hline & & & & & \multicolumn{2}{|c|}{ Nitrogen } & \multicolumn{2}{|c|}{ CARBON } \\
\hline & & & & & $\begin{array}{l}\text { Income } \\
\text { Grms. }\end{array}$ & $\begin{array}{l}\text { Outgo } \\
\text { Grms. }\end{array}$ & $\begin{array}{c}\text { Income } \\
\text { Grms. }\end{array}$ & $\begin{array}{l}\text { Outgo } \\
\text { Grms. }\end{array}$ \\
\hline \multirow{8}{*}{$\begin{array}{l}6988 \text { grms. timothy hay. } \\
400 \text { grms. linseed meal . } \\
\text { r66 I9 grms. feces . . } \\
4357 \text { grms. urine . . } \\
37 \text { grms. brushings } \\
4730 \text { grms. carbon dioxid } \\
\text { I42 grms. methan . } \\
\text { Gain by body. . . }\end{array}$} & & \multirow{8}{*}{$\begin{array}{l}\cdot \\
\cdot \\
\cdot \\
\cdot \\
\cdot \\
\cdot \\
\cdot \\
\cdot \\
.\end{array}$} & \multirow{8}{*}{\multicolumn{2}{|c|}{$\begin{array}{ll}\cdot & \cdot \\
\cdot & \cdot \\
\cdot & \cdot \\
\cdot & . \\
\cdot & . \\
\cdot & . \\
\cdot & . \\
. & .\end{array}$}} & $5^{6.4}$ & & \multirow{7}{*}{$\begin{array}{r}2831.7 \\
172.6\end{array}$} & \\
\hline & & & & & 21.9 & & & \\
\hline & & & & & & $\begin{array}{l}33.5 \\
32.4\end{array}$ & & $\begin{array}{r}\text { I } 428.7 \\
\text { I } 24.2\end{array}$ \\
\hline & & & & & & I. 3 & & 8.0 \\
\hline & & & & & & & & I 290.2 \\
\hline & & & & & & & & 106.6 \\
\hline & & & & & & I I.I & & 46.6 \\
\hline & & & & & 78.3 & 78.3 & $3004 \cdot 3$ & $3004 \cdot 3$ \\
\hline
\end{tabular}

The nitrogen balance shows that the animal gained I I.I $\times 6.0=$ 66.6 grams of protein. According to Köhler's results (88), the average protein of cattle contains 52.54 per cent of carbon. Consequently, the protein gained in this experiment contained $66.6 \times .5254$ $=35.0$ grams of carbon. The total gain of carbon, however, as shown by the carbon balance, was more than this, viz., 4.6.6 grams, and we accordingly have the following:-

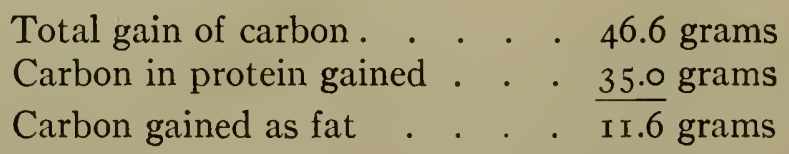

The elementary composition of animal fat was shown in Chapter I (34) to be very uniform, averaging 76.5 per cent of carbon. A gain of 0.765 gram of carbon in the form of fat, therefore, is equivalent to a gain of one gram of fat or a gain of one gram of carbon to I.3 I grams of fat, and accordingly the gain of I .6 grams of carbon in the form of fat shows a gain by the animal of Ir. $6 \div 0.765$, or I $1.6 \times$ I.3 I $=15.2$ grams of fat. Substantially the same method of 
computation can, of course, be applied when there is a loss of nitrogen or carbon or both. ${ }^{1}$

295. Gain or loss of glycogen. - The only non-nitrogenous organic substance other than fat present in the body in sufficient amounts to affect the foregoing computations is glycogen. It is generally assumed that under reasonably normal conditions of feeding the glycogen content of the body does not fluctuate materially, so that any considerable or long continued gain of carbon, other than that contained in protein, is in the form of fat. Probably this is not equally true in the case of a loss of carbon, and in any case the results of computations like that of the preceding paragraph are evidently subject to a degree of uncertainty as regards a possible gain or loss of glycogen by the body. While this is probably not serious in reasonably long periods it may be relatively important in short experiments. If, however, there can be added to the determination of the nitrogen and carbon balance that of the balances of hydrogen and oxygen the means are afforded for a more accurate calculation, since it is evident that the amounts of the latter two elements, especially of oxygen, retained in the body would be greater in the case of a storage of glycogen than in that of a storage of fat containing the same amount of carbon. The method of computation is, however, somewhat complicated and need not be gone into here. ${ }^{2}$

296. The respiratory quotient. - The respiratory quotient is the ratio of the volume of carbon dioxid excreted by an animal to the volume of oxygen taken up during the same time, i.e., it is $\frac{\text { Vol. } \mathrm{CO}_{2}}{\text { Vol. } \mathrm{O}_{2}}$. The respiratory quotient will obviously vary according to the kind of material which is being katabolized in the body. Thus in the oxidation of carbohydrates each liter of oxygen consumed gives rise to the production of one liter of carbon dioxid and the respiratory quotient therefore is I.o. On the other hand, when fat is oxidized, a portion of the oxygen unites with the hydrogen of the fat and only the remainder is available for the production of carbon dioxid. It is easy to compute, therefore, that each liter of oxygen consumed in the oxidation of fat will give rise to the production of

1 To avoid errors in computation it is convenient to regard losses in such computations as negative gains and to carry through the computation exactly as in the above experiment, using the algebraic sum or difference in every instance.

${ }^{2}$ See Atwater and Benedict, A Respiration Calorimeter, etc., Carnegie Institution of Washington, Publication No. 42 (I905). 
0.7 liter of carbon dioxid. Similarly, it may be computed that if protein of average composition be oxidized to urea, carbon dioxid and water, the respiratory quotient will be approximately 0.8 , although in reality the quotient for protein varies according to the nature of the nitrogenous products formed and the amount of carbon thereby withdrawn from oxidation to carbon dioxid. Ordinarily, however, the proportion of the gaseous exchange of the body due to the katabolism of protein is comparatively small, so that if, for example, the respiratory quotient closely approaches I.o, it is clear that the katabolism must be chiefly that of carbohydrates, while if, on the other hand, its value approaches 0.7 , it is equally evident that the katabolism must be chiefly that of fat. Values for the respiratory quotient intermediate between these extremes imply that the katabolism is in part that of fats (or proteins) and in part that of carbohydrates.

The respiratory quotient of course affords no information regarding the balance between income and outgo but its determination gives valuable information as to the nature of the material which is being katabolized in the body, particularly in short periods.

297. The respiration apparatus. - A determination of the gaseous exchange of an animal, such as is necessary in order to formulate the complete balance of matter, requires the use of some form of special apparatus known as a respiration apparatus.

In its simplest and earliest form the respiration apparatus consisted of a closed chamber of known capacity, such as was used by Crawford, Mayow, Black, Priestly, Lavoisier and others in their early experiments. The animal was placed in the hermetically sealed apparatus and the changes in the composition of the enclosed air which were brought about by its respiration were determined. Evidently, however, the method, while charmingly simple, is open to objections. The oxygen of the air is gradually consumed, while the carbon dioxid and other products of respiration accumulate. Even if the experiment be broken off before fatal results to the animal ensue, it is made under varying and increasingly abnormal conditions, while no very long trials are possible.

Two obvious methods of avoiding this difficulty at once suggest themselves; either to absorb the products of respira- 
tion and replace the oxygen consumed or to conduct a current of air through the apparatus. Correspondingly, two different types of respiration apparatus have been evolved, known respectively as the closed circuit and open circuit apparatus, or, from the names of the investigators who first developed them into practicable appliances, as the Regnault-Reiset and the Pettenkofer apparatus. Each of these two types may be subdivided into those intended to determine the total gaseous exchange of an animal and those which take account only of the pulmonary exchange.

298. The Regnault-Reiset apparatus. - In the closed circuit, or Regnault-Reiset apparatus, respiration takes place in

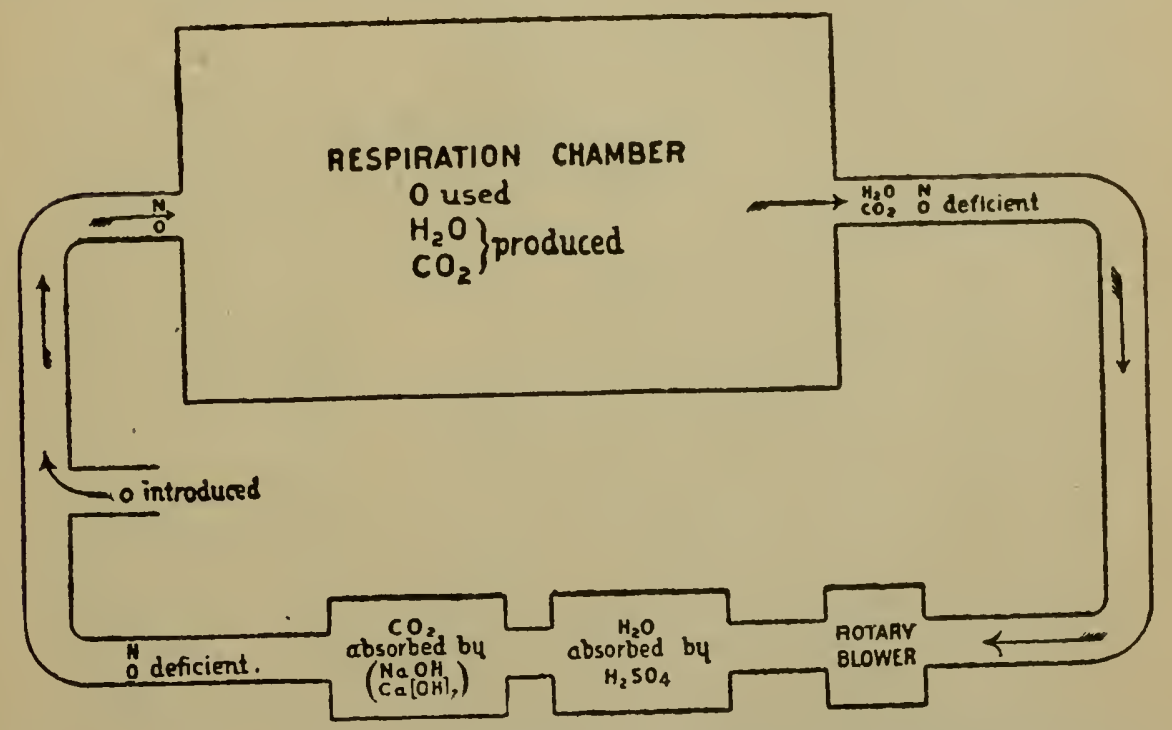

Fig. 24.- Scheme of closed circuit respiration apparatus. (Atwater and Benedict, Carnegie Institution of Washington, Publication No. 42.)

a confined volume of air, the possibility of any exchange between it and the outside atmosphere being carefully guarded against. By suitable mechanical means (a blower, for instance) the confined air is kept in circulation over suitable absorbents which take up the water and carbon dioxid given off, while the oxygen consumed is replaced from a gasometer or a cylinder of the compressed gas. The general scheme for such an apparatus is shown in Fig. 24. The increase in weight of the absorbents plus any increase in the amount of carbon dioxid and 
water contained in the air of the apparatus shows the amounts of these substances produced, while the amount of fresh oxygen admitted minus any increase of the oxygen contained in the air of the apparatus shows the quantity of this element absorbed.

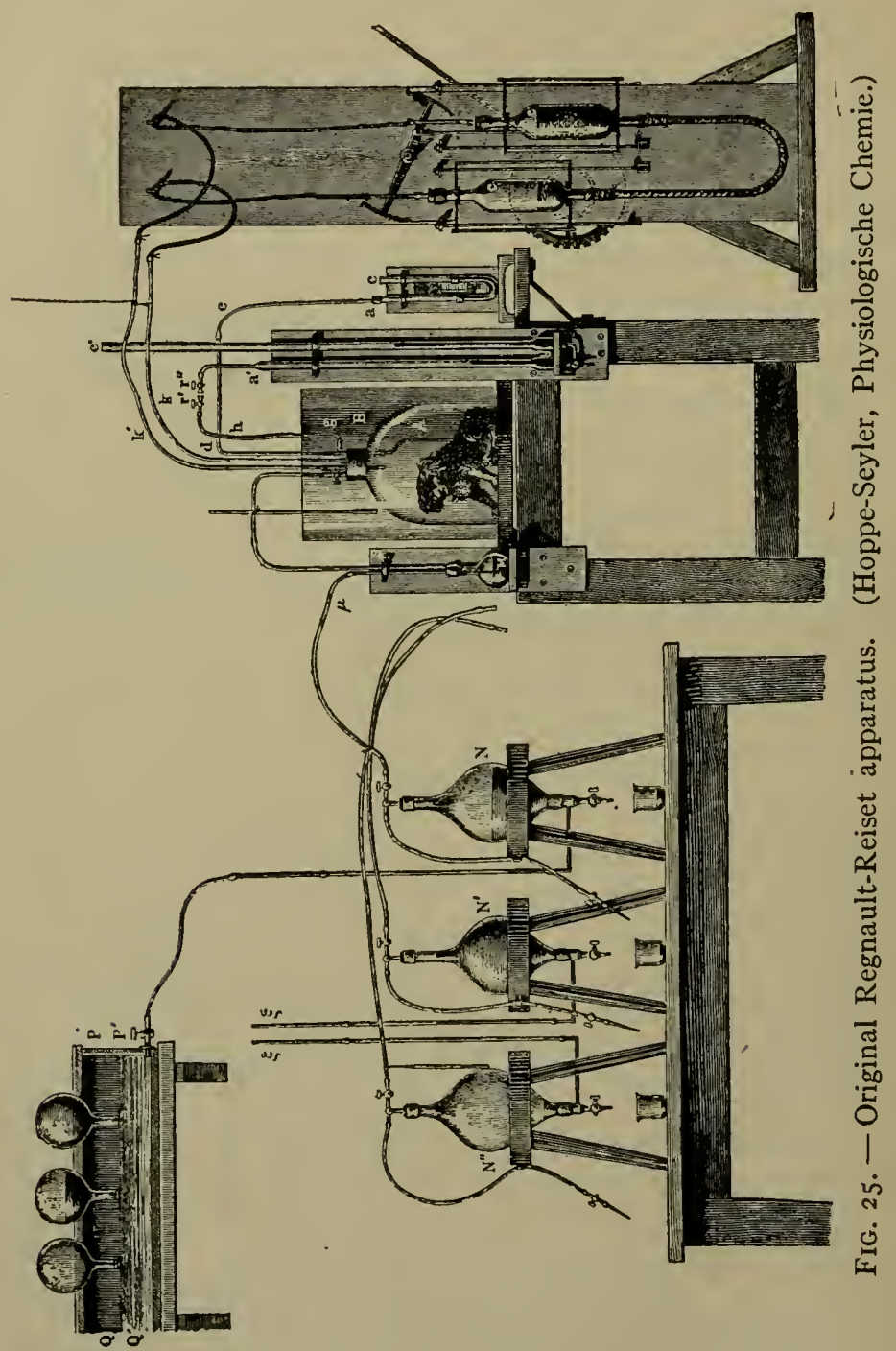

Any methane or hydrogen excreted accumulates in the apparatus and may be determined by an analysis of the contained air at the close of the experiment. The amount of nitrogen contained in the apparatus should, of course, remain unchanged if the apparatus is working properly. 


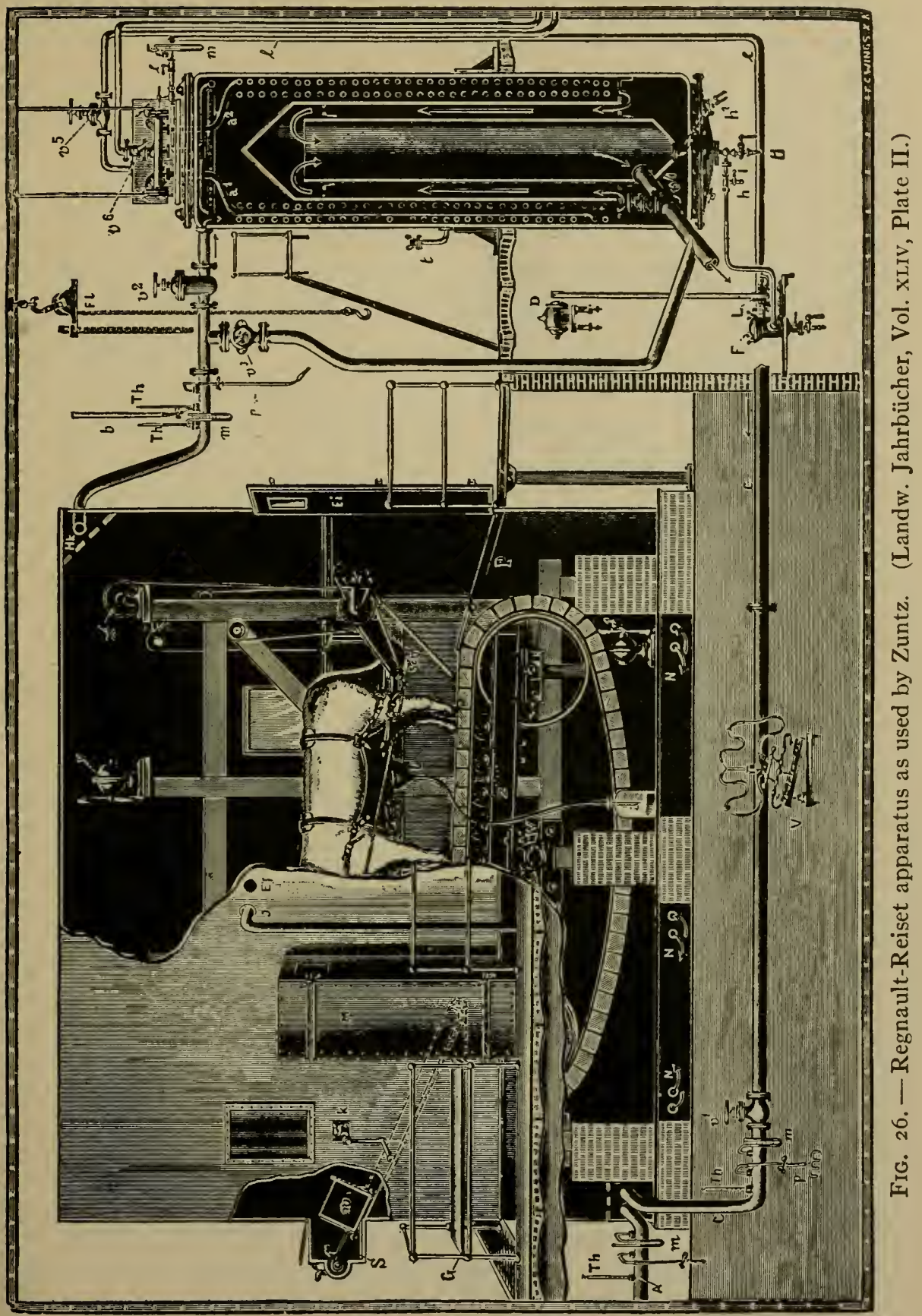


If the entire respiratory exchange is to be determined, the subject is placed in the respiration chamber represented in the diagram. If only the pulmonary exchange is under investigation, the respiration chamber is replaced by a mask or mouthpiece or even by a suitable cannula inserted in the trachea.

The original form of the Regnault-Reiset apparatus ${ }^{1}$ is shown in Fig. 25. The same investigators subsequently devised a larger one in which they made a number of experiments upon animals of various species including sheep, calves, swine and fowls. In theory this is the most perfect form of respiration apparatus, but numerous technical difficulties arise in its use. Various later forms have been devised but Atwater and Benedict ${ }^{2}$ were the first to construct one of a size suitable for man which was capable of a high degree of accuracy. Quite recently Zuntz ${ }^{3}$ has constructed a respiration apparatus of this type for experiments on domestic animals, a section of which is shown in Fig. 26, while for the determination of the pulmonary exchange, Benedict ${ }^{4}$ has devised a so-called "Universal" respiration apparatus.

299. The Pettenkofer apparatus. - In the Pettenkofer, or open circuit, respiration apparatus, the subject breathes in a continuous measured current of atmospheric air whose content of water, carbon dioxid and methane is determined before and after passing the animal, the difference, of course, showing how much of each gas the subject has added. In an apparatus suitable for small animals the entire amount of carbon dioxid and water in the incoming and outgoing air current may be determined, but in the larger forms it is necessary to measure the air current and make analyses upon relatively small samples, so that the analytical errors are multiplied by a large factor, while a determination of the oxygen balance has not as yet been found practicable. The general scheme of such an apparatus is shown in the diagram, Fig. 27. As in the case of the Regnault-Reiset apparatus, the respiration chamber may be replaced by a mask, mouthpiece or cannula for the investigation of the pulmonary exchange.

${ }_{1}$ Ann. de Chem. et de Physique, 3 ème Series, 26, 299.

${ }^{2}$ Carnegie Institution of Washington, Publication No. 42 (I905).

3 Landw. Jahrb., 44 (I9I3), 776.

${ }^{4}$ Deut. Arch. Klin. Med., 107 (I912), I56. 
The first practicable form of open circuit apparatus was devised by Pettenkofer ${ }^{1}$ for experiments on man. Its general appearance is shown in Fig. 28. The comparative simplicity of its operation and

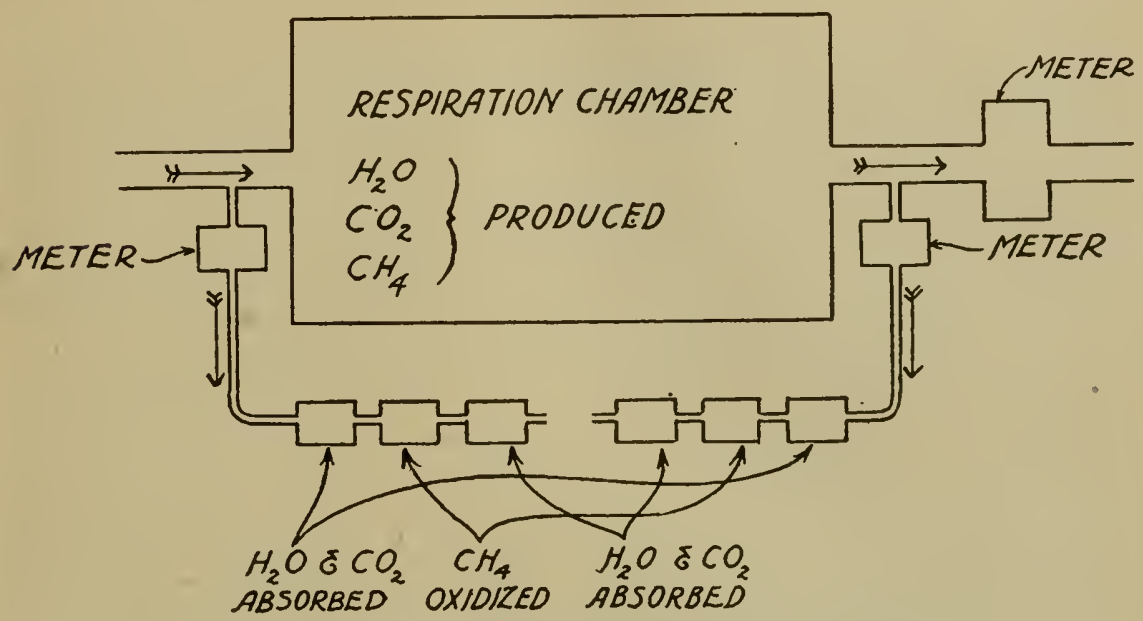

FIG. 27. - Scheme of Pettenkofer respiration apparatus.

the fact that it could be readily built of any desired size led to its extensive use in investigations upon agricultural animals, notably by Henneberg and Stohmann at Göttingen, Stohmann at Leipzig and G. Kühn and later Kellner at Möckern.

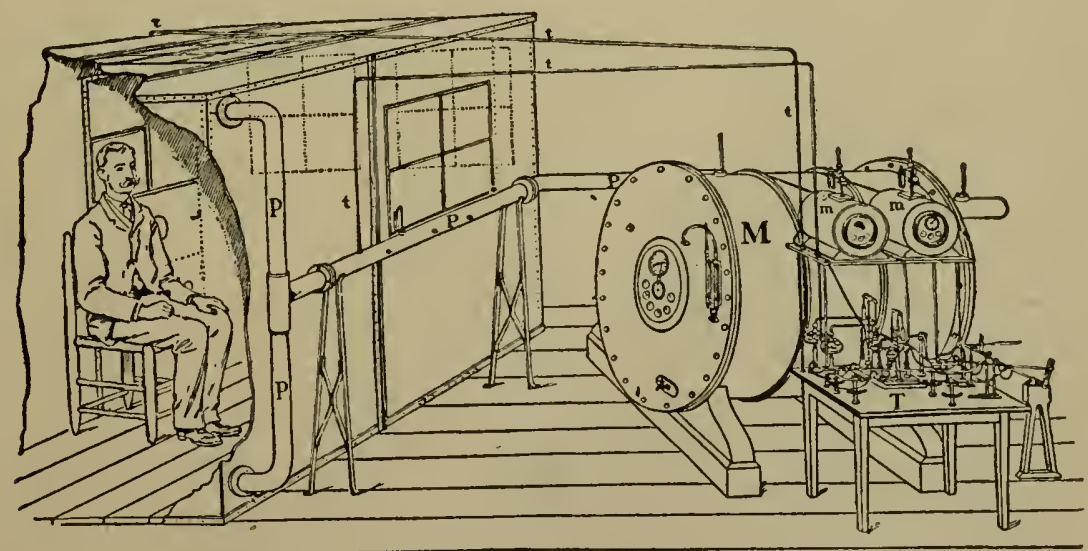

FIG. 28. - Pettenkofer respiration apparatus.

Explanatory sketch. (Atwater, U. S. Department of Agriculture, Office of Experiment Stations, Bulletin No. 21.)

${ }^{1}$ Ann. Chem. Pharm., Suppl. Bd. II, p. r. 
The principle of the Pettenkofer apparatus has also been very extensively used for the investigation of the pulmonary exchange, especially by Zuntz and his associates, to whom the development of this form is largely due. Figure 30 shows a horse equipped with a tracheal cannula for experiments with this type of apparatus. Owing to the fact that the excretory gases are not diluted with many times their volume of air, as is the case when a respiration chamber is used, the results are much sharper and it is possible to determine the amount of oxygen consumed as well as that of carbon dioxid given off.

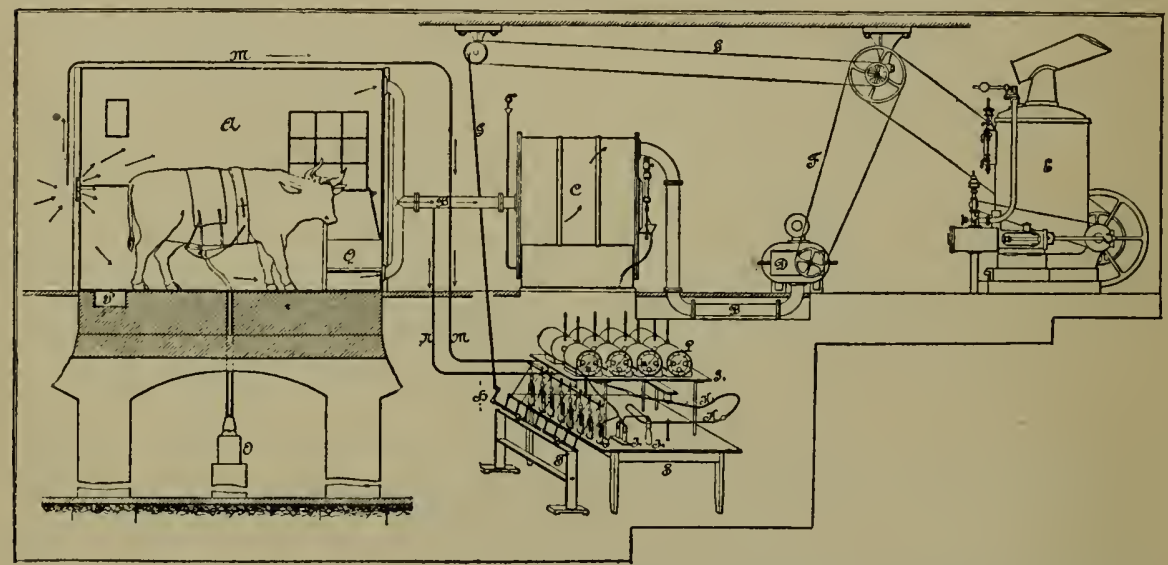

FIG. 29. - The Möckern respiration apparatus. (Bailey's Cyclopedia of American Agriculture.)

300. Investigation of pulmonary exchange. - For many purposes a determination of the gaseous exchange in the lungs, either with the Regnault-Reiset or the Pettenkofer type of apparatus, is preferable to determinations of the total exchange in a respiration chamber. The former method is especially adapted for short experiments. By its use, it is possible to trace sharply changes in the amount of the metabolism, the respiratory quotient, etc., produced by the administration of feed substances, drugs, etc., by experimental lesions, and especially by work, - changes whose amounts would often be relatively very small as compared with the total excretion for 24 hours as measured in the respiration chamber and which, therefore, if they did not escape detection altogether, could not be as accurately determined either quantitatively or chronologically. On the other hand, it is impracticable to continue its use through long periods, - a day, e.g., — and since it takes 
THE BALANCE OF NUTRITION

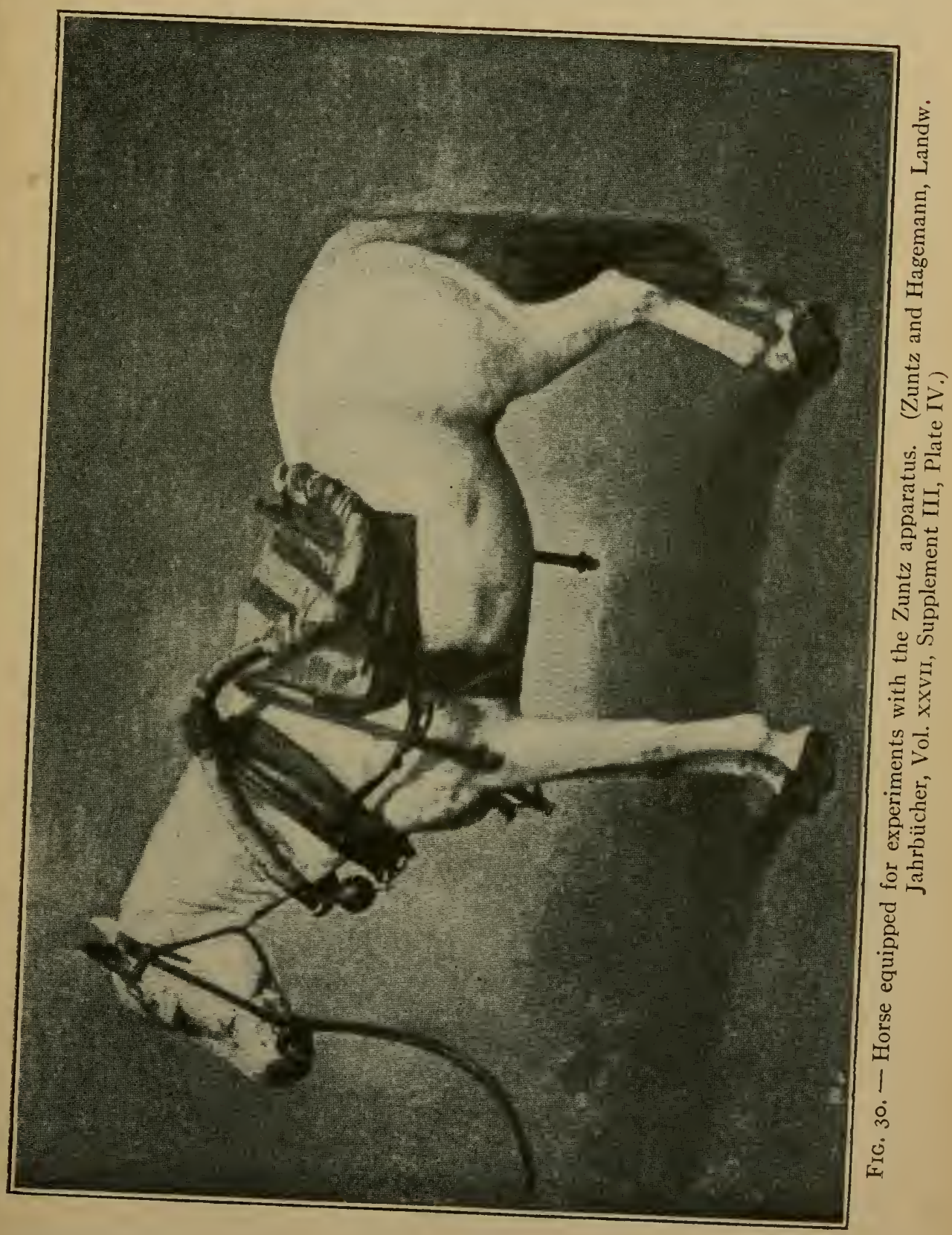


no account of the excretion through the skin and the alimentary canal, it is only by indirect methods that it is possible to compute the total balance of carbon, hydrogen and oxygen by its use.

301. Balance of water and of ash ingredients. - The respiration apparatus of either type serves to determine the excretion of water vapor by the subject as well as that of carbon dioxid and other gases and thus, in connection with the necessary analyses of the feed and visible excreta, to establish the gain or loss of hydrogen. Unfortunately, more or less difficulty is experienced in determining accurately the hydrogen balance owing in part to the liability to condensation of water in the apparatus and in part to the fact that the amount of organic hydrogen actually entering into the metabolism of the animal is small as compared with the amounts of water consumed and simply evaporated again.

The ash ingredients, of course, with the possible exception of minute amounts of sulphur, all leave the animal in the visible excreta and the balance of these elements may therefore be determined according to the same principles as the balance of nitrogen.

\section{§ 4. The Balance of ENERgy}

302. Balance of nutrition includes energy. - Since the animal body is essentially a transformer of energy (207), the balance of nutrition is not only concerned with the income and outgo of matter but also, corresponding to the dual function of feed (263), with the gain or loss of energy by the body. The study of the balance of energy is a method of investigating some of the important problems of nutrition which has been especially developed in recent years and which has proved fruitful of results. Before entering upon its consideration, however, a brief review of some of the elementary concepts of energetics as related to physiological processes may not prove superfluous.

\section{Elementary principles}

303. Energy. - Up to this point the word energy has been used without any precise definition. In a specific study of the 
balance of energy, however, it is important to have as definite a conception as possible of what is meant by the term. It is not altogether easy to give a simple general definition of energy, but for the present purpose that given by Noyes ${ }^{1}$ may be adopted, viz., "That which gives rise to changes in the properties of bodies and to the power to produce such changes." For the present purpose, however, the conception of energy may be more readily apprehended from illustrations than from definitions.

The subject may be conveniently approached from the side of mechanics. A moving body is capable of producing certain effects by virtue of its motion. The falling weight of a pile driver, for example, forces the pile downward against the resistance of the ground and at the same time produces heat at the point of impact. The projectile fired from a sixteen inch gun striking the side of the armored ship overcomes the cohesive force of the armor plate and deforms or penetrates it, while the blow also gives rise to an evolution of heat. The blows of the blacksmith, if rapid and heavy enough, may raise the iron on his anvil to a red heat. Accordingly, it is said that a moving body possesses energy in the form called kinetic energy, or energy of motion.

If a body suspended above the earth is set free it falls to the ground, and at the moment of contact with the earth possesses a certain amount of kinetic energy which was generated during its fall from something which was not energy of motion. This other form of energy, which the body possessed previous to its fall by virtue of its position, may be called gravitation energy. The same relation is illustrated by a swinging pendulum. During the downward swing, the gravitation energy which it possessed when at its highest point is converted into kinetic energy, while when it rises the kinetic energy which it possesses is reconverted into gravitation energy. When we lift a weight we are conscious of expending work, which is stored up as gravitation energy, to be liberated again as kinetic energy when the weight falls.

304. Forms of energy. - In general, whenever the rate of motion of a body is increased (or, to use a more familiar if less accurate expression, whenever motion is produced) it is to be

${ }_{1}^{1}$ General Principles of Physical Science, Ig02. 
inferred, as in the case of the falling body or the pendulum, that the kinetic energy produced has been derived from some other form of energy. In the examples thus far given this other form of energy was gravitation energy. In many familiar instances, however, this is not the case. The expanding steam in the cylinder of a steam engine parts with some of its heat to produce the motion of the piston. The electric current in the wire sets the armature of the motor in revolution. The combustion of gasoline in the cylinder of an engine produces motion of the engine as well as heat. Heat, electricity and chemical action may all be sources of kinetic energy and therefore the existence of heat energy, electrical energy and chemical energy is inferred.

The manifestations of energy are of the most varied character but its forms may be conveniently grouped under the following general heads:-

I. Kinetic energy

2. Gravitation energy

3. Cohesion energy

4. Volume energy

5. Electrical energy
6. Magnetic energy

7. Chemical energy

8. Heat energy

9. Rádiant energy

Of these, kinetic energy, chemical energy and heat energy are those of most importance in considering the balance of energy in the animal body.

305. Transformations of energy. - As is illustrated by the examples given in the previous paragraphs, and as has been assumed in speaking of energy changes in the animal body, the various forms of energy are capable of mutual transformations. Heat may be converted into motion in the heat engine. Motion in turn is converted into heat when a moving body is retarded by friction or stopped by contact with another body. When gasoline is burned freely, its chemical energy is converted into heat, but when it is exploded in the cylinder of an engine it yields also motion. This motion in turn may be stored in the form of gravitation energy in a lifted weight, or as cohesion energy in a coiled spring, or it may be made a source of electrical energy which in its turn gives rise to the radiant energy of light in the filament of a lamp.

In brief, all the physical phenomena of the universe of which we can take cognizance can be described in terms of changes of 
energy either as to form or intensity, and this fact has led some physicists to identify the concepts, of matter and energy and to maintain that the former can be fully interpreted in terms of the latter. Without entering here into this debated question, it will be convenient to follow for our present purpose the more familiar course of regarding matter and energy as two distinct although indissolubly connected entities.

306. The conservation of energy. - When a unit of kinetic energy is converted into heat energy it is found that the amount of heat obtained is always the same no matter what the process employed in effecting the conversion. Similarly, if a unit of heat be converted into kinetic energy the amount of the latter obtained is always the same and moreover is always equal to the quantity of kinetic energy which disappears when one unit of heat is produced.

What is true of heat energy and kinetic energy in this respect has been shown to be true of all the forms of energy. Not only are they convertible into each other but there is no loss or gain of energy in the conversion. When a quantity of energy of one form disappears an equivalent quantity simultaneously appears somewhere in some other form or forms. This great generalization, perhaps the most important in the history of physical science, is known as the law of the conservation of energy, or the first law of energetics. It was first clearly and distinctly formulated by Mayer in $\mathrm{r} 842$ and since that time has been verified by a great number of the most exact experiments and forms the basis of modern conceptions of physical processes. In substance, it asserts that the total energy of the universe as far as man knows it is a constant quantity, subject to continual changes of form but neither created nor destroyed.

That the law of the conservation of energy applies to the processes taking place in the body of the animal was exceedingly probable, a priori, and has been demonstrated experimentally by the researches of Rubner upon dogs, of Laulanié on various animals, of Atwater, Benedict, Lusk and their associates upon men and of Armsby and Fries upon cattle. ${ }^{1}$ The importance of this fact in relation to the study of energy changes in the body is obvious.

${ }^{1}$ Compare the writer's Principles of Animal Nutrition, pp. 263-268 and Penna. Expt. Sta., Bul. I 26. 
307. Heat energy unique. - In one respect heat energy occupies a peculiar position. Other forms of energy are in general readily and completely transformed into heat but there is no known method by which heat can be completely transformed into other forms, such as kinetic energy. Whatever portion of the heat is thus transformed obeys the law of the conservation of energy but part of it always remains in the form of heat. ${ }^{1}$

308. Units of energy. - Quantities of energy are measured by converting them into the same form and comparing them with some quantity of the same form of energy arbitrarily assumed as a unit.

Since quantities of kinetic energy can be expressed in terms of mass, space and time, a unit based on these concepts is taken as the fundamental unit of energy. The so-called C.G.S. (centimeter-gram-second) unit is the erg. An erg is a quantity of energy equal to twice the kinetic energy possessed by a mass of one gram moving with a velocity of one centimeter per second. Since this is a very small quantity, a unit called the joule, equal to ten million ergs, is often employed in practical measurements of energy, that is, $\mathrm{I}$ joule $=10^{7}$ ergs. For purposes where a still larger unit is desired the kilo-joule equal to one thousand joules is also employed.

In practice, however, heat is the form of energy which generally lends itself most readily to exact determination and, since other forms of energy are easily converted into heat, units of heat are extensively employed in the study of energy. The most common unit for this purpose is the calorie, which is the quantity of heat required to raise the temperature of one gram of water one degree centigrade. ${ }^{2}$

The foregoing is known as the small, or gram calorie (cal.). Where larger quantities of heat are to be measured the large,

1 This is, of course, one aspect of the second law of energetics. Theoretically, a perfect heat engine with a lower temperature limit of absolute zero would convert heat completely into kinetic energy. Since, however, we can neither obtain the temperature of absolute zero nor construct a perfect heat engine, this theoretical conception is simply a limit which may be more or less remotely approached in practice but never attained.

2 Since the specific heat of water varies at different temperatures, an exact definition of the calorie must specify the temperature at which it is measured. Practice differs in this respect but the preferable unit is the mean calorie, which is one onehundredth of the amount of heat required to raise the temperature of one gram of water from $0^{\circ}$ to $100^{\circ} \mathrm{C}$. 
or kilogram calorie (Cal.), equal to one thousand small calories, is employed, while for still larger quantities the Therm, equal to one thousand large calories, may be used. In the following pages the term calorie signifies the large, or kilogram, calorie, unless the contrary is expressly stated.

Certain units of gravitation energy are also frequently used, especially in mechanics, the more important ones being the gram meter, the kilogram meter and the foot pound. The gram meter is the energy required to raise a weight of one gram vertically through one meter in opposition to gravity, the kilogram meter is the energy required to raise a weight of one kilogram through one meter, and the foot pound is the energy required to raise a weight of one pound through one foot. Since the force of gravity varies at different points on the earth's surface these units as thus defined are not invariable. Taking the average force of gravity at sea level, however, as equal to 980.5 dynes, the relations between these various units are as follows:

EQUIVALENCE OF UNITS OF ENERGY

\begin{tabular}{|c|c|c|c|c|c|c|}
\hline & ERGS & $\begin{array}{c}\text { GRAM } \\
\text { METERS }\end{array}$ & $\begin{array}{c}\text { KILOGRAM } \\
\text { METERS }\end{array}$ & $\begin{array}{l}\text { Foot } \\
\text { Pounds }\end{array}$ & $\begin{array}{l}\text { Gram } \\
\text { Calo- } \\
\text { RIES }\end{array}$ & $\begin{array}{l}\text { KILOGRAM } \\
\text { CALORIES }\end{array}$ \\
\hline $\begin{array}{l}\text { I gram meter. } \\
\text { I kilogram meter } \\
\text { I foot pound . } \\
\text { I calorie } \\
\text { I Calorie }\end{array}$ & $\begin{array}{l}980.5 \times 10^{2} \\
980.5 \times 10^{5} \\
135.5 \times 10^{5} \\
4.184 \times 10^{7} \\
4.184 \times 10^{10}\end{array}$ & $\begin{array}{r}1000 \\
138.2 \\
426.6 \\
4266000\end{array}$ & $\begin{array}{l}0.001 \\
0.1382 \\
0.4266 \\
426.6\end{array}$ & $\begin{array}{l}0.007236 \\
7.236 \\
3.087 \\
3087\end{array}$ & $\mid \begin{array}{l}0.002344 \\
2.344 \\
0.3239 \\
1000\end{array}$ & $\begin{array}{l}0.2344 \times 10^{-6} \\
0.002344 \\
0.000324 \\
0.001\end{array}$ \\
\hline
\end{tabular}

309. Measurement of heat energy. - Quantities of heat are measured chiefly in two ways, viz., by their effects in raising the temperature of some substance or in changing its state of aggregation. Instruments for measuring quantities of heat are called calorimeters, i.e., heat measurers.

In the first method, as already implied in the definition of the calorie (308), water is ordinarily used as the calorimetric substance. ${ }^{1}$ For example, if the quantity of heat to be measured can be transferred without loss to a kilogram of water, and if the temperature of the water is thereby raised $2^{\circ} \mathrm{C}$., it is evident that the quantity of heat imparted to it is two large calo-

${ }^{1}$ Other substances than water may, of course, be employed, but water is usually the most convenient. 
ries. A calorimeter constructed after this principle is a water calorimeter. Such calorimeters have been devised in a great variety of forms according to the special purpose in view. The two essential requirements are that any escape of heat by conduction or radiation shall be either preventable or measurable and that the temperature increase be accurately determined.

In the second method the heat is caused to expend itself in changing the physical state of some substance as, for example, in melting ice or in evaporating some volatile liquid. The ice

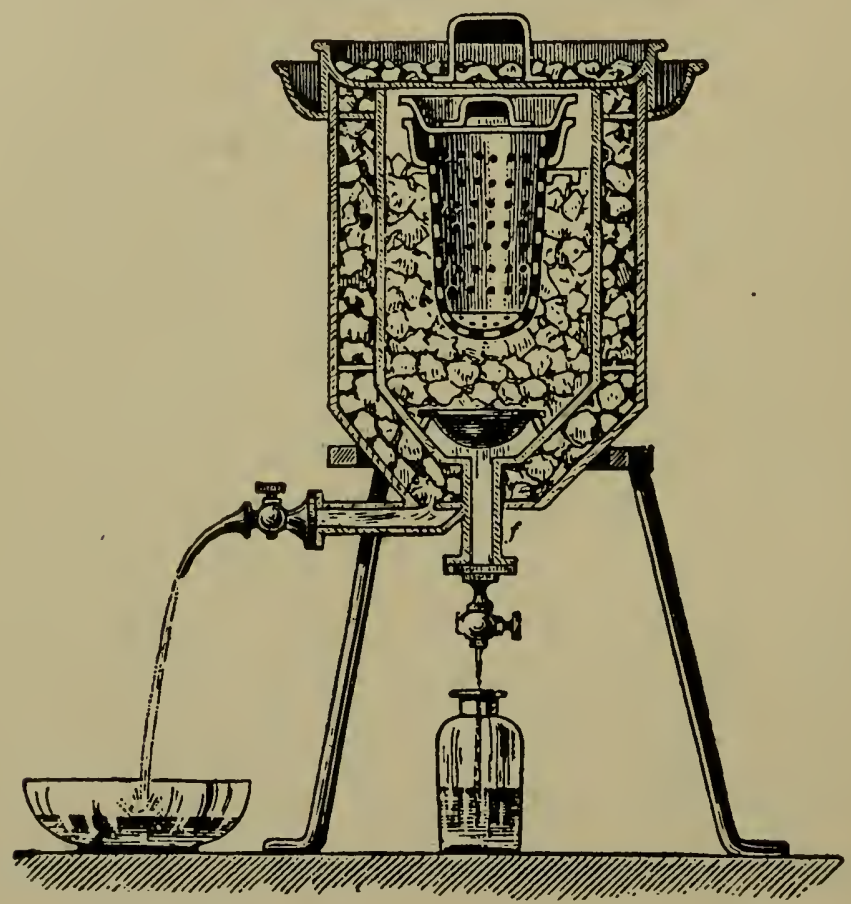

Fig. 31. - Lavoisier's ice calorimeter. (Schaefer, Text Book of Physiology.)

calorimeter is one of the oldest forms of calorimeter and has been extensively used for certain classes of work. Figure $3 \mathrm{I}$ shows a simple form of ice calorimeter used by Lavoisier. The source of heat is placed in the central vessel and imparts its heat to the surrounding ice, while the access of any extraneous heat is prevented by the outside jacket of ice.

In Lavoisier's calorimeter the amount of ice melted was determined by collecting and weighing the resulting water, but a much more accurate method of measurement is based upon the contraction which ice undergoes when converted into water. 
By means of the water calorimeter, it has been determined that the conversion of one gram of ice at $0^{\circ} \mathrm{C}$. into liquid water at the same temperature requires 79.24 gram calories of heat. By the use of this factor the indications of the ice calorimeter can be converted into calories.

310. Heats of Combustion. - As related to nutrition investigations, the chemical energy of an organic substance is most commonly measured by converting it in to heat by complete combustion with oxygen and measuring the heat by one of the methods just indicated, the result being called the heat of combustion of the substance. In the method most commonly used in nutrition investigations, the substance is burned in highly compressed oxygen (about 25 atmospheres) in a lined steel bomb, the heat being taken up by water. The method was first devised by Berthelot and subsequently modified by Mahler, Hempel and Atwater. One form of this calorimeter is shown in section in Fig. 32 .

311. Heats of combustion do not measure total energy. It should be clearly understood that the heat of combustion of an organic compound does not, as is sometimes erroneously stated, measure its total energy but simply the amount manifested in a particular chemical change. Thus, in the complete oxidation of one gram of starch to gaseous carbon dioxid and liquid water $4 \mathrm{I} 83$ gram calories of energy are transformed into heat. How much additional energy is still contained in the resulting carbon dioxid and water we do not know, nor is it necessary that we should. In using the heat of combustion as a measure of the chemical energy of starch the possible energy content of the carbon dioxid and water is simply assumed as an arbitrary zero, much as the engineer may assume a datum plane for his levels without regard to its height above sea level. In other words, the heat of combustion of starch or of any other substance shows how much chemical energy can be secured from it for conversion into other forms by processes of oxidation such as occur in the body.

312. Law of initial and final states. - In view of the very complicated nature of the metabolic processes, the question naturally arises whether the amount of chemical energy which a feed ingredient such as starch really puts at the disposal of the organism is the same as the amount of chemical energy which 


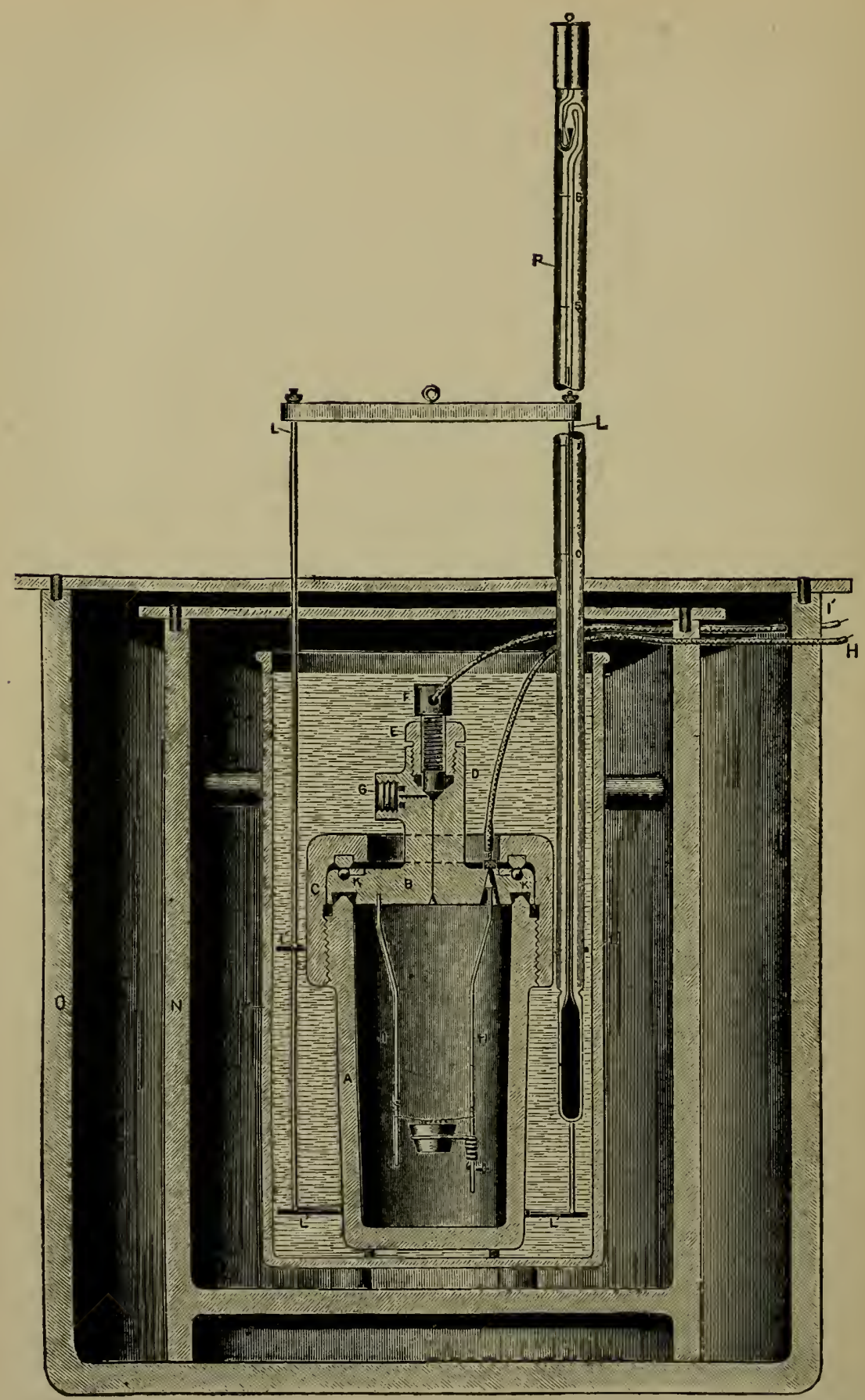

IIG. 32. - Section of bomb calorimeter. (Atwater, U. S. Department of Agriculture, Office of Experiment Stations, Bulletin No. 2I.) 
is transformed into heat by its almost instantaneous burning in oxygen. The answer to this question is found in what is called the law of initial and final states.

This law is that in any independent system the amount of energy transformed during a change in the system depends solely upon the initial and final states of the system and not at all upon the rapidity of the transformation nor upon the kind or number of the intermediate stages through which it passes. Although this law is true in the general form here stated, it was originally propounded as related to chemical reactions. If we start with starch and oxygen and end with the corresponding quantities of carbon dioxid and water, the amount of chemical energy converted into heat or other forms is the same, no matter whether the starch be burned almost instantaneously in pure oxygen or whether it be subjected to slow oxidation in the tissues of a plant buried in the soil; whether carbon dioxid and water are the immediate products of the action or whether the starch passes through intermediate stages like maltose, glycogen, dextrose, lactic acid, etc., etc., as in the body of the animal. It is simply necessary to determine the difference in chemical energy between the system in its initial and in its final state to obtain the amount of energy transformed during the change.

313. Measurement of kinetic energy. - The most common method of measuring the energy liberated by a machine or an animal as motion energy is its conversion, actually or virtually, into gravitation energy, which is measured by the units given on a previous page (308). In case of small amounts of energy a weight may be actually lifted, the product of weight into distance giving the number of gram centimeters or foot pounds of energy expended. In other cases, the subject may pull against a resistance produced, for example, by the friction of a brake, the traction being measured by some form of spring balance. In this case the kinetic energy is, as a matter of fact, converted into heat, but the tractive pull multiplied by the distance gives the equivalent number of gravitation units. In still another form the subject virtually lifts his own weight by climbing the inclined plane of a tread power, the body weight multiplied by the distance multiplied by the sine of the angle of ascent equaling the units of gravitation energy to be measured. 
Figure 33 shows a form of this apparatus used by Zuntz for work experiments upon horses.

Another method for measuring kinetic energy consists in converting it into electrical energy by causing the subject to

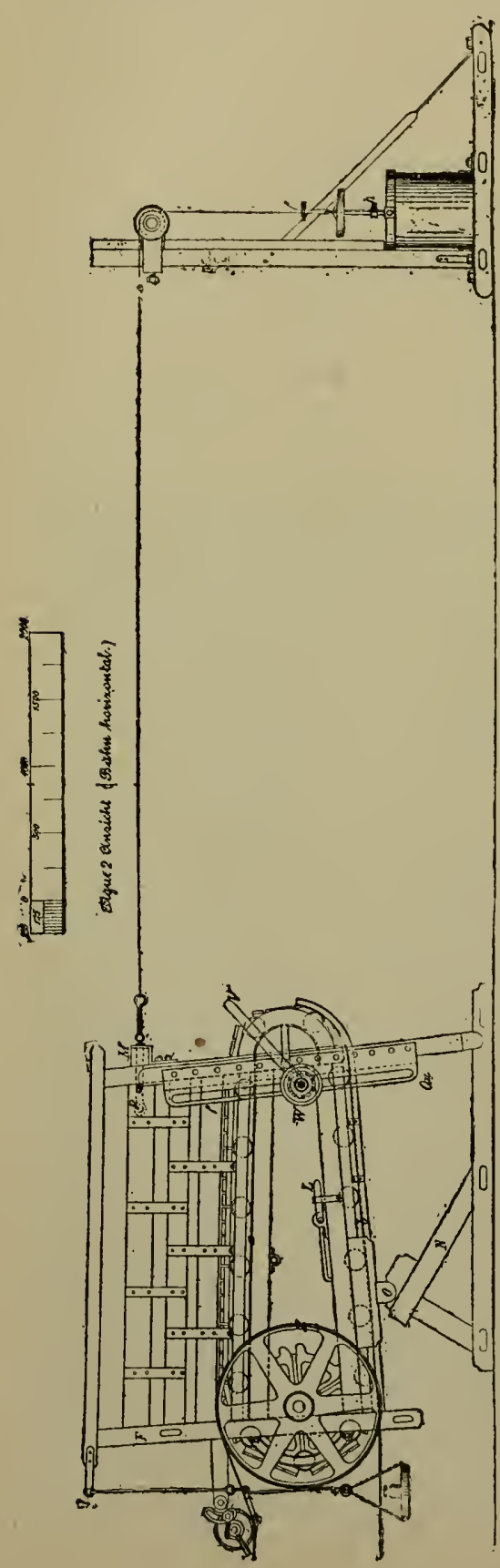
work against the resistance of a magnetic field. The amount of current thus generated can be measured in electrical units, or, as has been done by Atwater, Benedict and others, the electrical energy may be converted into heat and measured in calories.

The body's income of energy. - Gross energy

314. Only chemical energy can be utilized. - As was stated in the introductory paragraphs of this chapter, the animal body resembles an internal combustion motor in being a mechanism for the conversion of the chemical energy of certain compounds contained in the feed into kinetic energy. In considering the balance between income and outgo of energy, it is essential to recognize a further point of resemblance, viz., that neither the animal nor the motor can utilize other than chemical energy. There is no evidence that the animal body can use in any way any of the other forms of energy, such as heat, electricity or solar radiation 
which reach it from its environment, any more than the gasoline engine can use the energy of falling water or of an electric current. Chemical energy is not merely $a$ source but the only source from which the animal body can derive its supply.

315. Gross energy. - The income of energy may be ascertained, therefore, by determining the chemical energy contained in the various compounds present in the feed in the manner already indicated (309), viz., by converting it into heat and measuring the amount of the latter by means of a suitable calorimeter. In other words, the income of chemical energy is measured by the heat of combustion of the feed. In order to avoid the implication that this is the total amount of energy associated with the feed (311), it will be convenient to use the term gross energy as equivalent to the amount of energy manifested as heat when the feed is completely oxidized.

Since the chemical energy of a feeding stuff is converted into heat for purposes of measurement, its amount is usually expressed in heat units. It should be clearly understood, however, that this is simply a matter of convenience and that it is the chemical energy of feeding stuffs and not the heat produced by their combustion which is of use to the animal.

It is scarcely necessary to point out that the gross energy of the feed does not measure its nutritive value. Otherwise, anthracite coal, with a heat of combustion of some 7.9 Cals. per gram, would outrank most feeding stuffs, while hydrogen gas, with a heat of combustion of more than 34 Cals. per gram would stand still higher in the list. Obviously, the feed value of a substance depends not only upon its content of gross energy but upon the proportion of the latter which the body can utilize.

316. Heats of combustion. - The heats of combustion of a great variety of organic substances have been determined. Atwater ${ }^{1}$ in 1895 published a compilation of results upon a large number of compounds of importance in nutrition, Fries ${ }^{2}$ has prepared a rather more extensive list, and Benedict and Osborne ${ }^{3}$ have determined the heats of combustion of nineteen vegetable proteins.

1 U. S. Dept. Agr., Office Expt. Stas., Bull. 2 I (1895).

2 U. S. Dept. Agr., Bur. Anim. Indus., Búl. 94 (I907).

3 Jour. Biol. Chem., 3 (I907), i 19. 
The following tabulation may serve to give a general idea of the gross energy of some of the more important substances concerned in nutrition. It should be specially noted that the figures given are in most instances simply approximate averages.

Table 24. - Approximate Gross Energy

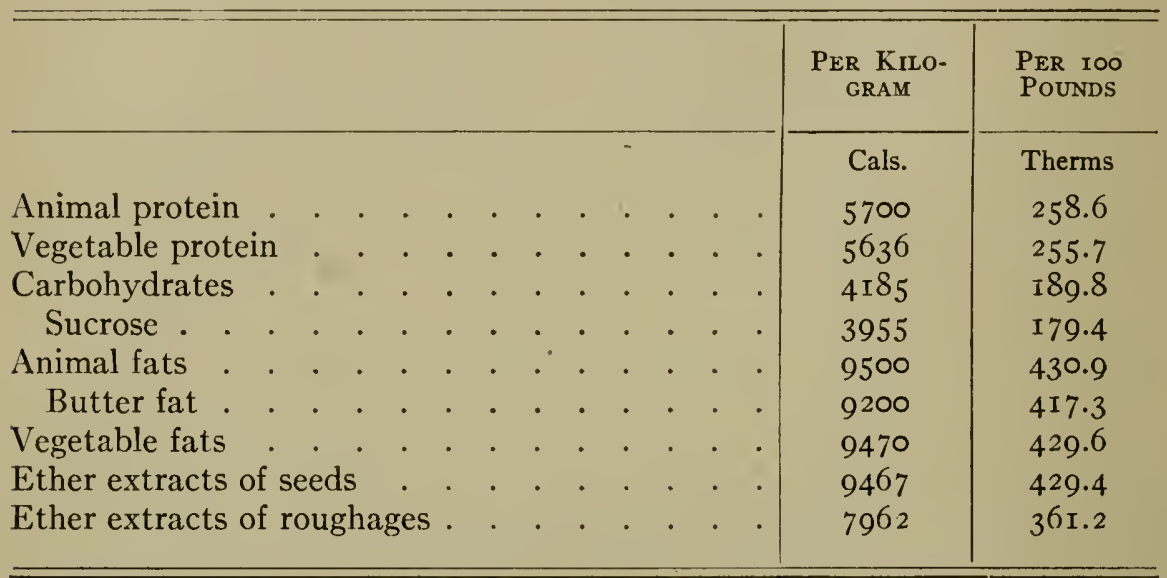

The following examples taken from the work of Kellner and of Armsby and Fries will serve to give a general idea of the gross energy of common feeding stuffs. It will be observed that the range of variation is relatively small in most instances.

Table 25.-Gross Energy of Feeding Stuffs

\begin{tabular}{|c|c|c|c|c|c|c|c|c|c|c|c|c|c|}
\hline & & & & & & & & & & & & $\begin{array}{c}\text { PER KILO- } \\
\text { GRAM }\end{array}$ & $\begin{array}{l}\text { PER IOO } \\
\text { PoundS }\end{array}$ \\
\hline & & & & & & & & & & & & Cals. & Therms. \\
\hline \multicolumn{14}{|l|}{ Roughage } \\
\hline Timothy hay & 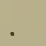 & . & . & & . & . & . & . & . & & . & $45 \mathrm{I} 8$ & 204.94 \\
\hline Red clover hay & & . & . & . & . & . & . & . & . & . & . & 4462 & 202.04 \\
\hline Mixed hay. & . & . & . & . & . & . & . & . & . & . & . & 4393 & I99.27 \\
\hline Alfalfa hay & . & . & . & . & . & . & . & . & . & . & . & 4372 & 198.31 \\
\hline Meadow hay & . & . & . & . & . & . & . & . & . & . & . & 4433 & 201.08 \\
\hline Corn stover & . & . & . & . & . & . & . & . & . & . & . & 4332 & I96.50 \\
\hline Oat straw & . & . & . & . & . & . & . & . & . & . & . & 4436 & 201.22 \\
\hline Wheat straw & . & . & . & . & . & . & . & . & . & . & . & 4444 & $201.5^{8}$ \\
\hline Straw pulp & . & . & . & . & . & . & . & . & . & . & . & $4 I 47$ & I 88. I I \\
\hline
\end{tabular}




\section{Concentrates}

Corn meal

Hominy chop

Wheat bran

Grain mixture No. $\mathrm{I}^{a}$

Grain mixture No. $2^{b}$

Beet molasses

Starch

Peanut oil

Wheat gluten .

$a$. Wheat bran, I4.28 per cent; corn meal, 42.86 per cent; old process linseed meal, 42.86 per cent.

$b$. Corn meal, 60 per cent; crushed oats, 30 per cent; old process linseed meal, Io per cent.

Some data are also available regarding the gross energy of the digested nutrients of feeding stuffs. The following averages are derived chiefly from Kellner's investigations.

\section{Table 26. - Gross Energy of Digested Nutrients}

\begin{tabular}{|c|c|c|c|c|c|c|c|}
\hline & & & & & & $\begin{array}{c}\text { PER KILO- } \\
\text { GRAII }\end{array}$ & $\begin{array}{l}\text { PER IOO } \\
\text { PoundS }\end{array}$ \\
\hline Protein of wheat gluten & & - & & & . & $\begin{array}{l}\text { Cals. } \\
5975\end{array}$ & $\begin{array}{c}\text { Therms. } \\
271.0\end{array}$ \\
\hline Protein - assumed average . . & 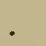 & 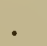 & 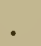 & . & . & 5700 & 258.6 \\
\hline Crude fiber $. \quad . \quad . \quad . \quad . \quad . \quad$. & . & . & . & . & . . & 4254 & I93.0 \\
\hline Nitrogen-free extract of hay & . & . & . & . & . . & 4232 & I92.0 \\
\hline Nitrogen-free extract of starch. & . & . & . & . & . . & 4185 & I 89.8 \\
\hline Ether extract of hay . . . . & . & 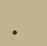 & . & . & . . & 8322 & $377 \cdot 5$ \\
\hline Peanut oil . . . . . . & . & ${ }^{\circ}$ & . & $\cdot$ & . . & 8821 & 400.1 \\
\hline Total organic matter of roughage & . & . & . & . & . . & 4473 & 202.9 \\
\hline
\end{tabular}

In the computation of energy balance, the factors commonly used are, for body protein 5.7 Cals. per gram and for body fat 9.5 Cals. per gram, although Köhler's average for the former is slightly lower, viz., 5.628 Cals. (88).

\section{The outgo of chemical energy}

Chemical energy supplied in the feed may escape unused for either of two reasons: first, the substances carrying it 
may fail to be incorporated into the body, or second, they may be incompletely katabolized.

317. The feces. - Since a greater or less proportion of the organic matter of most feeding stuffs fails of digestion and resorption by farm animals and so does not enter into the body proper (148), a considerable amount of unused feed energy escapes in the feces, while the excretory products which they carry (154) contain chemical energy which has failed of complete conversion in the body. The chemical energy of the feces of farm animals constitutes a very considerable item in their total outgo of energy. Its amount can be determined as in the case of feeding stuffs by burning a sample, after drying with suitable precautions, in a calorimeter and measuring the heat evolved.

318. Combustible gases. - The combustible gases produced by fermentation in the digestive tract also carry off relatively large amounts of unused chemical energy, the loss in this way being precisely analogous to that in the undigested matter of the feces except that it escapes in invisible products. These gases cannot well be separated from the other gaseous excreta for the purpose of making a direct determination of their energy. The amounts of carbon and hydrogen excreted in them, however, can be determined with the aid of the respiration apparatus and on the well-founded assumption that only methane and hydrogen are produced the amount of each excreted may be calculated. The heats of combustion of both these gases being known, the amount of chemical energy which they carry off can be readily computed.

319. Products of incomplete katabolism. - The heat of combustion of a substance, as already defined, is the amount of heat evolved when it is completely oxidized, that is, in the case of substances ordinarily occurring in feeding stuffs, when it is burned to $\mathrm{CO}_{2}, \mathrm{H}_{2} \mathrm{O}, \mathrm{N}_{2}$, and $\mathrm{SO}_{3}$. If the katabolism in the body stops short of these end products, the quantity of chemical energy transformed is clearly less than the gross energy of the substance by an amount equal to the heat of combustion of the incompletely oxidized products.

The proteins of the feed constitute the most important instance of this sort. All the nitrogen of the digested protein and part of its carbon, hydrogen and oxygen, are excreted in 
the urine in the form of crystalline nitrogenous products, of which urea is the most familiar and often the most abundant. When these products are burned they yield a certain amount of heat, thus showing that they still contain part of the gross energy of the protein, and that, therefore, only a portion of the latter has been transformed in the body.

320. The urine. - In the main, the urine is the vehicle for the removal from the body of the incompletely oxidized products of katabolism, although some unoxidized or partially oxidized material also escapes from the body in the form of the excretory products contained in the feces (317), and small amounts of chemical energy are contained in the cutaneous excretory products (198).

The energy of the katabolic products contained in the urine may be determined as in the case of the feces by burning the dried residue in the calorimeter, a small correction being usually necessary for unavoidable losses in drying.

An approximate calculation of the chemical energy of the urine may be based upon its nitrogen or better on its carbon content, using the average ratio found in experiments on the same species, but these ratios vary more or less in different cases, and in exact work direct determinations are called for.

321. Cutaneous excretion. - The amount of chemical energy removed in the perspiration is too small to be of any significance, except possibly in experiments on severe work.

In addition to the perspiration there is a continual small loss of matter with its accompanying chemical energy in the form of epidermal scales, hair, etc., sloughed off. These losses are comparable to the excretory products in the feces, since they consist essentially of incompletely katabolized body material. Their amount is small but is sufficient to be taken account of in exact experiments.

\section{Metabolizable energy}

322. General conception. - It has been shown in the foregoing paragraphs that more or less of the chemical energy of the feed escapes unused from the body, the total thus rejected being equal to the gross energy of the total excreta, solid, liquid 
and gaseous, as measured by their heats of combustion. If, then, the gross energy of the total excreta be subtracted from the gross energy of the feed, the remainder shows how much of the chemical energy of the feed can be metabolized, that is, converted into other forms in the organism. To this difference, the term metabolizable energy has been applied.

Metabolizable energy may be briefly defined as the gross energy of the feed minus the gross energy of the excreta. Thus in the experiment cited previously (294) to illustrate the method of determining the balance of matter, the energy content of the feed and excreta and the metabolizable energy of the total ration were as follows:-

Energy of feed

6988 grms. timothy hay . . . . . . . 27,727 Cals.

400 grms. linseed meal . . . . . . . 181 I Cals.

Energy of excreta

29,538 Cals.

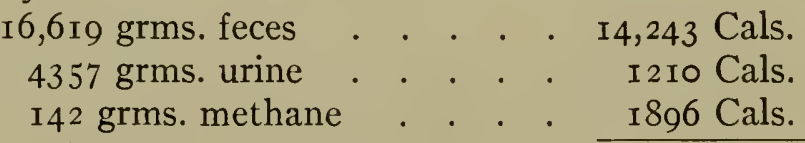

Metabolizable energy . . . . . . . . . . . $\frac{\mathrm{I} 2, \mathrm{I} 89 \mathrm{Cals}}{\text {. . }}$

It should be observed that the foregoing definition makes no assertion whatever as to the forms into which the metabolizable energy has been transformed nor as to the degree to which the transformation has been of service to the organism. Some of the energy, for example, may be retained in the body in a gain of fat or protein, as in the illustration just given, i.e., it may be temporarily set aside as a reserve to be used later, but it is still capable of transformation into other forms and therefore constitutes a part of the metabolizable energy. On the other hand, the feed might contain some substance capable of oxidation in the body but of no physiological value to it and which was simply burned to get rid of it. The heat thus generated might be entirely useless to the animal, yet this energy would be part of the metabolizable energy of the feed. Somewhat similarly, the energy liberated as heat in the methane fermentation constitutes part of the metabolizable energy, although it does not enter into the tissue metabolism. Metab- 
olizable energy means simply energy capable of transformation in the body. It is the maximum quantity which the feed can contribute to the energy changes in the organism. That it does not necessarily measure nutritive value is indeed suffciently apparent from the method used for its determination. As the example already given shows, this does not require any measurement of the gain or loss by the animal, but, like a digestion experiment, concerns itself simply with the feed and the excreta.

323. Synonyms for metabolizable energy. - Two other terms are frequently employed with substantially the same significance as metabolizable energy, viz., fuel value and available energy.

Fiel value. - The metabolizable energy of the feed is evidently capable of conversion into heat in the body. Since a considerable portion and sometimes all of it is actually thus converted, and since its amount is usually expressed as a matter of convenience in heat units, the term fuel value (or physiological heat value) has come into use as synonymous with metabolizable energy.

The term has the advantage of brevity, but has also certain disadvantages. In conjunction with the unit of measurement employed, it has a tendency to suggest that the purpose of the feed is to supply heat energy and that it is of value in proportion as it can do this, which is far from being the case. Moreover, there appears to be some danger of confusion due to the fact that the same term is used in a different sense in relation to fuels. The "fuel value" of a coal, for example, means the total amount of heat which it liberates when burned, and corresponds, therefore, to the gross energy of a feeding stuff, i.e., to its value if used as fuel under a boiler or in a heating plant. The fuel value of a feeding stuff, on the other hand, in the sense of its metabolizable energy, is the amount of heat which it can furnish when oxidized as it is in the body, i.e., more or less incompletely.

Available energy. - A much more unfortunate usage is the employment of the term available energy, equivalent to the German "Physiologischer Nutzwert," in the sense here assigned to metabolizable energy. This usage dates back to Rubner's investigations of the replacement values of nutrients in $188_{2}-$ 
I 885 and to his isodynamic values based upon them. In the light of the knowledge available at that time, this use of the term was perhaps justified, but as will appear later (369), it has since been shown that part of the metabolizable energy of the feed is virtually available for heat production alone, while only the remainder can be used for general body purposes. If the use of the term available energy is to be continued, therefore, it becomes necessary to distinguish two degrees of availability, using, for instance, the term gross available energy as equivalent to metabolizable energy and net available energy to signify that part of the metabolizable energy which is available for other purposes than heat production.

In the writer's judgment, simplicity and clearness of conception will be promoted by discontinuing altogether the use of the term available energy and employing the term metabolizable energy, or perhaps fuel value provided the latter is understood with the proper restrictions, to designate that portion of the gross energy of the feed which is capable of transformation in the animal organism.

324. Factors for metabolizable energy. - Rubner, and subsequently Atwater, have proposed factors by the use of which the metabolizable energy of the diet of man may be computed with a considerable degree of accuracy. ${ }^{1}$

Table 27. - Factors for Metabolizable Energy of Human Food

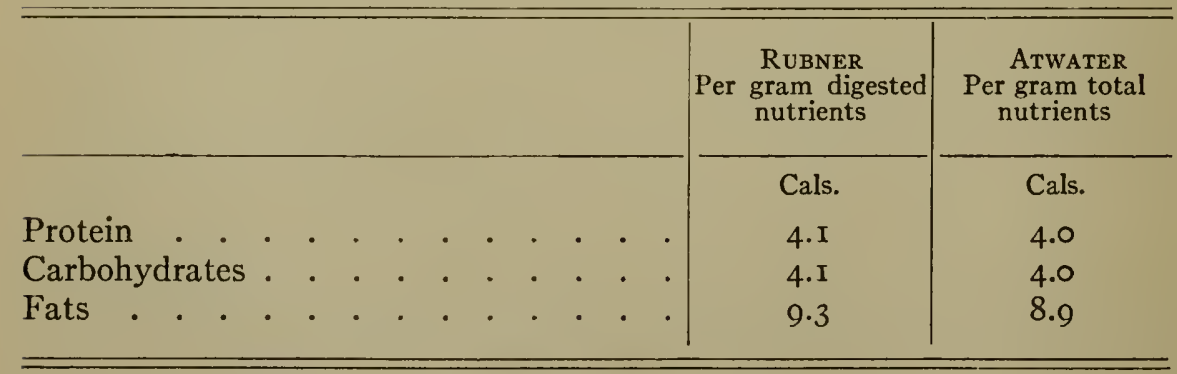

The use of these same factors yields approximately correct results for carnivora. They have sometimes been applied also to the digestible nutrients of the feed of herbivora but without sufficient warrant.

${ }^{1}$ Compare the writer's Principles of Animal Nutrition, pp. 272-28r. 


\section{The outgo of work and heat from the body}

325. Outgo of kinetic energy. - The feces, urine, combustible gases and cutaneous excreta carry off chiefly unused chemical energy. ${ }^{1}$ To recur to the illustration of the internal combustion motor, they are comparable with losses due to leakage or incomplete combustion of the fuel. The energy remaining after these losses have been met, i.e., the metabolizable energy, may be converted in part into mechanical work and in part into heat.

When an animal performs work, whether in drawing a load, carrying a rider, operating a tread power or simply lifting the weight of his own body at each successive step, a portion, although on the whole a relatively small percentage, of his total income of chemical energy is expended in moving objects, i.e., is converted into kinetic energy. The kinetic energy thus produced may be measured in accordance with the general methods described in a previous paragraph (313), usually by conversion into gravitation energy and measurement in gravitation units, i.e., the gram meter, kilogram meter or foot pound.

326. Outgo of heat. - The outgo of heat which common experience teaches is continually taking place from the bodies of men and of animals represents a very considerable share of the total income of chemical energy. It has been computed that if the heat produced by the average healthy man could be prevented from escaping from the body it would in a single day raise it to a pasteurizing temperature, while in the course of a month at the same rate, the temperature would be raised approximately to that of melting cast iron.

327. Animal calorimeters. - The great variety of animal calorimeters which have been devised for the purpose of measuring the heat production of living animals have been of three general types, which may be designated as water calorimeters, latent heat calorimeters and emission calorimeters.

Water calorimeters are those in which the heat is imparted to a known quantity of water, the rise of temperature of which is measured, i.e., they employ the first of the two methods of

${ }^{1}$ The heat which they also carry off is included in the total outgo of heat considered in the next paragraph. 
measuring heat previously described (309). Water calorimeters may be subdivided into those in which the heat is imparted to a stationary mass of water and those called flow calorimeters, in which it is taken up by a current of water.

Latent heat calorimeters make use of the second method of heat measurement, viz., causing it to effect a change in the physical state of the calorimetric substance. Thus Lavoisier employed an ice calorimeter in his experiments upon the relations between respiration and heat production. This type of calorimeter, however, is not well suited to experiments with animals and has been but little used.

Emission calorimeters may be said not to be in a strict sense calorimeters at all, i.e., they do not serve directly to measure quantities of heat but only to compare the rate of heat production by different sources, but they may be used indirectly to measure quantities. The principle of the emission calorimeter may be illustrated as follows: If a known source of heat (an electric resistance, for example) be placed in a closed receptacle located in a room kept at constant temperature, it will tend to heat the walls of the container. As the temperature of the walls rises, however, heat will be radiated from them with increasing rapidity until a balance is established between heat radiation and heat production and the temperature of the walls remains constant. If, now, a second source of heat, an animal, for example, be substituted for the first one, keeping the external conditions the same, and if it appears that, when an equilibrium is reached, the temperature of the walls is the same as in the first case, it is concluded that the rate of heat radiation is the same as in the first case, and that the animal is producing heat at the same rate as was the electric resistance, so that the amount of heat produced by the animal in a unit of time is thus indirectly measured.

The respiration calorimeter. - All animal calorimeters used for experiments of any length must necessarily be provided with ventilation. To prevent a loss of heat in the air current, it is introduced at the same temperature as that at which it leaves the apparatus. The ventilating air current, however, tends to remove water vapor from the chamber and the evaporation of this water, of course, absorbs a corresponding amount of heat as the so-called "latent heat of evaporation" of water. 
Either, therefore, evaporation must be prevented by keeping the air in the chamber saturated with water, thus introducing more or less abnormal conditions, or the amount of water carried away in the ventilating air current must be determined. If the latter course is followed, it is a relatively simple matter to include also determinations of the carbon dioxid and the combustible gases excreted, and perhaps of the oxygen con-

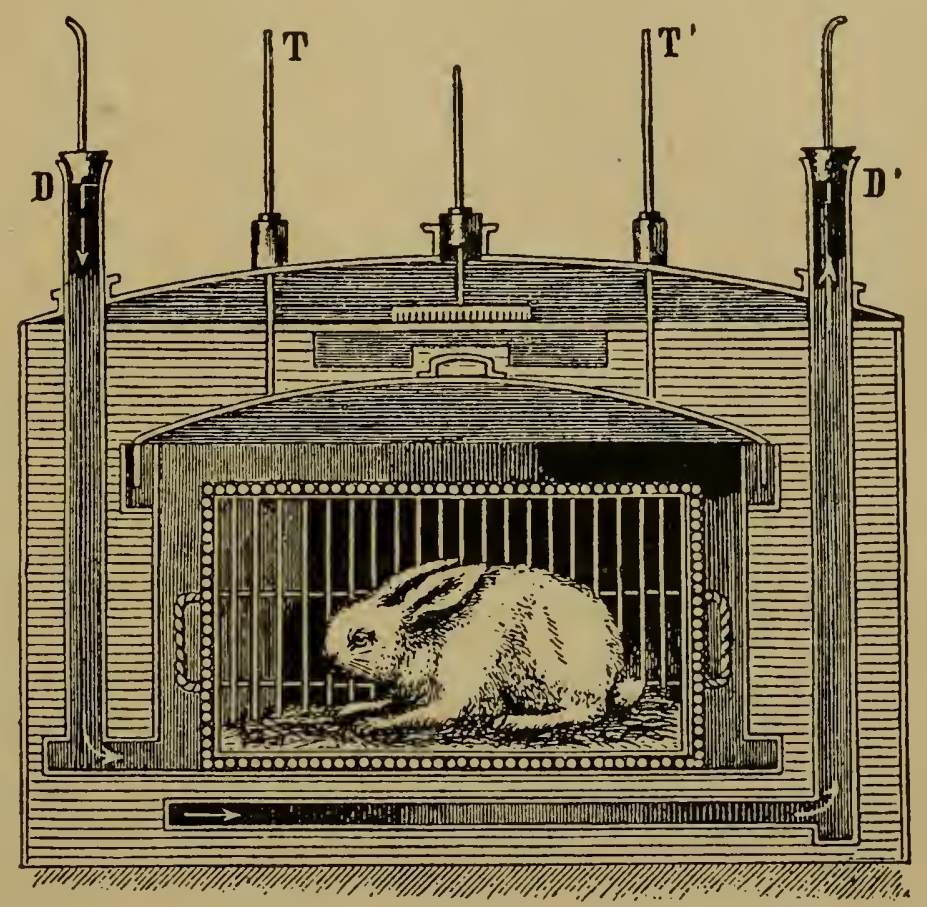

Fig. 34. - Dulong's water calorimeter (Schaefer, Text Book of Physiology).

sumed. The apparatus then becomes a combination of respiration apparatus and animal calorimeter and hence has been called a respiration calorimeter.

The apparatus used by Dulong in 1822 in his investigation of the source of animal heat, the construction of which is shown in Fig. 34, may serve to illustrate the form of calorimeter in which a stationary mass of water is used. This type of calorimeter has been used in various modifications, notably in the United States by Wood, ${ }^{1}$ Ott ${ }^{2}$

1 Smithsonian Contributions to Knowledge, No. 23 (1880).

${ }^{2}$ N. Y. Med. Jour., 49 (I 889 ), 342. 


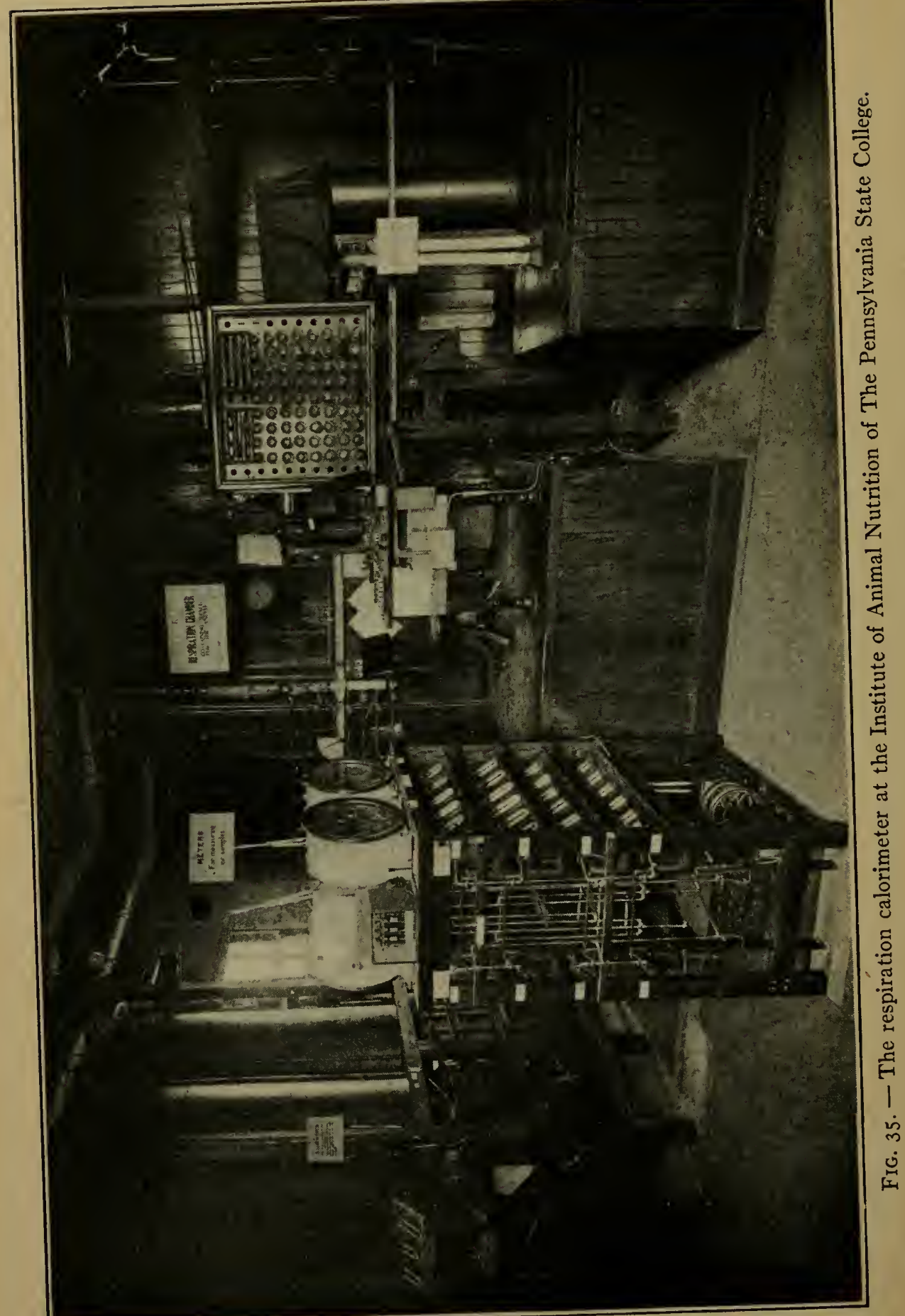


and Reichert. ${ }^{1}$ It is, however, not readily adapted for use with large animals, both on account of the difficulty in determining the true average temperature of a large mass of water and on account of the great weight of such an instrument.

The best known and most successful form of flow calorimeter for experiments upon animals is that devised by Atwater and Rosa ${ }^{2}$ and

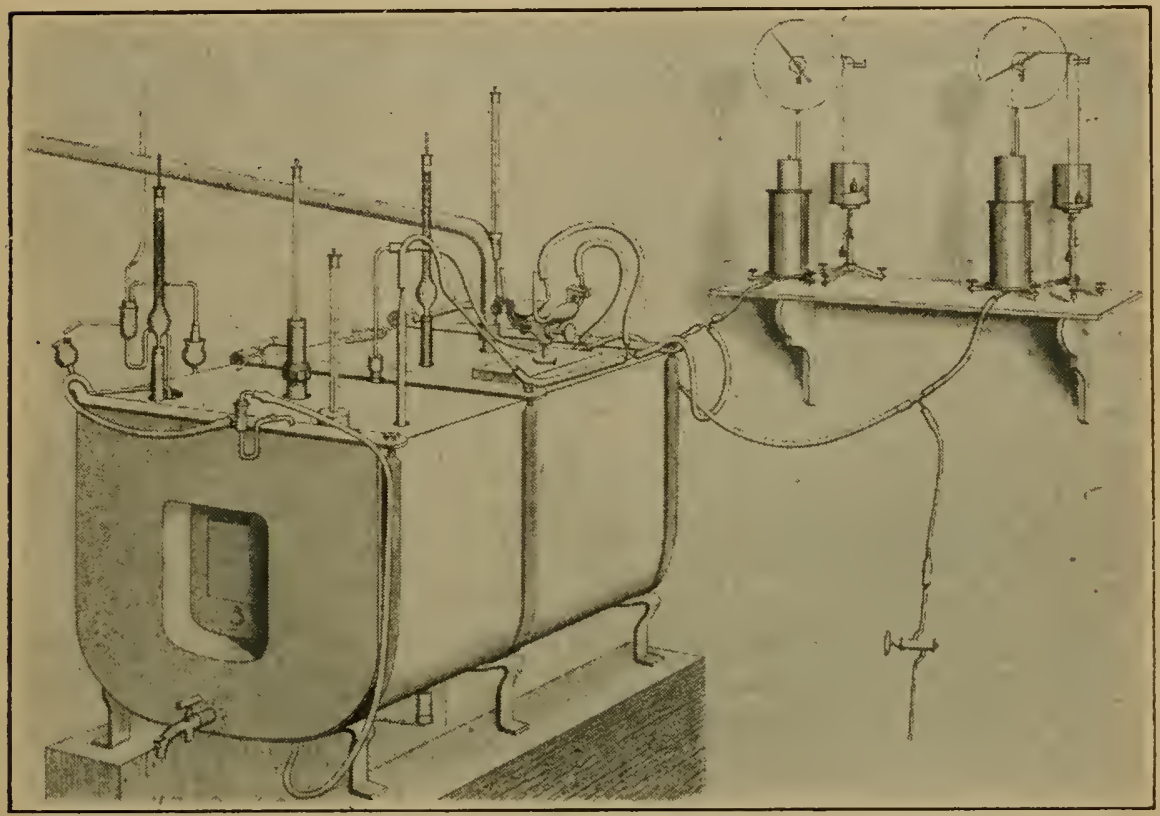

Fig. 36.

modified by Atwater and Benedict ${ }^{3}$ for experiments on man and adapted by Armsby and Fries ${ }^{4}$ and by Hagemann ${ }^{5}$ for experiments on the larger farm animals. Figure 35 shows the general appearance of the apparatus constructed by Armsby and Fries.

The most familiar form of emission calorimeter is that of Rubner, ${ }^{6}$ in which the changes in volume of the air enclosed between the double walls of the animal chamber constitutes the indicator. Figure 36 shows the general appearance of the Rubner apparatus. A very similar one has been devised by Rosenthal ${ }^{7}$ in which the pressure of the confined air at constant volume serves as the indicator.

1 University Med. Mag., I89o, ii, 173.

2 U. S. Dept. Agr., Office Expt. Stas., Bul. 63 (1899); Bul. I 36 (1903).

3 Carnegie Institution of Washington, Publication No. 42 (1905).

4 U. S. Dept. Agr., Bur. Anim. Indus., Bul. 5I, (1903); and Experiment Station Record, 15 (I903-r904), ro37.

${ }^{5}$ Landw. Jahrb., 41 (I9II), Erganzbd. I.

${ }^{6} \mathrm{Ztsch}$. Biol., 30 (1 894), 9 I.

${ }^{7}$ Arch. (Anat. u.) Physiol., I 894, p. 223. 
An interesting form of emission calorimeter is the so-called compensation calorimeter, in which the heat produced by the subject is balanced against that produced, for example, by burning pure hydrogen or by an electrical resistance in a precisely similar chamber. Calorimeters of this type have been described by Haldane, ${ }^{1}$ Bohr ${ }^{2}$ and recently by Tangl. ${ }^{3}$

328. Summary. - The foregoing facts may be summarized in the following tabular statement showing the several items of income and outgo of energy as well as the particular form of energy contained in each.

\begin{tabular}{ll|l} 
Income: & \\
Outgo: & Feed & \\
& Feces \\
& Urine \\
& Perspiration \\
& Combustible gases \\
& Work & \\
& Heat & Kinetic energy \\
& Heat energy
\end{tabular}

329. Exampie of an energy balance. - The same experiment upon a steer for which the nitrogen and carbon balance and the metabolizable energy $(290,294,322)$ have already been computed may also serve to illustrate the determination of the energy balance. In this experiment the animal performed no external work, so that no energy had to be measured in that form.

Table 28. - Daily Energy Balance of a Steer

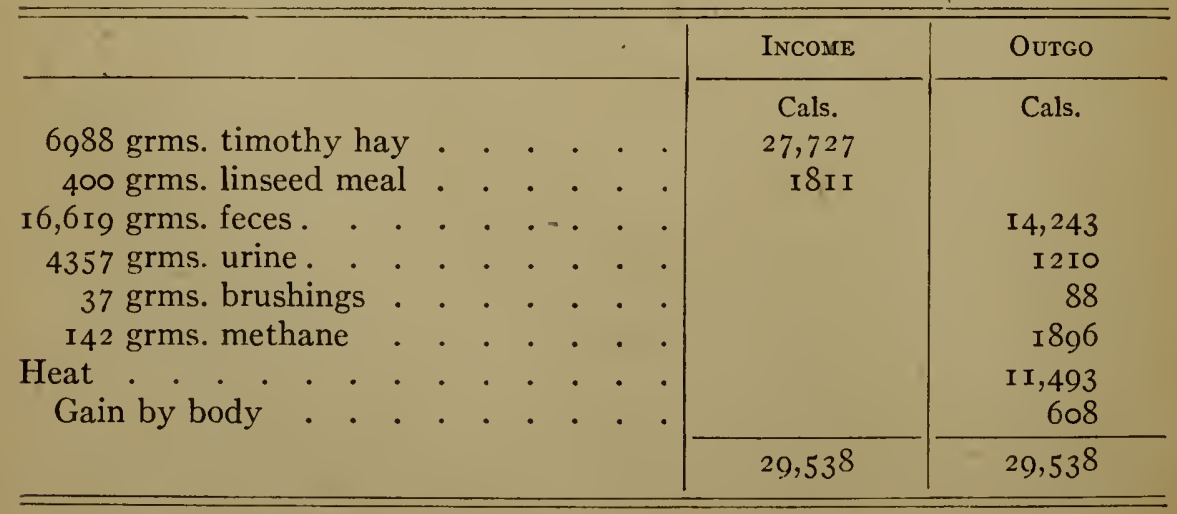

1 Jour. Physiol. (London), 16 (1 894), I 23.

2 Skand. Arch. Physiol., 14 (1903), 398.

${ }^{3}$ Biochem. Ztschr., 53 (I9I3), 2 I. 
According to the conception of the schematic body (280), these figures show that energy to the extent of 608 Cals. was stored up in the body as the chemical energy of either protein, fat or glycogen. Assuming that there was no change in the glycogen content of the animal, the nitrogen and carbon balance showed a computed storage of 66.6 grams of protein and 15.2 grams of fat (294). The average chemical energy of protein is 5.7 Cals. per gram and that of fat 9.5 Cals. per gram. The amounts of energy stored up in the fat and protein gained by the steer can therefore be computed as follows:

$$
\begin{aligned}
& \text { In protein } 5.7 \text { Cals. } \times 66.6=380 \text { Cals. } \\
& \text { In fat } \quad 9.5 \text { Cals. } \times 15.2=\underline{\mathrm{I} 44 \mathrm{Cals}} \text {. } \\
& \text { Total } 524 \text { Cals. } \\
& \begin{array}{l}
\text { Found from energy balance } \\
\text { Difference }
\end{array}
\end{aligned}
$$

It thus appears that in this experiment the gain of energy found by a direct determination of the energy balance and that computed from the balance of nitrogen and carbon agreed within 84 Cals., or 0.3 per cent of the total amount of energy involved. It is evident that determinations of the nitrogen and carbon balance on the one hand and of the energy balance on the other may serve as a mutual check, and also that the heat production of an animal may be computed quite accurately from determinations of the nitrogen and carbon balances (indirect calorimetry.)

\section{§5. Significance of Results}

Studies of the balance of nutrition have played a very prominent rôle in both physiological and agricultural investigation. Having considered in the foregoing pages the general methods of the balance experiment, a brief consideration of the significance of the results obtained by their use as compared with those reached by other methods seems called for.

330. Comparison with metabolism investigations. - The results of experiments like the one with a steer used as an illustration in previous paragraphs show, within the limits of experimental error, the loss or the storage of chemical energy 
resulting from the use of a certain feed or ration and approximately in what kind of material (protein, fat, glycogen) the energy lost or gained was contained. The balance experiment, therefore, is adapted to determine the total nutritive effect of a given substance, while if the comparative slaughter test be regarded as a form of balance experiment (284) the particular organs or tissues in which gain or loss took place can be determined.

The balance experiment, however, affords no insight into the details of the chemical mechanism by which the observed nutritive result is brought about. For example, balance experiments have demonstrated that starch may serve as a source of fat and have shown quantitatively the amount of fat formed from a given weight of starch. As applied to known chemical compounds, such a result as this is perfectly definite and of the highest value, but it gives absolutely no information as to the intermediate steps of fat formation, either in the processes of digestion, resorption or metabolism.

On the other hand, investigations of the intermediary metabolism, like those whose main results have been outlined in Chapter $\mathrm{V}$, have necessarily been to a large extent quàlitative. They have demonstrated some of the steps through which the various anabolisms and katabolisms occur, but as a rule have not attempted to deal directly with quantitative questions. ${ }^{1}$

Naturally the foregoing comparison is neither comprehensive nor exclusive. It aims simply to point out a broad general distinction between two types of nutrition investigation which in reality shade into each other.

Balance experiments have sometimes been characterized, with a certain half contemptuous implication, as "bookkeeping with the body." 'The characterization is a good one but the implication is unwarranted. It is perfectly true, as some critics of the balance experiment point out, that, for example, the most accurate record of the income of raw materials and outgo of finished products would of itself give a very incomplete notion of the operations of a great factory and that the successful conduct of such an enterprise requires as intimate a knowl-

${ }^{1}$ For a summary of some of the more important of these methods compare Dakin, Oxidations and Reductions in the Animal Body, Chapter III. 
edge as possible of the functions of each separate machine and of the changes undergone by the materials submitted to its action.

It may fairly be presumed, however, that these critics, even with the fullest knowledge of the technical details of such a factory, would hardly undertake to conduct it as a business enterprise without keeping account of the stock purchased and the output realized, i.e., exactly the sort of bookkeeping which the balance experiment attempts for the animal body. The truth is that both types of investigation are equally necessary and each aids in the interpretation of the other. The balance experiment has been especially prominent in the past, while at present attention is being directed to a greater extent to investigations of the intermediary metabolism, but neither can say to the other "I have no need of thee."

331. The balance experiment in agricultural investigations. - As already indicated, the methods of the balance experiment have been quite largely applied in agricultural investigations. Such investigations have been made, in the majority of cases, not with single chemical compounds, but with feeding stuffs or rations as a whole, and the effect observed in such an experiment is obviously a summation of the effects of all the ingredients contained in the feed consumed. The result, therefore, while entirely adequate to determine the total nutritive effect of the particular material experimented with is less capable of generalization than one obtained with a single chemical compound like starch or fat, and from this point of view may even be regarded as being in a sense empirical. A comprehensive knowledge of the nutritive value of a feeding stuff implies, first, a determination of the kinds and amounts of chemical compounds contained in it and, second, a determination of the exact physiological functions of each. Obviously, however, such a complete determination of the nutritive value of any considerable number of feeding stuffs is a work requiring a vast expenditure of time and labor. One justification, therefore, for the "short-cut" method of determining summarily by a balance experiment the effect of a feeding stuff or ration is that it appears possible to secure in this way within a reasonable time data which can be put to practical use in the comparison of feeding stuffs and rations. Moreover, it is to be anticipated 
that the conclusions as to the nutritive value of any material drawn from even the most elaborate chemical and physiological investigations will need finally to be checked and confirmed by the methods of the balance experiment.

The value of the balance experiment in relation to stock feeding, however, is far from being limited to the summary determination of the total nutritive values of feeding stuffs, although it has rendered important service in that field.

As will become apparent in Part III, the feed requirements for animals for various purposes, as well as the general physiological laws governing the processes of maintenance, growth, fattening, milk production, the performance of work, etc., can be successfully studied only with the aid of balance experiments, and the results obtained in such experiments are of general scientific value independent of the particular feeding stuff used. A striking illustration of the importance of such investigations on farm animals is afforded by the results obtained by Zuntz and his associates, by Kellner and others regarding the expenditure of energy in the digestion and assimilation of the feed (365-370). The marked differences between these animals and man or carnivora as regards the character of the feed and of the digestive processes have served to make prominent certain factors of the so-called "work of digestion" which were inconspicuous in the latter subjects and thus the investigations have yielded important contributions to comparative physiology.

332. Comparison with practical experiments. - Finally, it should be observed that the methods of exact feeding experiments based on a determination of the balance of matter and energy do not differ in their ultimate logical basis from those of so-called "practical" experiments. In both cases, the measure of the nutritive value of a feeding stuff, of the influence of changed conditions, or of the efficiency of the animal as a food producer, is the effect upon the animal. The difference lies in the accuracy and degree of detail with which that effect is determined. The reasons for the inadequacy of the live weight as a measure of nutritive effect have already been considered (281-283), while the experience of more than 50 years has sufficiently demonstrated that the attempt to measure nutritive effects by changes in the weight of the animal or by the gross 
product yielded fails to give results which are consistent with each other or which permit of the formulation of general principles. Only the laborious methods of the balance experiment or the refinements of physiological investigation can be relied upon to reveal those fundamental laws upon which the successful practice of stock feeding depends. 



\section{PART III}

\section{THE FEED REQUIREMENTS}





\section{CHAPTER VII}

\section{THE FASTING KATABOLISM}

333. Significance. - It is a familiar fact that in the absence of feed the life of the animal can be supported for a time at the expense of the materials of the body itself. If sufficient water and oxygen be supplied, those metabolic processes by which energy is liberated for the physiological activities of the body $(201,207)$ may continue for a considerable period, although, of course, they are ultimately halted by lack of material or impairment of the integrity of the protoplasm. The fasting animal in a state of rest, therefore, affords an opportunity to study the demands of the fundamental vital processes uncomplicated by the functions of digestion and resorption or by the requirements of growth, fattening or reproduction.

A qualitative and quantitative knowledge of the expenditure of matter and of energy by the fasting animal, then, is obviously an important step towards ascertaining the supply of feed necessary for various purposes.

334. Substances katabolized. - All the principal components of the body may be katabolized and yield energy for the support of the fasting organism.

Fat. - It is a familiar conception that fat formation is the body's method of disposing of surplus feed, and that the body fat is a store of reserve fuel material. The converse of this fact is equally familiar. The fasting or insufficiently fed animal loses fat, and may reach a stage of extreme emaciation before the active tissues fail to perform their functions. Obviously, the fasting animal lives largely upon its reserve of fat. These conclusions from common observation have been fully confirmed by comparative analysis of the carcasses of well-fed and of fasted animals as well as by the results of balance experiments in which the exact nature of the outgo from the body has been determined.

Carbohydrates. - In addition to fat the body contains more or less non-nitrogenous matter in the form of glycogen in the 
liver and muscles. During the first few days of fasting, this store of carbohydrates is also drawn upon, as is indicated by the fact that the respiratory quotient tends to approach unity, while later the amount of glycogen katabolized becomes very small.

Protein. - Balance experiments, however, while confirming the conclusion that the loss of tissue in fasting usually consists chiefly of fat together with some carbohydrates, show that there is also a continual katabolism of body protein and a corresponding excretion of urinary nitrogen. While the energy expended by the fasting animal is derived chiefly from the breaking down of non-nitrogenous material, the functional activities of the body necessarily involve the katabolism of a certain minimum amount of protein.

Ash. - Finally, in addition to those groups of substances whose katabolism yields energy to the body, the so-called mineral elements, or ash, of the body take part in the processes of katabolism and are continuously excreted in the urine of the fasting animal.

The foregoing facts are well illustrated by Benedict's ${ }^{1}$ investigations upon inanition. The average results of a number of experiments in which men fasted for from two to seven consecutive days were as follows:-

Table 29. - Average Katabolism of Fasting Men

\begin{tabular}{|c|c|c|c|c|c|c|c|c|c|}
\hline & & & \multirow{2}{*}{ 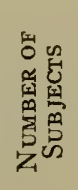 } & \multicolumn{3}{|c|}{ Total Katabolism } & \multicolumn{3}{|c|}{$\begin{array}{l}\text { KATABOLISM PER KILO- } \\
\text { GRAM BODY WEIGHT }\end{array}$} \\
\hline & & & & $\begin{array}{c}\text { Protein } \\
\text { Grms. }\end{array}$ & $\begin{array}{c}\text { Fat } \\
\text { Grms. }\end{array}$ & $\begin{array}{l}\text { Glycogen } \\
\text { Grms. }\end{array}$ & $\begin{array}{c}\text { Protein } \\
\text { Grms. }\end{array}$ & $\begin{array}{l}\text { Fat } \\
\text { Grms. }\end{array}$ & $\begin{array}{c}\text { Glyco- } \\
\text { gen } \\
\text { Grms. }\end{array}$ \\
\hline First day & . & & I4 & 60.2 & $\mathrm{I} 35 . \mathrm{I}$ & I 10.0 & 0.94 & 2.10 & I.69 \\
\hline Second day & 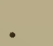 & . & I4 & 76.6 & $\mathrm{I} 65.9$ & $40.3^{*}$ & $\mathrm{I} .2 \mathrm{I}$ & $2.6 \mathrm{I}$ & $0.62 *$ \\
\hline Third day & . & . & 6 & 78.5 & I 55.2 & $2 \mathrm{I} .8$ & I. 28 & 2.54 & 0.36 \\
\hline Fourth day & . & . & 5 & 68.6 & $\mathrm{I} 47.2$ & $23 \cdot 3$ & I.I 5 & 2.47 & 0.40 \\
\hline Fifth day & . & . & 2 & 62.6 & $\mathrm{I} 46.4$ & $8.2 *$ & I. I I & $2.6 \mathrm{r}$ & $0.14^{*}$ \\
\hline Sixth day . & . & . & I & $64 \cdot 4$ & I 29.8 & 21.7 & I.I4 & 2.30 & $0.3^{8}$ \\
\hline Seventh day & . & . . & I & 60.8 & $\mathrm{I} 32.5$ & I 8.7 & I.08 & 2.36 & 0.33 \\
\hline
\end{tabular}

1 The Influence of Inanition on Metabolism; Carnegie Institution of Washington, Publication No. 77 (1907), pp. $45^{6-464}$.

* Omitting one case in which a small gain of glycogen was observed. 


\section{$\S$ I. The Protein Katabolism in Fasting}

335. Protein katabolism normally small. - In view of the structural functions of the proteins (264), it is of some importance to inquire what proportion of the total energy requirement is supplied by these substances.

This aspect of the subject has been considered especially by E. Voit, ${ }^{1}$ who has compiled and discussed the results of a considerable number of experiments upon fasting. While some of his computations are based on estimates, they are sufficiently accurate to outline definitely the main features of the fasting katabolism. They show that in what may be spoken of as the normal fasting animal, in which the influence of the previous feeding has disappeared and in which, on the other hand, the fat reserve has not been exhausted, the protein katabolism supplies a rather small proportion of the total energy transformed, the percentage with dogs, e.g., ranging in the majority of cases between ro and 17 .

336. Fasting protein katabolism variable. - It is not true, however, as has sometimes been loosely stated on the basis of C. Voit's experiments (338), that the protein katabolism of a fasting animal becomes constant within a short time. On the contrary, in the presence of an adequate amount of body fat, its amount tends to diminish with the progress of fasting. For example, in one of Benedict's fasting experiments (Table 29), the total urinary nitrogen upon the several days of the experiment was : -

Table 30. - Protein Katabolism of a Fasting Man - Benedict

\begin{tabular}{|c|c|c|c|c|c|}
\hline \multirow{2}{*}{ DAYS } & \multicolumn{2}{|c|}{ URINARY NITROGEN } & \multirow{2}{*}{ DAYS } & \multicolumn{2}{|c|}{ URINARY NITROGEN } \\
\hline & Total & $\begin{array}{c}\text { Per kilogram } \\
\text { weight }\end{array}$ & & Total & $\begin{array}{c}\text { Per kilogram } \\
\text { weight }\end{array}$ \\
\hline & Grams & Grams & & Grams & Grams \\
\hline I & I 2.24 & 0.206 & 5 & 10.87 & 0.191 \\
\hline 2 & I 2.45 & $.2 \mathrm{II}$ & 6 & 10.74 & .190 \\
\hline 3 & 13.02 & .223 & 7 & I0. I 3 & $.18 \mathrm{I}$ \\
\hline 4 & I I. 63 & .202 & & & \\
\hline
\end{tabular}

${ }^{1}$ Ztschr. Biol., 41 (I90I), I67. 
337. Influence of body fat. - E. Voit's compilation likewise showed clearly that the ratio of protein to total katabolism in fasting may vary considerably as between individuals, depending on the relative amount of fat contained in the body. So long as body fat is readily available as fuel, the amount of protein katabolized remains relatively small, but if the animal is originally deficient in fat, or if its content of fat becomes much reduced during fasting, more protein is katabolized to make up for the deficiency.

Usually, the store of fat in the body is less than that of protein, while in fasting its exhaustion is relatively more rapid. There comes a time, therefore, when the supply of non-nitrogenous material to the tissues begins to flag. When this happens, the protein katabolism begins to increase ; that is, when the supply of reserve fuel material runs low, the organism begins to use more of the protein of its tissues as a source of energy, and Voit ${ }^{1}$ has shown that this occurs whenever the ratio of fat to protein remaining in the body falls below a certain limit. If the animal was originally well nourished, this rise in the protein katabolism occurs only shortly before death, from which it has received the name premortal rise. In the case of very fat animals this point may never be reached, while, on the other hand, in a lean animal the protein katabolism may increase steadily from the very beginning of the fasting. The following three experiments upon a fat guinea pig, a medium fat dog and a lean rabbit, cited by Voit from Rubner's experiments, serve to illustrate these three types of fasting katabolism.

Table 3 I. - Fasting Protein Katabolism of Fat, Medium and Thin ANIMALS

\begin{tabular}{|c|c|c|c|c|c|}
\hline \multicolumn{2}{|c|}{ Guinea Pig } & \multicolumn{2}{|c|}{ Dog } & \multicolumn{2}{|c|}{ RABBIT } \\
\hline $\begin{array}{l}\text { Day of } \\
\text { Fasting }\end{array}$ & $\begin{array}{l}\text { Protein Katab- } \\
\text { olism in Per Cent } \\
\text { of Total Katab- } \\
\text { olism }\end{array}$ & $\begin{array}{l}\text { Day of } \\
\text { Fasting }\end{array}$ & $\begin{array}{l}\text { Protein Ka- } \\
\text { tabolism in Per } \\
\text { Cent of Total } \\
\text { Katabolism }\end{array}$ & $\begin{array}{l}\text { Day of } \\
\text { Fasting }\end{array}$ & $\begin{array}{l}\text { Protein Ka- } \\
\text { tabolism in Per } \\
\text { Cent of Total } \\
\text { Katabolism }\end{array}$ \\
\hline 2 & 10.4 & $2-4$ & I6.3 & 3 & I6.5 \\
\hline 3 & I I.I & IO-II & I $3 . \mathrm{I}$ & $5-7$ & 23.6 \\
\hline 4 & I I.O & I 2 & I $5 \cdot 5$ & $9^{-I} 2$ & 26.5 \\
\hline 5 & I I.9 & I3 & I 7.4 & $13-15$ & 29.8 \\
\hline 6 & I 1.8 & I4 & 20.0 & & 50.1 \\
\hline 7 & 6.9 & - & & I $7-18$ & 96.4 \\
\hline 8 & I I. 2 & & & & \\
\hline 9 & 10.9 & & & & \\
\hline
\end{tabular}

1 Ztschr. Biol., 41 (I901), 502. 
338. Influence of previous protein feeding. - The classic experiments of Carl Voit ${ }^{1}$ upon fasting dogs have shown that the protein katabolism in the early days of fasting may vary widely according to the amount of protein previously consumed. When the fasting follows a high protein ration, the protein katabolism on the first day of fasting may be relatively large, but it soon falls to a comparatively low level which is approximately the same whatever the initial ration. This behavior is well illustrated by the following results, all upon the same animal, which have been fully confirmed by numerous subsequent experiments.

Table 32. - Protein Katabolism of Fasting Dog - Voit

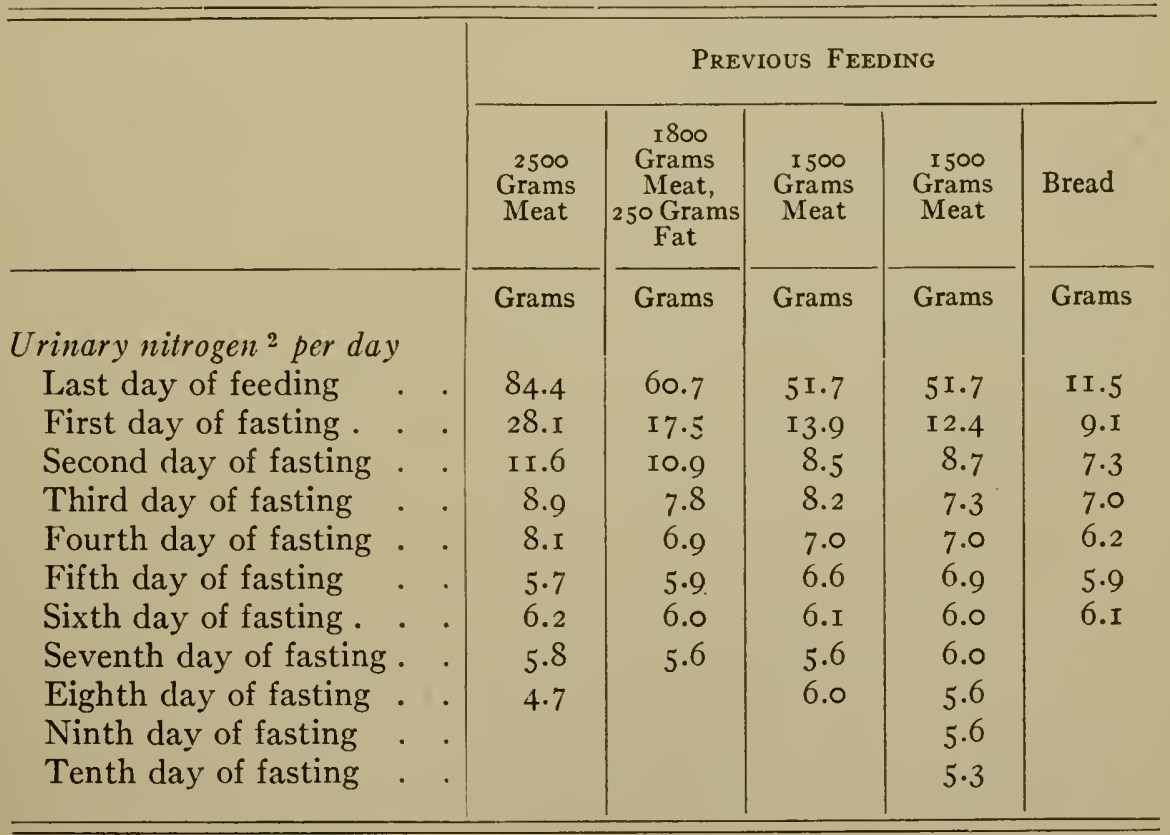

Furthermore, the high protein katabolism which is observed during the first two or three days of fasting after high protein feeding is accompanied by a relatively smaller katabolism of fat. Thus, in the first of the foregoing experiments respiration trials were made on the second, fifth and eighth days with the following results :-

${ }^{1}$ Ztschr. Biol., 2 (1 866), 307.

2 Computed from Voit's figures for urea. 
Table 33. - Total Katabolism of Fasting Dog

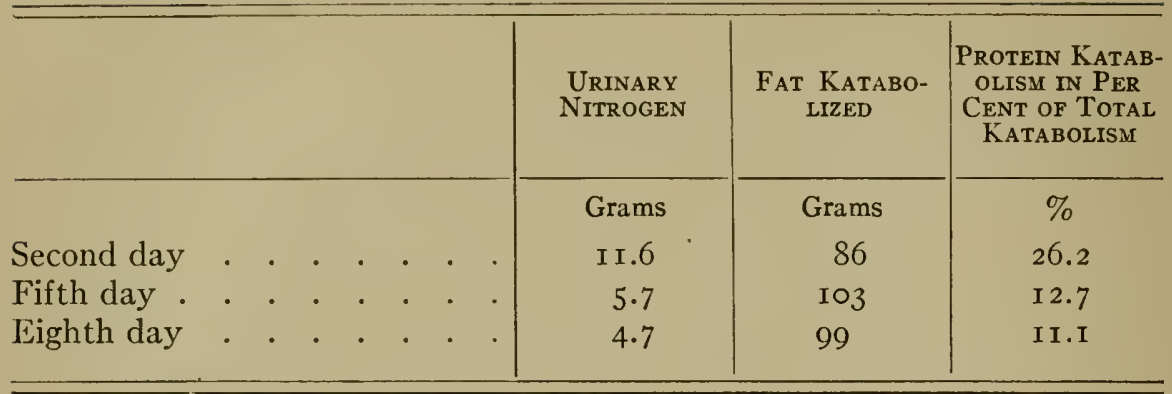

Obviously, we have here the reverse of what takes place in the later days of fasting, viz., a gradual substitution of fat for protein as the readily available supply of the latter in the body is reduced. Doubtless the effect would have been found to be still more marked on the first day of fasting, when the protein katabolism was equivalent to 28.I grams of nitrogen.

339. Physiological minimum of protein. - The facts recorded in the previous paragraphs render it evident that the lowest level of protein katabolism is not necessarily attained during complete fasting. Although the protein katabolism of a fasting animal soon reaches a comparatively low level which changes but slowly, nevertheless its amount may be greatly affected, on the one hand by the amount of protein previously consumed, and on the other hand by the stock of non-nitrogenous material (fat and glycogen) contained in the body. While normally some io to I 7 per cent of the energy metabolized in complete fasting is derived from protein (335), the proportion may rise to twice this amount on a day following heavy protein feeding, or to almost roo per cent in case of an animal whose stock of body fat is exhausted. In such cases it is evident that part of the protein is katabolized simply for the sake of supplying energy, since the smaller amounts katabolized in what may be called a normal or average condition of the fasting animal are at least sufficient to maintain all the vital functions, the latter proceeding for a considerable time in a substantially normal manner.

The level of protein katabolism being so dependent on the amount of non-nitrogenous material available as a source of energy, the question naturally arises whether by supplying an 
animal with liberal amounts of non-nitrogenous nutrients, but no protein, the protein katabolism might not be reduced to an amount even smaller than that observed in the absence of all feed. Experiments by C. Voit and by Rubner on dogs and by Landergren, Folin and Cathcart on man have shown this to be the case with these species.

Comparisons of this sort on farm animals are not readily made, especially with herbivora, and none have yet been reported, but McCollum and Steenbock ${ }^{1}$ have shown that the protein katabolism of the pig may be reduced by long continued feeding on a non-nitrogenous diet (starch) to an amount materially less than appears to be necessary in the feed of the animal under ordinary conditions to maintain nitrogen equilibrium (417), the average of all of their experiments being equivalent to $0.28 \mathrm{lb}$. of protein per rooo lbs. live weight.

340. Functions of protein in fasting. - The fact that a certain minimum katabolism of body protein persists even in the presence of the most abundant supply of non-nitrogenous nutrients has generally been interpreted in the past as showing that a certain amount of the protein of the cell is necessarily broken down in the performance of its physiological functions. This necessary minimum has been somewhat vaguely compared to the wear of a machine, Rubner especially designating it as the "wear and tear" quota of the protein katabolism. More recent investigations, however, have suggested the possibility of another explanation.

The actual nitrogenous nutriment of the body cells is not proteins as such, but substantially the simple amino acids out of which they are built up. As required, these amino acids may be synthesized to protein $(226,232)$, but there appears to be some reason for believing that they may also be necessary for other purposes in the body; that certain of them may, for example, as was suggested by Willcock and Hopkins, be essential to the production of the various internal secretions and hormones which apparently play so large a part in metabolism. If, however, the normal performance of the body functions calls for a supply of some particular amino acid, tryptophan e.g., this can be derived, in the fasting animal, only from the cleavage of body protein, since there is no evidence that tryptophan can 
be synthesized by the organism. It might very well be, therefore, that the minimum unavoidable protein katabolism in the absence of nitrogenous feed is due to such a demand for certain amino acids or other groupings, and only in part or not at all to a necessary breaking down of cell proteins as a condition of protoplasmic activity. Moreover, it is quite conceivable that both of these views may be true; that a part of the minimum protein katabolism represents a necessary destruction of cell protoplasm in the performance of its functions, while the other part represents protein broken down for the sake of securing certain constituents for specific purposes.

There will be occasion to consider these possibilities further in discussing the protein requirement for maintenance (398).

\section{$\S 2$. The Energy Katabolism in Fasting}

341. Internal work. - The body of an animal receiving no feed and doing no external work is still carrying on a great variety of internal activities, both mechanical and chemical. Of the former, the most prominent is the muscular work of circulation and respiration, together with the maintenance of muscular tonus (632), while the secretory and excretory activities of the various glands are typical of the latter. These various bodily activities, whose due performance is essential to the continued existence of the animal, may be conveniently summarized in the term internal work.

342. Measure of energy expended in internal work. - In the fasting animal, all the various forms of internal work indicated in the previous paragraph are performed by means of energy derived from the katabolism of the fats, carbohydrates and proteins contained in the tissues. The chemical energy thus utilized may undergo numerous transformations, but ultimately, since it does no work upon the surroundings of the animal, it assumes the form of heat. A determination of the heat produced by a fasting animal in a state of rest, therefore, furnishes a measure of the energy expended in internal work, or of what is often called the basal metabolism.

343. Relative constancy of energy katabolism. - The results recorded in $\S$ I regarding the nature of the material katabolized in fasting, and the way in which fat, carbohydrates and protein 
may mutually replace each other as fuel material according as one or the other is most available, render it evident that the controlling factor in the katabolism of the fasting body is the demand for energy for the performance of the internal work and can hardly have failed to suggest that this demand must be relatively constant in the same individual under like conditions. That such is in fact the case has been demonstrated by a large number of experiments. While not mathematically invariable, the fasting katabolism, expressed in terms of energy, tends to approach a uniform value in proportion as the experimental conditions are maintained constant. The fasting organism requires approximately the same quantity of energy from day to day for the performance of its necessary internal work, but seems more or less indifferent as to whether this energy is derived from the katabolism of fats, carbohydrates or proteins.

For example, in Voit's experiment cited in the previous section to illustrate the interrelations of protein and fat katabolism (Table 33), the computed energy of the protein and fat katabolized on each of the three days was as shown in the following table, from which it appears that the total energy katabolism, especially when computed per kilogram of live weight, was approximately the same on the different days.

Table 34. - Energy Katabolism of Fasting Dog

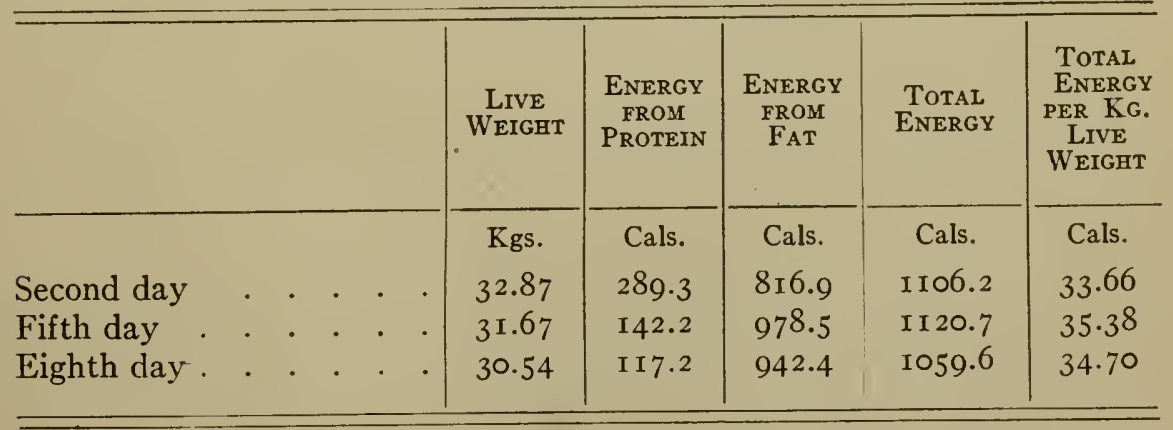

The same thing is true of Rubner's determinations of the fasting katabolism of a rabbit, a dog and a guinea pig, whose results as regards the protein katabolism have been already considered (337) and likewise of Benedict's investigations upon fasting men (334).

344. Energy expenditure in fasting a measure of maintenance requirement. - In the fasting animal in a state of 
complete rest and at moderate external temperature, the vital activities are evidently reduced to the minimum compatible with the continuance of life. Since the internal work of such an animal is performed at the expense of the chemical energy stored up in its tissues, the body's stock of energy is being constantly depleted by an amount equivalent to the internal work done and this loss of energy must be made good from the feed if the animal is to be maintained. The relatively constant total katabolism of the fasting animal, as expressed in its heat production, is therefore the measure of the amount of energy expended in carrying on the fundamental vital activities of the body, and consequently of the minimum quantity which must be supplied in a maintenance ration.

\section{§ 3. Conditions Affecting the Fasting Katabolism}

345. Size of animal. - That large animals katabolize more matter and produce more heat than smaller ones, and therefore require more feed for maintenance, needs no special proof. Experiment shows, however, that the difference is not proportional to size or weight, but that small animals have a more intense katabolism than large ones, its amount being approximately proportional to the body surface, which, of course, is relatively greater in the smaller animal.

The relation to body surface appears to have been first suggested by Bergmann (cited by Rubner) in $185^{2}$ and later by Münt $z^{1}$ in 1878 , but Rubner ${ }^{2}$ seems to have made the first, quantitative investigation of the question, determining the fasting katabolism of six dogs whose weights ranged from 3 to 24 kilograms.

While not mathematically constant, the ratio between the fasting katabolism and surface showed a close approximation to uniformity, and the same fact has been verified by a considerable number of investigators, although with some exceptions, and is now generally accepted. Moreover, it has been shown ${ }^{3}$ to be approximately true not only of animals of the same species but of animals ranging in size from man to domestic fowls and including also cold blooded animals.

346. Computation of katabolism per unit of surface. - It is a familiar fact that the surfaces of solids of the same shape,

1 Ann. Inst. Agron., III, p. 59.

${ }^{2}$ Ztschr. Biol., 19 (1883), 535.

${ }^{3}$ E. Voit; Ztschr. Biol., 41 (rgor), i I3. 
i.e., of those which are geometrically similar figures, are proportional to the two-thirds powers of their volumes. Since the specific gravity of animals varies but slightly, it may be said without material error that the body surfaces of animals of the same shape are proportional to the two-thirds powers of their weights. This relation may be expressed by the following formula, proposed by Meeh, ${ }^{1}$ in which $W$ equals the weight in grams, $S$ the surface in square centimeters, and $k$ is a factor which is constant for all animals of the same shape.

$$
S=k W^{\frac{2}{3}} \text {. }
$$

The value of the constant $k$ for the horse as reported by Hecker is 9.02. Trowbridge, Moulton and Haigh ${ }^{2}$ have determined the value of $k$ for 35 Hereford-Shorthorn cattle of various ages from birth up and in various conditions of fatness, using the empty weight as a basis. Dividing the animals into groups they found the following average values:-

\section{Table 35. - Values of $k$ for Beef Cattle}

Young and thin animals . . . . . . . . . . . . . 9.92

Animals in medium condition . . . . . . . . . 9.4 I

Fat animals 18 months old or less . . . . . . 8.57

Fat animals two years old or more . . . . . . . . . 7.65

According to these investigators the empty weight of cattle constitutes the following percentages of their live weight:-

Table 36. - Empty Weight as Percentage of Live Weight

Show cattle. . . . . . . . . . 92-94 per cent

Fat cattle . . . . . . . . . 9 9 I

Medium cattle . . . . . . . . 89-90

Thin cattle . . . . . . . . . . . . $87-89$

With the aid of the foregoing factors the total katabolism of beef cattle, and perhaps of other types, as determined by experiment may be computed per unit of body surface with reasonable accuracy. It is apparent that comparisons based upon the live weight instead of the empty weight would also be substantially accurate for thin and medium cattle. No similar data exist for other species of farm animals.

${ }^{1}$ Ztschr. Biol., 15 (I879), 425.

${ }^{2}$ Mo. Expt. Sta., Research Bul. I8. 
The principal cause of the difference between the groups of cattle appears to be the variation in the proportion of fat to active tissue, and Moulton ${ }^{1}$ has shown that if this be eliminated by making the fat-free empty weight the basis of computation, an average value of I0.34 for $k$ gives results very closely approximating those actually observed. He likewise finds that the body surface of thin and medium cattle is somewhat more closely proportional to the five-eighths than to the two-thirds power of the empty weight, while for fat cattle the five-ninths power gives the closest agreement, the corresponding values of $k$ being respectively 11.86 and $\mathrm{I} 3.40$.

347. Computation of katabolism to standard weight. - It is often desirable to compare the katabolism of animals of different weights or to compute experimental results to some convenient standard weight. Such comparisons should evidently be made on the basis of body surface rather than of body weight. Few actual determinations of the body surface of animals have been made, however, and with the exception of the horse and of beef cattle none on farm animals, so that it is in many cases impracticable to express the katabolism of the latter per unit of surface. For purposes of comparison between individuals of the same species and type, however, at least approximate results may be secured on the assumption that the animals to be compared are geometrically similar, so that their body surfaces are substantially proportional to the twothirds powers of their weights. For example, a steer weighing I 283 pounds was found to have a computed fasting katabolism (374) of 867 I Cals. It is often a matter of convenience to compute such a result to a weight of 1000 pounds. A steer weighing rooo pounds, other things being equal, would have a smaller katabolism in proportion to its smaller surface. The ratio between the surfaces of the two animals would be approximately $1000^{\frac{2}{3}}: 1283^{\frac{2}{3}}$, and the fasting katabolism of the smaller animal would therefore be $867 \mathrm{I}$ Cals. $\times\left(\frac{1000}{1283}\right)^{\frac{2}{3}}=7345$ Cals. In this way it is easy to compute the relative katabolism of different individuals without the necessity of expressing it per unit of surface.

Of course, such a comparison is only an approximation. In particular, as has just been shown (346), different animals are not of the same shape. The young animal differs in conforma-

$$
{ }^{1} \text { Jour. Biol. Chem., } 24 \text { (1916), } 299 .
$$


tion from the older one and the fat from the thin one and the beef steer and dairy cow, e.g., are far from being geometrically similar. Additional determinations of the relation of surface to weight in different species, types and ages of domestic animals would be of much interest but in their absence the method of comparison just outlined may probably be assumed to give a fair approximation to the truth and is certainly more accurate than a simple computation in proportion to weight.

348. Muscular activity. - As was implied in the introductory section of Chapter VI (274), and as will appear in greater detail in Chapter XIV, muscular work is done at the expense of energy derived from the katabolism of body substance, and no other single factor so largely influences the total katabolism. The minimum fasting katabolism which represents the demands of the indispensable life processes is exhibited only in a state of complete muscular rest. It is rarely the case, however, that an animal, even when at rest in the ordinary sense, does not maintain more or less muscular tension or execute more or less motions of various parts of the body, all of which, even when apparently slight, involve in the aggregate considerable expenditure of energy.

Zuntz and Hagemann, ${ }^{\mathbf{1}}$ for example, report a respiration experiment upon a horse in which the uneasiness caused by the presence of a few flies in the chamber of the apparatus caused an increase of ro per cent in the metabolism. Johansson ${ }^{2}$ found the hourly excretion of carbon dioxid by a fasting man when simply lying in bed (awake) to be 24.94 grams as compared with 20.72 grams when all the muscles were as perfectly relaxed as possible. Benedict and Carpenter ${ }^{3}$ have compared the metabolism of men during sleep with that of the same subjects lying quietly in bed immediately after waking. In the three cases which they regard as strictly comparable the increase in the heat production during the waking period ranged from 5.8 to 15.2 per cent, averaging I I .4 per cent. Benedict and Talbot, ${ }^{4}$ in experiments upon infants, found that even scarcely noticeable muscular activity produced a most marked effect on the carbon dioxid excretion, and Benedict and Pratt ${ }^{5}$ have noted similar results with dogs.

'Landw. Jahrb., 23 (1894), г6г.

2 Skand. Arch. Physiol., 8 (I 898), 85.

${ }^{3}$ Carnegie Institution of Washington, Publication No. 126 (Igro), p. 24 I.

${ }^{4}$ Amer. Jour. Diseases of Children, 4 (Igr 2), I 29.

5 Jour. Biol. Chem., 15 (1913), I. 
Since comparatively insignificant movements have such a striking effect upon the total katabolism, it is evident that the amount of muscular activity must be an important factor in determining the relative energy requirements of two animals even though their minimum katabolism in a state of absolute rest may be identical. In experiments of any considerable duration on normal animals, it is impossible to avoid more or less expenditure of energy in this incidental muscular work, while it is often a matter of difficulty to make the different periods of an experiment comparable in this respect.

349. Standing and lying. - Considerable muscular exertion is required during the waking hours to maintain the relative position of the different members of the body. This is especially true of standing. It has been shown that a man or an animal when standing excretes notably more carbon dioxid than when resting or lying down and produces correspondingly more heat. Differences of as much as 25 per cent have been observed in man and of 30 to 40 or more per cent, in cattle. It is evident, then, that if one animal lies down for twelve hours and another for only eight hours during the twenty-four, the former will, other things being equal, require less feed energy for actual maintenance than the latter.

350. External temperature. - Farm animals belong to that general class known as warm blooded, or homoiothermic, animals, whose bodies during health maintain a nearly constant temperature which is higher than that of their usual surroundings. The so-called "animal heat" is being continually generated by the katabolism going on in the body, while on the other hand the animal is continually imparting heat to its surroundings in four principal ways : viz., by conduction, by radiation, by evaporation of water, and as the sensible heat of the excreta.

Since the animal is both producing and losing heat continually, the maintenance of a constant body temperature implies the existence of some regulative mechanism by means of which the production and emission of heat may be adjusted to each other. This adjustment is effected in general in two ways which may be called, respectively, physical and chemical regulation.

351. Physical regulation of body temperature. - Changes in the temperature of its surroundings, in the relative humidity of the air, etc., tend to produce the same effect upon the animal 
as upon an inanimate body. A fall of temperature, for example, tends to increase the rate of outflow of heat and a rise of temperature to diminish it. In the so-called physical regulation, these tendencies are offset and the rate of heat emission maintained constant chiefly by means of changes in the temperature and state of moisture of the skin, brought about on the one hand by an adjustment of the blood flow and on the other through the perspiration.

The tendency of a rise of external temperature to check the outflow of heat is compensated for by a vaso-motor reflex which causes the arterioles leading to the surface of the body to relax (187), so that more blood flows through the skin capillaries, thus tending to raise the temperature of the surface and increase the outflow of heat. This phenomenon is readily observed in the flush which follows exposure to high temperatures. This method of regulation might be compared to opening the windows of a heated room to cool it.

If the external temperature continues to rise, visible perspiration occurs, or in the case of animals which have no sweat glands, like the dog, a peculiar form of breathing sets in and relatively large amounts of water are evaporated from the skin or from the tongue and the interior of the mouth and throat. In this way, large quantities of heat are carried off as the latent heat of vaporization of water, somewhat as an overheated room may be cooled by sprinkling the floor.

If the external temperature falls again, the process is reversed. Sensible perspiration decreases, the blood is diverted from the capillaries of the skin to the internal capillaries, and if the change takes place too rapidly, may even lead to congestion of the latter. The process is analogous to closing the 'windows of a room as the weather grows colder.

352. Chemical regulation of body temperature. - There are evidently limits to the possibilities of physical regulation. On the one hand, the external temperature may rise so high that it is impossible for the heat to escape from the body as fast as it is produced by the necessary katabolism, and heat apoplexy results. On the other hand, the temperature may fall so low that the utmost restriction of evaporation and the greatest possible diversion of the blood from the superficial capillaries is insufficient to conserve the body temperature. If the windows 
of the room are entirely closed, nothing more can be effected in this manner toward maintaining its temperature, and if the weather continues to grow colder, the fire in the room must be increased. Similarly, if the external cooling effect upon the animal becomes so great as to exceed the limits of physical adjustment, more fuel material is katabolized, that is, more heat is produced. This method of maintaining the body temperature is commonly called chemical regulation.

353. Mechanism of chemical regulation. - The chemical regulation is probably effected largely through muscular action, by visible motion or by an increase in the muscular tonus, either of which involves an increased heat production. This has been clearly shown to be true of man and probably applies also to other animals. Above the critical temperature, there appears to be a slight increase in the heat production with rising temperature, probably due to the additional energy expended in the various processes of physical regulation.

354. Critical temperature. - The temperature at which the physical regulation gives way to or begins to be supplemented by the chemical regulation has been called the critical temperature. $^{1}$ Above this temperature the radiating capacity of the body surface is varied to meet the varying conditions; below it, this method of regulation is largely exhausted and therefore the heat production is varied to meet the need. The critical temperature for man wearing ordinary clothing appears to be about $15^{\circ} \mathrm{C}$.; for the dog it is about $20^{\circ} \mathrm{C}$., for the guinea pig $30^{\circ}-35^{\circ}$, and for the hog, according to the results of Tangl ${ }^{2}$ and of Von der Heide and Klein, ${ }^{3}$ about $20^{\circ}-23^{\circ} \mathrm{C}$.

355. Other thermal conditions. - Any conditions tending to facilitate the escape of heat from the body will obviously act like a fall of temperature. Wind, for example, by removing the layer of partially warmed air next to the skin, tends to remove heat more rapidly from the body, so that the cold is felt more severely on a windy day, while, on the other hand, the effect of a high temperature is modified by wind. A high percentage humidity of the air on a warm day hinders the removal of heat by evaporation, so that a moist heat is more trying than a dry heat. Cold moist air, on the other hand, facilitates

1 The term refers, of course, to the temperature of the surroundings and not to that of the animal itself.

${ }^{2}$ Biochem. Ztschr., 44 (I9I2), 252.

Ibid., 55 (1913), 195. 
the escape of heat from the body by increasing the conducting power of the clothing, hair or fur, so that a damp cold is more severe than a dry cold. The direct rays of the sun may impart a considerable amount of heat to the body, thus moderating the effects of low temperatures and, on the other hand, increasing those of high temperatures. To be strictly accurate, then, one should speak of a critical thermal environment of the animal rather than simply of a critical temperature.

356. Influence on katabolism. - It is apparent from the foregoing facts that the energy katabolism of the fasting animal is affected by the external temperature and other thermal conditions to a considerably less extent than has been frequently imagined. It is by no means true that every fall in external temperature results in an increased katabolism in the animal for the sake of heat production, for if this were the case the converse would also be true, viz., that every rise in the external temperature would cause a corresponding decrease in the katabolism, so that finally, when the external temperature was equivalent to that of the body, the katabolism would be reduced to zero; that is, we should have life without katabolism, which is a contradiction in terms.

The fact that the heat production of an animal reaches a minimum at the critical temperature and that above that point it either remains unchanged or increases slightly shows that its extent is not determined by the needs of the organism for heat as such, since these diminish as the temperature rises. As a matter of fact, the production of heat in the body is not the purpose of katabolism but merely an incident of it. Heat is the form which the chemical energy of the katabolized material takes after it has served its purposes in the vital processes, and the nearly constant heat production above the critical temperature is simply due to the fact that the quantity of energy required for the internal work of the body is approximately constant and cannot be reduced simply by raising the external temperature. Heat is essentially an excretum to be gotten rid of. Incidentally, in warm-blooded animals, it serves also to maintain the body temperature necessary for the normal performance of the vital functions, but above the critical temperature there is a surplus over the amount required for this purpose which is disposed of by the processes of physical regu- 
lation already described. It is only when the external temperature sinks below this point that the katabolic processes are stimulated and more heat is produced, and only below this point, therefore, does the external temperature influence the energy requirement.

357. Effects of extremes of temperature. - The regulation of body temperature described in the foregoing paragraph is possible only within certain limits.

At very low temperatures the possibilities of chemical regulation may be exhausted, so that the animal is unable to produce heat as fast as it is abstracted and the body temperature begins to fall. An actual lowering of the body temperature, however, reduces the intensity of the katabolism exactly as it does in the case of a cold-blooded animal; the heat production sinks, bringing about a further fall in body temperature which again further diminishes the heat production, so that the animal speedily perishes from cold.

At very high temperatures the reverse process may take place. When the possibilities of physical regulation are exhausted, the body temperature rises. A very slight rise, however, has been shown to stimulate the katabolism and therefore the heat production, giving rise to a "vicious circle" which is the converse of that occurring at very low temperatures and which speedily leads to the animal being overcome by heat. 


\section{CHAPTER VIII}

\section{MAINTENANCE - THE ENERGY REQUIREMENTS}

358. Definition of maintenance. - Feed is supplied to farm animals either that they may yield products useful to man as materials for human food and clothing or that they may serve him by the performance of mechanical work.

But much as a factory must first be supplied with enough power to keep in motion the shafting, belting and machinery in general before any product can be turned out, so the animal mechanism must be provided with sufficient feed to maintain the processes essential to life before any continued production is possible. The amount required for this purpose is called the maintenance ration of the particular animal. It is the quantity necessary simply to support the animal when doing no work and yielding no material product. A balance experiment with an animal receiving precisely a maintenance ration would reveal an exact equality between income and outgo of ash, nitrogen, carbon, hydrogen and energy, showing that the body was neither gaining nor losing protein, fat, carbohydrates nor mineral elements. From this point of view, maintenance might be characterized as a state of labile equilibrium between the anabolic and katabolic processes of metabolism (203).

The word maintenance is sometimes used popularly in another sense to signify the total amount of feed required, for example, by a horse in order to perform his daily work or by a calf in order to make a normal growth. It is important to grasp the idea that, in its technical sense, the maintenance ration means the minimum required simply to maintain life. The total feed of the horse or calf would, from this point of view, be regarded as consisting of two portions; one of them the maintenance ration, which if fed by itself would just support the horse at rest or the calf without growth, and the other the productive portion of the ration, by means of which work is 
done or growth made. To recur to the illustration of the factory, the maintenance ration keeps the empty machinery running, while the additional feed furnishes the power necessary to turn out the finished product.

359. Significance of the maintenance ration in practice. It might seem at first thought that not much importance attaches to a determination of the maintenance ration. The animal kept on such a ration yields no direct economic return and hence simple maintenance feeding is to be avoided, so far as possible, while if it appears desirable to practice it the observation of the skilled stockman, especially if supplemented by occasional weighings, will usually suffice to determine whether or not the end is being attained. Nevertheless, the subject has much significance both for practice and for science.

A very considerable fraction of the feed actually consumed by farm animals - on the average probably fully one-half is required simply for maintenance. But if half of the farmer's feed bill is expended for maintenance, it is clearly important for him to know something of the laws of maintenance, - how its requirements vary as between different animals, how they are affected by the conditions under which animals are kept, how different feeding stuffs compare in value for maintenance, etc., - as well as to understand the principles governing the production of meat, milk, or work from the other half of his feed.

360. Bearing on interpretation of feeding experiments. From the point of view of the experimenter a knowledge of the maintenance requirement is likewise of great importance. In any rational study of the laws of nutrition, it is plainly inadmissible to attempt to establish general principles by a comparison of the feed with one of its effects, viz., production, while ignoring entirely its other effect, viz., maintenance. Failure to appreciate this fact is responsible for many misleading deductions from feeding experiments in the past.

It has been quite usual to compare the results of such experiments by computing the ratio of feed consumed to product yielded - i.e., either the feed consumed per pound of gain made or the gain produced per pound of feed consumed. Such a comparison, however, may give an entirely distorted idea of the real teachings of an experiment. Suppose, for example, 
that in a fattening experiment the quantities of two different rations consumed and the gains made were as follows:-

First Ration Second Ration

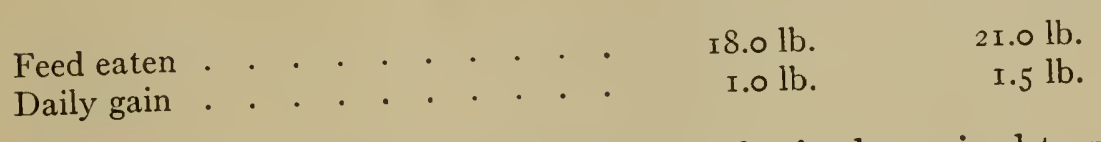

Compared in the way just indicated, the feed required to produce one pound of gain was i 8 pounds and i 4 pounds respectively, or the second ration appears to have been superior to the first by about 29 per cent. If, however, it was found that in each case 12 pounds of the feed were required simply to maintain the animal, a very different comparison is obtained, viz. 
requirements of the animal and the values of feeding stuffs and rations for that purpose may be considered from two points of view :-

First, we may inquire how much energy is necessary to support the quiescent animal and what amounts of it the various feeds and rations can supply in forms available for this purpose.

Second, we may ask what specific materials and how much of each must be supplied in the feed to make good the losses due to the continual katabolism of body substances. It is particularly the proteins, or rather the amino acids composing them, and the ash ingredients and perhaps the so-called vitamines which need to be considered in this respect, the body apparently possessing large powers of manufacturing other necessary ingredients from those supplied in ordinary feeding stuffs.

It' will be convenient to consider these two general classes of maintenance requirements in the order named, the present chapter dealing with the energy requirements.

362. Mutual replacement of organic nutrients. - The discussion; in Chapter V, of the functions of the principal groups of organic nutrients (262-267) showed that, besides certain specific values as sources of particular chemical compounds, they all serve as carriers of chemical energy for the needs of the organism. It would be anticipated, therefore, that the various digestible nutrients might mutually replace each other or the ingredients of the body, and numerous experiments have shown that such is indeed the case.

Fats fed to a previously fasting animal diminish or suspend the loss of body fat, while carbohydrates may be substituted for the feed fat with a similar result. As has already been shown (337), body protein may replace body fat in the katabolism of the fasting animal, while when protein is given to such an animal the non-nitrogenous portion of the molecule serves as a source of energy to the organism and can be substituted for body fat. On the other hand, an excess of feed protein above the minimum requirement can be replaced by fats or particularly by the carbohydrates, and likewise by organic acids.

In brief, the animal organism manifests a remarkable degree of flexibility as regards the nature of the material which it can utilize for its energy metabolism. Aside from the small minimum of protein required, the metabolic activities of the body 
may be supported, now at the expense of the stored body fat, now by the body protein, and again by the proteins, the fats, the carbohydrates, or the organic acids, of the feed. Whatever may be true economically, physiologically the welfare of the mature animal is not conditioned upon any fixed relation between the classes of nutrients in its feed supply, apart from the minimum requirements for protein and ash. But while the body may draw its energy from the most varied feed materials, it by no means follows that the gross energy of these materials is of equal value for the functions of the organism. On the contrary it has been shown that there are wide differences in this respect.

\section{§. Net Energy Values for Maintenance}

363. Method of determination. - The value of any nutrient or feeding stuff as a source of energy for maintenance is obviously measured by the extent to which it can diminish the loss of energy which the body would otherwise suffer. Suppose, for example, that a fasting dog was found to produce 600 Cals. of heat per day by the katabolism of his own tissues. If, in a subsequent experiment, fat be fed, this loss from the body will be diminished, more or less feed fat being virtually katabolized in place of body tissue. If fifty grams of fat are fed, and if a balance experiment shows that the loss of energy from the body is reduced from 600 Cals. to 200 Cals., it is plain that each gram of fat has reduced the loss by $400 \div 50=8 \mathrm{Cals}$. and the latter number shows the value of this particular fat for the maintenance of this particular animal.

364. Comparison with metabolizable energy. - As already defined (322), metabolizable. energy is that portion of the gross energy of the feed which is not carried off as chemical energy in the excreta but is capable of transformation in the body. It was natural to suppose, therefore, that the metabolizable energy of a substance would represent its value for maintenance and this was long believed to be true, but later investigations have shown that such is not the case.

For example, in balance experiments by Armsby and Fries a steer received in successive periods two different amounts of timothy hay, both insufficient for maintenance. The metab- 
olizable energy of the hay and the heat production per day, determined in the manner illustrated in Chapter VI $(322,329)$, were as follows :-

Table 37. - Determination of Net Energy Value of Timothy Hay

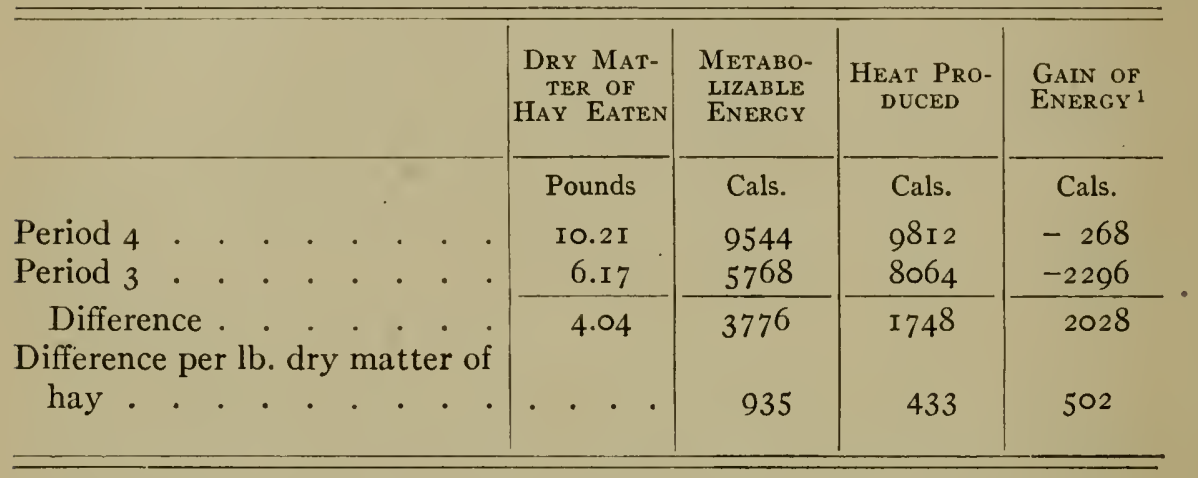

The 4.04 pounds of hay (water-free), added to the insufficient ration of Period III diminished the loss of energy from the body of the animal by 2028 Cals.; that is, they contributed this amount towards its maintenance. The net effect of the hay, therefore, computed exactly as in the supposed case of the dog in the preceding paragraph, was $2028 \div 4.04=502$ Cals. per pound of dry matter. But the added hay contained metabolizable energy to the amount of 935 Cals. per pound of dry matter. Clearly, therefore, by no means all of the metabolizable energy of the hay could be utilized for maintenance by the steer. Only 502 Cals. were used for this purpose, in place of energy previously derived from the katabolism of the fat and protein of the body, while the remaining 433 Cals. were, indeed, metabolized in the body, but resulted simply in increasing the heat production by this amount. ${ }^{2}$ The proportion of the metabolizable energy of this hay which was available for maintenance, then, was $502 \div 935=53.7 \%$. The foregoing result is typical of a large number of others which have been reached in experiments on various species of animals.

${ }^{1}$ Since submaintenance rations were fed, the gains were of course negative, i.e., chemical energy was lost from the body.

${ }^{2}$ Since gains of energy are computed from the difference between income and outgo, the figures of the last column of the table necessarily agree with those of the two preceding ones. They simply present a different aspect of the same facts. 
Even in the case of pure, or nearly pure, nutrients fed to carnivora, their maintenance values are less than their content of metabolizable energy.

365. Feed consumption increases heat production. - From a slightly different point of view, the experiment just cited furnishes a good illustration of the important fact that the consumption of feed tends to increase the heat production of the body. This is an observation as old as the time of Lavoisier. That investigator observed the oxygen consumption of a man to increase materially (about 37 per cent) after a meal, and a multitude of subsequent experiments by numerous investigators and on various species of animals have fully confirmed these earlier results, so that the fact of an increased metabolism consequent on the ingestion of feed is fully established. It is especially to the investigations of Zuntz and his associates ${ }^{1}$ that the demonstration of this fact and the recognition of its significance in relation to the nutritive values of feeding stuffs is due.

For example, Zuntz and Hageman, ${ }^{2}$ on the average of a number of experiments in which the respiratory exchange of a horse shortly before feeding in the morning, shortly after feeding, and some hours later was determined by means of the Zuntz form of the Pettenkofer apparatus (299), obtained the following results, computed per kilogram live weight per minute.

Table 38. - Heat Production by a Horse

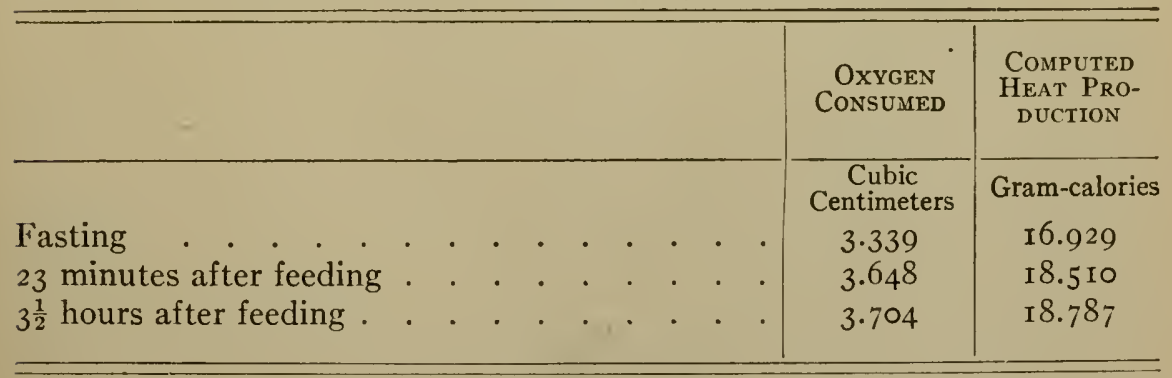

The same effect has been invariably observed with cattle in experiments by Armsby and Fries ${ }^{3}$ in which the heat production was determined directly by means of the respiration calorimeter. Thus in an

${ }^{1}$ Compare the writer's Principles of Animal Nutrition, pp. 377-385.

2 Landw. Jahrb., 27 (1898), Erganzbd. III, 282.

${ }^{3}$ Jour. Agr. Research, 3 (1915), 435. 
experiment in which three different amounts of alfalfa hay were fed to the same steer in different periods the results were as follows:-

Table 39. - Heat Production by a Steer

\begin{tabular}{ccccccccccccc}
\hline & & & & & & \\
\hline
\end{tabular}

Kellner's respiration experiments on fattening cattle have shown that the same effect is produced when feed is added to a basal ration. Thus when wheat gluten was added to a light fattening ration ${ }^{1}$ the heat production as calculated (329) from the balance of matter was as follows:

Table 40. - Heat Production by an Ox

Period I, Basal ration Period 4 , Same + gluten

For many years it was taught, in accordance with Rubner's theory of "isodynamic replacement," that with carnivora, and presumably with man, the nutrients were of value in proportion to their content of metabolizable energy. Rubner's own later investigations, ${ }^{2}$ however, as well as still more recent ones by Lusk and his associates $(367 e)$, have shown that what is true of the feeding stuffs consumed by horses and cattle is also true of nearly pure nutrients fed to dogs, viz., that if the experiment be made above the critical temperature for the animal there is in each case an increase in the heat production, so that the metabolizable energy is only partially available for maintenance. Thus the average of two of Rubner's experiments in which lean meat was fed gave the following results as compared with the fasting state:

1 Landw. Vers. Stat., 53 (1900), I30-I3I.

2 Die Gesetze des Energieverbrauchs bei der Ernährung, I902. 
Table 4i. - Heat Production by a Dog

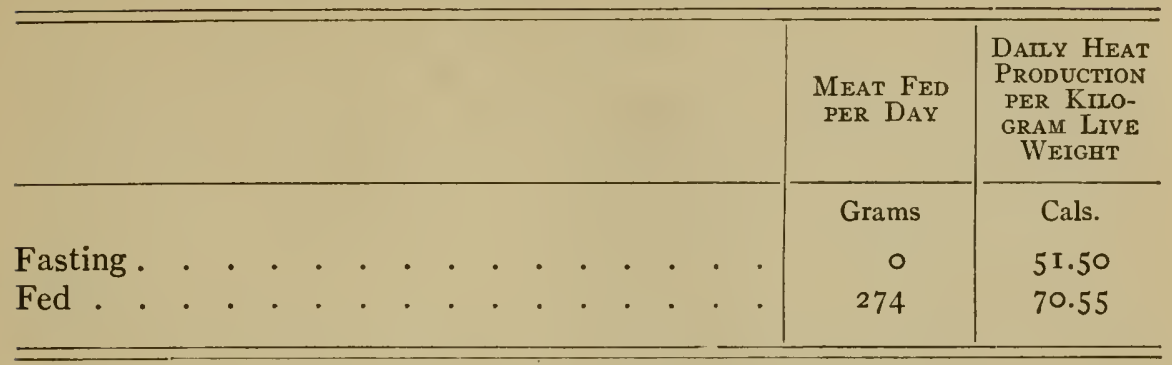

Proteins are especially efficient in stimulating the heat production but fats and carbohydrates produce the same effect although to a much less degree.

366. The specific dynamic action. - The effect of the various nutrients, notably of protein, in raising the heat production of the animal above the fasting level, as in the experiments just cited, has been called by Rubner their specific dynamic action. Kellner ${ }^{1}$ has proposed a different terminology. He divides the metabolizable energy of the feed into thermic and dynamic energy. Thermic energy, equivalent to Rubner's specific dynamic action, signifies that portion of the metabolizable energy which is of value to the organism only as a source of heat. Dynamic energy, equivalent to net energy as defined in a subsequent paragraph (370), on the other hand, is that portion of the metabolizable energy which can be utilized for the performance of the vital functions.

367. Causes of increased heat production. - The consumption of feed sets in operation (or increases) a variety of activities not manifested by the fasting organism.

a. Mechanical Work. - A not inconsiderable amount of muscular activity is expended by farm animals and especially by the herbivora in the prehension and mastication of their feed and in moving it through the alimentary canal. Since muscular work involves an expenditure of energy, all of which, in the case of internal work, finally takes the form of heat (342), the mechanical work of digestion is a considerable factor in increasing the metabolism of farm animals, although Armsby and Fries ${ }^{2}$ have presented reasons for believing that peristalsis

1 Ernährung landw. Nutzt., 6th Ed., p. ro5.

2 Jour. Agr. Research, 3 (19r5), 479. 
in cattle does not contribute very largely to the increased heat production consequent on the consumption of feed.

b. Glandular activity. - The increased metabolism required for the secretion of the digestive fluids and for the excretion of metabolic products is also to be reckoned among the causes of the heat production consequent on the ingestion of feed.

c. Fermentations. - The extensive fermentations, especially the methane fermentation, occurring in the digestive tract of herbivora $(128-130,132)$ result in a considerable evolution of heat. No entirely satisfactory determinations of its amount have yet been reported, but Von der Heide, Klein and Zuntz ${ }^{1}$ compute from Markoff's experiments that the methane fermentation in cattle gives rise to the evolution of 4.374 Cals. of heat per cubic centimeter of methane, equivalent to 6.07 Cals. per gram.

d. Intermediary metabolism. - The chemical changes which the nutrients undergo during digestion and resorption and especially in the intermediary metabolism (compare Chapters III and V) have been invoked to explain the increased heat production consequent on the consumption of feed, particularly of protein, but apparently without sufficient warrant, most of these reactions seeming to be substantially isothermic.

e. Direct stimulus to metabolism. - Recent investigations by Lusk and his associates ${ }^{2}$ upon the cause of the specific dynamic action, together with earlier experiments by Gigon, ${ }^{3}$ have gone far towards clearing up the subject. According to Lusk, the action of carbohydrates and fats is to be explained substantially as was done by C. Voit in I88I, viz., as the direct effect of a greater supply of non-nitrogenous material to the cells, i.e., as virtually a case of mass action. The products of protein katabolism, on the contrary, particularly the hydroxy and keto-acids resulting from the deaminization of the amino acids (233), act as direct stimuli to the katabolism of nonnitrogenous matter in the body cells.

That these actions play their part, along with mechanical work and fermentations, in bringing about the increased heat

1 Landw. Jahrb., 44 (I9r3), 795.

2 Jour. Biol. Chem., 12 (I9I 2), 349; 13 (I912), 27, I55, I85; 20 (I915), 555. Proc. Internat. Cong. Hygiene, I9I3. Arch. Inter. Medicine, 12 (I9I3), 485. Jour. Amer. Med. Asso., 63 (1914), 824.

${ }^{3}$ Skand. Arch. Physiol., 21 (I 909), 35r ; Arch. Physiol. (Pflüger), 140 (I9I I), 548. 
production resulting from the consumption of feed by herbivora cannot be doubted. Besides proteins, carbohydrates and fats, however, the feed of herbivora contains a great variety of other substances and the results upon steers obtained by Armsby and Fries ${ }^{1}$ seem to indicate that among these there may be compounds acting specifically as stimuli to the cell metabolism or to the minor muscular movements of the animal. Among the feeding stuffs examined, this appeared to be notably true of alfalfa hay and maize meal.

368. "Work of digestion." - The expenditure of energy by the body which results from the ingestion of feed has been somewhat loosely, and perhaps not altogether fortunately, designated as "work of digestion." While there may be objections to the term and while it must not be interpreted too literally, it may nevertheless serve a useful purpose as a collective expression for the energy cost to the organism of all the various processes involved in the digestion and assimilation of the feed. Its total amount is equal, of course, to the extra heat produced above that generated by the fasting animal. Rubner's specific dynamic action, or Kellner's thermal energy, is equivalent to the "work of digestion" in this broad meaning. The considerations presented in the previous paragraphs serve to indicate some of the factors of the "work of digestion" and render it evident that it is by no means all work in the mechanical sense. In herbivora this factor is an important one, while with man and carnivora it apparently plays a small part. A similar difference is strikingly shown in the case of the digestive fermentations, which are very extensive in ruminants but play a subordinate rôle in other animals.

369. Significance of expenditure of energy in feed consumption. - Whatever the part played by various factors in the increase of metabolism due to feed ingestion, the existence of that increase and the consequent augmented heat production is a fully established fact which has an important bearing upon the value of the feed as a source of energy.

Recurring once more to the comparison of the animal body with an internal combustion motor (274), if a gasoline engine has to obtain its supply of fuel by hoisting it from a lower level, it is evident that the energy spent in this way diminishes

1 Jour. Agr. Research, 3 (I915), 479. 
to just that extent the quantity of energy which the engine can deliver in other forms of work, so that the effect is virtually the same as if the energy content of the gasoline as delivered at the cylinder were diminished by the same amount.

In a precisely similar way, the energy expended in the so-called "work of digestion" and eliminated as heat does not serve the general purposes of the body. It cannot be said to be waste energy, like the chemical energy of the feces, for example, since part at least is expended for necessary purposes. The feed must be eaten and assimilated, just as the gasoline for the engine must be hoisted. The energy spent in so doing, however, constitutes virtually a deduction which must be made from the metabolizable energy of the feed in order to obtain the net amount of energy which it can contribute to the performance of the necessary internal work of the body (i.e., to its maintenance) or to such processes as the performance of external work or the storage of meat or fat.

370. Net energy values. - By means of balance experiments like that with a steer used as an illustration in a previous paragraph (364), the effect of a feeding stuff upon the heat production of an animal or the amount of energy which it contributes towards the maintenance of the body may be determined. The latter result has been called the net energy value of the substance because it shows the net result as regards energy obtained by its use. The net energy value of the hay in the illustration cited was $50_{2}$ Cals. per pound of dry matter. Net energy might be defined, therefore, as metabolizable energy minus the work of digestion, the latter term, of course, being understood in the very general sense already indicated as equivalent to the additional heat production caused by the consumption of the feed.

Stated in a slightly different way, the net energy value of a feeding stuff is the energy remaining after the losses of chemical energy in the various excreta and also the energy expended in the processes incident to the consumption of the material have been deducted from its gross energy. The amount of these deductions naturally varies as between different feeding stuffs. One containing much digestible matter, readily masticated and exerting little stimulating effect on the metabolic processes 
$(367 \mathrm{e})$, that is, causing little "work of digestion," will have a relatively high net energy value; while, on the other hand, materials of low digestibility, which undergo extensive fermentations, require much muscular work in their mastication and digestion, or stimulate the body metabolism, will have correspondingly low net energy values.

371. Net energy values for different purposes. - The net energy value of the same feeding stuff may differ according to the species of animal by which it is consumed and the purpose for which it is used.

The structure of the digestive organs of different species varies and, as is shown in Chapter XVI (713-717), specific differences in digestive capacity exist. In other words, the proportion of the gross energy of a feeding stuff which is lost in the feces differs as between different species, and therefore its net energy value tends to vary accordingly. Similarly, the extent to which bacterial fermentations occur in the digestive tract of an animal tends to influence the net energy value of its feed in two ways. The more extensive these fermentations, the less of the chemical energy of the feed is rejected in the feces but, on the other hand, the more chemical energy is given off in the combustible gases excreted or is transformed into heat in the process of fermentation and increases the "work of digestion." Finally, it appears not unlikely that the mechanical work required in mastication and digestion may vary as between different species.

The materials resorbed from maintenance or submaintenance rations may be regarded chiefly as fuel to be oxidized more or less directly, while in the fattening or growing animal a part of the digested nutrients is transformed into flesh or fat, or in the milking animal into butter fat, lactose, casein, etc. The net energy values for these purposes would evidently be equal to the amounts of energy contained in the gains made and might very well differ from the values for simple maintenance.

The net energy values of feeding stuffs for different species and for'the various purposes for which farm animals are kept, together with the methods for their determination or estimation, are discussed in Chapter XVII and the average results for a considerable number of feeding stuffs are tabulated in the Appendix. What is essential at this point is to acquire a clear idea of the general conception. 


\section{§ 2. The Maintenance Requirements of Farm Animals}

372. True maintenance and live weight maintenance. The maintenance of an animal in the strict sense signifies the preservation of the store of matter and of potential energy contained in the body, and only a ration which effects this is really a maintenance ration. As will appear in subsequent pages, however, much of the recorded information regarding the maintenance ration is derived from experiments in which the criterion of the sufficiency of the ration was its effect in maintaining the live weight of the animal. In experiments on mature animals and extending over a considerable period of time, it is unlikely that any gross error is involved, especially if determinations of the nitrogen balance show the protein supply to be adequate. In short periods, on the other hand, and especially in experiments on young animals, the live weight is a notoriously untrustworthy guide. The general reasons for this are familiar, but in young animals another very important factor enters into consideration. As is well known, the tendency to growth is one of the most marked characteristics of young animals. Waters ${ }^{1}$ has shown that this impulse to increase of tissue is so marked that it may apparently take precedence over the demand for maintenance, and that an animal may even maintain its weight and continue to increase in size of skeleton for a considerable time on a sub-maintenance ration.

Some ${ }_{5}$ immature cattle were fed for considerable periods on rations just sufficient to maintain their live weight. Under these conditions, the animals continued to grow in height, in depth of chest and length of head. At the same time, however, there was an evident falling off in the amount of fat tissue, both as judged by the eye and as shown by the appearance and by the chemical composition of the carcass. Histological studies, too, showed a reduction in the size of the fat cells and analyses of the adipose tissue showed a lower fat and higher water and protein content than in check animals. What occurred was evidently a consumption of body fat to supply energy, while at the same time an approximately equal weight of protein tissue was produced, which, on account of the relatively low energy value of protein and of the relatively large amount of water accompanying it, represented a much smaller quantity of energy than did the fat tissue which disappeared. In other words the rations were

${ }^{1}$ Soc. Prom. Agr. Sci., Proc. 29th Annual Meeting (Igo8), p. 7 I. 
not really but only apparently maintenance rations. It is perhaps hardly correct to say that in these experiments growth was maintained at the expense of the fat of the tissues. A more exact statement of the case would be that the increase of protein tissue and water masked the loss of fat. Presumably this effect would be less marked in more mature animals, in which the true maintenance and the live weight maintenance would doubtless approach each other closely when measured over long periods.

373. Methods of determining the maintenance requirement. - The most obvious method for determining the maintenance requirement of an animal is the method of trial. It consists of varying the amount of feed until constancy of live weight is attained or until a balance experiment shows equilibrium between income and outgo of matter and energy. This method, if extended over a considerable length of time, is particularly adapted to the determination of the live weight maintenance. When tested by the more refined method of the balance experiment, however, such a ration will only rarely and by accident be found to be exactly a true maintenance ration. Usually there will be revealed more or less gain or loss by the body for which a correction must be applied.

A second method consists of a comparison, like that used in a previous paragraph (364), to illustrate the determination of net energy values, between the effects of two different amounts of the same feeding stuff or ration upon the balance of energy. Such a comparison not only affords the means of computing the net energy value of the feed consumed but also serves to determine the energy requirement of the animal. The results in the case cited were as follows:-

Table 42. - Determination of Maintenance Requirement

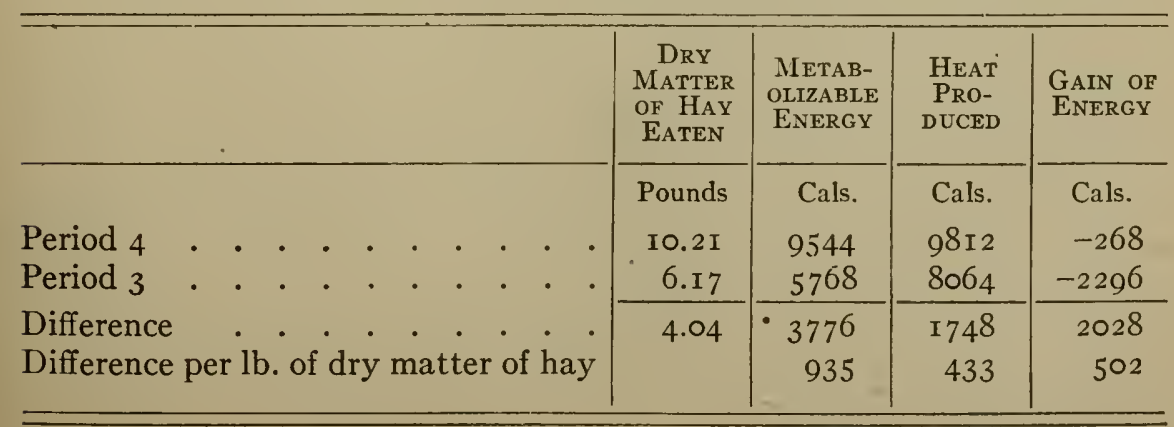


Each pound of dry matter of the hay decreased the loss of energy from the body by 502 Cals. The ration of 10.2 I lb. still permitted a loss from the animal of 268 Cals. To reduce this loss to zero would obviously require the addition of $268 \div 502$ $=0.53 \mathrm{lb}$. and an exact maintenance ration as regards energy would have been $10.2 \mathrm{I}+0.53=\mathrm{ro} .74$ pounds of the hay. In precisely similar fashion the metabolizable energy required for maintenance was

$$
9544+268 \times \frac{3776}{2028}=10042 \mathrm{Cal} .
$$

374. Computation of the fasting katabolism. - Another method of comparison, however, is of greater significance, since it affords results of more general value and also serves to bring out clearly the relations between the net energy values of feeding stuffs, the fasting katabolism and the maintenance requirement.

In the foregoing experiments each pound of hay withdrawn from the ration caused the heat production to decrease by 433 Cals. If, then, all the hay were withdrawn from Period 3 and the animal reduced to the fasting state, the heat production, or in other words the fasting katabolism, would be

$$
8064-(433 \times 6.17)=5392 \text { Cals. }
$$

The same result may also be computed from the losses of energy suffered by the animal. The withdrawal of each pound of hay increased this loss by 502 Cals. The withdrawal of all the 6.I7 pounds of Period 3, therefore, would increase the loss by $502 \times 6.17=3096$ Cals., making a total loss of 5392 Cals., equal to the fasting katabolism. In other words, by such a comparison as the foregoing it is possible to determine indirectly the fasting katabolism, which it is scarcely practicable to determine directly.

It was shown in Chapter VII (344), however, that the fasting katabolism is the measure of the maintenance requirement. To maintain the steer of this illustration it would be necessary to supply in his feed an amount of energy, after deducting the losses in the excreta (i.e., an amount of metabolizable energy), equal to the fasting katabolism, 5372 Cals., plus a sufficient additional amount to offset the additional heat production which the consumption of the feed would inevitably occasion, i.e., the work of digestion. 
But the difference between the metabolizable energy and the work of digestion is the net energy (370). Consequently the foregoing statement is equivalent to saying that the maintenance requirement of the steer was 5392 Cals. of net energy. Each pound of this particular hay had a net energy value of 502 Cals. To maintain the animal, therefore, there would be required $5392 \div 502=$ I0.74 lb., as previously computed.

375. Manner of stating the maintenance requirement. Evidently the maintenance requirement of an animal, such as the steer of the foregoing illustration, may be stated in a variety of ways - in terms of weight of feed, of amounts of digestible nutrients, of metabolizable energy or of net energy. So far as the results of a single experiment are concerned, it makes little difference which manner of statement is adopted, since they are all simply different ways of expressing the same facts. When it is desired to make general statements, however, there are very manifest advantages in stating the maintenance ration in terms of net energy.

It was shown in Chapter VII (343) that the fasting katabolism might be regarded as practically constant under uniform conditions. Consequently the net energy requirement for maintenance is equally constant, and in the foregoing example any ration having a net energy value of 5392 Cals. would have been a maintenance ration.

But since the net energy values of different feeding stuffs, as well as the proportion of their metabolizable energy which can be utilized for maintenance, may vary through a considerable range, the weight of feed or the amount of metabolizable energy which will suffice to maintain an animal will vary with the kind of material fed. For example, it is shown in subsequent paragraphs $(380,381)$ that a thousand-pound steer requires about 6.o Therms of net energy for maintenance. From the results of Armsby and Fries' determinations of net energy values (760), it is easy to compute that to supply this amount in timothy hay with a net energy value of 48.63 Therms per 100 pounds of dry matter would require $6.0 \div 0.4863=\mathrm{I} 2.34$ pounds of dry matter, but that if mixed hay with a net energy value of 43.37 Therms per rooo pounds were used, the amount of dry matter necessary would be $6.0 \div 0.4337=13.83$ pounds. The quantities of metabolizable energy contained in these main- 
tenance rations would likewise be different, as is shown in the following statement, in which are included for further illustration two mixed rations used by the same experimenters.

Table 43. - Examples of Maintenance Rations for a iooo-Pound STEER

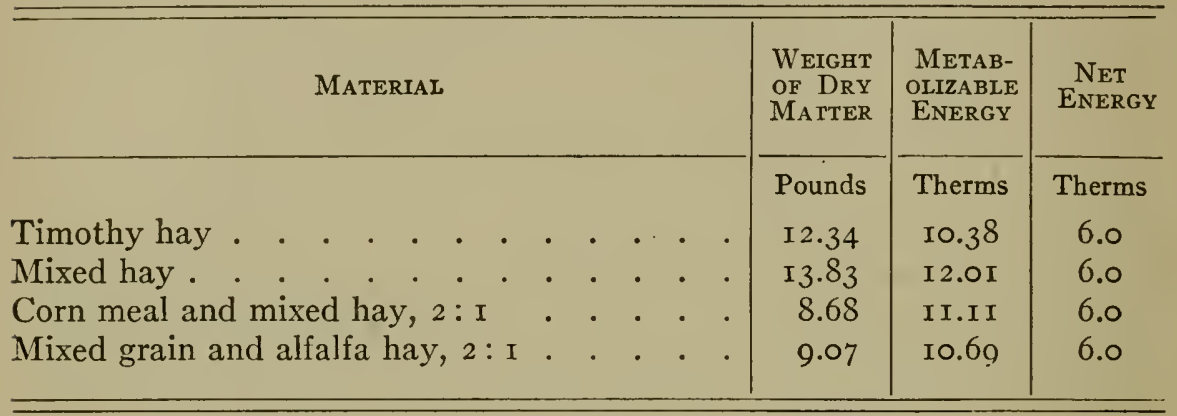

By stating the maintenance requirement in terms of net energy a single value is obtained for an animal, or a single average for a class of animals, which is a general expression of its maintenance requirement irrespective (substantially) of the particular feed or feeds which may be used to satisfy it, while a statement in terms of metabolizable energy or of weight of feed must also specify the particular kind of feed to which it applies. The greater convenience of the former method for the computation of actual rations is evident. To the extent to which the net energy values of feeding stuffs are known or can be estimated it is possible to make up an almost endless variety of combinations which will all be maintenance rations, i.e., will furnish the amount of net energy required by the animal.

In the following paragraphs, this method of expression will be followed so far as practicable, although unfortunately comparatively few determinations of the net energy requirements for maintenance have yet been reported except in the case of cattle.

376. Modified conception of energy requirement.-A study of the conditions, especially as regards muscular work, which influence the katabolism of the fasting animal makes it evident that the conception of the energy requirement outlined in Chapter VII requires some modification in its application to the actual feeding of animals.

As was there shown (344), the heat production of the fasting animal in a state of absolute muscular rest may be regarded as 
measuring the quantity of energy indispensable for its internal work. As a matter of fact, however, absolute muscular rest cannot be maintained for any considerable length of time, at least during the waking hours, even by voluntary effort. The horse or ox when at rest in the ordinary sense, i.e., when doing no external work, is still expending a not inconsiderable amount of energy in muscular activities of various sorts, some of which were indicated in $\S_{3}$ of the same chapter (348). In particular, it was stated (349) that standing as compared with lying causes a very marked increase in the heat production, especially in the case of cattle. When, therefore, the heat production of such an animal in the fasting state is taken as a measure of the energy required for its maintenance, it does not represent a state of absolute rest but simply with one of relatively less activity. The energy requirement for maintenance in the economic sense includes not only the absolute minimum required for the internal work but also the amount expended in various forms of incidental muscular work which are in a sense unnecessary physiologically but are unavoidable practically. Moreover, since the amount of this incidental work is more or less variable as between different individuals and in the same individual at different times, the energy requirement for maintenance is not a fixed, constant quantity whose exact value can be determined, but a variable one. The purpose of investigation is to show the range of variation which may be expected and to determine a general average value for the conditions of ordinary practice.

\section{The maintenance requirement of swine}

377. Net energy requirement. - With animals such as man, carnivora or swine, having a comparatively simple digestive apparatus and consuming relatively concentrated feed, the fasting energy expenditure can be determined without special difficulty by depriving the resting animal of feed during a relatively short period and measuring the katabolism with the aid of a respiration apparatus or calorimeter. The total amount of heat produced, determined either directly or by calculation, furnishes the measure of the energy expenditure and therefore of the net energy requirement for maintenance. 
Such an experiment must, of course, be made at a temperature above the critical temperature (354) for the animal, since otherwise the heat produced would be greater than that corresponding to the necessary internal work by the additional amount necessary to maintain the body temperature.

Numerous determinations of the fasting katabolism of man and of the smaller animals, such as the dog, cat, rabbit, guinea pig, etc., are on record, but the only experiments of this sort upon farm animals are those of Meissl, Strohmer and Lorenz ${ }^{1}$ and of Tangl ${ }^{2}$ upon swine.

Meissl's determinations were made at about $20^{\circ} \mathrm{C}$, a tem- " perature which, according to Tangl's later results, is well above the critical temperature for mature swine. In Tangl's experiments the animals spent most of the time lying; in Meissl's paper no statements are made on this point.

Excluding those of Tangl's experiments which were apparently below the critical temperature, the results, computed per too pounds in proportion to the two-thirds power of the live weight, were as follows: -

Table 44. - Net Energy for Matntenance of Swine

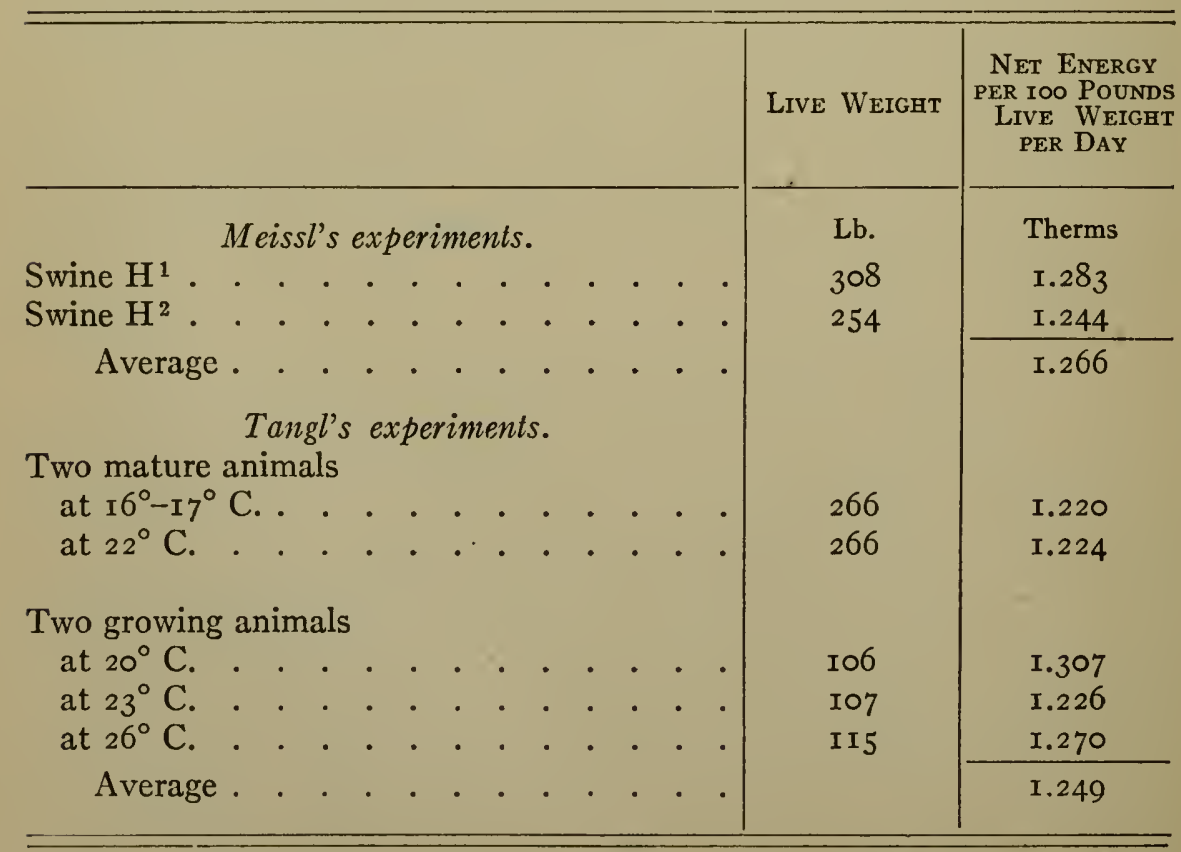

1 Ztschr. Biol., 22 (1886), 63.

2 Biochem. Ztschr., 44 (I9I 2 ), 252. 
From the foregoing results it appears that the average daily energy expenditure of fasting swine at rest and above the critical temperature is about $\mathrm{r} .25$ Therms per Ioo pounds live weight, and consequently that a maintenance ration must supply this amount of net energy. Assuming a value of 9.02 for the constant $k$ of Meeh's formula (346), this average is equivalent to I.089 Therms per square meter of body surface. Using an entirely different experimental method, Fingerling, Köhler and Reinhardt ${ }^{1}$ have computed the average energy requirement for maintenance of two growing pigs at almost the same amount, viz., I.045 Therms per square meter.

378. Metabolizable energy in maintenance rations. Unfortunately, few determinations of the net energy values of feeding stuffs for swine have been reported (761) and most of the data regarding the maintenance requirement of this species are expressed in terms of digestible matter or of computed metabolizable energy. The metabolizable energy contained in actual maintenance rations of swine has been determined in a single respiration experiment by Von der Heide and Klein ${ }^{2}$ and may be estimated more or less accurately in a number of live weight experiments. Such experiments have been reported by Taylor, ${ }^{3}$ Carlyle, ${ }^{4}$ Ostertag and Zuntz ${ }^{5}$ and Dietrich. ${ }^{6}$ The results show a very wide range, from a minimum of 0.897 . Therm per 100 pounds live weight for 50-pound pigs on a ration of one part meal and 4 parts skim milk to a maximum of $2.55^{8}$ Therms for roo-pound pigs on a ration of shorts, corn meal and oil meal. For this there may be a variety of reasons. Live weight results are notoriously uncertain (281283), and in growing animals especially the possibility of a maintenance of live weight by a substitution of water for fat (372) has to be borne in mind. The feeds used, too, were varied, and there seem to be indications that, in some cases at least, a smaller "work of digestion," especially in the case of rations containing much milk, may have contributed to reduce the amount of metabolizable energy necessary for maintenance.

The averages computed from all the experiments and those obtained by the omission of a few extreme results are as follows :-

1 Landw. Vers. Stat., 84 (I9r4), 149.

${ }^{3}$ Wis. Expt. Sta., Rpt. I90I, p. 67.

${ }^{6}$ Landw. Jahrb., 37 (I908), 226.
2 Biochem. Ztschr., 55 (r9r3), I95.

${ }^{4}$ Ibid., Bul. 104 (I903), p. 3I.

${ }^{6}$ Ills. Expt. Sta., Bul. I63 (I9I3). 


\section{Table 45. - Daily Maintenance Rations of Swine}

Metabolizable energy per 1oo lb. live weight

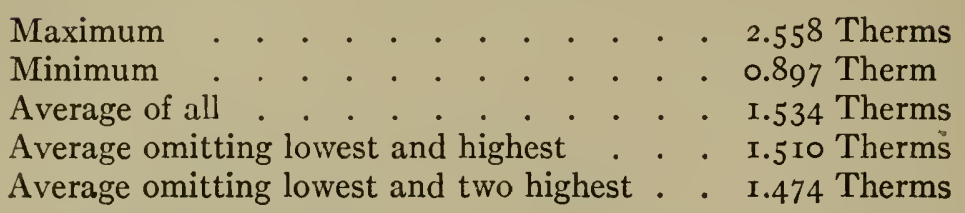

379. Comparison with net energy. - On the average of all the respiration experiments on fattening swine which are recorded in Chapter XVII (761), 78.I4 per cent of the metabolizable energy supplied may be computed to have been utilized for maintenance plus gain. If this may be assumed to represent approximately the percentage of the metabolizable energy available for maintenance, the foregoing maintenance rations contained, per Ioo pounds live weight, the following amounts of net energy:-

Table 46. - Daily Maintenance Rations of Swine

Computed net energy per roo lb. live weight

Minimum
Maximum
Average of all

The averarge requirement of net energy as thus computed does not differ greatly from the amount indicated by the experiments on fasting animals (377), but the enormous range in the results of the single experiments shows in a striking manner the need for further investigation.

\section{The maintenance requirement of cattle}

380. Net energy requirement. - In the case of ruminants, it is hardly practicable to determine directly the net energy requirement by measuring the katabolism of the fasting animal. Prolonged fasting would be required to free the voluminous and complicated digestive organs of these animals from feed residues, if this could be accomplished at all; and it would be difficult to determine when that point was reached, while it is questionable whether the results on such an animal could be regarded as normal. 
Table 47. - Net Energy Requirement for Maintenance of Cattle Corrected to I 2 hours standing

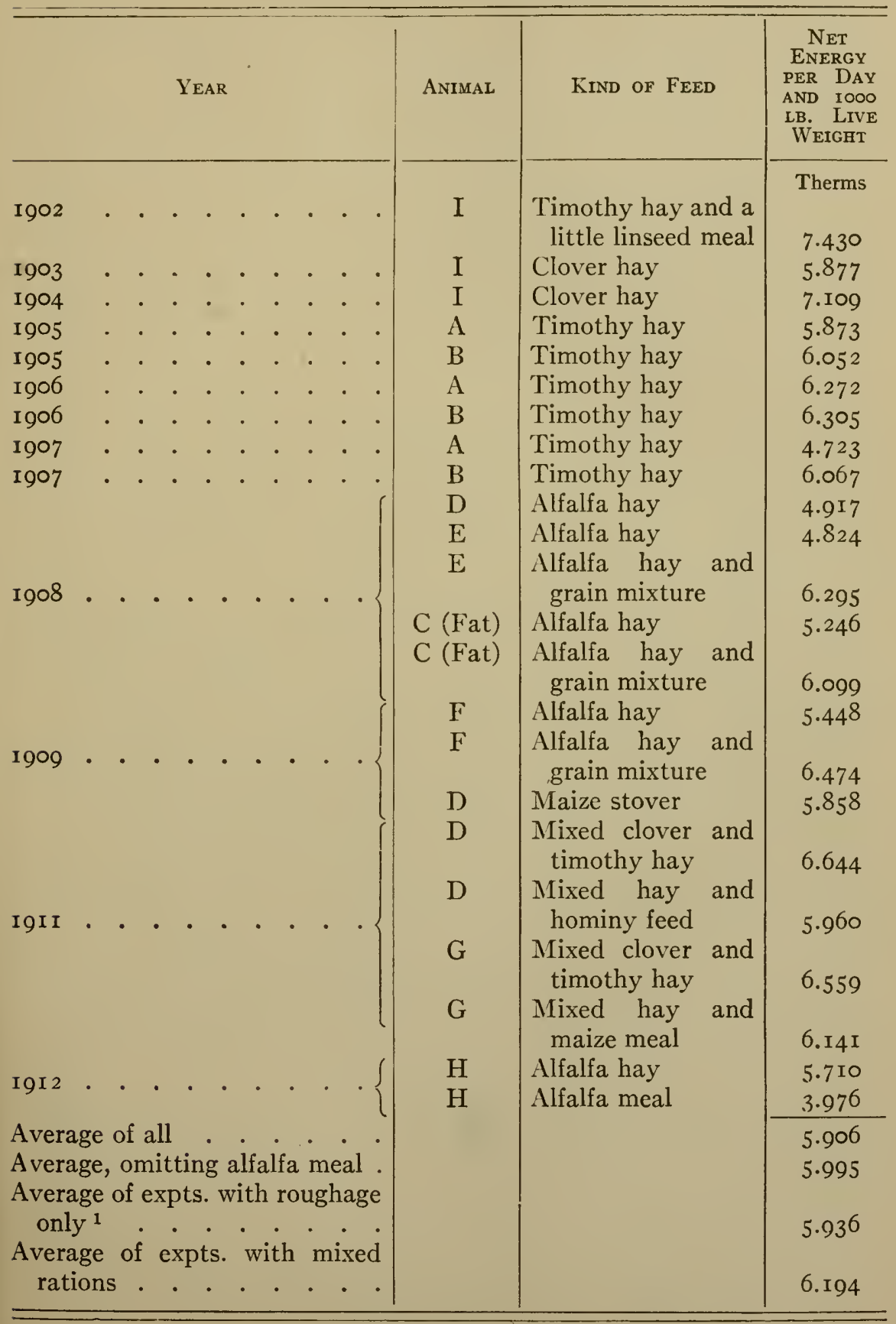

1 Omitting experiment on alfalfa meal. 
The fasting katabolism of such an animal may, however, be computed in the manner already described (374) from a comparison of two periods on different amounts of the same feed, or ration, both being less than that necessary for maintenance. Twenty-three experiments of this sort, on nine different steers, only one of which was fat, in which the relative metabolism of the animals when standing and when lying was determined, have been made by Armsby and Fries. ${ }^{1}$ Computed per rooo lb., in proportion to the two-thirds power of the live weight (347) and corrected ${ }^{2}$ to a uniform period of I $2_{2}$ hours standing out of the 24 , the net energy requirements were as shown in Table 47. No other experiments on precisely this plan have yet been reported.

Even if a few seemingly extreme results, like those of 1902 and I9I 2 be excluded, the figures show a wide range. The trials with mixed rations of roughage and concentrates show on the whole somewhat higher results than those with roughage only, but the experiments are hardly numerous enough to show whether this difference is significant.

381. Net energy in maintenance rations. - A considerable number of earlier experiments are also on record in which the amounts of net energy contained in actual maintenance rations of cattle may be computed with more or less accuracy.

The early experiments of Henneberg and Stohmann, on which was based Wolff's feeding standard for maintenance long current, as well as a considerable number of subsequent ones, ${ }^{3}$ have now chiefly an historic interest. Of the later investigations, by far the most important are those by G. Kühn and by Kellner ${ }^{4}$ in which approximate maintenance rations were fed. The small gains or losses of protein and fat by the animals were determined by means of a Pettenkofer respiration apparatus and corrected for upon the basis of results obtained in other respiration experiments on productive rations, and in this way the metabolizable energy required for maintenance was computed.

${ }^{1}$ Eight of them have been reported. U. S. Dept. Agr., Bur. Anim. Indus., Buls. 74, ror, and I 28 .

2 In the manner described in Jour. Agr. Research, 3 (I9I5), 454.

${ }^{3}$ Compare Penna. Expt. Sta., Bul. 42 (1898), pp. 8-2I.

4 Reported by Kellner: Landw. Vers. Sta., 53 (r900), pp. 6-16, 
In addition to these respiration experiments, investigations upon the live weight maintenance of cattle made by the writer, ${ }^{1}$ by Haecker, ${ }^{2}$ and by Evvard ${ }^{3}$ have been discussed elsewhere ${ }^{4}$ by the writer.

Table 48. - Net Energy in Daily Maintenance Rations of Cattle'

\begin{tabular}{|c|c|c|c|c|c|c|}
\hline \multirow{2}{*}{ 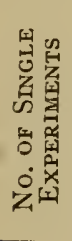 } & & \multirow{2}{*}{$\begin{array}{c}\text { Character of } \\
\text { FEED }\end{array}$} & \multirow{2}{*}{$\begin{array}{l}\text { Condition } \\
\text { OF ANIMALS }\end{array}$} & \multicolumn{3}{|c|}{$\begin{array}{l}\text { PER IOoo Pounds } \\
\text { LIVE WEIGHT }\end{array}$} \\
\hline & & & & 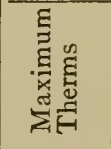 & 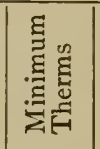 & 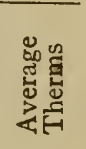 \\
\hline & $\begin{array}{l}\text { Respiration } \\
\text { experiments }\end{array}$ & & & & & \\
\hline I7 & Armsby and Fries . & Roughage 5 & Medium & 7.430 & 4.723 & 5.936 \\
\hline 5 & Armsby and Fries. & Mixed rations ${ }^{5}$ & Medium & 6.474 & $5.960 \mid$ & 6.194 \\
\hline 22 & Armsby and Fries. & Average of all ${ }^{5}$ & Medium & 7.430 & 4.723 & 5.995 \\
\hline 7 & Kellner . . . & Roughage & Medium & 6.780 & $4.92 \mathrm{I}$ & $5.74^{2}$ \\
\hline 29 & Average $^{6}$. & & & & & 5.934 \\
\hline & $\begin{array}{l}\text { Kellner . } \\
\text { Live weight } \\
\text { experiments }\end{array}$ & Mixed rations & Fat & $8.87 \mathrm{I}$ & 7.319 & 7.946 \\
\hline IO & Armsby . . . & (Hay only) & Thin & 7.044 & 6.136 & 6.505 \\
\hline 3 & Armsby . & (Mixed rations) & Thin & 6.039 & $4.7 \mathrm{I} 3$ & 5.423 \\
\hline 6 & Haecker . . & & Medium & 5.676 & 4.662 & $5.02 \mathrm{I}$ \\
\hline 3 & $\begin{array}{l}\text { Evvard, Ist 6o-day } \\
\text { expt. } \\
\text { Evvard, } \dot{3}_{32-\text { day }} \cdot\end{array}$ & Mixed rations & Medium & 7.850 & 6.450 & 7.18 \\
\hline & expt. . . . & Mixed rations & Medium & 一 & 一 & 8.090 \\
\hline 7 & $\begin{array}{c}\text { Eckles } \\
\text { Average }^{6}\end{array}$ & Mixed rations & Medium & 7.079 & $5.84 \mathrm{I}$ & $\frac{6.173}{6.18 I}$ \\
\hline 3 & $\begin{array}{c}\text { Evvard, 2d 6o-day } \\
\text { experiment }\end{array}$ & Mixed rations & $\begin{array}{c}\text { Partly } \\
\text { fattened }\end{array}$ & 10.620 & $8.150 \mid$ & 9.070 \\
\hline
\end{tabular}

For the purpose of computing the approximate net energy values of these rations it seems permissible to assume provisionally that the same proportion of their metabolizable energy

${ }^{1}$ Penna. Expt. Sta., Bul. 42 (I898).

${ }^{2}$ Minn. Expt. Sta., Bul. 79.

3 Thesis for degree of M. S., University of Missouri, Igog.

${ }^{4}$ U. S. Dept. Agr., Bur. Anim. Indus., Bul. I 43, 44-46.

${ }^{5}$ Omitting the experiment on alfalfa meal.

${ }^{6}$ Giving each experiment equal weight. 
was available for maintenance as in the case of the hays exclusive of alfalfa investigated by Armsby and Fries, ${ }^{1}$ viz., 52.8 per cent. For the mixed rations a percentage of 55 has been assumed. In Evvard's experiments the net energy was computed by that investigator. Eckles ${ }^{2}$ has also reported five determinations of the live weight maintenance of dry cows in which the net energy values of the mixed rations consumed were estimated from the writer's computed averages. ${ }^{3}$

The results of the computations are shown in Table 48 , Armsby and Fries' determinations of the net energy requirement being included for comparison.

For the medium and thin animals, the estimated net energy of Kellner's maintenance rations is distinctly less than the average maintenance requirement found in Armsby and Fries' experiments. The mean of the individual results of the two experimenters, on 16 different animals, is 5934 Cals. The average estimated net energy in the maintenance rations of the live weight experiments is somewhat greater, viz., 6r8r Cals., although if the one apparently exceptional result obtained by - Evvard be omitted, the average is reduced to 6riz Cals. The maintenance requirement of fat cattle is evidently distinctly greater than that in the unfattened state but the data are too few to permit the statement of a trustworthy average.

It appears, then, that the maintenance ration of mature cattle in thin to medium condition must supply, on the average, about 6000 Cals. of net energy per thousand pounds live weight, although with considerable variations from this average in individual cases. That the actual weight of feeding stuffs required to constitute a maintenance ration, as well as the quantities of metabolizable energy contained in it, will vary with the kinds of feeds used has already been pointed out (375) and is indeed sufficiently obvious.

\section{The maintenance requirement of sheep}

382. Metabolizable energy in maintenance rations. - Data regarding the maintenance ration of sheep are much less abundant than those for cattle and no experiments have been re-

${ }^{1}$ Jour. Agr. Research, 3 (I9I 5), 484-485.

2 Mo. Expt. Sta., Research Bul. 7, p. I 20.

3 U. S. Dept. Agr., Farmers' Bul. 346, p. 15. 
ported in which the net energy required for maintenance, i.e., the fasting katabolism, has been determined.

Respiration experiments upon sheep have been made by

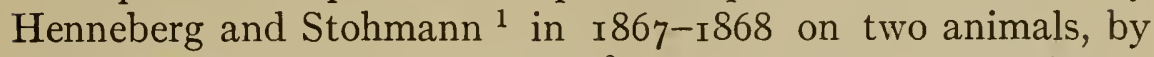
Henneberg, Fleisher and Müller ${ }^{2}$ in 1872 upon two animals, by Hagemann ${ }^{3}$ in 1899 on one animal, and by Kellner ${ }^{4}$ upon one animal. With the exception of the third, these were balance experiments with a Pettenkofer apparatus and included no direct determinations of energy so far as reported. The third investigation comprised a digestion and metabolism experiment in which the energy of the feed and the visible excreta was determined directly and also 42 determinations of the pulmonary respiration with the Zuntz type of apparatus. (299).

In addition to the foregoing respiration experiments there are a number of digestion experiments by Wolff in which the live weight of the animals was approximately maintained, ${ }^{5}$ and Henry ${ }^{6}$ reports a series of experiments by Carlyle and Kleinheinz with breeding ewes in which various mixed rations produced an average daily gain of 0.16 pound per head in animals averaging $\mathrm{I} 45$ pounds in weight.

In these experiments the metabolizable energy of the rations may be computed approximately from the digestible organic matter in the manner described in Chapter XVII (774). In the respiration experiments a correction for the gain or loss by the animal may be made as in the case of Kellner's experiments on cattle (381), while an approximate correction for the gain in the live weight experiments may also be made.

The results, computed per soo lb. live weight, are shown in Table 49 .

383. Net energy in maintenance rations of sheep. - As already stated, no direct determinations of the net energy required for the maintenance of sheep are on record and only unsatisfactory data are available for computing it from the metabolizable energy of maintenance rations. For the fore-

1 Neue Beiträge, etc., pp. 68-286.

2 Jahresber. Agr. Chem., 16-17 (I873-74), II, I45.

3 Arch. (Anat. u.) Physiol.; I899, Suppl., p. I38.

${ }^{4}$ Die Ernährung. landw. Nutzt., 6th Ed., p. 422.

${ }^{5}$ Compare U. S. Dept. of Agr., Bur. Anim. Indus., Bul. I 43 (I9I2), pp. 49-5I.

6 Feeds and Feeding, roth Ed., p. 482. 
going experiments, however, it may be permissible to assume, as in the case of cattle, that about 52.8 per cent of the metabolizable energy of roughage and 55 per cent of that of mixed rations was available for maintenance. The results of a computation upon this basis are contained in the last column of the following table. They possess a certain degree of interest, although obviously they are of uncertain value.

Table 49. - Energy in Daily Maintenance Rations of Sheep

\begin{tabular}{|c|c|c|c|c|c|c|c|c|c|c|c|c|c|c|}
\hline & & & & & & & & & & & & \multicolumn{3}{|c|}{ Per rooLb. Live Weight } \\
\hline & & & & & & & & & & & & & $\begin{array}{c}\text { Metaboliz- } \\
\text { able } \\
\text { Energy }\end{array}$ & $\begin{array}{c}\text { Net } \\
\text { Energy }\end{array}$ \\
\hline \multicolumn{13}{|c|}{ Respiration experiments } & Therms & Therms \\
\hline Henneberg and S & toh & mar & & & & & . & - & - & . & & & I. 475 & .779 \\
\hline Henneberg, Fleisc & che & $\mathrm{r}$ an & ad 1 & Mü & iller & & • & . & . & . & . & 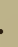 & 1.420 & $.78 \mathrm{I}$ \\
\hline Kellner . . . & . & . & . & . & . $\quad$. & • & - & • & - & . & $\cdot$ & & I. I IO & $.6 \mathrm{II}$ \\
\hline Hagemann . . & · & . & . & - & - & • & - & • & 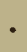 & . & - & • & 1.282 & .705 \\
\hline Average . . & . & . & . & . & . & • & . & - & - & $\cdot$ & ${ }^{\circ}$ & & I.322 & .719 \\
\hline \multicolumn{15}{|c|}{ Live weight experiments } \\
\hline Wolff, I87x, 6 exI & peri & imer & & & . & - & 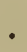 & . & . & - & & & I. 634 & .863 \\
\hline Wolff, r892-I 893 &, 8 & $\exp$ & erin & mer & nts & & . & . & . & . & . & & $\mathrm{I} .725$ & .863 \\
\hline Carlyle and Klier & nhe & inz & & 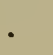 & . & & . & 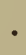 & . & 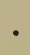 & 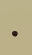 & & 1.513 & .832 \\
\hline Average - & . & . & . & . & . & 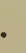 & - & • & - & - & . & & I. 624 & .853 \\
\hline Average of all & . & . & . & . & . & . & . & . & . & . & . & & I. 368 & .791 \\
\hline
\end{tabular}

384. Comparison of sheep with cattle. - It is of some interest to compare the maintenance requirement of sheep with that of cattle. Since the sheep is a much smaller animal than the steer it naturally requires relatively more feed in proportion to its weight (345), as the foregoing figures show to be the case. As compared with a steer weighing rooo pounds, ten sheep weighing roo pounds each would require for maintenance, according to the foregoing estimates, about 30 per cent more energy. If, however, the comparison be made in proportion to the twothirds power of the live weight, i.e., substantially in proportion to the body surface (347), a very different result is reached, the maintenance ration of the sheep as thus computed amounting to little more than 60 per cent of that of cattle. 
Table 50. - Maintenance Requirements of Cattle and Sheep. Computed in Proportion to Body Surface

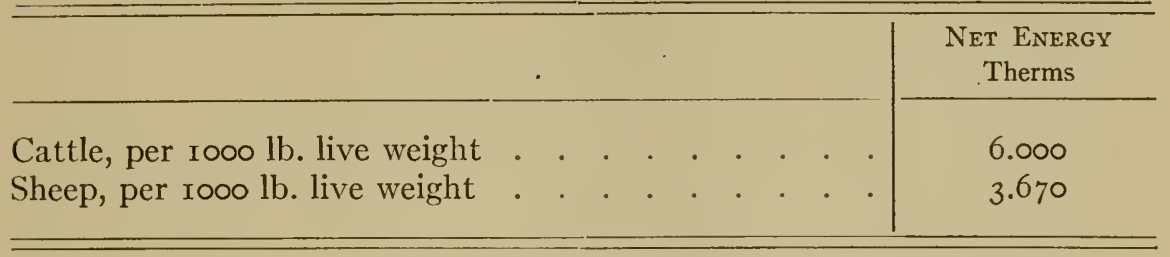

While such a comparison is of course but a rough approximation, it nevertheless seems to show conclusively that the metabolism of the sheep per unit of surface is distinctly lower than that of cattle, so that this animal apparently constitutes an exception to the results computed by E. Voit (345) for several other species. The thick coat of wool, of course, tends to reduce the rate at which heat is lost from the body and it seems at least a plausible conjecture that in the course of organic evolution the intensity of metabolism and the rate of heat radiation may have undergone mutual adjustment.

\section{The maintenance requirement of the horse}

385. Net energy requirement. - Zuntz and Hagemann ${ }^{1}$ have computed the fasting katabolism of the horse from the results of numerous short respiration experiments with the Zuntz type of apparatus (299) by means of a comparison identical in principle with that already described for cattle (374) but carried out by quite a different method, involving numerous estimates and computations.

They assume, on the basis of experiments on man, that 9 per cent of the metabolizable energy of the digestible nutrients consumed by a horse is converted into heat in the process of digestion, and compute from their own experiments that each gram of total crude fiber consumed increases the heat production by 2.086 Cals. additional, exclusive of that due to mastication. For the purpose of computing the fasting energy expenditure, those rest experiments on Horse III in which the feed consisted of oats, hay and straw, are used. On the basis of a number of short respiration experiments made within the first five hours after feeding, the total energy metabolism per day

${ }^{1}$ Landw. Jahrb., 27 (1898), Ergzbd, III, 27I-285 and 422-425. 
on the various rations is computed from the results of five previous balance experiments on similar rations by combining them in various ratios according to the proportions of oats, hay and straw consumed. From this is subtracted the heat computed to have been produced in the digestion of the feed (not including the work of mastication), the remainder showing, of course, the katabolism due to internal work, i.e., the net energy requirement. ${ }^{1}$

Table $5 \mathrm{I}$ shows for the eight experiments compared the total estimated heat production per day, the computed energy expenditure caused by the consumption of the feed, and by difference the energy expenditure in the fasting state, i.e., the net energy requirement for maintenance.

Table 51. - Net Energy Requirement for Maintenance of Horse

\begin{tabular}{|c|c|c|c|c|c|c|c|c|c|}
\hline \multirow[b]{2}{*}{ PERIODS } & \multirow[b]{2}{*}{ 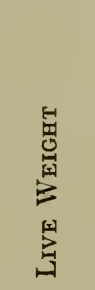 } & \multirow{2}{*}{ 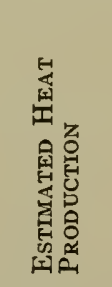 } & \multicolumn{3}{|c|}{ FEed } & \multirow{2}{*}{ 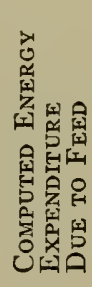 } & \multicolumn{2}{|c|}{$\begin{array}{c}\text { ENERGY } \\
\text { EXPENDITURE } \\
\text { IN FASTING }\end{array}$} & \multirow[b]{2}{*}{ SEASON } \\
\hline & & & $\stackrel{n}{\tilde{J}}$ & 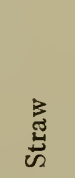 & 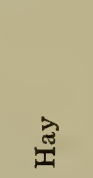 & & 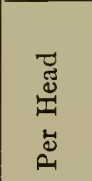 & 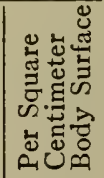 & \\
\hline & Kgs. & Cals. & Kgs. & Kgs. & Kgs. & Cals. & Cals. & Cals. & \\
\hline$a$ & 428. I & $\mathrm{I} 2,54 \mathrm{I}$ & 6 & I & 7 & 8403 & 4138 & 80.7 & Winter \\
\hline$b$ & 434.1 & I 1,674 & 6 & I & 6 & 7704 & 3970 & 76.7 & Summer \\
\hline$e$ & 450.4 & 12,364 & 6 & I & 6 & 7704 & 4660 & 87.9 & Winter \\
\hline$f$ & 449. I & I 1,783 & 6 & $\mathbf{I}$ & $4 \cdot 75$ & 6830 & 4953 & 93.6 & Summer \\
\hline$i$ & 440.1 & I I, 893 & 6 & I & 6 & 7704 & $4 \times 89$ & 80.2 & Winter \\
\hline$n$ & 448.2 & I I, 407 & 4.8 & 0 & $5 \cdot I$ & 5672 & 5735 & 108.5 & Summer \\
\hline$c$ & 442.2 & $12,45^{\circ}$ & 0 & 0 & 10.5 & 7340 & 5 I 10 & 97.6 & Summer \\
\hline No. I I $8 c$ & 434.6 & I I , O 2 I & 4.8 & 0.8 & I. 88 & 4I 22 & 6899 & I 33.3 & Winter \\
\hline
\end{tabular}

In the experiments with a standard ration of $6 \mathrm{kgs}$. of oats, one of straw, and six (or seven) of hay, the average computed fasting katabolism per day in three winter periods was 4.33 Therms, while in a single summer period it reaches the minimum of 3.97 Therms per head, or 4.08 Therms per rooo pounds live weight. Zuntz and Hagemann consider that the latter amount represents approximately the minimum requirement for the in-

${ }^{1}$ For a more complete account of the method, compare the writer's Principles of Animal Nutrition, pp. 386-387. 
ternal work and regard the higher figures obtained in the winter experiments as indicating a stimulation of the heat production by the low temperature to which the animal was exposed; i.e., they consider that the experiments were made below the critical temperature. The notably higher results obtained with lighter rations they ascribe to a similar cause, viz., that the heat arising from the work of digestion, together with that due to the necessary internal work (fasting katabolism), was insufficient to maintain the body temperature.

It must be confessed that, in view of the more active, temperament of the horse as compared with cattle this relatively low figure for the fasting katabolism is rather surprising, and the fact should not be overlooked that it is derived from short periods in which it is probable that the animal was unusually quiet. It perhaps represents more nearly the physiological than the economic minimum of net energy required for maintenance, and it would be of much interest to compare it with the results of 24 -hour experiments.

386. Metabolizable energy in maintenance rations. - A considerable number of experiments are on record in which the amount of total digestible matter required for the maintenance of the horse has been determined.

The maintenance rations of cattle and sheep may be determined with a good degree of accuracy by varying the quantity of feed given until equality between income and outgo or constancy of live weight is attained, but this method is not fully applicable to the horse. Owing to his more active temperament, feed seems to exert a greater stimulus upon his muscular activity than is the case with the more phlegmatic ruminants, so that a considerable excess over an actual maintenance ration may be consumed by a horse and expended in the various minor activities noted in Chapter VII (348), while the balance of income and outgo may show neither gain nor loss, i.e., may appear to show that the ration is a maintenance ration.

a. Wolff's determinations. - One method of avoiding this difficulty and determining the true maintenance ration is that employed by Wolff in his extensive investigations ${ }^{1}$ upon work production by the horse $(670,779)$. In these experiments the

${ }^{1}$ Compare the writer's Principles of Animal Nutrition, pp. 53I-535. 
horse performed a measured amount of work ${ }^{1}$ which was so adjusted in different periods as to be as nearly as possible in equilibrium with the feed consumed. This was considered to be the case when the live weight of the animal remained substantially unchanged for a considerable period and when the urinary nitrogen did not show an increase as a consequence of the additional work done (637).. By comparing the work performed on a basal ration with that which could be done with a heavier one, the ratio of the work done to the additional feed consumed was established within the limits of error of the method, this being the prime object of the experiments. This being determined, however, it was a simple matter to compute the amount of feed corresponding to the total work performed, while the difference between the latter and the total ration evidently was the maintenance ration. From the total digestible nutrients (inclusive of crude fiber) required for maintenance, as thus computed by Wolff, the equivalent amounts of metabolizable energy required for maintenance may also be computed approximately by the use of Zuntz and Hagemann's factor of 3.96 Cals. per gram (776).

In Wolff's earlier experiments and in those later ones in which approximately equal proportions of hay and grain were consumed, the maintenance ration was found to be approximately 4200 grams total nutrients per $500 \mathrm{kgs}$. live weight, equivalent to 16.63 Therms. In those later experiments (including the results of similar investigations by Grandeau as recomputed by Wolff) in which a larger proportion of grain was fed, the total nutrients required for maintenance ranged from 3600 to 3800 grams, equivalent to from I 4.26 to I 5.05 Therms. In other words, the amount of metabolizable energy required for maintenance varied with the proportion of roughage present, as would be anticipated from the results with cattle recorded on previous pages.

b. Zuntz and Hagemann's results. - From a respiration experiment at the Göttingen Experiment Station, Zuntz and Hagemann compute the metabolizable energy of the maintenance

${ }^{1}$ Wolff's experiments were made with a sweep-power arranged to serve also as a dynamometer. The actual measurements of the work performed, except in the later expetiments, proved to be too low, but Wolff believes them to be relatively correct, so that the ratio between the work as measured and the additional feed required to produce it may still serve as the basis of computation. 
ration of the horse by subtracting from the total digested nutrients the carbohydrate equivalent of the protein and fat gained by the animal, disregarding the possible stimulating effect of the feed. In this way, they find for the maintenance ration 2955.4 grams total digested nutrients per head, equivalent to II.70 Therms or I2.I Therms per Iooo pounds live weight, a result notably lower than Wolff's. This difference is ascribed by Zuntz and Hagemann to the larger content of crude fiber in Wolff's rations, the work of digestion of this ingredient as estimated by them (777) very nearly accounting for the difference.

c. Müntz's experiments. - Müntz ${ }^{1}$ in $1878-\mathrm{I} 879$ attempted to determine the maintenance ration of the horse by starting with an insufficient ration and gradually increasing it until an equilibrium between feed and live weight was secured, seeking in this manner to eliminate the stimulating effect of excess feed (392). The trials were made on the horses of the Paris Omnibus Company, their work ration being known from previous experiments. He found that a ration equal to $\frac{5}{12}$ of the work ration and which may be estimated to contain I2.I Therms of metabolizable energy per Iooo pounds live weight was slightly more than sufficient for maintenance.

d. Grandeau and LeClerc's results. - Grandeau and LeClerc, ${ }^{2}$ in addition to the experiments mentioned in connection with Wolff's results, fed five cab horses a ration of $8 \mathrm{kgs}$. of hay during a total of $\mathrm{i} 4$ periods of a month each (one to five periods for each animal) during each of which the digestibility of the ration was determined. On the average of all the periods, the results per day and head were as follows:-

Total digestible nutrients $($ fat $\times 2.4)$.

Equivalent metabolizable energy at 3.96 Cals. per

Daily gain in weight.

Average live weight

2783.7 grams

The foregoing ration, which was evidently somewhat more than a maintenance ration, is equivalent to $\mathrm{I} 3 . \mathrm{I}$ Therms of metabolizable energy per Iooo pounds live weight. This is

1 Annales de l'Institut National Agronomique, Tome 3, 1876-1879.

2 L'alimentation du Cheval de Trait, I883, III. 
materially less than was obtained in Wolff's earlier experiments with hay and about the same as that found by him and by Zuntz and Hagemann for rations containing much grain.

The following summary of the data regarding the metabolizable energy required for maintenance by the horse shows a considerable range of variation which is only partially explicable by the varying proportions of grain and roughage contained in the rations.

Table 52. - Maintenance Rations of the Horse

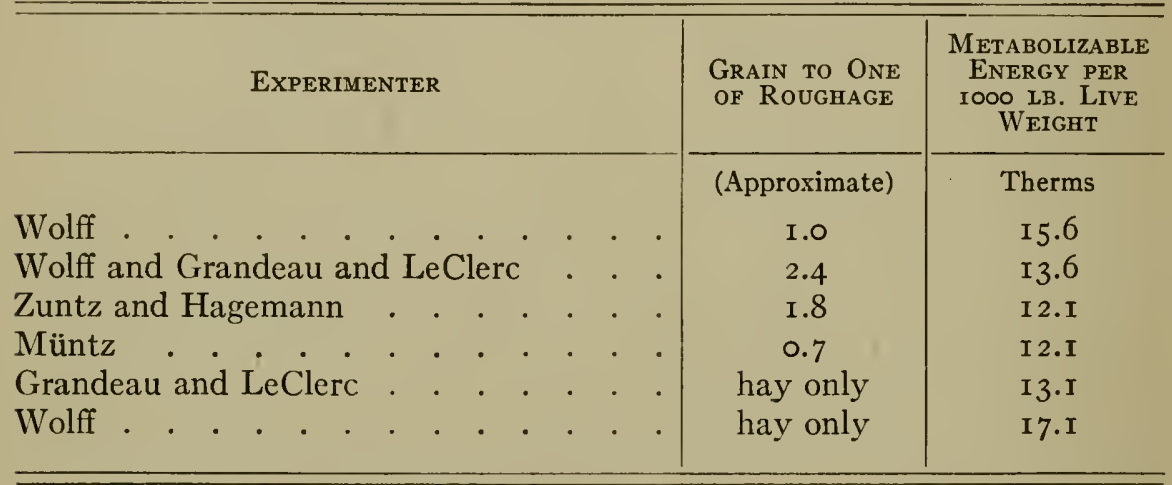

387. Metabolizable compared with net energy requirement. - The net energy required for maintenance, as with other animals, equals of course the fasting katabolism. This Zuntz and Hagemann compute, in the manner already described (385) to be 4.I Therms per thousand pounds live weight. As was there pointed out, however, those of their experiments in which the external temperature was lower or the amount of feed less gave higher results. The latter was also notably the case in earlier experiments in which still lighter rations were fed.

On the average of the eight most satisfactory experiments out of twelve ${ }^{1}$ on Horse II the total katabolism per day and head was I I.027 Therms upon a ration consisting of $3.5 \mathrm{Kgs}$. of oats, 0.5 of straw and 2.5 of hay. Computed in the same manner as in Table 5I, the expenditure of energy in the digestion of this ration is equal to 3782 Cals., which leaves a remainder of 7244 Cals., equivalent to r 4 0.3 Cals., per square centimeter of surface. This is a higher figure than any of those contained in Table $5 \mathrm{I}$, although the total katabolism was not notably different.

${ }^{1}$ Landw. Jahrb., 18, I ; 27, Ergzbd. III, 356-357. 
The authors conclude, therefore, that when the amount of heat liberated by the digestive work is small the lack is compensated for by an increased katabolism of body tissue. Their final result is that their animal required per head at least II.০o Therms of heat to maintain his body temperature. In other words, this is the minimum of metabolizable energy which must be contained in a maintenance ration, since if less be present, even although the ration supply the requisite amount of net energy, body tissue would still be katabolized for the production of the necessary heat. Computed per thousand pounds live weight, Zuntz and Hagemann's estimated maintenance requirement is: -

Net energy for internal work . . . . . . . 4.I Therms Additional required for heat production . . . . 7.8 Therms Total metabolizable energy required . . . . . . I I.9 Therms

In computing a ration for the actual maintenance of the horse at rest, it is necessary, according to these figures, to consider not only whether it supplies net energy equal to the fasting katabolism but also whether it contains sufficient metabolizable energy to support the necessary heat production. On the other hand no such allowance need ordinarily be made in computing work rations. The horse when at work is producing an excess of heat (compare Chapter XIV), and during the working hours no expenditure of feed energy for the sake of heat production would be called for, while any ordinary working ration would probably contain a considerable surplus of metabolizable energy over the maintenance demand during the hours of rest.

\section{The maintenance requirement of fowls}

388. Net energy requirements. - Gerhartz ${ }^{1}$ has measured the net energy requirement of fowls by means of a number of respiration experiments with the Regnault-Reiset type of apparatus (298) upon two fasting hens. He has also computed the fasting katabolism from a number of respiration experiments in which the animals were fed by subtracting from the total metabolism that computed to have been due to the

\footnotetext{
${ }^{1}$ Landw. Jahrb., 46 (1914), 797.
} 
consumption of the feed - i.e., by substantially the same general methods used by Zuntz and Hagemann for the horse (385). His results, computed per thousand square centimeters of body surface and also per 5 pounds live weight in proportion to the $\frac{2}{3}$ power of the latter, were as follows:-

Table 53.- Net Energy for Maintenance of Hens

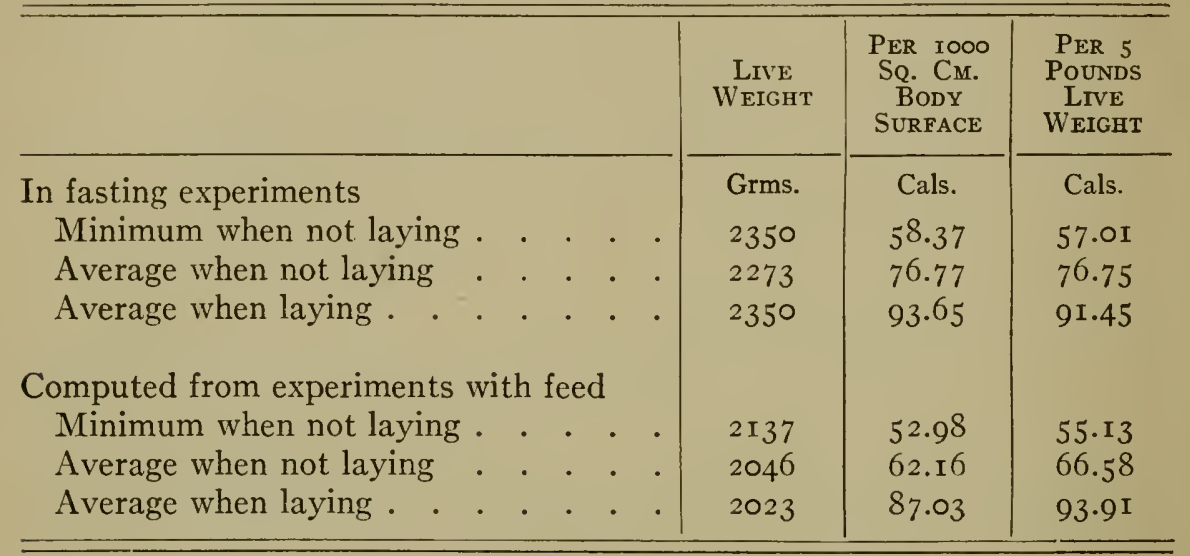

It would appear from the figures that the average maintenance requirement of a 5-pound hen may be estimated at approximately 72 Cals., while in periods of minimum muscular activity it may fall as low as $5^{6}$ Cals. The much higher figure (93 Cals.) obtained in the periods when the hen was laying does not represent maintenance simply, but includes also the energy expended in the formation of the egg. As with all small animals, the katabolism of the hen per unit weight is high, but when computed per unit of surface it does not differ greatly from that of other species.

389. Metabolizable energy in maintenance rations. - Gerhartz also determined the amount of feed required to maintain the live weight of his fowls and computes the corresponding amounts of metabolizable energy to have been per Iooo sq. $\mathrm{cm}$. body surface.

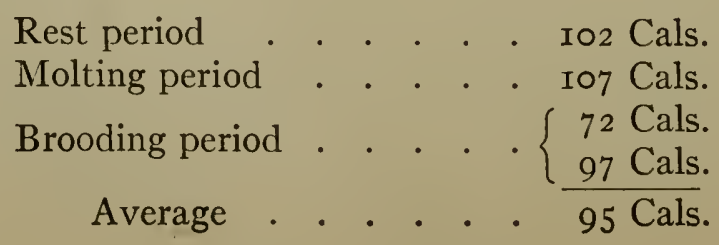




\section{Summary}

390. For convenience of reference, the average results regarding the energy required for the maintenance of the common species of farm animals as recorded in the foregoing pages are brought together in the following table. For live weights other than those stated the maintenance requirement may be computed in proportion to the surface in the manner described in Chapter VII (347).

Table 54.- Energy Requirements for Maintenance

Swine, per roo lb. live weight .

Cattle, per tooo lb. live weight

Unfattened

Fattened .

Sheep, per roo lb. live weight .

Horses, per rooo lb. live weight

Hens, per $5 \mathrm{lb}$. live weight

\begin{tabular}{|c|c} 
Net Energy & $\begin{array}{c}\text { Metabolizable } \\
\text { EnERGy } \\
\text { Therms }\end{array}$ \\
\hline 1.25 & 1.53 \\
6.00 & 10.47 \\
$7.95(?)$ & 13.55 \\
0.79 & 1.37 \\
4.10 & $\mathrm{II} .90$ \\
0.072 & 0.095 \\
\hline
\end{tabular}

As pointed out at the beginning of this section (376), the foregoing figures express an economic rather than a physiological requirement for energy. Besides the absolute energy requirement in a state of complete rest, they include the energy expended in divers forms of incidental muscular work, of which one of the most important, in farm animals, appears to be that of standing (349). The average for swine was obtained from experiments in which the animals were lying during most or all of the time. The average for cattle, as noted, has been computed to twelve hours standing, while that for the horse represents the katabolism when standing quietly.

Moreover, even with the limitations just indicated, the results represent averages from which the energy expenditure of the individual animal may differ considerably. Such averages are useful as a basis for computing feed requirements and rations, but it should be clearly understood that they are by no means physiological constants which can be applied to all animals 
indiscriminately or used as a basis for exact computations of the effects of feeds in individual cases.

\section{§ 3. Factors Affecting the Maintenance Requirement}

Certain conditions which affect the energy expenditure of the fasting animal, and therefore the amount of net energy required for maintenance, have already been discussed in Chapter VII (345-357), while a few others may be more conveniently considered at this point.

391. Temperament. - The nervous, restless animal is continually expending 'energy in a variety of unnecessary movements which may very materially increase the amount of energy needed for his maintenance as compared with that required by the quieter and more phlegmatic animal. There can be little question that those differences between the maintenance requirements of different animals which are ascribed somewhat vaguely to "individuality" are due to a large extent to varying amounts of muscular activity.

Thus in Armsby and Fries' determinations ${ }^{1}$ of the maintenance requirement of cattle $(380)$ the two animals designated as $\mathrm{A}$ and $\mathrm{B}$ were respectively a pure-bred beef animal and a scrub, the latter having more or less dairy blood and being of a decidedly more nervous disposition than the animal A. The difference in the requirements of the two animals, as shown by the following comparison, may be reasonably ascribed to this difference in temperament.

Table 55.- Net Energy Requirement for Maintenance

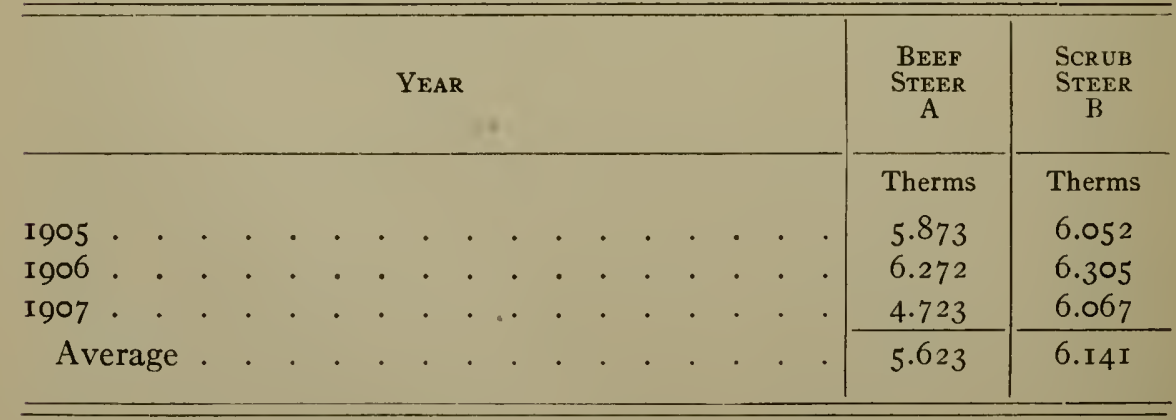

1 U. S. Dept. Agr., Bur. Anim. Indus., Bul. I 28 (I9I I), p. 53. 
Like the temperament, any external conditions tending to affect the degree of muscular activity will also tend to affect the maintenance requirement. The steer confined in a stall, for example, may take less muscular exercise, and therefore require less energy for maintenance, than one simply confined to a pen or open yard. The animal comfortably bedded and thereby induced to spend much of his time lying down will consume a smaller proportion of his feed for maintenance than one kept under less comfortable conditions. Any sort of excitement is likely to be paid for by increased muscular activity and correspondingly increased consumption of feed for maintenance.

392. The plane of nutrition. - It is somewhat generally believed that the amount of feed necessary for maintenance varies with the plane of nutrition on which the animal is kept. By this is meant that an animal which has been highly fed for some time will require a larger amount of feed for maintenance than a similar animal which has been sparsely fed and is in a more or less reduced condition. Thus, Waters ${ }^{1}$ writes: "Apparently the animal organism when kept for a long period of time on a low nutritive plane, as in the case of maintenance animals, gets on a more economical basis than when more liberally fed. For example, if we reduce the feed of an animal that has been previously liberally nourished to a point where for a month or more there is a small loss in weight, an equilibrium will later be established and subsequently the animal may increase in weight, the quantity and quality of the feed remaining the same. Thus a ration that was insufficient to sustain live weight at first may be capable later of maintaining the animal at a stationary body weight, and still later of causing an increase in weight. Digestion experiments with a number of animals indicate that a part of this is due to the more complete digestion of the feed by the animal on a low nutritive plane, but so far as the experiments have thus far progressed there does not seem to have been a sufficient increase in the degree to which the feed has been digested to account for all the increased efficiency in the ration noted." 2

Comparatively little experimental confirmation of these results has as yet been published, although respiration experi-

${ }^{1}$ Proc. Soc. Prom. Agr. Sci., I908, p. $96 . \quad{ }^{2}$ Compare Chapter XVI, 83 (722). 
ments on dogs by Kleinert ${ }^{1}$ and by Schlossmann and Murschhauser ${ }^{2}$ seem to point in the same direction.

Observations made by Zuntz and Hagemann ${ }^{3}$ on the horse appear suggestive in this connection. In a series of respiration experiments they have confirmed the common observation that a surplus of feed above the maintenance requirement stimulates the muscular activity and restlessness of this animal, so that a ration may be considerably more than sufficient to maintain the animal when standing quietly in the stall and yet give rise to no increase in weight under ordinary conditions. A similar stimulating effect of the feed upon the minor muscular movements of cattle, expecially while standing, seems to be indicated by the experiments of Armsby and Fries $(367 e)$. It seems possible that part, at least, of the diminution of the maintenance requirement observed by Waters may have been due to a voluntary restriction of motion on the part of the animals on the low nutritive plane.

In attempting to determine experimentally the minimum maintenance requirement it is evidently the safer method of procedure, especially with the horse, to approach the maintenance point by gradually increasing a sub-maintenance ration, as in Müntz's experiments on the horse $(386 c)$ and those of Armsby and Fries on cattle (374) rather than by the gradual reduction of a supermaintenance ration.

393. Fattening. - That fat animals have a relatively greater maintenance requirement than thin ones seems to be fairly well established for cattle by the experiments of Kellner and of Evvard, the results of which are recorded in Table 48 (381).

One obvious reason why the maintenance requirement per head should be greater for a fattened animal than for the same animal before fattening is the greater muscular effort expended in standing, due to the greater weight to be supported. Zuntz and Hagemann, in experiments upon the horse carrying weight on its back, found that this increase was proportional to the amount of weight added (665). If this be true generally, then that portion of the metabolism due to standing will increase more rapidly than the body surface. In Armsby and Fries' experiments on unfatted cattle, however, the energy expendi-

1 Ztschr. Biol., 61 (1913), 346.

2 Biochem. Ztschr., 53 (I9I3), 265.

${ }^{3}$ Landw. Jahrb. 27 (1898), Ergzbd. III, 2 II, 236. 
ture due to standing I 2 hours amounted to only about I 5 per cent of the total daily metabolism. The increase in the maintenance requirement per unit of surface which is indicated by Kellner's results is considerably greater than would be computed on this basis and the same is true of Evvard's fat animals, the difference becoming greater as the animals grew fatter.

394. Age. - The maintenance requirement of a young animal is naturally smaller per head than that of an older animal on account of the difference in size. Whether there is any difference in the relative requirement, that is, in the requirement computed to uniform weight or surface, is not altogether clear, few specific results on farm animals being on record. Evvard's results on yearlings (381) are somewhat higher than most of those which have been obtained with mature cattle, although, of course, these figures do not relate to the same individuals at different ages. Armsby and Fries ${ }^{1}$ in a series of respiration calorimeter experiments upon the same two animals in three successive years found with their full-blood steer a marked decrease in the maintenance requirement as a yearling and as a three-year old, when corrected to a uniform number of hours standing and computed in proportion to the two-thirds power of the weight. With the scrub steer, on the other hand, no distinct decrease of the maintenance requirement was observed.

Somewhat extensive data are on record regarding the metabolism of man at different ages. A summary of these by Tigerstedt ${ }^{2}$ seems to show clearly that the metabolism per unit of surface diminishes, although. not very rapidly, from youth to maturity. In view of the relatively slow growth of man, these results are comparable to such as might be obtained during the first six to twelve months of the life of ordinary domestic animals and for these ages there are no satisfactory determinations of the maintenance requirement.

If it be true that the maintenance requirement of a young animal is relatively greater than that of an older one, this may fairly be presumed to be due to a considerable extent to the greater muscular activity usually exhibited by young animals, which, as already pointed out, notably increases the body katabolism. .

1 U. S. Dept. Agr., Bur. Anim. Indus., Bul. I 28.

2 Nagel's Handbuch der Physiologie des Menschen, I, 469. 


\section{§ 4. The Relation of the Maintenance Requirement} to External Temperature

While the temperature to which an animal is exposed is but one among other factors which may affect its maintenance requirement, the somewhat complicated relations involved seem to warrant a separate discussion.

395. Feed consumption lowers the critical temperature. - In discussing the influence of temperature upon the fasting katabolism (350-357) it was shown that for the fasting animal there is a certain external temperature (or more strictly, thermal environment), called the "critical temperature," at which the heat production incidental to the necessary fasting katabolism just balances the unavoidable loss by radiation, conduction and evaporation, so that the body temperature is just maintained. Above this temperature, the fasting animal has a surplus of heat which it gets rid of by means of the physical regulation. Below the critical temperature, on the other hand, its katabolism is increased beyond that necessary for the internal work of the body in order to supply the necessary amount of heat; i.e., the energy expenditure is augmented.

As has been shown (365), however, the consumption of feed results in increasing the heat production of an animal. When an animal is fed, therefore, it has two sources of heat: first, as in the fasting state, the heat resulting from the katabolism incident to the necessary internal work of the body, and second, in addition to this, the heat generated by the so-called "work of digestion." Under these conditions, with more heat being produced in the body as the result of feed consumption, it is obvious that the animal can withstand a greater cooling effect of its surroundings without being compelled to katabolize body substance to maintain its body temperature. In other words, the "critical temperature" is lowered. Furthermore, the greater the amount of feed consumed the lower is the point to which the external temperature can fall without reaching the critical point, so that animals receiving heavy rations in productive feeding can withstand more cold than those on simple maintenance. Conversely, for any particular temperature there will be a definite amount of any given feed the consumption of which, together with the katabolism required for the internal 
work, will give rise to the production of an amount of heat just sufficient to balance the unavoidable loss of heat to the surroundings.

This influence of the quantity of feed is well illustrated by the following tabulation of Rubner's results upon a dog at different temperatures and consuming different amounts of meat.

Table 56. - Influence of External Temperature on Heat Production

\begin{tabular}{|c|c|c|c|c|c|}
\hline \multirow{2}{*}{ TEMPERATURE } & \multicolumn{5}{|c|}{ Heat Production per Kg. Body Weight } \\
\hline & Fasting & $\begin{array}{l}\text { Fed roo Grms. } \\
\text { Meat }\end{array}$ & $\begin{array}{l}\text { Fed } 200 \text { Grms. } \\
\text { Meat }\end{array}$ & Fed & $\begin{array}{l}320 \text { Grms. } \\
\text { Meat }\end{array}$ \\
\hline & Cals. & Cals. & Cals. & & Cals. \\
\hline $7^{\circ} \mathrm{C}$ & 86.4 & - & 77.7 & & 87.9 \\
\hline $\mathrm{I} 5^{\circ} \mathrm{C}$. & 63.0 & 一 & - & & 86.6 \\
\hline $20^{\circ} \mathrm{C}$ & $55 \cdot 9$ & 55.9 & 57.9 & & 76.3 \\
\hline $25^{\circ} \mathrm{C}$. & 54.2 & $55 \cdot 5$ & 64.9 & & 一 \\
\hline $30^{\circ} \mathrm{C}$. & 56.2 & 55.6 & 63.4 & & 83.0 \\
\hline
\end{tabular}

The amount of feed required to just offset the cooling effect of a low temperature might be called the critical amount of feed for that temperature. It will obviously be less the greater the proportion of its metabolizable energy which is dissipated as heat.

For example, in discussing the relative amounts of different feeds necessary for maintenance (375) it was stated that either I $3.83 \mathrm{lb}$. of mixed hay or $9.07 \mathrm{lb}$. of mixed grain and alfalfa hay would yield approximately 6.0 Therms of net energy, and would therefore constitute a maintenance ration for a rooopound steer. The amounts of metabolizable energy contained in these two rations, however, would be different, viz. :-

I3.83 lb. mixed hay

I 2.0I Therms

$9.07 \mathrm{lb}$. mixed grain and alfalfa hay . . . . 10.69 Therms

Since both are maintenance rations, the animal would neither gain nor lose energy and all the metabolizable energy of the feed would be finally converted into heat in both cases. The 
animal on the exclusive hay ration, therefore, would have at his disposal I.32 Therms more heat than the other and accordingly could withstand a lower temperature without drawing on his body for fuel.

396. Net energy below critical temperature. - Down to the critical temperature which corresponds to the particular amount and kind of feed consumed, in accordance with the facts brought out in the previous paragraphs, only part of the metabolizable energy serves to maintain the animal. The remainder is virtually expended in the "work of digestion" and converted into heat, and this heat, since not needed by the animal, becomes an excretum and is gotten rid of. If, however, the external temperature falls below the critical point the case is dif-. ferent. Heat resulting from the ingestion of feed is just as useful as heat from any other source for keeping the body warm. Under these conditions, therefore, all the metabolizable energy of the feed may be of use. Part of it (the net energy) is used directly for supporting the necessary internal work of the body, while the remainder prevents the necessity of katabolizing tissue for the sake of heat production and is therefore indirectly of use. In other words, the heat resulting from the consumption of feed may be substituted for heat which would otherwise have to be obtained by the katabolism of tissue. When the external temperature falls so low that all the heat produced by the digestive work is required for this purpose, obviously all the metabolizable energy of the ration is of use directly or indirectly to prevent loss of energy from the body and therefore all of it appears to be net energy.

Thus, if the ration of mixed grain and alfalfa hay used as an illustration in the previous paragraph be fed to a steer whose surroundings are kept at the critical temperature for the fasting animal, the 6.o Therms of net energy which the ration supplies will be used to support the internal work of the body, and the heat thus produced will be just sufficient to maintain the body temperature, while the remaining 4.69 Therms of metabolizable energy will be expended in superfluous heat production. Suppose, now, that the external temperature falls to a point at which the fasting katabolism would be I0.69 Therms instead of 6.0 Therms, i.e., at which this amount of heat is necessary to maintain the normal body temperature. The necessary internal work of the body still yields 6.o Therms, as before, 
while the additional 4.69 Therms of heat resulting from the "work of digestion" will be of use in keeping the animal warm and will obviate the necessity of its katabolizing body substance for that purpose. All the metabolizable energy of the ration, therefore, contributes to the maintenance of the animal under these conditions, part directly and part indirectly, and the availability is apparently roo per cent, while the real availability for the physiological processes in the body is only 56 per cent. If the experiment were made at an intermediate temperature at which the fasting metabolism would be 8.0 Therms, then 2.0 Therms of the heat due to the "work of digestion" would be of use in maintaining the body temperature and the apparent availability would be 75 per cent, i.e., the result would be a mixed one. Evidently, the actual expenditure of energy in the "work of digestion," and its complement, the net energy, can be determined only by experiments made above the critical temperature.

397. Bearing on maintenance ration. - The foregoing facts render it apparent that in the case of an animal on a maintenance ration the external temperature may fall considerably below the critical temperature for the same animal when fasting before there is any increase in the amount of feed actually required for maintenance. Only when the temperature falls so low that all the metabolizable energy of the ration is being utilized, directly or indirectly, to maintain the body heat will a further drop in the temperature call for greater feed consumption, i.e., for an increase in the maintenance ration. These considerations may affect the computation of actual maintenance rations. An example of this is afforded by Zuntz and Hagemann's results upon the maintenance requirements of the horse (387). According to these investigators, a horse weighing rooo pounds requires only 4.I Therms of net energy per day for maintenance, but the body also needs to be supplied with an additional 7.8 Therms of heat, making a total of II.9 Therms daily, to balance the loss of heat from the body. If, therefore, a maintenance ration be computed supplying the necessary 4.I Therms of available energy, it still remains to be considered whether the heat arising from the so-called "work of digestion " will supply the remaining 7.8 Therms of heat required. If it does not, the difference, according to Zuntz and Hagemann, will be made up by the katabolism of body tissue, as is illustrated in several of their experiments, and the ration will not maintain the animal although it contains net energy equal to the fasting katabolism. 
To put the matter in another way, Zuntz and Hagemann consider that when receiving the ordinary maintenance ration the critical external temperature for the horse is comparatively high, so that, for example, a ration which is sufficient for maintenance in summer may be insufficient in winter, not because it contains any less available energy but because it fails to meet the demand for heat.

Tangl's experiments (377) showed that the critical temperature for swine is likewise comparatively high $\left(68^{\circ}-73^{\circ} \mathrm{F}\right.$.), while the expenditure of energy in digestion by these animals, especially when fed chiefly or wholly on concentrates, is likely to be less than that of ruminants. Exposure to low temperatures, therefore, may be expected to increase the actual maintenance ration of swine, and this belief seems to be confirmed by the reported results upon the influence of exposure on the gains of fattening swine. ${ }^{1}$ It also seems possible that part of the very wide variations observed in the amount of metabolizable energy required for the maintenance of swine (378) may be due to differences in the temperature at which the experiments were made.

Experiments on cattle by Armsby and Fries have shown that at temperatures in the neighborhood of $63^{\circ} \mathrm{F}$., the feed may be reduced very considerably below the maintenance ration without any indication of an increased katabolism for the sake of heat production. A single series of comparisons at a somewhat lower temperature $\left(5^{\circ} \mathrm{F}\right.$.) also showed no increase in the katabolism, even on rations much below maintenance. No exact experiments at lower temperatures have been reported. Apparently, the critical temperature of ruminants is rather low, while the "work of digestion" is the source of a relatively large amount of heat, so that, under ordinary conditions of feeding, these animals are producing a surplus of heat and therefore a ration supplying net energy sufficient for maintenance is also ample as a source of heat.

${ }^{1}$ U. S. Dept. Agr., Bur. Anim. Indus., Bul. 108 (rgo8), pp. 84-86. 


\section{CHAPTER IX}

\section{MAINTENANCE (Continued) -- THE REQUIREMENTS OF MATTER}

As was pointed out in the introduction to the previous Chapter (361), the maintenance requirement is a twofold one, calling for the presence in the feed of adequate amounts of certain specific substances as well as for an adequate supply of energy. The former phase of maintenance in some of its broader aspects forms the subject of the present Chapter. These specific substances may be grouped for the purpose of this discussion as proteins or their constituents, ash ingredients and accessory constituents.

\section{§ I. The Protein Requirements for Maintenance}

398. Nature of protein requirement. - As was shown in Chapter VII (340), the loss of protein which the fasting body suffers may be interpreted in two ways. First, it may be regarded as due to the complete breaking down of a certain amount of protein as the necessary accompaniment of cell activity (Rubner's "wear and tear" quota). Second, it may be supposed that certain atomic groupings contained in the protein molecule may be indispensable for the normal functioning of the body, so that, if they are not contained in the feed, body protein may be katabolized for the sake of obtaining them.

In either case, it is clear that what the feed must supply in order to maintain the body in nitrogen equilibrium is not, strictly speaking, protein as such, but materials whose digestive cleavage will yield certain amounts and proportions of the constituent amino acids. On the first hypothesis, the requirements for the different " building stones" would be determined substantially by the quantities of each existing in the body tissues 
katabolized. According to the second hypothesis, it might be presumed that only certain of the atomic groups contained in protein, such as tryptophan, e.g., would need to be supplied. Moreover, it appears not unlikely that both hypotheses may be true and that body protein is katabolized both as a whole and at times as a means of obtaining certain amino acids. If such is the case, substantially all the "building stones" of the proteins, so far as they cannot be manufactured in the body, must be supplied in the feed, but relatively more of certain particular ones might be required than would be indicated by the make-up of the body proteins. Finally, it seems to be fairly well established that at least some of the amino acids can be manufactured in the body. This is almost certainly true of glycin and perhaps of prolin and arginin. If such be the case, it becomes even more clear that the protein requirement, so called, is really an amino acid requirement.

399. Amino acids required for maintenance. - Our actual knowledge of the amino acid requirement for maintenance is still meager, but it has been shown that a supply of tryptophan and probably of tyrosin is necessary for the maintenance of nitrogen equilibrium, while lysin is dispensable.

Willcock and Hopkins, ${ }^{1}$ for example, found that the zein of maize, which contains neither tryptophan nor lysin, was capable of supporting neither growth nor maintenance in mice, while the addition of tryptophan diminished although it did not altogether stop the loss of nitrogen from the body. Henriques ${ }^{2}$ obtained similar although less striking results in experiments with rats. It is to the work of Osborne and $\mathrm{Mendel}^{3}$ that we owe the most conclusive demonstration of the necessity of tryptophan for maintenance. They showed conclusively that zein as the sole source of protein was incapable of maintaining rats, while with the addition of tryptophan much better results were obtained and in two cases complete maintenance for a long time was secured. Miss Wheeler ${ }^{4}$ has reported similar results on mice. Furthermore, Osborne and Mendel have shown that the deficiencies of zein may be compensated for by the addition to the ration of other proteins containing the lacking amino acids.

1 Jour. Physiol. (London), 35 (I906), 88.

${ }^{2}$ Ztschr. Physiol. Chem., 60 (I909), I08.

${ }^{3}$ Carnegie Institution of Washington, Publication No. $5_{5} 6$ (rgrr); Jour. Biol. Chem., 13 (I9I 2), 233; 17 (I9I4), 325.

${ }^{4}$ Jour. Exp. Zoology, 15 (1913), 209. 
On the other hand, they have also shown that lysin is not essential to protein maintenance, they having been able to maintain animals for long periods on rations containing as their sole protein gliadin, which contains no lysin but does contain tryptophan.

What has been shown regarding the necessity of tryptophan and the dispensability of lysin for maintenance may doubtless prove to be true for other protein constituents, so that ultimately it may be possible to estimate the relative maintenance values of proteins on the basis of their chemical constitution. At present, however, this is far from being the case. The constitution of many of the proteins, particularly those of the roughages, is known inadequately or not at all, while the specific amino acid requirements for maintenance have still to be worked out and may conceivably vary as between different species.

400. Relative values of proteins for maintenance. - The facts regarding the variations in the constitution of the different proteins which are recorded in Chapter I (50) render it evident that these substances may be of quite unequal value as sources of amino acids to the organism. Thus, according to the data there tabulated, gliadin and zein would be about three or four times as valuable as legumin as a source of the amino acid prolin, while on the other hand legumin would be $2 \frac{1}{2}$ times as valuable as wheat glutenin as a source of lysin. The cereal proteins, especially those of wheat, are notably rich in glutamic acid and therefore relatively poorer in other constituents. If, then, the protein requirement for maintenance is in reality an amino acid requirement it would seem that these various proteins must be of unequal value for that purpose.

As regards single proteins, the experimental evidence just cited strongly supports this presumption, while Osborne and Mendel ${ }^{1}$ have likewise shown the existence of distinct differences in the values of lactalbumin, casein edestin, gliadin and milk proteins for the maintenance of rats. It must not be forgotten, however, that both man and domestic animals ordinarily consume a mixture of proteins, so that it may be presumed that deficiencies or excesses of particular "building stones" compensate for each other to a greater or less extent. On the whole the statement seems justified that while distinct differences

${ }^{1}$ Jour. Biol. Chem., 22 (I9I5), 24I. 
between mixed proteins from different sources as regards their value for maintenance have been shown to exist, they appear in many cases to be less than might be anticipated from the known differences in their chemical constitution. As a matter of practical necessity, then, pending the further investigations so greatly to be desired, the only course open for the present seems to be to follow established custom and deal with the protein of feeding stuffs and rations as a whole, with the consciousness that it is of unequal nutritive value in different materials but in the belief that such differences are not in all probability so great as to seriously invalidate the general usefulness of the results.

\section{Influence of feed supply on protein katabolism}

401. The minimum of feed protein. - The physiological minimum (339) below which the protein katabolism of the fasting animal cannot be reduced evidently constitutes a lower limit to the necessary supply of feed protein, but what surplus, if any, above this minimum must be supplied in order to secure actual protein maintenance is still an unsettled question. That the amount of feed protein necessary for maintenance is relatively small has been fully demonstrated. It appears to be well established also that on a diet containing an abundance of non-nitrogenous nutrients, especially of carbohydrates, a supply of protein materially less than the protein katabolism during complete fasting is sufficient to meet the needs of the organism, while it is possible that an amount little or no greater than that katabolized when abundance of carbohydrates is consumed will suffice.

Fats appear to be distinctly less efficient than carbohydrates in keeping the protein katabolism at the minimum. Precisely why this is the case has not been fully made out, although Landergren ${ }^{1}$ has advanced the explanation that a minimum of carbohydrates is essential to the chemical processes of metabolism and that when a sufficient amount is not supplied in the feed, protein is katabolized for the sake of producing carbohydrates, with the result that on a low protein diet nitrogen katabolism is increased.

${ }^{1}$ Jahresber. Tier. Chem., 32 (1903), 685. 
The facts recorded in Chapter VII (335-338), however, make it evident that the protein katabolism may be affected by the amount of both protein and non-nitrogenous material available in the body. For a correct interpretation of the results of experiments upon the maintenance requirement of protein, therefore, a knowledge of the influence of the feed supply upon the protein katabolism is essential.

402. Surplus protein katabolized. - While a relatively small quantity of digestible protein is sufficient, in the presence of an abundant supply of fuel material, to maintain the body in nitrogen equilibrium, an increase of the feed protein above this minimum does not result in any large or long-continued gain of protein tissue by the mature animal, but simply increases the protein katabolism, as is shown by the prompt appearance of a corresponding amount of nitrogen in the urine.

Table 57. - Protein Katabolism of Sheep per Day and Head

\begin{tabular}{|c|c|c|c|c|c|c|c|c|c|}
\hline & & & & & & \multicolumn{2}{|c|}{ SHEEP I } & \multicolumn{2}{|c|}{ Sheep II } \\
\hline & & & & & & $\begin{array}{l}\text { Nitrogen } \\
\text { digested }\end{array}$ & $\begin{array}{l}\text { Nitrogen } \\
\text { in urine }\end{array}$ & $\begin{array}{l}\text { Nitrogen } \\
\text { digested }\end{array}$ & $\begin{array}{l}\text { Nitrogen } \\
\text { in urine }\end{array}$ \\
\hline & & & & & & Grams & Grams & Grams & Grams \\
\hline Period I & . & & . & . & . & 8.18 & 7.48 & $7.8 \mathrm{I}$ & 6.98 \\
\hline Period 2 & . & . & . & . & . & I 7.86 & 16.82 & 17.72 & I6.37 \\
\hline Period 3 & . & . & . . & . . & . & 27.22 & $25 \cdot 75$ & $27 \cdot 33$ & 23.94 \\
\hline Period 4 & . & . & . . & . . & . & 36.99 & $32.7 \mathrm{I}$ & 37.07 & 32.09 \\
\hline Period 5 & . & 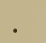 & . & . . & 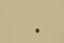 & 26.76 & 25.63 & $26.9 \mathrm{I}$ & 24.54 \\
\hline Period 6 & . & . & . & . . & . & 17.62 & I 6.64 & 16.94 & I 5.99 \\
\hline Period 7 & . & . & . & .. & . & 8.34 & 8.06 & 8.00 & 7.62 \\
\hline
\end{tabular}

The fact was demonstrated more than fifty years ago by C. Voit in collaboration at first with Bischoff ${ }^{1}$ and later alone and with Pettenkofer ${ }^{2}$ in experiments on carnivorous animals, and almost innumerable subsequent investigations have shown that it is true not only of these animals but of man and of herbivorous animals as well. Of the numerous investigations on herbivora in which the nitro-

1 Gesetze der Ernährung des Fleischfressers, I 860.

${ }^{2}$ Published chiefly in the Annalen der Chemie und Pharmacie and the Zeitschrift für Biologie. See also Voit, "Physiologie des Stoffwechsels," in Hermann's Handbuch der Physiologie. 
gen excretion has been determined, Table 57 may serve as an example. ${ }^{1}$ Two sheep were fed in periods $\mathrm{I}$ and 7 a basal ration of hay and barley meal. To this ration were added in the intermediate periods varying amounts of nearly pure protein in the form of conglutin (of lupins) or of flesh meal. A comparison of the nitrogen digested from the ration with the urinary nitrogen shows that the latter increased and diminished substantially parallel with the former.

403. Utilization of protein limited. - That the mere consumption of protein cannot cause a large storing up of it is indeed sufficiently obvious from daily experience. The muscles of the weakling cannot be converted into those of the athlete by feeding him upon a meat diet, nor the small man increased in size by a very abundant protein supply. The protein tissues of the mature animal have reached their natural limit of size and consequently the capacity of the body to store up protein is limited. Beyond the minimum required to make good the necessary katabolism in the cells, protein can be utilized in such an animal only to a small extent as protein, and it is therefore rapidly katabolized, its nitrogen appearing in the urine as urea and other familiar end products. Nor is the situation essentially different in the growing or the milk-producing animal. While these animals are able to utilize considerable amounts of feed protein, yet the limit to this utilization is set by the normal rate of growth of the protein tissues or the capacity of the mammary glands to manufacture the casein and other proteins of the milk. Any surplus of protein over what can be used for this purpose is katabolized precisely as is a surplus over the very small demand of the unproductive mature animal. (Compare Chapter XI, $\S 2$ and Chapter XIII, § 4.)

404. Protein as a source of energy. - This increased katabolism of protein, however, is not to be regarded as the total loss of so much feed material. In the presence of a surplus of protein, the amino acids resulting from its digestion are in large part deaminized (233), their nitrogen being excreted chiefly as urea, while a non-nitrogenous residue is left which contains the larger portion of the chemical energy of the protein which it represents and is in condition to be oxidized as fuel

${ }^{1}$ Henneberg and Pfeiffer; Jour. Landw., 38 (1890), 215. 
material (229). The increased nitrogen excretion on a high protein diet is simply the method by which the organism gets rid of surplus nitrogen while retaining the larger share of the energy of the protein for fuel purposes. It does not mean the total destruction of the corresponding amount of protein, but simply its transformation into compounds which can serve as sources of energy.

405. Fluctuations of body protein. - Although in the mature animal a surplus of feed protein is largely katabolized, so that a continued increase of the protein tissue of the animal cannot be brought about, as can that of the adipose tissue, simply by a surplus in the feed, the protein content of such an animal is not to be regarded as absolutely fixed, so that the protein supply has no effect upon it. On the contrary, a considerable range of variation is possible.

Thus it is a familiar fact that a fasting animal may live and continue to perform the essential bodily functions for some time while losing daily a not inconsiderable amount of protein. To cite a single striking example, Rubner observed in a fasting rabbit up to the time of death, on the nineteenth day, a loss of 45.2 per cent of the computed nitrogen of the body. While this is an extreme instance, nevertheless it is evident that there must be a relatively large loss of body protein in those more moderate cases in which the deprivation of protein is not continued so long as to cause death. Furthermore, the losses occurring in these latter cases may be made good by subsequent feeding and the animal restored to its original state. Illustrations of the same fact are familiar in the human subject in the emaciation due to illness and the restoration of the body during convalescence. In brief, it is evident that the body of the mature animal may fluctuate within somewhat wide limits as regards its protein content without necessarily causing any serious or permanent derangement of its functions.

406. Storage of feed protein. - Similar, although smaller, fluctuations in the protein content of the body appear to be caused by variations in the supply of feed protein, an increase in the latter giving rise to more or less storage of nitrogenous matter in the body, while a decrease has a contrary effect.

In other words, as regards its stock of nitrogenous materials the organism may exist and function at a higher or lower level 
according to the amount of protein supplied in the feed, while for each level of protein stock a certain supply in the feed is necessary - that is, the protein requirement for maintenance varies. With carnivora on a largely protein diet the adjustment of the body to the protein supply seems to take place rather promptly. In the case of herbivora, however, the adjustment appears to be more gradual, possibly owing to the relatively large supply of non-nitrogenous ingredients in their feed, and apparently some gain of protein may continue for a considerable time, although when expressed as a percentage of either the total feed protein or of the body protein the gain is relatively small.

407. Effect of deficiency of non-nitrogenous nutrients.The prime demand of the organism is for energy for the performance of its vital functions, and if necessary it will draw upon its own tissues for this purpose. No clear conception of the laws of protein metabolism can be reached without taking into consideration the energy relations.

As has already been shown, the proteins or at least the cleavage products of their digestion readily undergo a process of deaminization by which their nitrogen is split off and excreted, leaving a non-nitrogenous residue which is available as a source of energy. Ordinarily, however, the proportion of energy derived from the katabolism of protein is relatively small, the nonnitrogenous nutrients constituting its principal source.

But if, with an amount of protein in the feed just sufficient to sustain nitrogen equilibrium, the non-nitrogenous nutrients, especially the carbohydrates, be so reduced in amount that the total energy supply is insufficient for maintenance, not only is body fat drawn upon to make up the deficit, but the protein katabolism also increases, so that a supply of this nutrient which was previously adequate became insufficient and a loss of body protein occurs.

The effect is naturally most marked when the non-nitrogenous nutrients are withdrawn altogether. For example, Voit and Korkunoff ${ }^{1}$ found that when a dog was given an abundant supply of carbohydrates, protein equivalent to about 4.5 grams of nitrogen was sufficient to maintain him in nitrogen equilibrium. But when a similar amount

${ }^{1}$ Ztschr. Biol., 32 (1895), 67. 
of protein was given without non-nitrogenous nutrients it proved entirely insufficient for this purpose and about three times as much was required to attain protein maintenance, as the following table shows :-

Table 58. - Effect of Protein Supply on Protein Katabolism of DOG

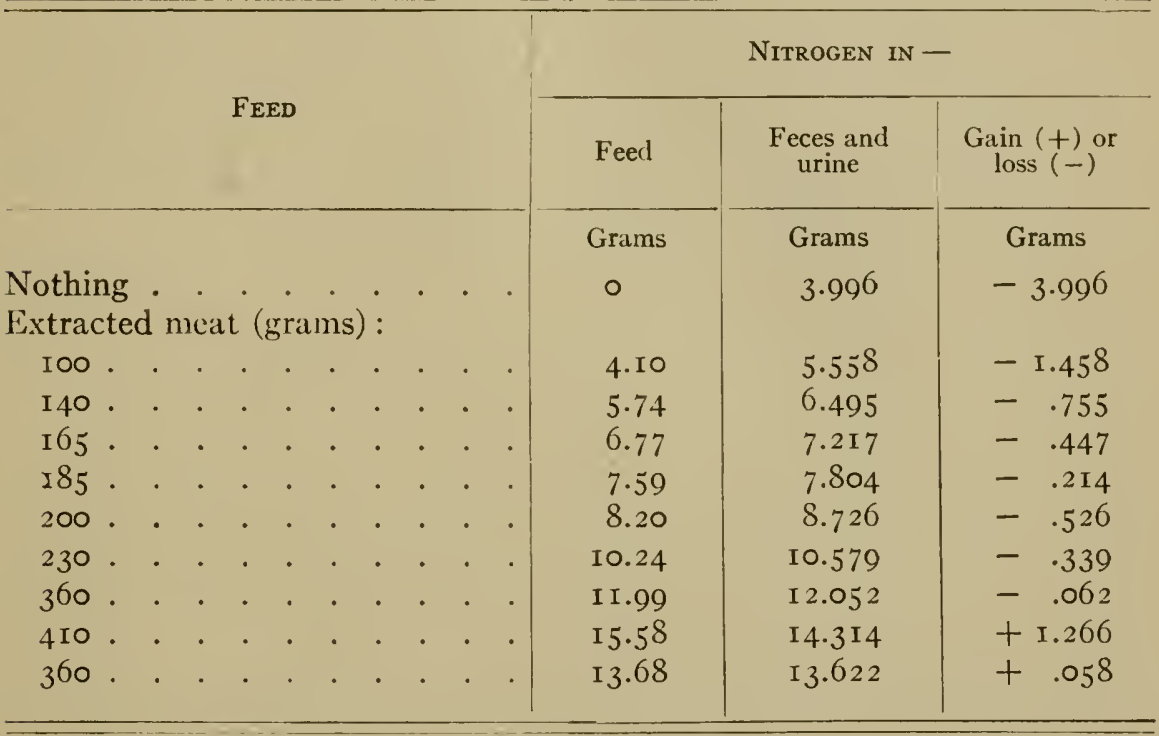

The results furnish also a striking illustration of the interesting relations between protein supply and protein katabolism which had been demonstrated more than 30 years earlier by the classic experiments of Bischoff and Voit (402), while rendering it evident that the quantity of protein required to produce nitrogen equilibrium when fed alone is very far from representing the minimum demand of the body.

What is so strikingly true in the total absence of non-nitrogenous nutrients holds good also in less degree in case of their relative deficiency. If a portion of the non-nitrogenous nutrients are withdrawn from a mixed ration, the protein katabolism usually increases.

408. Effect of surplus of non-nitrogenous nutrients. - If, on the contrary, non-nitrogenous nutrients be added to a ration, they tend to diminish the katabolism of protein.

As regards rations deficient in energy, this is, of course, only the converse of the statement of the preceding paragraph that 
the withdrawal of these materials tends to increase the protein katabolism, and as regards maintenance or submaintenance rations the two statements are equivalent. But even in the case of supermaintenance rations it has been found that the addition of a surplus of fat or, in particular, of carbohydrates, to a ration containing more than the minimum of protein tends to reduce the protein katabolism to a lower level. The effect is well illustrated, for example, by those of Kellner's respiration experiments on cattle ${ }^{1}$ in which starch was added to basal rations which were themselves sufficient to cause some fattening. The following table compares the urinary nitrogen upon the basal ration with that upon the augmented ration:-

Table 59. - Effect of Starch on Protein Katabolism of Cattle

\begin{tabular}{|c|c|c|c|c|c|c|c|c|c|c|c|c|c|c|c|}
\hline & & & & & & & & & & & & & & \multicolumn{2}{|c|}{ DaILX URINARy NItrogen } \\
\hline & & & & & & & & & & & & & & $\begin{array}{c}\text { On basal } \\
\text { ration }\end{array}$ & $\begin{array}{l}\text { With addition } \\
\text { of starch }\end{array}$ \\
\hline & & & & & & & & & & & & & & Grams & Grams \\
\hline $\mathrm{OxD}$ & • & - & - & . & - & . & . & . & & & & & & I 22.54 & 104.69 \\
\hline $\mathrm{OxF}$ & . & . & . & $\cdot$ & . & . & . & . & . & . & . & . & . & 106.03 & 81.18 \\
\hline $\mathrm{Ox} \mathrm{G}$ & . & . & . & . & . & . & . & . & . & . & . & . & . & 86.30 & 63.83 \\
\hline $\mathrm{OxH}$ & . & . & . & . & . & . & . & . & . & . & . & . & . & 109.28 & $8 \mathrm{I} \cdot 7 \mathrm{I}$ \\
\hline Ox J & . & . & . & . & . & . & . & . & . & . & . & . & . & I 22.62 & I03. I 3 \\
\hline
\end{tabular}

It has likewise been shown that this effect is produced not only by the true fats and by the soluble hexose carbohydrates, such as starch and the sugars, but likewise by the pentoses and, in the case of herbivorous animals, by those ill-known ingredients of feeding stuffs, especially of the crude fiber and the' nitrogenfree extract, which disappear in the passage of the feed through the alimentary canal and which are commonly spoken of as being digested. This statement covers also the organic acids, whether resulting from the fermentation of the carbohydrates or contained in the feed.

409. Protein katabolism depends chiefly on supply. - It should be clearly understood that even in the presence of a surplus of fat or carbohydrates the dependence of the protein

1 Landw. Vers. Stat., 53 (I900). 
katabolism upon the protein supply still holds true. Even the most liberal supply of non-nitrogenous nutrients cannot prevent the splitting-off and excretion of the nitrogen of surplus protein which was illustrated in previous paragraphs, but simply reduces it somewhat below the level which it would otherwise reach. To that extent, it helps to bring about, and probably to prolong somewhat, the temporary storage of protein mentioned on a previous page (406) and thus to bring the animal upon a higher plane of protein nutrition.

It is clear from the foregoing statements that no sharp distinction is to be conceived of between an insufficiency and a sufficiency of non-nitrogenous nutrients, but rather a tendency on the part of the latter to diminish the protein katabolism, a tendency more or less marked according to their abundance in the ration. It is not to be understood that no nitrogenous material is katabolized for fuel purposes as long as sufficient non-nitrogenous nutrients are present to supply the demands for energy, nor that even the largest quantities of the latter can prevent the katabolism of protein supplied in excess of its possible constructive use by the body.

\section{Protein requirements of farm animals}

410. Minimum and optimum of protein. - In considering the protein requirements of the different species of farm animals, it is important to distinguish between two points of view. On the one hand, it may be sought to determine the least amount of feed protein upon which the protein tissues of the animal can be maintained. On the other hand, the endeavor may be to formulate the most advantageous amount of protein to supply when an animal is actually to be maintained for a time and this amount may very possibly be greater than the physiological minimum. The first point of view, however, is plainly the fundamental one and should first receive consideration. Having determined the lower limit of protein supply, it will then be possible to consider intelligently the advantages, if any, of a surplus.

In considering the results of experiments directed toward the determination of the minimum of feed protein required by any individual or species, it is essential to bear in mind the facts 
regarding the influence of the feed supply upon the protein katabolism which have just been considered.

411. The plane of protein nutrition. - It has been shown in previous paragraphs that the protein katabolism adjusts itself more or less promptly to the supply in the feed. A surplus above the minimum requirement, while causing a small storage of protein, results chiefly in raising the plane of protein nutrition and so increasing the katabolism until income and outgo of nitrogen come into equilibrium. The mere fact, therefore, that an animal is in equilibrium with a certain supply of protein in its feed by no means proves the latter to be the least amount necessary for the maintenance of the animal, since it may be living upon an unnecessarily high plane of protein nutrition.

412. The supply of non-nitrogenous nutrients. - It has also been shown that the sufficiency of a given amount of protein depends not only upon the plane of protein nutrition of the body, but also, within certain limits, upon the amount of non-nitrogenous nutrients supplied with the protein. With an abundant supply of the former an amount of protein equal to the fasting katabolism, or perhaps even less, appears to be a sufficient minimum for maintenance. As the supply of nonnitrogenous materials is reduced a larger supply of feed protein seems to be required to reach equilibrium because more and more of it is diverted for use as fuel, so that in the total absence of non-nitrogenous nutrients a large excess of protein must be fed before equilibrium between income and outgo of nitrogen is reached. In interpreting experiments or formulating a maintenance ration, therefore, it is not sufficient to consider simply the amount of protein, but account must also be taken of the supply of non-nitrogenous materials, and only when the net energy content of the ration is ample for maintenance can it be concluded that a loss of body protein shows the protein supply to be insufficient.

413. Value of non-protein. - The crude protein of the feed of farm animals includes not only true protein but a great variety of other nitrogenous substances, grouped for convenience under the designation non-protein. In considering the results of experiments upon the protein requirements of these animals, therefore, it is necessary to determine whether the true protein should be the basis of comparison or whether 
the non-protein has some value for maintaining the protein tissues of the body.

It appears to have been demonstrated by recent experimental results, especially by those of Kellner, Morgen, and the Laboratory for Agricultural Research in Copenhagen, that the nonprotein of ordinary feeding stuffs is available for the maintenance of ruminants, probably indirectly through a conversion to protein by means of micro-organisms in the digestive tract (141). On the other hand, investigations have thus far failed to demonstrate that non-protein has any material value for other species or for production purposes (786-789). In the computation of rations for productive feeding, therefore, it appears desirable for the present to consider ordinarily only the digestible true protein, ignoring the non-protein. This implies, however, that the results of experiments upon the protein requirement shall be expressed in the same manner.

This will have two effects: First, it will make the protein requirement appear smaller than it really is. Suppose, for example, that a series of trials in which the ratio of digestible non-protein to digestible protein is $\mathrm{I}$ : Io shows that nitrogen equilibrium is reached with a ration supplying 500 grams protein and 50 grams nonprotein. Regarding the true protein only, the maintenance requirement is 500 grams, while including the non-protein it is 550 grams.

In the second place, however, this error will be largely compensated for when the actual computation of rations is also based on the true protein. Thus in the case just supposed, if a maintenance ration be computed from any feed or mixture in which the ratio of nonprotein to protein is the same as in the experiments from which the maintenance was deduced, viz., I : Io, it is obvious that the same final result will be reached whether the maintenance requirement be considered to be 500 grams of true protein or 550 grams of crude protein. Only when the proportion of non-protein to true protein varies widely from that existing in the rations used in determining the protein requirement will any significant error arise in computing rations.

In the results considered on succeeding pages, both the crude protein and true protein of the rations are stated when these are given in the reports of the experiments.

414. Computation to unit weight. - It was shown in Chapter VII (345) that the energy requirement for maintenance is substantially proportional to the body surface of the animal. 
No similar comparisons of the protein requirement appear to have been made. Since, however, the minimum protein requirement does not represent a demand for energy but for certain specific substances required for the normal functioning of the body, it seems plausible to suppose that its amount will depend rather upon the mass of active tissue than upon the body surface. If such be the case, the protein requirement may, with sufficient accuracy for practical purposes, be computed in proportion to the live weight and that course is followed in the succeeding paragraphs.

415. Protein requirement of cattle.-For obvious reasons it is impracticable to ascertain the fasting katabolism of ruminants as a basis for estimating their maintenance requirement as regards protein, but by a comparison of the recorded experiments in which the nitrogen balance upon small amounts of feed has been determined it is possible to fix approximately the limit of protein supply below which, even in the presence of an abundant supply of non-nitrogenous nutrients, a loss of body protein occurs.

Of the investigations upon the energy requirement for maintenance summarized in Chapter VIII (381) only those of Kellner and the live weight experiments of the writer, together with the early results of Henneberg and Stohmann, afford data regarding the minimum protein requirement. While protein maintenance was probably secured in the remaining instances there is no sufficient evidence to show that a surplus of protein was not being consumed $(402,411)$. In addition to the foregoing, the investigations by the Laboratory for Agricultural Research in Copenhagen ${ }^{1}$ upon the protein requirements for milk production (586) also afford approximate data as to the maintenance requirement, and Fingerling, ${ }^{2}$ in experiments upon the protein requirements of growing calves (471), obtained interesting indications regarding the quantity required for maintenance.

The lowest recorded amounts per Iooo pounds live weight upon which nitrogen equilibrium was reached were 0.2 I pound

${ }^{1}$ Denmark-Beretning fra den Kgl. Veterinaer of Landbohojskoles Laboratorium for landokonomiske Forsog. 6ode, 1906, and 63de, 1907, Kobenhavn. Translated by Mallèvre, Société de l'Alimentation Rationelle du Bétail. Compte Rendu de I İ̀me et I zème Congrès.

${ }^{2}$ Landw. Vers. Stat., 76 (IgI I), I. 
and 0.25 pound of crude protein in experiments on dry cows, while in experiments on steers almost as small a quantity, viz., 0.27 pound crude protein or 0.23 pound true protein, fell very little short of maintaining the nitrogen balance. Aside from these somewhat exceptional results, the lowest figures obtained were 0.43 pound crude protein and 0.38 pound true protein. If the few exceptionally low figures be omitted, the average and range of the results of the other experiments are as follows :-

Table 6o. - Average and Range of Protein Requirement of Cattle

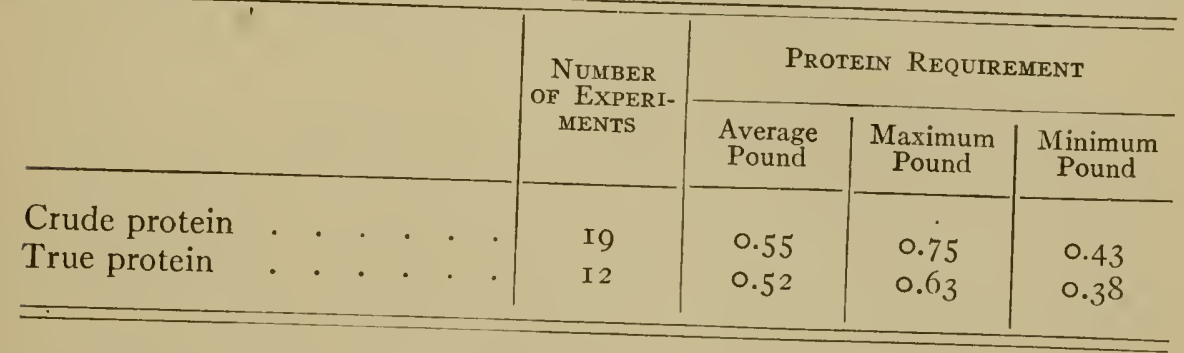

It seems safe, therefore, to estimate 0.6 pound of crude protein or 0.5 pound true protein per Iooo pounds live weight as representing in a general way the minimum protein requirement of cattle, with a range of perhaps O.I or 0.2 pound either way under varying conditions.

416. Protein requirement of sheep. - While a considerable number of experiments with sheep are on record in which live weight maintenance was secured, and a smaller number in which the nitrogen balance was maintained, few of them afford satisfactory data as to the minimum protein requirement.

A distinct difference between cattle and sheep, which affects the protein requirement, lies in the greater demand for protein incident to the growth of wool in the latter animals as compared with that of hair in the former. Determinations by Armsby and Fries on the same two steers in two consecutive winters showed an average production of epidermal tissue, including the growth of hair and the loss in brushings, equivalent to $0.0025 \mathrm{lb}$. protein per day and 1000 pounds live weight, an amount too small to materially affect the maintenance requirement. In the case of sheep, determinations by several investigators have shown the daily growth of wool per I0o0 pounds live weight to contain from o.Io to O.I $5 \mathrm{lb}$. of protein, the average being $0.135 \mathrm{lb}$. Although, as these figures show, the protein re- 
quirement of sheep for the growth of wool is considerably greater than that of cattle for the growth of hair, the absolute difference, after all, does not add very greatly to the total maintenance requirement.

The current feeding standards for the maintenance of sheep call for I.0-I.6 lb. of digestible crude protein per rooo pounds live weight, apparently upon the basis of Henneberg and Stohmann's early experiments (382) in which $\mathrm{I} .3 \mathrm{lb}$. of crude protein or $\mathrm{I} .04 \mathrm{lb}$. true protein produced but a slight gain of body protein in addition to the growth of the wool. There can be little doubt, however, that Henneberg and Stohmann's sheep received a surplus of protein above the actual maintenance requirement.

In a series of 20 digestion and metabolism experiments by Schulze and Märcker, ${ }^{1}$ decidedly smaller amounts of protein proved sufficient to maintain nitrogen equilibrium, the average of 6 experiments in which no loss of body protein was observed being $0.653 \mathrm{lb}$. digestible crude protein per 1000 pounds live weight. It is evident, then, that the protein supply of sheep can be reduced much below the amount fed in Henneberg and Stohmann's experiments without leading to a loss of body protein.

The most satisfactory data regarding the minimum requirement of sheep are afforded by Katayama's ${ }^{2}$ investigations, in which increasing amounts of nearly pure protein (" aleuronat") were added to a basal ration very poor in protein, consisting of hay, oat straw, starch and cane sugar. The protein in every case was substituted for a corresponding amount of starch, so that the total energy of the ration remained substantially-unchanged. On the average of two animals, $0.4 \mathrm{I} \mathrm{lb}$. digestible true protein per rooo pounds live weight was sufficient to maintain the nitrogen balance. Since, however, the growth of wool must have gone on, with a corresponding storage of nitrogen, there must have been an equivalent loss of protein by the active tissues of the body.

If to the minimum of $0.4 \mathrm{I}$ pound there be added $0.14 \mathrm{lb}$. per rooo pounds live weight for the growth of wool, it appears that the minimum protein requirement for the maintenance of mature sheep is in the neighborhood of $0.55 \mathrm{lb}$. It is interesting to note that the actual maintenance requirement for the body tissues is apparently quite as low relatively as for cattle.

1 Wolff; Die Ernährung der landwirtschaftlichen Nutztiere, p. zoo.

${ }^{2}$ Landw. Vers. Stat., 69 (I908), 32 I. 
417. Protein requirement of swine. - The determinations of the fasting katabolism of swine recorded in Chapter VIII (377) gave an average of $0.48 \mathrm{lb}$. per thousand pounds live weight for the fasting protein katabolism of swine, although with a considerable range in the individual results. McCollum ${ }^{1}$ has reported considerably lower figures for the protein katabolism of swine receiving no protein but fed liberal amounts of starch, the mean of twelve experiments being $0.26 \mathrm{lb}$. per Iooo pounds live weight with a range of $0.14 \mathrm{lb} .-0.33 \mathrm{lb}$. Whether, however, such small amounts as these are sufficient for actual maintenance, or if not, what excess above them is necessary, has not been certainly determined.

In the experiments of Von d. Heide and Klein (378), in one of which an approximate maintenance ration was fed to three young swine, there was a material gain of protein by the animals. The amounts actually katabolized, however, as shown by the amount of nitrogen excreted in the urine, were as follows for the three animals. together :-

\begin{tabular}{|c|c|c|c|c|c|c|c|}
\hline & & & & & \multirow{2}{*}{$\begin{array}{l}\text { Total Live } \\
\text { Weight }\end{array}$} & \multicolumn{2}{|c|}{ Protein Katabolism } \\
\hline & & & & & & Per Head & $\begin{array}{l}\text { Per rooo Lb. } \\
\text { Live Weight }\end{array}$ \\
\hline & & & & & $\mathrm{Kgs}$. & Grams & Lb. \\
\hline Period I Maintenance & . & . & . & . & 228.I & I 52.4 & 0.67 \\
\hline Period II Fattening . & . & . & . & . & 246.5 & $\mathrm{I}_{5} 6.2$ & 0.63 \\
\hline Period III Fattening . & . & . & . & . & $26 \mathrm{I} .5$ & I 5 I. 3 & $0.5^{8}$ \\
\hline
\end{tabular}

Dietrich, ${ }^{2}$ in his experiments upon maintenance ration of swine (378), found that 0.70 to $0.84 \mathrm{lb}$. of digestible protein per thousand pounds live weight sufficed to produce nitrogen equilibrium in two periods following an eight-day fasting period, but that about the same amounts ( 0.80 to 0.90 ) previous to the fasting period were insufficient, while in two trials in which respectively 0.94 and $\mathrm{r} .06 \mathrm{lb}$. were consumed protein maintenance was reached.

418. Protein requirement of the horse. - In the experiments by Grandeau and LeClerc described in Chapter VIII (386 $d$ ),

1 Wis. Expt. Sta., Research Bul. 21.

${ }^{2}$ Ills. Expt. Sta., Bul. I63 (I913). 
the nitrogen balance of the horses was determined during six of the periods. The following table shows the amounts of protein and of non-protein nitrogen digested in each period, the urinary nitrogen, and the small losses in epithelial tissue (epidermis, hoofs, hair, etc.) : -

Table 6r. - Nitrogen Balance of Horses

\begin{tabular}{|c|c|c|c|c|c|c|}
\hline & \multicolumn{2}{|c|}{ Horse No. I } & \multicolumn{2}{|c|}{ Horse No. 2} & \multicolumn{2}{|c|}{ Horse No. 3} \\
\hline & $\underset{\mathrm{I} 884}{\text { January, }}$ & $\begin{array}{l}\text { April, } \\
\text { I } 884\end{array}$ & $\begin{array}{l}\text { Novem- } \\
\text { ber, r } 88_{3}\end{array}$ & $\begin{array}{l}\text { May, } \\
\text { I } 884\end{array}$ & $\begin{array}{c}\text { December } \\
\text { I } 883\end{array}$ & $\underset{\text { I } 884}{\text { March }}$ \\
\hline \multirow{4}{*}{$\begin{array}{l}\text { Digested: } \\
\text { Protein nitrogen } \\
\text { Non-protein } \\
\text { nitrogen } \\
\text { Total nitrogen }\end{array}$} & Grams & Grams & Grams & Grams & Grams & Grams \\
\hline & 43.19 & 34.29 & 39.94 & 34.22 & $4 \mathrm{I} .82$ & 24.72 \\
\hline & 1.20 & $-\quad \mathrm{I} .0 \mathrm{I}$ & -3.23 & I0.78 & -2.09 & $-4.5^{8}$ \\
\hline & 44.39 & 33.28 & $35.7 \mathrm{I}$ & 35.00 & $39 \cdot 73$ & 20.14 \\
\hline $\begin{array}{c}\text { Nitrogen of epithe- } \\
\text { lial tissue }\end{array}$ & I.46 & 1.46 & I.46 & 1.46 & $\mathrm{I} .46$ & 1.46 \\
\hline Urinary nitrogen . & 35.17 & 38.75 & 30.70 & 41.92 & 37.62 & 32.70 \\
\hline Nitrogen gained & $7 \cdot 76$ & -6.93 & $3 \cdot 55$ & $\mathrm{I} .62$ & 0.65 & $-\mathrm{I} 4.02$ \\
\hline
\end{tabular}

Omitting the results upon horse No. 3 in March, when the digestible protein was exceptionally low, the other five periods show an average daily gain of nitrogen of 1.33 grams, while the average crude protein digested was 235 grams, or 0.59 .lb. per rooo pounds live weight, equivalent to about $0.50 \mathrm{lb}$. true protein.

419. The optimum of protein. - The data of the foregoing paragraphs seem to indicate a striking uniformity in the minimum protein requirement of the principal species of domestic animals with perhaps the exception of the hog when mature, 0.4 to 0.6 lb. per Iooo pounds live weight apparently sufficing to maintain nitrogen equilibrium under favorable conditions.

It should be clearly understood, however, that this figure represents a more or less accurately determined limit. It purports to be the amount below which the protein supply cannot be reduced without eventual protein starvation. The animal body, however, may adjust itself to a wide range of protein supply above the minimum, using some of it to increase 
the stock of protein in the body and katabolizing the remainder as fuel material. An increase of the protein supply above the minimum causes, after a relatively short time, the maintenance of the body protein at a higher level (411). The practical question in actual maintenance is far less as to the least amount of protein which may be used than as to the most advantageous level of protein nutrition; that is, as to the optimum of protein.

This question has been warmly debated in connection with human nutrition, having been brought to the fore especially by the investigations of Chittenden and his associates. ${ }^{1}$

On the whole it cannot be said that a considerable surplus of protein over the minimum requirement for maintenance that is, the maintenance of protein nutrition on a high plane - has been proved to be of any material advantage in the maintenance either of men or of domestic animals during periods covering several months. Whether a continued low protein diet through years or generations would show a different result is at present largely a matter of speculation. It is to be remarked, however, that the particular point under discussion is the protein requirement of the mature organism. That a deficiency of protein in the diet of a growing animal may have disastrous results is clear. If, however, the habitual food supply of a race of men or a group of animals is low in protein, the young are likely to share this deficiency with the mature, and it seems not impossible that this is an important factor in the alleged physical inferiority of certain races of men living on a low protein diet. This consideration warns us to exercise care in this respect in the management of the breeding herd.

420. Digestibility of low protein rations. - In the actual maintenance feeding of farm animals, the matter of the digestibility of the ration must also be considered. It has been shown $(723,724)$ that a relative deficiency of protein in the ration tends to depress the apparent digestibility of both the protein and non-nitrogenous nutrients, especially in the case of ruminants. A maintenance ration for these animals containing the minimum amount of protein together with the quantity of non-nitrogenous nutrients required to maintain the energy supply, would have a nutritive ratio (709), computed in the ordi-

1 Physiological Economy in Nutrition. 
nary way, of approximately $\mathrm{I}:$ I 2 . On such a ration there would, in all probability, be some loss of digestibility and an increase of its protein by 50 per cent might perhaps effect a gain in digestibility which would more than offset the increased cost, if any. Indeed, unless feeding stuffs especially poor in protein are used, it may often be difficult, even were it desirable, to reduce the protein content of a maintenance ration to the low level of absolute necessity.

\section{§ 2. The Ash Requirements for Maintenance}

421. Ash ingredients indispensable. - That a supply of the so-called mineral or ash ingredients, as well as of protein and of energy yielding materials, is necessary for the growth and maintenance of animals has been fully recognized since the time of Liebig, and was strikingly demonstrated by the wellknown experiments of Forster ${ }^{1}$ and of Lunin, ${ }^{2}$ which showed that animals supplied only with ash-free feed perished even sooner than when deprived of all feed.

Some of the reasons for these facts were indicated in the discussion of the functions of the nutrients in Chapter V (268-272), where it was shown that, besides their structural importance for both the skeleton and the soft tissues, the presence of ash ingredients in the body fluids is essential to the maintenance and regulation of the vital processes. Aside from the specific uses of single elements, such as iron, fluorin, iodin, etc., three general functions of the ash ingredients as a whole were there mentioned, viz., the maintenance in the body fluids and tissues of the normal osmotic pressure and of the relative concentration of the various ions, and, as a specific case of the latter, the preservation of neutrality.

422. Ash content of feed large. - Most feeding stuffs, however, and particularly the mixed rations of farm animals, contain what appear at first sight to be much larger amounts of ash ingredients than the body requires. Milk production, for example, causes an exceptionally large drain upon the ash content of the body, yet even rations made up of materials relatively poor in ash contain much larger amounts than are

${ }^{1}$ Ztschr. Biol., 9 (1873), 297.

${ }^{2}$ Ztschr. Physiol. Chem., 5 (188r), 3 I. 
found in the milk produced. Zuntz ${ }^{1}$ gives the following comparison of the ash ingredients in a ration recommended by Kellner for a cow producing 22 pounds of milk daily with the ash content of the milk yield :-

\section{Table 62. - Comparison of Ash Content of Ration and of Milk}

\section{In ration}

$88 \mathrm{lb}$. wet distiller's grain . I2

5.5 lb. meadow hay . . . I7

8.8 lb. straw . . . . . IO

$4.4 \mathrm{lb}$. dried potatoes . . 2

I.I lb. wheat bran . . . 7

2.2 lb. sesame cake

In $22 \mathrm{lb}$. milk .

\begin{tabular}{c|c|c|c|c|c}
$\begin{array}{c}\mathrm{CaO} \\
\mathrm{Grams}\end{array}$ & $\begin{array}{c}\mathrm{MgO} \\
\mathrm{Grams}\end{array}$ & $\begin{array}{c}\mathrm{K}_{2} \mathrm{O} \\
\mathrm{Grams}\end{array}$ & $\begin{array}{c}\mathrm{Na}_{2} \mathrm{O} \\
\mathrm{Grams}\end{array}$ & $\begin{array}{c}\mathrm{P}_{2} \mathrm{O}_{5} \\
\mathrm{Grams}\end{array}$ & $\begin{array}{c}\mathrm{Cl} \\
\mathrm{Grams}\end{array}$ \\
\hline & & & $\cdot$ & & \\
$\mathrm{I} 2$ & 24 & $\mathrm{I} 20$ & 20 & 52 & 8 \\
$\mathrm{I} 7$ & 8 & 43 & 2 & 9 & $\mathrm{I} 7$ \\
$\mathrm{IO}$ & 3 & 30 & 3 & 8 & 5 \\
2 & 4 & 45 & $\mathrm{I}$ & 9 & 3 \\
7 & 3 & 6 & 0.2 & $\mathrm{II}$ & 0.5 \\
25 & $\mathrm{I} 3$ & $\mathrm{I} 4$ & 4 & 32 & $\mathrm{I}$ \\
\hline 73 & 55 & 258 & 30.2 & $\mathrm{I} 2 \mathrm{I}$ & 34.5 \\
$\mathrm{I} 7$ & 2 & $\mathrm{I} 7$ & 4 & 20 & $\mathrm{IO}$ \\
\hline
\end{tabular}

Comparisons like the foregoing have tended to confirm the somewhat prevalent idea that rations adequate in other respects may be assumed to contain a sufficiency of ash ingredients. This is doubtless true of animals living in a state of nature but it is a questionable assumption under the artificial conditions to which many farm animals are subjected, as when receiving an excess of some single grain like Indian corn or of technical by-products, or when stimulated to a high degree of production.

423. Ash ingredients digestible. - It is true that of this relatively large supply of mineral matter in ordinary rations, a very considerable fraction, especially of certain elements, is found in the feces, and this fact has led to their being regarded as relatively indigestible. As was stated in Chapters III and IV $(164,199)$, however, this apparently low digestibility arises from the fact that the intestinal tract constitutes the normal path of excretion for certain elements, notably, in the case of herbivora, for calcium and phosphorus. Thus Forster has shown that in the dog the calcium of the feed is largely resorbed

${ }^{1}$ Jahrb. Deut. Landw. Gesell., I9I 2, p 570. 
in the upper part of the intestine where the contents are acid, while more or less of it is excreted again in the lower intestine. While it is impossible, therefore, to determine by means of the ordinary digestion experiment how much of such ingredients have actually been resorbed and excreted again and what proportion has escaped digestion, it appears safe to conclude that at least a considerable share of them has been dissolved and resorbed in the upper digestive tract and that the insufficiency of certain rations as regards mineral ingredients is not due to the indigestibility of the latter.

424. Contrast between organic and inorganic nutrients. There is an obvious distinction between organic and inorganic nutrients. The former may be said to be destroyed in the performance of their functions. A molecule of dextrose or of stearin, for example, can yield energy to the body only by being split up and oxidized step by step to carbon dioxid and water. The case is similar with protein so far as it is used for fuel purposes and even its specific functions seem to involve the cleavage and oxidation of its molecules. With the electrolytes contained in the body the case is different. A molecule of disodium phosphate, for example (or its ions), is not destroyed by the performance of its functions in maintaining neutrality no matter how long it serves that purpose and the molecule of sodium chlorid contributes its quota to the osmotic pressure of the blood serum as long as it remains dissolved in that fluid. Only as it escapes from the body will the need for a fresh supply arise.

\section{Losses of ash}

425. Causes of loss. - So far, therefore, as the maintenance of mature animals is concerned, the magnitude of the ash requirement will be substantially determined by the rate at which the various elements are eliminated from the body through the excretory organs. In growing animals there is in addition, of course, the demand for ash ingredients for structural purposes, both for the building up of the skeleton and to a less degree of the soft tissues, but even in this case the total ash requirement is determined in large degree by the rate of excretion. 
Some of the more important factors leading to the excretion of ash ingredients from the body and hence to the depletion of its stock are considered in the following paragraphs:-

426. Maintenance of osmotic pressure. - In the discussion of excretion in Chapter IV it was stated (198) that the essential function of the kidneys is to maintain a constant composition of the blood, those organs acting somewhat like an overflow valve by means of which any excess of a substance above the normal limit begins to be excreted. In this way the osmotic pressure in the body is regulated and an excess of any salt in the feed, sodium chlorid for example, is disposed of.

The matter is not quite so simple, however, as would appear from the foregoing statement. The action of the kidneys in eliminating surplus salts and so preventing an increase of osmotic pressure is not confined to the particular salt supplied in excess, but extends to others also. This is most strikingly shown in the case of the alkalies. If, for example, unusual amounts of potassium salts are consumed, an increased excretion of this element results in the urine, but the need of keeping the osmotic pressure at its normal level seems to be so great that more or less of the sodium salts are also excreted, even though their concentration in the blood may not be above the normal.

This relation as regards potassium and sodium has been shown by the well-known investigations of Bunge. ${ }^{1}$ who holds that the desire for common salt on the part of herbivora is due to the presence of relatively large amounts of potassium in their feed and the consequent tendency towards impoverishment of the body as regards sodium. The occurrence of salt hunger in animals receiving feed with an abnormal ratio of potassium to sodium has been explained in the same way.

In other words, the effort of the body to maintain the osmotic pressure of its fluids by removing a surplus of some one ingredient may bring about an impoverishment as regards other elements and so create a need for an increased supply of the latter in the feed.

427. Maintenance of neutrality. - Attention was called in Chapter V (271) to the fact that practical neutrality of the blood serum and lymph is necessary for the normal functioning

${ }^{1}$ Physiologie des Menschen, I905. 
of the body cells and to the important part played by the ash ingredients in maintaining this neutrality.

a. Acidosis. - A variety of chemical processes, both normal and pathological, occur in the body which tend to disturb its neutrality either by the addition of acid or the giving off of alkali so as to produce the condition known as acidosis, by which is meant a relative excess of acid over basic radicles.

A well-known example of pathological acidosis is that observed in diabetes. The perverted metabolism of this disease results in the production of large amounts of oxybutyric acid (266), which is neutralized to a certain extent by means of ammonia derived from the katabolism of protein but whose gradual accumulation finally results in the diabetic coma. Another example is the form of infantile acidosis in which an excess of fat in the food results in the formation of insoluble calcium salts of the fatty acids in the intestines and so removes basic ingredients in the feces. It has been suggested that the failure of young animals to thrive on milk exceptionally rich in fat may be due in part to the same cause.

Several sources of acid exist in the normal organism.

First, acids may be consumed as such, either in natural products or in fermented materials like silage. These acids are neutralized by the alkalies of the saliva or of the pancreatic juice, which are thus temporarily withdrawn from the body fluids. After resorption, however, the resulting alkali salts of the more common acids are readily oxidized, yielding carbon dioxid and water and restoring to the body fluids the bases previously withdrawn. Small amounts of some acids, such as tartaric and malic, however, tend to escape oxidization and to be excreted in the urine, carrying a corresponding amount of base with them. Oxalic acid and its salts are oxidized with difficulty and tend to impoverish the body in calcium by the formation of the insoluble calcium oxalate. This acid is liable to be especially injurious to horses and swine and to young ruminants, while in mature ruminants it seems to be largely destroyed by fermentation in the first stomach.

Second, the fermentations in the paunch of ruminants are a source of large amounts of organic acids which, like those contained in the feed, may cause a temporary withdrawal from the body fluids of alkali which is later restored when the salts are katabolized. 
Third, the considerable amount of hippuric acid produced by herbivora makes a very considerable draft upon the organism for bases. Thus, in four experiments by Diakow cited by Zuntz, it was equivalent to from $\frac{1}{4}$ to $\frac{1}{2}$ of the total excess of bases over inorganic acids in the urine.

Fourth, in the katabolism of the proteins, nucleo-proteins and other compounds containing sulphur and phosphorus, these elements are largely oxidized to sulphuric and phosphoric acids. The sulphur of one pound of protein having the composition of serum albumin, for example, if fully oxidized, would yield the equivalent of nearly one ounce by weight of concentrated sulphuric acid. High protein rations, therefore, tend to bring about a loss of bases from the body.

b. Neutralization of acids. - In all these various ways there is a constant tendency to disturb the neutrality of the body fluids and towards the establishment of an acidosis, to prevent which the acids must be neutralized. The significance of this was first shown by the experiments of Lunin already referred to (421), which showed that the life of animals fed on ash-free feed could be considerably prolonged by the addition of sodium carbonate to neutralize the acids produced in the body. Normally, this neutralization is accomplished in two general ways.

First, an excess of acid may be combined with the ammonia which is produced from the amino acids in the katabolism of protein (233) and is subsequently converted into urea in the liver. A part of this ammonia, however, may be diverted from this course and utilized to neutralize acids, the resulting ammonium salts being excreted in the urine in place of a corresponding amount of urea. The ammonia arising from the putrefactions in the lower intestines (140) may serve the same purpose. A small quantity of ammonium salts, arising from the neutralization of the acids produced especially in the protein katabolism, is normally found in the urine, while the feeding of inorganic acids or their injection into the blood stream, or a pathological acidosis, may greatly increase their amount.?

On the basis of early experiments upon rabbits it has been taught that the ability to neutralize acids by means of ammonia is peculiar to carnivora and omnivora and is present to a very limited extent in 
herbivora. It seems a priori unlikely that such a difference in the metabolic processes should exist, and later investigations have shown that there is no such fundamental distinction between the various species. It should be remembered, however, that the protein metabolism of herbivorous animals is often on a relatively low plane, and that consequently relatively less ammonia may be available than in the case of carnivorous animals.

Another phase of the matter, which has received little consideration, is the possibility that the long-continued presence of ammonium salts in the body may have an injurious effect. The possibility of injury through acid rations in this way could hardly be determined except by means of experiments, covering, if possible, the whole life cycle of the animal.

Second, an excess of acid may be disposed of by combination with the fixed bases present in the body. These are, in the first instance, those contained in the carbonates and phosphates of the blood and other fluids. Henderson, as already noted, has shown that these salts are present in the blood serum in such proportions that relatively large amounts of acids may be disposed of in this way without materially altering the reaction of the blood.

c. Excretion of acids. - The neutralization of acids produced in the body does not, however, necessarily involve the excretion of an equivalent amount of base. It is a familiar fact that the urine may possess a considerable degree of acidity. The work of Henderson shows that the kidneys are able to separate more or less of the phosphoric acid from the bases of the blood, excreting it as acid phosphates in the urine and retaining a corresponding amount of bases in the blood.

428. The skeleton as a reserve of ash ingredients. - The store of bases in the body fluids, however, is limited. The larger part of the ash of the body is contained in the skeleton, which constitutes a relatively large reserve of basic phosphates and carbonates which may be drawn upon to supplement the supply in the blood. This fact has an important bearing on the question of the necessary ash supply in the feed, while it must likewise be taken into account in experimental work. Long-continued maintenance on abnormal feeds or under conditions favoring acid production in the body may result in extracting from the body comparatively large amounts of mineral matter even to 
the extent, apparently, of bringing about pathological conditions, while on the other hand, normal feeding and conditions may enable such losses, if not too extensive, to be made good. The point which is of special importance is that these fluctuations of the ash content of the skeleton affect the ash as a whole. It was found by Aron that the composition of the bone ash as given in Chapter II (81) remains practically constant even when the skeleton has been greatly impoverished in total ash. In particular this has been shown to be true not only of the calcium and phosphoric acid of the bones but also of the minor ingredients such as carbonic acid, magnesium and even sodium. A draft upon the skeleton for sodium, for example, could be met only by the mobilization of an amount of total bone ash containing the requisite quantity of sodium, and this would result in throwing into the circulation relatively large amounts of calcium and phosphoric acid for which there may be no requirement, thus raising the percentage of these ingredients above the normal limit and leading to their excretion.

\section{Maintenance of ash balance}

429. Relation to feed. - The foregoing paragraphs clearly indicate that the ash requirements for maintenance depend chiefly on the amounts of the various ash ingredients which, for one reason or another, are thrown into the circulation in excess of the body's needs and are therefore removed by the excretory organs, and furthermore, that the nature of the feed consumed, particularly the relative proportions of its ash elements, is an important factor in determining these losses.

430. Deficiencies in ash ingredients. - Some feeding stuffs contain relatively little total ash and are especially deficient in particular elements. The most striking and familiar example of this is maize. According to Henry and Morrison ${ }^{1}$ average maize contains about I. 8 per cent of total ash, while its lime content is only 0.02 per cent and that of soda only 0.04 per cent. Some by-product feeds are similarly poor in particular ingredients. Obviously such feeds are not by themselves well adapted

${ }^{1}$ Feeds and Feeding, I 5 th Ed., p. 672. 
for growing or milking animals, in which a storage of ash in the product occurs. For the simple maintenance of mature animals, however, which is the topic under discussion, the question whether the small amount of lime present, for example, is adequate depends upon the rate at which lime is being lost from the body. If this loss could be reduced to zero, a feeding stuff containing no lime whatever would seem to be adequate for maintenance so far as that substance is concerned. In general, whether a feeding stuff or ration is to be regarded as containing an insufficient amount of some ash element for maintenance depends largely on how it affects those body functions which determine the rate of excretion of that element.

431. Acid and basic ash. - It is usually considered that the most important relation of feed in the respect just mentioned is that which it bears to the maintenance of neutrality in the body fluids. Feeding stuffs or rations containing in assimilable form much sulphur or phosphorus, for example, tend to cause the production in the body of corresponding amounts of sulphuric and phosphoric acids which must be neutralized. On the other hand, feeding stuffs containing large proportions of the bases tend to have the opposite effect. The relation of acid to basic elements has, therefore, an important bearing upon the suitability of a feeding stuff for ash maintenance. ${ }^{1}$

Feeding stuffs differ widely in this respect. In general it may be said that the concentrates contain relatively much phosphorus and sulphur, little calcium and only moderate amounts of potassium and sodium, while the roughages, especially those of better quality, are rich in calcium and alkalies and low in sulphur and phosphorus. A definite measure of these differences as related to the maintenance of neutrality in the body is obtained by converting the percentages of the several ash ingredients into chemical equivalents.

Alfalfa hay, for example, according to Henry and Morrison, ${ }^{2}$ contains in one kilogram the amounts of ash ingredients shown in the first column of the following statement. Dividing

${ }^{1}$ Evidently the sulphur and phosphorus present in organic combination must be included in such comparisons as well as the elements present in the form of electrolytes. In the older ash analyses the sulphuric acid represents only that part of the sulphur remaining after the material has been ashed, which, as is now known, is but a small part of the total sulphur.

${ }^{2}$ Feeds and Feeding, I 5 th Ed., p. 672. 
the amount of each by its equivalent weight gives the gram equivalents shown in the last two columns, showing that a kilogram of this feed contains I.300 gram equivalents of excess base.

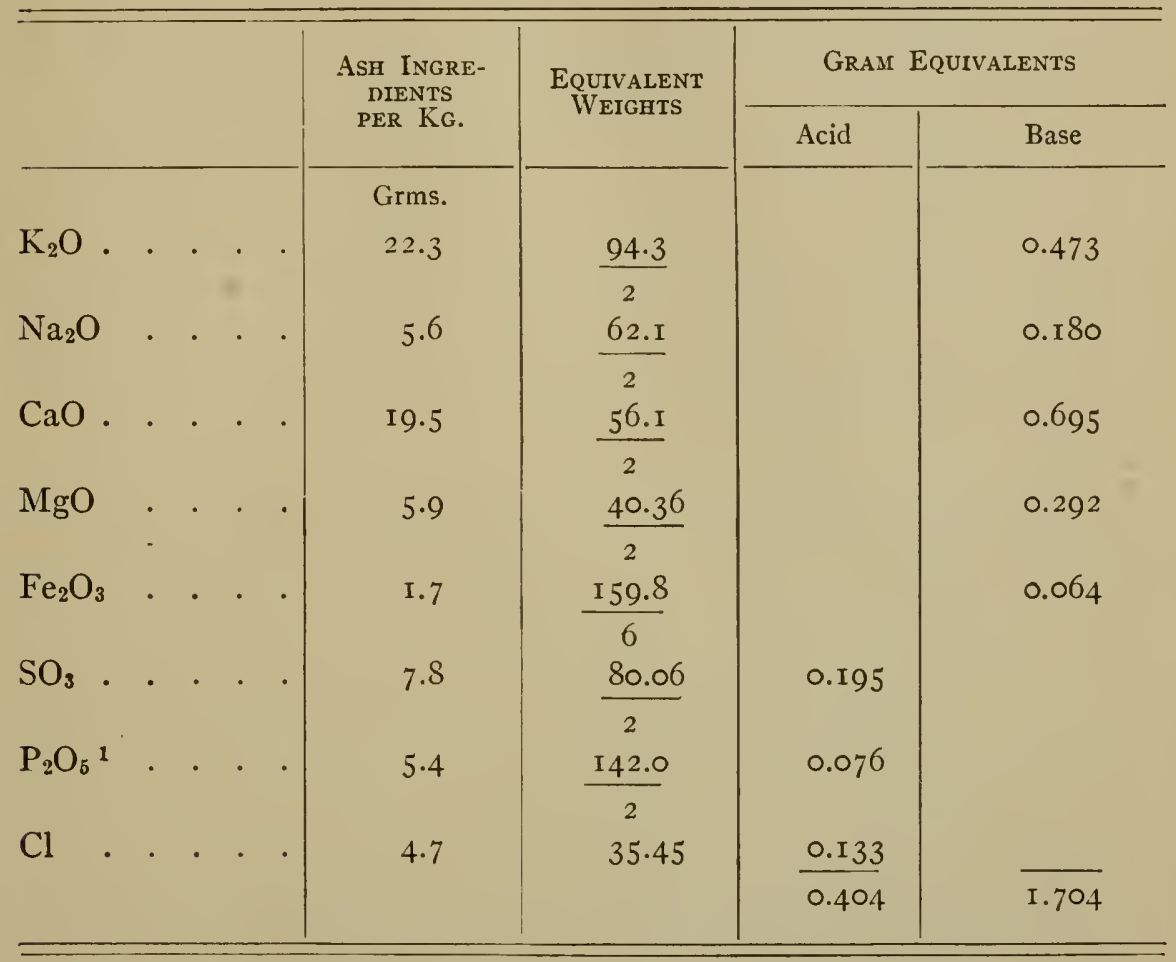

The results of a considerable number of computations of this sort by Forbes ${ }^{2}$ are contained in Table $\mathrm{X}$ of the Appendix, the equivalents of bases and acids being expressed in cubic centimeters of normal solution. The table shows clearly that some feeding stuffs, like the hays, for example, contain a considerable excess of basic ingredients, while others have an excess of acidforming elements. The ratio of phosphorus to calcium, too, which is a special case of the ratio of acids to bases, shows considerable variations.

432. Significance of acidity in ash. - Much stress had been laid on this distinction between feeding stuffs with acid or

1 Phosphoric acid is regarded as neutralized when two of its hydrogen atoms are replaced by basic elements.

2 Ohio Expt. Sta., Bul. 255. 
alkaline ash in discussions of both human nutrition and that of domestic animals. Doubtless the point is an important one but the assumption that all excess of acid over basic elements in the diet should be avoided seems hardly warranted, especially as regards maintenance. The fact that the body is to a certain extent provided with a means of defense against excessive acids through its ability to neutralize them by means of ammonia and through the power of the kidneys to separate acids and bases is sufficient to show that an excess of acid-forming elements in the feed is not necessarily injurious. It is only when the excess is so large as to exceed the capacity of these regulative arrangements and when it therefore begins to draw on the fixed bases of the body, or possibly when it causes the production of large quantities of ammonia, that it becomes a source of danger.

433. Alkali ratio of ash. - As indicated in previous paragraphs, the ratio of potassium to sodium in a feeding stuff may have an important bearing on the losses of ash from the body. It was there stated that while a surplus of potassium salts resorbed into the blood is promptly disposed of by excretion through the kidneys, it may carry along with it more or less sodium, so that a ration relatively rich in the former may tend to impoverish the body in the latter. Such a loss of sodium from the body, it would appear, might have serious indirect effects if continued long enough to cause a draft on the stock of sodium in the skeleton. Such a draft, as already said (428), involves the solution of a corresponding amount of the total ash of the skeleton, so that the bones would be impoverished in other ingredients, especially calcium and phosphoric acid, as well as sodium. In fact it has been found that fodders that cause malnutrition of the bones resulting in the disease known as rickets (Rachitis) usually show a misproportion of potassium to sodium. Zuntz ${ }^{1}$ cites the following comparisons of the ash of normal hay with that of hays causing the disease. Along with a somewhat greater ratio of phosphoric acid to calcium, the injurious hays show a very striking difference in the alkali ratio, as appears from the following table to which the corresponding figures for cow's milk have been added for comparison :-

\footnotetext{
${ }^{1}$ Jahrb. Deut. Landw. Gesell., I9I2, p. 577.
} 
Table 63.-Alkali Ratio of Ash

\begin{tabular}{|c|c|c|c|c|c|c|c|}
\hline . & & & & & $\mathrm{K}_{2} \mathrm{O}$ & $\mathrm{Na}_{2} \mathrm{O}$ & $\begin{array}{c}\text { Ratio } \\
\mathrm{Na}_{2} \mathrm{O} \text { TO } \\
\mathrm{K}_{2} \mathrm{O}\end{array}$ \\
\hline 1 & & - & & & $\%$ & $\%$ & I : \\
\hline Normal Brandenburg hay . . & . & . & . & & 20.00 & 5.43 & 3.68 \\
\hline Very injurious Brandenburg hay & & . & & & 37.65 & 1.74 & 21.64 \\
\hline Less injurious Brandenburg hay & & & • & & $33 \cdot 54$ & 2.50 & I 3.42 \\
\hline Normal Schwarzwald hay . . & . & . & 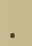 & & 20.88 & 4.40 & 4.75 \\
\hline Injurious Schwarzwald hay & . & . & . & & 37.40 & $0.2 \mathrm{I}$ & I78.10 \\
\hline Cow's milk . . . . . . . & · & - & · & & 34 & 8 & 4.25 \\
\hline
\end{tabular}

434. Balancing of ash ingredients in the ration. - While the animal body has a considerable degree of adaptability to variations in the ash supply, and while, during short periods, relatively large errors in this respect may be compensated for out of the comparatively large stock of ash in the body, nevertheless, it is clear from previous paragraphs that in the long run a reasonably close balance of the ash ingredients in the ration is necessary, and tables like that of the Appendix have been computed as guides for this purpose. That they convey useful information cannot be denied, but any attempt to base actual estimates regarding ash maintenance or the ash balance on such data overlooks some important considerations.

Ash not entirely digestible. - It must be remembered that not all of the ash ingredients of the feed can be assumed to be digested and resorbed. It is true that much of the ash found in the feces has really been digested and excreted again in the lower intestines but this is by no means true of the entire quantity. In the case of herbivora, especially, a considerable share of the dry matter of the feed escapes digestion and it can hardly be doubted that it carries with it into the feces more or less of its ash elements. This is particularly true of the sulphur and phosphorus of the proteins and the nucleoproteins. So far as these escape digestion, they carry their organic sulphur and phosphorus with them into the feces without giving it opportunity to contribute to acid production in the body. How far the same thing is true of the other ash elements it is impossible 
to say, since there is at present no way to determine how much of any particular ash ingredient found in the feces consists of undigested material and how much is to be regarded as an excretory product. This being the case, a table like that of the Appendix can give only a general and approximate idea of the total quantity of ash or of the balance of its basic and acid elements or of the alkalies in the materials actually resorbed and entering into the metabolism.

Influence of supply on excretion. - Moreover, it is necessary to take into consideration the influence discussed in previous paragraphs (429-433) of the nature and proportions of the ash ingredients actually resorbed upon their excretion. For example, potassium may lead to a loss of sodium and this in turn to losses of calcium and phosphoric acid, thus possibly affecting to a considerable extent the ratio of acid to basic elements in the excreta. Adding to this the facts that more or less of the acids produced in the body may be neutralized by ammonia instead of by fixed bases ( $427 b$ ), and that the kidneys have the power, in some species at least, to separate acids from bases (427 c), leading especially to excretion of acid phosphates, it is evident that the data of the table may fall considerably short of representing the actual value of the feed as regards maintenance of the ash balance.

435. The ash balance. - These considerations render it evident that the value of conclusions as to the balance of income and outgo of ash elements drawn from the composition of the feeding stuffs concerned must be more or less problematical, particularly as regards farm animals. Such conclusions are more or less probable deductions from the facts outlined in previous paragraphs regarding the functions of ash in the body and need to be checked by direct experiments. The actual effect of a feeding stuff or ration on the ash maintenance of herbivora can be determined with certainty only by means of direct comparisons of the income and outgo of all the ash elements, i.e., by determinations of the amounts contained in feed, feces and urine (metabolism experiments) or by comparative analyses of carefully selected test and control animals (comparative slaughter tests).

Data of this sort for mature animals are very scanty but some tentative conclusions may be drawn from experiments by 
Diakow ${ }^{1}$ and Cochrane, ${ }^{2}$ each on a single steer. Diakow's experiments include four periods on mixed rations containing much hay. In Cochrane's experiments alfalfa hay constituted the sole feed, supermaintenance, maintenance and submaintenance rations being consumed. Accordingly, the total ration contained in every instance a considerable excess of bases. Under those conditions, not only the feces but likewise the urine showed an excess of bases over acids, i.e., the animal was engaged in getting rid of excess bases. The net residue which was retained in the body consisted of basic material of rather constant composition even in the case of Diakow's nearly mature animal.

So far as they go, then, these experiments confirm the conclusion that with rations containing a large proportion of roughage, there is no reason to fear losses either specifically of fixed bases or in general of total ash. Such would almost always be the case with the ordinary maintenance rations of cattle, sheep and horses. Swine, on the other hand, if maintained entirely on grain, might very well receive rations not well balanced as regards ash, and experiments and observations which are discussed in Chapter XI (492-496) seem to indicate that even for maintenance the ordinary grain ration, especially if it consists largely of maize, should have its ash composition corrected. The effect of an acid ash in the mixed rations of herbivora, and the extent to which such acidity can be taken care of in the body without drawing on its reserves of ash, has still to be investigated. In view of the large amounts of surplus bases excreted under the conditions of Diakow's and Cochrane's experiments, it would seem likely that even a considerable excess of acid elements might be neutralized without drawing on the stock of fixed bases in the body.

In Diakow's experiments, the minimum quantities of O.II5 lb. calcium and $0.045 \mathrm{lb}$. phosphorus in the feed per Iooo pounds live weight sufficed to support not inconsiderable gains by the body. In Cochrane's experiments, a minimum of $0.147 \mathrm{lb}$. calcium per rooo pounds also resulted in a gain, while $0.039 \mathrm{lb}$. phosphorus was just sufficient for maintenance. In Henneberg's investigations ${ }^{3}$ upon the maintenance of cattle, however, dis-

${ }^{1}$ Landw. Jahrb., 44 ( $\operatorname{rg}_{3}$ ), 8.33. $\quad 2$ Penna. Inst. of An. Nutr., unpublished results. 1 Beiträge, etc., Heft, 1 (1860), p. II3. 
tinctly smaller amounts, viz., $0.090 \mathrm{lb}$. of calcium and $0.02 \mathrm{I} \mathrm{lb}$. of phosphorus proved adequate for maintenance. Weiske ${ }^{1}$ found that a mature sheep gained small amounts of ash ingredients on a ration of meadow hay containing, per Iooo pounds live weight, 0.I79 lb. calcium and $0.045 \mathrm{lb}$. phosphorus.

436. Correction of ash deficiencies. - As regards maintenance, it seems clear that the ash requirement is a qualitative rather than a quantitative one; i.e., that it is the proportions far more than the total amounts of ash ingredients that are important. If, then, there is reason to fear that the ash supply in the ration is inadequate for maintenance, any measures taken to remedy this must be directed chiefly toward the correction of the misproportion between different ingredients and only secondarily to an increase of their total quantity.

One method of effecting such a correction is by the direct addition of mineral matter. In attempting to correct deficiencies in this way, however, the simple addition of more ash material to the ration may not be effective. It is necessary also to take into account the nature of the defects to be made good. Maize, for example, has already been instanced (430) as a feeding stuff peculiarly low in ash, the exclusive use of which, even for maintenance, might readily lead to a loss of ash from the body. Maize is especially deficient in calcium and its exclusive use would be liable to cause a loss of this element. The attempt to supply additional calcium, however, by the addition of such materials as calcium phosphate or sulphate would not help the situation materially because the ash would still remain acid and thus capable of causing a loss of fixed bases irrespective of the additional amount of calcium present. On the other hand the addition of calcium in the form of carbonate, by the use of precipitated chalk or wood ashes, not only supplies additional calcium but remedies the acid condition which leads to a loss of that element, as has been well demonstrated in the numerous experiments on growing swine referred to in Chapter XI (496). The correction of the ash composition of hays causing malnutrition of the bones, like those instanced by Zuntz (433), presents quite different requirements. The very injurious Brandenburg hay, e.g., contained the following percentages of ash ingredients:-

${ }^{1}$ Landw. Jahrb., 9 (I880), 290. 
Table 64. - Ash of Hay Causing Rickets

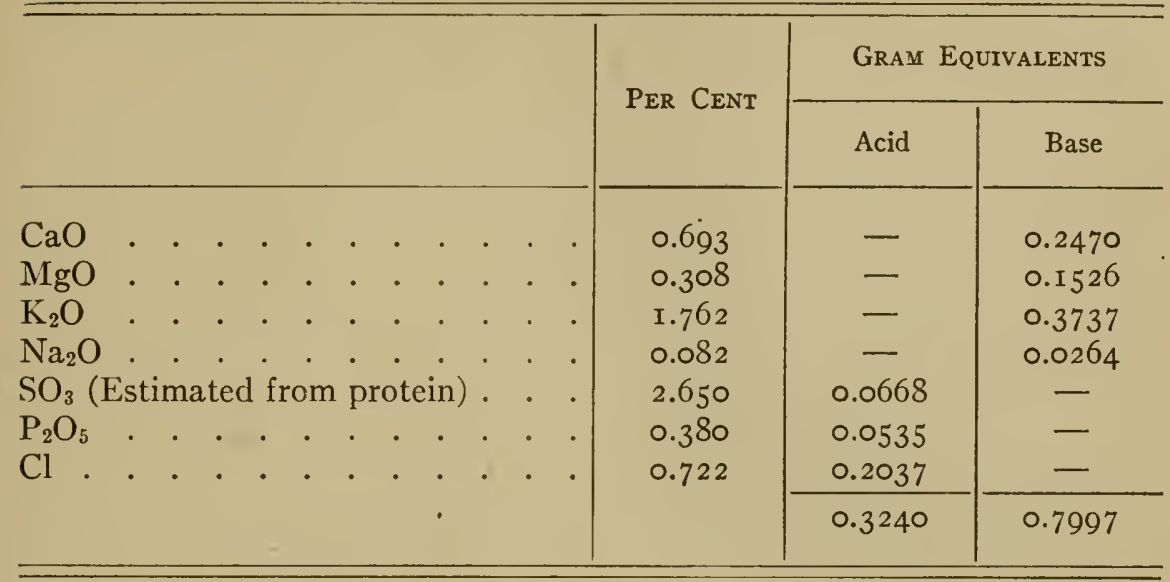

Such a hay is relatively deficient in calcium and phosphorus and would presumably be improved by the addition of calcium phosphate, while the addition of calcium carbonate would probably be unnecessary, since the hay contains an excess of basic over acid ingredients. In addition, however, to its deficiency in calcium and phosphorus, it shows a misproportion of potassium to sodium, which, as already explained, would tend to increase the excretion of calcium phosphate unless sodium salts, particularly the chlorid, were added.

In the mixed rations of herbivora, however, direct addition of mineral matter is seldom likely to be necessary unless roughage of abnormal quality is employed. Usually the surplus of bases in forage crops will more than balance the surplus of acid elements in the concentrates used, while the common salt ordinarily given as a condiment will more than balance any probable excess of potassium in the rations. Unusual rations, such perhaps as very heavy grain rations, those containing an unusual proportion of protein, or those made up of unusual feeds may form an exception to this general rule and require special consideration. In the case of swine, such a balancing of one feeding stuff against another as regards ash ingredients is less practicable, and the securing of a proper balance needs more attention. Until, however, more determinations of the actual ash balance of different species on different classes of rations are made (435) it is hardly possible to state with any 
definiteness what proportions of the different ash elements are required in maintenance rations and the whole subject offers a wide field for investigation.

\section{§3. AcCessory Substances}

Leaving out of account the fact that the proteins are a minor source of energy and considering only the requirements for matter, the proteins and ash elements of the feed are required substantially for maintenance, i.e., to make good losses of the structural elements of the body, especially if the ash content of the body fluids be included under this designation. Recent investigations, however, have revealed the presence in the feed of minute amounts of substances which appear to bear quite a different relation to nutrition and which may be called for convenience, accessory substances.

437. Vitamins. - Attention was first called to these accessory substances through investigations into the cause of the tropical disease known as beri beri. It has been shown that this is a nutritional disease, resulting from a preponderance in the diet of so-called "polished" rice, i.e., rice from which the seed coats have been removed. It is a tropical disease only in the sense that many inhabitants of the tropics subsist largely on rice. It has been shown that it can be produced in Europe by the excessive use of this grain.

Substantially the same disease (polyneuritis) may be induced in animals, especially in fowls, by an exclusive diet of polished rice and it is to experiments on these animals that most of our imperfect knowledge of the subject is due.

It has been shown that in man beri beri may be prevented by the use of a rational dietary, and especially by the substitution of rough rice or of other grains for polished rice. Experiments on animals have shown that a subject fed on polished rice until nearly at the point of death may be restored to normal condition in a short time by the administration of small amounts of an aqueous extract of rice bran, the improvement being so rapid as to appear almost miraculous. The generally accepted explanation is that the bran contains a small amount of a watersoluble substance or substances necessary for the normal functioning of the body, the lack of which in polished rice gives 
rise to the disease. Aqueous extracts of other substances, notably yeast, are capable of producing the same curative effects. The substances themselves have not yet been isolated but Funk ${ }^{1}$ who has been prominent in this line of investigation, has given them the general name of vitamins. It is thought that other nutritional diseases, such as scurvy and pellagra, as well as the cotton-seed poisoning of swine, are likewise due to the use of diets deficient in these vitamins.

438. Growth substances. - Very interesting facts of a somewhat similar character have been observed regarding growth, notably by Hart and McCollum, and by Osborne and Mendel. It seems to have been demonstrated that there are associated with certain fats, such as butter fat, the fat of egg yolks, cod liver oil, etc., substances whose absence from a ration otherwise adequate renders it incapable of permanently supporting growth. That this substance (or substances) differs from the vitamins of Funk seems apparent from the fact that normal maintenance may apparently be secured on rations from which it is absent.

The very interesting results obtained by Hart, McCollum, Steenbock and Humphrey ${ }^{2}$ with cows fed rations properly balanced according to the ordinary criteria but made up from the products of single plants (wheat, oats, maize) suggest that substances similar to the vitamins or the growth substances may play an important part in the nutrition of farm animals. These rations when continued for two or three years manifested specific differences in nutritive effect as regards growth and reproduction, although all of them seemed to be fairly adequate for the maintenance of live weight.

Most investigations upon these accessory substances, however, have been directed to their relation to growth and further discussion of their functions in nutrition may therefore be deferred until that subject is considered.

1 Ergeb. Physiol., 13 (I913), I 25.

${ }^{2}$ Wis. Expt. Sta., Research Bul., No. I7 (I9II). 


\section{CHAPTER X}

\section{THE FATTENING OF MATURE ANIMALS}

439. Disposal of surplus feed. - When an animal consumes feed in excess of that required simply for maintenance, a production of some sort results. The surplus feed may be transformed into material products as flesh, fat, milk, etc., which are stored up in the body or secreted, or it may be katabolized and its energy expended in the performance of work.

One of the simplest and most familiar examples of such production is afforded by the fattening of mature animals. Such fattening, it is true, is not of great economic importance, since the larger share of the world's meat supply is derived from animals which have not yet reached full maturity. Fattening, however, as well as growth, forms an essential part of the production of at least the better grades of meat, and while it is practiced largely on immature animals its feed requirements can be studied to better advantage in the mature animal. The purpose of the present Chapter is to consider the general nature of the fattening process and the demands which it makes upon the feed supply, leaving its economic aspects for discussion in connection with meat production.

440. Fattening requirements. - Just as the quantities of matter and energy required for maintenance depend, in the first instance, upon the amounts lost from the body during fasting, so the quantities which must be supplied in excess of maintenance to support the fattening process will depend primarily on the amount and composition of the gain made. The obvious first step in considering the feed requirements of the fattening animal, therefore, is a study of the composition of the increase.

\section{§ I. Composition of the Increase in Fattening}

441. Increase chiefly fat. - The discussions in previous chapters have rendered it evident that the chief function of 
the fat contained in the animal body is that of a reserve of energy for the vital activities, which may be drawn upon when the feed supply is insufficient and replaced when feed is abundant, while the protein of the mature animal is subject to much smaller fluctuations. It would be expected, therefore, that the gain made by a mature animal on a liberal ration would consist largely of fat. That such is indeed the case has been shown in two ways, viz., by means of comparative analyses of the carcasses of lean and fattened animals, i.e., comparative slaughter tests (284), and by means of balance experiments (285) from which the composition of the organic matter gained may be computed.

442. Comparative slaughter tests. - The classic example of this method is Lawes and Gilbert's well-known investigation ${ }^{1}$ into the composition of animals slaughtered for human food, the results of which are recorded in Chapter II (97).

The two pigs analyzed were from the same litter, and were believed to be very closely comparable at the beginning, so that it was possible to compute directly the composition of the increase during fattening. The other animals analyzed were not regarded as comparable. In order to estimate the composition of the increase in cattle and sheep, Lawes and Gilbert compute the weights of protein, fat, ash and total dry matter contained in the bodies of a large number of animals before and after fattening, using in the former case the analytical results obtained on the half-fat ox and the store sheep and in the latter those on the fat ox and fat. sheep. The differences, of course, show the gain of each ingredient. In the case of sheep and swine, they utilize the results of their own fattening experiments. In the case of cattle, the computations are based upon the results of experiments by others. The oxen whose composition was compared were mature animals. The sheep, on the other hand, were yearlings. Neither the age nor the weight of the pigs is stated, but their pig feeding experiments in general were made with animals ranging from somewhat over $100 \mathrm{lb}$. to $160 \mathrm{lb}$. in weight. The results as to this species, therefore, presumably relate to only partially mature animals.

In I $876-1877$, Henneberg, Kern and Wattenberg ${ }^{2}$ investigated the composition of the increase in weight of mature sheep in fattening. Their analyses were of the carcasses only but the

${ }^{1}$ Phil. Trans., II, r859, p. 493.

${ }^{2}$ Jour. Landw., 26 (1878), 545. 
weights of the offal parts were recorded, so that it is possible to compute approximately the weight of the fat-free body, exclusive of the contents of the digestive tract and of the wool.

Similar comparisons based on 75 and 82 day fattening periods with swine were reported by Soxhlet ${ }^{1}$ in $188 \mathrm{I}$ and the results of short fattening experiments ( 16 to 37 days) on geese by $\mathrm{B}$. Schulze $^{2}$ in 1882 and by Chaniewski ${ }^{3}$ in 1884 , the primary object of each case being a study of the sources of animal fat. Friske $^{4}$ in Igog and Pfeiffer and Friske ${ }^{5}$ in I9I I, in a study of the gain of protein by mature animals during a fattening period of about Ioo days, likewise reported a number of partial analyses of mature sheep similar to those made by Henneberg, Kern and Wattenberg.

Table 65. - Composition of Increase in Live Weight in Fattening

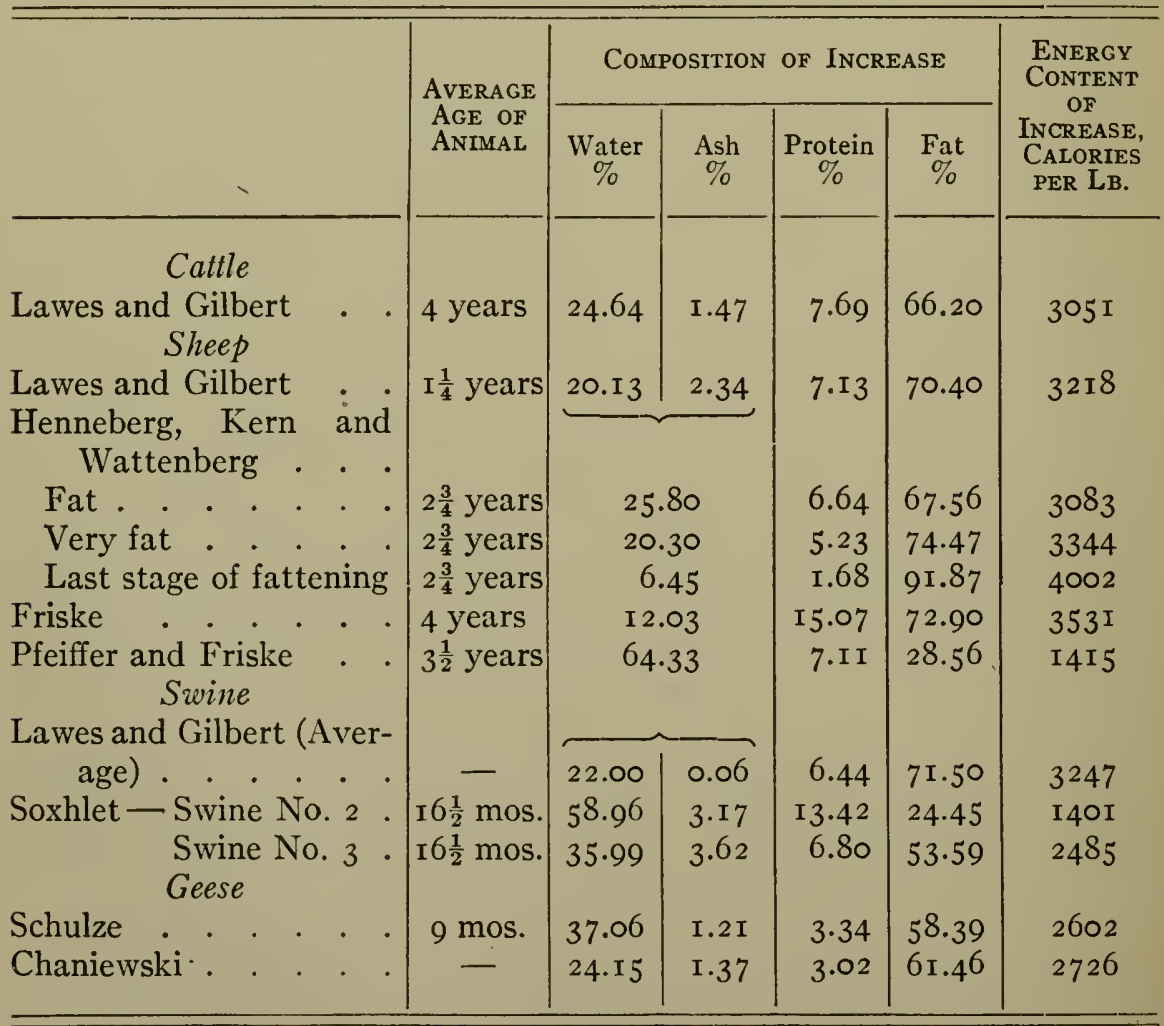

${ }_{1}^{1}$ Centbl. Agr. Chem., 10 (I88r), 674.

${ }^{2}$ Landw. Jahrb., 11 (1882), 57.
3 Ztschr. Biol., 20 (I 884), I79.

4 Landw. Vers. Stat., 71 (1909), 44r.

\section{Ibid., 74 (1911), 409.}


The results of these comparative slaughter tests, so far as they relate to the composition of the increase, are summarized in Table 65, which includes also the computed energy content of the increase.

443. Respiration experiments. - Respiration experiments on mature animals have fully confirmed the results of slaughter tests as 'regards the proportion of protein to fat in the increase, as appears from the summary of Table 66.

By far the most extensive respiration experiments are those made at the Moeckern Experiment Station ${ }^{1}$ by G. Kühn and by Kellner on mature fattening cattle. Of the 60 reported experiments in which there was a gain of both protein and fat, only 3 show less than 70 per cent of fat in the total organic matter gained and only 3 , a percentage above 95. Rejecting these 6 and grouping the remainder according to the percentage of fat gives the results shown in the first five lines of the table. To these are added the results of earlier experiments by Henneberg, Fleischer and Müller ${ }^{2}$ on sheep and by Meissl ${ }^{3}$ on swine. The gains of ash and of water were not determined in these experiments.

Table 66. - Proportions of Protein and Fat in Fattening Increase

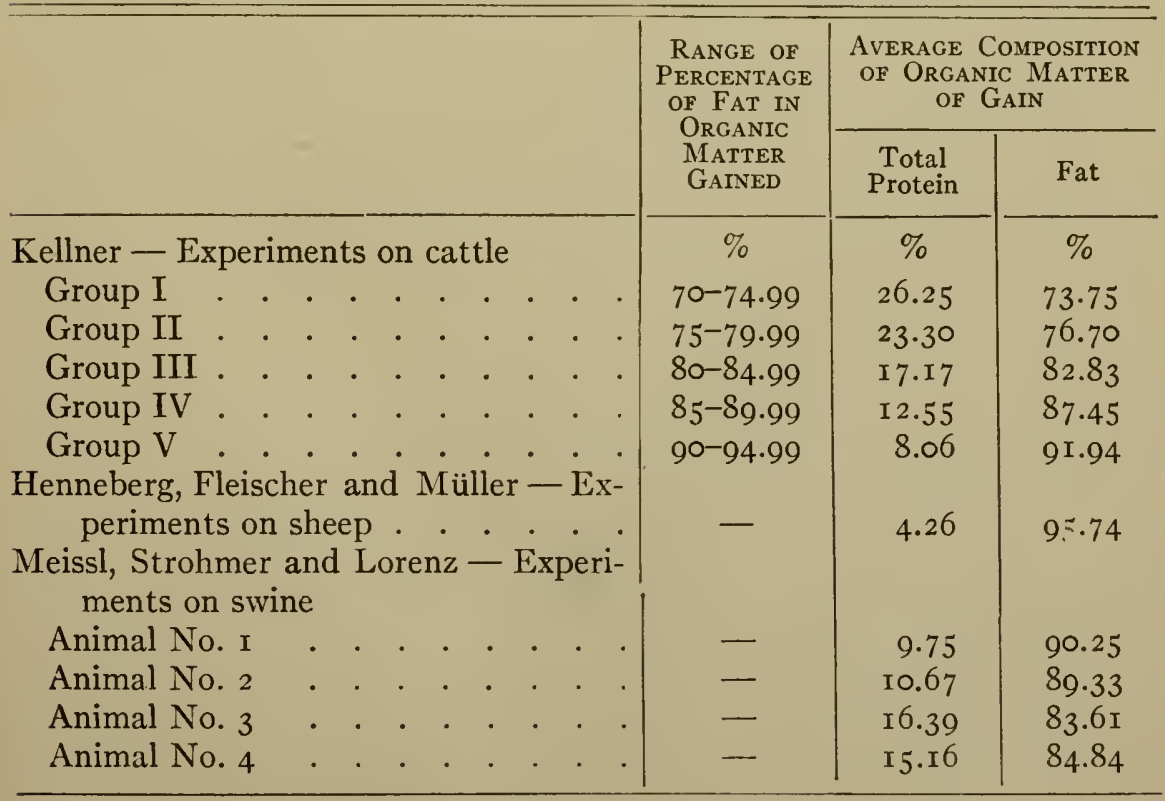

${ }^{1}$ Landw. Vers. Stat., 44 (I894), 370; 53 (I900), I.

2 Jahresber, Agr. Chem., 16-17 (I 876), II, I45.

${ }^{3}$ Ztschr. Biol., 22 (I 886), 63. 
Both the respiration experiments and the comparative slaughter tests demonstrate that the fattening of a mature animal is, as its name implies, largely a production of fat, which is deposited chiefly in the sub-cutaneous and internal adipose tissue and to a limited extent also in the muscles. A few of the comparative slaughter tests show a large storage of water but the organic matter gained in every case was chiefly fat. On the average of all the foregoing experiments by both methods, the composition of the organic matter stored up in the body of the fattening animal was as follows:-

Table 67. - Average Composition of Organic Matter Gained in FAtTening

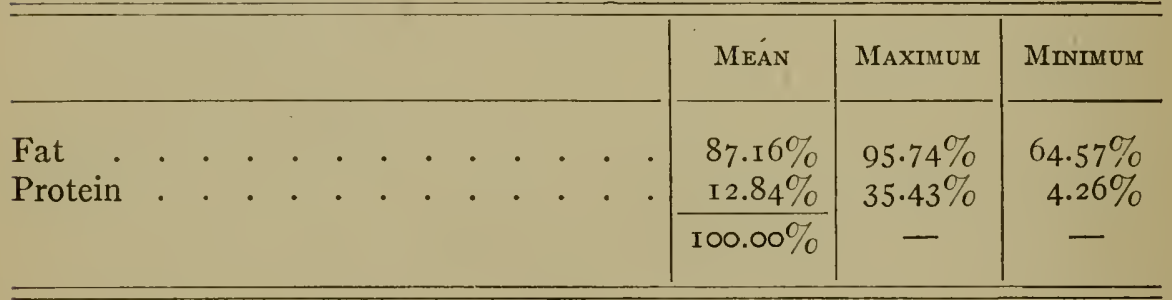

444. The gain of protein. - The foregoing data also show, however, that while the gain of dry matter in fattening consists chiefly of fat there is also a gain of more or less protein and of small amounts of mineral matter.

The actual gain of protein in some cases was not inconsiderable. This appears from Table 68, which includes both slaughter tests and metabolism experiments, most of which are identical with those from which the composition of the increase has been calculated.

It is probably safe to assume that in most of these experiments the feed contained a considerable surplus of protein over that necessary for maintenance. Such a surplus of protein, as was shown in Chapter IX (406), has a tendency to produce a somewhat limited storage of protein, which probably consists in an increase of the contents of the cells or of the protein held in solution in the body fluids rather than in an increase of the structural elements of the body. The observed gain, therefore, may represent in part an actual increase in the cell protoplasm or in the soluble protein of the body, while in addition, a relatively small amount is accounted for by the growth of hoof, horn, epidermis, etc., of the cattle and swine. Moreover, 
while the laying on of fat is accomplished largely by an increase in the fat-content of existing cells there appears to be also an increase in the number of cells in the adipose tissue, and the latter process may be assumed to require a supply of protein. The protein contained in one pound of subcutaneous adipose tissue of average composition would be equivalent to the storage of about $0.045 \mathrm{lb}$. of protein. Obviously, however, the growth of epidermal and adipose tissue can but partially account for the observed gain of protein in many of these instances and apparently a distinct increase of the nitrogenous tissue in fattening must be admitted, averaging, in these experiments, about 0.2 pound per day and Iooo pounds live weight or about 5.5 per cent of the total increase in live weight.

Table 68. - Gain of Protein by Mature Animals

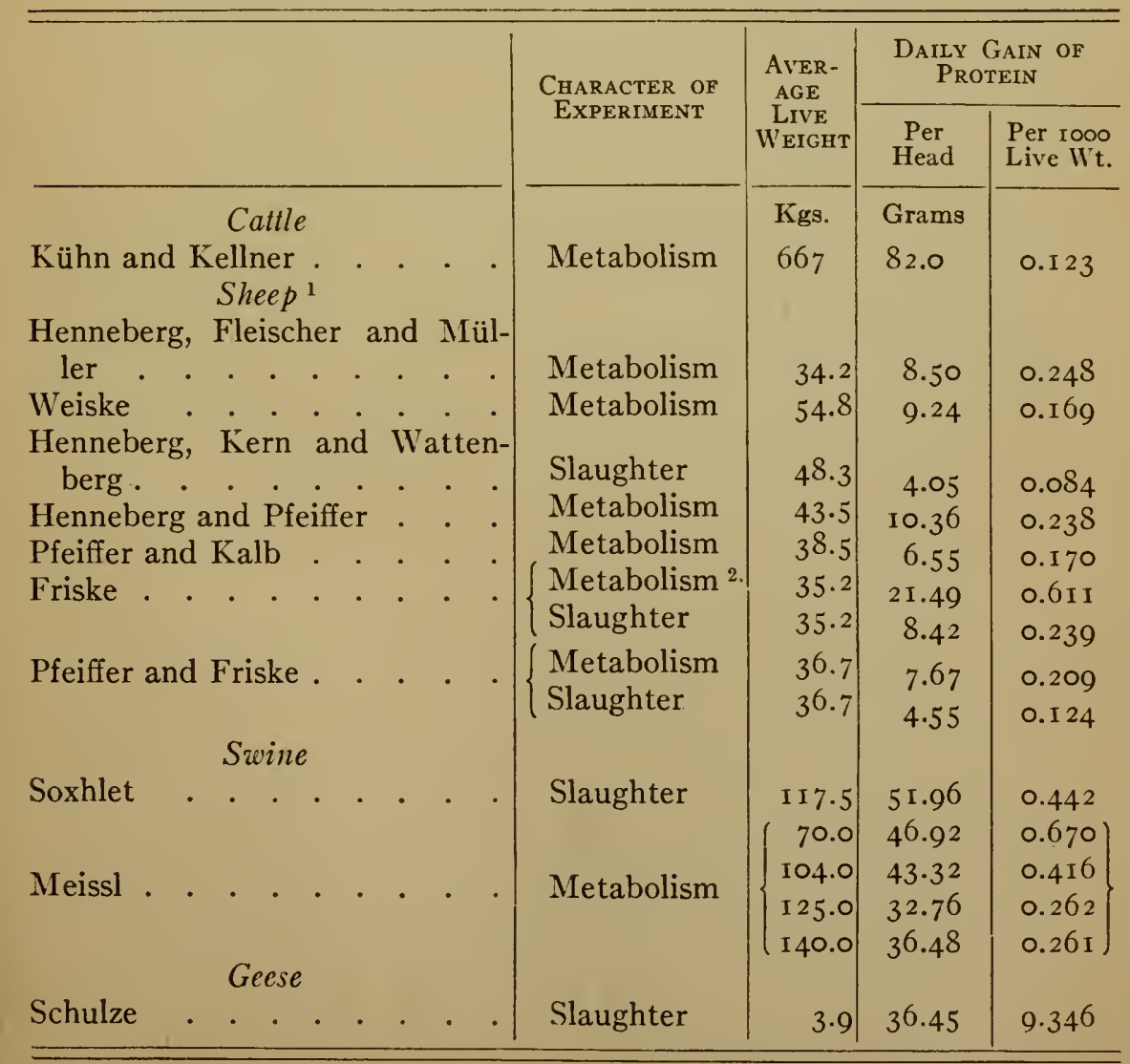

1 The nitrogen of the wool is not included in the gain.

2 The same animals were used also in the slaughter tests. 
445. Influence of fattening on the composition of the lean meat. - While fattening consists largely in an increase of adipose tissue in the ordinary sense, it has an important effect both upon the composition of lean meat in the commercial sense and upon that of the muscle tissue proper (fat-free lean meat).

Percentage of fat. - What is commonly spoken of as lean meat is by no means free from fat, since the term includes not only muscular fibers themselves with the relatively little fat

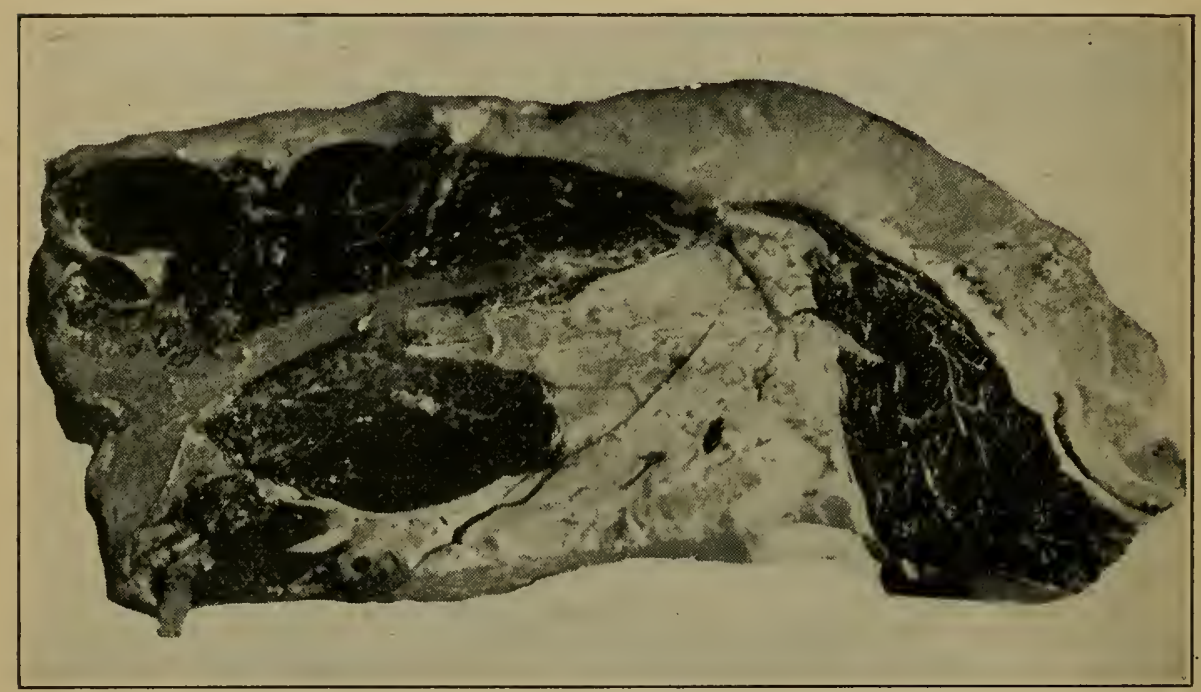

FIG. 37. - The marbling of meat. Porterhouse steak from a prime steer. (Illinois Experiment Station.)

which they contain, but the masses of connective tissue of all degrees of magnitude found between the muscle bundles and between the separate muscles (86). Fattening, especially intensive fattening, may cause a marked increase in the storage of intramuscular fat in the lean meat, as is evident to the eye in the so-called " marbling."

Such analyses of lean meat as are recorded confirm the evidence of the eye in this respect. A summary of the results on this point has been given by the writer ${ }^{1}$ elsewhere. The following example taken from that publication may serve to illustrate the point in question.

${ }^{1}$ U. S. Dept. Agr., Bur. Anim. Indus., Bul. I08 (Igo8), p. 33. 
Table 69. - Fat in Fresh Lean Meat - Leyder and Pyro

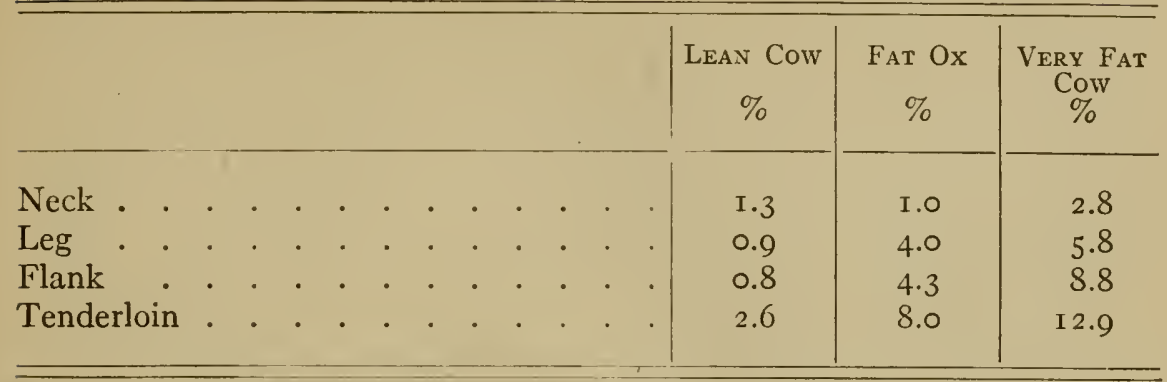

Similarly, Braman ${ }^{1}$ found the following percentages of fat in lean meat from a medium fat (common) and a well-fattened (prime) steer.

Table 70. - Fat in Fresh Lean Meat - Braman

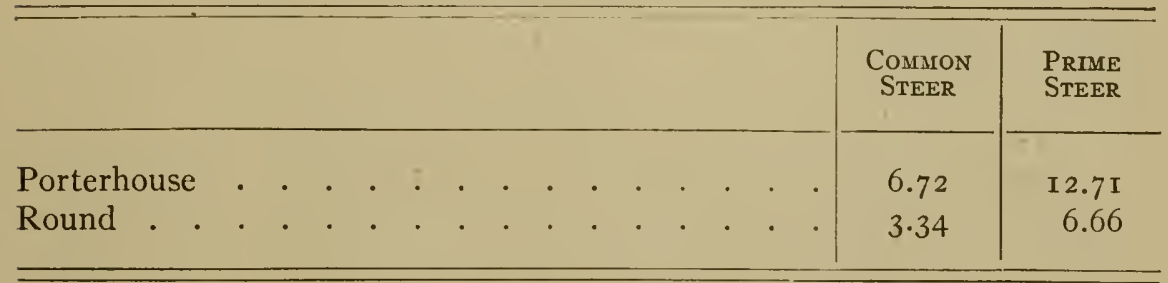

A practical difficulty in making such comparisons arises in the preparation of the sample. Obviously, the subcutaneous fat surrounding the meat should be discarded and the same is true of the large masses of fat found between the muscles, but just what part of the adipose tissue scattered through the meat of a fat animal should be regarded as mechanically separable and what part should be regarded as belonging to the meat proper is difficult to decide. Differences in the trimming of the pieces may account for some of the irregular results found by recent experimenters.

Extractives. - It appears to be established that fattening increases the nitrogenous extractives of the muscles as well as causes a deposition of fat in and about them. For example, in Henneberg, Kern and Wattenberg's experiments on sheep, included in Table 65, the composition of the meat from the lean and from the very fat animal (partially freed from connective tissue) computed to the fat-free state, was:-

${ }^{1}$ Ibid., Bul. I28 (Ig08), p. 86. 
Table 7 7 . - Composition of Fat-free Meat of Sheep

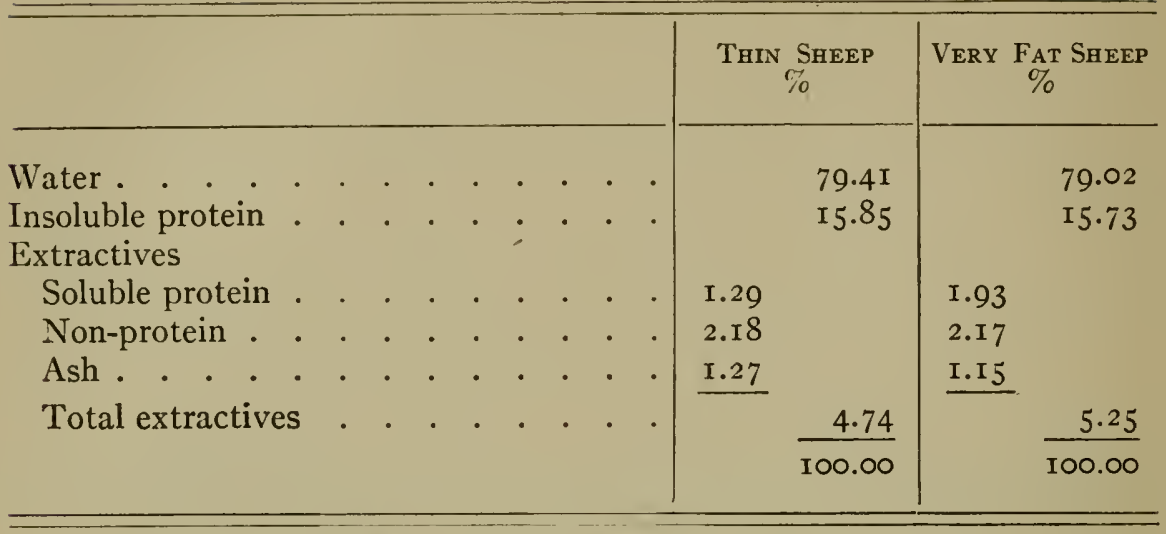

It is computed that the actual gains during the fattening of the fat animal were as follows:-

Insoluble protein . 38.7 Grms. Extractives :

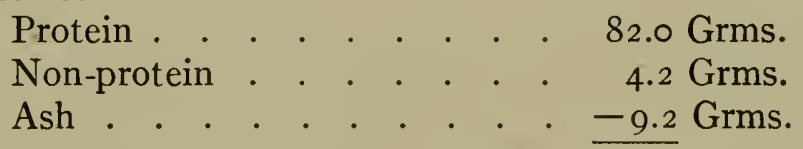

Total

77.0 Grms. II 5.7 Grms.

Somewhat similar results were obtained later by the same authors in experiments on fattening lambs. Evidently this increase in the soluble nitrogenous compounds of the muscles is one of the factors going to make up the observed gain of protein by fattening animals.

446. Object of fattening. - The fattening of animals as a commercial process is a practice based on experience, which has shown that the tenderness and palatability of the meat are materially increased thereby, so that the consumer is willing to pay a higher price for it. It is to this improvement in quality in the first instance, and only secondarily to the gain in weight, that the feeder looks for his profit.

The facts as to the composition of the increase in fattening recorded in the foregoing paragraphs serve to show what are the principal factors in this improvement in the quality of the meat. They are, first, the deposition of the intermuscular 
and intramuscular fat, and, second, an increase in the muscular tissues themselves, due in part at least to an increase in the soluble protein and in the nitrogenous extractives. The deposition of fat adds directly to the nutritive value of the meat, materially increasing its fuel value. Moreover, its mechanical effect in separating the fibers may be presumed to render the meat more tender, while the products of its decomposition in some forms of cooking (roasting and broiling) probably add to the flavor of the meat. The increase of the soluble protein is also doubtless one cause of the tenderness of the meat of fattened animals, while the other nitrogenous matters, though of little or no direct nutritive value, are an improvement through the added flavor and palatability which they bring about.

\section{$\S$ 2. FEed REQuirements FOR FAtTening}

447. Comparison with maintenance. - In the two preceding chapters it appeared that the maintenance requirements are determined substantially by the amounts of protein and of energy which are katabolized in fasting and which, therefore, must be made good from the feed in order to maintain the body. By analogy, the amounts of protein and energy stored up in the process of fattening may be taken as the measure of the requirements for fattening - i.e., of the amounts which the feed must be capable of supplying in an available form. In other words, the requirements for fattening are equivalent to the tissue produced just as the requirements for maintenance are equivalent to the tissue whose loss is to be prevented. The total feed requirement of a fattening animal, then, is to be regarded as made up of the maintenance requirement plus the fattening requirement.

In one important respect, however, the fattening requirement differs from the maintenance requirement. The latter, while not invariable, is still more or less constant for the same animal. In fattening, on the other hand, there may be a varying rate of production up to the limit set by the individuality of the animal and its capacity to eat and digesit food. Accordingly, as slow or rapid fattening is anticipated or desired, the daily requirement of the animal may be higher or lower. 


\section{Net energy values for fattening}

448. General conception. - Physiologically, the process of fattening may be regarded as a storing up by the animal, against a possible future scarcity, of feed energy supplied in excess of its immediate needs.

This storage of energy is not accomplished without some loss. As in maintenance feeding, so in fattening, a considerable portion of the feed energy escapes utilization for one reason or another. The conception of the net energy value as expressing that part of the feed energy which remains available after these various losses have been deducted, has been considered in Chapter VIII. The same conception may be extended to fattening rations. Just as the net energy value of a feed for. maintenance is measured by the loss of body energy which it prevents, so its net energy value for fattening is measured by the storage of body energy brought about.

449. Method of determination. - This conception, as well as the method of determining the net energy value for fattening, may be illustrated by the following respiration experiment by Kellner upon a mature ox, in which meadow hay was added to a mixed basal ration already sufficient to cause some gain. The second column of the table shows the metabolizable energy of the two rations, the third column the computed heat production, and the fourth the energy contained in the observed gain of protein and fat.

Table 72. - Determination of Net Energy Value for Fattening

\begin{tabular}{|c|c|c|c|c|c|c|}
\hline & & & $\begin{array}{c}\text { HAY } \\
\text { ADDED TO } \\
\text { BASAL } \\
\text { RATION }\end{array}$ & $\begin{array}{c}\text { METABO- } \\
\text { LIZABLE } \\
\text { ENERGY OF } \\
\text { RATION }\end{array}$ & $\begin{array}{c}\text { CoMPUTEd } \\
\text { HeAT } \\
\text { PRO- } \\
\text { DUCED }\end{array}$ & $\begin{array}{c}\text { ENERGY OF } \\
\text { FAT AND } \\
\text { PROTEIN } \\
\text { GAINED BY } \\
\text { BODY }\end{array}$ \\
\hline & & & $\mathrm{Lb}$. & Therms & Therms & Therms \\
\hline Basal ration + hay & . & . & 7.7 & 23.14 & I 8.90 & 4.24 \\
\hline Basal ration . . . & . & . & $=$ & I 7.64 & I 5.62 & 2.02 \\
\hline Difference . . . . & . & - & $\overline{7.7}$ & $5 \cdot 50$ & 3.28 & 2.22 \\
\hline Difference per lb. of hay & & . & - & 0.714 & 0.426 & 0.288 \\
\hline
\end{tabular}

Each pound of hay added to the basal ration resulted in a gain of protein and fat containing 0.288 Therm of energy. 
This was its net energy value for fattening. A comparison with Table 37 in Chapter VIII (364), showing the results of a determination of the net energy value for maintenance, renders evident the identity of the method employed in the two cases, the only difference being that in one case the comparison between the two rations is made below the point of maintenance and in the other case above it. It is evident that in fattening, as in maintenance feeding, there is a considerable expenditure of energy consequent upon the consumption of feed, so that only part of the metabolizable energy is actually stored up in the gain by the body. In the experiment given as an illustration, one pound of the hay contained 0.7 I4 Therm of metabolizable energy, of which only 0.288 Therm or 40.7 per cent was recovered in the gain.

450. Relative values for maintenance and for fattening. The same causes which were considered in Chapter VIII (367) are of course operative to bring about the increased expenditure of energy on the heavier rations of the fattening animal. In addition, it would appear that the chemical changes involved in the formation of fat from proteins and carbohydrates would result in more or less evolution of heat. Whatever expenditure of energy may be thus caused is additional to that caused directly by feed consumption under maintenance conditions and must evidently tend to reduce the net energy value of the feed by a corresponding amount; in other words, the net energy values of feeding stuffs for fattening would tend to be lower than those for maintenance. Such data as are available, however, do not appear to indicate that this difference is a considerable one in the case of farm animals, and it would appear that, in the case of cattle at least and presumably in that of other species, the net energy values of feeding stuffs may be regarded as being substantially the same for fattening as for maintenance. ${ }^{1}$

\section{Energy requirements for fattening}

451. Energy content of gain. - Since the net energy value of a feeding stuff or ration for fattening, as explained in the foregoing paragraphs, is that part of its total energy which can be stored up by the animal in the increase, it follows that the

1 Compare Armsby and Fries, Jour. Agri. Research, 3 (I915), 435. 
ration of such an animal must supply an amount of net energy equal to the maintenance requirement plus the quantity of energy contained in the gain made. The latter quantity, however, may be computed approximately from the data given in $\S$ I regarding the chemical composition of the increase in live weight during fattening. Estimating the energy content of protein at 2586 Cals. per pound (5.7 Cals. per gram) and that of fat at 4309 Cals. per pound ( 9.5 Cals. per gram), the energy content of one pound of increase was as shown in the last column of Table 65 (442).

Excluding two apparently questionable results, ${ }^{1}$ the range and average of the remainder are as follows. Although somewhat variable they indicate that on the average of an entire fattening period a pound of increase in live weight in cattle, sheep and swine is equivalent to about 3.25 Therms.

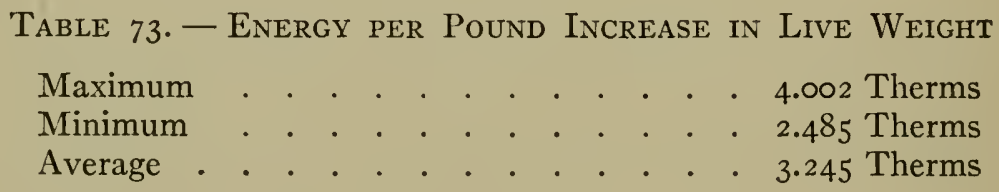

452. Influence of stage of fattening. - The results just cited are in most cases those of an entire fattening period. There can be little doubt, however, that the composition of the increase and its energy content vary materially as the fattening advances.

This appears clearly from Henneberg, Kern and Wattenberg's results upon sheep. The "fat" animal had been fed for ro weeks and was regarded as fat according to local standards. The "very fat" animal had been fed for 29 weeks, or until no further gain in live weight occurred. As the table shows, the total gain by the "very fat" animal contained materially lower percentages of water, ash and protein and a higher percentage of fat, and had a ro per cent higher energy content than the gain by the "fat" animal, while a comparison between the "fat" and the "very fat" animals shows the gain made by the latter during the last 19 weeks of fattening to have

${ }_{1}$ Pfeiffer and Friske's results appear exceptional, since the gain apparently consisted to an abnormally large extent of water, while the authors themselves point out that the gains of dry matter were notably less than should have been produced from the feed consumed. It would seem, therefore, that their omission is justified. Soxhlet's result upon swine No. I has also been omitted for a similar reason. 
contained nearly 92 per cent of fat and to have had an energy content of 4002 Cals. per pound. The same investigators also obtained entirely similar results in the fattening experiments on lambs cited in Chapter XI (458) although naturally the proportions of water and protein in the increase were greater than in the case of the mature sheep.

During the earlier stages of fattening, especially with thin animals, the storage of fat is accompanied by a considerable gain of water and by more or less increase in body protein. As the fattening progresses, however, the gain comes to consist to an increasing extent of fat accompanied by very little protein and a relatively small percentage of water. The energy content of a unit of gain in live weight, therefore, in the later stages of fattening is materially greater than in the earlier stages of the process. Evidently, then, more net energy will be required in a fattening ration to produce a pound of increase in live weight toward the close of the fattening process than at its beginning, a fact which is entirely in harmony with the experience of feeders that gains become increasingly expensive as the animals become fatter.

So far as definite conclusions are warranted from the rather scanty data available, it would seem that in the earlier stages of fattening a ration supplying (in addition to maintenance) about 2.5 Therms of net energy would be sufficient to support a gain of a pound of live weight, while in the later stages the requirement may rise to 4.0 Therms or perhaps even more.

\section{Protein requirements for fattening}

453. Protein unnecessary for fat production. - It was shown in Chapter V (247-249) that body fat, especially in the case of farm animals, is derived chiefly from the non-nitrogenous nutrients of the feed, protein playing but a subordinate rôle in its production, and Kellner has shown (769) that the proportion of the energy of protein which can be stored up by mature fattening animals is distinctly less than the corresponding percentage for the non-nitrogenous nutrients. So far as simple fat production is concerned, therefore, it would appear that a surplus of protein over that required for maintenance would be unnecessary and possibly disadvantageous on account 
of its tendency to stimulate the general metabolism of the body (365).

454. Protein in increase. - As appeared in § I (444), however, the actual increase in mature fattening animals has been found to contain a relatively small and rather variable proportion of protein, due in part to the growth of epidermal tissue, in part to an increase in the number of fat cells, and in part to an actual storage of protein and nitrogenous extractives in the muscular tissue (and in the internal organs?). It is to be remarked, however, that in most or all instances the rations consumed contained more protein than was necessary for maintenance, with, of course, an abundant supply of non-nitrogenous material, so that the conditions were favorable for such a storage of protein as that just mentioned. So far as the writer is aware, it has not yet been shown that the mature fattening animal actually requires any surplus of protein over the amount necessary for maintenance, although it can apparently utilize an excess, at least to some extent, to increase the stock of protein in its body.

At most, the requirement of the fattening animal as measured by the observed storage of protein is relatively small, one pound of increase in live weight containing in round numbers from $0.02 \mathrm{lb}$. to $0.08 \mathrm{lb}$. of protein.

455. Utilization of feed protein. - Assuming the observed gain of protein by the fattening animal to represent a real requirement, it is evident that a sufficient fattening ration must supply, in addition to the protein necessary for maintenance, an additional amount sufficient, after undergoing the various processes of digestion and metabolism, to yield the amount of body protein contained in the increase of body weight. As will appear more particularly in considering the subject of growth $(470,471)$, little is known regarding the amount of feed protein required to yield a unit of body protein. Doubtless this will differ as between different individual proteins, depending, for one thing, upon the proportions of the different amino acids which they contain, but adequate quantitative data are as yet unavailable.

456. Protein in fattening rations. - In the absence of definite knowledge regarding the availability of the protein of the feed, the question of the amount of this nutrient which should be 
supplied to fattening animals may be approached much as was the question of the amount necessary for maintenance in Chapter IX, i.e., by inquiring what is the least amount of digestible protein which, along with sufficient non-nitrogenous nutrients, has sufficed to support a satisfactory rate of fattening. If it appears that of two similar animals or lots of animals receiving equal amounts of feed, the one consuming the smaller amount of protein gave equally satisfactory gains, both as judged by the live weight and by the block test, it may be concluded that the smaller amount of protein was at least sufficient, although it cannot be determined whether it may not have been greater than was actually necessary.

Unquestionably, the protein requirements of mature fattening animals have been greatly overestimated in the past. Wolff's original feeding standards (791), published in I 864, recommended for fattening rations per thousand pounds live weight the following amounts of digestible protein:-

$$
\begin{aligned}
& \text { Cattle . . . . . . 2.5-3.0 lb. } \\
& \text { Sheep . . . . . 3.0-3.5 lb. } \\
& \text { Swine . . . . 2.7-5.0 lb. }
\end{aligned}
$$

Substantially these same figures have been repeated more or less uncritically from publication to publication, with a few exceptions, even up to the present time. It is clear, however, from Wolff's writings that his standards were based upon the then prevailing views of Voit and Pettenkofer (248) regarding the importance of protein as a source of animal fat rather than upon actual experimental results. Subsequent investigations, notably the respiration experiments of Kellner upon cattle (p. 367), have fully demonstrated that such large amounts of protein are neither necessary nor especially advantageous for fattening.

Sheep. - Indeed, Wolff himself has. demonstrated that his protein standard for sheep was unnecessarily high. In 1890 , he published ${ }^{1}$ the results of a comparison made in $1885^{-1} 886$ of maize and beans as feed for fattening sheep, using two lots of two mature sheep each. After a preliminary feeding, the following results were obtained in I07 days' feeding:-

1 Landw. Jahrb., 19 (I8go), 823. 
Table 74. - Influence of Protein Supply on Gain by Mature FatTENING SHEEP

Weight at beginning

Weight at close

Gain .

Digestible matter eaten per Ioo० kilograms live weight

Protein

Total digestible $($ fat $\times 2.4)$

$$
\begin{gathered}
\text { LOT I, FED } \\
\text { ON HAY } \\
\text { AND BEANS }
\end{gathered}
$$

LOT 2, FED

ON HAY

AND MAIZE

\begin{tabular}{r|r}
\multicolumn{1}{c|}{ Kgs. } & Kgs. \\
99.93 & $98.6 \mathrm{I}$ \\
$\mathrm{II} 8.75$ & $\mathrm{II} 8.56$ \\
\hline $\mathrm{I} 8.82$ & $\mathrm{I} 9.95$ \\
& \\
3.26 & $\mathrm{I} .8 \mathrm{I}$ \\
$\mathrm{I} 8.19$ & $\mathrm{I} 9.20$
\end{tabular}

Lot 2, receiving maize, produced about the same gain relatively to the digestible matter consumed as Lot I, notwithstanding the smaller amount of protein supplied. A block test tended to show a. slight superiority on the part of Lot 2. Subsequent experiments ${ }^{1}$ gave confirmatory results, barley being compared with beans on one animal each. The following table shows the actual digestible nutrients, computed per 1000 kilograms live weight, and the total gain for each period:-

\begin{tabular}{|c|c|c|c|c|c|c|c|}
\hline \multirow{3}{*}{ Period } & \multirow{3}{*}{$\begin{array}{l}\text { NUMBER } \\
\text { OF DAYS }\end{array}$} & \multicolumn{3}{|c|}{ SHEEP No. I, FED ON BARLEY } & \multicolumn{3}{|c|}{ Sheep No. 2, Fed on Beans } \\
\hline & & \multirow{2}{*}{$\begin{array}{l}\text { Total } \\
\text { Gain }\end{array}$} & \multicolumn{2}{|c|}{$\begin{array}{c}\text { Digested per rooo } \\
\text { Kilograms Live } \\
\text { Weight }\end{array}$} & \multirow{2}{*}{$\begin{array}{l}\text { Total } \\
\text { Gain }\end{array}$} & \multicolumn{2}{|c|}{$\begin{array}{l}\text { Digested per rooo } \\
\text { Kilograms Live } \\
\text { Weight }\end{array}$} \\
\hline & & & Protein & $\begin{array}{c}\text { Total } \\
\text { Nutrients }\end{array}$ & & Protein & $\begin{array}{c}\text { Total } \\
\text { Nutrients }\end{array}$ \\
\hline \multirow{5}{*}{$\begin{array}{l}\text { III } \cdot \\
\text { IV } \cdot \\
\text { V } \cdot \\
\text { Total }\end{array}$} & \multirow{5}{*}{$\begin{array}{l}29 \\
20 \\
38\end{array}$} & Kgs. & Kgs. & Kgs. & Kgs. & Kgs. & \multirow{5}{*}{$\begin{array}{l}\text { Kgs. } \\
\text { I6.1 } 2 \\
16.02 \\
16.32\end{array}$} \\
\hline & & I.6 & I. 62 & 14.69 & 2.4 & 3.13 & \\
\hline & & I.4 & 1.63 & I 5.23 & 0.9 & 2.95 & \\
\hline & & 4.0 & 2.03 & $17 \cdot 32$ & 3.6 & $3.6 \mathrm{I}$ & \\
\hline & & 7.0 & & & 6.9 & & \\
\hline
\end{tabular}

Table 75. - Influence of Protein Supply on Gain by Mature FatTENING SHEEP

${ }^{1}$ Landw. Jahrb., 25 (1896), 175. 
In the final period of an experiment by Weiske with lambs cited in Chapter XI (487) the animals, when two years old, received an exclusive hay ration from which they digested $1.22 \mathrm{lb}$. of protein per rooo pounds live weight. While no material fattening was possible on such a ration, there was still a gain of protein nearly as great per head as in earlier periods, thus rendering it probable that the protein supply was at least nearly sufficient for a moderate rate of fattening.

The foregoing results indicate that $\mathrm{I} .5 \mathrm{lb}$. of digestible protein per day and Iooo pounds live weight is at least sufficient for mature fattening sheep, while the experiments on cattle about to be mentioned suggest that the amount might even be reduced considerably below this limit.

Cattle. - In Kellner's respiration experiments upon fattening cattle (443), rations containing comparatively small amounts of protein produced as satisfactory a rate of fattening as those richer in that nutrient. Dividing the experiments into five groups according to the amount of digestible crude protein consumed gives the following averages:-

Table 76. - Influence of Protein Supply on Gain by Mature Fattening Cattle

\begin{tabular}{|c|c|c|c|c|c|c|c|c|c|}
\hline \multirow{2}{*}{\multicolumn{3}{|c|}{ GROUP }} & \multirow{2}{*}{$\begin{array}{c}\text { NUMBER } \\
\text { OF EX- } \\
\text { PERI- } \\
\text { MENTS }\end{array}$} & \multirow{2}{*}{$\begin{array}{c}\text { Aver. } \\
\text { LIVE } \\
\text { WEIGHT }\end{array}$} & \multicolumn{2}{|c|}{$\begin{array}{l}\text { Rations PER IOOO } \\
\text { KGS. LIVE WEIGHT }\end{array}$} & \multicolumn{3}{|c|}{$\begin{array}{l}\text { GAINS PER } 1000 \\
\text { KGS. LIVE WEIGHT }\end{array}$} \\
\hline & & & & & $\begin{array}{c}\text { Digestible } \\
\text { Protein }\end{array}$ & $\begin{array}{c}\text { Metaboliz- } \\
\text { able } \\
\text { Energy }\end{array}$ & Protein & Fat & $\begin{array}{c}\text { Com- } \\
\text { puted } \\
\text { Energy }\end{array}$ \\
\hline & & & & Kgs. & Kgs. & Therms & Grams & Grams & Therms \\
\hline I & . & • & 7 & 656 & $0.5^{23}$ & 31.54 & 87.2 & 6Iо.I & 6.29 \\
\hline II & . & . & I4 & $65 I$ & 0.745 & 33.89 & 82.3 & 619.8 & 6.36 \\
\hline III & . & • & I 8 & 667 & x.069 & $34 \cdot 50$ & I3I.O & 661.0 & 7.03 \\
\hline IV & . & . & I I & $67 I$ & I. 332 & $35 \cdot 32$ & I 54.0 & 756.8 & 8.07 \\
\hline V & . & . & IO & $69 x$ & 2.168 & $43 \cdot 36$ & I 57.0 & 754.0 & 8.06 \\
\hline
\end{tabular}

The greater gains obtained in the experiments in which the larger amounts of protein were fed are not to be ascribed to this fact but to the greater consumption of total feed, since it has been shown that protein is no more available than non-nitrogenous nutrients for fat production. The point of the comparison is that rations containing amounts of protein little if at all greater than the maintenance requirement gave relatively quite as large gains per unit of energy supplied as did those containing three or four times as much protein. 
The periods having been short in these experiments, the gain in live weight cannot be satisfactorily determined, but on the basis of Lawes and Gilbert's determinations of the composition of the increase (442) it may be estimated to have been approximately one pound per day.

Loges ${ }^{1}$ reports the results of experiments undertaken at Pomritz to test Kellner's conclusions, in which a nutritive ratio of $I:$ I0.3 gave as great gains in weight with mature cattle as one of $\mathrm{I}: 5.7$, but the absolute amounts of protein consumed are not stated in the abstract.

Apparently from 0.75 to $\mathrm{I} \mathrm{lb}$. of digestible protein per Ioo० pounds live weight is sufficient to meet the requirements of fully mature fattening cattle.

Swine. - Such experiments on the fattening of mature swine as are on record show that these animals, like cattle and sheep, need at most but a comparatively small surplus of protein over the amount necessary for maintenance.

The respiration experiments by Meissl, Strohmer and Lorenz upon the sources of fat by swine (443) afford a general illustration of this. The following table shows the digestible protein and the metabolizable energy of the feed and the gain of energy by the animal. No distinct superiority of the high protein ration of Experiment IV over the low protein rations of Experiments I and III appears, while the greatest gain was realized in Experiment II with a moderate protein supply but relatively high energy content.

Table 77. - Influence of Protein Supply on Gain by Mature FatTENING Swine

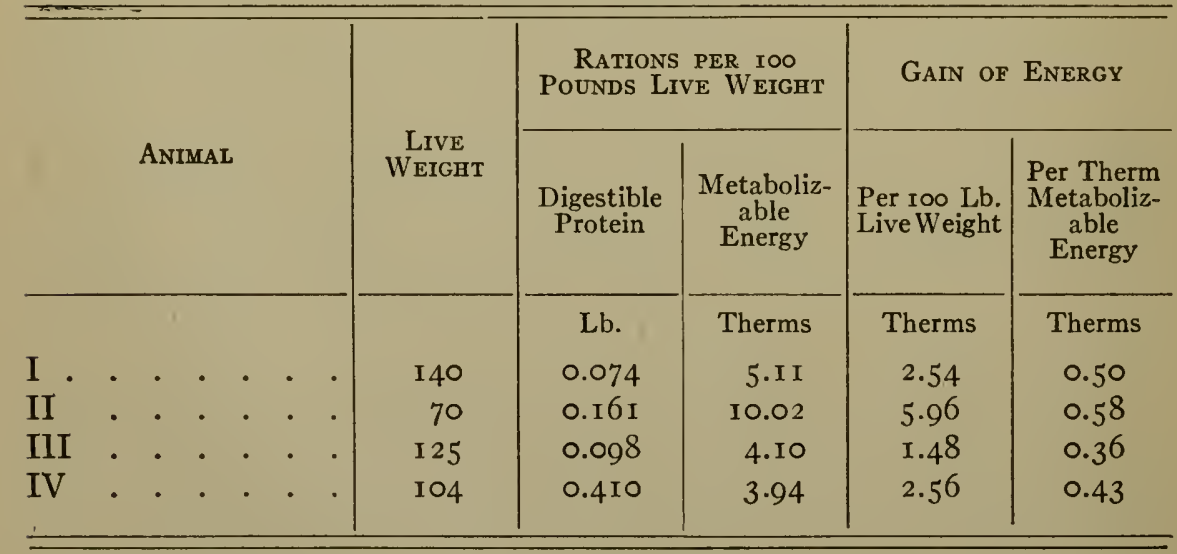

Soxhlet, in his experiments on the same subject (442), fed two swine I 6 months old and weighing about 200 pounds each at the beginning ${ }^{1}$ Centbl. Agr. Chem., 31 (1902), 646. 
of the experiment, a low protein ration consisting exclusively of rice for 75 and 82 days, respectively. The protein content of the ration and the average daily gain in live weight per head were as follows:-

Table 78. - Influence of Protein Supply on Gain by Mature FatTENING SWINE

\begin{tabular}{|c|c|c|c|c|c|c|c|c|}
\hline & \multirow{2}{*}{ ? } & & & & \multirow{2}{*}{$\begin{array}{c}\text { INITIAL Live } \\
\text { Weight }\end{array}$} & \multicolumn{2}{|c|}{ Digestible Protein $(\mathrm{N} \times 6.25)$} & \multirow{2}{*}{$\begin{array}{c}\text { DAILY GAIN } \\
\text { IN LIVE } \\
\text { WEIGHT PER } \\
\text { HEAD }\end{array}$} \\
\hline & & & & $t$ & & $\begin{array}{l}\text { Per Day and } \\
\text { Head }\end{array}$ & $\begin{array}{l}\text { Per Day and } \\
\text { roo Lb. Live } \\
\text { Weight }\end{array}$ & \\
\hline $\begin{array}{l}\text { II } \\
\text { III }\end{array}$ & • & · & $\dot{\cdot}$ & . & $\begin{array}{l}\text { Lb. } \\
220 \\
2 \mathrm{I} 3\end{array}$ & $\begin{array}{c}\text { Lb. } \\
0.265 \\
0.269\end{array}$ & $\begin{array}{c}\text { Lb. } \\
\text { O.I } 2 \text { I } \\
\text { O.I } 26\end{array}$ & $\begin{array}{l}\text { Lb. } \\
\text { I.I } 5 \\
\text { I.O4 }\end{array}$ \\
\hline
\end{tabular}

These few results upon mature swine are of interest as showing the possibility of considerable fattening on low protein rations. In practice the results are of comparatively little significance since the commercial fattening of swine is usually carried out upon the immature animal.

The recorded experiments show that in the fattening of mature animals as satisfactory results have been obtained with rations containing 0.75 to $\mathrm{I} .5$ pounds of digestible protein per Iooo pounds live weight as with those containing a much more abundant supply. Even these amounts, however, are from 50 to roo per cent higher than is necessary for maintenance, but with the exception of a small group of Kellner's experiments in which approximately the maintenance requirement of protein was consumed the results fail to show whether it is practicable or advisable to reduce still further the protein content of fattening rations. As regards the simple question of protein supply, it appears likely that an amount of this nutrient but little superior to the maintenance requirement is all that is absolutely necessary. In practice, however, the inferior digestibility of low-protein rations $(\mathbf{7 2 3}, \mathbf{7 2 4})$ as well as the fact that such rations are likely to be less palatable than those furnishing a more liberal supply have to be considered. The simple addition of non-nitrogenous nutrients to a maintenance ration 
might furnish ample material for the production of body fat and yet not convert it into a practicable fattening ration. The economic aspects of the question, however, will be considered in connection with the subject of meat production (Chapter XII), the present chapter dealing more especially with the physiological aspects of the fattening process. 


\section{CHAPTER XI}

\section{GROWTH}

\section{§ I. General Nature of Growth}

457. Cell multiplication. - The animal originates in a single microscopic germ cell. Its advance from this insignificant beginning to the size and complexity of maturity is effected by a multiplication of the number of cells, together with a progressive differentiation of function, the whole constituting the process of growth. Growth, then, may be characterized briefly as consisting in an increase of the structural elements of the body, chiefly by cell multiplication, resulting in a gain in size and weight.

\section{The increase during growth}

458. Composition of increase. - As with fattening animals, so in a study of the feed requirements of growing animals, a prime factor to be considered is the amount and composition of the gain made at different ages. The nature of the gain made during growth may be investigated either by means of comparative slaughter tests or by means of respiration experiments. Of the former there are on record a study by Wilson ${ }^{1}$ on the growth of pigs for the first I 6 days after birth, an investigation by Tschirwinsky ${ }^{2}$ on pigs between the ages of 2 and 6 months, one by Kern and Wattenberg ${ }^{3}$ on the growth of lambs between the ages of 6 and 28 months, one by Jordan ${ }^{4}$ upon the growth of cattle between the ages of 23 and 33 months and one by Wellmann ${ }^{5}$ on young pigs. Data regarding dogs and cats are also on record in investigations by Thomas ${ }^{6}$ and by Gerhartz. ${ }^{7}$

${ }^{1}$ Amer. Jour. Physiol., 8 (I903), I97. $\quad{ }^{2}$ Landw. Vers. Stat., 29 (1883), 3 I 7.

3 Jour. Landw., 28 (1880), 289.

${ }^{4}$ Maine Expt. Sta., Rpt. I895, Vol. 2, pp. 36-77.

${ }^{5}$ Landw. Jahrb., 46 (I9I4), 499.

${ }^{6}$ Arch. (Anat. u.) Physiol., rgr I, p. 9.

${ }^{7}$ Arch. Physiol. (Pflüger), 135 (I910), I63. 
Respiration experiments by Soxhlet ${ }^{1}$ on three young calves included determinations of the gain or loss of ash, while the live weights of the animals are also recorded. The feed being exclusively milk, the variations in the contents of the digestive tract were probably slight and a computation of the composition of the increase based upon the live weights seems justified.

The results of both the slaughter and respiration experiments are contained in the following table, the energy content being computed from the fat and protein.

Table 79. - Composition of Increase of Live Weight in Growth

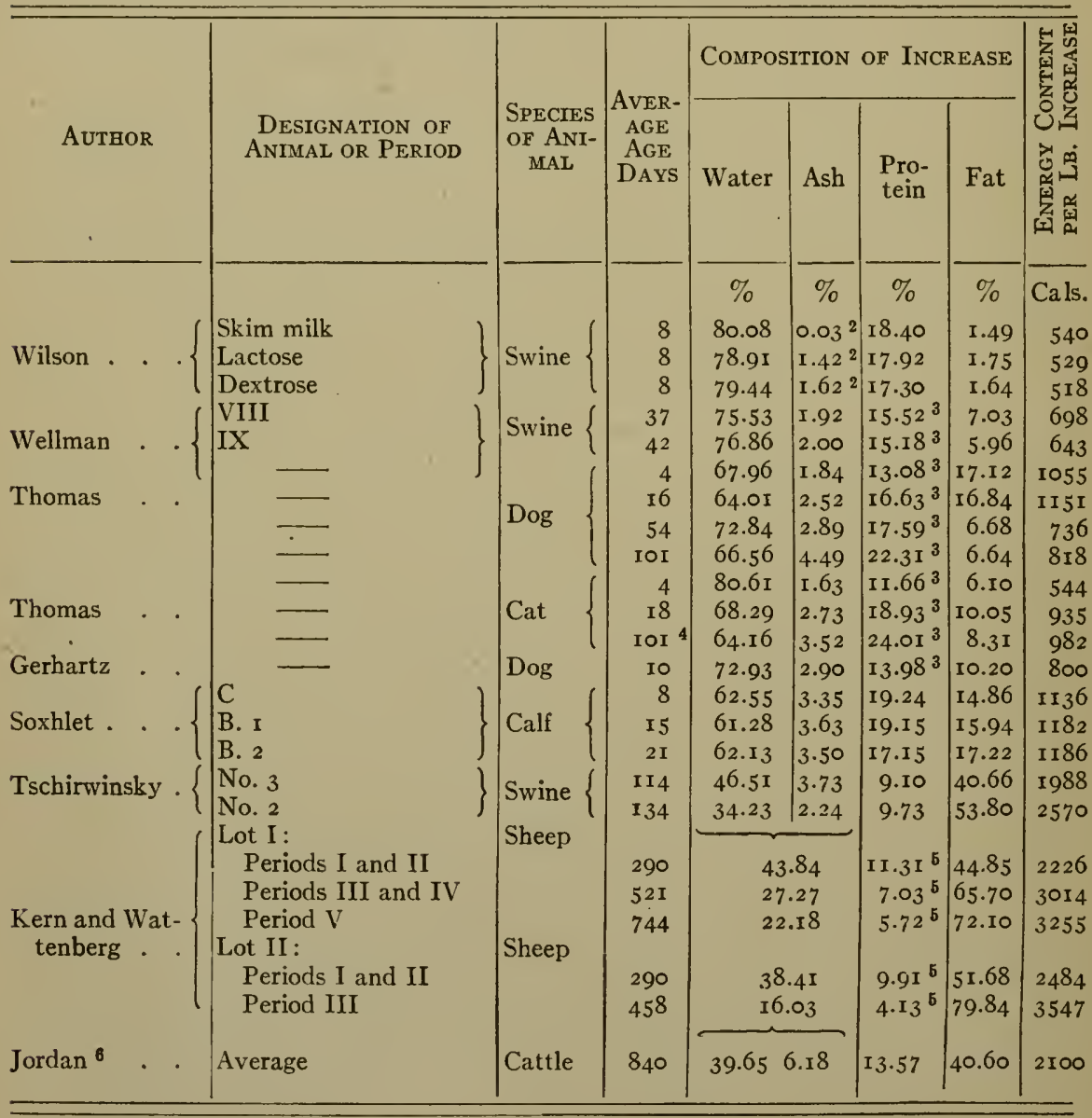

${ }_{1}$ er Ber. Versuchs-Station Wien, pp. IoI-r 55 .

2 By difference.

${ }^{3}$ Fat- and ash-free dry matter.

${ }^{4}$ Two periods.

"Computed from "fat-free body."

'The figures differ slightly from those reported by the author. 
In spite of irregularities and gaps in the table two general facts are clearly shown; first, that the percentage of water in the gain decreases and that of dry matter increases with advancing age of the animal, and second, that of the dry matter gained, an increasing proportion is fat as the animal matures. The latter fact becomes especially clear if the composition of the dry matter of the ash-free gain be computed.

The result of investigations by Waters, Mumford and Trowbridge as reported by Henry and Morrison ${ }^{1}$ are quite in accord with the teaching of Table 79, the percentage composition of the first and the second 500 pounds gained by young fattening steers being as follows:-

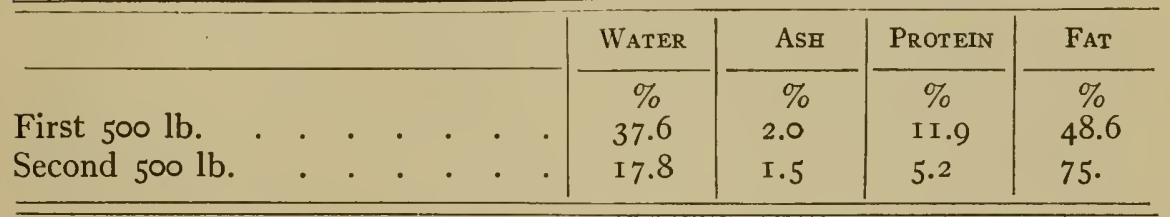

459. Energy content of gain. - The amount of energy stored in a unit of increase in live weight shows a fairly regular and notable increase as the animal grows older, due to the smaller percentage of water and the higher percentage of fat which it contains. The rate of increase in the energy content per unit in those cases in which no considerable fattening of the animal was attempted seems to be fairly regular up to about 3.0 Therms per pound and the same thing is also true of most of the results upon fattening animals up to about 3.5 Therms per pound, although the actual energy content per unit at the same age is naturally greater in the fattening animal and the limit is therefore reached earlier in life. In both cases the limit seems to correspond in a general way with the average energy content of the gain made by mature fattening animals as estimated in Chapter X (451), viz., about 3.25 Therms per pound. The data, however, are few and further investigation is much to be desired.

\section{Relation of growth to age}

460. The rate of growth. - If the successive weights or dimensions of a growing animal be platted, there are obtained

\footnotetext{
${ }^{1}$ Feeds and Feeding, I5th Ed., p.84.
} 
what might be called the curves of weight or of stature. These rise rapidly at first and afterwards more slowly as the animal approaches maturity. Or in like manner the increments of weight or size observed in successive equal periods (day, week, month or year) may be platted, showing at what periods the absolute growth is most rapid.

It is evident, however, that an increase of a pound in weight by an animal weighing 500 pounds is relatively much less than the same increase in a roo-pound animal. For many purposes, a better expression of the relation of growth to age is afforded by a computation of the rate of growth, by which is meant the increment in a given unit of time expressed as a fraction of the amount present at the beginning of that time. Thus in the instance just supposed the rate of growth in weight per day would be in the first case one five-hundredth and in the second one one-hundredth. In the second case the small animal, in proportion to its weight, is growing five times as fast as the larger and may be regarded as showing five times the energy of growth. An evident advantage of this manner of expression is that it permits of a comparison between animals of very different weights, as, for example, of sheep with cattle.

461. Rate of growth at different ages. - Somewhat extensive observations, both on man and the lower animals, show that the rate of growth as just defined diminishes from birth onward, the diminution being more rapid at first and slower as maturity is approached. This subject has been discussed in a most illuminating manner by Minot ${ }^{1}$ on the basis of his own and others' observations on guineá pigs, rabbits, chicks and other animals as well as on/man. Graphically the rate of growth is expressed by a descending curve, steep at first, but gradually becoming more and more nearly horizontal, while the same curve extends backward without material break into intrauterine life. Foster says: "It seems as if the impetus to growth given at impregnation gradually dies out." In the early stages of growth, therefore, the anabolic processes, which tend to build up tissue, predominate, while as time goes on the katabolic processes gain more and more over the anabolic until at maturity the two tend to become substantially balanced.

${ }^{1}$ C. S. Minot: Age, Growth and Death, Chapter III. 
462. The measure of growth. - The most familiar and obvious measure of growth is the increase in size or weight of the body. While for many purposes this is an entirely adequate standard, it is not a strictly accurate expression of growth proper.

In the first place the facts regarding the composition of the increase in growth which have just been considered render it evident that a unit of gain in live weight has a very varying significance. In the very young animal as much as 80 per cent of it may consist of water, while its dry matter is chiefly protein. In the nearly mature animal, on the contrary, its percentage of water may fall to between 30 and 40 , while its dry matter consists largely of fat. Moreover, a surplus of feed over the maintenance ration may lead to a deposition of fat in the young as well as in the mature animal, resulting in a greater increase in weight than that due to normal growth. On the other hand, as was shown in Chapter VIII (372), growth in the sense of increase in size may continue on a ration barely sufficient or even insufficient to maintain a stationary weight, i.e., growth when expressed in terms of weight may be masked by a loss of fat.

The essential structural elements of the body, the increase of which constitutes growth proper, consist (aside, of course, from water) mainly of protein and mineral matter (98). Growth, therefore, in this view of it, is equivalent to a gain by the body of protein and ash, especially the former. The increase of protein, therefore, may be regarded as constituting a more accurate measure of growth in the narrower sense than mere increase in weight.

463. Rate of increase of protein. - What is true of the weight or size of the growing animal is true also of growth in the somewhat narrower sense of increase of protein tissue.

The writer has elsewhere ${ }^{1}$ collated the results of a number of experiments, including those whose results regarding the composition of the increase are recorded in Table 79 , in which the gain of protein by growing animals has been determined with more. or less accuracy. In addition the results of experiments by Fingerling ${ }^{2}$ on calves, of Ostertag and $\mathrm{Zuntz}^{3}$ upon pigs,

1 U. S. Dept. Agr., Bur. Anim. Indus., Bul. I08 (I908), pp. I3-I7.

${ }^{2}$ Landw. Vers. Stat., 68 (I g08), I4I ; 76 (I9I 2), I.

${ }^{3}$ Landw. Jahrb., 37 (I908), 231. 
and of Just ${ }^{1}$ on lambs have been included in the table which follows.

In those cases in which the experiments were made by the method of comparative slaughter tests, the composition of the control animals gives an approximate measure of the initial protein content of the body. When no control animal was analyzed the initial protein content has been estimated as well as possible from the live weight. Since this was the case in the majority of the experiments it seems desirable also to compute the gain of protein per rooo live weight. Except in the case of very young or very fat animals, the results are likely to correspond substantially with those computed in the other way, while they have the advantage of being expressed in the manner usually adopted for formulating feeding requirements.

Table 80. - Rate of Gain of Protein

\begin{tabular}{|c|c|c|c|c|c|c|c|c|c|}
\hline & & & & & & & \multirow{2}{*}{$\begin{array}{c}\text { Average Age } \\
\text { Days }\end{array}$} & \multicolumn{2}{|c|}{ DaIly Gain of Protein } \\
\hline & & & & & & & & $\begin{array}{c}\text { Per roo Body } \\
\text { Protein }\end{array}$ & $\begin{array}{l}\text { Per Iooo Live } \\
\text { Weight }\end{array}$ \\
\hline & & $t t l$ & & & & & & & \\
\hline Soxhlet & . & . & . & . & . & . & 8 & 2.347 & 3.994 \\
\hline Soxhlet & . & . & . & . & . & . & $I_{5}$ & 2.076 & $3.55^{2}$ \\
\hline Soxhlet & . & 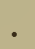 & . & . & . & . & I8 & I. 644 & 2.803 \\
\hline Soxhlet & . & . & . & . & . & . & 21 & I. 722 & 3.024 \\
\hline Fingerling . & . & . & . & . & . & . & $2 I$ & I.974 & 3.085 \\
\hline Soxhlet . & . & . & . & . & . & : & 32 & I. 693 & 2.755 \\
\hline De Vries Jzn & & . & . & . & . & : & 37 & I.335 & 2.276 \\
\hline De Vries Jzn & . & . & . & . & . & . & 38 & I. 246 & 2.124 \\
\hline Neumann & . & . & . & . & . & . & 40 & I.795 & 2.945 \\
\hline Neumann . & . & . & . & . & . & . & 45 & I. 449 & 2.419 \\
\hline De Vries Jzn & . & . & . & . & . & . & 45 & I. 272 & 2.169 \\
\hline Fingerling . & . & . & . & . & . & . & 47 & I. 248 & $2.16 I$ \\
\hline De Vries Jzn & . & . & . & . & . & . & 50 & 0.880 & I.500 \\
\hline Neumann . & - & - & . & . & . & . & 50 & 1.082 & I. 844 \\
\hline Neumann . & . & - & . & . & . & . & 54 & 1.026 & - \\
\hline Neumann & . & . & . & . & . & . & 57 & I. 320 & 2.284 \\
\hline Neumann & . & - & . & . & . & . & 62 & 0.939 & I.6II \\
\hline De Vries Jzn & . & - & . & . & . & . & 63 & 0.678 & I. 209 \\
\hline De Vries Jzn & . & . & . & . & . & . & 64 & 0.655 & 1.209 \\
\hline De Vries Jzn & . & . & . & . & . & . & 65 & I.O2O & I. 723 \\
\hline Fingerling . & 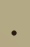 & 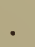 & . & . & . & . & 68 & 0.948 & I.719 \\
\hline Neumann . & • & $\cdot$ & . & . & . & . & 69 & 1.062 & I. 823 \\
\hline De Vries Jzn & . & . & . & . & . & . & 74 & $0.7 \mathrm{I} 3$ & $\mathrm{I} .27 \mathrm{I}$ \\
\hline
\end{tabular}

${ }^{1}$ Landw. Vers. Stat., 69 (1908), 393, results of periods 3, 5, 7 and 9. 


\begin{tabular}{|c|c|c|c|c|c|c|c|c|c|}
\hline & & & & & & & & DaILY GaIN & of Protein \\
\hline & & & & & & & Days & $\begin{array}{c}\text { Per roo Body } \\
\text { Protein }\end{array}$ & $\begin{array}{l}\text { Per rooo Live } \\
\text { Weight }\end{array}$ \\
\hline & & Cattle & & & & & & & \\
\hline De Vries J & Jzn & . . & . & . & . & . & 100 & 0.7 I I & I.I92 \\
\hline Fingerling & . & . . & . & . & . & . & I5O & 0.48 & 0.83 \\
\hline Fingerling & & .. & . & . & . & . & 182 & $0.4 \mathrm{I}$ & 0.76 \\
\hline Fingerling & . & . . & . & . & - & . & $2 \mathrm{I} 4$ & 0.33 & 0.64 \\
\hline Fingerling & . &. & . & . & . & . & 297 & 0.22 & 0.47 \\
\hline Jordan & - & Sheep & . & . & . & . & 840 & 0.064 & 0.089 \\
\hline Weiske & . & $\cdot$ & - & . & . & . & 140 & 0.372 & $0.65 I$ \\
\hline Weiske . & . & . . & . & . & . & . & 177 & 0.307 & 0.499 \\
\hline Weiske & . & . . & . & . & . & . & $2 \mathrm{I} 4$ & 0.219 & 0.360 \\
\hline Weiske . & . & .. & . & - & - & . & 254 & 0.288 & 0.449 \\
\hline Just . . & . . & . & . & . & . & . & 285 & 0.233 & 0.475 \\
\hline Kern and & Wat & ttenb & erg & . & . & . & 290 & 0.272 & 0.303 \\
\hline IVeiske . & . &. & . & . & - & . & 293 & O. I 79 & 0.284 \\
\hline Just . . & . . & . . & . & . & . & . & 315 & 0.182 & 0.370 \\
\hline Weiske . & . . & . . & . & . & . & . & 328 & 0.160 & 0.264 \\
\hline Just . . & . & . . & . & . & . & . & 360 & 0.180 & 0.360 \\
\hline Weiske & . . & . . & . & . & . & . & 366 & 0.238 & 0.382 \\
\hline Just . . & . & . . & . & . & . & . & 390 & $0.15^{8}$ & 0.315 \\
\hline Weiske. & . . & . . & . & . & . & . & 405 & 0.178 & 0.301 \\
\hline IVeiske & . . & . $\cdot$ & . & - & . & . & $43^{6}$ & 0.033 & $0.06 \mathrm{I}$ \\
\hline Kern and & Wat & ttenb & erg & . & . & . & $45^{8}$ & 0.068 & 0.074 \\
\hline Kern and & Wat & ttenb & erg & . & . & . & $52 I$ & 0.087 & 0.096 \\
\hline Kern and & Wat & $\begin{array}{l}\text { ttenbe } \\
\text { Swine }\end{array}$ & & · & . & . & 745 & 0.067 & 0.069 \\
\hline Ostertag a & nd 2 & Zuntz & & . & - & $\cdot$ & $5 \frac{1}{2}$ & $5 \cdot 553$ & 9.029 \\
\hline Sanford an & ad L & usk & . & . & . & . & 7 & 7.269 & $5.62 \mathrm{I}$ \\
\hline Wilson . & & $\cdot$ & . & . & . & . & 8 & 6.852 & 5.757 \\
\hline Ostertag a & nd 2 & Zuntz & & . & . & . & I3 & 4. I 29 & 6.675 \\
\hline Ostertag a & nd 2 & Zuntz & & . & . & . & $2 \mathrm{I}$ & I. 840 & 3.257 \\
\hline Ostertag a & nd 2 & Zuntz & & - & . & . & 26 & 0.757 & I. 470 \\
\hline Tschirwin & sky . & . . & . & . & . & . & I I 4 & 0.442 & 0.663 \\
\hline Tschirwin & sky. & Dog & . & . & . & . & I34 & 0.483 & 0.740 \\
\hline Thomas & . . & & . & - & - & • & 4 & 5.94 & $7 \cdot 73$ \\
\hline Gerhartz & . . & . . & . & • & . & . & IO & 6.44 & 7.67 \\
\hline Thomas & . . & . . & . & . & . & . & I6 & $6.7 \mathrm{I}$ & 8.73 \\
\hline Thomas & . . & . . & . & . & . & . & 54 & 1.70 & 2.35 \\
\hline Thomas & . & Cat & . & . & . & . & IOI & 1.82 & 2.93 \\
\hline Thomas & . . & . . & . & • & - & . & 4 & 6.10 & $7 \cdot 57$ \\
\hline Thomas & . . & .. & - & - & - & - & I 8 & 5.89 & $7.9 \mathrm{I}$ \\
\hline Thomas & . . & . . & . & . & . & . & IOI & I.6o & 3.05 \\
\hline
\end{tabular}


It is obvious that the error in single results obtained in this way may be very considerable, but the general teaching of the table is perfectly clear, viz., that the rate of growth of protein tissue, like the increase in size or in weight, whether expressed per unit of body protein or per rooo live weight, is relatively high in the new-born animal and decreases rapidly at first and more slowly later, tending to be asymptotic to the zero line.

Letting the $g$ equal the gain of protein per day per 1000 live weight and $a$ the age in days, a curve represented by the empirical equation ${ }^{1}$

$$
g=\frac{135}{a+20}
$$

corresponds fairly well with the general average of the observed results on cattle and sheep. With swine, the few results appear to indicate a greater rate of growth during the first three months. This is shown clearly in the accompanying graph (Fig. 38) in which the individual results on the different species are shown by the light lines, while the heavy curve is that represented by the foregoing equation. Of course considerable individual variations are to be expected, and no particular significance attaches to the mathematical form of the curve, but it would seem that this formula may be used tentatively to express in a broad general way the average rate of protein growth of farm ruminants at different ages. The few results on the dog and cat seem to indicate a higher rate of growth in the young of these species.

464. Rate of gain of energy. - While the rate of increase of protein, as discussed in the foregoing paragraphs, may be regarded as the measure of growth in the more restricted sense, and while it is of importance as an indication of the amount of protein which must be supplied in the feed, the actual gain in normal growth includes more or less production of fat, as is clearly shown by the data regarding the composition of the increase already considered (458). Growth, therefore, in practice involves a storage of energy in the body not merely in the protein gained but also in the accompanying fat laid on, while it is difficult to draw an exact line between the growth and the fattening of young animals.

${ }^{1}$ The equation of a rectangular hyperbola. 


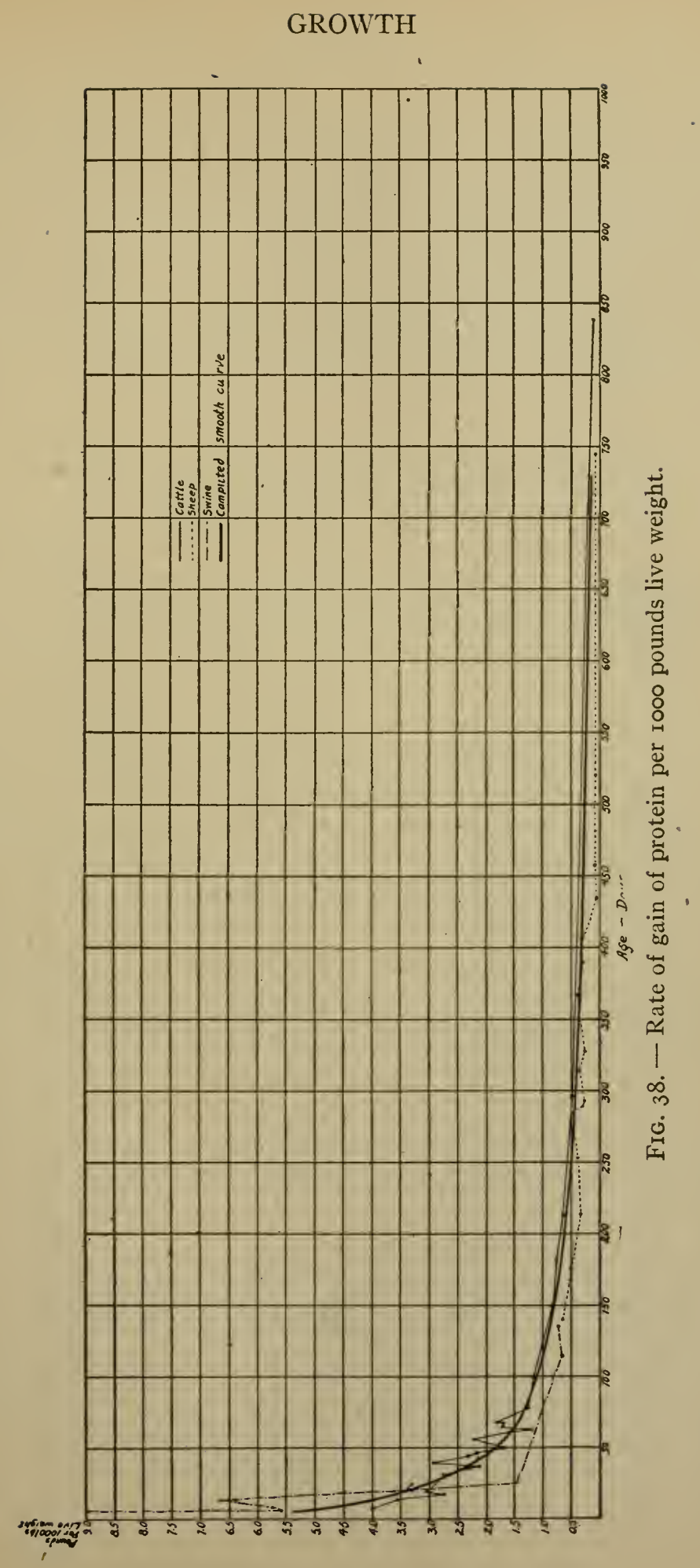


If it may be assumed that in those of the experiments recorded in Table 79 in which no considerable fattening was attempted the increase in weight was approximately that due to normal growth, the amount of energy contained in the increase and the daily rate of gain of energy per rooo pounds live weight may be computed. The following table shows the results of such a comparison, the figures per Iooo pounds being computed in direct proportion to the weight.

Table 81. - Energy Content of Daily Growth

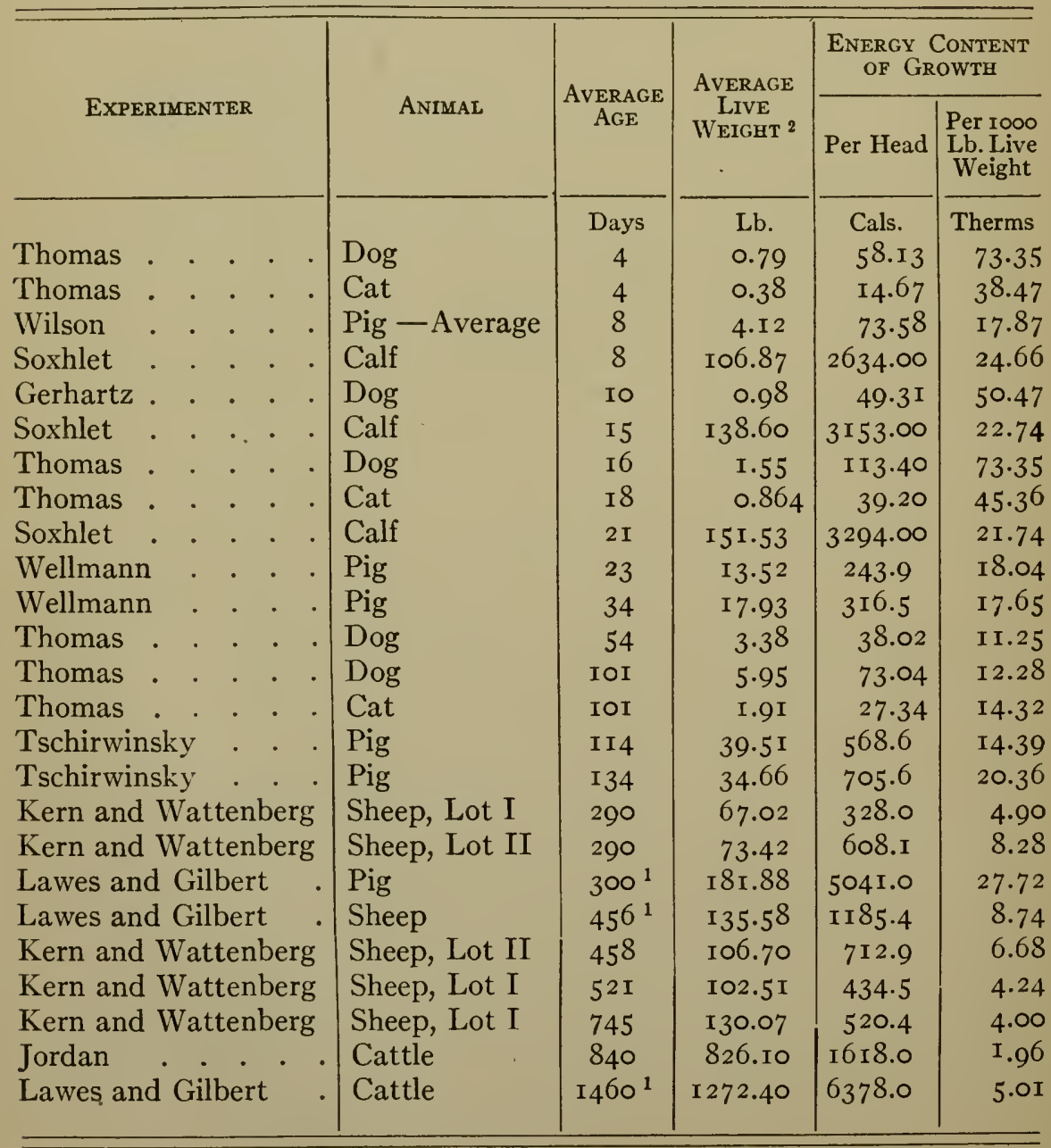

1 Approximate.

${ }^{2}$ All data refer to empty weight, exclusive of hides. 
The rate of gain of energy as thus computed is notably greater for young carnivora (dogs and cats) during the first two or three weeks than that of pigs or calves. Aside from this, the results on farm animals, although more or less irregular, present in general the same picture as those on the rate of gain of protein, viz., a diminishing energy of growth with advancing age. The few instances showing a wide divergence from the majority may probably be assumed to be due to rapid fattening.

\section{§ 2. The Utilization of Feed in Growth}

\section{The utilization of protein}

465. Relative values of proteins for growth. - A consideration of the utilization of protein in growth necessarily raises the question of the relative values of different individual proteins in this respect.

As was pointed out in Chapter IX (398), it appears probable that the protein requirement for maintenance is essentially an amino acid requirement and that the relative values of proteins for maintenance may prove to depend largely or wholly on their ability to supply certain specific "building stones" required for the performance of specific functions. In the growing animal there is, in addition to this requirement for functional purposes, a demand for amino acids out of which new body proteins may be built up. In growth, therefore, the amino acid requirements may differ from those for maintenance not only in being quantitatively greater but in being qualitatively different. A striking illustration of this is afforded by the investigations of Osborne and $\mathrm{Mendel}^{1}$ on the relation of lysin to growth.

In common with other investigators they have found that tryptophan is indispensable for maintenance (399). Wheat gliadin contains tryptophan but only a minute amount of lysin. While they have repeatedly secured maintenance for long periods on rations containing gliadin as the sole protein, they have been unable to secure growth with such rations, but the simple addition of lysin enabled growth to proceed at a normal rate. The body proteins contain lysin, ox muscle, for example, yielding 7.6 per cent (50). Evidently this

${ }^{1}$ Jour. Biol. Chem., 12 (I9I 2), 473; 17 (I9I3), 325; 26 (I9I6), 293. 
amino acid cannot be synthesized in the body but must be supplied in the feed in order to permit the construction of the new protein molecules in the tissue, while for maintenance (399) it appears to be dispensable. Moreover, they have shown that the addition to inadequate proteins like gliadin of other proteins containing lysin permits growth to take place and furthermore that the proportion of the second protein which must be added in order to support normal growth is less in proportion as it is richer in lysin.

Osborne and Mendel's conclusions have been strikingly confirmed by the results obtained by Buckner, Nollau and Kastle ${ }^{1}$ from feeding young chicks grain mixtures of high and low lysin content.

It appears that the lack of lysin in a protein renders it incapable of supporting growth, although it may still be adequate for maintenance (399), and that the proportion of lysin in those proteins containing it constitutes a limiting factor for the amount of growth which they can support. Tryptophan is obviously another limiting factor in this respect, while it must be regarded as altogether probable that other amino acids belong in the same category and may become limiting factors if the supply of them is deficient. In other words, the amount of some particular amino acid which is available may become the minimum factor which determines the rate of growth, just as the minimum supply of potassium, for example, may determine the rate of growth of a crop. The unsatisfactory results obtained in practice with maize as the sole feed for young animals may well be due in large part to the poverty of the mixed proteins of this grain in tryptophan and lysin, it having been shown that as the sole source of protein they can support but slow growth (783).

Unfortunately the knowledge available on these points is as yet chiefly qualitative in character and affords no sufficient foundation on which to base a quantitative discussion of the relative values of proteins in farm practice. Accordingly, in the case of growth as in that of maintenance it appears necessary for the present to consider questions regarding the protein requirement upon the basis of total protein, largely irrespective of its nature. (Compare Chapter XVII, § 4.)

466. Percentage retention of feed protein. - In the mature animal, the katabolism of protein substantially keeps pace

${ }^{1}$ Amer. Jour. Physiol., 39 (I915), I62. 
with the supply in the feed (402), as indeed is really implied in the conception of maturity. By a mature animal is meant one which has completed its growth, and growth consists essentially in an increase of the nitrogenous structural elements of the body. Obviously, therefore, if the capacity for growth has been exhausted, no material storage of protein can occur and an excess of this material above the maintenance requirement will serve chiefly or wholly as a source of energy to the organism.

With the young animal the case is different. Its rapidly growing cells and tissues demand a liberal supply of protein, and if this is afforded by the feed it is largely utilized to build up tissue instead of undergoing nitrogen cleavage. Consequently, other things being equal, a much larger percentage of the feed protein is retained in the body.

The investigations whose results have been considered on previous pages (463), especially those upon the younger animals, afford striking illustrations of this fact, Soxhlet's experiments upon calves being the earliest and most familiar. Their results are summarized in the following table, the feed consisting of fresh whole milk ad libitum.

Table 82. - Percentage of Feed Protein Retained - Soxhlet

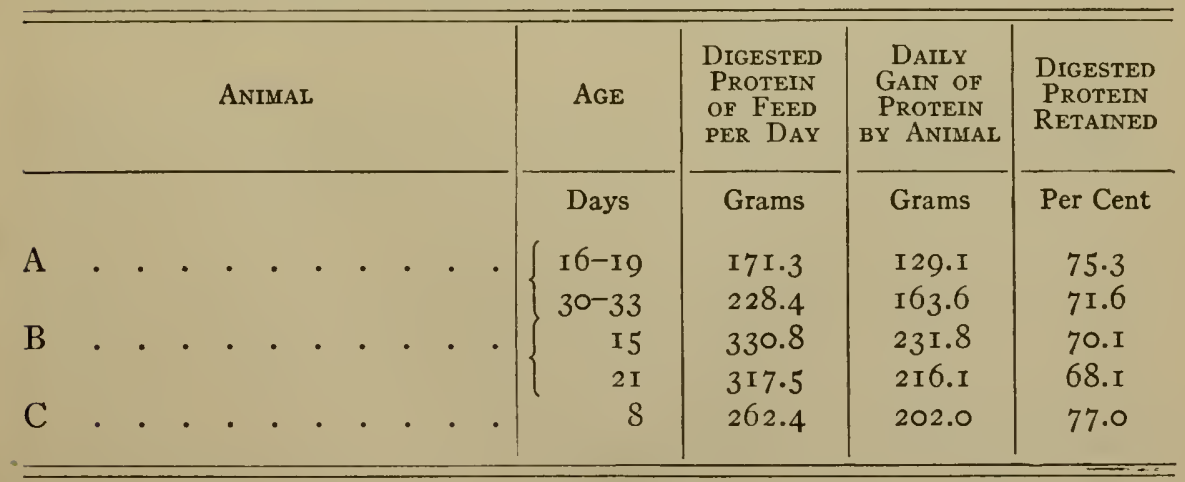

More recent and even more striking illustrations of the same fact are afforded by Fingerling's experiments. Thus, in one instance a calf averaging 9 days old received whole milk and in a succeeding period milk with the addition of butter fat and lactose, and retained the percentages of digested protein shown in the following table. 
Table 83. - Percentage of Feed Protein Retained - Fingerling

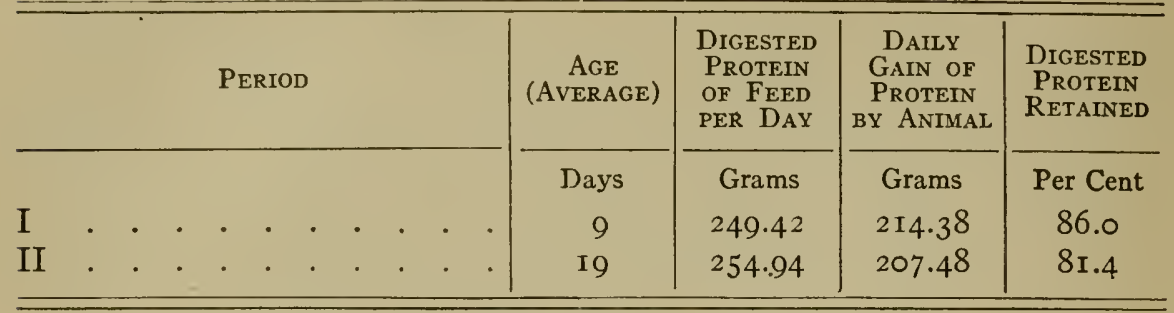

With advancing age, a relatively smaller retention is observed. Thus Neumann obtained for calves 40 to 70 days old percentages varying from 38.7 to 48.3 , and Tschirwinsky, experimenting on pigs Ioo to 120 days old, observed a retention of 20.7 to 33.6 per cent of the digested protein. With still older animals a yet smaller percentage retention has been observed, diminishing to nearly zero with fully mature animals.

467. Does not measure utilization. - On the basis of this greater percentage retention it has been customary to say that the utilization of feed protein is high in the case of the young animal and diminishes rather rapidly as it grows older. This statement is made essentially from a commercial standpoint and in that sense it is true. Only the growing animal is capable of using any large amount of feed protein to increase its stock of body protein and the ability to do this is the more marked the younger the animal.

The percentage retention of the feed protein, however, is necessarily variable and neither affords a measure of the effciency with which the animal converts it into body protein nor permits a comparison of that efficiency at different ages. The comparison is disturbed by two important factors to which attention has been especially called by Fingerling, ${ }^{1}$ viz., the influence of the total amount of protein supplied and the effect of a deficient energy supply.

468. Influence of protein supply. - As has already been implied, growth is primarily dependent upon biological factors. The feed supplies material for growth but does not determine its maximum rate. The rate of increase of protein as formulated in the previous section (463) represents (so far as the results are trustworthy) the capacity of the animal for protein

${ }^{1}$ Landw. Vers. Stat., 74 (I910), I. 
storage at different ages, but the percentage of the feed protein which is retained will depend upon the relation between this capacity and the amount of protein actually supplied. For example, suppose a calf weighing roo pounds to be capable of storing up per day 0.25 pound of protein and to require 0.05 pound for maintenance. If it receives 0.35 pound digestible protein in its feed and is able to store up the maximum amount of 0.25 pound on this ration, $7 \mathrm{I} .4$ per cent of the digestible protein would be retained, while 28.6 per cent would katabolize and its nitrogen excrete in the urine. But if the feed of the animal supplied 0.45 pound digestible protein, the gain would still be 0.25 pound, since this is the maximum possible for the animal, but the percentage of the feed protein retained would be only 55.6 , while 44.6 per cent of it would be katabolized. The organism is unable to use the added one-tenth pound for constructive purposes and therefore it is katabolized as shown in Chapter IX (402-404) and serves simply as a source of energy. In other words, the greater the excess of protein supplied in the ration over the minimum required by the demands of growth and maintenance, the lower will be the percentage retained in the body. On the other hand, with rations deficient in protein the percentage retention will increase with the protein supply up to the minimum amount necessary to utilize the growth capacity of the animal.

Fingerling's experiments afford striking confirmation of the truth of the foregoing deductions from the general laws of protein katabolism.

A calf received daily in one period $8 \mathrm{kgs}$. of whole milk with an addition of butter fat and lactose, while in the succeeding period whole milk alone was fed in amounts proportional to the age of the calf, averaging $1 \mathrm{r} .875 \mathrm{kgs}$. per day. The results as regards protein, expressed in terms of nitrogen, were as follows:-

Table 84. - Influence of Protein Supply on Percentage Retention gF Nitrogen

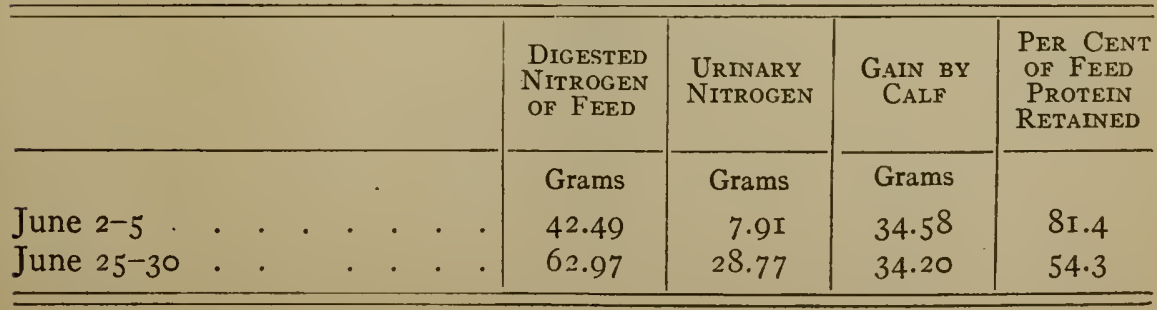


Evidently the protein supply was sufficient in the first period to ensure normal growth. The additional supply in the second period, therefore, had no effect on the gain but simply increased the protein katabolism, i.e., the added protein was used as a source of energy for maintenance or for the production of fat.

On the other hand, a supply of protein notably insufficient to permit normal gain may yet show a comparatively high percentage retention. Thus the same calf received in an intermediate period only $4 \mathrm{kgs}$. per day of whole milk together with sufficient butter fat and lactose to supply the necessary energy. As compared with the first period only about one-half of the normal gain of protein was secured, yet the percentage retention is but slightly reduced.

Table 85. - High Percentage Retention of Nitrogen on InsuffiCient Protein

\begin{tabular}{|c|c|c|c|c|c|c|c|c|c|c|}
\hline & & & & & & & $\begin{array}{l}\text { DIGESTED } \\
\text { NITROGEN } \\
\text { OF FEED }\end{array}$ & $\begin{array}{l}\text { URINARY } \\
\text { NITROGEN }\end{array}$ & $\underset{\text { CaLF }}{\text { GaIN BY }}$ & $\begin{array}{l}\text { PER CENT } \\
\text { OF FEED } \\
\text { RETAINED }\end{array}$ \\
\hline & & & & & & & Grams & Grams & Grams & \\
\hline June $2-5$. & - & - & . & . & . & . . & 42.49 & 7.91 & $34 \cdot 5^{8}$ & $8 \mathrm{I} .4$ \\
\hline June $13-$ I 8 & . & - & . & . & . & . . & I9.95 & $5 \cdot 5$ & 14.90 & $74 \cdot 7$ \\
\hline
\end{tabular}

469. Influence of deficient energy supply. - But not only may a surplus of protein be utilized as a source of energy in the manner just illustrated, but if the energy supply in the feed is inadequate protein may be diverted from growth to serve as fuel material, precisely as in the case of maintenance (412), thus lowering both the observed gain and the percentage retention.

This effect is well illustrated by the following experiment by Fingerling upon a calf receiving in the first two periods a limited quantity (ro kgs. per day) of whole milk. As the animal grew older the energy supply became insufficient and protein was diverted to fuel purposes so that the actual gain and the percentage retention both diminished. When, in a third period, one-half of the milk was replaced by butter fat, the protein supply being kept at nearly the same level by the addition of egg albumin, the actual gain rose nearly to its original level and the percentage retention became even higher than at first on account of the somewhat reduced protein supply. 
Table 86. - Influence of Energy Supply on Percentage Retention of Nitrogen

\begin{tabular}{|c|c|c|c|c|c|c|c|c|}
\hline & & & & & $\begin{array}{l}\text { DIGESTED } \\
\text { NITROGEN } \\
\text { OF FEED }\end{array}$ & $\begin{array}{l}\text { URINARY } \\
\text { NITROGEN }\end{array}$ & $\begin{array}{c}\text { GaIN BY } \\
\text { CALF }\end{array}$ & $\begin{array}{l}\text { PER CENT } \\
\text { OF FEED } \\
\text { Protein } \\
\text { RETAINED }\end{array}$ \\
\hline & & & & & Grams & Grams & Grams & \\
\hline Sept. $29-$ Oct. I . & . & • & . & . & $5 \mathrm{I} .84$ & I 2.62 & 39.22 & $75 \cdot 7$ \\
\hline Oct. $7-9 .$. & . & . & . & . & 51.87 & I9.99 & 31.84 & 61.5 \\
\hline Oct. $19-27$. & . & . & . & . & $45 \cdot 76$ & 8.10 & 37.66 & 82.3 \\
\hline
\end{tabular}

470. Meaning of utilization. - The percentage of the digestible protein of the feed which is retained in the body of the growing animal, then, is not in itself a measure of the efficiency of the animal organism in converting feed protein into body protein, since the proportion retained is affected both by the magnitude of the protein supply in the feed and by the energy content of the ration. What then is the correct conception of the utilization of the feed protein?

As appeared in the previous section, the amount of protein which a growing animal can store up seems to be a function of its age (463), and the attempt was made to formulate approximately the capacity for growth in this sense at different ages. The percentage utilization of the feed protein in the physiological sense, as distinguished from the percentage retention, is the ratio between the body protein thus stored up and the least amount of feed protein in excess of the maintenance requirement which is necessary to support this growth under the most favorable conditions, especially as to energy supply. Suppose, for example, that an animal three months old actually has the capacity, as computed by the formula on page 378 , to store up daily I.23 pounds protein per Iooo pounds live weight, and that it has been shown that it can just reach this capacity on a ration supplying 2 pounds of digestible protein per day. Deducting 0.5 pound for maintenance (415), there remains $\mathrm{I} .5$ pounds of protein in the ration out of which is produced 1.23 pounds of body protein. The utilization is therefore $1.23 \div$ I. $5=82$ per cent. If, on the other hand, it was found that 2.5 pounds of protein had to be supplied in the ration in order 
to bring the gain of protein up to the capacity of the animal, the percentage utilization would be only $1.23 \div 2.0=62$ per cent, while on the other hand if the maximum growth could be secured with I.73 pounds of digestible protein, the utilization would evidently be roo per cent.

471. Experimental results. - The writer is not aware of any exact determinations of the percentage utilization in the sense just defined, that is, of the maximum amount of protein tissue which can be produced either from single proteins or from the mixed proteins of feeding stuffs, but interesting data regarding the utilization of protein by growing animals are furnished in experiments by Fingerling ${ }^{1}$ upon calves and by Just ${ }^{2}$ on lambs in which the influence of a varying protein supply upon the nitrogen balance was determined.

Reckoning the maintenance requirement for protein at 0.5 pound per rooo pounds live weight, Fingerling's results for those periods in which the estimated capacity for growth appears to have been fully utilized were as follows :-

Table 87. - Computed Utilization of Protein by Calves

\begin{tabular}{|c|c|c|c|c|c|c|c|c|c|c|c|}
\hline & \multirow{2}{*}{\multicolumn{2}{|c|}{ ANIMAL }} & & \multirow{2}{*}{ Period } & \multirow{2}{*}{$\begin{array}{c}\text { AVER- } \\
\text { AGE } \\
\text { AGE } \\
\text { DAYS }\end{array}$} & \multicolumn{2}{|c|}{$\begin{array}{c}\text { GAIN OF PRO- } \\
\text { TEIN PER } \\
\text { IOOO }\end{array}$} & \multicolumn{2}{|c|}{$\begin{array}{c}\text { FEED PROTEIN } \\
\text { IN EXCESS OF } \\
\text { MAINTENANCE } \\
\text { PER IOOO }\end{array}$} & \multicolumn{2}{|c|}{$\begin{array}{l}\text { PeRCentage } \\
\text { Utilization }\end{array}$} \\
\hline & & & & & & $\begin{array}{l}\text { Capac- } \\
\text { ity for } \\
\text { Gain }\end{array}$ & $\begin{array}{c}\text { Ob- } \\
\text { served } \\
\text { Gain }\end{array}$ & $\begin{array}{c}\text { True } \\
\text { Protein }\end{array}$ & $\begin{array}{c}\text { Crude } \\
\text { Protein }\end{array}$ & $\begin{array}{c}\text { True } \\
\text { Protein }\end{array}$ & $\begin{array}{l}\text { Crude } \\
\text { Protein }\end{array}$ \\
\hline \multirow[t]{2}{*}{ B } & \multirow{2}{*}{. } & \multirow{2}{*}{ • } & & I & I 72 & 0.70 & 0.67 & I.94 & 2.00 & 34.5 & 33.5 \\
\hline & & & & 2 & I7 5 & 0.63 & 0.63 & 0.62 & 0.84 & IOI.6 & 75.0 \\
\hline \multirow{3}{*}{$\mathrm{C}$. } & \multirow{3}{*}{$\cdot$} & \multirow{3}{*}{ - } & \multirow{3}{*}{ • } & I & I 57 & 0.76 & 0.85 & 2.15 & 2.44 & 39.5 & 34.8 \\
\hline & & & & 2 & I84 & 0.66 & 0.75 & 0.79 & 1.04 & 94.9 & 72.1 \\
\hline & & & & 3 & 2 I I & $0.5^{8}$ & 0.68 & 2.59 & 2.85 & 26.3 & 23.9 \\
\hline \multirow{3}{*}{ G. } & \multirow{3}{*}{\multicolumn{2}{|c|}{$\cdot \quad \cdot$}} & & I & 237 & 0.53 & 0.57 & I.08 & I. 29 & 52.8 & 44.2 \\
\hline & & & & 2 & 262 & 0.48 & 0.54 & I.I 3 & I. 37 & 47.8 & 39.4 \\
\hline & & & & 4 & 309 & $0.4 \mathrm{I}$ & 0.50 & $0.3 \mathrm{I}$ & 0.49 & I $6 \mathrm{I} .3$ & 102.0 \\
\hline \multirow{3}{*}{$\mathrm{H}$. } & \multirow{3}{*}{\multicolumn{2}{|c|}{$\cdot \quad \cdot$}} & & 5 & 339 & $0.3^{8}$ & 0.44 & 0.25 & $0.4^{2}$ & I 76.0 & 104.8 \\
\hline & & & & Prelim- & I 35 & 0.87 & 1.07 & 2.42 & $2.8 \mathrm{I}$ & 44.2 & $3^{8.1}$ \\
\hline & & & & $x-6$ & 187 & 0.05 & 0.80 & 0.74 & I.OI & I08. I & 79.2 \\
\hline
\end{tabular}

${ }^{1}$ Landw. Vers. Stat., 76 (I9I2), г.

2 Ibid., 69 (I 908), 393. 
From these figures it appears that in the low protein periods the estimated capacity of the animals for growth was fully utilized with a surplus of digestible true protein over the maintenance requirement equal to or even less than that actually recovered in the growth, while a much larger supply of protein failed to secure any additional growth but simply forced up the protein katabolism. In other words, if the estimate for the maintenance requirement is approximately correct, the utilization of the digestible protein in the low protein periods must have approached roo per cent. Indeed, in at least two cases it is necessary to admit either that the estimate for maintenance is too high or that non-protein was used for maintenance.

Table 88. - Computed Utilization of. Protein by Lambs

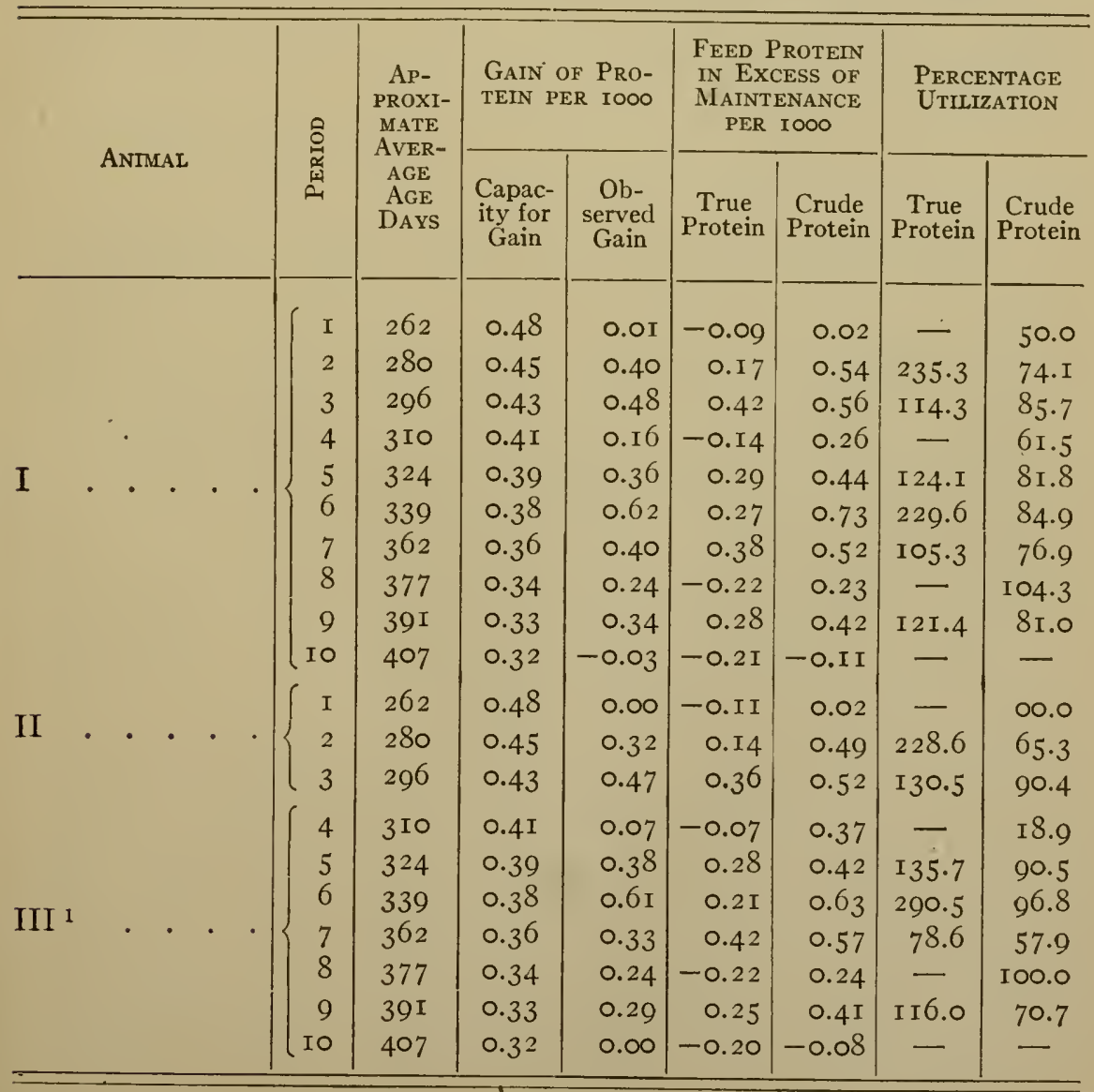

I Substituted for No. II. 
Interesting data pointing in the same direction are contained in the investigation by Just, in which the nutritive value of non-protein for lambs was compared with that of protein. Estimating the maintenance requirement at 0.5 per thousand and computing the results of the protein periods as in Fingerling's experiments, it appears that in nearly every case the actual gain of protein was only slightly less than the surplus of digestible crude protein above the maintenance requirement, while in many cases it was distinctly greater than the digestible true protein available. Apparently the non-protein must at least have contributed to the maintenance of the animal if not to its growth, while the utilization of the digestible true protein - must have been very high (Table 88).

Neither Fingerling's nor Just's investigations are adequate to solve the general problem of the maximum possible utilization of protein in growth, but their results indicate that it may be very high and should lead to caution in the interpretation of experiments upon the protein requirements for growth.

\section{Utilization of energy - net energy values for growth}

472. General conception. - The conception of net energy values for growth is entirely analogous to that of net energy values for maintenance or for fattening. They represent that portion of the feed energy supplied in excess of the maintenance requirement which the animal is able to store up in the gain made. It is important to keep this conception clearly in mind when considering the utilization of feed in growth and not to be misled by the greater economic efficiency of the young animal as a producer of live weight increase.

It is a familiar fact that the young animal gains in weight relatively much faster than when more mature and this has led to the general impression that the young animal utilizes its feed more perfectly than the older animal, or in other words, that the net energy value of a feeding stuff for growth is greater than that for maintenance or for fattening. It is true that the gain in live weight is different in character in the young animal, containing more water and protein and less fat and therefore less energy $(458,459)$, but on the other hand the results recorded in $\S$ I show a greater rate of growth as regards both protein and 
energy $(463,464)$ in the young animal as compared with the more mature one. Is this difference to be ascribed to a specifically higher percentage utilization on the part of the younger animal, or is it due to a relatively greater consumption of feed or the relatively high net energy values which usually characterize the feeds given the young animal, particularly milk? The mere fact, for example, that a young animal consuming milk utilized a higher percentage of the feed energy than did the same animal later upon a mixed ration would not necessarily show any physiological superiority on the part of the younger animal but might be due solely to the difference in the kind of feed consumed. So, too, the mere ability to consume relatively large amounts of highly concentrated feed in the form of milk and thus to secure a large surplus above the maintenance requirement might $(360,510)$ give the younger animal a marked economic advantage without indicating any more efficient conversion of the surplus energy supplied than in the older animal.

Unfortunately, investigations regarding the utilization of feed at different ages have been few in number and the available data regarding net energy values for growth are exceedingly meager. To a large extent it is necessary to be content with comparisons of a very general nature, leading to probabilities only.

473. Experiments on suckling animals. - The experiments by Soxhlet on calves and those by Wilson on pigs cited on previous pages (458) and likewise an investigation by Rubner and Haubner ${ }^{1}$ on infants afford some data for approximate estimates of the percentage of the metabolizable energy of milk utilized by growing animals.

The computations involve a number of uncertain assumptions, particularly as regards the maintenance requirement, and none of them afford a satisfactory basis for comparing the utilization of the metabolizable energy of milk at different ages. It is of some interest, however, to compare the average utilization computed from these experiments with that estimated by the use of Rubner's factors for the "specific dynamic action " of equal amounts of pure nutrients on mature animals.

1 Ztschr. Biol., 36 (I898), I ; 38 (I899), 31 5. 
As explained in Chapters VIII and XVII (366, 759), Rubner's "specific dynamic action" is synonymous with the energy expenditure caused by the consumption of feed, and if it be subtracted from the metabolizable energy the remainder is the net energy. The percentage utilization is of course the net energy divided by the metabolizable energy.

Estimated in this way, the percentage utilization would be as shown in the first column of the following table, the second and third columns of which show the utilization as computed by the writer both with and without a ro per cent addition to the fasting katabolism as estimated from the data on mature animals.

Table 89. - Estimated Utilization of Metabolizable Energy of MILK

Rubner's experiments Soxhlet's experiments Wilson's experiments

\begin{tabular}{|c|c|c|}
\hline \multirow[b]{2}{*}{$\begin{array}{l}\text { COMPUTED, } \\
\text { USING } \\
\text { RUBNER'S } \\
\text { FACTORS }\end{array}$} & \multicolumn{2}{|c|}{ COMPUTED BY WRITER } \\
\hline & $\begin{array}{l}\text { Fasting } \\
\text { Katabolism } \\
\text { Same as in } \\
\text { Mature } \\
\text { Animals }\end{array}$ & $\begin{array}{c}\text { Fasting } \\
\text { Katabolism } \\
\text { Io\% } \\
\text { Greater } \\
\text { than in } \\
\text { Mature } \\
\text { Animals }\end{array}$ \\
\hline$\%$ & $\%$ & $\%$ \\
\hline 84.08 & 73.10 & \\
\hline 86.18 & 73.77 & $75 \cdot 5^{6}$ \\
\hline 83.99 & $70.3 I$ & 75.47 \\
\hline
\end{tabular}

While it is clear that no final conclusions can be based upon small differences between figures obtained as these have been, it seems suggestive, nevertheless, that the actual experiments with growing animals show a lower average utilization than would be expected from Rubner's results upon mature animals. Moreover, Wilson's results are apparently lower than those which may be computed from Meissl's and Kornauth and Arche's respiration experiments upon mature swine consuming grain (757). Certainly these comparisons afford little support to the notion that the utilization of energy in the physiological sense by young animals is much higher than that by mature animals. 
474. Experiments on older animals. - Of experiments upon older animals those of Kern and Wattenberg on lambs and of Tschirwinsky on pigs (458), permit an approximate computation of the utilization of the feed energy during growth and afford data for some comparisons, although in neither case were very young animals employed, the lambs being between 6 and 7 months old at the beginning of the experiment and the pigs between 9 and ro weeks.

On the whole, the results of these experiments seem to indicate, if anything, a rather lower percentage utilization by the younger animals as compared with the older. At any rate they fail to show any superiority on the part of the former. The same is true of the results of experiments by Armsby and Fries ${ }^{1}$ upon steers ro to 27 months old in which the availability was determined by the use of the respiration calorimeter. While not decisive, the results seem to indicate a slightly lower availability of the mixed grain and possibly of the hay for the younger animals.

475. Embryonic growth. - Several experimenters, especially Tangl and his associates ${ }^{2}$ and Bohr and Hasselbalch, ${ }^{3}$ have determined the energy expended in the development of the embryo in oviparous animals, i.e., in the organization of the substances of the egg into embryonic tissue. These investigations have shown that a relatively large proportion of the chemical energy contained in the egg is evolved as heat during the process of development, so that the percentage recovered in the embryo, ranging from 60 to 68 per cent, is distinctly lower than the utilization of the energy of milk by suckling animals as computed in a previous paragraph (473). Moreover, they show that the utilization of the energy of the egg is notably less in the earlier than in the later stages of incubation, as low a figure as 28 per cent having been observed after ro days' incubation.

The method may be illustrated by the results of two experiments by Tangl and Mituch, each upon three hens' eggs. From analyses of similar eggs from the same hen, it was computed that the three used contained respectively 229.72 Cals. and 291.38 Cals. chemical energy.

1 U. S. Dept. Agr., Bur. Anim. Indus., Bul. I 28 (I9I I), 5 I.

${ }^{2}$ Arch. Physiol. (Pflüger), 93 (Ig03), 327; 98 (Ig03), 490; 104 (Ig04), 624; 121 (I908), 423 and 437.

${ }^{3}$ Skand. Arch. Physiol., 10 (I900), I49 and 353; 14 (I903), 398. 
At the end of incubation the embryo ${ }^{1}$ was separated from the yolk sack and its contents and the energy of each determined with the following results : -

Table 90. - Utilization of Energy in Incubation

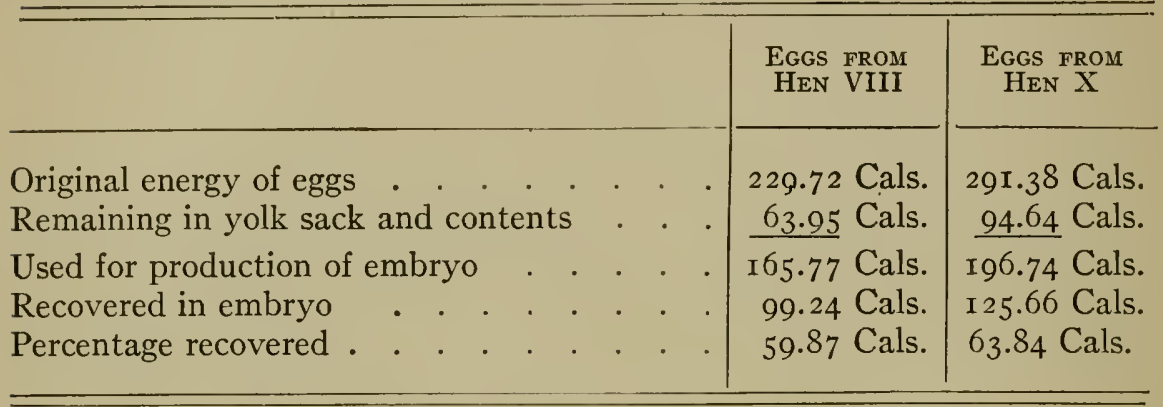

In other words, 35 to 40 per cent of the energy of the egg substance used was not recovered but escaped as heat. Comparison with the loss of dry matter showed that the material thus katabolized consisted substantially of fat. No loss of nitrogen was observed.

Experiments on mammalian and reptilian embryos, especially by Bohr, ${ }^{2}$ by Murlin ${ }^{3}$ and by Carpenter and Murlin, ${ }^{4}$ appear in accord with the foregoing conclusion, since they show that the metabolism of the embryo per unit of weight is as great or greater than that of the mature animal, despite the fact that the maintenance requirement of the former must be decidedly less.

The growth of the embryo consists essentially of the organization of protein tissue. The fact that there is no loss of nitrogen during incubation would indicate that chemically the process is effected by a cleavage and resynthesis of protein which appears to be a nearly isothermic process $(233,367 d)$. Apparently the organization of the protein into structure is what calls for the large expenditure of energy.

476. Summary. - The experimental results mentioned in the foregoing paragraphs may be briefly summarized in the following statements :

In the case of suckling animals, while no direct comparisons of the same animal at different ages are available, the utilization of the metabolizable energy of milk for growth appears to be

1 Including the egg membranes.

2 Skand. Ärch. Physiol., 10 (I g00), 4I3; 15 (I904), 23.

${ }^{3}$ Amer. Jour. Physiol., 26 (1910), I 34.

${ }^{4}$ Arch. Inter. Med., 7 (I9I I), I 84 . 
distinctly less than would be expected from Rubner's results on the utilization of pure nutrients by mature animals. In the case of swine, moreover, the utilization appears to be even less than that of the metabolizable energy of grain by mature animals, although the contrary would naturally have been anticipated. The results with older animals, while far from conclusive, seem, if anything, to indicate a lower utilization by younger animals as compared with older ones and at any rate fail to show that it is any greater in the former case.

The results on embryonic growth show a relatively large expenditure of energy in development and indicate a comparatively low utilization of energy. This large expenditure of energy in development seems to be required chiefly for the organization, in the broader sense, of the embryonic structure rather than for the mere chemical transformation of egg substances, and it seems to be relatively greater in the young as compared with the more mature embryo.

477. Provisional hypothesis. - While it would be rash to draw any final conclusions from the foregoing data, it may be permissible to formulate a working hypothesis to the effect that the conversion of feed protein (including the protein of the egg) into tissue requires a considerably greater relative expenditure of energy than does the conversion of surplus feed into fat, the difference representing what might be called the work of organization, i.e., the formation of organized structure in the young animal and especially in the embryo. It has been shown (463) that the rate of growth decreases rapidly with increasing age. Accordingly, the work of organizing new protein tissue, so far as this is measured by the storage of protein, must constitute a steadily diminishing proportion of the total energy expenditure of the organism, since as the animal grows older the increase consists to a diminishing extent of protein and to an increasing extent of fat. The percentage utilization of the feed energy would therefore, upon this hypothesis, tend to increase. It would be least immediately after birth and after two to four months would become relatively small, corresponding to the changing character of the gain. Probably by the time an animal has been weaned and is consuming the normal feed of its species, the percentage utilization of the feed energy might be assumed to be not much less than that ex- 
hibited by the mature animal and at any rate to be practically proportional to it. This would mean, of course, that the net energy values of feeding stuffs for maintenance and fattening might be used also to measure at least their relative if not their absolute net energy values for growing animals.

The determination of the validity of this provisional conclusion offers an interesting and profitable field for investigation.

\section{§3. The Feed Requirements for Growth}

478. Contrast with fattening. - In the case of fattening animals the conception of the feed requirement, particularly as regards energy, is somewhat artificial, since the extent of the fattening depends, within the limits of the animal's capacity, largely upon the amount of feed supplied. Growth, on the other hand, unless the feed fails to supply the necessary materials and thus becomes a limiting factor, goes on at a rate substantially determined by other conditions, the most obvious of which are the species, individuality and age of the animal. Indeed, it may be said that, within normal limits, the capacity for growth determines the feed consumption rather than the reverse. Heavy feeding may cause fattening but it does not appear, at least in the case of the higher animals, to materially accelerate growth, although Eckles ${ }^{1}$ observed the growth of dairy calves to be somewhat more rapid upon heavy as compared with scant rations. In growth, therefore, as in maintenance, there is a real requirement to be satisfied, its measure being the amount and character of the increase which the young animal is capable of making under normal conditions.

Mention has been made (372) of the interesting results of experiments by Waters ${ }^{2}$ upon growth under adverse conditions, while Osborne and $\mathrm{Mendel}^{3}$ have shown that growth which has been suspended for a time because of inadequate feed supply may be resumed when this deficiency is made good (deferred growth). Neither of these possibilities, however, invalidates the statement just made that the continued maintenance of a normal rate of growth requires a definite supply of matter and energy.

1 Mo. Expt. Sta., Bul. I35, I9I5.

2 Soc. Prom. Agr. Science, Proc. 29th Annual Meeting, I908, p. 7 I.

${ }^{3}$ Jour. Biol. Chem., 18 (I9I4), I95; 23 (I915), 439; Amer. Jour. Physiol., 40 (19I6), I6. 
479. Total increase in normal growth at different ages. The feed requirements of the growing animal as regards protein and energy depend in the first place on the amounts which such an animal is capable of storing up in normal growth. From the data regarding the rate of growth recorded in $\S \mathrm{I}$ of this chapter, even though they are somewhat fragmentary, it seems possible to derive average figures regarding the storage of protein and energy in growth at different ages which may be of some value as a guide in estimating the feed requirements of the growing animal.

As regards protein, it was shown that the rate of gain per Ioo0 live weight apparently does not vary widely as between cattle, sheep and swine, and an empirical formula (463) was given by which its amount at any age may be approximately estimated. As regards energy, fewer data are available, especially for farm animals, but the graphic representation in Fig. 39 of the results recorded in Table 8I (464) shows a diminishing rate of gain of energy as the animal grows older.

In the following tabulation the daily gain of protein at different ages has been calculated by means of the formula just mentioned and the gain of energy estimated from the smoothed graph of Fig. 39. The two together may be taken as an approximate expression of the normal increase in growth at different ages.

Table 9i. - Daily Increase in Growth per iooo Pounds Live Weight

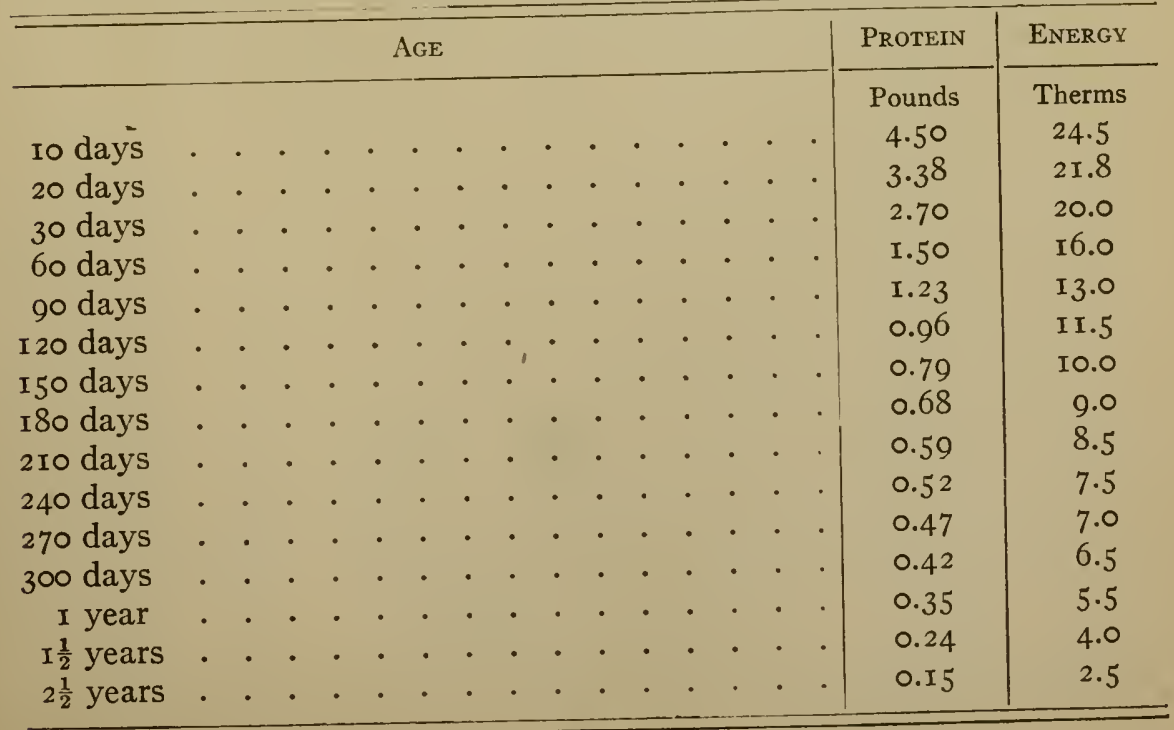



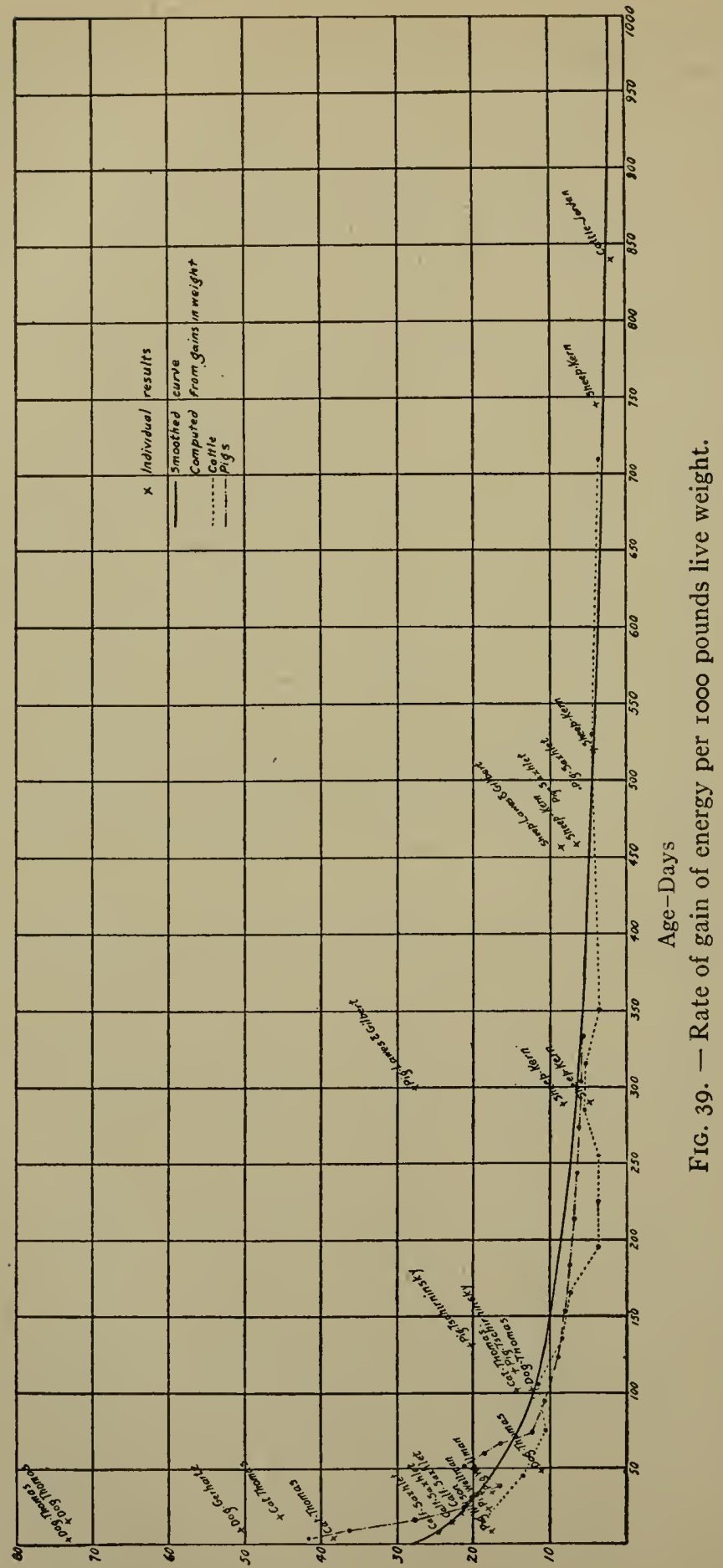

Therms per 1000

pounds live weight 


\section{Energy requirements}

480. Computation from daily increase. - The estimates contained in the foregoing table of the amount of energy stored up in growth, although unfortunately based on scanty data, show, to the extent to which they can be relied on, the amounts of net energy which are necessary for the support of growth without any considerable fattening. Since the results described in $\S 2$ render it probable that the net energy values of feeding stuffs for growth do not differ widely from those for maintenance or for fattening (477), the figures of the table, with the addition of the maintenance requirement, would afford a basis for computing rations for young animals.

481. Computation from gain in live weight. - Another method of computation furnishes to a certain degree a check upon the results recorded in the last table. The amount of energy stored up in the increase at different ages may be estimated by applying the results regarding the energy values per unit of increase which are recorded in Table 79 (458) to the gain of live weight actually observed in the growth of animals under normal conditions.

a. Cattle. - From data secured at the Missouri Experiment Station ${ }^{1}$ regarding the rate of growth of 5 Hereford, 6 Jersey, 2 Ayreshire and 5 Holstein calves, the writer has computed the following figures for the daily gain of energy per iooo pounds live weight by calves.

For an animal one month old, it is assumed that the energy content of the increase in live weight was the same as the average of Soxhlet's respiration experiments, viz., Ir70 Cals. per pound, and that this increased at a uniform rate to a maximum of 3000 Cals. per pound at 18 months old. The observed daily gain of energy has been computed per rooo pounds live weight to eliminate the influence of the varying size of the different breeds. The average daily gains per rooo pounds live weight are computed for each breed separately, and these means are again averaged, i.e., each breed has been given equal weight. None of the animals were fattened to any great extent.

${ }^{1}$ Private communications from Professors P. F. Trowbridge and C. H Eckles. Data for the dairy breeds have been published in Bul. I35 of the Missouri Station. 
Table 92. - Average Gains by Growing Calves per iooo Pounds Live WeIght

\begin{tabular}{|c|c|c|c|}
\hline APProximate AGE & $\begin{array}{c}\text { DaILy Gain IN Live } \\
\text { WEIGHT }\end{array}$ & $\begin{array}{c}\text { ESTIMATED ENERGY } \\
\text { CONTENT OF I LB. } \\
\text { OF INCREASE }\end{array}$ & $\begin{array}{c}\text { DaILY GAIN OF } \\
\text { ENERGY }\end{array}$ \\
\hline Months & Pounds & Therms & Therms \\
\hline $0-I$ & I 2.73 & I.I 70 & 14.89 \\
\hline$O-I^{1}$ & 19.54 & I. I 70 & 22.86 \\
\hline$I-2$ & 10.69 & I. 272 & 13.60 \\
\hline $2-3$ & $7 \cdot 3^{2}$ & I. 374 & 10.06 \\
\hline $3-4$ & 7.84 & 1.476 & I I. 57 \\
\hline $4-5$ & 5.29 & I. 578 & 8.35 \\
\hline $5^{-6}$ & 4.53 & 1.680 & 7.61 \\
\hline $6-7$ & 2.05 & I. 782 & 3.65 \\
\hline $7-8$ & 1.99 & I. 884 & 3.75 \\
\hline $8-9$ & I.94 & I. 986 & 3.85 \\
\hline 9-IO & 2.68 & 2.088 & 5.60 \\
\hline IO-I I & 2.57 & 2.190 & 5.63 \\
\hline I I-I 2 & $1.5^{8}$ & 2.292 & 3.62 \\
\hline I $2-18$ & 1.64 & 2.904 & 4.76 \\
\hline I $8-24$ & 1.25 & 3.000 & 3.75 \\
\hline
\end{tabular}

b. Swine. - Similar approximate estimates may be made for the pig from data reported by Henry. ${ }^{2}$ At weekly intervals, up to 70 days old, the computation is based on the live weights and gains shown in Henry's table. The gains by the older animals are estimated on the basis of his statement ${ }^{3}$ that the larger breeds should weigh 250 pounds at one year, it being assumed that the rate of gain would decrease at an approximately uniform rate.

It is evident that the results on the heavier animals as compiled by Henry were obtained by heavy feeding for fattening, since it may be computed from the figures for the weights and gains that the animals would reach a weight of about 320 pounds at about io months old. The energy content per pound of gain is estimated on the assumption that it would increase uniformly from the average of about 500 Cals. obtained by Wilson and by Wellmann for young pigs (458) up to 2485 Cáls. as computed in Table 65 of Chapter X (442) for Soxhlet's swine No. 3 at 500 days old.

1 Average of Soxhlet's experiments. $\quad 2$ Feeds and Feeding, Ioth Ed., p. 499.

${ }^{3}$ Loc. cit., p. 553 . 
Table 93. - Average Gains by Growing Pigs per iooo Pounds Live WEIGHT

\begin{tabular}{|c|c|c|c|}
\hline APPRoximate AGE & $\begin{array}{c}\text { Daily Gain IN Live } \\
\text { Weight }\end{array}$ & $\begin{array}{l}\text { ESTIMATED ENERGY } \\
\text { CONTENT OF I LB. } \\
\text { OF INCREASE }\end{array}$ & $\begin{array}{l}\text { DaILY GaIN of } \\
\text { ENERGY }\end{array}$ \\
\hline Days & Pounds & Therms & Therms \\
\hline $0-7$ & 78.55 & 532 & 41.79 \\
\hline $7-\mathrm{I} 4$ & 65.09 & 559 & 36.38 \\
\hline$I 4-2 I$ & 47.62 & $5^{85}$ & 27.86 \\
\hline $2 I-28$ & 34.62 & 6 I2 & 2 I.I9 \\
\hline $28-35$ & $3 I .53$ & 639 & 20.15 \\
\hline $35-42$ & 25.09 & 665 & I6.69 \\
\hline $42-49$ & 27.72 & 693 & I9.2I \\
\hline $49-5^{6}$ & $29 \cdot 48$ & 720 & 21.23 \\
\hline $56-63$ & 24.92 & 750. & I8.69 \\
\hline $63-70$ & $2 \mathrm{I} \cdot 54$ & 776 & I6.7 I \\
\hline $70-90$ & I 4.92 & 827 & . $\quad 12.34$ \\
\hline $90-120$ & I I. 45 & 945 & 10.82 \\
\hline $\mathrm{I} 20-\mathrm{I} 50$ & 8.43 & 1063 & 8.96 \\
\hline I $50-180$ & 6.79 & I I 8 I & 8.02 \\
\hline I $80-2$ IO & 5.68 & I300 & $7 \cdot 38$ \\
\hline $210-240$ & 4.87 & I 4 I 8 & $6.9 \mathrm{I}$ \\
\hline $240-270$ & 4.26 & I 536 & 6.54 \\
\hline $270-300$ & 3.78 & I654 & 6.25 \\
\hline $300-330$ & $3 \cdot 40$ & I 772 & 6.03 \\
\hline $330-360$ & 3.08 & I89I & 5.82 \\
\hline
\end{tabular}

482. Estimated averages. - The foregoing results have been plotted in Fig. 39 (479) for comparison with those derived from the computations of $\S \mathrm{I}$, regarding the rate of storage of energy. By drawing smooth curves through the results for calves and for pigs, respectively, the following approximate estimates of the average rate of gain of energy by these two species have been obtained. No similar data appear to be available for other species of farm animals. The graph would seem to indicate that the results for cattle may apply fairly well also to sheep, although no figures for the latter species are recorded below 300 days old. Preliminary computations on the basis of the live weights assumed by Kellner for lambs at different ages seem to indicate higher figures for the first six months, especially for animals of the mutton breeds. 
Table 94. - Estimated Rate of Gain of Energy per Day and 1000 Pounds Live Weight

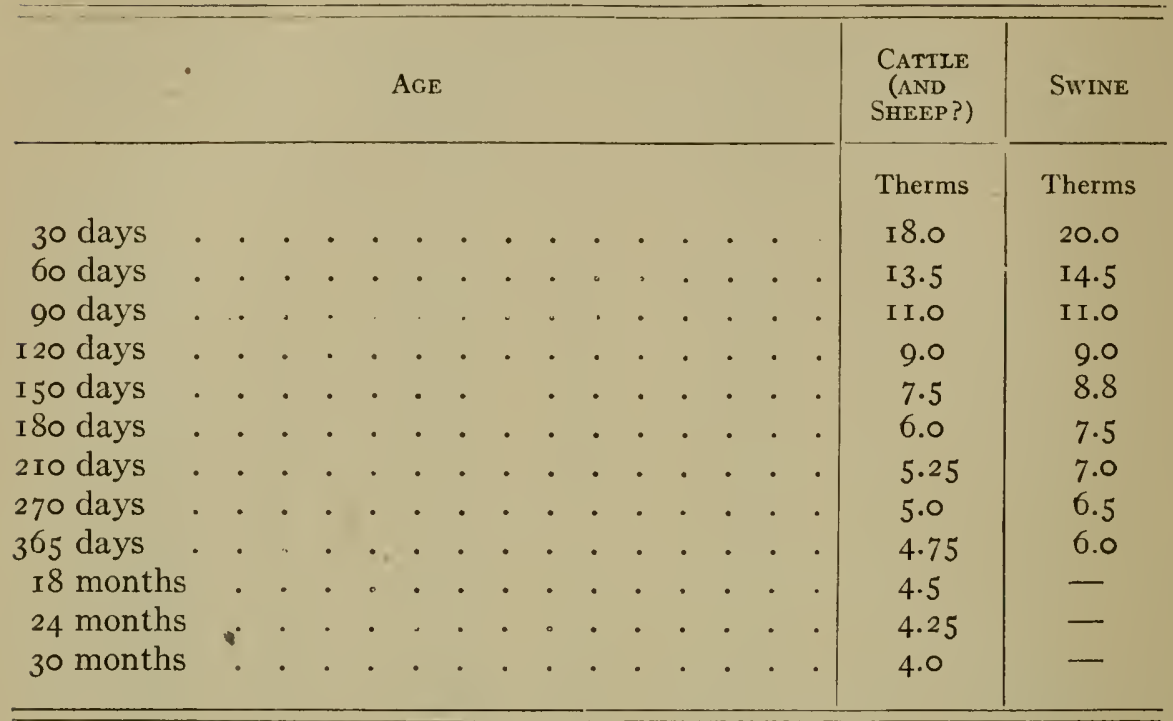

It cannot be claimed that the foregoing computations are particularly satisfactory. The data are scanty, and the element of personal judgment unavoidably enters, especially into the estimates of the energy value of a unit of increase in live weight. Nevertheless, while there are very considerable divergencies at certain points, there is after all a certain general agreement in the results, and they may perhaps serve as a first approximation towards an expression of the growth capacity of farm animals in terms of energy storage. It is much to be regretted that such a fundamental factor in determining the feed requirements for growing animals is so imperfectly known and the determination of the amount and composition of the increase in growth at different ages, whether by means of comparative slaughter tests or with the aid of respiration or calorimetric experiments, offers an interesting field for investigation. With the smaller animals, such as pigs, lambs and particularly fowls, it would appear that such determinations might be made without great difficulty.

483. Total energy requirements. - The foregoing figures attempt to show approximately the actual storage of energy per rooo pounds of live weight by growing animals at various ages. An adequate ration for such an animal, however, must 
not only supply net energy equal to that contained in the growth made, as indicated by the foregoing table, but in addition sufficient net energy for maintenance, the sum of the two being the total net energy required by the animal. Computing from Table 94 and from the estimated live weight at different ages ${ }^{1}$ the energy storage per head and adding the maintenance requirement computed in proportion to the two-thirds power of the live weight (347) gives the total energy requirements shown by Table IV $b$ of the Appendix.

\section{Protein requirements}

484. Minimum requirement. - As with the energy of the feed, the protein supply of the growing animal is essentially a limiting factor. A deficient supply or one lacking certain essential "building stones" (465), may check growth temporarily or permanently through simple lack of material, but it does not appear that a surplus of protein can materially stimulate the rate of growth.

Granting the approximate accuracy of the estimates of the actual gain of protein in normal growth made on previous pages $(463,479)$, the quantity of digestible feed protein required in the ration of the growing animal at any particular age will depend upon what proportion of the latter can be converted into body protein and stored up, i.e., upon the percentage utilization of the feed protein (470). As was shown in the preceding section, however, this is very imperfectly known. If, on the basis of Fingerling's and Just's results (471), it be assumed that the utilization may approach Ioo per cent, then the amounts estimated in Table 9I (479), with the addition of about 0.5 per rooo for maintenance, would be the least amounts of digestible protein which must be supplied to support the normal increase of protein tissue.

485. Results in practice. - As a matter of fact, however, experience seems to show that a more liberal supply of feed protein than is indicated by these estimates is at least advantageous if not necessary in the actual rearing of animals. While there are few investigations on record directed specifically to the determination of the minimum protein requirements of

${ }^{1}$ In direct proportion to the live weight. 
growing animals, there are a considerable number of experiments, especially upon immature fattening animals, in which the increase of live weight has been determined upon rations otherwise reasonably similar but containing varying proportions of protein.

In the immature fattening animal, it seems safe to assume that the feed protein (in excess of maintenance) is applied substantially to the support of growth and that this growth goes on parallel with the fattening process but more or less independent of it. There appears to be no evidence that protein specifically stimulates or aids fattening, so that conclusions regarding the protein supply drawn from fattening experiments may be regarded as applicable to growth without fattening.

If in such an experiment, in which the total amounts of feed consumed do not differ widely, it appears that the smaller amount of protein has been as efficient as the larger as regards 'gain in live weight, and if the gain appears to be normal in amount, there is a strong presumption that the lesser amount of protein was at least sufficient for the needs of the animal for growth and maintenance, while if a block test shows a normal character of increase this presumption is further strengthened. Obviously, results of this sort cannot be relied on to fix definitely the lower limit of protein supply, but they may furnish indications regarding it.

486. Experiments with cattle. - In the experiments upon calves by Soxhlet, De Vries Jzn and Neumann, included in Table 8o (463), showing the rate of gain of protein, the amounts of digestible protein consumed as compared with the actual gains were as follows:-

Table 95. - Protein Consumed by Calves

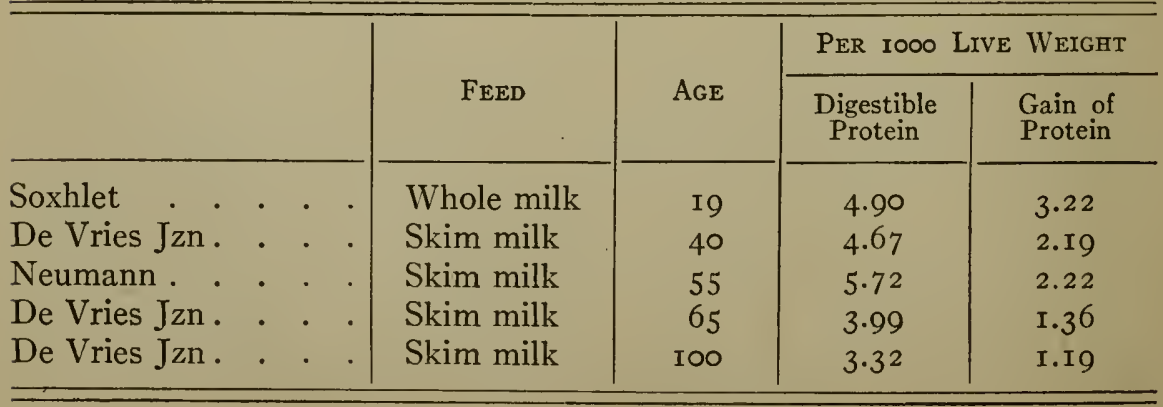


In the light of Fingerling's results upon suckling calves (466), however, there can be little doubt that the protein supply in these experiments was unnecessarily great, especially with the older animals.

The writer ${ }^{1}$ has elsewhere discussed some of the earlier live weight results bearing upon the protein supply of immature fattening cattle which seem to indicate much higher requirements than might be deduced from the actual gains of protein at the several ages. Those results are here tabulated in a slightly altered form and with the addition of a subsequent experiment by Schneidewind. ${ }^{2}$ The summary of course represents to a degree the judgment of the writer and the figures are to be interpreted as indications rather than as determinations.

Table 96. - Estimated Protein Requirements of Cattle

\begin{tabular}{|c|c|c|}
\hline $\begin{array}{c}\text { AGE } \\
\text { Years }\end{array}$ & EXPERIMENTER & $\begin{array}{l}\text { Digestible Protein per } \\
\text { IOOO Live Weight }\end{array}$ \\
\hline $\begin{array}{l}\mathrm{I} \\
\mathrm{I} \frac{1}{3} \\
\mathrm{I} \frac{1}{2} \\
2 \\
2 \\
2 \\
2 \\
2-2 \frac{1}{2} \\
2 \frac{1}{2} \\
3 \\
3\end{array}$ & $\begin{array}{l}\text { Waters } \\
\text { Jordan } \\
\text { Schneidewind } \\
\text { Schneidewind } \\
\text { Jordan } \\
\text { Frear } \\
\text { Waters } \\
\text { Schneidewind } \\
\text { Mumford } \\
\text { Frear } \\
\text { Jordan }\end{array}$ & $\begin{array}{l}2.00 \\
\text { I. } 63 \\
2.00 \\
1.60 \\
1.50 \\
1.50 \\
\text { I.50 to } 2.00 \\
\text { I. } 67 \\
\text { I. } 50 \text { to } 2.00 \\
\text { I. } 00 \\
\text { I. } 26\end{array}$ \\
\hline
\end{tabular}

The foregoing estimates correspond in general with the protein requirements for growing cattle as formulated in the WolffLehmann and Kellner feeding standards (790-793). In experiments upon two steers, directed principally to other questions, Armsby and Fries ${ }^{3}$ observed a normal rate of increase in weight upon rations containing amounts of digestible protein much smaller than are called for by current feeding standards, although still in excess of the estimated normal gain of protein for the cor-

${ }^{1}$ U. S. Dept. of Agr., Bur. Anim. Indus., Bul. Io8 (I go8), pp. 6o-65.

${ }^{2}$ Landw. Jahrb., 36 (I907), 687.

${ }^{3}$ U. S. Dept. of Agr., Bur. Anim. Indus., Bul. I 28 (IgI I), pp. 88-9o. 
responding ages. The experiments do not show, however, that these amounts might not have been still further reduced.

Table 97. - Digestible Protein per iooo Pounds Live Weight. ARMSBY AND FrIES

\begin{tabular}{c|c|c|c}
\hline \multicolumn{2}{c|}{ Steer A } & \multicolumn{2}{|c}{ SteER B } \\
\cline { 1 - 2 } Approximate Age & Digestible Protein & Approximate Age & Digestible Protein \\
\cline { 2 - 4 } Months & Pounds & Months & Pounds \\
$9 \frac{1}{2}$ & I.42 & I 2 & I.64 \\
I $2 \frac{1}{2}$ & I.40 & I $4 \frac{1}{2}$ & I.77 \\
I8 & I.09 & I9 & I.25 \\
$20 \frac{1}{2}$ & 1.03 & 22 & 1.23 \\
$25 \frac{1}{2}$ & 0.72 & 27 & 0.85 \\
\hline
\end{tabular}

Henry and Morrison ${ }^{1}$ likewise report the results of unpublished experiments by Haecker in which growing fattening steers made satisfactory gains on amounts of digestible protein intermediate between those recommended by Kellner for beef and for dairy breeds.

On the other hand, Fingerling's investigations on calves $4 \frac{1}{2}-$ I I months old, already cited in a discussion of the utilization of feed protein (471), indicate that a much lower level of protein supply may be adequate to support normal growth.

The experiments ${ }^{2}$ were made upon four grade or full-blood Simmenthaler calves from four to seven months old at the beginning of the trials, and belonging to early-maturing strains. The rations fed consisted of a basis of hay or straw, or both, to which were added in varying proportions wheat gluten, peanut oil and starch with the necessary amount of salt. The protein supply was varied by varying the amount of wheat gluten, the energy values of the rations being kept as nearly identical as possible by corresponding changes in the starch and oil. The experiments were intended to test the necessity for the relatively large amounts of protein called for by the current standards and also the influence of a deficient energy supply upon the gain of protein.

As appears from Table 87 , the medium rations, supplying in the neighborhood of I.2 pounds of protein per rooo pounds live weight, were clearly sufficient to meet the demands of the maximum possible protein gain, since an increase of the

\footnotetext{
${ }^{1}$ Feeds and Feeding, I 5 th Ed., p. 670.

- 2 Landw. Vers. Stat., 76 (rgr 2), r.
} 
digestible protein to more than double that amount failed to produce any greater gain but simply increased the protein katabolism. That such was the case is likewise indicated by the fact that the actual gains of protein per rooo live weight in these cases agree very well with those computed by the use of the formula on page 378 , tending to be greater rather than less. ${ }^{1}$

487. Experiments with sheep. - The amounts of digestible protein necessary for growing sheep as formulated by Wolff in his original feeding standards were based upon experiments of his own ${ }^{2}$ in which the digestibility of the feed and the gain in live weight were determined. Later Weiske $^{3}$ made a series of ten determinations of the nitrogen balance of two lambs at ages ranging from four to twenty-four months. The rations consumed were meadow hay with a decreasing proportion of grain (peas) in Periods I to VII and of hay alone in the remaining periods, and the rate of increase in live weight was somewhat greater than that of similar animals on pasture. The following table contains the results of both investigations.

Table 98. - Protein Consumed by Growñng Sheep

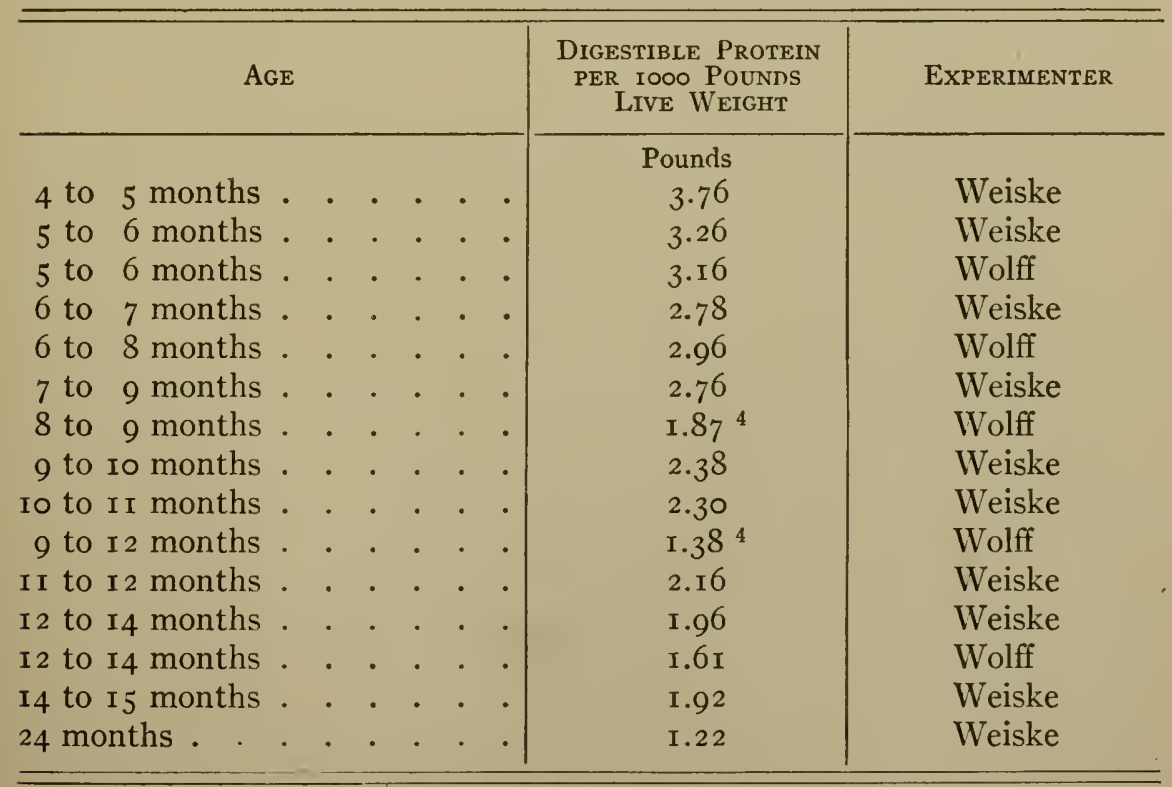

1 The one exception to the above statement is the case animal G in Period III, in which the energy content of the ration was somewhat low.

2 Landw. Jahrb., 2. (1873), 221.

${ }^{3}$ Ibid., 9 (1880), 205.

1 Believed by Wolff to be too low. 
Bull and Emmett ${ }^{1}$ have compiled the results of fifty American experiments on fattening lambs, comprising 5127 animals, and computed the protein and net energy content of the rations consumed. They divide the animals into four classes according to the live weight, and subdivide these classes into groups according to the amount of digestible protein consumed. A comparison of these groups shows in general that in each class, even with a liberal supply of feed energy, the rate of growth increased as the supply of protein increased up to a certain fairly well-defined amount, beyond which a further increase of protein had in general little or no effect. The authors estimate the amounts of digestible protein necessary to ensure satisfactory gains by fattening lambs as follows:-

Table 99. - Estimated Protein Requirements of Fattening Lambs

\begin{tabular}{c|c|c}
\hline Live Weights & Estimated Age & $\begin{array}{c}\text { Digestible Protein PER } \\
\text { rooo LB. Live Weight }\end{array}$ \\
\cline { 2 - 3 } Pounds & Months & Pounds \\
$50-70$ & 5 & $3.1-3.3$ \\
$70-90$ & 7 & $2.5-2.8$ \\
$90-110$ & 9 & $2.2-2.4$ \\
I I0-150 & I 5 & I.4-1.9 \\
\hline
\end{tabular}

On the other hand, Just's results on lambs recorded in Table 88 (471), like those of Fingerling in calves, point to a much lower protein requirement.

488. Experiments with swine. - As is illustrated in Table $93(481 b)$, the swine is distinguished above other farm quadrupeds by its very rapid growth, especially in the earlier stages. The young pig is able to double his weight in little more than a week and to nearly treble it in two weeks, a rate of growth reached or exceeded by no farm animal with the possible exception of young fowls.

Such a rapid rate of growth implies, of course, a correspondingly large storage of protein, a conclusion fully confirmed by the investigations of Ostertag and Zuntz, of Wilson, and of Sanford and Lusk, cited in $\S$ I (463) which showed an average

$$
1 \text { Ills. Expt. Sta., Bul. } 166 \text { (1914). }
$$


daily gain of from six to nine pounds of protein per thousand live weight during the first sixteen days after birth. Plainly, young pigs need a relatively large supply of protein in their feed, but unfortunately no attempts have thus far been reported to determine the minimum of feed protein necessary at different ages and especially by older pigs, simply to ensure normal growth. There are on record, however, a considerable number of experiments in which rations supplying varying amounts of protein have been fed to fattening pigs and the effects upon the make-up of the carcass and upon the rate of increase in live weight observed. These experiments have served to demonstrate in a striking manner the practical advantages of a liberal protein supply and while in many instances the minimum protein requirement may have been considerably exceeded, nevertheless, the results as a whole are perhaps no less useful as a guide in practice.

It is impossible to include here even an enumeration of the large number of experiments of this sort. For a summary of earlier investigations the student may be referred to the summary published by Wolff in $1876 .^{1}$ A considerable number of earlier experiments in the United States as compiled by the writer gave results of the same general nature.

The later experiments upon this subject may be divided into those directed more specifically to the determination of the influence upon quality and chemical composition of the carcass and those in which the increase in live weight was the principal criterion.

\section{Effect of insufficient protein upon the carcass of pigs. -} Striking results as to the make-up of the carcass in young pigs have been reported by several investigators in experiments in which exclusive maize feeding was compared with the use of mixed rations supplying much more protein and ash. The trials have been popularly spoken of as "Feeding for fat and for lean." In reality they are a study of the effect of inadequate protein (and ash?) supply in limiting growth. The subject was first taken up by Sanborn ${ }^{2}$ and soon after by Henry. $^{3}$ In general it was found that in the pigs receiving the

1 Ernährung der landwirtschaftlichen Nutztiere, pp. 465-496.

2 Mo. Agr'l College, Bul. Io, I 4 and 19.

${ }^{3}$ Wis. Expt. Sta., Rpts 4, 5, 6, I7, I8, 19 and 21 . 
low protein (maize) rations the weights of blood, of internal organs and in some cases of certain individual muscles were relatively less than with comparable animals receiving the high protein (mixed) rations, while on the other hand, the deposits of adipose tissue appeared notably greater in the maizefed animals.

Since these investigations were made it has become a well-recognized fact that the mixed proteins of maize are inadequate to support rapid growth (783) and the results reached are to be regarded as being to a considerable degree the expression of this qualitative deficiency.

As regards the quantitative aspect of the experiments it is to be remarked that in most instances the difference between the rations as regards protein was purposely made large. While the experiments have made it clear that exclusive maize feeding fails to afford an adequate supply of protein for growing pigs, it does not follow that as large quantities of protein as were contained in the contrasting rations were necessary. In the later Wisconsin experiments especially, as the writer has pointed out, ${ }^{1}$ the gain in live weight was often little greater on the high protein than on the low protein rations and sometimes even less.

Furthermore, while the animals were compared more or less extensively as to the weights of the various organs at the close of the feeding, and in one instance at least the carcasses were analyzed, the experiments were not of the nature of comparative slaughter tests and did not afford data for computing the actual amount of protein gained. Moreover, the results upon the carcasses analyzed ${ }^{2}$ seem to indicate that the rations affected the adipose tissue as to its distribution through the carcass rather than as to its total amount.

Finally, the striking results as to general thrift, and especially as to the growth and strength of the bones, are probably to be attributed to differences in the ash supply (496), quite as much as to differences in the protein supply.

On the whole, while these investigations are valuable from the standpoint of practice as a demonstration of the ill effects of a deficient amount or quality of protein, it cannot be said that this class of experiments affords very definite information as to the actual protein requirements of pigs.

1 U. S. Dept. Agr., Bur. Anim. Indus., Bul. ro8 (I908), p. 74.

${ }^{2}$ Iowa Expt. Sta., Bul. 48 (I900), pp. 373-45I ; U. S. Dept. Agr., Bur. Anim. Indus., Bul. 108, p. 75. 
490. Fattening experiments with pigs. - Of the more recent experiments upon the influence of the protein supply upon the rate of gain of immature fattening pigs, four series made by the Halle Experiment Station at Lauchstädt ${ }^{1}$ and a series of cooperative experiments at a number of the German experiment stations under Kellner's general direction are of special interest. While these relate primarily to fattening, the comparative results with rations of equal energy content should furnish some indications as to the sufficiency of the protein supply, since the rate of increase of protein tissue can hardly be supposed to differ materially from that in simple growth without fattening.

In the Halle experiments it is interesting to note the gradual lowering of the average protein supply from the high level of the first series. The final series seems to show that satisfactory results may be obtained from rations whose protein content per Iooo pounds at the different weights of the animal is as follows, although the earlier trials seem to indicate somewhat higher figures.

Table ioo. - Estimated Protein Requirements of Fattening Pigs

At weight $77^{-100 ~} \mathrm{lb}$. At weight $110-165 \mathrm{lb}$. At weight $165-220 \mathrm{lb}$. At weight over $220 \mathrm{lb}$.

In 1906 coöperative experiments were initiated by the German Agricultural Council at a number of German experiment stations upon the value of potatoes as feed for fattening pigs and especially upon the protein supply necessary for their most complete utilization. The results of experiments upon this point at eleven stations, upon a total of I 84 animals, have been discussed by Kellner ${ }^{2}$ under whose general direction the work was done. According to Kellner ${ }^{3}$ young fattening pigs should receive per 1000 live weight the following amounts of protein :-

${ }^{1}$ Landw. Jahrb., 28 (I899), $947 ; 31$ (I902), 916; 36 (I907), 679; 39, Ergzbd. III (1910), I79.

2 Ber. Deut. Landw. Rat, Heft 3 (1908).

${ }^{3}$ Die Ernährung der landw. Nutztiere, 5th Ed., p. 488. 
Table ioi. - Kellner's Standards for Fattening Pigs

\begin{tabular}{|c|c|c|}
\hline AGE & Average Live Weight & $\begin{array}{l}\text { Digestible PROTEIN PER } \\
1000\end{array}$ \\
\hline Months & Kgs. & \\
\hline $2-3$ & 20 & 6.2 \\
\hline $3-5$ & 50 & $4 \cdot 5$ \\
\hline $5^{-6}$ & 65 & $3 \cdot 5$ \\
\hline $6-8$ & 90 & 3.0 \\
\hline $9-12$ & I 30 & 2.4 \\
\hline
\end{tabular}

The outcome of the coöperative experiments tended to confirm these standards, indicating that any considerable departure from them will fail to meet the requirements of rapid growth in the best strains of animals or to secure the largest returns. On the other hand, the fourth Lauchstädt series perhaps points in the direction of lower standards, especially for the more mature animals. For breeding animals Kellner recommends somewhat smaller amounts of protein, viz. :-

Table ro2. - Kellner's Standards for Growth of Pigs

\begin{tabular}{c|c|c}
\hline AGE & LIVE WEIGHT & $\begin{array}{c}\text { PROTEIN PER } \\
\text { IOOO }\end{array}$ \\
\hline Months & Kgs. & \\
$2-3$ & 20 & 6.2 \\
$3-5$ & 40 & 4.0 \\
$5-6$ & 55 & 3.0 \\
$6-9$ & 80 & 2.3 \\
$9-$ I 2 & I 20 & 1.7 \\
\hline
\end{tabular}

Dietrich ${ }^{1}$ recommends notably larger amounts of protein, although he too recommends less for breeding than for fattening animals. His figures for fattening pigs are 6.0 to 7.0 per 1000 up to the age of about 6 months, followed by a gradual reduction to 3.3 per thousand at 7 to 8 months. For breeding animals his figures are 5.0 to 5.5 up to 6 months of age, gradually diminishing from that point to 2.0 at maturity.

491. General conclusions. - It is apparent from the foregoing paragraphs that the evidence regarding the protein re-

${ }^{1}$ Ills. Expt. Sta., Circulars 126, I33 and I 53. 
quirements for growth is fragmentary and more or less conflicting. On the one side are investigations like Fingerling's on cattle and Just's on sheep $(486,487)$ which appear to show that it is possible for the animal to support what seems a normal rate of growth upon a supply of protein little greater than the maintenance requirement plus the amount actually stored. On the other side stand the results of experiments and observations upon the fattening of immature animals, in which rations at least approximately equal as to their content of net energy, and therefore presumably equally effective for simple fattening, have produced a greater increase in weight when they contained relatively much more protein than the results of the other class of experiments would indicate to be necessary. Further consideration of this apparent conflict of evidence, however, shows that the two classes of experiments are hardly comparable:

For one thing, the experiments in which a relatively high protein supply seemed advantageous were all fattening experiments. The effect of the feed was measured by the gain in live weight, which itself is a somewhat uncertain criterion, while a considerable share of this increase was due to a storage of fat rather than of protein. Fingerling's and Just's experiments, on the contrary, relate distinctly to growth and the comparisons are based on the actual amounts of protein tissue produced, although it must be admitted that any experimental errors would probably tend to make the excretion of nitrogen appear too low, and therefore the gain of protein too high.

Another important difference between the two classes of experiments lies in the nature of the rations. In the metabolism experiments they were composed largely of commercially pure nutrients such as starch, oil, etc., with only the amount of roughage necessary to supply bulk, and in particular, the variations in the protein supply were effected by changes in the amount of commercially pure wheat gluten. In the fattening experiments, on the contrary, the higher protein content of the rations was obtained by the use of the ordinary proteinrich feeding stuffs. What these experiments really show is that a larger proportion of these feeding stuffs was advantageous, but it does not necessarily follow that this advantage was due to the added protein. For one thing such a modification of the rations must have affected the ash supply to a certain extent. 
In particular, however, the influence of those accessory substances or "growth substances" $(498,499)$ which recent investigations have shown to play such an important part in conditioning growth is to be considered. It seems not impossible that high-protein feeds may in some such way have a stimulating effect upon the capacity for growth quite independently of their protein content. On the other hand, however, any such stimulating effect upon growth would be absent in experiments made with pure nutrients added to a basal ration of hay or straw, and yet a fairly normal rate of increase seems to have been maintained.

On the whole, one can hardly fail of the impression that the requirements for protein as such in growth have been overestimated and that the organism may utilize its protein supply more economically than the current feeding standards would indicate; in other words, that the actual protein supply may be made considerably smaller than has been supposed before it becomes a limiting factor in growth. Until this impression is confirmed by more extensive investigation, however, it appears the safer course to adhere provisionally to the accepted standards, and the protein requirements for growth as estimated in Table IV $b$ of the Appendix are based upon those formulated by Kellner.

\section{Ash requirements}

492. Growth involves storage of ash. - The growing animal, like the mature one, requires mineral ingredients for the purposes enumerated in Chapter $\mathrm{V}$ (268-272), but in addition to this the formation of new tissue and especially of the skeleton involves the storage of ash ingredients which must be derived from the feed. This is shown clearly by the data recorded in $\S$ I (458) regarding the composition of the increase, its ash content, aside from one exceptional case, ranging from 1.42 per cent to 6.18 per cent.

493. Rate of storage in growth. - Data regarding the rate of storage of mineral elements in growth are not very numerous and are largely confined to experiments on the two important elements calcium and phosphorus. The principal investigations are those of Soxhlet, ${ }^{1}$ Neumann, ${ }^{2}$ Lehmann, ${ }^{3}$ and Weiske ${ }^{4}$

$1_{\text {I }}$ er Ber. Versuch-Station Wien, pp. IoI-I 55.

${ }^{3}$ Landw. Vers. Stat., 1 (1859), 68.

2 Jour. Landw., 41 (I893), 343.

4 Jour. Landw., 21 (I873), I39. 
on calves, those of Weiske ${ }^{1}$ on lambs, and those of Forbes ${ }^{2}$ and of .Weiser ${ }^{3}$ on pigs.

Arranging the available results upon the retention of calcium and phosphorus per rooo live weight in order of the age of the animals, irrespective of the species, gives the following showing of the effect of age upon the rate of gain of these two elements. It appears that in suckling animals, the rate of gain of the ash ingredients, like that of protein and energy $(463,464)$, is relatively high, while there is a distinct falling off in the rate as the animal grows older, although not to the same extent as in the case of the organic nutrients.

Table 103. - Dally Retention per rooo Live Weight

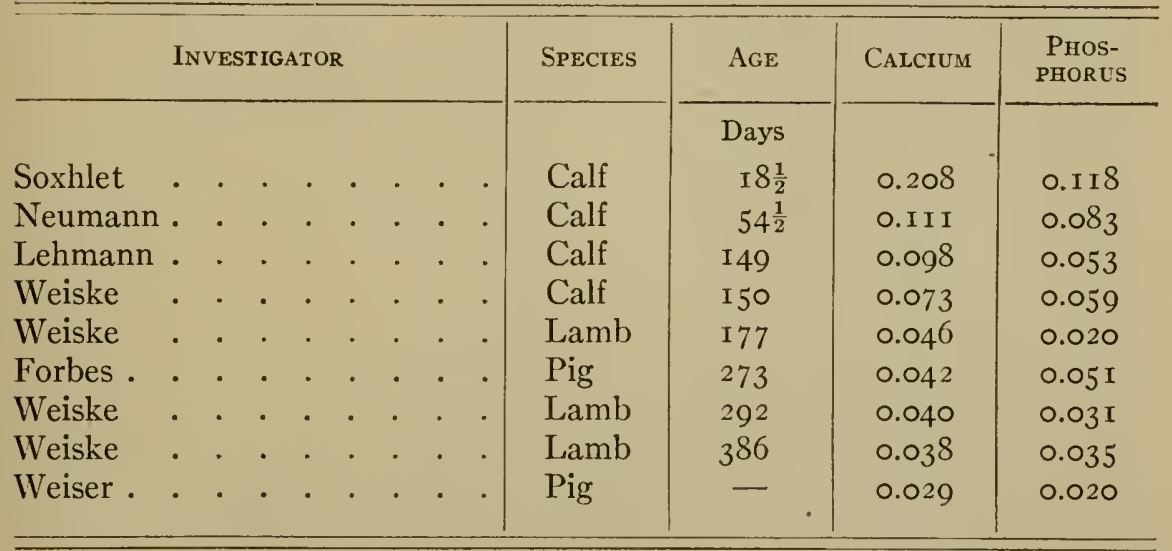

The data of the foregoing experiments hardly afford an adequate basis for estimating the ash requirements at different ages. As compared with the amounts of mineral elements consumed in or assimilated from the feed, the body of even a very young animal contains a large stock of these substances which can be drawn upon to a certain extent to meet any temporary deficiency in the feed. It is possible, therefore, that the amounts retained in the relatively short periods of the foregoing experiments may be less than are necessary or desirable for continuous normal growth. On the other hand it would appear from Forbes' results ${ }^{4}$ that, under favorable conditions, ash may be stored in the bones in excess of the actual maintenance needs and constitute a reserve of mineral matter in the body. The

1 Landw. Jahrb., 9 ( 1880 ), 205.

${ }^{3}$ Biochem. Ztschr., 44 (1912), 279.

2 Ohio Expt. Sta., Technical Bul. 5, p. 378.

${ }^{4}$ Loc. cit., p. 371 . 
fact shown in Chapter IX (435) that more or less storage of ash elements may occur even in the mature animal points in the same direction. The organism appears far less sensitive to fluctuations of its daily supply of ash than to those of the organic nutrients because it has relatively a much larger reserve to draw upon.

494. Total retention during growth. - Some notion of the total amounts of mineral elements assimilated during growth may be secured by computing from Lawes and Gilbert's analyses of the ash of the entire bodies of farm animals the weights of each ash ingredient contained in them. Thus if the live weight at one year old be assumed to be for cattle 400 kilograms (880 pounds) and for sheep 50 kilograms (I ro pounds) and for a six months' old pig 50 kilograms, then, applying the analyses of the half-fat ox, store sheep and store pig respectively, the total amounts of mineral elements in the bodies and the average daily retention per head (including the stock contained in the bodies at birth in the case of the sheep and pig) would be as follows:-

Table io4. - Total Retention of Ash Ingredients During Growth

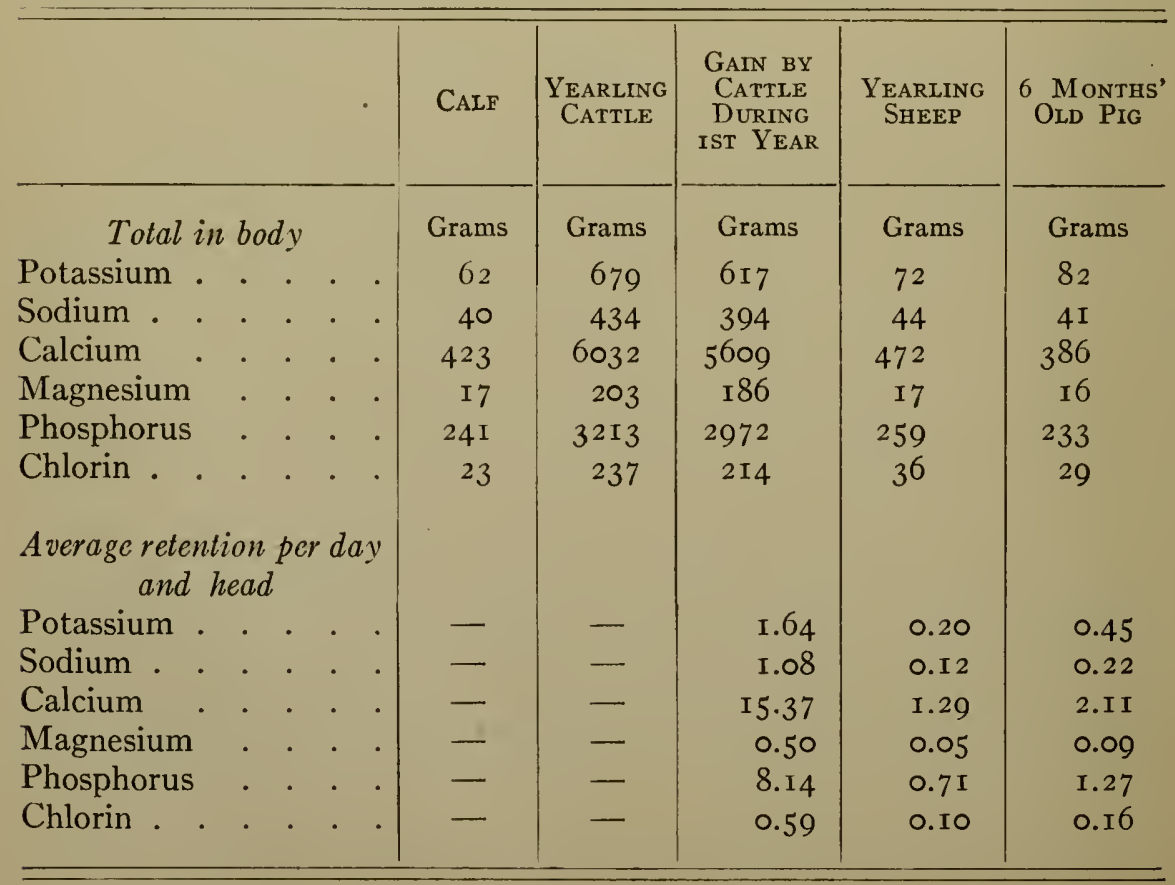


In order to compare the figures thus obtained with the results recorded in the previous paragraph for the retention during short periods, it is necessary to eliminate the influence of varying weight by computing the results per Iooo. The retention of calcium and phosphorus as thus computed agrees very well with that found in the balance experiments with the exception of Forbes' high result for phosphorus with the pig. On the other hand the computed retention of the alkalies is strikingly less, a fact for which no obvious reason appears.

Table 105. - Average Daily Retention per 1000 Live Weight

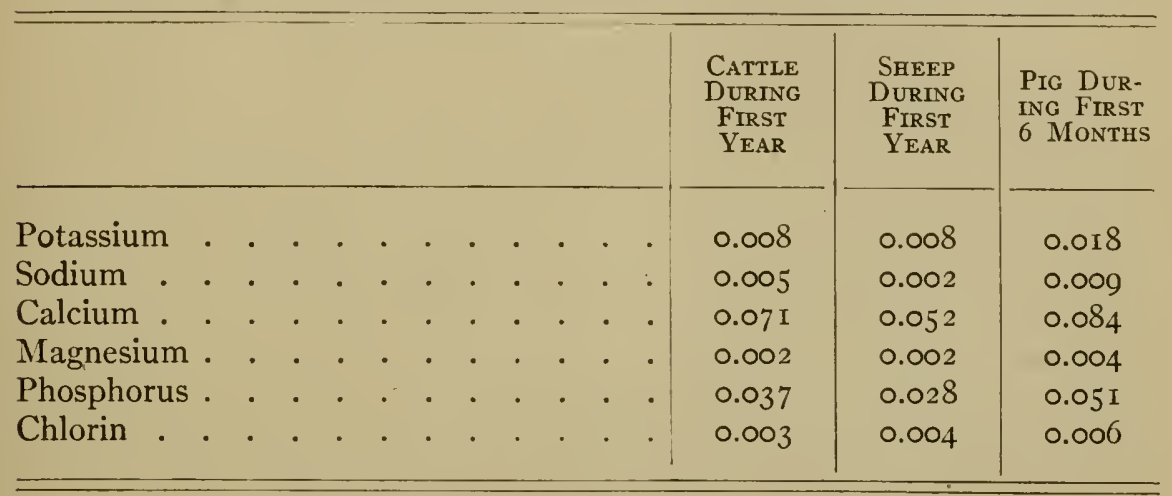

On the whole it appears that our knowledge of the ash requirements of growing animals, that is, of the actual amounts stored up in normal growth, is quite fragmentary and unsatisfactory.

495. Availability of ash ingredients of feed. - If it is difficult to formulate from existing data any trustworthy estimates of the ash requirements of growing animals, it is even more difficult to make any definite statements regarding the total amount of any particular element which must be supplied in the feed in order to meet those requirements, although to the extent to which the results recorded in the previous paragraphs are trustworthy, it is possible to formulate the minimum supply. Thus, Weiske's results on sheep show a retention of from 20 to 35 mgrs. of phosphorus per kilogram of live weight. If these figures represent the normal requirements, it is evident that a ration containing less than this amount would not supply enough phosphorus for normal growth. What surplus above this amount is necessary in the feed would depend on the proportion of the feed phosphorus which is capable of solution in 
the digestive tract, and still more upon the effect of other elements on the elimination of phosphorus. An amount of this element amply sufficient to meet the normal demand when supplied in one ration might be quite inadequate in another of a different character.

Since the intestines are the normal path of excretion for some ash elements $(164,199)$, a computation of the digestibility of these elements in the ordinary sense, by comparing the amounts in feeds and feces, gives an entirely false idea of their availability. Moreover, it was shown in Chapter IX (429-433) that the rate at which mineral elements are lost from the body depends to a large degree upon the qualitative composition of the ash of the feed, variations in the supply of one element sometimes affecting materially the gain or loss of another. In particular it was pointed out that the proportion of acid and basic elements and to a less degree the ratio of potassium to sodium may have striking effects of this sort. For example, in Weiser's experiments on pigs (493), the addition of 5 grams of calcium carbonate to a ration of 1000 grams of maize not only changed a loss of calcium into a gain but also produced the same effect on the phosphorus balance, so that a phosphorus supply which was previously insufficient to maintain the body was able to support a material gain.

Table ro6. - Ash Balance of Swine with and without Calcium Carbonate

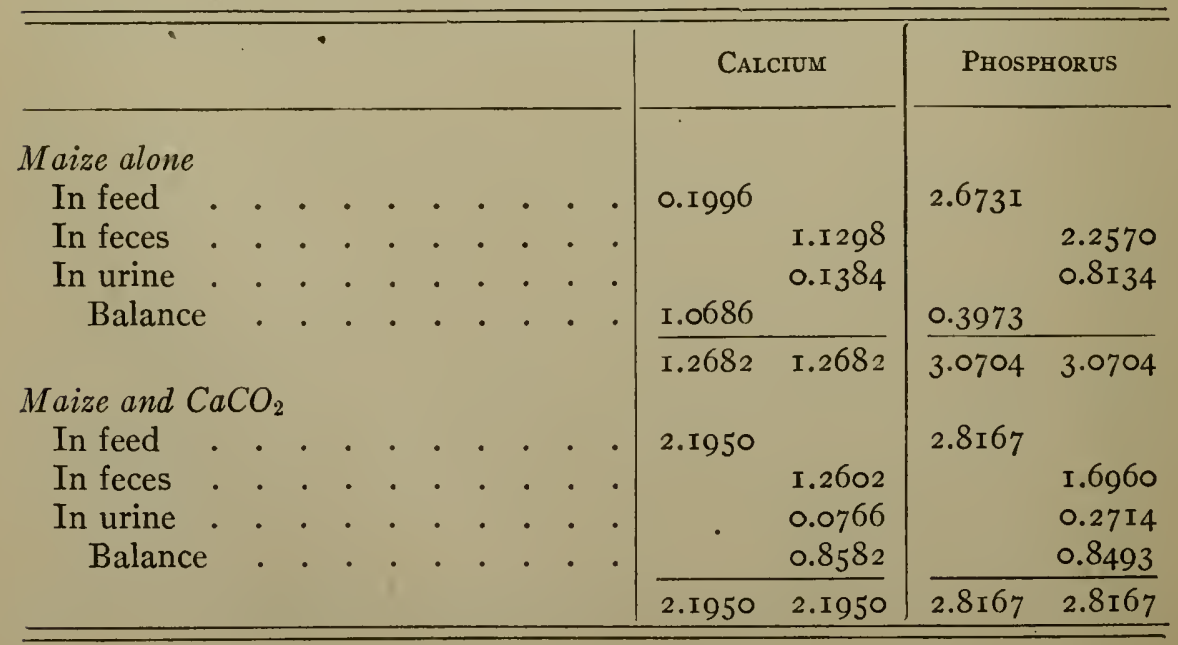


It has been maintained, principally on the basis of Soxhlet's experiments on calves (493), that the availability of the ash ingredients of milk, and particularly of its calcium and phosphorus, is especially high. The percentages retained in the body on the average of the five trials were:-

\begin{tabular}{|c|c|c|c|c|c|c|c|c|}
\hline Cotal ash & & & & & & & & \\
\hline Potassium & & & & & & & & \\
\hline Sodium & & & & & & & & \\
\hline Calcium & & & & & & & & \\
\hline Mag & & & & & & & & \\
\hline Pho & & & . & & & & & \\
\hline Chlorin & & & & & & & & \\
\hline
\end{tabular}

Neumann's experiments with somewhat older calves (493), however, render it evident that the cause of the high retention of calcium and phosphorus was the large demand for these elements in the body. It can hardly be supposed that these elements are less assimilable in the skim milk used in Neumann's experiments, yet the percentage retention was scarcely more than one-half as great as in Soxhlet's experiments, viz., in the experiments on skim milk alone.

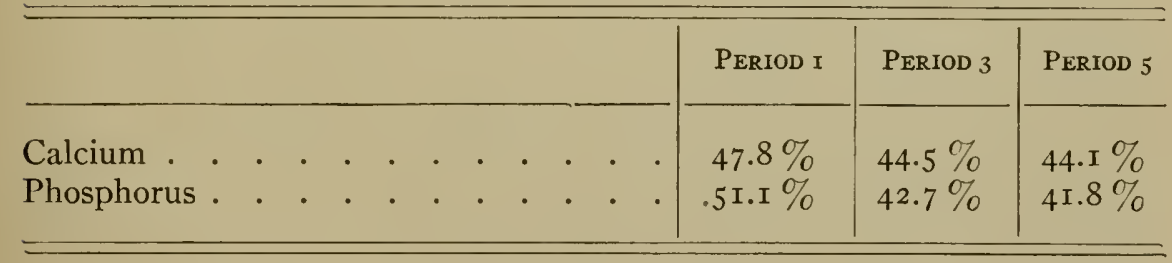

The older animals obviously required less of these elements and therefore excreted the excess, the phosphorus in the urine and the calcium in the feces.

Lehmann's and Weiske's experiments (493) with older calves on mixed rations showed a percentage availability of the phosphorus and calcium fully as great as that observed for skim milk in Neumann's experiments, and here too the natural conclusion is that the demand for these elements in the body, rather than any lower availability per se, is the cause of the less assimilation. It is well established that the inorganic phosphates may be quite completely assimilated, and Fingerling ${ }^{1}$

\footnotetext{
${ }^{1}$ Landw. Vers. Stat., 79-80 (I9I3), 847; 86 (I9I5), 75.
} 
has shown the same thing to be true of a variety of organic phosphorus compounds, as well as of the phosphorus of concentrated feeding stuffs, while in case of roughages ${ }^{1}$ an availability of approximately 50 per cent was observed. These facts throw some doubt on Kellner's conclusion that the feed should contain two or three times the quantities of minera! elements which would normally be stored in the body.

496. Effects of deficiency of ash. - But while it seems scarcely possible to make any definite quantitative statements regarding the necessary ash supply of growing animals there is abundant evidence of the evil effects of an insufficient supply. In particular, a deficiency of calcium, as already indicated, may have serious consequences both directly and on account of the fact that such a deficiency generally connotes an acid ash (431).

Kellner ${ }^{2}$ cites experiments by. Roloff and by Voit, in which young dogs and pigs receiving feed poor in calcium showed deficient growth and developed severe pathological symptoms, the skeleton showing a notable deficiency in ash ingredients (Rachitis). Forbes ${ }^{3}$ has collected a large number of experiments on this subject in some of which marked effects on the composition of the bones were observed while in others these effects were not very distinct. In still more recent experiments by Weiser ${ }^{4}$ upon pigs, a diet deficient in calcium restricted the growth and produced a skeleton containing an excess of water and organic matter and deficient in ash. Contrary to the results of Aron (428) the bone ash on the calcium-poor rations was deficient in calcium and contained an excess of alkalies, especially sodium.

Of farm animals, pigs are most likely to suffer in this way, partly because their growth is relatively rapid and.partly because they often receive almost exclusive grain rations which are apt to be low in calcium (431). Henry ${ }^{5}$ has shown that supplementing such rations with calcium phosphate or carbonate results in the production of heavier and stronger bones, and Burnett ${ }^{6}$ has confirmed these results. Hart and $\mathrm{McCol}$ -

1 Biochem. Ztschr., 37 (I9I I), 266.

2 Ernährung der landw. Nutztiere, 6th Ed., p. 177.

${ }^{3}$ Ohio Expt. Sta., Tech. Bul. 5, pp. 384-390.

${ }^{4}$ Biochem. Ztschr., 66 (r9r4), 95.

5 Wis. Expt. Sta., 6th Rpt., pp. 6-4I ; Bul. 25.

${ }^{6}$ Neb. Expt. Sta., Buls. 94 and 107 and $23 \mathrm{~d}$ Rpt. 
lum $^{1}$ found that confined pigs on a ration of maize alone and drinking distilled water failed to grow, while the addition of an artificial mixture of salts enabled nearly normal growth to be made.

With cattle and sheep a deficiency of calcium is not usually to be feared, since roughages are usually rich in this element. Straw and roots, however, are rather low in calcium and so are certain by-product feeds, especially those like gluten feed and meal, distiller's grains, etc., which have been subject to extraction with water.

497. Forms of phosphorus. - A much discussed question is that of the relative value of organic and inorganic phosphorus compounds. It was stated in Chapter V (258), that the animal body is apparently able to synthesize its organic phosphorus compounds from inorganic phosphorus. Forbes ${ }^{2}$ has given a very complete review of the literature of this subject. His general conclusion is that it has not been proven that a supply of organic phosphorus is essential, although he regards the proof that inorganic phosphorus can serve all the purposes for which any animal needs phosphorus as being incomplete. As regards the relative efficiency of the two, the facts already noted in Chapter IX $(437,438)$ and in the following paragraphs, regarding the importance of accessory substances, in particular the so-called growth substances, in nutrition strongly suggest that the apparent superiority of organic phosphorus which has been observed in some experiments may have been due to the presence of such substances accompanying the organic phosphorus compounds and not to the latter as such.

\section{Accessory substances}

498. Relation of fats to growth. - It was mentioned in Chapter V (265), in considering the functions of the nutrients, that it had apparently been shown that the presence of a certain minimum amount of fat (or at least of ether-soluble substances - lipoids) was necessary for growth. Later investigations, however, have led to a different interpretation of these 
earlier results. As the technique of experimentation with isolated nutrients has been developed by the work of Röhmann, McCollum, Osborne and Mendel and others, it has become evident that it is not the lipoids as such but some substance or substances associated with them which are essential tò continued growth. On the one hand, growth has been maintained, for a considerable time at least, on a practically fat-free diet, while on the other hand it has been shown that by no means all fats are capable of exerting this favorable effect on growth.

Both McCollum and Osborne and Mendel have found that rats fed mixtures of purified nutrients containing no fat may grow normally for a considerable time, but after about 75 or 100 days, and after reaching perhaps $\frac{2}{3}$ of the mature weight, there is a more or less abrupt cessation of growth followed by a speedy decline in weight. Substantially the same result ensues when certain forms of fat (lard, beef fat, olive oil, almond oil) are added to the ration, but if, on the other hand, purified butter-fat, cod liver oil or certain other fats be added to the ration of an animal which has ceased to grow and begun to decline in weight this decline is promptly stopped and practically normal growth resumed. These results indicate the existence of two groups of fats, one of which aids growth while the other does not. Evidently, therefore, the growth supporting property does not reside in the glycerids themselves but in some accompanying substances.

499. Growth substances. - On the basis of later investigations, McCollum ${ }^{1}$ rejects Funk's hypothesis of the existence of numerous specific "vitamins" and distinguished only two growth substances (or classes of substances), both of which are essential to growth. One, lipoid-soluble, which he calls fatsoluble $A$, is associated with certain fats, while the other, called water-soluble $\mathrm{B}$, is soluble in water and apparently never associated with fats. The fat-soluble $A$ is absent from all vegetable fats thus far examined. It is present in small but insufficient amounts in the grains but appears to be relatively abundant in the leaves of plants.

That other factors than these specific growth substances may markedly influence growth is, however, apparent from recent experiments by Hart and McCollum ${ }^{2}$ who found that the freedom

\footnotetext{
1 Jour. Biol. Chem., 23 (I9I 5), I8I and 23I ; 25 (1916), I05; Amer. Jour. Physiol., 41 (rgr6), 333 and 36r.

${ }^{2}$ Jour. Biol. Chem., 19 (rgr 4), 373.
} 
of a small paddock in which they can root stimulates the growth of pigs to a degree quite out of proportion to the amount of actual feed thus obtained. Furthermore, they have shown that, with both pigs and cows (438), rations consisting exclusively of wheat products seem to have a direct effect in hindering growth. The subject is one which is hardly ripe for discussion, but it opens up an interesting field for investigation, while it emphasizes the importance of variety in rations.

No data have been published regarding the percentage utilization of the feed actually consumed in these experiments as measured by the amount of growth actually made on the inadequate rations. 


\section{CHAPTER XII}

\section{MEAT PRODUCTION ${ }^{1}$}

\section{$\S$ i. Nature of Meat Production}

500. Definitions. - By "meat" is understood in a general way the flesh of an animal as distinguished from the skeleton on the one hand and the internal organs, hide and other offal on the other. Meat in this general sense is separable mechanically into adipose tissue ("fat") and lean meat, both of which, but especially the latter, are of somewhat complex composition.

The adipose tissue (94) consists of connective tissue in which a greater or less accumulation of fat has taken place and is essentially a reserve of non-nitrogenous, energy-yielding material. The lean meat, or meat in the narrower sense (86), consists primarily of muscular tissue along with more or less fat, and its characteristic ingredients are the proteins.

501. Proportion of fat and lean in carcass. - The proportion of lean meat to fat tissue in the carcass is naturally quite variable, depending somewhat upon the age but chiefly on the feeding of the animal, insufficient nutrition reducing the store of fat in the body to a minimum while heavy feeding may cause the production of large amounts of it. Thus Lawes and Gilbert found the proportion of fat in the carcasses of the ten animals analyzed by them (97) to vary from I5.3 to 48.3 per cent. Jordan observed a range of 18.80 to 24.62 per cent in steers $2-2 \frac{1}{2}$ years old. Tschirwinsky reports the extremes of 10.39 and 40.92 per cent in pigs, while Wilson found a minimum of I.3I per cent in new-born pigs. Atwater ${ }^{2}$ gives the following as the average composition of a side of beef of medium fatness :-

1 The discussions in this chapter follow, to a considerable extent, those presented by the writer in U. S. Dept. Agr., Bur. Anim. Indus., Bul. Io8 (Igo8).

${ }^{2}$ U. S. Dept. Agr., Office Expt. Stas., Bul. 2 I (I895), p. 35. 
Table 107. - Average Composition of a Side of Beef of Medium Fatness

\begin{tabular}{|c|c|c|c|c|c|c|c|c|c|c|c|c|c|c|c|c|c|c|c|}
\hline & & & & & & & & & & & & & & & & & & & $\%$ \\
\hline \multirow{5}{*}{\multicolumn{2}{|c|}{$\begin{array}{l}\text { Water } \\
\text { Protein } \\
\text { Fat. } \\
\text { Ash. }\end{array}$}} & \multirow{5}{*}{. } & \multirow{5}{*}{\multicolumn{2}{|c|}{$\begin{array}{ll}\cdot & \cdot \\
\cdot & \cdot \\
\cdot & \cdot \\
\cdot & \cdot\end{array}$}} & \multirow{5}{*}{$\cdot$} & \multirow{5}{*}{\multicolumn{2}{|c|}{$\begin{array}{ll}\cdot & \cdot \\
\cdot & \cdot \\
\cdot & \cdot \\
\cdot & \cdot\end{array}$}} & \multirow{5}{*}{$\stackrel{.}{.}$} & \multirow{5}{*}{\multicolumn{2}{|c|}{$\begin{array}{ll}\cdot & \cdot \\
\cdot & \cdot \\
\cdot & \cdot \\
\cdot & \cdot\end{array}$}} & \multirow{5}{*}{$\begin{array}{l}\dot{ } \\
\dot{ } \\
\dot{ }\end{array}$} & \multirow{5}{*}{\multicolumn{2}{|c|}{$\begin{array}{lll}\cdot & \cdot & \cdot \\
\cdot & \cdot & \cdot \\
\cdot & \cdot & \cdot \\
\cdot & \cdot & \cdot\end{array}$}} & \multirow{5}{*}{\multicolumn{2}{|c|}{$\begin{array}{ll}\cdot & . \\
\cdot & . \\
\cdot & . \\
\cdot & .\end{array}$}} & \multirow{5}{*}{$\begin{array}{ll}\cdot & \cdot \\
\cdot & \cdot \\
\cdot & \cdot \\
\cdot & \cdot\end{array}$} & \multirow{5}{*}{\multicolumn{2}{|c|}{$\begin{array}{lll}\cdot & \cdot & \cdot \\
\cdot & \cdot & \cdot \\
\cdot & \cdot & \cdot \\
\cdot & \cdot & \cdot\end{array}$}} & 54.77 \\
\hline & & & & & & & & & & & & & & & & & & & 17.20 \\
\hline & & & & & & & & & & & & & & & & & & & 27.07 \\
\hline & & & & & & & & & & & & & & & & & & & 0.96 \\
\hline & & & & & & & & & & & & & & & & & & & 100.00 \\
\hline
\end{tabular}

Lean cuts of meat, however, may contain much less fat than is indicated by the foregoing statement. Thus Grindley and Emmett ${ }^{1}$ analyzed seven samples of beef round from which the visible fat had been removed. The minimum figure for fat was 3.I9 per cent in the fresh substance, or I2.29 per cent of the dry matter. The average of the seven analyses was as follows:-

Table io8. - Average Composition of Seven Samples of Beef Round with Visible Fat Removed

\begin{tabular}{|c|c|c|c|c|c|c|c|c|c|c|c|c|c|}
\hline & & & & & & & & & & & & $\begin{array}{l}\text { IN FRESH } \\
\text { SubSTANCE }\end{array}$ & $\begin{array}{l}\text { IN WATER-FREE } \\
\text { SUBSTANCE }\end{array}$ \\
\hline $\begin{array}{l}\text { Water } \\
\text { Ash } \\
\text { Protein } \\
\text { Extractives } \\
\text { Fat. }\end{array}$ & $\begin{array}{l}\cdot \\
\cdot \\
\text {. } \\
\text {. } \\
\text {. }\end{array}$ & $\begin{array}{l}\cdot \\
\cdot \\
\cdot\end{array}$ & $\begin{array}{l}\cdot \\
\cdot \\
\cdot \\
\cdot\end{array}$ & $\begin{array}{l}\cdot \\
\cdot \\
\cdot \\
\cdot \\
\cdot\end{array}$ & $\begin{array}{l}\cdot \\
\cdot \\
\cdot \\
\dot{ } \\
\cdot\end{array}$ & $\begin{array}{l}\cdot \\
\cdot \\
\cdot \\
\cdot \\
\cdot\end{array}$ & $\begin{array}{l}\cdot \\
\cdot \\
\cdot \\
\cdot \\
\cdot\end{array}$ & $\begin{array}{l}\cdot \\
\cdot \\
\cdot \\
\cdot \\
\cdot\end{array}$ & $\begin{array}{l}\cdot \\
\cdot \\
\cdot \\
\cdot \\
\cdot\end{array}$ & $\begin{array}{l}\cdot \\
\cdot \\
\cdot \\
\cdot \\
\cdot\end{array}$ & $\begin{array}{ll}\cdot & \cdot \\
\cdot & \cdot \\
\cdot & \cdot \\
\cdot & \cdot \\
\cdot & .\end{array}$ & $\begin{array}{r}\% \\
73.30 \\
1.13 \\
\text { I8.79 } \\
2.98 \\
4.88 \\
\end{array}$ & $\begin{array}{c}\% \\
- \\
4.27 \\
70.67 \\
\text { I I.07 } \\
17.8 \mathrm{I} \\
\end{array}$ \\
\hline & & & & & & & & & & & & 100.00 & 100.00 \\
\hline
\end{tabular}

Voit found in the carefully prepared lean meat which he used as representing substantially protein feed, and which had been most painstakingly freed from all visible fat, 0.9 I per cent of ether extract in the fresh substance, equal to 3.77 per cent of the dry matter.

The term meat commonly suggests to the mind the muscular tissue of the animal, and has become almost synonymous with

1 Ills. Expt. Sta., Bul. I62. 
a protein diet. It is evident, however, that the commercial growing of meat may involve the production of considerably more fat than protein and that, in so far as this fat is actually consumed, meat is far from being the distinctively protein food which it is. ordinarily considered. Thus the so-called "nutritive ratio" of the average side of beef, calculated in the usual manner, is about $I: 3.5$. On the other hand, however, it is equally true that the proteins of meat are the distinctive ingredients for the sake of which it is produced and eaten, while the fat, although a valuable nutrient, is to a certain extent subsidiary and accidental.

502. Processes involved. - Corresponding in a general way to the two main constituents of commercial meat, viz., muscular tissue and adipose tissue, two distinct physiological processes are involved in meat production, viz., growth and fâttening.

Growth. - The animal at birth is usually regarded as unfit to serve as human food. Moreover, even were this not the case it would be in the highest degree uneconomic to fail to utilize the marked assimilative powers of the young animal for the production of body tissue (meat) from feed. Consequently the production of meat involves more or less growth in all cases. This may, for special reasons, be concluded early, as in the production of lamb or veal, but as a whole the world's commercial meat supply is derived from animals at least approaching maturity. This growth of animals from birth to approximate maturity consists essentially of an increase in the protein tissues (457), the rate of which is essentially determined by the nature and individuality of the animal and can at most be but slightly stimulated by an increased protein supply $(403,484)$.

Fattening. - Fattening, on the contrary, is a process which, in a given animal at least, is largely under the control of the feeder. Substantially it is dependent on the quantity of feed consumed by the animal in excess of the requirements for maintenance and growth, and there is lacking any definite proof that the actual storage of energy in the form of gain for a given amount of excess feed is seriously affected either by the age or the individuality of the animal. Fattening, therefore, may take place at any age, although of course the greater demand 
for material for growth in the young animal tends to reduce the proportion of the feed available for fattening.

The prime object of fattening (446) is an improvement in the quality of the meat by the deposition of fat between the fibers of the meat, and to some extent by increasing the extractives of the meat itself. The large deposits of fat about the internal organs and under the skin are incidental to this and are to a certain extent a waste. The subcutaneous fat affords a convenient index to the quality of the meat, and of course the adipose tissue of the carcass is of some value, but these fat deposits largely represent the price paid for the improved quality of the meat proper. It is not impossible that the traditions of the market may cause the process of fattening to be pushed beyond what is necessary.

This improvement in quality may be, and to a considerable extent is, secured by a comparatively short period of high feeding after growth has been nearly completed. It is obvious, however, that no sharp line can be drawn between the processes of growth and fattening. A calf or yearling may be fattened while growing, and a two-year-old steer will continue to grow to some extent while being fattened. The two processes shade into each other and economic considerations will decide whether they shall be carried on more or less simultaneously by a single producer or at different times by two different individuals.

In brief, meat production may be defined as a combination of growth and fattening, which may be either simultaneous or successive, but the production of protein tissue is the primary object in view, while the accumulation of fat, although adding to the nutritive value and to the palatability of the meat, is more or less a secondary matter. The purpose of the present chapter is to consider the application of the principles of growth and fattening discussed in the two preceding chapters to this branch of food production.

503. Factors of meat production. - From the economic point of view, the meat producing animal may be looked upon as a mechanism by means of which the raw material contained in the various feeding stuffs is converted into the finished product for human consumption. Regarding meat production, then, as a manufacturing process, the amount and quality of 
the production obtained is plainly dependent upon three factors : first, the efficiency of the mechanism ; second, the amount and quality of the raw material supplied; third, the conditions under which the mechanism is operated.

\section{§ 2. The Animal as a Factor in Meat Production}

Of the three factors just mentioned, the animal may fairly. be said to be the one of prime importance. The success of the feeder depends primarily upon the capacity of his animals to convert profitably large amounts of raw materials into a finished product of high quality.

\section{Early maturity}

504. Definition of maturity. - Much stress is rightly laid upon the importance of early maturity in meat production, although the term is used in two more or less distinct senses.

Strictly speaking, a mature animal is one which has completed its growth - i.e., one in which the increase of protein tissue has reached its natural limit. In this sense, that one of two animals which reaches this natural limit first is the earlier maturing. With animals which reach substantially the same limit of size, this conception of early maturity is, of course, synonymous with a greater absolute rate of protein growth (460), while if the latter be expressed relatively to the weight of the animal, as in previous pages, the same thing is true regardless of size.

The term early maturity, however, is used also in a quite different sense, referring to the conformation of the animal rather than to completed growth. Thus, if a steer at 22 months has attained the typical beef form and reached sufficient size to meet the demands of the market, he is said to be mature. Obviously, this does not mean that he has completed his growth, but simply that he has made sufficient growth to furnish marketable meat. This conception of maturity, in other words, is commmercial rather than physiological. It is important to note, however, that it involves a physiological element. A certain size of carcass as well as a certain conformation is demanded, and to reach this at an early age almost necessarily implies a greater rate of growth, whether measured physiologi- 
cally by increase of protein tissue or practically by gain in weight.

In whichever sense the term maturity is used, however, the matter reduces itself to the question of rate of growth. The greater the initial impulse to growth, the sooner, other things being equal, will the animal complete his growth, while if the rate of growth can be made sufficiently rapid the desired accumulation of meat, and consequent weight, may be reached before physiological maturity. In other words, the rate of growth may be looked upon as expressing the capacity of the machine, since, as was stated in Chapter XI, it is substantially determined by biological factors and is apparently little affected by the feed supply, provided only that the latter is adequate.

505. Economic significance. - There seems no reason to suppose that there is any material difference as regards physiological economy between rapid growth and slow growth; that is, there is no reason to suppose that the storing up of certain amounts of protein and energy in the body of an animal in one month requires any greater or any less total feed supply, in addition to the maintenance requirement, than the storing up of the same amounts in the two months' time, except as heavy feeding may diminish the percentage digestibility of the ration (722). In other words, it may be assumed that if a gain of one pound in live weight contains 2500 Calories of energy, the ration must supply that amount of net energy above the maintenance requirement within the time required to make the gain, whether that time be one day or three.

From the economic point of view, however, there is a very important difference which explains the stress laid upon early maturity in meat-producing animals. It is plain that, other things being equal, the animal which inherits the greater initial impulse to growth, and in which that impulse dies out the more slowly, will reach either physiological maturity or a given size and weight sooner than the one in which that impulse is less. It makes a very material difference, however, to the producer of beef cattle, for example, whether a calf weighing roo pounds at birth has the capacity to reach a weigh of 200 pounds at two years old, or whether he requires three years to do it. This is not, however, because there is any material difference in the amount of feed which the animal requires to manufacture the 
I Ioo pounds of increase. The difference as regards feed cost comes in the expenditure for maintenance, since each pound of gain, as well as the original roo pounds, must be maintained from the time it is laid on until maturity. The animal, then, which has the higher rate of growth and which matures in two years costs the owner a notably less expenditure for feed than the one maturing in three years, to say nothing of the saving in cost of attendance and in interest on the investment.

\section{Age}

506. Influence on cost of production. - It is an undisputed fact that gain is made more rapidly and more cheaply by the younger as compared with the older animal. This is true both in growth proper and in the commercial fattening of partly mature animals. ${ }^{1}$

On the other hand, it was shown in Chapter XI (472-476) that there is no experimental evidence that the capacity of the young animal for making a more rapid gain is due to any greater physiological economy in the conversion of surplus digestible material into tissue, while it has also been established (720) that the digestive power of the young animal is not materially different from that of the mature animal. As regards protein, the indications are that the loss of nitrogenous material in the actual conversion of feed protein into body protein is not ordinarily great and is no greater in the old than in the young animal, while as regards energy it was shown that the probabilities are in favor of the view that its utilization is less rather than greater in the younger than in the older animal.

507. Causes of greater economy. - More or less confusion of thought has resulted from this apparent conflict of evidence, while feeding experiments like those cited by Henry and Morrison have been made the basis of unwarranted inferences as to the greater digestive and assimilative powers of young animals. This confusion has arisen to a large degree through failure to distinguish between physiological and commercial economy and it is important to secure a clear conception of the elements of the commercial superiority of the younger animal.

${ }^{1}$ Compare Henry and Morrison, Feeds and Feeding, I 5 th Ed., pp. $431-434,5$ I 2 , $568-572$. 
508. Difference in feeding stuffs. - The difference in the character of the feed consumed by the animal at different ages must not be overlooked. The very young animal subsists on milk (or milk substitutes). As it grows older and begins to consume solid feed, the latter must be at first of a rather concentrated character and highly digestible while, with advancing maturity, the ration is likely to consist to an increasing extent of coarser and more bulky materials. It is evident that to make a direct comparison between animals receiving such different rations on the basis of the dry matter of the latter is to ascribe to differences in the animals what is really due to differences in the feed. The ration of the younger animal will usually have the higher percentage digestibility, while at the same time it may cause a smaller expenditure of energy in the processes of digestion and assimilation, so that the net energy values of the rations per unit of dry matter are unequal. That an animal shows a greater rate of gain on milk than later on a mixed ration of grain and roughage does not necessarily show that the younger animal made any more efficient use of the materials actually resorbed, but may be simply because it receives more actual feed (net energy) in a unit of dry matter.

509. Difference in composition of gain. - It must also be remembered that the cheaper gain made by the younger animal means gain in live weight and that, as shown in Chapter XI (458), this increase is of inferior food value as compared with that of the more mature animal and represents the storage of less energy, since it contains more water and a larger proportion of protein to fat in its dry matter. A greater increase in live weight, even on perfectly comparable rations, therefore may be compensated for by the lower quality of that increase. Gain by the younger animal is, so to speak, more dilute.

510. Feed consumption. - A third important factor, especially when the animal is not pushed to the limit of his capacity, is the relatively greater consumption of feed by the younger animal. While the individual consumes more feed per head as it grows older, the consumption per unit of live weight and in particular per unit of body surface decreases. For example, in Henry's averages for swine and in Weiske's experiments on growing lambs cited in Chapter XI $(481 b, 487)$, the total feed consumption was:- - 
Table iog. - Relation of Weight of Pigs to Feed Consumed

\begin{tabular}{|c|c|c|c|c|}
\hline \multirow{3}{*}{ RANGE OF WEIGHT } & \multirow{3}{*}{$\begin{array}{l}\text { ACTUAL AVER- } \\
\text { AGE WEIGHT }\end{array}$} & \multicolumn{3}{|c|}{ Daily Feed } \\
\hline & & \multirow[b]{2}{*}{ Per Day } & \multicolumn{2}{|c|}{ Per roo Lb. Live Weight } \\
\hline & & & $\begin{array}{l}\text { In Proportion } \\
\text { to Weight }\end{array}$ & $\begin{array}{l}\text { In Proportion } \\
\text { to Surface }\end{array}$ \\
\hline Pounds & Pounds & Pounds & Pounds & Pounds \\
\hline 15 to 50 & $3^{8}$ & 2.2 & 6.0 & $4 \cdot 9$ \\
\hline 50 to 100 & 78 & $3 \cdot 4$ & $4 \cdot 3$ & \\
\hline Ioo to I 50 & I 28 & 4.8 & 3.8 & \\
\hline I 50 to 200 & 174 & $5 \cdot 9$ & $3 \cdot 5$ & \\
\hline 200 to 250 & 226 & 6.6 & 2.9 & \\
\hline 250 to 300 & $27 I$ & $7 \cdot 4$ & 2.7 & \\
\hline 300 to 350 & 320 & $7 \cdot 5$ & 2.4 & 3.45 \\
\hline
\end{tabular}

Table i io. - Feed Consumption by Lambs

\begin{tabular}{|c|c|c|c|c|c|c|c|c|c|}
\hline \multirow{2}{*}{\multicolumn{2}{|c|}{ - }} & & & & & & \multirow{2}{*}{ Live Weight } & \multicolumn{2}{|c|}{$\begin{array}{l}\text { DIGESTIBLE ORGANIC MATTER } \\
\text { EATEN PER } 50 \text { KGS. LIVE } \\
\text { WEIGHT }\end{array}$} \\
\hline & & & & & & & & $\begin{array}{l}\text { In Proportion } \\
\text { to Weight }\end{array}$ & $\begin{array}{l}\text { In Proportion } \\
\text { to Surface }\end{array}$ \\
\hline & & & & & & & Kgs. & Grams & Grams \\
\hline Period I & & & - & & . & . & 20.5 & 1059 & 787 \\
\hline Period II & . & . & . & . & & . . & $25 \cdot 5$ & 1029 & \\
\hline Period III. & . & . & . & 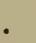 & . & . . & 28.9 & 870 & \\
\hline Period IV & . & . & . & . & . & . & 32.6 & 850 & \\
\hline Period V & . & - & • & . & . & . . & 35.0 & 757 & \\
\hline Period VI & . & . & . & . & . & . . & $35 \cdot 3$ & 755 & \\
\hline Period VII & & . & . & . & . & . . & 38.0 & 710 & \\
\hline Period VIII & . & . & . & . & . & . & 40.5 & $68 I$ & \\
\hline Period IX . & . & • & - & . & . & . . & 39.0 & 690 & \\
\hline Period X . & . & ${ }^{\circ}$ & & 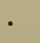 & & . & 57.5 & 549 & 575 \\
\hline
\end{tabular}

But the maintenance requirement is approximately proportional to the body surface. Consequently the feed consumption as the animal grows older does not keep pace with the increase in its maintenance requirement, so that a constantly diminishing proportion of its feed is available for productive purposes. For example, in Periods $\mathrm{I}$ and $\mathrm{X}$ respectively of Weiske's experiment it may be computed that the metaboliz- 
able energy of the rations consumed and the approximate maintenance requirements per day and head were:-

Table iil. - Diminishing Availability of Feed

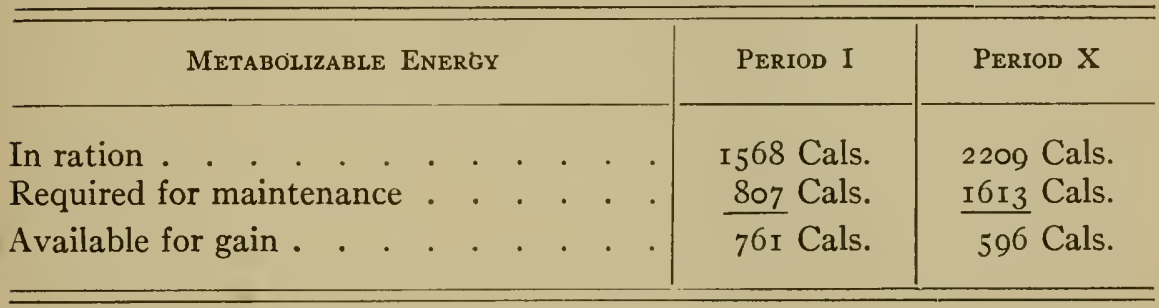

In Period I, 48.5 per cent of the metabolizable energy of the ration was available for growth as compared with only 27 per cent in Period X.

In brief, then, the undisputed superiority of the young animal as regards the amount of feed required to produce a unit of increase may be reasonably ascribed:

First, to the fact that his feed is often of a more concentrated nature, containing a greater proportion of digestible matter and perhaps causing a smaller expenditure of energy in connection with its digestion and assimilation.

Second, to the fact that the gain of live weight in the young animal 'contains a less percentage of dry matter and especially of fat and therefore represents the storage of less energy than the same increase in the older animal.

Third, that the total feed consumption of the animal, especially upon the more bulky feeds generally used for simple growth, may not increase as rapidly as the maintenance requirement, so that an increasing proportion of the feed is required simply for maintenance and is unavailable to produce increase.

511. Production of lean meat. - The difference in the nature of the gain made at different ages which, as has just been shown, is a material factor in determining the cost of gain in live weight, is of even greater importance in another aspect of the matter.

As shown in Chapter XI (460-463), the capacity for growth in the stricter sense, i.e., for increase of protein tissue, is especially characteristic of the young animal and decreases rapidly as he grows older, while it does not appear that it can be materially 
stimulated by the protein supply in the feed. It is of the highest economic importance, therefore, to utilize to the full the ability of the young animal to lay on protein tissue. In the early stages of growth, he is able to utilize a relatively abundant supply of feed protein which, if given to an older animal, would largely undergo protein katabolism and be lost so far as growth is concerned, while at the same time the total feed per head required for maintenance is smaller. The feeder cannot afford to stint the protein supply of the young animal, while the earlier the process of growth can be completed or approach nearly enough to completion to satisfy market demands, the more economically will it be conducted.

The conclusions regarding the rate of increase of protein tissue considered in Chapter XI are, however, derived chiefly from determinations of the gain or loss of total nitrogenous matter, including, besides the edible portion, the protein of the skin, hair, hoofs, horns and other epidermal tissue, of the internal organs and of the skeleton. It is important, therefore, to inquire into the rate of increase of the edible portion of the carcass.

Table il 2. - Gain of Fat-free Lean Meat and Total Protein by LAMBS

\begin{tabular}{|c|c|c|c|c|c|c|c|c|c|c|}
\hline \multirow{2}{*}{\multicolumn{2}{|c|}{$\overline{7}$}} & \multirow{2}{*}{\multicolumn{3}{|c|}{ Lot }} & \multirow[b]{2}{*}{ Period } & \multirow[b]{2}{*}{$\begin{array}{l}\text { Length } \\
\text { OF } \\
\text { PERIOD }\end{array}$} & \multirow[b]{2}{*}{$\begin{array}{l}\text { CHARACTER } \\
\text { OF RATION }\end{array}$} & \multirow{2}{*}{$\begin{array}{c}\text { Aver- } \\
\text { AGE } \\
\text { AGE } \\
\text { OF } \\
\text { ANI- } \\
\text { MALS }\end{array}$} & \multicolumn{2}{|c|}{$\begin{array}{c}\text { GAIN PER WeEk } \\
\text { AND HEAD }\end{array}$} \\
\hline & & & & & & & & & $\begin{array}{l}\text { Fresh } \\
\text { Fat- } \\
\text { free } \\
\text { Meat }\end{array}$ & $\begin{array}{l}\text { Total } \\
\text { Dry } \\
\text { Protein } \\
\text { (Esti- } \\
\text { mated) }\end{array}$ \\
\hline & & & & & & Days & & Days & $\begin{array}{l}\text { Kilo- } \\
\text { grams }\end{array}$ & $\begin{array}{l}\text { Kilo- } \\
\text { grams }\end{array}$ \\
\hline & & & & & I and II & 203 & Growing & 290 & O.II4 & 0.056 \\
\hline I & • & $\cdot \cdot$ & - & . & III and IV & 259 & Growing & $52 I$ & .053 & .031 \\
\hline & & & & & & I 75 & Fattening & 745 & .042 & .029 \\
\hline 2 & • & • & • & $\cdot$ & $\int \mathrm{I}$ and II & 189 & Fattening & 290 & .130 & .077 \\
\hline & • & $\bullet^{\circ}$ & & & III & I 47. & Fattening & $45^{8}$ & .040 & .026 \\
\hline
\end{tabular}

While it may be fairly assumed that the increase of edible meat will be in a general way proportional to the increase of total protein, it is equally clear that there may be considerable departures from the average. Unfortunately, however, the data upon this point are 
scanty, owing to the laborious and expensive nature of such experiments. About the only results available are those of Kern and Wattenberg on lambs and those of Jordan on steers, that is, these are the only ones which permit of a comparison of the rate of growth of similar animals in successive periods. Both these experiments have been outlined in Chapter XI (458). Table I I 2 shows the gain of fatfree lean meat in Kern and Wattenberg's experiments as compared with the estimated gains of total protein. The term "meat" refers only to the meat of the "butcher's pieces," freed from sinews and coarser connective tissue by passage through a meat grinder. The production of fat-free lean meat was, in general, parallel to that of total protein, diminishing with advancing maturity. Apparently, however, the rate of gain of total protein diminished less rapidly than that of edible meat.

Jordan's experiments include a comparison of the weights and chemical composition of two pairs of animals at the end of twentyseven months' and seventeen months' feeding respectively. The protein of the lean meat after mechanical separation from the fat tissue, and the total body protein, were as follows:-

\section{Table ti3. - Gain of Protein by Cattle}

\begin{tabular}{|c|c|c|c|}
\hline No. of ANimal & Average Age & $\begin{array}{l}\text { Protein of } \\
\text { LEan MEat }\end{array}$ & $\begin{array}{c}\text { Total Protein } \\
\text { OF Body }\end{array}$ \\
\hline & & Pounds & Pounds \\
\hline 2 & 32 months & 42.13 & I67.94 \\
\hline \multirow[t]{2}{*}{ I } & 22 months & $37.96^{1}$ & I36.30 \\
\hline & $\begin{array}{l}\text { Gain in Io months } \\
\text { Gain per day }\end{array}$ & $\begin{array}{l}\text { 4.17 } \\
.0128\end{array}$ & $\begin{array}{r}31.64 \\
.0971\end{array}$ \\
\hline \multirow{3}{*}{$\begin{array}{l}3 \\
4\end{array}$} & 32 months & 43.24 & I6I.38 \\
\hline & 22 months & 35.08 & I 26.30 \\
\hline & $\begin{array}{l}\text { Gain in to months } \\
\text { Gain per day }\end{array}$ & $\begin{array}{l}8.16 \\
.026 \mathrm{I}\end{array}$ & $\begin{array}{l}35.08 \\
. \text { II } 23\end{array}$ \\
\hline
\end{tabular}

So far as conclusions can be safely drawn from these few results, it would appear that the rate of gain of lean meat runs parallel to that of total protein and like the latter diminishes with age, the diminution being somewhat more rapid in the former case than in the latter. At all ages the storage of total

${ }^{1}$ Nos. I and 4 were somewhat lighter animals than Nos. 2 and 3 . The protein content has been computed to the live weight of the heavier animal in each case. 
protein (or, more exactly, the increase in the fat-free body) considerably exceeds the gain of lean meat proper and with increasing maturity this difference seems to become relatively greater.

512. Best age for fattening. - While meat production in the narrower sense of increase of protein tissue is confined to the immature animal, the improvement of its quality by the fattening process is an essential part of commercial meat production. Fattening, however, may be effected at practically any time in the life of the animal. Assuming that an animal is to be in the hands of the same owner from birth until slaughter, at what stage should the distinctively fattening process as distinguished from growth be begun?

It is evident that the beginning of the fattening process may be delayed too long. To take the extreme case, it would be obviously uneconomical first to grow an animal to full maturity and then to add a fattening period. While there is no reason to suppose that the amount of feed actually expended in the production of a unit of fat would be materially greater than if the fattening were conducted during the latter part of the growing period, the expenditure for maintenance, care, interest, etc., would be simply so much added to the cost of production. On the other hand, heavy fattening rations, containing large amounts of non-nitrogenous nutrients, even if they do not interfere with the growth of young animals, are uneconomical, tending either to overload the meat with fat or toward the accumulation of cheap internal fat, and making the animal ripe for the butcher before his capacity for producing lean meat has been properly utilized. A limited market exists, of course, for fat lambs and veals; but for the production of the world's meat supply it is important to utilize the capacity for growth up to a point at least approaching maturity. Too early fattening tends to produce an animal which, even if not of inferior quality, must be maintained in a fat condition until the growth of lean meat has had an opportunity to overtake that of fat. Plainly the beginning of the fattening should be so timed that it will be completed by the time the rate of gain of lean meat ceases to be profitable under the existing market conditions.

The period in the life of the animal at which fattening should begin, then, will depend upon its inherited capacity for growth, 
i.e., its rate of growth as defined on previous pages. If this is rapid, as, for example, in the improved breeds of swine especially and to a somewhat less extent with cattle and sheep, it may be practicable to begin the fattening almost from birth, the innate tendency to growth assuring sufficient size and weight by the time good marketable condition is attained. To secure this result, however, it is necessary to use rations containing large amounts of easily digestible feed in a small bulk and such rations are necessarily comparatively expensive. Moreover, growth as well as fattening requires an expenditure of feed energy, and as appeared in Chapter XI (473-476) a not inconsiderable one. The capacity of an animal to consume feed, however, is limited and when a relatively young animal is put on full feed, the more growth he makes the less feed will remain for fattening. This corresponds with the experience of practical feeders that mature animals will reach a higher condition in a given time than the young ones.

Under present economic conditions, as a rule, only the best grade of animals having to a high degree the quality of early maturity can be profitably handled in the way just indicated. With animals inferior in this respect, the more economical procedure usually is a period of growth upon comparatively cheap rations, consisting to a considerable extent of roughage, followed by a relatively short period of intensive fattening, beginning, however, before the capacity for growth has been entirely lost. The economy lies, of course, in the possibility of supporting growth and maintenance upon relatively cheap feeds during the longer time necessary in the case of inferior animals and will depend to a large extent upon the relative costs of feeding stuffs. The actual feed cost of the fattening itself is likely to be about the same in either case.

For the individual who raises and fattens his own animals, then, it would appear to be economical, so far as the feed cost is concerned, to use as early maturing animals as possible and to push them so as to fit them for market at as early an age as they are capable of.

When, however, as is notably the case in beef production, the rearing of animals and their fattening for market are in different hands, other important economic considerations enter in to modify this conclusion. In this case the business of 
the feeder is substantially to enhance the quality of the meat, and the profit of the transaction depends to a considerable extent upon the difference between buying and selling prices, and includes a large element of speculation. While it is true that the animal which still retains more or less capacity for growth will make the cheaper gains, nevertheless, if the market price of such animals is relatively high compared with that of more mature animals it may be more profitable as a business proposition to feed older animals, even though the feed cost per pound of gain is higher.

\section{Condition}

513. Decreasing gains in fattening. - It is generally admitted that in the case of the nearly mature fattening animal the rate of gain in live weight decreases as the fattening progresses until a limit is reached beyond which the increase, if obtained at all, is slow and very costly. Several causes are responsible for this :

First, the maintenance requirement of the animal increases with its gain in weight (393). The capacity of the digestive organs, however, undergoes no corresponding increase, and consequently the amount of excess feed is correspondingly reduced and its proportion in the ration made less, so that the total feed requirement per unit of gain will be greater.

Second, the appetite of well-fattened animals not infrequently diminishes, resulting in a lessened consumption of feed. This again has a double effect, diminishing the total amount of excess feed available and reducing the ratio of excess feed to total feed.

Third, a unit gain in live weight toward the close of the fattening period represents a larger storage of energy than the same gain at the beginning (452).

514. Effect on economy of gain. - For all these reasons it is natural that the "condition" of the animal - that is, its state of fatness - should have a marked effect on the rate and economy of gain. Georgeson ${ }^{1}$ reports the following results for a lot of 3 -year-old grade Shorthorn steers, the number of days stated in the table meaning in each case the number from the beginning of the feeding:-

$$
{ }^{1} \text { Kansas Expt. Sta., Bul. 34, p. } 95 .
$$


Table i 4 . - Grain Consumed by Steers per Pound of Gain

Number of Days Fed

Grain Consumed

PER POUND OF

GAIN

Pounds

$7 \cdot 30$

8.07

8.40

9.01

9.27

I0.00

Henry ${ }^{1}$ reports the following similar results for fattening swine :

Table ir 5. - Influence of Length of Fattening Period on the Feed Consumption and Gain of Hogs

First week . . . . 222

Second week . . . 235

Third week . . . 246

Fourth week . . . 257

Fifth week . . . 270

Sixth week . . 28I

Seventh week . . 294

Eighth week . . 303

Ninth week . . 3 3 3

Tenth week . . . 322

Eleventh week . . 332

Twelfth week .

340
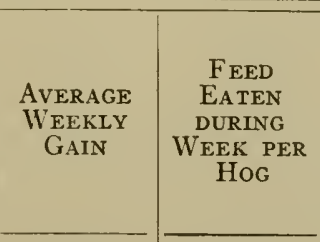

FeEd for IOO Pounds
OF GAIN

On the other hand, Mumford, ${ }^{2}$ in large-scale feeding experiments with cattle, has failed to note any such marked diminution

${ }^{1}$ Feeds and Feeding, Ioth Ed., p. 5 ro.

${ }^{2}$ Ills. Expt. Sta., Bul. I03, p. 57. 
of the gain during the later stages of feeding as had been generally found by other experimenters.

There is no sufficient evidence on record to show whether or not the actual percentage utilization of the excess feed diminishes with the advance of fattening.

\section{Breed and Individuality}

That both those inherited qualities which characterize the recognized meat breeds and the individual differences between single animals are important factors in the economy of meat production is generally recognized. It is a fact of common observation that marked differences exist between individual animals as regards the return which they yield for the feed consumed, but the reasons for these differences have not always been clearly seen, and in particular there has been a tendency to assign them to physiological causes, such as difference in digestive or assimilative power, and some unwarranted conclusions on these points have gained currency.

515. Digestive power. - The superiority of one breed or animal over another as regards feeding capacity is often ascribed to a difference in the extent to which the feed is digested, although those who make this assertion often understand by digestion what is more properly termed "utilization." Undoubtedly there are differences in digestive power between different animals, but except in the case of manifestly abnormal animals they have been found to be comparatively slight and quite insufficient to account for the marked differences in production $(\mathbf{7 1 8}, \mathbf{7 1 9})$. Neither is there any evidence that the improved breeds of meat-producing animals possess any superiority in this respect over the ordinary unimproved animals.

An illustration of the latter fact is afforded in experiments by Armsby and Fries, ${ }^{1}$ in which no material difference was observed in the digestive powers of a pure-bred beef animal and a "scrub" at the approximate ages of one, two and three years. The same experiments also failed to show any material differences in the losses of energy in urine and methane, so that the percentage of the feed energy which was metabolizable, especially when computed on the

1 U: S. Dept. of Agr., Bur. Anim. Indus., Bul. I28 (IgII). 
energy of the digested matter, was substantially the same for the two animals. The figures for the digestibility of the dry matter and for the percentage of the digestible energy metabolizable, each being the average of two periods, were as follows :-

Table i 6. - Digestibility by Pure-bred and Scrub Steers

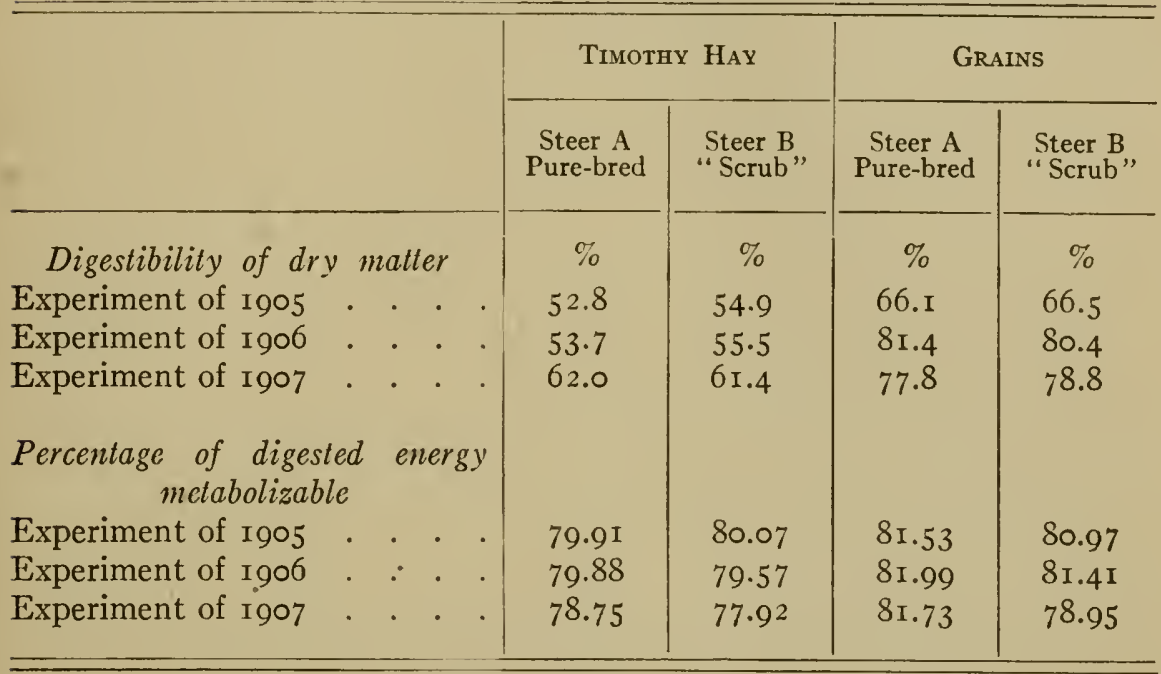

516. Assimilative power. - This term may be used to designate broadly the ability of the organism to convert the digested nutrients of the feed into body tissue. Is the good meat producer able to form from a unit of digested feed of a given kind more new tissue than can the inferior animal? In other words, is the net energy value of the feed affected by the individuality of the animal? As yet there has been scarcely any scientific investigation bearing upon this question, but such evidence as is available does not indicate the existence of material differences in this respect.

Such of Kellner's determinations of net energy values for fattening (449) as were made upon similar feeding stuffs with different animals show a generally good agreement as regards the utilization of the energy of the feed, although it does not appear from the accounts of the experiments whether or not the animals used differed materially in type.

The experiments by Armsby and Fries, just referred to, were directed more specifically to the investigations of this question. They failed to demonstrate any decided advantage on the side of the pure-bred 
animal so far as the percentage utilization of the energy supplied in excess of the maintenance requirement was concerned, the slight difference observed, especially in the earlier years, being perhaps accounted for by the greater tendency of the pure-bred steer to lay on fat.

In the aggregate a considerable number of breed tests of cattle, sheep and swine have been made by the American experiment stations, the results of some of which have been summarized by Henry ${ }^{1}$ so as to show the quantity of feed consumed per unit of gain. While in individual cases considerable fluctuations are to be found, nevertheless, the results as a whole certainly fail to indicate any marked superiority of one breed over another in this respect, and later experiments have not given materially different results. When we come to consider the other possible factors, such as differences in live weight, in maintenance requirement, in total feed consumed, etc., we must conclude that the recorded results give no clear evidence of any specific individual or breed differences in the actual physiological processes involved in the conversion of feed into tissue, although it is equally true, of course, that they fail to prove the absence of such differences.

It seems clear that it is necessary to look elsewhere than to a supposed greater digestive and assimilative capacity of the typical meat-producing animal for an explanation of his economic superiority over the less specialized individual.

517. The maintenance requirement. - It was shown in Chapter VIII $(376,391)$ that not inconsiderable differences may exist between different individuals as regards the maintenance requirement. Thus in the case of cattle the extreme figures of 4.72 Therms and 7.43 Therms of net energy per Ioo0 pounds live weight were observed for thin animals. Of the various factors affecting the maintenance requirement, it was pointed out that one of the most important is the degree of muscular activity of the animal even when in the state of socalled rest, and the decidedly lower maintenance requirement found by Armsby and Fries for a pure-bred beef steer as compared with a scrub was there interpreted as probably due to the more nervous disposition and greater restlessness of the latter.

It is clear, however, that of two animals receiving identical rations the one which has the lower maintenance requirement

${ }^{1}$ Feeds and Feeding, roth Ed., pp. 328 and 5 II. 
will have the larger surplus for growth or fattening and, other things being equal, will make the greater increase per unit of total feed. To what extent a lower maintenance requirement is characteristic of high-bred meat-producing animals remains to be determined. If it appears to be a general fact, it would go far toward explaining any superiority on the part of the latter.

It is not impossible, also, that differences in the amount of muscular activity may play a more important part in fattening than in the experiments on maintenance hitherto reported. In the latter, the experimental conditions necessitated considerable restriction of the freedom of motion, while under the conditions of practice a wider scope may perhaps be afforded to the individuality of the animal in this respect.

518. Feed consumption. - Another important element of individual superiority is the ability of an animal to consume regularly large amounts of feed. Of two animals otherwise similar, it is clear that the one which is able to consume day after day the heavier ration is the better meat producer. It is not always realized, however, that the heavier feeder makes a relatively more profitable use of his feed because, as pointed out in Chapter VIII (360), assuming the maintenance requirement to be the same, the productive part of the ration forms a larger part of the total ration in the case of the large eater. Consequently, since all the feed must be paid for, this animal makes the more economical gain, not because he utilizes his excess feed better but simply because he is able to consume more of it.

There are doubtless marked differences between individual animals in this respect. Whether the specific meat-producing breeds as a whole possess any advantage in this respect appears doubtful in view of the results on record regarding the feed cost of gain with different breeds. Apparently the quality is one to which the attention of breeders has not been specially directed, yet it is one which, it would seem, might well repay attention.

519. Type and conformation. - It is a well-recognized fact that the conformation of a meat animal is a very important factor in determining his selling price. The improved meat breeds as a rule show a higher ratio of dressed to live weight, a better distribution of fat in the finished carcass, a somewhat 
larger proportion of the higher priced cuts and a higher quality of meat. They are all important factors in the economic production of meat, but there is no evidence that their possession renders an animal any more efficient as a converter of feed into meat.

520. Early maturity. - The economic importance of a rapid rate of growth and of the consequent early maturity has been considered in previous paragraphs $(504,505)$.

It is a matter of common experience that there exist marked differences between individuals of the same species both as to the weight finally attained by the mature animal and as to the rate of growth at the same age. It is natural to interpret this fact as indicating corresponding individual differences in the rate of growth, especially of protein tissue, but the writer is not aware of any recorded experiments bearing specifically on this point. It is true that the quality of early maturity is popularly attributed to the meat breeds, but as regards cattle at least Henry ${ }^{1}$ has shown that the data at hand fail to prove that the beef breeds as such show a greater rate of gain in live weight or a greater weight at maturity than do the dairy breeds, although it is likewise true that other elements than simply the weight enter into the economic conception of maturity. If it is correct to ascribe the individual differences noted above to variations in the rate of growth of protein tissue, it suggests a field for investigation of much interest both to the breeder and the feeder.

\section{§ 3. Feeding for Meat Production}

521. Feeding as related to individuality. - The facts considered in the previous section relate to the capacity of the animal as a mechanism for the conversion of vegetable products into meat. They (and other less important ones) determine the degree to which, from the commercial standpoint, the animal is able to utilize the feed given it. Favorable modifications of any of these factors are of advantage because they enable a larger and more profitable production to be secured.

Feeding stands in a somewhat different relation, in that its purpose is to supply the material upon which the mechanism

\footnotetext{
${ }^{1}$ Feeds and Feeding, Ioth Ed., p. 329.
} 
works. It is of prime importance to the feeder that his animals shall have the largest possible productive capacity, but while the maximum which the animal can produce is determined by its breed and individual characteristics and cannot be materially affected by feeding, the amount which it actually does.produce in any given case must depend upon the amount of material supplied to it in its feed. Production may be limited by a deficient feed supply, although it cannot be forced above a certain maximum by increasing the ration.

522. Feed requirements. - Since feed is to be looked upon as a supply of raw material for the animal mechanism, it is clear that the kind and amount required will depend primarily upon the capacity of the animal. The young animal, with his marked capacity for growth, will require relatively more of the specific materials for growth, viz., protein and ash, than will the older animal. The early maturing animal, with his greater rate of growth, will require more total feed per day than the one maturing more slowly. The animal with the capacity to consume and utilize large total amounts of feed must be given these larger amounts in order that his advantage in this respect may be fully utilized.

As already pointed out, meat production is a combination of growth and fattening, the latter process being superimposed upon the former. The feed requirements of the meat-producing animal, therefore, include in the first place the requirements for normal growth, to which are added during a longer or shorter time according to circumstances the requirements for the production of fat. The feed requirements for these two purposes have already been considered in the two previous chapters, but may be conveniently recapitulated here with more particular emphasis on economic relations.

\section{Protein requirements for meat production}

523. Relation to age. - It was shown in Chapters X and XI that it is only during growth that any considerable production of meat in the narrower sense, i.e., of muscular tissue, takes place, and likewise that the energy of growth is greatest in the young animal and diminishes, at first rapidly and then more and more slowly, until physiological maturity, when but a slight 
increase of the total protein and still less of meat proper can be secured. Evidently the question of the necessary protein supply in the rations of meat-producing animals is of special importance during the early stages of growth.

524. Minimum protein supply for growth. - The meat-producing animal, then, in order to utilize fully his capacity for growth must be supplied in his feed at each stage of that growth, in addition to his maintenance requirement, with at least as much digestible protein as he is capable of storing up in his growth. Whether any greater quantity than this is necessary or advantageous is, as has been shown (491), still to some degree an unsettled question. Some experiments, especially with cattle and sheep, indicate that any considerable surplus is unnecessary for normal growth, while, on the other hand, feeding experiments with pigs and to some extent with ruminants indicate that amounts considerably in excess of those thus computed assure at least greater gains of live weight.

525. Protein requirements in fattening. - While growth and fattening may be regarded physiologically as distinct processes, it is economically important in the practice of meat production that they should go on more or less simultaneously. The growth of even the very young animal is not simply a production of protein tissue, but normally includes more or less fat production, while in proportion as one has to deal with early maturing animals it is desirable to begin the fattening proper at a comparatively early stage of growth (512).

There appears to be no reason for regarding the actual fattening process as being essentially different in the growing and in the mature animal. It has been shown, however $(\mathbf{4 5 3}, \mathbf{4 5 6})$ that in the latter case no material excess of protein over that required for maintenance is necessary. So far as the mere supply of building materials is concerned, therefore, there seems no reason to suppose that the actual protein requirement for combined growth and fattening is any greater than that for normal growth without fattening. The conclusions regarding the protein requirements for growth recorded in Chapter XI (482), therefore, may be regarded as applicable also to young animals that are being fattened, especially since they were derived in part from results on immature fattening animals, and from this point of view the increased feed supply required for 
the fattening might consist exclusively of non-nitrogenous nutrients.

526. Influence on digestibility. - In the actual compounding of rations for fattening, however, whether for mature or for growing animals, account must be taken of the fact that such a considerable addition of non-nitrogenous nutrients to a maintenance or growth ration may have an unfavorable effect upon its digestibility. In particular, it has been shown (723) that a large proportion of easily digestible carbohydrates in a ration (i.e., a "wide" nutritive ratio) tends to depress the apparent digestibility of the protein. Accordingly, if, starting with a ration just adequate to support the normal rate of growth, the attempt be made to convert it into a fattening ration by simply increasing its digestible carbohydrates, the effect may be to virtually diminish the amount of protein available so that the ration, while containing abundant material for fat production, may fail to supply enough protein to utilize fully the animal's capacity for growth.

The increase due to growth, however, is an important factor of the cheaper gains made by immature animals (509). In increasing the total feed supply in order to secure the fattening of the young animal, therefore, it is important to avoid the danger of so decreasing the apparent digestibility of the protein by the too free use of feeding stuffs rich in carbohydrates as to reduce the protein supply below that needed for growth. Moreover, it has been found (723-727) that a relative deficiency of nitrogenous matter in a ration also decreases the digestibility of the carbohydrates, particularly of those less soluble forms which are acted upon chiefly by the fermentative processes in the rumen or cœcum, and so tends to reduce the energy value of the ration.

It is difficult, however, to make any very definite statements regarding the practical significance of these effects in actual feeding. Kellner recommends that the nutritive ratio (709) of a fattening ration, computed in the usual way, be not made wider than about $I: 8-9$ for cattle and sheep and $I:$ IO- 2 for swine. Ordinarily, there will be little difficulty in compounding rations conforming to this rule, especially when home grown protein feeds are available, and such rations when fed in sufficient amounts to support reasonably rapid fattening would 
supply more digestible protein than is called for by the estimates in Appendix Table IV $b$. When protein feeds are especially expensive an even smaller proportion of protein might doubtless be used to economic advantage, even though at the expense of some loss of digestibility, without unduly curtailing the protein supply, especially in the case of animals approaching maturity.

527. Specific effects of feeding stuffs. - Account must be taken also of the fact that in most of the experiments upon the protein requirement thus far reported the variation in the protein supply was effected by varying the proportions of certain concentrates in the ration, such, e.g., as substituting cottonseed meal for maize. As was suggested in Chapter XI (491), however, such a substitution may not only affect the ash balance of the ration but may serve to introduce substances which stimulate the growth process or perhaps the fattening process. While we are not to suppose that such substances can take the place of actual nutrients (738), they might enable the protein in the ration to be more fully utilized, or they might, by stimulating the fattening process, create an appetite for more feed.

At any rate it seems to be the general experience of stockmen that the addition of certain feeds rich in protein, especially the oil meals, to the rations of fattening animals tends to induce them to consume feed more freely and thus (518) to yield more profitable gains.

\section{Energy requirements for meat production}

528. Combined growth and fattening. - An attempt was made in Chapter XI (480-483) to estimate approximately the net energy values required at different ages for normal growth without material fattening. To the extent to which the fattening process is to be carried on at the same time, these requirements must evidently be increased by amounts equal to the additional net energy stored up in the increase of adipose tissue desired or expected. Subject to the limitations indicated in previous paragraphs, this additional energy may be supplied by the addition of either nitrogenous or non-nitrogenous materials to the growth ration. 
It was estimated in Chapter $\mathrm{X}$ that a pound of increase in live weight in the mature fattening animal is equivalent to about 3.25 Therms of energy. If it is allowable to apply this average to the fattening of younger animals, this would be equivalent to saying that for each pound of increase in weight above that due to growth proper, about 3.25 Therms of net energy should be added to the requirements for growth as estimated in Chapter $\mathrm{XI}$ (480-483). The energy requirement of the meat animal, therefore, will obviously depend on its capacity to produce gain of flesh or fat and the extent to which it is desired to utilize this capacity, and no specific and invariable requirements can be formulated.

529. Total amount of feed. - If, for the reasons given in previous paragraphs, the proportion of digestible protein in the ration is kept above a certain limit, the question of the amount of net energy to be supplied resolves itself into the question of the most profitable total amount of feed to be given and this depends upon a variety of conditions.

It has already been pointed out (512) that only with animals having a rapid rate of growth and maturing early is it advisable to begin intensive feeding before a fair degree of maturity is reached. With ordinary animals the major portion of their growth may be more cheaply supported upon pasture and the ordinary roughages with relatively small amounts of concentrates, since the growth process cannot be materially hastened by heavy feeding. When, however, the time for beginning the fattening process involving the use of expensive concentrates is reached (533), whether this be early or late, it is important to hasten it as much as practicable in order to reduce the cost of maintenance, attendance, etc., and the question of the most profitable amount of feed becomes an important one.

530. Heavy feeding profitable. - That comparatively heavy feeding of fattening animals is economically advantageous is shown by the experience of practical feeders, and is evident from the fact, to which attention has already been called several times, that a less proportion of the heavy ration is required for the maintenance of the animal. Were this the only factor involved, it would follow mathematically that the greater the amount of feed consumed the greater would be the growth per unit of feed and therefore that the appetite of the fattening animal should 
be stimulated to the greatest extent possible. In fact, however, other considerations come in to modify this conclusion.

531. Influence on digestibility. - Overfeeding to the extent of causing digestive disturbances and throwing the animal " off feed" is of course to be avoided, since the resulting disturbance and the subsequent lessened consumption of feed may outweigh any advantage from the increased amount eaten. It is the regular uniform feeder that is likely to be the profitable animal rather than the one with a capricious appetite.

But aside from this danger, it seems well established that the percentage digestibility of mixed rations, such as would be used in productive feeding, decreases more or less as the quantity consumed increases. The results on record in this respect (722) are scarcely sufficient for any quantitative estimate of the magnitude of this effect, but it is evident that it must tend to diminish the efficiency of the rations.

532. Influence on net energy values. - Such a decrease of digestibility as that just noted is, of course, equivalent to a decrease in the net energy value of the rations. There appears to be a somewhat general impression, however, that in addition to this effect on digestibility, the matter and energy actually resorbed from the ration become less efficient in producing gain as the amount of the ration is increased - in other words that when the organism is flooded with the resorbed products of digestion, the katabolic processes are stimulated and a larger share of the energy of the digested matter escapes as heat. . As appears in Chapter XVII (764), the evidence on this point as yet seems hardly sufficient to warrant positive statements. The net energy values of feeding stuff.s which have thus far been reported have been obtained chiefly in experiments on rations ranging from submaintenance to only moderately heavy fattening rations, and the results show no distinct indication of a decrease with increasing amounts of feed. On the other hand, physiological considerations render it quite conceivable that the effect of the feed in stimulating metabolism and so increasing the heat production (365) may be relatively greater on a high than on a low nutritive plane.

Apparently more or less falling off in the nutritive effect of a fattening ration as its amount is increased must be anticipated, whether on account of decreasing digestibility or of 
lessened utilization of the digestible matter or a combination of the two. Whether this diminution, within the limits of the animal's capacity to consume feed, is sufficient to offset the economic advantage of such increased consumption remains to be shown, although Morgen ${ }^{1}$ reports experiments on sheep in which very heavy rations. actually produced smaller gains in live weight than lighter ones. Finally it should be remembered that it is the actual gain of chemical energy by the animal which is believed to bear a tolerably constant relation to the feed energy. It has been repeatedly pointed out that the gain in live weight is a very uncertain indication of the amount of energy stored up. It is quite conceivable that the larger gain to be expected on the heavier ration may contain less water and more dry matter or less protein and more fat than that produced on the lighter ration, and that consequently the increase in weight may not be proportional to the increase in feed. In that case, unless the higher quality of the gain were recognized by the market, the economic advantage attached to heavier feeding would be diminished or wiped out.

533. Proportion of concentrates to roughage. - The foregoing considerations apply in the first instance to varying, amounts of the same mixture of feeding stuffs. In the case of herbivora, however, heavy feeding must necessarily be effected by increasing the proportion of concentrates or of roots to roughage, the higher cost of the former per unit of net energy being more than offset by the economic advantage incident to the much larger amount which can be consumed. When such an addition of concentrates contains a large proportion of carbohydrates (as in the case of maize or roots) it would appear (724) that the digestibility of the rations would suffer to a certain extent owing to the low protein content of the ration, while common observation indicates a more rapid passage of the feed through the digestive tract of heavily grained ruminants and suggests a decrease in total digestibility which has not, however, been experimentally confirmed.

534. Standards. - It is clear from the foregoing that under ordinary conditions mature or nearly mature fattening animals, such as the cattle ordinarily fattened in the United States, should be fed as heavily and pushed as rapidly as the capacity

\footnotetext{
${ }^{1}$ Fütterung und Schlachtergebnisse, pp. 22 and 33.
} 
of the animals and the skill of the feeder will permit. This conclusion was reached long ago by practical feeders, so that the results of experience and of investigation appear quite in harmony. Such an intensive feeding can be effected only by a free use of concentrates and unless the latter are very expensive as compared with roughages, it is economy to use them to the largest practicable extent.

Under these conditions it is evident that there is very little significance in a feeding standard in the ordinary sense, so far at least as the amount of feed is concerned. It may, it is true, afford a basis for preliminary computation of the amount of feed required for a season's feeding, if this is of any importance, but in actual feeding the problem is to induce the animals, by means of the art of the skilled feeder, to consume large amounts of feed without injury to their appetites or digestive capacity, and this is largely a question of the individuality of the animal or lot. The one thing to be kept in mind is to see that the supply of protein in the ration is sufficient to ensure the normal growth of protein tissue, since this causes a relatively rapid increase in weight.

For younger fattening animals, somewhat more definite requirements might be formulated in the manner indicated in a previous paragraph (528) on the basis of the requirements for fattening and for growth as estimated in Chapters X and XI.

The compilation by Bull and Emmett ${ }^{1}$ of American experiments on fattening lambs referred to in Chapter XI (487) included data regarding the computed net energy content of the rations. They conclude that the production of satisfactory gains required the following amounts of digestible protein and net energy per Iooo pounds live weight.

Table i 7. - Requirements of Fattening Lambs per 1000 Lb. Live WEIGHT

\begin{tabular}{|c|c|c|c|}
\hline LIVE WeIGHT & Estimated Age & Digestible Protein & Net Energy \\
\hline Pounds & Months & Pounds & Therms \\
\hline $50-70$ & 5 & $3 \cdot I-3 \cdot 3$ & I7-I9 \\
\hline $70-90$ & 7 & $2.5^{-2.8}$ & I $8-20$ \\
\hline $90-$ I IO & 9 & $2.2-2.4$ & I $7-20$ \\
\hline I IO-I 50 & I 5 & I.4-I.9 & $16-19$ \\
\hline
\end{tabular}

${ }^{1}$ Ills. Expt. Sta., Bul. I66 (I914). 
No similar compilations for other species of farm animals have yet been reported, although much valuable material in the publications of the experiment stations awaits such discussion.

\section{§ 4. Influence of External Conditions}

While the capacity of the animal as a meat producer and a supply of feed sufficient in quantity and quality to fully utilize that capacity are the two great factors in meat production, yet the conditions under which the animal is kept are not without influence on the results obtained.

\section{Temperature}

535. Teachings of practice. - Since the temperature of the animal body is maintained by the katabolism of materials derived from the feed, it seemed natural to conclude that cold surroundings would lead to a wasteful oxidation of feed for simple heat production, and considerable emphasis has been laid in the past upon the economic importance of providing fairly warm quarters for live stock. At the same time, however, great numbers of cattle, in particular, were being successfully fattened in sheds and open feed lots and more recently a considerable amount of experimental work has been reported showing that this supposedly uneconomic practice actually gives better returns than feeding in warm quarters. The results of a considerable number of such comparisons have been summarized by the writer ${ }^{1}$ and leave no doubt as to the validity of this conclusion, while it is entirely in harmony with the principles governing the influence of external temperature upon metabolism which were discussed in previous chapters.

536. Critical temperature. - As was shown in Chapter VII (354), there is a certain approximate temperature, called the critical temperature, at which the minimum outflow of heat just balances the necessary heat production resulting from the internal work and below which more or less oxidation of tissue is required to maintain the normal temperature of the body. Furthermore, it has been shown (395-397) that the digestion and assimilation of feed and its conversion into tissue result in

${ }^{1}$ U. S. Dept. Agr., Bur. Anim. Indus., Bul. 108 (1908), pp. 79-86. 
the evolution of relatively large amounts of heat, especially in the ruminants, and that the effect of this internal production of heat is virtually to lower the critical temperature as compared with that of the fasting animal. In other words, there is for each animal and for each ration a certain temperature above which the heat produced becomes in part an excretum, to be gotten rid of by radiation and evaporation.

It appears likely that a certain excess of heat production over that absolutely required to maintain the body temperature is advantageous, both as promoting the comfort of the animal and especially as affording a margin in case of temporary fluctuations of temperature. On the other hand, both our own personal sensations and the observations of practical stock feeders show that an unnecessarily high temperature is debilitating, affecting both appetite and general health. In practice, then, it is desirable to keep the thermal surroundings of the animal within the range above indicated - somewhat above the critical point but not so much so as to affect the appetite and thrift. It is evident that the limits of this range may vary widely with the kind of animal and with the amount of the ration.

537. Amount of ration. - The influence of this factor upon the requirements for protection from cold is clearly indicated by what has already been said. The heavier the ration, other things being equal, the more heat will be evolved during its digestion and conversion into tissue. Mature animals on full feed thus have at their disposal a large amount of surplus heat and naturally can thrive under conditions of exposure which might be seriously detrimental to young, growing animals on relatively light rations. Thus one of Kellner's experiments on a fattening ox gave the following results :-

Table ir 8. - Excess Heat Production in Fattening

Metabolizable energy of ration . . . . . . 26,600 Cals.

Energy stored as gain . . . . . . . . 5,920 Cals.

Energy evolved as heat . . . . . . . . 20,740 Cals.

Computed maintenance requirement . . . . 15,060 Cals.

Excess of heat . . . . . . . . . 5,680 Cals.

Excess over maintenance . . . . . . . . $37.7 \%$

538. Age and weight of animals. - The internal work of like animals of different sizes, under like conditions, appears to 
be approximately proportional to their body surface (345), and there is even good ground for believing that this law applies in a broad way to animals of the most diverse species and size. Since the action of external temperature is also approximately proportional to the surface, it would be expected that the size of the animal would not be an important factor. In fact, however, the other conditions are rarely alike. The young animal in particular is likely to be getting a relatively lighter ration than the animal which is being pushed for the butcher, and thus to have less surplus heat at its disposal, while the indefinable factor of "hardiness" would also seem to be in favor of the older animal.

539. Humidity. - The relative humidity of the air is an important factor in the temperature relations of the animal. Moist air tends to increase the conductivity of the hair or wool, just as it does that of the clothing of man, thus facilitating the escape of heat and raising the critical temperature. Accordingly, it is to be anticipated that in a dry climate, like that of the northwestern United States, animals might be safely exposed to a greater degree of cold than in a damp climate, like the winter of the seaboard States.

540. Temperature of drinking water. - In general, the same considerations adduced in discussing the influence of the temperature of the air apply to that of the drinking water. Under heavy feeding, especially, unless in very cold quarters, the animal has a surplus of heat which it can apply to warming its drink. If, then, the latter is at such a temperature as to be consumed freely, there would seem to be no occasion for heating it further, except for one important consideration. The temperature of the air acts continuously and with approximate uniformity. That of the water, on the other hand, acts only at intervals, often only two or three times or even once per day. If, now, the animal consumes within a short time a large amount of cold water, a correspondingly rapid expenditure of heat is required to warm this water to the body temperature, and this demand may for a time exceed the supply of surplus heat and cause an increased oxidation of tissue or food material for the sake of heat production only. Such a loss can never be made good at a later hour since, once converted into heat, the energy has escaped from the grasp of the body. Other things being 
equal, then, it will clearly be desirable to have the water consumption approximate as nearly as possible a continuous consumption by having it constantly accessible, while if the stock are watered only at intervals the temperature of the water may need to be rather higher than in the other case.

\section{Shelter}

A protection from rain or snow and from wind may be of quite as much importance as protection from low temperatures simply.

541. Precipitation. - An important factor in the case is the amount of precipitation (rain or snow) to be expected during the feeding period. In cold weather the low temperature of the water which penetrates to the skin of animals is the cause of a loss of heat which may be regarded as practically an addition to that due to the cold air, the extent of both losses being affected by the thickness of the animal's coat. Far more important than this, however, is the expenditure of heat required to dry out the coat after it is wet, and this, as it would seem and as some of the experiments with sheep seem to indicate, would be greater with the heavier coated animal when it has once become thoroughly wet. Still greater, relatively, is the heat required to melt the snow falling on the animal or that upon which it is compelled to lie.

These effects, it will be observed, are largely independent of the indications of the thermometer, and it is clear that the nature of the climate as regards humidity and precipitation is quite as important a factor as the temperature in its bearing on the question of shelter, and that in many localities a roof to shelter the animals from storms may be as efficient as a tight barn. One-advantage of the roof, already mentioned incidentally, is that it provides the possibility of a dry bed, thus not only adding to the comfort of the stock but avoiding expenditure of energy in warming up or evaporating water or melting snow or ice.

542. Wind. - All are familiar with the greater severity of a windy day as compared with a still one of the same temperature. A large part of the protective value of the clothing of man or the coat of an animal resides in the air entangled between the fibers of the material. Wind tends to replace this air with fresh, 
cold air and thus greatly reduces the protective effect. A windbreak, therefore, may have a distinct economic value in stock feeding.

543. Insolation. - The effects of the weather are appreciably modified by the exposure of stock to direct sunlight. Aside from any direct effect of the light as such, a not inconsiderable amount of heat is imparted to the body by the sun's rays. During cold weather this is likely to be a distinct advantage, but during the hot months the reverse is true. Since the animal cannot reduce its heat production below that resulting from its internal work and the digestion and assimilation of its feed, it may seriously tax its powers to dispose of the additional heat imparted by the direct sunlight. In this case shelter of some sort may be required for opposite reasons to those obtaining during the cold months. For similar reasons a supply of cool, fresh water and exposure to the wind may be of great advantage in helping the animal to get rid of its surplus heat.

\section{Other conditions}

544. Exercise. - The well-known fact that muscular exertion is accomplished at the expense of the katabolism of tissue and ultimately, therefore, at the expense of the feed, would seem at first thought to indicate that the activity of the meat-producing animal should be restricted as much as practicable. In the case of the growing animal, however, another very important element enters into the case, namely, the fact that moderate exercise tends to stimulate the growth of the muscular system, or, in other words, the production of lean meat. Since this is the essential object sought, a normal and reasonable amount of muscular activity on the part of the growing animal should be allowed and encouraged, even though the muscular exercise involves the consumption of more feed. Accordingly, young stock should be given the freedom of the pasture or range to as great an extent as practicable, while at the same time care should be taken to supply abundant feed containing a sufficient supply of protein in order that enough material may be present to supply the demand for growth stimulated by the exercise.

In the case of breeding stock, especially, a most important consideration is that of the health and stamina of the animal, 
which can hardly fail to suffer through overconfinement. The above principles apply in a general way to all classes of stock. In particular, hogs should be given an opportunity for more movement and exercise than is frequently allowed.

In the case of animals which have reached the fattening stage, on the other hand, there is comparatively little growth of protein tissue, while it is only necessary to maintain sufficient health to ensure a normal appetite and assimilation of feed. In proportion, then, as this stage is reached, the endeavor should be to reduce the amount of exercise taken and to keep the fattening animal as quiet as possible. To this end comfortable quarters should be provided, with plentiful bedding, and the animals should be kept as undisturbed as possible, so that they may " eat and lie down." This is particularly important in the case of the sheep on account of its timid nature. For similar reasons it is desirable to have the water supply of fattening animals close at hand.

545. Water supply: - It should never be forgotten that rapid production, involving the utilization of relatively large amounts of feed, requires the consumption of a corresponding amount of water for the physiological purposes of the animal. For this reason, as well as for the one previously mentioned (540), it is desirable that stock should have ready access to water, if possible, at all times and that the water supplied should not be too cold to be consumed freely by the animals. 


\section{CHAPTER XIII}

\section{MILK PRODUCTION}

\section{$\S$ i. The Physiology of Milk Production}

546. Components of milk. - In addition to water, milk contains representatives of the four great groups of nutrients, viz., proteins, fats, carbohydrates and ash.

Proteins. - The principal protein of milk is casein, a substance belonging to the group of phosphoproteins (55). This protein is peculiar to milk, not being found elsewhere in the body.

In addition to casein, milk contains also a lact-albumin and a paraglobulin in small amounts. Their presence may be demonstrated by precipitating the casein by means of acid and heating the filtrate. Traces of peptones, possibly due to the presence of a proteolytic enzym, are also found in milk.

According to König, the casein content of milk has been observed to vary from I.79 per cent to 4.23 per cent and that of the other proteins from 0.25 per cent to $\mathrm{r} .44$ per cent.

Fats. - Fats occur in milk in the form of microscopic globules varying greatly in size and held in suspension in the colloidal solution of casein. In cow's milk the diameter of these fat globules may be stated in a general way to range from 0.0016 to 0.01 millimeter and in a single cubic centimeter of average milk their number runs into the millions. The fat globules were formerly described as surrounded by a membrane of a protein nature, but the supposed membrane is now regarded as simply a condensation of the protein of the milk, due to surface tension.

Milk fat, like other animal fats, is a mixture of a number of simple fats or triglycerids. As compared with body fats, the fat of milk is relatively rich in olein and consequently has a relatively low melting point. It is especially distinguished from 
body fat, however, by the presence of a considerable proportion of fatty acids of low molecular weight, as already noted in Chapter I (30), where a list of the principal constituents is given. The presence of these so-called "volatile fatty acids" (i.e., acids which can be distilled in a current of steam) affords an important means for the detection of adulterations of butter.

The percentage of fat in milk varies widely. For the cow a minimum of 1.67 per cent is reported by König. Six per cent, on the other hand, is a high figure, although occasionally 7 per cent is reached. Babcock states that 9 per cent is the maximum observed for a cow giving as much as 15 pounds of milk daily.

The milk fat carries traces of lecithins and cholesterins and also varying amounts of coloring matter, derived, as Palmer and Eckles ${ }^{1}$ have shown, chiefly from the carotin of the feed.

Carbohydrates. - Milk contains in solution a disaccharid peculiar to itself, namely, lactose, or milk sugar (13). In distinction from fat, the percentage of lactose in fresh milk shows comparatively small variations, averaging about 5 per cent in cow's milk. The souring of milk is brought about by a fermentation of the milk sugar by which its molecule is split into four molecules of lactic acid.

Among the organic ingredients of milk should also be mentioned citric acid, which occurs in appreciable quantities in the form of calcium citrate.

Ash. - The total mineral matter in cow's milk averages about 0.7 per cent according to Van Slyke. ${ }^{2}$

Qualitatively, the ash of milk contains the same ingredients found in all animal substances. Its quantitative composition, however, as compared with the blood serum, on the one hand, and with that of the tissues on the other, shows some interesting relations. Bunge ${ }^{3}$ gives the following figures for the composition of the ash of the serum of cattle blood and of the ash of cow's milk. To these have been added Lawes and Gilbert's figures for the ash of a calf for the sake of comparison.

1 Jour. Biol. Chem., 17 (I9I4), I9I-264.

2 Jordan, The Feeding of Animals, 1908, p. 305.

${ }^{3} \mathrm{Ztschr}$. Biol., 10 (1874), 30r ; 12 (1876), r9r. 
Table i 19. - Percentage Composition of Ash

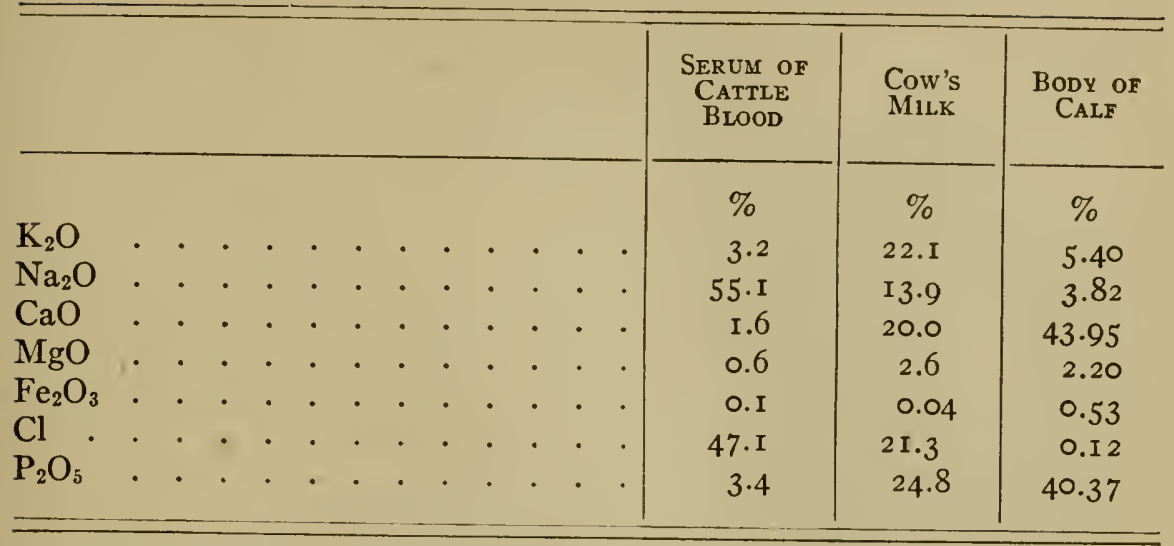

With smaller animals, having a shorter period of growth, the relations are even more striking. Thus, for the rabbit Bunge ${ }^{1}$ reports the following results.

Table i20. - Percentage Composition of Ash

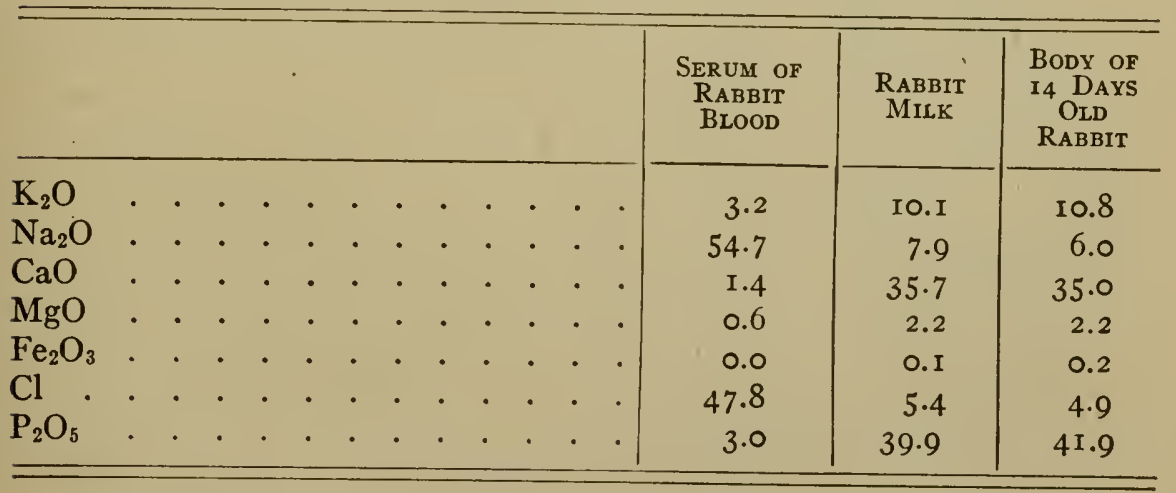

It appears that while sodium and chlorin are the predominant ingredients of the blood serum, these elements are present in milk in relatively small proportions, while potassium, calcium and phosphorus predominate in the latter, the ash of milk closely resembling that of the body of the same species.

547. Average composition. - Wing ${ }^{2}$ cites the following figures as showing approximately the average composition of cow's milk ${ }^{3}$ according to various authorities.

1 Quoted by Sellheim in Nagel's Handbuch für Physiologie, II, 188.

2 Milk and its Products, 1897 , p. 17.

${ }^{3}$ For data regarding the composition of the milk of other species than cattle, see Schaefer's Text Book of Physiology, Vol. I, p. I25. 
Table i21. - Average Composition of Cow's Milk

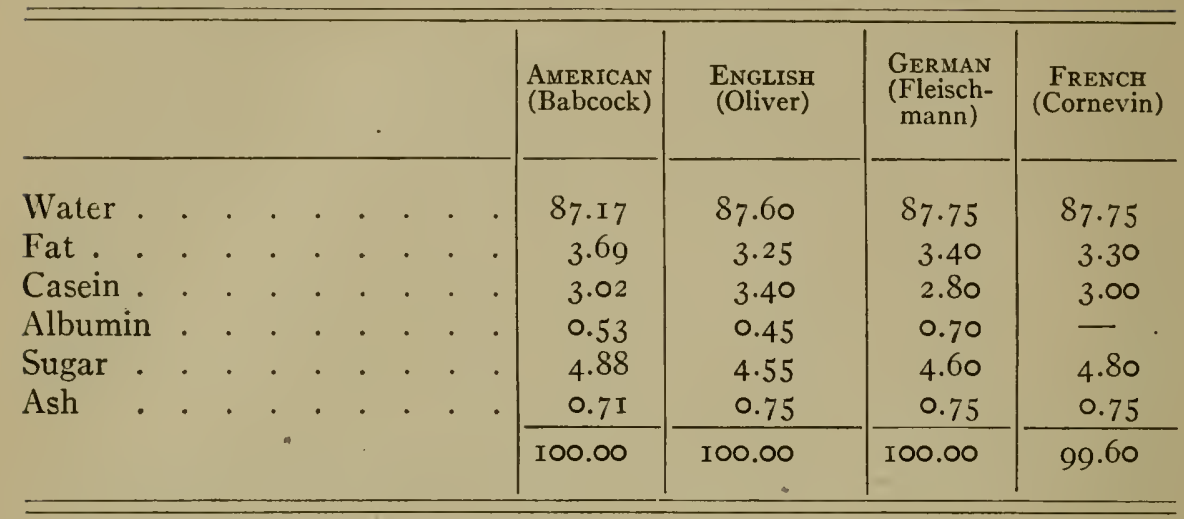

548. Milk glands. - The milk glands, properly speaking, are two in number, one on each side of the median line of the body, although in many animals each gland is subdivided into

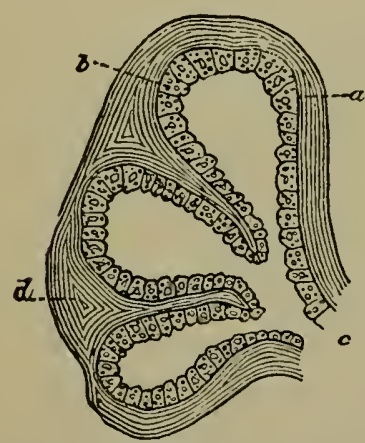

FIG. 40. - Lobule of milk gland. (Armsby, Manual of Cattle Feeding.) two or more lobes having separate outlets or teats. Thus in the horse and sheep each gland has two lobes, in the cow two or three, and in the hog from ten to fourteen. The milk gland is classified as a compound tubulo-acinous gland. Its structure may be roughly compared to that of a bunch of grapes. It consists of a great number of acini or alveoli, three of which are shown schematically in Fig. 40, corresponding to the single berries of the grape cluster. Each alveolus consists of an outer layer of connective tissue carrying capillary blood vessels, nerves and lymphatics. These alveoli are about $\frac{1}{30}$ of an inch in diameter and are united in groups of 3 to 5 to form lobules having a common outlet as shown in the figure. Internally, the alveoli are lined with a single layer of epithelial cells (Fig. 4I), which are the active agents in secreting milk. The ducts or passages leading from the alveoli are also lined with epithelial cells but of a different sort and which do not produce milk. These ducts unite to form larger ones, as shown in Fig. 42, which lead finally to the teat, emptying first into the so-called " milk cistern," a cavity lying near the base of the teat. In compound 
milk glands there is more or less connection through these milk ducts between the several lobes, but none between the two glands on either side of the body.

The milk gland, therefore, consists of a framework of connective tissue carrying more or less fat, of alveoli, milk ducts, veins, arteries, lymph vessels and nerves, the whole forming a reddish gray spongy mass. In the cow the two glands constituting the udder are separated by a band of fibrous tissue which serves to support the organ. The udder may vary widely in the proportion of connective and fatty tissue on the one hand and of true secreting tissue (alveoli) on the other. A large proportion of the former gives what is commonly known as a fleshy

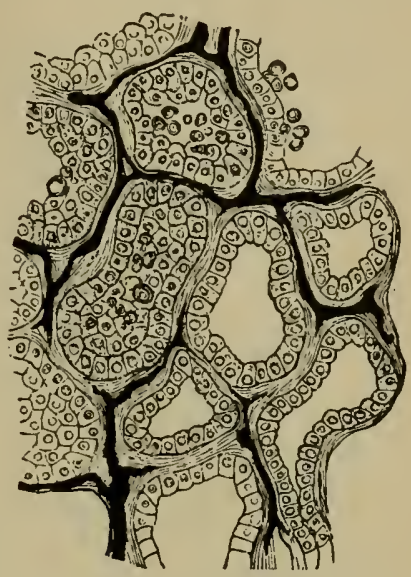

FIg. 4I. - Alveoli of milk gland. (Wilckens, Form und Leben der Landwirthschaftlichen Hausthiere.) udder. The size of the udder, therefore, is not the sole criterion

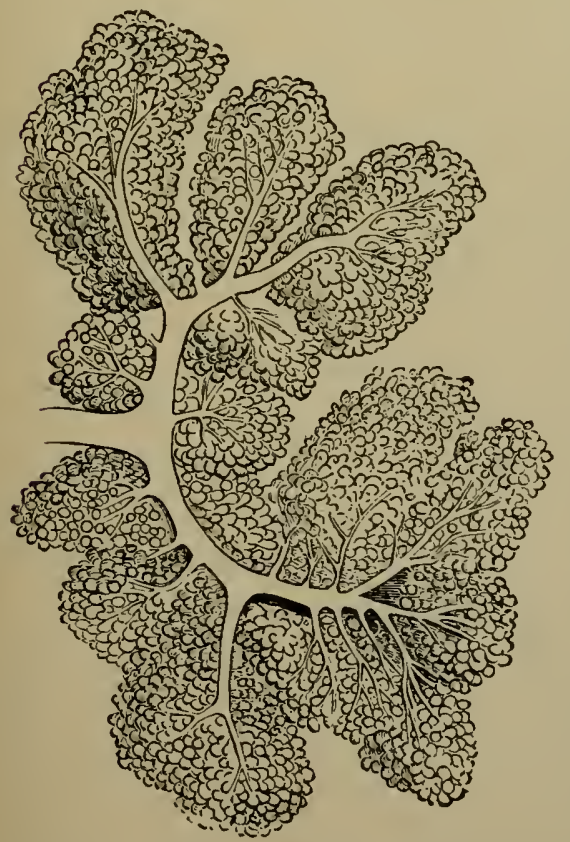

FIG. 42. - Structure of milk gland. (Wilckens, Form und Leben der Landwirthschaftlichen Hausthiere.) of its capacity as a milk producing organ.

At the branches of the milk ducts are located sphincter muscles which are more or less under the control of the animal and the contraction of which interferes with the flow of milk, enabling the animal, as the phrase goes, to "hold up " her milk.

549. Development of milk glands. - In the young animal, the milk glands are rudimentary and in the male remain so. during life, except in extraordinary cases. In the female, however, as sexual maturity approaches, a considerable formation of glandular tissue takes place, but the glands 
reach their full-development only in the later stages of pregnancy. At that time, a rapid growth of the alveoli and perhaps the formation of new ones occurs, the stimulus to this growth being, according to Bayliss and Starling, the formation of certain stimulating substances (Hormones) in the fetus which pass into the blood of the mother and so reach the milk glands. That other causes may at least coöperate, however, is shown by the apparently well-established fact that the regular removal of the fluid found in the glands of the virgin animal, or even mechanical stimulation, may lead to the formation of considerable quantities of milk, in some instances even in the male.

550. The secretion of milk. - That milk formation is a true secretion and not a mere filtration of material from the blood is clearly shown by the facts already stated regarding the composition of milk. As was pointed out, all the principal organic ingredients of the milk are peculiar to it. Casein and lactose are not found elsewhere in the animal body, and while the principal simple fats of milk are also found in the body fat, their proportions are different in the milk fat and the latter is specially characterized by the presence of glycerids of the lower acids of the aliphatic series. Furthermore, even more marked quantitative differences exist between the mineral elements of the milk and those of the blood serum. From all these facts, it is clear that the milk gland is a producing or secreting organ and that the solid ingredients of the milk are largely manufactured in it out of materials derived from the blood.

A theory of milk secretion first propounded by Virchow found wide acceptance. According to this theory, milk production consists essentially of a physiological fatty degeneration of the epithelial cells of the alveoli. The microscope shows that the cells of the actively secreting gland are larger than those in the resting gland and more or less filled with fat globules, especially on the side toward the cavity of the alveolus. It was held that while this process went on the cell divided, forming two or more, and that finally the cell next to the cavity liquefied, setting free the fat globules which it contained and, perhaps with the addition of more or less water, constituted the milk. Milk production was thus regarded as a form of the growth of tissue. 
Subsequent investigation, however, has generally failed to show satisfactory evidence of cell division. A modification of Virchow's theory still held is that while there is no cell division, the outer portion of the protoplasm is sloughed off and dissolved, forming the milk, and is again renewed by the growth of new protoplasm. The weight of opinion, however, regards milk production as a true secretion, entirely analogous to that observed in other glands. It is not believed that there is normally a breaking down of cells, but that the latter extrude their secreted materials into the alveolus precisely as do the secreting cells of other glands. This is held to apply to the fat globules as well as to the other ingredients of milk. The process is in many ways analogous to that of the resorption of digested material by the epithelial cells of the small intestine, the obvious difference being the direction in which the materials move.

The secretion of milk in the active udder is a more or less continuous process, the product accumulating in the cavities and passages of the gland. Fleischmann long ago showed, however, that the cavities of the udder cannot possibly contain the amount of milk produced in a single milking by a reasonably productive cow, and it is well recognized that a rapid secretion of milk occurs during suckling or milking. In other words, the milk gland, like other glands, reacts to a specific stimulus.

551. Sources of ingredients of milk. - While the ultimate source of the material contained in the milk is of course the feed, the milk gland draws its supply of material for milk production immediately from the blood, while at the same time it brings about extensive chemical transformations in the substances thus supplied. Probably all the ingredients of the milk should be regarded as products of the chemical activity of the epithelial cells of the glands, although the extent to which the original material is modified varies.

552. Origin of milk proteins. - The albumin and globulin of milk are quite similar to the corresponding substances in the blood. The casein, on the other hand, is radically different. In the first place, it is, as already stated, a conjugated protein containing some phosphorus-bearing radicle. Whether the latter is derived exclusively from the organic phosphorus compounds of the feed has not been demonstrated, although it appears probable that inorganic phosphorus compounds (phos- 
phates) may be utilized as sources of the phosphorus of the milk (257, 258, 497).

The production of casein, however, is not simply a conjugation of a simple protein with a phosphorus group. The constitution of casein is markedly different from that of the proteins of the blood serum or of the muscles, as is shown by the proportions of its various cleavage products as given in Chapter I (50), so that if casein is formed from the protein of the blood or tissue, a considerable reconstruction of their molecules is necessary. On the other hand, if the casein of the milk is built up in the epithelial cells of the udder, in the manner suggested in Chapter $\mathrm{V}(\mathbf{2 2 6}, \mathbf{2 2 7})$, from the simpler cleavage products in the blood, the process is specific for the milk gland.

553. Origin of milk fats. - It was stated in Chapter V (247249) in discussing the sources of body fat that although the latter may be derived in part from the fat of the feed and show some of its characteristics, nevertheless, the production of fat must be regarded as due essentially to the activity of the fat cells, and not to a simple deposition.

In the first place, it has been demonstrated by the researches of Jordan and others that milk fat as well as body fat may be formed from the carbohydrates of the feed.

In Jordan's ${ }^{1}$ experiments cows were fed either with an ordinary ration or with one very poor in fat and the production of fat in the milk determined. After deducting the maximum amounts of fat which could possibly be accounted for by the protein and fat of the feed, a considerable balance was left which could only have been produced from the carbohydrates. The following table gives a summary of the results: -

Table I 22. - Production of Fat by Cows

\begin{tabular}{|c|c|c|c|c|c|}
\hline $\begin{array}{l}\text { NUMBER } \\
\text { OF DAYS }\end{array}$ & $\begin{array}{l}\text { Total Pro- } \\
\text { TEIN } 2 \text { METAB- } \\
\text { OLISM }\end{array}$ & $\begin{array}{c}\text { EQUIVALENT } \\
\text { FAT }\end{array}$ & $\begin{array}{l}\text { FAT of } \\
\text { FEED }\end{array}$ & $\begin{array}{c}\text { TOTAL FROM } \\
\text { FAT AND } \\
\text { PROTEINS }\end{array}$ & $\begin{array}{l}\text { Fat Actually } \\
\text { Produced }\end{array}$ \\
\hline 59 & $\begin{array}{l}\text { Grams } \\
\text { I } 5, \text { IO9 }\end{array}$ & $\begin{array}{r}\text { Grams } \\
7,766\end{array}$ & $\begin{array}{l}\text { Grams } \\
\mathrm{I}, 490\end{array}$ & $\begin{array}{c}\text { Grams } \\
9,256\end{array}$ & $\begin{array}{l}\text { Grams } \\
\text { I } 7,5^{8} 5\end{array}$ \\
\hline 74 & 34,66 I & I 7,8 r 6 & $2,2 \mathrm{II}$ & 20,027 & 37,637 \\
\hline 4 & 2,209 & I, I 3 I & $\mathrm{I}, 5 \circ 4$ & 2,635 & 3,289 \\
\hline
\end{tabular}

${ }^{1}$ N. Y. (Geneva) Expt. Sta., Buls. 132 and 197.

2 Digested protein of feed less gain of protein by the animal. 
It should perhaps be pointed out that the formation of fat from carbohydrates in these experiments may not necessarily have occurred in the milk gland itself. It is entirely conceivable that the main portion of the synthesis of the fat may have taken place elsewhere and that the fat or its precursors were simply transferred to the milk gland.

Second, it has also been shown by a considerable number of experiments that, as in the case of body fat, the fat of the feed may sensibly affect the properties of the milk fat. Not only have changes in the melting point, iodin number, and other properties of butter fat been found to follow in a general way similar changes in the feed fat, but characteristic ingredients of foreign fats given in the feed have been detected in the milk. While it is not necessary to conclude, and is indeed unlikely, that the feed fat is simply transferred, as it were mechanically, to the milk, it is clear, on the other hand, that relatively large fragments of the fat molecule are able to pass through the epithelial cells into the milk. These facts render it evident that feed fat is a source of milk fat. Not only so, but experiments by Morgen and his associates, to be mentioned later (613), seem to show that a certain amount of fat in the feed (in herbivorous animals at least) conduces to the most efficient production of milk fat.

The idea that the fat of milk is produced synthetically to a considerable extent is perhaps supported also by the presence in it of the lower acids of the aliphatic series, which may be intermediate steps in the synthesis of fat from simpler carbon compounds, or, on the other hand, may arise during the partial breaking up of the carbon chain in the feed fat which probably precedes its transformation into milk fat.

As a general conclusion, therefore, it may be stated that the fat of milk may have its origin either in the fat or in the carbohydrates of the feed, or in both. Whether it may also be produced from protein has not been demonstrated experimentally, but reasoning by analogy with the formation of body fat, it must be regarded as at least very probable.

554. Origin of lactose. - The lactose of milk is a disaccharid yielding upon hydration dextrose and galactose. Dextrose or its derivatives are abundant in the feed of herbivorous animals and it is also a constant ingredient of the blood. On the other 
hand, while the ordinary feed of herbivora contains carbohydrates yielding galactose, the latter is apparently transformed into glycogen quite promptly and at any rate has not been found in the blood, while animals receiving feed containing no galactose (carnivora, e.g.) produce lactose in their milk. The probability seems to be that the galactose half of the lactose is manufactured in the milk gland from the dextrose of the blood.

555. Sources of ash. - The ash ingredients of the milk, including its sulphur and phosphorus, are, of course, derived ultimately from the corresponding ingredients of the feed. In liberal milk production on ordinary winter rations containing a sufficiency of organic nutrients, however, it appears from investigations by Forbes ${ }^{1}$ that considerable amounts of calcium, magnesium and phosphorus may be drawn from the relatively large store contained in the body, presumably to be replaced in later stages of lactation.

556. Character of milk production. - While the statement that milk production is a form of tissue growth is probably incorrect anatomically, it is essentially true so far as the chemical composition of the product and the demands which it makes on the feed supply are concerned. This is clearly shown by comparing the ratio of protein to fat in the organic matter of milk and in that of the increase in weight of growing animals. In the solids of milk, it is evident that in order to make a fair comparison its milk sugar should be reduced to the equivalent amount of fat. Taking Babcock's figures (547) as representing the average composition of milk, the 4.88 per cent of sugar divided by 2.25 is equivalent to $2 . \mathrm{I} 7$ per cent of fat, which added to the 3.69 per cent of fat present as such makes a total fat equivalent of 5.86 per cent, while if milk sugar were thus replaced by fat the total organic matter would amount to $9.4 \mathrm{I}$ per cent. On this basis, roo parts of organic matter would contain 37.73 per cent of protein and 62.27 per cent of fat. Comparing these figures with those given in Chapter XI (458) for the composition of the increase in growth, it appears that the proportion of protein to fat is greater than that computed for young animals except in the earliest stages of growth.

The computed energy content of average milk solids is 2620 1 Ohio Expt. Sta., Bul. 295 (I9I6). 
Cals. per pound. This is greater than the energy content of the dry matter gained by very young animals, but less than that computed in later stages of growth. In a general way, then, it may be said that milk solids correspond in proportion of protein and in energy value per pound to the gains made by growing animals when in the neighborhood of three months old.

557. Rate of production of milk solids. - A beef calf three months old may be assumed to make a growth of approximately I.5 pounds per day, containing perhaps three-fourths of a pound of dry matter with an energy content of about 2200 Cals. The very moderate yield of $I_{5}$ pounds of average milk per day would contain about 1.92 pounds of total solids equivalent to 5030 Cals. of energy. In other words, considerably more than twice as great a production would be effected by the relatively small bulk of the secreting cells in the udder as by the whole body of the calf. When it is further considered that the product of the dairy cow is all edible, her great economic value as a producer of human food becomes obvious. On this point Jordan says: ${ }^{1}$ "A cow yielding 6000 pounds of average milk per year is not regarded as an unusual animal. This means, however, the annual production of not less than 780 pounds of milk-solids, an amount at least double the dry matter in the body of a cow weighing 900 pounds. When we consider that this manufacture of new material is carried on not only during a single year, but through the entire adult life of the animal, we begin to realize how extensive are the demands upon the food supply. Still more striking is the case of high-grade cows yielding annually over half a ton of milk solids, and when we remember the performance of Clothilde, whose 26,000 pounds of milk produced in a year certainly contained more than 2500 pounds of solid matter, we must regard the cow as possessing wonderful powers of transmutation. Her capacity for the rapid and economical production of human food of the highest quality is not equaled by any other animal."

558. Factors of milk production. - Milk production differs from meat production in one very essential particular. In the latter, broadly speaking, an increase in the whole body of the animal is what is sought, and while the product may vary in

\footnotetext{
1 The Feeding of Animals, I908, p. 308.
} 
market quality, all the feed consumed in excess of the maintenance requirement is available for the production of gain. In milk production, on the contrary, what is desired is the secretion of a single set of glands. An increase in weight in the mature dairy cow is not sought. At best it represents a diversion of feed to other purposes than the one in view, while any considerable fattening tends to check the activity of the milk glands. In feeding for milk production, therefore, it is necessary to consider not only the surplus feed above the maintenance requirement but the factors affecting the distribution of that surplus between milk production on the one hand and growth or fattening on the other hand. The art of feeding for milk consists in stimulating the milk production to the greatest economically possible extent and in supplying othe feed material necessary for this production, while avoiding, in the mature animal, any material increase of body tissue.

The factors governing milk production are essentially the same as in other branches of animal production, viz., the animal, the environment and the feed supply.

In milk production, however, the relative importance of the first and second conditions is greater than in other forms of production for the reason that they may materially influence the distribution of the excess feed between milk production and tissue increase.

\section{§ 2. The Animal as a Factor in Milk Production}

559. The prime factor in successful dairy production is the animal. Unless the latter possesses abundant secreting tissue which is capable of being stimulated to a normal rate of activity and of yielding a secretion of good quality, the most scrupulous care and the most abundant feeding will inevitably fail to yield satisfactory returns.

\section{Individuality}

560. Includes breed differences. - The influence of individuality may be said to include that of breed, since a breed is simply an aggregate of more or less similar and genetically related individuals. It is outside the scope of this work to discuss problems of breeds and breeding, and this branch of the 
subject will therefore be considered mainly from the point of view of individual differences.

561. Influence on yield of milk. - While the actual quantity of milk produced is affected by feed, care and other circumstances, the capacity of the animal as a milk producer is an individual characteristic. Just as the maximum speed of which a horse is capable is dependent primarily upon his conformation, spirit and other individual characteristics, while the actual rate at which he travels at any given time is largely dependent upon his driver, so the maximum capacity of the milk cow constitutes an individual limit beyond which she cannot be pushed by any amount of care or feed.

Striking illustrations of the importance of individuality are afforded by the various public tests of dairy cows. For example, in the World's Columbian Exposition of 1893 , the conditions of the so-called ninety-days test were such as to induce liberal feeding and the best of care on the part of the exhibitors. The cows, numbering 74 , were of three different breeds and presumably represented the best available specimens of each breed.

The following table shows the average daily product of the best ${ }^{1}$ and the poorest cow of each breed in that test.

Table 123. - Average Daily Yield of Cows in Ninety-Days Test, World's Columbian Exposition ${ }^{2}$

\begin{tabular}{|c|c|c|c|}
\hline & MrLK & FAT OF MILK & $\begin{array}{l}\text { Total SoLIDS } \\
\text { OF MILK }\end{array}$ \\
\hline $\begin{array}{l}\text { Best Jersey } \\
\text { Poorest Jersey } \\
\text { Poorest in per cent of best }\end{array}$ & $\begin{array}{l}40.4 \mathrm{lb} . \\
22.9 \mathrm{lb} . \\
56.7 \%\end{array}$ & $\begin{array}{l}\text { т. } 98 \mathrm{lb} . \\
\text { r.09 lb. } \\
55.1 \%\end{array}$ & $\begin{array}{l}5.67 \mathrm{lb} . \\
3.21 \mathrm{lb} . \\
56.6 \%\end{array}$ \\
\hline $\begin{array}{l}\text { Best Guernsey } \\
\text { Poorest Guernsey } \\
\text { Poorest in per cent of best }\end{array}$ & $\begin{array}{l}39.0 \mathrm{lb} . \\
\mathrm{I} 9.3 \mathrm{lb} . \\
49.5 \%\end{array}$ & $\begin{array}{l}\text { I.70 lb. } \\
0.97 \mathrm{lb} \text {. } \\
57.1 \%\end{array}$ & $\begin{array}{l}5.39 \mathrm{lb} . \\
2.75 \mathrm{lb} . \\
51.0 \%\end{array}$ \\
\hline $\begin{array}{l}\text { Best Shorthorn . } \\
\text { Poorest Shorthorn . } \\
\text { Poorest in per cent of best }\end{array}$ & $\begin{array}{l}40.9 \mathrm{lb} . \\
23.9 \mathrm{lb} . \\
58.4 \%\end{array}$ & $\begin{array}{l}\mathrm{I} .49 \mathrm{lb} \text {. } \\
0.80 \mathrm{lb} \text {. } \\
53.7 \%\end{array}$ & $\begin{array}{l}5.29 \mathrm{lb} . \\
2.87 \mathrm{lb} . \\
54.3 \%\end{array}$ \\
\hline
\end{tabular}

${ }^{1}$ By best and poorest cows is meant those which showed the greatest and least net profit under the rules of the test.

${ }^{2}$ Jersey Bulletin, Dec. 12, 1893. 
Even the lowest of these records are remarkably good considering the unfavorable conditions necessarily incident to a public test. In each of these three picked herds, however, the production of the poorest animal was only from 50 to 60 per cent of that of the best animal. Moreover, the differences between individuals of the same breed were much greater than the differences between the averages for the three breeds. That even greater differences exist among the common cows of the country has been shown by numerous statistical investigations, some of the most striking of which have been collected by Eckles. ${ }^{1}$

562. Influence on economy of feeding. - While it is unlikely that the utilization of the feed in the narrower sense (i.e., the amount of milk solids of a given composition manufactured in the udder from equal amounts of nutritive substances supplied) is materially affected by the individuality of the animal, the feed utilization in the broader economic sense is very largely dependent upon this factor. It must be constantly borne in mind that, as already stated (558), efficiency in milk production is in large part a question of the distribution of the feed supplied in excess of maintenance. Some animals, by virtue of individual or inherited peculiarities, are able to transform large amounts of excess feed into milk without storing up any considerable portion of it in the form of body tissue. Such animals tend to remain spare in body and if well fed produce large amounts of milk. They are the typical dairy animals. Other individuals, on the contrary, have a wellmarked tendency in the opposite direction, viz., toward the production of body tissue. When fed heavily, they utilize the additional feed chiefly in this direction and show little or no tendency toward an increase in milk production. These are typical meat-producing animals. The two types, of course, shade into each other by imperceptible gradations.

The important bearing of these facts upon the nutrition of dairy animals will be further considered later (606-610). Here it may simply be noted that the superiority of certain individuals which has been illustrated in the preceding paragraphs is doubtless due to a considerable extent to the ability to consume large amounts of feed and convert the surplus into milk rather than into body tissue.

${ }^{3}$ Dairy Cattle and Milk Production, I9II, pp. I18-126. 
563. Influence on course of lactation. - That individuality plays an important part in determining the rate at which the milk yield falls off with advancing lactation is shown more specifically in a subsequent paragraph (568).

564. Influence on composition of milk. - It is a matter of common observation that cows vary as regards the richness of their milk, that is, as regards the amount of cream or butter which can be obtained from a given weight of milk. Various breed tests at experiment stations have served to define more exactly the influence of individuality and breed upon the composition of milk. The results cited in Table 124 are intended to illustrate this influence and not primarily to compare different breeds. The table shows the averages of the results obtained at three different experiment stations ${ }^{1}$ for several breeds in tests covering periods of time ranging from eight months to two years. The results from each station are given weight in the average in proportion to the number of cows under test, and the average results are arranged in the order of the fat content of the milk.

Table i24. - Average Composition of Milk of Different Breeds

\begin{tabular}{|c|c|c|c|c|c|c|c|c|c|c|c|c|c|}
\hline & & & & & & & & ASH & $\begin{array}{c}\text { CASEIN } \\
\text { AND } \\
\text { ALBU- } \\
\text { MIN }\end{array}$ & $\begin{array}{l}\text { LAC- } \\
\text { TOSE }\end{array}$ & FAT & $\begin{array}{l}\text { Total } \\
\text { SoLIDS }\end{array}$ & $\begin{array}{c}\text { NUM- } \\
\text { BER OF } \\
\text { COWS } \\
\text { AVER- } \\
\text { AGED }\end{array}$ \\
\hline & & & & & & & & $\%$ & $\%$ & $\%$ & $\%$ & $\%$ & $\%$ \\
\hline Holderness & 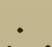 & . & - & & & • & . & 0.68 & 3.14 & $5 . \mathrm{II}$ & 3.46 & I 2.39 & 2 \\
\hline Holstein-F & ciesi & ian & . & . & . & . & . & 0.67 & 3.22 & 4.89 & $3 \cdot 5^{I}$ & I 2.29 & 6 \\
\hline Ayrshire & . & & - & - & . & - & . & 0.67 & $3 \cdot 38$ & $5 \cdot 13$ & 3.64 & I 2.82 & 9 \\
\hline Shorthorn & . & - & . & . & . & . & . & 0.73 & 3.27 & 4.80 & 3.65 & I 2.45 & 3 \\
\hline Devon . & - & - & - & - & . & . & . & 0.76 & $3 \cdot 74$ & $5 \cdot 3^{2}$ & $4 \cdot 54$ & I 4.36 & 2 \\
\hline Guernsey & . & - & - & - & . & - & . & 0.75 & 3.78 & 4.94 & 4.96 & I 4.43 & 5 \\
\hline Jersey & . & . & - & . & . & . & . & 0.75 & 3.92 & 4.98 & 5.25 & I 4.90 & 8 \\
\hline
\end{tabular}

Fat is evidently the most variable ingredient of milk, its maximum exceeding its minimum in these averages by more than $5^{\circ}$ per cent. Along with the increase of fat there is

${ }^{1}$ Maine Expt. Sta., Rpt. r89o, p. 29; New Jersey Expt. Sta., Rpt. r890, pp. 223224; New York (Geneva) Expt. Sta., Rpt. I89r, pp. 94-104. 
also an increase of the total protein and of the total solids, but these are relatively smaller than that of the fat, the totals being 25 per cent and 2 I per cent, respectively. There is also an increase of $\mathrm{I} 2$ per cent in the proportion of ash, while the lactose, on the contrary, shows comparatively small and irregular changes, the extreme range of the differences being I I per cent, while it does not increase regularly with the increase of the other ingredients. The lactose is evidently the most constant ingredient of the milk.

565. Influence on composition of milk solids. - The real nature of the differences in composition, however, is rendered clearer by computing the percentage composition of the waterfree total solids, with the results shown in Table 125 :-

Table i25. - Average Composition of Milk Solids of Different BREEDS

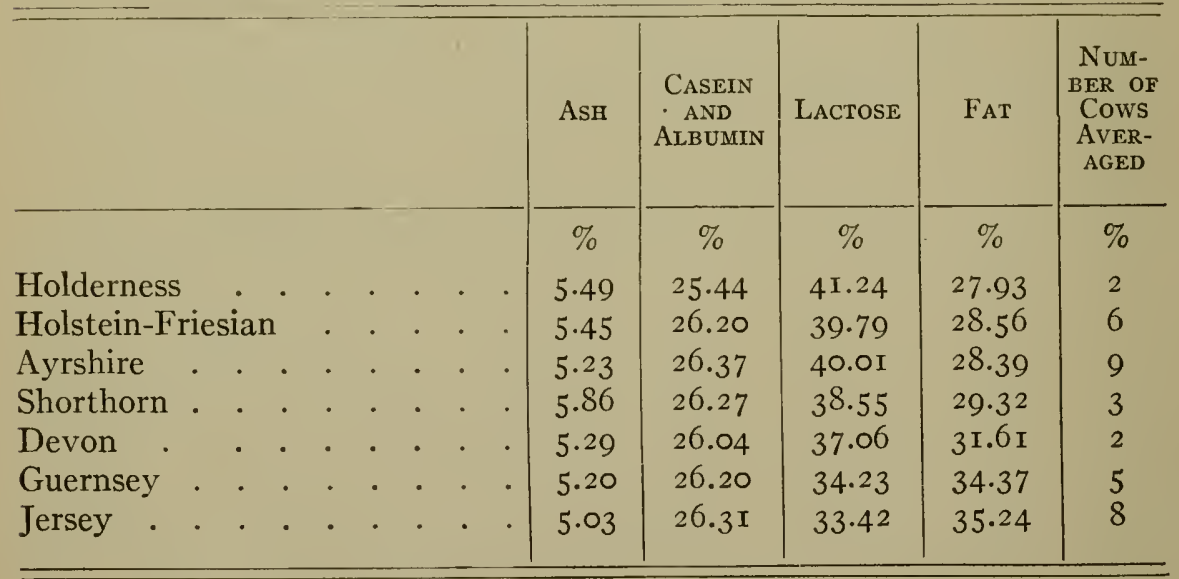

From the foregoing table, it appears that the percentages of ash and of total protein in the milk solids are very constant, the single figures differing but very slightly from the averages of 5.36 and 26.10, respectively. The essential difference in the composition of the milk solids lies in the proportion of lactose to fat, the former decreasing as the latter increases, while the total percentage of the two taken together is practically constant, varying less than 0.7 per cent from the average of 68.53 per cent. In other words, it appears from these figures that in cows producing milk rich in fat the secreting cells form relatively less lactose and correspondingly more fat, while, as 
Table 124 shows, this difference is accompanied by a relatively smaller secretion of water, so that the percentage of total solids in the resulting milk is greater.

Cooke, ${ }^{1}$ in 1890 , drew the same conclusion from a study of over 2400 analyses of milk reported by the experiment stations of the United States up to that date, and more recently Haecker $^{2}$ has reached substantially the same result from analyses of 544 individual samples of milk from the Minnesota Station herd.

566. Variability of composition in same animal. - It should be noted that the foregoing conclusions are drawn from the average composition of the milk of the same individuals for comparatively long periods. The composition of the milk of the same cow, however, may and frequently does vary quite widely from one milking to another without affecting its average composition as computed from analyses of a number of milkings.

This has been observed especially in the case of the fat because far more determinations have been made of this constituent than of any other, the fat being both the most valuable and the most easily determinable ingredient. Variations as great as I per cent in the fat content of successive milkings of the same cow are not uncommon and differences of 2 and even 3 per cent not very rare. Whether there is a correlated variation in the proportion of lactose, as in the averages compared in the previous paragraph, does not appear. It is presumed that these variations are due largely to external influences but no definite connection with any specific factors of environment has been traced with certainty, although Spier ${ }^{3}$ believes them to be due to incomplete milking (575). It is evident that correct comparisons of the yields of different animals, or of the same animal at different times, can be made only on the basis of the average yield and composition for a number of days.

The extent of this variability in the composition of milk from one milking to another appears to be an individual peculiarity, the milk of some cows being much more uniform in daily composition than that of others. An interesting example of this has been reported by Farrington. ${ }^{4}$

1 Vt. Expt. Sta., Rpt. I89o, pp. 97-100.

2 Minn. Expt. Sta., Bul. I 40 (19I4), p. 5 I.

${ }^{3}$ Jour. Highland and Agr. Soc., I 909, p. 287.

${ }^{4}$ Ills. Expt. Sta., Bul. I 7 (I89I), p. 9. 


\section{Stage of lactation}

567. Milk production a periodic function. - Milk production has as its object the nourishment of the offspring. In a state of nature it is a periodic function, beginning at the birth of the young or shortly before, while as the young animal gradually becomes less dependent on the mother it diminishes in intensity and finally ceases. Although man has greatly prolonged the period of milk production of the cow, so that the time during which she goes dry is relatively short and in some instances is eliminated altogether, nevertheless, milk production still retains its periodic character and undergoes marked changes during the progress of a lactation.

568. Influence on yield of milk. - The most evident effect of advancing lactation is the gradual decrease of the amount of milk produced, but the rate of decrease may differ widely at different stages in the same animal and in different animals at the corresponding period in lactation. As a rule, the amount of milk does not reach its maximum immediately after the birth of the young, but shows an increase for one or two weeks in the case of the cow. Following this maximum, there is typically a slow falling off for several months followed by a more rapid decrease as the time of the next calving approaches, all but exceptional cows going dry for a longer or shorter time. In the case of farrow cows, the milk production may continue to show a comparatively slow decrease for a much longer time.

The curves of lactation as they may be called, however, vary greatly from cow to cow and from year to year with the same animal and show marked irregularities often not readily explained by any observed conditions.

569. Influence on composition of milk. - In general the percentages of total solids and of fat tend to increase, especially toward the end of lactation when the quantity of milk falls off rapidly. Like the changes in quantity, these variations in composition are often irregular and sometimes are scarcely manifest at all until the rapid falling off in quantity sets in toward the end of the lactation.

570. Bearing on experimental methods. - The unavoidable changes in the yield and composition of milk with the advance of lactation must be taken account of in all experiments on 
milk production, and render the interpretation of their results peculiarly difficult. It is obvious, e.g., that if a change of the ration of a cow is accompanied by a decrease in her milk yield part at least of the decrease may be due to the progress of lactation and not to the change of feed. On the other hand an increase of the milk yield in a later period of the experiment may be partly offset by the natural shrinkage in milk. In brief the later periods of an experiment are at a disadvantage compared with the earlier periods.

Two methods for eliminating or attempting to eliminate this influence of lactation have been used, viz., the period system and the group system.

571. The period system. - In the period system, as introduced by Wolff, Kühn and others of the earlier experimenters, the animal receives an identical ration in two or more periods well removed from each other in point of time - usually the first and last periods - and from the results of these periods the average daily rate of decrease in the yield of milk and its ingredients is calculated. On the assumption that had the same ration or treatment been continued unchanged this rate would have been uniform throughout the experiment, it may be computed what yields, would have been secured in the intermediate periods. A comparison of these computed yields with those actually observed is taken as the measure of the effect of the change in feed or other conditions. The accuracy of this method depends of course on the correctness of the assumption that the yields would have decreased at a uniform rate.

572. The group system. - The use of the group system was introduced by Fjord and his successors in the Copenhagen Experiment Station in connection with their determinations of the so-called feed units (702). The period system seeks to compare each animal with itself. The group system, on the other hand, attempts to compare an animal or group with another check animal or group. In a long preliminary period both groups receive the same ration or treatment and their relative production is determined. One of the groups is then continued on the same treatment while with the other group the factor to be tested is introduced. Finally, in a concluding period, both groups are again treated as in the initial period. 
A combination of the two systems may also be used, one group of animals being fed varying rations in successive periods, while the other receives a uniform ration throughout the entire experiment.

A very complete discussion of the methods of eliminating the influence of advancing lactation in the interpretation of the results of experiments on milk production is to be found in a recent article by Morgen. ${ }^{1}$

\section{§ 3. The Influence of Environment on Milk Produc- TION}

The word environment is here used loosely as a convenient term to summarize all those external influences other than feed which may affect milk production. The dairy cow appears to be particularly sensitive to external conditions, some of the more important of which are considered in the following paragraphs.

\section{Milking}

Milking is but an imperfect imitation of the suckling of the young, and naturally its efficiency in securing the milk is likely to be affected by a variety of circumstances.

573. Frequency of milking. - As already stated, the cavities of the udder in heavy milkers cannot hold all the milk produced at one milking. Between milkings there evidently may be a considerable accumulation of matter in the alveoli and canals which appears to have the effect of diminishing the secreting activity of the epithelial cells through what might be crudely called "back pressure." Suckling or milking would have the effect of relieving this "pressure" and perhaps rendering secretion more easy, while at the same time it seems to act as a direct stimulus to secretion. At any rate it is a fact that more frequent milking tends to increase the yield of milk, especially in the case of good cows and in the earlier stages of lactation. The effect of frequent milking is strikingly illustrated in the following experiments by Kaull. ${ }^{2}$ The abrupt falling off in the milk yield when the milking was made very frequent may per-

1 Landw. Vers. Stat., 77 (I912), 35 I.

2 Cited by Kellner, Die Ernährung der landw. Nutztiere, 6th Ed., p. 52 I. 
haps be interpreted as due to overstimulation or mechanical irritation of the udder.

Table i26. - Effect of Frequent Milking

\begin{tabular}{|c|c|c|c|c|c|c|c|c|}
\hline & & & & & & & $\begin{array}{c}\text { Total Quantity } \\
\text { OF MILK PER } \\
\text { MILKING }\end{array}$ & $\begin{array}{l}\text { Total QUantity } \\
\text { OF MILK IN } 24 \\
\text { Hours }\end{array}$ \\
\hline $\begin{array}{l}\text { Milking every } 24 \mathrm{hr} \text {. . } \\
\text { Milking every } 2 \mathrm{hr} \text {. } \\
\text { Milking every } 4 \mathrm{hr} \text {. } \\
\text { Milking every } 2 \mathrm{hr} . \\
\text { Milking every } 65 \mathrm{~min} \text {. } \\
\text { Milking every } 50 \mathrm{~min} \text {. }\end{array}$ & $\begin{array}{l}\cdot \\
\cdot \\
\cdot \\
\cdot \\
\cdot \\
\cdot\end{array}$ & $\begin{array}{l}\cdot \\
\cdot \\
\cdot \\
. \\
.\end{array}$ & $\begin{array}{l}\cdot \\
\cdot \\
\cdot \\
\cdot \\
\cdot \\
\cdot\end{array}$ & $\begin{array}{l}\dot{ } \cdot \\
\dot{.} \\
\dot{.} \\
\dot{ }\end{array}$ & $\begin{array}{l}\cdot \\
\cdot \\
. \\
. \\
. \\
-\end{array}$ & $\begin{array}{l}\cdot \\
\cdot \\
\cdot \\
. \\
. \\
.\end{array}$ & $\begin{array}{l}3.8 \mathrm{I} \mathrm{Kg} \text {. } \\
2.46 \mathrm{Kg} \text {. } \\
2.06 \mathrm{Kg} \text {. } \\
\text { I. I I Kg. } \\
0.66 \mathrm{Kg} \text {. } \\
0.07 \mathrm{Kg} \text {. }\end{array}$ & $\begin{array}{r}7.62 \mathrm{Kg} . \\
9.84 \mathrm{Kg} . \\
\mathrm{I} 2.36 \mathrm{Kg} . \\
\mathrm{I} 3.32 \mathrm{Kg} . \\
\mathrm{I} 4.62 \mathrm{Kg} . \\
2.02 \mathrm{Kg} .\end{array}$ \\
\hline
\end{tabular}

Results obtained in short experiments, however, give an altogether exaggerated idea of the practical advantage of frequent milking. As Fleischmann has shown, the capacity of the udder adjusts itself quite definitely to its productive activity and in the measure in which this takes place the gain due to more frequent milking diminishes or disappears. He estimates the increased yield obtained by three as compared with two daily milkings at about 6 or 7 per cent. In many cases, therefore, it will be questionable whether the additional milk obtained by a third milking will be at all sufficient to pay for the extra labor involved. In the case of very productive cows in the earlier stages of lactation more frequent milking may be necessary, not so much for the sake of obtaining the extra milk as for the sake of avoiding inflammatory conditions in the udder and especially for preventing the permanent depression of the secreting power which would follow incomplete milking and which would mean a loss of milk throughout the whole lactation.

574. Influence of frequent milking on composition of milk. Frequent milking tends to increase the percentage of solids and of fat in the milk. This effect is manifest especially when the intervals between the milkings are of unequal length.

When milking takes place at regular intervals, the several milkings tend to have about the same average composition. If the intervals vary in length, the milk obtained after the shorter interval on the average contains a higher percentage of 
solids and of fat, i.e., it is more concentrated than that yielded after the longer interval. The differences in the composition of the night's and morning's milk, which have been the subject of so much discussion, appear explicable upon this basis, the interval between the morning's and night's milking being usually less than that between the night's and morning's.

575. Completeness of milking. - If successive portions of the same milking be analyzed, the percentage of fat in the later portions will be found to be greater than in the earlier ones, while the percentage of solids-not-fat varies comparatively little. The fact of the greater richness of the so-called "strippings" is well known. This difference was at one time explained as caused by an actual rising of the cream on the milk contained in the udder. The fact, however, that but a comparatively small amount of milk is held in the milk cistern, as well as the entire anatomy of the udder, renders this explanation untenable. The difference is probably due to a partial retention or stagnation of the fat globules in the alveoli and canals, they being afterward washed out by the portions of milk secreted during the latter part of the milking. Incomplete milking not only fails to get this fat, thus lowering the quality of the milk actually obtained (compare 566), but it appears that the retention of the fat in the alveoli tends to check the secretion of the milk. In all forms of milking, therefore, it is important that the cow be milked out as completely as practicable. The advantages of various methods of manipulating the udder, such as the Hegelund method, are probably due largely to this influence. Similarly, in the use of milking machines it seems to be necessary with most cows to remove the last portions, or strippings, by hand.

\section{Muscular exertion - exercise, fatigue}

The influence of muscular exertion upon milk secretion has been much discussed upon a comparatively slender experimental basis. In the United States the question has usually been as to the desirability of allowing freedom of motion and exercise to dairy cows, while in Europe, especially among small farmers, cows are used for draft to a not inconsiderable extent. 
576. Feed cost of exercise. - Attention was called in the discussion of the maintenance requirement in Chapter VIII (391) to the very marked effect of muscular exertion in increasing the katabolism, especially of body fat or of the non-nitrogenous ingredients supplied by the feed. It has been frequently argued from this fact that the amount of exercise allowed to dairy cows should be restricted as much as possible. Not a few dairymen indeed have gone so far as to confine their cows entirely, reasoning that since the object of their business is to convert feed into milk any diversion of it to the support of muscular exertion was a waste. This, however, is a very narrow and inadequate view of the subject. Most authorities on dairying regard a moderate amount of exercise for dairy cows as beneficial. Thus Martiny ${ }^{1}$ in 187 I cites five authorities on this point and expresses the opinion that exercise and moderate work increase rather than decrease the yield of milk, while severe work has an unfavorable effect upon both the yield and quality. Similar opinions are expressed later by C. F. Müller, Fleischmann, Kirchner, König and Von Klenze. These earlier data are of the nature of more or less empirical observations rather than of actual experiments.

577. Morgen's investigations. - Of actual experiments upon the influence of muscular exertion upon milk production, those of Morgen ${ }^{2}$ at the Hohenheim Experiment Station are the most convincing because they were made under strictly comparable conditions and especially because the relative amounts of work performed in the different periods were determined.

The two Simmenthal cows employed were accustomed to being used for draft. The work was done at a slow walk upon the sweep power dynamometer used by Wolff in his experiments upon work production by the horse $(386 a, 670,779)$, the amount of work performed being regulated in part by the resistance of the dynamometer and in part by the number of hours of work required, so that approximately single, double and quadruple work was done. The ration fed, which was a liberal one, was unchanged throughout the trials. The experiment consisted of II periods, approximating two weeks ${ }^{3}$ each of alternate rest

Die Milch, Part I, pp. 345-435.

2 Landw. Vers. Stat., 51 (I899), I 7.

3 Eleven to twenty-six days. 
and work periods, beginning with a rest period, so that each work period was preceded and followed by a rest period.

The rather moderate amount of work performed caused some decrease in the volume of milk produced, the effect tending to be a little greater in the periods in which most work was done. The decrease, however, was chiefly a decrease in the amount of water secreted, although a slight diminution in the yield of total milk solids, ranging from ro to 85 grams per day, was observed. In other words, the effect of the work was to render the milk somewhat more concentrated. The most notable effect, however, was upon the yield of fat, which showed an actual increase in every case but two. This increase was compensated for by a decrease of the fat-free solids, so that analyses of the milk showed a higher percentage of fat and of total solids, while the percentage of fat-free solids remained practically unchanged.

578. Confirmatory results. - Quite similar results, although obtained in some cases by less rigorous methods, have been reported by Dornic, ${ }^{1}$ Stillich, ${ }^{2}$ Backhaus, ${ }^{3}$ Torssell ${ }^{4}$ and Dolgich. ${ }^{5}$

Observations by Sturtevant, ${ }^{6}$ Henkel $^{7}$ and Hills ${ }^{8}$ upon the effect of fatigue on the yield and composition of milk are also in accord with the results of experiments upon work and exercise in showing a tendency to reduce the quantity of milk and at the same time to increase both the percentage and the actual yield of fat.

Aside from the question of the effects of overexertion, it appears clear that a considerable amount of work may be performed by cows without any serious diminution of the volume of their milk and with an actual increase in the yield of fat, its most valuable ingredient. The lightest work in Morgen's experiments was roughly equivalent to hauling a load of a ton $\mathbf{I} \frac{\mathbf{3}}{\mathbf{4}}$ miles over a smooth level road. This is certainly much more labor than the ordinary cow will perform when turned loose in a comfortable yard or paddock.

${ }^{1}$ Milch Ztg., 25 (1896), 331.

2 Jahresber. Agr. Chem., 39 (1897), 529.

3 Centbl. Agr. Chem., 28 (r899), 492; Expt. Sta. Rec., 10 (I899), 85.

${ }^{4}$ Expt. Sta. Rec., 12 (I90I), 38I. $\quad{ }^{5}$ Jahresber. Tier Chem., 33 (I904), 382.

${ }^{6}$ N. Y. (Geneva) Expt. Sta., Rpt. I882, p. 25.

7 Landw. Vers. Stat., 46 (I896), 329.

${ }^{8}$ Vt. Expt. Sta., Rpts. I 894 , p. I62, I898, p. 367 and 1899 , p. 309. 
It is still true, of course, that the energy for all muscular exertion is ultimately supplied by the feed. In the instance just mentioned the extra feed required for this purpose may be approximately estimated, on the basis of the data contained in Chapter XIV, at two-thirds of a pound of digestible matter per day, equal to about eight-tenths of a pound of maize. The feed cost of the exercise ordinarily taken by cows turned out in the yard must be insignificant and be far outweighed by the tonic effects of fresh air, sunshine and freedom on their health and general condition, while in the case of heavily fed cows some exercise may possibly be of advantage in diminishing the tendency to fatten. The question of turning out dairy cows for exercise, then, virtually reduces itself to the question whether the cost of the labor involved is repaid by the effect upon the health of the animals.

\section{Temperature. Shelter}

579. Air temperature. - The general principles regarding the relations between external temperature, heat production and feed supply, already discussed in Chapters VII (350-356), VIII (395-397) and XII (535-543), apply also to the dairy cow. Like the beef steer, the well-fed dairy cow in full flow of milk is consuming a large excess of feed above her maintenance ration and is producing a correspondingly large amount of heat.

For example, in an experiment reported by Jordan ${ }^{1}$ the computed heat production of two cows (disregarding slight changes in weight) and the estimated amount of heat which would have been produced on a maintenance ration were as follows:-

\section{Table i 27. - Estimated Heat Production of Cows}

Cow No. ro

Weight

Computed heat production . .

Estimated heat production on maintenance ration . . . .
$775 \mathrm{Lb}$.

I 8.67 Therms

I0. Io Therms
Cow No. 12 I $200 \mathrm{Lb}$.

21.10 Therms

I3.70 Therms

The heat production was greater in one case by 85 per cent and in the other by 54 per cent than the estimated amount on maintenance, which is a considerably greater excess than that computed (537) for Kellner's fattening steers.

${ }^{1}$ The Feeding of Animals, The Macmillan Co., New York, I908, p. 3 Io. 
So far as mere maintenance of body temperature goes, then, no reason appears why a cow might not be subjected to comparatively low temperatures without causing any increased katabolism for the sake of heat production solely. That the same factors of size and weight, humidity of air, and the amount and character of ration as in the case of the steer enter into the question is obvious.

580. Shelter, etc. - The question of shelter does not differ in principle with the cow and with the steer. The influence of precipitation, wind, insolation and temperature of drinking water are the same qualitatively on the cow as on the steer and the same reasons which render shelter desirable in the one caze apply in the other.

581. Modifying factors. - The foregoing facts, however, are scarcely sufficient to justify the conclusion that a dairy cow may be treated in this respect like a beef steer. In making a quantitative application of these facts in practice, certain modifying factors require consideration.

Relative body surface. - Even the most casual comparison of the dairy cow with the beef steer is sufficient to show that they differ materially in form and to raise the supposition that the ratio of body surface to weight may vary considerably in the two types. The writer is not aware of any measurements of body surface of cows but one can hardly avoid the impression that the spare angular form of the typical dairy cow exposes relatively more surface than the compact, rounded form of the beef animal.

Condition. - Outdoor winter feeding of cattle is practiced largely with fattening animals and it is with them that most experiments have been conducted. With such an animal a considerable covering of fat is usually acquired before the onset of extreme cold weather, while the typical dairy cow devotes her feed to milk production and carries very little body fat. There are no definite data as to the protective value of a fat covering but doubtless it is a poor conductor of heat and it would seem that it might have considerable influence in reducing radiation.

Skin and hair. - The skin of the dairy cow is reputed to be thinner than that of the steer and may therefore be a better radiator of heat. The coat of hair of the cow, too, is apt to be shorter and lighter than that of the steer, whether as a result 
of breeding or of continuous shelter and warm quarters, and is to that extent a poorer protection against loss of heat.

For all these reasons, it is clear that the loss of heat from the dairy cow may well be more rapid than that from the steer of like weight under the same external conditions and that consequently the minimum limit of external temperature below which additional katabolism is caused may be higher for the former than for the latter.

582. The direction of production. - Another important consideration in connection with the question of temperature and shelter for dairy cows is that of their possible influence upon the direction of production. Stress was laid at the outset of this discussion of the factors of milk production (558) upon the essential difference between beef production and milk production due to the fact that in the latter it is simply the secretion of a single gland and not a general increase of the whole body which is desired. The activity of the milk gland, however, is much more sensitive to external influences than, for example, that of adipose tissue. It is quite conceivable, therefore, that a degree of cold or exposure which, from the standpoint of heat production merely, might not require any additional katabolism to maintain the body temperature, might nevertheless check the formation of milk, especially if the cow were subjected to it suddenly. In such a case it would be anticipated, either that feed previously used for milk production would be stored up as body fat or else, if the cow continued to eat the same amount, would lead to a stimulation of the general body katabolism and so to an unnecessary increase in heat production.

In other words, exposure to cold might conceivably neither increase the feed consumption nor diminish the total utilization of surplus feed but might, nevertheless, be a disadvantage because it diverted the current of productive activities from the formation of milk to other and undesired forms of production.

583. Results in practice. - Only meager experimental evidence is available regarding the practicable or desirable limits of temperature for dairy cows.

Plumb ${ }^{1}$ compared the feed consumption and milk yield of two lots of purchased cows, one of which was turned out into the yard about one hour per day on sunny days while the other was turned 1 Ind. Expt. Sta., Bul. 47 (1893), pp. 89-96. 
out for eight hours every day without regard to the weather but with some shelter from the wind. The cows consumed feed ad libitum. The exposed lot ate much more grain but somewhat less hay than the sheltered lot and produced r6r.I pounds more milk. No proof of the comparability of the two lots is given.

Brooks ${ }^{1}$ exchanged two lots of cows between an artificially heated stable kept at $55^{\circ} \mathrm{F}$. and a cooler, unheated one, the temperature of which is not reported. Rather more milk was produced in the warm stable but its percentage of fat was lower.

Richards and Jordan ${ }^{2}$ recorded the milk yield of a number of cows upon uniform feed in alternate periods in which the stable temperature was maintained, respectively, at about $45^{\circ}$ and $55^{\circ} \mathrm{F}$. More milk was produced in three cases out of four and more butter fat in two cases out of four at the higher temperature.

Spier ${ }^{3}$ reports experiments at four farms, on a total of 88 animals upon the relation of stable temperature and ventilation to milk yield. He calls attention to the fact that both these factors are involved in experiments upon the influence of shelter. The following table shows the results obtained in two specially cold periods as compared with the average of warmer preceding and following periods and likewise the average results for the entire experiments. The average rations consumed are stated but there is no record of actual feed consumed during the several periods nor of the live weights of the animals.

Table i28. - Influence of Ventilation and Stable Temperature on Milk Production

\begin{tabular}{|c|c|c|c|c|c|c|}
\hline & \multicolumn{3}{|c|}{ FREE VENTILATION } & \multicolumn{3}{|c|}{ RESTRICTED VENTILATION } \\
\hline & $\begin{array}{l}\text { Milk per } \\
\text { Day and } \\
\text { Head }\end{array}$ & $\begin{array}{l}\text { Fat in } \\
\text { Milk }\end{array}$ & $\begin{array}{l}\text { Stable } \\
\text { Temper- } \\
\text { ature }\end{array}$ & $\begin{array}{l}\text { Milk per } \\
\text { Day and } \\
\text { Head }\end{array}$ & $\begin{array}{l}\text { Fat in } \\
\text { Milk }\end{array}$ & $\begin{array}{l}\text { Stable } \\
\text { Temper- } \\
\text { ature }\end{array}$ \\
\hline Dec. $20-J a n .4$ & Lb. & $\%$ & ${ }^{\circ} \mathrm{F}$. & Lb.: & $\%$ & ${ }^{\circ} \mathrm{F}$. \\
\hline Warm periods . & 29.0 & $3 \cdot 55$ & $53 \cdot 76$ & 28.9 & $3 \cdot 48$ & 61.73 \\
\hline $\begin{array}{l}\text { Cold period . } \\
\quad \text { Feb. I4-Mar. } 27\end{array}$ & 29.0 & $3 \cdot 5 \mathrm{I}$ & 41.20 & 29.0 & 3.53 & 52.30 \\
\hline Warm periods . & $25 \cdot 3$ & 3.63 & 50.31 & 25.4 & $3 \cdot 48$ & 6o.II \\
\hline $\begin{array}{l}\text { Cold period } \\
\text { Entire Experiments }\end{array}$ & 25.4 & 3.69 & 46.07 & $25 \cdot 5$ & $3 \cdot 5^{\mathrm{I}}$ & 56.67 \\
\hline Nov. 22-Mar. 27 . & $27 \cdot 5$ & $3 \cdot 55$ & 49.82 & $27 \cdot 3$ & 3.49 & $59 \cdot 40$ \\
\hline
\end{tabular}

1 Mass. (Hatch) Expt. Sta., Rpt. I895, p. 39.

${ }^{2}$ Wis. Expt. Sta., 2 Ist Rpt., I903-I904, p. I 43.

3 Jour. of Highland and Agr. Soc., r 909, pp. 255-306. 
The foregoing results show no perceptible effects from the temperature fluctuations within the range of these experiments either on the lots kept in the cooler stables from the start nor on those in warmer quarters when the temperature of the latter fell.

Davis ${ }^{1}$ reports experiments at the Pennsylvania Station, covering three seasons, in which comparable lots of cows were kept in an open shed and in an ordinary "bank" barn. It was found that the milk yield of both was similarly affected by sudden drops of temperature but that the milk yield of the exposed group decreased more rapidly during the winter than did that of the sheltered group, the difference in the average daily yield for the entire season varying from practically nothing in I9I I-I9I 2 to about three pounds in I9I3-I9I4. It was observed that the exposed cows had the keener appetites and consumed more roughage than did the sheltered animals. Both groups maintained good health. The amounts of milk produced per Therm of estimated net energy contained in the feed and also per Therm of net energy in excess of the estimated maintenance requirement were as shown in the following table from which it appears that the production by the sheltered lot was slightly the more economical.

\section{Table I 29. - Milk Yields of Sheltered and Exposed Cows}

\begin{tabular}{|c|c|c|c|c|c|c|c|c|c|c|c|c|c|c|c|}
\hline & & & & & & & & & & & & & & $\begin{array}{c}\text { PER Therm } \\
\text { Net ENERGy } \\
\text { OF Total } \\
\text { FEEd }\end{array}$ & $\begin{array}{c}\text { PER ThERM } \\
\text { Net ENERGy } \\
\text { IN EXCESS } \\
\text { OF MAINTE- } \\
\text { NANCE }\end{array}$ \\
\hline \multicolumn{14}{|c|}{ Exposed lot } & Lb. & $\mathrm{Lb}$. \\
\hline I9I I-I 2 & . & - & • & . & - & - & . & • & . & . & - & . & . & I. 266 & 2.244 \\
\hline I9I $2-I 3$ & . & . & . & . & . & • & - & • & - & . & . & . & $\cdot$ & I. 683 & 2.570 \\
\hline I9I3-I 4 & . & . & . & . & - & . & 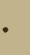 & • & . & - & . & . & $\cdot$ & I. $45^{8}$ & 2.515 \\
\hline Avera & age & . & - & . & . & & & • & • & . & . & . & . & 1.469 & 2.443 \\
\hline I9I I-I 2 & . & . & - & . & - & • & • & • & - & . & . & . & . & I. 354 & 2.499 \\
\hline I9I 2-I 3 & . & . & - & . & - & • & • & • & . & . & . & . & . & I. 737 & 2.825 \\
\hline I9I $3^{-I} 4$ & . & . & - & - & . & - & - & • & . & . & . & . & $\cdot$ & 1.402 & 2.639 \\
\hline Avera & & . & - & . & • & • & & $\cdot$ & • & . & . & . & . & $\overline{1.498}$ & $\overline{2.654}$ \\
\hline
\end{tabular}

A number of instances have also been reported in which the substitution of a single thickness of muslin in cow stables in place of glass windows has proved satisfactory.

${ }^{1}$ Penna. Expt. Sta., Rpt. I9I3-I9I4, pp. I83-226. 
On the whole it may be said that such experiments as are on record agree with the deductions from physiological data and indicate that the need for warm quarters for dairy cows has been overemphasized, but are insufficient to establish the limits within which stable temperature does not affect yield. Much doubtless depends, as Spier points out, upon the previous treatment of the cows. Warmly stabled animals carry a summer rather than a winter coat and a low temperature seems likely to have more effect on such animals than on those gradually accustomed to it as the weather grows colder.

Where cows are kept in the stable most of the time the question of temperature is of special interest in its relation to ventilation. Practically, a cow stable must be warmed in most cases simply by the heat derived from the animals themselves and a high temperature can be obtained only by means of more or less restricted ventilation. If low temperatures can be used, more perfect ventilation, with its beneficial effects upon the health and vigor of the animals, is possible.

\section{§4. The Utilization of Feed in Milk Production}

\section{The utilization of protein}

584. Meaning of utilization. - The conception of the utilization of protein in milk production as here considered is substantially identical with that of its utilization in growth already discussed (470). It is the ratio of the protein contained in the milk to the least amount of feed protein which is required to produce it under the most favorable conditions.

585. Surplus protein katabolized. - While it is evident that milk production requires a liberal supply of protein in the ration, the amount actually secreted in the milk is determined primarily by the individuality of the animal, precisely as is the storage of protein in the case of the growing animal. It is not possible to increase at will the amount of protein secreted in the form of milk by increasing the supply of protein in the feed. While it appears to be true that the activity of the milk glands can be stimulated somewhat by an abundant protein supply (599), it is nevertheless true that the animal produces an amount of milk determined essentially by its capacity and any surplus of protein over that necessary for this purpose is katabolized 
just as is the case with a surplus supplied to a young animal, or for that matter to a mature animal. Feed protein is substantially a supply of material and not a cause of production.

This is strikingly illustrated in experiments by Jordan ${ }^{1}$ in which the protein supply of two cows, beginning with a liberal amount, was gradually diminished to about one-half and then gradually increased again to the original quantity. The following table shows the average nitrogen balances of Cow No. I 2 of the second series of experiments, the daily results being grouped into periods as indicated.

\section{Table i3o. - Average Daily Nitrogen Balance of Cows}

\begin{tabular}{|c|c|c|c|c|c|c|c|}
\hline & & & $\begin{array}{c}\text { No. of } \\
\text { Days }\end{array}$ & $\begin{array}{l}\text { NItROGEN } \\
\text { DigEsted }\end{array}$ & $\begin{array}{l}\text { NITROGEN } \\
\text { OF MILK }\end{array}$ & $\begin{array}{l}\text { NITROGEN } \\
\text { OF URINE }\end{array}$ & $\begin{array}{c}\text { GAIN BY } \\
\text { BODY }\end{array}$ \\
\hline Tan. $30-$ Feb. 6 & . & & 7 & $\begin{array}{l}\text { Grams } \\
\text { I } 86.6\end{array}$ & $\begin{array}{c}\text { Grams } \\
8 \mathrm{I} .7\end{array}$ & $\begin{array}{r}\text { Grams } \\
87.0\end{array}$ & $\begin{array}{l}\text { Grams } \\
+\quad \text { I } 7.9\end{array}$ \\
\hline Feb. 6-Feb. I6 & & 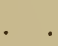 & IO & I 85.2 & $8 \mathrm{I} \cdot 4$ & 87.5 & $\begin{array}{r}+16.3 \\
+16\end{array}$ \\
\hline Feb. I6-Feb. 26 & . & . & IO & I6I.6 & $77 \cdot 5$ & $8 I .9$ & +2.2 \\
\hline Feb. 26-Mar. 8 & • & . & IO & I30.8 & 74.0 & $5^{6.5}$ & +0.3 \\
\hline Mar. 8-Mar. I8 & & . & IO & I I 7.2 & 66.6 & $43 \cdot 7$ & +6.9 \\
\hline Mar. I8-Mar. 28 & & . & I0 & I 43.6 & 69.6 & $6 \mathrm{I} .8$ & + I 2.2 \\
\hline Mar. 28-Apr. 7 & • & . & IO & I 7 I.4 & 71.6 & 89.2 & +10.6 \\
\hline Apr. 7 -Apr. 14 & . & . & 7 & 185.7 & 71.9 & 104.4 & +9.4 \\
\hline
\end{tabular}

The yields decreased in quite a normal way with the advance in lactation, the yield of protein, like that of total milk solids, diminishing, while the percentage of protein in the latter remained about the same. On the low protein rations of the middle periods there seems to have been some falling off in the amount of milk protein produced in comparison with what might have been expected on an unchanged ration, but the difference is small except in one or two periods in which the protein supply reached the lowest limit. Aside from this, the principal effect of the variations in the amount of digestible protein supplied was to increase or diminish the amount of nitrogen excreted in the urine, which, as the table clearly shows, rose and fell with the supply of nitrogen in the food.

586. Estimates of utilization of protein. - In attempting to reach conclusions regarding the utilization of feed protein for the production of milk protein, then, it is evidently necessary to avoid an excess of protein in the ration, since such an excess

${ }^{1}$ N. Y. (Geneva) Expt. Sta., Buls. I32 (I897) and I97 (I901). 
is subject to rapid katabolism, so that high protein rations will necessarily show a low apparent utilization of the protein for milk just as they do for growth (468). On the other hand, too small a supply of protein may cause the tissue proteins of the body to be mobilized and utilized as a source of milk protein so that a direct comparison of feed protein and milk protein would give too high a result. To determine the utilization of feed protein, therefore, it is necessary, while maintaining a sufficient energy supply, to reduce the protein content of the ration as nearly as possible to that which is just sufficient to prevent a loss of body protein and then to compare the feed protein minus the maintenance requirement with the milk protein.

Such an experiment obviously requires a determination of the nitrogen balance of the animal, and relatively few of the reported investigations on milk production include such a determination, while in none yet reported has the sufficiency of the energy supply been demonstrated by means of respiration experiments. There are, however, a not inconsiderable number of experiments on record in which the live weights of the animals have been well maintained and in which amounts of digestible protein but little greater than those found in the milk plus those estimated to be necessary for maintenance have been adequate for the production of at least moderate amounts of milk without drawing on the body protein.

Naturally an exact balance of the income and outgo of nitrogen will rarely be secured. In most cases it is necessary to compare the feed protein with the algebraic sum of the milk protein and the gain or loss of body protein, the comparison being more nearly correct as the latter factor becomes smaller.

Table I3I shows the computed utilization of the protein of a number of low protein rations, the daily maintenance requirement of crude protein being estimated as 0.6 pound per I000 pounds live weight in direct proportion to the latter. It includes the experiments by Jordan upon the sources of milk fat, the results of one of which as regards protein have just been cited, an experiment by Hayward ${ }^{1}$ the results of which as regards the nitrogen balance are still unpublished, the extensive experiments upon the minimum protein requirements

1 Penna. Expt. Sta., Rpt. I90I-I902, pp. 314 to 396. 
Table i3i. - Utilization of Protein in Milk Production

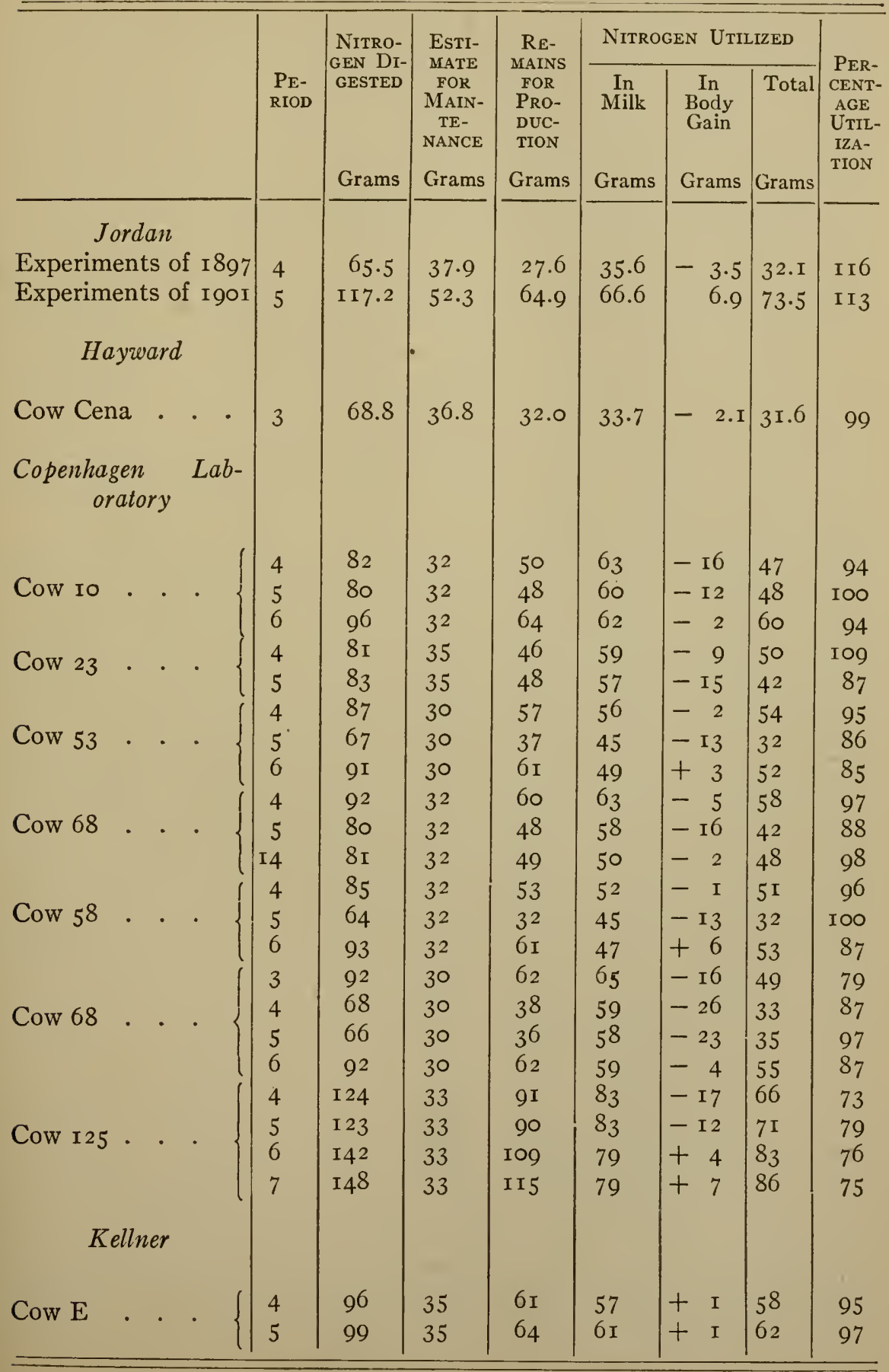


of dairy cows carried on at the Laboratory for Agricultural Research in Copenhagen ${ }^{1}$ and unpublished respiration experiments by Kellner. ${ }^{2}$ The experiments of Hart and Humphrey mentioned in the next paragraph, when computed in the same way, also show a high percentage utilization of the digested protein, although the gains and losses of body protein are relatively so considerable as to disturb the comparison. Haecker's low protein rations in $1902-3-4-5$, as noted on a subsequent page (602), seem to afford another example of the high utilization of feed protein.

While too much weight should not be attached to the results of comparisons like the foregoing, especially since they include a more or less uncertain estimate of the protein requirement for maintenance, they nevertheless seem to indicate beyond reasonable doubt that on low protein rations the protein of at least some feeding stuffs may be converted into milk protein without any very large loss.

587. Relative values of proteins for milk production. - The considerations advanced in preceding chapters $(400,465)$ regarding the relative values of different proteins for maintenance and for production render it altogether probable that they also differ in value as sources of milk protein. No experiments on this point have as yet been reported, but Hart and Humphrey ${ }^{3}$ in two series of experiments on cows have compared the mixed proteins of maize, wheat, gluten feed, oil meal and distillers' grains with proteins prepared from milk (784), using maize stover and silage as roughage. They found the average percentage of the resorbed nitrogen which was recovered in the milk yield plus the gain (or minus the loss) of the body protein to be

\begin{tabular}{|c|c|c|c|c|c|c|}
\hline lk powde & & & & & & \\
\hline Casein & & & . & & & \\
\hline Ia & & & & & & \\
\hline Vh & & & & & & \\
\hline feed & & & & & & \\
\hline il & & & & & & \\
\hline istillers' grains & & & & & & \\
\hline
\end{tabular}

1 Denmark-Beretning fra den Kgl. Veterinear of Landbohojskoles Laboratorium for landokonomiske Forsog. 60de, 1906, and 63de, 1907, Kobenhavn. Translated by Mallèvre, Société de l'Alimentation Rationale du Bétail. 'Compte Rendu de I I ème et I zème Congrés.

2 Die Ernährung der landw. Nutztiere, 6th Ed., I9I2, p. 55 I.

3 Jour. Biol. Chem., 21 (I9I 5), 239; 26 (I9I6), 457. 
If the probable requirement for protein maintenance be deducted from the total resorbed nitrogen, the utilization of the remaining protein, calculated as in the experiments of the previous paragraph, was notably higher, approaching or reaching too per cent in several instances.

The differences observed were largely due, however, to fluctuations in the gain or loss of body protein, the formation of milk protein being quite uniform from period to period, and this fact seems to render the results of somewhat questionable relevance as regards the special question of comparative values as sources of milk protein, although they do show marked differences in total efficiency.

\section{The utilization of energy}

588. Net energy values for milk production. - The net energy value of a feeding stuff or ration for milk production is identical in conception with that for fattening (448) or for growth (472) already considered. It is that part of the feed energy supplied in excess of the maintenance requirement which is recovered in the product. For example, if a cow produces per day $20 \mathrm{lb}$. of four per cent milk, "containing (604) 336 Cals. of energy per pound, the total of 6720 Cals. would be the net energy value which must be supplied in the ration in addition to that required for maintenance.

As pointed out in Chapter VIII (371), it cannot be assumed that the net energy values for maintenance, fattening or growth apply to milk production, but the values for the latter purpose must be determined by direct experiment. As yet, very scanty data are available on this point, the only results yet reported being three contained in a brief preliminary paper by Kellner.

589. Complete energy balances. - Kellner ${ }^{1}$ reports the nitrogen, carbon and energy balances, determined as in his experiments on oxen, of three cows receiving mixed rations and varying considerably in their milk yield. By the method described in Chapter XVII (768-772), it is estimated that the net energy values of the rations and the percentage utilization of their metabolizable energy for fattening would be : -

${ }^{1} 5^{\text {ter }}$ Internat. Kongress für Milchwirtschaft, I 9 I I. 


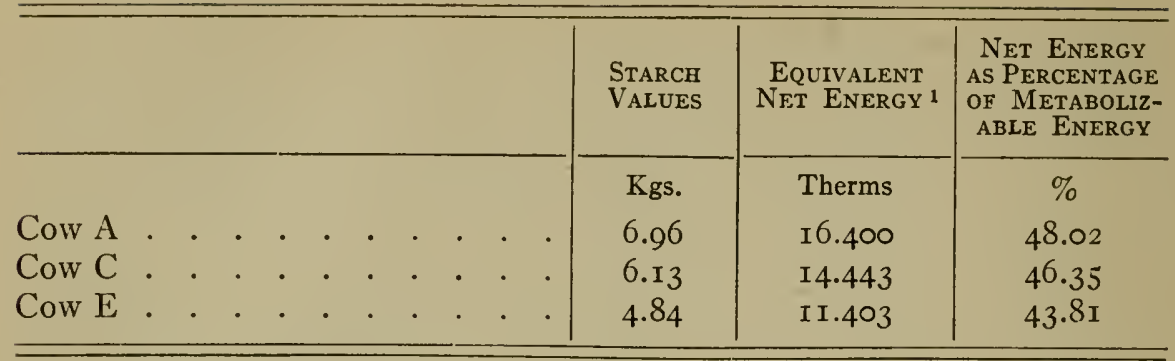

Estimating the maintenance requirements of the animals from their live weights on the basis of his average results on the maintenance of oxen (381), Kellner obtains the following energy balances showing a considerably higher utilization for milk production than that computed for fattening.

Table 132. - Energy Balances of Dairy Cows

\begin{tabular}{|c|c|c|c|}
\hline & Cow A & Cow $\mathrm{C}$ & Cow $\mathrm{E}$ \\
\hline Income & Therms & Therms & Therms \\
\hline In feed $\quad$. & 63.309 & 59.096 & 46.536 \\
\hline Outgo & & & \\
\hline $\begin{array}{l}\text { In feces and urine } \\
\text { In methane }\end{array}$ & $\begin{array}{r}25 \cdot 353 \\
3.803\end{array}$ & $\begin{array}{r}23.783 \\
4.149\end{array}$ & $\begin{array}{r}\text { I6.996 } \\
3.508\end{array}$ \\
\hline Total . . . & 29.156 & $27.93^{2}$ & 20.504 \\
\hline Metabolizable & & & \\
\hline $\begin{array}{l}\text { Total } \cdot \dot{\cdot} \text { Estimated mainte- }\end{array}$ & $34 \cdot 153$ & 31.164 & 26.032 \\
\hline nance $\cdot \cdot$ & IO. II 4 & II.303 & I0.586 \\
\hline $\begin{array}{r}\text { Available for pro- } \\
\text { duction } .\end{array}$ & 24.039 & I9.86I & I 5.446 \\
\hline Production & & & \\
\hline $\begin{array}{l}\text { Milk } \\
\text { Body fat } \\
\text { protein }\end{array}$ & $\begin{array}{r}13.907 \\
1.782 \\
\end{array}$ & $\begin{array}{r}10.617 \\
2.447 \\
\end{array}$ & $\begin{array}{l}8.919 \\
0.928 \\
\end{array}$ \\
\hline Total . . . . & $\overline{1_{5} .689}$ & $\overline{13.064}$ & 9.847 \\
\hline $\begin{array}{l}\text { Utilization of metab- } \\
\text { olizable energy } \\
\text { Computed utilization } \\
\text { for fattening }\end{array}$ & $\begin{array}{r}\% \\
65 \cdot 3 \\
48.0\end{array}$ & $\begin{array}{r}\% \\
65.8 \\
46.4\end{array}$ & $\begin{array}{r}\% \\
63.8 \\
43.8\end{array}$ \\
\hline
\end{tabular}

${ }^{1}$ I Kilogram starch value $=2.356$ Therms net energy. 
The percentage utilization in milk production alone may also be approximately estimated from Kellner's figures by subtracting from the metabolizable energy available for production the amounts estimated to be required for the production of the observed gain of body tissue. The results of this calculation are shown in the following table. A similar computation by Kellner based on his estimated starch values gives substantially the same results.

Table i33. - Utilization of Metabolizable Energy in Milk ProDUCTION

\begin{tabular}{|c|c|c|c|}
\hline & Cow A & Cow $\mathrm{C}$ & Cow $\mathbf{E}$ \\
\hline $\begin{array}{l}\text { Metabolizable energy } \\
\text { Available for total } \\
\text { production }\end{array}$ & 24.039 The & I0.86I Therms & \\
\hline Required for body gain & $\underline{3.7 \mathrm{II}}$ & $\underline{5.279} \quad$, & $\begin{array}{r}2.118 \\
\end{array}$ \\
\hline $\begin{array}{l}\text { Available for milk } \\
\text { production }\end{array}$ & 20.328 & I 4.582 & 13.328 \\
\hline $\begin{array}{l}\text { Recovered in milk } \\
\text { Utilization }\end{array}$ & $\begin{array}{r}\text { I3.907 “ } \\
68.4 \mathrm{I} \%\end{array}$ & $\begin{array}{l}10.617 \text { " } \\
72.80 \%\end{array}$ & $\begin{array}{l}8.919 " 6 \\
66.91 \%\end{array}$ \\
\hline
\end{tabular}

590. Partial energy balances. - Partial energy balances of two cows which made but slight gains in live weight are reported by Jordan, ${ }^{1}$ the maintenance requirement being estimated from the live weight and the excretion of methane computed from the digestible carbohydrates. Assuming that there was no gain or loss of fat or protein by the body, the following comparisons can be made:

Table i34. - Utilization of Metabolizable Energy in Milk ProDUCTION

\begin{tabular}{|c|c|c|c|c|}
\hline & \multirow{2}{*}{ Cow ro } & \multicolumn{3}{|c|}{ Cow 12} \\
\hline & & Period I & Period 2 & Period 3 \\
\hline $\begin{array}{l}\text { Metabolizable energy . } \\
\text { Estimated maintenance }\end{array}$ & $\begin{array}{l}27.320 \text { Therms } \\
\text { I0.152 “ }\end{array}$ & $\begin{array}{l}\text { 32.I I } 8 \text { Therms } \\
\text { I3.846 "“ }\end{array}$ & $\begin{array}{l}31.718 \text { Therms } \\
\text { I3.846 " }\end{array}$ & $\begin{array}{l}\text { 30.335 Therms } \\
\text { 13.846 " }\end{array}$ \\
\hline $\begin{array}{l}\text { Energy in milk : : } \\
\text { Utilization } .\end{array}$ & 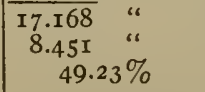 & $\begin{array}{l}18.272 \\
\text { II.176 “ } \\
61.16 \%\end{array}$ & 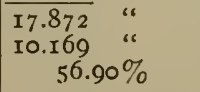 & $\begin{array}{r}6.489 \\
10.547 \\
63.96 \%\end{array}$ \\
\hline
\end{tabular}

${ }^{1}$ N. Y. (Geneva) Expt. Sta., Bul. I97, pp. $24-32$ and 2oth Rpt. (I90I), p. 29. 
Eckles ${ }^{1}$ has likewise determined partial energy balances of ten milking cows for an entire year on rations just sufficient to maintain their live weight. In these experiments the percentage digestibility of the rations is computed for eight of the cows on the basis of results obtained in digestion trials on five of the animals, while the maintenance requirement of all but one of the cows was determined in live weight experiments after the cows were dried off, with the results reported in Chapter VIII (381).

Estimating the metabolizable energy of the rations at 3.7 Therms per kilogram of digestible organic matter (753) and computing the results, exactly as in Jordan's experiments, on the assumption of no gain or loss by the body, the following values for the percentage utilization in milk production are obtained.

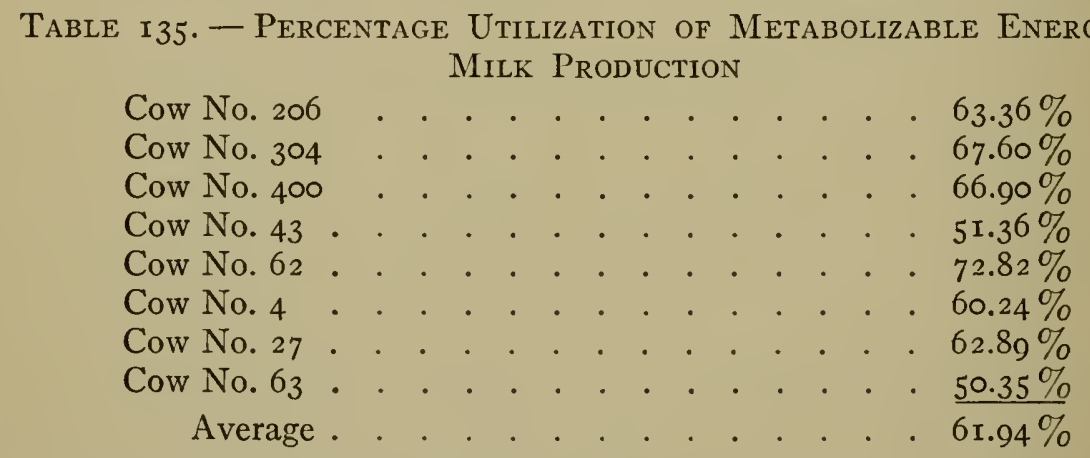

Haecker, ${ }^{2}$ in discussing the results of extensive experiments with the dairy herd of the Minnesota Experiment Station, has compared the digestible nutrients of the feed and the solids of the milk by reducing both to their carbohydrate equivalent. ${ }^{3}$ Subtracting the estimated maintenance requirement from the total carbohydrate equivalent (" nutriment") of the feed, he finds that of the remainder from 50.25 per cent to 66.22 per cent was recovered in the milk, the general average for nine years being 54.65 per cent, while the live weights of the cows were in general maintained. This seems to indicate a decidedly lower utilization of energy than that computed in Kellner's, Jordan's and Eckles' experiments. It must be noted, however,

${ }^{1}$ Mo. Expt. Sta., Research Bul. 7.

${ }^{2}$ Minn. Expt. Sta., Bul. I 40 (I9I4), p. 45.

3 The fat of the feed is multiplied by the factor 2.2 and that of the milk by 2.25 and the product added to the carbohydrates and protein. The sums, which are called " nutriment," are, of course, approximately proportional to the energy content of the milk and the metabolizable energy of the feed respectively. 
that the digestibility of the rations in Haecker's experiments was estimated from average figures which, according to Eckles' results (722), are probably too high for cows in milk, although on the other hand Haecker's estimate for the maintenance requirement also seems high.

591. Net energy values for milk probably greater than for fattening. - A comparison of Kellner's results (589) with those obtained by the same author ${ }^{1}$ and by Armsby and Fries ${ }^{2}$ for the utilization of metabolizable energy in either maintenance, growth or fattening seems to indicate clearly that the net energy values for milk production are distinctly higher than those for the latter purposes, although no direct comparisons on the same feeding stuff or ration can be made:

Both Jordan's and Eckles' results tend to confirm this conclusion, which is further strengthened by the fact, to which Eckles calls attention, that with one exception the actual energy content of the milk in his experiments was greater than the net energy value available in the ration producing it as computed by the use of Kellner's factors.

Unfortunately, no results upon the net energy values of single feeding stuffs or nutrients for milk production have yet been reported, so that it is impossible at present to make any exact quantitative comparisons.

592. Cause of higher net energy values for milk production. - The apparently higher net energy values for milk production as compared with tissue production may be plausibly ascribed to the difference in the composition of the products. As shown in Chapters $\mathrm{X}$ and XI, the organic matter of the increase in fattening consists chiefly of fat (441-443) and even in the case of growth fat makes up a considerabie proportion of it (458) except in extreme youth. In average milk, on the comtrary, protein and milk sugar constitute two-thirds of the total organic matter and carry over one-half of the total energy.

It seems not improbable that the conversion of digestible protein into milk protein, or of digestible carbohydrates into milk sugar, may involve a comparatively small expenditure of energy as compared with the synthesis of fat from carbohydrates or protein. If such be the case, the organic matter

1 Landw. Vers. Sta., 53 (I900), I.

${ }^{2}$ Jour. Agr. Research, 3 (I9I5), $435 ; 7$ (I9I6), 379. 
of the milk would retain a larger percentage of the chemical energy of the digestible matter from which it was formed than would the increase of body tissue which that same digestible matter could produce.

593. Computation of equivalent net energy values for fattening. - Let it be assumed that the digestible protein and carbohydrates of the feed may be converted into the corresponding compounds of milk without loss and that the expenditure of energy in the production of milk fat from carbohydrates is the same as that observed by Kellner (769) for the production of body fat. Then each gram of protein or carbohydrates in the milk would require the supply in the feed of one gram of digestible protein or carbohydrates respectively, while each gram of milk fat if manufactured from carbohydrates would require about 3.9 grams of the latter.

The corresponding amounts of energy recovered in milk production and in fattening respectively would, according to the foregoing assumptions, be as follows:-

Table 136. - Computed Energy Recovered in Milk Production and in Fattening

\begin{tabular}{|c|c|c|c|}
\hline Supplied in Feed & Produced in MilK & $\begin{array}{c}\text { ENERGY } \\
\text { RECOVERED } \\
\text { IN MILK }\end{array}$ & $\begin{array}{c}\text { ENERGY } \\
\text { RECOVERED } \\
\text { IN FATTEN- } \\
\text { ING }^{1}\end{array}$ \\
\hline I gram protein & I gram protein & 5.7 Cals. & 2.24 Cals. \\
\hline I gram carbohydrates & I gram carbohydrates & 4.I Cals. & 2.37 Cals. \\
\hline 3.9 grams carbohydrates & I gram fat & 9.23 Cals. & 9.23 Cals. \\
\hline
\end{tabular}

On this basis, it is easy to compute approximately the amount of net energy for fattening which would be required for the production of a given amount of milk of known composition. Thus average four per cent milk, according to Table I44 (604), contains 3.08 per cent of protein, 4.85 per cent of carbohydrates and 4.0 per cent of fat. The actual amount of energy contained in a pound of such milk would be 336 Cals., while the amount of energy which would have been recovered from the same feed if used for fattening would have been only 252 Cals. Conversely, an amount of feed containing $25^{2}$ Cals. of net energy as computed from the results of fattening experi-

${ }^{1}$ Kellner's factors. 
ments would suffice to support the storage of 336 Cals. of energy in four per cent milk. The method of computation is shown in the following table.

Table i37. - Energy Recovered in Four Per Cent Milk and in FATTENING

\begin{tabular}{|c|c|c|}
\hline & $\begin{array}{l}\text { ENERGY RECOVERED IN } \\
\text { MILK }\end{array}$ & $\begin{array}{l}\text { EQUIVALENT ENERGY RE- } \\
\text { COVERED IN FATTENING }\end{array}$ \\
\hline $\begin{array}{l}\text { Protein . . } \cdot \text {. } \\
\text { Carbohydrates . } \\
\text { Fat } \cdot \text {. } \cdot \text {. } \\
\text { Total per roo grams } \\
\text { Total per pound . }\end{array}$ & $\begin{aligned} 5.7 \times 3.08= & 17.5 \text { Cals. } \\
4.1 \times 4.85= & 19.8 \text { Cals. } \\
9.23 \times 4.00= & \frac{36.9}{74.2} \text { Cals. } \\
& 336 \text { Cals. }\end{aligned}$ & $\begin{aligned} 2.24 \times 3.08= & 6.9 \text { Cals. } \\
2.37 \times 4.85= & \text { I } 1.5 \text { Cals. } \\
9.23 \times 4.00= & \frac{36.9}{55.3} \text { Cals. } \\
& 252 \text { Cals. } \\
& \text { Cals. }\end{aligned}$ \\
\hline
\end{tabular}

594. Kellner's results. - Confirmation of this hypothesis is afforded by the results of Kellner's respiration experiments (589). In substantially the way just outlined, Kellner computes that while the actual chemical energy of the milk solids produced by his cow A was I3.907 Therms, this was equivalent to only 10.367 Therms of net energy value for fattening, and therefore, that a ration supplying this amount in excess of that required for maintenance and body gain should be sufficient to support the observed milk production. For the three cows for which results are reported, the requirements for net energy as thus computed compared with the estimated net energy values of the rations were as follows:-

Table 138. - Net Energy Values for Fattening in Kellner's ExPERIMENTS

\begin{tabular}{|c|c|c|c|}
\hline & Cow A & Cow $\mathrm{C}$ & Cow $\mathrm{E}$ \\
\hline & Therms & Therms & Therms \\
\hline Total in ration & 16.400 & 14.443 & II. 403 \\
\hline Required for maintenance & 4.594 & $\underline{5.137}$ & 4.806 \\
\hline & I I. 806 & $9 \cdot 306$ & 6.597 \\
\hline Required for body gain & I. 782 & 2.447 & .928 \\
\hline Available for milk production $\cdot \cdot \cdot \cdot$ & 10.024 & 6.859 & 5.669 \\
\hline $\begin{array}{l}\text { Computed requirement for milk produc- } \\
\text { tion. } . . \\
\end{array}$ & 10.367 & 6.975 & 6.079 \\
\hline
\end{tabular}


The amounts of net energy actually available for milk production correspond quite closely with the amounts computed to be required according to the foregoing assumptions, and Kellner states that this was also the case in a considerable number of his unpublished experiments, although in others, especially those in which a surplus of feed was given, the agreement was far from being so good, the difference in one case reaching 24 per cent.

Quite in harmony with the general conclusions of the foregoing paragraphs is the statement by Eckles ${ }^{1}$ that in his experiments "A therm of "energy in the feed produced more energy in milk when the per cent of fat was low than when it was high. Apparently a given amount of feed is more efficient when used to produce milk medium to low in fat. It appears from this that the production of fat is a greater tax upon the animal than is the production of other constituents of the milk carrying equal energy value."

\section{§5. Feeding for Milk Production}

595. Feeding a secondary factor. - As has already been urged, the feeding of a milking animal is in a certain sense a secondary factor in dairying. The possibilities of successful milk production depend primarily upon the capacity of the animals as milk producers and upon the maintenance of such an environment as will give free play to this capacity. Feed, on the other hand, while equally necessary, is after all essentially the supply of raw material upon which the animal mechanism works and cannot greatly stimulate production, though it may limit it for lack of material.

The same thing is substantially true, of course, of all forms of productive feeding, but it is especially the case in the feeding of dairy animals for the reason already noted (558), that it is the product of a single gland and not a general increase of body tissue which is desired. Improper rations, therefore, may in this case not only limit the total production but, even if sufficient in quantity, may if deficient in quality deflect production from milk to fattening, or possibly to greater muscular activity, and thus fail to utilize fully the milk-producing capacity of the 
animals. Feeding, therefore, while in a sense a secondary factor is nevertheless an important one.

596. Feed requirements. - Regarded solely as a source of material for the formation of milk, the daily ration must, of course, contain an adequate amount of protein and ash and a quantity of non-nitrogenous nutrients sufficient to furnish material for the manufacture of the non-nitrogenous ingredients of the milk, while it must also supply enough energy for the physiological activities of the body, including maintenance and the energy expended in the processes of milk formation.

In addition to this, however, there must be taken into consideration the possibility of the presence or absence in the feed of substances which may have a specific effect on the milk gland, either by stimulating or depressing its action as a whole or by affecting qualitatively the character of its action and so the composition of the milk. There is, of course, a possibility of such specific effects in other forms of production, but it is most obvious in milk production for evident reasons and has been most studied in that connection.

\section{Protein requirements for milk production}

597. Milk rich in protein. - The physiological purpose of milk production is, of course, to support the growth of the young.

The essential feature of growth, however (462), is the production of new protein tissue, which, in the suckling animal, is relatively rapid, and in order to support this growth the milk must contain protein in amount more or less proportional to the rate of growth of the species. Cow's milk is decidedly protein in character, the ratio of protein to non-nitrogenous ingredients corresponding roughly with that of the increase made by an animal three months old (556). Moreover, in the case of the cow, man has been able to increase greatly the natural milk-producing capacity, with, of course, a corresponding increase in the total amount of milk protein formed. Even the moderate daily yield of 20 pounds of milk of average composition contains over 0.6 pound of protein, while the extraordinary yields of champion cows contain several times this amount.

598. Minimum protein requirement. - Just as in the case of growth, it is evident that the least amount of digestible protein 
which can possibly meet the requirements of the milk-producing animal is the quantity required for the maintenance of the body protein plus the actual amount of protein contained in the milk yielded. For example, if a rooo-pound cow is to produce daily 25 pounds of milk containing 3.2 per cent of total protein, it is evident that her ration must contain in digestible form at least the 0.8 pound of protein contained in the milk plus the approximate 0.6 pound presumably required for body maintenance, or a total of approximately 1.4 pounds. A less supply than this must evidently result either in a falling off in the milk yield or in a conversion of body protein into milk protein.

How much more than this minimum amount must be supplied by an adequate ration will depend upon the percentage utilization of the feed protein in the sense already discussed in $\S 4$ of this chapter (584-586), i.e., upon the proportion of it capable of conversion into milk protein. Thus in the illustration just employed, if 80 per cent of the surplus feed protein can be utilized the protein requirement would be 1.0 pound for milk production plus 0.6 pound for maintenance, or 1.6 pound instead of I.4 pound. The case is parallel with that of the protein requirement for growth discussed in Chapter XI (484491) and in both instances the experimental data available are insufficient for a final conclusion, although the probabilities appear to indicate the possibility of a high percentage utilization under favorable conditions.

599. Protein as a stimulus to the milk glands. - The foregoing considerations do not, however, exhaust the subject. In them it has been tacitly assumed that the amount of milk protein manufactured by the milk glands is substantially fixed. It seems well established, however, that in addition to furnishing material for the manufacture of milk protein, the nitrogenous matter of the feed may act to some extent as a stimulus to the glands, causing a more active secretion not only of protein but of all the milk solids. In other words, it would appear that a greater or less surplus of protein over the amount indicated by calculations like the foregoing is necessary if it is desired to take full advantage of the milk-producing capacity of the animal or to delay as much as possible the natural shrinkage in milk due to advancing lactation. 
That such is the case has long been taught, but many of the early experiments upon which this teaching was based are inconclusive in that they relate to the effect of adding proteinrich feeding stuffs to relatively light rations low in protein. ${ }^{1}$ The total digestible matter (energy supply) as well as the protein in the rations was thus increased, sometimes by a considerable amount, the quality of the protein sometimes improved, and the proportions of the ash ingredients more or less altered, while the possibility of the presence in the added feed of specific stimulating substances (617-621) must be reckoned with. It is illogical, therefore, to ascribe the beneficial effect entirely to the increase in digestible protein, although this was doubtless one of the factors.

Jordan's investigations. - Of more recent investigations in which these sources of uncertainty were largely avoided those of Jordan, some of the results of which as regards the utilization of protein have already been cited (585), afford a good example and may serve to illustrate the general method of such experiments. Beginning with a ration fairly high in protein, the proportion of this nutrient was gradually reduced to a comparatively low figure and then gradually increased again to the original amount by an exchange in the rations between a nearly pure protein (wheat gluten) and either maize or rice meal. The total digestible matter was thus kept practically constant while the probability of any specific effect was reduced to a minimum.

The actual yields of total milk solids and of milk protein in the several periods are recorded in Table I $39_{9}$ and together afford a fairly accurate measure of the amount of production. Before drawing conclusions as to the influence of the varying protein supply, however, it is necessary to take account of the natural shrinkage in milk. Assuming that the rate of falling off in milk due to advancing lactation, as shown by the difference between the first and last periods, was uniform (571), the actual yields of solids and of protein as compared with those which would have been anticipated had the feed remained unchanged, were as follows:-

${ }^{1}$ Compare Wolff's summary in Ernährung der landw. Nutztiere, I876, pp. 500550. 
Table I39. - Influence of Protein Supply on Milk Production (Results per day and head)

\begin{tabular}{|c|c|c|c|c|c|c|c|}
\hline & \multirow{2}{*}{ PERIOD } & \multirow{2}{*}{$\begin{array}{c}\text { CRUDE } \\
\text { PRO- } \\
\text { TEIN } \\
\text { DI- } \\
\text { GESTED }\end{array}$} & \multicolumn{2}{|c|}{$\begin{array}{l}\text { YIELD OF MILK } \\
\text { SoLIDS }\end{array}$} & \multicolumn{2}{|c|}{$\begin{array}{l}\text { YIELD OF MILK } \\
\text { PROTEIN }\end{array}$} & \multirow{2}{*}{$\begin{array}{l}\text { GAIN } \\
\text { OF } \\
\text { BODY } \\
\text { PRO- } \\
\text { TEIN }\end{array}$} \\
\hline & & & $\begin{array}{l}\text { Com- } \\
\text { puted }\end{array}$ & $\begin{array}{l}\text { Ob- } \\
\text { served }\end{array}$ & $\begin{array}{l}\text { Com- } \\
\text { puted }\end{array}$ & $\begin{array}{c}\text { Ob- } \\
\text { served }\end{array}$ & \\
\hline \multirow{7}{*}{ Experiment of 1897} & & Lb. & Lb. & Lb. & Lb. & Lb. & Lb. \\
\hline & I & 1.70 & 2.72 & 2.72 & 0.64 & 0.64 & +0.03 \\
\hline & 2 & I. 42 & 2.59 & 2.28 & 0.62 & 0.62 & +0.05 \\
\hline & 3 & $\mathbf{I} .85$ & 2.49 & 2.22 & 0.60 & 0.59 & +0.40 \\
\hline & 4 & 0.90 & 2.38 & I. 87 & $0.5^{8}$ & 0.49 & -0.05 \\
\hline & 5 & $0.4 \mathrm{I}$ & 2.24 & I. 67 & 0.56 & 0.44 & -0.43 \\
\hline & 16 & $1.5^{8}$ & I.96 & I.96 & $0.5 \mathrm{I}$ & $0.5 \mathrm{I}$ & +0.46 \\
\hline \multirow{8}{*}{ Experiment of 1901} & I & 2.57 & $4 \cdot 55$ & 4.55 & I.I 2 & I.I2 & +0.25 \\
\hline & 2 & 2.63 & $4.4 \mathrm{I}$ & 4.20 & I.IO & I.I 2 & +0.30 \\
\hline & 3 & 2.20 & 4.28 & 4.02 & 1.08 & I.07 & $\circ$ \\
\hline & 4 & 1.80 & $4 \cdot I 4$ & 3.77 & 1.06 & 1.02 & $\circ$ \\
\hline & 5 & I.60 & 4.01 & $3 \cdot 5^{\circ}$ & 1.05 & $0.92^{\circ}$ & +0.08 \\
\hline & 6 & I. 98 & 3.87 & 3.57 & $\mathrm{I} .03$ & 0.96 & +0.17 \\
\hline & 7 & 2.35 & 3.73 & 3.65 & I.OI & 0.99 & +0.13 \\
\hline & 8 & 2.55 & 3.60 & 3.60 & 0.99 & 0.99 & +0.12 \\
\hline
\end{tabular}

Although the low protein rations were able to support a considerable milk production without causing the body protein to be drawn upon materially, nevertheless, a more liberal supply of digestible protein was accompanied by a distinctly greater production of both total milk solids and milk protein.

Morgen's investigations. - The extensive investigations of Morgen and his associates ${ }^{1}$ upon milk production by sheep include a large number of trials in which an exchange between comparatively pure protein on the one hand and starch or oil on the other was made in the rations. The results, therefore, afford valuable data regarding the influence of the protein supply as distinguished from the possible effects of associated factors. In nearly all instances the ration of the low protein period contained a considerable surplus of digestible protein above the total of milk protein plus maintenance protein. In the following table, computed by the writer, the experiments

${ }^{1}$ Landw. Vers. Stat., 61 (1904), I ; 62 (1905), 25 I; 64 (I906), 93 ; 66 (1907), 63. 
have been grouped according to the amount of this surplus, and the average percentage increase in the yield of milk solids and of milk fat which resulted from an increase of the feed protein has been computed for each group.

Table i40. - Influence of Protein Supply on Milk Production

\begin{tabular}{|c|c|c|c|c|c|c|}
\hline & \multirow{3}{*}{$\begin{array}{l}\text { NUM- } \\
\text { BER OF } \\
\text {.EX- } \\
\text { PERI- } \\
\text { MENTS }\end{array}$} & \multicolumn{3}{|c|}{$\begin{array}{l}\text { SuRplus }{ }^{1} \text { ProteIN as Per } \\
\text { CENT OF MILK ProteIN }\end{array}$} & \multirow{2}{*}{\multicolumn{2}{|c|}{$\begin{array}{c}\text { PERCENTAGE IN- } \\
\text { CREASE OF YIELD } \\
\text { ON HIGH PROTEIN } \\
\text { RATIONS }\end{array}$}} \\
\hline 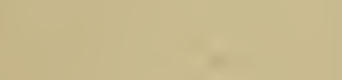 & & \multicolumn{2}{|c|}{$\begin{array}{l}\text { In Low Protein } \\
\text { Rations }\end{array}$} & \multirow{2}{*}{$-\mid \begin{array}{c}\text { In High } \\
\text { Protein } \\
\text { Rations }\end{array}$} & & \\
\hline & & Range & Average & & $\begin{array}{l}\text { Milk } \\
\text { Solids }\end{array}$ & $\begin{array}{l}\text { Milk } \\
\text { Fat }\end{array}$ \\
\hline $\begin{array}{l}\text { Protein substituted } \\
\text { for fat. . . }\end{array}$ & $\begin{array}{l}\mathrm{I} \\
5 \\
9 \\
2 \\
2 \\
\end{array}$ & $\begin{array}{l}\text { Less than } \\
\text { I } 5^{\circ} \\
15^{\circ}-249 \\
25^{\circ}-349 \\
35^{\circ}-449 \\
45^{\circ}-549\end{array}$ & $\begin{array}{l}\text { I } 2 \text { I } \\
\text { I } 94 \\
299 \\
4 \text { I I } \\
45^{\circ}\end{array}$ & $\begin{array}{l}231 \\
334 \\
549 \\
525 \\
412\end{array}$ & $\begin{array}{r}+27.4 \\
+\quad 7.2 \\
+\quad 2.1 \\
+\quad 8.9 \\
-\quad 9.6\end{array}$ & $\begin{array}{l}+23.5 \\
-\quad 7.6 \\
-10.1 \\
-\quad 1.3 \\
-\quad 14.5\end{array}$ \\
\hline $\begin{array}{l}\text { Protein substituted } \\
\text { for carbohydrates. }\end{array}$ & $\begin{array}{r}\text { I9 } \\
7 \\
6 \\
8 \\
4 \\
\text { I } \\
2 \\
3 \\
3 \text { I }\end{array}$ & $\begin{array}{l}\text { I } 50-249 \\
25^{\circ}-349 \\
35^{\circ}-449 \\
45^{\circ}-549 \\
55^{\circ}-649 \\
65^{\circ}-749 \\
75^{\circ}-\end{array}$ & $\begin{array}{l}2 \text { I } 4 \\
3 \text { I I } \\
374 \\
492 \\
637 \\
669 \\
802\end{array}$ & $\begin{array}{l}348 \\
437 \\
429 \\
641 \\
742 \\
534 \\
897\end{array}$ & $\begin{array}{r}+12.8 \\
+18.9 \\
+\quad 7.1 \\
+\quad 8.8 \\
+25.6 \\
+69.5 \\
+20.3\end{array}$ & $\begin{array}{r}+6.6 \\
+\quad 7.1 \\
+\quad 5.5 \\
+\quad 5.6 \\
+27.6 \\
+54.7 \\
+18.4\end{array}$ \\
\hline
\end{tabular}

On the whole, Morgen's investigations seem to furnish conclusive evidence of a stimulating effect of protein on milk production. Even when the protein supply already largely exceeded the minimum demand, a further addition was in most instances followed by a distinct increase in the yield of milk solids and usually in that of milk fat. It should be said, however, that a respectable minority of the individual experiments failed to show this effect. Of the nineteen single trials in which protein was substituted for fat, eleven showed an increased yield of milk solids and six an increased yield of milk fat. Out of the

1 Digestible protein minus requirements for maintenance and for growth of wool. 
thirty-one trials in which protein was substituted for carbohydrates, twenty-six showed an increased yield of milk solids and twenty-one an increased yield of milk fat. In thirteen out of the entire fifty experiments, therefore, the presence of additional protein failed to cause an increase in the milk solids, while in twenty-three trials it failed to produce an increase of milk fat.

600. Effect of protein-rich feeds. - In addition to investigations like those noted in the last paragraph, in which the effect of an interchange of practically pure nutrients was studied, a considerable number of experiments are on record in which an enrichment of a ration in digestible protein has been effected by an interchange of feeding stuffs, as for example, by the substitution of cottonseed meal for maize meal.

In all these experiments the low protein rations contained, with one or two exceptions, a surplus of digestible protein above the milk protein plus the estimated maintenance, yet a further increase of digestible protein was followed by a larger yield of milk per unit of organic matter digested, the increase ranging from I per cent to 39 per cent. As in the experiments described in the previous paragraph, the results appear somewhat capricious, showing no consistent relation between the excess of protein supplied and the relative increase of milk production secured.

It should be added that in many of these experiments the proteins of the low-protein rations consisted to a considerable extent of maize protein, which has since been shown to be of inferior nutritive value (783).

601. Protein fed in American practice. - On the basis of experiments and observations, Wolff ${ }^{1}$ recommended a standard for dairy feeding calling for 2.5 pounds of digestible protein daily per rooo pounds of live weight. Although later modified by Lehmann, this standard was for many years almost universally accepted on Wolff's authority, supported by the undoubted fact that in many instances the addition of protein-rich feeding stuffs to ordinary farm rations materially increased the milk yield. Later observations, however, seem to indicate that while protein is important the amount necessary in practice has been somewhat overestimated.

${ }^{1}$ Die Ernährung der landw. Nutztiere, I876, p. 548. 
Woll ${ }^{1}$ was the first to make an extensive study of dairy practice in the United States as regards the protein supply, finding that very many successful dairymen were using rations supplying materially less protein than was called for by Wolff's standard. The average of all the rations reported as compared with Wolff's standard was as follows :-

Table i4r. - Digestible Matter in Dairy Rations

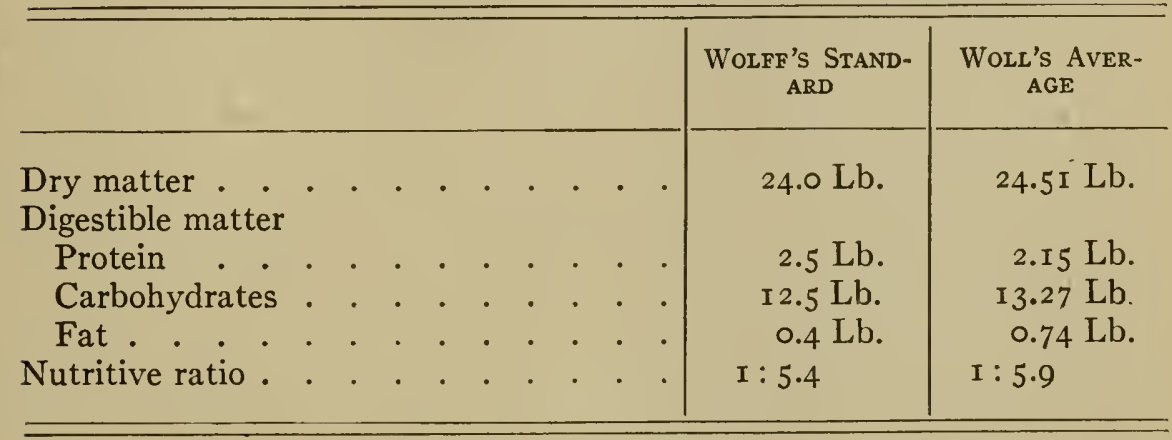

Woll points out that this average, while it does not represent any scientific investigation of milk production, expresses the results of American feeding experience, and although it does not demonstrate either that less protein would not be sufficient or that more would not be advantageous, it does afford a safe guide for practice, and indicates that rations containing less protein than the Wolff standard calls for are probably more profitable.

Somewhat similar observations were reported by Phelps $^{2}$ in $1892-1893$ with the additional feature that in several instances the rations fed were subsequently modified at the suggestion of the experimenter and the yield on the new ration determined. Phelps recommends a supply of 1.9 to 2.5 pounds digestible protein per head according to the productiveness of the cow, the amount to be based on the yield of milk rather than on the live weight, and believes such rations will give more economical production than those containing less protein.

602. Experiments on herds. - Haecker ${ }^{3}$ has reported extensive observations and experiments on the protein supply

1 Wis. Expt. Sta., Buls. 33 (1892) and 38 (1894).

2 Conn. (Storrs) Expt. Sta., Rpt. I897, pp. I7-66.

${ }^{3}$ Minn. Expt. Sta., Buls. 7 I, 79, and I40. 
of the dairy herd of the Minnesota Station, leading to the conclusion that the Wolff-Lehmann standard calls for unnecessarily large amounts of protein.

During nine years, yields which were regarded as normal and satisfactory, either on the basis of total amounts produced or of feed consumed per unit of milk, were secured on rations containing, with the exception of the year I895-1896, about 2 pounds of digestible protein per rooo pounds live weight. ${ }^{1}$

During three of these years, comparisons were also made between a group of cows receiving about 2 pounds of digestible protein per day and rooo pounds live weight and one receiving about I. 5 pounds. In the earlier years the low protein rations appeared as efficient as the higher ones, but toward the end of the three years the low protein group showed deficient vitality, apparently indicating a lack of protein.

In all nine years, the (estimated) digestible protein in the high protein rations supplied a considerable surplus over the protein of milk plus maintenance. Estimating the maintenance requirement of protein at 0.7 per ı०o०, Haecker makes the following comparisons: ${ }^{2}$ -

\section{Table I42. - Protein Supply of Dairy Herd}

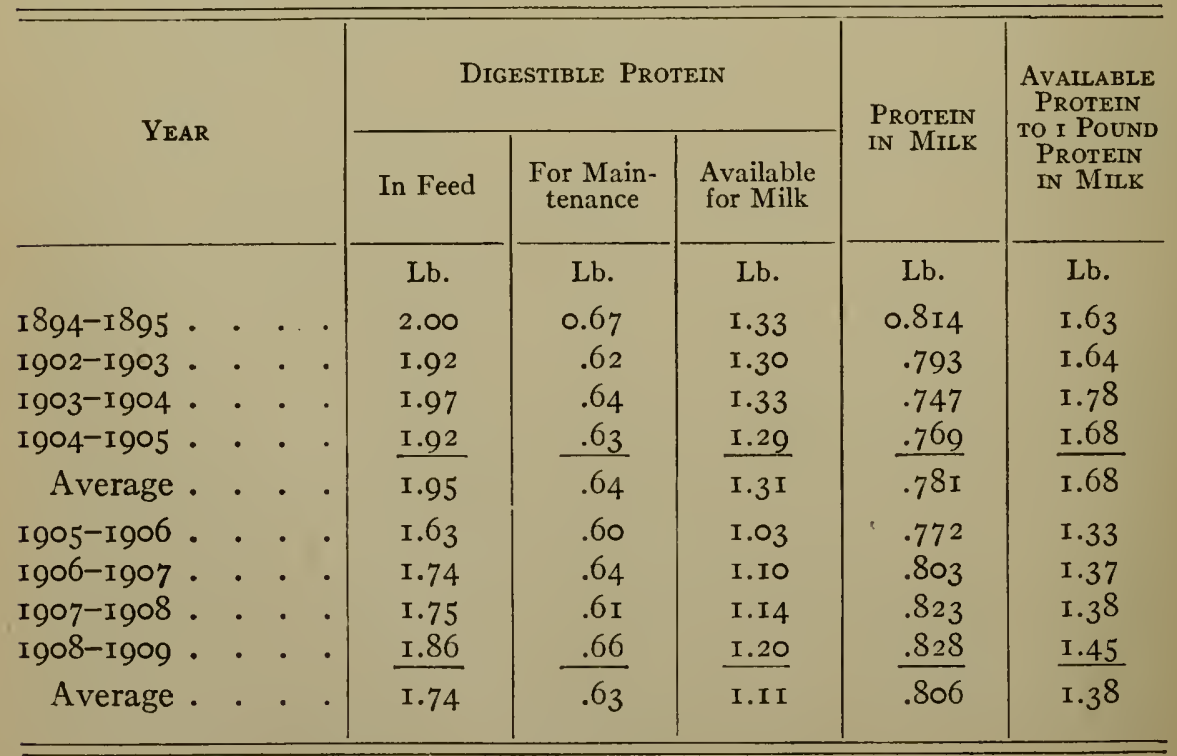


The protein content of the milk from the low protein groups is not reported, but an approximate estimate indicates that it could not have been much less than the surplus of feed protein over maintenance, thus furnishing further instances of an apparently high percentage utilization of feed protein (586). While the indications are that such very low protein rations were inadequate, it seems clear that a surplus of 40 or 50 per cent of available protein over that contained in the milk was ample to support normal production.

Woll ${ }^{1}$ has reported a nine-year series of observations on the dairy herd of the Wisconsin Station, the time being divided into three periods of three years each, during the first and third of which the rations had a nutritive ratio of $\mathrm{I}: 7$, while during the second three years it was $I: 6$. The estimated digestible protein consumed per day by cows weighing slightly over Ioo० pounds was

Average of periods $\mathrm{A}$ and $\mathrm{C} \mathbf{1 . 7 6}$ pounds. Average of period B I.97 pounds.

Table 143. - Surplus of Available Protein in Herd Rations

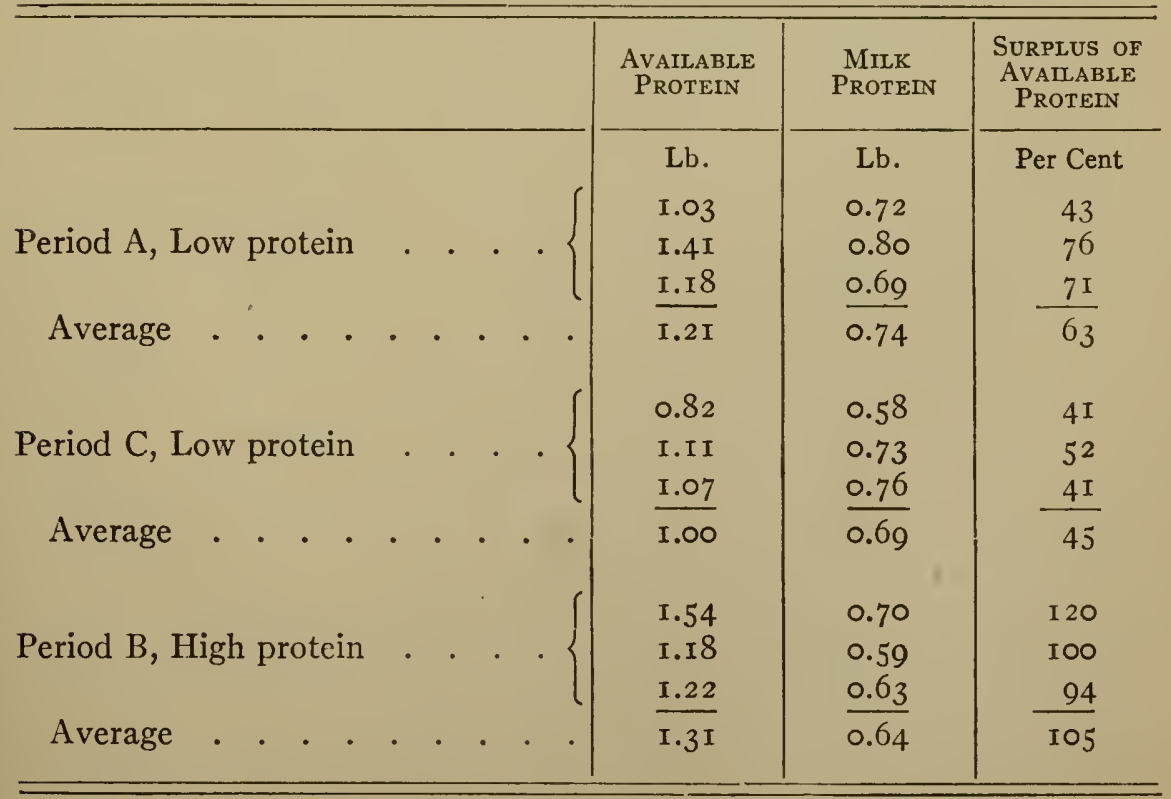

1 Wis. Expt. Sta., Research Bul. I3. 
The results for the entire year and likewise for the winter rations showed on the whole a somewhat greater and a decidedly more economical average production on the smaller supply of protein. The protein content of the milk is not stated, but estimating it at 3.38 per cent and allowing 0.6 pound per rooo for protein maintenance, the approximate surplus of the available protein (digestible protein minus maintenance requirement) over the milk production in the winter rations was as shown in Table 143 in each of the nine years.

603. Summary. - In view of the great differences between individual cows both as to yield and composition of milk, it is clear that no one figure can express the protein requirement for milk production per day and head, but that it must vary with the amount and character of the milk produced.

It appears to be fairly well established (586) that the digestible feed protein of ordinary mixed rations may be converted into milk protein without any very great loss and that consequently a moderate rate of milk production may be maintained, at least for a time, on rations furnishing a comparatively small surplus of digestible protein over the milk protein plus the requirement for maintenance.

On the other hand, however $(599,600)$, both experiments with pure proteins and those in which an increase in the protein content of rations has been secured by the use of protein-rich feeds seem to indicate clearly a stimulating influence of excess protein on milk production, although in the majority of cases the effect was not very large. Contrary to what might have been anticipated, however, an increase in the digestible protein of the ration appears to have been on the whole quite as effective with animals already on a high plane of protein nutrition, i.e., receiving a large surplus over the minimum requirement as with those on a much lower level of protein supply. This appears with especial clearness in Morgen's experiments on sheep. The results therefore fail to indicate the limits within which this stimulating effect is manifest or to establish any quantitative relation between the surplus protein supplied and the additional milk yielded. They afford no basis, therefore, for any estimate of the extent to which a stimulation of milk production by means of excess protein will be economically profitable under any given conditions. 
The experiments by Haecker and by Woll on herds (602) seem to justify the conclusion that in commercial milk production in the United States a ration supplying, in addition to the maintenance requirement, digestible protein equal to 150 to I6o per cent of the milk protein yielded is ample in this respect to sustain a normal rate of milk production and may be distinctly more profitable than a ration richer in protein. It is not impossible, however, that when circumstances warrant the effort to secure the maximum production possible to the animal a more liberal supply of protein would be advantageous.

\section{Energy requirements for milk production}

604. Energy content of milk. - As in other forms of stock feeding, the principal factor in determining the energy required in the feed is the amount of chemical energy contained in a unit of product. In the case of milk production this factor varies through a wide range on account of the large differences in composition of milk due to individual and breed differences, stage of lactation, etc.

Haecker ${ }^{1}$ has arranged the results of analyses of 543 samples of milk in ten groups according to the fat content. His averages, together with the energy content per pound of milk as computed by the writer, are as shown in the following table:-

Table i44. - Composition and Energy Value of Milk

\begin{tabular}{|c|c|c|c|c|c|}
\hline \multicolumn{2}{|c|}{ NUMBER OF } & \multicolumn{3}{|c|}{ CoMPOSITION } & \multirow{2}{*}{$\begin{array}{c}\text { Total } \\
\text { ENERGY PER } \\
\text { POUND }\end{array}$} \\
\hline Samples & Milkings & Fat & Protein & $\begin{array}{c}\text { Carbohy- } \\
\text { drates }\end{array}$ & \\
\hline & & $\%$ & $\%$ & $\%$ & Cals. \\
\hline - & - & 2.5 & 2.55 & $4 \cdot 45$ & 253 \\
\hline 47 & 658 & 3.0 & 2.68 & 4.60 & 278 \\
\hline 55 & 770 & $3 \cdot 5$ & $2.8 \mathrm{I}$ & $4 \cdot 75$ & 306 \\
\hline 57 & 798 & 4.0 & 3.08 & 4.85 & 336 \\
\hline I I6 & I6 24 & $4 \cdot 5$ & 3.27 & 4.97 & 365 \\
\hline 103 & I 442 & 5.0 & 3.45 & 4.99 & 390 \\
\hline 89 & I 246 & $5 \cdot 5$ & 3.65 & 4.92 & $4 \mathrm{I} 5$ \\
\hline 39 & 546 & 6.0 & 3.82 & $4.9 I$ & 440 \\
\hline 24 & 336 & 6.5 & 4.02 & 4.90 & 467 \\
\hline I3 & I82 & 7.0 & 4.22 & 4.84 & $49^{2}$ \\
\hline
\end{tabular}

${ }^{1}$ Minn. Expt. Sta., Bul. I40, p. 5 I. 
If there were available definite knowledge regarding the net energy values of feeding stuffs for milk production, the foregoing figures for the total energy of the milk would serve as a basis for estimating the net energy supply required for the production of a given yield of milk for any one of the ten grades, 25 pounds of 4 per cent milk, for example, requiring $336 \times 25=8400$ Cals. of net energy in the feed.

605. Equivalent energy values for fattening. - In the absence of determinations of the net energy values of feeding stuffs for milk production (588) it is impossible to make direct use, in the manner just indicated, of the foregoing data regarding the energy content of milk. Pending such determinations, however, it appears possible to estimate the net energy requirements in the feed of dairy cows in another way, viz., by computing from the composition of the milk, in the manner already described (593), the amount of fattening which is equivalent in energy requirement to a unit of milk yield. Thus it was estimated, on certain assumptions, that the amount of feed energy required for the production of one pound of average 4 per cent milk would, if applied to fattening, have produced a gain of only $25^{2}$ Cals. in place of the 336 Cals. actually present in the milk. Accordingly, a ration containing, in excess of maintenance, $25^{2}$ Cals. of net energy for fattening would have been adequate to produce a pound of milk containing 336 Cals. of energy. In this way the amount of net energy required for the production of one pound of milk of each of the grades included in the previous table may be computed.

By this device of reducing the total energy content of the milk to the equivalent amount of net energy for fattening, it appears possible to utilize the net energy values of feeds obtained by Kellner and others in maintenance or fattening experiments as a basis for computing rations for milk. Such a method is, of course, provisional, and the basis for it at present is somewhat slender, but it seems the best one now available. In its actual use for computing rations, however, it appears necessary also to take into account the fact shown by Eckles (722) that with well-fed cows the digestibility of the rations is on the average some 5 per cent lower than the average digestion coefficients which are used in computing net energy values. Accordingly, the figures for the equivalent energy for fattening as computed 
for the several grades of milk have been increased by 5 per cent, giving the following results, which may be used provisionally to compute from the figures of Table VII of the Appendix the rations required for the production of milk of different grades.

Table 145. - Equivalent Energy Values for Fattening

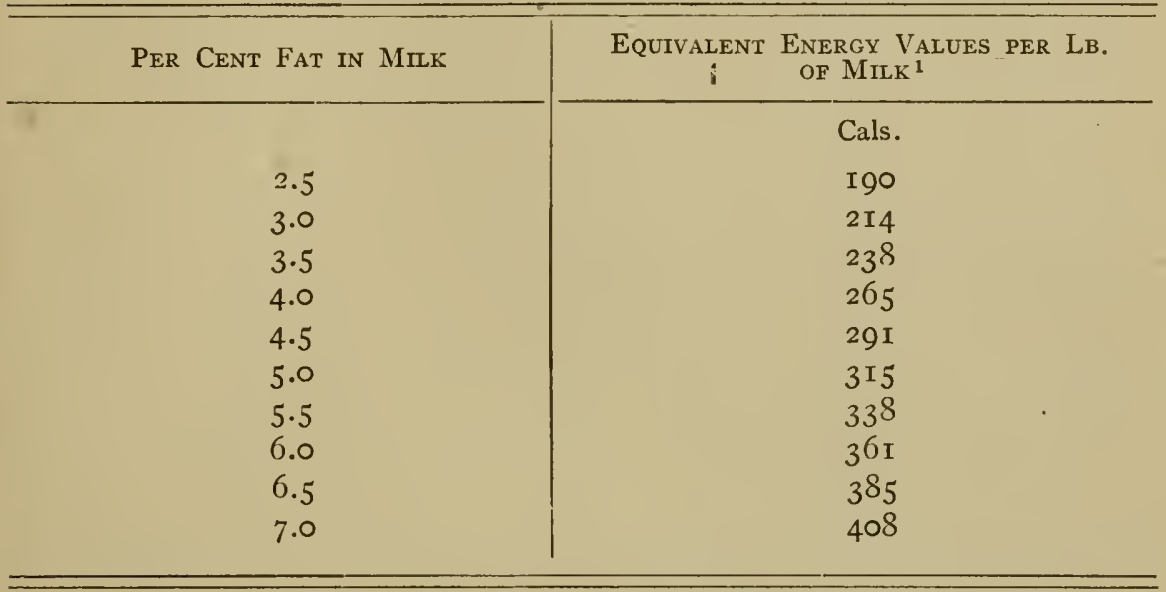

606. Concurrent fattening. - Were all the surplus feed above the maintenance requirement applied to milk production, it would be a comparatively simple matter to compute the amount of feed energy required in a daily ration. Thus, if a cow weighing rooo pounds were capable of producing 25 pounds of 4.5 per cent milk daily, the net energy required in her ration would be computed as follows : -

For milk production 25 lb. of milk @ 29x Cals. For maintenance

Attention has been called several times, however, to the fact that in the milking animal at least two forms of production are possible, viz., milk and increase of body tissue (fattening), only the former of which is usually desired. To these may perhaps be added, as a third form of production, a possible stimulation of the incidental muscular activity of the animal by heavy feeding (609). Evidently if conditions are such that

${ }^{1}$ Including 5 per cent allowance for difference in digestibility. 
part of the feed energy is diverted to these other purposes, the ration must supply more net energy per pound of milk than would be necessary if all the latter were utilized for milk production.

607. Influence of plane of nutrition. - It appears to be well established both by common experience and by direct experiment that such a diversion of energy from milk production to other forms may in fact take place before the maximum capacity of the milk glands is reached. On moderate rations, the net energy, after satisfying the maintenance requirement, may apparently be utilized entirely for milk production. As the feed is increased, however, the animal does not continue to utilize all the available net energy for milk production up to the limit of its capacity and then suddenly begin to utilize any surplus for fattening. On the heavier rations the concentration of the digested nutrients in the body fluids increases, the organism reaches a higher plane of nutrition, and at a point varying with different individuals this greater concentration of available material causes fattening to begin, which, so to speak, robs the milk glands of feed intended for milk production.

608. Influence of individuality. - The individuality of the animal is a most important factor in this connection. With cows having an inherited tendency toward fattening, as in the so-called beef breeds, this point at which energy begins to be divided between milk production and fattening may be reached on comparatively light rations. Such animals can be brought up to their maximum milk-producing capacity only at the expense of a considerable expenditure of feed for concurrent fattening and are likely to be unprofitable for dairy purposes. On light rations, giving a moderate yield of milk, the maintenance requirement constitutes too large a proportion of the feed cost, while with heavier feeding production is directed too largely to fattening.

With the typical dairy animal, on the other hand, having but a slight tendency to fatten, the feed may be increased well towards the amount required to support the maximum capacity of the milk glands, or in exceptional cases even up to that point, without causing any material diversion to fattening. Such animals, especially if of large milk-producing capacity, are the profitable dairy animals so far as the cost of feed is concerned. 
The relations between feed supply, milk production and fattening outlined in the foregoing paragraphs have been clearly demonstrated in a number of investigations on dairy feeding, such as those of Waters, Caldwell and Weld ${ }^{1}$ and of Waters and Hess ${ }^{2}$ at the Pennsylvania station, those by Woll and Carlyle $^{3}$ at the Wisconsin station, and especially those by Haecker ${ }^{4}$ at the Minnesota station.

609. Stimulation of katabolism. - But while the diminishing returns obtained from the feed of the dairy cow as its amount is increased beyond a certain maximum may be explained in part by a diversion of net energy from milk production to fattening, it seems to be true also that heavier feeding may cause a larger proportion of the digested organic matter to be oxidized, either as the result of greater muscular activity or by a direct stimulation of the katabolic processes. This is especially evident in breed tests in which heavy rations have been consumed. Striking illustrations of it are afforded by the results of the tests of dairy breeds at the Louisiana Purchase Exposition in I 904 as computed by Haecker ${ }^{5}$ and by the extensive comparisons of German breeds reported by Hansen. ${ }^{6}$

610. Diminishing returns from feed. - It is evident from the foregoing that, with the possible exception of cows of a very pronounced dairy type, the maximum yield of milk can be secured only at the expense of a simultaneous production of more or less body fat and perhaps also of a stimulation of the katabolic processes of the body. Consequently, beyond the point at which this fattening or stimulation begins, the milk production per unit of net energy in the feed must necessarily be a diminishing one, and it is clear that the determination of the net energy requirements for milk production is to a considerable extent an economic problem.

Milk will be produced at the least feed (energy) cost per pound when the ration is so adjusted as to produce as great a yield of milk as is possible without causing fattening. ${ }^{7}$ If the

1 Penna. Expt. Sta., Rpt. I893, p. 24-36.

${ }^{3}$ Wis. Expt. Sta., I 7 th Rpt. (I900), p. 37-6r.

${ }^{4}$ Minn. Expt. Sta., Buls. 79 and I40. ${ }_{5}$ Minn. Expt. Sta., Bul. Io6, p. I 58.

${ }^{6}$ Landw. Jahrb., 35 (I906), Ergzbd. IV, I47-236; 37 (I908), Ergzbd. III, 236$410 ;{ }_{2}$ er Ber. vom Dikopshof (I9II), $210,430$.

${ }^{7}$ It may be presumed that the stimulating effect upon the katabolism occurs chiefly in heavy feeding which causes fattening also. 
energy supply is decreased below this point the milk yield will tend to fall off while the maintenance requirement remains practically constant. The maintenance, therefore, will consume a larger percentage of the total feed energy so that, exactly as in growth or in fattening, while the net energy requirement for the formation of a unit of milk remains approximately constant, the total net energy necessary to support both maintenance and milk production increases relatively per unit of product.

On the other hand, if the feed is increased so as to cause fattening or to stimulate katabolism, it is clear that the energy requirements per unit of milk produced will be apparently increased for the reasons already explained. Such an increase in the feed cost, however, may be economically justifiable for the same reasons as in the case of any form of intensive production. In average commercial milk production, it may be doubted whether the rations should be made heavy enough to cause any considerable fattening, and so far as this is the case, the estimated net energy values per unit of milk in Table 145 may serve as the basis for computing rations. If, however, feed is relatively cheap and dairy products high in price, the diminishing returns due to heavier feeding may still be profitable up to a certain point even though more energy per unit of milk must be supplied in order to support concurrent fattening, while the fact that more or less of the fat stored in the body may be utilized for the support of milk production in the early stages of the next lactation is also to be considered.

\section{Fat requirement for milk production}

611. Is fat essential ? - It was noted in discussing the functions of the nutrients (265) and also in connection with the requirements for growth $(498,499)$ that the presence in the feed of certain fats or of substances associated with them appears to be essential to growth. Since milk production is in many respects analogous to growth it is of interest to inquire whether the fats of the feed exert any such specific effect, either on milk production as a whole or on the production of milk fat.

That milk fat as well as body fat may be manufactured in the body in large amounts from other nutrients has been shown 
beyond question by the experiments of Voit, Kühn and Fleischer, M. Fleisher, Wolff and especially by those of Jordan, ${ }^{1}$ while the latter investigator demonstrated that milk fat can be formed from carbohydrates (553). Jordan's experiments on cows, as well as the later ones of Morgen ${ }^{2}$ on sheep and goats, likewise show that relatively large amounts of milk may be produced on rations made up of feeding stuffs very poor in fat or from which the larger part of the fat has been extracted. It is scarcely feasible to prepare absolutely fat-free rations for such animals and the writer is not aware of any experiments on milk production with such rations, but it is clear that at most but very small amounts of fat can be regarded as indispensable.

612. Addition of fat to rations. - Experiments in which the fat content of ordinary rations has been increased, either by the direct addition of fat in one form or another or by the substitution of fat for carbohydrates, have given very contradictory results. An increased percentage of fat in the milk has been very frequently observed, sometimes accompanied by an increase in the actual yield of fat and sometimes not, while in other cases the results have been entirely negative. In many instances the experiments are complicated by the fact that the fat was simply added to a basal ration, thus increasing the total amount of feed. ${ }^{3}$ The most recent investigations are those undertaken upon a common plan under the auspices of the German Agricultural Council at ten German experiment stations with, in all, 196 cows, the results of which have been reported by Kellner. ${ }^{4}$

The increase in the fat of the rations was effected by the substitution of rice feed ${ }^{5}$ for rye meal and starch, so that fat replaced an equivalent amount of carbohydrates. The results, therefore, included any "specific" effects of these two feeding stuffs, if such there were (618). Per Iooo pounds live weight, the fat-poor rations contained 0.25 to 0.50 pound digestible fat and the fat-rich 0.47 to I.ro pounds.

1 N. Y. (Geneva) Expt. Sta., Buls. I32 (I897) and I97 (I901).

${ }^{2}$ Landw. Vers. Stat., 61 (I g04), I ; 62 (I g05), 25 I ; 64 (I906), 93.

${ }^{3}$ Compare Kellner, Die Ernährung der landw. Nutztiere, 6th Edition, pp. 564-566.

${ }^{4}$ Reichsamt des Innern; Berichte über Landwirtschaft, Heft I and 2.

5 According to Hansen rice feed has the specific effect of depressing the fat production, although this effect did not appear manifest in most of these experiments nor in those of Fingerling (613). 
Grouping the results regarding the fat content of the milk according to the total amount of milk yielded, it appears that an increase in the percentage of fat in the milk was in general associated with a decrease in the total yield and vice versa.

Table i46. - Effect of Increasing Fat of Rations

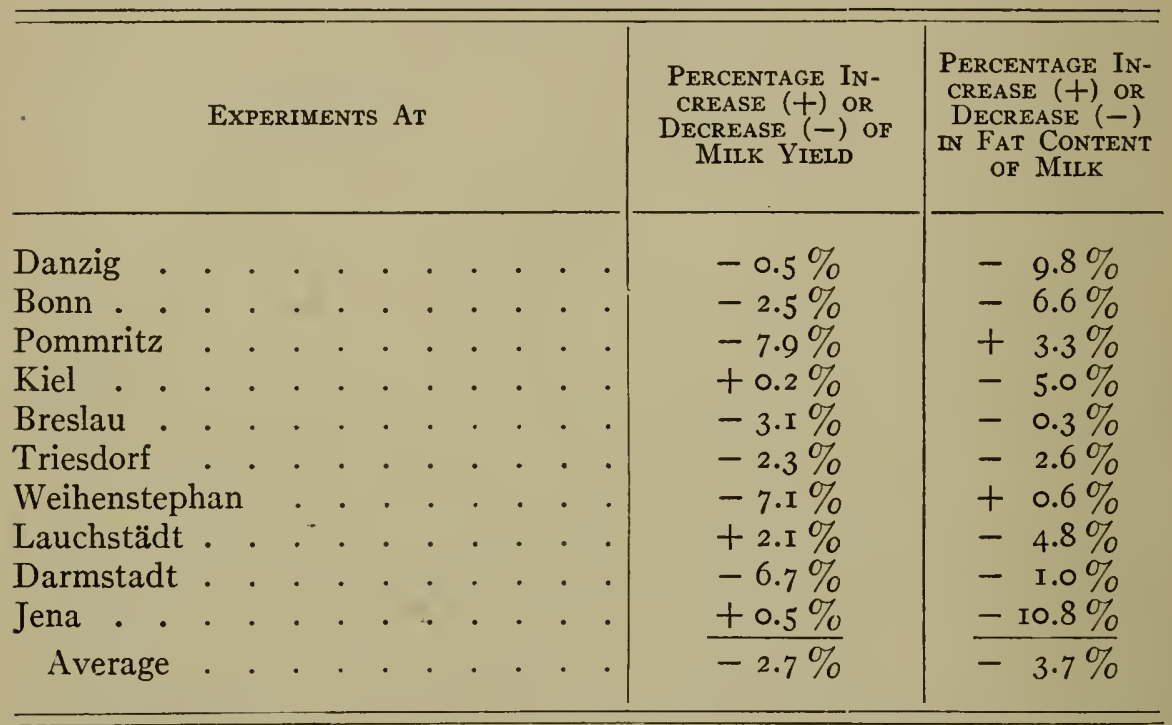

Striking individual differences in cows, however, were observed. For example, in two of the experiments the range of increase or decrease for the individual animals consequent on the substitution of fat for carbohydrates was as follows:-

Table 147. - Influence of Individuality on Effects of Fat Increase

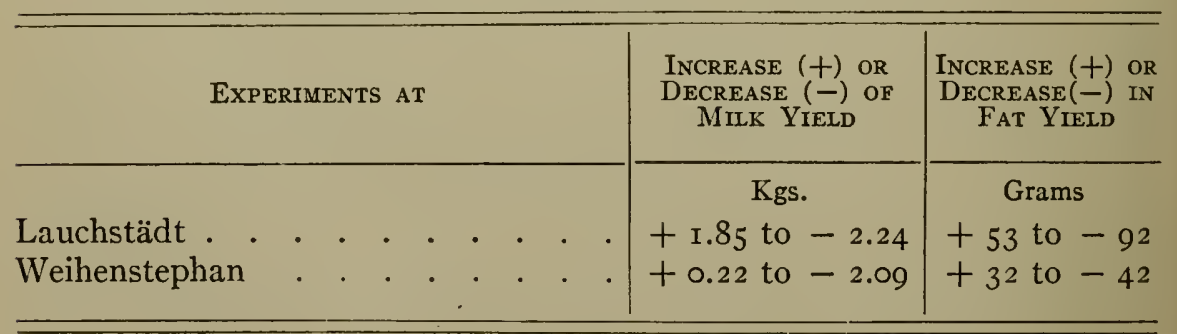

It seems clear from the foregoing results that under the average conditions of practice no material advantage can be expected from increasing the digestible fat of dairy rations 
above 0.4 to 0.5 pound per Iooo pounds live weight, although a gain may result with individual cows.

613. The minimum of feed fat. - On the other hand, the extensive investigations by Morgen and his associates on sheep and goats, already referred to (599), have shown that with these animals an increase of the fat content of rations exceptionally deficient in this ingredient results in most cases in an increased yield of milk solids and especially in a specific increase of the fat content of the milk.

The rations consisted of a basis of roughage poor in fat ${ }^{1}$ to which various commercially pure nutrients were added. Fat in various forms was added to scant basal rations and likewise substituted for carbohydrates or protein in heavier rations. Experiments of the latter sort, in which the energy content of the rations was kept substantially unchanged, are especially convincing. An increase of the fat content of the fat-poor rations, either by direct addition or by substitution, up to 0.5 to $\mathrm{r} .0 \mathrm{lb}$. per $1000 \mathrm{lb}$. live weight not only resulted in a distinct increase in the yield of milk and of milk solids but likewise in an increased percentage of fat in the fresh milk and in the milk solids. This specific influence of fat as compared with protein is illustrated in Table I40, which shows that while a substitution of protein for fat or for carbohydrates increased the yield of solids, the yield of fat was decreased in the former case. Fingerling ${ }^{2}$ has likewise shown that increasing the fat content of a ration by substituting a feed rich in fat for one rich in carbohydrates (rice meal in place of barley meal) likewise increases the fat yield.

This specific effect of feed fat on the production of milk fat appears to be more marked in the case of sheep and goats than in the case of cows. It was observed up to a limit of approximately I.o pound per rooo pounds live weight, but above that the results were if anything negative, while with cows, as already shown, an increase of the digestible fat above 0.4 pound per Iooo pounds live weight generally produces little or no effect. Morgen ascribes the difference to the greater relative production of fat per unit of weight by the smaller animals.

In ordinary dairy rations fat will not often fall below the apparent limit of 0.4 to 0.5 pound. Only when feeds unusually poor in fat are used, such as straw or inferior grades of hay or

${ }^{1}$ In part artificially extracted.

${ }^{2}$ Landw. Vers. Stat., 64 (rgo6), 299. 
by-products containing a minimum of fat, may a favorable effect upon the yield of milk and its percentage of fat be anticipated from an increase in the supply of digestible fat.

614. Influence on utilization of energy. - None of the experiments on the influence of the fat supply upon milk production afford any exact data regarding the concurrent gain or loss of tissue, since no determinations of the gaseous excreta were made. It is impossible, therefore, to determine whether the observed effect of the feed fat was brought about by a stimulation of milk production at the expense of fattening, i.e., by modifying the direction in which the energy of the feed was utilized, or whether, under its influence, the metabolism in the milk gland was actually effected more economically.

\section{Ash requirements for milk production}

Practically no data are on record upon which a trustworthy estimate of the ash requirements of the dairy cow can be based.

615. The outgo in the milk. - It is true that the outgo of mineral elements in the milk may be determined without special difficulty and that reasonably accurate figures are available from which it may be estimated. This, however, is but a single element in the problem. It became evident in considering the ash requirements for maintenance in Chapter IX (421-436) and those for growth in Chapter XI (492-497) that neither the actual availability of the mineral elements of feeding stuffs nor the influence of the amount and quality of the ash supply upon the losses in feces and urine has been sufficiently investigated to permit any satisfactory conclusions as to the influence of these factors.

Kellner ${ }^{1}$ has, however, computed the approximate requirements for calcium and phosphorus from the outgo in the milk. Accepting Henneberg's estimate of $7 \mathrm{I} .4$ grams of calcium and 2 I.8 grams of phosphorus per rooo kilograms live weight for the maintenance requirements, he adds to these three times the average amounts found in the milk upon the somewhat questionable assumption that only one-third to one-half the feed ash is available. Computed for a yield of 20 pounds of milk per day by a thousand pound cow, his results are as follows :-

1 Ernährung landw. Nutztiere, 6th Ed., p. 595. 
Table i48. - Estimated Requirements for Milk Production

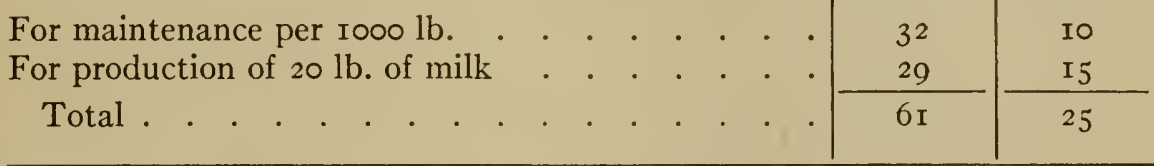

616. The supply in the feed. - Kellner states that ordinary dairy rations will usually meet the requirements just stated and that only in exceptional cases will it be necessary to supplement the calcium supply.

Forbes ${ }^{1}$ has shown, however, that rations fully adequate, so far as organic nutrients are concerned, to support a considerably greater milk production than that on which Kellner's requirements are based may nevertheless permit very material losses of mineral ingredients, especially of calcium, magnesium and phosphorus. Complete ash balances are reported for six animals on three different rations, all of which maintained the live weights of the cows and resulted in gains of body protein. With an average live weight of about 935 pounds and an average daily milk yield of about 36 pounds ( $6.38 \mathrm{Kgs}$.), the calcium and phosphorus requirements as computed according to Kellner's method and the actual amounts supplied in the rations were :-

Table i49. - Calcium and Phosphorus for Milk Production

\begin{tabular}{lllll|cc|c}
\hline \hline & & & & & & & \\
\hline
\end{tabular}

1 Ohio Expt. Sta., Bul. 275 (19r6). 
Whether such relatively large losses by fresh cows usually accompany copious milk production and are made up again in the later stages of lactation, and whether this depletion of the mineral reserves of the body is one of the factors in the natural shrinkage of milk production, as suggested by Forbes, are matters for future investigation.

It is evident, however, that none of the foregoing data afford much information regarding the real ash requirements of dairy cows.

\section{Feed as a stimulus to milk production}

617. Flavoring substances. - By flavoring substances is meant those whose presence in small amounts improves the odor or taste of a feeding stuff or ration while not adding materially to its content of protein or energy. In other words, they are substances which do not yield matter or energy to the body in the ordinary sense, but which may nevertheless affect the course or rapidity of metabolism.

That the flavor or aroma of feeding stuffs is not an insignificant element in determining their commercial value, not only for milk production but for other purposes, is well established by practical experience. This superiority is doubtless due largely to the fact that a palatable feed is consumed more freely than one lacking in flavor. In the case of milk production, however, it appears that, within certain rather narrow limits, various flavoring materials may act as a direct stimulus to the milk gland, causing a greater yield of milk and especially of fat.

In Morgen's experiments on milk production cited on previous pages extensive use was made of rations consisting largely of almost flavorless materials. With such rations it was found to be impossible to secure yields equal to those obtained from rations supplying equal amounts of protein and energy but made up of normal feeds. The addition to these flavorless rations, however, of such substances as fennel, anise or hay distillate, or the introduction of malt sprouts, caused a distinct increase in the milk yield, so that, with rations containing a sufficiency of fat, almost or quite normal results were secured. ${ }^{1}$ Moreover, a distinct effect was observed in increasing the fat production and the percentage of fat in the milk.

${ }^{1}$ Landw. Vers. Stat., 61 (I904), I. 
Subsequent experiments by Fingerling ${ }^{1}$ fully confirmed these results. The addition to the flavorless rations, or to damaged hay, of salt, hay distillate, fennel, or even the impregnation of rations with the odor of the latter substances, caused a marked increase in the yield of milk and in its content of fat as well as in the percentage of fat in the milk solids, while similar additions to normal rations were without effect. Fingerling's experiments likewise show clearly, however, that this effect of flavoring materials, while of much physiological interest, can rarely be of much economic importance and they lend no support to the claims of the numerous condimental feeds, milk powders, etc., so largely advertised. It was also shown that certain feeding stuffs (malt sprouts, palmnut cake, cocoa cake and beet molasses) when added to a ration of damaged hay and pure nutrients increased the milk and fat yields to about the same extent as flavoring with fennel. Whether these effects are due to some form of nerve stimulus, either general or specific, or to an increased production of the hormones of milk production (549) does not appear.

618. Specific effects of feeds. - The fact just noted that certain feeds stimulate the production of milk and of milk fat, appparently by their influence on the flavor of rations, leads naturally to a consideration of the so-called "specific" effects of feeds in general. The belief has long been held in practice that feeding stuffs may promote milk production and improve the quality of the milk to an extent not fully explained by the amounts of digestible matter or of energy which they supply. On the other hand, there has been no general agreement as to what particular feeding stuffs possess this power, and scientific investigators have been led to question the existence of such effects, particularly upon the composition of milk. A discussion of the literature of the subject up to 1903 by Lemmermann and Linkh ${ }^{2}$ affords striking instances of the discrepancies between different experiments. The effects of such feeds as palmnut meal, cocoa meal, and cottonseed meal, for example, are reported by different experimenters as favorable, unfavorable or indifferent.

${ }^{1}$ Landw. Vers. Stat., 62 (I905), I ; 64 (I906), $357 ; 67$ (I907), 253; 71 (I909), $373 ; 74$ (I9II), I63.

${ }^{2}$ Landw. Jahrb., 33 (1903), 564. 
Defective planning of experiments is doubtless responsible for much of this confusion. In many instances the experimenters have simply added the feed to be tested to a light basal ration, as in the familiar experiments by G. Kühn ${ }^{1}$ on palmnut meal so frequently referred to. Others, while substituting one feeding stuff for another, have failed to show that the total amount of digestible matter supplied was unchanged. In some extensive investigations, for instance, oil meals and similar feeds have been interchanged in amounts supplying equal quantities of protein without regard to other ingredients. Under such conditions concordant results could not be expected, and one can but agree with Lemmermann and Linkh that the evidence is inconclusive, while their own experiments, although indicating specific effects for various feeding stuffs, are scarcely more convincing.

Similar negative evidence is afforded by the extensive feeding trials with dairy herds carried out in Denmark by Fjord, Friis and Storch and which afford the basis for the so-called "feed unit" or Scandinavian system of comparing rations (702). In these trials a variety of feeding stuffs, including many reputed to have specific effects on milk production, were compared with ordinary farm grains and failed to exert any material influence on the milk secretion other than what may be plausibly explained by the variations in the protein content and the total nutrients of the rations incident to the experimental method. In particular, indications of a specific effect on the production of milk fat are lacking.

More positive results have been reached, however, in two recent investigations, viz., in a series of investigations by Hansen at the Agricultural Academy Bonn-Poppelsdorf and in a series of coöperative experiments on palmnut meal made under the auspices of the German Agricultural Council.

619. Hansen's experiments. - Hansen's experiments ${ }^{2}$ included nine series on 63 cows, extending over 5 years, in which the various feeding stuffs to be tested were substituted in a comparison ration for others which appeared to be indifferent in this respect. Care was taken to keep the total digestible nutrients in the rations, or after the first 3 years, the estimated net energy values (starch values), unchanged.

1 Jour. Landw., 22 (I874), I 78.

${ }^{2}$ Landw. Jahrb., 35 (I906), I 25; 35 Ergzbd. Bd. IV, 327 ; 37 (I908), Ergzbd. Bd. III, I 7I ; 40 (I9I I), Ergzbd. Bd. I, I 29. 
The results show distinct effects of certain feeding stuffs on the milk yield which were apparently quite independent of the supply of digestible nutrients or of energy values, or of the protein supply, and which were consistent when the experiments were repeated.

Hansen ${ }^{1}$ distinguishes three groups of these feeding stuffs. Those of the first group, including "maizena " (apparently gluten feed), maize and oats, increase the quantity of milk but depress the percentage of fat, so that the total yield of fat is not materially changed. Those of the second group, including palmnut meal, cocoa residues, maize distillers' grains, and to a less degree linseed and cottonseed meal and the legumes, increase the total yield of fat without materially affecting the quantity of milk, so that the percentage of fat in the milk is increased. Those of the third group, including poppy cake, "false flax" 2 cake, rice feed and to a less degree sesame cake, diminish the yield of fat but do not sensibly affect the quantity of milk, so that the percentage of fat is decreased.

In a subsequent investigation ${ }^{3}$ on substantially the same plan, Hansen has compared the effects of palmnut cake containing respectively 5.55 and $\mathrm{I} 2.42$ per cent fat when fed in different amounts. He concludes that the specific effect increases with the proportion of palmnut cake in the ration and with the percentage of fat contained in the cake. He finds that to secure significant results in practice, about 2 pounds per rooo pounds live weight of fat-rich cake and $2 \frac{1}{2}$ to 3 pounds of the poorer grades are necessary. Different individual animals have different degrees of susceptibility to the effects of palmnut cake but the result can be obtained if sufficient is fed.

The principal criticism to be made of Hansen's experiments is that the experimental periods were so short - usually 7 days preliminary and 7 days for the experiment proper. It is not an unusual experience in dairy feeding experiments to see a change of rations followed by a temporary stimulation of the milk production which is not sustained, and the question naturally arises whether the "specific" effects which seem to be demonstrated in the first week or two would have continued for a longer time.

1 Loc. cit., Bd. 40, pp. I87-r88.

2 Camelina Sativa.

${ }^{3}$ Landw. Jahrb., 47 (I9I4), 30. 
620. Coöperative experiments. - The coöperative experiments under the auspices of the German Agricultural Council ${ }^{1}$ relate to the influence of palmnut cake or meal and were made according to a common plan at seven different institutions with, in all, I 86 cows. The experimental periods covered about one month each, of which the first 5 to 7 days were regarded as a preliminary feeding. The comparison was between 4 pounds of palmnut meal per Iooo pounds live weight and an amount of a mixture of maize meal and peanut meal supplying equal protein and energy values (computed). After correcting for the advance of lactation, the substitution of palmnut meal resulted not only in each of the 7 experiments as a whole, but with nearly all the individual cows in a distinct increase of the fat production. The total quantity of milk yielded was substantially unaffected, so that the percentage of fat in the milk was increased. The average effects of the palmnut meal were as shown in the following table.

Table i50. - Effects of Palmnut Meal on Milk Production

\begin{tabular}{|c|c|c|c|c|c|c|c|c|c|c|}
\hline & & & & & & & \multicolumn{2}{|c|}{$\begin{array}{l}\text { DAILY INCREASE }(+) \\
\text { OR DECREASE }(-) \text { IN } \\
\text { YIELD }\end{array}$} & \multicolumn{2}{|c|}{$\begin{array}{c}\text { Percentage of Fat in } \\
\text { Milk }\end{array}$} \\
\hline & & & & & & & $\begin{array}{l}\text { Milk } \\
\text { Kgs. }\end{array}$ & $\begin{array}{c}\text { Milk Fat } \\
\text { Grams }\end{array}$ & $\begin{array}{l}\text { On PaIm- } \\
\text { nut Meal }\end{array}$ & $\begin{array}{l}\text { On Check } \\
\text { Ration }\end{array}$ \\
\hline Bonn . . . & . & • & . & . & . & . & -0.29 & +62 & $3 \cdot 58$ & 3.24 \\
\hline Danzig . & - & . & - & . & 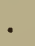 & & +0.58 & +48 & 3.25 & 2.97 \\
\hline Griefswald . & . & . & . & . & . & $\bullet$ & +0.28 & +22 & 3.17 & 3.05 \\
\hline Hamburg . . & . & . & - & . & . & . & +0.09 & +64 & $3.5 \mathrm{I}$ & 3.17 \\
\hline Jena $\cdot$. & . & . & . & . & . & . & -0.04 & $+I_{5}$ & 3.85 & 3.68 \\
\hline Friesdorf . & . & . & . & . & . & & +0.06 & +25 & 3.78 & $3 \cdot 5 \mathrm{I}$ \\
\hline Weihenstephan & - & . & . & - & . & . & +0.02 & +13 & $4.2 \mathrm{I}$ & 4.05 \\
\hline
\end{tabular}

In general, cows that were good milkers seemed more susceptible to the effects of the palmnut meal than those yielding smaller amounts. It was also observed that the effect did not immediately follow the change of feed but developed gradually, reaching its maximum in the course of one or two weeks, and

1 Berichte über Landwirtschaft, Heft 21 and 23. 
continued for a time after the feeding of palmnut meal was discontinued. This fact is of particular interest in its bearing upon the interpretation of Hansen's results.

The evidence of these two series of experiments seems to put the possibility of a "specific" effect of certain feeding stuffs upon milk production beyond doubt. They open up an interesting field for further investigation, both as regards the physiological explanation of the fact and as to its practical significance.

621. Specific effects associated with fats. - In view of what is known regarding the significance of certain fats (or of substances associated with them) for growth (498), it is of interest to note that these "specific" effects on milk production seem to be associated to a considerable extent, although not exclusively, with the fat consumed. Morgen's investigations (613) show that the addition of fat to his flavorless rations had such a stimulating effect up to a certain limit. Many of the feeding stuffs believed to exert such "specific" effects are relatively rich in fat, notably palmnut meal for which the result seems best established. Moreover in the case of the latter material, as just noted, Hansen finds the influence most marked with samples rich in fat. Whether these effects are due to the fat as such or to associated substances, as is believed to be the case in growth, is a matter for future investigation.

622. Influence on utilization of energy. - In conclusion, it may not be superfluous to point out that the stimulating effects of feed on milk production do not necessarily imply any higher utilization of the feed energy supplied. Certain feeds apparently "speed up" the metabolic processes in the udder, but whether the increased production is effected with an increased or a decreased efficiency cannot be determined from experiments of the type thus far made. (Compare Chapter XVII, § r, 737, 738.)

623. Influence of feed on composition of milk. - The results outlined in the last few pages have an obvious bearing on the much discussed question of the effect of feeding on the composition of milk. That such an influence exists has long been the belief of practical dairymen, while the tendency of scientific investigation has been on the whole to throw doubt upon it. Some writers have gone so far as to practically deny that the feeding has any significant influence upon the composition of 
the milk, while others, more conservative, have contented themselves with pointing out the conflicting nature of the evidence.

624. Influence on percentage of fat in milk. - Since fat is the specially valuable ingredient of milk, the discussion has centered around this substance. An increase in the percentage of fat in the milk may result from an increase in the percentage of total solids, i.e., a decrease in the water content, as well as from a specific increase in the fat. The conservative view on this point was thus summed up by Jordan in $1908 .{ }^{1}$

"This question has been much discussed and much investigated from the work of Kühn in I868 down to the present day. Many experiments have been conducted for long periods and short periods in which very moderate rations have been compared with very large ones, highly nitrogenous foods with those of a low protein content, dry with green or succulent materials, and grains of the same class with one another, and, in a great majority of cases, the verdict has been that 'no consistent relation appears to exist between the quantity or character of the ration and the composition of the milk.' The writer has examined the results of nearly all the important experiments of this character of which he could find a record; and in but few cases could he discover that there was a material increase or decrease in the proportion of milk solids which bore a logical relation to variations in the ration. In some cases a temporary change appeared in the milk immediately after a violent change in the ration, but in most instances of this kind there was very soon a return to the animal's normal product. In a small proportion of experiments, the milk appeared to sustain a permanent though not extensive modification. The weight of testimony bears out the statement that the quality of milk cannot be changed at will by the farmer, but is largely determined by causes not under his control, such as breed and individuality, although feeding and treatment, especially the latter, have more or less influence upon the character of the milk secreted."

Much of the alleged effect of feeding stuffs upon the composition of milk is associated with the question of the so-called "specific" effects of feeding stuffs (618). As was pointed out

1 The Feeding of Animals, 5th Edition, The Macmillan Co., New York, rgo8, pp. 3 I $7-3$ I 8 . 
in considering that question, both the planning and execution of many of the older experiments were defective and their results must be regarded as inconclusive. The more recent experiments of Hansen and of the German Agricultural Council, on the other hand, as well as the investigations of Morgen, Fingerling and their associates upon the influence of feed fat and of condiments upon milk production, afford numerous apparently unquestionable instances of an effect of the ration upon the fat content of the milk.

For example, the experiments of the German Agricultural Council on palmnut meal (620) showed an average increase in the percentage of fat ranging in the different experiments from 4 per cent to I I per cent, while individual cows showed even more striking differences. Morgen's results on the specific effect of feed fat when added to fat-poor rations (613) and likewise Fingerling's results regarding the influence of condiments (617) afford, even more striking examples of the same effect. Apparently it must be admitted that, under some conditions, the fat content of milk may be distinctly affected by the feeding and that this effect appears to be associated with the fat supply of the ration.

625. Influence on percentage of fat in solids. - Furthermore, it appears from such of these latter experiments as included determinations of the total solids of the milk that the increase in fat content was essentially a " one-sided" increase, i.e., that the proportion of fat to other solids in the milk was increased. This was notably the case in the majority of experiments on fat-poor rations, in which the proportion of fat in the milk solids was increased by from I 2.5 per cent to 23.5 per cent by the addition of fat to the feed. Similar although much less marked results were also obtained in Fingerling's experiments upon the influence of condiments.

In Hansen's experiments, too, the influence on the fat content of the milk was due, in the majority of instances, largely to an increase or decrease of the percentage of fat contained in the milk solids, the increase or decrease over the comparison rations being over 5 per cent in fully one-third of the experiments, while Lindsey ${ }^{1}$ has confirmed Hansen's results as regards cocoa meal. 
It seems clear from the foregoing facts that the proportion of fat in the milk solids, as well as the total yield of fat and its percentage in the fresh milk, may be influenced, temporarily at least, by the nature of the feed, and it may be presumed that some of the results obtained on this point in the earlier and less conclusive experiments did, as a matter of fact, represent a real effect of this sort.

626. Significance in practice. - Too much stress must not, however, be laid on the physiological facts apparently established by the evidence just considered. It still remains true that those major differences in the composition of milk from different sources which are of commercial importance are due to breed and individual differences in animals (564). As has been repeatedly insisted, the prime factor in successful dairying is the capacity of the animal as a milk producer. The quality of milk best suited to meet the demands of a particular market is most easily and certainly secured by intelligent breeding and selection, while any influence of the feed is essentially a secondary factor. At the same time the results seem to indicate that while feed is a secondary factor it is not altogether a negligible one. If it is possible by suitable selection of feed to permanently increase the fat yield to any such extent as has been observed in short experiments, or if, on the other hand, it may be depressed by an unsuitable choice of feeding stuffs, the matter is one of considerable importance and might well be made the subject of large scale coöperative investigations similar to those of the German Agricultural Council on palmnut meal. 


\section{CHAPTER XIV}

\section{WORK PRODUCTION}

627. Prime purpose of excess feed. - Aside from reproduction, the prime purpose for which a mature animal consumes feed in excess of its maintenance requirement is the production of the external mechanical work required for its diverse activities, either natural in the wild animal or enforced in the domesticated work animal. It is true that more feed may be consumed than is required for this purpose and that a fattening of the animal may result. The latter, however, is simply a laying aside of reserve material which may be utilized later and, however important economically, may be regarded as physiologically incidental. Any considerable fattening of the work animal is not only a diversion of energy from the main purpose of the feeding but constitutes an extra weight to be carried by the animal, while if too extensive it may interfere with heart action and respiration.

Since horses or mules are substantially the working animals of the United States, the following discussion will have reference chiefly to these animals.

\section{$\S$ I. The Physiology of Work Production}

\section{Nature of muscular work}

628. The muscles. - Mechanical work is performed by an animal by means of its muscles $(84,85)$, of which there are two kinds called, respectively, striped, or striated, and smooth, or non-striated, muscles from the appearance of the microscopic fibers of which they are composed. The skeletal muscles, by means of which external work is performed, are striated muscles. They are also called voluntary muscles because they are inner- 
vated from the cerebro-spinal system and are under the control of the will. The muscles of the internal organs are chiefly non-striated muscles, the heart being the conspicuous exception, and are to a very limited degree subject to the will, being innervated from the sympathetic nervous system. In the study of work production, therefore, we have to do chiefly with the phenonena of striated voluntary muscles.

The physiology of the muscle and of muscular contraction is a very complex subject and wide differences of opinion exist regarding many aspects of it. All that is attempted here is to outline such general features as seem necessary for a proper comprehension of its relations to nutrition.

629. Contraction. - When a suitable stimulus, which in the living animal is usually a nerve stimulus, is applied to a muscle it contracts, that is, it tends to grow shorter and thicker. This change is brought about by a shortening and thickening of the individual fibers of which the muscle is built up. A single stimulus, such, for example, as that caused by the making or breaking of an electric circuit, gives rise to what is known as a simple muscular contraction or twitch. If such a stimulus is repeated with sufficient frequency it produces a series of simple contractions which fuse together, resulting in a state of contraction which continues, subject to the effects of fatigue, as long as the stimulus acts. This form of muscular contraction has received the name of "tetanus." In the living animal the ordinary contractions of the muscles, brought about by the nervous system, even those that seem but momentary, are essentially tetanic in their character.

The term contraction as used in connection with the physiology of muscle does not, however, necessarily imply an actual shortening of the muscle. Contraction may either be isotonic or isometric. When the muscle in contracting overcomes a constant resistance, as, for example, in raising a weight, the contraction is said to be isotonic. When, on the other hand, the points of attachment of the muscle are fixed, evidently no work can be done in the mechanical sense but the muscle still contracts in the physiological sense, i.e., exerts a pull. Such a contraction is called an isometric contraction.

630. Chemical changes in contraction. - In a muscular contraction, either isotonic or isometric, there occurs a rapid 
katabolism of materials contained in the muscle or brought to it by the circulation together with a corresponding transformation of their chemical energy. This katabolism is in effect an oxidation, yielding chiefly carbon dioxid and water, but as to the details of the process, the views of physiologists differ.

Certain general features of muscular katabolism are fairly well made out. First the immediate accompaniment of contraction is not an oxidation but a rapid, almost explosive, breaking down of a substance or substances present in the muscle, causing the production of carbon dioxid. It has been shown, according to Zuntz and Loewy, that the muscle contains no free oxygen. Nevertheless, it contracts instantaneously when stimulated, while the effects upon the blood supply follow later, circulation and respiration being stimulated by the carbon dioxid and other products formed. Furthermore, it has been shown that, under certain conditions at least, a muscle may continue to contract and give off carbon dioxid in the entire absence of oxygen.

With continued activity of the muscle, there is established more or less distinctly a state of equilibrium with the increased blood supply, oxygen being taken up by the muscle and carbon dioxid given off, while, according to a number of experimenters, the dextrose of the blood also disappears during its passage through the muscle. Other products of muscular katabolism, notably lactic acid and potassium mono-phosphate - the so-called fatigue products - tend to accumulate in the muscle and diminish and finally suspend its ability to respond to a stimulus. Fatigue of the muscles usually results from a gradual accumulation of these substances and not from lack of material to be katabolized.

631. Energy transformations. - The katabolism of matter which takes place in muscular contraction implies an equivalent conversion of chemical energy into kinetic energy. The energy thus transformed appears finally in the two forms of heat and visible motion (work) though the ratio between the two may vary widely under different conditions. As regards the intermediate stages of this process, relatively little certain knowledge is yet available. Broadly, it may be said that there are two possible general views. The first of these considers that the potential energy of the material katabolized is first converted into heat, and that subsequently a portion of this heat is converted into mechanical motion. The second 
general view considers that heat and work are simultaneously produced, a portion of the energy taking one form and a portion the other. The former view has been supported by no less distinguished an authority than Englemann, but nevertheless it has not been generally accepted by physiologists. In particular, it is difficult to conceive of the existence in a muscle of sufficient temperature differences to account for its observed efficiency. In other words, the muscle is not in general regarded as being a heat engine. The prevailing view, stated in the broadest outline, is that in the chemical changes consequent on a stimulus, energy is in part liberated as heat and in part expended in producing or maintaining tension of the muscle fibers. To use a simple illustration, it is as if by some process the elasticity of a cord supporting a weight were to be suddently increased. The cord would contract and the weight would be lifted for a certain distance. In isotonic contraction, that is, when the muscles are free to shorten, the increased tension set up does mechanical work. In isometric contraction this increased tension is also finally converted into heat, as for example in the case of muscular contraction applied to simply sustaining a weight. In this case no work in the mechanical sense is done, but energy is expended in what has sometimes been called "static work." A familiar illustration of "static work " is the muscular effort required in standing.

632. Tonus. - In the foregoing paragraphs it has been tacitly assumed that before and after a contraction the muscle is absolutely relaxed. Such is not normally the case. Even in a state of rest, so-called, there is a greater or less degree of tension of the muscles, especially during the wakening hours, known as tonus or tonic contraction. In other words, the living muscle is slightly on the stretch, as is shown by the fact that it gapes open when cut or shortens when its connections with the bone are severed. This tension, like the much greater one set up in active contraction, is maintained, in part at least, by a continual katabolism in the muscle, which respires, taking up oxygen and giving off carbon dioxid. In other words, the "resting" muscle is in a state of slight isometric contraction and is doing "static work." According to the principles just enunciated, all the energy transformed in such a muscle finally takes the form of heat, so that, as indicated in Chapter VII 
(348), muscular katabolism is the most important source of heat in the animal body. The degree of tonus and consequently the rate of heat production seems to vary at different times and in different bodily conditions. During profound sleep it is much reduced. It is probably increased by all conditions which favor the development of a vigorous muscular system. What is ordinarily spoken of as a muscular contraction, therefore, and especially a tetanic contraction, is in a sense an enormous increase of a condition already existing in the muscle.

\section{Secondary effects of muscular exertion}

The great increase of the muscular katabolism during the performance of work gives rise to important secondary effects, particularly upon the circulation and respiration. It is a familiar fact that in active exercise the heart action is largely increased and the breathing becomes deeper and more rapid, and that ordinarily the limit to muscular exertion is set, not by the power of the muscles themselves but by the ability of the heart and lungs to keep pace with the demands upon them.

633. Circulation. - The circulating blood is the medium by which oxygen is conveyed to the muscles and carbon dioxid and other products of their katabolism removed. The latter function is of special importance because an accumulation in the muscle of the products of its own katabolism speedily reduces and ultimately suspends its power to contract. In muscular exercise, therefore, an increase in the rate of circulation is essential to the continued activity of the muscles. For example, in experiments by Chauveau and Kaufmann ${ }^{1}$ the ratio between the circulation in the resting as compared with the active muscle in the living animal varied between $I: 3.35$ and $\mathrm{I}: 6.60$. Zuntz and Hagemann, ${ }^{2}$ in their investigations upon the work of the heart, found the average amount of blood passing through the heart of a horse per minute to be during rest 29.I6 liters and during work 53.03 liters. By this increase in the rate of circulation through the muscles the carbon dioxid and other injurious products of the muscular katabolism

1 Comptes rend., 104, I I 26, I352, I 409.

2 Landw. Jahrb., 27 (I898), Supp. III, 405. 
are rapidly removed and an abundant supply of oxygen is ensured. In fact, it is usually true that during work which is not excessive the venous blood contains less carbon dioxid and more oxygen than during rest.

Since the heart is a muscular organ, it is obvious that this increase in the circulatory activity must add materially to its metabolism. In the performance of work, therefore, there is an expenditure of matter and energy, not only for the work of the skeletal muscles, but likewise for the additional work of the heart. Zuntz and Hagemann in their experiments upon the horse just mentioned compute that during moderate work the katabolism due to the work of the heart amounts to 3.8 per cent of the total katabolism of the body.

634. Respiration. - The greater activity of the circulation consequent upon muscular exertion would be futile were not provision made for more efficient aëration of the blood in the lungs through an increased activity of the respiration. Under the stimulus of the carbon dioxid and other katabolic products of muscular activity which enter the blood, the respiratory movements are increased in frequency or depth or both, as described in Chapter IV (194), thus making possible a more rapid gaseous exchange between the blood and the air in the lungs. This action is usually so efficient that the expired air during work contains a smaller proportion of carbon dioxid than it does during rest, notwithstanding the fact that the total quantity eliminated is much greater. Since respiration, like circulation, is maintained by muscular action, it is true in the former case as in the latter that a greater activity of the function necessitates a greater metabolism for that purpose.

\section{Effect of work upon protein katabolism}

As already indicated, knowledge of the details of muscular katabolism is still meager. The student of nutrition, however, is less directly interested in these details than he is in knowing the aggregate effect of the performance of work upon the expenditure of matter and energy by the body under varying conditions, since it is this latter which must be made good by the feed supply. Much effort has therefore been devoted to studies of the influence of muscular exertion upon the kind and 
amount of material broken down in the body during work. It will be convenient to consider the effects of muscular work, first upon the protein katabolism and second upon the katabolism of non-nitrogenous material.

635. Early views. - Since the muscles, by means of which work is performed, consist largely of protein, it was not unnatural for the early physiologists to suppose that the substance of the muscle itself was consumed and yielded the energy for the work done. This was Liebig's view, although it does not seem to have been based upon any actual experimental results. He taught that work was performed at the expense of a katabolism of protein in the muscles, causing an in. creased excretion of nitrogenous by-products and an increased demand for protein in the feed, while the carbohydrates and fats of the feed were regarded as simply heat and fat producing materials.

636. Analogy with engine. - The analogy drawn in Chapter VI (274-276) between the body and an engine, however, might of itself lead one to question the truth of this view. An engine does not do work by burning up its own substance but by burning fuel material, and if it is well constructed the wear due to the work imposed upon it is comparatively slight. It might be reasonably expected, therefore, that the machinery of the animal body would prove to be at least as perfectly constructed as an artificial machine and at least equally capable of converting the energy of fuel material into work without destroying the materials entering into its own structure. That such is indeed the case under normal conditions was first shown by Carl Voit, whose results have been fully confirmed by later investigators.

Voit's first investigation ${ }^{1}$ was upon a dog alternately resting and doing considerable work on a treadmill both when fasting and upon a liberal meat diet. The results are shown in the first of the two following tables, while the second contains the average results of a later series of similar experiments by Pettenkofer and Voit ${ }^{2}$ on a man.

1 Untersuchungen über den Einfluss des Kochsalzes, des Wassers, und der Muskelbewegungen auf den Stoffwechsel. r860. Summarized by E. v. Wolff in Die Ernährung der landw. Nutzthiere, pp. 386-388.

2 Ztschr. f. Biol., 2 (1866), 478. 
Table i5I. - Effect of Work on Protein Katabolism of a Dog

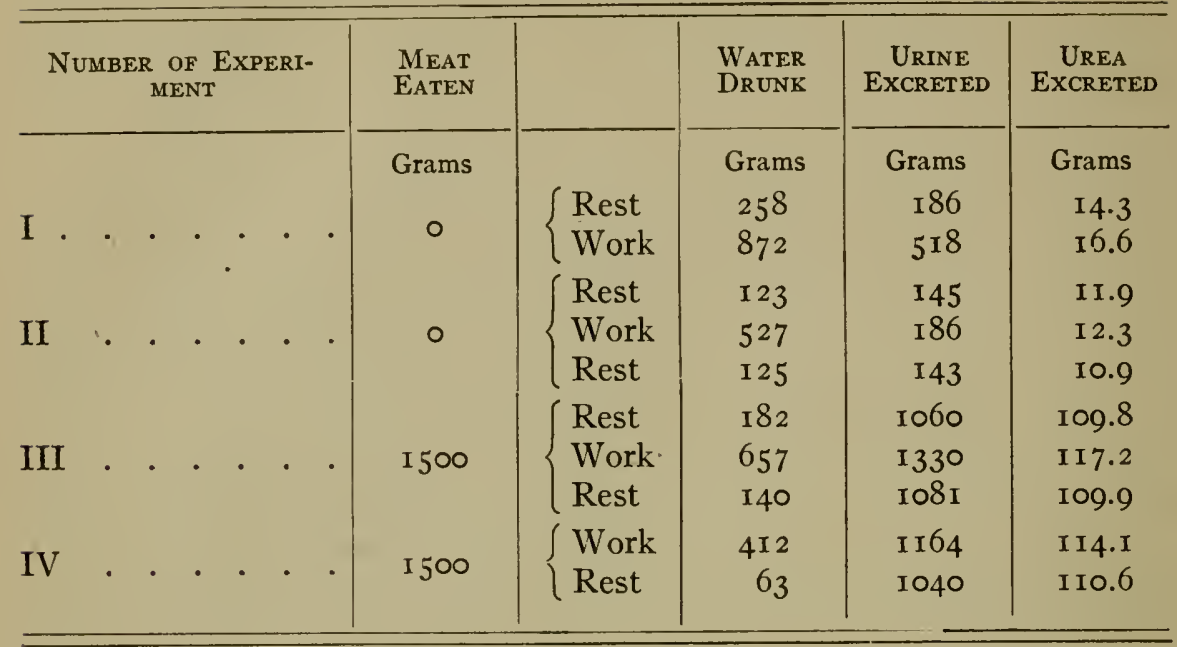

Table 152. - Effect of Work on Protein Katabolism of a Man

\begin{tabular}{|c|c|c|c|c|c|c|c|c|c|}
\hline & & & & & & & & $\begin{array}{l}\text { NUMBER OF } \\
\text { EXPERTMENTS }\end{array}$ & $\begin{array}{c}\text { UREA EX- } \\
\text { CRETED }\end{array}$ \\
\hline Fasting & & & & & & & & & Grams \\
\hline Rest & . & . & . . & . . & . & . . & & 2 & 26.5 \\
\hline Work & . & . & . . & . . & . . & . . & . . & I & 25.0 \\
\hline Average diet & & & & & & & & & \\
\hline Rest & . & . & . . & . . & . . & . . & . . & 3 & 33.6 \\
\hline Work . & 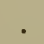 & . & . . & . . & . & . . & . & 2 & 36.8 \\
\hline
\end{tabular}

In the case of the man, while there was a great increase in the amount of carbon dioxid and water excreted, there was practically no increase in the excretory nitrogen. With the dog fasting or on a meat diet only, there was in every case a small increase which Voit attributes to a deficiency of non-nitrogenous nutrients and not to the direct effect of muscular exertion.

637. Influence of non-nitrogenous nutrients. - While Voit's results seem quite in harmony with present conceptions of the animal organism as essentially a converter of energy, they aroused considerable criticism at the time and led to an extended controversy as to the source of muscular energy. The effect 
of work upon the protein katabolism was repeatedly investigated under the most varied conditions with results which appeared upon their face to be conflicting. Some observers found a marked increase in the excretion of nitrogen during or following work, while in other investigations no such effect was apparent. The key to these conflicting results seems to have been first discovered by Kellner in 1879 in experiments upon the work horse. ${ }^{1}$ He found that so long as the total amount of feed was ample, variations in the quantity of work performed were without effect upon the protein katabolism. If, however, the work was increased to an amount sufficient to cause a falling off in the weight of the animal, thus indicating that the energy supply was insufficient, the excretion of nitrogen in the urine increased promptly. Furthermore, it was found that if either carbohydrates or fat were added to a ration which was just sufficient to enable the animal to perform a given amount of work, the demands upon the animal could be correspondingly increased without causing any increase in the protein katabolism. This may be illustrated by the following summary of an experiment in which the addition to the ration consisted of starch and in which the amount of work performed is expressed in the number of revolutions of the sweep power dynamometer used.

Table I53. - Effect of Starch on Protein Katabolism of Working Horse

\begin{tabular}{|c|c|c|c|c|c|c|}
\hline & \multirow{2}{*}{\multicolumn{2}{|c|}{ Period }} & \multirow{2}{*}{$\begin{array}{l}\text { WORK, REVO- } \\
\text { LUTIONS OF } \\
\text { DYNAMOMETER }\end{array}$} & \multicolumn{2}{|c|}{ NItRogeN } & \multirow{2}{*}{$\begin{array}{c}\text { LIVE } \\
\text { WEIGHT }\end{array}$} \\
\hline & & & & Digested & In Urine & \\
\hline & \multirow{6}{*}{ Without starch } & \multirow{6}{*}{..} & & Grams & Grams & Kgs. \\
\hline $\mathbf{I}$ & & & 300 & I 2 I.I & 107.2 & 540.0 \\
\hline II-a & & & 600 & I 2 I.I & I 10.2 & $53^{8.3}$ \\
\hline $\mathrm{II}-\mathrm{b}$ & & & 600 & I 2 I.I & I 15.6 & 533.1 \\
\hline III & & & 500 & I 2 I.I & IO9.4 & 532.5 \\
\hline IV & & & 400 & I 2 I.I & iog. 6 & 530.7 \\
\hline I $\}$ & \multirow{2}{*}{ With starch } & \multirow{2}{*}{..} & 800 & I 20.I & I I 5.5 & 517.1 \\
\hline II) & & & 600 & I 20.1 & 109.6 & 5 I 5.4 \\
\hline
\end{tabular}

${ }^{1}$ Landw. Jahrb., 8 (1879), 701 ; 9 (1880), 65 I. 
The effect of even a small excess of work in increasing the nitrogen excretion of the horse was so sharp that Kellner even attempted to determine how much work could be performed at the expense of a given weight of starch or fat by increasing the demand upon the animal up to the point where it just failed to cause an increase in the nitrogen excretion and a fall in live weight.

A considerable number of more recent experiments have fully confirmed Kellner's conclusion that a deficiency of non-nitrogenous nutrients is the chief cause of the increased protein katabolism which sometimes occurs during work and have shown that in the presence of a sufficient amount of fats and especially of carbohydrates even severe work can be performed without increasing the nitrogen excretion. Indeed, moderate work continued for a number of days has in some cases been accompanied by a gain of nitrogen, a fact apparently quite in accord with the common experience that the muscles are strengthened by exercise. It is clear that the body normally uses non-nitrogenous materials as the source of the energy expended in muscular work, exactly as it does in the case of the energy required for its internal activities. Only when the supply of non-nitrogenous materials is inadequate does it resort to the katabolism of protein as a source of energy for external work, precisely as it does during fasting or on exclusive protein feeding as a source of energy for internal work $(339,407)$.

\section{Effect of work upon the katabolism of non-nitrogenous matter}

638. Gaseous exchange increased. - In striking contrast with the minimal effect of work upon the excretion of nitrogen is its very marked effect in increasing the consumption of oxygen and the excretion of carbon dioxid and water. This increase is too obvious from common experience and too well established scientifically to require more than an illustration. The fact of such an increase was shown in the researches of Lavoisier and confirmed by the earlier experimenters in this field, such as Scharling in 1843 , Hirn in 1857 and especially Smith in I859. The investigations of Pettenkofer and Voit in I866, however, appear to have been the first to be executed according to modern methods. Their results regarding the influence of work upon 
the protein katabolism have already been cited (636) but may be repeated in connection with those obtained with the aid of the respiration apparatus.

Table 154. - Influence of Work on Gaseous Exchange of Man

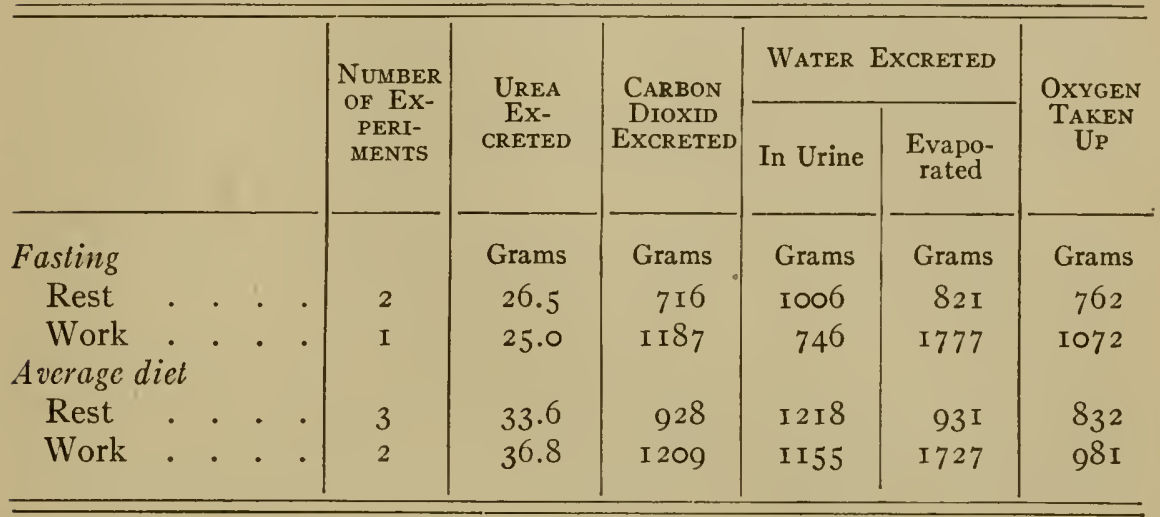

639. Effects are immediate. - Experiment confirms the common observation that the increased pulmonary exchange consequent upon muscular exertion begins almost immediately, reaches its maximum in a very short time and disappears promptly when the work ceases. This is especially true of the absorption of oxygen, of which no considerable amount appears to be stored up in the body in the free state. In the case of the excretion of carbon dioxid, more or less of this gas can be held in solution in the blood and lymph and there is consequently some slight lag in its excretion.

In view of this prompt adjustment of the respiration to the amount of work, determinations of the pulmonary exchange by some one of the forms of respiration apparatus described in Chapter VI (297-299) are especially useful in studying the effects of work upon the katabolism. The use of this method renders it possible to compare the gaseous exchange during periods of work with that of the same animal at rest and thus to determine very sharply the additional oxygen consumption and carbon dioxid excretion caused by a measured amount of work. The comparative simplicity of the apparatus required, the ease with which the respiratory changes can be followed in short periods, and the fact that both oxygen and carbon dioxid 
can be determined, have led to the extensive use of this method for investigations upon work production.

640. Nature of non-nitrogenous material katabolized. Since under normal conditions muscular exertion does not increase the protein katabolism, it follows that the substances oxidized for the performance of work must be substantially either carbohydrates or fats. If the former, each volume of carbon dioxid given off will correspond to an equal volume of oxygen taken up; that is, the respiratory quotient (296) will be I.o. On the other hand, if the material oxidized consists solely of fat, the respiratory quotient will be approximately 0.7 , while if both are being consumed, it will have an intermediate value. Moreover, it is comparatively simple to calculate from the respiratory quotient the proportions in which the two are being katabolized. Investigations of this sort show that the proportions of fat and carbohydrates katabolized for the performance of work may vary within wide limits, both groups being readily available as sources of energy.

\section{Sources of energy for muscular work}

641. Proteins vs. non-nitrogenous matter. - Liebig's assumption (635) of an increase of the protein katabolism in muscular contraction implied that the proteins were the source of the energy manifested, and this view prevailed for many years. When Voit, in I860, showed (636) that muscular exertion is not necessarily accompanied by any material increase in the protein katabolism, the inference seemed unavoidable that non-nitrogenous materials were the main sources of muscular energy. This conclusion, however, was too radical to be at once accepted in opposition to Liebig's authority and numerous ingenious, but not always convincing, hypotheses were advanced to explain the observed phenomena on the assumption that the proteins were, nevertheless, the source of the energy expended.

642. Fick and Wislicenus' experiment. - The first attempt, however, at a quantitative comparison of the work performed with the energy available from the protein katabolized during its performance was the famous experiment of Fick and Wislicenus ${ }^{1}$ in 1866 . These observers made an ascent of the Faul-

1 Vrtljschr. Naturf. Gesell. Zurich, 10, 317. 
horn, a Swiss mountain 64I 8 feet high, after having abstained from nitrogenous food for 17 hours, and found that the amount of protein katabolized during the six hours occupied by the ascent and the seven succeeding hours of rest, as measured by the urea excreted, was insufficient, according to their computations, to account for more than about one-third of the energy required to lift their bodies to the height of the mountain, making no allowance for the work of the internal organs nor for those muscular exertions which did not contribute directly to the work done. They observed no considerable increase in the urinary nitrogen over that excreted before the ascent.

643. Protein insufficient as source of energy. - It is true (637) that with an insufficient supply of non-nitrogenous materials in the feed muscular exertion may lead to an increase in the protein katabolism, but in the many comparisons which have been made since the time of Fick and Wislicenus by far more refined methods than were available to them, this increase has been shown to be entirely inadequate to furnish the energy for the work performed. Moreover, even the supposition that the energy of the total protein katabolized was all applied to work production usually fails to account for the energy expended.

The facts, then, first, that the chief, and often the only, effect of muscular work is to increase the katabolism of non-nitrogenous material; second, that even the total protein katabolism is in most cases insufficient to supply the energy expended in work; and third, that, as Kellner (637) has shown, the addition of non-nitrogenous nutrients to the ration enables more work to be done; demonstrate beyond cavil that under ordinary conditions of nutrition it is the non-nitrogenous ingredients of the body and of the feed which supply most or all of the energy expended in the performance of work.

644. Functions of proteins. - The foregoing statements should not be understood as an assertion that the proteins play no part in the production of muscular work. In the first place, their katabolism furnishes a considerable amount of nonnitrogenous products $(\mathbf{2 2 9}, \mathbf{2 3 3})$ and that these products are available to supply energy for work has been strikingly shown by Pflüger. He maintained a dog for about nine months on an exclusive diet of almost fat-free meat and showed that on this diet the animal was capable of performing large amounts of 
work. Aside from the small quantities of fat and glycogen contained in the meat the energy for work under these conditions could have been derived only from the proteins or their cleavage products. These results show clearly that protein may be used to a large extent as a source of muscular energy, but it is nevertheless true that under ordinary conditions, and particularly with farm animals, the main supply of energy is, as already stated, through the non-nitrogenous ingredients of the feed.

It is by no means impossible, however, that a certain amount of protein katabolism may be necessary in a muscular contraction. Such a contraction is a function of the protoplasm of the muscle fibers and it is conceivable that a portion of the energy arising from the katabolism of the proteins and nucleoproteins of the muscle and ordinarily appearing as heat in the resting muscle may be switched off, so to speak, to aid in producing the contraction. In other words, it is possible that a certain level of protein metabolism may be necessary in order to maintain the most favorable conditions for transforming the potential energy of non-nitrogenous materials into work. ${ }^{1}$ Such a fact would, of course, have an important bearing upon the amount of protein required for a working animal, but at present the matter belongs in the realm of speculation.

\section{§ 2. The Efficiency of the Body as a Motor}

\section{General results}

645. Body substance is immediate source of energy. While the energy expended in work production is of course derived ultimately from the feed consumed, its immediate source, as stated in $\S \mathrm{I}(\mathbf{6 3 0})$, is the katabolism of body substance, and an animal may perform a considerable amount of labor in the fasting state at the expense of stored-up material. It will aid in the discussion of the somewhat complicated question of the efficiency of the animal as a prime motor to consider first the efficiency with which the body utilizes this stored-up energy, i.e., to inquire what percentage of the total energy of the body material katabolized for work production is recovered in the

1 Compare Armsby, Principles of Animal Nutrition, pp. 207-209. 
work done, deferring to the following section a study of the efficiency of the animal as a converter of feed energy into useful work and of the feed requirements of work animals.

646. Mechanical efficiency of muscle. - A muscle may be regarded as a machine for the conversion of chemical energy into mechanical work and one may, therefore, speak of its efficiency in somewhat the same sense as of that of a steam engine or an electric motor. By efficiency in this sense is meant the proportion of the total energy mobilized during a contraction which is recovered in the work done. Thus if an isolated muscle lifts a weight of ten grams through one centimeter, it does Io gram centimeters of work, equivalent (308) to $0.2344 \times 10^{-4}$ gram calories. If the increased katabolism caused in the muscle by its contraction were shown to be $0.4688 \times 10^{-4}$ gram calories, the efficiency of the muscle would be $0.2344 \div 0.4688=50$ per cent, that is, 50 per cent of the total energy mobilized would be recovered as mechanical work.

Much experimental work has been devoted to the study of the single muscle as a machine. The subject is a complicated one, and unanimity of views upon it, especially as to the mechanism of muscular contraction, has by no means been reached. As regards the efficiency of the muscle as a converter of energy, however, one fact is perfectly well established, viz., that it varies within quite wide limits, depending especially upon the load as related to the capacity of the muscle and upon the degree of shortening.

647. Mechanical efficiency of the body as a whole. - If the amount of energy mobilized in each muscle concerned in the performance of a certain form of work were known, it is conceivable that, assuming each muscle to act with its maximum efficiency, an average theoretical efficiency might be computed for the whole group of muscles. The conditions for the maximum efficiency of a muscle, however, seldom or never obtain in the working animal, and consequently this hypothetical efficiency is not attained. Of its many muscles, some serve largely or wholly to maintain the relative positions of the different parts of the body, i.e., their contractions are isometric (629) and consequently have an efficiency approaching zero. Others contract to a varying extent and under loads less than the maximum. Some muscles, owing to their anatomical re- 
lations, work at a less mechanical advantage than others, while the extent to which a group of muscles is called into action will vary with the nature of the work. Moreover, the performance of labor by an animal sets up various secondary activities, notably of the circulatory and respiratory organs $(633,634)$, which consume their share of energy and yet do not contribute directly to the performance of the work, and the extent of these secondary activities varies with the nature and the severity of the work. Some of these sources of loss of energy are analogous to the radiation losses from the cylinder of a heat engine, while others are comparable with the internal resistances of the engine itself.

Determinations of the efficiency of the isolated muscle, therefore, afford no adequate means of estimating the efficiency of the body as a whole and the latter must be determined by direct experiment. Such a determination is made by causing the animal to perform a measured amount of work under conditions which also permit the measurement, either directly as heat or by the methods of indirect calorimetry described in Chapter VI, of the total body energy metabolized.

Thus in experiments by Benedict and Cathcart ${ }^{1}$ upon a man riding a bicycle ergometer, the subject breathed through the mouthpiece of a Benedict universal respiration apparatus (298), by means of which the oxygen consumption and the carbon dioxid elimination could be determined. From these data the amount of energy metabolized in the body was computed and compared with the amount of mechanical work done as measured by the ergometer. For example, in one of these tests the energy output per minute as computed from the respiratory exchange was 6.32 Cals., while the mechanical work done per minute was equivalent to 1.02 Cals. In other words, I.02 $\div 6.32=16$. I per cent of the total energy output was recovered as useful work, the remainder taking the form of heat.

648. Gross and net efficiency. - Comparisons like that of the preceding paragraph give what is called the gross efficiency of the body, i.e., they show what proportion of the total energy metabolized during work is recovered in the useful work done. It is analogous to the efficiency of an engine as computed from

${ }^{1}$ Muscular Work; Carnegie Inst. of Washington, Publication No. I87 (I913). 
a comparison of the brake horse power with the steam consumption.

But the body katabolizes matter and liberates energy for other purposes than the performance of external work, - i.e., it has a maintenance requirement for the support of its internal work $(341,342)$ analogous in some respects to the energy required to run an engine without load. The subject of Benedict and Cathcart's experiment produced during rest (lying on a couch) r.o9 Cals. of heat per minute. If this maintenance requirement be subtracted from the total energy output during work there is left 5.23 Cals., as the additional energy output required for the performance of the 1.02 Cals. of measured external work. Computed in this way an efficiency of $1.02 \div 5.23$ $=$ I 9.5 per cent results. This has been called the net efficiency. It shows the utilization of that portion of the energy output which is expended in the physiological processes required for the production of external work as distinct from the various forms of internal work included in the maintenance requirement. In computing the net efficiency in this way difficulty arises in deciding upon the proper deduction to be made. Thus in an experiment like that just cited, one may subtract from the total energy output of the body during work, not only the energy expenditure for maintenance during rest but likewise that caused by sitting on the ergometer and causing it to rotate without load, and the remainder may be regarded as the energy metabolized for the performance of the useful work. The total output of energy being 6.32 Cals. per minute during the work, it was determined that the same subject metabolized I.I3 Cals. more energy per minute when riding without load than when at rest. The added load in the work experiment, therefore, required the expenditure of $6.32-($ I.O9 + I.I 3$)=4.10$ Cals. per minute for the performance of 1.02 Cals. of work, from which an efficiency of 24.9 per cent may be computed. Similarly, in experiments with the work horse one may subtract the energy expended during horizontal locomotion instead of that metabolized during rest and compare the remainder with the useful work done. ${ }^{1}$

1 For a discussion of the various base lines for the computation of efficiency, compare Benedict and Cathcart's publication already cited, pp. II2-136, and also. Reach, Biochem. Ztschr., 14 (I908), 430; Landw. Jahrb., 37 (I908), I053. 
This method of computation is unlike any usually employed by the engineer, and Schreber ${ }^{1}$ has criticized it severely. The engineer is accustomed to estimate the losses due to the internal resistance of an engine by a comparison of brake horse power and indicated horse power. No method exists, however, for determining the indicated horse power of the animate motor, if indeed it permits of any corresponding conception, and only the method of comparison just outlined is available. It is as if the engineer had no indicator and estimated the efficiency of his engine by deducting from the total steam consumption that required to run the engine empty and compared the remainder with the external work done. The internal work of the animal, however, like that of the engine, is largely mechanical. If, on the basis of Zuntz's computation of the efficiency in locomotion (652), it may be assumed that this internal work is performed with approximately the same efficiency as the external work, then the net efficiency of the animal will be somewhat analogous to the efficiency of the steam in the cylinder of the engine.

649. Gross efficiency variable. - It should be observed that while the net efficiency may be regarded as substantially constant under a considerable variety of conditions, the gross efficiency will vary with the ratio of work done (load) to maintenance requirements. Thus if Benedict and Cathcart's subject had done. only half as much work per minute with the same net efficiency, his gross efficiency would have been only I3.7 per cent instead of I6.I per cent.

\section{Total energy expended per minute.}

For mechanical work, $0.5 \mathrm{I} \mathrm{Cal.} \div 0.195=2.62$ Cals.

For maintenance

$$
\text { Total }
$$

Recovered in work done

Gross efficiency

1.09 Cals.

3.7 I Cals.

$0.5 \mathrm{I}$ Cals.

I3.7 per cent

Up to the point at which the net efficiency begins to be affected, the gross efficiency will increase with increasing load as Benedict and Cathcart show experimentally to be the case. This influence of the maintenance requirement upon the computation of the utilization of energy is identical with that to which attention has already been called in connection with the utilization for material products. If the useful work performed 
be reduced to zero, as for example in horizontal locomotion, the gross efficiency of course also becomes zero.

650. Efficiency per day. - The figures for either gross or net efficiency show the efficiency for the time during which the work is being done. Since, however, it is not practicable to stop the animal machine when the demand for work ceases, the efficiency for the entire 24 hours, i.e., the degree to which the body energy is utilized in practice, will evidently vary with the number of hours work done per day. Thus if Benedict and Cathcart's subject had been able to work 8 hours per day at the same rate as in the experiment just cited, his gross efficiency for the 24 hours would have been as follows :-

Energy expended

$$
\begin{aligned}
& 480 \text { minutes work @ } 6.32 \text { Cals. = } 3034 \text { Cals. } \\
& 960 \text { minutes rest @ r.09 Cals. }=\frac{\text { ro46 Cals. }}{4080 \text { Cals. }}
\end{aligned}
$$

Work done

480 minutes @ I. 02 Cals. $\quad=490$ Cals.

Efficiency per day

$$
\text { I2.0I per cent }
$$

On the other hand, if he had worked only one hour per day, it may be presumed that both the net and gross efficiency of the work production during the hour of work would have been substantially the same but the efficiency for the 24 hours would have been much lower, viz.,

Energy expended

60 minutes work @ 6.32 Cals. = 379 Cals.

$$
\text { I380 minutes rest @ } 1.09 \text { Cals. }=\frac{1504 \text { Cals. }}{1883 \text { Cals. }}
$$

Work done

60 minutes work @ r.02 Cals.=6r Cals.

Efficiency per day

3.24 per cent

In discussions of the efficiency of a man or an animal as a motor, and particularly in comparisons with artificial motors, it is essential to distinguish clearly whether the net, or the gross efficiency is meant and likewise to base the comparisons upon the performance per day. Since the net is apparently less affected than the gross efficiency by variations in the intensity and duration of the work, it appears to be the most logical 
method of comparison in the case of the animal as well as being the most convenient in practice.

651. Analysis of total work. - A quadruped performs work by means of locomotion, with or without draft, either horizontally or on an inclined plane. The work which it performs may therefore be subdivided into work of locomotion, work of draft and work of ascent and the efficiency for each form computed separately. The same is of course true of man, but in addition other forms of work, such as turning a crank with the hands or with the feet (stationary bicycle) or lifting a weight directly may be performed. The method of analyzing the work of a quadruped has been worked out especially by Zuntz and may be conveniently illustrated from Zuntz and Hagemann's investigations on the work horse. ${ }^{1}$ The methods of indirect calorimetry were used, carbon dioxid production and oxygen consumption being determined with the Zuntz apparatus (279) and the corresponding energy output calculated. The work was done upon a special tread-power located in the open air, and during the rest experiments the animal likewise stood in the tread power. The inclination of the platform of the power could be varied, and it could also be driven by a steam engine, so that by setting it horizontal the work performed by the animal was reduced to that of locomotion alone. The distance traveled was measured by a revolution counter and in the experiments on draft the animal pulled against a dynamometer. The apparatus used is illustrated in Chapter VI, Fig. 33 (313).

652. Horizontal locomotion. - This is an important factor in work production, since it requires the expenditure of considerable energy in successive liftings of the body at each step and the overcoming of internal resistances. The energy thus expended does not ultimately produce any work in the mechanical sense, but all appears as heat. The work of locomotion, therefore, is in a sense not useful work although necessarily incident to the performance of the work.

If the tread power be set horizontal and driven by a motor, the total energy output by the subject will measure what may be called, by analogy with the gross efficiency (648), the gross expenditure in locomotion. Subtracting the energy output during rest (standing) from the total output during locomotion

${ }^{1}$ Landw. Jahrb., 18 (I889), I ; 23 (I894), I25; 27, Ergzbd. III, (I898). 
shows of course the expenditure in the latter exclusive of that required to maintain the body upright (work of standing), or what may be called the net expenditure.

On the average of thirty-five trials upon locomotion at a walk, Zuntz and Hagemann found the net expenditure of energy by the horse per meter of horizontal locomotion (after correcting in the manner described in the next paragraph for the small amount of work of ascent due to the fact that the tread power was not exactly horizontal) to be as follows per kilogram of weight:

At a speed of 78 meters per minute . . 3256 gram calories At a speed of 90.16 meters per minute . . 3666 gram calories At a speed of 98.I I meters per minute . . . 3929 gram calories

The actual amount of mechanical work done in horizontal locomotion and converted into heat cannot be measured directly. Zuntz and Hagemann, however, have computed it by means of a formula proposed by Kellner ${ }^{1}$ and by comparison with the figures just given, compute a net efficiency of about 35 per cent.

653. Work of ascent. - The animal may also perform work by drawing or carrying a load up a hill. Taking the simpler case of carrying a load, the total output of energy would be expended for three purposes, viz., maintenance (resting value), locomotion, and lifting the weight of the load plus body in opposition to gravity. Zuntz separates the two latter factors from each other by a comparison of two experiments in which the ratio of distance traveled to ascent, i.e., the angle of ascent, differs.

Thus in the thirty-five trials with nearly horizontal locomotion the average energy output per kilogram of live weight, after deducting the maintenance requirement, was 0.4035 gram calories per meter traveled. During the same time, however, the body was lifted through 0.4395 centimeters, equivalent to 0.004395 kilogram meters of work of ascent per kilogram of live weight. In thirteen experiments on ascending a moderate grade, the average energy expended in excess of maintenance per kilogram live weight was I.0795 gram calories per meter traveled, while the work of ascent was 0.10704I kilogram meters per kilogram. Letting $x$ equal the energy per kilogram required for one meter of horizontal locomotion and $y$ the energy required for the

${ }^{1}$ Landw. Jahrb., 9 (1880), 658. 
performance of one kilogram meter of work of ascent, the two following equations may be formulated:-

$$
\begin{aligned}
& x+0.004395 y=0.4035 \text { cals. } \\
& x+0.10704 \text { I } y=1.0795 \text { cals. }
\end{aligned}
$$

From these equations the values of $x$ and $y$ can be computed to be as follows:-

$$
x=0.3746 \text { cals. } y=6.5856 \text { cals. }
$$

Since one kilogram meter is equivalent to 2.344 cals., it follows that the net efficiency in the work of ascent was $2.344 \div 6.5^{8} 5^{6}=35.73$ per cent. In effect, the net efficiency in work of ascent is computed by deducting from the total energy output the amounts expended for maintenance and for horizontal locomotion and comparing the remainder with the measured work of ascent. The results given in the previous paragraph for the energy expended in locomotion were computed according to this scheme.

654. Work of draft. - The net efficiency in draft was computed by a similar method. The tread power was set nearly horizontal. On the average of sixteen trials the total energy output in excess of maintenance per kilogram live weight and per meter traveled was I.502I cals., the work of ascent 0.005 II 5 kilogram meters and the work of draft 0.I53 27 kilogram meters. Letting $z$ equal the energy expended in the performance of $\mathrm{I}$ kilogram meter of work of draft, the following equation may be formulated:-

$$
x+0.005 \text { II } 5 y+0 . \text { I53I } 27 z=\text { I.502 I cals. }
$$

Substituting average values for $x$ and $y$, the value of $z$ is 7.143 cals., equivalent to a net efficiency of 32.84 per cent.

655. Correction for speed. - In experiments made at a walk it was found that the expenditure of energy per meter increased materially as the speed increased, as is illustrated by the averages already cited (652) and is shown more fully in a succeeding paragraph (663). In computing the efficiency of work of ascent or draft, it is necessary to take account of this fact. The method of doing so is a method of approximation, the details of which need not be gone into here. ${ }^{1}$

1 Compare Armsby, Principles of Animal Nutrition, pp. 507-508. 
656. Summary. - The following table contains a summary of Zuntz and Hagemann's results regarding the efficiency of the body of the horse as a motor. As is apparent from the foregoing explanations, the table shows the net efficiency in the various forms of work into which the total work done can be separated in the manner just described $(651,654)$.

Table 155. - Net Efficiency of the Horse in Different Forms of WORK

\begin{tabular}{|c|c|c|c|c|c|c|}
\hline & \multicolumn{3}{|c|}{ WORK AT A WALK } & \multicolumn{3}{|c|}{ WORK at a SLOW Trot } \\
\hline & \multicolumn{2}{|c|}{$\begin{array}{l}\text { Net Expendi- } \\
\text { ture of Energy }\end{array}$} & $\begin{array}{l}\text { Net } \\
\text { Effi- } \\
\text { ciency }\end{array}$ & \multicolumn{2}{|c|}{$\begin{array}{l}\text { Net Expenditure } \\
\text { of Energy }\end{array}$} & $\begin{array}{l}\text { Net } \\
\text { Effi- } \\
\text { ciency }\end{array}$ \\
\hline $\begin{array}{c}\text { For I } \mathrm{kgm} \text {. work of ascent, without } \\
\text { load: }\end{array}$ & cals. & Kgm. & $\%$ & cals. & Kgm. & $\%$ \\
\hline 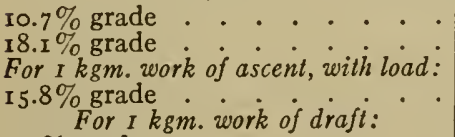 & $\begin{array}{l}6.8508 \\
6.9787 \\
6.502\end{array}$ & $\begin{array}{l}2.9116 \\
2.9660 \\
2.7634\end{array}$ & $\begin{array}{l}34.3 \\
33.7 \\
36.2\end{array}$ & $7 \cdot 3647^{1}$ & 3.1300 & 31.961 \\
\hline $\begin{array}{l}\text { I. } 5 \% \text { grade } \\
\text { Locomotion per kg. mass per meter } \\
\text { without load: }\end{array}$ & $\begin{array}{r}7.5190 \\
10.3360\end{array}$ & $\begin{array}{l}3.1960 \\
4.3930\end{array}$ & $\begin{array}{l}31.3 \\
22.7\end{array}$ & $\begin{array}{r}7.4240^{1} \\
10.0780^{2}\end{array}$ & $\left|\begin{array}{l}3.1550^{1} \\
4.2820^{2}\end{array}\right|$ & $\begin{array}{l}31.7^{1} \\
23.4^{2}\end{array}$ \\
\hline $\begin{array}{l}\text { Speed of } 2.9 \mathrm{r} \text { miles per hr. } \\
\text { Speed of } 3.36 \text { miles per } \mathrm{hr} . \\
\text { Speed of } 3.66 \text { miles per } \mathrm{hr} . \\
\text { The same with load on back: } \\
\text { Speed of } 3.36 \text { miles per } \mathrm{hr} \text {. }\end{array}$ & $\begin{array}{l}0.3256 \\
0.3666 \\
0.3929 \\
0.3914\end{array}$ & & & $\begin{array}{l}0.5478^{3} \\
0.6007^{3}\end{array}$ & & \\
\hline
\end{tabular}

657. Relative utilization of fats and carbohydrates. - In view of Chauveau's theory ${ }^{4}$ that fat must first be converted into dextrose, with the elimination as heat of a considerable portion of its energy, before it can serve directly as a source of energy for the physiological processes, it becomes of much interest to inquire to what relative extent the energy of fats and carbohydrates is utilized in muscular work.

In Zuntz and Hagemann's extensive investigations, particularly in those upon the horse, there were very considerable variations in the proportions of fat and carbohydrates katabolized. 'The individual trials in which the same kind of work was per-

1 Single experiment.

2 Two experiments. Work probably excessive.

3 Independent of speed.

${ }^{4}$ Compare Armsby, Principles of Animal Nutrition, pp. I53-154 and 399-405. 
formed also show in many cases similar variations. Notwithstanding this, however, the percentage of energy utilized did not vary materially in these instances and there is no indication of any such differences as would be expected according to Chauveau's theory.

The question has also been investigated directly by Zuntz and his associates in experiments on dogs and on man. In these experiments, the feed consisted as largely as possible of the nutrient to be tested (protein, carbohydrates or fat, respectively), so that the body metabolism was largely at its expense. The method of investigation was substantially the same as that which has just been described. The final results for the energy metabolism per kilogram and meter traveled were:-

TABle 156. - Comparison of Nutrients for Work Production

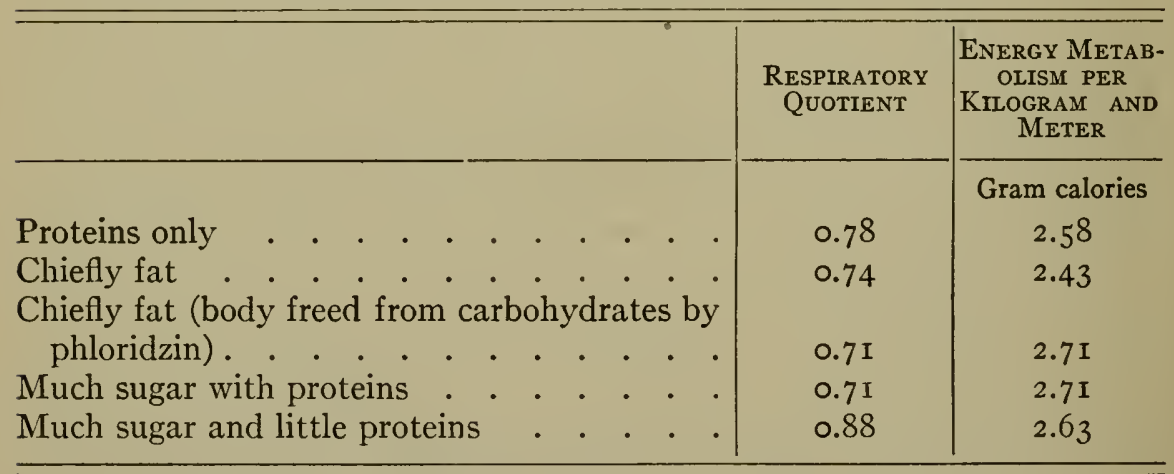

The differences are quite small, while, as Zuntz points out, if 2.6 cals. represent the demand for energy per unit of work when carbohydrates are the source it should, according to Chauveau's theory, rise to about 3.68 cals. when the energy is derived exclusively from fat.

Later and more elaborate experiments on man led to the same conclusion. Atwater and Benedict, ${ }^{1}$ Benedict and Milner, ${ }^{2}$ and Benedict and Cathcart $^{3}$ also report experiments upon men which, while not regarded as conclusive, indicate a possible slight inferiority of fats but one not at all comparable with that demanded by Chauveau's theory. On the whole, then,

1 U. S. Dept. of Agric., Office Expt. Stas., Bul. I36 (I903), I 82.

2 U. S. Dept. of Agric., Office Expt. Stas., Bul. I75 (I907), 234.

${ }^{3}$ Muscular Work; Carnegie Institution of Washington, Publ. No. 187 (1913), 145. 
the conclusion seems warranted that if any difference exists in the utilization of the energy of fats and of carbohydrates it is too small to be of much practical significance.

\section{Conditions affecting effciency}

658. Efficiency varies. - As appears from the foregoing summary (656), the net efficiency of the animal body as a motor is comparatively high in the case of the horse, considerably exceeding in most instances 30 per cent. It may be said in a broad general way that with this animal about one-third of the energy metabolized for a specific form of muscular exertion (i.e., in excess of maintenance or of maintenance plus locomotion) is recovered in the mechanical work done. It is also evident, however, that the organism has no one fixed degree of efficiency but that the latter may vary through a somewhat wide range under different conditions.

659. Forms of work done. - The experiments thus far cited refer largely to work done by walking horizontally or up a grade, with or without draft. Of all the forms of work yet investigated, the ascent of a moderate grade or, in other words, the lifting of the body by the legs, appears to be the one which is performed most economically, a net efficiency of over 36 per cent being reported both for the horse and for man. This percentage, however, decreases considerably as the angle of ascent is increased. For horizontal locomotion, as already noted (652), Zuntz computes an efficiency of 35 per cent. Draft up a slight grade was performed somewhat less efficiently in the case of the horse, the percentage being approximately $3 \mathrm{I}$, while draft up an $8 \frac{1}{2}$ per cent grade was done with an efficiency of less than 23 per cent.

Other forms of work appear to be performed with a less degree of efficiency. Thus experiments on man in which the work was done by turning a crank with the hands have shown decidedly lower efficiencies than those in which the work was done on a treadmill. The same was true in the experiments of Benedict and Cathcart on man, in which the work was done upon a stationary bicycle, the maximum figures computed ${ }^{1}$ for the net efficiency of 6 subjects ranging from 20.4 to 25.2 per cent.

$$
{ }^{1} \text { Loc. cit., p. } 125 .
$$


Species. - The difference just noted between the efficiency of the human body and that of the horse is evidently due largely to differences in the kind of work performed, since the work of ascent is done with about equal efficiency in both cases. Klein ${ }^{1}$ finds that the work of ascent is performed by the ox with about the same net efficiency as by the horse but that the former animal expends much more energy in horizontal locomotion per unit of distance traveled than does the horse, viz., about 0.53 to 0.55 gram calories, per meter distance and kilogram live weight.

660. Individuality. - Zuntz and Hagemann's experiments upon the horse show interesting individual differences between animals, presumably due to differences in conformation. For example, Horse No. XIII carried a given load on his back with less expenditure of energy than did Horse No. III. Horse No. II expended more energy than Horse No. III in horizontal locomotion at a walk but less in trotting. No. II likewise utilized energy to a slightly less extent than No. III in ascending a grade and to a considerably less extent in horizontal draft but, on the other hand, like No. XIII, carried a load on his back more economically than No. III.

The possible bearing of these facts upon questions of heredity and breeding opens up. an interesting field of speculation.

661. Training and fatigue. - It is a familiar experience that any unaccustomed form of work is much more fatiguing at first than it is later. This is due in part to the fact that in making unfamiliar motions more accessory groups of muscles are called into activity than are necessary later when more skill has been acquired. The experience of a learner on the bicycle is an excellent example of this. In the second place, however, simple exercise of a group of muscles in a particular way seems to increase their average mechanical efficiency.

This effect may be illustrated by the results of two series of experiments by Gruber upon himself in which he determined the carbon dioxid excreted during work. Thus in hill climbing the amounts excreted in twenty minutes were:-

\section{Series I:}

Hill climbing without practice . . . . . . 40.98 grams

Hill climbing after I 2 days' practice . . . . 32.22 grams

1 Zentbl. Physiol., 26 (I9I2), 722. 
Series II :

Hill climbing without practice . . . . . 38.83 grams

Hill climbing after I4 days' practice . . . . 31.00 grams

That the less use of accessory muscles is not the only cause of this increase in efficiency is indicated by experiments upon convalescents, which have shown that the gradual strengthening of the muscles results in a more economical performance of their work, largely independent of any special training for a particular kind of work.

Conversely, fatigue has been shown by numerous observers to materially increase the relative amount of metabolism per unit of work. Schnyder ${ }^{1}$ summarizes the matter in the statement that it is not the work itself but the muscular effort required which determines the amount of metabolism, a conclusion which seems to have anticipated Hill's results ${ }^{2}$ regarding the relation of muscular tension to metabolism.

662. Intensity of Work. - It has already been shown (649) that the gross efficiency of the body tends to increase with the intensity of work, i.e., with the number of units of work performed in a unit of time, for the reason that the proportion of the total energy expended which is devoted to useful work increases. On the other hand, common observation tends to show that this can be true only within limits, and that excessive work is performed uneconomically.

The intensity of the work may be increased by increasing either the speed, the load moved, or the angle of ascent. It would be anticipated, therefore, that an undue increase of any one of these factors would result in a diminished net efficiency.

663. Influence of speed. - That great speed in horizontal locomotion involves a largely increased expenditure of energy is evident. The race horse or the track athlete traveling a mile at top speed obviously metabolizes vastly more energy than one traveling the same distance at a moderate rate.

In the case of the horse, Zuntz and Hagemann's results on horizontal locomotion at a walk (652) show an increased net expenditure of energy per kilogram weight and meter distance with increased speed, while locomotion at a trot showed no distinct increase up to a speed of about $7 \frac{1}{2}$ miles an hour.

${ }^{1}$ Ztschr. Biol., 33 (1896), 28 g.

2 Jour. Physiol. (London), 42 (I9II), I. 
Much study has been expended upon horizontal locomotion in man. The somewhat extensive literature of the subject as summarized by Benedict and Murschhauser ${ }^{1}$ shows clearly a marked increase in the net expenditure of energy per unit of locomotion as the speed increases. Brezina and Reichel ${ }^{2}$ found that beyond a certain maximum speed (about 80 meters per minute) it became an exponential function of the velocity, while below that speed only slight variations were shown.

The influence of speed upon the net efficiency in work of ascent seems to be much less marked than that upon the expenditure in locomotion. No results upon the horse are available. With man, Brezina, Kolmer and Reichel ${ }^{3}$ in experiments on a tread power found that the net expenditure per kilogram and meter distance in walking up a grade was substantially independent of speed at velocities considerably below the maximum just indicated. Since this was found to be true also of horizontal locomotion, it follows that the efficiency in work of ascent must also have been nearly independent of the speed.

Benedict and Cathcart ${ }^{1}$ found that both the net and gross efficiency in work done on their bicycle ergometer, which might be regarded as a form of draft, decreased as the speed increased. In none of the various experiments cited was there any air resistance, the work being done on a stationary apparatus. In actual practice this is an important factor at high speeds, increasing very much more rapidly than the speed.

664. Influence of gait. - According to Zuntz and Hagemann's results (656) an increase of speed in the horse obtained by a change of gait from a walk to a trot involves a notable increase in the net energy expended for locomotion per unit of weight and distance, although an increase in the trotting speed up to a moderate limit causes no further increase. With man, on the contrary, Benedict and Murschhauser find that locomotion at a given speed is performed more economically in running than in walking.

Such differences are doubtless brought about to a considerable extent by differences in the height to which the body is lifted at each step and the degree to which extraneous motions, such as swinging the arms in rapid walking, are brought into play.

1 Carnegie Institution of Washington, Publication No. 23I (I9I5), pp. 12-28.

${ }^{2}$ Biochem. Ztschr., 63 (I9I4), i 70.

${ }^{3}$ Biochem. Ztschr., 65 (r9i4), r6 and 35.

${ }^{4}$ Carnegie Institution of Washington, Publication No. 187 (I913), I38. 
665. Influence of load. - With the horse, Zuntz and Hagemann find that carrying a load on the back causes a distinct increase in the net expenditure for horizontal locomotion per unit of mass moved. The work of ascent, on the contrary, was performed with at least as high an efficiency by an animal carrying a weight as by one without load. With man, Benedict and Cathcart find the net efficiency but little affected by the amount of resistance in their bicycle ergometer. Brezina and Reichel find that at moderate speeds the load carried by a man affects but slightly the net expenditure per kilogram and meter distance but that above the point at which the speed begins to affect the latter, the increase is greater as the load is increased.

666. Influence of grade. - The net efficiency with which work of ascent is done decreases as the grade is made steeper. Zuntz and Hagemann in their experiments upon the horse observed a decrease of the efficiency from 34.3 per cent to 33.7 per cent as the grade was increased from I0.7 per cent to I8. I per cent, while for work of draft the efficiency was $3 \mathrm{I} .3$ per cent on a 0.5 per cent grade but only 22.7 per cent on an 8.5 per cent grade.

That the same is true in the case of man is illustrated in experiments by Loewy, who obtained the following results on three different individuals.

Table 157. - Influence of Grade on Net Efficiency in Work Production

\begin{tabular}{l|c|c|c}
\hline & \multicolumn{3}{|c}{ Net Efficiency } \\
\hline & A. L. & J. L. & L. Z. \\
\cline { 2 - 4 } 23 & $\%$ & $\%$ & $\%$ \\
30.5 & 34.3 & 36.1 & 36.6 \\
36.6 & 34.3 & 32.6 & 36.6 \\
& 29.0 & 32.3 & 32.2 \\
\hline
\end{tabular}

The same conclusion was reached in the recent investigations of Brezina and his associates ${ }^{1}$ on man. From an extensive series of experiments they compute the net efficiency to have been approximately:-

${ }^{1}$ Biochem. Ztschr., 63 (I914), 170; 65 (I9r4), I6. 
Grade

$10 \%$

$20 \%$

$30 \%$
Net Effictency

$39 \%$
$31 \%$
$27 \%$

\section{§ 3. FEed Requirements FOR WORK}

As is the case in feeding for other purposes, the working animal needs to be supplied with an adequate amount of energy in available form and with certain specific forms of matter, particularly proteins and ash ingredients.

\section{The requirements of matter}

667. Functions of protein. - As shown in Chapter IX (418), the daily protein requirement of the horse for simple maintenance is apparently about the same as that of other farm animals, viz., approximately, 0.6 pound of digestible crude protein per rooo pounds live weight, although the experimental data are rather scanty.

It appeared in $\S$ I of this chapter (641-643) that the energy expended in muscular work is practically derived from the katabolism of carbohydrates and fats, the protein katabolism being unaffected by work so long as an ample supply of non-nitrogenous nutrients is available. One might at first thought be inclined to conclude, therefore, that the simple addition of nonnitrogenous material to a maintenance ration would suffice to enable it to support a corresponding amount of work production and that a maintenance ration of digestible protein would also be a sufficient supply for the working horse. Such a conclusion would, however, be premature. It is quite conceivable that in order to maintain the muscle as an efficient instrument for converting chemical energy into mechanical work, a higher plane of protein metabolism may be necessary than is required to support it in nitrogen equilibrium when doing no work, while the possibility, for example, of a favorable influence of an abundant protein supply on the blood circulation and on the nervous system should not be overlooked. In fast work especially, the demand of the animal for oxygen reaches a high level. Since the blood is the vehicle by which oxygen is introduced into the body, an adequate stock of blood, or 
more particularly of hæmoglobin, would be necessary and a liberal supply of protein seems to assist in securing this. If any of these conjectures should prove to be true the proteins may play a not insignificant rôle in the production of muscular work without any evidence of the fact appearing in the total nitrogen excretion.

668. The protein requirement. - No specific investigations regarding the minimum protein requirement of the work horse seem to have been made, but the extensive experiments of Wolff, Grandeau, Müntz and others referred to on previous pages afford numerous instances in which entirely satisfactory results were obtained from rations comparatively low in protein, although in none of them was the supply reduced to the maintenance requirement. Similarly, in Langworthy's extensive compilation ${ }^{1}$ of rations fed in practice, numerous examples of low protein rations are to be found.

In fact, it would be difficult to compound from ordinary feedstuffs a ration sufficient to support any considerable amount of work without introducing more protein than is presumably required for simple maintenance. Such being the case, the principal point to be taken into consideration is the effect of a reduced protein supply upon the digestibility of the ration (723725). Any ration carrying sufficient protein to ensure normal digestion would doubtless furnish ample protein for work production in all ordinary cases, with the possible exception of work at high speed. A nutritive ratio, computed in the usual way (709), of I : Io or I : I 2 would unquestionably ensure ample protein for slow work, and probably for moderately rapid work also. In the case of man, as is well known, experience or tradition have led to the general employment of high protein rations by athletes. On the other hand, however, Chittenden ${ }^{2}$ has shown that the protein supply of athletes and soldiers, as well as that of men of sedentary occupations, may be reduced much below the usual level without loss of efficiency. Even in these experiments, however, the protein supply was much higher than the amounts which recent experiments have shown to suffice for the maintenance of nitrogen equilibrium in man at rest or doing only light work.

1 U. S. Dept. Agri., Office Expt. Sta., Bul. I25 (1903).

${ }^{2}$ Physiological Economy in Nutrition, I904.

20 
669. Ash requirements. - As pointed out in previous chapters, the ash requirements of an animal deserve greater consideration than they generally receive, while in the case of growth, at least, the presence of certain accessory substances in the ration is necessary. So far as the working horse is concerned, however, no sufficient data seem available for a discussion of these topics.

\section{The energy requirement}

670. Economic, or over-all efficiency. - In the preceding section certain comparisons were made between the efficiency of the animal body as a prime motor and that of artificial engines. The animal body, however, is not only a prime motor but includes also the furnace in which the fuel is burned and resembles in this respect a complete power plant, such as a locomotive, for example, rather than an engine.

Just as the energy of the fuel of a locomotive is subject to certain losses due to incomplete combustion and to radiation of heat before the steam reaches the cylinders, so portions of the energy of the feed escape in the excreta or are expended in the various processes incident to the formation in the body of those substances whose katabolism yields the energy for a muscular contraction.

Since these losses and expenditures are largely unavoidable, they constitute part of the energy requirements of the work animal, and from the economic point of view the efficiency of the animal is measured by a comparison of the total feed energy with the work done. This might be called the economic efficiency, comparable to the over-all efficiency of a steam plant as computed by a comparison of coal consumption with the brake horse power obtained. Any such comparisons, of course, must take account of the maintenance requirement of the animal when doing no work and must therefore be made on the basis of the 24-hour output of work (650).

Few satisfactory direct determinations of the economic efficiency of work animals in the foregoing sense, i.e., of the relation of the work done to the feed (or total feed energy) required for its performance, have yet been reported. 
The extensive investigations on the work horse initiated at Hohenheim by Kellner and continued under Wolff's direction, and which have been referred to in Chapter VIII (386 a) in their bearing upon the maintenance requirement, were intended primarily to determine the energy requirements for work. Unfortunately, however, as there noted, the measurements of the work done in the earlier experiments were subsequently discovered to be inaccurate. In the comparatively few later experiments of $189 \mathrm{I}-94$, various mixed rations were fed. While, therefore, the total energy consumed per unit of work could be computed, only few and uncertain data for individual feeding stuffs can be deduced and the results are therefore of small general value for the particular phase of the subject under discussion here.

671. Net energy values for work production. - The question of the energy requirement of the work animal may, however, be approached in a somewhat different way.

The energy expended in work production, as already stated $(630,631)$, is derived primarily from the katabolism of body substances. The function of the feed so far as energy is concerned is to replace in the body the energy thus expended. The net energy value of a feeding stuff for work production, then, is measured by the amount of body energy which it can thus replace. The case is precisely parallel to that of maintenance as discussed in Chapter VIII (370). The net energy value of a feeding stuff for the latter purpose is measured by the extent to which it prevents loss of body energy as a consequence of internal work, while the net energy value for the former purpose is measured by the extent to which it prevents or makes good a loss of body energy due to external work. Conversely, the working animal requires in addition to maintenance a supply of net energy in its ration equal to the amount of body energy katabolized for work production.

In view of this close similarity between the functions of feed in maintenance and in work production, the assumption seems warranted that the net energy values of feeding stuffs for these two purposes are substantially the same. Thus in an experiment with a steer already described (364), it was found that one pound of timothy hay contributed 502 Cals. to the maintenance of the animal. If the same animal had been required to do I67 Cals.of external work and had performed it with the same average net efficiency as the horse, viz., about one-third, he would have 
katabolized body substance containing $167 \div \frac{1}{3}=502$ Cals. of energy and it would be anticipated that one pound of timothy hay would have been sufficient to replace this energy in the body. Similarly, the performance of 1000 Cals. of external work by a horse would cause the mobilization of about 3000 Cals. of body energy, and the feed necessary to support this work would have to supply about 3000 Cals. of net available energy.

In brief, the net energy values for maintenance, determined in the manner described in Chapter XVII and tabulated in the Appendix, may be regarded as also net energy values for work production, and the energy requirements of the work animal may be expressed in terms of these net energy values.

672. Net energy requirements. - It is plain, in the light of the foregoing discussion, that the amount of net energy required by an animal for work production may be regarded as equal to the body energy metabolized in the performance of the work.

From the data contained in $\S 2$, it is possible to estimate approximately how much energy in excess of its maintenance requirement must be mobilized in the body of a horse, e.g., to perform a known amount of mechanical work of a specific kind. Thus a horse in hauling a load having a draft of 100 pounds 20 miles on a level road would do 10,560 foot tons of mechanical work, equivalent to 342 I Calories. Table 155 (651) shows the net efficiency of the horse in draft to be about $3 \mathrm{I} .3$ per cent. Accordingly, the animal would have to mobilize in his body for the performance of this work $4321 \div 0.313=10,929$ Calories, and his feed must therefore supply this amount of net energy in addition to the requirements for locomotion, maintenance or other purposes.

The total expenditure of body energy during the performance of work by the horse, as appears from $\S 2$, includes substantially four factors in varying proportions, viz., the expenditure for maintenance, for horizontal locomotion, for ascent (or descent) and for draft. A fairly accurate estimate of the net energy required to do a certain piece of work may therefore be obtained by computing the requirement for each of these factors separately from the data for net efficiency already recorded and adding the results.

For example, let it be supposed that a horse weighing I roo pounds hauls a load of 2000 pounds, having a horizontal draft 
of Ioo pounds, 15 miles per day, including 5 miles up a I per cent grade, at a speed of $3 \frac{1}{2}$ miles per hour. The mechanical work performed consists of lifting the weight of the animal plus the load 264 feet and in overcoming a draft resistance of 100 pounds through 79,200 feet. The total mechanical work, therefore is as follows:-

\section{Table 158. - Example of Computation of Work Done}

Draft 100 $\times 5280 \times 15=7,920,000$ foot pounds $=2.565$ Therms

Ascent 3 100 $\times 264=8$ 818,400 foot pounds $=0.265$ Therms

Total $8,738,400$ foot pounds $=2.830$ Therms

The amount of body energy which the horse must metabolize in the performance of this daily task will be that corresponding to the mechanical work of draft and of ascent, computed from the percentages in Table I55, together with the energy expended in locomotion according to the same table and the energy requirement for maintenance. The total requirement of net energy per day, therefore, will be as follows: ${ }^{1}-$

Table i59. - Example of Computation of Net Energy Requirement

\begin{tabular}{|c|c|c|}
\hline $\begin{array}{l}\text { For draft } \\
\text { For ascent } \\
\text { For locomotion } \\
\text { For maintenance }\end{array}$ & $\begin{array}{l}2.565 \div 0.3 \mathrm{I} 3 \\
0.265 \div 0.343 \\
0.262 \times 15 \\
(\mathbf{3 8 5})\end{array}$ & $\begin{array}{l}\text { 8.195 Therms } \\
\text { 0.773 Therms } \\
\text { 3.930 Therms } \\
\text { 4.356 Therms }\end{array}$ \\
\hline Total & & $\overline{17.254}$ Therms \\
\hline
\end{tabular}

673. Calculation of rations. - Having in some such way as that just illustrated determined the net energy requirement of the work horse it is evident that the corresponding ration may be computed if the net energy values of the feeding stuffs to be used are known. Unfortunately, in the case of the horse, the principal work animal of the United States, such net energy values of feed stuffs as we now possess have not been directly determined but are the results of somewhat complicated calculations (775-778). For the ox, on the contrary, fairly satisfactory data regarding the net energy values of feed stuffs are available $(760,773,774)$, but in this case very few determinations of the efficiency of the animal's body in work production have yet been

${ }^{1}$ The computation could be somewhat simplified by assuming a uniform net efficiency of $\frac{1}{3}$ for all forms of work. 
reported, although the indications are (659) that it is not widely different from that of the horse.

Using the net energy values for the horse obtained by Zuntz and Hagemann's method of computation and contained in Table VIII of the Appendix, rations may readily be computed for this animal in the same general manner as for any other, their accuracy depending upon the accuracy of the net energy values used. Thus, in the case just supposed, the requirement of net energy was I 7.254 Therms. From the figures of the table it is easy to compute that the following ration would meet the requirement.

TABLE i60. - EXAMple of Ration For Work

Net ENERGY

Io lb. meadow hay .

3.270 Therms Io lb. oats 4.I lb. maize .

8.820 Therms 5.164 Therms I 7.254 Therms

The principal difficulty in practice lies in the determination of the amount of work done. With farm animals doing a variety of work at more or less irregular intervals, it seems hardly possible to make any computation of the mechanical work performed which would be trustworthy or which would justify the time consumed. The sufficiency of the ration of the farm horse will ordinarily be judged of by the live weight and condition of the animal, and the principal use of tables of net energy values will be as an aid in securing the necessary feed energy at the cheapest rate per unit.

On the other hand, where a large number of horses or mules are used for the same kind of work under uniform conditions it would seem possible to make fairly reliable estimates of the work done and to compute feed requirements with a reasonable degree of accuracy. It appears not unlikely that such computations might lead to considerable economy, since, as was pointed out in considering the maintenance requirements of the horse (392), a surplus of feed seems especially apt to stimulate this animal to restlessness and an unnecessary expenditure of energy in minor muscular activities.

674. Feeding standards for the horse. - More or less arbitrary estimates for light, medium, and heavy work may also be formulated, as has been done by various writers. 
According to Wüst ${ }^{1}$ a horse weighing rooo pounds is capable of performing daily about two million kilogram meters of work, inclusive of that of locomotion. Allowing for the work of locomotion, this seems to agree well with Thurston's statement : ${ }^{2}$ "It is customarily assumed that a horse may develop 22,500 foot-pounds per minute throughout a day's work of eight hours." If this may be regarded as full work and if the average net efficiency of the animal be taken as one-third, the net energy requirements for the work itself and the total requirements, inclusive of maintenance, would be as follows:-

Table r6i. - Net Energy Requirements of the Horse

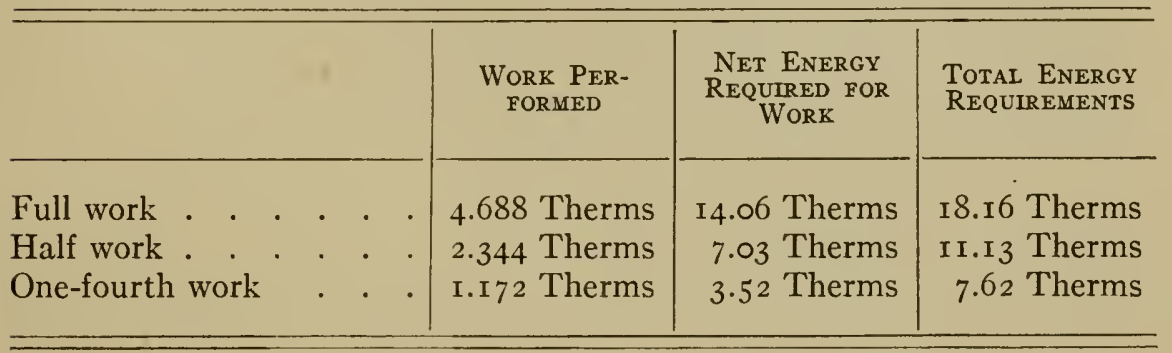

It should be noted that the discussions of the foregoing pages apply specifically to the work horse and the results have only a limited application to the feeding of pleasure or race horses. With such animals, the cost of feed is economically a very minor factor and success depends on experience and skill rather than on mathematical computations. That a fairly liberal supply of protein in rations for fast work is indicated by physiological considerations has been already pointed out (667).

675. Comparison with power plant. - As stated (670), no satisfactory direct determinations of the over-all efficiency of work animals are recorded, but it may be computed in a case like that used as an illustration on a previous page $(672,673)$. There the total useful work was 2.830 Therms, while the gross energy of the computed ration would be approximately 55.800 Therms and the over-all efficiency, therefore, $2.830 \div 55.800=$ 5.I per cent. As was shown in $\S 2$ (649) to be the case with the

${ }^{1}$ Cited by Kellner, Die Ernährung der landw. Nutztiere, 6th Ed., p. 465.

2 The Animal as a Machine and a Prime Motor, I894. 
gross efficiency of the body, however, this percentage will vary from case to case. It will increase with the intensity of the work and decrease with the number of hours the animal is idle per day, i.e., it will vary as the ratio of useful work to maintenance requirement varies. In the case supposed, the animal worked 6 hours per day. If we imagine his bodily machinery stopped for the remaining 18 hours, as an engine might be, and charge him with only $\frac{1}{4}$ of his 24 -hour maintenance requirement, the total feed energy necessary would be reduced to about 45.230 Therms and the over-all efficiency during the hours of work, computed on this basis, would be 6.26 per cent, or about that of a modern steam locomotive. In actual practice, the conditions with an animal are very much as if it were necessary to keep up a full head of steam for 24 hours or as if an internal combustion motor were to be run continuously although actual work was being done for only a portion of the time. 


\section{PART IV}

\section{THE FEED SUPPLY}





\section{CHAPTER XV}

\section{THE FEEDING STUFFS}

676. Sources of feeding stuffs. - In the several chapters of Part III the feed requirements of different classes of farm animals and for different forms of production have been considered.

In the more primitive forms of animal husbandry, such as the pastoral husbandry of ancient times or the range feeding of the western United States, these requirements were met by the consumption of the natural products of the soil. Increasing population and rising land values, however, inevitably tend to the displacement of pastoral agriculture by more intensive forms in which a much greater variety of feeding stuffs is available for domestic animals. Forage crops are grown for use in the winter and to supplement the deficiencies of the pasture; grain is produced in excess of the effective demand for human consumption and utilized as stock feed; finally, as this surplus of grain decreases with the growing requirement for human food, a great variety of residues from the preparation of the crude products of the farm for man's use, the by-product feeds, becomes available to the stockman.

It does not fall within the province of the present work to consider either the problems of agronomy connected with the production of feeding stuffs or the technical details of the manufacturing processes which yield the various by-product feeds, but a brief consideration of the general properties of the principal classes of feeding stuffs seems desirable as an introduction to the discussion of the principles determining their nutritive values

677. Classification. - The three main classes of feeding stuffs, as already stated in Chapter II (111), are the coarse fodders, or roughages, consisting of the vegetative organs of plants, the roots and tubers, and the concentrates, the latter comprising both the grains and similar farm products and the by-products of divers industries. The members of these three classes of 
feeding stuffs may be variously grouped for different purposes, but the following scheme, although not strictly consistent, may serve the purpose of this discussion.

\section{Classification of feeding stuffs}

Roughages, or coarse fodders.

Dried

Grasses

Legumes

Straws

Fresh

Grasses

Silage

Legumes

Roots and tubers

Concentrates

Farm products

Cereal grains

Leguminous grains

Oil seeds

Dairy products

By-products

By-products of milling

By-products of fermentation industries

By-products of oil extraction

By-products of starch and glucose manufacture

By-products of sugar manufacture

By-products of the packing house

The following characterization of these various classes of feeding stuffs is reproduced without material change from an earlier article by the writer. ${ }^{1}$

\section{$\S$ I. Roughages, OR COARse Fodders}

678. General characters. - The roughages are characterized chemically by a relatively large percentage of crude fiber, which forms the framework of the plant. They usually do not contain very much protein, although in some this ingredient shows a fairly high percentage. The proportion of crude

1 Bailey's Cyclopedia of American Agriculture, rgo8, Vol. III, pp. 58-92. 
fat is small and includes much besides true fat. The nitrogenfree extract, along with more or less starch and sugar, includes a great variety of less familiar carbohydrates and of other substances whose nutritive value is problematical. By far the larger proportion of the roughages in common use is supplied by two classes of plants, - the grasses (Gramineæ), including maize, and the legumes (Leguminosæ). Furthermore, crops belonging to both these classes may be used for fodder when but partially mature (hay, maize forage), or they may be allowed to ripen, the grain may be removed, and the residue (straw, stover) used for feeding.

679. The grasses. - The larger share of the hay crop and of the pasturage of the United States is supplied by plants known in a restricted and popular sense as grasses, such as timothy, blue-grass, red-top. To these must be added, as a most important source of forage in the United States, maize, or Indian corn, which botanically is a grass, although not commonly so called. The forage supplied by these plants has a very wide range of nutritive value, depending on a variety of conditions. Chief among these is the stage of maturity at which the crop is utilized. In young, growing vegetation the cell walls are thin and consist of nearly pure cellulose, while the cells are filled with active protoplasm whose chief ingredients are proteins. Hence, forage cut at this stage shows a relatively low percentage of crude fiber and a high percentage of proteins. Young and tender pasture grass, relatively rich in protein and low in crude fiber, may even approach the concentrates in value, as illustrated by the following comparison of the dry matter of a sample of young pasture grass with that of average oats:-

Table i62. - Comparison of Pasture Grass and Oats

\begin{tabular}{|c|c|c|c|c|}
\hline & \multicolumn{2}{|c|}{ Pasture Grass } & \multicolumn{2}{|c|}{ OATS } \\
\hline & $\begin{array}{l}\text { Percentage } \\
\text { composition }\end{array}$ & $\begin{array}{l}\text { Digestible } \\
\text { matter }\end{array}$ & $\begin{array}{l}\text { Percentage } \\
\text { composition }\end{array}$ & $\begin{array}{c}\text { Digestible } \\
\text { matter }\end{array}$ \\
\hline Ash . . . . . & 9.23 & 一 & 3.37 & - \\
\hline Crude protein . . . & 21.89 & I 3.42 & 13.26 & 10.39 \\
\hline Crude fiber . . . . & $\mathbf{1} 8.25$ & 606 & Io.67) & \\
\hline Nitrogen-free extract & $44 \cdot 39\}$ & 40.00 & 67.08 & $54 \cdot 3^{2}$ \\
\hline Ether extract . . & 6.24 & $3 \cdot 59$ & 5.62 & 4.70 \\
\hline
\end{tabular}


As the plant matures, the cell walls grow thicker and become more and more impregnated with tough, woody material. At the same time, more soluble carbohydrates, as starch and sugar, are being produced, while the protoplasm comes to occupy but a small part of the cell. The fully mature forage, therefore, is rich in crude fiber of a tough, resistant sort, contains much carbohydrate material in general and tends to be poor in proteins. For example, three samples of meadow-grass, cut at different dates, had the following composition, reduced to a uniform percentage of water:-

Table i63. - Composition of Hay Cut at Different Dates

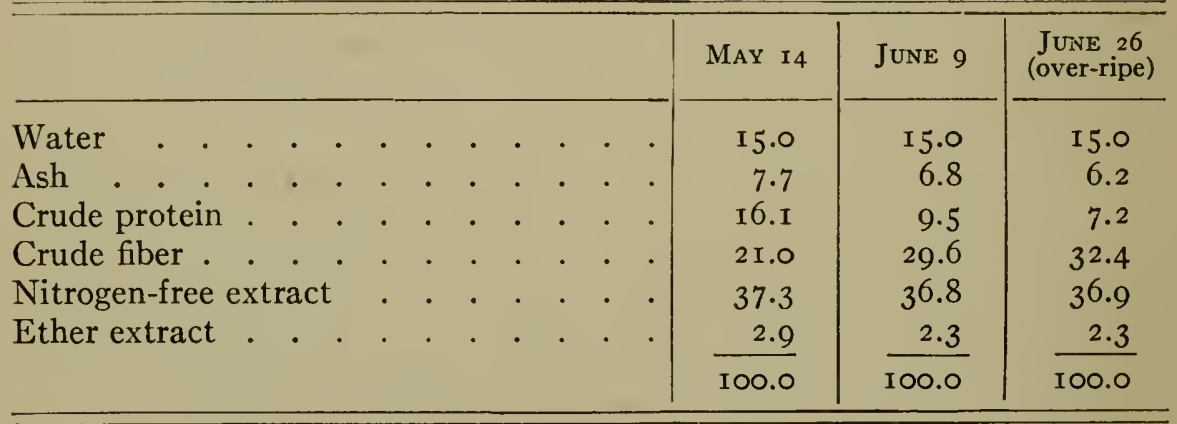

Accompanying this change in composition goes a decrease in digestibility. In the first place, the crude fiber becomes more resistant to the action of the digestive organs. Furthermore, the less soluble crude fiber seems to have a tendency to protect the contents of the cells from digestion. At any rate, the percentage digestibility of the protein, and, to a less degree, that of the other ingredients also suffers. The percentage digestibility of the several ingredients of the above samples of grass, omitting the ash, was found to be as follows: -

Table i64. - Percentage Digestibility of Hay Cut at Different DATES

Crude protein

Crude fiber.

\begin{tabular}{c|c|c} 
MAY I4 & JUNE 9 & JUNE 26 \\
\cline { 1 - 2 }$\%$ & $\%$ & $\%$ \\
73.3 & $72 . \mathrm{I}$ & 55.5 \\
79.5 & 65.7 & $6 \mathbf{I . I}$ \\
75.7 & 61.9 & 55.7 \\
65.4 & 51.6 & 43.3 \\
\hline
\end{tabular}


No determinations of the energy values of these samples were made, but it may be fairly assumed that the increasing woodiness not only diminished the total amounts of digestible nutrients contained but also increased the relative expenditure of energy in digestion and assimilation, so that the lesser amount of digestible matter in the more mature samples was probably less valuable per unit than that of the younger samples.

When the seeds of grasses begin to form, there is a rather rapid transfer of nutritive materials to them from the stalks and leaves. The seeds of the ordinary hay grasses, however, are so small and so well protected by their seed-coats that they either shell out and are lost or largely escape mastication and digestion. Grass harvested after the seeds have formed practically furnishes straw rather than hay.

680. Maize. - A somewhat important exception to the general rule regarding the influence of maturity is observed in the case of maize. While advancing maturity produces its normal effects on the stalks and leaves, such large amounts of easily digestible material are stored up during ripening in the grain, and the latter makes up so large a percentage of the total weight of the crop, that it outbalances the effect of increasing maturity, and the ripe or nearly ripe crop, taken as a whole i.e., as used for silage or as field-cured forage - is more digestible than at earlier stages of growth. For example, the dry matter of maize forage at three different stages had the following composition and digestibility :-

Table i65.- Composition and Digestibility of Maize Forage at Different Stages

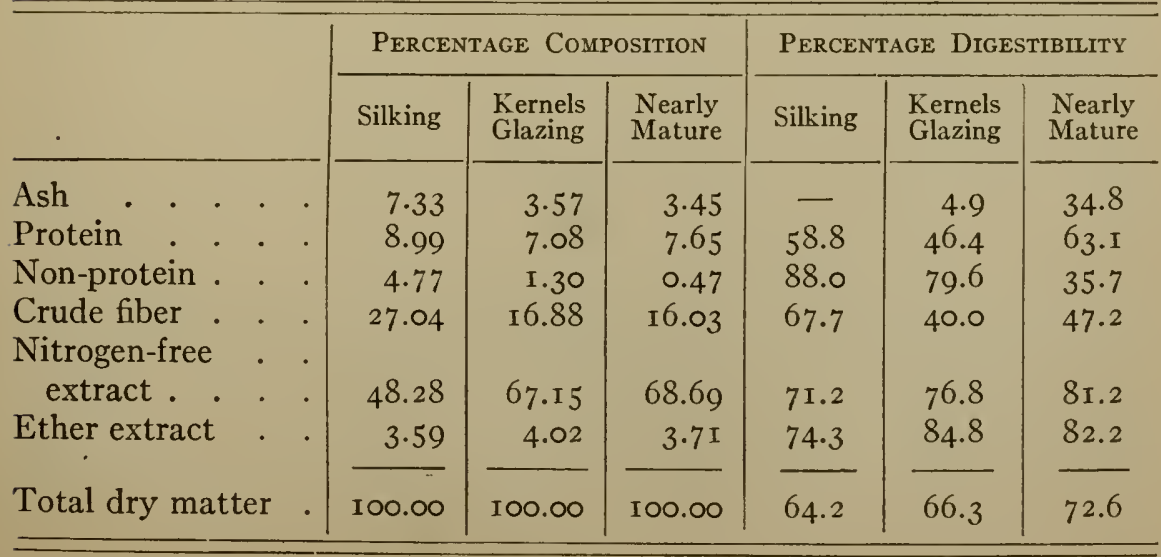


On the other hand, of course, the digestibility of the stalks and leaves alone (stover) diminishes as in the case of other grasses as the plant grows older.

681. Proportions of vegetative organs. - The composition and digestibility of the grasses is also materially affected by the proportions of the various vegetative organs. The influence of the large proportion of seed in the maize plant has already been mentioned. In general, the leaves of the grasses, and of other forage plants as well, are more tender and contain less crude fiber and more proteins than the stems. Leafy species and varieties therefore tend to have a higher feeding value than those which consist more largely of stems, and any influences, such as thickness of planting, manuring, season, and the like, affecting the relative proportion of leaves, tend also to affect the value of the crop. The combined result of all these factors is to make the composition of grass, or of the hay or silage made from it, extremely variable. American analyses of timothy hay, for example, show total protein ranging from 3.8 per cent to 9.8 per cent and fiber varying from 22.2 per cent to 38.5 per cent. The corresponding variations in hay from a few other grasses are as follows:-

Table i66. - Protein and Fiber in Various Grasses

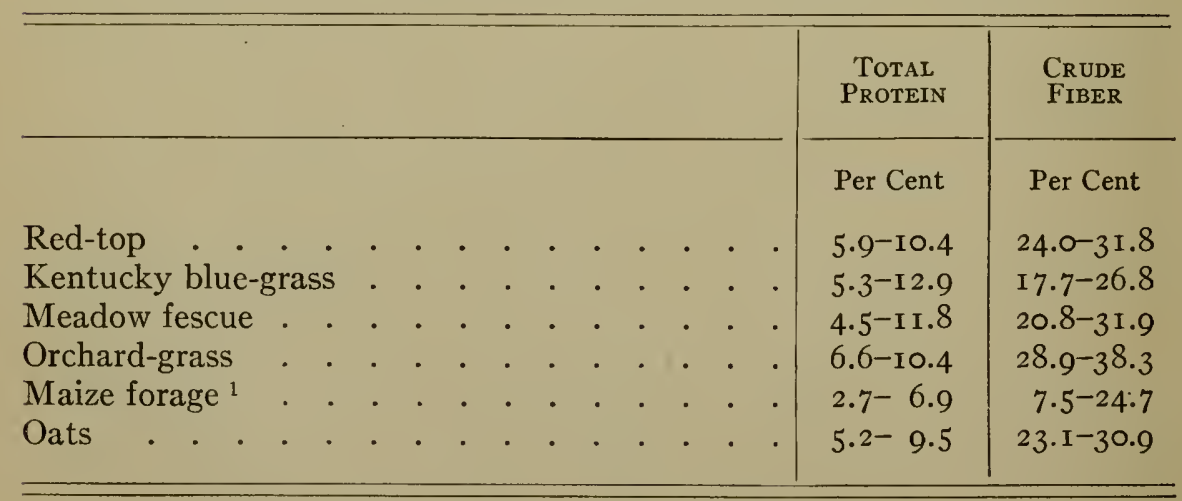

That these variations in composition are accompanied by corresponding differences in digestibility has already been pointed out. Moreover, the percentage of crude fiber in roughage appears to be a fairly accurate index of the relative expendi-

${ }^{1}$ Entire plant, usually containing considerably more water than hay. 
ture of energy in digestion (770). Not only does coarse, woody forage contain less digestible matter, but what it does contain is less valuable to the animal, pound for pound, than that derived from forage of a better quality.

682. The legumes - the clovers, alfalfa, peas, beans, vetches, and the like - constitute a source of forage second only to the grasses in importance, while their value as renovating crops gives them a peculiar position in agriculture. Broadly speaking, leguminous forage may be said to differ from that of the grasses in two main points. First, under like conditions it is notably richer in proteins than the latter. Second, there is a more marked difference between the physical properties of the stems and the leaves in the legumes, the rather coarse stems increasing relatively to the leaves with advancing maturity. Hay from somewhat mature legumes is therefore likely to be bulky, to have a higher percentage of crude fiber than grass hay, and relatively to be less digestible. For the same reason it is more subject to mechanical losses in curing, which likewise lower its quality. For all these reasons, the composition and digestibility of leguminous forage show an even greater range than those of the grasses, and the importance of timely cutting is still more marked. In brief, the influences which affect the composition and digestibility of the grasses affect those of the legumes in substantially the same way but to an even greater extent.

683. Straw consists of the vegetative organs of the plant after the removal of the ripe or nearly ripe seeds. Since the ripening of the seed consists largely in the transfer to it of soluble materials from the leaves and stems, it follows that the straw will be poor in digestible materials in proportion to the extent of seed formation and the degree to which the seeds ripen. Furthermore, those parts of the plant most distant from the seed are found to be most completely exhausted of food material. The straw of the common small grains is relatively very poor in proteins and fat, while still containing not inconsiderable amounts of digestible carbohydrates and related substances. Its tough, woody character, however, as indicated by its high percentage of crude fiber, points to a relatively large expenditure of energy in its digestion, and its real nutritive value is therefore low. Wheat and rye straw stand at the 
foot of the list, while oat and barley straw are more valuable. Sheep are especially adapted to utilize straw, consuming the upper and more valuable parts and rejecting the coarser parts. The straw of maize (stover) constitutes a valuable feeding stuff. It is relatively less woody than that of the small grains, has a relatively high degree of digestibility, and is more palatable than ordinary straw. To secure its complete consumption, however, it is necessary to cut or shred it, and it has been questioned whether the additional material eaten in the cut fodder is worth the labor of cutting. The straw of the legumes is richer in protein than that of the cereals and lower in fiber, with correspondingly higher digestibility. On the other hand, it is usually coarse and unpalatable, and liable to contain molds and other fungi.

\section{§ 2. Roots, Tubers and Fruits}

684. Contain much water. - Roots and tubers constitute a distinct class of feeding stuffs, differing markedly in their properties from the coarse fodders on the one hand and the concentrated feeding stuffs on the other. With them may be included for convenience certain fruits, notably pumpkins and other cucurbita. They are characterized especially by their large proportion of water. In the root crops proper (beets, turnips, carrots, mangels and the like) the percentage of water may vary from 80 to 95 . The tubers (of which potatoes are the chief representative) contain less water, the range being approximately 66 to 82 per cent. A second equally marked characteristic of these feeding stuffs is the low percentage of crude fiber in their dry matter. Their percentage of crude protein is also low, and a large share of it consists of non-protein of inferior nutritive value.

685. A source of carbohydrates. - The dry matter of these crops consists largely of the more readily soluble carbohydrates. In the tubers starch is the predominant carbohydrate, while in beets, especially sugar beets, cane sugar occupies this position, and this substance has been shown to have a distinctly lower nutritive value, for ruminants at least, than starch. In other root crops, the carbohydrates consist largely of gums, pectin substances, and other compounds, including the pentose car- 
bohydrates, whose exact nutritive value is still uncertain. There are also present in roots, and particularly in fruits, more or less organic acids whose nutritive value is low. In consequence of their succulent and tender nature, tubers, and especially roots, have a high degree of digestibility and may be presumed to require little energy for their digestion. They are therefore a valuable source of carbohydrate material, even though some of their ingredients are of somewhat inferior value. In general, the dry matter of tubers is more valuable than that of roots. On the other hand, the dietetic effects of roots are especially prized, but the considerable amount of labor required for their cultivation tends to restrict their use.

\section{§ 3. The Concentrates}

686. Comparison with roughage and with roots. - The concentrated feeding stuffs, or "concentrates," as their name implies, are those which contain a large amount of nutriment in a small weight and bulk. They stand in contrast, on the one hand, with roughage, in which the real nutriment is accompanied by a large proportion of woody fiber and other indigestible matter which adds to the weight and bulk without materially increasing the nutritive value. On the other hand, they excel the roots and tubers because, while the dry matter of the latter is very valuable, it is largely diluted, so to speak, with water. The concentrates are therefore the main reliance for the rapid, intensive production of meat, milk or work. The concentrates may be subdivided into farm products and the by-product feeding stuffs.

\section{Farm products}

687. The cereal grains. - The grains were, until comparatively recent times, the main reliance of users of concentrates, and indeed are still in many sections of the United States. Corn, oats, barley, rye, peas, beans, rice and at times even wheat, are feeding stuffs whose value needs no advocate. These seeds contain, stored away for the use of the young plantlet, proteins, fats and carbohydrates of the most valuable character and "representing the highest type of vegetable food." Their 
nitrogenous matter is chiefly in the form of true proteins of recognized nutritive value, their carbohydrates are largely starch, and their ether extract chiefly true fat. Being closely related to the nutrition of the young plant, the composition of the properly matured seed shows much smaller variations than that of the coarse fodders. The degree of maturity of the seed, however, materially affects its composition and in much the same way as it does that of the coarse fodders. In the early stages of seed formation, the protein and ash flow abundantly from the vegetative organs to the seed, while later the ripening of the seed is largely an accumulation of carbohydrates. Any influences, therefore, which check the normal development of the seed, such as drought or lodging of the grain, tend to produce a seed richer in protein and poorer in carbohydrates. Light, shriveled grain, therefore, tends to be high in protein. Moreover, the ingredients of unripe seeds differ to a considerable extent from those of ripe seeds. The nitrogen, for example, is to a larger extent in the form of non-protein rather than true protein, and the carbohydrates are in the form of sugars of one sort or another rather than starch, as in the ripe grain.

688. Composition and digestibility of cereals. - The cereal grains are characterized by a medium percentage of protein ( 8 to 14 per cent), chiefly composed of true protein, a rather low percentage of fat (I.5 to 6 per cent) and a high percentage of carbohydrates, largely starch. Their ash is small in amount and in it potassium and phosphorus acid are prominent, while but little calcium is found. Maize contains rather less protein than the other cereal grains, with correspondingly high percentages of starch and of fat. While it has been shown that the protein content of corn can be notably increased by selection and breeding, the effects of the latter have not yet sensibly affected the character of the commercial crop. The naked grains (maize, rye, wheat) show a comparatively high percentage digestibility, and both in this respect and as regards their composition exhibit less variation than the hulled grains (oats, barley). In the latter, the variable proportion of the relatively valueless hulls to the kernel causes both composition and digestibility to vary greatly. Oats, for example, have shown the extremes of 6 and 17 per cent protein and 3 to 7 per cent of fat. The hulls resemble straw in composition and value. They therefore in- 
crease the proportion of crude fiber in the grain, and correspondingly diminish its digestibility and nutritive value.

689. Uses of cereals. - The place of the cereal grains in feeding practice is clearly indicated by the foregoing statements. They enable the feeder to introduce into his rations, without unduly increasing their bulk or weight, large amounts of easily digestible and highly nutritious ingredients. Of themselves, they contain a fair proportion of protein for many purposes, especially for mature animals, but they are not capable of offsetting a deficiency of protein in the other ingredients of the ration, nor do they supply enough of this ingredient to meet fully the demands of the rapidly growing animal or the highly productive dairy cow.

690. Leguminous grains. - The leguminous grains share the general physical properties of the naked cereal grains, and like them contain feed materials (proteins, carbohydrates, fats) of the highest grade. They are especially characterized, in contrast with the cereal grains, by their relatively high percentage of protein, ranging according to American analyses from 20 to 42 per cent. Some of them, as the soybean and the lupine, also carry notable amounts of fat, but the more common ones are not richer in this substance than the cereals. They are richer in ash than the cereals, notably as regards phosphoric acid and lime. Their digestibility is generally high. Like the cereals, they are valuable as sources of total digestible feed in a concentrated form, but unlike these they serve also to enrich rations in protein. Aside from certain technical by-products, they are the most available materials for this purpose, and the culture of leguminous feeding crops, both for this purpose and for their effects on the soil, deserves careful consideration.

691. Oil seeds. - The oil seeds, such as flax, cotton and rape, are not commonly used directly as feeding stuffs because of their commercial value. These seeds contain a high percentage of protein, while in place of much of the carbohydrates of the cereals and legumes a large percentage of oil is found. Flaxseed contains a considerable quantity of so-called " mucilage," which swells up with water to a slimy mass and has a very soothing effect on the digestive organs. Cottonseed is fed to cattle to some extent, usually either boiled or roasted, but is regarded as dangerous for growing swine. 


\section{By-products}

692. Nature. - The by-product feeding stuffs are the residues of technical processes by which the products of the soil are prepared for man's use, either as food or for other purposes. The more important of these technical processes are: the milling of grains; the manufacture of cereal foods; the manufacture of alcoholic liquors; the manufacture of starch and glucose ; the manufacture of sugar; and the extraction of oils.

693. By-products of milling. - Milling residues, particularly of wheat, are among the most familiar of the by-product feeding stuffs. They include the screenings secured in cleaning the grain for milling and the bran and middlings secured in the grinding proper. The screenings are an exceedingly variable mixture according to the quality of the grain, containing, besides light and broken grains, a great variety of weed seeds, fragments of straw, sand and earth, as well as spores of numerous fungi, and dirt of all sorts. While some of these have undoubted feeding value, the possible danger to the health of the animals, and of the infestation of the fields with weed seed through the manure, demand great caution in the use of

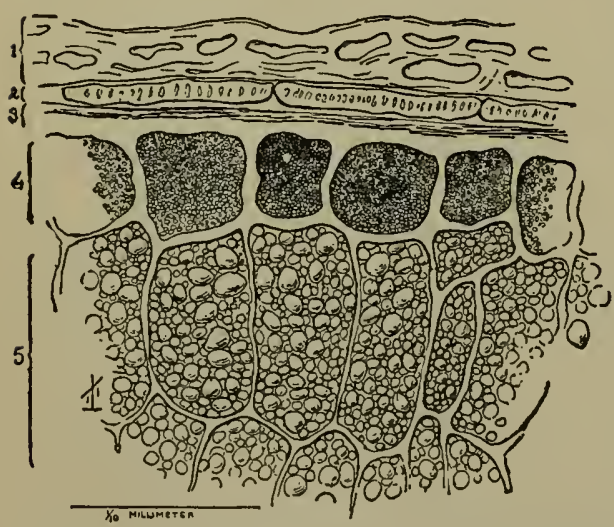

FIG. 43. - Partial section of wheat grain. (Bailey's Cyclopedia of American Agriculture.)

I, Seed pod. 2, Outer seed coat. 3 , Inner seed coat. 4, Gluten cells. 5, Starch cells. (Jordan.) screenings as feed. Its addition to bran or middlings is to be regarded as an adulteration.

Bran. - The bran of wheat or rye consists essentially of the seed-coats of the grain, the layer of so-called gluten cells immediately beneath them, and a proportion of the inner, floury part of the grain varying with the perfection of the milling. The seed-coats of the grain contain most of its crude fiber, while the gluten cells are richer in proteins than the inner part of the kernel. In proportion, therefore,'as the bran is more perfectly separated from the flour, does it become at once richer in protein and in crude 
fiber and poorer in easily digestible carbohydrates. Such bran is more valuable as a source of protein than the more floury bran, but at the same time contains less total digestible matter, and probably has an inferior value as a source of energy.

Middlings, as the name indicates, are intermediate products between bran and flour. In modern/methods of milling, various grades are produced, in the names of which there is a considerable lack of uniformity. The "brown" middlings contain more of the seed-coats (bran) than the "white" middlings, which approach the low-grade flour (" red dog" flour) in character. Shorts seem to be substantially the same as middlings. Because of their smaller content of hulls, middlings are decidedly more digestible than bran, while scarcely inferior to it in percentage of protein.

Buckwheat middlings, a by-product from the milling of buckwheat, contains nearly twice as much protein and fat as average wheat middlings, and correspondingly less carbohydrates. It is sometimes called buckwheat bran, but this name is also applied to the tough, innutritious hulls of the buckwheat, which have little feeding-value and which are not infrequently used as an adulterant of the middlings. The middlings are credited with a tendency to ferment or become rancid when stored in bulk, and also with producing a soft, oily butter-fat when fed in large amounts.

Rice bran resembles wheat bran, but contains less protein and fully twice as much fat. The pure bran is sold largely under the name of rice meal, while the commercial bran contains an admixture of varying amounts of rice hulls. The hulls, which are separated from the kernel as the first process in the milling, contain about 40 per cent of fiber, and are heavily impregnated with silica and covered with hard, silicified fibers which are liable to cause severe and even fatal irritation of the digestive organs. Their presence in the bran to any large extent is to be regarded as a dangerous adulteration.

Rice polish results from the polishing of the rice grains after the removal of the bran and germ. It contains somewhat less fat and protein than the pure bran, but is considerably more digestible.

All these rice by-products contain more or less grits or fragments of the kernel, which have been found to be rather difficult of digestion. The rice products are also rich in fat, 
which becomes rancid rather easily and often renders the material unpalatable. It is asserted that this rancidity can be prevented by kiln-drying the bran or polish as soon as produced.

Uses of milling by-products. - There has been a tendency to regard the milling by-products largely as sources of protein. While it is true that the bran and middlings are richer in protein than whole wheat or other cereal grains, the difference is not sufficient to enable them to offset to any marked degree the deficiencies of other ingredients of the ration in this respect. They are to be regarded primarily as sources of digestible matter as a whole, with a tendency to increase somewhat the proportion of protein in the ration. Familiarity with the good qualities of wheat bran in particular, its comparative safety as a feed in inexperienced hands, and its good dietetic effect have tended to an exaggerated idea of its feed value. When it rules high in price it is usually possible to substitute other feeding stuffs for it, partially or wholly, which will furnish both protein and energy more cheaply. Buckwheat middlings, on the

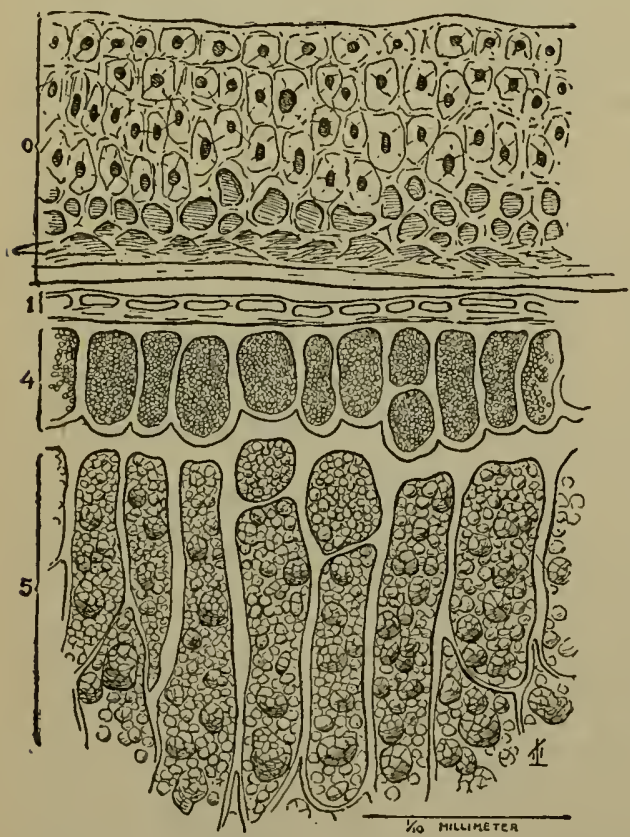

FIg. 44. - Partial section of oat grain. (Bailey's Cyclopedia of American Agriculture.)

o, Hull. I, Seed coat. 4, Gluten cells. 5, Starch cells. (Jordan.) contrary, often furnish a cheap source of protein for a ration otherwise deficient in it.

694. Breakfast food residues. - In the manufacture of the great variety of so-called cereals, or breakfast foods, now on the market, a considerable quantity of by-products accumulates. In the case of the most common of these, oatmeal, the residue consists chiefly of the hulls of the oats together with some of the lighter grains.

Oat hulls. - The hulls themselves have scarcely more feeding value than the straw, which they resemble in composition, 
while the proportion of light oats is not sufficient materially to raise the value. Oat hulls are rarely offered as such in the market but are usually disposed of in one of two ways. First, they are made the basis of various proprietary feeds, cheap by-products of various sorts being added, usually including a small amount of the protein-rich by-products shortly to be described. These feeds are offered under various names and with abundant advertising testimonials. While they are by no means worthless, it is evident that the oat hulls themselves are no more valuable because of the addition to them of other materials, while the consumer ultimately pays the cost of mixing, transportation and advertising. The second use to which oat-hulls are put is the adulteration of the mixed feeds, especially corn and oat feeds, which are freely offered on the market. Since it is difficult to recognize even a considerable adulteration of this sort, such mixed feeds should be purchased only from manufacturers of known integrity or under a satisfactory guarantee as to purity.

Barley feed, a by-product of the manufacture of pearled barley, is similar in feeding value to oat hulls.

Hominy feed. - In the manufacture of hominy from corn, the hull, the germ and the more starchy parts of the kernel are rejected and constitute hominy feed, or hominy chop, which is similar to the whole kernel in composition and digestibility, except that its percentage of fat is greater. Consequently it has a somewhat higher feeding value, although the fat is likely to become rancid on long keeping and thus lower its quality.

695. By-products of the fermentation industries. - The manufacture of alcoholic liquors consists essentially in the conversion of the starch of grains or potatoes into sugar and the subsequent fermentation of this sugar by means of yeast. The resulting liquor may be consumed directly (beer, ale) or it may be distilled, yielding the more concentrated distilled liquors or commercial alcohol.

Malt sprouts. - The first step in the process is the preparation of malt, by allowing moistened barley to germinate. The growth of the sprouts is stopped by drying when they are about one-third inch long, and these dried sprouts, separated from the grain, constitute malt sprouts. Being young roots of barley, they have the general properties of all young 
plant growth, containing a high percentage of nitrogen, much of it in the form of non-protein, and a low percentage of crude fiber.

Brewers' grains. - The next step in the process is the mashing of the ground malt and other grain with warm water. In this process, the diastase of the sprouted barley acts on the starch of the grain, transforming it into sugar. In the manufacture of beer or ale, the resulting liquid is drawn off and fermented separately, leaving a residue known as brewers' grains, which is used extensively as a dairy feed. In the fresh state it is valuable, but is subject to the disadvantage of fermenting or souring very readily, and tending in this state to injure the quality of the milk. Somewhat recently, economical processes for drying it have been perfected, and the dried brewers' grains constitute a valuable feed which can be shipped like any other dried feed.

Distillers' grains. - In the preparation of distilled liquor or alcohol, the liquid is fermented in contact with the grains and the alcohol then distilled off, leaving a residue known as distillers' grains or distillery slop. This residue is much wetter than brewers' grains, but is less subject to fermentation, since the sugar has been more completely removed. Large quantities of it are now put on the market in the dried form, both under its own name and various trade names, some of which contain no suggestion of the real nature of the material. It constitutes a valuable source of stock feed. The grains produced from rye are regarded as the poorest and those from maize as of the best quality.

In all these processes the object is to convert the starch of the grain as completely as possible into sugar and then into alcohol. This results in increasing the percentage of all the other ingredients in the residues. They contain accordingly a high percentage of protein with also a somewhat greater percentage of crude fiber than the ordinary grains. They serve, therefore, not only to supply digestible matter as a whole but also to correct a deficiency of protein in the ration.

696. By-products of oil extraction. - The extraction of commercial oils from various oil-bearing seeds leaves by-products, called oil cake or oil meal, some of which have a high feeding value. Of these, cottonseed and linseed meal are the only ones extensively used in the United States and are typical of the 
others. The seeds of cotton and flax are rich in both fat and protein. Hulled cottonseed contains about 30 per cent of each and flaxseed about 22 per cent protein and 35 per cent fat, the latter percentage, however, being somewhat variable. The oil is extracted from the seeds either by pressure or by the use of solvents, leaving a residue still containing some fat and very rich in protein.

Cottonseed meal. - At present cotton oil is extracted only by pressure, the resulting hard cake being ground to cottonseed meal. The highest grade of cottonseed meal is made from the hulled seed and contains 40 to 44 per cent of crude protein and 8 to 9 per cent of fat. It should be nearly free from the hulls and therefore contain little crude fiber. Cottonseed meal is adulterated extensively with the tough, black hulls of the cottonseed, which have a very low feeding value. This is especially true of the inferior grades of commercial cottonseed meal, which are sold at a lower price than the standard grade.

Linseed meal. - Linseed oil is extracted from the flaxseed both by pressure and by means of naphtha, the latter being completely removed from the resulting oil-meal and recovered for use again. The "new process" of extraction removes the fat more completely than the "old process" of pressure, and the resulting linseed meal is somewhat poorer in fat and contains somewhat more protein than the old-process meal. The process of extraction by pressure has been so far perfected in recent years, however, that the difference between the old-process and new-process meal is distinctly less than formerly. The protein of the new-process meal appears to be slightly less digestible than that of the old-process meal, which tends still further to reduce the difference between the two.

Other oil meals. - Oils are also manufactured commercially from the seeds of the common peanut, the soybean, the oil palm and the cocoa palm. The resulting oil cakes or meals are extensively used as feeding stuffs in European countries but do not appear to have as yet found access to the feed market of the United States to any considerable extent.

The corn-germ meal mentioned in connection with the gluten feeds may also be classed as an oil-meal.

697. By-products of starch and glucose manufacture. Starch and glucose are made in the United States chiefly from 
maize. The starch is separated by coarse grinding and the use of water, the starch being carried off in suspension and allowed to settle out. Glucose is manufactured by further treatment of the starch with acid. In the preparation of the starch, the parts of the kernel which are rejected are the hull, the germ

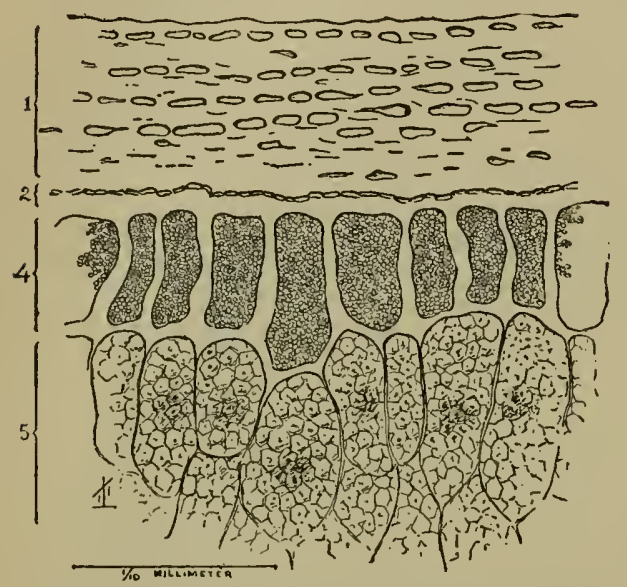

FIG. 45- - Partial section of maize kernel. (Bailey's Cyclopedia of American Agriculture.)

I, Outer layer of skin. 2, Inner layer of skin. 4, Gluten cell. 5 . Starch cells. (Jordan.) and the more glutinous part of the interior of the grain from which the starch cannot be completely separated.

Corn (maize) bran. - The hulls are comparatively low in protein and contain considerable fiber. When sold separately they are called corn bran, although the composition of commercial samples indicates some admixture of the germs.

Germ meal. - The germ contains about 30 per cent of oil, which has a commercial value and is secured by pressing the germs. The residue constitutes germ meal, which still contains about 7 per cent of oil, and in the neighborhood of I I per cent of crude protein.

Gluten meal and feed. - The glutinous residue of the kernel constitutes gluten meal, containing, in general, 30 to 40 per cent of crude protein with a comparatively low percentage of fat and fiber. Some factories mix the gluten meal and the hulls, and sell the mixture under the name of gluten feed, which contains approximately 24 per cent of crude protein, 6 per cent of crude fiber and 6 per cent of fat. Sometimes the hulls and germs are sold together under the names "sugar feed" or "starch feed," either wet or dry. In fact, various mixtures of the three main products are made and sold under diverse commercial names. These various glucose products should invariably be purchased on a guarantee as regards composition and purity.

698. By-products of sugar manufacture. - Sugar has come to be manufactured from sugar-beets to a considerable extent in 
the United States, while in certain regions the manufacture from sugar cane is an important industry.

Sugar-beet pulp. - The sugar is extracted from the finely cut beets by means of water in what is known as the diffusion process. The residue from this constitutes what is commonly known as beet pulp, which is essentially sugar beets minus the sugar and some of the other soluble substances. In the fresh state it contains 90 to 95 per cent of water, which may be reduced to about 85 to 87 per cent by pressing. Its general properties are similar to those of roots and it occupies much the same place in the ration. Its digestible matter consists chiefly of carbohydrates belonging to the group of pectins and gums, somewhat inferior to the sugar of the beets but, according to recent investigation, fully as valuable as the digestible matter of mangels. The wet beet pulp is too heavy to bear long transportation, but may be preserved in the neighborhood of the factory by ensiling. It is now, however, dried and put on the market as dried beet pulp, containing not more than 5 to io per cent of water. The dried pulp is relatively about equally valuable with the wet pulp, especially if soaked in water, as it should be before feeding.

Molasses. - In the further manufacture of sugar either from sugar beets or sugar cane, there remains, as a final residue, the molasses. This contains 20 to 25 per cent of water, approximately 50 per cent of sugar, scarcely more than one-half per cent of true protein, and 8 to ro per cent of non-protein, along with other substances of doubtful nutritive value. It is essentially a source of easily soluble carbohydrates, principally sugar. Beet molasses, in particular, has a marked laxative action, commonly ascribed to the potassium salts present in it but perhaps due quite as much to the sugar. For this reason, care is required to accustom animals to it gradually and not to overfeed with it. Its laxative qualities are said to be valuable when used in small amounts for horses in preventing attacks of colic.

Molasses feeds. - Owing to its physical properties, molasses is an inconvenient material to handle. To avoid this difficulty, the so-called molasses feeds have been put on the market. These consist of molasses dried down on some suitable material. A large number of concentrated feeding stuffs have been used 
for this purpose, and it has also been dried together with the beet pulp, forming molasses pulp. All these feeds are of value in proportion to the materials out of which they are made.

699. By-products of the packing house. - The slaughtering of meat animals on a large scale in the modern packing house yields a number of highly nitrogenous by-products which are of especial value in the feeding of swine and poultry.

Dried blood is especially rich in protein, of which it contains over $80 \%$, practically all of which is digestible. It contains a small amount of fat and but little ash.

Tankage consists essentially of the residue left after the rendering of the meat scraps, trimmings and scrap bones of the packing house. Tankage contains much less protein than dried blood but, on the other hand, contains a considerable percentage of fat, while the bone which it contains renders it relatively rich in ash ingredients, especially calcium and phosphorus. As is obvious from the method of its manufacture, tankage is likely to vary widely in composition and should always be bought on a guarantee. 


\section{CHAPTER XVI}

\section{RELATIVE VALUES OF FEEDING STUFFS}

As soon as live stock husbandry emerged from the pastoral stage and man began to store up forage for the winter or to utilize the products of his cultivated land for feeding his domestic animals, the question of the relative values of the different feeding stuffs necessarily arose. As agriculure has gradually become more intensive and as the variety of natural materials and of technical by-products available has increased, the question has grown in importance, the traditions of practice based on the experience of earlier investigations have been recognized to be insufficient guides, and much effort has been put forth to replace these traditions by exact knowledge.

\section{$\S$ I. Direct Comparisons of Feeding Stuffs}

700. Hay values. - A natural and logical method of investigation was to feed the materials in question to animals and compare the amount of increase or of milk which was secured. Good meadow hay was universally regarded as a complete feed, suitable for practically all purposes. Hence it was naturally taken as the standard and the effort was made to establish from the results of experience and experiment what amounts of different feedstuffs would replace a unit weight of hay. In this way arose the tables of so-called hay values. ${ }^{1}$ The first of these was that published by Thaer in Germany in 1809 , based chiefly on the early chemical analyses of Einhof in which the constituents soluble in water, alcohol, dilute acids and dilute alkalies were determined. The sum of all these ingredients, without distinction as to kind, was taken to represent the nutritive value, and the hay values were computed in proportion to them.

1 Compare Henneberg, Über den Heuwert der Futterstoffe; Beiträge zu Fütterung der Wiederkäuer, Heft I, I860, pp. I-I6; and von Gohren, Naturgesetze der Fütterung, I872, pp. 286-305. 
The system had the advantage of simplicity. Experience had afforded a fairly definite idea of the quantity of hay required for a given amount of production. It was only necessary to compute from the hay values what weights of the available feeding stuffs would produce equal effects. The simplicity of the calculations, due especially to the fact that the relative value of a feed was expressed by a single fixed number, led to a rapid adoption of the system. "To each feeding stuff a definite hay value was assigned and in a short time one had a beautiful table constructed which gave the most exact information regarding the value of the most diverse feeding materials in comparison with hay. Anything which appeared in any way suited for feeding found its place in the table and each new feeding stuff which the progress of agronomy provided, directly or indirectly, was likewise quickly incorporated. It went so far that even the salt supplied to the animals was computed in hay values." 1

Thaer himself based his figures in part on the results of practical experiments. Numerous subsequent investigators carried out direct comparisons of feeding stuffs on an extensive scale and not one but several tables of hay values were formulated. Unfortunately, these tables differed widely from each other, some of them giving two or three times as great a hay value as another to the same feed. It was evident also that the unlimited substitution of different classes of feeds, as for instance of grain or roots for hay, was impossible. Such discrepancies and limitations led to various modifications of the methods of estimating the hay values. Boussingault regarded the protein content of the feed as the principal factor, while Nathusius took into account also the content of crude fiber and Wolff ${ }^{2}$ worked out a somewhat elaborate method in an attempt to retain the convenience of reckoning with a single number for a feed. The impossibility of this, however, gradually came to be recognized, and the hay values have now only a historical interest.

701. Practical feeding trials. - But while the system of hay values has become obsolete the idea of determining the relative nutritive values of feeding stuffs on the basis of direct comparisons of the results obtained in practice has survived in

1 Settegast, Die Fütterungslehre, I879, p. 4.

${ }^{2}$ Die landwirtschaftliche Fütterungslehre, I86r, pp. 455-456. 
full vigor. A very considerable share of the investigations in stock feeding during the last two decades, especially perhaps in the United States, has consisted of experiments intended to determine the effects of the substitution of one feed for another in a ration.

Undoubtedly the so-called practical trial has an important part to play in the development of a sound theory of feeding as well as in relation to the economic aspects of the subject. Regarded, however, simply as a means for the quantitative determination of the relative values of feeding stuffs it is subject to precisely the same limitations and uncertainties as the old attempt to determine hay values, and in this respect has in general led to scarcely more satisfactory or concordant results. It is as true in the later as in the earlier experiments that the effect of a feeding stuff may vary widely with the combination in which it is fed and the conditions under which it is used.

702. Feed units. - An interesting attempt to revive the fundamental conception of hay values in a modified form and within a restricted field, and thus to retain the advantage of expressing the relative value of a feed by a single number, is found in the so-called feed unit system devised by Fjord and his associates in Denmark and extensively used also in Sweden. ${ }^{1}$

The feed unit system, like that of hay values, is essentially a system of empirical equivalents according to which feeding stuffs may replace each other. Instead of hay, the basis of comparison is a unit weight of grain (corn, barley, wheat or rye or a mixture of grains). This is called a feed unit and the amounts of other feeds required to equal the feed unit have been determined in very extensive coöperative feeding experiments by the group system (572) with swine and especially with dairy cows. The experiments themselves have been executed with every precaution to ensure accuracy. The results for dairy cows, as revised by Woll for American feeding stuffs, and the Danish values for swine and for the horse are given by Henry and Morrison ${ }^{2}$ as follows :-

1 For a more complete discussion of the feed unit system compare Woll; Wisconsin Expt. Sta., Circular No. 37.

2 Feeds and Feeding, I5th Edition, p. I27. 
Table i67. - Amount of Different Feeds Required to Equal One FEED UNIT ${ }^{1}$

\begin{tabular}{|c|c|c|}
\hline \multirow{2}{*}{ FEED } & \multicolumn{2}{|c|}{$\begin{array}{l}\text { FEED REQUIRED TO } \\
\text { EQUAL I UNIT }\end{array}$} \\
\hline & Average & Range \\
\hline FOR DAIRY COWS & & \\
\hline \multicolumn{3}{|l|}{ Concentrates } \\
\hline $\begin{array}{l}\text { Corn, wheat, rye, barley, hominy feed, dried } \\
\text { brewers' grains, wheat middlings, oat shorts, } \\
\text { peas, molasses beet pulp, dry matter in roots }\end{array}$ & 1.0 & - \\
\hline Cottonseed meal & 0.8 & 一 \\
\hline Oil meal, dried distillers' grains, gluten feed, soy- & & \\
\hline $\begin{array}{c}\text { beans } \\
\text { Wheat bran, oats, dried beet pulp, barley feed, }\end{array}$ & 0.9 & - \\
\hline malt sprouts . . & I.I & - \\
\hline Alfalfa meal, alfalfa molasses feeds . . . . & 1.2 & 一 \\
\hline Hay and straw & & \\
\hline 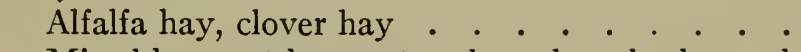 & 2.0 & $1.5-3.0$ \\
\hline $\begin{array}{l}\text { Mixed hay, oat hay, oat and pea hay, barley and } \\
\text { pea hay, red-top hay } . .\end{array}$ & 2.5 & $2.0-3.0$ \\
\hline Timothy hay, prairie hay, sorghum hay . . . & 3.0 & $2.5-3 \cdot 5$ \\
\hline Corn stover, stalks or fodder, marsh hay, cut straw & 4.0 & $3 \cdot 5^{-} 6.0$ \\
\hline Soiling crops, silage and other succulent feeds & & \\
\hline 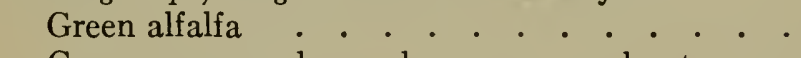 & 7.0 & $6.0-8.0$ \\
\hline $\begin{array}{l}\text { Green corn, sorghum, clover, peas and oats, can- } \\
\text { nery refuse } \\
\end{array}$ & 8.0 & $7.0-10.0$ \\
\hline 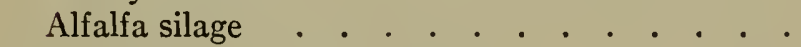 & 5.0 & - \\
\hline Corn silage, pea vine silage . . . . . . . . & 6.0 & $5.0-7.0$ \\
\hline Wet brewers' grains . . . . . . . . . . . & 4.0 & 一 \\
\hline Potatoes, skim milk, buttermilk . . . . . & 6.0 & 一 \\
\hline Sugar beets . . . . . . . . . . . . . . . & 7.0 & - \\
\hline 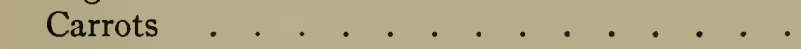 & 8.0 & - \\
\hline 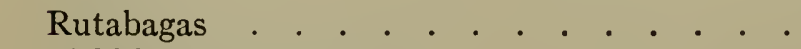 & 9.0 & 8.0-10.0 \\
\hline 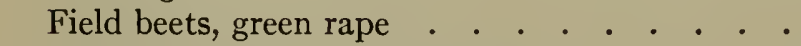 & 10.0 & - \\
\hline Sugar beet leaves and tops, whey . . . . . & I 2.0 & 一 \\
\hline Turnips, mangels, fresh beet pulp. . . . . . & I 2.5 & $10.0-15.0$ \\
\hline $\begin{array}{l}\text { The value of pasture is generally placed at } 8 \text { to ro } \\
\text { units per day, on the average, varying with } \\
\text { kind and condition . } . . .+. . .\end{array}$ & & \\
\hline
\end{tabular}

1 The values for pigs and horses are those given in the Danish valuation table and those for dairy cows the values as revised by Woll for American feeding stuffs in Wisconsin Circular, No. 37. 
Table 167.- Amount of Different Feeds Required to Equal One Feed Unit (Continued)

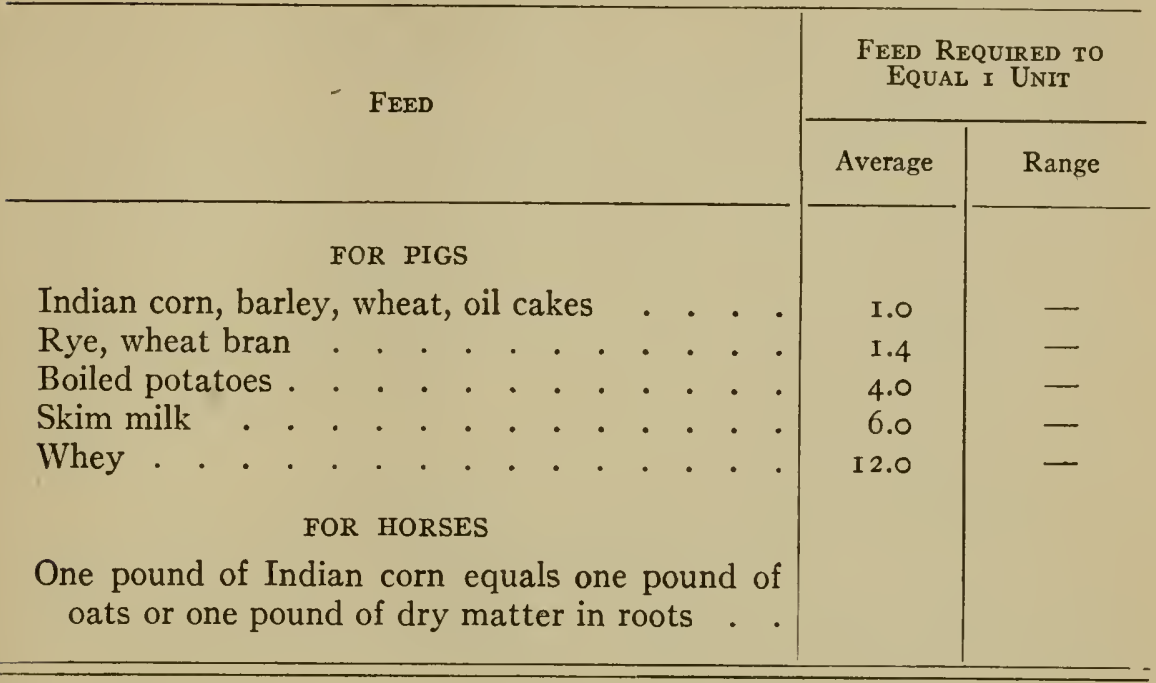

703. Logical basis of feed unit system. - The Scandinavian feed unit values have a broad experimental basis. The results of the experiments have been reasonably consistent and in general the feed unit values correspond well with the relative net energy values discussed in the following chapter except that they ascribe somewhat higher values to proteinrich feeds.

Nevertheless, the logical basis of the system has the same defect that is inherent in all such systems. As was shown in Chapter V (263), feed has two distinct functions and these functions are incommensurable. It is as impossible to combine the value of a feed as a source of protein or other structural material with its value as a source of energy, and to express the result in a single number, as it is to compare the relative values of food and water to a starving man. A protein-rich feed like cottonseed meal, for example, will necessarily produce a greater effect when added to a ration deficient in protein than when added to one containing an abundance of that ingredient; with a material deficient in protein precisely the reverse would be true. As a matter of fact the feed units are only claimed to be equivalent values, " under ordinary conditions of feeding these animals, when fed in mixed rations that would contain over a certain 
minimum of digestible protein." 1 As Henry and Morrison have pointed out, "The feed unit system has been evolved in a comparatively small region where similar crops are grown on the different farms and the price of purchased feeds does not vary widely throughout the district."

704. Comparison of feed units and net energy values. The writer is not able to agree with those who would introduce the feed unit system in this country with its wide variety of feeding stuffs and conditions. The applicability of the feed units, as just pointed out, is conditioned upon the presence of sufficient protein in the rations. As thus limited, however, they practically attempt to measure the relative values as sources of energy, and for this purpose the use of the net energy values to be considered in the next chapter is just as simple arithmetically and equally accurate, while it has two immense advantages. First, the net energy values are rational and not empirical values. They are based on physiological investigations and their very imperfections tend to stimulate further investigation which may lead to their great improvement or to the discovery of new and still better methods of comparison. The feed unit, on the other hand, constitutes a dead end so far as investigation is concerned, leading to nothing beyond some increase in numerical accuracy, while it is far inferior in pedagogic value. Second, the feed units are purely relative values, based on direct comparisons of the results with different materials with no attempt to discover the causes of the observed differences. They show to what extent one feeding stuff is better or worse than others, but establish no relation between feed and product. Energy values, on the other hand, aim to show the amount of product which may be expected from a unit weight of the feeding stuff - i.e., the amount of energy which it can contribute to the maintenance of the body or to the building up of new tissue. Thus, if average maize meal, for example, has an energy value of 85 Therms per hundred pounds, this means that one hundred pounds of it, fed as part of a maintenance ration, would conserve in the body of the animal an amount of fat and protein having an energy value of 85 Therms, which would otherwise be burned up to support the vital activities. Furthermore, it means that, if added to the maintenance ration, the maize will furnish ma-

${ }^{1}$ Woll, loc. cit. p. 13. 
terial sufficient to produce a quantity of milk or of meat having an energy value of 85 Therms. Still further, the investigations by which these facts are established also show that out of the approximately I 87 Therms gross energy of roo pounds of maize meal, about 50 escape unused in the various excreta, while about $5^{2}$ are expended in the various processes connected with the consumption and assimilation of the feed. In other words, they show the nature of the losses suffered as well as the final amount of product to be expected. Such data as these have an independent value and are of an entirely different nature from those expressed in the feed units.

\section{$\S$ 2. Relative Values Based on Composition and DigestibiLity}

705. Chemical composition. - Even before the rise of the system of hay values, attempts were made by Davy, Einhof, Sprengel and others to compare feeding stuffs on the basis of chemical analyses, and indeed the earlier hay values were based in part on such comparisons (700). The methods for the chemical analysis of feeding stuffs were gradually improved, although they still remain quite imperfect, but along with this improvement came a clearer recognition of the fact that the problem of relative values is at bottom a physiological and not a chemical question.

706. Physiological functions of nutrients. - In particular the teachings of Liebig and the investigations of Bischoff and Voit ${ }^{1}$ on the nutrition of carnivora served to establish those basal facts regarding the functions of proteins, carbohydrates, fats and ash in nutrition which. have been confirmed and extended by later investigations and have been outlined in Chapter V. Haubner appears to have been the earliest to suggest the application of these principles to comparisons of feeding stuffs and the feeding of farm animals, while to Grouven ${ }^{2}$ belongs the credit of having first formulated the requirements of animals and the values of feeding stuffs in terms of the different classes of nutrients. His tables, however, were based on the total nutrients found by chemical analysis and were comparatively

1 Gesetze der Ernährung des Fleischfressers, I860.

2 Vorträge über Agriculturchemie, 1858. 
soon replaced by more accurate data based on determinations of the digestible nutrients.

707. Henneberg's and Stohmann's investigations. - It is to the fundamental investigations of Henneberg and Stohmann ${ }^{1}$ at the Weende Experiment Station, near Göttingen, that we are indebted for the inauguration of a system of comparing the values of feeding stuffs which has endured with little material change up to the present time. These investigators were the first to apply systematically in studying the nutrition of herbivora the physiological principles already demonstrated for other classes of animals and to base their determinations upon the outgo as well as upon the income of the body. Their earlier experiments deal chiefly with the digestibility of feeding stuffs and rations. Later a comprehensive scheme of investigation, including determinations of the gaseous excreta, was laid out ${ }^{2}$ and begun but never completed.

\section{Table i68. - Example of Computation of Digestible Nutrients}

\begin{tabular}{|c|c|c|c|c|c|c|}
\hline & & & & & Clover Hay & MaIze MeaL \\
\hline \multicolumn{5}{|c|}{ Chemical composition } & & \\
\hline Water . . & & & & & 15.03 & I 3.73 \\
\hline Ash . . . . . & . & . & & · . & 5.49 & 1.25 \\
\hline Protein . . . . . & . & & & .. & 10.24 & 8.80 \\
\hline Non-protein . . . & . & & sa & - . & $\mathbf{I} .36$ & 0.25 \\
\hline Crude fiber. . . . & . & & & . . & $28.6 \mathrm{I}$ & 1.89 \\
\hline Nitrogen-free extract & . & & & · . & 36.98 & 70.44 \\
\hline \multirow[t]{2}{*}{ Ether extract . . } & . & & & & 2.29 & 3.64 \\
\hline & & & & & 100.00 & $\overline{100.00}$ \\
\hline \multicolumn{5}{|c|}{ Percentage digestibility } & & \\
\hline Ash . . . . . & . & & & & $46.48 \%$ & $18.40 \%$ \\
\hline Protein . . . . & . & • & , & • . & $53.19 \%$ & $66.43 \%$ \\
\hline Non-protein . . & . & . & & & $100.00 \%$ & $100.00 \%$ \\
\hline Crude fiber . . . . & . & & & & $50.27 \%$ & $32.40 \%$ \\
\hline Nitrogen-free extract & . & & 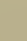 & & $68.94 \%$ & $97.75 \%$ \\
\hline Ether extract . . & . & & & . . & $65.02 \%$ & $95.74 \%$ \\
\hline Digestible nut & ien & & & & & \\
\hline Ash . . . . & . & & & & $5.49 \times 0.4648=2.55 \%$ & $1.25 \times 0.1840=0.23 \%$ \\
\hline Protein . . . . . & . & . & . & . . & $10.24 \times 0.5319=5.45 \%$ & $8.80 \times 0.6643=5.85 \%$ \\
\hline Non-protein . . . & . & 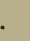 & 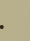 & . & $1.36 \times 1.000=1.36 \%$ & $0.25 \times 1.000=0.25 \%$ \\
\hline Crude fiber & . & • & & . & $28.61 \times 0.5027=14.38 \%$ & $1.89 \times 0.3240=0.61 \%$ \\
\hline Nitrogen-free extract & . & . & & . & $36.98 \times 0.6894=25.49 \%$ & $70.44 \times 0.9775=68.85 \%$ \\
\hline Ether extract . . . & . & . & & . & $2.29 \times 0.6502=1.49 \%$ & $3.64 \times 0.9574=3.48 \%$ \\
\hline
\end{tabular}

1 Beiträge zur Begründung einer rationellen Fütterung der Wiederkäuer, I860 and 1864 .

2 Neue Beiträge, etc., 1870. 
708. The digestible nutrients. - The methods of digestion experiments as used by Henneberg and Stohmann and modified by later experimenters were outlined in Chapter III (157-161). A vast number of determinations of digestibility have been made, upon a great variety of materials, and the results have served as the basis for computing the relative values of feeding stuffs. The method of comparison may be illustrated by means of the digestion experiment on clover hay and maize meal used in Chapter III to illustrate the method. (Table I68.)

Simplified statement. - Since the digestible crude fiber and digestible nitrogen-free extract have been shown $(168,169)$ to have the elementary composition of starch, they have been commonly added together and called carbohydrates. Considering the digestible ether extract to be substantially fat, and omitting the ash on the assumption that an average ration contains a sufficient supply, the amounts of the three principal groups of digestible nutrients may be stated more concisely as follows :-

Table i69. - Simplified Statement of Digestible Nutrients

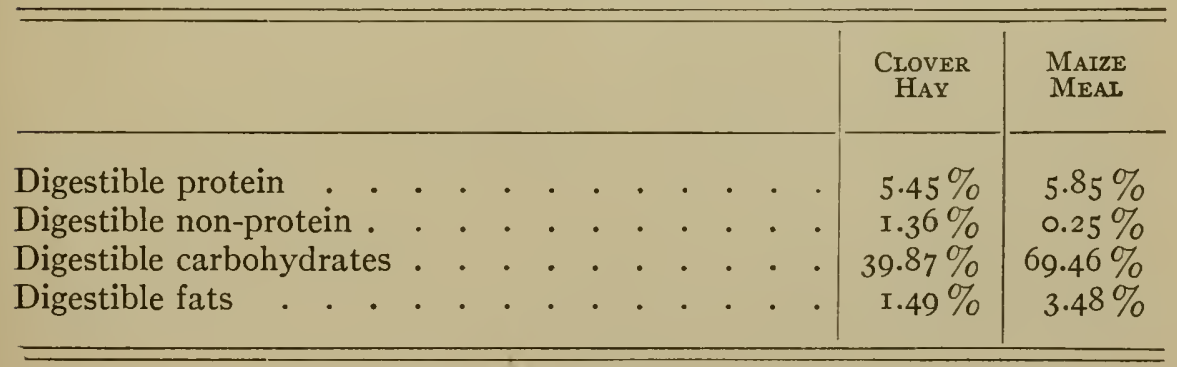

This statement may be still further simplified. A pound of fat produces when burned about 2.25 times as much heat as the same weight of carbohydrates. The non-proteins have approximately the same heat value as the carbohydrates, while it is still questioned whether they help to build up protein tissue. By multiplying the digestible fat by the factor 2.25 and adding the digestible carbohydrates and non-protein we obtain the carbohydrate equivalent for the digestible matter other than protein and the digestible nutrients may be expressed in the following still more concise form:- 
Table i70. - Digestible Nutrients Reduced to Carbohydrate Equivalent

Digestible protein

Digestible carbohydrates equivalent to non-nitrogenous nutrients

Total nutrients

709. The nutritive ratio. - By the method just illustrated the content of a feeding stuff in digestible matter is expressed by two numbers which correspond to the two functions of the nutrients already described (263). The digestible protein shows what the feeding stuff can contribute towards the structural needs of the body, while the carbohydrate equivalent of the digestible non-nitrogenous nutrients shows what portion of the digestible nutrients can serve only as a source of fat or of energy. The ratio between these two quantities gives a useful indication as to whether a feeding stuff or mixture of feeding stuffs is suited for forms of production like growth or milk production, which require a considerable supply of protein, or whether it is better adapted for those which, like work or fattening, make special demands for fuel material. This so-called "nutritive ratio" (better, nutrient ratio) is obtained by a simple proportion. Thus in the two instances just given, it is computed as follows, the second half of the proportion constituting the nutritive ratio:-

For clover hay, $5.45: 44.58=1: 8.2$

For maize meal $5.85: 77.54=\mathrm{I}: 13.3$

710. Significance of results. - Under the stimulus of Henneberg and Stohmann's pioneer work and under the leadership of Wolff, investigation of the digestibility of feeding stuffs was actively taken up in Germany and later in the United States and other countries, and as the result of much labor expended during the last fifty years a fairly complete knowledge of the amounts and proportions of the digestible nutrients supplied by most of the ordinary feeding stuffs has been accumulated. Extensive tables of averages have been published by 
various authors, including many of the agricultural experiment stations, and it is an easy matter for the feeder to learn what amounts and kinds of digestible nutrients any given feed or ration will supply.

In view of the extensive use of such tables it is important that the exact significance of the results which they embody should be understood. As a mere matter of logical conception, the comparison of feeding stuffs on the basis of their digestible nutrients is inferior to that based on hay values or on feed units. In these methods the attempt is made, however crudely, to compare the actual effects produced by the feeding stuffs in the animal body. A determination of digestibility, on the contrary, affords no direct information whatever as to the nutritive effect of the materials digested. It is not even necessary to weigh the animal in a digestion trial. The comparison of feeding stuffs on this basis is between what they contain and not between what they accomplish.

Nevertheless, tables of digestible nutrients have been of great value in promoting more rational and profitable feeding, but it is becoming increasingly evident that they express but part of the truth. The essential feature of the newer methods of comparison outlined in this volume is not that they employ units of energy as a basis of comparison but that they constitute a return to the logical conceptions which were at the basis of the early methods and which were discussed in so illuminating a manner by Henneberg and Stohmann in the introduction ${ }^{1}$ to their "Neue Beiträge" in I870. These newer methods seek to determine, by more elaborate and accurate methods than were available to the earlier experimenters, the actual effect of the feed on the body of the animal as well as its content of matter and energy.

\section{§ 3. Conditions Affecting Digestibility}

711. Digestibility variable. - Not only is the current method of estimating the relative values of feeding stuffs, as described in the previous section, based largely on the digestibility of the materials in question, but the latter is also a most important fac-

1 Über das Ziel und die Methode der auf den landwirtschaftlichen Versuchsstationen auszufuhrenden thier-physiologischen Untersuchungen. 
tor in determining the actual production values discussed in the next chapter, since, as there shown (742), the excretion in the feces constituted the greatest, although not the only, loss of chemical energy suffered by the feed.

The percentage digestibility of a feeding stuff or of its several constituents, however, has not a fixed and invariable value, analogous to the solubility of a chemical compound, but may be affected more or less by a variety of conditions, although to a less extent than is frequently supposed. This arises from the fact noted in Chapter III (155) that portions of ingredients capable per se of solution and resorption in the digestive tract actually escape digestion for various reasons and reappear in the feces. Any conditions which influence the digestibility in this way, however, necessarily affect the value of the feeding stuff by whichever method determined, and the more important of them may be conveniently considered in this connection.

The conditions which affect, or which are supposed to affect, the degree of completeness with which the potentially digestible ingredients of a feeding stuff are actually digested may be divided into those relating to the animal itself and those relating to the feed.

\section{Conditions relating to the animal}

712. Variation at different times. - An important fact, which must be borne in mind in studying the influences of various factors upon digestion, is that the percentage digestibility of the same feeding stuff by the same individual has been found to vary more or less at different times.

This has been shown especially by G. Kühn. ${ }^{1}$ In experiments upon the digestibility of meadow hay by cattle the variations in the percentage digestibility of the dry matter, which is the one least subject to error, ranged from 0.6 to 2.1 , averaging $\mathrm{I} \cdot 3$, and the digestibility of the organic matter showed about the same variations. That for the nitrogen-free extract averaged $\mathrm{I} .8$, while in the case of the crude fiber, protein and ether extract it reached $3 \cdot 3$. These variations were shown to be materially larger than the possible errors of experiment. Similar, although relatively somewhat smaller, variations were observed on rations of hay and bran. Moreover, Kühn points out that the maximum differences were found in those cases in which the larger

${ }^{1}$ Landw. Vers. Stat., 29 (I883), I29, I47 and 153. 
number of single trials were made. No connection could be traced between the variations in digestibility and the condition of the animals.

The writer ${ }^{1}$ observed a similar difference in two experiments upon one sheep with clover hay while the other sheep of the pair showed no significant difference. In later experiments ${ }^{2}$ in which the feces of three steers were quantitatively collected daily for periods of 56 and 27 days on identical rations, it was shown that the digestibility of the air dry matter ${ }^{3}$ and nitrogen computed from overlapping tenday periods, varied at times from the average for the whole experiment by amounts greater than the estimated experimental error. Mumford, Grindley, Hall and Emmett ${ }^{4}$ likewise observed distinct fluctuations in the digestion coefficients obtained with cattle in successive weekly periods following preliminary periods of from two to four weeks.

All the foregoing experiments were upon dry feed and the writer is inclined to attribute them, to a considerable degree at least, to irregular voiding of the feces.

713. Species. - The differences in the anatomy of the digestive organs of different species might naturally be expected to result in differences in the extent to which the feed of these species is digested. This is true especially of those ingredients of the feed whose so-called digestibility is due to the action of organized ferments and which, therefore, will be more or less dependent upon the opportunities which the digestive tract affords for the stagnation of the feed and so for the activity of these organisms.

714. Species of ruminants. - Few direct comparisons of the digestibility of the same feeding stuff by different species of ruminants are on record. In view of the similarity of the alimentary canal in these species, one would naturally expect to find comparatively small differences in the extent to which identical feeding stuffs are digested. In a general way this expectation is borne out by the average results of a large number of recorded digestion experiments upon feeds bearing the same name, although not of identical composition. Thus Wolff, in 1874 , compared the results of about 40 German experiments

${ }^{1}$ Amer. Jour. of Science, 28 (1885), 368.

2 Penna. Expt. Sta., Bul. 42 (1898), pp. I 29-141.

3 The daily excretion of dry matter was not determined and there is a possibility of a small error due to lack of exact uniformity in the air drying.

${ }^{4}$ Ills. Expt. Sta., Bul. I72 (I9I4). ${ }^{5}$ Landw. Fütterungslehre. 
on cattle and sheep and Jordan and Hall ${ }^{1}$ have made similar comparisons of nine American experiments.

On the basis of comparisons of this sort it has been generally considered that digestion coefficients obtained with one species of ruminants may be applied to others without material error and the sheep or goat has been the favorite experimental animal. Such direct evidence as is available, however, leads to some modification of this conclusion.

Comparisons of the digestive powers of cattle and sheep for identical feeding stuffs have been reported by Frear, ${ }^{2}$ the Mississippi Station, ${ }^{3}$ Bartlett, ${ }^{4}$ Kellner, ${ }^{5}$ Tangl and Weiser, ${ }^{6}$ Zuntz, and Völtz. ${ }^{8}$

The experimental results, while not extensive and not altogether consistent, seem, when taken in connection with the general comparisons previously made, to warrant the conclusion that as regards the better grades of roughages the difference in digestive power between cattle and sheep is not marked and, with the exception of Zuntz's rather remarkable results, the same would seem to be the case as regards concentrates. On the other hand, it would appear that in the case of the coarser and less digestible forms of forage a distinct difference exists in favor of cattle. Kellner is inclined to ascribe this difference to the greater percentage of water in the contents of the lower intestine of cattle as compared with sheep, which favors a more extensive action of the organized ferments.

715. The horse compared with ruminants. -- A number of comparisons have been made of the digestibility of identical feeding stuffs by horses and by sheep as representing ruminant animals. The most extensive trials of this sort were made by Wolff $^{9}$ in Hohenheim from I 877 to 1884 but Tangl and Weiser ${ }^{10}$ have also compared the digestibility of several samples of hay by horses and by sheep or cattle and Langworthy ${ }^{11}$ has compiled the results of a large number of digestion experiments

${ }^{1}$ U. S. Dept. Agr., Office Expt. Stas., Bul. 77 (1900), 90.

${ }_{2}^{2}$ Penna. Expt. Sta., Rep. I89o, 58.

3 Eighth Rpt. (1895), 79.

4 Maine Expt. Sta., Bul. r ro (1904).

${ }^{5}$ Landw. Vers. Stat., 63 (1906), 313.

6 Landw. Jahrb., 35 (I906), 205.

${ }^{7}$ Jahrb. Ver. Spiritus Fabrikanten in Deutschland, XII (I9I2), 324.

8 Landw. Jahrb., 45 (1913), 422.

9 Grundlagen für die rationelle Fütterung des Pferdes, 1886.

${ }^{10}$ Landw. Jahrb., 35 (1905), 159.

11 U. S. Dept. Agr., Office Expt. Stas., Bul. 125, p. 44. 
Table i7I. - Digestibility by Sheep and by Horses

\begin{tabular}{|c|c|c|c|c|c|c|c|c|c|c|c|c|c|c|c|}
\hline & & & & & & & & & \multirow[b]{2}{*}{$\begin{array}{c}\text { NUMBER } \\
\text { OF } \\
\text { EXPERI- } \\
\text { MENTS }\end{array}$} & \multicolumn{6}{|c|}{ Percentage Digestibility } \\
\hline & & & & & & & & & & $\begin{array}{l}\text { Dry } \\
\text { Mat- } \\
\text { ter }\end{array}$ & $\begin{array}{l}\text { Organic } \\
\text { Matter }\end{array}$ & $\begin{array}{l}\text { Crude } \\
\text { Pro- } \\
\text { tein }\end{array}$ & $\begin{array}{c}\text { Crude } \\
\text { Fiber }\end{array}$ & $\begin{array}{l}\text { Nitro- } \\
\text { gen- } \\
\text { free } \\
\text { Extract }\end{array}$ & $\begin{array}{l}\text { Ether } \\
\text { Ex- } \\
\text { tract }\end{array}$ \\
\hline & & RO & JGL & $A G$ & & & & & & & & & & & \\
\hline & & $h c$ & at s & tra & $w^{1}$ & & & & & & & & & & \\
\hline Sheep & . & . & $\cdot$ & • & · & - & $\cdot$ & . & 2 & 45 & 48 & 一 & 59 & 37 & 一 \\
\hline $\begin{array}{l}\text { Horse } \\
\qquad M e c\end{array}$ & $\dot{a d o}$ & & - & - & inf & $\dot{e r} i$ & or & - & 2 & 20 & 23 & - & 27 & I8 & 一 \\
\hline Sheep & $\cdot$ & - & - & • & - & - & $\cdot$ & - & 7 & 57 & 59 & 54 & 68 & 62 & 46 \\
\hline $\begin{array}{l}\text { Horse } \\
\qquad M e\end{array}$ & $\dot{a d c}$ & w & ay & - & b & ra & ge & - & 4 & 47 & 47 & 57 & 39 & 56 & 23 \\
\hline Sheep & - & - & . & - & · & . & . & . & 8 & 56 & 59 & 57 & 56 & 62 & $5 \mathrm{I}$ \\
\hline $\begin{array}{l}\text { Horse } \\
\qquad M e a\end{array}$ & $\dot{a d o}$ & • & 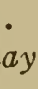 & - & su & ber & ior & - & 4 & 47 & 48 & 57 & 36 & 55 & 24 \\
\hline Sheep & . & • & . & . & . & · & - & . & IO & 62 & 64 & 65 & $6_{3}$ & 65 & 54 \\
\hline $\begin{array}{l}\text { Horse } \\
D\end{array}$ & $\dot{r i c}$ & & is & : & - & ass & · & - & 6 & 50 & $5 \mathrm{I}$ & 62 & $4^{2}$ & 57 & 20 \\
\hline Sheep & $\cdot$ & • & • & • & - & . & . & . & 2 & 65 & 76 & 73 & 8o & 76 & 65 \\
\hline Horse & & $a$ & ior & & iay & • & - & $\cdot$ & I & 54 & 62 & 69 & 57 & 66 & I3 \\
\hline Sheep & . & . & - & . & . & - & $\cdot$ & • & 8 & 55 & $5^{6}$ & 56 & 50 & $6 \mathrm{I}$ & $5^{6}$ \\
\hline Horse & • & . & . & $h$ & & - & - & $\cdot$ & 5 & 51 & $5 \mathrm{I}$ & 56 & 37 & 63 & 29 \\
\hline Sheep & - & . & - & & • & • & • & - & I 2 & 58 & 59 & $7 \mathrm{I}$ & 45 & 66 & $4 \mathrm{I}$ \\
\hline Horse & - & • & $\dot{O} a$ & & • & - & - & - & 6 & $5^{8}$ & $5^{8}$ & 73 & 40 & 70 & I4 \\
\hline Sheep & - & . & - & . & - & - & · & $\cdot$ & I3 & 70 & $7 \mathrm{I}$ & 80 & 30 & 76 & 83 \\
\hline Horse & - & • & $\dot{B e a}$ & & - & - & - & · & 8 & 66 & 68 & 86 & 2 I & 74 & 71 \\
\hline Sheep & - & • & $\cdot$ & & · & - & $\cdot$ & $\cdot$ & 6 & 87 & 90 & 87 & 79 & $9 I$ & 84 \\
\hline Horse & . & . & $\dot{P e}$ & & - & - & - & - & 6 & $8_{5}$ & 87 & 86 & 65 & 93 & I3 \\
\hline Sheep & . & . & - & • & - & - & - & $\cdot$ & 2 & 88 & 90 & 89 & 66 & 93 & 75 \\
\hline Horse & - & - & & & • & - & - & . & I & 77 & 80 & $8_{3}$ & 8 & 89 & 7 \\
\hline Sheep & . & · & $\cdot$ & • & • & - & $\cdot$ & $\cdot$ & 2 & 88 & 88 & 88 & 97 & 78 & 78 \\
\hline Horse & . & . & & & - & - & . & . & I & $7 \mathrm{I}$ & 72 & 94 & $5 I$ & $5^{I}$ & 27 \\
\hline Sheep & - & - & $\cdot$ & . & $\cdot$ & - & $\cdot$ & $\cdot$ & 2 & 88 & 88 & 79 & 62 & $9 \mathrm{I}$ & 85 \\
\hline Horse & $\cdot$ & - & • & - & - & . & . & - & I & 90 & 9 I & 78 & 100 & 94 & 63 \\
\hline
\end{tabular}

${ }^{1}$ Results regarded by Wolff as of questionable accuracy. 
on both horses and ruminants. The results of Wolff's comparisons are contained in Table $\mathrm{I} 7 \mathrm{I}$.

In general, the comparisons have shown a distinct superiority of ruminants over horses in the digestion of roughages, especially as regards those ingredients (crude fiber and nitrogen-free extract) whose so-called digestion is wholly or in part a fermentation. Even in the better grades of forage the crude fiber was on the whole considerably less digestible by horses than by ruminants, although three of Tangl's experiments are exceptions, while less difference appears as regards the nitrogen-free extract and scarcely any as regards the crude protein. On the other hand, little difference was observed in most cases in the digestibility of the total organic matter and nitrogen-free extract of concentrates. In the latter the digestibility of the crude fiber was also relatively low but in view of its small amount and the consequent uncertainty in the results little significance attaches to this difference. The notably lower figures for the digestibility of the ether extract by horses arise in all probability from a larger excretion of ether-soluble excretory products in the feces of these animals rather than from any real difference in digestibility.

716. Swine compared with ruminants. - Comparisons of the digestibility of identical feeds by swine and by sheep have been reported by Honcamp, Neumann and Müllner, ${ }^{1}$ the feeding stuffs being wheat, rye and the by-products of their milling. Although the results upon the individual animals of the same species fluctuated somewhat, as is not unusual (718), the average results showed no material superiority on the part of either species.

Owing to the small percentage of crude fiber contained in the feeds, the results upon this ingredient are naturally quite variable and of no especial significance. Aside from this, there seems to have been a slight superiority on the part of the swine in the case of the rye products (with the exception of the germ), while with the wheat products the reverse was the case, especially with the coarser milling products. The swine seem to have digested the crude protein fully as well as the sheep in all the experiments.

Fingerling, Bretsch, Lösche and Arndt, ${ }^{2}$ in experiments designed especially to test the relative digestive powers of sheep and

${ }^{1}$ Landw. Vers. Stat., 81 (I9I3), 205. ,

${ }^{2}$ Ibid., 83 (I913), r8I. 
swine for crude fiber, added straw pulp, young grass and wheat chaff to basal rations. Their average results were as follows:-

TAble 172. - Digestibility by Sheep and by Swine

\begin{tabular}{|c|c|c|c|c|c|c|c|c|}
\hline & & & $\begin{array}{l}\text { DRY } \\
\text { MAT- } \\
\text { TER }\end{array}$ & $\begin{array}{c}\text { OR- } \\
\text { GANIC } \\
\text { MAT- } \\
\text { TER }\end{array}$ & $\begin{array}{l}\text { CRUDE } \\
\text { PRO- } \\
\text { TEIN }\end{array}$ & $\begin{array}{l}\text { CRUDE } \\
\text { FIBER }\end{array}$ & $\begin{array}{c}\text { NITRO- } \\
\text { GEN- } \\
\text { FREE } \\
\text { EX- } \\
\text { TRACT }\end{array}$ & $\begin{array}{c}\text { ETHER } \\
\text { Ex- } \\
\text { TRACT }\end{array}$ \\
\hline & & & $\%$ & $\%$ & $\%$ & $\%$ & $\%$ & $\%$ \\
\hline Sheep & Straw pulp & & & 73.10 & - & 77.27 & 72.23 & - \\
\hline Swine & 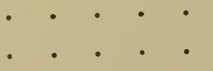 & . & 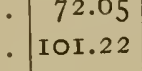 & 88.85 & - & 94.81 & 63.75 & - \\
\hline Sheep . & Grass & & & 60.77 & 76.85 & 69.49 & 67.29 & 66.93 \\
\hline Swine & $\begin{array}{llll}\cdot & \cdot & \cdot & \cdot \\
\cdot & \cdot & \cdot & \cdot\end{array}$ & - & 49.58 & 5 I. 86 & 52.05 & $39 \cdot 39$ & 52.07 & 84.35 \\
\hline Sheep. & Wheat chaff & & 40.33 & 46.93 & $55 \cdot 5^{6}$ & 30.34 & 51.54 & - \\
\hline Swine . &. & . & 20.53 & 22.95 & & & 27.86 & - \\
\hline
\end{tabular}

The amount of straw pulp added to the basal ration was comparatively small, so that the results on this material are subject to relatively large errors (161), but the conclusion seems indicated that pure cellulose, freed from encrusting matter, can be readily digested by swine, and this conclusion is fully supported by the later determinations of Fingerling, Köhler and Reinhardt. ${ }^{1}$ For the crude fiber of ordinary feeding stuffs, on the contrary, the digestive power of the swine was decidedly inferior to that of the sheep.

A general idea of the relative digestive power of swine and ruminants may also be gained by a comparison of the average results obtained for the two species on feeding stuffs of the same name although not of identical composition, as shown by compilations like those by Kellner ${ }^{2}$ and by Henry and Morrison. ${ }^{3}$

The recorded results do not indicate that there is any material difference between swine and ruminants as regards their digestive power for concentrates. As in the case of the horse, there seems to be a tendency toward a lower percentage digestibility of ether extract by swine, due most likely to the presence

1 Landw. Vers. Stat., 84 (I9I4), I 49.

2 Ernährung landw. Nutztiere, 6th Ed., p. 45.

Feeds and Feeding, I5th Ed., pp. 647-652. 
of more ether-soluble excretory products in the feces of these animals. The figures for the crude fiber of concentrates are also materially lower with swine in some cases, but in others equal to or even higher than those obtained for ruminants. In view of the small percentage of crude fiber in the concentrates and the corresponding range of possible error, however, the results on this point are of little significance. The crude fiber of roughage is but imperfectly digested by swine. Crude protein would appear, on the whole, to be rather more completely digested by swine than by ruminants, possibly indicating the presence of more nitrogenous excretory products in the feces of the latter.

717. Fowls compared with swine. - Owing to the difficulty of collecting the feces of fowls separately from the urine, comparatively few determinations upon these animals have been made. Bartlett, ${ }^{1}$ who has reported a number of such experiments, gives the following as the average digestion coefficients obtained in all recorded experiments up to I9Io.

Table I73. - Digestibility by Fowls

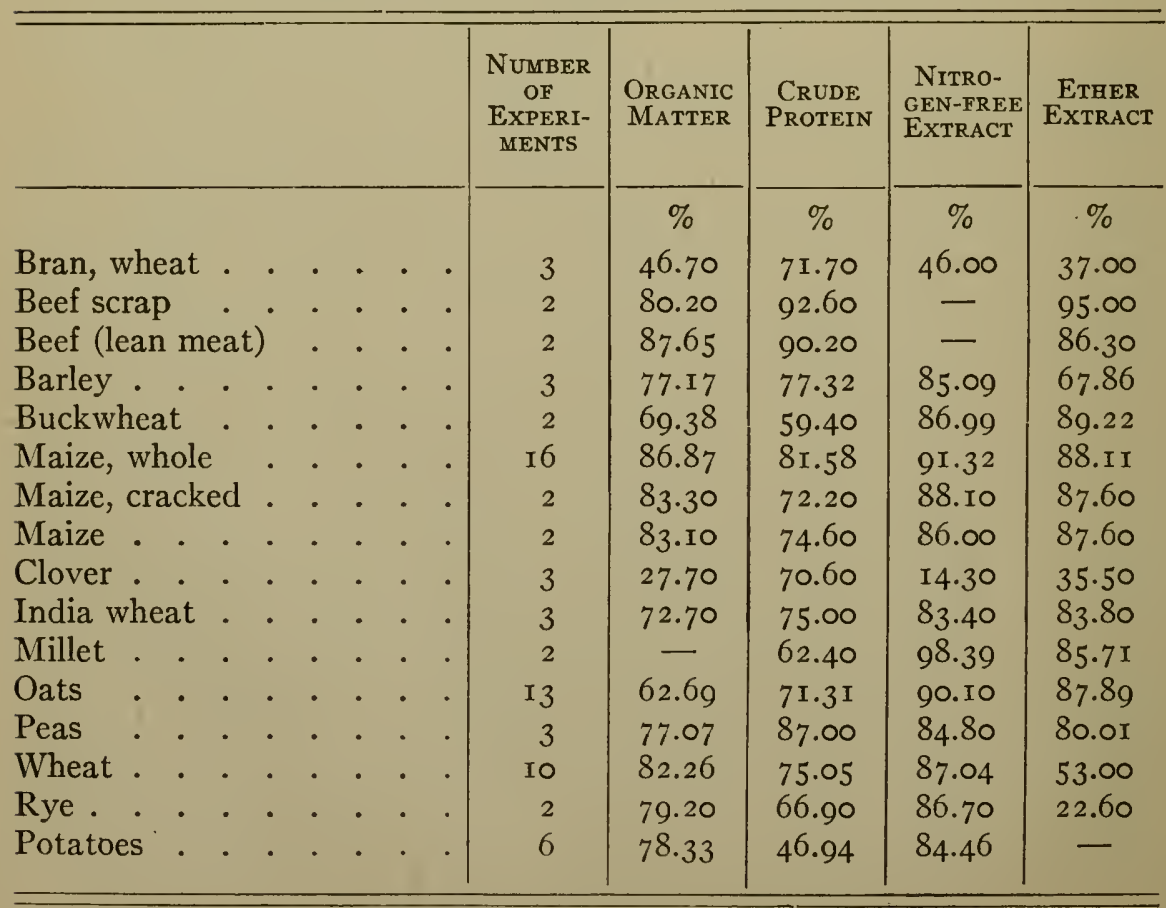


Crude fiber appears to be relatively difficult of digestion by fowls and the results obtained upon this ingredient were variable and apparently capricious. Aside from this, a comparison of results with those for swine shows quite a close general agreement between the two classes of animals.

718. Individuality. - In addition to the specific differences just considered, differences have likewise been observed in the digestibility of the same feeding stuff by individuals of the same species. To some extent this may be due to abnormalities, such as defective teeth or chronic diseases of the digestive organs, but in normal animals distinct individual differences also seem to occur.

In their compilation of the results of American digestion experiments, Jordan and Hall ${ }^{1}$ were unable to find conclusive evidence of such differences in digestive power and are inclined to attribute the apparent variations which were observed largely to the variability at different times already considered (712). The experiments by G. Kühn, ${ }^{2}$ however, which were cited in the discussion of the latter possibility, seem also to afford indubitable instances of individual differences in cattle and the same is true of experiments by the writer $^{3}$ in which three grade Shorthorn steers were under observation at different times for five years. Carmichael, Newlin and Grindley ${ }^{4}$ have likewise observed significant differences in the digestive powers of individual pigs. On the other hand, Christensen and Simpson ${ }^{5}$ made three series of digestion trials on alfalfa hay for two successive years, using four range steers each year, and failed to find any consistent individual differences.

The existence of time variations in digestibility (712) renders it somewhat difficult to decide whether an observed difference in the digestion of the same feeding stuff by two animals is really an expression of individuality or whether it is in a sense accidental. A comparison based on a single digestion trial as ordinarily made is liable to be misleading, and to secure correct results requires either a number of trials or a trial extending over a longer period than is ordinarily employed. On the

1 U. S. Dept. Agr., Office Expt. Stas., Bul. 77 (I900), 88.

${ }^{2}$ Landw. Vers. Stat., 29 (I883), I29, I47, 153.

${ }^{3}$ Penna. Expt. Sta., Bul. 42 ( 898 ), I 24.

${ }^{4}$ Science, July 2 , I 9 I 5 , p. 38 .

${ }^{5}$ New Mexico Expt. Sta., Bul. 9r (Igr4). 
whole, however, the conclusion seems justified that animals of the same species may differ to some extent in their digestive power but that these individual differences are probably less than appear to be indicated by the results of single digestion trials and are certainly much too small to account in any degree for the economic differences in animals. Even the differences observed in the results of short digestion trials rarely exceed three or four per cent and are usually materially less than this.

719. Breed. - The foregoing facts are sufficient of themselves to render improbable the existence of any considerable breed differences as regards digestion, and this conclusion has been confirmed by the experiments of Haubner and Hofmeister, ${ }^{1}$ of Wolff ${ }^{2}$ and of Armsby and Fries. ${ }^{3}$ The recorded data taken together fail to indicate any material difference in the digestive power of different breeds or between pure-bred and scrub animals.

There exists a somewhat general impression that animals which show themselves superior as producers of meat, milk, etc., whether as the result of breed, heredity, or of individual variation, owe that superiority, in part at least, to a superior digestive power; that is, it is supposed that the improved breeds of farm animals and the superior individual animals within a breed are able to extract more nutriment from a given weight of a feed than can inferior animals. The reasons for the undoubted economic superiority of some individuals over others have been considered in previous chapters. So far as differences in digestive power are concerned, however, the experimental evidence gives little support to the popular impression.

720. Age. - Comparisons of the digestive power of the same animals (lambs) at different ages were made by Wolff 4 in $187 \mathrm{I}-72$ which led to the conclusion that between the ages of six and fourteen months the percentage digestibility of the feed remained practically unchanged and this conclusion is confirmed by the results of an experiment by Weiske ${ }^{5}$ undertaken primarily for another purpose.

721. Work. - Investigations on the effect of the performance of work upon the digestibility of rations have naturally been

${ }^{1}$ Landw. Vers. Stat., 12 (1869), 8.

3 U. S. Dept. Agr., Bur. Anim. Indus., Bul. I 28.

${ }^{4}$ Landw. Jahrb., 2 (1873), 22 I.

${ }^{2}$ Landw. Jahrb., 1 (1872), 533.

${ }^{5}$ Ibid., 9 (I880), 205. 
made upon the horse. Experiments upon this subject have been reported by Wolff and his associates at Hohenheim and by Grandeau and LeClerc at Paris.

Wolff's ${ }^{1}$ experiments were upon a single animal, a draft horse weighing about $550 \mathrm{kgs}$. (I 200 pounds). The ration remained the same in all the periods and was insufficient to maintain the weight of the animal in the periods of heavier work. The work was that of draft, done at a slow walk (about r.9 miles per hour) with in most instances a draft of $60 \mathrm{kgs}$., the total work per day (not including that of locomotion) ranging from 475,000 to $1,800,000$ kilogram meters. Under these conditions, no effect on the digestibility of the mixed rations employed was observed.

Grandeau and LeClerc's investigations ${ }^{2}$ were made upon several different horses of the Paris Cab Company and included experiments upon work and others upon simple locomotion both at a walk and a trot together with rest experiments.

The plan of the experiments differed from that of Wolff's in some important particulars. The animals used were lighter (about $400 \mathrm{kgs}$. as compared with $55^{\circ} \mathrm{kgs}$.) and apparently of a more active temperament as indicated by their more rapid walk, the velocity of which varied from 2.6 to 3.0 miles per hour. The work, which was that of draft, was done on a dynamometer similar to Wolff's. The draft was about half of that of Wolff's experiments and the total amount of work ${ }^{3}$ was considerably less, ranging in most cases from 400,000 to 600,000 kilogram meters per day, with a maximum in one experiment of 785,000 . Its amount was approximately the same in all the experiments in each series and was not greater at a trot than at a walk. ${ }^{4}$ Finally, corresponding to the main purpose of the experiment, which was to study the feed requirements of cab horses, the rations in the periods of work or of walking exercise were heavier than in those in the periods of rest, the increase being one-tenth in the experiments on locomotion and, in most cases, one-half in the experiments on work. The proportions

1 Landw. Jahrb., 8, Ergzbd. I (1879), 73; 16, Ergzbd. III (I887), 53-71.

${ }^{2}$ L'alimentation du cheval de trait; Berger-Levrault et cie, I882-89.

${ }^{3}$ Not including that of locomotion.

${ }^{4}$ The total work in the former case was, of course, somewhat greater on account of the greater expenditure of energy in trotting as compared with walking (664). 
of the different feeding stuffs in the rations, however, remained the same, except in a very few cases.

On the whole, and despite some irregularities in the results on single ingredients, Grandeau and LeClerc's results agree with Wolff's in showing that work even at a somewhat rapid walk does not materially affect the digestibility of rations. On the other hand, they show a distinct decrease of the percentage digestibility in the periods in which the work was done at a trot. It scarcely seems that this effect can be ascribed to the work as such, since the measured amount was less than in Wolff's experiments and was not greater at a trot than at a walk. Moreover, mere horizontal locomotion at a trot, in some instances at least, seems to have produced the same effect, which apparently is due to the difference in gait. It is true that the rations were heavier in the work periods and that this (722) may possibly have affected the digestibility, but no reason is apparent why it should have produced a greater effect in the trotting periods than in the walking periods.

The influence of work has also been investigated in a different way by Tangl ${ }^{1}$ and by Scheunert. ${ }^{2}$ A weighed amount of oats was fed after 36 hours of fasting and the animal was killed from one to five hours later and the contents of the stomach and small intestines weighed and analyzed. On the assumption that none of the crude fiber of the oats was digested in this portion of the alimentary tract, the results show that work delays the passage of the feed from the stomach to the intestines, especially during the first one or two hours. As a consequence, the gastric juice penetrates the larger mass of the feed more slowly and more of it is neutralized by the saliva, so that the stage of starch digestion is prolonged and that of protein digestion shortened, the result being that more carbohydrates and less protein are digested. In the later stages of digestion the differences tend to equalize themselves, while the effect of work upon the intestinal digestion was found to be small. Scheunert computes that the total digestibility was considerably increased by the performance of work. As noted, however, the results cover only the first five or six hours of digestion. The method of comparison is confessedly an approximate one and the results show very considerable variations among themselves. Only actual digestion experiments suffice to decide the question of the total effect of work upon digestion.

1 Arch. Physiol. (Pflüger); 65 (1 896), 545.

2 Arch. Physiol. (Pflüger); 109 (I905), I45; Landw. Jahrb., 34 (I905), 805. 


\section{Conditions relating to the feed}

722. Quantity of feed. - Current methods of computing rations regard the digestibility of feeding stuffs as unaffected by the amounts consumed. As regards exclusive feeding with roughage, the results of a considerable number of experiments by various investigators appear to justify this view. With mixed rations of roughage and concentrates fewer experiments have been made, but the results indicate a distinct decrease in the percentage digestibility when the amount of the ration is increased considerably above that required for maintenance.

Roughage. - The early experiments of Henneberg and Stohmann on cattle include several cases in which varying amounts of clover and meadow hay were fed and in which the digestibility was substantially unaffected by the quantity consumed, and the same was found to be true by Wolff at Hohenheim in a number of experiments on sheep. ${ }^{1}$ Later comparative experiments by the same investigator ${ }^{2}$ on sheep and horses have confirmed his earlier results, as have also those of Tangl and Weiser ${ }^{3}$ upon sheep fed alfalfa hay or alfalfa silage, while Müntz and Girard ${ }^{4}$ likewise found no distinct effect of the quantity consumed upon the digestibility of alfalfa hay by the horse. In three experiments by Armsby and Fries ${ }^{5}$ on each of two steers a submaintenance ration of timothy hay was slightly better digested than a maintenance ration in five cases out of six, but the differences were small, amounting to from r.o to 2.7 per cent on the dry matter. Later unpublished experiments have given similar results. In earlier experiments ${ }^{6}$ by the same authors on different amounts of clover hay, practically no differences were observed.

Mixed rations. - Kellner ${ }^{7}$ obtained the following results in four periods in which varying amounts of a mixed ration consisting of meadow hay, dried molasses beet pulp, rye bran and cottonseed meal were fed to cattle.

${ }_{1}$ Compare Wolff, Die Ernährung der landwirtschaftlichen Nutztiere, pp. 63 and 64 .

${ }^{2}$ Landw. Jahrb., 1 (1872), 533; Landw. Vers. Stat., 21 (1878), 19.

${ }^{3}$ Landw. Vers. Stat., 74 (I9II), 277 and 282.

${ }^{4}$ Centbl. Agr. Chem., 27 (I898), 756.

5 U. S. Dept. of Agri., Bur. Anim. Indus., Bul. I28 (Igr I), p. 27.

${ }^{6}$ U. S. Dept. of Agri., Bur. Anim. Indus., Bul. 74 (1905), pp. I2-13; Bul. No. IOI (I go8), pp. I I-I3.

7 Ernährung landw. Nutztiere, 6th Ed., p. 49. 
Table i74. - Effect of Amount of Mixed Ration Consumed on Digestibility

\begin{tabular}{|c|c|c|c|c|c|c|c|c|}
\hline & & & \multirow[b]{2}{*}{$\begin{array}{l}\text { DAILY } \\
\text { RATION }\end{array}$} & \multicolumn{5}{|c|}{ DigestibILITY } \\
\hline & & & & $\begin{array}{l}\text { Organic } \\
\text { Matter }\end{array}$ & $\begin{array}{c}\text { Crude } \\
\text { Protein }\end{array}$ & $\begin{array}{l}\text { Crude } \\
\text { Fiber }\end{array}$ & $\begin{array}{c}\text { Nitrogen- } \\
\text { free } \\
\text { Extract }\end{array}$ & $\begin{array}{c}\text { Ether } \\
\text { Extract }\end{array}$ \\
\hline & & & Kgs. & $\%$ & $\%$ & $\%$ & $\%$ & $\%$ \\
\hline Period I & . & . & 10.84 & 76.1 & 71.0 & 62.8 & 82.0 & 63.5 \\
\hline Period II . & . & . & 13.01 & 74.7 & 68.3 & $6 \mathrm{r} .2$ & 80.8 & 64.4 \\
\hline Period III & . & . & I 5.18 & 72.8 & 65.8 & 59.2 & 79.0 & 64.2 \\
\hline Period IV & . & . & 10.84 & 75.8 & 71.2 & 62.6 & $8 \mathrm{I} .2$ & 67.6 \\
\hline
\end{tabular}

Eckles ${ }^{1}$ determined the digestibility of mixed rations sufficient for maximum milk production, and thirteen months later, when the cows were dry, that of a maintenance ration of the same mixture of feeding stuffs. About thirty per cent of the dry matter of the ration was derived from hay, thirty-six per cent from silage and thirty-four per cent from grain.

Table i75. - Digestibility of Mixed Rations by Cows

\begin{tabular}{|c|c|c|c|c|c|c|c|}
\hline & \multirow{2}{*}{$\begin{array}{c}\text { MIXED } \\
\text { RATION } \\
\text { PER } \\
\text { HEAD }\end{array}$} & \multirow{2}{*}{$\begin{array}{c}\text { DRX } \\
\text { MATTER } \\
\text { EATEN } \\
\text { PER } \\
\text { IOOO } \\
\text { LIVE } \\
\text { WEIGHT } 2\end{array}$} & \multicolumn{5}{|c|}{ Percentage Digestibility } \\
\hline & & & $\begin{array}{l}\text { Organic } \\
\text { Matter }\end{array}$ & $\begin{array}{l}\text { Crude } \\
\text { Protein }\end{array}$ & $\begin{array}{l}\text { Crude } \\
\text { Fiber }\end{array}$ & $\begin{array}{l}\text { Nitro- } \\
\text { gen-free } \\
\text { Extract }\end{array}$ & $\begin{array}{l}\text { Ether } \\
\text { Ex- } \\
\text { tract }\end{array}$ \\
\hline Cow No. 27 & Kgs. & & & & & & \\
\hline Full ration - $\cdot$ & 24.95 & $3 I \cdot 30$ & 66.3 & $5^{8.8}$ & 53.8 & 72.6 & 67.0 \\
\hline $\begin{array}{c}\text { Maintenance ration } \\
\text { Cow } \mathrm{No} .62\end{array}$ & $8.7 \mathrm{I}$ & 10.95 & 73.8 & $67 \cdot 3$ & $55 \cdot 3$ & 82.1 & 73.2 \\
\hline Full ration . . & I5.88 & I9.88 & 67.0 & 60.6 & 53.9 & 73.6 & 59.8 \\
\hline Maintenance ration & 7.62 & $9 \cdot 5^{8}$ & 72.2 & $65 \cdot 5$ & $52 . I$ & 8т.о & 73.9 \\
\hline
\end{tabular}

Mumford, Grindley, Hall and Emmett ${ }^{3}$ determined the digestibility of four different mixed rations of hay and grain by four pairs of cattle receiving respectively slightly more than a maintenance ration, one-third feed, two-thirds feed and full feed. One animal of the fullfed pair showed a distinctly lower digestive power than the other in all the periods and the same was true of one animal receiving the two-thirds feed in the last two periods. The results upon the other animal in this case are shown in the table in parenthesis:-

${ }_{1}$ Mo. Expt. Sta., Research Bul. 4.

2 Approximate.

${ }^{3}$ Ills. Expt. Sta., Bul. I72 (I9I4). 
Table i76. - Digestibility of Dry Matter by Cattle

\begin{tabular}{|c|c|c|c|c|c|}
\hline & PERIOD I & PERIOD 2 & Period 3 & PERIOD 4 & AVERAGE \\
\hline $\begin{array}{l}\text { Ratio of hay to } \\
\text { grain . . }\end{array}$ & $\begin{array}{c}\text { I : I } \\
\text { Per Cent }\end{array}$ & $\begin{array}{c}\mathrm{I}: 3 \\
\text { Per Cent }\end{array}$ & $\begin{array}{c}\mathrm{I}: 5 \\
\text { Per Cent }\end{array}$ & $\begin{array}{c}\text { I : } 5 \\
\text { Per Cent }\end{array}$ & Per Cent \\
\hline $\begin{array}{l}\text { Maintenance } \\
\text { One-third feed } \\
\text { Two-thirds feed } \\
\text { Full feed }\end{array}$ & $\begin{array}{l}69.9 \\
67.12 \\
65.62 \\
63.03(64.50)\end{array}$ & $\begin{array}{l}77.28 \\
72.06 \\
69.07 \\
64.56(69.64)\end{array}$ & $\begin{array}{l}78.79 \\
75.74 \\
73.62(75.57) \\
70.11\end{array}$ & $\begin{array}{l}79.99 \\
77.14 \\
75.10(77.80) \\
76.12(79.53)\end{array}$ & $\begin{array}{l}76.51 \\
73.02 \\
70.83(72.02) \\
68.46(72.12)\end{array}$ \\
\hline
\end{tabular}

In these experiments the digestibility of the maintenance ration appears to have been distinctly higher than that of the heavier rations in Period I, in which the largest proportion of hay was fed. The differences became much less marked as the total feed was progressively increased, and as between the two-thirds and full-feed ration was scarcely significant, especially in view of the individual differences in this pair. The effect also decreased as the proportion of grain in the ration was increased, so that on the average of the four periods but little difference is shown with certainty among the three heavier rations.

Unpublished experiments by Armsby and Fries, in which varying amounts of a uniform mixture of two-thirds grain and one-third hay were fed to steers, afford confirmation of the foregoing results:-

Table 177. - Digestibility of Mixed Rations by Steers

\begin{tabular}{|c|c|c|c|c|c|c|c|c|c|c|c|}
\hline & & & & \multirow{2}{*}{\multicolumn{2}{|c|}{$\begin{array}{c}\text { AMOUNT } \\
\text { OF } \\
\text { MIXED } \\
\text { RATION }\end{array}$}} & \multirow{2}{*}{$\begin{array}{c}\text { DRY } \\
\text { MATTER } \\
\text { EATEN } \\
\text { PER } \\
\text { IOOO } \\
\text { LYVE } \\
\text { WT. }\end{array}$} & \multicolumn{5}{|c|}{ Percentage Digestibility } \\
\hline & & & & & & & $\begin{array}{l}\text { Organic } \\
\text { Matter }\end{array}$ & $\begin{array}{c}\text { Crude } \\
\text { Protein }\end{array}$ & $\begin{array}{l}\text { Crude } \\
\text { Fiber }\end{array}$ & $\begin{array}{c}\text { Nitro- } \\
\text { gen-free } \\
\text { Extract }\end{array}$ & $\begin{array}{l}\text { Ether } \\
\text { Extract }\end{array}$ \\
\hline \multicolumn{4}{|c|}{ Steer C } & & Kgs. & & & & & & \\
\hline Period 2 & 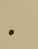 & 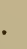 & . & & 2.55 & 8.26 & 68.8 & 67.2 & 25.2 & 79.2 & 75.7 \\
\hline $\begin{array}{r}\text { Period } 3 \\
\text { Ste }\end{array}$ & & & . & & 1.80 & $5.8_{3}$ & 74.9 & $73 \cdot 7$ & $39 \cdot 5$ & $83 \cdot 4$ & 78.9 \\
\hline Period I & . & . & . & & 3.75 & 15.74 & 68.9 & 66.9 & 40.6 & 76.3 & 70.6 \\
\hline Period 2 & . & & . & & 2.04 & $8.5^{8}$ & $75 . \mathrm{I}$ & 65.2 & $49 \cdot 9$ & 83.0 & 78.0 \\
\hline $\begin{array}{r}\text { Period } 3 \\
\text { Ste }\end{array}$ & $r$ & & . & & $\mathrm{I} .45$ & 5.64 & 74.1 & 68. I & $44 \cdot 7$ & 82.4 & 78.4 \\
\hline Period I & . & 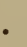 & . & & $5 \cdot 4$ & I 5.25 & 68.4 & 65.0 & 35.6 & 76.7 & 77.6 \\
\hline Period 2 & . & . & . & & 3.2 & $9 \cdot 36$ & $72 . \mathrm{I}$ & 66.9 & 37.0 & $8 \mathrm{I} . \mathrm{I}$ & 80.6 \\
\hline Period 3 & . & . & . & & I.9 & $5 \cdot 59$ & 74.2 & 70.2 & 40.4 & 82.7 & 80.0 \\
\hline
\end{tabular}


One would naturally be inclined to ascribe the lower digestibility of heavier rations to their greater bulk and relatively more rapid passage through the digestive tract, and in part to the consequent lessened extent of the bacterial fermentations. It would seem that on liberal rations material potentially digestible and resorbable may thus escape digestion and appear in the feces. Some of the reasons for this have already been indicated in discussing the feces as a feed residue (155). The presence of considerable amounts of undigested grains or fragments of grain in the feces of heavily fed animals is readily demonstrated by washing out the finer portions, but actual digestion experiments to determine the extent of this loss have not yet been reported.

723. Excess of carbohydrates. - It has been established by numerous experiments that an undue proportion of carbohydrates in a ration tends to reduce its digestibility, especially by ruminants. The effect is most distinct when pure digestible carbohydrates are added to a ration, but is manifest also when large amounts of feeding stuffs rich in carbohydrates are introduced.

An example of the former is afforded by an experiment by G. Kühn ${ }^{1}$ on two oxen. It was divided into three periods, in the first of which the animals received a daily ration of $9 \mathrm{kgs}$. of hay to which, in the second and third periods, $2 \mathrm{kgs}$. and 3.5 kgs. of starch, respectively, were added. Assuming the starch to have been completely digested ${ }^{2}$ the following amounts of the several ingredients were computed to have been digested from the hay by $\mathrm{Ox} \mathrm{V}$.

TABLE i 78. - Nutrients Digested from Hay, with AND without STARCH

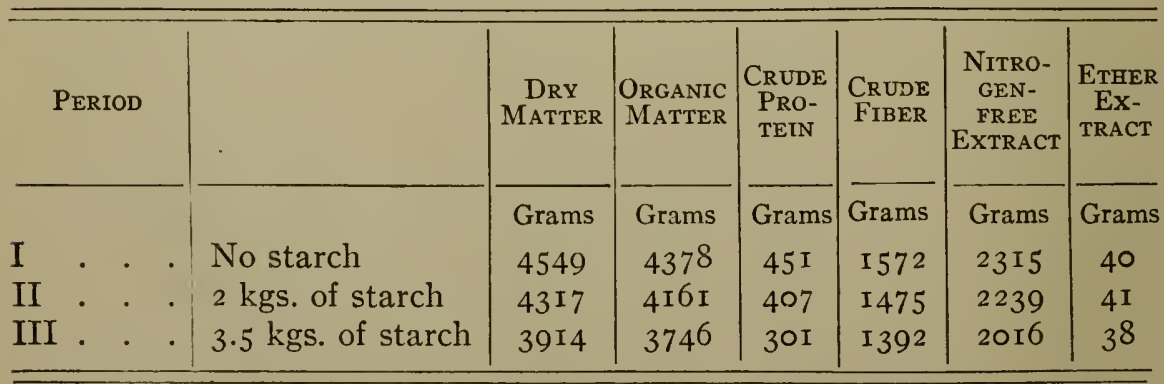

1 Landw. Vers. Stat., 44 ( 1894 ), 470-472.

${ }^{2}$ No starch could be detected microscopically in the feces. 
Expressed in another way, the feces in Periods II and III contained the following amounts of hay ingredients which, according to the results of Period I, must be regarded as digestible, but which under the influence of the addition of starch escaped digestion.

Table i79. - Digestible Nutrients of Hay Escaping in Feces

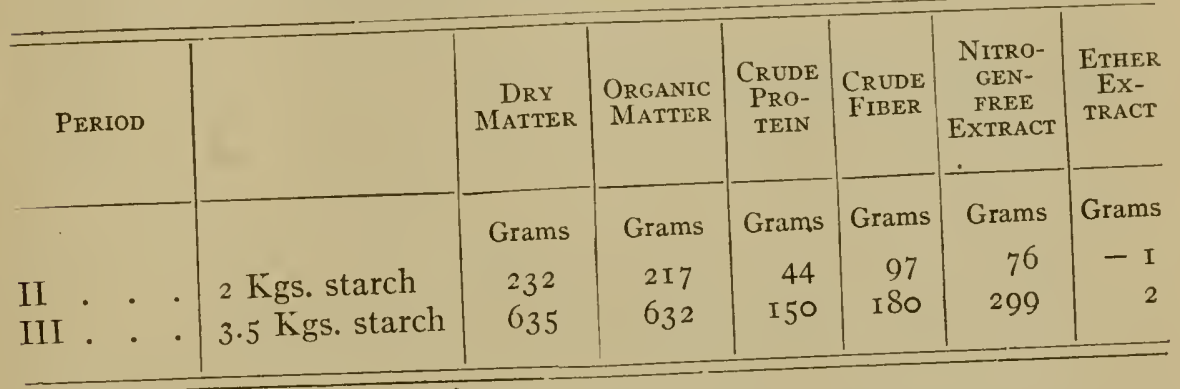

The foregoing is a typical example of the results of numerous similar experiments on ruminants in which starch, sugar, pectin substances and even cellulose have been added to hay and to mixed rations. Other things being equal, the magnitude of the effect has usually increased, as in this instance, with the quantity of carbohydrates added. Its total amount has varied considerably in different experiments, but qualitatively the result has been uniformly the same. This constitutes the so-called "depression of digestibility," since, of course, the digestion coefficients are lowered by the escape of potentially digestible matter in the feces. It should be noted, however, that in some of these instances more or less of the added carbohydrate (starch) has itself escaped digestion. According to experiments by Wolff, ${ }^{1}$ swine appear to be much less sensitive to this influence of carbohydrates than are ruminants and a few observations by Grandeau and Alekan ${ }^{2}$ seem to indicate that the same may be true of the horse.

724. Feeding stuffs rich in carbohydrates, as well as such materials as starch or sugar, may apparently likewise cause a decrease of digestibility, although the quantitative relations cannot always be so clearly followed as in experiments with pure carbohydrates. Thus six experiments with beet molasses by

1 Landw. Vers. Stat., 19 (I876), 273.

2 Jahresber. Agr. Chem., 49 (I906), 350; Ann. Sci. Agron., I904, I, 30, 330. 
Lehmann ${ }^{1}$ and one by Kellner ${ }^{2}$ showed that this substance, like the pure carbohydrates, caused a depression in the digestibility of all the ingredients of a basal ration, but in two later trials by Kellner ${ }^{3}$ the only effect was on the digestibility of the crude fiber.

The question has been especially investigated, however, by Wolff in regard to the feeding of tubers and roots. Heavy feeding of these materials is generally stated, on the strength of his experiments, to result in a pronounced decrease in the digestibility of the remainder of the ration, although, as Wolff himself points out, the evidence is by no means conclusive.

In Wolff's extensive series of experiments on sheep ${ }^{4}$ increasing quantities of roots or potatoes were fed along with hay whose digestibility had been previously determined, and it was found that as the amount of roots added to the ration was inçreased, the feces contained increasing amounts of undigested nutrients. For example, in one experiment with meadow hay and sugar beets, the percentage digestibility of the hay, computed on the assumption that the sugar beets were completely digestible, was as follows:-

Table i80. - Computed Percentage Digestibility of Hay with and without Sugar Beets

\begin{tabular}{|c|c|c|c|c|c|c|}
\hline & $\begin{array}{c}\text { DRY } \\
\text { MATTER }\end{array}$ & $\begin{array}{l}\text { ORganic } \\
\text { MatTER }\end{array}$ & $\begin{array}{c}\text { CRUde } \\
\text { PROTEIN }\end{array}$ & $\begin{array}{l}\text { CRUdDE } \\
\text { FIBER }\end{array}$ & $\begin{array}{c}\text { NITRO- } \\
\text { GEN-FREE } \\
\text { EXTRACT }\end{array}$ & $\underset{\text { EXTRRACT }}{\text { ETHER }}$ \\
\hline & $\%$ & $\%$ & $\%$ & $\%$ & $\%$ & $\%$ \\
\hline Fed alone & $55 \cdot 9$ & 59.2 & 57.6 & 55.2 & 62.1 & 60.0 \\
\hline Fed with sugar beets & 43.9 & 50.0 & 51.0 & 51.9 & 50.0 & 29.4 \\
\hline
\end{tabular}

What seems a more reasonable method of comparison, however, is to compute the digestibility of the roots in the first instance on the assumption of unaltered digestibility of the hay, just as in the case of concentrates (161) and to see whether the coefficients thus obtained show any decrease as the proportion of roots fed is increased. Wolff has carried out the computation in this manner for his entire series of experiments, numbering in all I Io single trials. The aver-

1 Landw. Jahrb., 25 Erzgbd. II (I896), II7.

${ }^{2}$ Landw. Vers. Stat., 53 (r900), r99.

${ }^{3}$ Ernährung landw. Nutztiere, 5th Ed., pp. 158-175.

${ }^{4}$ Landw. Jahrb., 8 Ergzbd. I (1879), I23. 
age results for total organic matter and for nitrogen-free extract are fairly uniform in each series of experiments, although there was somewhat more variation in the individual trials, and fail to give any decided indication of a diminished digestibility with the increasing amounts of roots consumed. The computed digestibility of the crude protein is more or less variable, but on the whole a decreased digestibility of this ingredient as the proportion of roots to hay increased seems to be plainly shown.

It is, of course, impossible to determine in a digestion experiment in which roots are added to a basal ration what proportion of the fecal matter is derived from the roots and what from the remaining ingredients, and such results as those of Wolff may be interpreted either as showing a fairly constant digestibility of both the hay and the roots (aside from crude protein), or, on the assumption of complete digestibility of the roots, as showing a progressive depression in the digestibility of the hay. To the writer, the former appears on the whole the more reasonable course, although it should be added that in some of the experiments the absolute amount of crude fiber in the feces was increased by an amount greater than that contained in the roots consumed, thus demonstrating a depression of the digestibility of this constituent of the hay. Probably the truth lies between the two views. It is unlikely that roots are entirely digestible and, on the other hand, it is probable that a large proportion of them may diminish to some extent the digestibility of other feeding stuffs consumed with them. It is to be remembered, however, that roots contain not altogether inconsiderable quantities of crude protein which, as shown in a following paragraph (727), tends to offset the effects of their carbohydrates.

725. Cause of diminished digestibility of protein. - Attention has already been called (163-167) to the influence of the excretory products contained in the feces on the apparent digestibility of the nutrients and especially of protein. According to Kellner's and Pfeiffer's results, the digestion of each roo grams of dry matter, whether protein or nitrogen-free material, results in the excretion in the feces of approximately 0.4 gram of nitrogen in the form of these excretory products. If, then, a kilogram of dry starch be added to a basal ration, the nitrogenous excretory products in the feces are increased by ap- 
proximately 25 grams, so that apparently 25 grams less of protein is digested from the basal ration, while in reality the true digestion may not have been affected. Thus in Kühn's experiment with hay and starch (723) the nitrogenous excretory products corresponding to the 1646 grams dry matter of the $2 \mathrm{kgs}$. of starch consumed, would be approximately 40 grams, while the excess actually found was 44 grams, the difference being insignificant.

The agreement is by no means always so close as in this instance and in none of the experiments on the addition of carbohydrates which have been cited was the true digestibility (166) of the protein determined. Nevertheless, the general conclusion seems justified that at least the larger part of the influence of carbohydrates and of feeding stuffs rich in carbohydrates on the apparent digestibility of the protein of the feed is due to the fact that, when added to a basal ration, they increase the nitrogenous excretory products in the feces. On the other hand, however, it must be remembered, that while the true digestibility may not be lowered, it is, as already pointed out (167), the apparent digestibility which measures the real advantage derived by the animal from the digestion of its feed. Whether the increased excretion of nitrogenous matter in the feces after carbohydrate feeding be due to an apparent or a real depression of digestibility, or to both combined, it is none the less a loss of protein from the body.

In general the depression in the percentage digestibility of the protein is greater the poorer the basal ration is in this ingredient. As Kellner ${ }^{1}$ has pointed out, however, this does not justify the statement frequently made that the magnitude of the depression is dependent upon the nutritive ratio of the feed. The difference is purely a mathematical one. A decrease of the digestibility of the protein by 50 grams, for example, is relatively very much greater in a basal ration of oat straw, containing only I 40 grams of apparently digestible crude protein than in a basal ration of meadow hay containing 430 grams of digestible protein.

The fact that the addition of protein tends to decrease the apparent digestibility of the protein of a basal ration is also readily explicable from this point of view. Pfeiffer's exper-

${ }^{1}$ Landw. Vers. Stat., 44 (1894), 344. 
iments (162) showed that the increase in the nitrogenous excretory products in the feces was about the same whether the added digestible matter consisted of carbohydrates or of protein. Consequently, the addition of protein to a ration would tend to diminish the apparent digestibility of the protein just as would the addition of carbohydrates.

The non-proteins, especially when given in the form of green vegetable material and roots, likewise increase the nitrogen content of the feces, but a review of the literature of the subject 1 shows that, as in the case of the proteins, the increase consists, at least in large part, of metabolic products and does not indicate any decrease in the true digestibility of the protein, although it does, of course, decrease the amount available to the organism.

726. Cause of diminished digestibility of carbohydrates. The depression of digestibility of the non-nitrogenous ingredients of the feed of ruminants appears to be due to an entirely different cause, viz., to a modification in the fermentation processes in the rumen, and the fact that these effects are observed chiefly on this class of animals lends strong support to this view.

It has already been stated (128-132) that the disappearance of more or less of the comparatively insoluble carbohydrates of the feed during its passage through the alimentary tract is due, particularly in ruminants, to a bacterial fermentation, occurring principally in the first stomach and yielding chiefly carbon dioxid, methane and organic acids. Furthermore, it has been shown that when the more soluble carbohydrates, like starch and sugar, are introduced into the ration they are attacked by the organisms and undergo the same fermentation, yielding corresponding amounts of the characteristic gaseous product, methane. It can scarcely be doubted that the decreased digestibility of the less soluble carbohydrates under these circumstances is due to a partial diversion of the activity of the ferment organisms to the maltose resulting from the action of the saliva on the starch or to the sugar directly added, since these substances are presumably more readily attacked than cellulose and the like.

The action of nitrogenous substances in counteracting this effect of an excess of readily soluble carbohydrates is plausibly explained as due to its supplying more nitrogenous food

1 Compare U. S. Dept. of Agr., Bur. Anim. Indus., Bul. I39. 
for the organisms and so stimulating their multiplication and activity, and the fact that readily soluble nitrogenous materials like amino acids or ammonium salts seem to be particularly effective is quite in harmony with this view. The action of nitrogenous materials in stopping the excretion of undigested starch, on this view, would be explained as due to an increase of the proportion fermented, leaving less to be acted on by the digestive juices of the intestines.

727. Effect of addition of protein. - It was shown in the last paragraph that rations containing a large portion of carbohydrates and therefore relatively deficient in protein, i.e., those having a wide nutritive ratio, are likely to show an impaired digestibility, especially by ruminants. Correcting this condition by increasing the protein content of such rations tends, as would be expected, to increase their digestibility.

Trials have been made by several investigators of the effect of the addition of nearly pure protein (wheat gluten with 78 per cent of crude protein, or fish meal with 96 per cent of crude protein in the organic matter) to a basal ration. In general, such an addition has had little effect on the digestibility of the protein of the basal ration, but in several experiments on ruminants an increased digestibility of crude fiber, and, in some cases of the nitrogen-free extract, has been observed, especially, with basal rations poor in protein. In other instances, however, particularly when the deficiency in protein was less marked, this effect has been either slight or entirely absent and the same is true of such experiments on swine as have been thus far reported. Experiments are also on record in which the addition of feeding stuffs rich in protein, such as oil cake or legumes, has distinctly increased the digestibility of the crude fiber of a basal ration and others in which such an addition has stopped an excretion of undigested starch in the feces.

728. Effect of non-protein. - The addition to the basal ration of ruminants of digestible non-protein in the form of plant extracts as a rule tends to diminish the apparent digestibility of the protein of the basal ration, i.e., to increase the excretion of nitrogen in the feces, while the simpler forms of non-protein, such as asparagin or ammonium salts, have not usually produced this effect. ${ }^{1}$

${ }^{1}$ Compare U. S. Dept. Agr., Bur. Anim. Indus., Bul. 139 (19II), pp. 14-28. 
On the other hand, the effect of protein in increasing the digestibility of the non-nitrogenous ingredients of rations containing an excess of carbohydrates is shared also by the non-proteins, such comparatively simple substances as asparagin or even ammonium salts having in a number of instances exerted a marked influence of this sort.

729. Influence of drying. - The simple removal of water from a feeding stuff affects its digestibility but slightly. Weiske ${ }^{1}$ compared the digestibility of green and dried alfalfa and esparcet by sheep. In these experiments the forage was mowed daily, one-half of it fed and the other half dried without loss, which was a comparatively easy task with the relatively small amounts to be handled. In the second half of the experiment the portions of dried forage were fed to the same animals in the

Table i8i. - Percentage Digestibility of Forage, Green and Dried

\begin{tabular}{|c|c|c|c|c|c|c|c|c|}
\hline & & & $\begin{array}{l}\text { Total } \\
\text { ORGANIC } \\
\text { MATTER }\end{array}$ & $\begin{array}{c}\text { Crude } \\
\text { Protein }\end{array}$ & $\begin{array}{c}\text { CRUdDE } \\
\text { FIBER }\end{array}$ & $\begin{array}{l}\text { NitRo- } \\
\text { GEN-FREE } \\
\text { EXTRACT }\end{array}$ & $\begin{array}{c}\text { ETHER } \\
\text { EXTRACT }\end{array}$ & AsH \\
\hline Alfalfa & & & & & & & & \\
\hline Green . . & . & . & 67.40 & 83.08 & $45 \cdot 15$ & 72.79 & & 51.46 \\
\hline Dried . . & . & • & 66.69 & 82.73 & 44.83 & 71.71. & & 51.30 \\
\hline $\begin{array}{l}\text { Difference } \\
\text { Esparcet }\end{array}$ & . & . & $-0.7 \mathrm{I}$ & -0.35 & $-0.3^{2}$ & $-1.08^{\circ}$ & & -0.16 \\
\hline Green . . & . & . & 66.35 & 72.50 & 42.16 & 78.29 & 66.68 & $50.2 \mathrm{I}$ \\
\hline Dried . . & . & . & 62.12 & 69.98 & 36.40 & 74.35 & 66.24 & $45 \cdot 59$ \\
\hline $\begin{array}{c}\text { Difference } \\
\text { ARMSBY AND } \\
\text { CALDWELL } \\
\text { Grass }\end{array}$ & D & . & -4.23 & $-2.5^{2}$ & -5.76 & -3.94 & -0.44 & -4.62 \\
\hline Green . . & . & $\cdot$ & 68.87 & 65.66 & $74 \cdot 37$ & 73.18 & 54.73 & 50.05 \\
\hline Dried . . & . & - & $7 \mathrm{I} \cdot 3 \mathrm{I}$ & 71.66 & 76.78 & 72.95 & 60.06 & $55 \cdot 5^{6}$ \\
\hline $\begin{array}{r}\text { Difference } \\
\text { MorGEN }\end{array}$ & . & . & +2.44 & +6.00 & $+2.4 \mathrm{I}$ & -0.23 & +5.33 & $+5.5 \mathrm{I}$ \\
\hline Grass & & & & & & & & \\
\hline Green . & ${ }^{\circ}$ & $\cdot$ & $66.4^{2}$ & 69.6 & 65.6 & 77.8 & $65: 6$ & 30.7 \\
\hline Dried . . & ${ }^{\circ}$ & . & $63.4^{2}$ & $55 \cdot 7$ & 66.3 & 73.8 & 66.3 & 12.5 \\
\hline Difference & . & . & $-3.0^{2}$ & -3.9 & +0.7 & -4.0 & +0.7 & $-\mathrm{I} 8.2$ \\
\hline
\end{tabular}

${ }^{1}$ Jour. f. Landw., 25 (I877), 170.

2 Total dry matter. 
same order that the green forage was. Armsby and Caldwell ${ }^{1}$ subsequently made a similar experiment upon a cow by substantially the same plan, using mixed grasses cut while still young and corresponding substantially to pasture grass, and Morgen ${ }^{2}$ has reported comparisons of the same sort on three sheep. The average results of the four comparisons are shown in Table $\mathrm{I} 8 \mathrm{I}$. While the earlier experiments are open to criticism in some particulars, on the whole the conclusion appears warranted that the digestibility of forage is not very materially diminished by the simple removal of water and that the lower value of ordinary dry roughage as compared with green forage is largely due to differences in maturity and composition.

730. Cutting of roughage. - The digestibility of coarse fodders is not increased by cutting, and, indeed, it would be difficult to conceive how that process could have such an effect, since in either case the feed is comminuted during mastication to practically the same extent. This is strikingly shown in experiments by Kellner ${ }^{3}$ in which the preparation of straw and chaff was carried to the extent of grinding it to a fine meal. Table I 82 shows his comparison between wheat straw and barley straw cut into inch and a half lengths or finely ground.

Table 182. - Digestibility Cut and Finely Ground

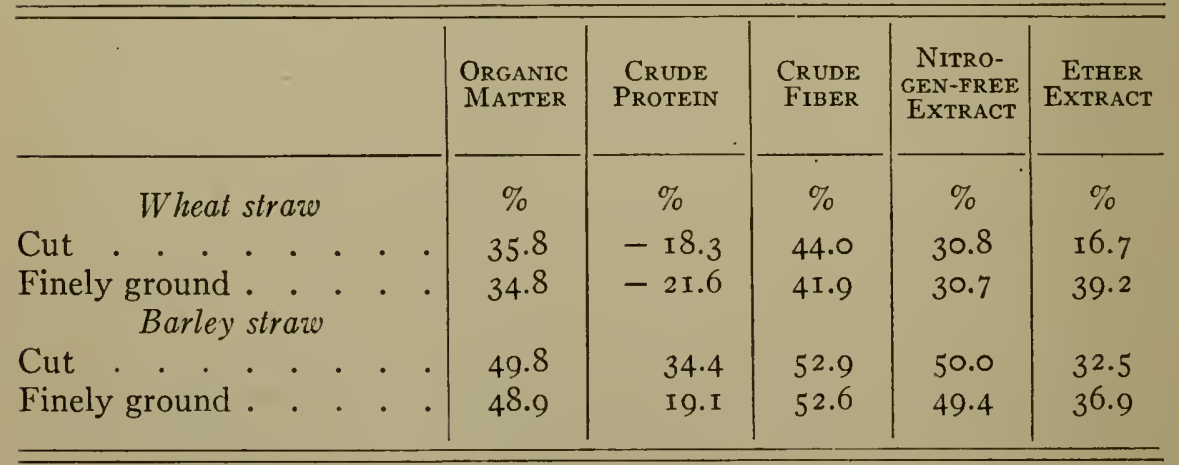

731. Grinding of grain. - The outer coats of seeds are resistant to solvents, their purpose being to protect the seeds from external influences. When whole grain is fed, especially in large

1 Penna. Expt. Sta., Rpt. I888, p. 60; Agricultural Science, 3, 295.

2 Landw. Vers. Stat., 75 (I9II), 321.

${ }^{3}$ Ernährung landw. Nutztiere, 6th Ed. p. 266. 
amounts to greedy feeders or to animals with imperfect teeth, more or less of it escapes mastication and, protected by the outer coats, passes through the digestive tract relatively unacted upon. Such apparently intact grains of corn, oats, etc., still capable of germination, are a familiar sight in the droppings of heavily fed animals.

Such visible losses, however, are not confined to the feeding of whole grain but, although less obvious, extend to cracked or crushed grain as well. If, for example, the feces of full-fed cattle receiving cracked corn or other grain be washed out, a considerable amount of fragments of grain may be recovered, the amount depending upon the total quantity fed and the consequent rapidity with which it passes through the digestive tract. Moreover, it is evident that the mechanical separation by washing is necessarily imperfect. Not only may the sieve hold back other things than fragments of grain, but it is likewise clear that any undigested fragments of the latter which are smaller than the meshes of the sieve will pass through and be lost, so that fine meal or well-masticated grain might suffer a greater loss through incomplete digestion than would be indicated by such tests. While it is to be supposed that smaller fragments will undergo more rapid solution in the digestive tract than larger ones, it is evident that the rapidity of passage through the organs is an important factor and that even comparatively small bits may, under some circumstances, escape complete digestion, while on the other hand, with light feeding, whole grain might be almost as well digested as when ground. Qualitatively, the results reached by washing out the feces are of great interest, but they may readily be misleading as regards the actual advantage of grinding.

Surprisingly few investigations upon the relative digestibility of ground and unground grain have been reported. Jordan and Hall, ${ }^{1}$ in their compilation of American digestion experiments up to I900, present two comparisons with horses and two with swine, all of which show the ground grain to be more digestible than the unground, the difference with respect to the dry matter ranging from 3.3 to 14 per cent.

Gay, ${ }^{2}$ in experiments upon oats with a horse weighing about

1 U. S. Dept. Agr., Office Expt. Stas., Bul. 77, p. 97.

2 Centbl. Agr. Chem., 25 (I896), 729. 
340 kilograms ( $750 \mathrm{lb}$.$) and receiving per day 3$ kilograms of oats and 2 kilograms of hay, obtained the following results:-

Table 183. - Percentage Digestibility of Oats by a Horse

\begin{tabular}{|c|c|c|c|c|c|c|}
\hline & $\begin{array}{c}\text { DRY } \\
\text { MATTER }\end{array}$ & AsH & $\begin{array}{c}\text { CRUDE } \\
\text { PROTEIN }\end{array}$ & $\begin{array}{l}\text { CRUDE } \\
\text { FIBER }\end{array}$ & $\begin{array}{c}\text { NITRO- } \\
\text { GEN-FREP } \\
\text { EXTRACT }\end{array}$ & $\begin{array}{c}\text { ETHER } \\
\text { ExTRACT }\end{array}$ \\
\hline Whole . & 64.53 & 27.78 & 71.30 & 42.00 & 74.70 & 40.90 \\
\hline Crushed . & $68.5^{8}$ & 31.97 & 79.15 & 48.87 & 74.99 & 59.46 \\
\hline Ground . & 72.73 & $42.7 \mathrm{I}$ & 94. I I & 63.60 & 75.19 & 54.78 \\
\hline Gain by crushing & 4.05 & 4.19 & 7.85 & 6.87 & 0.29 & $\mathrm{r} 8.56$ \\
\hline Gain by grinding & 8.20 & I 4.93 & $22.8 \mathrm{I}$ & 21.60 & 0.49 & 13.88 \\
\hline
\end{tabular}

While the results just cited are more or less variable, and while the small differences in the digestibility of the nitrogen-free extract in Gay's experiments seem peculiar, the results as a whole clearly show an increased digestibility by swine and horses as a result of grinding, while they also show that the difference is apparently not very great - less perhaps than would have been expected.

Gay also reports the following results of similar experiments upon a sheep weighing 8I kilograms and eating 500 grams of oats and $75^{\circ}$ grams of alfalfa hay:-

Table i84. - Percentage Digestibility of Oats by a Sheep

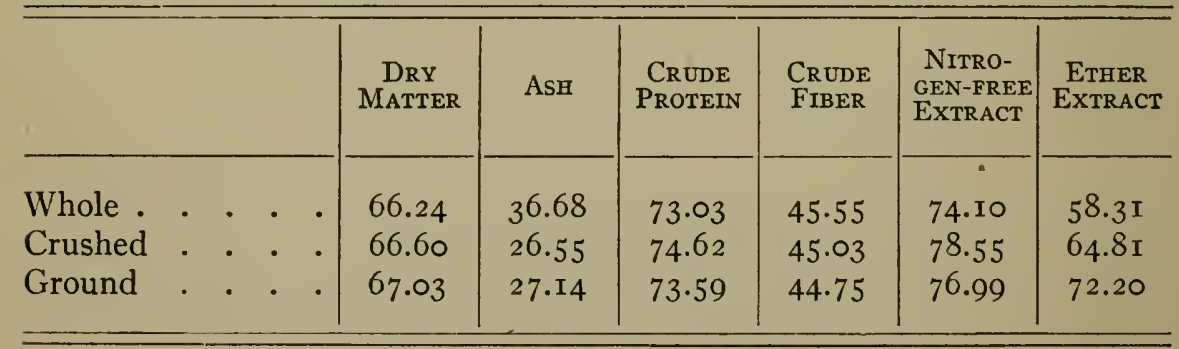

With the exception of the ether extract, whose digestibility it is difficult to determine accurately (165), the percentage digestibility is practically identical in the three cases. So far as a single experiment goes, therefore, it indicates that there is no advantage in grinding oats for sheep. Experiments upon 
other ruminants and with other feeding stuffs are lacking, but it does not appear surprising that a ruminant should digest whole grain more completely than a non-ruminant. As a whole, the results upon the influence of grinding on digestibility are comparatively meager and in particular they afford no information as to the effect of variations in the amount fed upon the relative digestibility of whole grain and of coarse or fine meal.

732. Acids. - The extensive use of silage lends interest to the question of the influence of acids on the digestibility of feeding stuffs.

Weiske ${ }^{1}$ compared the digestibility of meadow hay with and without the addition of sulphuric acid ( 0.75 per cent $\mathrm{SO}_{3}$ ) by one sheep, using two periods on each ration, and obtained almost absolutely identical results, with the exception of a slight increase in digestibility of the ash and ether extract of the acidified hay. Kellner ${ }^{2}$ added a much larger proportion of lactic acid ( 2.67 per cent) to a ration of hay and maize fed to a sheep and likewise observed practically no effect on the digestibility.

Apparently, then, such amounts of organic acids as are ordinarily consumed in silage and other feeds are without effect on digestion in the case of ruminants and this conclusion is to a certain extent supported by the general results of experiments which have shown that ensiled forage is fully as digestible as the same material carefully dried. The amounts of acid consumed under normal conditions are after all not large as compared with the quantities produced in the rumen and neutralized by the saliva. That excessive amounts of acids may stimulate peristalsis and so produce scouring is doubtless true, and it may be presumed that other species, such as the horse, for example, may be more sensitive to acids than ruminants.

733. Condiments. - One of the exaggerated claims made for the various proprietary condimental feeds is that they are able to increase materially the digestibility of rations to which they are added. Not the slightest scientific basis for this claim exists. All experimenters agree that they are without influence in this respect. Recent investigations by Fingerling, ${ }^{3}$ for example, in which fennel, anise, fenugreek and malt sprouts were

1 Jour. Landw., 33 (1885), 21. $\quad{ }^{2}$ Ernährung landw. Nutztiere, 6th Ed., p. 56.

${ }^{3}$ Landw. Vers. Stat., 62 (I905), 4 I-57. 
added both to ordinary feeds and to a ration made up of abnormally flavorless materials showed no effect upon the percentage digestibility.

734. Water drinking. - Stress has been laid by numerous writers on the supposed effect of water drinking on digestion, particularly by the horse. It has been asserted that drinking after feeding tends to dilute the gastric juice and to wash the feed out of the stomach and the feeder has been advised to water his animals before feeding rather than after feeding.

Even were the supposed facts true, it is questionable whether the conclusions drawn would be warranted, since the stomach, far from being the sole organ of digestion, serves largely as a sort of preliminary reservoir (119), and the extensive intestines of farm animals afford ample opportunity for the digestion of any substances which may escape action in the stomach. As a matter of fact, however, no such washing out or degree of dilution occurs as has been supposed. As has already been stated (131), the contents of the stomach are semi-solid rather than liquid and, as shown by their stratification, much less mixing of them takes place than is sometimes imagined. Scheunert ${ }^{1}$ has shown that in the horse the larger part of the water drunk passes along the walls of the stomach and around its contents and is quite promptly discharged into the small intestine. This is especially the case when the stomach is well filled with feed. In the contrary case more water is retained, but in no case did the total dilution of the entire stomach contents exceed about ro per cent. Moreover, the water which enters the duodenum is rather rapidly resorbed and has no material effect in the transportation of feed into the large intestine.

In view of these facts it is not surprising to find that the few digestion trials which have been made show no evidence of a decrease in digestibility as a result of drinking after eating.

Gabriel and Weiske ${ }^{2}$ in experiments on two sheep found no significant difference in the percentage digestibility of a ration of oats and hay, whether the water was given before or after feeding or kept constantly before the animals. The percentage digestibility of the organic matter was:-

1 Arch. Physiol. (Pflüger), 144 (I9I2), 4I I ; 151 (IgI3), 396.

${ }^{2}$ Landw. Vers. Stat., 45 (1895), 3 II. 


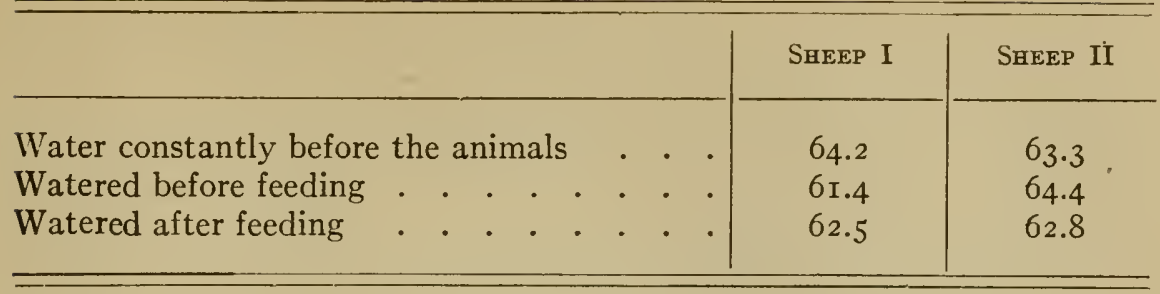

Tangl, ${ }^{1}$ in a number of experiments upon four different horses, found that when watered before drinking, the consumption of water was irregular and was less than when they were watered during or after feeding, and that the corresponding digestibility was also less in nearly every case. Suggestive in this connection are the results of Foster and Lambert, ${ }^{2}$ who found that in the dog a restricted supply of water tended to decrease the secretion of gastric juice. The foregoing results on animals seem to be in general accord with those of Hawk's extensive studies on the effects of water drinking in man.

1 Jahresber. Agr. Chem., 28 (1899), 661.

2 Expt. Sta. Rec, 25 (IgI I), I6. 


\section{CHAPTER XVII}

\section{THE PRODUCTION VALUES OF FEEDING STUFFS}

\section{$\S$ i. General Considerations}

735. Definition. - By the production values of feeding stuffs, as distinguished from the relative values discussed in the last chapter, is meant the actual effect produced by a unit weight of the substance in maintaining an animal or in supporting the processes of growth and fattening or of milk or work production. That such production values will also express relative values scarcely needs mention.

Even at their best, comparisons based on the "digestible nutrients," such as have been in vogue for many years and have become familiar to all students of the subject, can show only the relative and not the absolute values of feeding stuffs. It is true that to the extent to which it may be assumed that the digestible nutrients as determined by analyses and digestion experiments actually consist of proteins, carbohydrates and fats, their amount may furnish a useful clue to the nutritive value of the material consumed. Even then, however, it affords no quantitative measure of the results to be expected, while in the case of most feeding stuffs, as appeared in Chapters II and III, the actual nature of the digested material has been but very incompletely investigated. Neither the chemistry of feeding stuffs nor the behavior of their various constituents in metabolism is sufficiently well known to serve as the basis for any trustworthy estimate of their actual nutritive effect. The latter can be determined only by a direct trial with the animal, and during the past two decades considerable progress has been made in this direction.

736. Determination of production values. - By definition the production value is the effect produced upon the animal by a unit of the feed under consideration. The general methods 
for ascertaining this effect have been considered in Chapter VI. It was there shown that neither the gain nor loss of live weight or the gross weight of product is a sufficiently accurate measure of it (281-283, 604) and that the attainment of exact results requires the employment of some form of the balance experiment (285), based on the conception of the balance of nutrition. According to this conception, the production values of a feeding stuff for various purposes are measured, either by the extent to which it can prevent a loss of protein, ash and fat from the body during maintenance or work or can support the storage of these ingredients in the body or the milk. It was also pointed out in the same chapter that the investigations of the last thirty years have shown that the problem may be advantageously studied from the standpoint of energetics and that in this way the expression of the results may be notably simplified and unified. From this standpoint the feed is regarded as a supply of ash and protein (or amino acids) on the one hand and of energy on the other and its effect is similarly expressed by the gain or loss by the body of protein and ash and of chemical energy respectively. We may distinguish, therefore, between production values for protein, for ash, and for energy.

737. Two aspects of feed supply. - For a clear conception of the nature and significance of the production values of feeding stuffs, however, it is essential to distinguish between two aspects or functions of the feed supply.

In the past the feed has been regarded chiefly as the source of the material necessary for the constructive processes going on in the body and of the energy required to support its metabolic activities. It supplies ash to maintain or increase the mineral matter of the body, protein (or amino acids) to build up its tissues or supply the protein of milk, energy to support the vital activities of the various organs. This aspect of the matter has been the prominent one in the preceding chapters of this work.

Recent investigation, however, is bringing into prominence another classs of influences exerted by the feed upon the organism. The studies upon the "vitamins," "accessory ingredients," "growth substances," "stimulating substances," "specific effects of feeds," etc., which have been several times 
referred to in Part III are rendering it increasingly evident that, quite aside from its value as a supply of structural material and of energy, the nature of the feed may profoundly influence the course and intensity of the metabolic processes. In particular it appears that the absence of certain as yet ill-defined substances may constitute a limiting factor, particularly in growth, or may lead to the development of specific diseases, while, on the other hand, McCollum's observations on the exclusive use of wheat products (499) seem to indicate that similar effects of a more or less toxic character may follow the excessive consumption of feeding stuffs ordinarily regarded as healthful. It is important, therefore, to secure as definite a conception as possible of the significance of these new facts in their relation to the older conceptions of production values.

738. Significance of " accessory ingredients." It is clear that the "accessory ingredients" (using this simply as a convenient summary term for the various classes of substances indicated in the last paragraph) influence the nutritive value of a feeding stuff in an essentially different fashion than does the quantity of available ash, protein, and energy which it supplies. The latter limits the amount of production which the feeding stuff can support; the presence or absence of the former may determine the extent to which this potential value is actually realized. Thus in Chapter XI, experiments by Osborne and Mendel and by Hart and McCollum $(498,499)$ were described which show that a mixture of pure nutrients may be prepared which shall contain an abundant supply of complete. proteins, of ash and of energy but upon which young animals (rats) fail to grow, while the addition to such a mixture of minute amounts of substances associated with certain fats enables the rations to support normal growth. In some aspects of the matter, these " accessory ingredients" might be crudely compared with the lubricants of a machine, which of themselves furnish neither power nor material, but which enable power derived from the consumption of fuel to be more efficiently used and therefore conduce to the production of a larger output.

A lack of lubricants in the case just supposed might conceivably affect the output of a machine in one or both of two ways. The undue friction might slow down the machine as a whole so that less raw material would pass through it in a given time, 
or it might affect specifically certain more delicate parts of the machine and so reduce the efficiency of the machine and cause it to yield less finished product per unit of raw material consumed.

In which of these two ways a deficiency in " accessory substances" affects the nutrition of an animal does not appear to have been determined. It would seem probable, however, that, in the case of a young animal, for example, a deficient dietary acts to slow down or stop the whole group of anabolic processes involved in growth. ${ }^{1}$ The organs would thus be rendered incapable of converting a normal daily amount of feed into body substances and a corresponding decrease in feed consumption would presumably follow. In such a case it is quite conceivable that such feed as was actually eaten in excess of the maintenance requirement might be just as efficient in producing gain and have as great a production value per unit as in a normal ration. In other words, it is conceivable that lack of the " accessory substances" may, in a sense, affect the economic rather than the physiological efficiency of the ration. The writer has failed to -note any experiments in which this aspect of the matter has been considered. In practically all reported investigations upon the influence of "accessory substances," the feed consumption has been regulated by the appetite of the animal and in many instances has not even been reported.

The undoubted importance of the accessory ingredients of feeding stuffs has led, on the part of some writers, to a tendency which as yet appears hardly justified to minimize the significance of the production values in the older sense. The subject is too new and the field too broad to warrant dogmatic conclusions, but it still remains true that the prime function of a feeding stuff is to supply structural material and energy for the body, and its potentialities in this respect are expressed in its production values. That the results attained by its use in practice are affected by other considerations has long been recognized. Thus, if a feeding stuff is unpalatable for some reason and is not eaten freely, the portion consumed may show a high nutritive effect per unit and yet the use of the feed be inadvisable. The presence of toxic substances might

1 Naturally such an effect might be brought about by a retardation of certain specific metabolisms upon which the whole growth process depended and the specific metabolisms affected might differ in different cases. 
prevent the use of a feed in sufficient amounts to be profitable and yet the nutritive effect of the feed within the limits of tolerance might be considerable.

Production values, then, if determined by means of balance experiments made under normal conditions, are to be regarded as showing the potential values of feeding stuffs as sources of matter and energy, i.e., their worth as constituents of a ration which contains sufficient amounts of whatever " accessory ingredients " are necessary to ensure the normal course of metabolism. The study of "accessory substances" in the broadest sense of the term has revealed an additional and apparently very important group of factors influencing the extent to which the potentialities of feeding stuffs are actually utilized. It is possible that in the future there must be added to the requirements already outlined for ash, protein (or amino acids) and energy for the various purposes of feeding, the requirements for the "accessory substances" necessary to secure the most efficient functioning of the cells and organs of the body.

\section{§ 2. Production Values as Regards Energy - Net ENERGY VALUES}

739. Recapitulation. - The consideration of the processes of nutrition in Part II, and in particular the study of metabolism and of the balance of nutrition in Chapters V and VI, has shown that the animal body is primarily a transformer of chemical energy and that quantitatively the most important function of the feed is to supply this energy. In the several chapters of Part III the conception of net energy values was developed and the requirements for net energy by different species of farm animals and for different purposes were discussed. It is apparent from those discussions that the net energy value is only another name for the production value of a feeding stuff as regards energy as defined in the preceding section. It appears desirable at this point, therefore, to recapitulate the general facts regarding the energetics of the animal body which are contained in previous chapters, and to consider in greater detail their bearing upon the production values of feeding stuffs, even at the expense of a certain amount of repetition. 
740. Gross energy. - The energy supply of an animal is contained in its feed as chemical energy, and the maximum amount which any substance can furnish for the vital activities by its oxidation in the body is measured by its heat of com. bustion. This has been called its gross energy (315) to avoid the implication that it represents the total amount of energy associated with the substance.

741. Losses of energy. - It rarely, if ever, happens, however, that this maximum effect is realized. In practically every case a larger or smaller proportion of the chemical energy of the feed escapes unutilized. These losses of energy are of two general classes.

First, a portion of the chemical energy of the feed fails to be transformed at all, leaving the body as chemical energy in the visible excreta and in the combustible gases arising from gastric and intestinal fermentations.

Second, another portion of the chemical energy of the feed is indeed transformed, but at ordinary temperatures virtually results merely in a superfluous heat production. It is true that the metabolism consequent upon feed consumption is not only unavoidable but may be regarded as a necessary expenditure of energy for the support of the activities connected with digestion and assimilation. Nevertheless, from the standpoint of the net gain or loss by the organism this portion of the feed energy, which ultimately takes the form of heat and escapes from the body, must be regarded as a loss.

\section{The losses of chemical energy}

742. Losses in feces. - Chemical energy escapes in the feces both in the undigested feed residues which they contain and in the excretory products which they carry. While the latter portion is not derived immediately from the feed consumed, but constitutes a loss of incompletely katabolized matter, it must, none the less, be included in estimating the net effect of a ration on the energy balance of the body.

With herbivorous animals, the excretion in the feces constitutes the greatest loss of chemical energy, and the one which varies most as between different feeding stuffs or different species of animals, as is apparent from the results recorded in Table 187 
(749). This is especially true of the energy of roughages, which contain much indigestible matter, but even with the more digestible materials the loss through the feces is relatively considerable. With swine it is relatively less than with herbivora because the former animals are usually largely fed on concentrates. The influence of various conditions upon the losses in the feces, i.e., upon digestibility, has already been discussed in $\S 3$ of the previous chapter.

743. Losses in urine. - The urine is especially the vehicle for the removal from the body of the end products of protein katabolism, of which urea is the most familiar and frequently the most abundant. Numerous other nitrogenous substances, however, are contained in the urine, particularly the purins and, in herbivorous animals, hippuric acid. Moreover, as stated in Chapter V $(224,225)$, the urine of herbivora in particular may contain relatively considerable quantities of non-nitrogenous excretory products regarding the nature of which little is known. All these substances carry off a portion of the gross energy of the feed as unused chemical energy, the amount of the loss being measured by their heats of combustion. That the extent of these losses cannot be satisfactorily computed from the nitrogen content of the urine has already been pointed out. The loss of chemical energy in the urine, as appears from Table I87, constitutes a relatively small percentage of the total loss. As would be expected, it is quite variable, being higher as the feed supply is richer in protein, and lower with relatively indigestible substances where the loss in the feces is larger.

744. Fermentation losses. - The gaseous products, chiefly methane, of the fermentation of the carbohydrates in the digestive tract of herbivora, especially of ruminants, carry off considerable amounts of unused chemical energy, one gram of methane, for example, having a heat of combustion of 13.344 Calories.

The extent to which the carbohydrates are attacked by the methane fermentation appears to be somewhat variable. Armsby and Fries ${ }^{1}$ have observed that with cattle it is distinctly greater on light than on medium or heavy rations and the same authors likewise observed a single instance of indi-

1 Jour. Agr'l Research, 3 (Igís), 445. 
vidual difference in this respect between animals. Recently Zuntz and his associates ${ }^{1}$ have reported striking instances in which the extent of the methane fermentation in particular has been markedly affected by the make-up of the rations and especially by the order in which the feeds were consumed, while Völtz and his associates ${ }^{2}$ have laid much stress upon the practical importance of these results. No such marked differences were observed in Armsby and Fries' experiments but the range of feeding stuffs used was not so wide. It is perhaps too early to judge of the full significance of Zuntz's results, but they should at least serve to correct the notion, more or less subconsciously held by not a few, of digestion as a perfectly definite process and of a digestion coefficient as a sort of chemical constant. On the other hand, however, it seems quite possible to overestimate the effect of such variations in the digestive process upon the net energy values of feeding stuffs. On the whole, they appear to be of far less significance than other factors to be considered later.

The percentage of the gross energy which is lost in the fermentation gases, as appears from Table I88 (749), is not usually very large. It is naturally greatest in the case of feeding stuffs rich in carbohydrates, especially the easily soluble carbohydrates.

745. Computation of fermentation losses. - While the experimental determination of the energy losses in feces and urine is a comparatively easy task, requiring relatively simple appliances, the determination of the fermentation losses necessitates the use of the somewhat complicated and costly respiration apparatus. In the absence of such an apparatus, however, it is possible to compute the fermentation losses with a fair degree of accuracy from the results of the ordinary digestion experiment. The methane fermentation attacks chiefly or wholly the carbohydrates $(135,140)$ and in the case of cattle, in particular, it has been shown that the amount of methane produced is in general proportional to the amount of total carbohydrates (crude fiber and nitrogen-free extract) digested.

Kellner $^{3}$ in forty-four experiments with cattle on mixed rations found the average methane excretion to be 4.2 grams

${ }^{1}$ Landw. Jahrb., 44 (1913), 765 ; Landw. Vers. Stat., 79-80 (1913), 78I.

2 Landw. Jahrb., 44 (I913), 685; 45 (I913), 325.

3 Landw. Vers. Stat., 53 (Ig00), 4I5. 
for each Ioo grams of total carbohydrates digested, and the estimated results for ruminants recorded in Table I 88 for the losses in methane were computed on that basis. With the addition of later unpublished experiments, Kellner's ${ }^{1}$ average was increased to 4.3 grams. Later experiments by Armsby and Fries ${ }^{2}$ have given slightly higher averages, viz., 4.8 grams for roughages and 4.7 grams for concentrates. No similar results for the smaller ruminants have been reported but probably it may be safely assumed that the average for cattle is substantially applicable to these species also.

In the horse the principal seat of the methane fermentation is the colon and cœcum (128). Since the more soluble carbohydrates of the feed are largely or entirely digested before reaching these organs, methane is much less copiously produced than in the case of ruminants and may be regarded as derived chiefly from the fermentation of crude fiber.

In respiration experiments on mixed rations of oats, hay and straw, Lehmann, Zuntz and Hagemann ${ }^{3}$ observed as the result of eight rather discordant experiments an average total excretion of methane of 4.73 grams per 100 grams digested crude fiber and in addition an average excretion of 0.203 gram of hydrogen per 100 grams digested crude fiber. In more recent experiments, Von der Heide, Steuber and Zuntz, ${ }^{4}$ using a Regnault-Reiset respiration apparatus (298), obtained for the methane excretion per 100 grams digested crude fiber 9.06 grams on hay and 2.28 grams on straw pulp. Using the average of these rather discordant experiments, the fermentation losses in the case of the horse may be approximately computed from the amount of crude fiber digested.

Swine with their simpler alimentary canal suffer but small losses from fermentation in the digestive tract.

Fingerling, Köhler and Reinhardt ${ }^{5}$ found the amounts of combustible gases excreted too small to be determined with their form of Pettenkofer apparatus. Von der Heide and Klein ${ }^{6}$ in three experiments with a Regnault-Reiset apparatus obtained the following results:-

1 Ernährung landw. Nutztiere, 6th Ed., p. 94.

2 Jour. Agr'l Research, 3 (I915), p. $450 .{ }^{3}$ Landw. Jahrb., 23 (I894), I 25.

4 Biochem. Ztschr., 73 (1916), I6r.

${ }^{6}$ Biochem. Ztschr., 55 (1913), I95.

${ }^{5}$ Landw. Vers. Stat., 84 (I914), 197. 
Table 185. - Excreted by Swine per ioo Grams Digested CARBOHYDRATES

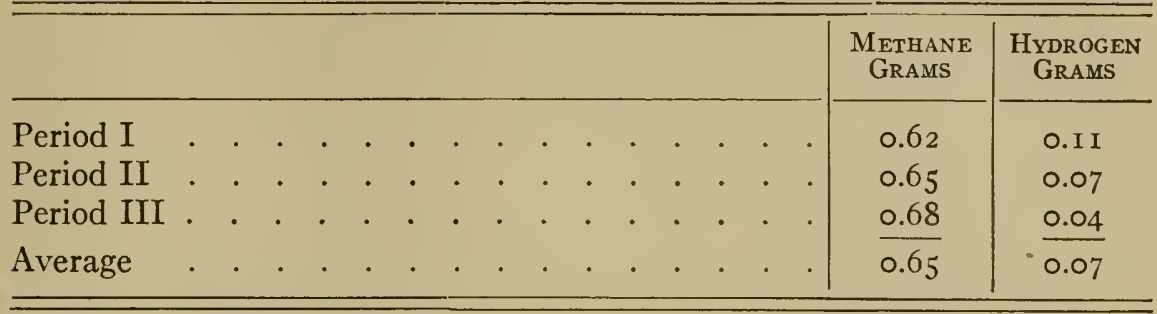

Although there is considerable range in the results of individual experiments, and while those on non-ruminants are few in number, nevertheless, the foregoing figures afford a basis for an approximate estimate of the losses of chemical energy in the combustible gases. Summarizing the available data and computing the equivalent quantities of energy, it appears that the following average deductions may be made from the gross energy of the feed for the fermentation losses.

Table i86. - Factors for Computing Fermentation Losses

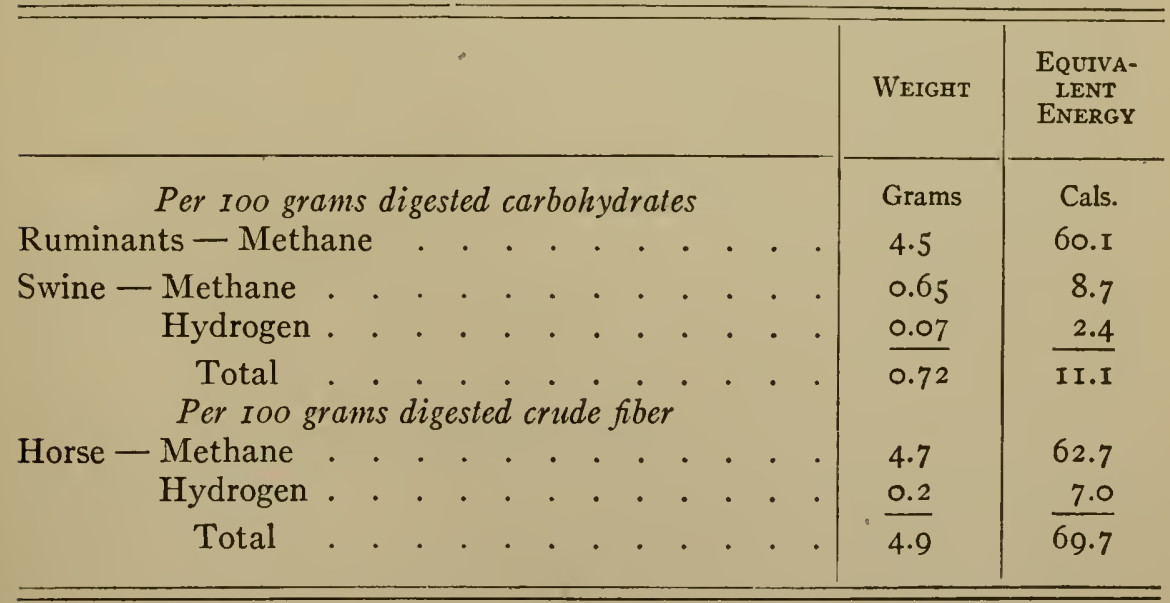

\section{Metabolizable energy}

746. Definition. - The difference between the chemical energy of the feed and that lost in the excreta shows how much of the former is capable of being converted into other forms in the body, either during the changes which the feed undergoes in 
the digestive tract or in the course of metabolism in the tissues. As stated in Chapter VI (323), this convertible portion of the feed energy has been given various names by different investigators, such as "physiological heat value," "fuel value," "available energy," etc., but following a suggestion made earlier by the writer it is here designated as "metabolizable energy."

747. Method of determining. - As is apparent from the foregoing paragraphs, the direct determination of the metabolizable energy of a feeding stuff or ration requires the measurement of the amounts and heats of combustion of the feed and of the solid, liquid and gaseous excreta by the methods outlined in Chapter VI. These quantities being known, a simple subtraction gives the metabolizable energy. Thus the results of the experiment used as an illustration in Chapter VI (322), put in a somewhat more detailed form, were as follows :-

Table i 87. - Example of Determination of Metabolizable Energy

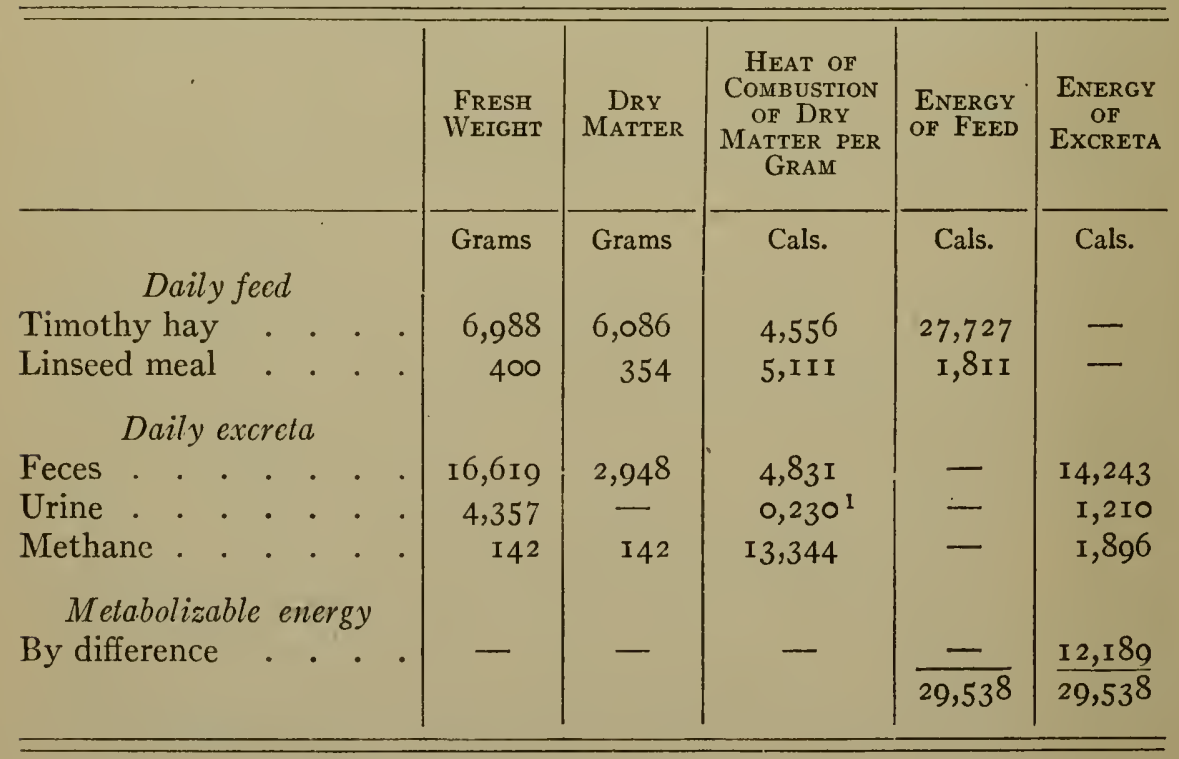

748. Correction for gain or loss of protein. - In the foregoing experiment the animal gained 15.2 grams of fat and 66.6 grams of protein and therefore stored up in its body equivalent amounts of energy, viz.,

In protein, 5.7 Cals. $\times 66.6=380$ Cals.

In fat, $\quad 9.5$ Cals. $\times 15.2=$ I 44 Cals.

${ }^{1}$ Per gram fresh urine. 
The 144 Cals. of energy contained in the fat, however, although not actually transformed into other forms of energy, were capable of such transformation had the demands of the organism required it, and therefore constitute part of the metabolizable energy of the feed. With the 380 Cals. contained in the protein stored up, however, the case is different. Had these 66.6 grams been katabolized, part of their energy would have escaped in the resulting nitrogeneous metabolic products. According to Rubner each gram of urinary nitrogen derived from lean meat is equivalent to 7.45 Cals. of chemical energy. The katabolism of the 66.6 grams of protein, therefore, would have increased the chemical energy of the urine by 83 Cals., while only 297 Cals. would have been transformed. This amount of 83 Cals. must consequently be added as a correction to the urinary energy as measured in computing the metabolizable energy. In case of a loss of protein from the body a similar correction must evidently be subtracted.

When a respiration apparatus for the determination of the combustible gases is not available, their amount may be estimated from the digestible carbohydrates in the manner already outlined (745), so that it is possible to estimate the metabolizable energy with a considerable degree of accuracy from the results of an ordinary digestion experiment to which has been added the collection of the urine and determinations of the heats of combustion of the visible excreta. The additional labor thus required is so small that it is to be hoped that in future digestion experiments it may be undertaken whenever possible and that in this way more extensive data may be secured regarding the metabolizable energy of feeding stuffs. While such results do not show the production values of the rations (750), they constitute an important step toward their more exact determination.

749. Experimental Results. - There are on record a somewhat limited number of experiments with cattle and a few with swine in which the losses of energy in the feces, urine and methane respectively have been determined directly, while in a considerably larger number the losses of methane have been estimated from the digestible carbohydrates (crude fiber plus nitrogen-free extract) in the manner just described. The results of these experiments are recorded in Table $188,{ }^{1}$ which shows the percentages of the gross energy which were carried

1 This table is not claimed to be an exhaustive compilation of data, but is believed to be fairly complete. 
off in the several excreta and, by difference, the percentages which were metabolizable. The metabolizable energy per gram of digestible organic matter is also added, since, as will appear subsequently, it forms a convenient basis for the computation of metabolizable energy when direct determinations of it are not available.

Table i88. - Apparent Metabolizable Energy

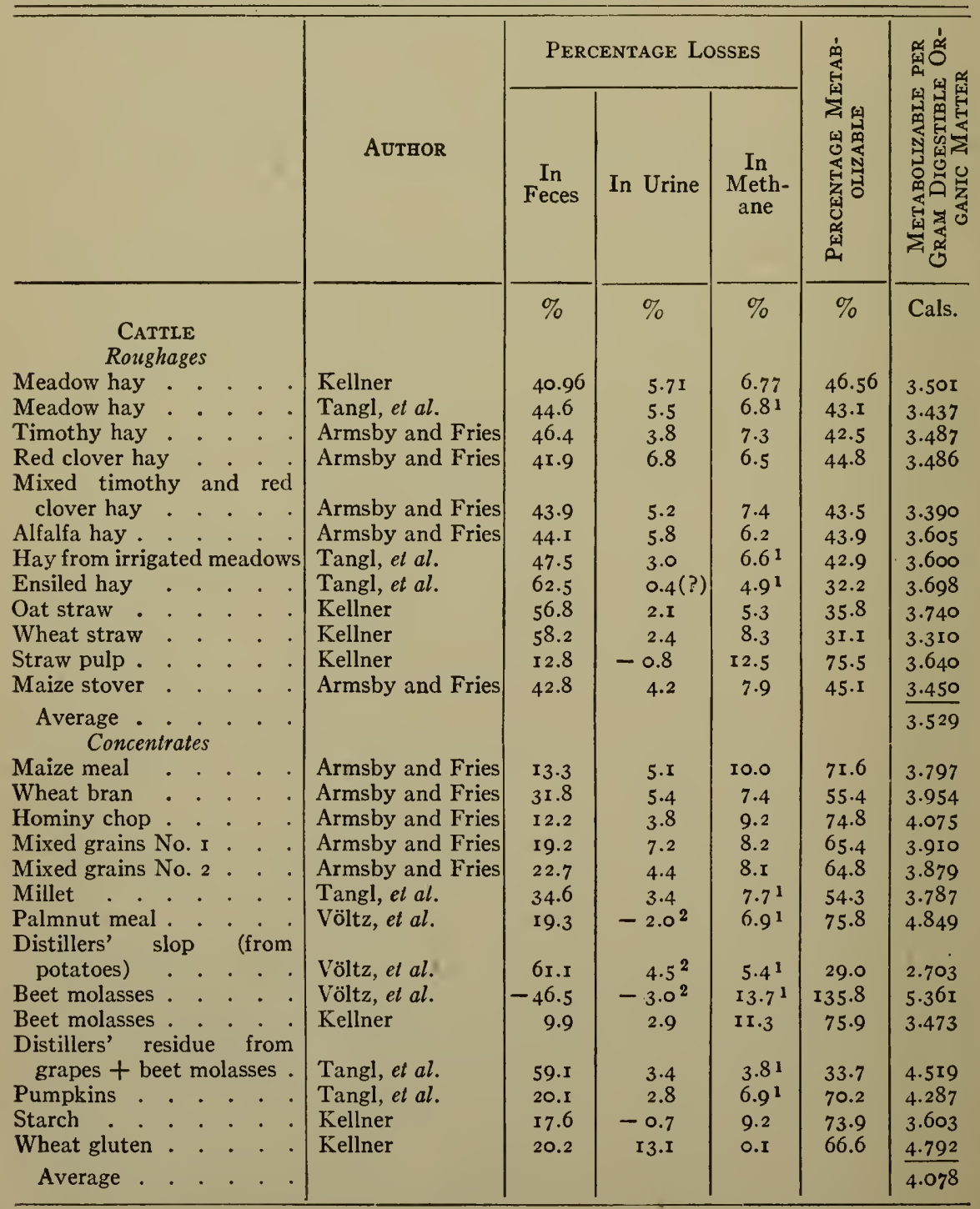


Table i88-Apparent Metabolizable Energy (Continued)

\begin{tabular}{|c|c|c|c|c|c|c|}
\hline & \multirow[b]{2}{*}{ AUTHOR } & \multicolumn{3}{|c|}{ Percentage Losses } & \multirow{2}{*}{ 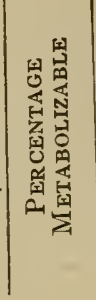 } & \multirow{2}{*}{ 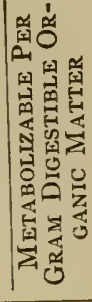 } \\
\hline & & $\begin{array}{l}\text { In } \\
\text { Feces }\end{array}$ & $\underset{\text { Urine }}{\text { In }}$ & $\begin{array}{c}\text { In } \\
\text { Meth- } \\
\text { ane }\end{array}$ & & \\
\hline & \multicolumn{6}{|c|}{$\begin{array}{l}\text { SHEEP } \\
\text { Roughages }\end{array}$} \\
\hline $\begin{array}{l}\text { Meadow hay }: \cdot . \\
\text { Meadow hay }: \cdot .\end{array}$ & $\begin{array}{l}\text { Tangl, et al. } \\
\text { Völtz, et al. }\end{array}$ & $\begin{array}{l}46.6 \\
43.5\end{array}$ & $\begin{array}{l}4.8 \\
4.8^{2}\end{array}$ & $\begin{array}{l}6.2^{1} \\
6.3^{1}\end{array}$ & $\begin{array}{l}42.4 \\
45.4\end{array}$ & $\begin{array}{l}3.559 \\
3.6 \mathrm{II}\end{array}$ \\
\hline Hay from peat meadows & Tangl, et al. & 59.4 & 4.1 & $4.8^{1}$ & $\begin{array}{l}43.4 \\
3 \mathrm{I} .6\end{array}$ & 3.544 \\
\hline Hay from alkali soil. & Tangl, et al. & 52.9 & $4 . I$ & $5 \cdot 5^{1}$ & $37 \cdot 5$ & $3.60 \mathrm{r}$ \\
\hline $\begin{array}{l}\text { Hay from same, irrigated. } \\
\text { Alpine hay. }\end{array}$ & Tangl, et al. & 40.4 & 4.1 & II. 41 & 44.1 & 3.288 \\
\hline $\begin{array}{r}\text { Alpine hay . } . ~ \\
\text { Average } . \\
\end{array}$ & Tangl, et al. & 39.2 & 4.1 & $6.9^{1}$ & 49.8 & $\frac{3.765}{35.6 \mathrm{I}}$ \\
\hline $\begin{array}{r}\text { Alfalfa hay . . . . . } \\
\text { Average . }\end{array}$ & Tangl, et al. & 32.5 & 4.1 & $5.4^{1}$ & 58.0 & $\frac{4.467}{3.691}$ \\
\hline $\begin{array}{l}\text { Dried potato vines } \\
\text { Same with fruit }\end{array}$ & Völtz, et al. & $\begin{array}{l}43.2 \\
45.9\end{array}$ & $\begin{array}{l}3.5^{2} \\
3.8^{2}\end{array}$ & $\begin{array}{l}5 \cdot 3^{1} \\
4.8^{1}\end{array}$ & $\begin{array}{l}48.0 \\
45.5\end{array}$ & 4.182 \\
\hline Same with fruit & \begin{tabular}{|l} 
Völtz, et al. \\
Völtz, et al.
\end{tabular} & $\begin{array}{l}43 \cdot 9 \\
41 \cdot 3\end{array}$ & $5 \cdot 2^{2}$ & $6.3^{1}$ & $\begin{array}{l}45 \cdot 5 \\
47.2\end{array}$ & $\begin{array}{r}4.319 \\
3.620\end{array}$ \\
\hline $\begin{array}{l}\text { Hay and ensiled potato } \\
\text { vines . . . . . }\end{array}$ & Völtz, et al. & 42.2 & $5 \cdot 9^{2}$ & $5.9^{1}$ & 46.0 & 3.526 \\
\hline $\begin{array}{l}\text { Wheat straw } \\
\text { Average }\end{array}$ & Völtz, et al. & 74.9 & $4 \cdot \mathrm{I}^{2}$ & $4.6^{1}$ & I6.4 & $\frac{2.378}{3.655}$ \\
\hline Concentrates & Tangl, et al. & $35 \cdot 5$ & 4.I & $6.6^{1}$ & 53.8 & \\
\hline $\begin{array}{l}\text { Oats } \\
\text { Millet }\end{array}$ & Tangl, et al. & 30.2 & 7.1 & $8.2^{1}$ & 54.5 & $\begin{array}{l}3.973 \\
3.405\end{array}$ \\
\hline Corn-and-cob meal . . & Tangl, et al. & 31.0 & 2.9 & $8.2^{1}$ & 57.9 & 3.836 \\
\hline Palmnut meal . . . & Völtz, et al. & 35.0 & $\begin{array}{r}4 \cdot 5^{2} \\
-120^{2}\end{array}$ & $6.3^{1}$ & 54.2 & 3.977 \\
\hline $\begin{array}{l}\text { Lentils } \\
\text { Distillery slop from pota- }\end{array}$ & Völtz, et al. & 7.0 & $12.9^{2}$ & $8.7^{1}$ & $7 \pi \cdot 4$ & 4.079 \\
\hline $\begin{array}{l}\text { Distillery slop from pota- } \\
\text { toes }\end{array}$ & Völtz, et al. & 23.2 & $7.6^{2}$ & $6.3^{1}$ & 62.9 & 4.383 \\
\hline Beet molasses . . . & Völtz, et al. & $\mathbf{1} 8.6$ & 17.3 & 7.9 & 56.29 & 3.124 \\
\hline Average . . - . & & & & & & 3.825 \\
\hline $\begin{array}{c}\text { HoRSES } \\
\text { Roughages }\end{array}$ & & & & & & \\
\hline Meadow hay . . & Tangl, et al. & 55.1 & 3.6 & $1.9^{1}$ & 39.4 & $\begin{array}{l}3.707 \\
3.854\end{array}$ \\
\hline $\begin{array}{l}\text { Hay from peat meadow } \\
\text { Hay from alkali soil. }\end{array}$ & $\begin{array}{l}\text { Tangl, et al. } \\
\text { Tangl, et al. }\end{array}$ & $\begin{array}{l}66 . \mathrm{I} \\
59.3\end{array}$ & $\begin{array}{l}3.7 \\
3.7\end{array}$ & $\begin{array}{l}0.8^{1} \\
1.6^{1}\end{array}$ & 29.4 & $\begin{array}{l}3.854 \\
3.803\end{array}$ \\
\hline & $\begin{array}{l}\text { Tangl, et al. } \\
\text { Tangl, et al. }\end{array}$ & $\begin{array}{l}59 \cdot 3 \\
50.4\end{array}$ & $\begin{array}{l}3.7 \\
3.7\end{array}$ & $\begin{array}{l}1.0^{2} \\
2.0^{1}\end{array}$ & $\begin{array}{l}45.4 \\
43 .\end{array}$ & $3.74 \mathrm{I}$ \\
\hline & $\begin{array}{l}\text { Tangl, et al. } \\
\text { Tangl, et al. }\end{array}$ & 50.1 & 3.7 & r. $6^{1}$ & $\begin{array}{l}43 \cdot \\
44.6\end{array}$ & 3.915 \\
\hline Sour meadow hay . & Tangl, et al. & 66.4 & 3.7 & $1.5^{1}$ & 28.4 & 3.607 \\
\hline Silage from same . & Tangl, et al. & 70.0 & 3.7 & I. $6^{1}$ & 24.7 & 3.352 \\
\hline Average & & & & & & $3.7 \times 2$ \\
\hline Concentrates & Tangl, et al. & $4 \mathrm{I} \cdot 4$ & 3.7 & $0.2^{1}$ & 54.7 & 4.493 \\
\hline $\begin{array}{l}\text { Oats } \\
\text { Distillery residue from }\end{array}$ & & 660 & & & & \\
\hline $\begin{array}{l}\text { grapes and beet molasses } \\
\text { Average . . . . }\end{array}$ & Tangl, et al. & 66.9 & 1.4 & $0.8^{1}$ & 30.9 & $\frac{4.76 \mathrm{I}}{4.627}$ \\
\hline
\end{tabular}

1 Estimated.

${ }^{2}$ Not corrected to N. equilibrium. 
Table i88-Apparent Metabolizable Energy (Continued)

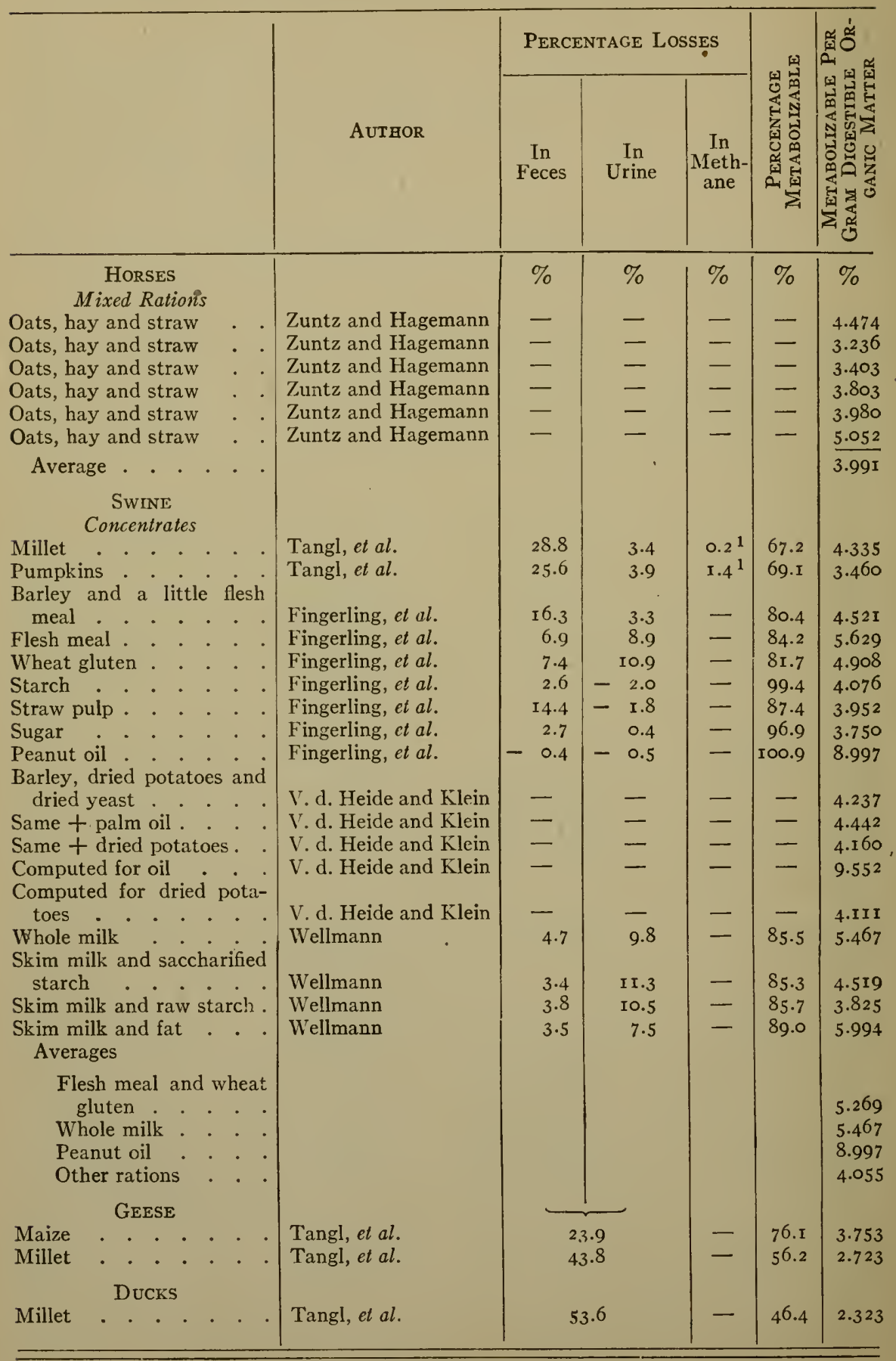

1 Estimated. 
750. Significance of metabolizable energy. - By metabolizable energy, as already explained, is meant simply the energy capable of transformation in the body, with no implication as to the proportion of the energy thus transformed which can be utilized by the organism. The heat evolved during the methane fermentation, for example, constitutes part of the metabolizable energy as thus defined, although it does not enter into the tissue metabolism.

The metabolizable energy of a feeding stuff does not measure its production value, since it takes account of only one of the two classes of losses to which its chemical energy is subject. Obviously, however, it is an essential factor in fixing that value, since frequently from one-fourth to one-half or more of the feed energy is thus rejected unused. The determination or estimation of the metabolizable energy of a feeding stuff is, therefore, an important step in ascertaining its production value as regards energy, and constitutes an advance over the simple determination of digestibility, since it takes account of the losses in urine and methane as well as of those in the feces.

751. Real and apparent metabolizable energy. - The metabolizable energy of a feeding stuff as determined experimentally in the manner illustrated in a preceding paragraph (747) is the aggregate effect as regards energy of all the influences which the feeding stuff exerts on the digestive processes.

For example, in one of Kellner's experiments beet molasses added to a basal ration diminished the amount of energy carried off in the methane by 135.8 Cals., while at the same time it so depressed the digestibility of the basal ration that the amounts lost in the feces and urine were increased by 1865.9 Cals. and 272.3 Cals. respectively. By the method of computation here used, the algebraic sum of these amounts is virtually regarded as representing the losses of energy from the molasses and is subtracted from the gross energy of the latter to obtain its metabolizable energy. The metabolizable energy as thus computed expresses the net increase in the amount of energy available for conversion in the body and may be called the apparent metabolizable energy.

On the other hand, the results for the metabolizable energy of the digestible nutrients recorded in the next paragraph include corrections for these secondary effects. They aim to show 
the actual amounts of metabolizable energy supplied by the digested portions of the feed irrespective of its secondary effects - i.e., to express its real metabolizable energy. Such figures give a more accurate idea of the store of metabolizable energy contained in the feeding stuff regarded by itself, while the apparent metabolizable energy is better adapted for use in a discussion of questions of feeding. ${ }^{1}$ The distinction is similar to that already discussed in Chapter III (167) between real and apparent digestibility.

752. Computation of metabolizable energy from digestible nutrients. - While, in the absence of a respiration apparatus, the metabolizable energy of a feeding stuff or ration may be estimated with a fair degree of accuracy by the method outlined in previous paragraphs, not every experimenter is equipped to determine the heats of combustion of the feed and the visible excreta, and no satisfactory method of computing them is available. Various attempts have accordingly been made to compute the metabolizable energy of feeding stuffs from chemical data.

One such method is that employed by Rubner and by Atwater for estimating the metabolizable energy of the food of man and of carnivora as described in Chapter VI (324), their factors for protein, carbohydrates and fat being applied directly to the digestible nutrients of feeding stuffs, and several tables of energy values as thus computed have been published. Later investigations, however, showed that the results thus obtained were much too high in the case of herbivorous animals, especially of ruminants. To cite but a single instance, experiments on cattle by the writer ${ }^{2}$ gave the results shown in Table I 89 for metabolizable energy as compared with those computed by the use of Rubner's factors, and Kellner's somewhat earlier results ${ }^{3}$ led to the same general conclusion.

There are two principal reasons for this discrepancy. The first is the extensive fermentation of the carbohydrates in the digestive tract of ruminants, leading to a relatively larger loss of energy in the combustible gases excreted. The second reason is the fact that the urine of herbivora carries off much more non-nitrogenous material (224) than is the case with man or carnivora. The results of direct determinations on swine

1 Compare Armsby, Principles of Animal Nutrition, pp. $29 \mathrm{I}-293$ and 333-335.

2 Penna. Expt. Sta., Bul. 71, p. 7.

${ }^{3}$ Landw. Vers. Stat., 53 (I904), 440-449. 
show much smaller differences between the observed and computed results, the fermentation losses in particular being notably less with swine than with cattle or sheep (745).

Table r89. - Comparison of Metabolizable Energy per Pound

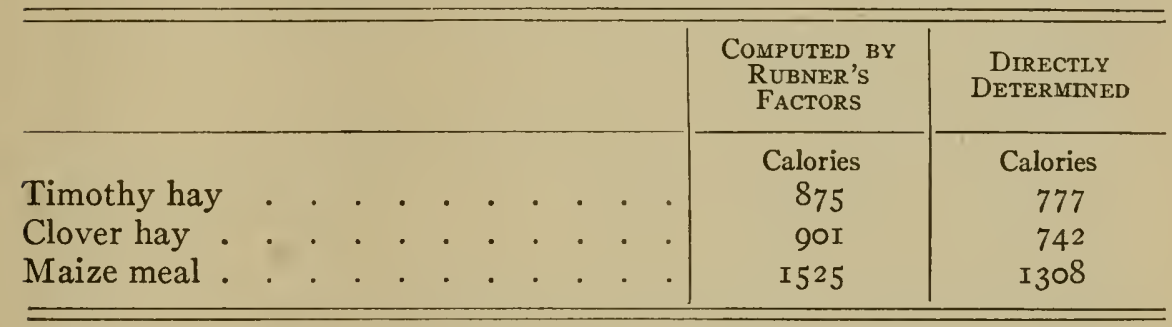

Kellner has attempted to secure factors for cattle similar to those of Rubner for men and carnivora by means of experiments in which approximately pure nutrients (starch, sugar, oil, gluten) were added to a basal ration. In the case of starch, for example, the increase in the amount of nitrogen-free extract digested was compared with the increase in the total metabolizable energy of the ration, the losses of energy in feces, urine and methane being determined with the aid of a respiration apparatus by the method of indirect calorimetry (329). The results are corrected for the effects of the starch upon the digestibility of the several nutrients of the basal ration and upon the losses from the latter in urine and methane, i.e., the real metabolizable energy is computed. A few similar determinations on other species have also been reported.

In an earlier publication ${ }^{1}$ the writer has discussed in considerable detail the recorded experiments regarding the metabolizable energy of the nutrients digested by farm animals with the results summarized in the following table. To the extent to which satisfactory factors can be selected, this table may be used to compute the metabolizable energy of feeding stuffs or rations whose digestibility is known, but it should be noted that the results will include no allowance for the secondary effects of the feed on the digestive processes and will probably be higher than the "apparent" metabolizable energy obtained by direct experiment. 
Table igo. - Metabolizable Energy of Digestible Nutrients per Gram

\begin{tabular}{|c|c|c|c|}
\hline & Cattle & Horse & SwINE \\
\hline 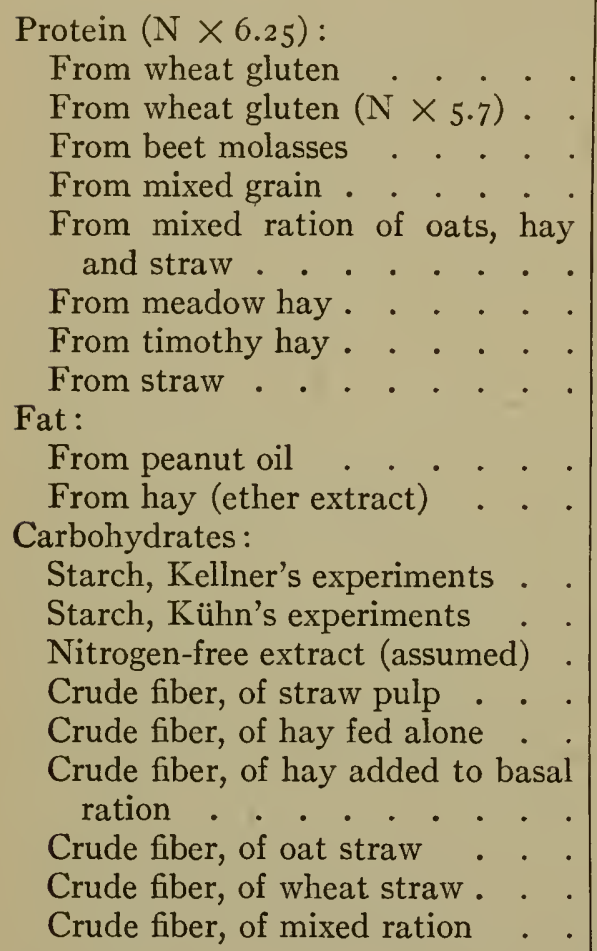 & $\begin{array}{l}\text { Cals. } \\
4.894 \\
4.95^{8} \\
3.984 \\
- \\
- \\
1.272 \\
3.057(?) \\
(?) \\
8.821 \\
8.322 \\
3.763 \\
3.648 \\
- \\
3.606 \\
3.311 \\
3.606 \\
3.437 \\
3.001 \\
-\end{array}$ & $\begin{array}{l}\text { Cals. } \\
- \\
- \\
- \\
3.228 \\
- \\
- \\
- \\
- \\
- \\
4.185 \\
- \\
- \\
- \\
3.523\end{array}$ & $\begin{array}{c}\text { Cals. } \\
- \\
- \\
\overline{4} .083 \\
- \\
- \\
- \\
- \\
- \\
- \\
- \\
-\end{array}$ \\
\hline
\end{tabular}

753. Computation of metabolizable energy from digestible organic matter. - A more simple and direct method of computation may, however, be employed, based on the total digestible organic matter of the ration. As already pointed out, the differences shown in Table I 88 between the percentages of the gross energy of different feeding stuffs which are metabolizable are due chiefly to differences in the proportion of the chemical energy carried off in the feces, while the losses in urine and methane are far more uniform. Accordingly, the metabolizable energy per unit of digestible organic matter necessarily exhibits much smaller variations than that per unit of dry matter, and in fact shows a striking degree of uniformity. Selecting those averages which appear most trustworthy, the results may be summarized as follows:- 
Table igi. - Metabolizable Energy per Kilogram Digestible ORganic Matter

\begin{tabular}{|c|c|c|c|c|c|c|}
\hline & & & $\begin{array}{c}\text { NuMBER } \\
\text { OF SINGLLE } \\
\text { TRIALS }\end{array}$ & MaXimum & Minimum & MEAN \\
\hline & Roughage & & & Therms & Therms & Therms \\
\hline Cattle & . . . . . & . & 73 & $3 \cdot 74$ & $3.3 \mathrm{I}$ & 3.53 \\
\hline Sheep & . . . . . . & . & 33 & 3.77 & 3.29 & 3.56 \\
\hline Horse & Concentrates & $\cdot \cdot$ & I3 & 3.92 & $3 \cdot 35$ & $3.7 \mathrm{I}$ \\
\hline Cattle & . . . . . & . & $3 I$ & 4.85 & 3.79 & 4.04 \\
\hline Sheep & . . . & . . & 25 & 4.08 & $3.4 \mathrm{r}$ & 3.85 \\
\hline Horse & . . . . & . . & 8 & 4.76 & 4.49 & 4.62 \\
\hline Swine ${ }^{1}$ & . . & . & 36 & 5.63 & 3.46 & 4.40 \\
\hline
\end{tabular}

A similar degree of uniformity appears when the results on mixed rations are compared, as the following summary shows :-

$$
\begin{gathered}
\text { Table 192. - Metabolizable Energy per Kilogram Digestible } \\
\text { Organic Matter in Mixed Rations }
\end{gathered}
$$

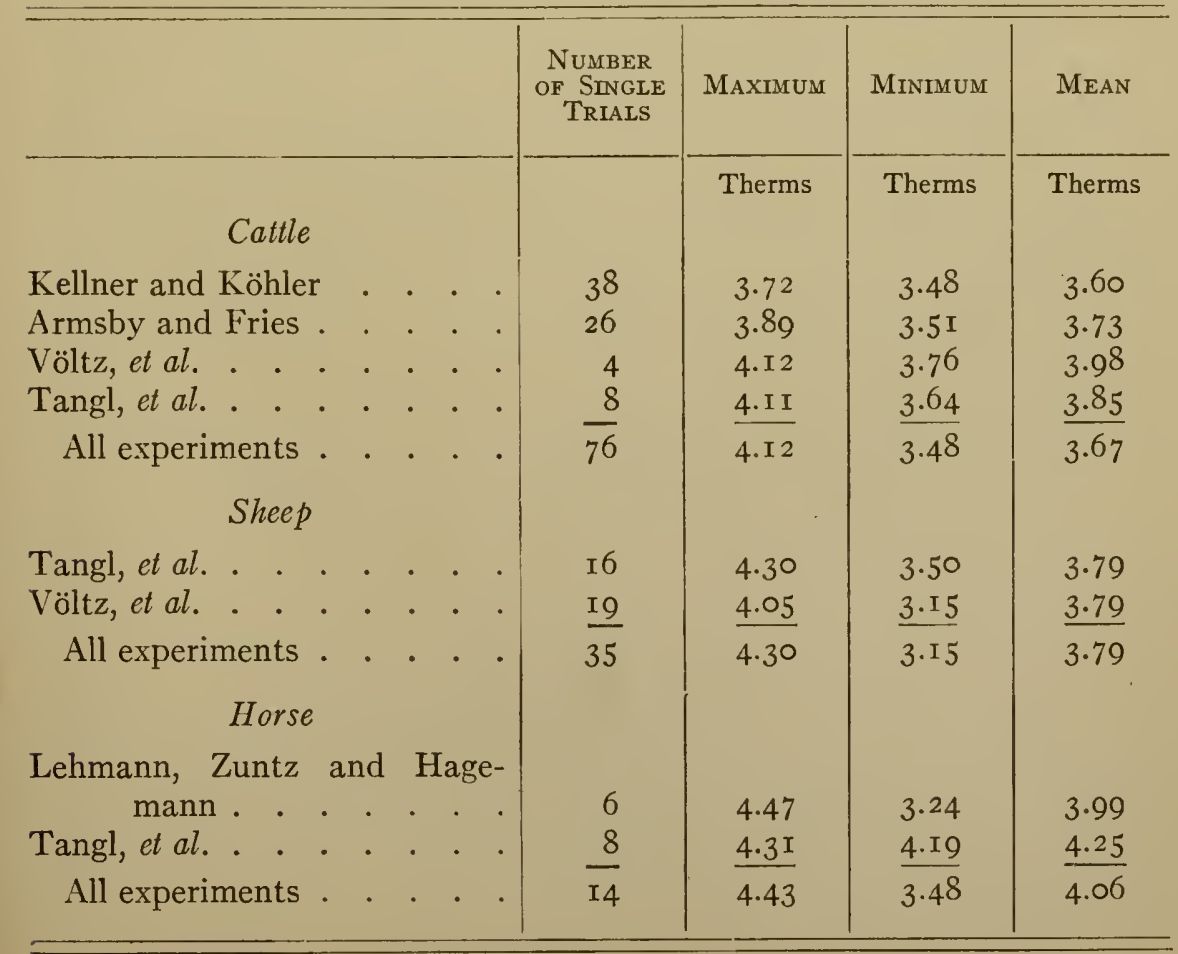

1 Excluding feeds containing much oil. 
Feeding stuffs, rich in protein and fat, especially the latter, naturally give higher values, as is illustrated by the following results, likewise taken from Table 188.

Table 193. - Metabolizable Energy per Kilogram Digestible Organic Matter

Palmnut meal

Cattle. . . . . . . . . 4.85 Therms

Sheep . . . . . . . . 3.98 Therms

Wheat gluten

Cattle . . . . . . . . . 4.79 Therms

Flesh meal

Swine . . . . . . . . 5.63 Therms

Taking the pound as the unit for reasons of practical convenience, it is believed that for the approximate computation of the metabolizable energy of ordinary feeding stuffs or rations whose content of digestible organic matter is known or can be estimated, the following factors may be used, at least for ruminants, without serious error:-

Table 194. - Metabolizable Energy per Pound Digestible ORGanic Matter

\begin{tabular}{l|c|c|c}
\hline \hline & Ruminants & SwINe & Horses \\
\hline Roughage . . . . . . . . . . . & Therms & Therms & Therms \\
Mixed rations - roughage and concentrates & - & - & I.683 \\
Concentrates & & & \\
Grains and similar material & & & \\
With less than 5 per cent digestible fat . & $\left.\begin{array}{l}\text { I.769 } \\
\text { With more than 5 per cent digestible fat . } \\
\text { I.8I4 }\end{array}\right\}$ & I.935 & 2.096 \\
Oil meals and materials high in protein & I.996-2.I77 & 2.390 & - \\
\hline
\end{tabular}

\section{Losses of energy in heat production}

It was stated in a previous paragraph (741) that the gross energy of a feeding stuff is subject to two classes of losses, viz., losses of untransformed chemical energy in the excreta and losses through conversion into heat. The losses of chemical energy have been discussed in the preceding paragraphs. The second class of losses has now to be considered. 
754. Influence of feed consumption on metabolism. - As is evident from $\S$ I of Chapter VIII (365), the fact that the consumption of feed tends to increase the heat production of an animal has become a commonplace of physiology. The magnitude of the effect varies within rather wide limits according to the species of animal and the chemical and physical properties of the feed, while there is still more or less difference of opinion as to its causes. Zuntz and his associates have called it "work of digestion" and have attributed it largely to increased muscular and glandular activity of the digestive and excretory organs. Numerous investigations in this field have been made on carnivora or on man, in which the increase of the metabolism is not usually very large except when much protein is consumed. The more recent experiments on these species appear to have shown that the mechanical work of the digestive organs is but a small factor and that the term "work of digestion " is not a fortunate one. With herbivora and especially with ruminants, on the other hand, the total effect on the heat production is quantitatively much more marked, and the mechanical factor is of greater significance.

755. Results on cattle. - As illustrated in an earlier chapter $(364,449)$ the effect of feed consumption upon the metabolism of ruminants may be determined by comparing two periods in which different amounts of the same feeding stuff or ration are consumed, the increment of heat production on the heavier ration being compared with the additional amount of feed consumed. The experiments thus far reported have been almost exclusively upon cattle, the principal ones being the pioneer investigations of Kellner and Köhler ${ }^{1}$ and the later ones of Armsby and Fries. ${ }^{2}$

Most of Kellner and Köhler's experiments were made on supermaintenance rations. The heat production was not measured directly, but computed from the balance of carbon and nitrogen in the manner indicated in Chapter VI (329), i.e., by indirect calorimetry. Only a few of their results have as yet been published in full, but from data regarding a few of the other experiments contained in Kellner's book, the increments of heat production may be computed.

${ }^{1}$ Landw. Vers. Stat., 47 (I896), 275; 50 (I898), 245; 53 (I900), I-474. Die Ernährung der landwirtschaftlichen Nutztiere, 6th Ed., Berlin, I9I2.

${ }^{2}$ Jour. Agr. Research, 3 (I9I5), 435. 
Armsby and Fries' experiments included both submaintenance and supermaintenance rations and the heat was measured directly with a respiration calorimeter.

As elsewhere summarized by the writer, the average results derived from the two series of experiments are as follows:-

Table i95. - Increment of Heat Production by Cattle

\begin{tabular}{|c|c|c|c|c|c|c|c|c|c|c|c|c|c|}
\hline & & & & & & & & & & & & $\begin{array}{l}\text { EXPERI- } \\
\text { MENTERS }{ }^{1}\end{array}$ & $\begin{array}{l}\text { HEAT INCRE- } \\
\text { MENT PER IOO } \\
\text { LB. DRY } \\
\text { MATTER EATEN }\end{array}$ \\
\hline \multicolumn{12}{|c|}{ Roughage } & & Therms \\
\hline Timothy hay . & . & . & . & . & & . & • & . & . & - & . & $A \& F$ & 35.47 \\
\hline Red clover hay & . & . & . & . & . & . & . & . & . & . & . & $A \& F$ & 44.13 \\
\hline Red clover hay & . & . & . & . & . & . & . & . & - & . & . & $\mathrm{K} \& \mathrm{~K}$ & 42.27 \\
\hline Mixed hay & . & . & . & . & . & . & . & . & . & . & .. & A \& F & 44.45 \\
\hline Alfalfa hay . & - & . & . & . & . & . & . & . & . & . & . . & A \& F & 53.03 \\
\hline "Grass hay" . & . & . & . & . & . & . & . & . & . & . & & $\mathrm{K} \& \mathrm{~K}$ & 47.40 \\
\hline Meadow hay . & $\cdot$ & - & - & - & - & - & . & $\cdot$ & . & . & . & $\mathrm{K} \& \mathrm{~K}$ & 56.88 \\
\hline Rowen . . & . & . & . & . & . & . & . & . & . & . & . & $\mathrm{K} \& \mathrm{~K}$ & 43.46 \\
\hline Maize stover & . & . & . & . & . & . & . & . & . & . & . & A \& F & 48.31 \\
\hline Barley straw & . & . & . & . & . & . & . & . & . & . & : & $\mathrm{K} \& \mathrm{~K}$ & 39.78 \\
\hline Oat straw . & . & . & . & $\cdot$ & . & . & . & . & . & $\therefore$ & . & $\mathrm{K} \& \mathrm{~K}$ & 46.00 \\
\hline Wheat straw & - & - & . & $\cdot$ & . & . & . & . & . & $\cdot$ & . & $\mathrm{K} \& \mathrm{~K}$ & 51.62 \\
\hline Straw pulp & • & $\dot{C} 0$ & ice & itr & & . & . & . & . & · & . & $\mathrm{K} \& \mathrm{~K}$ & 52.62 \\
\hline Maize meal & - & . & . & . & . & . & . & . & . & . & . & $A \& F$ & $5^{8.33}$ \\
\hline Hominy feed . & . & . & . & . & . & . & . & $\cdot$ & . & - & . & A \& F & 61.92 \\
\hline Wheat bran & $\therefore$ & . & . & . & . & . & . & . & . & . & . & $\mathrm{A} \& \mathrm{~F}$ & $53 \cdot 39$ \\
\hline Grain mixture I & No. & $I^{2}$ & & . & . & . & . & . & . & . & & A \& F & 60.19 \\
\hline Grain mixture I & No. & $2^{3}$ & & $:$ & . & . & . & . & . & . & .. & A \& F & 51.76 \\
\hline Cottonseed mea & & - & . & . & . & . & . & . & . & . & . . & $\mathrm{K} \& \mathrm{~K}$ & $44 \cdot 36$ \\
\hline Linseed meal . & . & . & . & $\cdot$ & . & . & . & . & - & . & .. & $\mathrm{K} \& \mathrm{~K}$ & 54.79 \\
\hline Palmnut meal & · & • & • & - & . & . & . & - & - & - & . & $\mathrm{K} \& \mathrm{~K}$ & 45.68 \\
\hline Peanut meal & . & . & . & . & . & . & . & . & . & . & . & $\mathrm{K} \& \mathrm{~K}$ & 52.57 \\
\hline Beet molasses & . & . & - & . & . & . & . & . & . & - & . & $\mathrm{K} \& \mathrm{~K}$ & 44.82 \\
\hline Starch . . & . & . & . & . & . & . & . & . & . & . & & $\mathrm{K} \& \mathrm{~K}$ & $5^{6.6} \mathrm{I}$ \\
\hline Peanut oil. & . & - & $\cdot$ & . & . & . & . & . & . & . & . & $\mathrm{K} \& \mathrm{~K}$ & 78.34 \\
\hline Wheat gluten & . & . & . & . & . & . & . & . & . & . & .. & $\mathrm{K} \& \mathrm{~K}$ & 95.08 \\
\hline
\end{tabular}

${ }^{1}$ In this and following tables, A \& $\mathrm{F}$ signifies Armsby and Fries and $\mathrm{K} \& \mathrm{~K}$ Kellner and Köhler.

${ }^{2}$ Wheat bran, I4.28 per cent; corn meal, 42.86 per cent; old process linseed meal, 42.86 per cent.

${ }^{3}$ Corn meal, 60 per cent; crushed oats, 30 per cent; old process linseed meal, ro per cent. 
756. Results on sheep. - In a series of respiration experiments upon two sheep by Kern and Wattenberg, reported by Henneberg and Pfeiffer, ${ }^{1}$ varying amounts of nearly pure protein in the form of conglutin or of flesh meal were added to a basal ration of hay and barley meal. The writer ${ }^{2}$ has computed from the recorded results of these experiments the metabolizable energy of the additions to the basal ration and the energy of the resulting gain. The difference between the two shows the amount of energy lost as heat.

Table ig6. - Increment of Heat Production by Sheep

\begin{tabular}{|c|c|c|c|c|c|c|c|c|c|}
\hline & & & & \multirow[b]{2}{*}{ PERIOD } & \multirow{2}{*}{$\begin{array}{c}\text { DRY } \\
\text { MATTER } \\
\text { OF } \\
\text { ADDED } \\
\text { FEED }\end{array}$} & \multirow{2}{*}{$\begin{array}{c}\text { METABO- } \\
\text { LIZABLE } \\
\text { ENERGY } \\
\text { OF } \\
\text { ADDED } \\
\text { FEED }\end{array}$} & \multirow[b]{2}{*}{$\begin{array}{c}\text { ENERGY } \\
\text { OF RE- } \\
\text { SULTING } \\
\text { GAIN }\end{array}$} & \multicolumn{2}{|c|}{ HEAT INCREMENT } \\
\hline & & & & & & & & Total & $\begin{array}{c}\text { Per roo } \\
\text { Lb. Dry } \\
\text { Matter } \\
\text { Eaten }\end{array}$ \\
\hline \multirow{4}{*}{ Conglutin } & \multirow{4}{*}{\multicolumn{2}{|c|}{$\cdot$}} & \multirow{4}{*}{. } & & Grams & Cals. & Cals. & Cals. & Therms \\
\hline & & & & II & I 17.6 & 588.4 & 517.8 & 70.6 & 27.24 \\
\hline & & & & III & 234.8 & I 100.3 & 741.8 & $35^{8.5}$ & 69.26 \\
\hline & & & & IV & 350.8 & I639.2 & I 106.9 & 532.4 & 68.86 \\
\hline \multirow{2}{*}{ Flesh-meal . } & \multirow{2}{*}{\multicolumn{3}{|c|}{$\cdot$}} & V & 258.0 & I I31.7 & 672.5 & 459.2 & $69 \cdot 31$ \\
\hline & & & & VI & 63.5 & 454.9 & $315 \cdot 7$ & I39.2 & 49.71 \\
\hline
\end{tabular}

The results are notably lower than those obtained by Kellner for wheat gluten fed to cattle, although in the three middle periods they are higher than those found with that species for other concentrates, but there are several points of uncertainty in the experimental results and the method of computation is an approximate one. On the whole, pending further investigation, it appears probable that the results obtained with cattle may, without very serious error, be regarded as applicable to other species of ruminants.

757. Results on swine. - The data regarding the increment of heat production consequent on the consumption of feed by swine, although more abundant than those for sheep, are still rather meager. Respiration experiments made by Meissl, Strohmer and Lorenz $z^{3}$ in a study of the sources of animal fat,

1 Jour. Landw., 38 ( 1890 ), $215 . \quad 2$ Principles of Animal Nutrition, pp. 463-465.

3 Ztschr. Biol., 22 ( 1886 ), 63. 
and by Kornauth and Arche ${ }^{1}$ upon the nutritive value of cockle may be made the basis of estimates of the energy expenditure due to feed consumption, while the later investigations of Von der Heide and Klein, ${ }^{2}$ of Fingerling, Köhler and Reinhardt ${ }^{3}$ and of Wellmann, ${ }^{4}$ were directed more specifically toward a study of the energy relations.

Neither of the two investigations first mentioned included any energy determinations, but by substantially the same method as that applied in the previous paragraph to experiments with sheep, assuming an average maintenance requirement, the heat increment per unit of feed may be computed.

Von der Heide and Klein, in Zuntz's laboratory, have measured with the aid of a respiration apparatus of the Regnault-Reiset type (298) the metabolism of three swine on a basal ration slightly more than sufficient for maintenance and consisting of barley meal, dried potatoes and dried yeast, and also the effect of the addition to this basal ration of dried potatoes and of palm oil. The energy of the feed and excreta was determined. Estimating the fasting katabolism of the three animals from the body surface, the results may be computed as in the two previous experiments. A computation from the total heat increments above the basal ration (i.e., without correction for the differences in live weight) gives somewhat higher results.

Fingerling, Köhler and Reinhardt, in experiments on two growing swine about eight months old, added approximately pure nutrients (starch, peanut oil, straw pulp, wheat gluten, flesh meal and sugar) to a basal ration consisting of ground barley with a little flesh meal. The animals gained steadily in weight. By a comparison of the first and last periods, on the basal ration, the authors compute the average fasting katabolism per square meter of body surface to have been ro44.67 Cals., which agrees fairly well with the average computed in Chapter VIII (377), viz., ro89 Cals. per square meter. Taking the average of the first and last periods as the basal ration, in order to eliminate the effects of the increase in live weight, and subtracting it from the results of the intermediate periods, the fasting katabolism being estimated in proportion to the surface of the animal, the heat increment due to the added nutrients may be computed in the manner illustrated for starch in the following table, while by correcting the results obtained in the first and last periods for the small amount of flesh meal included in the ration, the energy expenditure per gram of dry matter in the barley may likewise be estimated.

${ }^{1}$ Landw. Vers. Stat., 40 (I892), I 77.

${ }^{3}$ Landw. Vers. Stat., 84 (I914), I49.
? Biochem. Ztschr., 55 (I913), I95.

${ }^{4}$ Landw. Jahrb., 46 (I9I4), 499. 
Table 197. - Example of Computation of Heat Increment in SWINE

\begin{tabular}{|c|c|c|c|c|c|c|c|}
\hline \multirow[b]{2}{*}{ Starch } & \multirow{2}{*}{$\begin{array}{c}\text { TOTAL } \\
\text { DRY } \\
\text { MATTER } \\
\text { CON- } \\
\text { SUMED }\end{array}$} & \multirow{2}{*}{$\begin{array}{l}\text { METAB- } \\
\text { OLIZA- } \\
\text { BLE } \\
\text { ENERGY }\end{array}$} & \multirow[b]{2}{*}{$\begin{array}{l}\text { GAIN BY } \\
\text { ANIMAL }\end{array}$} & \multirow{2}{*}{$\begin{array}{l}\text { COM- } \\
\text { PUTED } \\
\text { HEAT } \\
\text { PRODUC- } \\
\text { TION }\end{array}$} & \multirow{2}{*}{$\begin{array}{c}\text { ESTI- } \\
\text { MATED } \\
\text { FASTING } \\
\text { KATAB- } \\
\text { OLISM }\end{array}$} & \multicolumn{2}{|c|}{$\underset{\text { INCREMTENT }}{\text { HEAT }}$} \\
\hline & & & & & & Total & $\begin{array}{c}\text { Per } \\
\text { Gram } \\
\text { Dry } \\
\text { Matter }\end{array}$ \\
\hline 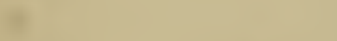 & Grams & Cals. & Cals. & Cals. & Cals. & Cals. & Cals. \\
\hline Period 2. & I 582.2 & 5990.32 & 2508.19 & 3482.13 & $2200.7 \mathrm{I}$ & I $28 \mathrm{I} .42$ & $0.8 \mathrm{Io}$ \\
\hline Average of periods I and 6 & I 180.3 & 4368.40 & 1041.03 & 3327.37 & 2328.68 & 998.69 & 0.846 \\
\hline Starch by difference & 401.9 & 1621.92 & I 467.16 & $\mathbf{I} 54.76$ & -I 27.97 & 282.73 & 0.704 \\
\hline
\end{tabular}

Wellmann, in the course of experiments on the rearing of calves and pigs on skim milk and modified skim milk, determined by means of comparative slaughter tests the gain of flesh and fat by two pigs during twenty-three and thirty-four days respectively, and also collected the feces and urine quantitatively during the entire period of feeding. The energy of the feed and excreta was determined directly. Assuming a basal katabolism of I 100 Cals. per square meter of surface (377), the heat increment due to the feed may be computed as in previous cases.

The results of the foregoing investigations are summarized in Table I98. One of Wellmann's results, obtained with a very restless animal, may be regarded as probably too high and has been excluded. In Von der Heide and Klein's experiment on palm oil the quantity of fat consumed was relatively large as compared with that in Fingerling's experiment on peanut oil, although the total ration was not excessive.

Despite some irregularities, a comparison of these results with those for cattle (755) shows clearly that with swine the energy expenditure consequent on feed consumption is decidedly less than with ruminants. Fingerling's results with approximately pure nutrients are especially interesting in this respect. As regards the more soluble carbohydrates (starch and sugar) one can hardly err in ascribing the difference largely to the fact that in the comparatively simple digestive organs of swine fermentations occur only to a limited extent, while in cattle they have been estimated to account for from 9 to 16 per 
cent of the total increment in heat production. Straw pulp, on the contrary, caused fully as great an increase in the heat production of swine as in that of cattle. Fingerling explains this upon the supposition that the straw pulp was fermented rather than digested. He failed, however, to find any corresponding excretion of methane (745), and Von der Heide, Steuber and Zuntz ${ }^{1}$ have observed only a relatively small evolution of combustible gases from this material in case of the horse. The differences as regards oil and protein are not readily explicable since, according to Kellner, they are not subject to the methane fermentation.

Table 198. - Increment of Heat Production by Swine

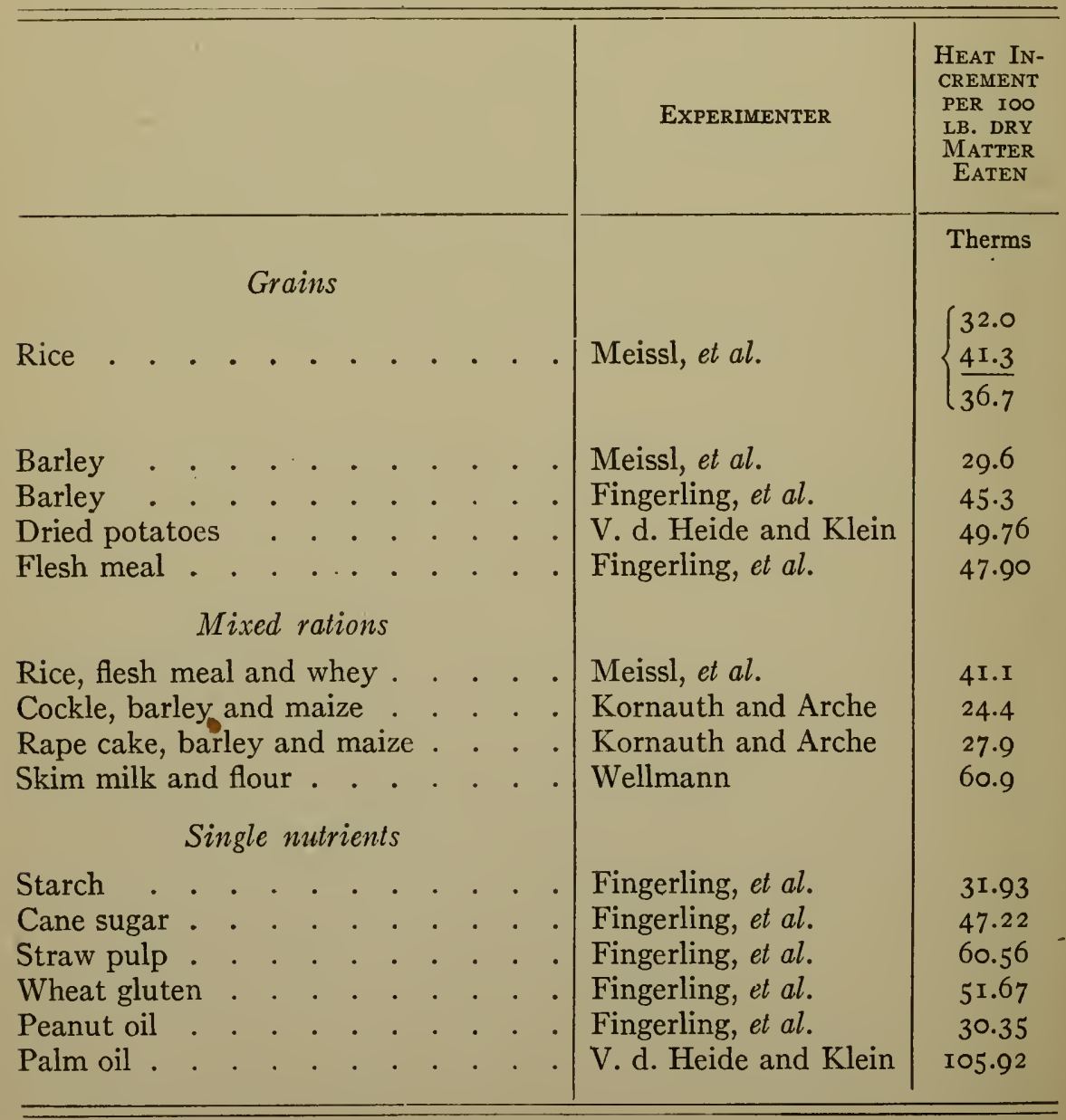

${ }^{1}$ Biochem. Ztschr., 74 (I9I6), I6r. 
758. Experiments on the horse. - No experiments on this animal have been reported in which the energy expenditure due to the consumption of a single feeding stuff has been determined. Practically the only data available are those derived from the extensive investigations of Zuntz and Hagemann, the results of which regarding the fasting katabolism have been considered in Chapter VIII (385). On the basis of their experiments they compute the energy expenditure and the net energy value from the composition and digestibility of the ration by a method identical in principle with that employed in the experiments on cattle already described. The experiments were conducted so differently, however, as to constitute practically a distinct method and they may be more conveniently considered in connection with the computation of net energy values discussed in subsequent paragraphs (775-778).

759. Results on carnivora. - Mention was made in Chapter VIII $(365,366)$ of the fact that in carnivora, as well as in herbivora and omnivora, the consumption of feed stimulates the heat production, the increase having been called by Rubner the specific dynamic action. It is evident that experiments like those of Rubner and of Lusk were virtually determinations of net energy values for these species. While having no direct bearing on the question of the nutritive values of feeding stuffs for farm animals, these data have been extensively quoted in related physiological writings and it seems desirable to include them here. Rubner's later experiments were made at about $33^{\circ} \mathrm{C}$., or considerably above the critical temperature for the dog, a fact which is of importance in the interpretation of the results (395-397).

A balance experiment with a respiration calorimeter in which nearly enough fat was fed to supply the requirement for energy gave

Table i99. - Increment of Heat Production by Dog on Fat Diet

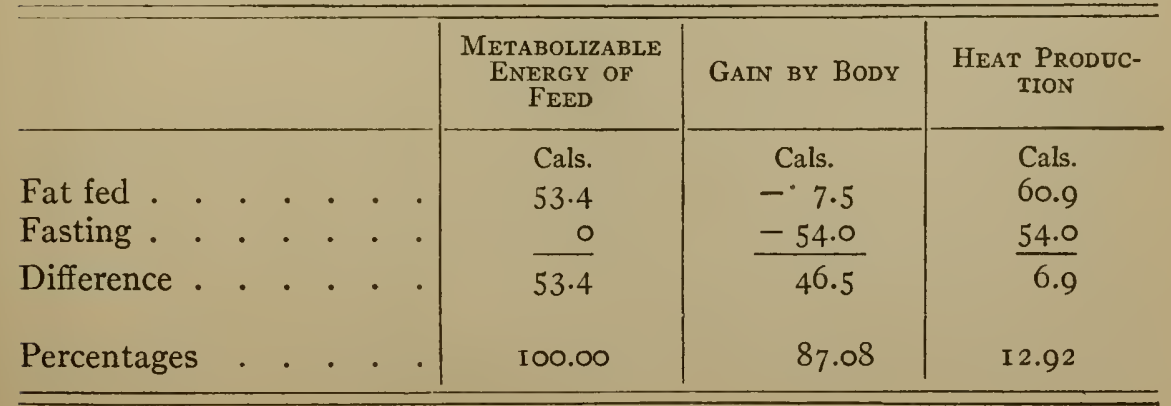


per kilogram live weight the results shown in Table 199 , which are stated in a form somewhat different from that used by Rubner but which in substance are identical with his.

These figures appear somewhat remarkable in view of the fact that the comparison is virtually with body fat. Literally interpreted, it means that the energy of feed fat is only 87 per cent as valuable for maintenance as the energy of mobilized body fat plus a little protein. If this be true, it implies a larger expenditure of energy in the digestion of fat or a greater stimulating effect of the resorbed fat upon cell activity than now seems probable, since the katabolism of resorbed feed fat can hardly differ greatly from that of body fat. Rubner's figure is the result of a single experiment and unfortunately it enters into the computation of all the other results. It is a matter of much interest, therefore, that Lusk ${ }^{1}$ has found a much lower heat increment for fat. In two calorimetric experiments in which an emulsion of olive oil was given to a dog he found the additional heat elimination to be 0.92 per cent and $\mathrm{r} .49$ per cent of the energy of the oil, so that on the average 98.8 per cent of energy of the fat was available for maintenance, a much higher figure than Rubner's.

Both Rubner and Lusk find the most marked effect to be produced by protein. In two other experiments by Rubner an amount of lean meat nearly sufficient to maintain the dog was fed. The meat contained a small amount of fat, the average metabolizable energy of the feed per kilogram live weight being as follows:-

In protein 56.70 Cals.

In fat

$$
\frac{4.95}{61.65} \text { Cals. }
$$

Using the data afforded by the experiment on fat, the heat increment due to the protein may be computed as follows:-

Table 200. - Increment of Heat Production by Dog on Meat Diet

\begin{tabular}{|c|c|c|c|}
\hline & $\begin{array}{c}\text { METABOLIZABLEE } \\
\text { ENERGY OF } \\
\text { FEED }\end{array}$ & GaIN By Body & $\begin{array}{l}\text { Heat Produc- } \\
\text { tION }\end{array}$ \\
\hline 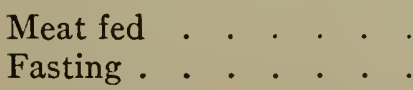 & $\begin{array}{l}\text { 6r.65 Cals. } \\
0\end{array}$ & $\begin{array}{l}-\quad 8.90 \text { Cals. } \\
-51.50 \text { Cals. }\end{array}$ & $\begin{array}{l}\text { 70.55 Cals. } \\
5 \text { I.50 Cals. }\end{array}$ \\
\hline $\begin{array}{l}\text { Difference } \\
\text { Difference due to fat }\end{array}$ & $\begin{array}{r}61.65 \text { Cals. } \\
4.95 \text { Cals. }\end{array}$ & $\begin{array}{r}42.60 \text { Cals. } \\
4.31 \text { Cals. }\end{array}$ & $\begin{array}{l}\text { I9.05 Cals. } \\
0.64 \text { Cals. }\end{array}$ \\
\hline $\begin{array}{l}\text { Difference due to protein } \\
\text { Percentages }\end{array}$ & $\begin{array}{l}56.70 \text { Cals. } \\
\text { I00.00 }\end{array}$ & $\begin{array}{l}38.29 \text { Cals. } \\
67.53\end{array}$ & $\begin{array}{l}\overline{\text { I } 8.4 \mathrm{I}} \text { Cals. } \\
3^{2.47}\end{array}$ \\
\hline
\end{tabular}

1 Jour. Biol. Chem., 13 (I912), 38. 
Williams, Riche and Lusk report results agreeing substantially with those of Rubner when computed in the same way, although they regard his method of computation as erroneous. Rubner's and Lusk's averages are contained in the following table. It should be clearly understood that these figures are not applicable to the "digestible nutrients" of the feed of herbivora.

Table 201. - Percentage of Metabolizable Energy Available Average Results for Dogs

\begin{tabular}{|c|c|c|c|c|c|c|c|}
\hline & \multicolumn{3}{|c|}{$\theta_{0}$} & \multicolumn{2}{|c|}{ RUBNER } & \multicolumn{2}{|c|}{ LUSK } \\
\hline & & & & $\begin{array}{c}\text { Increment of } \\
\text { Heat Pro- } \\
\text { duction } \\
\%\end{array}$ & $\begin{array}{c}\text { Available for } \\
\text { Maintenance } \\
\%\end{array}$ & $\begin{array}{c}\text { Increment of } \\
\text { Heat Pro- } \\
\text { duction } \\
\%\end{array}$ & $\begin{array}{c}\text { Available for } \\
\text { Maintenance } \\
\%\end{array}$ \\
\hline Body protein & 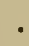 & & - & 31.9 & 68.I & - & - \\
\hline Meat protein & & & . & 30.9 & 69.1 & 36.0 & 64.0 \\
\hline Gelatin . . & . & & . & 28.0 & 72.0 & - & - \\
\hline Fat . . & . & & 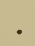 & I 2.7 & $87 \cdot 3$ & I. 2 & 98.9 \\
\hline Cane sugar . & . & . & $\cdot$ & 5.8 & 94.2 & - & - \\
\hline Dextrose & - & . & . & - & - & $4 \cdot 9$ & $95 . \mathrm{I}$ \\
\hline
\end{tabular}

\section{Net energy values}

In the previous paragraphs there have been considered the losses of energy in the excreta and those due to the increased heat production which results from the consumption of feed. As pointed out in the introductory paragraphs of this section, that portion of the gross energy of a feeding stuff which remains after deducting these two classes of losses constitutes its net energy value, or its production value as regards energy. Stated in a slightly different way, the net energy value is equal to the metabolizable energy minus the increment of heat production. It differs from the relative value, based on the digestible nutrients or the metabolizable energy, in taking account of all the losses of energy to which the feed is subject.

760. Net energy values for cattle. - It is apparent from the foregoing discussions that the data regarding losses of energy and net energy values are much more abundant for cattle than for any other species of farm animals. Combining the 
data of Table I95 (755) regarding the losses due to increased heat production with those regarding the losses of chemical energy in the excreta recorded in another form in Table 188 (749) gives the results contained in the following tables, ${ }^{1}$ the

Table 202. - Net Energy Values of Feeding Stuffs for Ruminants Per Hundred Pounds of Dry Matter

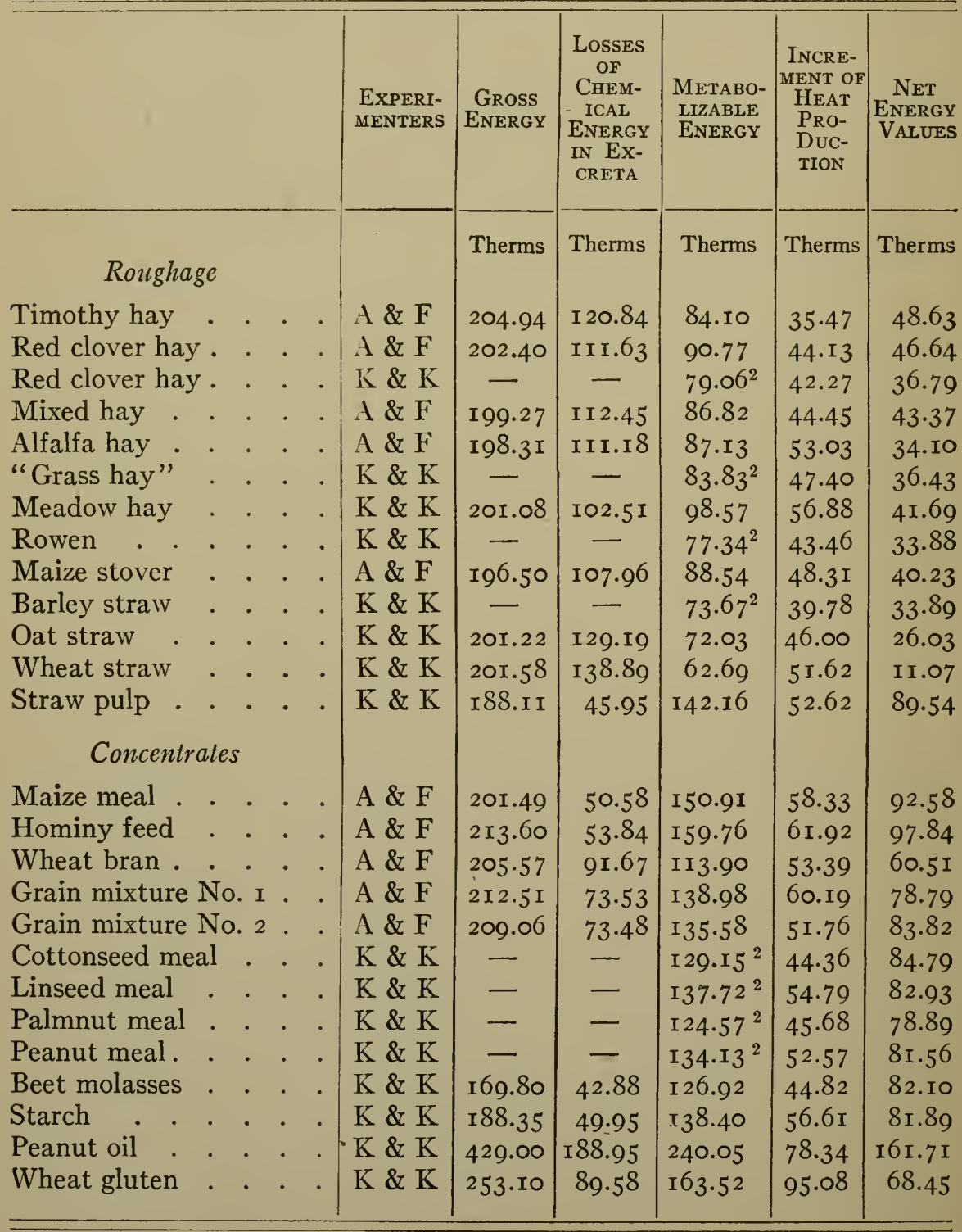


first showing the losses of energy and the net energy values per Ioo pounds of dry matter and the second the percentage distribution of the gross energy of the feeding stuffs between the various losses and the net energy values. As already indicated $(745,749,756)$, it appears probable that these values may be used also for other classes of ruminants without serious error.

Table 203. - Distribution of Energy of Feed in Ruminants

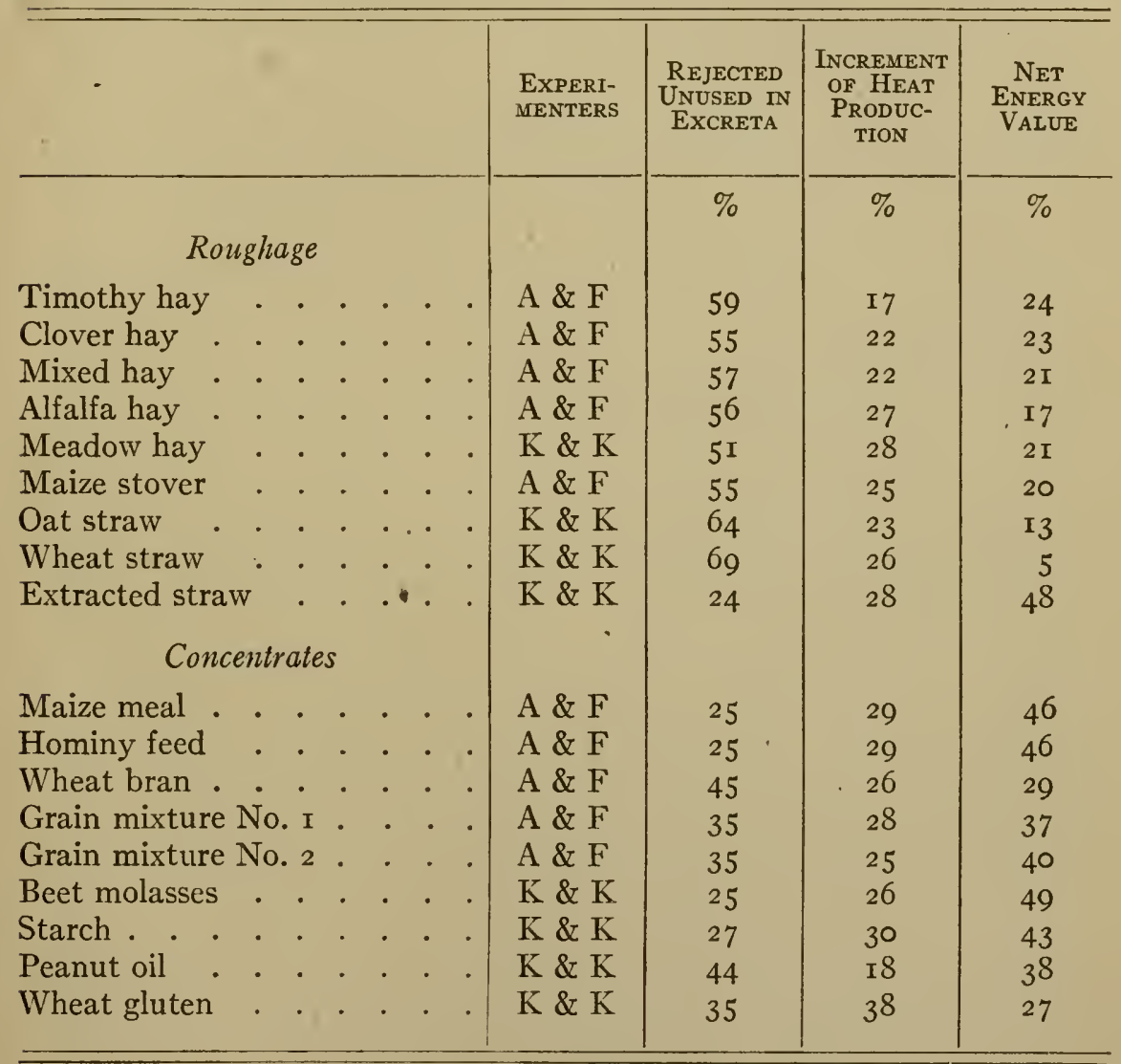

761. Net energy values for swine. - Combining in the same form the results of the determinations of the heat increment caused by the consumption of feed by swine (757) which are recorded in Table I98 and such data regarding the losses of energy in feces, urine and methane as are contained in Table 188 (749) yields the net energy values shown in Table 204 :- 
Table 204. - Net Energy Values of Feeding Stuffs for Swine Per .Ioo Pounds Dry Matter

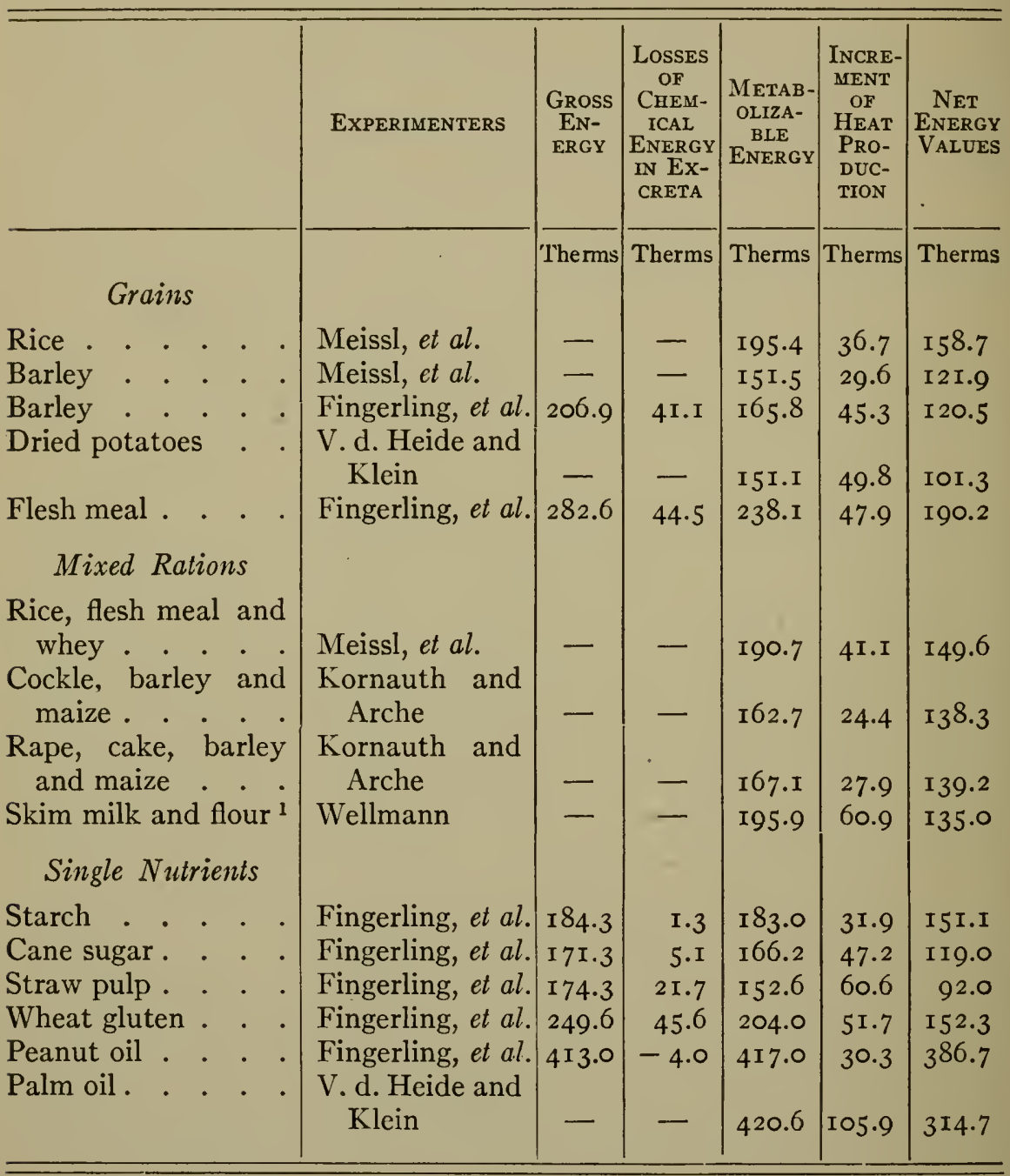

762. Comparison of roughage and concentrates. - The average results recorded in the foregoing tables for the total increase in metabolism resulting from the consumption of a unit of dry matter - i.e., for the so-called "work of digestion" in the widest sense - are scarcely in accord with common conceptions. Unconsciously misled by an unfortunate terminology, we have been accustomed to think of the more coarse

1 Omitting one very restless animal. 
and woody feeding stuffs, like hay, straw, stover, etc., as requiring a greater expenditure of energy in their digestion and assimilation than the more concentrated and highly digestible grains, for example. It may be somewhat surprising, therefore, to note the relatively small differences in this respect between different classes of feeding stuffs, as well as the fact that, in case of cattle, the average is distinctly higher for the concentrates than for the roughages, viz., 58.75 Therms per Ioo pounds dry matter as compared with 46.54 . While the mechanical work required for the digestion of concentrates is presumably less than that necessary in case of roughages on account of the greater expenditure for the mastication of the latter, this difference appears to be more than compensated for by other factors, so that on the whole fully as great an increment of the heat production results from the consumption of the concentrates. As a class, concentrates are superior to roughage, not because their consumption involves a less expenditure of energy but because they contain more metabolizable energy, so that more remains available for body use after the expenditure has been met.

763. Differences between feeding stuffs. - But while the foregoing results do not show the existence of the great contrast between the two chief classes of feeding stuffs in their effects on the energy expenditure of the body which seems at times to have been assumed, they nevertheless reveal distinct differences even between feeding stuffs of the same class. Thus, among the hays a distinct increase is found from timothy hay with an average heat increment of 35.47 Therms through mixed hay and clover hay up to alfalfa, with an average of 53.03 Therms. Apparently the legumes cause a distinctly greater increase in the metabolism than do the grasses. The chief difference between the two seems to lie either in their effect upon the work of peristalsis or in the degree to which they stimulate the general metabolism. One can hardly doubt that the latter is the chief cause and is naturally inclined to associate it with the higher percentage of protein in the legumes. That other causes may also be operative, however, is indicated by the result on maize stover, which is nearly as high as in the case of alfalfa and shows a similar distribution among the several factors.

Among the concentrates there may be noted in particular the 
marked effect of maize in noticeably increasing the metabolism, especially during standing. This result is of interest in view of Zuntz and Hagemann's observations on the stimulating effect of maize upon the metabolism of the horse, which were also made on the standing animal, although no increase in the minor muscular activity was reported. Grain mixture No. I, containing 43 per cent of maize meal, likewise showed a similar effect, although with grain mixture No. 2, containing 60 per cent of maize, it was much less marked, possibly on account of the lower content of protein ( 2.5 as compared with 17.5 per cent).

764. Influence of amount of feed consumed. - In the discussions of the foregoing paragraphs it has been tacitly assumed that both the losses of chemical energy in the excreta and the increment of heat production consequent upon feed consumption are proportional to the quantity of feed ingested, i.e., that the net energy values per unit of feed are substantially unaffected by the amount consumed or by the plane of nutrition of the animal.

This seems not to accord with the general belief that heavy rations are relatively less effective than lighter ones and that the fat animal utilizes its feed less efficiently than the thin one. It became clear, however, in the course of the study, in Part III, of the feed requirements for various forms of production, that a variety of factors are influential in determining the actual outcome of feeding operations and that diminishing returns from heavy or long continued feeding do not necessarily imply a diminishing efficiency of the feed as a source of body material or energy. On the other hand, however, surprisingly little specific investigation appears to have been devoted to this fundamental question.

Obviously, differences in the amount consumed might influence the net energy value of a feeding stuff either by affecting the extent to which chemical energy is lost in the excreta (i.e., the metabolizable energy) or by affecting the magnitude of the losses due to increased heat production.

Influence on metabolizable energy. - That in mixed rations the digestibility may suffer more or less on heavy feeding has already been shown in Chapter XVI (722), notably in Eckles' and Armsby and 
Fries' experiments in which decreases of 8 to ro per cent were observed on rations varying in amount by from 42 to I 86 per cent, although it should be noted that in Armsby and Fries' later experiments only submaintenance or moderate production rations were used.

On the other hand, however, it was found in the latter experiments that the losses of energy in the methane were distinctly greater on the lighter rations so that the differences in the amount of feed consumed, within the limits of these experiments, failed to show any unmistakable effect upon the quantity of energy actually liberated in the body from a unit weight of feed. Moreover, it must be borne in mind that a considerable amount of the additional energy secured by the more extensive fermentation of the lighter ration is liberated in the digestive tract as heat of fermentation and does not enter into the energy exchange of the body tissues, so that the difference in the net nutritive effect is likely to be less than that in the metabolizable energy as ordinarily defined. How far such a compensation would occur in more liberal feeding is difficult at present to say.

Influence on heat production. - It is believed by some, however, that, aside from differences in digestibility, etc., the metabolizable energy actually derived from the feed is less efficiently utilized on heavy than on light rations and by fat than by thin animals, i.e., that a unit of metabolizable energy supplied yields less product. This does not appear exactly probable, a priori. So far as the increased heat production is due to mechanical work of digestion, it would appear that it would be substantially proportional to the amount of dry matter consumed, except possibly on extremely heavy rations. So far as it is due to a stimulation of the body metabolism by the digestive products resorbed $(367 e$ ) it would appear more likely that, in accordance with the general laws of mass action, it would be a diminishing function of the quantity present. Certain authors, especially Grafe and Müller, have, it is true, reported experiments which are claimed to demonstrate a so-called "luxus consumption" on heavy rations of carbohydrates, but their results scarcely appear to the writer entirely conclusive.

It has already been shown in Chapter X (450) that any heat production arising from a synthesis of body substance, such as that of fat from carbohydrates, for example, and which might be supposed to result in a decreased efficiency of the feed energy on supermaintenance as compared with submaintenance rations, is apparently not sufficient in amount as to materially affect the net energy values of feeding stuffs. As regards cattle, the writer has elsewhere ${ }^{1}$ discussed the results of Kühn's and Kellner's respiration experiments in their bearing on this question, reaching the conclusion that their general

1 Principles of Animal Nutrition, pp. 466-47I. 
tendency seems to be in favor of the hypothesis that the proportion of the metabolizable energy utilized is substantially independent of the quantity of feed, provided that the changes in the latter are not so great as to materially modify the course of the fermentations in the digestive tract. Armsby and Fries' results on the same species ${ }^{1}$ tend to confirm these conclusions, since they afforded no distinct evidence of an increase in the heat production per unit of feed as the amount of the latter was increased.

On the whole the probabilities seem to be that the limit to the most efficient use of feed energy, in herbivora at least, is set by the capacity of the alimentary canal to digest and assimilate feed rather than by the capacity of the organism to utilize the material transmitted to it by the organs of resorbtion. If this proves to be the case, the net energy values may be regarded as being, if not strictly constant, at least nearly so over a wide range of feeding.

765. Influence of age, breed and individuality. - That differences due to age, breed or " individuality" may exist between animals as regards the efficiency with which they utilize the energy of their feed and consequently as regards the net energy values of the latter does not appear particularly probable $a$ priori. Such data on these points as are available have been referred to in Chapters XI and XII, the general conclusion being reached that the evidence is insufficient to establish the existence of any marked differences of this sort except, perhaps, in the growth of very young animals.

766. Influence of kind of production. - It will not have escaped notice that the foregoing data regarding net energy values relate entirely to the production of body tissue, whether directly in growth or fattening or indirectly in maintenance or in work production. While it is perhaps unlikely that the values for these various purposes are stricly identical, the discussions in Chapters VIII, X, XI, XIV and in the present chapter seem to render it probable that the differences are not of sufficient magnitude to interfere seriously with the use of these net energy values for the computation of rations in practice.

As regards an important branch of animal husbandry, however, viz., milk production, as was shown in Chapter XIII, scarcely any accurate data regarding net energy values are yet

\footnotetext{
${ }^{1}$ Jour. Agr. Research, 3 (I915), 472-476 and Fig. 2.
} 
available, although it appears probable that they are higher than the corresponding values for tissue production. A tentative method of utilizing the present net energy values in computing the requirements of dairy cows was there proposed (605), but definite experimental data are much to be desired.

\section{§3. The Computation of Net Energy Values}

767. Importance. - It is apparent from the foregoing paragraphs that the number of actual experimental determinations of net energy values as yet recorded is comparatively small and that it can hardly be increased very rapidly, while it is obviously impracticable to apply the laborious method of respiration and calorimeter experiments to all the great number of feeding stuffs now in use. Determinations of the metabolizable energy, in which at least the energy of the feeding stuffs and of the visible excreta has been determined, are rather more numerous, while there are on record the results of a great number of digestion experiments in which no determinations of energy were made. It is highly important that the mass of statistical data thus accumulated, and summarized in tables of the composition and digestibility of feeding stuffs, should not be incontinently thrown overboard simply because a newer point of view has revealed more clearly its deficiencies. On the contrary, it should be utilized to the fullest extent possible, in connection with the as yet rather meager results of the more recent experimental methods, for computing the net energy values of such feeding stuffs as have not yet been subjected to direct investigation.

\section{Computation from digestible mutrients}

768. Kellner's investigations. - To Kellner is due the first attempt to make practical application of the conception of the feed as the source of energy to the body. In 1880 , in his investigations upon the relations between muscular activity and metabolism in the horse (637) he determined the additional amount of work. which the animal was able to perform as a result of the addition to his rations of starch and of fat. $\mathrm{He}$ expressed his results in terms of the percentage of the energy 
of the starch or fat which was recovered as useful work and called attention to the desirability of determinations of the heats of combustion of nutrients and feeding stuffs. Sixteen years later, after Rubner had published his fundamental work on the replacement values of nutrients, and Zuntz and his associates $(385,651-656)$ had begun their investigations upon the metabolism of the horse from the standpoint of energy, Kellner was able to return to the subject and undertake the extensive investigations with cattle frequently cited on previous pages.

769. Energy values of digestible nutrients. - Taking as his point of departure the digestible nutrients of feeding stuffs, Kellner sought first to determine the net energy values of the digestible protein, carbohydrates and fats for cattle by adding these substances in as pure form as possible to a basal ration in the manner already described (449). The results with cattle obtained in this way on starch, straw pulp, sugar, wheat gluten and oil are included in Table 202 (760). In that table, however, these materials are regarded in the light of feeding stuffs and the energy losses and net energy values relate to the substance as a whole and include all its effects. For his purpose, however, Kellner computed the net energy values, not of these substances as a whole but of the protein, carbohydrates and fat which determinations of digestibility showed to have been resorbed from them, with the following results. ${ }^{1}$

Table 205. - Net Energy Values of Digestible Nutrients for Cattle

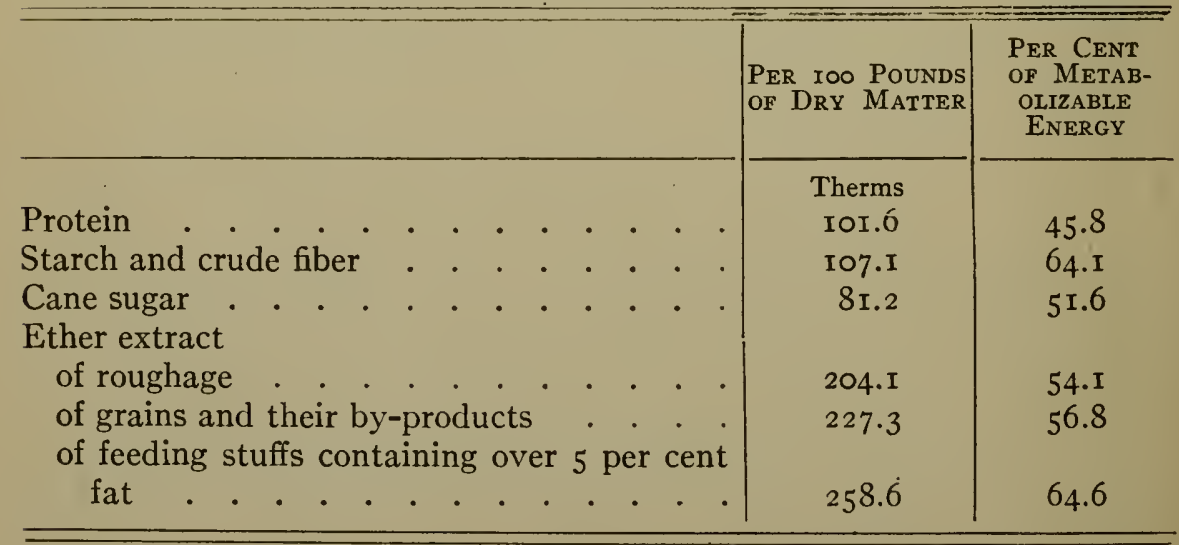

${ }^{1}$ Landw. Vers. Stat., 53 (I900), I-474; Ernährung landw. Nutztiere, 6th Ed., 95-159. 
Obviously the percentages in the last column are analogous to those obtained by Rubner and by Lusk (759) in experiments on dogs, and the differences between the two sets emphasize the differences in the nutritive processes of the two species.

770. Correction for crude fiber. - Kellner then proceeded to test the applicability of these factors to the ordinary feeding stuffs of cattle. With a certain number, notably the oil meals, ${ }^{1}$ the net energy values as computed by the use of his factors from the amounts of protein, carbohydrates and fat actually digested showed a close agreement with those found in direct experiments with the respiration apparatus. The digestible nutrients of these materials were of full value as compared with pure starch, gluten or oil.

Table 206. - Net Energy Values of Oil Meals for Cattle Per Ioo Pounds Dry Matter

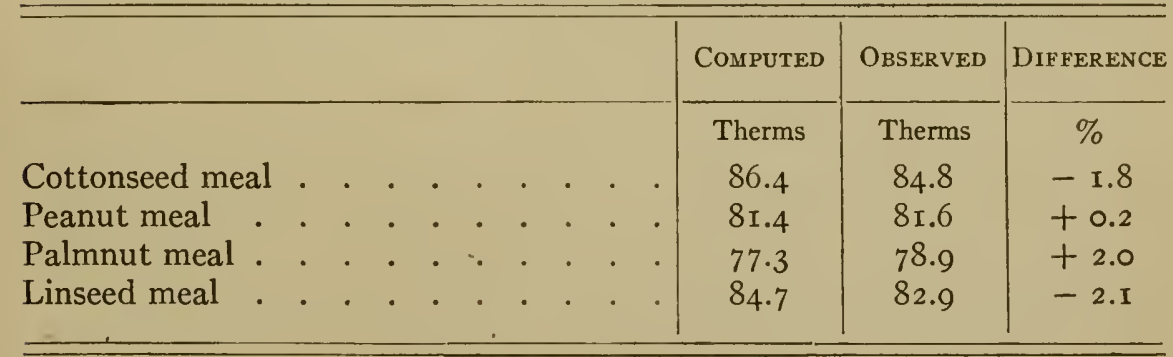

On the other hand, a striking contrast with the oil meals is afforded by the roughages, whose net energy values as directly determined were much lower than those computed, the deficit ranging from 30 per cent to 80 per cent and being greatest with the coarsest and least digestible materials. ${ }^{2}$ Kellner found this deficit to be more nearly proportional to the crude fiber than to any other ingredient of the feeding stuffs, ranging from 46.3 Therms to 76.7 Therms per 100 pounds of total fiber. By subtracting the average of 61.7 Therms from the computed net energy values, results were obtained which agreed well with those secured in direct experiments in the case of the hays but still showed considerable discrepancies for the straws, as follows:-

$$
{ }^{1} \text { Loc. cit., p. I60. } \quad{ }^{2} \text { Ibid., p. } 162 .
$$


Table 207.- Net Energy Values of Roughages for Cattle Per Ioo Pounds Dry Matter

\begin{tabular}{|c|c|c|c|c|c|c|c|c|c|c|c|c|}
\hline & & & & & & & & & - & $\begin{array}{c}\text { COMPUTED } \\
\text { WITH COR- } \\
\text { RECTION } \\
\text { FOR CRUDE } \\
\text { FIBER }\end{array}$ & OBSERVED & $\begin{array}{l}\text { DIFFER- } \\
\text { ENCE }\end{array}$ \\
\hline & & & & & & & & & & Therms & Therms & Per Cent \\
\hline \multicolumn{13}{|l|}{ Wheat straw } \\
\hline Sample $a$. & . & . & . & . & . & . & . & . & . & I6.I & $9 . \mathrm{I}$ & -43.4 \\
\hline Sample $b$. & . & . & . & . & . & . & . & . & . & $7 \cdot 5$ & 10.4 & +38.0 \\
\hline Oat straw & . & . & . & · & . & . & . & . & . & 23.0 & 28.5 & +24.1 \\
\hline Barley straw & . & . & . & . & . & . & . & . & . & 28.4 & 33.9 & +19.5 \\
\hline \multicolumn{13}{|l|}{ Meadow hay } \\
\hline Sample $a$. & . & . & . & $\cdot$ & . & . & . & . & . & 35.4 & 35.0 & - $\quad 1.3$ \\
\hline Sample $b$. & . & . & . & . & . & . & . & . & . & 48.3 & 47.1 & -2.5 \\
\hline Clover hay . & . & . & . & - & - & . & . & . & . & $35 \cdot 3$ & 36.8 & +4.1 \\
\hline "Grass hay" & . & . & . & . & . & . & . & . & . & 39.4 & 36.7 & -7.0 \\
\hline Rowen . . & . & . & . & . & . & . & . & . & . & 36.1 & 33.9 & -6.0 \\
\hline
\end{tabular}

For finer materials like chaff, presumably requiring a less expenditure for mastication, 3 I.8 Therms per Ioo pounds of total crude fiber was deducted. For green forage containing 16 per cent or more of crude fiber the same deduction was made as for dry forage and for that containing 4 per cent or less of crude fiber, the same as for chaff, while between these limits a sliding scale was used. This correction for crude fiber was applied only to roughage.

Kellner ascribed this apparent effect of crude fiber largely to the mechanical work required for its mastication and transportation through the alimentary canal, but in part also to the fermentations to which it is subject; in other words, he ascribed it to the so-called "work of digestion." In reality, however, the crude fiber can be regarded only as a convenient empirical measure of the differences between concentrates such as the oil meals and roughage. It has already been shown from Kellner's own experiments and from others (762) that the loss of energy in this way, far from being greater, is on the whole rather less with roughages than with concentrates and that the mechanical work of the digestive organs is probably a rather small factor in it. Roughages have relatively less net energy value, not 
because they contain much crude fiber which causes much mechanical work but because the feeding stuff as a whole stimulates the metabolism and causes a loss of energy which, though not greater, or, it may be, even less, than in the case of concentrates, is deducted from a much smaller amount of metabolizable energy supplied by the less amount of substances digested.

771. Relative values for concentrates. - That the crude fiber is far from being the only determining factor of the amount of energy expended in consequence of feed consumption is clearly shown by the majority of Kellner's experiments on concentrates and roots. Although with the oil meals a close agreement of the observed and computed results was obtained, in most instances the observed net energy value fell considerably short of that computed by the use of the factors of Table 205. The following table contains the results of the comparisons thus far reported. ${ }^{1}$ They show clearly that the digestible organic matter has a very unequal value in different classes of feeding stuffs, but a comparison with the percentages of crude or of digestible nutrients also shows that the crude fiber fails in these cases as a measure of the differences.

Table 208. - Observed Net Energy Values for Cattle as Per Cent of Computed

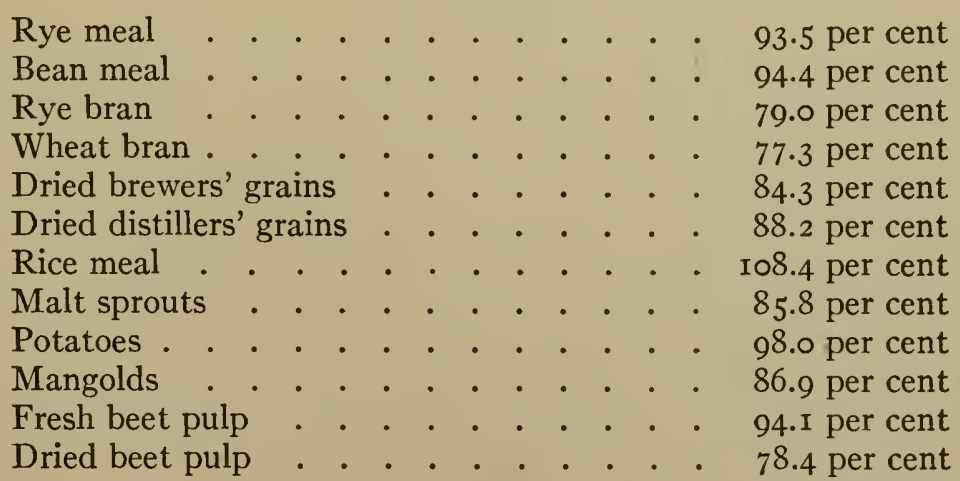

In computing the net energy values of concentrates, therefore, Kellner made no correction for the crude fiber, but instead corrected in each case the value computed from the digestible nutrients by multiplying it by a percentage (Wertigkeit) taken directly from the foregoing table when possible or estimated

${ }^{1}$ Ernährung landw. Nutztiere, pp. I65-167. 
from it. For example, the net energy values of alfalfa hay and of wheat bran having the composition and digestibility given by Allen ${ }^{1}$ would be computed as follows :-

Table 209. - Computation of Net Energy Values per ioo Pounds ACCORDING TO KELLNER

\section{Alfalfa Hay}

Digestible protein . . . . . $10.58 \times 1.016=10.75$ Therms

Digestible carbohydrates . . . . . $37.33 \times 1.071=39.98$. Therms

Digestible ether extract. . . . . . I.38 $38.04 \mathrm{I}=\underline{2.82}$ Therms 53.55 Therms

Total crude fiber . . . . . . $25.00 \times 0.617=\underline{15.43}$ Therms

Net energy value . . . . . . . 38.12 Therms

Wheat Bran (Relative Value $77 \%$ )

Digestible protein . . . . . . . I $2.0 \mathrm{I} \times \mathrm{I} .0 \mathrm{I} 6=\mathrm{I} 2.20$ Therms

Digestible carbohydrates . . . . . $41.23 \times 1.07 \mathrm{I}=44.16$ Therms

Digestible ether extract. . . . . . $2.87 \times 2.273=\frac{6.52}{62.88}$ Therms

Net energy value . . . . . . $62.88 \times 0.77=48.42$ Therms

772. Starch values. - Kellner's results were in reality net energy values, as is evident from the method by which they were obtained. In order, however, to avoid the use of the large numbers required to express the net energy values of rations in Calories, , and also to avoid the introduction of unfamiliar terms, he converted them for practical use into what he called "starch values." The starch value of a feeding stuff may be briefly defined as the amount of pure starch (assumed to be perfectly digested) which has the same net energy value. Thus, Kellner's table gives the starch value of maize meal as 8I.5 kilograms per Ioo kilograms, or 8I.5 pounds per roo pounds. One pound of starch, according to Kellner's results (769), has a net energy value of 107I Cals. The starch value of 81.5 given for maize meal, therefore, is equivalent to a net energy value of $107 \mathrm{I} \times 8 \mathrm{r} .5=87,286$ Cals., or 87.29 Therms, per 100 pounds and conversely the starch values of the alfalfa hay and wheat bran of the previous paragraph would be 35.59 and 45.21 , respectively. ${ }^{2}$

1 U. S. Dept. Agr., Farmers' Bulletin 22 (Rev.), rgor, pp. 8-9.

${ }^{2}$ In other words, Kellner's starch values multiplied by $1.071=$ net energy values per $100 \mathrm{lb}$. 
Kellner's starch values yield numbers of the same order of magnitude as those already familiar in tables of digestible nutrients and avoid unfamiliar units. They accomplish these ends, however, by ignoring the whole conception on which the system is built up, while some striking instances in recent literature have shown that it is not always easy, even for experts, to avoid confusion of thought in connection with their use. It appears to the writer to have been an unfortunate concession to attempt to express quantities of energy in terms of matter. He believes the intelligent feeder can readily learn to use units of energy in his computation of rations, as not a few have already done, and that there are manifest advantages in going over frankly and boldly to a system based on energy, while the objection to the use of large numbers is readily avoided by the employment of a larger unit of energy, the Therm (308). Net energy values expressed in Therms per roo pounds are of the same order of magnitude as the familiar figures for digestible nutrients, and even if roo kilograms be made the basis of calculation they are not inconveniently large. For these reasons, energy values of feeding stuffs in the present volume are expressed in Therms per roo pounds.

\section{Computation from digestible organic matter}

773. Independent of chemical composition. - It is apparent from the foregoing description of Kellner's somewhat complicated method that it is essentially based on the digestible protein, carbohydrates and fats of the older relative values (705-710), while it involves in its execution certain more or less empirical corrections which are at bottom simply methods of applying the average net results on typical feeding stuffs to other materials. Armsby and Fries ${ }^{1}$ have proposed a method which seeks to attain the same end more directly and simply, relating the energy content and the necessary deductions to the total dry matter or total digestible matter of the feeding stuff independently of its chemical composition.

The energy content of a feeding stuff is just as definite a quantity as its content of protein, carbohydrates, or fats, and

1 Jour. Agr. Research, 3 (I915), 486. 
it is entirely possible to trace the distribution of that energy in the body quite independently of any knowledge of the chemical composition of the material. Not only so, but it is believed that in discussing energy values there are distinct advantages as regards simplicity, and perhaps also as regards accuracy, in cutting loose as far as possible from the conventional data regarding chemical composition and digestion coefficients and in dealing directly with quantities of energy.

This statement is by no means to be understood to stigmatize comparisons based on chemical methods as either valueless or superfluous. The problems of nutrition are too complex and too difficult for us to refuse any light that can be thrown on them by any method, and the energy relations touch only one phase of them. The point is that in whatever degree their energetic aspects can be separated from their chemical aspects, to that extent we possess two independent methods of approach to them.

774. Method of computation. - As already pointed out, the net energy value of a feeding stuff is equal to its metabolizable energy minus the heat production caused by its consumption. It has been shown (753) that the metabolizable energy of a feeding stuff, when not determined directly, may be computed approximately from the total digestible organic matter by multiplying by a proper factor. If from this result there be subtracted the energy expenditure due to feed consumption, either as directly determined or as estimated from that of similar feeds, the remainder is approximately the net energy value. Thus in the same two feeding stuffs just used to illustrate Kellner's method each pound of digestible organic matter, according to the averages on previous pages (753-755), would contain 1.60 Therms of metabolizable energy in the hay and $\mathrm{I} .77$ Therms in the bran; the average losses of energy in heat production per pound of dry matter would be for the hay 0.5303 Therm and for the bran 0.5339 Therm, and the computation of the net energy values would be as follows : ${ }^{1}$ -

1 The digestible protein, carbohydrates and fats enter into the calculation simply as a means of obtaining the total digestible organic matter when, as is usually the case, this is not reported separately. If the latter is the case, then the computation is, as stated above, independent of the chemical composition. 
Table 2 io. - Computation of Net Energy Values per ioo Pounds ACCORding to ARMSBY AND Fries

\begin{tabular}{|c|c|c|}
\hline & ALfalfa Hay & Wheat Bran \\
\hline $\begin{array}{l}\text { Total dry matter } \\
\text { Digestible }\end{array}$ & 9r.6 lb. & 88.5 lb. \\
\hline Protein . . . . . & 10. $58 \mathrm{lb}$. & I2.0r lb. \\
\hline Carbohydrates . . & $37.33 \mathrm{lb}$ & $4 \mathrm{I} .23 \mathrm{lb}$. \\
\hline Fats . . • & r. $38 \mathrm{lb}$. & $2.87 \mathrm{lb}$. \\
\hline $\begin{array}{c}\text { Total digestible organic } \\
\text { matter. } . .\end{array}$ & $49.29 \mathrm{lb}$ & 56. I I lb. \\
\hline $\begin{array}{l}\text { Metabolizable energy } \\
\text { Loss in heat production }\end{array}$ & $\begin{array}{l}49.49 \times 1.60=78.86 \text { Therms } \\
91.60 \times 0.5303=48.58 \text { Therms }\end{array}$ & $\begin{array}{l}56.11 \times \text { I.77 }=99.3 \mathrm{I} \text { Therms } \\
88.50 \times 0.5339=47.25 \text { Therms }\end{array}$ \\
\hline Net energy value & $\overline{30.28}$ Therms & $\overline{52.06}$ Therms \\
\hline
\end{tabular}

The same method of computation is of course applicable to other species than cattle, so far as the meager data at hand permit. The results of such computations, based upon the average composition and digestibility of American feeding stuffs, are contained in the tables of the Appendix.

\section{Computation of net energy values for the horse}

775. Zuntz and Hagemann's method. - The method employed by Zuntz and Hagemann ${ }^{1}$ for computing net energy values for the horse (758) is substantially similar to that just illustrated for cattle. The metabolizable energy is estimated from the digestible nutrients and from it is subtracted the computed energy expenditure due to the consumption of the feed.

776. Metabolizable energy. - From the results of five digestion and metabolism experiments on rations of oats, hay and straw in different proportions made at intervals between I 888 and I89I, they compute the metabolizable energy of the total digestible nutrients (including the digested fat multiplied by 2.4 ) to average $3.96 \mathrm{Cals}$. per gram, corresponding to 3.99 Cals. per gram digestible organic matter as computed by the writer in Table 188 (749). In the respiration experiments, the digestible nutrients were not determined directly but were estimated by combining the results of the same five digestion and metabolism experiments in various ratios according to the proportion of oats, hay and straw consumed.

777. Increment of heat production. - Experiments upon man, made by Magnus-Levy in Zuntz's laboratory, had previously shown

${ }^{1}$ Landw. Jahrb., 27 (I898); Ergzbd. III, 2II-236, 276-279, 4I8. 
that food consumption increased the total metabolism by about 9 per cent of the metabolizable energy of the food eaten. Zuntz and Hagemann assume that this result is applicable to the digestible nutrients of the feed of the horse.

In addition, it was found that hay produced a much more marked effect than did grain in augmenting the heat production of the horse as estimated from the respiratory exchange, which was determined by means of the Zuntz apparatus in short periods at various intervals after the consumption of more or less diverse rations, a small correction being added for cutaneous and intestinal respiration. This difference is ascribed to the crude fiber of the hay and its amount is computed to be 2.086 Cals. per gram. The energy expended in the mastication of the feed is likewise related to its crude fiber content, being estimated at 0.565 Cals. per gram. The total heat increment per gram of crude fiber, therefore, is estimated at $2.65 \mathrm{Cals}$. per gram.

778. Computation of net energy value. - In brief, Zuntz and Hagemann compute the heat production due to the consumption of feed by the horse to be equal to 9 per cent of the metabolizable energy, estimated at the rate of 3.96 Cals. per gram of digestible nutrients, plus 2.65 Cals. for each gram of total crude fiber present, and by subtraction of these amounts from the metabolizable energy obtain the net energy value.

The method of computation may be conveniently illustrated from the data given by Langworthy ${ }^{1}$ for timothy hay. Zuntz and Hagemann's factors, recalculated per Ioo pounds for convenience, become for metabolizable energy I.796 Therms and for crude fiber I.202 Therms. On this basis the calculation of the heat production due to the hay would be as follows :-

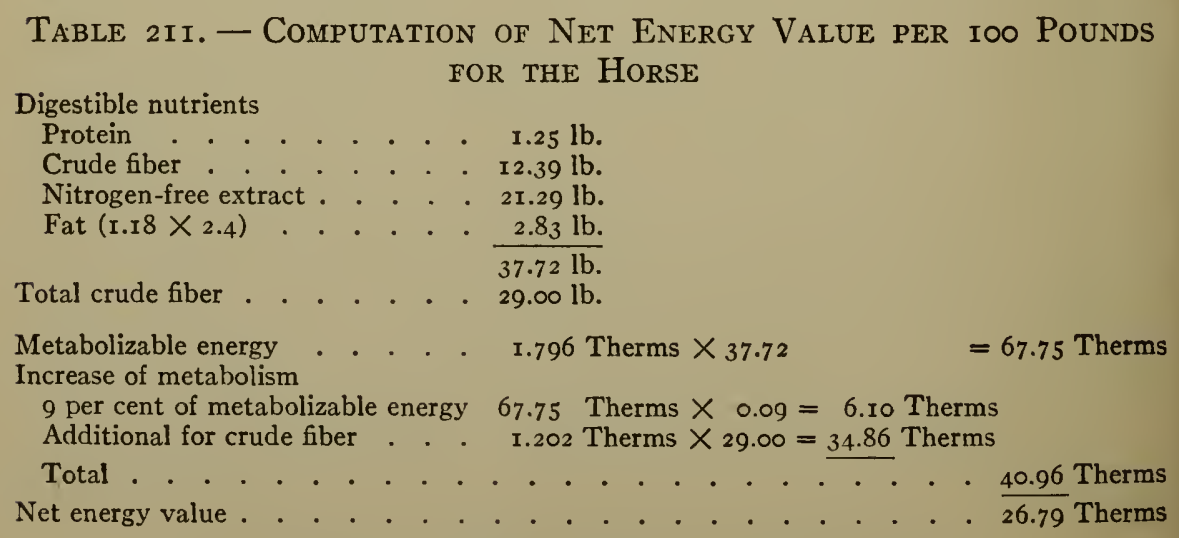

${ }^{1}$ U. S. Dept. Agr., Office of Expt. Stas., Bul. $₫ 25$, p. I4. 
As is evident from the methods by which the factors were reached, this method of calculation is not strictly exact, but the authors believe it to be a sufficiently close approximation on which to base computations of rations in practice.

Zuntz and Hagemann's method of computation has been the subject of considerable criticism, the two principal points being, first their estimate, based upon the results of experiments on man, of 9 per cent for the effect of the digestible nutrients, and second, and more especially, the assumption that the metabolism for 24 hours may be computed from the results of comparatively short respiration experiments. Qualitatively, Zuntz and Hagemann have clearly demonstrated the very considerable increase of energy metabolism in the horse during the digestion of his feed, as well as the fact that this increase is relatively greater with roughage than with grain, and they were the first to point out that this effect must be taken into account in estimating the values of feeding stuffs. There may be a difference of opinion as to the quantitative accuracy of their figures and certainly investigations by more direct methods, involving fewer assumptions and complex calculations, are greatly to be desired, but until such results are obtained, we may continue to use provisionally those reached in the manner just described.

779. Wolff's method of computation. - His extensive investigations upon the working horse made at Hohenheim in I877 to I894 and antedating the investigations thus far mentioned, led Wolff to a still simpler approximate method of estimating the relative net energy values of feeds for the horse.

It was shown, on the average of a considerable number of comparisons, that the digestible nutrients from roughage were less efficient both for work production and for maintenance than were those derived from grain. Wolff found, however, that if the digestible crude fiber were omitted from the comparisons, the ratio between the fiber-free nutrients and the work performed was comparatively uniform and also that this assumption yielded uniform results for the amount of fiber-free nutrients necessary for maintenance. He therefore concluded that the crude fiber in the rations of the horse was apparently valueless and that the remaining digestible nutrients might be regarded as of equal value whether derived from grain or from roughage. Expressed in the light of our present conceptions, this is practically equivalent to saying that the net energy value is proportional to the amount of fiber-free nutrients.

Wolff is careful to say that the digestible crude fiber is apparently valueless, and virtually regards the amount of crude fiber as furnishing a convenient empirical measure of the difference in the value of 
the digestible nutrients of roughage as compared with those of grain. That such is the case is doubtless explained in part by the rather limited variety of feeding stuffs employed in the experiments. The roughage was meadow hay with, in some cases, a small addition of straw, while the grain was usually oats, partially replaced in some instances by other feeds. Whether the same relation between fiberfree nutrients and work done would hold in widely different rations is not apparent.

Wolff's results are relative only. They do not show the actual amount of net energy in the rations but only that it was proportional to the fiber-free nutrients. The energy content of the latter would differ considerably from the net energy as computed by Zuntz and Hagemann's method, first because it does not include the deduction of 9 per cent of the metabolizable energy, and second, because it assumes a uniform value of zero for crude fiber, while Zuntz and Hagemann's method gives the crude fiber a negative value if it has a digestibility of less than 55 per cent. Values computed according to Wolff's method from the fiber-free nutrients would therefore considerably exceed Zuntz and Hagemann's figures.

\section{§ 4. Production Values as Regards Protein}

\section{Relative values of proteins}

780. Differences in proteins. - As appears from the discussions of the preceding section, the production values of feeding stuffs as regards energy may already be formulated with some degree of accuracy, although further investigation is much needed.

Concerning the production values as regards protein, the situation is far less satisfactory. For years the protein of feeding stuffs has been treated as if it were a single chemical substance; i.e., the different proteins known to exist in feeding stuffs have been assumed to have substantially equal nutritive values. The more recent investigations into the chemistry and physiology of the proteins, however, have resulted in an entire change in the point of view. As has been fully shown in previous chapters $(340,398,465,552)$, it is the constituent amino acids into which the proteins are split in digestion which are the materials out of which body protein is constructed, and the processes of maintenance, growth or milk production require for their support, not proteins as such, but certain amounts and proportions of such of the amino acids as cannot 
be synthesized in the body. In place of a single requirement for protein, it would appear that there must be substituted a number of separate amino acid requirements, a deficiency as regards any one of which may constitute a limiting factor.

781. Incomplete and unbalanced proteins. - As appeared in Chapter I (50) certain vegetable proteins may be classed as incomplete proteins in the sense that they lack entirely one or more of the amino acids characteristic of proteins in general. The classic example of an incomplete protein is gelatin, which lacks tyrosin and tryptophan and which has long been known to be incapable by itself of maintaining the stock of body protein in an animal. A similar case among the vegetable proteins which has been much discussed is the zein of maize, which yields neither lysin, glycin nor tryptophan on hydrolysis and which is incapable of supporting either maintenance (399) or growth (465). Still another instance is afforded by the gliadin of wheat (465), which lacks lysin and which, while adequate for maintenance, is unable to support growth. Furthermore, the proteins of the cereal grains in general, while not incomplete in the sense of absolutely lacking certain amino acids, may, from the standpoint of animal nutrition, be called unbalanced in that, as compared with the body proteins, they are relatively rich in glutamic acid and therefore correspondingly deficient in other constitutents, including those ingredients which, like lysin in particular, appear to be essential to growth. It appears evident that in the conversion of a unit weight of such a protein into body protein, a considerable portion of the amino acid present in excess must undergo deaminization (233) and be substantially a waste product so far as the protein requirement of the body is concerned, although it may of course serve as a source of energy. Quantitative results as to the maximum percentage utilization of individual proteins, however, are not yet available.

782. Application of results. - But while the general validity of the newer point of view seems well established, it does not appear possible as yet to utilize it in establishing net protein values for feeding stuffs comparable to the net energy values discussed in $\S 2$ of this chapter. For this there are three principal reasons.

First, sufficient knowledge of the proteins of feeding stuffs is lacking. Although the constituents of a considerable number 
of vegetable proteins derived from seeds is known, those contained in roughages and in roots have not yet been investigated, although a beginning has been made ${ }^{1}$ in determining the proportions of the different groups of amino acids which are yielded by the total nitrogenous matter (crude protein) of various feeding stuffs.

Second, as has appeared in previous chapters, such information as is available respecting the protein requirements of farm animals has been derived from experiments in which only the total protein supplied was considered without reference to its kind. Practically no knowledge is available as to the amino acid requirements of the various farm animals for different purposes.

Third, even were the production values of the various single proteins known, it would not be possible to estimate from them the production values of the mixed proteins of feeding stuffs, since a deficiency in one protein might be compensated by a surplus in another and the mixture show a much higher production value than either of its ingredients separately. Thus, as already noted, the value of wheat gliadin, which lacks lysin, is practically zero for growth, while as part of a mixture with other proteins supplying lysin it may have a high value, the replacement of 25 per cent of it by lactalbumin, for example, rendering the mixture fully adequate to support normal growth. Each particular mixture of proteins would have its own production value, which might differ widely from the mean of the values for the individual constituents.

The qualitative differences in proteins are doubtless of much significance, and the researches in progress can hardly fail ultimately to lead to a more rational method of valuation than that now in use, but as yet they do not afford an adequate basis for expressing the values of feeding stuffs in general as sources of protein. For the purposes of the stock feeder, therefore, it still seems necessary to adhere to the older method which regards the digestible protein of a feeding stuff as expressing approximately its production value in this respect, thus virtually assuming that in ordinary mixed rations the protein deficiencies of the different ingredients will largely balance each other, and this method has been followed in the tables of the

1 Grindley, Joseph and Slater; Jour. Amer. Chem. Soc., 37 (I915), 1778 and 2762 : Nollau; Jour. Biol. Chem., 21 (I915), 6r r. 
Appendix. This should be done, however, with a distinct consciousness of the inadequacy of the method and with the hope that it may ultimately be replaced by one having a more scientific basis.

Meanwhile, notice should be taken of the results of several recent investigations upon the mixed proteins of a few feeding stuffs, particularly those of the cereal grains.

783. Low value of maize proteins. - The demonstration of the insufficiency of the zein of maize for either maintenance or growth (781) has tended not unnaturally to produce the impression that this important feeding stuff is relatively valueless as a source of protein. Zein, however, is not the only protein of maize. According to Osborne and $\mathrm{Mendel}^{1}$ the mixed proteins of maize are made up approximately as follows :-

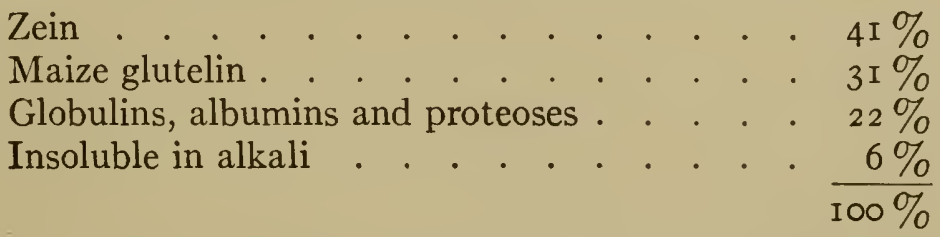

Glutelin yields all the amino acids which zein lacks and the same is probably true of the other proteins of maize. Evidently the results of experiments on zein do not show maize to be valueless as a source of protein, although they do indicate a relatively low value and this conclusion has been confirmed by the experiments of Osborne and Mendel on rats and of Waters on pigs. On the other hand, however, Hart and McCollum ${ }^{2}$ were able to obtain a normal growth of pigs on rations supplying only maize protein but supplemented by salts.

Osborne and Mendel ${ }^{1}$ have investigated the nutritive value of the mixture of proteins contained in the "corn gluten" produced in the manufacture of starch from maize and consisting chiefly of zein and glutelin in the proportion of approximately roo to 44 . In such a mixture, the deficiencies of the zein are to a greater or less extent compensated for by the glutelin, and the mixed proteins not only proved adequate for maintenance but were able to support rather slow growth. The addition to them of lactalbumin or of casein, however, rendered them much more efficient and induced normal growth.

${ }^{1}$ Jour. Biol. Chem., 18 (Igr4), I.

${ }^{2}$ Ibid., 19 (I9I4), 373. 
Waters ${ }^{1}$ in experiments on growing pigs has shown in a striking manner the practical significance of Osborne and Mendel's results. In each of the four trials reported, one lot of animals received only maize. The others were given maize with the addition of ash ingredients, either by direct additions of salts or in the form of the socalled protein-free milk, while still others received an addition of complete proteins, as nearly ash-free as possible, derived in some cases from blood and in others from milk. The growth of the lots receiving only maize was either very slow or practically zero and the same was true when ash was added, showing that the failure to grow was not due to a lack of mineral matter. When, however, complete proteins were added to the maize, steady and normal growth took place and comparative analyses of the carcasses showed a corresponding storage of body protein by the animals. The total results as to live weights were as follows :-

Table 212. - Influence of Nature of Protein Supply on Growth of Pigs

\begin{tabular}{|c|c|c|c|c|c|}
\hline & & $\begin{array}{c}\text { LENGTH OF } \\
\text { TRIAL }\end{array}$ & $\begin{array}{l}\text { INITIAL }{ }^{2} \\
\text { WEIGHT }\end{array}$ & $\begin{array}{c}\text { FINAL } \\
\text { WEIGHT }\end{array}$ & $\begin{array}{l}\text { DaILY } \\
\text { GAIN }\end{array}$ \\
\hline Second trial & & Days & Lb. & Lb. & Lb. \\
\hline Maize alone . . . . . & . . & 280 & 50 & 108 & $0.2 \mathrm{I}$ \\
\hline Maize and ash . . . & . . & 280 & 50 & 102 & 0.19 \\
\hline Maize and blood albumin & & 280 & 50 & 330 & 1.00 \\
\hline $\begin{array}{c}\text { Maize, blood albumin and } \\
\text { Third trial }\end{array}$ & ash & 280 & 50 & 362 & I.II \\
\hline Maize alone & . . & I87 & 50 & $5 \mathrm{I}$ & o. \\
\hline Maize and ash . . . . & . . & I87 & 50 & 50 & o. \\
\hline Maize and protein-free milk & & I 87 & 50 & 38 & -0.06 \\
\hline $\begin{array}{c}\text { Maize and milk protein } \\
\text { Fourth trial }\end{array}$ & .. & I 87 & 50 & 334 & 1.50 \\
\hline Maize alone . . . . & 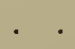 & ISo & 50 & II 7 & 0.37 \\
\hline Maize and ash . . . . & . . & 180 & 50 & 108 & 0.32 \\
\hline Maize and protein-free milk & & I 80 & 50 & I $4 \mathrm{I}$ & 0.51 \\
\hline Maize and milk albumin & & I80 & 50 & 239 & I.05 \\
\hline $\begin{array}{l}\text { Maize and casein } . \dot{\text { Fifth trial }}\end{array}$ & . . & 180 & 50 & $29 I$ & I. 34 \\
\hline Maize alone . . . . & - & 200 & 30 & 79 & 0.25 \\
\hline Maize and milk ash & . . & 200 & 30 & 55 & 0.13 \\
\hline Maize and tryptophan . & .. & 200 & 30 & 74 & 0.22 \\
\hline Maize and milk albumin & .. & 200 & 30 & 268 & I.I9 \\
\hline Maize and casein. & .. & 200 & 30 & 232 & I.Or \\
\hline
\end{tabular}

1 Proc. Soc. Prom. Agr. Sci. (1914), p. 7.

${ }^{2}$ Approximate. The exact initial weights are not given in the report cited. 
784. Values of other cereal proteins. - Investigations at the Wisconsin Experiment Station led to the conclusion that not only the proteins of maize but the unbalanced proteins of other cereal grains are distinctly inferior to milk proteins as sources of protein for growth and milk production.

Hart, Humphrey and Morrison ${ }^{1}$ in two comparisons of maize and alfalfa proteins for growing heifers observed a retention of approximately 20 to 24 per cent of the maize nitrogen as compared with much higher figures obtained for milk proteins in later experiments at the same institution.

McCollum ${ }^{2}$ reports a series of trials on young pigs in which the effects of the proteins of maize, wheat and oats, of casein and of skim milk on the nitrogen balance were compared. The protein supply varied in the different trials but the author presents reasons for believing that in no case did it exceed the amount the animal was capable of utilizing in growth, so that the results are not affected in the manner discussed in Chapter XI (468) by surplus protein being katabolized. On the higher protein rations, from 20 to 34 per cent of the resorbed nitrogen was retained in the body of the animal, while, contrary to what would naturally be expected, the percentage retention was decidedly lower on rations supplying less protein. The milk proteins, on the other hand, showed a decidedly higher percentage retention, viz., for casein $5^{I}$ per cent and for skim milk proteins 66 per cent.

Hart and Humphrey ${ }^{3}$ have compared the proteins of maize, wheat, gluten feed, oil meal, distillers' grains and milk as sources of protein for milking cows (587). Unfortunately, the effects were chiefly on the body protein, so that the only comparison possible is between the algebraic sums of body protein and milk protein. Computed in this way, the average percentage efficiency for three animals was, for milk proteins, 59, for maize 40 , for wheat 36 , for gluten feed 45 , for oil meal $6 \mathrm{I}$ and for distillers' grains 60.

785. Alfalfa proteins. - Hart, Humphrey and Morrison in their comparisons of maize and alfalfa proteins just mentioned (784), found the total nitrogen of alfalfa to show about the same percentage retention in both growth and milk production as did the total nitrogen of maize.

In none of these Wisconsin experiments is the maintenance requirement of the animals taken into account in computing

1 Jour. Biol. Chem., 13 (I912), I33.

3 Ibid., 21 (I9I5), 239; 26 (I9I6), 457.

${ }^{2}$ Ibid., 19 (I9I4), 323. 
the percentage efficiency of the protein. If this be done, using the approximate data contained in Chapter IX (415-417), the percentages of the proteins supplied in excess of maintenance which were retained would be distinctly increased in every case. It cannot be concluded, therefore, that the low percentages computed by the Wisconsin investigators show that only these rather small proportions of the cereal proteins are capable of transformation into body proteins. On the other hand, however, such a conjectural correction would result in making the relative differences between the different proteins appear greater than those shown by the method of calculation used.

No other studies upon the relative values of the mixed proteins of feeding stuffs have come to the writer's notice.

\section{Value of non-protein}

In a previous paragraph (782) the conclusion was reached that for the present the only available measure of the protein values of feeding stuffs is the total amount of digestible protein which they contain. In the application of this method it becomes necessary to decide whether the basis of comparison shall be the "crude" protein or the "true" protein as determined by existing conventional methods (104-107); in other words, to decide what value, if any, shall be assigned to the non-protein.

786. Early investigations. - Following the recognition of the fact that the substances grouped under the collective term non-protein make up a considerable share of the nitrogenous matter of numerous feeding stuffs, much labor has been expended in efforts to determine their nutritive value as compared with that of the true proteins. These investigations have been summarized by the writer elsewhere. ${ }^{1}$ While much diversity of opinion has prevailed, the general tendency has been to consider the non-protein as of questionable value. Kellner, the leading German authority, in particular, regarded it as valueless.

787. New viewpoint. - With advancing knowledge of the chemistry of the proteins and of the chemical mechanism of

${ }_{1}$ Principles of Animal Nutrition, pp. 52-58; U. S. Dept. Agr., Bur. Anim. Indus., Bul. I39 (I9II). 
protein nutrition, however, it has become increasingly evident that many of these earlier results are of little real significance and that the question of the nutritive value of non-protein must be approached from a different standpoint. It has become evident, for example, that attempts to replace proteins completely by a single amino acid or even by two or three of them must necessarily fail, since the formation of body protein requires the presence of all its constituent building stones in proper proportions. For the same reason the addition of an amino acid to a ration can be effective only if the proteins of that particular ration happen to be deficient in that one constituent.

Furthermore, experiments with ingredients of the non-protein which do not form part of the protein molecule are of questionable significance. For example, asparagin, which has been a favorite subject of investigation for reasons of convenience, is not found among the cleavage products of the proteins but belongs to the class of acid amides. So far as appears, it could contribute to the formation of protein only after conversion into the related aspartic acid (47) and it has not yet been shown that the body can undo the amide linkage of nitrogen. Moreover, as appeared in Chapter I (60-67), the non-protein includes, in addition to acid amides like asparagin, a great variety of nitrogenous substances which are but remotely related chemically to the proteins and whose nutritive value is at best doubtful.

It would appear that the value of the non-protein of a feeding stuff as a source of body protein must be determined by precisely the same thing which is believed to measure the value of an individual protein or of the mixed proteins of feeding stuffs, viz., the kinds and proportions of amino acids which it can yield, since there is no evident reason why an amino acid existing ready formed in a feeding stuff should differ in value from the same substance split off from protein in the process of digestion. If this be admitted, however, the distinction made in recent years between protein and non-protein in feeding stuffs becomes rather meaningless. If the value of each is measured by its amino acid content, then what is needed to fix the production values of feeding stuffs as regards protein is a knowledge of the kinds and amounts of these compounds which the feeding 
stuff as a whole (i.e., its crude protein) can furnish, irrespective of whether they exist in a soluble, as it were predigested, form or are first produced in the digestive tract of the animal.

788. Indirect utilization of non-protein by herbivora. - In the case of herbivora, especially of ruminants, another factor enters into the consideration of the value of the non-protein, viz., its relation to the ferment organisms which play so large a part in the digestive processes of these animals.

It was stated in Chapter III (141) that the presence of soluble nitrogenous compounds in the feed tends to stimulate the multiplication and activity of these organisms, thus bringing about an increase in the excretion of methane and in the proportion of carbohydrates apparently digested. It was likewisè indicated that the protein produced at the expense of non-protein in the multiplication of the microörganisms might serve as a source of protein to the body and thus bring about an indirect utilization of the non-protein. Much experimental evidence supporting this view is on record, particularly the extensive investigations of Morgen and his associates, which have been discussed elsewhere ${ }^{1}$ by the writer. Three general conclusions regarding the behavior of non-protein in the body were drawn, viz. :-

In ruminants, a conversion of non-protein into protein appears to be effected by the microörganisms of the digestive tract. The extent of this conversion appears to be relatively greater in the case of ammonium salts and asparagin than in that of the non-protein of vegetable extracts.

The protein thus formed from non-protein seems to be digested subsequently. The apparent formation of indigestible protein observed by some investigators appears to be due to an increase in the metabolic products contained in the feces, "caused by the specific action of the vegetable extracts upon the digestive tract.

By means of its conversion into bacterial protein, the nonprotein in the feed of ruminants may serve indirectly for maintenance and also as a source of protein for milk, and probably for growth, in rations deficient in protein.

Quantitatively, however, the various forms of non-protein used in these experiments were much inferior to protein and a substitution of the former for the latter caused a marked falling

${ }^{1}$ U. S. Dept. Agr., Bur. Anim. Indus., Bul. 139 (IgII). 
off in production. For maintenance alone, non-protein seemed quite effective, but neither for growth nor for milk production could it equal protein. It seems probable that the limiting factor in this indirect utilization of non-protein is the extent to which it can be synthesized into protein by the microörganisms rather than any inferiority in the nutritive value of the resulting protein.

789. Conclusions. - It seems clear that the evidence is insufficient to warrant any general conclusions regarding the nutritive value of non-protein, if indeed any general statement regarding such a heterogeneous group is possible. Ultimately, it may be that studies of the amino acid yields of the total nitrogenous matter (crude protein) of feeding stuffs, or comparisons of its relative efficiency in supporting maintenance or growth, will lead to the formulation of production values for the crude proteins of different materials, but for the present the writer feels that the safer course is to make the digestible "true " protein, so-called, the basis of comparison.

While some experiments, notably the Copenhagen experiments on dairy cows (586), seem to indicate a relatively high value for the non-protein of roots especially, most investigators, particularly Morgen and his associates, have, as already noted, found them decidedly inferior to protein. It is true that the non-protein contains amino acids which may at times be utilized indirectly by herbivora through the agency of the microörganisms of the digestive tract, but even this indirect utilization seems to be rather limited in extent in most instances. The conventional "true" protein, on the other hand, may be regarded as representing approximately the real proteins of a feeding stuff and it would seem that these mixed proteins are likely to supply more nearly a balanced amino acid mixture in digestion than would result from the inclusion of the non-protein. Investigations of the protein values of feeding stuffs should doubtless take account of whatever amino acids the non-protein supplies, i.e., they should relate to the crude protein. With continued study of these relations, it may be hoped that greater clarity may be attained, but until that end is reached, the digestible "true" protein seems the safer basis for the formation of tables of the production values of feeding stuffs and for the computation of rations. Whatever error is 
thus involved tends to make the protein content of the rations somewhat higher than if the crude protein were made the basis of the computation. It is, therefore, an error on the safe side, since a deficiency of protein may limit production while a surplus at worst simply tends to increase the cost of the ration, and the difference in the latter respect is seldom considerable. 


\section{CHAPTER XVIII}

\section{THE COMPUTATION OF RATIONS}

\section{§ I. Feeding Standards}

790. Origin. - As the hay values described in Chapter XVI (700) gradually gave place to new methods of comparing the values of feeding stuffs based upon improved methods of chemical analysis and upon investigations into the general laws of nutrition, an attempt naturally followed to express the nutritive requirements of animals in a similar manner instead of in terms of gross weight of feed or of hay values. Thus originated the feeding standards for different species of farm animals which later came to be popularly regarded more or less in the light of prescriptions or recipes for successful feeding.

791. Early standards. - The earliest suggestion along this line seems to have originated with Haubner ${ }^{1}$ about 1840 . Lingethal, ${ }^{1}$ in 1857 , amplified the suggestion, but Grouven ${ }^{2}$ in I 858 was the first to formulate specifically the requirements of farm animals, expressing them in .terms of dry matter, total protein, total fat (ether extract), and "carbohydrates" (total material soluble in acids and alkalies). In other words, the crude nutrients were the basis of Grouven's standards.

Wolf took the next step in advance by making the digestible nutrients as determined by the methods of Henneberg and Stohmann (707-710) the basis for comparisons of feeding stuffs and for expressing feed requirements. His feeding standards were first published in I864 in Mentzel and von Lengerke's Landwirtschaftlicher Kalender and were also incorporated in his widely read book, Die landwirtschaftliche Fütterungslehre, in I874. These standards attempted to formulate the amounts of digestible protein, carbohydrates and fats which should be

${ }^{1}$ Quoted by Grouven; Kritische Darstellung aller Fütterungs-Versuche. Kassel, I863, p. 327 .

2 Vorträge über Agricultur-Chemie, 1858. 
contained in rations for various purposes in order to secure satisfactory results under average conditions. Thus, the Wolff standard for dairy cows was:-

Feeding Standard for Milk Cows per Day and tooo Pounds Live WEIGHT

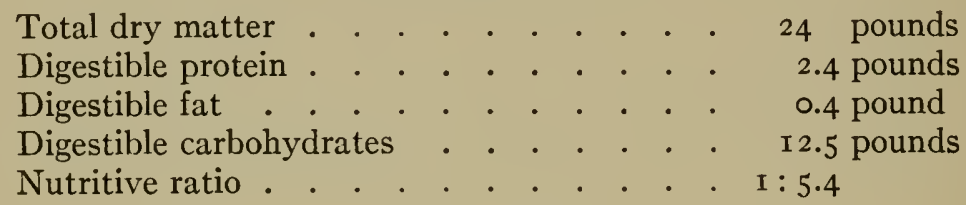

This means that any mixture of suitable feeding stuffs from which a cow can digest 2.5 pounds of protein and $\mathrm{r} 3$ pounds of non-nitrogenous nutrients per day will constitute a suitable ration and produce a good flow of milk.

The Wolff standards were introduced into the United States a few years later through the writings of Johnson, Atwater and others, and by the writer's translation of Wolff's book, ${ }^{1}$ and found wide currency among students of stock feeding and with popular writers.

792. Modifications of the Wolff standards. - That with the progress of investigation modifications should be made in standards formulated fifty years ago was to be expected. From I 864 to I 896 Wolff's standards were published annually in Mentzel and von Lengerke's Kalender practically unchanged. From 1897 to 1906 they were continued under the charge of Lehmann, who introduced some additions and modifications, the principal ones being the subdivision of the standard for dairy cows according to milk yield and the distinction between meat and milk or wool breeds in the standards for growing animals. These constitute the well known Wolff-Lehmann standards.

793. Kellner's standards. - Both the Wolff and the WolffLehmann standards, as already noted, were expressed in terms of the so-called digestible nutrients. Kellner, in 1905, in the first edition of his Ernährung der landwirtschaftlichen Nutztiere, proposed the system of calculation by means of starch values (772) which has since been associated with his name, and formulated a table of feeding standards expressed according to this new method.

${ }^{1}$ Manual of Cattle Feeding, I880. 
In one respect Kellner's standards differ radically from preceding ones and constitute a notable advance. While the earlier standards, like the earlier tables of feeding stuffs, assume digestible protein, carbohydrates and fats from different sources to be of substantially equal nutritive value, Kellner's figures take account of those differences in the values of nutrients as sources of energy which have been revealed by recent investigations and express the needs of animals in this respect in what are, in fact, although not in form, net energy values. In addition, his standards regard only the true protein as of value and they reduce somewhat the very high requirements of fattening animals for protein as postulated by early authors. In other respects, however, they are on substantially the plan of the Wolff-Lehmann standards, i.e., they are in form prescriptions or recipes for rations for different purposes.

\section{§ 2. FeEd Requirements}

794. Limitations of feeding standards. - From the outset it was necessary to guard against misconceptions arising from the very definite form in which the feeding standards were presented. Their authors insisted from the first that they were intended as general guides and not as fixed rules to be rigidly adhered to. But the human mind craves a recipe and there has been a persistent tendency to substitute for the study of the principles of nutrition a series of exercises in applied arithmetic. Others again, perhaps misled by the name, have interpreted the feeding standards as representing a physiological demand of the animal; - a sort of moral ideal in feeding, to be aimed at, but concerning which concessions have to be made to human fallibility and the pressure of circumstances.

The difficulty inherent, more or less, in all forms of feeding standards, but especially in the earlier ones, is that they fail to take sufficient account of the fact that the feeding of farm animals is an economic problem. A manufacturer would not buy some average amount of raw material which might be regarded as the norm for his business, irrespective of the capacity of his own factory or of the market for the finished product. When high prices prevailed he might find it profitable to handle a maximum amount of raw material and so to reduce the 
percentage of his overhead costs, even at the risk of some loss of efficiency in the manufacturing process. In the contrary case, he might find it necessary to run considerably below his maximum capacity in order to tide over a bad season. In somewhat similar fashion it is necessary for the stock feeder to adapt his rations to the economic conditions under which he works. While the animal cannot be handled like a machine in a factory, nevertheless, as has appeared in previous chapters, it shows a large degree of flexibility in its requirements both quantitatively and qualitatively. No single fixed standard is either physiologically or economically necessary for productive feeding.

795. The feeder's problem. - As the feeder looks at his animals, the problem which they present is a threefold one.

First, he must furnish them with sufficient repair material and energy to keep the body machinery running, i.e., he must supply a maintenance ration. The requirements for this purpose, although subject to individual variations, have been worked out with some degree of accuracy and this part of his problem is relatively simple.

Second, in addition to a maintenance ration, he must supply his animals with the amounts of matter and of energy necessary for the production of the meat, milk or work which he desires them to yield. Here his task is much less simple.

It is evident in the first place, as has been emphasized in previous chapters, that the producing capacity of the animal is the prime factor in the problem. No argument is necessary to show that a cow producing thirty pounds of milk daily requires a greater addition to her maintenance ration than does one having a capacity of only fifteen pounds, or that a steer which can gain three pounds daily needs more surplus feed than one capable of making only one pound of gain. Good business economy demands that the better animal be given feed sufficient in amount and kind to permit its producing capacity to be fully utilized, thus reducing the relative cost of maintenance. On the other hand, it would be an obvious waste to give a mediocre or poor producer a ration adequate for two or three times the production of which it is capable.

Third, the feeder, like the manufacturer, must adapt his practice to market conditions. As prices of feeding stuffs fall and those of animal products rise, he will tend to feed more in- 
tensively, but here he encounters the law of diminishing returns. The dairy cow affords, perhaps, the most striking illustration of this. An increase in the quantity of her feed above a moderate ration may be expected to cause an increase in milk secretion but at the same time an increasing proportion of the extra feed will be diverted to fattening (606-610). Similarly, a rather small protein supply appears adequate to support moderate milk production but larger amounts seem to act as a stimulus to the activity of the milk glands and to increase the yield of milk (603), but presumably at a diminishing rate. The dairyman's problem is to utilize these stimulating effects up to the point at which the increase in yield is offset by the added cost of the ration, and the solution of this problem requires experience and good judgment and is one in which little aid can be afforded by feeding standards.

What is so emphatically true of dairy feeding applies in. greater or less degree to all forms of animal production. Even though there may be no decrease in the utilization of the feed in the strict physiological sense, diminished digestibility, stimulation of incidental bodily activity; or changing composition of increase tend to make heavy rations or high protein rations relatively less effective than more moderate ones.

What the feeder needs in order to meet this situation intelligently is not so much a fixed standard, or group of standards, as a knowledge of the amount and kind of feed required under various conditions for the manufacture of a unit of producta pound of increase in live weight, for example, or a pound of milk of a given quality. To the extent to which this information is available he can, knowing his animals, proportion the feed to the capacity of each and thus go far toward securing the most efficient production. It appears desirable, therefore, to assume a somewhat different point of view from that which has largely prevailed in the past and to substitute for the conception of feeding standards the modified conception of feed requirements.

796. Feed requirements. - Haecker ${ }^{1}$ appears to have been the first to apply this idea to milk production and to formulate the feed requirements for the production of a pound of milk

${ }^{1}$ Minn. Expt. Sta., Bul. 79 (1903), pp. I04-107. 
of different grades. As modified by his subsequent experiments, this statement of requirements ${ }^{1}$ has become known as the Haecker standard, although, strictly speaking, it is not a standard in the older sense. The writer ${ }^{2}$ subsequently published a tentative statement of the protein and energy requirements per pound of milk containing four per cent of fat and illustrated the computation of rations on this basis, without, however, attempting similar estimates for other grades of milk. Later Woll and Humphrey, ${ }^{3}$ Savage ${ }^{4}$ and Eckles ${ }^{5}$ have adopted various forms of the same conception. Henry and Morrison ${ }^{6}$ have included in their modified Wolff-Lehmann standards Haecker's requirements for milk production and also similar data, based on unpublished results by the same experimenter, for growing fattening steers, and have also widened somewhat the range of the standards for other purposes and introduced minimum and maximum figures.

On the other hand, however, all of the foregoing requirements and standards, with the exception of Eckles', are expressed in terms of digestible nutrients and are therefore open to the criticism of ignoring differences in the relative values of nutrients from different sources.

797. Requirements in terms of protein and energy. The several chapters of Part III were devoted primarily to a consideration of the feed requirements of farm animals in terms of digestible protein and of net energy. In the case of maintenance, these requirements may be regarded as to a certain degree fixed and capable of computation upon the basis of the size of the animal, being related either to its weight or to its body surface. In the case of productive feeding, on the contrary, the obvious method of comparison is that of feed (in excess of maintenance) with yield, and an attempt was therefore made to estimate the feed requirements per unit of product. The results of these estimates have been brought together in Tables I-VI of the Appendix, which include also for convenience estimates of the total requirements per day and head for normal growth at different weights and ages.

1 Minn. Expt. Sta., Bul. 140, p. 56.

2 U. S. Dept. Agr., Farmers' Bul. 346 (rgog), pp. r9-25.

${ }^{3}$ Wis. Expt. Sta., Research Bul. 13. ${ }^{4}$ N. Y. (Cornell) Expt. Sta., Bul. 323.

${ }^{5}$ Mo. Expt. Sta., Research Bul. 7.

${ }^{6}$ Feeds and Feeding, r 5 th Ed., p. 669. 
That the requirements there tabulated resemble more or less the earlier feeding standards and share to some degree their limitations is undeniable and likewise unavoidable. No finite number of formulas, however accurate, can cover specifically all the various conditions of practice, and in particular it is scarcely possible for them to include any consideration of the financial aspects of the matter. The most that seems possible is, first, to formulate the average requirements under ordinary circumstances and then to indicate as definitely as present knowledge permits, as has been attempted in Chapters VII$\mathrm{XIV}$, the influence of various conditions in modifying these requirements. The difference between the older and newer formulas lies far more in the point of view than in the completeness or exact numerical accuracy of the figures and neither can be utilized as infallible recipes which shall spare the user the trouble of observing and thinking.

798. Defects of the tables. - That not a few of the estimates of feed requirements contained in the Tables of the Appendix rest on quite meager data is apparent from the discussions in Part III. This is particularly true of the requirements for growth, as will be evident from a study of Chapter XI. To a somewhat less degree the same is true of those for milk production, the energy requirements in particular being based on an hypothesis regarding the cause of the higher net energy values for milk production $(593,605)$ which has not yet been submitted to experimental test.

The estimates of the protein requirements are particularly unsatisfactory for two reasons.

In the first place, they virtually assume all proteins to be of equal value. That such is not the case has been repeatedly stated in previous pages, but it has also been shown that present knowledge of the constitution of the vegetable proteins and of the amino acid requirements of the body is insufficient to serve as the basis of a more satisfactory system.

In the second place, there has been very little systematic investigation of the minimum protein requirements of farm animals for different purposes or of the percentage of different proteins capable of utilization for the production of body protein or of milk protein. The requirements given in the tables are, to a large extent, based on observations in practice, 
and it is quite possible that they may, with safety, be considerably reduced in some instances.

Furthermore, the tables of the Appendix include no estimates of the ash requirements. This is not because the latter are unimportant, for it is not improbable that they may at times be a controlling factor, but simply because study in this field has not progressed far enough to permit of their formulation.

But while it has seemed desirable to emphasize here certain defects of the feeding requirements as formulated, as a precaution against their uncritical use, they are by no means to be rejected as worthless but are capable of affording valuable aid to the intelligent feeder. By their use he can get a general idea of the feed requirements of his animals and can compute rations which will approximately supply the requisite amounts of protein and energy. His ability as a feeder will be shown, first, in his power to estimate the conditions which will modify the feed requirements of his particular animals and cause his feeds to vary from the average, and second, in the skill with which he can interpret the daily results and modify his feeding in accordance with them.

799. Dry matter. - The amount of dry matter which the ration contains must also be taken into consideration. The total volume of feed which an animal requires, although rather variable, has its limits. In computing rations the most convenient indication of the bulk of the feeds is the percentage of dry matter shown in the first column of Tables VII, VIII and IX of the Appendix. In very general terms it may be said that a rooo-pound ruminant should be given from 20 to 30 pounds of dry matter per day, 25 pounds being perhaps a fair average, while for the horse smaller amounts will be appropriate.

An examination of the tables shows that concentrated feeding stuffs contain much more protein and energy in proportion to their dry matter than do the forage crops. Evidently, then, in heavy feeding, where the purpose is to give the animal all the feed possible, the ration should consist as largely as practicable of concentrated feeding stuffs, because only in that way can the required amount of nutriment be obtained without unduly increasing the bulk of the ration. In light feeding, on the contrary, 
roughage may predominate, because it is usually relatively cheaper and can supply the required amount of feed in a bulk which the animal can consume.

\section{§ 3. Method of Computation ${ }^{1}$}

The examples given on the following pages are intended simply as illustrations of the method of using the tables of the Appendix and not as model rations. Limitations of space forbid the multiplication of examples, but the reader who grasps the method will have no serious difficulty in applying it to his own conditions, while facility will be acquired with surprising rapidity by practice. It will be observed that the form of these tables and the methods of computation do not differ materially from those which have been used for many years in computing rations on the basis of "digestible nutrients," although the significance of some of the figures is different. It may be added that the digestible protein in the tables is true protein - that is, it does not include the non-protein. Consequently the percentages, as well as the amounts estimated in the rations on succeeding pages, are somewhat smaller than in the older tables.

800. Total feed required. - A bunch of "feeders" 2 to 3 years old, averaging rooo pounds per head and in better than average condition are to be fattened on clover hay and cornand-cob meal. Such cattle, if of good grade, should weigh I 400 pounds each when ready for market and should not require over 200 days to make the gain of 400 pounds. They should therefore make an average gain of 2 pounds per day.

It may be estimated (Table III) that a gain of I pound live weight by animals of this grade will require about 3.5 Therms of net energy value in the feed; for a daily gain of 2 pounds, therefore, the requirement would be 7 Therms. To this must be added the maintenance requirement, which will increase as the animals grow heavier. For the average weight of $\mathrm{I} 200$ pounds it is sufficiently accurate to use the maintenance requirement computed (Table I) for 1250 pounds, viz., 7 Therms. This

1 The contents of this section are reproduced by permission of the Honorable Secretary of Agriculture, from Bulletin No. 459 of the U. S. Department of Agriculture, prepared by the writer. 
makes the total net energy requirement per day i4 Therms on the average of the whole feeding period.

If we assume that 2 pounds of grain will be fed for each pound of hay, it is easy to compute from the figures in the last column of Table VII the amount of feed required to supply i4 Therms of net energy, as follows:-

THERMS

In roo pounds of average clover hay . . . . . . . 38.68

In 200 pounds of corn-and-cob meal . . . . . . . 15 I.60

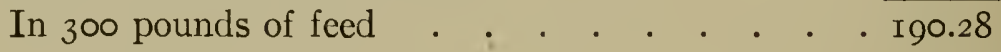

In I pound of feed . . . . . . . . . . $\quad .634$

To supply I4 Therms requires I4 $\div 0.634=22.08$ pounds of total feed, consisting of 7.36 pounds of clover hay and $\mathrm{I} 4.72$ pounds of corn-and-cob meal, or, in round numbers, $7 \frac{1}{2}$ pounds of hay and 15 pounds of meal.

This, of course, represents the average ration for the whole feeding period. At the beginning the feed will naturally be lighter and consist to a larger extent of hay, while the amount of feed, and especially the proportion of grain, will be gradually increased until, toward the end of the feeding, the animals are consuming all the grain they will take, with only enough hay to insure the necessary bulk and proper digestion. Naturally, too, the form in which the corn is given will usually be varied in the course of the feeding.

801. Improvement of a ration. - In the foregoing example it was assumed that the feeding stuffs to be used had been decided upon and attention was directed simply to the quantity required. Let us now take up the question from the other end and see whether a study of the ration may not yield some suggestion of possible improvement.

According to Table VII, clover hay and corn-and-cob meal, respectively, contain in roo pounds:-

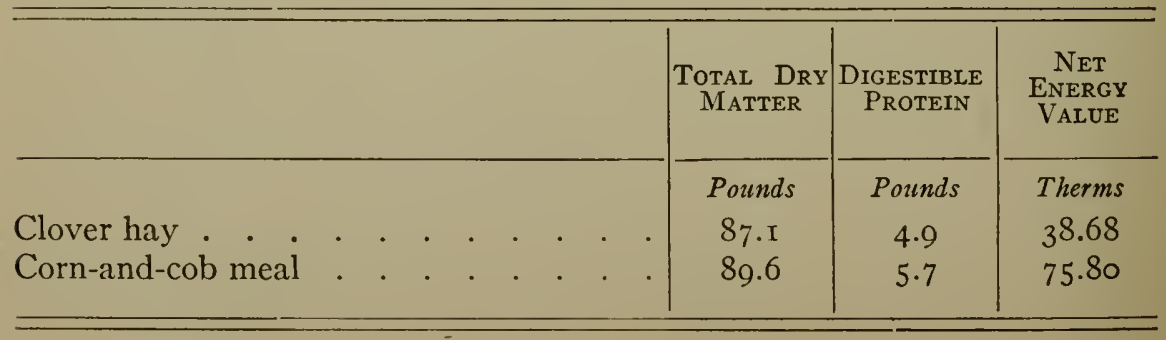


The $7 \frac{1}{2}$ pounds of clover hay in the ration will evidently contain :-

$$
\begin{aligned}
87.1 \times 0.075 & =6.53 \text { pounds of dry matter. } \\
4.9 \times 0.075 & =0.37 \text { pound of digestible protein. } \\
38.68 \times 0.075 & =2.90 \text { Therms of net energy value. }
\end{aligned}
$$

A precisely similar computation for the corn-and-cob meal gives the following results:-

$$
\begin{aligned}
89.6 \times 0.15 & =13.44 \text { pounds of dry matter. } \\
5.7 \times 0.15 & =0.85 \text { pound of digestible protein. } \\
75.8 \times 0.15 & =11.37 \text { Therms of net energy. }
\end{aligned}
$$

\begin{tabular}{|c|c|c|}
\hline $\begin{array}{l}\text { Total DRy } \\
\text { MatTER }\end{array}$ & $\begin{array}{l}\text { Digestible } \\
\text { ProteIN }\end{array}$ & $\begin{array}{c}\text { NET } \\
\text { ENERGY } \\
\text { VALUE }\end{array}$ \\
\hline Pounds & Pounds & Therms \\
\hline 6.53 & 0.37 & 2.90 \\
\hline I3.44 & .85 & II.37 \\
\hline I9.97 & I. 22 & I 4.27 \\
\hline
\end{tabular}

Adding these amounts, we find that the total ration contains :

Clover hay, $7 \frac{1}{2}$ pounds .

Corn-and-cob meal, 15 pounds

Total

The quantity of energy, of course, corresponds with that estimated to be necessary, because the amounts of feed were fixed upon on that basis. We observe, however, that the amount of digestible protein in the ration is less than that estimated in Table IV to be needed by beef cattle of this age and weight. A ration like the above might produce fair gains, but it probably would fail to take full advantage of the capacity of such cattle for growth and the gain would most likely fall below that which was anticipated. An increase in the protein might be expected to make the ration more efficient.

To make any marked change in the ration in this respect, it is evident that we must introduce into it some feed much richer in protein than either of those composing it. On consulting Table VII it is evident that what we need is one of the by-product feeds, like gluten feed or meal, the oil meals, etc., and also that only a small amount of one of these will be needed to effect a marked change in the ration. Thus, if we substitute 2 pounds of old-process linseed meal for 2 pounds of the corn-and-cob meal, the ration will foot up as follows:- 


\begin{tabular}{|c|c|c|c|c|}
\hline & & $\underset{\text { Total DRy }}{\text { MatTER }}$ & $\begin{array}{c}\text { Digestible } \\
\text { Protein }\end{array}$ & $\begin{array}{c}\text { NET } \\
\text { ENERGY } \\
\text { VALUE }\end{array}$ \\
\hline & & Pounds & Pounds & Therms \\
\hline Clover hay, $7 \frac{1}{2}$ pounds . . . & - $\cdot$ & 6.53 & 0.37 & 2.90 \\
\hline Corn-and-cob meal, I3 pounds . . & . . & II .65 & .74 & 9.85 \\
\hline Old-process linseed meal, 2 pounds . & . & $\mathrm{I} .82$ & .57 & 1.78 \\
\hline Total . $. \quad . \quad . \quad . \quad . \quad . \quad . \quad$. & . . & $\overline{20.00}$ & $\overline{1.68}$ & $\overline{I 4.53}$ \\
\hline
\end{tabular}

Thus at a comparatively small additional expense we are able to improve the ration materially by adding the lacking protein, and there is little doubt that the improved ration would produce a more rapid gain and, under ordinary conditions, a more profitable one as well, either by increasing the total gain or shortening the feeding period.

802. Computing a ration from given feeding stuffs. - There are available for a dairy herd field-cured corn forage (including the ears), clover hay, corn meal, wheat bran and gluten feed. Table VII shows that these feeding stuffs, if of good average quality, will furnish in roo pounds:-

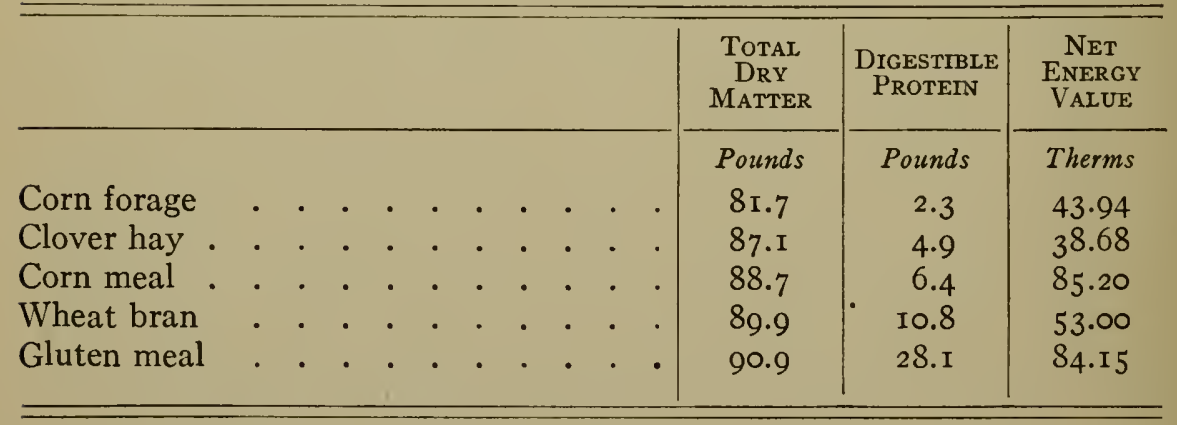

The cows average $85^{\circ}$ pounds per head and have produced in previous years an average of 20 pounds of milk per day testing 4 per cent of fat. According to Table I, the maintenance requirement of such animals per day and head would be approximately :-

Digestible protein . . . . . . . 0.43 pound

Net energy . . . . . . . . . . 5.40 Therms 
For the production of 20 pounds of 4 per cent milk there would be needed, according to Table $\mathrm{V}$ :-

Digestible protein $(0.05 \times 20)$. . . . 1.0 pound

Net energy $(0.27 \times 20)$. . . . 5.4 Therms

The total feed requirements per day and head are therefore:

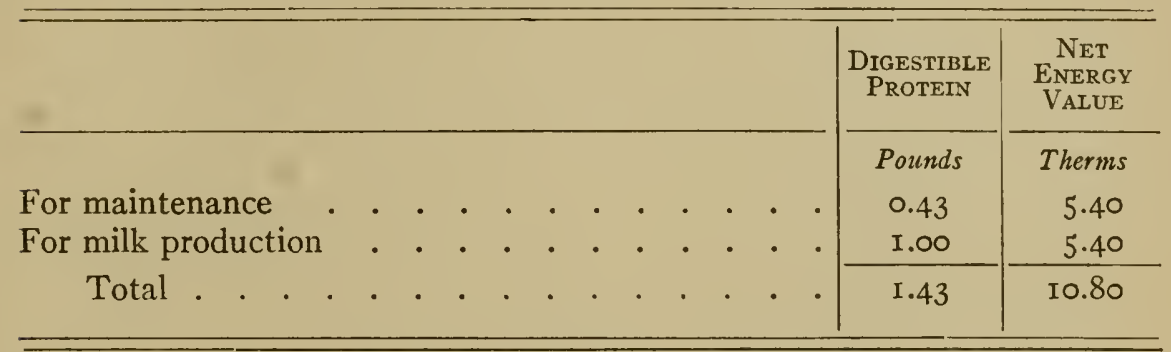

The problem, then, is to find a mixture of the available feeding stuffs which will yield these amounts of digestible protein and of energy, and which shall have a suitable bulk.

The first step in the construction of a ration is to fix upon the amounts of coarse fodders. It is usually desirable to use as large a proportion of these as possible, since they are usually cheaper sources of feed than grain. On the other hand, the amount of them which an animal can consume is limited. Much depends upon the individual animals, and the proper amount can only be told by trial, but we should probably aim to get from I 2 to 14 pounds of dry matter in the form of coarse fodder. Corn forage being a cheap feeding stuff, we shall naturally use this freely, with probably some hay for variety. By a little trial, we find that ro pounds of corn forage and 6 pounds of clover hay will give us I3.4 pounds of dry matter and the amounts of digestible protein and of energy shown below :-

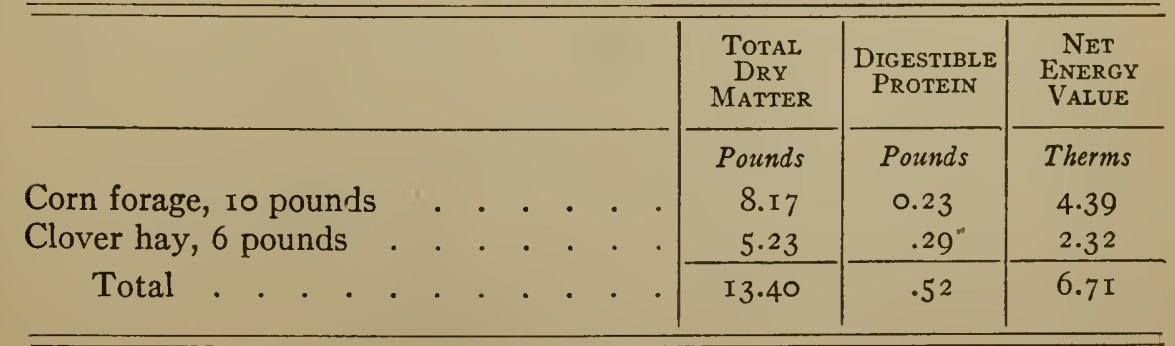


To this we have to add sufficient grain to bring the ration up to the requirement. The proper amount we must ascertain by trial. We will take, at a venture, 4 pounds of corn meal and 2 pounds of wheat bran. Adding this to the ration we have:-

Corn forage, ro pounds Clover hay, 6 pounds Corn meal, 4 pounds Wheat bran, 2 pounds

Total

\begin{tabular}{|c|c|c}
$\begin{array}{c}\text { Total } \\
\text { Dry } \\
\text { Matter }\end{array}$ & $\begin{array}{c}\text { Digestible } \\
\text { Protein }\end{array}$ & $\begin{array}{c}\text { Net } \\
\text { ENERGy } \\
\text { VALUE }\end{array}$ \\
\cline { 1 - 2 } Pounds & Pounds & Therms \\
8.17 & 0.23 & 4.39 \\
5.23 & .29 & 2.32 \\
3.55 & .26 & $3.4 \mathrm{I}$ \\
$\mathrm{I.80}$ & .22 & $\mathrm{I.06}$ \\
\hline $\mathrm{I} 8.75$ & $\mathrm{I.00}$ & $\mathrm{I} \mathrm{I} . \mathrm{I} 8$ \\
\hline
\end{tabular}

Comparing these totals with the requirement as computed, we find that the ration is ample as regards energy, but considerably low in digestible protein. The rather low figure for dry matter shows that more feed may be added to the ration if desirable, but the total for net energy makes it evident that what is needed is not more feed, but feed of a different composition, supplying more protein along with rather less energy. Gluten meal answers this requirement, and substituting 2 pounds of it for 2 pounds of corn meal gives a ration which, while still a trifle high in energy, agrees as closely as necessary with the computed requirements. Thus :-

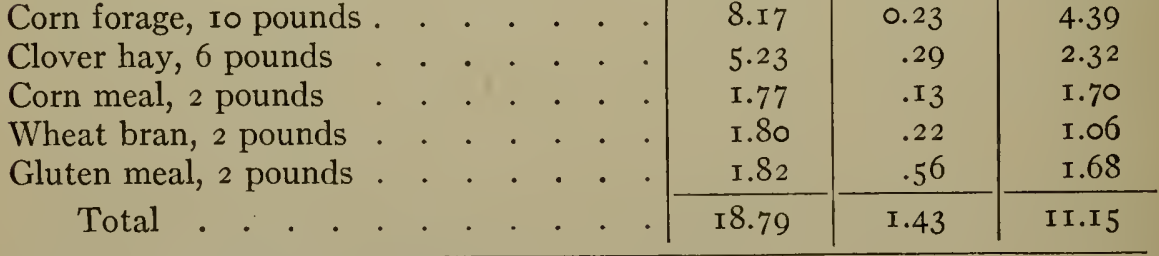

This ration corresponds with the average requirement of the whole herd, since it is based on its average performance. It hardly need be said that it should be modified to suit the requirements and capacities of the individual cows, the heavy milkers getting more and the lighter ones less. 
By proceeding in this manner, with a little patience we can usually get a ration corresponding as closely as is necessary to the requirement, provided the feeds available admit of it. With a little experience one very soon learns to guess pretty closely, and with some practice the computations become very easy. An exact agreement with the requirement need not be sought for, since in practice the composition of the feeds will probably vary more or less from the average of the tables.

803. The choice of feeding stuffs. - When, as in the last example, feeding stuffs must be purchased in order to get the desired relation between the protein and the energy of the ration, it is evident that often a wide range of choice may be offered. In such a case the question at once arises which of the various feeds available is it most economical to purchase, it being evident, of course, that this is not necessarily the one offered at the lowest price.

No simple method of determining this point is possible, because, as we have seen, the food serves two entirely distinct purposes in the body. Sometimes the supply of protein is the specially important point, and in other cases what is needed is a supply of energy without special reference to whether its source be protein or non-nitrogenous material. Consequently, the relative values of two feeding stuffs may vary under different circumstances. Some writers have based their comparisons of the values of by-product feeds solely upon their content of protein, for the reason that such feeds are often bought especially to supply this ingredient while the fats and especially the carbohydrates are usually produced in abundance upon the farm. They regard that purchased feeding stuff as the most economical which furnishes a pound of digestible protein at the lowest cost, ignoring any value in the other ingredients. It is obvious, however, that this is a one-sided view. The other ingredients have a value, and this is especially true in the case of a feeder who buys a considerable part of his grain supply and depends upon it as a source of energy as well as of protein. The method of comparison illustrated in the following pages is based primarily upon the cost per unit of energy because this is on the whole the most important function of the feed, but the method takes account also of the amount of protein present. 
Let us suppose the following feeding stuffs are available to a dairyman at the prices named:-

Prices of feeds per ton

Oats (40 cents per bushel) . . . . . . . $\$ 25$

Corn meal . . . . . . . . . . . . 25

Wheat bran . . . . . . . . . . . . 2 I

Wheat middlings (flour) . . . . . . . 24

Dried brewers' grains . . . . . . . . . . 23

Gluten meal . . . . . . . . . . . . . 27

Cotton seed meal (prime) . . . . . . . . 30

Old-process linseed meal . . . . . . . . . 33

The supply of coarse feed on the farm is sufficient to furnish each animal per day 32 pounds of silage and 8 pounds of clover hay; the cows average Iooo pounds each and may be expected to produce per day about 24 pounds of milk testing 4.5 per cent fat.

The first step is to compute, in precisely the same way as in the previous example, the estimated requirements of these cows per day as follows :-

For maintenance

For 24 pounds of milk:

Protein $24 \times 0.052$

Net energy $24 \times 0.29$

Total requirement

The amount of silage and clover hay available will furnish, according to Table VII, the following amounts of dry matter, digestible protein, and net energy value:-

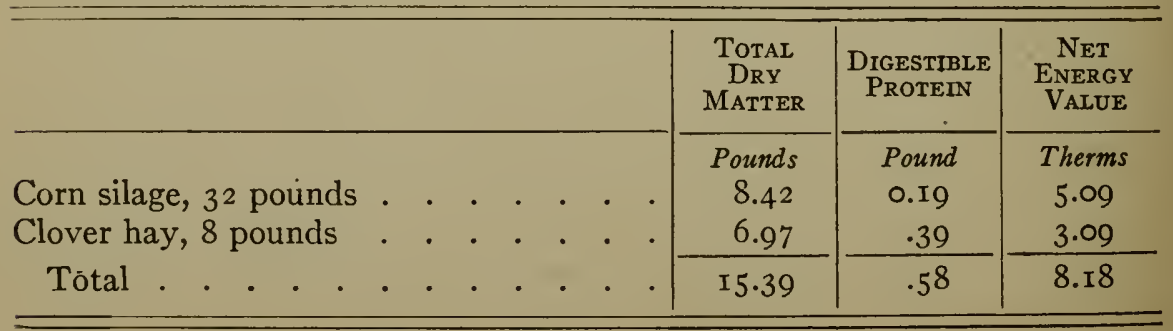


The question now is what feeding stuffs is it most economical to buy (or to refrain from selling if in stock) to complete the ration. The first step in deciding this question is to compare the various feeds as sources of energy and see which one furnishes a unit of net energy value at the lowest price. This computation gives the following results :-

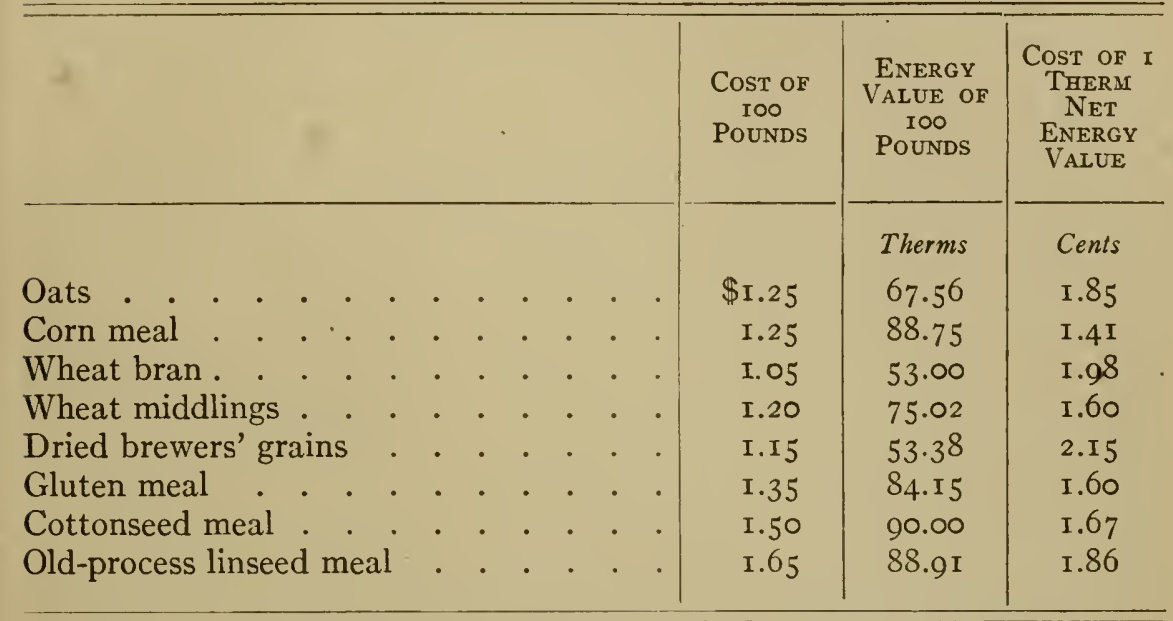

Evidently, if it were simply a question of supplying energy to the animals, we should use corn meal, since that supplies a unit of energy at a much lower price than any of the other feeding stuffs. If it were thought desirable to add variety to the ration, wheat middlings would obviously be our next choice.

It is evident, however, without going through the labor of computation, that while corn meal and wheat middlings may be used in the ration, neither will supply enough protein if used exclusively. Of the available feeding stuffs which are rich in protein and which may therefore serve to balance the deficiency of this ingredient, gluten meal is relatively the cheapest, and cottonseed meal comes next. While the difference between the two is not great, we shall naturally try the cheaper one. It is not difficult to determine by a few trials that $2 \frac{1}{2}$ pounds of corn meal and $3 \frac{1}{2}$ pounds of gluten meal, in addition to the coarse fodder available, will give a ration corresponding very closely to the requirements, as the following table shows :- 


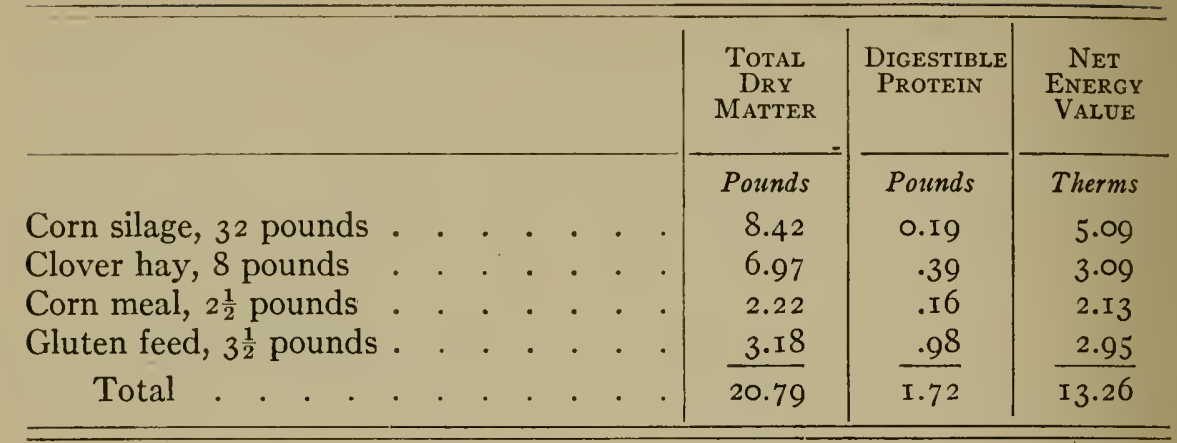

This ration shows as close an agreement with the computed protein requirement as could be desired, but contains a slight surplus of energy. The comparatively low figure for dry matter indicates that more coarse fodder might have been used had it been available, with the probable effect of cheapening the ration. As it is, we have used the feeds relatively lowest in price and apparently have a very economical ration.

Cottonseed meal, however, is nearly as cheap as a source of energy as gluten meal, while it contains considerably more protein. It seems worth while, therefore, to see whether it may not be possible to secure the necessary protein more cheaply by using a smaller amount of the former feed in place of the gluten meal. Three pounds of cottonseed meal will supply almost exactly the same amount of protein as $3 \frac{1}{2}$ pounds of gluten meal. Making this substitution, the ration stands as follows:-

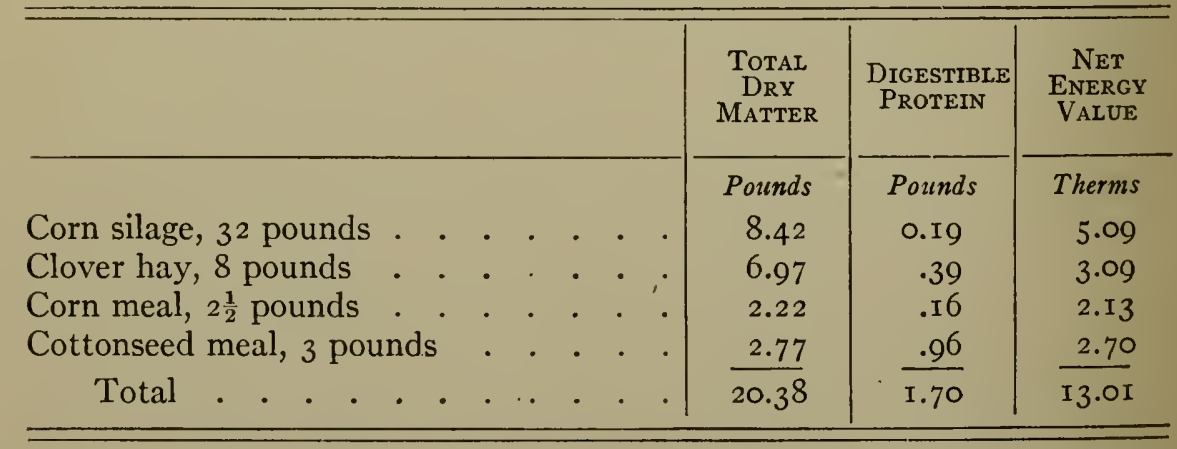

This ration agrees with the computed requirements even better than the previous one, while a simple comparison shows that it is a trifle cheaper. The grain portion of the two rations costs as follows:- 


\begin{tabular}{|c|c|c|c|c|c|c|c|c|c|c|c|c|}
\hline & & & & & & & & & & & $\begin{array}{c}\text { FIRST } \\
\text { RATION }\end{array}$ & $\begin{array}{l}\text { SECOND } \\
\text { RATION }\end{array}$ \\
\hline & & & & & & & & & & & Cents & Cents \\
\hline Corn meal & & . & & . & & & & & & . & 3.13 & 3.13 \\
\hline Gluten meal & . & . & . & . & . & . & . & . & . & . & 4.73 & - \\
\hline Cottonseed meal & . & - & . & . & . & • & . & . & . & . & - & $\underline{4.20}$ \\
\hline Total & . & . & . & . & . & . & . & . & . & . & 7.86 & 7.33 \\
\hline
\end{tabular}

It thus appears that the ration made up with the somewhat more expensive cottonseed meal is actually the cheaper. The difference, to be sure, is small, yet for 30 cows fed for 200 days it would amount to $\$ 30$. Such a difference is only likely to be found, however, when, as was assumed in this instance, some feed very high in protein can be had at a relatively cheap rate. In general, it may be said that when there are no very marked differences in the cost of a Therm of energy value in the feeding stuffs constituting the bulk of the ration, that one of the various high-protein feeds which supplies energy at the lowest cost should ordinarily be used, although it is always wise to check up this point, as in the example just given.

804. The compounding of rations. - While in the foregoing examples an exact daily ration is computed, it would, of course, be utterly impracticable in most cases to weigh out separately each day's ration for each animal. Individual weighings of feeds at intervals would often yield valuable information and might profitably be undertaken, but for the ordinary routine of feeding, simpler methods must be used.

When practicable, the grain feed may be advantageously mixed in advance in the desired proportions in as large quantities as the storage capacity available and the proper preservation of the materials will permit. Where facilities are available, the whole amount of grain required for all the animals may be weighed out daily, or even for each feeding, without much additional labor. In distributing the grain to the individual animals, regard, of course, should be paid to their productive capacity and their individual peculiarities. The ration as computed is for the average animal. The skill of the feeder is shown in adapting it in quality and in amount to the individual. 
Doubtless individual weighings at intervals, as already suggested, would be useful as a control on the accuracy of the distribution.

The weighing of coarse fodder is usually a more difficult problem on account of its bulk. When, however, silage or cut fodder is handled in trucks, the matter is still comparatively simple. Long fodder, on the contrary, is not readily weighed. Nevertheless, even here an occasional weighing, if practicable, as a control upon the feeding, is very desirable.

In all these and similar matters common sense is necessary. The computed ration expresses the best estimate that can be made of the actual average requirements, but it is at best more or less of an approximation. It would be foolish, therefore, to seek extreme exactness in realizing it or to go to more expense in the weighing and apportioning of the feed than the saving in the latter would amount to. The scale upon which the feeding is conducted will play an important part. Where scores or hundreds of animals are being fed, an exactness may profitably be sought which would be absurd in the case of two or three animals. Finally, it should be remembered that these computed rations are guides and not recipes. They may aid the feeder in wisely using the resources at his command, but they cannot take the place of experience and good judgment. 
APPENDIX 



\section{APPENDIX}

\section{ESTIMATED PROTEIN AND ENERGY REQUIREMENTS OF FARM ANIMALS}

Compare Chapter XVIII, § 2 .

Table I. - Maintenance Requirements of Cattle and Horses, Per DAY AND HEAD

\begin{tabular}{|c|c|c|c|c|c|}
\hline \multirow{2}{*}{$\begin{array}{c}\text { Live } \\
\text { WEIGHT }\end{array}$} & \multicolumn{2}{|c|}{ Cattle } & \multicolumn{3}{|c|}{ HoRSES } \\
\hline & $\begin{array}{l}\text { Digestible } \\
\text { Protein }\end{array}$ & Net Energy & $\begin{array}{l}\text { Digestible } \\
\text { Protein }\end{array}$ & Net Energy & $\begin{array}{l}\text { Metaboliz- } \\
\text { able Energy } 1\end{array}$ \\
\hline Pounds & Pounds & Therms & Pounds & Therms & Therms \\
\hline I 50 & 0.08 & г.69 & 0.08 & I.I6 & $3 \cdot 36$ \\
\hline 250 & O.I3 & 2.38 & 0.13 & I. 63 & 4.72 \\
\hline 500 & 0.25 & 3.78 & 0.25 & $2.5^{8}$ & $7 \cdot 50$ \\
\hline 750 & 0.38 & 4.95 & 0.38 & 3.39 & 9.82 \\
\hline 1000 & 0.50 & 6.00 & 0.50 & 4.10 & I I.90 \\
\hline $125^{\circ}$ & 0.63 & 6.96 & 0.63 & 4.76 & I3.80 \\
\hline$I_{5} 50$ & 0.75 & 7.86 & 0.75 & $5 \cdot 37$ & I5.59 \\
\hline
\end{tabular}

Table II. - Maintenance Requirements of Sheep and Swine, per - Day and Head

\begin{tabular}{|c|c|c|c|c|}
\hline \multirow{2}{*}{$\underset{\text { WIVE }}{\text { WIGHT }}$} & \multicolumn{2}{|c|}{ SHEEP } & \multicolumn{2}{|c|}{ SWINE } \\
\hline & Digestible Protein & Net Energy & Digestible Protein & Net Energy \\
\hline Pounds & Pounds & Therms & Pounds & Therms \\
\hline 20 & $0.01 \mathrm{I}$ & 0.27 & 0.010 & 0.43 \\
\hline 40 & 0.022 & 0.43 & 0.019 & 0.68 \\
\hline 60 & 0.033 & 0.56 & 0.029 & 0.89 \\
\hline 80 & 0.044 & 0.68 & 0.038 & 1.08 \\
\hline 100 & 0.055 & 0.79 & 0.048 & I. 25 \\
\hline 120 & 0.066 & 0.89 & $0.05^{8}$ & I. $4 \mathrm{I}$ \\
\hline 140 & 0.077 & 0.99 & 0.067 & I. $5^{6}$ \\
\hline 160 & 0.088 & I.09 & 0.077 & $1.7 \mathrm{I}$ \\
\hline I80 & 0.099 & I.I 7 & 0.086 & I. 85 \\
\hline 200 & O.IIO & I. 25 & 0.096 & I.99 \\
\hline
\end{tabular}

${ }^{1}$ To support heat production of animal at rest (387). 
Table III. - Requirements for Fattening with no Considerable - Growth - All Species - in Addition to the Maintenance REQUIREMENT

\begin{tabular}{|c|c|c|c|}
\hline \multirow{2}{*}{ - } & \multicolumn{2}{|c|}{$\begin{array}{l}\text { Per Pound of Increase in } \\
\text { LIVE WeIGHT, IN ADdition } \\
\text { To the MAINTENANCE RE- } \\
\text { QUIREMENT }\end{array}$} & \multirow{2}{*}{$\begin{array}{l}\text { Digestible PROTEIN } \\
\text { PER IOOO LB. LiVE } \\
\text { WEIGHT, IN ADDI- } \\
\text { TION TO THE MAIN- } \\
\text { TENANCE REQUIRE- } \\
\text { MENT }{ }^{2}\end{array}$} \\
\hline & $\begin{array}{l}\text { Digestible } \\
\text { Protein }^{1}\end{array}$ & Net Energy & \\
\hline & Pounds & Therms & Pounds \\
\hline In early stages . . . & $0.1_{5}$ & 2.50 & \\
\hline In late stages & 0.05 & 4.00 & $0.25-0.75$ \\
\hline $\begin{array}{l}\text { Average for entire fatten- } \\
\text { ing period }\end{array}$ & 0.10 & & 0.25 .0 .15 \\
\hline
\end{tabular}

Table IV. - Requirements for Growth with no Considerable FATTENING

a. Per Pound of Increase in Live Weight, in Addition to the Maintenance Requirement

\begin{tabular}{|c|c|c|c|c|}
\hline \multirow[t]{2}{*}{$\mathrm{AGE}$} & \multicolumn{2}{|c|}{ Cattle (aNd SheEP?) } & \multicolumn{2}{|c|}{ SwINE } \\
\hline & $\begin{array}{c}\text { Minimum of } \\
\text { Digestible } \\
\text { Protein }{ }^{3}\end{array}$ & Net Energy & $\begin{array}{c}\text { Minimum of } \\
\text { Digestible } \\
\text { Protein }^{3}\end{array}$ & Net Energy \\
\hline Months & Pounds & Therms & Pounds & Therms \\
\hline $0-1$ & 0.23 & I. I 70 & 0.17 & 0.65 \\
\hline$I-2$ & 0.22 & 1.272 & 0.16 & 0.77 \\
\hline $2-3$ & 0.22 & I. 374 & 0.15 & 0.88 \\
\hline $3-6$ & $0.2 \mathrm{I}$ & 1.680 & O.I4 & I.23 \\
\hline $6-9$ & $0.2 \mathrm{I}$ & I.986 & 0.12 & 1.59 \\
\hline $9^{-12}$ & 0.20 & 2.292 & O.IO & 1.96 \\
\hline I $2-18$ & 0.18 & 2.904 & 0.07 & 2.66 \\
\hline I 8-24 & 0.16 & 3.000 & 一 & 一 \\
\hline $24-30$ & O.I4 & 3.250 & - & - \\
\hline
\end{tabular}

1 Estimated from protein content of increase.

2 Estimated from experiments on fattening (456).

${ }^{3}$ Estimated protein content of increase. 
APPENDIX

b. Per Day and Head, Including Maintenance

(s) Cattle

\begin{tabular}{|c|c|c|c|c|c|c|}
\hline \multirow{2}{*}{ AgE } & \multicolumn{3}{|c|}{ BeEF BreEds } & \multicolumn{3}{|c|}{ DAIRY BREEDS } \\
\hline & $\begin{array}{c}\text { Live } \\
\text { Weight }\end{array}$ & $\begin{array}{c}\text { Digestible } \\
\text { Protein }{ }^{1}\end{array}$ & $\begin{array}{c}\text { Net } \\
\text { Energy }\end{array}$ & $\begin{array}{c}\text { Live } \\
\text { Weight }\end{array}$ & $\begin{array}{c}\text { Digestible } \\
\text { Protein } 1\end{array}$ & $\begin{array}{c}\text { Net } \\
\text { Energy }\end{array}$ \\
\hline Months & Pounds & Pounds & Therms & Pounds & Pounds & Therms \\
\hline I & 125 & 0.70 & 3.7 & 100 & 0.40 & 3.1 \\
\hline 2 & I 75 & 0.85 & 4.2 & I 35 & 0.45 & 3.4 \\
\hline 3 & 200 & 0.90 & 4.2 & I65 & 0.55 & 3.6 \\
\hline 6 & $35^{\circ}$ & I. I 5 & 5.0 & 275 & 0.70 & 4. I \\
\hline 9 & 450 & I. 25 & $5 \cdot 7$ & 325 & 0.75 & $4 \cdot 4$ \\
\hline I 2 & $55^{\circ}$ & 1.40 & 6.5 & 400 & 0.80 & 5.1 \\
\hline I8 & 750 & 1.40 & 8.2 & 550 & 0.85 & 6.4 \\
\hline 24 & 900 & 1.30 & $9 \cdot 3$ & 700 & 0.85 & 7.6 \\
\hline 30 & 1000 & I. 30 & 9.9 & 800 & 0.85 & 8.2 \\
\hline
\end{tabular}

(2) Sheep

\begin{tabular}{|c|c|c|c|c|c|c|}
\hline \multirow{2}{*}{$\dot{A G E}$} & \multicolumn{3}{|c|}{ WOOL BREEDS } & \multicolumn{3}{|c|}{ MUTTON BREEDS } \\
\hline & $\begin{array}{c}\text { Live } \\
\text { Weight }\end{array}$ & $\begin{array}{l}\text { Digestible } \\
\text { Protein } 1\end{array}$ & $\begin{array}{c}\text { Net } \\
\text { Energy }\end{array}$ & $\begin{array}{c}\text { Live } \\
\text { Weight }\end{array}$ & $\begin{array}{l}\text { Digestible } \\
\text { Protein } 1\end{array}$ & $\begin{array}{c}\text { Net } \\
\text { Energy }\end{array}$ \\
\hline Months & Pounds & Pounds & Therms & Pounds & Pounds & Therms \\
\hline 3 & 37 & O.I 3 & 0.78 & 40 & 0.22 & 0.84 \\
\hline 6 & 65 & 0.18 & 0.95 & 72 & 0.30 & I.O3 \\
\hline 9 & 82 & 0.17 & I.06 & 98 & 0.28 & $\mathrm{I} .22$ \\
\hline I 2 & 90 & 0.15 & I.I 2 & I 5 & 0.25 & 1.36 \\
\hline I 8 & 100 & O.I 2 & I.I9 & I 50 & 0.22 & I. 64 \\
\hline
\end{tabular}

(3) Swine

\begin{tabular}{c|c|c|c}
\hline \hline Age & Live Weight & Digestible Protein 1 & Net Energy \\
\cline { 1 - 2 } Monihs & Pounds & Pounds & Therms \\
I & I5 & 0.10 & 0.65 \\
2 & 30 & 0.20 & 1.00 \\
3 & 52 & 0.30 & I.38 \\
6 & II & 0.40 & 2.28 \\
9 & I83 & 0.50 & 3.06 \\
I 2 & 250 & 0.55 & 3.80 \\
\hline
\end{tabular}

1 Based on Kellner's standards. 
TAble V. - Requirements For Milk Production

Add to the maintenance requirement the following amounts for each pound of milk of the several grades.

\begin{tabular}{c|c|c}
\hline \hline Grade of MilK & Digestible Protein & Net Energy \\
\cline { 1 - 3 } Per Cent Fat & Pounds & Therms \\
2.5 & $0.04 \mathrm{I}$ & 0.190 \\
3.0 & 0.043 & $0.2 \mathrm{I} 4$ \\
3.5 & 0.045 & 0.238 \\
4.0 & 0.049 & 0.265 \\
4.5 & 0.052 & $0.29 \mathrm{I}$ \\
5.0 & 0.055 & 0.315 \\
5.5 & 0.058 & 0.338 \\
6.0 & $0.06 \mathrm{I}$ & $0.36 \mathrm{I}$ \\
6.5 & 0.064 & 0.385 \\
7.0 & 0.068 & 0.408 \\
\hline
\end{tabular}

Table VI. - Requirements for Work Production by the Horse (674) Per Iooo Pounds Live Weight

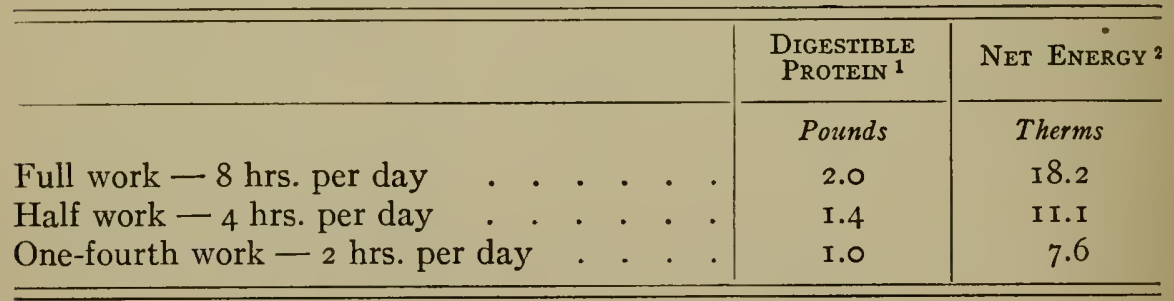

AVERAGE DRY MATTER, DIGESTIBLE PROTEIN AND NET ENERGY VALUES OF FEEDING STUFFS PER IOO POUNDS

Henry and Morrison ${ }^{3}$ have recently published a very valuable compilation of American analyses of feeding stuffs and of the results of American digestion experiments, and on this basis have calculated the content of digestible nutrients in a great variety of feeding stuffs.

With the permission of these authors and with the coöperation of Assistant Professor Fred Silver Putney, of The Pennsylvania State College, the writer has computed from their tables the net

${ }^{1}$ Kellner's standards.

2 To be computed from Table VIII.

${ }^{3}$ Feeds and Feeding, I 5 th Edition, pp. 633-666. 
energy values of the more important feeding stuffs in the manner described in Chapter XVII $(\mathbf{7 7 3}, \mathbf{7 7 4})$ with the results regarding ruminants reported in Bulletin No. I42 of the Pennsylvania Experiment Station and in Bulletin No. 459 of the U.S. Department of Agriculture. Those results, with a few additions and corrections, are here reproduced and the computation has also been extended, as well as the meager basis now available will permit, to the data regarding swine supplied by Henry and Morrison's tables. The figures for the horse are derived in part from the same source and in part from Zuntz and Hagemann's investigations, the net energy values being computed according to the method proposed by those investigators (775-778). The tables show primarily the net energy values for maintenance or fattening. There seems good reason for believing, however, that they may be taken without serious error to represent also the net energy values for growth and for work production and at least the relative values for milk production.

Henry and Morrison's tables include only the crude protein $(\mathrm{N} \times 6.25)$. The amount of non-protein has been estimated from the crude protein by the writers on the basis of Kellner's averages.

Table VII. - VAlues Per ioo Pounds for Ruminants

\begin{tabular}{|c|c|c|c|c|}
\hline & \multirow{2}{*}{$\underset{\text { MATTER }}{\text { Dry }}$} & \multicolumn{2}{|c|}{ Digestible } & \multirow{2}{*}{$\begin{array}{c}\text { NET } \\
\text { ENERGY } \\
\text { VALUE }\end{array}$} \\
\hline & & $\begin{array}{l}\text { Crude } \\
\text { Protein }\end{array}$ & $\begin{array}{l}\text { True } \\
\text { Protein }\end{array}$ & \\
\hline Dried Roughage & & & & \\
\hline Hay and fodder from cereals & Pounds & Pounds & Pounds & Therms \\
\hline $\begin{array}{l}\text { Brome grass, smooth } \\
\text { Corn (maize) fodder (ears included, medium }\end{array}$ & 91.5 & 5.0 & $3 \cdot 5$ & 40.83 \\
\hline $\begin{array}{l}\text { dry) } \\
\text { Corn (maize) stover (ears removed, medium }\end{array}$ & $8 \mathrm{r} \cdot 7$ & 3.0 & 2.3 & 43.94 \\
\hline $\begin{array}{llllllllllll}\text { dry) } & . & . & . & . & . & . & . & . & . & . & \end{array}$ & 81.0 & $2 . \mathrm{I}$ & I.6 & 31.62 \\
\hline Kafir fodder, high in water & 71.7 & 3.0 & 1.8 & 34.28 \\
\hline Kafir stover, high in water & 72.7 & $\mathrm{x} \cdot 3$ & r.o & 27.65 \\
\hline Millet, Hungarian . . . & $85 \cdot 7$ & 5.0 & $3 \cdot 9$ & 46.96 \\
\hline Mixed timothy and clover . . . . . . & 87.8 & $5 \cdot 3$ & 3.6 & 40.85 \\
\hline Oat hay . . . . . . . . . . . & 88.0 & $4 \cdot 5$ & 3.9 & 32.25 \\
\hline
\end{tabular}


Table VII. - Values per roo Pounds for Ruminants (Continued)

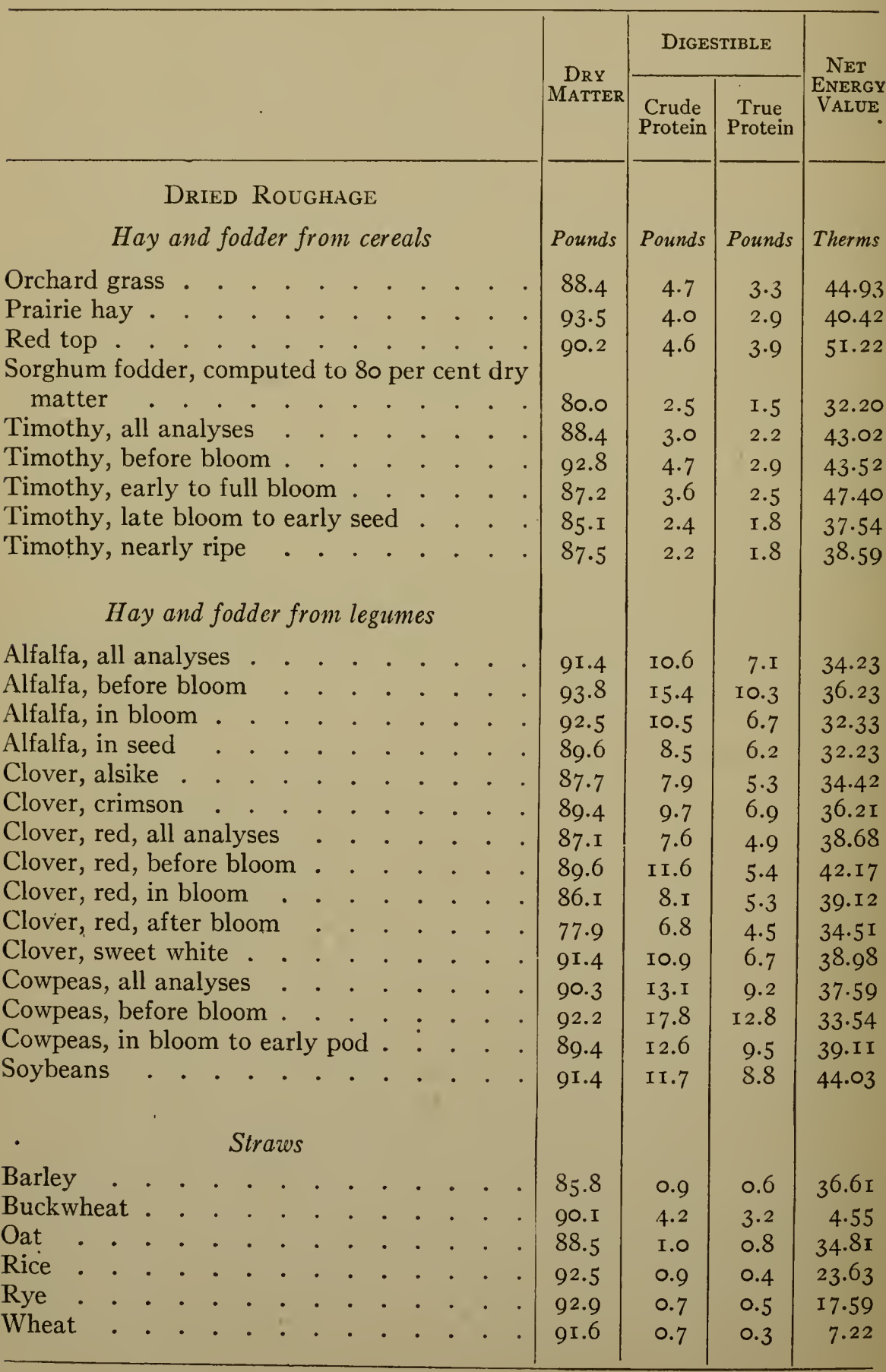


Table VII. - Values per ioo Pounds for Ruminants (Continued)

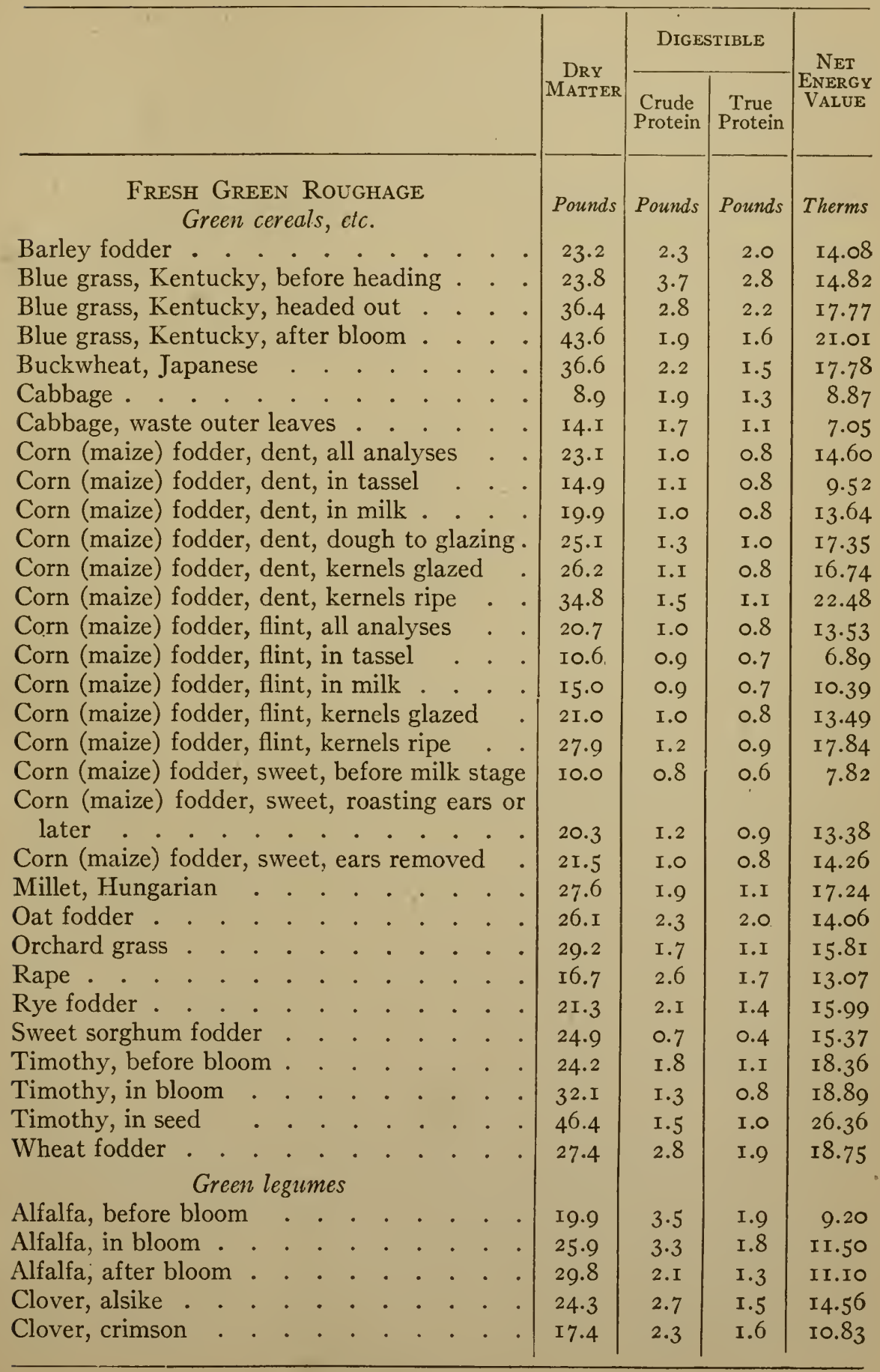


Table VII. - Values per ioo Pounds for Ruminants (Continued)

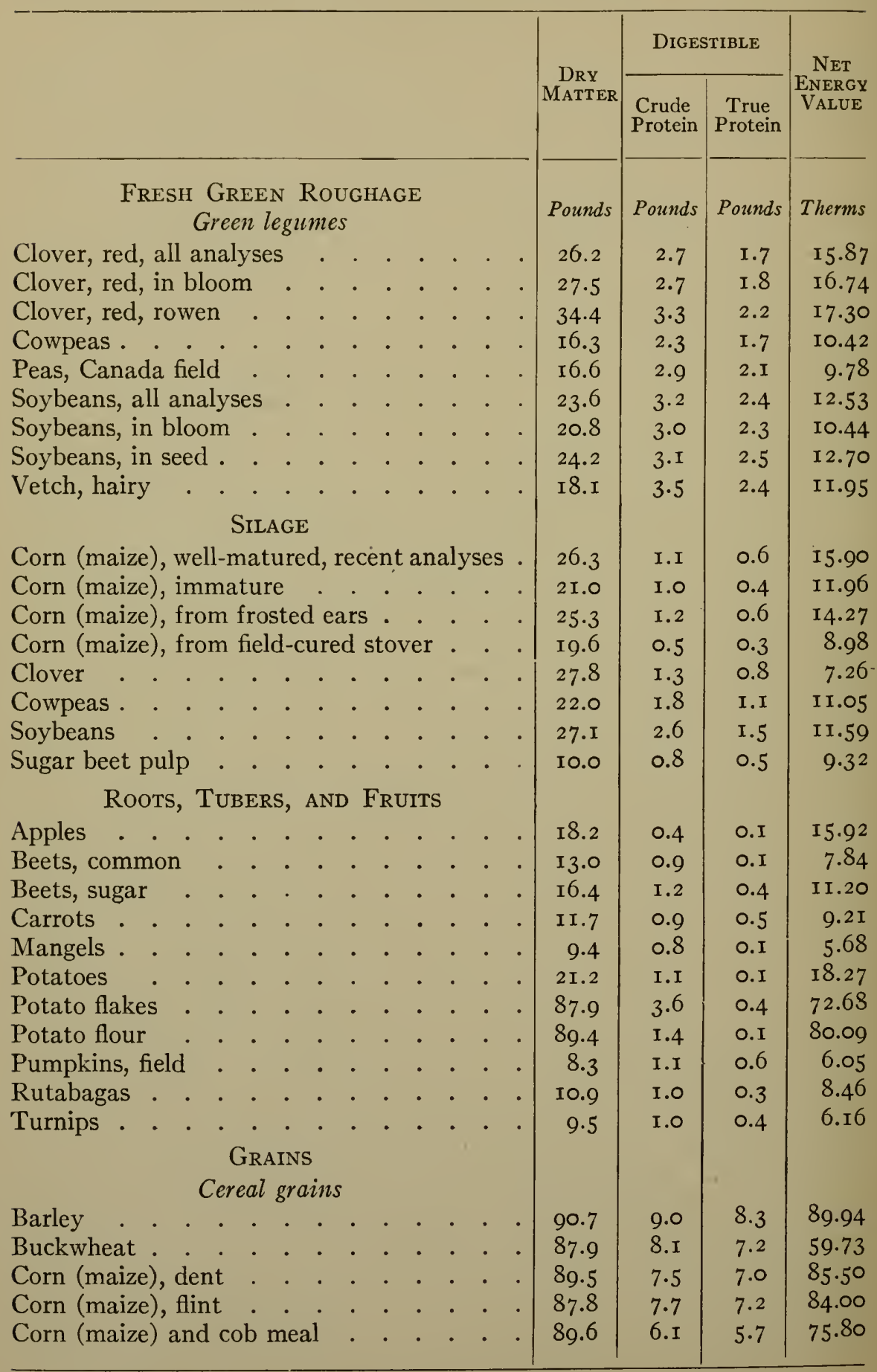


Table ViI. - Values Per ioo Pounds for Ruminants (Continued)

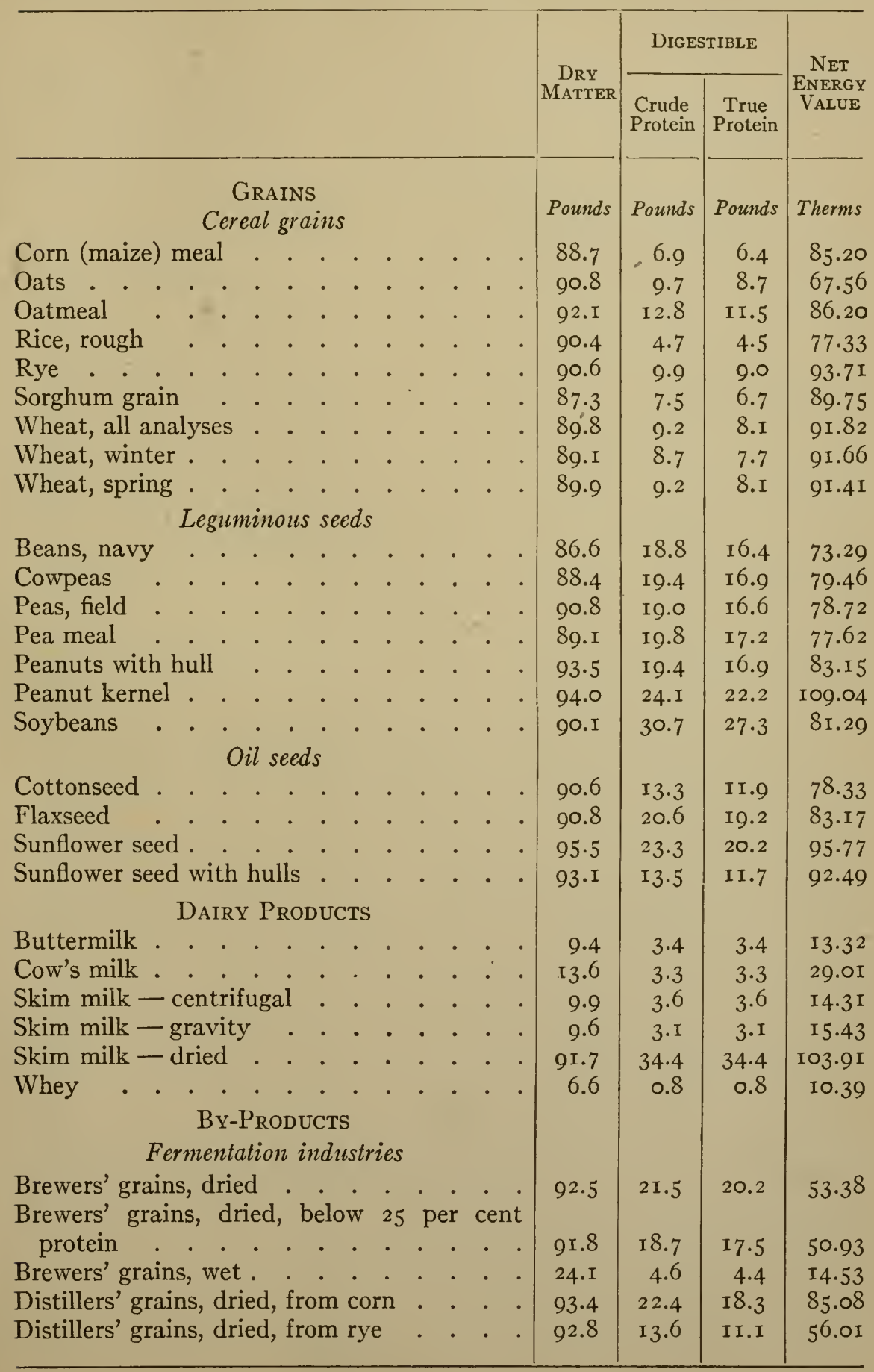


Table VII. - Values per ioo Pounds for Ruminants (Continued)

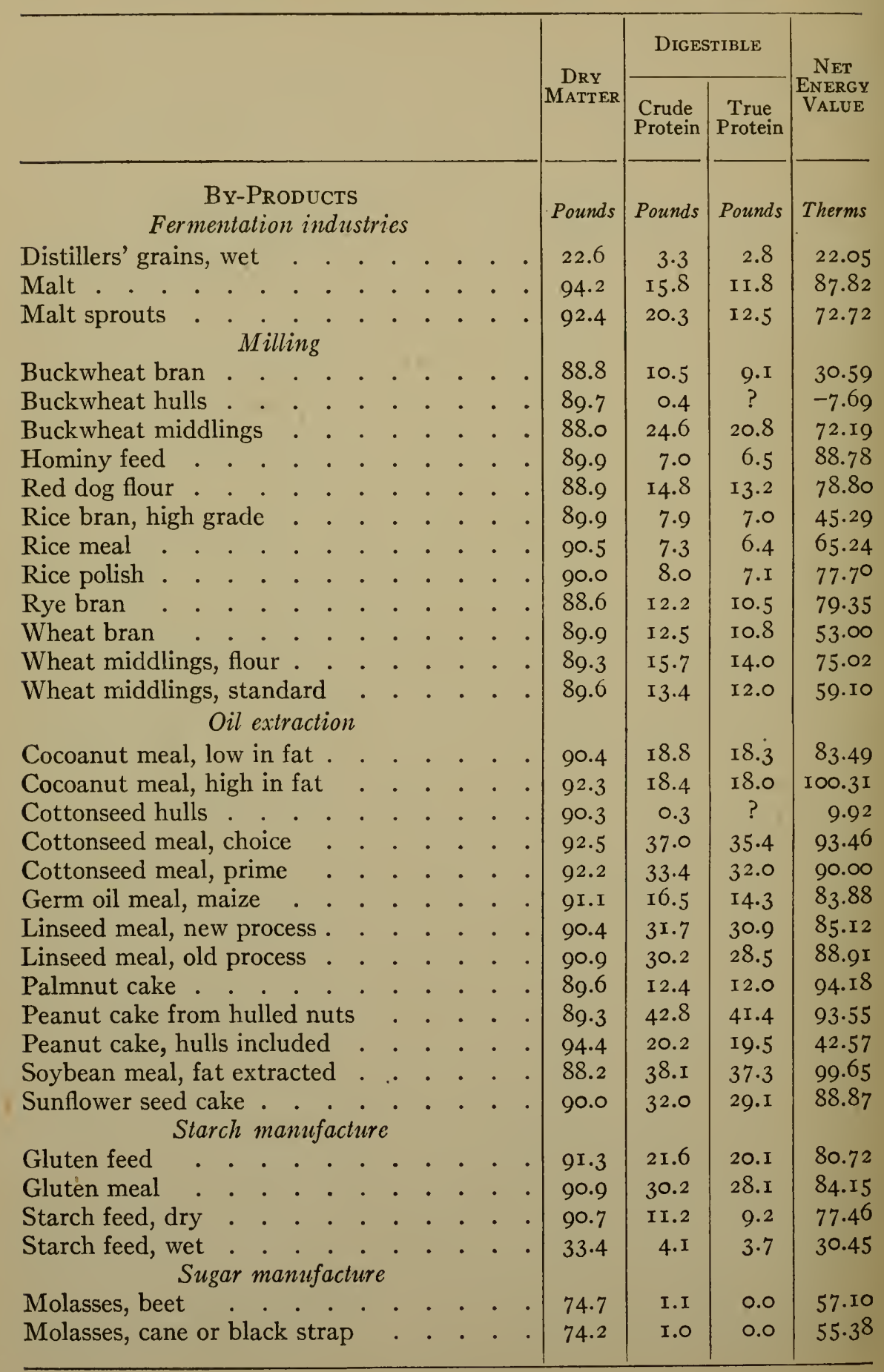


Table VII. - Values per ioo Pounds for Ruminants (Continued)

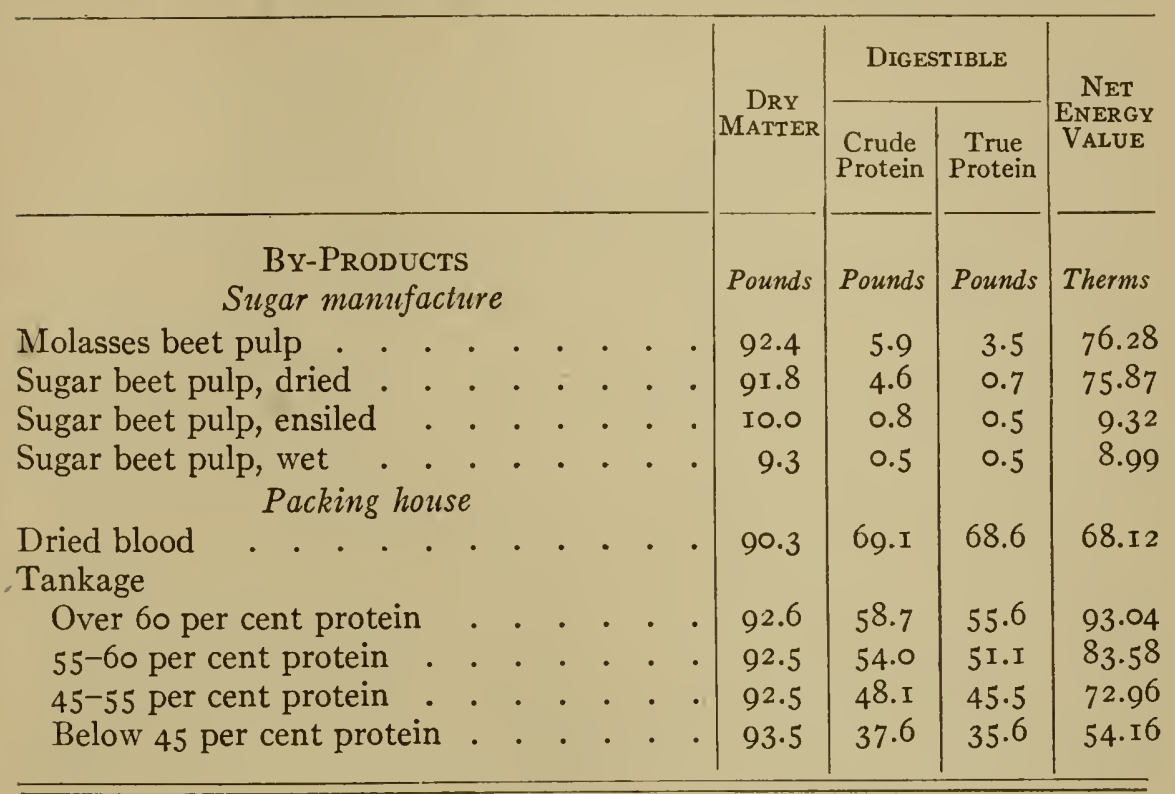

Table VIII. - Values per ioo Pounds for the Horse

\begin{tabular}{|c|c|c|c|c|c|c|c|c|c|c|c|}
\hline & & & & & & & & \multirow{2}{*}{$\underset{\text { Matter }}{\text { Dry }}$} & \multicolumn{2}{|c|}{ Digestible } & \multirow{2}{*}{$\begin{array}{c}\text { NET } \\
\text { ENERGY } \\
\text { VALUES }\end{array}$} \\
\hline & & 4 & & & & & & & $\begin{array}{c}\text { Crude } \\
\text { Protein }\end{array}$ & $\begin{array}{c}\text { True } \\
\text { Protein }\end{array}$ & \\
\hline & & & & & & & & Pounds & Pounds & Pounds & Therms \\
\hline Alfalfa hay &. & . & • & . & . & . & . & $9 \mathrm{I} .4$ & 10.9 & $7 \cdot 4$ & 48.82 \\
\hline Red clover hay & & . & . & . & . & . & . & 87.1 & 7.2 & $4 \cdot 5$ & 39.94 \\
\hline Timothy hay & .. & . & . & • & . & . & - & 88.4 & I.3(?) & $0.5(?)$ & 26.64 \\
\hline Wheat straw & . . & . & . & • & . & . & . & 9 г.6 & 0.8 & 0.4 & -20.90 \\
\hline Beans . & . . & . & . & . & . & . & . & - & I9.5 & I 7.I & 109.40 \\
\hline Corn (maize), & dent & . & . & . & . & . & . & 89.5 & 5.9 & $5 \cdot 4$ & I I 2.80 \\
\hline Corn (maize), & meal & 1 . & . & . & . & . & . & 88.7 & $7 . I$ & 6.6 & 132.70 \\
\hline Oats. . &. & . & . & . & . & . & . & 90.8 & 9.9 & 8.9 & 93.44 \\
\hline Peas. . & . . & . & . & ${ }^{\circ}$ & - & . & . & 90.8 & I 8.7 & I6.3 & 105.20 \\
\hline Linseed cake & . . & . & . & . & . & . & . & 90.9 & 29.5 & 27.8 & Ior.60 \\
\hline Carrots . . & . . & . & . & . & . & . & . & II. 7 & I. 2 & 0.8 & I6.60 \\
\hline Potatoes & . . & . & . & . & . & . & . & $2 \mathrm{I} .2$ & I.9 & 0.9 & 35.70 \\
\hline
\end{tabular}


Table IX. - Values per ioo Pounds for Swine

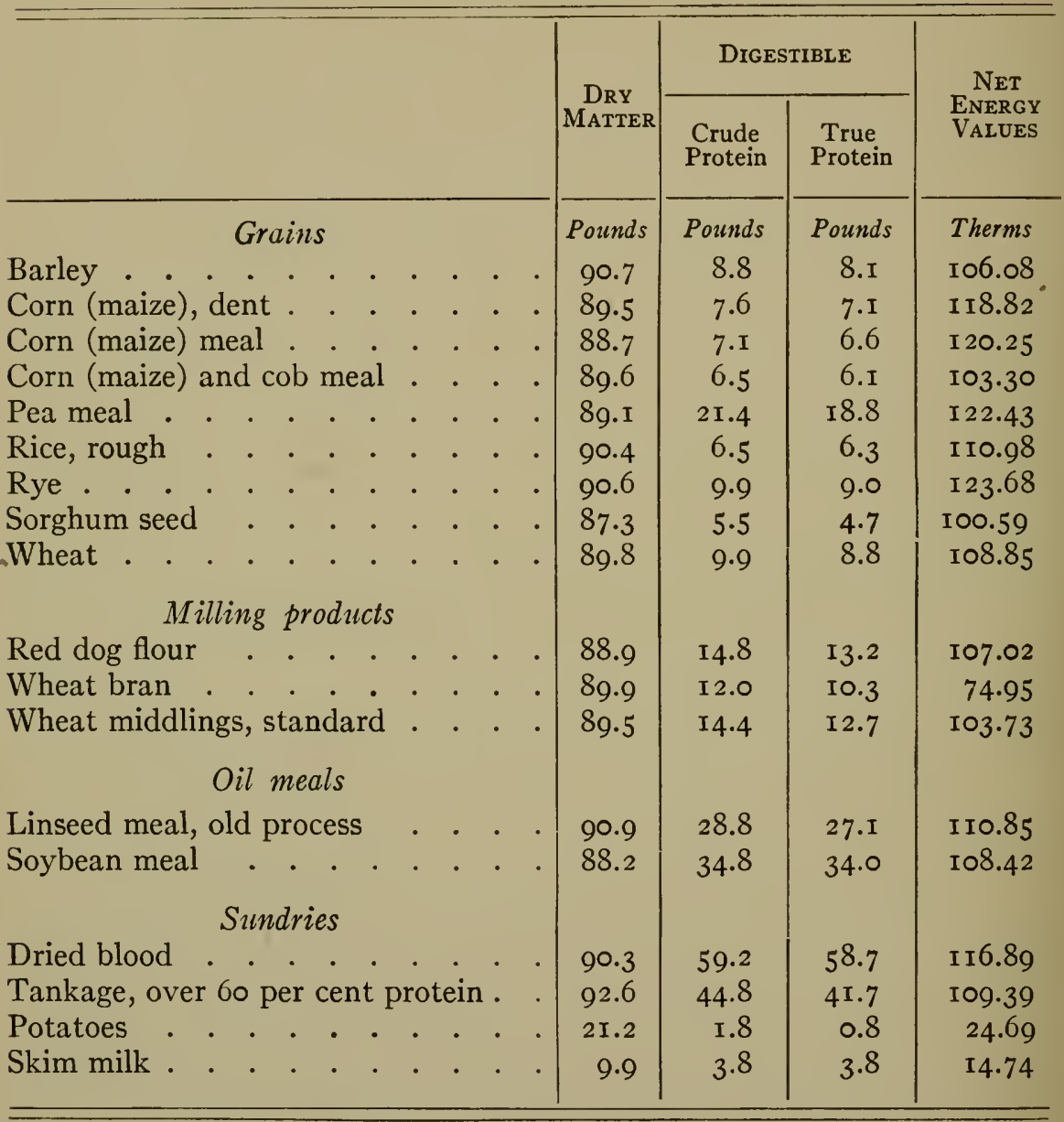




\begin{tabular}{|c|c|}
\hline 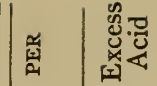 & 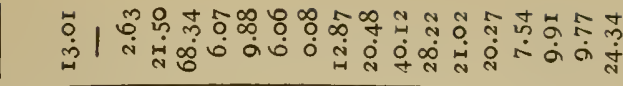 \\
\hline 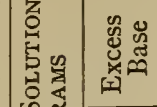 & 11111111111111 \\
\hline 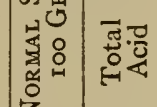 & 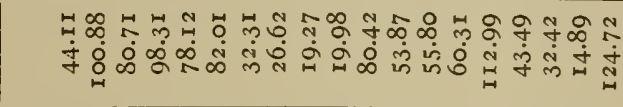 \\
\hline 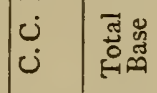 & 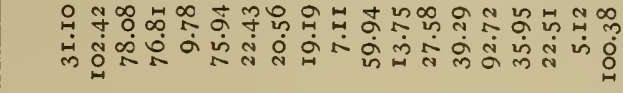 \\
\hline 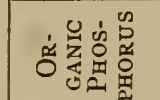 & 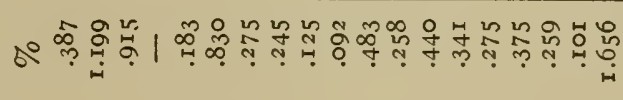 \\
\hline 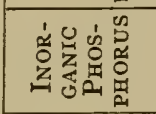 & จீ \\
\hline 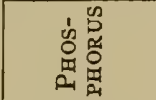 & 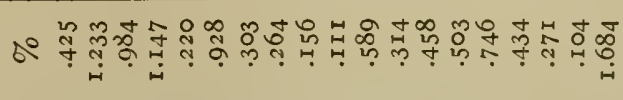 \\
\hline 苞照 & 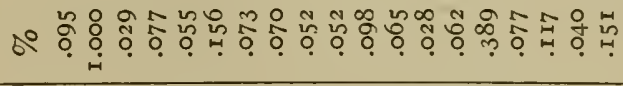 \\
\hline 嵓罢 & 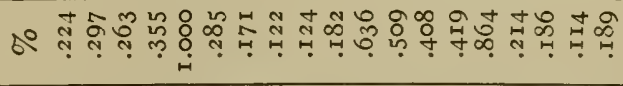 \\
\hline 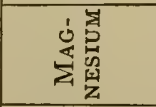 & 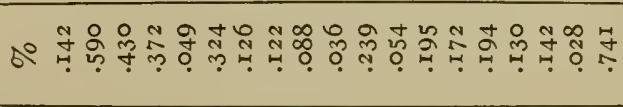 \\
\hline 递 & 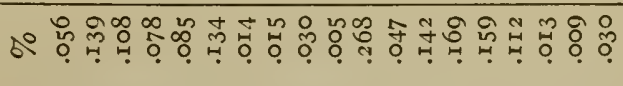 \\
\hline 施 & 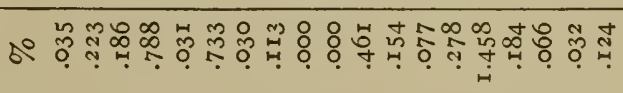 \\
\hline 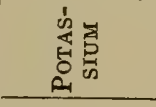 & 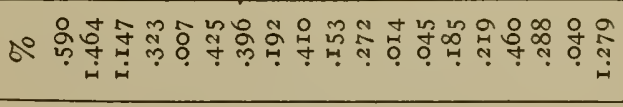 \\
\hline 異 & 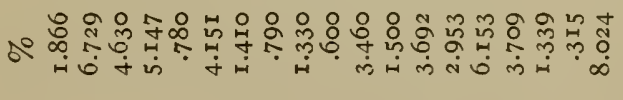 \\
\hline & 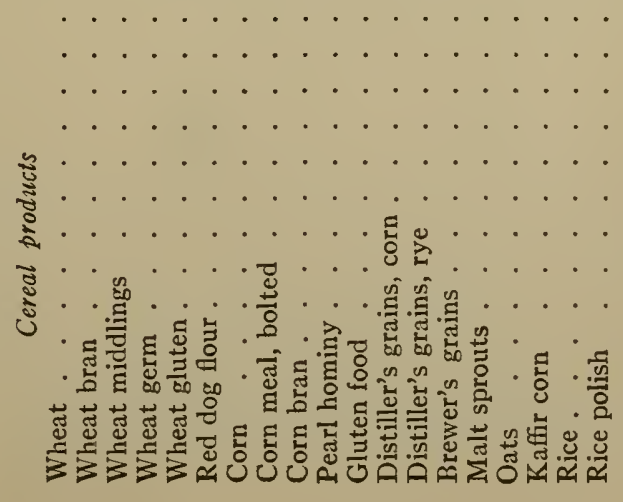 \\
\hline
\end{tabular}




\begin{tabular}{|c|c|c|}
\hline 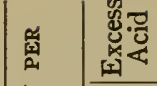 & 11111111111111111111111110 & 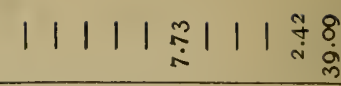 \\
\hline 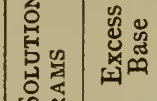 & 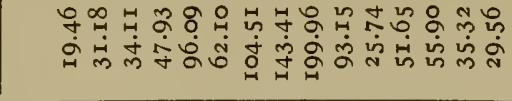 & 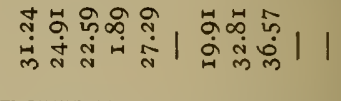 \\
\hline 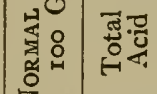 & 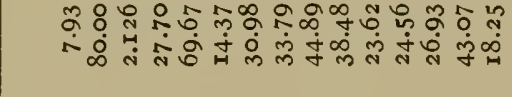 & 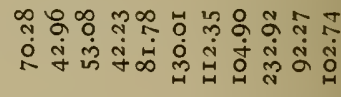 \\
\hline 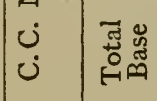 & 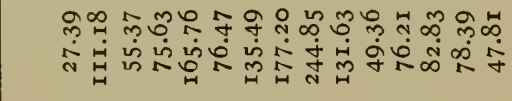 & 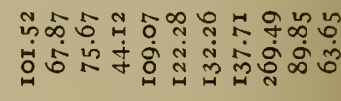 \\
\hline 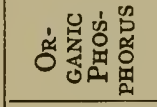 & 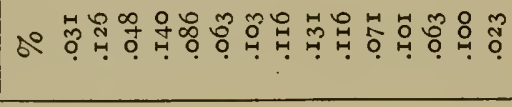 & 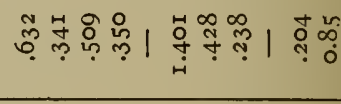 \\
\hline 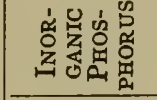 & 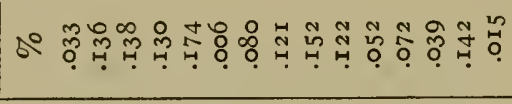 & 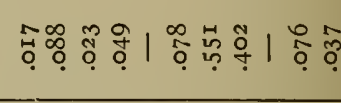 \\
\hline 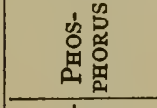 & 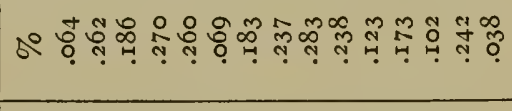 & 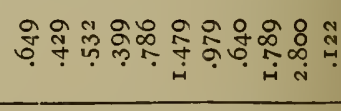 \\
\hline 总罢 & so & 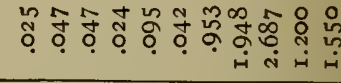 \\
\hline 㸃宽 & 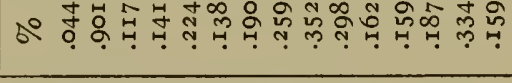 & 导势。 \\
\hline 总窟 & 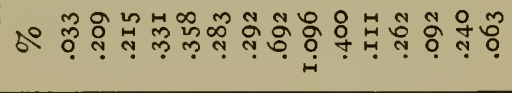 & 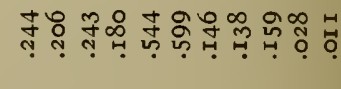 \\
\hline 它卷 & 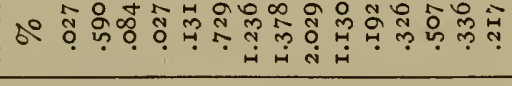 & 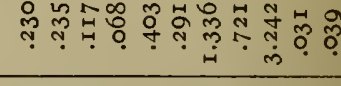 \\
\hline 䪪 & 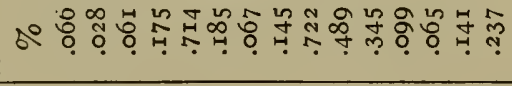 & 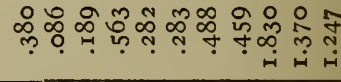 \\
\hline 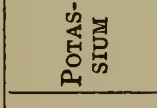 & 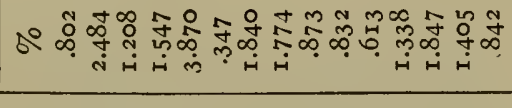 & 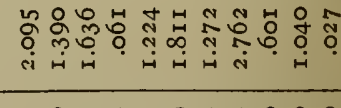 \\
\hline 㚻 & 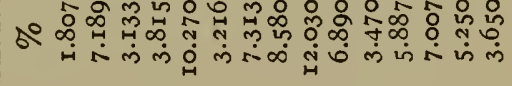 & 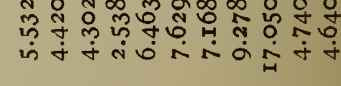 \\
\hline & 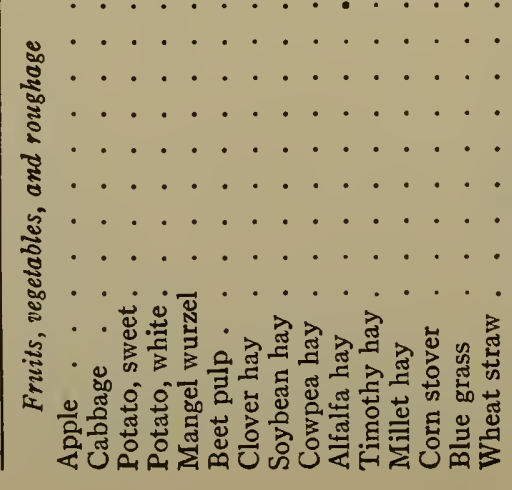 & 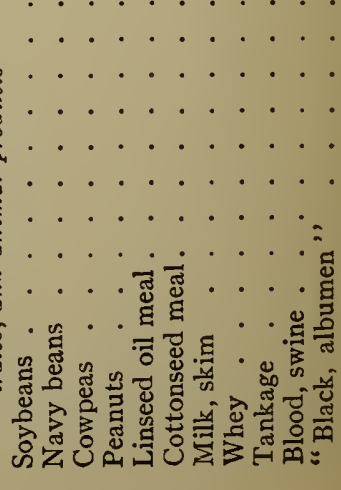 \\
\hline
\end{tabular}




\section{INDEX}

Abomasum, 80

Accessory substances, 348,42 I significance of, 632

Acid :

hippuric, I 63

hippuric, synthesis of, $I 63$

uric, synthesis of, I 7 I

Acidity in ash, significance of, 34I

Acidosis, 336

Acids :

excretion of, 338

influence on digestibility, 627

neutralization of, 337

nucleic, 34

anabolism, I 68

autogenesis, 169

cleavages, I 70

deaminization, I 7 I

katabolism, I 70

metabolism, 168

synthesis, 169

organic, 40

formation of, in digestion, 40,158

in feeding stuffs, 40

metabolism of, I59

Adipose tissue, 58, 424

composition of, 59

Age :

best for fattening, 436

influence on

composition of gain, $43 \mathrm{I}$

cost of production of meat, 430

digestibility, 6ro

effects of temperature, 454

energy requirements for maintenance, 307

feed consumption, 43I

net energy values, 666

production of lean meat, 433

relation of growth to, 373

relation of protein requirements to, 445

Albuminoids, 33

Albumins, 33
Alfalfa proteins, value of, 683

Alkali ratio of ash, 342

Alkaloids, 37

Amids, 37

occurrence in plants, 38

Amino acids, 37

from simple proteins, 28,29

occurrence in plants, 38

relative values for growth, $38 \mathrm{I}$

required for maintenance, $3 \mathrm{I} 4$

Ammonia, formation of, in katabolism of proteins, I 65

Amount of feed, influence on

effects of temperature, 454

meat production, 443, 449

metabolizable energy, 664

milk production, 5 I 5

net energy values, 664

production, 269 of methane, 665

Amylase, 78, 86

Amylopsin, 78, 86

Anabolism, I45

of fats, I7 I

nucleic acids, I 68

phosphorus, I80

simple proteins, 160

Animal :

as factor in meat production, 428

milk production, 470

as prime motor, 192

Araban, I5

Arabinose, 9

Arteries, I 26

Ascent, work of, $55 \mathrm{I}$

efficiency of body in, 55 I

Ash, 5

acid and basic, 340

alkali ratio of, 342

balance, 2 I 6

maintenance of, 339, 344

body, proportion of in bone, 48 in offal, 56

bone, composition of, 48 


\section{Ash, - continued}

content of feed, 332

correction of deficiencies in, 346

determination of, in feeding stuffs, 67

digestion of, ror

effects of deficiency of, 420

ingredients, 6

availability of, $4 \mathrm{I} 7$

balancing of, in ration, 343

deficiencies in, 339

digestibility of, I $18,333,343$

excretion of, I4I

functions of, 187

indispensable, 332

metabolism of, I 78

skeleton as reserve of, 338

losses of, 334

causes of, 334

of milk, 460

sources of, 468

outgo of, in milk, 520

proportion of, in animal, 5

in feeding stuffs, 5

rate of storage of, in growth, 414

requirements for growth, $4 \mathrm{I} 4$

maintenance, 332

milk production, 520

work production, 562

significance of acidity in, $34 \mathrm{I}$

supply in dairy rations, $52 \mathrm{I}$

total retention of, during growth, $4 \mathrm{I} 6$

Assimilative power :

influence of breed on, 44I

individuality on, 44I

Autogenesis of nucleic acids, 169

\section{Balance:}

of ash, 216

carbon, 205

example of, 206

energy, 2 I 6

example of, 240

income and expenditure, I94

matter, 202

nitrogen, 202

determination of, 203

example of, $2 \circ 3$

nutrition, 192, 201

includes energy, 216

water, 2 I 6

Balance experiments, 200

comparison with metabolism investigations, 24I

practical experiments, 244
Balance experiments, - continued

in agricultural investigations, 243

significance of results of, 24 I

Barley feed, $5^{85}$

Bases, organic, 37

Bile, 86

Blood, 123

coagulation of, 125

corpuscles, red, 124

white, I 24

course of, 127

plasma, I 25

platelets, I 24

Body :

comparison with power plant, 567

composition of entire, $6 \mathrm{I}$

fat- and ash-free, 65

fat-free, 64

efficiency of. (See Efficiency)

expenditure by, 192

schematic, I95

substances sources of energy for work, 544

temperature, chemical regulation of, 263

physical regulation of, 262

Bone, 47

ash composition of, 48

composition of, 47

proportion of body ash in, 48

protein in, 48

Bones as reserve of ash ingredients, 338

Bran, 582

rice, 583

rye, 582

wheat, 582

Breakfast food residues, 584

Breathing :

mechanics of, 134

regulation of rhythm of, 137

Breed, influence on

assimilative power, 441

composition of milk, 473

composition of milk solids, 474

digestive power, 440, 6ro

early maturity, 44I

feed consumption, 443

maintenance requirements, $44^{2}$

meat production, 440

net energy values, 666

Brewers' grains, 586

By-products, 582

nature of, 582

- of fermentation industries, $5^{85}$

milling, 582 
By-products, -- continued

uses of, 584

oil extraction, 586

starch and glucose manufacture, 587

sugar manufacture, 588

the packing house, 590

\section{Calcium :}

metabolism of, I8I

occurrence of, 6

Calorimeters, 22I

animal, 235

emission, 236

latent heat, 236

respiration, 236

water, 236

Calves:

energy requirements, 399

gains by, in growth, 400

protein requirements, 404

Capillaries, 126

Carbohydrates, 7

cause of diminished digestibility of, 62 I classification of, 8

digestible, $\mathbf{1} 2 \mathbf{I}$

digestion of, 89

formation of fat from, I55, I 74

from fats, 178

proteins, 167

formed in the body, 155

functions of, I 86

influence of excess of, on digestibility, $6 \mathrm{r} 6$

feeds rich in, on digestibility, 6I7

katabolism of, I 56

intermediary, 157

metabolism of, 152

occurrence of, 7

of milk, 460

origin of, 467

pentose, 9,15

metabolism of, 157

relative utilization of fats and, for work production, 553

Carbon balance, 205

example of, 206

Carbon dioxid :

excretion of, I39

determination of, 208

through the skin, 139

formed in respiration, 136

product of metabolism, I 44

Carnivora, influence of feed consumption

Cartilage, 49
Cattle:

energy requirements for growth, 339 maintenance, 288

influence of feed consumption on heat production, $65 \mathrm{I}$

maintenance requirements of, compared with sheep, 294

net energy values for, 659 computation of, 667,673

protein requirements for growth, 404 maintenance, 326

Cell, 42

enclosures, 45

nucleus, 42

structure, 42

wall, 44

Cellulose, I 2

digestion of, 89

fermentation of, 90

Cereal grains, 579

composition and digestibility of, 580

uses of, 58I

values of proteins of, 682

Cerebrosids, 23

Changes in digestion, summary of, IOI

Chemical changes in muscular contraction, 532

Chlorin, occurrence of, 7

Choice of feeding stuffs, 703

Cholesterins, 22

Chymosin, 83

Circulation, I 23

adjustment of, I3I

influence of work on, 535

mechanics of, $\mathrm{I} 28$

scheme of, I 27

Cleavage products, proportions of, in simple proteins, $3 \mathrm{I}$

Coagulation of blood, I 25

Coarse fodders, 72,572

general character of, 572

proportion of vegetative organs in, 576

Cœcum, 84

Collagens, 33

Colloids, conversion of, into crystalloids

Colon, 85 in digestion, 102

Combustible gases, outgo of chemical energy in, 230,636

Combustion, heat of, 223, 227

Compounding of rations, 707

Computation:

of improvement of a ration, 698 rations, 689

from given feeding stuffs, 700 
Computation, - conlinued method of, 697

of total feed required, 697

Concentrates, 72, 579

comparison with roots, 579

roughage, 662

determination of digestibility of, II 5 proportion of, to roughage, $45 \mathrm{I}, 696$ relative values for, $67 \mathrm{I}$

Condiments, influence of, on digestibility, 627

Condition, influence of, on economy of gain, 438

meat production, 438

rate of gain in fattening, 438

Conditions affecting digestibility, 6or, $602,6 \mathrm{I}_{3}$

Conditions, external, influence of, on meat production, 453 milk production, 478

Conformation, relation of, to meat production, 443

Conservation of energy, 219

Contraction, muscular, 532 chemical changes in, 532 energy transformations in, 533

Corn bran, 588

Cottonseed meal, 587

Critical temperature, 264,453 lowered by feed consumption, 308

Crude fiber, $\mathbf{I} 3$

composition of digested, I 20

correction of net energy values for, 669

determination of, in feeding stuffs, $7 \mathrm{I}$ influence of, on heat production of horse, 676

proportion of pentosans in, $7 \mathrm{I}$

Cutaneous excretion, outgo of chemical energy in, 23I

Cutting of roughage, influence of, on digestibility, 624

Cytoplasm, 42

Dairy rations:

addition of fat to, $5 \mathrm{I} 7$

ash in, 521

protein in, 506

Deaminization :

of nucleic acids, $\mathbf{I} 7 \mathrm{I}$

simple proteins, 165

reversible, 166

Deficiencies in ash :

correction of, 346

effects of, 420
Dextrins, 14

Dextrose, 8

Digestible nutrients. (See Nutrients)

Digestibility, I I I, 60 I

apparent, I 20

by horse compared with ruminants, 604

species of ruminants, 603

swine compared with ruminants, 606

conditions affecting, 601

definition of, II I

determination of, II I, II4

influence of excretory products, I 8

influence on,

of acids, 627

addition of protein, 622

age, 6 ro

breed, 6ro

condiments, 627

conditions relating to the animal, 602

feed, $6 \mathrm{I}_{3}$

cutting of roughage, 624

drinking, 628

drying, 623

excess of carbohydrates, 6 I6

feeds rich in carbohydrates, $6 \mathrm{I} 7$

grinding of grain, 624

heavy feeding, 450

individuality, 609

non-protein, 622

protein supply, 447

quantity of feed, 613

roots, 6 I 8

species, 603

tubers, 6r 8

water drinking, 628

work, 6 Io

laboratory determination of, I I 6

of ash ingredients, I I $8,333,343$

carbohydrates, diminished, cause of, 621

cereal grains, 580

concentrates, determination of, II 5

ether extract, I I9

grasses, influence of maturity on, 574

maize forage, influence of maturity on, 575

nitrogenous substances, II9

protein, diminished, cause of, 6r9

variable, 6or

variation of, at different times, 602

Digestion, 77

changes in, IOI

chemistry of, 89 
Digestion, - continued

conversion of colloids into crystalloids in, 102

experiments, example of, II4

methods of, II 2

time required for, II 3

extent of protein cleavage in, 98

intestinal, 87

molecular simplification in, 103

of ash, IOI

carbohydrates, 89

cellulose, 89

disaccharids, 95

electrolytes, IOI

fats, 88,95

hemicelluloses, 92

non-proteins, 96, 100

nucleic acids, 99

pentosans, 91

phosphorus, I0I

proteins, $83,88,95$

by erepsin, 98 pepsin, 96

trypsin, 97

starch, 79, 88, 92

in intestines, 94 stomach, 93

sulphur, IOI

organs of, 77

general plan of, 77

solution of nutrients in, IOI

uniformity of nutritive material, I03

work of, 277

differences between feeding stuffs, $66_{3}$ roughage compared with concentrates, 662

Digestive power, influence of breed, 440 individuality, 440

Diminishing returns from feed in milk

Disaccharids, 10 production, 515

digestion of, 95

general properties of, II

Distillers' grains, 586

Draft, work of, 552

efficiency of body in, $55^{2}$

Dried blood, 590

Drinking, influence of, on digestibility, 628

Dry matter, 3

of body, composition of fat- and ashfree, 65

requirements of, 695

Drying, influence of, on digestibility, 623

Duodenum, 84
Economy of feeding, influence of individuality on, 472

Efficiency of body, 544

as motor, 544

compared with power plant, 567

conditions affecting, 555

economic, 562

gross and net, 546

in work of ascent, $55 \mathrm{I}$

draft, 552

influence on,

of fatigue, 556

forms of work, 555

gait, $55^{8}$

grade, 559

individuality, 556

intensity of work, 557

load, 559

speed, 552,557

training, 556

mechanical, 545

over-all, 562

per day, 549

variable, 548,555

Efficiency of muscle, 545

Electrolytes, digestion of, IOI

Embryo, net energy values for growth of, 393

Emulsification of fats in digestion, 95

Emulsion of fats, I9

Energy :

available, 233

balance of, 216

example of, 240

in milk production, 493,495

chemical, 218

outgo of, 229,635

conservation of, 219

definition of, 216 .

expenditure in,

internal work, measure of, 256

feed consumption, 275

significance of, 277

locomotion, $55^{\circ}$ '

influence of speed on, 552, 557

for work, body substance as source of, 544

protein as source of, 542

forms of, $2 \mathrm{I} 7$

gross, 227,635

income of, 226

katabolism of, in fasting, 256

constancy of, 256

kinetic, 218

measurement of, 325 
Energy, - continued

outgo of, 235

losses of, $229,235,635$

chemical, 299, 635

in feces, 230,635

fermentation, 230,636

computation of, 627

heat production, 235,650

urine, $23 \mathrm{I}, 636$

metabolizable, 231, 639

comparison of net energy values with, 27 I

computation of,

from digestible nutrients, 646 organic matter, 648

factors for, 234

for the horse, 675

general conception of, 23 I

influence on, of amount of feed, 664

method of determining, 640

of digestible nutrients, 648

feeding stuffs, 642

real and apparent, 645

significance of, 645

synonyms for, 233

net. (See Net Energy)

outgo of, $229,235,635,650$

in combustible gases, 230,636

feces, 230,635

heat production, 235,650

urine, 231,636

production values as regards, 634

protein as source of, $3 \mathrm{I} 8$

rate of gain of, in growth, 378

requirements

for fattening, $36 \mathrm{I}$

growth, 399

of cattle, 399

sheep, 401

swine, 400

maintenance, 267

factors affecting, 304

influence on

of age, 307

fattening, 306

plane of nutrition, 305

stage of fattening, 362

temperature, 304

manner of stating, 283

methods of determining, $28 \mathrm{I}$

modified conception of, 284

of cattle, 288

farm animals, 280, 303

fowls, 301

horses, 295
Energy, - continued
sheep, 292
swine, 285

relation of temperature to, 308

meat production, 448

milk production, 5 I I

work production, 562,564

sources of, for work, 542

supply, influence of, on retention of protein, 386

total, not measured by heat of combustion, 223

transformations of, 218

in muscular contraction, 533

units, 220

utilization of,

in growth, 390

milk production, 493

work production, 544

values, net. (See Net Energy Values)

Environment, influence of, on milk production, 478

Enzym reactions reversible, I 50

Enzyms as agents in metabolism, 148

digestive, 78

extracellular, 148

intracellular, I 49

in the body, 150

Epithelium, 105

Erepsin, 79, 87, 98

action of, on proteins, 98

Esophagus, 79

Ether extract:

digested, I 22

digestibility of, I 19

of feeding stuffs, 70

Excretion, 123, I 39

functions of kidneys in, 140

of ash ingredients, I4I

carbon dioxid, I39

nitrogenous products, I40

water, $\mathrm{I}_{42}$

Exercise :

feed cost of, $48 \mathrm{I}$

influence of, on meat production, 457

milk production, 480

yield of milk fat, 482

Expenditure, balance of income and, I94 of energy in horizontal locomotion, 550

Extractives, percentage of, in lean meat, 357

Farm animals, composition of bodies of, 62 
Fasting:

energy katabolism in, $25 \mathrm{I}$

functions of protein in, 255

katabolism, 249

computation of, 282

conditions affecting, 258

energy expended in, 25I, 257

influence on,

of body fat, 252

external temperature, 262,265

muscular activity, 26I

previous feeding, 253

size of animal, $25^{8}$

standing and lying, 262

substances katabolized, in, 249

protein katabolism in, $25 \mathrm{I}$

normally small, $25^{\text {I }}$

variable, $25 \mathrm{I}$

Fat and lean, proportions of in carcass, 424

Fat:

addition of, to dairy rations, $5^{\text {I } 7}$

animal, sources of, 173

body, influence of on fasting katabolism, 252

proportion of in offal, 56

computation of gain or loss of, 205 crude, determination of, in feeding stuffs, 70

gain or loss of, 205

manufacture of, 172

minimum of, for milk production, 5 I 9

mobilization of reserve, I 77

of feed, resynthesis of, I 7 I

of milk, influence of exercise on yield of, 482

origin of, 466

percentage of, in lean meat, 356

milk, influence of feed on, 528

milk solids, influence of feed on, 529

production, protein unnecessary for, 363

proportion of, in meat, 425

relative utilization of carbohydrates and, for work production, 553

requirements of, for milk production, 5 I 6

storage of, 172

Fatigue, influence of, on efficiency of body, 556

milk production, 482

Fats, I 6

anabolism of, I $7 \mathrm{I}$

animal, elementary composition of, 2 I chemical changes in resorption of, 108
Fats, - continued

chemical reactions of, 18

digestion of, 88, 95

distinction between oils and, 19

emulsification of, in digestion, 95

emulsion of, I9

formation of carbohydrates from, 178

from carbohydrates, I 55, I 74

protein, I68, I73

functions of, 186

hydrolysis of, 18

katabolism of, I7 6

melting points of, I9

metabolism of, I 7 I

molecular structure of, I7

native, 19

occurrence of, I

of milk, 459

oxidation of, at $\beta$ carbon atom, I 77

physical properties of, I 8

relation of, to growth, $42 \mathrm{I}$

saponification of, in digestion, 96

specific effects of feeds associated with, 527

Fattening, 350

best age for, 436

composition of increase in, 350, 352, $353,354,364$

concurrent, in milk production, $5^{\mathrm{I}} 3$

contrast with growth, 396

during growth, 448

energy content of gain in, $36 \mathrm{I}$

energy requirements for, $36 \mathrm{r}, 448$

equivalent energy values for, 572

gain of protein in, 354, 364

influence of condition on, 438

on composition of lean meat, $35^{6}$

energy requirements for maintenance, 306

net energy values for, 360

object of, 358,427

of mature animals, 350

pigs, protein requirements of, $4 \mathrm{II}$

protein requirements for, 363,446

rations, protein in, 364

requirements, $350,359,36 \mathrm{r}, 363,446$, 448

stage of, influence of, on energy requirements, 362

utilization of protein in, 364

Fatty acids, I 7

Feces, 105, 109

as excretory product, 109

feed residue, Io9

composition of, I I I 
Feces, - continued

losses of energy in, 635

outgo of energy in, 230

Feed :

as stimulus to milk production, 522

consumption,

energy expended in, 275

increases heat production, 273

influence of,

on heat production, $65 \mathrm{I}$

by the horse, 675

on metabolism, $65 \mathrm{I}$

influence on,

of age, $43 \mathrm{I}$

breed, 443

individuality, 443

significance of energy expenditure in, 277

diminishing returns from, in milk production, 5 I 5

dual function of, 183

influence of, on composition of milk, 527

quantity of, influence of, on digestibility, 6г 3

requirements, $69 \mathrm{I}, 693,694$

for growth, 396

maintenance, 280,313

meat production, 445

milk production, 500

supply, 569

two aspects of, 63 I

surplus, disposal of, $35^{\circ}$

total amount of, for meat production, 449

unit system, logical basis of, 595

units, 593

comparison of, with net energy values, 596

utilization of, in milk production, 488

Feeding as related to individuality, 444

Feeding standards, 689

early, 689

for meat production, $45 \mathrm{I}$

the horse, 566

Kellner's, 690

limitations of, $69 x$

origin of, 689

Wolff's, 689

modifications of, 690

Feeding stuffs, 57 I

accessory ingredients of, 632

significance of, 632

choice of, 703

classes of, 72
Feeding stuffs, - continued

classification of, $57 \mathrm{I}$

composition of, 66

determination of

ash in, 67

crude fat in, $70,7 \mathrm{I}$

crude protein in, 68

nitrogen-free extract in, $7 \mathrm{I}$

non-protein in, 69

protein in, 67

true protein in, 67

water in, 67

direct comparisons of, $59 \mathrm{I}$

ether extract of, 70

metabolizable energy of, 639,642

production values of, $630,634,678$

relative values of, 59 I, 597

rich in carbohydrates, influence of, on digestibility, 6 I 7

specific effects of, 448

associated with fats, 527

on milk production, 523

sources of, $57 \mathrm{I}$

sundry ingredients of, 39

Feeding trials, practical, 592

Fermentation industries, by-products of, 585

Fermentation, losses of chemical energy in, 636,639

computation of, 637

Flavoring substances, $4 \mathrm{I}$

influence of, on milk production, 522

Fluids, digestive, 78

Forms of work, influence of, on efficiency of body, 555

Fowls :

digestibility by, compared with swine, 608

energy requirements for maintenance of, 301

Fruits, 579

Fuel value, 283

Functions :

of ash ingredients, I 87, I 90

carbohydrates, 186

fats, 186

feed, dual, 183

non-nitrogenous nutrients, 187

nutrients, I 82

physiological, 597

proteins, I 85

water, I90

Gain in fattening, energy content of, $36 \mathrm{I}$ 
Gain in growth, energy content of, Growth,-continued

$$
373
$$

rate of, in fattening, influence of condition, 438

Gait, influence of, on efficiency of body, $55^{8}$

Galactans, 14

Galactolipins, 23

Galactose, 9

Gaseous exchange increased by work, 540 through the skin, I39

Gastric juice, 82

Gelatinoids, 33

Germ meal, 588

Glands :

parotid, 79

salivary, 79

sublingual, 79

submaxillary, 79

Globulins, 33

Glucose manufacture, by-products of, 587

Glucosids, Io

nitrogenous, 37

Glutelins, 33

Gluten feed, 588

meal, 588

Glycogen, I4

computation of gain or loss of, 207

content of body, 6r

conversion of, to dextrose in the liver, I 53

formation of, in liver, 153

gain or loss of, 205

muscle, I 54

storage, $6 \mathrm{I}$

Glycoproteins, 35

Grade, influence of, on efficiency of body, 559

Grain, influence of grinding on digestibility of, 624

Grasses, 573

influence of maturity on composition of, 573

digestibility of, 574

Grinding of grain, influence of, on digestibility, 624

Gross energy, 635

Group system, 477 .

Growth, 37 I

ash requirements for, 414

contrast with fattening, 396

energy requirements for, 399

fattening during, 448

feed requirements for, 396

increase in, $37 \mathrm{I}$

composition of, $37 \mathrm{I}$

involves storage of ash, 4I 4

measure of, 375

minimum of protein for, 446

nature of, $37 \mathrm{I}$

net energy values for, 390

of cattle, energy requirements for, 399 protein requirements for, 404

sheep, energy requirements for, $40 \mathrm{I}$ protein requirements for, 407

swine, effect of insufficient protein on, 409

energy requirements for, 400

protein requirements for, 408

protein requirements for, 403

results in practice, 403

rate of, 373

at different ages, 374

rate of gain of energy in, 378

protein in, 375

storage of ash in, $4 \mathrm{r}_{4}$

relation of fats to, $42 \mathrm{I}$

relation of, to age, 373

relative values of amino acids for, $38 \mathrm{r}$

proteins for, $38 \mathrm{I}$

retention of ash during, $4 \mathrm{I} 6$

retention of protein in, 382

influence of energy supply on, 386

protein supply on, 384

substances, 4I, 348, 422

total increase in, at different ages, 397

utilization of energy in, 390

feed in, $38 \mathrm{I}$

Gums, I5

protein in, $384,387,388$

Hæmoglobin, I35

Hæmoglobins, 35

Hay values, 59I

Heart, I 25

Heat energy, measurement of, $22 \mathrm{I}$

unique, 220

of combustion, 223,228

outgo of, 235

production, causes of increase in, 275

increased by feed consumption, 273

influence on, of amount of feed, 665 crude fiber, 676 feed consumption, $6_{5}$ I

by the horse, 675

roughage compared with concentrates, 662

losses of energy in, $65^{\circ}$ 
Heavy feeding, influence of, on digestibility, 450

net energy values, 450

profitable in meat production, 449

Hemicelluloses, I3

digestion of, 92

Hexosans, I 2

Hexoses, 8

Hominy feed, $5^{85}$

Horse :

computation of net energy values for, $675,676,677$

digestibility by, compared with ruminants, 604

energy requirements for maintenance of, 295

feeding standards for, 566

influence of feed consumption on heat production by, 675

metabolizable energy for, 675

protein requirements of, for maintenance, 329

Humidity, influence of, on effects of temperature, 455

Hydrolysis of simple proteins, 164

Hydrogen, losses of energy in, 639

Ileum, 84

Improvement of a ration, computation of, 698

Income, balance of expenditure and, 194 of energy, 226

Increase :

composition of, 198

influence of age on, 43I

in fattening,

composition of, $350,352,353$, 354,364

energy content of, 352

protein in, 364

growth,

composition of, $37 \mathrm{I}$

energy content of, 373

total at different ages, 397

Individuality :

feeding as related to, 444

influence of

on assimilative power, 44I

course of lactation, 473

digestibility, 609

digestive power, $44 \mathrm{I}$

economy of feeding, 472

efficiency of body, 556

feed consumption, 443

maintenance requirements, 442
Individuality - continued

meat production, 440

milk production, 5 I 4

net energy values, 666

yield of milk, 47 I

Ingredients of milk, sources of, 465

Initial and final states, law of, 223

Intensity of work, influence of, on effciency of body, 557

Intercellular substance, 46

Intestine, large, 85

small, 84

Inulin, 14

Invertases, 79, 78

Investigation, methods of, I94 of details of metabolism, I94

Ionic concentration, maintenance of, $-\mathbf{8} 88$

Iron, metabolism of, I8I occurrence of, 6

Isolation, shelter from, 457

Jejunum, 84

Juice, intestinal, 87

pancreatic, 86

Katabolism, $\mathrm{r} 45$

computation of per unit of surface, 258 to standard weights, 250

fasting, 249

conditions affecting, 258

computation of, 282

influence on,

of body fat, 252

external temperature, 262, 265

muscular activity, $26 \mathrm{I}$

previous feeding, 253

size of animal, 258

standing and lying, 26r

of protein variable, $25 \mathrm{I}$

substances katabolized in, 249

of carbohydrates, 156

intermediary, 157

energy in fasting, $25 \mathrm{I}$

constancy of, 255

fats, 176

non-nitrogenous matter, influence of work on, 540

nucleic acids, 170

phosphorus, I 80

proteins, 162

formation of ammonia in, 165

nitrogenous end products of, 162

two stages of, I6 4

sulphur, 179

products of incomplete, 230 
Katabolism, - continued

protein,

dependent on supply, 322

in fasting, $25 \mathrm{I}$

normally small, $25 \mathrm{I}$

influence on, of feed supply, 3 I 6

work, 536

in work, influence of non-nitrogenous nutrients on, 538

stimulation of, in milk production, 5I5

Keratins, 33, 57

Kidneys, functions of, 140

Kind of production, influence of, on net energy values, 666

Kinetic energy, 2 I 8

measurement of, 225

outgo of, 235

Lactase, 79, 87

Lactation:

course of, influence of individuality on, 473

stage of, bearing on experimental methods, 476

influence on composition of milk, 476 milk production, 470

yield of milk, 476

Lactose, II

origin of, 467

Lean meat, 424

influence of age on production of, 433

fattening on composition of, 356

percentage of extractives in, 357

fat in, 356

Lecithins, 22

Lecithoproteins, 35

Legumes, 577

Leguminous grains, $58 \mathrm{I}$

Levulose, 9

Ligament, 49

Lignin, 13

Linseed meal, 587

Lipases, 79, 86

Lipoids, 16

cell, formation of, 172

nitrogenous, 37

Live weight as measure of nutritive effect, 196

fluctuations of, 197

influence of, on effects of temperature,

Liver, 86 454

glycogenic function of, ${ }_{52} 2$

Load, influence of, on efficiency of body,

559
Locomotion, energy expenditure in, 550 influence of speed, 552,557

Magnesium, metabolism of, I8I occurrence of, 6

Maintenance, 267

amino acids required for, 314

ash requirements for, 332

definition of, 267

energy requirements for, $267,280,303$

factors affecting, 304

influence on

of age, 307

fattening, 306

plane of nutrition, 305

temperament, 304

manner of stating, 283

method of determining, 28I

modified conception of, 284

relation of temperature to, 308

matter requirements for, 313

minimum of protein for, 3I 6, 323

net energy values for, $27 \mathrm{I}$

of ash balance, 339, 344

cattle, energy requirements for, 288 protein requirements for, 326

fowls, energy requirements for, 301

horses, energy requirements for, 295 protein requirements for, 329

neutrality, 335

osmotic pressure, 335

sheep, energy requirements for, 292 protein requirements for, 327

swine, energy requirements for, 285 protein requirements for, 329

optimum of protein for, 323

protein requirements for, $3 \mathrm{I} 3,323$

nature of, $3 \mathrm{I} 3$

relative values of proteins for, 315

requirements, 269

influence of breed, 442

individuality, 442

significance of, in interpretation of feeding experiments, 268

in practice, 268

true and live weight, 280

value of non-protein for, 324

Maize, influence of on metabolism, 664

proteins, low value of, $68 \mathrm{I}$

Maize forage, 575

influence of maturity on composition

of, 575

digestibility of, 575

Malt sprouts, 585 
Maltase, 79,87

Maltose, I I

Manifolds, 8o

Mannose, 9

Matter :

balance of, 202

dry, 3

requirements of,

for fattening, 363

growth, 403, 4I4

meat production, 445

milk production, $50 \mathrm{I}, 520$

work production, 560

Maturity :

definition of, 428

early, 428

economic significance of, 429

influence of breed on, 444

influence of,

on composition of grasses, 573

maize forage, 575

digestibility of grasses, 574 maize forage, 575

Meat, definition of, 424

fat-free, composition of, 52

proportion of fat in, 425

Meat production, 424

animal as factor in, 428

combined growth and fattening in, 448

energy requirements for, 448

factors of, 427

feed requirements for, 445

feeding for, 444

feeding standards for, $45 \mathrm{I}$

heavy feeding profitable in, 449

influence on,

of age, 430

condition, 438

drinking water, 455

exercise, 457

external conditions, 453

shelter, 456

temperature, 453

nature of, 424

processes involved in, 426

protein requirements for, 445

relation of conformation to, 443

type to, 443

total amount of feed for, 449

Metabolism, $\mathbf{r} 44$

a gradual process, 147

analytic, 146

definition of, I 44

enzyms as agents in, 148

general conception of, I 44
Metabolism, - continued

general scheme of, 182

influence on, of feed consumption, $65 \mathrm{I}$

investigations, comparison of, with

balance experiments, $24 \mathrm{I}$

of details of, I94

of ash ingredients, 178

calcium, I8I

carbohydrates, $I^{2} 2$

fats, I 7 I

iron, I8I

magnesium, I 8 I

nucleic acids, $\mathrm{I} 68$

nucleoproteins, I 68

organic acids, 159

pentosans, I 58

pentose carbohydrates, $\mathbf{I} 57$

phosphorus, I 80

potassium, I8I

- proteins, $\mathrm{I} 60$

sodium, I8I

sulphur, I79

oxidative, I 46

Metabolizable energy. (See Energy)

Metaproteins, 35

Methane, heat of combustion of, 636

influence of amount of feed on production of, 665

losses of energy in, 637,639

production of, in digestion, 90,94

Methods of investigation, I94

Middlings, buckwheat, 583

wheat, 583

Milk:

ash, 460

sources of, 468

average composition of, $46 \mathrm{r}$

carbohydrates, 460

origin of, 467

components of, 459

composition of, $46 \mathrm{I}$

influence on,

of breed, 473

completeness of milking, 480

feed, 527

frequency of milking, 479

stage of lactation, 476

variability in same animal, 475

energy content of, 5 I I

fat, influence of exercise on yield of,

fats, 459 482

origin of, 466

glands, 462

development of, 463 
Milk, - continued

protein as stimulus to, 502

influence of feed on percentage of fat in, 528

proteins, 459

origin of, 465

secretion of, 464

solids, composition of, influence of breed on, 474 .

influence of feed on percentage of fat in, 529

rate of production of, 469

sources of ingredients of, 465

yield of, influence on

of completeness of milking, 480

frequency of milking, 478

individuality, $47 \mathrm{I}$

stage of lactation, 476

Milk production, 459

a periodic function, 476

animal as a factor in, 470

ash requirements for, 520

character of, 468

concurrent fattening in, $5 \mathrm{I} 3$

diminishing returns from feed in, 5 I 5

energy balances in, 493, 495

energy requirements for, 5 I I

factors of, 469

fat requirements for, $5_{1} 6$

feed as stimulus to, 522

feeding a secondary factor in, 500

feeding for, 500

influence on,

of environment, 478

exercise, 480

fatigue, 482

flavoring substances, 522

frequency of milking, 478

individuality, $5 \mathrm{I} 4$

plane of nutrition, $5^{\mathrm{I}} 4$

protein-rich feeds, 506

protein supply, 504, 507

shelter, 484

stage of lactation, 476

temperature, 483

modifying factors, 484

minimum of fat for, 519

protein for, $50 \mathrm{I}$

net energy values for, 493,497

equivalent fattening values, 498

outgo of ash in, 520

physiology of, 459

protein as stimulus to, 502

requirements for, 5 or

relative values of proteins for, 492
Milk production, - continued

requirements for, 5 or

specific effects of feed on, 523

stimulation of katabolism in, 5 I 5

supply of ash in, 52 I

utilization of,

energy in, 493

feed in, 488

protein in, 488

estimate of, 489,49 I

Milking, completeness of, influence of on

yield, 480 composition, 480

frequency of, influence of on composi-

yield, 478 tion, 479

Milling, by-products of, 582

uses of, 584

Mineral matter, 5

Molasses, 589

Molasses feeds, $58 \mathrm{~g}$

Molecular simplification in digestion, 103

Monosaccharids, 8

composition of, 8

Motion, tissues of, 50

Motor, efficiency of body as, 544

Mouth, 79

Muscle extractives, 37

fat-free, composition of, 52

mechanical efficiency of, 545

Muscles, 50, 53I

composition of, $5 \mathrm{I}$

structure of, 50

Muscular work, nature of, $53 \mathrm{I}$

Net energy below critical temperature, 310

Net energy values, 271, 278, 634, 659

comparison of feed units with, 596

with metabolizable energy, 272

computation of, $667,673,677$

for the horse, $675,676,677$

from digestible nutrients, 667

organic matter, 673

independent of chemical composition, 673

importance of, 667

correction of, for crude fiber, 669

determination of, 272

for cattle, 659

different purposes, 279

fattening, 360

growth, 390

of embryo, 393

older animals, 393 
Net energy values, - continued suckling animals, 39I

maintenance, $27 \mathrm{I}$

milk production, 494, 497

equivalent fattening values, 498

ruminants, 660

swine, $66 \mathrm{I}$

work production, 563

influence on,

of age, 666

amount of feed, 664

breed, 666

heavy feeding, 450

individuality, 666

kind of production, 666

method of determination, 27 I

of digestible nutrients, 668

relative, for maintenance and fattening, 361

Neutrality, maintenance of, I89, 335

Nitrogen balance, 202

determination of, 203

example of, 203

Nitrogen factors, 69

Nitrogen-free extract:

composition of digested, . 12 I

constituents of, 72

determination of, in feeding stuffs, 7 I

Nitrogen, free, not excreted, 202

Nitrogenous products, excretion of, 140

Non-nitrogenous matter:

influence of work on katabolism of, 540 katabolized in work, nature of, $54^{2}$

of urine, I 59

origin of, 160

Non-proteins, 36

determination of, in feeding stuffs, 69 digestion of, 96 , 100

general properties of, 36

groups of, 36

indirect utilization of, 622

influence of, on digestibility, 622

nitrogen factors for, 70

occurrence of, 36

value of, 324,684

for maintenance, 324

Nucleoproteins, 34

metabolism of, $\mathrm{I} 68$

Nucleus of cells, 42

Nucleic acids, 34

digestion of, 99

Nutrients :

digestible, 599

computation of, 598

of metabolizable energy from, 646 Oxyhæmoglobin, I36
Nutrients, - continued

net energy values from, 667

metabolizable energy of, 648

net energy values of, 668

significance of, 600

functions of, 182

mutual replacement of, 270

non-nitrogenous,

effect of deficiency of, 320,324 surplus of, $32 \mathrm{I}, 324$.

functions of, 187

physiological functions of, 597

solution of, in digestion, IOI

Nutrition, balance of, I92, 201

includes energy, 216

Nutritive effect, live weight as measure of, 196

total, I95

Nutritive ratio, 600

Oat hulls, 584

Offal, composition of, 55

proportion of body ash in, 56

fat in, 56

protein in, 56

Oil extraction, by-products of, 586

Oil meals, $5^{87}$

seeds, $58 \mathrm{I}$

Oils, distinction between fats and, I 9

ethereal, 40

Omasum, 8o

Organic acids, production of in digestion, 90

Organic matter, 4

digestible, computation of metabolizable energy from, 648

net energy values from, 673

subdivision of, 4

Osmotic pressure, maintenance of, I 88 335

Outgo of chemical energy, 229

in cutaneous excretion, 23I

feces, 230

urine, $23 \mathrm{I}$

heat, 235

kinetic energy, 235

work, 235

Over-all efficiency of body, 562

Oxygen :

absorption of, by blood, 135

through skin, I 39

consumption of, in metabolism, 146 determination of, 208

supply of, I 32 
Packing house, by-products of, 590

Pancreas, 86

Parotid glands, 79

Passage of feed from stomach, 83

Paunch, 80

Pectins, I 5

Pentosans, 15

digestion of, 9I

fermentation of, 9 I

metabolism of, 158

proportion of, in crude fiber, $7 \mathrm{I}$

Pentoses, 9

metabolism of, 158

Pepsin, 79, 82, 96

Peptids, 30, 35

Peptones, 35

Period system, 477

Pettenkofer respiration apparatus, 2 I 2

Phosphatids, 22, 23

Phospholipins, 22

Phosphoproteins, 35

Phosphorus :

anabolism of, 180

digestion of, IOI

forms of, $7,180,421$

inorganic, value of, $42 \mathrm{I}$

katabolism of, $\mathrm{r} 80$

metabolism of, iso

occurrence of, 7

Pigs :

energy requirements of, 400

feeding standards for, 4I 2

gains by, in growth, 4 or

protein requirements of, 4 I I

Plane of nutrition, influence of, on energy requirements for maintenance, 305

milk production, $5 \mathrm{I} 4$

Plasma, blood, 125

Polypeptids, 3 I

Polysaccharids, I I

chemical structure of, I I

terminology of, $\mathrm{r} 2$

Potassium, metabolism of, I8I occurrence of, 6

Power plant, comparison of body with, 567

efficiency of, compared with body, 567

Practical feeding trials, 592

Precipitation, shelter from, 456

Prime motor, animal as, 192

Production values, as regards protein, 678 energy, 634

definition of, 630
Production values, - continued

determination of, 630

of feeding stuffs, $630,634,678$

Prolamins, 33

Proteans, 35

Proteases, 79, 86, 87

Protein :

addition of, influence of, on digestibility, 622

as source of energy, 318,542

stimulus to milk glands, 502

body, fluctuations of, 319

proportion of, in bone, 48

offal, 56

cause of diminished digestibility of, 6 ro

cleavage, extent of, in digestion, 98

computation of gain or loss of, 204

consumed by calves, 404

lambs, 407

crude, determination of, in feeding stuffs, 68

digestibility of, I I 9

functions of, in fasting, 255

work production, 543,560

gain of, in fattening, 354

or loss of, 202

in dairy rations, 506

fattening rations, 364

increase in fattening, 364

influence of, on digestibility of rations, 331

insufficient, effect of, on growth of swine, 409

katabolism,

dependent on supply, 322

in fasting, $25 \mathrm{I}$

variable, $25 \mathrm{I}$

normally small, 25 I

in work, influence of non-nitrogenous

- nutrients on, 538

influence on, of feed supply, 316

work, 536

minimum of,

for growth, 446

maintenance, 316, 323

milk production, 5 or

nitrogen factors for, 69

nutrition, plane of, 324

of feed, storage of, 319

optimum of, for maintenance, 323, 330

physiological minimum of, 254

production values as regards, 678

rate of increase of, in growth, 375

retention of, in growth, 382 
Protein, - continued

influence on, of energy supply, 386 protein supply, 384

requirements,

computation of, to unit weight, 325

for fattening, $363,4 \mathrm{I} 6,446$

growth, 403

of cattle, 404

sheep, 407

swine, 408

results in practice, 403

maintenance, 3 I 3,323

nature of, 3 I 3

meat production, 445

milk production, $50 \mathrm{I}$

work production, $56 \mathrm{I}$

of cattle, $326,367,404,501$

horses, $329,56 \mathrm{I}$

sheep, $327,365,407$

swine, $329,368,408$

relation of, to age, 445

rich feeds, influence of, on milk production, 506

supply, influence of, on digestibility, 447

milk production, 504, 507

retention in growth, 384

surplus, katabolized, 3I 7, 488

true, determination of, in feeding stuffs, 68

unnecessary for fat production, 363

utilization of, in fattening, 364

growth, $384,387,388$

milk production, 488 , 49 I

limited, 3 I 8

Proteins, 24

alfalfa, values of, 683

cereal, values of, 683

chemical changes in resorption of, IO7

coagulated, 35

coagulation of, 26

conjugated, 25,34

derived, 25,35

primary, 35

secondary, 35

digestion of, $83,88,95$

by erepsin, 98

pepsin, 96

trypsin, 97

formation of fat from, 173

functions of, 185

incomplete, 679

maize, low value of, $68 \mathrm{I}$

nomenclature of, 24

of milk, 459
Proteins, - continued

origin of, 465

physical properties of, 25

putrefaction of, 99

relative values of, 678

for growth, $38 \mathrm{I}$

maintenance, $3 \mathrm{I} 5$

milk production, 492

simple, 25, 26

anabolism of, $\mathrm{I} 60$

classification of, 32

cleavage products of, 28

composition of, 26

deaminization of, 165

reversible, 166

formation of, ammonia in katabolism of, 165 carbohydrates from, 167

fat from, 168

hydrolysis of, 28, I64

katabolism of, 162

metabolism of, 160

nitrogenous end products of katabolism of, $\mathrm{I} 62$

non-nitrogenous residue of, 163

proportions of cleavage products in, 3 I

structure of, 27

synthesis of, 30

from digestive products, $I 60$

two stages in katabolism of, I64 unbalanced, 679

\section{Proteoses, 35}

Protoplasm, 42

composition of, 44

Ptyalin, 78, 79

conditions of action of, 92

Pulmonary exchange, investigation of, $2 \mathrm{I} 4$

Putrefaction of proteins, 99

Pylorus, 83

Quantity of feed, influence of, on digestibility, 6r 3

Quotient, respiratory, 207

Raffinose, II

Rate of growth, 373

at different ages, 374

Rations:

compounding of, 707

computation of, $565,689,697$

from given feeding stuffs, 700

improvement of, 698

for work production, calculation of, 565 
Rectum, 85

Regnault-Reiset respiration apparatus, 209

Relative values of feeding stuffs, 59r, 597

Requirements :

for fattening, 350, 359, 36r, 363

growth, 396, 399, 403, 4I4

maintenance, 269, 280, 3I 3,332

meat production, $444,445,448$

milk production, $500,50 \mathrm{I}, 5 \mathrm{II}, 520$

work production, 560,562

of ash,

for growth, 4I4

maintenance, 332

milk production, 520

work production, 562

dry matter, 696

energy for fattening, 36I

growth, 399

maintenance, 280, 303

meat production, 448

milk production, 5 I I

work production, 562,564

feed, 69г, 693, 694

fat for milk production, $5 \times 6$

protein for fattening, 363

growth, 403

maintenance, 3i3, 323

meat production, 445

milk production, $50 \mathrm{I}$

work production, 56 I

Residue, non-nitrogenous, of simple pro-

Resorption, 105 teins, $r 63$

chemical changes in, I07

mechanism of, ro6

paths of, ro7

rôle of osmosis in, ro6

Respiration, I 23, I32

apparatus, 208

Pettenkofer, 2 I 2

Regnault-Reiset, 209

calorimeters, 236

influence of work on, 536

of tissues, 136

regulation of, $\mathrm{I} 37$

Respiratory quotient, 207

Reticulum, 80

Reversible reactions, I50, r66

Reversibility of metabolic reactions, 152 ,

Rhamnose

$$
\text { I53 }
$$

Rice bran, 583

polish, 583
Roots, 73, 579

influence of, on digestibility, 618

Roughage, 72, 572

comparison of, with concentrates, 662

general character of, 572

influence of cutting on digestibility of, 624

proportion of, to concentrates, $45 \mathrm{I}$, 696

proportion of vegetative organs in, 576

Rumen, 8o

Ruminants, digestibility by, compared with horses, 604 swine, 606

species of, 603

net energy values for, 660

Rumination, 8I

Rye bran, 582

Saliva, 79

action of, in stomach, 73

on starch, 92

Saponification of fats in digestion, 95

Schematic body, I95

Scleroproteins, 34

Sheep :

digestibility by, compared with horse, 604

swine, 606

energy requirements for growth, $40 \mathrm{I}$ maintenance, 292

influence of feed consumption on heat production by, 653

maintenance requirements of, compared with cattle, 294

protein requirements for growth, 407 maintenance, 456

Shelter from precipitation, 456

sun, 457

wind, 456

influence of, on meat production, 456

milk production, 484

Size of animal, influence of, on fasting katabolism, 2.58

Skeleton as reserve of ash ingredients, 338

Skin, gaseous exchange through, $\mathrm{I} 39$

Slaughter tests, comparative, I99, 35 I

Sodium, metabolism of, I8I

occurrence of, 6

Solution of nutrients in digestion, ror

Species, influence of, on digestibility, 603

of ruminants, digestibility by, 603

Specific dynamic action, 275 
Specific effects of feeds, 448 associated with fats, 527 on milk production, 523

Speed, influence of, on efficiency of body, $55^{2}, 557$

on energy expenditure in locomotion, $55^{2}, 557$

Standing and lying, influence of, on fasting katabolism, 26r

Starch, I3

digestion of, 79, 88, 92

in intestines, 94

stomach, 93

fermentation of, in digestion, 94

manufacture, by-products of, 587

values, 672

Steapsin, 79, 86

Stomach, 79

of hog, 8I

horse, $8 \mathrm{I}$

ruminants, 80

sheep, 80

passage of feed from, 83

Straw, 577

Suckling animals, net energy values for

Sucrase, 79,87

growth of, 39 I

Sucrose, to

Sugar beet pulp, 589

Sugar manufacture, by-products of, 588

Sulphur,

digestion of, IOI

katabolism of, I 79

metabolism of, I 79

occurrence of, 7

Sun, shelter from, 457

Sundry ingredients of animals, 39 plants, 40

Surface, computation of, computation of katabolism per unit of, 258

Surplus feed, disposal of, 350

Swine :

digestibility by, compared with fowls, 608

ruminants, 606

effect of insufficient protein on growth of, 409

energy requirements for growth, 400 maintenance, 285

influence of feed consumption on heat production by, 653

net energy values for, $66 \mathrm{I}$

protein requirements for growth, 408

maintenance, 329
Synthesis

of hippuric acid, 163

nucleic acids, 169

simple proteins from digestive products, $\mathrm{I} 60$

seat of, I6I

uric acid, I 7 I

Synthetic processes in the body, I 46

Tankage, 590

Temperature,

body, chemical regulation of, 263

physical regulation of, 262

critical, 264, 453

lowered by feed consumption, 308

effects of extremes of, 266

external, influence of age, on effects of, 454

amount of ration on effects of, 454

humidity, on effects of, 455

live weight on effects of, 454

influence of, on fasting katabolism, 262,265

energy requirements for maintenance, 304

meat production, 453

milk production, 483

modifying factors, 484

of drinking water, influence of, on meat production, 455

relation of, to energy requirements for maintenance, 308

Tendon, 49

Tissue, adipose, 58

composition of, 59

Tissues:

animal, 45

classification of, 45

connective, 49

elastic, 49

epidermal, 57

composition of, 57

functions of, 57

of alimentation, 54

chemical composition of, 55

motion, 50

reserve, 58

supporting, 46

Tonus, 534

Total feed required, computation of, 697

Training, influence of, on efficiency of body, 556

Triglycerids, 17,19

elementary composition of, 20 
Trisaccharids, II

Trypsin, 79, 86, 97

Tubers, 73, 579

influence of, on digestibility, 61 8

Type, relation of, to meat production, 443

Units of energy, 220

equivalence of, $22 \mathrm{I}$

Urea, I 62

antecedents of, $\mathrm{I}_{2}$

Urine, losses of chemical energy in, 636 non-nitrogenous matter of, 159

origin of, 160

outgo of chemical energy in, 23 I

Utilization :

of energy in growth, 390

milk production, 493

feed in growth, $38 \mathrm{I}$

milk production, 488

non-proteins, 686

proteins, in growth, $384,387,388$

milk production, 488

estimates of, 489 , 49 I

meaning of, 488

relative, of fats and carbohydrates for work production, 553

Veins, 126

Villi, I05

Vitamins, 4I, 348

Water:

balance of, $2 \mathrm{I} 6$

determination of, in feeding stuffs, 67

drinking, influence of, on digestibility, 628

meat production, 455

excretion of, $\mathrm{I}_{42} 2$

functions of, 3,190

supply, $45^{8}$

Waxes, 2 I

Weight. (See Live weight)

Wheat bran, 582

Wind, shelter from, 456
Work :

analysis of, 500

body substance source of energy for, 544

forms of, influence of, on efficiency of body, 555

influence of,

on circulation, 535

digestibility, 610

gaseous exchange, 540

katabolism of non-nitrogenous matter, 540

protein, 536

respiration, 536

intensity of, influence of, on efficiency of body, 557

internal, 256

measure of energy expended in, 256

muscular, nature of, 531

nature of non-nitrogenous matter katabolized in, 542

of ascent, $55 \mathrm{I}$

efficiency of body in, $55 \mathrm{I}$

of digestion, 277

differences between feeding stuffs, $66_{3}$ roughage compared with concentrates, 662

draft, $55^{2}$

efficiency of body in, $55^{2}$

outgo of, 235

protein as source of energy for, $54^{2}$

secondary effects of, 535

sources of energy for, 542

Work production, 53 I

ash requirements for, 565

calculation of rations for, 565

energy requirements for, $5^{62}$

feed requirements for, 560

functions of protein in, 543, 560

net energy values for, 563

physiology of, 53 I

protein requirements for, $56 \mathrm{I}$

Xylan, I 5

Xylose, 9 

•

$\mathrm{HE}$ following pages contain advertisements of
books by the same author or on kindred subjects 



\title{
Principles of Feeding Farm Animals
}

\author{
By SLEETER BULL \\ Associate in Animal Nutrition, University of Illinois \\ I2mo, illustrated, $\$ I .75$
}

This volume is an outgrowth from a class manual written for the author's students in a general, elementary course in stock feeding. The scientific facts underlying the art of feeding animals have been presented in such a manner that the book will not only be suitable for use as a text for college courses in general feeding, but will also be valuable to the farmer who has not had the opportunity of a technical education in agriculture.

The author first discusses the scientific aspects of the subject, presenting them in a simple, concise manner. Following that are presented rather definite rules regarding the feeding of the different classes of livestock which, taken in connection with the feeding standards and the discussion of the nutritive value of the different feeds, should enable the inexperienced feeder to formulate satisfactory rations. Also, in treating of the feeding values of the different feeds, the statements regarding the amounts, proportions and combinations of feeds in the rations of different classes of farm animals are specific rather than general. A large number of valuable illustrations and tables have been included.

Instead of devoting separate chapters to the feeding of the different classes of farm animals the author has, in order to avoid duplication, discussed separately the use of each of the principal feeds for the different species and classes of livestock. For example, under the discussion of corn, its use is given in the rations of growing cattle, colts, pigs and lambs; fattening cattle, hogs and sheep; breeding cattle, horses, hogs and sheep, dairy cows, and work horses.

In addition to the discussion of the nutritive value of feeds and rations, the author has also given particular attention to their fertilizing values, a phase which is often neglected both by the student and the stockman.

"The book ought to be in the library of every farmer." - Farmer's Mail, Topeka.

"It cannot fail to find wide range by the two classes of people the author desires to reach, namely, the student and the feeder." - Journal American Chemical Society, Washington, D.C.

THE MACMILLAN COMPANY 


\section{The Feeding of Animals}

\section{BY WHITMAN HOWARD JORDAN}

Director of the New York Agricultural Experiment Station at Geneva

New Edition, Revised, and Entirely Reset

I2mo, illustrated, 463 pages, $\$ 1.75$

This volume has been revised to incorporate the more recent knowledge concerning animal nutrition and to organize the text into a more convenient form for student use.

As with the former edition, the text is divided into two parts: Part I deals with the general principles of biochemistry that bear upon animal nutrition. Part II gives the practical side of feeding animals with such attention to principles as relate specifically to the nutrition of the various classes of farm animals. By this arrangement the volume will be useful for classroom work with those students who have given little or no attention to bio-chemistry as such, at the same time serving the interest of those students who have given considerable attention to chemical studies. The farmer and general reader will also find the treatise helpful in the practice of animal husbandry.

"A valuable contribution to agricultural literature. Not a statement of rules or details of practice, but an effort to present the main facts and principles fundamental to the art of feeding animals." - New England Farmer.

"A book to take down and use day after day, the year around." - Farm Stock Home, Minneapolis.

\section{THE MACMILLAN COMPANY}




\title{
The Scientific Feeding of Animals
}

\author{
By Professor O. KELLNER
}

\author{
Authorized Translation by \\ WILLIAM GOODWIN, B.Sc., Рн.D.
}

Lecturer on Agricultural Chemistry, and Head of the Chemical Department, South-Eastern Agricultural College (University of London), Wye, Kent.

Cloth, I2mo, $\$$ I.75

An authorized English translation of the valuable work of Dr. O. Kellner. It explains in simple language the general laws which underlie the feeding of animals and the scientific foundations upon which the principles of animal nutrition rest.

"I wish to say that it is one of the most valuable books in the English language on Feeding Farm Animals. The author is extremely lucid in expression and concise in statement. He covers his field in a manner that is well planned and such as will give the reader a most excellent knowledge of the general principles of feeding." - Professor Charles S. Plumb, Ohio State University.

"Dr. Kellner's standing as a student and investigator in this subject is too high for any words of commendation to be needed, and I feel sure that the translator and publisher have done a service in rendering this work available to English and American students." - ProfEssor Henry P. Armsby, Pennsylvania State College.

\section{THE MACMILLAN COMPANY}




\section{The Breeding of Animals}

\section{By F. B. MÚMFORD, M.S.}

Professor of Animal Husbandry, Dean of the College of Agriculture, and Director of the Experiment Station of the University of Missouri.

I2mo, illustrated, 304 pages, $\$ \mathbf{I . 7 5}$

This text-book deals first with the fundamental questions of inheritance common to plants and animals, but emphasizes the principles, methods and practices which are peculiar to animal breeding.

The improvement of the domestic animal resulting in the development of highly specialized qualities useful to man is one of the most notable achievements in modern agriculture. How, through man's efforts, these highly specialized and valuable qualities have been acquired, and how these qualities have come to be represented in the constitution of the germplasm and thus transmitted from parent to offspring, is a subject of great scientific and practical interest.

The scientific principles which govern the practice of animal breeding may all be classified under inheritance, reproduction and development. The text emphasizes particularly those scientific principles which are recognized as the basis of heredity and which have been sufficiently well established to afford a real basis for the practice of animal breeding. The physical basis of heredity in the germplasm of the cell and the significant changes resulting in transmission are described and illustrated.

The physiology of reproduction and its applications to the practice of breeding is the subject of an important chapter. The interrelations of heredity and development which constitute the real basis of the breeder's art are discussed. The practical questions of inbreeding, crossbreeding, grading, fertility, sterility, and sex are considered in the light of the most modern development of biological science.

THE MACMILLAN COMPANY

Publishers 64-66 Fifth Avenue New York 




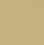



1 








\section{LIBRARY OF CONGRESS \\ ||||||||||||| ||||||||||||||||||||||||||||

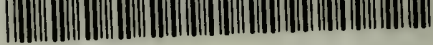 \\ 00008948197}

